\title{
Final Program \\ Forty Third Annual Meeting \\ International Neuropsychological Society
}

\author{
February 4-7, 2015 \\ Denver, Colorado, USA
}

9:00 AM-12:00 PM

1. SILVER, JM

9:00 AM-12:00 PM

1. PENNINGTON, BF

9:00 AM-12:00 PM

1. LAIRD, AR

1:00-4:00 PM

${ }^{\star}$ SEE FINAL ADDENDUM

1. YURGELUN-TODD, D

1:00-4:00 PM

1. FEINSTEIN, A

1:00-4:00 PM

1. HERMANN, BP

\section{WEDNESDAY, FEBRUARY 4, 2015}

CE 1: Persistent Neuropsychiatric Symptoms After Concussion: Evaluation, Effort, and Ethics

Presenter: Jonathan M. Silver

Centennial A

Persistent Neuropsychiatric Symptoms After Concussion: Evaluation, Effort, and Ethics

CE 2: Genes, Brain, and Behavior in Neurodevelopmental Disorders: Science and Practice

Presenter: Bruce F. Pennington

Centennial B-C

Genes, Brain, and Behavior in Neurodevelopmental Disorders: Science and Practice

CE 3: Neurocognitive Networking: Modern Neuroimaging Methods for Understanding Neurocognition

Presenters: Angela R. Laird, Jennifer Robinson

Centennial G-H

Neurocognitive Networking: Modern Neuroimaging Methods for Understanding Neurocognition

CE 4: Impact of Marijuana on the Developing Brain

Presenter: Deborah Yurgelon-Todd

Centennial A

Impact of Marijuana on the Developing Brain

CE 5: The Neuropsychiatry of Multiple Sclerosis

Presenter: Anthony Feinstein

Centennial B-C

The Neuropsychiatry of Multiple Sclerosis

CE 6: Primary and Treatment Related Comorbidities in Pediatric and Adult Epilepsies: Revising our Understanding of the Relationships Presenters: Bruce P. Hermann, Madison M. Berl, David W. Loring Centennial G-H

Primary and Treatment Related Comorbidities in Pediatric and Adult Epilepsies: Revising our Understanding of the Relationships 
1:00-4:00 PM

4:15-4:30 PM

4:30-5:30 PM

1. FEINSTEIN, A

5:30-6:30 PM

6:00-7:30 PM

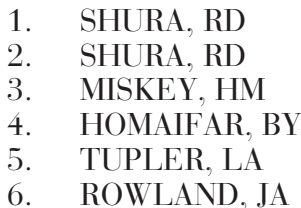

6:00-7:30 PM

1. HIPLOYLEE, $\mathrm{C}$

2. REYNOLDS, M

3. TROYANSKAYA, M

4. KISSER, JE

5. MERZ, Z

6. LEQUERICA, A

7. JUNG, S

8. YANG, C

9. WILLIAMSON, JB

10. CHIOU, KS

11. TWAMLEY, EW

12. SUNDERARAMAN. P

13. LEMONS, A

14. YEE, MK
INS Student Liaison Committee Workshop: Brain-Behavior Relationships in the Developing Child: A Primer in Pediatric Neuropsychology

Presenters: H. Gerry Taylor, E. Mark Mahone

Centennial F

Welcome Address

Program Chair: Derin J. Cobia

Centennial Ballroom (D-E)

Invited Address: Looking Behind the Smokescreen: Cannabis, Cognition and Multiple Sclerosis

Presenter: Anthony Feinstein

Centennial Ballroom (D-E)

Looking Behind the Smokescreen: Cannabis, Cognition and Multiple Sclerosis

INS Awards Ceremony With Opening by Phamaly Theatre Company Centennial Ballroom ('D-E)

Poster Symposium: Cognitive and Neuropsychiatric Functioning of OIF/ OEF/OND Veterans

Chair: Robert D. Shura

Centennial Ballroom Foyer

\section{Acquired Brain Injury (TBI/Cerebrovascular Injury \& Disease - Adult)}

Cognitive and Neuropsychiatric Functioning of OIF/OEF/OND Veterans

The Behavioral Dyscontrol Scale-II: A Unique Measure of Executive Functioning

The Relationship of Self-Reported Disinhibition and Posttraumatic Stress to Objective Performance

The Relationship of Suicidal Ideation to Objective and Subjective Executive Functioning Olfactory Deficits in Veterans Serving Post-9/11 Reporting TBI: A Potential Biomarker of Injury

Alterations in Resting-State Brain Network Structure Associated with mTBI and PTSD

\section{Poster Session 1: ABI-Adult \& Emotional Processes Centennial Ballroom Foyer}

\section{Acquired Brain Injury (TBI/Cerebrovascular Injury \& Disease - Adult)}

Recovery From Postconcussion Syndrome

Biological and Psychosocial Factors Associated with Post-Stroke Emotional Functioning

Everyday Functioning, Symptom Reporting, and Cognition Following Injury During Combat Deployment

Lifetime Prevalence of Head Injury in a Demographically Diverse Community Sample

The Prevalence of Sport Concussion Citations in Blast Concussion Publications

Relationship Between Disturbed Sleep and Executive Functioning in Traumatic Brain Injury: A Pilot Study

Ecological Validity of Traditional Neuropsychological Tests: Role of Memory, Executive Skills, and Learning in Predicting Everyday Functioning in a Clinical Population

Anxiety Symptoms as the Major Contributing Factor to Post-Concussion Syndrome: A Prospective Study

The Identification of Emotional Facial Expressions in People with Mild Traumatic Brain Injury and symptoms of PTSD

Performance Differences and Predictors of Learning After Moderate and Severe Traumatic Brain Injury

Multivariate Predictors of Social Reintegration and Satisfaction in Veterans with Histories of Traumatic Brain Injury

Examining the Neuropsychological correlates of Trail Making Test Based on the Chronicity of Brain Injury

Post-War Traumatic Brain Injuries are Influenced by Number of Pre-War, but not DeploymentRelated Brain Injuries in Gulf War Veterans

History of Pre-war Brain Injuries Influences Total Current Health Symptoms in a Cohort of 19901991 Gulf War Veterans 


\section{HANTKE, N \\ 16. KAUP, AR \\ 17. CZIPRI, SL \\ 18. GREEN, C}

19. YORK, C

20. WRIGHT, MJ

21. WRIGHT, MJ

22. WRIGHT, MJ

23. WRIGHT, MJ

24. WRIGHT, MJ

25. BRADFORD, LS

26. HERSHAW, J

27. O'BRIEN, T

28. LANGE, R

29. DE GUISE, E

30. DE GUTSE, E

31. PASTOREK, NJ

32. KENNEDY, Q

33. BERNIER, RA

34. KRENGEL, M

35. FROST, RB

36. DENBOER, J

37. SEICHEPINE, DR

38. STEED, D

39. LENGENFELDER, J

40. SEIBERT, L

41. AGBAYANI, K

42. LEFEBVRE, $\mathrm{G}$

43. KAPLAN, D

44. WONG GONZALEZ, D

45. LOGAN, DM

46. BANKS, SJ

47. ONEIL, ME

48. YUTSIS, M

49. PONSFORD, JL

50. CHAMARD, E

51. HAYS, C

52. GENOVA, HM

53. AMICK, M

54. KIM, RT

55. RAKIC, J

56. COTHRAN, TP

57. PAGULAYAN, K
Diagnosis of PTSD Predicts Worse Delayed Free Recall Performance in Veterans with Complex Medical Problems: Results from the War Related Illness and Injury Study Center

A Novel Video Game to Assess the Cognitive Impact of Traumatic Brain Injury in Older Adulthood: A Pilot Study

The Impact of Psychiatric Distress on Neuropsychological and Daily Functioning in a Veteran Population with Mild Traumatic Brain Injury

Demographic and Clinical Characteristics of a Traumatic Brain Injury Sample upon Admission to Brain Rehabilitation Clinic: A Descriptive Study Utilizing the Mayo Classification System of Injury Severity

Somatization and PTSD Symptoms Predict Persistent Postconcussive Symptoms in a Veteran Outpatient Polytrauma Clinic

Activity Memory and Subjective Workload Following Traumatic Brain Injury

Event-Based Prospective Memory and Subjective Cognitive Workload Following Traumatic Brain Injury

Functional Outcome and Subjective Cognitive Workload Following Traumatic Brain Injury Functional Outcome and Symptoms of Depression and Anxiety following Traumatic Brain Injury The Item Specific Deficit Approach to Memory Dysfunction and Subjective Workload Following Traumatic Brain Injury

Misconceptions about Traumatic Brain Injury Among US Army Behavioral Health Professionals Mild TBI and the Aging Brain: Eye Tracking Evidence from a Novel Neurocognitive Assessment Tool Impact of psychoeducation on perceptions of injury severity and cognitive complaints in veterans with TBI

Neuropsychological Outcome from Military-related Traumatic Brain Injury (TBI): Preliminary Analyses of the Role of Resilience, TBI Severity, and Blast Exposure

Stakeholder Perceptions on the Roles Clinical Neuropsychologists Can Play in Rehabilitation Service Delivery for Victims of a Traumatic Brain Injury with Mental Health Disorders

Usefulness of the Rivermead Post-Concussion Symptoms Questionnaire for Outcome Prediction in Patients with Mild Traumatic Brain Injury

Association of Family Distress to Health Outcomes in Veterans with a History of Combat-Related Mild Traumatic Brain Injury

Can a Simple Wargame Provide an Unobtrusive Indicator of TBI? A Case Study

Functional Status at Discharge Is Higher Among Alcohol-Positive Older Adults after TBI

Examination of NSI-symptoms in OEF/OIF Veterans with Multiple Concussions

Cross-sectional Analysis of Cognition, Time Since, and Number of Concussions in Division I Athletes Utilizing Serial Neuropsychological Assessment to Evaluate Recovery from Traumatic Brain Injury:

Acute to 2 Years Post-Injury

Frequency of Traumatic Brain Injuries in a Cohort of 1990-1991 Gulf War Veterans

Predicting Development of Dementia: A Parallel Process Latent Growth Curve Analysis

Apathy and Quality of Life in Traumatic Brain Injury

Effect of Body Orientation to Blast on Risk of Post Concussive Symptoms among Active Duty Service Members

Cognitive profile and rate of impairment comparisons between cerebellar and frontal lobe strokes

Neuropsychological Impact of Repeated Sub-Concussive Blows to the Head in Male and Female

Athletes

Multidisciplinary Concussion Assessment: The Relationship Between Neuropsychological and

Vestibular Physical Therapy Measures

Prospective Memory Following Traumatic Brain Injury: A Meta-Analysis

Cognitive Control of Conscious Error Awareness: Variable Task Performance in Moderate-to-Severe

Traumatic Brain Injury

Verbal Fluency and Brain Health in Boxers and Mixed Martial Arts Fighters

Visual Dysfunction in Patients with Traumatic Brain Injury: A Systematic Review

Treatment Effect Versus Natural Recovery in Moderate to Severe Traumatic Brain Injury: Efficacy of Postacute Rehabilitation

Mortality Following Traumatic Brain Injury and Rehabilitation

White matter long-term abnormalities in the corpus callosum of female concussed athletes

Predictors of Academic and Employment Status in Veterans with a History of Traumatic Brain Injury Facial Affect Recognition in Traumatic Brain Injury: an fMRI study

Number and Severity of Mild TBIs and PTSD Symptoms Predict Neuropsychological Performance in OEF/OIF Veterans

Physical Fatigue and Vegetative Symptoms Best Predict Quality of Life (QoL) in Veterans with Mild to Moderate Traumatic Brain Injury

Pre and Post Daily Functioning Comparison in Mild and Moderate TBI

The Relationship Between Sleep Variability and Reaction Time Performance Across CPT-II Blocks in Traumatic Brain Injury Inpatients

Differential Effects of Repeated Blast-Related mTBI on Limbic and Higher Level Cognitive Systems: A Resting State fMRI Study 


\author{
58. GRAJZEL, K \\ 59. HANSON, KL \\ 60. RAU, HK \\ 61. CLARK, AL \\ 62. VAS, A \\ 63. COHEN, J \\ 64. BUDISIN, B \\ 65. EVANS, SA \\ 66. TOMASZCZYK, JC \\ 67. MEYERS, K \\ 68. MARTIN, R \\ 69. BOSWORTH, CG
}

70. ASKEN, B

71. UDERMAN, J

72. GRANADER, Y

73. REVUELTAS, AM

74. WEISS, LR

75. VANUK, JR

76. VANUK, JR

77. HAZAMY, AA

78. TWAITE, JT

79. KILLGORE, WD

80. KILLGORE, WD

81. BRUCE, SE

82. ALKOZEI. A

83. SZELES, D

84. DEL PIERO, LB

85. ANDERSON, LB

86. ABEARE. C

87. KELLOGG, EJ

88. JOHNSON, PL

89. BAULDRY, RM

90. SHDO, SM

91. CONSIDINE, CM

92. GARCIA, R

6:30-7:30 PM
Psychological Distress Has a Larger Effect on Baseline Concussion Test Performance than Brain Injury History

Alcohol-Related Psychosocial Problems are Associated with TBI Injury Characteristics, Greater PostConcussive Symptomatology, and Poorer Cognition in Veterans with a History of Mild TBI

Comorbid Pituitary Dysfunction and mTBI Characterized by Specific Weaknesses in Verbal Memory: Evidence from Combat Veterans with Repeated Blast-Related mTBI

Mild Traumatic Brain Injury (mTBI) Moderates the Association Between White Matter Lesion Burden and Memory

Expanding Measurement of Abstract Thinking in Adults with Traumatic Brian Injury

Differential Predictors of Stroke and Cardiovascular Disease as They Relate to Brain Structure and Function: Implications for the Human Connectome

Preliminary Evaluation of the Diagnostic Utility of Diffusion Tensor Imaging (DTI) for Detecting Mild Traumatic Brain Injury (mTBI) in Spinal Cord Injury (SCI) Patients

Evidence for a Central Disorder of Pain in PD and Its Relevance to PD Cognition

Recovery of Variability in Attention Task Performance after Moderate-Severe Traumatic Brain Injury Computer-Based Neuropsychological Performance in Retired Professional Football Players

The Influence of Headache Impact on Life Satisfaction and Community Reintegration in Veterans with Mild Traumatic Brain Injury

Factors Associated with Default Mode Network Functional Connectivity in Traumatic Brain Injury

\section{Acquired Brain Injury (TBI/Cerebrovascular Injury \& Disease - Child)}

Pre-morbid Characteristics of Adolescent Student-Athletes: a Sport-Related Concussion Perspective

\section{Assessment/Psychometrics/Methods (Child)}

Psychometric Assessment of Social Cognition in Pediatric Populations

\section{Autism Spectrum Disorders}

Updated BRIEF Profiles in Children with Autism Spectrum Disorders

\section{Emotional Processes}

Analysis of Mexican Children's Recognition of Facial Expression of Emotions

Changes in Blood Oxygen Level-Dependent (BOLD) Response to Affective Picture Viewing After Acute Exercise

Napping in Conjunction with Brief Internet-Based Training as a Means of Enhancing Emotional Intelligence

Engaging in Meditation and Internet-Based Training as a Means of Enhancing Emotional Intelligence Emotional Sentence Processing in Persons with Parkinson's Disease

Musical Training is Associated With Lower Levels of Alexithymia and Greater Introspective Thought

Enhancing Emotional Intelligence via Brief Internet-Based Training

Emotional Intelligence is Associated with Coordinated Resting State Activity Between Emotion Regulation and Interoceptive Experience Networks

An fMRI investigation of visual cortex activity in response to fearful faces in participants with PTSD Looking for Evil Intent: Emotional intelligence and the use of socially relevant facial cues during an emotional decision making task

The Influence of Spatial Presentation on the Emotional Perception of Pictures

Cognitive and Emotional Correlates of Family Aggression

Emotional Intelligence Deficits in Agenesis of the Corpus Callosum

The Role of Stress and Emotion Regulation in Post-Concussive Symptom Reporting in Healthy Adults Investigating the Relationship Between Emotional Dysregulation, Impulsivity, and Executive Functions In a Non-Clinical Sample

The Impact of Self-Reported Difficulty in Emotion Regulation on Emotional Memory and Emotional Processing of Pictures

Generalized Anxiety and Major Depressive syndrome measured by the SCL-90-R in Two Manganese

(Mn) Exposed Ohio Towns

Specific Right-Temporal Contributions to Distinct Behavioral Subcomponents of Empathy in Neurodegenerative Disease

Objective \& Subjective Sleep Quality Differentially Relate to Depressive, Cognitive, and CognitiveAffective Processes in an Obstructive Sleep Apnea Referral

Psychological Symptoms and Quality of Life Among Residents Exposed to Long-Term, Low-Dose Environmental Manganese (Mn)

\section{Welcome Reception}

Centennial Ballroom Foyer 


\section{THURSDAY, FEBRUARY 5, 2015}

7:20-8:50 AM

1. KIRKWOOD, M

7:20-8:50 AM

1. STERN, Y

9:00-10:00 AM

1. BARCH, DM

10:00-10:15 AM

10:15-11:15 AM

${ }^{\star}$ SEE FINAL ADDENDUM

10:15-11:45 AM

1. PRESSON, $\mathrm{N}$

2. ADAMSON, MM

3. BUDISIN, B

4. KLIPFEL, $\mathrm{K}$

10:15-11:45 AM

1. OKONKWO, OC

2. JOHNSON, S

3. BENDLIN, BB

4. SCHULTZ, SA

5. BRATZKE, L

6. CLARK, LR

10:15-11:45 AM

1. CHEUNG, Y
CE 7: Pediatric Mild TBI: Who Gets Better, Who Doesn't, and What's Neuropsychology Got to Do with It

Presenter: Michael Kirkwood Centennial A

Pediatric Mild TBI: Who Gets Better, Who Doesn't, and What's Neuropsychology Got to Do with It

CE 8: Cognitive Reserve, From Theory to Intervention

Presenter: Yaakov Stern

Centennial B-C

Cognitive Reserve, From Theory to Intervention

Invited Address: Connectomics and Cognition: A Tale of Many Regions Presenter: Deanna M. Barch Centennial Ballroom (D-E)

Connectomics and Cognition: A Tale of Many Regions

Coffee Break

Centennial Ballroom Foyer

INS Early Career Award Presentation : Brain, Behavior and Beyond:

Tracing the Social Landscape of Pediatric TBI

INS Early Career Award Winner: Miriam H. Beauchamp

Centennial Ballroom (D-E)

Paper Session 1: TBI - DTI

Moderator: Elisabeth Wilde

Centennial A

Advanced White Matter Imaging with High Definition Fiber Tractography Predicts

Neuropsychological Test Performance in Adults with Traumatic Brain Injury

DTI Metrics from the Right Inferior Longitudinal Fasciculus and Thalamic Tract Best Discriminate TBI in Patients and Controls

Diagnostic Uncertainty and Speculations Regarding Mild Traumatic Brain Injury (mTBI): Diffusion Tensor Imaging (DTI) Versus Conventional Neuroimaging

Microstructural, Functional-Connectivity, and Neurocognitive Disruption in Pediatric Traumatic

Brain Injury: A DTI and Resting-State fMRI Study

Symposium 1: Investigating Preclinical Alzheimer's Disease: The Wisconsin Registry for Alzheimer's Prevention Experience Chair: Ozioma Okonkwo Centennial B-C

Investigating Preclinical Alzheimer's Disease: The Wisconsin Registry for Alzheimer's Prevention Experience

Amyloid imaging and CSF biomarkers in the Wisconsin Registry for Alzheimer's Prevention Connectivity loss in preclinical Alzheimer's disease: insights from diffusion tensor imaging Cardiorespiratory capacity attenuates the influence of age and amyloid- $\beta$ on cognition Multimorbidity and cognition: Latent class analysis within the WRAP cohort

Psychometric definitions of mild cognitive impairment and applications to identifying early cognitive change in the Wisconsin Registry for Alzheimer's Prevention (WRAP) study

Paper Session 2: Pediatric Neuropsychology \& Neuroimaging Moderator: Dalin Pulsipher Centennial F

Association between Acute Treatment-related Neurotoxicities and Executive Dysfunction in Longterm Survivors of Childhood Acute Lymphoblastic Leukemia 
2. ROSENQVIST, JE

3. FOX, ME

4. MRAKOTSKY, C

5. FITZER, KR

6. WILLIAMS, VJ

10:15-11:45 AM

1. GROTE, C

2. BODIN, D

3. BUTTS, AM

4. PONSFORD, JL

5. HOKKANEN, L

10:15-11:45 AM

1. SANZ, J

2. SANZ, J

3. KRIVITZKY, L

4. HARDY, KK

5. WALSH, KS

10:15-11:45 AM

1. ZANINOTTO, AC

2. CHOI, S

3. LOPEZ, WD

4. CRANSTON, CC

5. WIENER, JR

6. WELSH, M

7. QUINTIN, EC

8. WHITESIDE, DM

9. FRANCIS, T

10. GUNTHER, S

11. DENNEY, DA

12. VAN MOORLEGHEM, K

13. PICK. L

14. HOLCOMBE, BD

15. HUNTBACH, BA

16. KAUR, S
Neurocognitive Development in 3- to 11-Year-Old Children: An International Comparison Dorsal Anterior Cingulate-Based Functional Connectivity in Adolescents with Negative Attentional Bias

Reduced Cortical Thickness and Neuropsychological Function in Children with Crohn's Disease Cognitive Hypothesis Testing for Targeted Reading Disability Subtype Interventions: Impact on Reading Competency and White Matter Connectivity

Lateral Ventricular Volume, White Matter Integrity, and Intellectual Outcomes in Spina Bifida and

Shunted Hydrocephalus

\section{Symposium 2: International Perspectives on Education and Training in Clinical Neuropsychology \\ Chair: Christopher Grote \\ Centennial G-H}

International Perspectives on Education and Training in Clinical Neuropsychology

A Review of the Hstory and Crrent Issues and Challenges in North American Postdoctoral Training in Clinical Neuropsychology

A Fellow's outlook on recruiting and matching for postdoctoral training in clinical neuropsychology in North America

Australian Models of Ttraining in Clinical Neuropsychology

Neuropsychology Training Models and Issues in Europe

\section{Poster Symposium: Executive Function in Pediatric Medical Conditions Chair: Jacqueline Sanz Centennial Ballroom Foyer}

\section{Executive Functions/Frontal Lobes}

Executive Function in Pediatric Medical Conditions

Executive Function as a Predictor of Quality of Life in School Age Children with Congenital Heart Disease

The Relationship Between Executive functioning and Age/disease factors in Pediatric Stroke Computerized Cognitive Training for Children with Cancer and Neurofibromatosis type 1

Patterns of Executive Functioning in Pediatric Medical Disorders affecting the Central Nervous System

Poster Session 2: EF/Frontal, Forensic, \& Malingering Centennial Ballroom Foyer

\section{Executive Functions/Frontal Lobes}

Neuropsychological Assessment in Traumatic Diffuse Axonial Injury Patient Before and After Repetitive Transcranial Magnetic Stimulation - A Pilot Study

Neurobiological characteristics associated with impulse control impairment of Internet Addiction: A resting state EEG study

English as a Second Language and Performance on Tests of Executive Functioning

A Case for the Use of Action Fluency in Medial-Frontal Lesions

The Neurobehavioral Exam: A Measure of Mental Status and Frontal Lobe Function

Homeless Men in Transitional Housing Receiving the BrainWise Curriculum: Baseline Data on

Executive Functions, Problem Solving and Coping Self-Efficacy

Association Between Executive Functions and Loneliness Across the Adult Lifespan

The Relationship Between Cognitive Reserve and Executive Functioning

Gender Effects on Self-Awareness of Executive Functioning Deficits in Adolescents with ADHD

Exploring the Pencil Tapping Task: Analyzing Receptive Vocabulary and Obedience as Correlates of Inhibitory Control

Relationships Between the Questionnaire for Impulsive-Compulsive Disorders in Parkinson DiseaseRating Scale and Measures of Executive Function

Anxiety Predicts Performance on Stroop Interference but not Stroop Switching

Verbal Fluency Performance among Deaf Readers

Exploring Differences in Gender and Disease Risk on Executive Function in Pediatric Survivors of Acute Lymphoblastic Leukemia (ALL)

Differences in Self-Reported Executive Functioning between Pedophilic and Non-Pedophilic Sex Offenders

Serum Brain Derived Neurotrophic Factor mediates the relationship between Central Adiposity and Executive Function in middle age 
17. SPAT, J

18. MONCRIEF, GG

19. KLIPFEL, K

20. RAAK, J

21. KAYLEGIAN, J

22. SWAN, N

23. MEISTER, J

24. LUKOSE, A

25. GERST, EH

26. DE GUISE, E

27. MOORE, WR

28. HANNA, S

29. ROHRBACHER, C

30. KORNBLITH, ES

31. CHILD, A

32. THOMPSON, LI

33. WALKER, KA

34. SIEBENMORGEN, M

35. MCGAULEY, S

36. BRANSON, R

37. TURNER, E

38. BLINKOFF, DC

39. CIRINO, PT

40. COMBS. HL

41. MORAIS, HB

42. FARRER, TJ

43. DUGGAN, EC

44. SKEEL, RL

45. FEIGON, M

46. SULLAN, M

47. SANTOS, OA

48. BARRASH, J

49. POMMY, J

50. KIELY, T

51. LIGHT, SN

52. BIRATH, J

53. GORGENS, KA

54. ETHERTON, J

55. COOLIDGE, FL

56. PADULA, CB

57. CURTIS, KL

58. CURTIS, KL

59. WHITESIDE, D
The Nature of Perseverative Errors on the Auditory Consonant Trigram Test: Relationship to Other Measures of Frontal Lobe Dysfunction

Cognitive Reserve and Neuropsychological Test Performance in Blast Exposed Operation Enduring Freedom/Operation Iraqi Freedom (OEF/OIF) Veterans

Executive Functions and Dating Aggression in Young Adults

The Impact of Working Memory Deficits on In-group versus Out-group Helping Behavior

Academic Achievement and Executive Functioning is Mediated by Parental Involvement in Homeless Urban Youth

DKEFS Color Word Interference Switching and Learning Ability

Obesity Confers Greater Risk for Cognitive Difficulties Associated with Poor Sleep Quality

Correlates and Predictors of Endogenous-Cue Prospective Memory in Schizophrenia

Prediction of Academic Performance with Rating Scale and Cognitive Measures of Executive Function

Olfactory and Executive Dysfunctions Following Orbito-basal Lesions in Traumatic Brain Injury

Examination of the bilingual advantage in young adults using two behavioral rating scale measures of executive function

Performance on the Tower Test in Agenesis of the Corpus Callosum

Executive Functions and Hypothetical Risk-Taking as Predictors of Developmental Tasks in

Emerging Adulthood: Academic Self-Efficacy and Career Decision Making

Tremor, Motor, and Executive Function Profiles in Adult Residents Environmentally Exposed to

Manganese

Executive Functions and ADHD Symptomatology

Profiles of Executive Function in the Prediction of Alcohol Use Among Emerging Adults in NYC

Components of Executive Functioning Differentially Relate to Estimates of Intelligence

Streamlining Assessment of Inhibition: The Value of Multi-Method Assessment

Neuropsychological Improvement Following a Novel Inpatient Treatment for OEF/OIF Veterans with PTSD and Multiple Comorbidities

Examining an Alternate Version of the Baycrest Multiple Errands Test

The Relationship between Hot and Cold Executive Functioning and Childhood Maltreatment in College Students

Apathy and Working Memory in a Non-Clinical Population

Executive Functions, Self-Regulated Learning, and Reading Intervention

Higher Heart Rate Variability Predicts Better Performance on Executive Functioning Measures in

Older Adults

Network connectivity and Proverb test performance

Association between White Matter Hyperintensities and Alternate Scoring for Trail Making Test Part B

Examining Executive Behavior of Young Adults: Convergent Validity Analyses of an Executive

Functions Screener

Sex Differences in Impulsivity Factor Structures of Behavioral and Self-Report Measures

Executive Dysfunction in Adults with Sickle Cell Disease: Depressed versus Non-Depressed Groups

Adiposity and Cognitive Functioning among Older Adults in the San Luis Valley Health and Aging Study (SLVHAS)

Comparison of Ex-Gaussian Analysis of Reaction Time on Non-Executive and Executive Elementary

Cognitive Tasks in ADHD and Control Subjects Versus Schizotypal and Control Subjects

Types of Acquired Personality Disturbances Following Brain Damage: Exploration with Cluster

Aanalysis

Neuropsychological Subtypes of Executive Function in Schizophrenia and Healthy Controls Using A

Community Detection Approach

The Effect of Depression Chronicity and Severity on Executive Function

Anhedonia, Executive Function, and Endogenous Opioids in Lateral Prefrontal Cortex in Major

Depressive Disorder (MDD): A PET Study

\section{Forensic Neuropsychology}

Performance Validity and Workload in Healthy Adults and Adults with Traumatic Brain Injury Identifying and Treating the Superfecta: TBI, Mental Illness, Substance Abuse in a County Jail Controlled Oral Word Association Test Performance is Not Impaired by Induced Pain Assessment of DSM-5 Neurocognitive Disorder in 3,090 Adult Prison Inmates

Cognition and Trial Competency Restoration: Using the RBANS as a Treatment Indicator for Patients Deemed Incompetent to Stand Trial

A Comparison of Mild Traumatic Brain Injury and Chronic Pain Patients on Symptom Self-Report,

Objective Measures of Cognitive Functioning, and Psychological Factors

The Influence of Exaggeration and Presence of Spinal Pathology on Select MMPI-2-RF RC Scales in a Chronic Spine-Related Pain Sample

California Verbal Learning Test, Second Edition (CVLT-II) Recognition Measures as embedded

Performance Validity Tests in a Mild Traumatic Brain Injury sample 
60. STIKA, MM

61. LEPPO, R

62. PIERCE, C

63. ZENISEK, $\mathrm{R}$

64. LAU, L

65. BAR-HEN, M

66. TEAGUE, A

67. IAMPIETRO, M

68. VASSERMAN, M

69. TRAHAN, DE

70. PULSIPHER, DT

71. AMEDORO, S

72. WARDIN. L

73. WARDIN, L

74. PLOETZ, D

75. PROTO, D

76. GAVETT, BE

77. GAVETT, BE

78. MACALLISTER, WS

79. PARIKH, SA

80. ROBINSON, J

81. COLLINS, R

82. WYMAN-CHICK, K

83. LICHTENSTEIN, J

84. COPELAND, C

85. STENCLIK, J

86. BRICKELL, TA

87. SHARLAND, MJ

88. PARKS, AC

89. SHURA, RD

90. SEMLA, M

91. ROTHONG, N

92. SILK-EGLIT, G

93. SILK-EGLIT, G

94. LANGE, R

95. PATRICK, R

12:00-1:00 PM

1. FEIN, D

\section{Malingering/Effort Testing}

Relationship between Symptom and Performance Validity Test Performance in a Sample of Criminal Defendants

Preliminary Examination of TOMM Performance in A Clinically-Referred Sample of Deaf and Hard of Hearing Children

Failure Rates of Alternative TOMM Indices and Cutoffs in a General Medical Population

Reliable Digit Span as a Measure of Effort in Dementia

Effects of Symptom- and Test-Coaching on the Detection of Feigned Neuropsychological Deficits

Validation in Patients of an Algorithm for Effort Assessment Embedded in a Neuropsychological

Computerized Battery

Debunking the "Amotivational Syndrome" in Chronic Cannabis Users With Objective Measures of Effort

Word Memory Test Findings in a Pediatric Mixed Clinical Sample

Utility of CVLT-C Recognition Discriminability as a Measure of Effort in Clinically Referred Children with Developmental Disorders

Specificity of the CVMT Symptom Validity Scale in Normal Adults

Effort Test Failure, Intervention, and Degree of Neuropsychological Impairment in Children/ Adolescents with Concussion or Epilepsy

Exploratory Analysis of Mood/Anxiety and Effort Following Pediatric Concussion

Classification Accuracy of the Wisconsin Card Sorting Test (WCST) in Detecting Noncredible

Cognitive Performance in Neuropsychological Outpatients

Trail Making Test (TMT) as a Performance Validity Test (PVT) in Neuropsychological Outpatients Using the Automatized Sequences Task as a performance validity test in youth with neurological

diagnoses

Effect Sizes of Neuropsychological Performance Decrements as a Function of Sample-Derived PVT Hit Rate

Do "Effort" Tests Measure Effort? A Call to Abandon this Misleading Term

Linking Standalone Performance Validity Test Scores

Adventures in Pediatric and Adolescent Performance Validity Testing: A Case Series

Using the Personality Assessment Inventory to Predict Non-Credible Cognitive Performance in

Patients with Mild Traumatic Brain Injury

Examination of the Severe Impairment Profile in Dementia Evaluations

The Relation between the WMT and CVLT-2 in a Sample of Patients Evaluated for Dementia Performance Invalidity in Non-Clinical Undergraduate Research Participants: A Follow-Up Study Introducing a Forced Choice Recognition Task to the CVLT-C (FCR-Child): Preliminary Findings Relative Utility of Performance and Symptom Validity Tests for Assessing Cognitive Performance and Symptom Report

Increasing Classification Accuracy of the TOMM: Comparison of the Albany Consistency Index and the Invalid Forgetting Frequency Index

Influence of Symptom Validity Test Performance on the Traumatic Brain Injury Quality of Life (TBIQOL) Scale in U.S. Military Service Members

Examination of Embedded Performance Validity Indicators for the Conners' Continuous Performance Test (CPT-II) and Brief Test of Attention (BTA) in a Large Clinical Sample Using the Structure Inventory for Malingered Symptomatology (SIMS) to Detect Feigned

Postconcussional and Posttraumatic Stress Symptoms in Simulators

Embedded Performance Validity Measures with Post-Deployment Veterans: Cross-Validation and Efficiency with Multiple Measures

Classification Accuracy for Non-Credible Performance of the Personality Inventory (PAI)

Psychosocial Scales in Traumatic Brain Injury

Relationships between Word Memory Test Scores and Neuropsychological Performance in Patients with Epilepsy

Use of the Impairment Index and Neuropsychological Deficit Scale as Indicators of Performance Validity

Use of Abnormal Score and Overall Test Battery Mean Intra-Individual Variability Scores as

Measures of Performance Validity

Examination of the 'Mild Brain Injury Atypical Symptoms' and 'Validity-10' scales to Detect Symptom Exaggeration in U.S. Military Service Members

Psychological Characteristics of Individuals who put forth Inadequate Cognitive Effort

\section{The Birch Memorial Lecture: Optimal Outcome in Autism Spectrum Disorders \\ Presenter: Deborah Fein Centennial Ballroom (D-E)}

Optimal Outcome in Autism Spectrum Disorders 
1:30-3:00 PM

1. YURGELUN-TODD, D

2. YURGELUN-TODD, D

3. SOLOWIJ, N

4. TAPERT, $\mathrm{S}$

1:30-3:00 PM

1. KAMPER, J

2. GARNER, AA

3. BRINKMAN, TM

4. MZAYEK, Y

1:30-3:00 PM

1. HOROWITZ, T

2. PADGETT, LS

3. HOROWITZ, T

4. HARDY, KK

5. COSMAN, J

\section{1:30-3:15 PM}

1. HILLARY, FG

2. HILLARY, FG

3. WYLIE, G

4. WILDE, EA

5. CALHOUN, V

\section{1:30-3:00 PM}

1. SUMIDA C

2. MARTIN CS

3. ROGERS, $\mathrm{S}$

4. ROGERS. S

5. SOTO-AÑARI, M

6. MAY, PE

7. LANE, EM

8. BREWSTER, $\mathrm{P}$

9. BARULLI. D

10. MALLYA, S
Invited Symposium: Cannabis Effects in Vulnerable Populations

Chair: Deborah Yurgelon-Todd

Centennial Ballroom (D-E)

Cannabis Effects in Vulnerable Populations

Abberant Orbitofrontal Connectivity in Adolescent Marijuana Smokers

Cannabis Effects on the Brain in Schizophrenia and as a Vulnerability Toward Psychosis Phenotypes

Neuropsychological and Neuroimaging Findings in Adolescent Marijuana Users: Longitudinal Results

\section{Paper Session 3: Sleep \\ Moderator: Angela Jefferson \\ Centennial A}

The Validity of Actigraphy as a Sleep Correlate in the TBI population

Impact of Experimentally Manipulated Sleep on Adolescent Simulated Driving

Sleep disturbance contributes to attention and memory problems in adolescent and young adult survivors of childhood acute lymphoblastic leukemia (ALL)

Neurocognitive Function, Oxidative Stress and Sleep Disturbances in Long-Term Survivors of

Childhood Acute Lymphoblastic Leukemia (ALL)

\section{Symposium 3: A New Look at Chemobrain: Conceptualizing and Measuring Cognition in Cancer Patients \\ Chair: Todd Horowitz \\ Centennial B-C}

A new look at chemobrain: Conceptualizing and measuring cognition in cancer patients Looking At Cancer Related Cognitive Impairment Through The Lens Of CHC Theory: Does It Improve Our Vision?

Attention And Chemobrain: The View From Cognitive Psychology

Neuropsychological Assessments within the Children's Oncology Group: A Model for Success

Mobile Tests For Assessing Cognition In Aging And Disease

\section{Symposium 4: Using Neuroimaging and Connectivity Modeling to} Understand Network Plasticity After Brain Injury: Advancing Theory and Methods

Chair: Frank G. Hillary

Centennial G-H

Using neuroimaging and connectivity modeling to understand network plasticity after brain injury: advancing theory and methods

When physical disruption increases brain connectivity: the "hyperconnectivity hypothesis" after brain trauma

Investigation of information flow during a novel working memory task in individuals with traumatic brain injury

Integration of structural and functional imaging data in understanding connectivity

Functional network connectivity as a marker of brain injury

\section{Poster Session 3: Aging \& Epilepsy}

Centennial Ballroom Foyer

\section{Aging}

Who, When, and Where: Age-Related Differences on a Novel Episodic-Like Memory Task Association of Subjective Fatigue Complaints with Objective Cognitive Performance in Older Adults Ease Up, Study Up, or Forget About It: How Neuroticism Relates to Learning, Memory, and Cognitive Decline in Older Adults

Are Older Couples More Similar or Different in Cognition?

Inhibitory Control and Response Suppression as Latent Variables in Older Illiterate Bilinguals Engagement in Activities and Cognitive Functioning Among Older Adults in the Health and Retirement Study

Abnormal Nocturnal Fluctuations in Ambulatory Blood Pressure Relate to Worse Cognitive Performance in Older Adults: The Vanderbilt Memory \& Aging Project

Influence of Physical and Mental Health on Long-Term Cognitive Training Intervention Outcomes Cognitive Reserve is associated with Strategy Selection Independently of Executive Abilities

The Association between Global Health Habits and Cognitive Function in Non-Demented Older Adults 


\section{SCOTT, BM}

12. TRIFILIO, E

13. ROGERS, S

14. SNITZ, BE

15. MCINERNEY, K

16. MOLDOVAN, CP

17. KARIMIAN, A

18. GRIGSBY, J

19. EPPIG, J

20. DAVIS, HP

21. XU, Y

22. FRANCHOW, EI

23. MORGAN, B

24. COOLIN, A

25. TSAPANOU, A

26. GARCIA. S

27. BROWN, DS

28. NORMAN, AL

29. GENESER, AC

30. PORAT, S

31. STEWART, CC

32. STEWART, JJ

33. BUTTERFIELD, LC

34. GROSS, EZ

35. VAN PATTEN, $R$

36. STEPHENS, M

37. O' SHEA, DM

38. MEDINA, LD

39. MILLER, JS

40. MOORE, C

41. WOOLVERTON, CB

42. WALD, D

43. COOK, $\mathrm{AH}$

44. COOK, АH

45. COOK, $\mathrm{C}$

46. FEDOR, A

47. BLOCK, CK

48. GIOVANNETTI. T

49. DEKHTYAR, M

50. SHAW, EE

51. ZEC, RF

52. SANDERSON-CIMINO, M

53. MAYE, JE

54. MAYE, JE

55. PADULA, CB
Walking as a Stressor in Cognitively Normal Older Adults: Post-Exercise Pulse Pressure Better Predicts Executive Function than Pre-Exercise Pulse Pressure

Age Related Changes in Apathy, but not Anticipatory Anhedonia in Cognitively Normal Older Adults

The Counterintuitive Relationship between Sleep Quality and Neuropsychological Functioning in Older Adults

Temporal Dynamics of Subjective and Objective Memory Change in Aging

Neurocognitive Correlates of Hazard Perception in Normal Aging

The Impact of Age, Education, and Spiritual Well-Being on Executive Functioning in Healthy Older Adults

The Interactive Effects Of Age And Stress On Cognition And Functional Abilities Among HIV+ Individuals

Sensory Loss and Cognitive Decline among Older People: The San Luis Valley Health and Aging Study (SLVHAS)

Superior Verbal Memory in SuperAgers: Generalizability to Learning, Visual Memory, Language, and Executive Functioning

Performance on a Manual and Computerized Tower of London across the Life Span

Depression: The Effect of Mood on Cognitive Functioning in a Sample of Healthy Older Adults Complex Motor Planning: Sensitivity to Cognitive Concerns of Community-Dwelling Older Adults Longitudinal Prediction of Driving Risk in an Older Adult Sample

Inhibitory Control Underlies Recollection and Reconstruction Processes in Older Adults' Hindsight Judgments

Relationship of Self-reported Sleep Problems to Cognition in Aging

Sleep as a Mediator for the Effects of an Exercise Regimen on Cognition in Older Adults

Relationship of Metabolic Syndrome to Rate of Cognitive Decline in Normal Controls, Mild Cognitive Impairment and Alzheimer's Disease

Baseline Structural MRI Volumes and Five-year Follow-up Neuropsychological Performance in Cognitively-Intact Older Adults

Subjective Memory Complaints in Older African Americans

Personal Experience with Dance and Cortical Gray Matter Thickness in the Cognitively Normal and Mild Cognitive Impaired Elderly

Diverse Resources Contribute to Decision Making in Non-demented Older Adults

Verbal IQ as an Intervening Variable in Age-related Memory Decline

Apathy and Fatigue are Better Predictors of Cognitive Performance than Other Mood Variables in a Sample of Healthy Older Adults

Detection of Age- and Alzheimer's Disease Risk-Related Compensatory Strategies Using Ex-Gaussian Response Time Parameter Estimates

Effects of Context Maintenance on Cued-Stroop Performance in Healthy Aging

Examining Interleukin- 6 as a Predictor of Change in Processing Speed in Healthy Older Adults

The differential influence of extraversion on the association of age with cognition and the brain

The Impact of Dysexecutive Symptoms on Prospective Memory in Healthy Aging, Mild Cognitive Impairment, and Dementia

The Appraisal of Subjective Cognitive Complaints to Detect Severities of Cognitive Decline

Erectile Dysfunction and Cognitive Change Over Time

Self-imagining Improves Memory in Older Adults

Is Exercise More Important for Cognitive and Mood Functioning among Parkinson's Disease Patients than Normal Elderly?

Reduced Cortical Atrophy in Cognitively Successful Elderly Adults

Psychological Well-Being in Cognitively Successful Elderly Adults

Hypertension and Driving Risk: Neuropsychological Ability as Mediator

The Effects of a Brief, Water-Based Exercise Intervention on Cognitive Function in Older Adults

The Interaction Between Medical Burden and Anticholinergic Cognitive Burden on

Neuropsychological Function in a Geriatric Primary Care Sample

Relations between Multiple Collateral Reports of Everyday Functioning and Performance-Based

Assessment in MCI

Optimal Cognitive Aging in those 75+ is Associated with Greater Brain Reserve (larger hippocampal volumes) and Reduced Genetic Risk (E4-)

Functional Connectivity Within and Across Cortical Networks is Associated With Cognition During Aging

Effects of Age and Education on MMSE Scores

Age Moderates the Effect of Elevated Pulse Pressure on White Matter Lesion Burden in Older Adults Nightly Sleep and Cognitive Performance in Older Adults With and Without Amnestic Mild Cognitive Impairment

Cardiovascular Disease Risk Factors and Cognitive Performance in Cognitively Normal VITAL Study Participants

Longitudinal Cognitive Trajectories of Women Veterans from the Women's Health Initiative Memory Study 


\author{
56. SAPKOTA, S \\ 57. ZAHODNE, LB \\ 58. GRAVANO, J \\ 59. ROTBLATT, LJ \\ 60. HUNT, I \\ 61. LIEBEL, SW \\ 62. SALMINEN, L \\ 63. SULLIVAN, E \\ 64. MCNEELY, J \\ 65. NICODEMUS, NE \\ 66. SEELYE, A \\ 67. CORREA, LN \\ 68. CAMPBELL. L
}

69. LOGUE, E

70. SCARISBRICK, DM

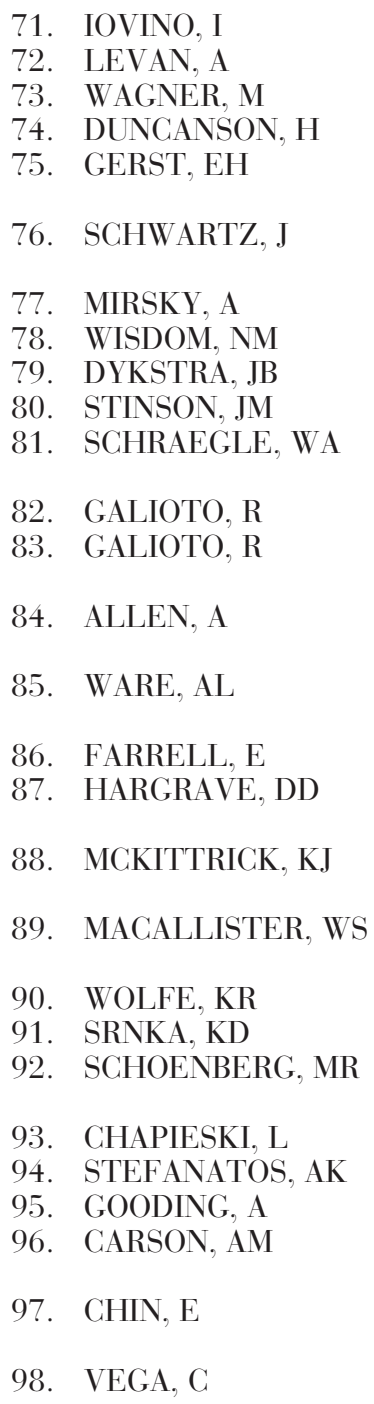

Genetic Risk for Executive Function Decline in Non-Demented Aging is Associated with APOE and Selectively Intensified by BDNF and COMT

Longitudinal Application of a Novel Method for Quantifying Cognitive Reserve in Aging Based on the Decomposition of Episodic Memory Variance

White Matter Lesion Burden and Lateralized Recognition Memory Abilities in Old Age

Temporal Order Memory Declines Across the Adult Lifespan: Insights into Middle and Old Age Performance Monitoring in Older Adults: A Meta-Analytic Review

Evaluating the Reliability and Construct Validity of an FMRI-Compatible Symbol Search Task

Age-related Changes in Gray Matter and White Matter Diffusivity among Healthy Older Adults

The Effect of Distractions on Geriatric and College-Aged Samples within a Virtual Apartment Stroop Task

Blood Glucose Mediates the Relationship between Cognitive Function and Sleep Quality in MiddleAged Adults

Does Anxiety Modulate the Effect of Education on Older Adults' Cognitive Abilities?

Unobtrusively Measured Sleep Disturbance and Sleep Variability Impact Neuropsychological

Performance in Cognitively Intact Older Adults

On Target: Fitt's Law and Aging

The Relationship between SuperAging and APOE Genotype, Vascular Risk and Brain Morphology

\section{Assessment/Psychometrics/Methods (Adult)}

Criterion-Validity of the WAIS-IV Cognitive Proficiency Index (CPI)

\section{Dementia (Alzheimer's)}

The Relationship between Neurocognitive and Functional Status: The Utility of Neurocognitive Performance in Predicting Collateral Informant Symptom Ratings

\section{Epilepsy/Seizures}

Academic Performance and Attention in Pediatric Epilepsy

Preliminary Investigation of the SSIS in Children with Epilepsy

Episodic Misperception of Time Associated with Temporal Lobe Epilepsy

Case Series of CBT in Reducing Seizure Frequency

The Impact of Anticonvulsant Medication on Academic Skills as Mediated Through Processing Speed and Working Memory

Executive Functioning and Behavioral Profiles in Childhood Absence Epilepsy and Juvenile

Myoclonic Epilepsy

Gender Differences in Sustained Attention in Idiopathic Generalized Epilepsy

Chaining Likelihood Ratios to Detect Psychogenic Non-epileptic Events (PNEE)

Utility of Routine Screening for Mental Health Problems in an Outpatient Pediatric Epilepsy Clinic Psychometric Properties of the Patient Competency Rating Scale in a Seizure Disorder Population The Neuropsychologist's Users Guide for Language Lateralization Using Functional Imaging and Dichotic Listening: A case study

Self-Awareness of Cognitive Deficits in Older Adults with Epilepsy and Mild Cognitive Impairment Subjective Cognitive Complaints vs. Objective Neuropsychological Performance in Older Adults with Epilepsy

Varying Impairments in Children with Landau-Kleffner Syndrome: Short-term Auditory Memory and Attention

The Intracarotid Amobarbital Procedure Predicts Change in Verbal Memory in Patients With Good Pre-operative Memory Who Undergo Temporal Lobectomy in the Language Dominant Hemisphere Use of the Repeatable Battery of Neuropsychological Status (RBANS) in Patients with Epilepsy Appropriateness of Rey 15-Item Test with recognition trial and Dot Counting Test for Assessing Performance Validity in Adults with Epilepsy

The Impact of Illness-related, Psychopathological, and Demographic Variables on Families of Children with Epilepsy

Sensitivity of the Wisconsin Card Sorting Test-64 versus the Tower of London for Detecting Executive Dysfunction in Children with Epilepsy

Executive Functions following Pediatric Epilepsy Surgery: Reliable Change Index Analysis Intra-Individual Variability in Children with Recent Onset Epilepsy

Improved Surgical Treatment for Temporal Lobe Epilepsy? Neuropsychological Outcome Following the Inferior Temporal Gyrus Approach for Selective Amygdalohippocampectomy

Do Memory Tests Provide any Localizing Information in Pediatric Epilepsy? Clinically Meaningful Change in Psychosocial Functioning Following Pediatric Epilepsy Surgery The relationship between age, executive functioning, and verbal learning and memory in epilepsy Social Functioning in Pediatric Patients with Intractable Epilepsy: Relative Contributions of SeizureRelated Variables, Cognitive Functions and Parental Anxiety about Epilepsy

The Utility of a Lateralization Rating of Cognitive Dysfunction in Pediatric Epilepsy Presurgical Evaluations

Verbal Memory Decline After Left Temporal Lobe Epilepsy Surgery in a Pediatric Sample 
99. EICHSTAEDT, KE

3:00-3:15 PM

3:15-4:45 PM

1. ROGALSKI, E

2. ROGALSKI, E

3. CHAPMAN, S

4. JAGUST, WJ

\section{3:15-4:45 PM}

1. BEAUCHAMP, МH

2. BEAUCHAMP, МH

3. ROBINSON, KE

4. RUSHBY, JA

5. MCDONALD, S

\section{3:15-4:45 PM}

1. POULSEN, C

2. POULSEN, $\mathrm{C}$

3. KUO, C

4. LUU, P

\section{3:15-4:45 PM}

\section{${ }^{\star}$ SEE FINAL ADDENDUM}

\author{
1. GARRETT, KD \\ 2. KORINEK, L \\ 3. GARRETT, KD \\ 4. PERRY, W \\ 5. PERRY, W \\ 6. GRACE, ES
}

\section{3:15-4:45 PM}

1. SUDIKOFF, EL

2. HEITZER, A
Verbal Fluency Performance in Temporal Lobe Epilepsy: General Verbal Ability Accounts for Lateralizing Effect of Phonemic but Not Semantic Fluency

Coffee Break

Centennial Ballroom Foyer

Invited Symposium: Refining Our Expectations and Understanding of Cognitive Aging Chair: Emily J. Rogalski Centennial $\mathbf{A}$

Refining Our Expectations and Understanding of Cognitive Aging Neurobiologic Features of Cognitive SuperAging

Brain and Cognitive Enhancement in Aging through Complex Reasoning and Aerobic Training Factors Associated With Age-Related Cognitive Decline and Compensation

\section{Symposium 5: Biological Markers of Social and Emotional Impairment after Traumatic Brain Injury \\ Chair: Miriam H. Beauchamp Centennial B-C}

Biological Markers of Social and Emotional Impairment after Traumatic Brain Injury Theory of Mind and Emotional Face Processing after Early Mild Traumatic Brain Injury Executive Function and Theory of Mind as Predictors of Social Adjustment in Childhood TBI: Regional Brain Injury as a Moderator Diminished Arousal and Emotional Responsivity after Severe Traumatic Brain Injury Heart Rate Variability as an Index of Emotional Processing Disturbance in People with Traumatic Brain Injury

Symposium 6: Functional Mapping for Presurgical Planning Using dEEG Source Localization and Transcranial Stimulation Chair: Catherine Poulsen Centennial F

Functional Mapping for Presurgical Planning Using dEEG Source Localization and Transcranial Stimulation

Dense-array EEG Source Localization of Language Function

Dense-array EEG Source Localization of Motor Function

Neuromodulation of Primary Motor Cortex with Transcranial Direct Current Stimulation

Poster Symposium: Assessment of Physicians: From Prospective Screening to Rehabilitation

Chair: Kelly D. Garrett

Centennial Ballroom Foyer

Forensic Neuropsychology

Assessment of Physicians: From Prospective Screening to Rehabilitation

Overview and Scope of Neuropsychological Performance Among Physicians of Advanced Age

Systematic Prospective Cognitive Screening Programs for Medical Staff

Neuropsychological Assessment of Late Career Physicians

Professional Issues and Ethics for Neuropsychologists in Screening, Evaluation, and Rehabilitation of Physicians

Signed, Sealed, and Delivered: What Referring Organizations Need in Neuropsychological Screening and Testing Reports on Aging and Other Physicians

Poster Session 4: Cross Cultural, Drugs, Genetics, HIV/AIDS, \& MS/ALS Centennial Ballroom Foyer

\section{ADHD/Attentional Functions}

Medication Effects on ADHD as Revealed by a Novel Continuous Performance Test Hyperactivity and Disinhibition Among Preschool Age Children Born Prematurely 
3. PAEK, E

4. WONG, A

5. NITZAN-TAMAR, O

6. MOSS, NC

7. GALUSHA-GLASSCOCK, JM

8. PETRANOVICH, CK

9. ANDREOTTI, C

10. BRYANT, KR

11. RIDER, G

12. LEON, A

13. LEON, A

14. FLORES, I

15. CARRION, C

16. KAYLEGIAN, J

17. BENNETT, J

18. AVILA, J

19. SHAKED, D

20. BURRELL, L

21. HAWKSHEAD, BE

22. EHRLER, MR

23. AASE, D

24. BOWLER, RM

25. PETRIE, J

26. ALI, S

27. CÁ́CERES-LUNA, G

28. HAMMERS, DB

29. HUCKANS, M

30. MAHONEY, JJ

31. REGNER, MF

32. CHERNER, M

33. SCOTT, TM

34. ARENIVAS, A

35. RAO, R

36. LEAFFER, EB

37. FONG, MW

38. HINTON, VJ

39. FEE, RJ

\section{Cognitive Intervention/Rehabilitation}

Intervention of Developmental Dyslexia through Working Memory Treatment

Analyzing the Relationship of Disrupted Cognitive Function on Social Health and Participation after Neurological Disorders: a Structural Equation Modeling Approach

\section{Cognitive Neuroscience}

Eye-Tracking Patterns as a Tool to Identify and Classify Cognitive Styles

\section{Cross Cultural}

The Relationship Between Primary Language, Socioeconomic Status, and Executive Function in Prechoolers Born Very Low Birthweight (VLBW)

Comparison of ANAM4 with Common Neuropsychological Tests in a Racially Diverse Sample Differing Aspects of Social Information Processing and Parent-reported Social Competence in Internationally Adopted Girls with a History of Institutionalization

Role of Age, Health Status, and Education on RBANS Performance in an Older African American Community Sample

Education Attainment and Premorbid Ability Affects Boston Naming Test Performance in a Rural Sample

Cognitive Outcomes of Bilingual Preterm Children at Ages Three and Six

The Effect of Educational Attainment on Assessment of Adaptive Functioning in a Sample of Monolingual Spanish-speaking Hispanics

Analyzing the Efficacy of the Spanish Version of the Frontal Systems Behavior Scale (FrSBe) in Assessing the Executive Functioning of a Sample of Monolingual Spanish-speaking Hispanics Performance of Hispanics and Non-Hispanic Whites on the NIH Toolbox Cognition Battery: The Roles of Ethnicity and Language Backgrounds

Cognitive and Behavioral Predictors of Adaptive Functioning in a Monolingual Spanish-Speaking Hispanic Sample

Executive Functioning, Risk Behaviors, and Time Homeless: The Significance for LGBTQ Homeless Youth

Socio-cultural Factors Outweigh Language Factors in WRAT Reading Performance

The Relationship Between Cultural Factors and Executive Functioning in English-speaking and Farsi-speaking Iranian individuals

\section{Drug/Toxin-Related Disorders (Including Alcoholism)}

The Influence of Substance Intake on Neuropsychological Performance

Effects of Cannabis Consumption on Verbal Fluencies

Neurocognitive Markers of Relapse Risk Assessed Using FMRI

Lifetime Methamphetamine and Cigarette Consumption Differentially Impact Executive Functioning in Men and Women

Alcohol Use History is Associated with Social Perception Task Performance

Manganese in Air: Associations in Residents with Tremor and Motor Function

Visual Attention, Object Recognition Processing, Cognition, and Physiological Differences in Males and Females with Substance Abuse and Opioid Addiction

Investigating Attention and Executive Functions Impact on Intraindividual Variability in Children with Fetal Alcohol Spectrum Disorder (FASD)

Analysis of predictive factors for psychoactive substances consumption among college students in Peru

Bad Air, Bad Cognition: The Effects of Wintertime Inversions on Executive Functioning Among Elderly Residents

The Association Between Plasma Inflammatory Markers and Depression, Anxiety, and Cognition in Adults with and without Methamphetamine Dependence

The Impact of Premorbid IQ on Cognitive Functioning in Individuals with a Cocaine Use Disorder Substance Dependence Demonstrates Altered Efficiency and Increased Bidirectional Network Causal Connectivity

COMT Val158Met Val/Val Genotype May Mitigate Methamphetamine-related Executive Dysfunction Neuropsychological Function is Improved among Opioid Dependent Drug Users Who Adhere to Opiate Agonist Treatment with Buprenorphine-Naloxone

\section{Genetics/Genetic Disorders}

Neuropsychological Profile of Pediatric Beta Thalassemia Major: A Case Study Neuropsychological Outcome in Russell-Silver Syndrome

The impact of sleep quality on executive function deficits observed in children with dystrophinopathy Neuropsychological Profile in Hereditary Spastic Paraplegia (HSP): A Case Report

Verbal Working Memory Selectively Contributes to Academic Achievement in Children with

Dystrophinopathy

The Role of Socioeconomic Status in the Dystrophinopathies 


\section{BERNIER, FP \\ 41. GERTSBERG. AG 42. SCHWARZ, G \\ 43. MCFALL, G \\ 44. MORSE, C}

\section{THALER, NS \\ 46. STEINER, A \\ 47. BAKER, L \\ 48. AMBROZIAK, AR}

49. FAZELI, PL

50. SHEPPARD, DP

51. WILLIAMS, C

52. MOORE, DJ

53. KEUTMANN, M

54. VAN DYK. K

55. KAMAT, R

56. CASALETTO, KB

57. SAYEGH, P

58. HINKLE, CD

59. ARCE RENTERIA, M

60. SEIDER, T

61. IUDICELLO, J

62. MAROUINE, MJ

63. PAUL, R

64. EAGAN, D

65. EAGAN, D

66. SAKAMOTO, M

67. DEVLIN, KN

68. KULKARNI, A

69. UKUEBERUWA, D

70. POTVIN, D

$\begin{array}{ll}\text { 71. } & \text { LOPES COSTA, SM } \\ \text { 72. } & \text { RIVERA, PM } \\ \text { 73. } & \text { BANERJEE, NS } \\ 74 . & \text { FORTE, M } \\ \text { 75. } & \text { STROBER, L } \\ 76 . & \text { NEIDINGER, S } \\ \text { 77. } & \text { MILLER, AK } \\ \text { 78. } & \text { HANCOCK, L } \\ \text { 79. } & \text { EVANKOVICH, KD }\end{array}$

Complex Neurodevelopmental Profile, Reminiscent of Williams Syndrome, in a Child with EP300related Syndromic Intellectual Disability

Neuropsychological Predictors of Driving Outcomes in Patients with Huntington's Disease

Relations between Lab-Based and Parent-reported Executive Functioning in Children and Adolescents with Williams Syndrome

An Alzheimer's Genetic Risk Composite Interacts with Diabetes Status to Predict Neurocognitive Speed Level and Change in Non-Demented Older Adults

Neurocognitive Functioning in Patients with 22q11.2 Deletion Syndrome: A Meta-Analytic Review

\section{HIV/AIDS/Infectious Disease}

Behavioral dysregulation is associated with increased neurocognitive variability Working Memory Deficits in Spanish-speakers with HIV-Associated Neurocognitive Disorders Cognitive Predictors of the Medication Management Task-Revised in an HIV+ Racial/Ethnic Minority Cohort

Does Depression Prevalence Contribute to Neurocognitive Disorder Overdiagnosis in HIV?

Moderate Physical Activity is Associated with Better Neurocognitive and Everyday Functioning in Older Adults with HIV Disease

Gender Differences in the Risk of Mild Cognitive Impairment in HIV Disease

Components of Executive Functioning and Visuospatial Memory in a Sample of HIV Positive

Individuals with a History of Alcohol Abuse

Neuropsychological functioning is associated with multitasking in older HIV+ adults

Sex Differences in Visuospatial Memory Impairment among HIV+ Drug Users

Covert Orienting in HIV: the Effects of Self-Reported Physical Health and Aging

Neurobehavioral changes in acute and early HIV infection

Metacognition Partially Mediates the Relationship between Neurocognitive and Everyday Functioning among Older HIV+ Adults

The Interactive Effects of Body Mass Index and Depression on Neurocognitive Functioning Among HIV+ Adults

Neuropsychological Profile of Anti-NMDA Receptor Encephalitis

Reaction Time Variability in HIV+ Adults with and without Current Substance Use Disorders

Clinical Factors Affecting Cerebral White Matter Damage in HIV

Does HIV Disease Accelerate Neurocognitive Aging?

Neurocognitive Decline in HIV-infected Hispanics: Rates and Predictors

Impact of the HIV Tat C30C31S dicysteine substitution on neuropsychological function in patients with clade $\mathrm{C}$ disease

Herpes Simplex-1 Infection is Associated with Reduced Right Hippocampal Volume Among Middle Aged Individuals with Genetic Risk for Alzheimer's Disease

Infection with Herpes Simplex-1 is Associated with Reductions in Executive Function and Full Scale

IQ (FSIQ) Among Healthy Middle Aged Adults with Genetic Risk for Alzheimer's Disease

Development of a brief iPad-based screening tool for detection of HIV-related neuropsychological impairment

IP-10 Mediates HIV-Associated Neuropsychological Dysfunction

\section{Imaging (Functional)}

Frontal Lobe Inefficiency After TBI Detected Using fNIRS During Stroop Task

\section{Imaging (Structural)}

Diffusion Tensor Imaging Links Regional Brain Integrity to Fatigue Severity in MS

\section{Medical/Neurological Disorders/Other (Child)}

The Effects of ADHD on the Cognitive Profiles of Children with NF1

\section{Multiple Sclerosis/ALS/Demyelinating Disorders}

Saccadic Eye Movements and Cognition in Multiple Sclerosis: a Case Study Affective Disorders and Cognitive Performance in Multiple Sclerosis

Is Vitamin D Insufficiency Related to Neuropsychological Function in Multiple Sclerosis?

Moderate Caffeine Intake and Verbal Memory in Multiple Sclerosis

Should I Stay or Should I Go? Employment Concerns Among Individuals Diagnosed with Multiple Sclerosis (MS) Within the Past Five Years

Conscientiousness and Depression are Related to the Ability to Delay Gratification in Multiple Sclerosis

Neuropsychological Correlates of Time-Based Versus Event-Based Prospective Memory in Multiple Sclerosis

A Case Series of Aging Multiple Sclerosis Patients Presenting with Dementia

Longitudinal Evaluation of Cognitive, Academic, and Adaptive Functioning in Pediatric Multiple Sclerosis 


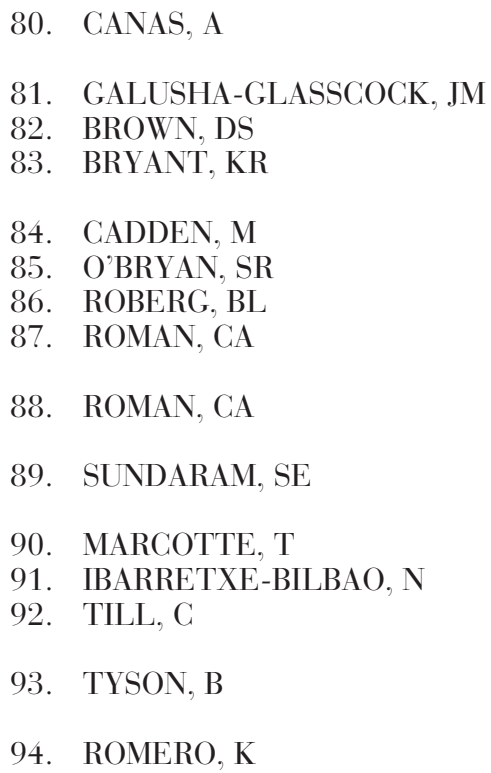

1. KESSELS, RP

9:00-10:00 AM

1. HOKKANEN. L
Cognitive Decline without Neuroimaging Evidence of Disease Progression in an Adolescent Patient with MS

Test-Retest Stability of a Novel Executive Function Measure in Patients with Multiple Sclerosis The Impact of Fatigue, Depression, Sleep and Daytime Sleepiness on Cognition in Multiple Sclerosis The Relationship between Depression and Cognitive Dysfunction in Individuals with Multiple Sclerosis

Beyond Binary: Exploring the Merits of Multiple Depression Groups

Slowed Saccadic Eve Movements in Multiple Sclerosis

Differences in Theory-of-Mind Abilities between Multiple Sclerosis Subtypes

Performance on the Symbol Digit Modalities Test Predicts Decreased White Matter Integrity a Decade Later in Multiple Sclerosis

Cognitive Reserve Moderates the Effect of Depression on Working Memory Performance in Multiple Sclerosis

The Effects of Aging on White Matter Integrity in Relapsing-Remitting Multiple Sclerosis: A Diffusion-Tensor Imaging Study

Driving Performance at Clinically Effective Cannabis Doses for the Treatment of MS Spasticity White matter injury and its relation with compensatory cortical activation in multiple sclerosis Perceived Parental Support and Social Stress as Moderators of Cognitive Decline in Pediatric-Onset Multiple Sclerosis Patients

Structural Changes in Thalamic Subnuclei Associated with Pain and Attention Performance in Multiple Sclerosis

Effects of Cannabis Use on Gray Matter Volume and Cognition in Patients with Multiple Sclerosis

Invited Address: Tales from Both Sides of the Brain

Presenter: Michael S. Gazzaniga

Centennial Ballroom (D-E)

Tales from Both Sides of the Brain

INS Town Hall

Centennial Ballroom (D-E)

Student Social, Hosted by the INS Student Liaison Committee Location TBA

\section{FRIDAY, FEBRUARY 6, 2015}

CE 9: Sleep: A Silent Contributor to Cognitive Problems

Presenter: Mark S. Aloia Centennial A

Sleep: A Silent Contributor to Cognitive Problems

CE 10: Learning from Your Mistakes? Errorless Learning in Amnesia and Dementia

Presenter: Roy P. Kessels

Centennial B-C

Learning from Your Mistakes? Errorless Learning in Amnesia and Dementia

Invited Address: Lifetime Trajectories of Cognition - From Birth Cohorts to Aging Studies

Presenter: Laura Hokkanen Centennial Ballroom (D-E)

Lifetime Trajectories of Cognition - From Birth Cohorts to Aging Studies 
10:00-10:15 AM

10:15-11:15 AM

${ }^{\star}$ SEE FINAL ADDENDUM

10:15-11:45 AM

1. CHOI. J

2. HAMSPTEAD, BM

3. NATION, DA

4. ALMEIDA, RP

5. GUZMAN, VA

6. BOTT, NT

\section{0:15-11:45 AM}

1. DEWEY, D

2. BERNIER, FP

3. ANDERSON, PJ

4. THORNTON, SK

5. TEN EYCKE, KD

6. DEWEY. D

\section{0:15-11:45 AM}

1. RASKIN, S

2. WALKER, KA

3. WOZNIAK, JR

4. MIGLIORINI, $\mathrm{R}$

5. GLASS, L

\section{0:15-11:45 AM}

1. ARNETT, $\mathrm{P}$

2. MERRITT, VC

3. MEYER, J

4. CADDEN, M

5. UKUEBERUWA, D
Coffee Break

Centennial Ballroom Foyer

INS Mid-Career (Benton) Award Presentation : A Glimpse Behind the Veil: Multimodal Assessment and Rehabilitation of Memory and Executive Functioning

Benton Award Winner: Brian Levine

Centennial Ballroom (D-E)

\section{Paper Session 4: Alzheimer's Disease \\ Moderator: Munro Cullum \\ Centennial A}

Self-efficacy for cognitive rehabilitation in Alzheimer's disease

Evidence of Transfer following Mnemonic Strategy Training in Patients with Mild Cognitive Impairment

Cognitive profiles of tau pathology and amyloidosis in prodromal AD

Cognitive Reserve Modifies Age-Related Alterations in CSF Biomarkers of Alzheimer's Disease

Regional White matter Hyperintensities and Fibrillar Amyloid Deposition

Altered Sense of Humor Comprehension in Neurodegenerative Disease: Neuroanatomical Correlates

\section{Symposium 7: Developmental Motor Disorders: From Genes to Brains to Behavior \\ Chair: Deborah Dewey \\ Centennial B-C}

Developmental Motor Disorders: From Genes to Brains to Behavior

Copy-number Variation in Canadian Children with Developmental Coordination Disorder Implicates

Neurodevelopmental Genes

Neonatal MRI Predicts Motor Impairment in Very Preterm School-aged Children

Functional Brain Activation during a Motor Inhibition Task in Children with Developmental

Coordination Disorder and Attention Deficit/ Hyperactivity Disorder

Executive Function Deficits in Children with Developmental Coordination Disorder and Attention

Deficit Hyperactivity Disorder: Same or Different

Developmental Brain Dysfunction: Co-occurrence is Associated with Impaired Neuropsychological

Functioning

\section{Paper Session 5: Alcohol-Related Dysfunction}

Moderator: William MacAllister

Centennial F

Effect of Drinking Behavior on Cognitive Functions in College Students

Executive Functioning Deficits in Offspring of Alcohol Dependent Probands Predate Alcohol

Dependence

Choline supplementation improves memory performance in preschool children with fetal alcohol spectrum disorders: A randomized controlled trial

Anterior Cingulate Cortex Structure Relates to Behavioral Inhibition in Children with Heavy Prenatal

Alcohol Exposure

Academic Achievement Deficits in Children with Heavy Prenatal Alcohol Exposure: Presence,

Prevalence. Neural Correlates

Symposium 8: Behavioral Genetics in Neuropsychology: Exploring New Frontiers in MS and mTBI

Chair: Peter Arnett

Centennial G-H

Behavioral Genetics in Neuropsychology: Exploring New Frontiers in MS and mTBI

Identifying the Role of the Apolipoprotein E (APOE) Gene in Concussion Outcome in Collegiate Athletes

Influence of Serotonin Transporter Genotype Status on Affective Bias Post-concussion

APOE $\varepsilon 4$ Allele Alters Depression Course in MS

Cognitive Functioning in MS and Relationship to Serotonin Transporter Genotype 
10:15-11:45 AM

1. BLACKWELL, LS

2. MISKEY, HM

3. SMITH, JM

4. LIM, AF

5. MALFAIT, D

6. MILLER, NK

7. PASTOREK, NJ

8. THOMPSON, J

9. TAKEDA, C

10. PUTCHA, D

11. VENKATESAN, UM

12. ROY, A

13. WOODARD, JL

14. DUDA, B

15. YUAN, J

16. STEWART, JJ

17. ZIMMERMANN, KM

18. GONZALES, M

19. PRICE, JS

20. HERHOLZ, P

21. SCHUSTER, $\mathrm{V}$

22. RAO, JA

23. ALKOZEI, A

24. CRANE, NA

25. ZLATAR, ZZ

26. ALFINI, AJ

27. KRISHNAN, K

28. MEDAGLIA, JD

29. POMMY, J

30. MCINTOSH, E

31. CHOI, A

32. MCLAREN, ME

33. REITER, K

34. SMITH WATTS, AK

35. BUCHHOLZ, JL

36. HIZEL, L

\section{Poster Session 5: Imaging (Structural \& Functional) \& Psychopathology/ \\ Neuropsychiatry \\ Centennial Ballroom Foyer}

\section{Acquired Brain Injury (TBI/Cerebrovascular Injury \& Disease - Child)}

Gender Differences in Children and Adolescents with Head Injury

\section{Acquired Brain Injury (TBI/Cerebrovascular Injury \& Disease - Adult)}

Personality Assessment Inventory Profiles of Post-Deployment Veterans: Differential Effects of Mild Traumatic Brain Injury and Psychopathology

\section{Acquired Brain Injury (TBI/Cerebrovascular Injury \& Disease - Child)}

Genetic and Environmental Influences on Executive Functioning after Pediatric Traumatic Brain Injury

\section{Imaging (Functional)}

Neural Correlates for a New Cognitive Flexibility Measure Using Functional Near-Infrared Spectroscopy

Children with Benign Epilepsy with Centro-temporal Spikes: an fMRI, Cognitive and Behavioral Study

Neuroanatomical Contributions to Planning and the Relationship Between Resting State fMRI and DKEFS Tower Test

Altered Brain Activation Associated with Posttraumatic Stress Symptoms

Social Outcome and Fractional Anisotropy of the Frontal Lobe in Children with Chronic Traumatic Brain Injury

Effects of order of task execution on Trail-Making Test performance

Altered Intrinsic Functional Coupling Between Core Neurocognitive Networks in Parkinson's Disease Organization of Functional Network Representations within Posterolateral Parietal Cortex

A novel voxel-based approach to examine network plasticity during recovery from Traumatic Brain Injury

A Novel Method for Assessing Behavioral and Neural Correlates of Levels of Semantic Knowledge Cognitive Reserve Predicts Bilateral Compensatory Brain Activation in Older Adults During a Working Memory Task

Intrinsic Functional Connectivity of Gait and Executive Functioning in Older Adults

Diagnostic Accuracy of SPECT Scans: Examining Specific Brain Areas of Hypoperfusion at Baseline in Alzheimer's Disease

Connectivity of the bilateral FFA during eye gaze perception

Aerobic Fitness and the Brain at Midlife: Evidence for the Role of the Cerebellum

Thalamo-cortico-thalamic Circuitry Differences in Emerging Adult Nicotine Users Seeking Treatment for Substance Dependence

Lateralization Paradigms in fMRI Studies - Robustness and Reliability

In Search of a Robust and Reliable Paradigm to Assess Visuospatial Functions: A Functional MRI Study

Cognitive Control Network Disruption and its Relationship with Gray Matter Volume in Late Life Depression

Emotional intelligence is differentially correlated with prefrontal cortical responses to backward masked fearful and angry faces

Independent Component Analysis of Cognitive Control as Treatment Predictors for Major Depressive

Disorder

Sedentary Time is Associated with Higher Tau Protein Load in Healthy Aging

The Effects of Exercise Training Cessation on Cerebral Blood Flow: an Arterial Spin Labeling

Analysis

Longitudinal Change in Resting-State Networks Over the Course of Recovery from TAI

\section{Imaging (Structural)}

Addressing Brain and Cognitive Reserve with Network Control Theory

Intact Cerebellar Structure and Fine Motor Function with Religious Use of Ayahuasca

Metabolic Syndrome and the Entorhinal Cortex: A Cortical Thickness Study

Comparison of Manual Versus FreeSurfer Calculated Intracranial Volumes in Older Adults

Symptom Dimensions of Depression and Frontal Brain Volume in Older Adults

Walking intervention increases VO2 peak and cortical thickness in MCI and healthy older adults

Pilot Test of the Utility of a Visual Rating System for Identifying Artifacts in Diffusion-Tensor Imaging (DTI) Data

Anxiety Sensitivity Correlates with Left Anterior Insula Volume in Posttraumatic Stress Disorder Organizational and Neuroanatomical Contributions to the Rey-Osterrieth Complex Figure in Nondemented Older Adults with Parkinson's Disease 
37. HOOD, A

38. BURCIAGA. J

39. LANCASTER, M

40. TANNER. JJ

41. TANNER, JJ

42. FLEMING, JC

43. TROTTER, BB

44. SHOLLENBARGER, SG

45. BRADSTREET, LE

46. SZYMKOWICZ, SM

47. COOLEY, S

48. BIRTCHER, K

49. CROWLEY, SJ

50. IBARRETXE-BILBAO, N

51. STEWARD, K

52. THAMES, AD

53. HAN, D

54. HARRISON. TM

55. GULLETT, JM

56. SULLAN, M

57. MERKLEY, TL

58. HAUT, MW

59. WATSON, C

60. BLANKEN, AE

61. WU, TC

62. DELBENE, $\mathrm{V}$

63. KILLGORE, WD

64. KUHN, T

65. THOMPSON, J

66. THOMPSON, J

67. THOMPSON, J

68. SHURA, RD

69. SAK. T

70. MILLER, IN

71. JERARD, T

72. HARLEY, A

73. WILDE, EA

74. NORRIS-BRILLIANT, A

75. CZEPIELEWSKI. LS

76. IP R

77. CARRATHERS, T

78. LOJEK, E

79. MATSUI. M

80. ROGERS, S

81. FROST, RB
White Matter Integrity Mediates the Relationship Between Prolonged Exposure to High and Variable Phenylalanine Levels Over the Lifetime and Strategic Processing in Children with Phenylketonuria The Association of Cognitive Functioning and Cortical Atrophy in Late Life Depression Diffusion Tensor Imaging Predictors of Cognitive Decline in Healthy Older Adults

Reduced Temporal White and Gray in Non-demented PD with Verbal Memory Deficits

Template Matters in Tract-Based Spatial Statistics

The Relationship between Hippocampal Dentation and Neuropsychological Memory Performance in Healthy Adults

Effect of Adolescent Binge Drinking on Frontal White Matter Circuitry in Veterans of OEF/OIF/OND Impact of Sleep Quality on Prefrontal Gyrification in Cannabis Using Emerging Adults

The Examination of White Matter Microstructure, Autism Traits, and Social Cognitive Abilities in Neurotypical Adults

Subthreshold Depressive Symptoms are Associated with Age-related Structural Brain Changes Impact of Blood Pressure on White Matter Tracts in Healthy Older Adults

Hippocampal, Medial Temporal Lobe, and Ventricle Volumes: Longitudinal follow up of Amnesic Subjects

A Majority Rule Approach for Segmenting the Corticospinal Tract from High Angular Resolution Diffusion Imaging

Distinct Brain Volume, Diffusivity and Activation in Parkinson`s Disease MCI Subtypes

Peripheral Inflammation Does Not Relate to Cortical Thickness in Middle Aged Adults

Using Multimodal Neuroimaging to Understand Neurocognitive Functioning and Fatigue in Patients With Hepatitis C

Grey Matter Correlates of Susceptibility to Scam in Community-Dwelling Older Adults

The Relationship between Common Cognitive Reserve Measures and Brain Structure in Nondemented Older Adults

The Gating Role of the ACC in PTSD Re-Experiencing: A Connectivity Study

Proof-of-concept for in vivo mapping of the human locus coeruleus using Fast Gray Matter

Acquisition T1 Inversion Recovery (FGATIR) Imaging Data

Persistent Post-concussive Symptoms and Cortical Thinning in Mild Traumatic Brain Injury

Primary Motor Cortex Thickness Correlates with the Ability for Motor Learning

Perceived Stress and Corpus Callosum Integrity in Older Adults

Cognitive Decline, Ventricular Enlargement, and Hippocampal Atrophy in Mild Cognitive Impairment

Volumetric and DTI Analyses of Sports Concussion

Association Between Autism-Associated Polymorphism in CNTNAP2 and Bilateral Caudate Nucleus

Volume Reductions in Healthy Adults

Fractional Anisotropy of Frontoparietal Connections Predicts Individual Resistance to Sleep

Deprivation

Altered White Matter Connectivity in Adjacent Medial Temporal Circuits in Temporal Lobe Epilepsy

\section{Psychopathology/Neuropsychiatry (Including Schizophrenia)}

Altered Neurocognitive Profiles on the WJ-III-Cognitive in a Middle-Aged Sample of Adult Depression: A Preliminary Analysis

Neurocognitive differences between Schizophrenia and Depression as measured by CNS Vital Signs Sensitivity of Computer-Based Assessment in the Identification of Neurocognitive Differences between Schizophrenia and Bipolar Disorder

Assessment and Treatment of a Veteran with Conversion Myoclonus and PTSD

The Relationship Between Executive Functioning and the Personality Assessment Inventory Validity and Clinical Scales in a Mixed Neuropsychological Sample

Utility of the MMPI-2-RF Fs Validity Scale in Determining Interpretability of Somatic/Cognitive Scales in a Veteran Sample

Personality Predictors of Cognitive Performance in Young Adults with Remitted Major Depressive Disorder

Is ACTH or Cortisol a Better Predictor of Story Memory Deficits in Psychotic Major Depression? Improvement in Psychiatric Status Following a Novel Inpatient Treatment for OEF/OIF Veterans with PTSD and Multiple Comorbidities

Neurocognitive Predictors of Eating Disorders

Improvement of Verbal Learning in Schizophrenia: Results from a Randomized, Double-Blind.

Placebo-Controlled Trial of Amantadine Adjunctive to Antipsychotics

Self-awareness of Patients on a Neuropsychiatric Unit

Examining Single Item Maintenance Impairment in Schizophrenia After Intact Updating

Recovery from Depression: the Value of Executive Functions and Coping

Cognitive remediation therapy focusing upon the strategy coaching in schizophrenia

How Does a History of Depression Influence the Current Neuropsychological Functioning of Older

Adults?

The Relationship Between Symptom Severity and Performance on the MATRICS Consensus Battery in Symptomatic Schizophrenics Compared to Matched Controls 
82. ZILBERFAYN, I

83. KEILP JC

84. HUNTER, M

85. DALY, M

86. BODAPATI, AS

87. POTHIER, W

88. RICHMOND, CE

89. ESTEVIS, E

90. ESTEVIS, E

91. UMEKUBO, KA

92. R.-MERCIER, A

93. GORLYN, M

94. PLANTE, W

95. LA, DD

96. JANG, K

97. GALLEGOS RODRÍGUEZ, YE

98. CAVACO, S

99. TWAMLEY, EW

12:00-1:00 PM

1. CATANI, M

1:00-3:00 PM

1. FILLEY, CM

2. HEILMAN, KM

3. FILLEY, CM

4. KERTESZ, A

5. DENCKLA, M

6. YEO, RA

1:30-3:00 PM

1. MABBOTT, D

2. JACOLA, LM

3. CONKLIN HM

4. MABBOTT, D

5. HARDY, KK
White Matter Integrity and its Relationship to PTSD Symptoms and Sleep Quality in OEF/OIF Veterans

Evidence Against Ventral Prefrontal Dysfunction in Suicidal Behavior

Dynamic causal modeling of selective attention predicts relapse in patients recovering from addiction Characterization of Somatosensory Processing in Relation to Schizotypal Traits in a Sample of Nonclinical Young Adults

Cognitive Correlates of a Social Attentional Bias in Schizophrenia

Methodological Guidelines to Improve Research Designs on Cognition in Schizophrenia

What Do Drawing Tasks Measure In Serious Mental Illness? A Preliminary Analysis Using The

Boston Qualitative Scoring System For The Rey Complex Figure Copy

Neuropsychological dysfunction and informed consent capacity among depressed inpatients

Memory dysfunction in major depression: Not an artifact of poor effort

Self-Reported Impulsivity Mediates the Relationship Between Schizotypy and Performance on the

Wisconsin Card Sorting Test

The effect of Maltreatment on Neuropsychological functioning of Children, Adolescents and Adults with Psychiatric disorders: Meta-analysis

Semantic Fluency Deficit Is a Marker for High-Lethality Suicide Attempt Risk in Major Depression Contributors of Executive Deficits in Incarcerated Youth

Posttraumatic Stress Disorder and Obstructive Sleep Disorder Effects on Cognition in Older Veterans Source localization of P300 in college students with schizotypal traits

Cognitive functioning outcomes in Cognitive Behavioral Social Skills Training (CBSST) for racial/ ethnic minority individuals diagnosed with schizophrenia spectrum disorders

Impulse Control Disorders and Apathy in Parkinson's Disease

Neurocognitive Insight among Individuals with Schizophrenia

Invited Address: Disconnection in the Connectome Era

Presenter: Marco Catani

Centennial Ballroom (D-E)

Disconnection in the Connectome Era

\section{Invited Symposium: Norman Geschwind and the Lasting Influence of Disconnection \\ Chair: Chris M. Filley \\ Centennial Ballroom (D-E)}

Norman Geschwind and the Lasting Influence of Disconnection

Geschwind and Apraxia

Disconnection and White Matter

Disconnexion Syndromes and Aphasia

Geschwind's Impact on Developmental Dyslexia and Related Disorders

Geschwind's Impact on Clinical and Experimental Neuropsychology

\section{Symposium 9: The Role of Physiological Factors and Novel Interventions in Mitigating Poor Neuropsychological Outcomes in Pediatric Brain Tumor Survivors \\ Chair: Donald Mabbott \\ Centennial B-C}

The Role of Physiological Factors and Novel Interventions in Mitigating Poor Neuropsychological Outcomes in Pediatric Brain Tumor Survivors

The Impact of Excessive Daytime Sleepiness on Neurocognitive Performance and Brain Activation in Children Diagnosed with Craniopharyngioma

Aerobic Fitness in Relation to Cognitive Performance and Brain Function among Children Newly Diagnosed with Craniopharyngioma

Exercise training alters functional connectivity in children treated for posterior fossa brain tumors Neuropsychological Functioning Following Computerized Working Memory Training with Survivors of Pediatric Brain Tumors 
1:30-3:00 PM

1. THAMES, AD

2. BYRD, DA

3. CAGIGAS, XE

4. SUAREZ, PA

5. MADORE, MR

6. FERNANDEZ, AL

1:30-3:00 PM

1. MAXWELL, E

2. SINOPOLI, K

3. WILKINSON, A

4. HARIK. L

5. TREBLE-BARNA, A

6. TREBLE-BARNA, A

7. SIMIC, $\mathrm{N}$

8. HUBER, J

9. EDELSTEIN, K

10. BRADLEY, KA

11. FERNANDEZ, V

12. SIMIC, N

13. ARRINGTON, C

14. CIRINO, PT

15. KULESZ, PA

1:30-3:00 PM

1. DE GYVES, G

2. RAI, JK

3. SOUBBOTINA, M

4. FERENC. L

5. HERTING, $\mathrm{N}$

6. BERL, M
Symposium 10: Stepping Out from Silence: Initiating Difficult Dialogues about Diversity in the Context of Neuropsychological Education, Training, and Leadership

Chair: April Thames

Discussant: Mariana Cherner

Centennial G-H

Stepping Out from Silence: Initiating Difficult Dialogues about Diversity in the Context of Neuropsychological Education, Training, and Leadership

Demystifying the Process of Engaging in Difficult Dialogues at the Organizational Level of

Neuropsychological Training and Practice

Cross-Cultural Neuropsychology: A Difficult Dialogue

Culturally Meaningful Feedback: A Difficult Dialogue in the Making

Unequal Positions of Power: Initiating Difficult Dialogues in the Trainee and Supervisor Relationship

Translating measures for use across international settings: A critical analysis of current approaches

\section{Poster Symposium in Honor of Maureen Dennis Centennial Ballroom Foyer}

\section{Acquired Brain Injury (TBI/Cerebrovascular Injury \& Disease - Child)}

The Role of Anxiety, Family Burden, and Executive Functioning on Mathematical Performance in Children with Traumatic Brain Injury

"Is My Child Self-Aware?" Metacognitive Knowledge of Post-Injury Sequelae Following Childhood Traumatic Brain Injury

Predicting Attention Problems in the First Year Following Pediatric Traumatic Brain Injury (TBI) The Impact of Pediatric Traumatic Brain Injury on Written Expression: a Diffusion Tensor Imaging Study Utilizing Tract-Based Spatial Statistics

Classroom Functioning Following Traumatic Brain Injury in Young Children

Parenting Practices as a Time-Varying Moderator of Executive Dysfunction Following Traumatic

Brain Injury in Young Children

Predicting Executive Function Following Pediatric Traumatic Brain Injury (TBI)

\section{Autism Spectrum Disorders}

Toward Social and Cultural Competence in Developmental Pediatrics: Parents' Perceptions of The Autism Diagnostic Observation Schedule

\section{Cancer}

Longitudinal Effects of Radiation on Neurocognitive Functions in Adults with Primary Brain Tumors Cognitive Neuroscience

Corpus Callosum Microstructure and Auditory Interhemispheric Transfer in Spina Bifida Myelomeningocele

\section{Learning Disabilities/Academic Skills}

Cortico-cerebellar connectivity in reading impaired children: A probabilistic tractography study

\section{Medical/Neurological Disorders/Other (Child)}

White and Grey Matter Relations to Simple, Choice, and Cognitive Reaction Time in Spina Bifida The Effects of Shunt Revisions on Intelligence in Congenital Hydrocephalus: A Meta-Analysis Predictors of Academic Fluency in Spina Bifida Myelomeningocele

Attention in Spina Bifida Myelomeningocele: Relations with Brain Volume and Integrity

Poster Session 6: Assessment-Child, Dementia (AD), \& Medical/

Neurological Disorders-Child

Centennial Ballroom Foyer

\section{Assessment/Psychometrics/Methods (Child)}

Exploring decision-making in children from 6 to 9 years old using a risky choice task The ecological validity of neuropsychological tests of executive function in children with Fetal Alcohol Spectrum Disorder (FASD)

Clinical Utility of the BDEFS-CA ADHD-EF Index in Classifying Children with ADHD

Construct Validity of the BRIEF-P for Teacher-Rated Executive Functions

Neuropsychological, Academic Achievement, and Behavioral Differences Between the CBCL Pediatric Bipolar Profile, ADHD, and Emotionally Normal Controls

Everyday Executive functioning in Focal Pediatric Epilepsy on the Revised BRIEF 
7. RIEGER, RE

8. ERICKSON, KT

9. BUCHHOLZ, B

10. WALSH, KS

11. WALSH, KS

12. MESSER, M

13. MESSER, M

14. SNYDER, AR

15. WALD, D

16. TSUI-CALDWELL, Y

17. GRABYAN, JM

18. SMITH, TJ

19. MARREIRO, CL

20. GURNANI, A

21. SANDERS, CL

22. HEGDE, $\mathrm{K}$

23. FARLEY, SV

24. FLOWERS, AT

25. CLEM, M

26. JENNETTE, KJ

27. VILA-CASTELAR, C

28. TRAVIS SEIDL, JN

29. DURANT, J

30. HOWIESON, D

31. MIZUKI, BM

32. PERRY, CE

33. PERRY, CE

34. HASSENSTAB, J

35. FARRELL, M

36. NATION, DA

37. BUTTS, AM

38. MELROSE, RJ

39. TRIVEDI, MA

40. BOOTS, EA

41. ALVERSON, WA

42. GIFFORD, K

43. WEISSBERGER, G

44. ELIASSEN, CF
Associations among Maternal Depressive Symptoms, Interactive Behaviors, and Child Development in Very Low Birth Weight and Normal Birth Weight Preschoolers

A Comparison of Social Cognitive Deficits in Children with Autism Spectrum Disorder and At-Risk for Pediatric Bipolar Disorder

Verbal Memory Evaluation by the Immediate Post-Concussion Assessment and Cognitive Testing (ImPACT) and the California Verbal Learning Test Children's Version (CVLT-C) in Patients Presenting With Concussion

Executive Function Profiles in Children with Neurofibromatosis Type 1 Compared with Healthy Children on the Behavior Rating Inventory of Executive Functions-2

Executive Function Profiles in Pediatric Cancer Survivors Compared with Healthy Children on the Behavior Rating Inventory of Executive Functions-2

Development and Reliability of a New Academic Achievement Battery

Development and Reliability of a Revised Behavior Rating Inventory of Executive Function (BRIEF)

Normative Values and the Impact of Demographics at Baseline for the Sport Concussion Assessment Tool (SCAT3) in Teenagers

\section{Dementia (Alzheimer's)}

Spatial Disorientation in Mixed Alzheimer's Disease and Vascular Dementia

Schelten et al. Ratings for Mesial Temporal Lobe Atrophy in the Cache County Study on Memory Health and Aging

Enhancing Prediction of Longitudinal Decline in Instrumental Activities of Daily Living in

Alzheimer's Patients Using Functional and Cognitive Pre-progression Estimates

Effects of a 12-week Exercise Intervention on Default Mode Network Connectivity in MCI and Healthy Elders

The Effect of Depression and Behavioral Disturbance on Cognitive Change in Diverse Older Persons

The Differential Effects of Alzheimer's Disease on Cognitive Performance: A Meta Analysis

Nutritional Status and Neuropsychological Functioning in Persons with Dementia: The Cache County Dementia Progression Study

Rethinking the File Drawer Problem of Null Findings: Vascular Burden Did Not Predict Dementia in a Nationally Representative Sample of Older Adults

RBANS Total and Memory Scores Differentiate AD from MCI and Other Dementia Subtypes

Effects of Demographic and Cognitive Factors on an Observation-Based Daily Functional Test

Baseline Neuropsychiatric Symptoms and Stability of Mild Cognitive Impairment

The Association of Cognitive Endophenotypes and Risky Single Nucleotide Polymorphisms of Alzheimer's Disease within the Alzheimer's Disease Neuroimaging Initiative (ADNI) Database

Short-Term Efficacy of Cholinesterase Inhibitors in Alzheimer's Disease Measured by Sensitive Tasks of Attention

Cognitive and Functional Correlates of Neuropsychiatric Syndromes in Mild Alzheimer's Disease

Relationship between the Activities of Daily Living Questionnaire and the Montreal Cognitive

Assessment

Stability and Prognostic Value of Memory Complaints in Older Adults

DRS-2 Construction and Memory Scores Differentiate Between Alzheimer's Disease and Parkinsonian Dementias

Association between cognition and latent toxoplasmosis in healthy older adults and in Alzheimer's disease

Association between latent toxoplasmosis and Alzheimer's disease

Cognitive Performance in the Dominantly Inherited Alzheimer Network (DIAN)

Windows of Awareness in Alzheimer's disease: Insight into Word-finding Difficulty Brings Memory

Deficits to Light

Elevated pulse pressure is associated with tau-mediated neurodegeneration, cerebral amyloidosis, and progression to dementia in the very-old

Amyloid PET confirmed Alzheimer's disease: Neurocognitive differences in Logopenic Progressive Aphasia vs. Dementia of the Alzheimer's Type

Distinct Patterns of Structural and Functional Connectivity in Amnestic and Non-amnestic Early Onset Alzheimer's Disease

The relationship between oral versus written discrepancy scores on the symbol digit modalities test (SDMT) and fMRI activation during SDMT performance and white matter integrity in middle aged adults with a parental history of Alzheimer's disease (AD): Influence of APOE genotype

Occupational Complexity and Cognitive Reserve in Middle-aged Adults at Risk for Alzheimer's

Disease

Characteristics Associated with Cognitive Asymmetry in a Large Sample of Alzheimer's Disease Patients

Ambulatory blood pressure variations relate to greater subjective cognitive decline in older adults:

The Vanderbilt Memory \& Aging Project

Longitudinal Semantic Fluency Performance in Hispanic Older Adults in the Early Stages of

Alzheimer's Disease

Hippocampal subfield atrophy in multi-domain, but not in amnestic mild cognitive impairment 


\author{
45. EDMONDS, EC \\ 46. KIELB, S \\ 47. HAYS, C \\ 48. BETTCHER, BM \\ 49. WERHANE, ML \\ 50. KINZER, A
}

\section{PEACH, RK}

52. AGOSTON, A

53. FISCHER, MP

54. ANTONINI, T

55. SALONEN, E

56. COHEN, J

57. ELLEFSON, SE

58. LUNGREN, E

59. PHOONG, MA

60. SALAMA, CH

61. BEAN JAWORSKI, J

62. NEUMANN, J

63. WISE, S

64. HARRELL, M

65. ALLEN, A

66. PIERCY, JC

67. DIQUATTRO, ME

68. GRAHAM, DM

69. COLBERT, AM

70. LUCCHETTI, A

71. RAU, S

72. BADALY, D

73. COOL, DL

74. HARDY, SJ

75. CASNAR, C

76. WHITING, S

77. OLSON, K

78. SANZ, J

79. SANZ, J

80. WESONGA, E

81. DINH, KL

82. GREIF, SM
Characterizing Subtle Cognitive Decline and Biomarker Staging in Preclinical Alzheimer's Disease Subjective Cognitive Complaints and Early Cognitive Features in Preclinical Alzheimer's Disease Resting Cerebral Blood Flow Measured with Arterial Spin Labeling MRI is Associated with

Cerebrospinal Fluid Biomarkers of Alzheimer's Disease

Proinflammatory Chemokines Selectively and Negatively Associate with Episodic Memory in Alzheimer's Disease Phenotypes

Dissociation of Alzheimer's Disease Neuropathological Burden and Vascular Pathology on Cognition by CDR Stage: Results from the Framingham Brain Donation Program

Dementia Worry is Related to (In)accuracy of Self-reported Memory Complaints

\section{Language and Speech Functions/Aphasia}

Treatment for Aphasia Associated with Attentional Impairment: A Comparative Effectiveness Study

\section{Medical/Neurological Disorders/Other (Child)}

Executive Functioning Predicts Interpretation of Social Intents in Children with Fetal Alcohol Spectrum Disorder

Differential Neuropsychological Dysfunction Associated with Age of Treatment Onset in Three Relatives with Congenital Hypothyroidism

Neurocognitive Functioning in Infants Undergoing Cardiac Transplantation

Can a Computed Tomography Examination of the Head Affect Later Cognitive Functions? Follow-up of a Randomized Controlled Trial

A Comparison of the Intellectual and Adaptive Behavior Functioning of Children with Prenatal Exposure to Methamphetamine with Children with ADHD

Intellect, Spatial Planning and Executive Functioning in Children with Chiari Malformation Type I

Visual and Verbal Memory Performance in Children wtih Fetal Alcohol Spectrum Disorders

Case Study: Neuropsychological Functioning in a 12-Year Old With Unilateral Right Cerebellar Hypoplasia

Functional Outcomes of Children with Brain Tumor following Inpatient Rehabilitation

Differential Impact of Visuospatial Integration on Academic Skill Development in Children and

Adolescents with Critical Cyanotic Congenital Heart Disease

Neuropsychological Functioning, Fatigue, and Educational Support in Pediatric Transverse Myelitis Sluggish Cognitive Tempo and Executive Functions in Children with Sickle Cell Disease Childhood Narcolepsy and ADHD Comorbidity

Language Deficits in Children; An Investigation into Left Hemisphere Cortical Dysplasia Infertility Treatment and Neuropsychological Functioning in Preterm-Born Preschoolers A Case Study: Neurocognitive Trajectory of Adolescent with a Complex Medical History Deficits in Encoding but not Retention of Facial Stimuli in Children with Heavy Prenatal Alcohol Exposure

Effects of Processing Speed and Memory on Academic Outcomes in Children Following Allogeneic Hematopoietic Stem Cell Transplantation

Psychosocial Profiles in Pediatric Patients with Median Arcuate Ligament Syndrome

An Examination of Factors Contributing to Sluggish Cognitive Tempo (SCT) in Spina Bifida (SB) and ADHD, Inattentive Type (ADHD-I)

Cognitive, Behavioral, and Socioemotional Functioning in Children with Congenital Heart Disease Compared to Children Diagnosed with Attention-Deficit/Hyperactivity Disorder

Pre- and Post-Cerebral Bypass Neuropsychological Functioning of a 17-year-old Female with Moyamoya Disease and Atypical Cerebral Organization

Neurocognitive Functioning in Pediatric Sickle Cell Disease: A Cumulative Risk Perspective Parent and Teacher Perspectives on BASC-II Content Scales in Young Children with NF1 Cognitive and Psychosocial Functioning in Children with Sickle Cell Disease versus ADHD Predictive Validity of Parent-Reported Working Memory in Pediatric Sickle Cell Disease Emotional and Behavioral Outcomes in School Age Children with Congenital Heart Disease Emotional and Behavioral Outcomes in School Aged Children with Congenital Heart Disease (CHD) and Autism

Difference in the Relationship Between Age and Mean Diffusivity of White Matter in Children with Phenylketonuria

Neuropsychological Sequelae of Opsoclonus-Myoclonus Syndrome: A Case Series

Cognitive, Emotional, and Behavioral Features as Predictors of Adaptive Functioning in a Clinical Pediatric Population 
3:00-3:15 PM

3:15-4:45 PM

1. FLETCHER, JM

2. TURKSTRA, L

3. SCHACHAR, RJ

4. JURANEK, J

5. TAYLOR. HG

\section{3:15-4:45 PM}

1. HAMMEKE, TA

2. JIVANI, S

3. ZHAVORONKOVA, L

4. ELLIS, M

\section{3:15-4:45 PM}

1. ROYALL, DR

2. ROYALL. DR

3. PALMER, RF

4. GAVETT, BE

5. KOPPARA, A

6. ROYALL, DR

3:15-4:45 PM

3:15-4:45 PM

1. PADGETT, LS

2. PADGETT, LS

3. HOROWITZ, T

4. AHLES, T
Coffee Break

Centennial Ballroom Foyer

Invited Symposium: The Young Damaged Brain: A Symposium in Honor of Maureen Dennis

Organizers: Jack M. Fletcher, Brenda Spiegler

Discussant: Erin D. Bigler

Centennial Ballroom (D-E)

The Young Damaged Brain: A Symposium in Honor of Maureen Dennis

Different Routes to Pragmatic Communication Impairment in Adolescence

Etiology of Attention Deficit Hyperactivity Disorder (ADHD): Lessons from Cognitive Function and

Traumatic Brain Injury

The Cerebellum in Neurodevelopmental Disorders

Plasticity of Function After Childhood Brain Injury

\section{Paper Session 6: TBI - Functional Imaging \\ Moderator: Michael Larson \\ Centennial A}

Recovery of Functional Brain Networks Following Sport-Related Concussion

Effects of Injury Severity on Default Mode Network Volume in Pediatric Traumatic Brain Injury and the Relationship to Attention

Brain Functional Connectivity in Traumatic Brain Injury Patients and Healthy Persons

Differential Outcomes in Neural and Cognitive Functioning in Children with Moderate-to-Severe

Traumatic Brain Injuries: The UCLA RAPBI Study of Event-Related Potentials

\section{Symposium 11: The Psychometric Assessment of Dementia and Related} Conditions using the Latent Variable " $\delta$ "

Chair: Donald Royall

Centennial B-C

The Psychometric Assessment of Dementia and Related Conditions using the Latent Variable " $\delta$ " Welcome Back to Your Future: $\delta$ 's Rationale and Very Ancient History

Future Dementia Status is Almost Entirely Explained by the Latent Variable $\delta$ 's Intercept and Slope

Neuropathology Mediates Age, Race, and APOE effects on $\delta$ in Pathologically Confirmed AD

Validation of the latent dementia phenotype $\delta$ in a German Sample with clinical data, CSF and

neuroimaging biomarkers

The 'd' Evolution of Cognitive Assessment

Panel Discussion: The Use of Neuropsychological Instruments in Research, Presented by the INS Student Liaison Committee

Presenters: David Libon, Cecil Reynolds, Robert K. Heaton, Sandra Weintraub Centennial F

Symposium 12: How Your Network Shapes Your Science and Vice Versa: New Ways to Think about Advancing Your Research Career and Obtaining Funding

Chair: Lynne Padgett

Centennial G-H

How Your Network Shapes Your Science and Vice Versa: New Ways to Think about Advancing Your Research Career and Obtaining Funding

Portfolios and Project Officers: Learning about Grant Funding at the National Cancer Institute

I Went from Principal Investigator to Program Officer, and You'll Never Guess What I Learned about the Granting Process!

Obtaining Grant Funding: Taking Advantage and Creating Opportunities 
3:15-4:45 PM

1. WILLIAMS, J

2. WILLIAMS, J

3. OSIPOWICZ, K

4. MCWILLIAMS, K

5. PATRICK, K

6. TART-ZELVIN, A

3:15-4:45 PM

1. HARRELL, KM

2. GOLDSWORTHY, R

3. HARMS, $\mathrm{V}$

4. ADAMS, SW

5. UPSHAW, J

6. GHILAIN, CS

7. BAYNARD, J

8. ELLISON, RD

9. ANDERSON, SA

10. ROBINSON, KE

11. DEYOUNG, N
12. FONG, CH
13. MURRAY, L
14. ORD, AS
15. ORD, AS
16. PABON, R
17. HESSEN, E
18. MECHANIC-HAMILTON, D
19. STEED, D
20. SELIGMAN, SC
21. THORGUSEN, SR
22. FYOCK, CA
23. SCHAEFER, LA
24. BISS, RK
25. BANGEN, KJ
26. LOBUE, C

\author{
Poster Symposium: Anterograde Memory Disorder As Disconnection \\ Syndrome \\ Chair: J. Michael Williams \\ Centennial Ballroom Foyer
}

\section{Memory Functions}

Anterograde Memory Disorder As Disconnection Syndrome

The Hippocampus As a Cortical Interface Derived From An Analysis of Its Structural Anatomy and Function

Neuroimaging Methods Used to Examine the Hippocampus, Memory and Emotion Results of Resting State Functional Connectivity Studies of the Hippocampus Results of Diffusion Tensor Imaging studies of the Hippocampus and Emotion Results of fMRI Studies of the Hippocampus and Emotion

\section{Poster Session 7: Behavioral Neurology, Dementia (Subcortical), \& Medical/Neurological Disorders-Adult Centennial Ballroom Foyer}

\section{Assessment/Psychometrics/Methods (Adult)}

Clinical Video Telehealth Compared to Face-to-Face Assessment Using the Montreal Cognitive Assessment: Is Clinical Video Telehealth Reliable?

Are Assumed Differences in Delay Discounting and Delayed Gratification Due to Procedural Variance?

\section{Behavioral Neurology/Cerebral Lateralization/Callosal Studies}

Put Your Best Side Forward: The Effect of Facial Attractiveness Asymmetry on Posing Biases Movement Disorder Symptoms Associated with Unified Parkinson's Disease Rating Scale (UPDRS) in Two Manganese (Mn)-Exposed Communities

Connecting the Disconnected: Importance of an Integrated Approach to Neuropsychological Evaluation in a Patient with ACC

Assessing DBS Candidacy in Idiopathic PD: Do Emotional Factors Influence Outcome?

The Effect of Social Isolation on Aggressive Behavior and Cannabinoid Receptors in the Amygdala Late-Onset Rasmussen's Encephalitis: A Neuropsychological Case Study

Assessing DBS Candidacy in Idiopathic Parkinson's Disease: The roles of education and ethnicity

\section{Cancer}

Psychosocial Functioning Following Pediatric Brain Tumor: Contribution of Executive Function, Brain Activation, and Coping to Predicting Outcome

\section{Dementia (Subcortical, Specific Disorders, MCI, etc.)}

Classifying patients with and without dementia using cardiovascular risk factors Procedural-Based learning in Parkinson's Disease Motor Subtypes

Behavioral and Neural Correlates of Word Retrieval Treatment for Dementia

Adaptive Behavior, Practical Judgment, and Cognitive Functioning in Older Adults

A Factor Analysis Cross-Validation of the Dementia Rating Scale-2

Stability of MCI Patient Centered-Outcomes Following Intervention

Cognitive profiles in MCI and newly diagnosed and unmedicated Parkinson disease

Executive Function, Memory and Partner Report of Everyday Functioning in Healthy Controls, MCI and $\mathrm{AD}$

Differential Predictive Influence on the Development of Dementia: Depression and Neuropsychiatric Symptoms

Differences in Subtle and Overt Everyday Action Error Patterns in Healthy Aging, Mild Cognitive Impairment, and Alzheimer's Disease

Examining a Theoretical Model of Practice Effects and Cognitive Decline: Contributions of Learning and Response to Task Novelty

Comparing the Relationship between the Memory Assessment Clinic Memory Scales, Objective Memory Performance, and Medial Temporal Lobe Volumes in MCI patients

Imaging-Diagnosed Normal Pressure Hydrocephalus in a Patient Initially Presenting with Psychiatric Symptoms

A Novel Method to Improve Face-Name Memory in Older Adults with Age-Normal Memory and aMCI

Impact of cerebrovascular risk on brain amyloid- $\beta$, cerebral blood flow, and neuropsychological functioning in normal aging and mild cognitive impairment

Self-reported History of Head Injury and Age of Diagnosis in Dementia 
27. TIMPANO SPORTIELLO, MR

28. WEAKLEY, AM

29. WEAKLEY, AM

30. SEIDENBERG, M

31. KELLY, DA

32. FLOWERS, AT

33. PIROGOVSKY TURK, E

34. DECK. BL

35. LASSEN-GREENE, CL

36. CHAN, ML

37. EBERT, P

38. GARRETT, R

39. HOLDEN, HM

40. STAFFARONI, AM

41. DE LEON. F

42. DE LEON, F

43. TIERNEY, S

44. SHERMAN, JC

45. RADKE, A

46. PANDYA, S

47. BURKE, MM

48. REYNOLDS, M

49. NGUYEN, L

50. KARANTZOULIS, S

51. NICCOLAI. LM

52. COHEN, ML

53. CERBONE, B

54. KIRTON, JW

55. BONO, AD

56. PERSAD, C

57. SCHWAB, N

58. DEMIAN, M

59. MANDERINO, L

60. THOMPSON, J

61. PATERSON, TS

62. WAGNER, M

63. YEN, K

64. TAN, A

65. HARCIAREK, M

66. HARCIAREK, M

67. IKANGA, JN

68. FLODEN, D
Italian Network Study Group on Parkinson's Disease-Mild Cognitive Impairment (INPM):

Preliminary Results

Automated Classification of Mild Cognitive Impairment and Dementia

Neuropsychological Measures Essential for Cognitive Impairment Classification Using Machine

Learning

Longitudinal Investigation of Recent and Remote Famous Names in MCI and Healthy Participants

Semantic Specificity and Memory Age of Famous Names Reflect Progression of Alzheimer's Disease

Memory Performance in MCI Using Memory and ADL Tasks

Volumetric Correlates of Episodic Memory in Nondemented Parkinson's Disease

Everyday Functioning in Mild Cognitive Impairment: Understanding Impairment Through Analysis of Action and Language Errors

Mild Cognitive Impairment and Everyday Function: Longitudinal Changes in Speed versus

Performance

The Clinical Utility of a Behavior Rating Scale in Distinguishing Alzheimer Disease (AD) and

Behavioral Variant Frontotemporal Dementia (bvFTD)

Proactive and Retroactive Memory Interference in Adults with Amnestic Mild Cognitive Impairment and Typically Aging Adults

Relationship Between Specific Cognitive Domains and Quality of Life in Parkinson's Disease with

Mild Cognitive Impairment (PD-MCI)

Verbal Learning and Memory in Premanifest and Manifest Huntington's Disease: Evidence from the California Verbal Learning Test-II

Longitudinal Assessment of Cognition in Semantic Variant Primary Progressive Aphasia

Prospective Memory and Functional Abilities in Healthy Aging and Mild Cognitive Impairment

Contingency-Based Prospective Memory in Mild Cognitive Impairment and Dementia

Memory for Intentions Screening Test Reveals Similar Prospective Memory Performance in

Huntington's and Parkinson's Disease

Pronoun Problems in MCI: New Research Begins to Reveal the Source of Difficulty

Classification Accuracy of the Dynamic Affect Recognition Test (DART) in Neurodegenerative

Disease

Predictors of Reversion from Mild Cognitive Impairment to Normal Cognition

Detrimental Impact of Traumatic Brain Injury on Visuospatial Processing and Executive Function in

Parkinson's disease

Frontotemporal Dementia: Two Case Studies

Predictors of Health-Related Quality of Life Decline in Parkinson's Disease

Sensitivity of the RBANS in Parkinson's disease with and without cognitive impairment

Neuropsychological Predictors of Declining Financial Capacity in Persons with Mild Cognitive

Impairment Due to Alzheimer's Disease

The Unique and Combined Effects of Apathy and Depression on Cognition in Patients with

Parkinson's Disease

The Relationship between MOCA Scores and Performance on the RBANS and NAB in Parkinson's

Disease with and without Cognitive Impairment

\section{Medical/Neurological Disorders/Other (Adult)}

Moderating effects of age and education on the relationship between body mass and memory function in community adults

Demographic and Clinical Predictors of Facial Expressivity Improvement for Individuals with

Parkinson's Disease (PD) Receiving the Lee Silverman Voice Treatment (LSVT)

Relationship between depression and apathy post Deep Brain Stimulation Surgery in patients with

Parkinson's disease

Preliminary Data: White Matter Burden and Processing Speed Relative to Acute Pre to Post Surgery

Decreases in Default Mode Network

Health Literacy and Cognition in Kidney Transplant Recipients

Traditional Cognitive Deficiencies Predict Impaired Performance on an Emotion Recognition Task in Bariatric Surgery Candidates

Additive Effects of Comorbid Psychiatric Disorders Post-TBI on Aspects of Cognition

Ill-Structured EPS Ability Predicts Medication Adherence Above and Beyond Well-Structured EPS

and Traditional Measures of Intelligence in Renal Transplant Patients

Too quiet: A case of auditory Charles Bonnet Syndrome

Effects of Glucose Levels on Information Processing in College-Aged Adults

Effects of High Blood Pressure on Executive Function in College-Aged Adults

What do the event-related potentials tell us about the anterior attentional system in dialyzed patients with end-stage renal disease?

Anterior attentional/executive system in adequately hemodialyzed patients with end-stage renal

disease. Evidence from the ROBBIA

The Association Between the years of Essential Hypertension and Memory

Mattis Dementia Rating Scale-2 Does Not Predict Quality Of Life After Subthalamic Nucleus Deep

Brain Stimulation 


\author{
69. COOK, SE \\ 70. BRENNAN, L \\ 71. MISKEY, HM \\ 72. ALTMANN, LJ \\ 73. KRAUSKOPF, EE \\ 74. JONES, J \\ 75. RENN, BN \\ 76. DODDS, A \\ 77. CHERRY, BJ \\ 78. MILLER, JS \\ 79. CALVO, D \\ 80. WYMAN-CHICK, K \\ 81. COPELAND, JN \\ 82. KIEWEL, NA \\ 83. ROCHETTE, AD \\ 84. RYAN, AB \\ 85. ALOSCO, M \\ 86. PIPER, L \\ 87. BUTTERFIELD, LC \\ 88. FISCHER, M \\ 89. SORDAHL, J \\ 90. GRANT, M \\ 91. REYNOLDS, GO \\ 92. HOTH, KF \\ 93. ZIEGLER, D
}

\section{5:00-6:00 PM}

1. BIGLER, ED

6:00-6:30 PM

6:30-7:30 PM

:20-8:50 AM

1. NADEAU, SE

7:20-8:50 AM

1. RANKIN, KP
Characterizing the Neurocognitive Profile of Parkinson Disease with Freezing of Gait

Effects of Memantine on Cognition in Parkinson's Disease Dementia and Dementia with Lewy Bodies: A Meta-Analytic Review

Neuropsychological Assessment of a Veteran with a Large Arachnoid Cyst

Effects of an Aerobic Exercise Intervention in Parkinson's Disease

The Impact of Cerebral Microemboli on Cognitive Function Following Bubble-Contrast Transcranial Doppler for Evaluation of Cardiac Shunt

Latent Growth Curve Analysis Reveals Worsening Parkinson's Disease Quality of Life is Driven by

Depression

Screening for Cognitive Impairment in a Diabetic Primary Care Sample

The Relation Between Apathy and Quality of Life in Parkinson's Disease

Cognitive Clusters in Fibromyalgia, A Chronic Pain Condition

A Clinical Profile of Dysautonomia in Parkinson's Disease for Individuals with and without Deep Brain Stimulation

Obesity does not influence effects of fasting on attention and executive function

Effects of bilateral deep brain stimulation on verbal fluency in patients with Parkinson's disease: A meta-analysis

Accuracy of Patient And Care Partner Reports Regarding Specific Cognitive Functions: Implications for Diagnosis of Mild Cognitive Impairment in Parkinson's Disease

Can We Evaluate Visual Memory in Parkinson's Disease?

Gender is Associated With Memory Task Performance in Older Adults With Heart Failure

Influence of Depression on the Different Aspects of Quality of Life in Parkinson's

Preliminary Evidence for the Adverse Impact of Depressive Symptoms on Driving Fitness in Older

Adults with Heart Failure

Neuropsychological Profiles in Adults with Sickle Cell Disease

The Parkinson's Active Living (PAL) Program: A Behavioral Intervention Targeting Apathy in

Parkinson's Disease

Profound Memory Impairment as Rare Complication Following Bariatric Surgery

The Relationship Between Body Mass Index and Processing Speed

Examining the Efficacy of Brief Adherence Interventions for Individuals with Phenylketonuria

Gender Differences in Verbal Learning in Parkinson's Disease

Nocturnal Oxygen Desaturation and Cognitive Performance in COPD

DRD2 Polymorphisms Confer Increased Risk of Behavioral Impulsivity in Parkinson Disease

INS Presidential Address: Networks, Neural Connectivity and

Neuropsychology

INS President: Erin D. Bigler

Centennial Ballroom (D-E)

Networks, Neural Connectivity and Neuropsychology

INS Business Meeting

Centennial Ballroom (D-E)

Friday Evening Reception

Capitol Ballroom (4th Floor)

\section{SATURDAY, FEBRUARY 7, 2015}

\section{CE 11: How Neurons Enable Language and Cognition Presenter: Stephen E. Nadeau Centennial A}

How Neurons Enable Language and Cognition

\author{
CE 12: Neurobiology of Socioemotional Behavior in Health and Neurologic \\ Disease \\ Presenter: Katherine P. Rankin \\ Centennial B-C
}

Neurobiology of Socioemotional Behavior in Health and Neurologic Disease 
9:00-10:30 AM

1. ANDREWS-HANNA, J

2. GRADY, C

3. IRISH, M

4. GABRIELI, $\mathrm{S}$

9:00-10:30 AM

1. PAGULAYAN, K

2. SORG, S

3. JAK. AJ

4. GRANDE, LJ

5. SADEK, JR

6. WISDOM, NM

9:00-10:30 AM

1. TULSKY, DS

2. GERSHON, R

3. TULSKY, DS

4. CARLOZZI, NE

5. CASALETTO, KB

6. HEINEMANN AW

9:00-10:30 AM

1. JOANNETTE, M

2. MANNING, KJ

3. RENTZ, DM

4. HARRISON, CE

9:00-10:30 AM

1. ALOSCO, M

2. CARRATHERS, T
Invited Symposium: Exploring the Function and Dysfunction of the Brain's Default Network Chair: Jessica Andrews-Hanna Centennial Ballroom (D-E)

Exploring the Function and Dysfunction of the Brain' Default Network Age Differences in the Functional Connectivity of the Default Network

The Wandering Mind Standing Still - Exploring the Functional Properties of the Default Network in the Dementias

The Default Mode Network and Psychopathology

\section{Paper Session 7: Veteran Populations}

Moderator: David Tate

Centennial A

The Impact of Repeated Blast-Related mTBI on Brain Activation During a fMRI Working Memory Task

Frontothalamic Structural Connectivity in Veterans with Mild Traumatic Brain Injury: Associations with Executive Functions

Neuropsychological performance in treatment seeking OEF/OIF/OND Veterans with a history of mild TBI

Impaired Verbal Memory Associated with Close Blast Exposure in OEF/OIF Veterans

Changes in Executive Functioning with Successful PTSD Treatment

An Examination of Cognitive Deficits after Controlling for Respondent Validity in Veterans with Chronic PTSD

Symposium 13: Using the NIH Toolbox for Neuropsychological and Behavioral Functioning in Individuals who have Disabilities Chair: David Tulsky

\section{Centennial B-C}

Using the NIH Toolbox for Neuropsychological and Behavioral Functioning in Individuals who have Disabilities

Overview and update on what is new with Toolbox

Using the NIH Toolbox Cognitive Battery (NIHTB-CB) in individuals who have had Traumatic Brain Injury (TBI)

An Examination of the NIH Toolbox Motor, Sensory and Emotion Batteries in Individuals with Disabilities

Uncorrected versus Demographically-Adjusted Scores on the NIH Toolbox Cognition Battery: What is the Difference?

Relationships between NIH Toolbox Cognition and Emotional Measures and Indicators of Participation for Community-Dwelling Persons with Stroke, Spinal Cord Injury and Traumatic Brain Injury

Paper Session 8: Aging

Moderator: Katherine Gifford Centennial F

Neuropsychological Performance of Normal Elderly with Significant $\beta$-amyloid Deposition: a PiBPET Imaging Study

Navigation Driving and Vehicle Control in Healthy Older Adults and Subjective Cognitive Impairment

Cognition and biomarker abnormalities in clinically normal older adults

How Do Exercise and Leisure Differentially Relate to Frontal Functions of Older and Younger Adults?

Poster Session 8: ABI-Child, Autism, Cognitive Neuroscience, \& Electrophysiology/EEG Centennial Ballroom Foyer

\section{Acquired Brain Injury (TBI/Cerebrovascular Injury \& Disease - Adult)}

Reductions in Physical Activity Predict Cognitive Decline in Older Adults with Heart Failure

Acquired Brain Injury (TBI/Cerebrovascular Injury \& Disease - Child)

Impact of Age on Neuropsychological Measures Among Youth with Acute Concussions 


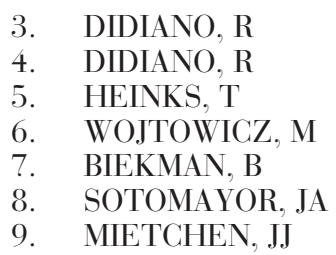

10. WISE, J

11. COMBS, HL

12. BERNSTEIN. J

13. GAGNER, C

14. GAGNER, C

15. MCNALLY, KA

16. PETERSON, RL

17. PEARCE, $\mathrm{K}$

18. FARRER, TJ

19. BLAHA, RZ

20. KEENER, W

21. RANSOM, D

22. RANSOM, D

23. ZAYAT, M

24. VARGAS, G

25. NARAD, M

26. GRETENCORD, AA

27. LALONDE, G

28. SUFRINKO, A

29. ROBERTS, RM

30. AMAYA-HODGES, M

31. AMAYA-HODGES, M

32. HARTLEY, N

33. GIOIA, GA

34. ADACHI. S

35. ACOSTA, MT

36. CASTELLUCCIO, B

37. STEPHENSON, K

38. HEFFELFINGER, A

39. MAISEL, M

40. MACMULLEN FREEMAN, L

41. CARLEW, AR

42. PAGE, MJ

43. BRADBURY, K

44. MOULTON, E

45. LEE, C

46. PATRICK, K
Gender Differences in BASC-2 Self and Parent Rating Scales after Concussion

Gender Differences in Naming Tasks Following Concussion

Sleep is related with post-acute socio-behavioral outcome after pediatric mild traumatic brain injury

Concussion History in High School Athletes with Attention Deficit Hyperactivity Disorder

Resilience and Brain Imaging in Pediatric Traumatic Brain Injury

Variability of Recovery in a Pediatric Concussion Sample

Fractional Anisotropy of the Ventral Striatum Following Moderate to Severe Traumatic Brain Injury in Children

Word Fluency and Reading Comprehension in Young Children with Orthopedic and Traumatic Brain Injury

Irritability and Aggression as Indicators of Persistent Post-Concussive Symptoms in Adolescent Athletes

Benefits of Aerobic Training for Concussion in Adolescents: a Preliminary Report of a Pilot

Randomized Clinical Trial

Are Behavioural Problems Following Mild TBI in Preschoolers Associated with Theory of Mind?

The Relationship between Emerging Behavioral Problems and Parental Distress following Mild

Traumatic Brain Injury in Early Childhood

Effectiveness of a Brief Cognitive Behavioral Intervention for Children and Adolescents with

Prolonged Post-Concussive Symptoms

Pre-Injury Anxiety and Postconcussive Problems in a Pediatric Sample

Near Point Convergence and Concussion: Demographic and Neurocognitive Findings

Chronic MRI Findings, Ventromedial Prefrontal Cortex (vmPFC) Volume, and Behavioral

Functioning in a Pediatric Sample with Complicated Mild Traumatic Brain Injury

PTSD Symptoms After Complicated Mild to Severe TBI in Adolescence: What Role does Memory of Injury Play?

The Relationship Between Post-Concussion Symptoms and Psychological Status in Children and Adolescents With Prolonged Recovery Following Mild Traumatic Brain Injury

Evidence-Based Assessment of Cognitive Exertion and Academic Problems during Concussion

Recovery

Modeling Academic Effects of Concussion with Evidence-Based Assessment of Executive Functions

The Effectiveness of Concussion Education in Youth: A Pilot Study

Correlates of Prolonged Recovery After Pediatric Concussion

Changes in Parent-Teen Interactions and Family Functioning During the Initial 24 Months Post

Traumatic Brain Injury

Gender Differences in Postconcussive Symptoms of Sport-related Concussions in Children and

Adolescents

The Quality of Mother-Child Interactions Six Months Post-TBI in Preschool Children

The Influence of Sleep Duration and Sleep-related Symptoms on Baseline Neurocognitive

Performance among Male and Female High School Athletes

What White Matter Tracts are Most Affected by Pediatric TBI? A Meta-analysis of Diffusion Tensor Imaging (DTI) Research

Factor Structure of BRIEF Parent and Self-Report Forms for Monitoring Recovery in Children with Concussion

Relations between Adolescents' Anxiety and Post-Concussion Symptom Reports

The Mediating Role of Traumatic Brain Injury on the Relationship between Temperament and Psychopathology in Urban Homeless Youth

Evidence-Based Approach to Detecting Concussion in Children: Combining Symptom Reports and Cognitive Performance

\section{Autism Spectrum Disorders}

Language Development in School-aged Children with Pervasive Developmental Disorders: Examining Relationships among Syntactic, Vocabulary, and Speech Abilities

Autism Spectrum Disorders (ASD) in Neurofibromatosis Type 1 (NF1): Clinical and Neurobiological correlations

Characterizing the White Matter Tract Integrity of Youth with a History of Autism Spectrum

Disorder Who Have Achieved Optimal Outcome

Alexithymia as a Predictor of Dimensional Scales of Autism Symptoms

Factors Influencing Age of Evaluation for Autism

Dispositional Mindfulness Predicts Anxiety in People Diagnosed with Autism Spectrum Disorder

The Association between Executive Functions and Social Skills in Children with Autism Spectrum Disorder

Title: The Virtual Reality Classroom for the Assessment of Autism Spectrum Disorders

Executive Functioning Profiles in a Clinical Sample of Youth With and Without Autism

A Comparison of High- and Low-Risk Children with Autism Spectrum Disorder

Developmental Trajectories in Toddlers with ASD

Visuomotor adaptation in children with Autism Spectrum Disorders

Driving Behaviors of Young Adults with Autism Spectrum Disorders 

47. WAGNER, AE
48. RICHARD, AE
49. SEPPÄ, ER
50. DUFFIELD, T
51. HAISLEY, LD
52. ABRAMS, DN
53. KENWORTHY, L

\author{
54. BROOKS HOLLIDAY, S \\ 55. SABBAH, LE \\ 56. SMITH, AL \\ 57. KENNEDY, Q \\ 58. TOPPING, KM \\ 59. CONSIDINE, CM \\ 60. CONSIDINE, CM \\ 61. JENKINS, LM \\ 62. SAAVEDRA, F \\ 63. MOON, C \\ 64. SHANE, B \\ 65. CARBINE, K \\ 66. ZIEGLER, D \\ 67. KAEMMERER, T \\ 68. KARPOUZIAN, T \\ 69. PISNER, D \\ 70. LETZEN, JE \\ 71. GRILLI, MD \\ 72. MARKOWSKI, S \\ 73. ROSEN, AC \\ 74. LAMAR, M \\ 75. REINEBERG, A \\ 76. SALAZAR, R \\ 77. TARHAN, LY \\ 78. BURKAUSKAS, J \\ 79. STEFANATOS, G \\ 80. KAIS, LA \\ 81. MANGAL, P \\ 82. NIERMEYER, M \\ 83. CLAYSON PE \\ 84. CLAWSON, A \\ 85. MITCHELL, MB
}

10:30-10:45 AM

\section{0:45 АМ-12:15 РМ}

1. VAN ZANDVOORT, MJ
Neurocognitive Subtypes of ASD

Visual Attention Shifting in Autism Spectrum Disorders

The Association between Autism Spectrum Traits and Visual Processing in Young Adults with Very Low Birth Weight: The Helsinki Study of Very Low Birth Weight Adults

Trails Performance in ASD

Executive Functioning Deficits Associated with Parenting Stress: Parents of Children with ASD Early Child Characteristics Predict Transition from ASD to Non-ASD

The Executive Function Challenge Task (EFCT): A Lab-based Observational Measure of Flexibility and Planning in Typically Developing Children and Those with Autism Spectrum Disorder

\section{Cognitive Neuroscience}

Specialized Training in Neuropsychology: A Review of Doctoral-Level Programs

Distinction Between Dopamine and Norepinephrine Related Tasks

Cognitive Dysfunction among Adults with Hyperglycemia

The STEP Model: Characterizing Simultaneous Time Effects on Practice for Flight Simulator

Performance Among Middle-Aged and Older Pilots

Gender Role Beliefs Mediate Priming Effects of Gender-Congruent Verbal Fluency Task

The Influence of Sleep Quality and Health Behaviours on Mood and Attention

Objective and Subjective Sleep Quality Determinants of Cognitive and Negative Affective Processing Performance in Good vs. Bad Sleepers

Effects of Music on Pleasure and Cognition During Functional Brain Imaging

Verbal Cognition as a Predictor of Adaptive Behavior in Children with Cochlear Implants

Sleep Apnea and Grey Matter Volume in Individuals with Heart Failure: A Voxel-Based

Morphometric Analysis

The Contribution of General Intelligence and Emotional Intelligence to the Ability to Appreciate Humor

A Comparison of Neuropsychological Functioning in Major Depressive Disorder, Generalized Anxiety Disorder, Obsessive Compulsive Disorder, and Healthy Controls

Dynamic Interplay Between Distractions and Internally- and Externally-directed Attention

Age-Related Differences in Implicit Motor Sequence Learning: Exploring the Influence of Embedded Associative Structure

Neural Correlates of Working Memory Capacity

Visuospatial reasoning mediates the relationship between emotion recognition and emotional intelligence

Temporal Differences in the Relationship Between Dorsal and Ventral Attention Networks Based on Pain Intensity

The Contribution of Memory to the Self-Concept in Amnesia

Sleep Onset Latency and Duration are Associated with Self-Perceived Invincibility

Effect Of Phonemic Cuing On Recall Of Personally Relevant Names Derived From Email

Cognitive and Neural Efficiency: What Planning and Organization Behaviors Reveal About Brain Connectivity and the Human Connectome

Meta-analytic Function of Brain Regions Predicts Resting-state Connectivity

Effects of Cognitive Deficits on Gait in Parkinson's Disease

Action Understanding and Production: Common and Distinct Neural Substrates

Cardioselective Beta-blockers Effect Cognitive Functioning in Coronary Artery Disease Patients

\section{Electrophysiology/EEG/ERP}

Disassociation of steady-state and transient evoked response findings in Word Deafness

Anxiety Influences Neural Correlates of Inhibitory Control

Emotional Memory, the Late Positive Potential, and Parkinson's Disease

Effects of Task Novelty on Cognitive Contributions to Planning Times and EEG Activity During

Complex Motor Sequencing

Effects of Dopamine Precursor Depletion on Feedback-Related Performance Monitoring

Reversal Learning in Autism: Neural and Behavioral Performance Monitoring

ERP Age Effects during Face-Name Recognition: Relation to Neuropsychological Test Performance

\section{Coffee Break}

Centennial Ballroom Foyer

\section{Paper Session 9: Cognitive Neuroscience \\ Moderator: Scott Langenecker Centennial A}

Stimulation of Supplementary Eye Fields induces temporary neglect during awake neurosurgery 

2. IARIA, G
3. RYMAN. SG
4. TRACY, JI
5. JAKOBSEN, E
6. YEO, RA

10:45 AM-12:15 PM

1. HAALAND, KY

2. VERFAELLIE, M

3. HAALAND, KY

4. O'CONNOR, MG

5. DELUCA. J

6. BAUER, RM

7. KOPELMAN, M

10:45 АM-12:15 РМ

1. TAYLOR, JA

2. CAVANAUGH, J

3. HUNTBACH, BA

4. OHLHAUSER, L

5. THOMPSON, J

6. THOMPSON. J

7. TOMASZCZYK, JC

8. WARD, A

9. GOVEROVER, Y

10. TWAITE, JT

11. RUBINFELD, L

12. SCOTT, BM

13. STABLER, AR

14. PENNINGTON, CR

15. MUSIL, S

16. LEGARRETA, M

17. CAIRNCROSS, M

18. DUNGANSON, H

19. PIERCY, JC

20. DUNN, CB

21. LOCKWOOD, CA

22. GILL. SK

23. BARRASH, J

24. DEYOUNG, N

25. VINCENT, A

26. FERNANDEZ, AL

27. FISCHER, BL
Developmental Topographical Disorientation is associated with Decreased Functional Connectivity between the Hippocampus and Prefrontal Cortex

Structural Network Hub Connectivity Predicts Intelligence

Pre-surgery resting-state graph-theory measures predict neurocognitive outcomes after brain surgery in temporal lobe epilepsy

Individual In Vivo Sub-parcellation of Broca's Region Using Functional Connectivity Glyphs

Rich Club connectivity and executive function in healthy controls and individuals with schizophrenia

\section{Symposium 14: The Many Faces of Memory Disorders: Video Case Examples and Neuroanatomical Correlations Organizers: Kathleen Y. Haaland, Russell M. Bauer \\ Discussant: Michael Kopelman Centennial B-C}

The Many Faces of Memory Disorders: Video Case Examples and Neuroanatomical Correlations The Many Faces of Memory Disorders in the Context of Contemporary Memory Theory Medial Temporal Lobe Svndrome: Amnesia in an Anoxic Patient after Cardiac Arrest Wernicke Korsakoff's Syndrome: Contributions to Models of Memory

Profound Amnesia and Confabulation following Anterior Communication Artery Aneurysm When Working Memory Isn't Working: Profound Retrieval Deficits in a Case of Early-Onset Pick's Disease

Discussion

\section{Poster Session 9: Assessment-Adult, Cognitive Intervention/Rehab, \& Visuospatial/Neglect Centennial Ballroom Foyer}

\section{Assessment/Psychometrics/Methods (Adult)}

A Psychometric Examination of Pain Scales Commonly Used in the Management of Chronic Pain Examining the Differential Effects of Natural and Synthetic Aromas of Lavender and Peppermint on Cognition, Mood, and Subjective Workload

Predicting Performance on Sequencing and Backward Span Tasks Using a Verbal and Non-Verbal n-Back Task

Comparability of the Full and Short Forms of the Personality Assessment Inventory in a Stroke Population

Grooved Pegboard and RBANS Performance: Links between Motor and Cognitive Functioning Relationship of Finger Tapping to General Neurocognitive Functioning

Development of a Scale to Measure Patient Acceptability of Cognitive Therapy

A Critical Evaluation of the Validity and Clinical Implications of Episodic Future Thinking Actual Reality: Using the Internet to Assess Everyday Functioning after Traumatic Brain Injury Intellectual and Cognitive Performance of Musicians and Healthy Controls: Differences on the Montreal Cognitive Assessment (MoCA) and Other Measures

Validity and Reliability of the Dalhousie Computerized Attention Battery in Healthy Older Adults Comparison of Psychogenic Movement Disorder Patients with Non-epileptic Seizures vs Other Hyperkinetic Motor Manifestations: An Integrated Model of Psychosocial and Neuropsychological Functioning

Cross-Cultural Sensitivity of the Mini-Mental State Exam (MMSE) as a Screener for Memory Impairment

Personality Traits Influence Processing Speed Performance in a Neurologically Intact Population MMPI-2 Profiles in an Academic Medical Center: The Defended and the Defenseless

Neuropsychological Performance in Veterans with Chronic Pain

Neuropsychological Assessment of Decision-Making Capacity in Patients With Barriers to

Communication

Error Analysis of the TMT: Relevance to Driving Safety

Comparison of the Buschke Selective Reminding Test and the California Verbal Learning Test Second Edition in a Stroke Population

Sensitivity and Specificity of the Benson Figure as a Cognitive Screen in Older Adults Effect of Pain, Emotional Distress, and Cognition on Social Role Outcome Measures

Visual Naming, Age, Educational Level and WAIS-III Factorial Components Mediate Level and Pattern of Performance on the Judgment of Line Orientation Test

Iowa Scales of Personality Change: Normative Data From Healthy Older Adults

The reliability and accuracy of administering The Montreal Cognitive Assessment over telehealth Test-Retest Reliability, Practice Effects, and Base Rates of Change for the ANAM-GNS Battery Construct and Concurrent Validity of the Spanish Adaptation of the Boston Naming Test Tele-Neuropsychology at the Madison VA Hospital: An Extension of GRECC Connect 
28. YOCHIM, B

29. GELDMACHER, DS

30. TYRRELL, C

31. LEVY, S

32. AHN, SS

33. BREWSTER, P

34. HARDY, DJ

35. CONSIDINE, CM

36. GAVETT, BE

37. PAULS, CD

38. DINH, KL

39. HORTON, DK

40. MILLER, B

41. CALAMIA, M

42. LEITNER. D

43. SILVERMAN, J

44. MIETCHEN, JJ

45. TYLER, $\mathrm{H}$

46. HAJ-HASSAN, S

47. DAVIS, HP

48. BAILIE, JM

49. GURNANI, A

50. GURNANI, A

51. BENSON. LM

52. CARLOZZI, NE

53. DONDERS, J

54. HILL-JARRETT, T

55. K THIRUSELVAM. I

56. K THIRUSELVAM, I

57. LANGE, R

58. DAY, EF

59. PIERS, RJ

60. OMALLEY, K

61. LUU, H

62. MALIHIALZACKERINI. S

63. TAYLOR, L

64. MENDOZA, HJ

65. NEUGNOT-CERIOLI, M

66. KINNE, E

67. CHOWDHRY, S

68. KERNS, KA

69. MORGAN, KN

70. THOMAS, KR
A Verbal Naming Test for Use with Older Adults: Development and Initial Validation

Level of Impairment in Instrumental Activities of Daily Living Predicts Alabama Brief Cognitive Screener Scores in a Memory Disorders Clinic

Demographic Variables Relating to Scores on the Trail Making Test in Middle-Aged and Older Adults Assessing Executive Function in Parkinson's disease: A Comparison between Sample-Matched Norms and Population-Based Norms

The Relationship between Social Desirability and Subjective Self-Reported Cognitive Functioning in patients with Bipolar Disorder and Healthy Volunteers

Telephone Administration of the Mental Alternation Test: Sensitivity to Cognitive Status in

Community-Dwelling Older Adults

The Concept of Workload: An Illustration with the Tower of Hanoi Test

Predicting Differences in Mood, Cognitive Performance, and Cognitive-Affective Processes among

"Good" versus "Bad" Sleepers Based on Subjective and Objective Reports of Sleep Quality

Preliminary Development of an Adaptive Digit Span Test: Effect of Item Presentation Order

The Columbia Quality of Life Inventory (CQOL): A measure to assess meaningful outcomes in ECT trials

Comparing RBANS Performance Using Randolph and Oklahoma Norms in a Geriatric Inpatient Sample

Diagnostic Accuracy of Standard and Abbreviated Forms of the MoCA

Added Value of Neuropsychological Assessment to the Clinical Dementia Rating Scale in Assessing Mild Cognitive Impairment

Factor Structure and Clinical Correlates of the Wender Utah Rating Scale (WURS)

Examining the Convergent Validity of Neuropsychological Evaluation with Functional Outcome in a Stroke Population

Evaluation of the Modified Telephone Interview for Cognitive Status as a Screen for Mild Cognitive Impairment

Convergent, Discriminant, and Predictive Validity of the Neuropsychological Assessment Battery Shape Learning Subtest

Comparability of In-Person versus Remote Proctoring for Neuropsychological Test Administration Empirically-Derived Subtypes of Mild Cognitive Impairment: The Vanderbilt Memory \& Aging Project

Performance and Convergent Validity across the Life Span for a Computerized and a Manual Trails Making Test

Exploratory and Higher-Order Factor Analysis of the Automated Neuropsychological Assessment Metrics V4.0 (ANAM4)

Base rates for annual neuropsvchological test score changes in the Uniform Data Set

Reliable change on neuropsychological tests in the Uniform Data Set

Does the Boston Naming Test - 2 Function Equivalently for African American and Caucasian Adults? Psychometric support for the validity of PROMIS and Neuro-QOL in Huntington disease

Discrepancies between Self and Informant Reports on the BRIEF-A after mTBI

Measurement Invariance of the Brief Test of Adult Cognition by Telephone (BTACT) in Caucasian and African American Adults

Application of Item Response Theory (IRT) to Trial 1 of the California Verbal Learning Test Second Edition

Application of Item Response Theory (IRT) to the Standardized Assessment of Concussion Clinical Utility and Psychometric Properties of the Traumatic Brain Injury Quality of Life (TBI-QOL) Scale in U.S. Military Service Members

The Five Point Test: Age and Education on Test Taking Strategy

Clock Drawing in a Healthy Community Sample: A Principal Component Analysis

Impact of Demographic Variables on the Brief Visuopatial Memory Test-Revised

\section{Cognitive Intervention/Rehabilitation}

The influence of demographic variables on executive functioning after cognitive rehabilitation Meta Cognitive Training (MCT) to improve neuropsychological functioning in patients with surgically treated frontal lobe tumor and in patients affected by schizophrenia

The Effects of Vitamin D Supplementation on Cognition in a Rescreened Sample

Attention Concerns of Cancer Survivors Participating in a Cognitive Training Intervention

A game-based approach to cognitive remediation in children with borderline intelligence

Rehabilitation Needs of Female Veterans with Post-Traumatic Stress Disorder and Mild Traumatic

Brain Injury

Adults with Severe Traumatic Brain Injury Show Greater Cognitive Improvements than Adults with

Mild/Moderate Traumatic Brain Injury on Computerized Cognitive Training: A Pilot Study

Efficacy of the "Caribbean Quest" Computer Intervention in Children with ASD and FASD

Can Lumosity Assessment Tools Determine Functional Change? An Independent Investigation of

Reliability

Compensatory Cognitive Training for People with Severe Mental Illness 
71. THOMAS, KR

72. TOMASZCZYK. JC

73. SÉGUIN, M

74. KANG, J

75. MECHANIC-HAMILTON, D

76. ERCOLI, LM

77. JONES. J

78. LAFO. JA

79. SOL, K

80. NOVAKOVIC-AGOPIAN. T

81. FAIR, JE

82. SANDRY, J

83. DEYOUNG, N

84. CURCIO. N

85. BOGDANOVA, Y

86. JURICK. SM

87. ZAHODNE, LB

88. TWAMLEY, EW

89. HARRISON, CE

90. RODRIGUEZ. JA

91. MOSQUERA, DM

92. DIAZ-SANTOS, M

93. BAUGHMAN, BC

\section{2:30-2:00 PM}

1. DUVALL. S

2. VAN STEENBURGH, JJ

3. LAJINESS-O'NEILL, R

4. LUONG-TRAN, C

5. MURDAUGH, D

6. SOUTH, M

12:30-2:00 PM

1. EWING-COBBS, I
Effects of Wii. Aerobic Exercise and Cognitive Interventions on Older Adults' Self-Evaluation of Intellectual Aging

Feasibility and Preliminary Efficacy of an Online Cognitive Environmental Enrichment Intervention for Patients with Moderate-to-Severe Traumatic Brain Injury

Ready! Set? let's Train! : Efficacy of an intensive attention training program and its association with executive functioning after childhood traumatic brain injury

Memory Skills for Older Adults with PTSD: Update on a Clinical Demonstration Project for Veterans The Cognitive Fitness Program: A Novel and Comprehensive Approach to Successful Cognitive Aging Association of baseline cognition and change in verbal memory among breast cancer survivors receiving a cognitive rehabilitation intervention

Influence of Everyday Walking on Cognition: Multilevel Modeling Analyses Results from the Village Interactive Training and Learning (VITAL) study

Effects of Combined Cognitive Training and Physical Exercise on Spatial Navigation and Learning in Older Adults

Somatization Predicts Self-Efficacy in mTBI, and Both Predict Post-Concussion Symptom Reporting Goal-Oriented Attentional Self-Regulation Training in Veterans with Chronic TBI - Long Term Outcomes

Errorless and Errorful Learning in Moderate-to-Severe TBI: The Impact of Neuropsychological Functioning and Mood on Performance

What underlies the link between cognitive reserve and long-term memory impairment? Exploratory insight from two memory impaired populations

Should it be done? Establishing the equivalence of a telehealth versus in-person memory workshop Cognitive Behavioral Therapy to Enhance Cognitive Rehabilitation Efficacy in Alzheimer's Disease

TMS Improves Cognitive and Neuropsychiatric Symptoms in Veterans with Blast TBI

Mental Health Treatment Reduces Post-concussive Symptoms and Symptom Overreporting in Iraq and Afghanistan Veterans

External Control Beliefs Explain Racial Disparities in Reasoning Training Gains in ACTIVE

Effects of Compensatory Cognitive Training for Veterans with Traumatic Brain Injuries: Do Comorbid Posttraumatic Stress Disorder and Depressive Symptoms Limit Improvement?

\section{Cognitive Neuroscience}

Effects of Leisure on Cognition Among Older Adults

\section{Visuopatial Functions/Neglect/Agnosia}

Background Distraction During Vertical Character Line Bisection

The Center of Mass and the Edge of Attention

The Effect of Visual Cues on the Resolution of Perceptual Ambiguity in Parkinson's Disease and Normal Aging

I'm Pretty Sure I had a Stroke: A Case of Focal Posterior Hemispheric Dysfunction Overlooked as Non-Organic Pathology

\section{Paper Session 10: Autism}

Moderator: Melissa Armstrong Centennial A

Examining Gender Differences in Cognition and Phenotype in Young Children with Autism Spectrum Disorder

Transcranial Direct Current Stimulation Enhances Working Memory and Selective Attention in Highfunctioning Autism

Aberrant Neural Synchrony in Autism Spectrum Disorders Revealed with Magnetoencephalography (MEG) During Resting State: Relationship with Core Behaviors

It Takes Two to Tango: Executive Control in Students with Autism Spectrum Disorder Is Affected by Teacher Behavior

The Impact of Reading Intervention on Brain Structure and Function Underlying Language in Children with Autism Spectrum Disorders

A Step Behind: Atypical Amygdala Activation during Fear Conditioning and Extinction in Autism Spectrum Disorders

Symposium 15: Disruption of Neural Connectivity After Traumatic Brain Injury in Children: Contribution of Neuroimaging to Understanding LongTerm Cognitive and Behavioral Outcomes Chair: Linda Ewing-Cobbs Centennial B-C

Disruption of Neural Connectivity After Traumatic Brain Injury in Children: Contribution of Neuroimaging to Understanding Long-Term Cognitive and Behavioral Outcomes 


\author{
2. RYAN, N \\ 3. JOHNSON, CP \\ 4. WILDE, EA \\ 5. EWING-COBBS, L
}

12:30-2:00 PM

\author{
1. SMITH. J \\ 2. SMITH, J \\ 3. ERICKSON, KI \\ 4. LIU-AMBROSE, T
}

12:30-2:00 PM

1. FERNANDEZ, AL

2. COLE, PG

3. THOMPSON, J

4. PETRANOVICH, CK

5. JASINSKI, L

6. PASSAROTTI, AM

7. NOONAN, N

8. BITTON, A

9. SIMONE, AN

10. SHIN, M

11. VASSERMAN, M

12. KIM, M

13. KINGERY, KM

14. ISQUITH, PK

15. SITARENIOS, G
16. MCGURDY, MD
17. WITHROW, S
18. KRYZA-LACOMBE, M
19. NA, S
20. NA, S
21. VAN DYK, K
22. APPLE, A
23. GIOIA, AR

The Emergence of Age-Dependent Social Cognitive Deficits after Generalized Insult to the Developing Brain: a Longitudinal Prospective Study using Susceptibility-Weighted Imaging Reading Skills After Traumatic Brain Injury in Children: Relation with White Matter Pathways

Neuroimaging and Long-term Recovery after Early TBI in Children: What can Tractography Reveal about Post-TBI Development?

Pathway Integrity and Neuropsychological Outcomes During the First Two Years after Pediatric TBI: Recovery or Persistent Deficit?

\section{Symposium 16: Exercise as Brain Medicine: State of the Science Chair: J. Carson Smith Discussant: Stephen Rao Centennial G-H}

Exercise as Brain Medicine: State of the Science

Exercise and Physical Activity Along the Alzheimer's Risk Continuum

Moderators of Exercise-Related Brain Plasticity

Neuroimaging Evidence for "Central Benefit Model" of Exercise in Falls Prevention

\section{Poster Session 10: ADHD/Attention, Cancer, Language/Aphasia, Learning Disabilities/Academic, \& Memory Centennial Ballroom Foyer}

\section{Assessment/Psychometrics/Methods (Child)}

Validity of a Test for the Early Detection of Dyslexia in Spanish-speaking children: Test de Velocidad de Denominación

\section{ADHD/Attentional Functions}

WISC-IV IQ and Index Score Variability According to Severity of ADHD Symptomatology ADHD and Incidental Learning: Demonstrated weaknesses on the Rey Complex Figure Test The Influence of Neuropsychological Aspects of Attention on Parent-reported Behavior and School Competence in Internationally Adopted Girls with a History of Institutionalization

The Clinical Utility of the Repeatable Battery for the Assessment of Neuropsychological Status (RBANS) in the Assessment of Adult ADHD

Do Children with ADHD and Bipolar Disorder Differ in Terms of Executive Dysfunction? The Contribution of Sluggish Cognitive Tempo to Executive and Adaptive Functioning Executive Functioning and the Role of Basic Cognitive Processes in 8-year-olds With and Without ADHD

Preschool Inattentive but not Hyperactive-Impulsive Symptoms Predict Working Memory at 8-yearsold

Effect of Mobile-based Neurofeedback with Cognitive Training Program for Children

Comparing Three Methods of Stroop Interference Calculations in Clinically Referred Children Deficits of decision-making in college students with ADHD traits

The Role of Verbal and Spatial Working Memory in Predicting Academic Performance in Children with ADHD

Diagnostic Accuracy of a Revised Behavior Rating Inventory of Executive Function (BRIEF) for Children with ADHD

A Revised Continuous Performance Test (CPT) for the Assessment of Attention Processes in 4-7 year-old Children

\section{Cancer}

Treatment Intensity Predicts Self-Awareness of Executive Functioning in Young-Adult Survivors of Childhood Brain Tumor

The Association of Cognitive Changes with Quality of Life in Men with Prostate Cancer Undergoing Androgen Deprivation Therapy

Cognitive Functions in Patients with Ovarian Cancer treated with First-Line Chemotherapy

Dorsolateral Prefrontal Cortex Activation in Adult Survivors of Pediatric Brain Tumors Relative to Controls on a fMRI Vigilance Task Over Time

Cumulative Neurological Factors Predict Long-term Outcomes in Adult Survivors of Childhood Brain Tumors

Association of Self-Reported Cognitive Complaints and Neuropsychological Performance Among Younger and Older Breast Cancer Survivors

Hippocampal shape deformity associated with cognitive variability in breast cancer patients

Relationships between Parent-, Teacher-, and Self-Reported Behavior Ratings and

Neuropsychological Outcomes in Childhood Cancer Survivors 


\section{SCHAGEN, S \\ 25. LUCCHETTI. A \\ 26. GRIECO, J \\ 27. GRIECO, J \\ 28. LEWIS, W \\ 29. HEFLIN, L \\ 30. MARIANI, M \\ 31. KIMBERG, C \\ 32. ASHFORD, JM \\ 33. NOLL, KR \\ 34. YAO, C \\ 35. RAGHUBAR, KP \\ 36. SCHREIBER, JE \\ 37. CHERRIER, M \\ 38. BRINKMAN, TM \\ 39. SADIGHI. ZS \\ 40. SHERMAN, JC \\ 41. BANERJEE, $P$ \\ 42. AMIDI. A}

\author{
43. MCCULLAGH, J \\ 44. STRASSER, A \\ 45. STOOP, MC \\ 46. REHMEL, JL \\ 47. RAGHUBAR, KP \\ 48. GARCIA, A \\ 49. BALDO, J \\ 50. AILION, A \\ 51. DRONKERS, NF \\ 52. PILLAY, SB
}

\author{
53. YOUN, T \\ 54. HARRELL, M \\ 55. CROCKER, $\mathrm{N}$ \\ 56. MIYAKE, Y \\ 57. HALE, JB \\ 58. FRITZ, C
}

59. MACALLISTER, WS

60. PLOURDE, V

61. HUSTON-WARREN, EA
Late Effects of Cancer Treatment on Cognitive Function and Brain White and Gray Matter in Testicular Cancer Survivors

Comparisons of Parent and Child Reports of Pediatric Pain, Coping, and Psychosocial Functioning in Newly Diagnosed Pediatric Cancer Patients

A Matched Comparison Study Assessing Longitudinal Outcome of Children with Posterior Fossa Syndrome versus those Without Complication Following Posterior Fossa Tumor Resection and Proton Radiation Therapy

Intellectual and Executive Functioning in Pediatric Brain and CNS Tumor Patients After Proton

Radiation Therapy

Association of Caloric Intake with Executive Function and Attention in Long-Term Survivors of Childhood Cancer

Longitudinal Neuropsychological Functioning in Cancer Patients Using a Pre-Cancer Baseline

Neuropsychological Assessment

Executive functioning and motor deficits in WHO grade 4 primary brain tumours

End-of-Therapy Attention Predicts Long-Term Executive Function Outcomes in Survivors of Childhood Acute Lymphoblastic Leukemia (ALL)

Identifying Risk Factors for Adaptive Functioning Deficits among Children Diagnosed with Craniopharyngioma

Neurocognitive Functioning, Gender, and Inflammatory Markers in Patients with Colorectal Cancer Prior to Chemotherapy

Pretreatment Reaction Time Intraindividual Variability in Women Diagnosed with Breast Cancer The Relationships among White Matter Integrity and Attention in Pediatric Brain Tumor Posterior Fossa Syndrome and Long-term Neurocognitive Problems among Children Treated for Medulloblastoma on a Multi-institutional, Prospective Study

Neural and Behavioral Response to Cognitive Training in Cancer Survivors with Cognitive Symptoms Impaired executive function and reduced social integration among adult survivors of pediatric central nervous system (CNS) tumors

Effect of Seizure Morbidity on Neurocognitive Outcome, Quality of Life, and Social Attainment in Adult Survivors of Childhood Central Nervous System (CNS) and Non-CNS Cancers

Preserved Cognitive Function following Proton Radiation in Adults with Low Grade Glioma

Voxel-Based Lesion-Symptom Mapping of Expressive and Receptive Language in Brain Tumor Patients

Brain Connectivity and Neuropsychological Functioning in Testicular Cancer Patients - exploring the effect of chemotherapy

\section{Language and Speech Functions/Aphasia}

Dichotic Listening Training for Auditory Processing Deficits in Aphasia

Paired-Associate Learning in Deaf Readers

Susceptibility to Phonological Interference in a Spoonerism Elicitation Task

Comprehension of Common \& Uncommon Proverbs in Individuals with Agenesis of the Corpus Callosum

Neurocognitive Predictors of Academic Functioning in Children with Cochlear Implants

Neural Correlates of Semantic Processing Across Input Modalities

A Voxel-based Lesion Analysis of Reading in a Large Cohort of Left Hemisphere Stroke Patients

Long-Term Survivors of Cerebellar Tumors: Impact of Processing Speed and Working Memory on Phonemic and Semantic Fluency Performance

Grey and White Matter Involvement in Classic Aphasia Syndromes

Neural Correlates of Impaired Definition Naming Relative to Picture Naming Using Voxel-Based Lesion-Symptom Mapping

\section{Learning Disabilities/Academic Skills}

A Comparison of Quantitative EEG Between Cooperative Learning And Lecture-Based Learning In Adult

Children with Identified Reading Weakness: Significant Deficits in Phonological Processing?

Evaluation of Symbolic and Nonsymbolic Magnitude Judgment and Its Relationship with

Mathematics Achievement in Children with Heavy Prenatal Alcohol Exposure

Systematic review of $\mathrm{N} 400$ waveform processing of semantic words in specific language impairment Neuropsychological and Behavioral Profiles of Written Expression SLD Subtypes: Implications for Differentiated Instruction

Differential Relation between Reading Comprehension and Neurocognitive Constructs for Dyslexic and Typical Readers at Different Ages

A comparison of Woodcock Johnson III and WIAT III scores in clinically referred children and adolescents

Neuropsychological Mediators of the Association between Inattention Symptoms and Reading Abilities in Childhood

Path Models for Middle School Mathematical Outcomes 
62. APPLEMAN, ER

63. VINGERHOETS, G

64. ZANINOTTO, AC

65. OMALLEY, K

66. PRINCE, CE

67. ANDERSON, DM

68. GAVETT, BE

69. FLORES, A

70. MAULE, AL

71. DUNN, CB

72. PANOS, AH

73. HAMILTON, J

74. MCGAULEY, S

75. RHODES, E

76. LEIBEL, DK

77. NICHOLAS, CR

78. FAMA, R

79. ENGLAND, HB

80. MCFARLAND. CP

81. ROSSETTI, M

82. QUTROZ, YT

83. SHEPPARD, DP

84. BAKER, CA

\section{Memory Functions}

Effects of Sleep Quality, Assessed by Actigraphy, on Motor Learning in Healthy Young Adults The Link Between S100ß and Audioverbal Memory Perfomance in Patients Undergoing Carotid Revascularization

Improvement on Visuospatial Memory Test After Diffuse Axonal Injury: One-Year Follow Up Study Effects of Age and Sex on Verbal Memory

Neuropsychological Performance and Response to Prompting for Delayed Visuospatial Memory Age-Related Differences in Free Recall and Mnemonic Strategy Use

The Effects of Age on the Learning and Forgetting of Primacy, Middle, and Recency Components of a Multi-Trial Word List

The Effects of Negative Mood and Rumination on Specificity of Autobiographical Memory

A Meta-analysis of Self-reported Neurological and Neuropsychological Symptoms in Gulf War Veterans

Spatial Learning and Memory: Visually Scanning the Environment Predicts Performance on a Virtual Water Maze

Memory in Left and Right Hemispherectomy

Isolated Memory Impairment following Bilateral Hippocampal Damage Secondary to Human Herpes Virus 6 Encephalitis (HHV6) in the Context of Bone Marrow Transplant for Pediatric Leukemia Prospective Memory in OEF/OIF Veterans with PTSD with or without Mild Traumatic Brain Injury using Virtual Week

Serial Order Position Effects in Alzheimer's and Vascular Dementia: A Clinical Application of Competitive Queuing

Higher Levels of Perceived Stress Are Associated with Better Verbal Memory Performance in Older Men

Longitudinal assessment of self-reported memory function in cognitively normal, middle-aged adults: Findings from the WRAP study

Temporo-parietal Contribution to Visual Episodic Memory Performance in HIV Infection Modulating Categorical and Coordinate Spatial Processing Using Transcranial Direct Current Stimulation

Prospective Memory among Veterans: The Role of PTSD in Forgetting Intentions

The Relationship among Adiponectin Isoforms, Memory Performance, and Gender

Adaptation and validation of the Spanish version of the Face Name Associative Memory Exam (FNAME) in cognitively normal older individuals

A Comparison of Prospective Memory Capacity in Huntington's Disease and HIV Infection

Musical Working Memory in Musicians and Non-musicians within Baddeley's Multicomponent Working Memory Model 


\title{
Abstracts Presented at the Forty Third Annual Meeting International Neuropsychological Society
}

\author{
February 4-7, 2015 \\ Denver, Colorado, USA
}

WEDNESDAY MORNING, FEBRUARY 4, 2015

CE 1:

Persistent Neuropsychiatric Symptoms After Concussion: Evaluation, Effort, and Ethics

Presenter: Jonathan M. Silver

$$
\text { 9:00 a.m.-12:00 p.m. }
$$

\footnotetext{
J.M. SILVER. Persistent Neuropsychiatric Symptoms After Concussion: Evaluation, Effort, and Ethics.

Approximately 1.5 million Americans experience traumatic brain injury each year, the vast majority of which are mild. In the moments following a TBI, postconcussive symptoms are nearly universal. These include alterations of consciousness, disturbances of attention, slow processing speed, impaired declarative memory, and executive dysfunction, and frequently are accompanied by emotional and behavioral disturbances as well as sensory and motor problems. Over the days to weeks after mild TBI, recovery usually proceeds rapidly and typically is complete. When early symptoms are unrecognized, misunderstood, and/or inadequately addressed, early postconcussive symptoms may become chronic and engender secondary psychological health and psychosocial consequences. Pre-injury health and psychosocial factors also influence the short- and long-term effects of TBI. Understanding and improving outcomes after TBI therefore requires consideration not only of the effects of external physical forces on the brain but also the person sustaining that injury and the events preceding and following it. Ethical issues exist regarding the professionals participation in the legal and independent evaluations, as well as decisions as to return to sports and work. This session will provide participants with new and emerging perspectives on mild TBI. A heuristic with which to understand the influences of pre-injury, injury-related, and post-injury factors on postconcussive symptoms will be presented.

Correspondence: Jonathan M. Silver, M.D., NYU School of Medicine, 40 East 83rd Street Ste 1E, New York, NY 10028. E-mail: Jonathan. Silver@nyumc.org.
}

CE 2:

Genes, Brain, and Behavior in Neurodevelopmental Disorders: Science and Practice

Presenter: Bruce F. Pennington

9:00 a.m.-12:00 p.m.

B.F. PENNINGTON. Genes, Brain, and Behavior in Neurodevelopmental Disorders: Science and Practice.

Only a few decades ago, disorders like dyslexia, ADHD, and autism were poorly understood and the target for considerable unscientific speculation and questionable treatment approaches. Now we have an emerging neuroscience of atypical development, but paradoxically, controversial therapies for these disorders are as prevalent as ever, and many children with these disorders are not receiving the help they deserve. Closing this gap between science and practice is an important goal for scientists, clinicians, educators, and policy makers to pursue. In this workshop, I will provide a multi-level update of the science of these disorders, that includes etiology (genes and environment), brain mechanisms, cognition, and symptoms. I will then review how this new science informs best practices for diagnosing and treating these disorders.

Correspondence: Bruce F. Pennington, Ph.D., Department of Psychology, University of Denver, Frontier Hall, Room 242, 2155 S. Race St., Denver, CO 80208.E-mail:bpenning@du.edu

CE 3:

Neurocognitive Networking: Modern Neuroimaging Methods for Understanding Neurocognition

Presenters: Angela R. Laird, Jennifer Robinson

$$
\text { 9:00 a.m.-12:00 p.m. }
$$

A.R. LAIRD \& J. ROBINSON. Neurocognitive Networking: Modern Neuroimaging Methods for Understanding Neurocognition.

Recent advances in functional neuroimaging have emphasized the benefits of a network approach to better elucidate cognitive operations in the brain. This course will provide a foundation for understanding functional brain connectivity methodologies. First, standard and advanced techniques for investigating neurocognitive networks in functional magnetic resonance imaging (fMRI) time series data (e.g., seed-based correlation, independent component analysis, graph theory) will be reviewed. Second, new developments and applications will be discussed and demonstrated for employing neuroimaging meta-analysis methods 
to (a) interrogate task-based and task-free functional networks, (b) examine clinically-relevant disease states, and (c) identify regions of interest as a basis for model building. Third, in the rising era of "big data", many databases and neuroinformatics resources have evolved to meet the needs of cognitive neuroscientists; these sources of data and their availability to researchers will be reviewed.

Correspondence: Angela R. Laird, Ph.D., Department of Physics, Florida International University, 11200 SW 8th Street, Miami, FL 33199.

E-mail:neurolab@fiu.edu

\section{WEDNESDAY AFTERNOON, FEBRUARY 4, 2015}

\section{CE 4: \\ Impact of Marijuana on the Developing Brain}

\section{Presenter: Deborah Yurgelon-Todd}

$$
\text { 1:00-4:00 p.m. }
$$

\section{YURGELUN-TODD. Impact of Marijuana on the Developing Brain. \\ Marijuana (MJ) is the most commonly used illicit drug by adolescents in the US. Research suggests that the integrity of the prefrontal cortex may be particularly salient for understanding both the risk for onset of MJ abuse and the transition into chronic use. Specifically, the frontal system has been shown to play a major role in self-regulation, inhibi- tory function, decision-making and initiation of behavioral responses. Observed deficits in frontally mediated executive functions in MJ users have raised the debate as to whether alterations in these frontal brain circuits are due to neurodevelopmental changes or the neurotoxic effects of cannabis exposure. This course will review the development of frontal circuits and their relationship to psychiatric disorders. Second, it will summarize recent findings on the effects of MJ in adolescents. Third. the course will present basics of magnetic resonance methods including MR spectroscopy often applied to study the effects of MJ. \\ Correspondence: Deborah Yurgelun-Todd, PhD, Department of Psychiatry, University of Utah School of Medicine, 383 Colorow Drive Rm 323, Salt Lake City, UT 84108.E-mail: Deborah.Yurgelun-Todd@ hsc.utah.edu}

\section{CE 5: The Neuropsychiatry of Multiple Sclerosis}

\section{Presenter: Anthony Feinstein}

$$
\text { 1:00-4:00 p.m. }
$$

\section{A. FEINSTEIN. The Neuropsychiatry of Multiple Sclerosis.}

Neuropsychiatric difficulties in multiple sclerosis may be divided into two broad categories, namely disorders of mood and affect on the one hand and cognitive dysfunction, on the other. The first category is dominated by depression which may affect up to $50 \%$ of MS patients over the course of their lifetime. Recent MRI data suggest a link between depression and atrophy and lesions affecting medial frontal and temporal brain regions. MRI findings can, however, account for little more than $40 \%$ of the depression variance suggesting a prominent role for psychosocial factors in the pathogenesis of depression. Depression exerts negative effects on quality of life and cognition and is also associated with an increased suicide rate in MS patients. For that reason, not missing the diagnosis and providing effective treatment are essential. A Cochrane Review suggests that cognitive behavior therapy is the treatment of choice. Pseudobulbar affect may affect up to $10 \%$ of MS patients. MRI data can account for $75-80 \%$ of the PBA variance. The svndrome responds well to a number of medications, including the tricyclic and SSRI antidepressants. Bipolar Affective Disorder is twice as common in MS patients as the general population. Euphoria is present in $9-13 \%$ of MS subjects. There are no treatment trials for either of these two syndromes. Impaired cognition is present in $40-70 \%$ of MS patients, depending on disease course. The hallmark deficits are slowed information processing speed, impaired memory and executive dysfunction. Structural and functional brain MRI studies have revealed a robust correlation between cognitive deficits and the imaging data. Cognitive reserve, as in other neuropsychiatric disorders and the aging literature, is considered protective. Pharmacotherapy is not effective in treating cognitive dysfunction, but cognitive rehabilitation is going through a renaissance and may offer enduring benefits to a subgroup of patients. Preliminary evidence suggests that smoking cannabis, which might relieve pain and spasticity in some MS patients, may be associated with a further deterioration in cognitive function.

Correspondence: Anthony Feinstein, MPhil, PhD, FRCPC, University of Toronto Department of Psychiatry, Sunnybrook Health Sciences Centre, Psychiatry, 2075 Bayview Ave., FG 16, Toronto, ON M4N 3M5, Canada.E-mail: ant.feinstein@utoronto.ca

CE 6:

Primary and Treatment Related Comorbidities in Pediatric and Adult Epilepsies: Revising our Understanding of the Relationships

\section{Presenters: Bruce P. Hermann, Madison M. Berl, David W. Loring}

\section{1:00-4:00 p.m.}

B.P. HERMANN, M. BERL \& D. LORING. Primary and Treatment Related Comorbidities in Pediatric and Adult Epilepsies: Revising our Understanding of the Relationships.

Epilepsy is the fourth most common neurological disorder affecting people throughout the lifespan with epidemiological peaks in childhood and older adulthood. In addition to the underlying disease substrate giving rise to seizures, the epilepsies can be complicated by diverse cognitive, behavioral, and social comorbidities that diminish quality of life and lifespan achievement. Recent major advances include a revised classification of seizures and epilepsy syndromes, improved understanding of the epidemiology of the comorbidities including their timing and course, improved interventions including epilepsy surgery, and an enhanced understanding of the diverse influences of the epilepsies and their treatment on normal neurodevelopmental and aging processes. This workshop will present an integrated overview of the epilepsies, its treatment, and problematic neurobehavioral comorbidities and their underlying neurobiological substrate in children, adolescents and adults. Correspondence: Bruce P. Hermann, University of Wisconsin School of Medicine and Public Health, Department of Neurology Box 5132 CSC H4,600 Highland Ave., Madison, WI 53792.E-mail: hermann@ neurology.wisc.edu 


\section{Invited Address: \\ Looking Behind the Smokescreen: Cannabis, Cognition and Multiple Sclerosis}

\section{Presenter: Anthony Feinstein}

$$
\text { 4:30-5:30 p.m. }
$$

\section{A. FEINSTEIN. Looking Behind the Smokescreen: Cannabis, Cognition and Multiple Sclerosis.}

Multiple sclerosis may impair cognition in $40-70 \%$ of patients depending on the disease course. The deficits are typically those of information processing speed, working memory and executive function. An estimated $14-18 \%$ of MS patients smoke or ingest cannabis for help with pain, spasticity and insomnia. Given concerns that cannabis may impair cognition in healthy subjects the use in patients with MS begs the question of whether it may further compromise cognition. There are a paucity of data that address this, but findings from three studies will be presented suggesting that regular cannabis smoking is associated with greater cognitive impairment in MS patients. fMRI and structural MRI data from one these studies that underpin the cognitive findings will also be presented. As with any medication, it is important for those who use it or prescribe it that the benefits and risks be weighed. This is starting to take place with cannabis and MS but more data are needed to inform the discussion.

Correspondence: Anthony Feinstein, MPhil, PhD, FRCPC, University of Toronto Department of Psychiatry, Sunnybrook Health Sciences Centre, Psychiatry, 2075 Bayview Ave., FG 16, Toronto, ON M4N 3M5, Canada.E-mail:ant.feinstein@utoronto.ca

\section{Poster Symposium: Cognitive and Neuropsychiatric Functioning of OIF/OEF/OND Veterans}

\author{
Chair: Robert D. Shura
}

6:00-7:30 p.m.

\section{Acquired Brain Injury (TBI/ \\ Cerebrovascular Injury \& Disease - Adult)}

\section{R.D. SHURA \& J. VASTERLING. Cognitive and Neuropsychiatric Functioning of OIF/OEF/OND Veterans.}

Symposium Description: The increasing number of Veterans returning from recent conflicts in Iraq and Afghanistan comprise a unique cohort facing complex challenges, including TBI, PTSD, and depression. The MA-MIRECC multi-site Neurocognition Lab, which focuses on post-deployment Veterans, is conducting an ongoing study in this population using a six-hour neuropsychological assessment battery. The purpose of this symposium is to review studies from this sample across a variety of methods, with a focus on cognitive complaints and neuropsychiatric impairments that might complicate successful reintegration following deployment. Data were mostly drawn from a sample of 212 Veterans who completed the study, with a mean age of 35 years and education of 14 years. The sample was $89 \%$ male and $72 \%$ Caucasian. Dr. Tupler's study used additional participants from two other sites. Dr. Robert Shura will present findings from studies on the Behavioral Dyscontrol Scale-II, an executive functioning measure originally intended to predict functional independence in geriatric populations. Dr. Holly Miskey will present evidence that self-reported disinhibition does not necessarily reflect objective performance, but that distress related to PTSD might constitute a better predictor. Dr. Beeta Homaifar will present data on the relationship among executive functioning, depressive symptom severity, and suicidal ideation, and also comment on the disparity between self-report and objective findings. Dr. Larry Tupler will present on olfaction (using the UPSIT) as a biomarker for TBI. Finally, consistent with this year's meeting theme, Dr. Jared Rowland will present a network analyses of whole-brain resting-state activity measured using magnetoencephalography (MEG) demonstrating the effects of mTBI and PTSD on the small worldness of the alpha and delta networks in a sub-sample of participants $(n=28)$. Dr. Jennifer Vasterling will serve as our discussant.

Correspondence: Robert D. Shura, PsyD, Hefner VAMC, MA-MIRECC, 11M-2/MH\&BS, 1601 Brenner Ave., Salisbury, NC 28144. E-mail: shura@marshall.edu

R.D. SHURA, J.A. ROWLAND \& R.E. YOASH-GANTZ. The Behavioral Dyscontrol Scale-II: A Unique Measure of Executive Functioning.

Objective: The Behavioral Dyscontrol Scale (BDS) was initially created to predict functional impairment in geriatric populations. The test measures dynamic motor organization, alphanumeric sequencing, and insight. A revised scoring system (BDS-II) resulted in improved psychometric properties, but few studies have examined the construct validity of the BDS-II or its application in non-geriatric populations. This study examined the BDS-II factor structure, construct validity. and relationship to mTBI and psychopathology in Iraq and Afghanistan Veterans. Participants and Methods: Participants were 164 post-deployment Veterans who passed the Green's Word Memory Test. Convergent and divergent validity were established using correlations with other cognitive measures. Scale structure and construct validity were examined using exploratory factor analyses with oblique rotations. T-tests were used to compare BDI-II scores with mTBI and psychopathology status. Results: Factor analysis identified two distinct factors of the BDS-II, which contrasted to previous research on the BDS identifying three factors. The BDS-II factors were correlated with specific executive functioning measures but not with tests of memory, motor dexterity, or olfaction. Factor analysis of the two BDS-II factors and 16 other cognitive measures resulted in a six-factor solution, with BDS-II Factor 1 loading with measures related to working memory and Factor 2 loading with CPT-II error measures. BDS-II scores were not significantly different based on current psychopathology or history of mTBI. Conclusions: The BDS-II appears to measure executive functions related to working memory and monitoring. Although the BDS-II might show utility in identifying executive impairments in non-geriatric populations, there were no differences in BDS-II scores based on psychopathology or mTBI status in this sample.

Correspondence: Robert D. Shura, PsyD, 1601 Brenner Ave, Salisbury, NC28144.E-mail: Robert.Shura2@va.gov

H.M. MISKEY, R.E. YOASH-GANTZ \& K.H. TABER. The Relationship of Self-Reported Disinhibition and Posttraumatic Stress to Objective Performance.

Objective: We investigated the relationship of self-reported disinhibition to PTSD-related distress, PTSD diagnosis, and mild TBI (mTBI) in the prediction of performance on objective measures of disinhibition. Studies suggest self-report of executive impairment does not predict performance after controlling for psychopathology. Additionally, individuals with $\mathrm{mTBI}$ have been found to overestimate dysfunction. Hypotheses: 1) mTBI would moderate the relationship between reported disinhibition (FrSBe) and performance on objective measures, 2) PCL-M would predict performance on objective measures above and beyond FrSBe, and 3) PTSD diagnosis would predict performance above and bevond FrSBe. Participants and Methods: Of the 135 Veterans passing symptom and performance validity tests, 23 met ACRM criteria for mTBI only, 28 met SCID-I criteria for PTSD only, and 17 met criteria for both mTBI and PTSD. Participants completed the FrSBe Disinhibition subscale, PCL-M, CPT-II, and Stroop. Results: Hierarchical regressions demonstrated that FrSBe predicted omissions ( $p=.016 ; 2.8 \%$ of the variance) but not commissions or Stroop performance. When PCL-M 
was added, it significantly predicted omissions $(p<.001 ; 12.6 \%$ of the variance) and FrSBe was no longer a significant predictor. PCL-M also significantly predicted commissions ( $p=.004 ; 5.9 \%$ of the variance) but not Stroop. PTSD diagnosis did not predict performance on any objective measure. Contrary to expectations, mTBI did not moderate the relationship between self-reported disinhibition and performance on any objective measure. Conclusions: Disinhibited performance was best explained by elevated PTSD-related distress. Neither PTSD diagnosis nor mTBI status was related to performance on objective measures of disinhibition, and self-reported disinhibition did not predict performance after accounting for self-reported PTSD distress.

Correspondence: Holly M. Miskey, PhD, 1601 Brenner Avenue, 11M-2/ MH\&BS, Salisbury, NC 28144.E-mail:Holly.Miskey@va.gov

B.Y. HOMAIFAR, R.D. SHURA, H.M. MISKEY, R.E. YOASHGANTZ \& J.A. ROWLAND. The Relationship of Suicidal Ideation to Objective and Subjective Executive Functioning.

Objective: We used objective and subjective executive functioning (EF) measures to examine the differences in Veterans with/without suicidal ideation (SI), as most studies only use objective measures. Hypotheses: 1) those with SI (Ideators) will perform poorer than those without SI (Non-Ideators) on EF measures: 2) level of depression will moderate the relationship between SI and EF. Participants and Methods: 114 Veterans completed the SCID-I, a TBI interview, BDI-II, Beck Scale for Suicide Ideation (BSI), and EF measures (FrSBe and CPT-II). Ideators: BSI score $>0$. Non-Ideators: BSI score $=0$. Results: Hypothesis 1: Ideators reported significantly greater impairment on the FrSBe-E subscale (self-report of executive dysfunction) and committed more CPT-II commission errors. Differences disappeared when current MDD diagnosis and $\mathrm{mTBI}$ history were entered as covariates. Hypothesis 2: The model including commissions and BDI-II was significant, explaining $28.1 \%$ of the variance in SI, yet more accurately classifying Non-Ideators $(91.3 \%$ compared with $52.9 \%$ of Ideators). There was an interaction effect: as Non-Ideators' BDI-II scores increased, they made more errors of commission. The model including FrSBe-E and BDI-II was significant, explaining $24.6 \%$ of the variance in SI, yet more accurately classifying Non-Ideators (92.5\% compared with $35.3 \%$ of Ideators). There was no interaction; only BDI-II was a significant predictor. The FrSBe-E subscale added no unique variance and was highly correlated with the BDI-II $(r=.71)$. Both instruments appeared to assess psychological distress with the BDI-II being the stronger predictor of SI. Conclusions: Although Hypothesis 1 was initially supported, differences disappeared after accounting for MDD and mTBI. For Hypothesis 2, our models more successfully classified Veterans who denied current SI, frequently classifving Ideators as Non-Ideators.

Correspondence: Beeta Y. Homaifar, ECHCS/VISN19 MIRECC, 1055

Clermont St.,Denver, CO 80220.E-mail:Beeta.Homaifar@va.gov

L.A. TUPLER, R.E. YOASH-GANTZ, T.A. CAMPBELL, S.D. MCDONALD, C.L. MCCORMICK, R.D. SHURA, H.M. MISKEY, T.C. PICKETT \& W.C. WALKER. Olfactory Deficits in Veterans Serving Post-9/11 Reporting TBI: A Potential Biomarker of Injury. Objective: Assessment of olfaction holds promise as a biomarker of traumatic brain injury (TBI). Chemosensory stimulation in the olfactory mucosa of the nasal cavity is communicated ipsilaterally via the first cranial nerve through the foramina of the cribriform plate to the olfactory bulb, which lies beneath the orbitofrontal cortex. The cribriform plate is a jagged, bony structure which can easily fragment from bluntforce trauma, and thus anosmia and hyposmia are common clinically observed sequelae of TBI, particularly from coup and contracoup forces to frontal and occipital lobes. Olfactory deficits in TBI, however, have received relatively little formal study. Participants and Methods: We examined 215 Veterans serving post 9/11, most of whom were deployed to Iraq or Afghanistan and reported history of TBI $(\mathrm{n}=96)$, no TBI ( $\mathrm{n}$ = 87), or uncertainty regarding TBI status $(\mathrm{n}=32)$. The University of Pennsylvania Smell Identification Test (UPSIT), yielding a maximum score of 40 , was used to assess olfaction. Results: Borderline significance was observed $(\mathrm{F}[2,212]=2.87, \mathrm{p}=0.0587)$ indicating poorest performance for subjects reporting TBI $(32.7 \pm 5.1)$ compared with those without history of TBI $(34.0 \pm 3.1)$ and those uncertain $(34.1$ \pm 2.8 ). Number of TBIs reported was highly significantly correlated with olfactory performance $(r=-0.23, p=0.0014)$. Conclusions: These results support use of the UPSIT and other tests of olfaction to support an inference of TBI history. The proximity of the olfactory bulb to orbitofrontal lobe furthermore suggests that olfactory impairment may provide a marker of behavioral disinhibition meriting further study. Correspondence: Larry A. Tupler, Durham Veterans Affairs Medical Center, 508 Fulton Street, Durham, NC27701.E-mail:Larry:Tupler@ va.gov

J.A. ROWLAND, J. STAPLETON-KOTLOSKI, K.H. TABER \& D.W. GODWIN. Alterations in Resting-State Brain Network Structure Associated with mTBI and PTSD.

Objective: To examine the effects of mild traumatic brain injury (mTBI) and posttraumatic stress disorder (PTSD) on resting-state brain networks measured using magnetoencephalography (MEG). Participants and Methods: Participants included 28 right-handed, male, postdeployment Veterans (mean age $=37.6$; mean education $=13.8$ : 75\% Caucasian). Diagnoses included: mTBI without PTSD $(n=6)$, PTSD without mTBI $(n=6)$, mTBI with PTSD $(n=6)$, without mTBI or psychiatric diagnosis $(n=10)$. Network analysis was performed in the alpha and delta bandwidths. Alpha is the bandwidth of predominate activity at rest. Increases in delta bandwidth activity have been reported in individuals with mTBI. Network metrics included average degree, density, average clustering coefficient, average path length, diameter, global efficiency, small worldness, and modularity. Group differences were examined using 2 (mTBI) x 2 (PTSD) univariate ANOVAs covarying for education. Results: There was a significant interaction between mTBI and PTSD for small worldness of alpha networks $(\mathrm{p}<.041)$ suggesting participants with PTSD without mTBI had lower small worldness than other participants. There was a significant main effect of mTBI for small worldness of delta networks $(p<.025)$ suggesting higher small worldness associated with mTBI. Conclusions: These results demonstrate mTBI and PTSD alter network structure as measured by small worldness, a metric associated with efficiency of information transfer. These results extend previous findings of pathological activity in the delta bandwidth associated with mTBI by demonstrating alterations in the delta resting-state network. Additionally, these results extend previous findings of altered default-mode network connectivity in individuals with PTSD to the whole-brain resting-state network. These findings may help clarify how these common postdeployment conditions affect brain function. Correspondence: Jared A. Rowland, Ph.D., 1601 Brenner Ave, 11M-2, Salisbury,NC28144.E-mail:.Jared.Rowland@va.gov

\section{Poster Session 1: ABI-Adult \& Emotional Processes}

$$
\text { 6:00-7:30 p.m. }
$$

\section{Acquired Brain Injury (TBI/ Cerebrovascular Injury \& Disease - Adult)}

\section{HIPLOYLEE, P. DUFORT, H. DAVIS \& C. TATOR. Recovery From Postconcussion Syndrome.}

Objective: Those who do not recover from a concussion after 3 months are said to have postconcussion syndrome (PCS). PCS consists of a constellation of somatic, affective and cognitive symptoms that can affect work/school/play. This exploratory study seeks to determine whether those who recover from PCS are different from those who do not recover - particularly in demographics and nature of symptoms. 
Participants and Methods: A total of 285 PCS sufferers were seen by C.T. from January 1997 - June 2013 at the Toronto Western Hospital, and were then contacted via mail for follow up. Only those with updated mailing addresses were sent a questionnaire regarding the concussion assessed by C.T. and their course of recovery.

Results: A total of 257 patients were contacted and 142 responded (response rate: $55 \%$ ). After exclusions (presence of hemorrhage/contusion or recovery $\leq 3$ months), 119 respondents remained. One-way ANOVAS were employed for continuous variables and chi-squares for categorical variables to compare those that recovered from their index concussion and those who did not. Respondents who recovered $(n=30)$ showed a trend to be younger $(22.93$ years $\pm 13.1 \mathrm{SD}, \mathrm{p}=0.054)$ than those who did not recover $(29.19$ years $\pm 15.87 \mathrm{SD}, \mathrm{n}=89)$. The recovered group did not differ from the non-recovered group in sex, the number of previous concussions, cause of concussion or whether they experienced a subsequent concussion $(p \geq 0.512)$. Both groups also experienced similar numbers of somatic, affective, and cognitive symptoms at the time of clinic visit $(p \geq 0.158)$. Complete recovery from PCS was seen by 3 years with average recovery by 11.65 months $\pm 7.71 \mathrm{SD}$. For the 89 that did not recover, average PCS duration was 53.73 months \pm 46.9 SD.

Conclusions: Recovery from PCS may be seen in younger individuals and within 3 years. Further investigation is required to determine which factors can predict recovery from PCS.

Correspondence: Carmen Hiploylee, University Health Network, 750 Dundas St W, Suite 3-313, MedWest Building, Toronto, ON M6J 3S3, Canada.E-mail: carmen.hiploylee@uhn.ca

M. REYNOLDS, J.C. PIERCY, L. OHLHAUSER, D. LEITNER, J. UPSHAW, H. MILLER \& E. CONCEPCION. Biological and Psychosocial Factors Associated with Post-Stroke Emotional Functioning.

Objective: We examined the biological and psychosocial factors related to emotional functioning following stroke. Vascular localization of the stroke, level of social support, and disability level were considered as possible determinants of post-stroke adjustment as measured by anxiety, depression, and well-being.

Participants and Methods: Inpatients $(\mathrm{n}=35)$ completed questionnaires assessing their emotional functioning, level of social support, and disability level at the time of their neuropsychological evaluation within two weeks of sustaining a stroke.

Results: Of the biological factors, the vascular territory of the stroke was moderately correlated with emotional functioning. Specifically, right posterior lesions were associated with greater feelings of unhappiness, lower levels of life satisfaction, and more somatic complaints. Of the psychosocial factors, high social support was associated with lower levels of both depression and anxiety. Cohabitation (living with another person) after discharge was correlated with higher levels of anxiety. Conversely, living at home - cohabitating or alone - after discharge was associated with higher levels of life satisfaction.

Conclusions: We compared whether biological or psychosocial factors were associated with emotional functioning following stroke. For the biological factors, right posterior lesions were associated with greater feelings of unhappiness, lower levels of life satisfaction, and more somatic complaints. For the psychosocial factors, it makes intuitive sense that high social support was associated with lower levels of both depression and anxiety; however, the relationship between marital status, discharge location, and emotional functioning is more convoluted. Cohabitation is associated with more anxiety, while living at home after discharge is associated with more life satisfaction. While not evaluated in this study, quality of marital relationship may play a role in this relationship. Correspondence: Meredith Reynolds, University of Montana, 301 Kiwanis Street, Apartment 9, Missoula, MT 59802. E-mail: meredith. reynolds@umontana.edu
M. TROYANSKAYA, N.J. PASTOREK, R.S. SCHEIBEL, N.J. PETERSEN \& H. LEVIN. Everyday Functioning, Symptom Reporting, and Cognition Following Injury During Combat Deployment.

Objective: To investigate if a history of any deployment-related injury or of concussion only has a persistent effect on individuals' everyday functioning, symptom reporting, and cognitive performance years after deployment.

Participants and Methods: Participants, who all were combat-deployed service members and veterans, included 89 individuals who had been exposed to blast and reported symptoms consistent with mild traumatic brain injury (mTBI), 26 control participants who had no history of blast exposure or mTBI, but sustained extracranial injury during deployment, and 37 control participants who had no history of blast exposure or any injury. Twenty-six indicators of everyday functioning, current neurobehavioral symptoms (e.g., pain, insomnia, anxiety, impulsiveness, depression, and hostility), and cognitive abilities were assessed using self-report questionnaires and objective neuropsychological testing. Demographics, injury characteristics, overall intellectual functioning, and intensity of combat exposure were also collected. The analyses of variances were conducted to identify main effects of injury group status. When models were significant, the Scheffe post-hoc method was used to determine between group differences.

Results: Participants in all three groups did not differ significantly in age, education, or current level of intellectual functioning and demonstrated adequate effort. Participants with history of deployment-related mTBI were significantly different in their current symptom reporting and everyday functioning compared to uninjured controls. There were no significant differences in cognitive performance among three groups. Conclusions: Individuals with deployment-related injuries demonstrate problems across a range of outcome variables, with limited evidence that individuals with a history of concussion report more problems than those with non-concussion injuries

Correspondence: Maya Troyanskaya, MD, PMR, Baylor College of Medicine,6550 Fannin \#NB 410, Houston, TX 77025.E-mail: mayat@ bcm.edu

J.E. KISSER, S.R. WALDSTEIN, M.K. EVANS \& A.B. ZONDERMAN. Lifetime Prevalence of Head Injury in a Demographically Diverse Community Sample.

Objective: Examine the prevalence of head injury in a demographically diverse, urban-dwelling sample.

Participants and Methods: History of head injury was examined in 2,801 African-Americans and Whites in Wave 1 of the Healthy Aging in Neighborhoods of Diversity across the Life Span study - a community-based, epidemiological investigation of adults. The present sample had a mean age of 48 years, was $44 \%$ male, $42 \%$ White, $59 \%$ below the $125 \%$ poverty line, and $11 \%$ had a history of head injury with loss of consciousness reported during a physician interview. Logistic regression analyses examined the odds of head injury history as a function of sex, race, poverty status, age, quadratic age, and their interactions.

Results: A significant 3-way interaction was noted among race, poverty status, and age $(\mathrm{OR}=1.087,95 \% \mathrm{CI} 1.025-1.153, \mathrm{p}=.005)$. Among Whites living in poverty, younger individuals had greater odds of head injury than older individuals, whereas older African-Americans living in poverty had greater odds of head injury. Additionally there was a significant 3 -way interaction among sex, race, and quadratic age (OR $=1.007,95 \%$ CI $1.000-1.013, \mathrm{p}=.040)$. Among White men and African-American women, those closer to the mean age (48 years) had lower odds of head injury than those nearer the minimum (30 years) or maximum (64 years) ages.

Conclusions: History of head injury varies substantially with age, sex, race, and poverty status. Further investigations using longitudinal cognitive data may provide insight into the role of head injury in cognitive decline and risk of dementia among sociodemographically diverse adults. 
Correspondence: Jason E. Kisser, MA, Psychology, UMBC, 1000 Hilltop Circle, Baltimore, MD 21250.E-mail: jk6@umbc.edu

\section{Z. MERZ. The Prevalence of Sport Concussion Citations in Blast Concussion Publications.}

Objective: Despite many physiological and psychological differences between sport and military-specific (blast) concussions, much of blast concussion research is borrowed from existing sport literature. This creates the potential for inappropriate neuropsychological test interpretations and the utilization of inadequate treatment methodologies. The purpose of this analysis is to describe differences between sport and blast concussions and determine the prevalence at which sport-specific citations appear in blast concussion publications.

Participants and Methods: No active participants were involved. This study utilized bibliometrics, which is commonly used to analyze citations in the overall evaluation of scientific activity.

Results: Of 65 military-specific articles that met inclusion criteria, $40 \%$ included at least one sport-specific citation. Of this $40 \%, 38.5 \%$ cited only one sport-specific article, leaving the remaining $61.5 \%$ as citing two or more sport-specific articles. Furthermore, an analysis of the Military Acute Concussion Evaluation (MACE), self-described as a tool for the identification of military-specific concussions, showed that $50 \%$ of references contained sport-specific research focusing on high-school and collegiate athletics.

Conclusions: Vastly different injury biomechanics, as well as physiological and psychological consequences exist between sport and blast concussions. Despite observed differences, literature involving returnto-duty guidelines continues to be pulled from sport-specific literature. These differences point to the need for unique return to duty/play guidelines, treatment methodology, and neuropsychological testing for each condition. Additional neuropsychological testing implications, as well as future research avenues, will be discussed in greater detail. Correspondence: Zachary Merz, Saint Louis University, 9906 heritage drive, apt c, Saint Louis, MO 63123.E-mail:merzz@slu.edu

\section{A. LEQUERICA \& N.D. CHIARAVALLOTI. Relationship Between Disturbed Sleep and Executive Functioning in Traumatic Brain Injury: A Pilot Study.}

Objective: A pilot study was conducted to examine the relationship between sleep characteristics and performance on measures of executive functioning in individuals with TBI complaining of sleep disturbance over the course of one week.

Participants and Methods: This cross sectional study included 8 males and 5 females at least 6 months post-TBI living in the community. All participants complained of sleep disturbance as indicated by a score above 5 on the Pittsburgh Sleep Quality Index. Mean age of participants was $42.5(\mathrm{SD}=17.7)$ years and average time since injury was 5.2 years $(\mathrm{SD}=7.6)$. Injury severity ranged from mild to moderate for this pilot study but all individuals were capable of completing a Shifting Attention (SA) Test administered using CNS-Vital Signs computer software after the seventh day of monitoring. Number of awakenings $(\mathrm{NWK})$ and time awake after sleep onset (WASO) were derived by having participants complete daily sleep logs, and through actigraphy using a wrist accelerometer or motion sensor to measure rest/activity cycles throughout the week.

Results: Regression analyses showed subjective WASO and NWK across the week of monitoring significantly predicted performance on the SA Test, each accounting for over $40 \%$ of the variance (WASOr $=-0.66, \mathrm{p}$ $=0.014, \mathrm{NWKr}=-0.70, \mathrm{p}=0.008)$.

Conclusions: Two measures of sleep disruption showed a moderate, negative relationship with a test of executive function. No significant relationships were found between these sleep parameters when only the night before testing was examined for its impact on testing the following day. This suggests that habitual sleep patterns captured across the week may have an impact on executive functioning among individuals with TBI who complain of sleep disturbance.
Correspondence: Anthony Lequerica, Kessler Foundation Research Center, 1199 Pleasant Valley Way, West Orange, NJ 07052. E-mail: alequerica@kesslerfoundation.org

S. JUNG, D. LAPORTE, S. ZIMNY, L. KNIGHT \& M. FRANZEN. Ecological Validity of Traditional Neuropsychological Tests: Role of Memory, Executive Skills, and Learning in Predicting Everyday Functioning in a Clinical Population.

Objective: Traditional neuropsychological tests are increasingly being used to predict a patient's real-world functioning with little empirical evidence to support this practice. Past research examining the ecological validity of neuropsychological tests for predicting functional capacity has resulted in mixed findings.

Participants and Methods: The current study examined the role of measures of executive functioning, memory, and learning in predicting functional skills among individuals with psychological disturbance (PSY) versus individuals with neurocognitive disorder (NCD). Functional capacity was assessed using the Independent Living Scales (ILS), a performance-based measure of instrumental activities of daily living. Archival data were collected and analyzed on 57 outpatients who were referred for neuropsychological evaluation at Allegheny General Hospital. Another sample of 198 patients was included for a confirmatory factor analysis, which provided empirical justification for aggregating tests into index scores based on the cognitive construct assessed.

Results: Using forced-entry, hierarchical multiple regression analyses, results revealed that the Executive Functioning Index significantly predicted ILS performance for the NCD group. In contrast, the Delaved Memory Index was a significant predictor of ILS scores for the PSY group. After accounting for age, education, and depression, Logical Memory II and Trail Making Test Part B were significant predictors of ILS performance for the PSY group. For the NCD group, Trail Making Test Part B significantly predicted ILS scores even after controlling for the effects of age, education, and depression.

Conclusions: These results suggest that some commonly used neuropsychological tests can demonstrate useful levels of ecological validity among certain populations.

Correspondence: Sharon Jung, Indiana University of Pennsylvania, 41 Burroughs St. Unit \#102, Detroit, MI 48202. E-mail: s.jung@iup.edu

Y. YOU, S. HUANG, S. HSIAO, Y. TSAI, W. LIN \& C. YANG. Anxiety Symptoms as the Major Contributing Factor to Post-Concussion Syndrome: A Prospective Study.

Objective: Post-concussion syndrome (PCS) is a cluster of symptoms in patients with mild traumatic brain injury (mTBI). PCS usually includes a set of physical, cognitive, and emotional symptoms, and may cause widespread disturbances and affects the patients' daily life. Although anxiety has been demonstrated as one of the most important influencing factors to contribute the presence of PCS, studies that focus on the association between anxiety and PCS are still limited. This study thus aims to prospectively uncover the relationships between the anxiety and PCS in patients following mTBI.

Participants and Methods: A total of 149 participants, including 53 healthy adults and 96 patients suffering from mTBI, were recruited. All participants were evaluated with the specific neuropsychological tests, the Checklist of Post-Concussion Symptoms (CPCS) and the self-rating scales (including the BDI-II, \&BAI) for investigating emotion status. Participants were prospectively evaluated at 2 weeks, 1 month, and 3 months post-injury.

Results: Patients' PCS reporting and emotional status were significantly worse than healthy controls at 1 month post-injury, while patients' PCS and anxiety symptoms significantly improved at 3 month post-injury. Regardless of 1 month or three months post-injury, PCS and emotional status of high-anxiety patients were significantly worse than those of the low-anxiety and none-anxiety ones. Most importantly, anxiety symptoms at 3 months post-injury significantly correlated with PCS manifestations at 3 months post-injury. 
Conclusions: This study prospectively demonstrates that patient's anxiety could be one of the most important contributing factors to the presence of PCS. The future research thus may further explore the related factors of anxiety symptoms after mTBI, and provide the appropriate interventions for those patients to decrease the persistent PCS. Correspondence: , . E-mail:

J.B. WILLIAMSON, D. LAMB, L. SALAZAR BEJARANO, M. HARCIAREK, S. PORGES \& K.M. HEILMAN. The Identification of Emotional Facial Expressions in People with Mild Traumatic Brain Injury and symptoms of PTSD.

Objective: To learn if mild traumatic brain injury (mTBI) influences emotional cognition. mTBI and PTSD are common in combat veterans. mTBI has been associated with varying degrees of acute alterations in emotions, most of which resolves within a week to a month post injury. Some patients with mTBI, however, have persistent impairments of their emotions. This persistent dysregualtion may in part be related to impairments in perception of emotions and it is unclear to what extent, if any, the neurological injury caused by mTBI influences performance. Participants and Methods: The participants were 37 combat veterans. 17 experienced a mTBI-with a loss of consciousness (LOC) and $20 \mathrm{did}$ not. These veterans presented with a range of symptoms of PTSD, from no symptoms to severe PTSD, as measured by PTSD Check List - Military (PCL-M). All TBI injuries were remote (>6 months). Participants were shown 47 morphing emotional faces, starting at neutral and slowly morphing into one of the six primary emotions. They were asked to interrupt the morphing faces when they recognized the emotional expression. Accuracy and reaction time for each type of emotional facial expression was measured.

Results: Using a multivariate general linear model with a fixed effect of LOC and covariate of PCL-M score, it was demonstrated that patients who experienced LOC with mTBI, independent of PTSD severity, identified faces that depicted anger less accurately than did patients with no LOC. Further, patients with LOC, independent of PTSD severity, were significantly slower in the identification of high arousal emotions including surprise, fear, and happiness.

Conclusions: Effectively processing emotional facial expressions is dependent on intact connectivity across brain regions including visual networks, the right hemisphere's temporal-parietal cortex and the amygdala. Though not available in this preliminary dataset, white matter damage to specific structures connecting occipital, and temporoparietal areas with the amygdala may have induced this disorder.

Correspondence: John B. Williamson, PhD, Neurology, University of Florida, 8114 SW 53rd Place, Gainesville, FL 32608. E-mail: john. williamson@neurology.ufl.edu

K.S. CHIOU, J. SANDRY \& N.D. CHIARAVALLOTI. Performance Differences and Predictors of Learning After Moderate and Severe Traumatic Brain Injury.

Objective: Learning deficits after traumatic brain injury (TBI) are well-documented and suggested to be moderated by poor executive functioning (EF). This study examined differences in learning ability within a TBI sample and the predictive nature of $\mathrm{EF}$ on learning, testing the hypotheses that: 1) adults with TBI meeting a pre-determined learning criterion outperform those unable to meet the learning criterion on tests of EF and 2) EF predicts learning ability after TBI.

Participants and Methods: 51 adults with moderate/severe TBI (GCS $\leq 12$; $\geq 1$ year post-injury) completed neuropsychological tests. Participants were divided into 2 groups [learning-intact (L-int) and learning-impaired (L-imp)] based on achievement of 2 perfect repetitions of a 10-word list presented over a maximum of 15 trials. Cognitive performance between groups was compared using independent samples t-tests and predictors of learning ability were identified using logistic regression.

Results: L-int ( $\leq 15$ learning trials; $n=25$ ) and L-imp (learning criterion not achieved; $n=26$ ) groups did not differ in age, education, or time post-injury. The L-int group scored significantly higher in verbal recall, working memory, verbal fluency, and inhibition. No between-group differences were found in attention, recognition memory, premorbid intelligence, set-shifting, or emotional functioning. Inclusion of working memory performance in a regression model increased the probability of accurately predicting learning ability.

Conclusions: Results reveal stronger executive skills in the L-int than the L-imp group. Furthermore, working memory performance served as a significant predictor of learning ability in our sample. Together, these findings support the contribution of executive processes to learning after TBI. Applications of findings to cognitive rehabilitation are discussed. Correspondence: Kathy S. Chiou, PhD, Kessler Foundation, 1199 Pleasant Valley Way, West Orange, NJ 07052.E-mail:kchiou@ kesslerfoundation.org

H.J. ORFF, C. HAYS, A. JAK \& E.W. TWAMLEY. Multivariate Predictors of Social Reintegration and Satisfaction in Veterans with Histories of Traumatic Brain Injury.

Objective: Approximately $20 \%$ of Iraq/Afghanistan Veterans have experienced a traumatic brain injury (TBI). TBI can result in persistent postconcussive symptoms such as sleep disturbance, fatigue, pain, mood disturbance, and cognitive symptoms. These chronic problems may also disrupt social and family functioning. We explored psychiatric, postconcussive, and cognitive factors as predictors of subjective and objective satisfaction with social and family relationships.

Participants and Methods: Participants included 50 unemployed Veterans with a history of mild to moderate TBI and cognitive impairment in the context of valid neuropsychological assessment. Bivariate correlations were performed to explore associations of psychiatric, postconcussive, and cognitive factors with subjective and objective measures of social and family contact. A series of multivariate stepwise regressions were then performed to determine the best predictors of objective and subjective social and family contact.

Results: Higher levels of depressive symptomatology were the strongest predictors of lower levels of social/family contact and satisfaction. Worse performance on a Category Fluency task predicted having fewer social contacts, whereas worse performance on a Digit Symbol task and Trails switching task predicted higher levels of subjective satisfaction with family contact.

Conclusions: Depressive symptoms and aspects of cognitive functioning may impact Veterans' social and family contact and satisfaction. Evidence-based interventions addressing depression and cognitive symptoms may improve community reintegration and subjective satisfaction with social and family relationships.

Correspondence: Henry J. Orff, PHD, Center of Excellence for Stress and Mental Health, VA San Diego Healthcare System, 3350 La Jolla Village Drive, 151B, San Diego, CA 92161. E-mail: horff@ucsd.edu

P. SUNDERARAMAN, A. JAMES \& M. SCHULTHEIS. Examining the Neuropsychological correlates of Trail Making Test Based on the Chronicity of Brain Injury.

Objective: The utility of traditional (time) and derived scores from Trail Making Test (TMT) after chronic brain injury is not known. The current study sought to investigate the relationship of these scores with different neuropsychological tests in two groups of individuals with moderate/severe brain injury.

Participants and Methods: Twenty individuals (Group 1: $n=10$, injury period $\leq 11$ years, $\mathrm{M}=7.1, \mathrm{SD}=3.21$ and, Group $2: \mathrm{n}=10$, injury period $>11$ years, $M=23.45, \mathrm{SD}=4.08$ ) were recruited from a day treatment rehabilitation setting. Along with the Time and Error scores for Parts $A$ and $B$, derived scores such as the Trails Total $(A+B)$, Interaction $([\mathrm{A} * \mathrm{~B}] / 100)$, Difference (B-A) and Ratio (B/A) scores were calculated. For both groups, these eight TMT scores were correlated with a neuropsychological battery.

Results: No significant differences between the two groups for age and education were found. Fewer and differential pattern of 
neuropsychological correlates were found for Group 1 compared to Group 2. In Group 1, Trails B time was correlated to Digit Span Sequencing $(\mathrm{r}=.67, \mathrm{p}<0.05)$, Oral SDMT with Trails Total $(\mathrm{r}=.-79, \mathrm{p}<.01)$ and Interaction $(r=.-75, p<0.01)$, and Ratio score with WAIS-IV Similarities $(\mathrm{r}=-.66, \mathrm{p}<.05)$. In Group 2, Arithmetic was correlated with Trails $\mathrm{A}(\mathrm{r}=.-78, \mathrm{p}<.01)$ and B Time $(\mathrm{r}=-.82, \mathrm{p}<.01)$, Trails Total $(\mathrm{r}=-.86$, $\mathrm{p}<0.001)$ and Interaction $(\mathrm{r}=-.80, \mathrm{p}<.01)$, Oral SDMT with Trails $\mathrm{B}$ time $(\mathrm{r}=-.70, \mathrm{p}<.05)$ and Difference score $(\mathrm{r}=-76, \mathrm{p}<.01)$, and Animal fluency with Trails $\mathrm{B}(\mathrm{r}=.64, \mathrm{p}<.01)$ and Trails Total $(\mathrm{r}=-.67, \mathrm{p}<.01)$. Immediate and delaved verbal memory was also correlated with TMT performance.

Conclusions: In the first decade of injury, TMT performance is influenced by executive functioning abilities such as processing speed. Following that period, additional abilities such as math, memory, and fluency influence performance. Derived TMT scores seem to tap into varied cognitive abilities. Clinicians may consider using TMT derived scores in lieu of administering longer batteries thus reducing test-related fatigue.

Correspondence: Preeti Sunderaraman, M.S., Psychology, Drexel University, 2215 Arch St., Apt 403, Philadelphia, PA 19103. E-mail: preetis.ps@gmail.com

A. LEMONS, D.R. SEICHEPINE, M.K. YEE, P.A. JANULEWICZ, K. SULLIVAN \& M. KRENGEL. Post-War Traumatic Brain Injuries are Influenced by Number of Pre-War, but not DeploymentRelated Brain Injuries in Gulf War Veterans.

Objective: Self-reported traumatic brain injury (TBI) is associated with an increased risk for future brain injury in athletes, but it is unknown if deployment-related TBIs in Veterans predict the incidence of post-war brain injuries. The present study examined this question in a cohort of Gulf War Veterans (GWV). It was hypothesized that deployment-related TBIs would predict post-war TBIs.

Participants and Methods: Two-hundred and thirteen male GWV (mean age $=54.9 \pm 8.4$ ) from the Ft. Devens, MA cohort completed a survey that included questions on the number of TBIs incurred before, during and after the war. TBI was defined using the American Academy of Neurology's current guidelines. Logistic regressions were performed with pre-war or deployment-related TBIs as the predictor variable and presence of post-war TBI as the dependent variable. Additional regressions using deployment-related TBIs as the predictor variable were performed for Veterans with $(\mathrm{n}=115)$ and without $(\mathrm{n}=98)$ pre-war TBI. Results: Pre-war TBIs predicted occurrence of post-war TBI $(B=1.5$, $\mathrm{p}<.000)$. For the overall sample, TBIs during the war significantly predicted occurrence of post-war TBI $(B=1.5, p=.007)$. However, this relation was only significant for Veterans with pre-war TBI history $(B=2.3$. $\mathrm{p}=.002)$, but not for Veterans without pre-war history $(\mathrm{B}=1.1, \mathrm{p}=.118)$. Conclusions: Similar to findings in athletes where pre-season brain injuries are predictive of TBIs during the season, in GWV, TBIs before and during war predicted post-war brain injury. However, the relation between deployment-related TBIs and post-war TBIs was modified by prior brain injury. Taken together, these findings indicate that pre-war TBIs are more influential on post-war TBIs than deployment-related brain injuries in GWV.

Correspondence: Daniel R. Seichepine, Boston VA Research Institute, 150 S Huntington Ave, Building \#9, Room 330, Boston, MA 02130. E-mail:daniel13@bu.edu

M.K. YEE, D.R. SEICHEPINE, A. LEMONS, P.A. JANULEWICZ, K. SULLIVAN \& M. KRENGEL. History of Pre-war Brain Injuries Influences Total Current Health Symptoms in a Cohort of 19901991 Gulf War Veterans.

Objective: Traumatic brain injury (TBI) has not been considered a contributing factor to chronic health symptoms experienced by Gulf War (GW) Veterans, but has been connected to poorer health in other populations. The aim of the present study was to examine the relation between pre-war and war-related TBIs and current health symptoms in GW Veterans.

Participants and Methods: Two hundred and thirteen male 19901991 GW Veterans (mean age $=54.9 \pm 8.4$ ) from the Ft. Deven's Cohort self-reported frequency of mild TBIs before and during war. A definition consistent with the American Academy of Neurology's current guideline was utilized. Veterans also endorsed the absence or presence of 34 health symptoms over the last 30 days. Linear regressions were performed with pre-war or war-related TBIs as the predictor variable and total number of health symptoms as the dependent variable. Additional regressions using war-related TBIs as the predictor were performed for Veterans with $(\mathrm{n}=115)$ and without $(\mathrm{n}=98)$ pre-war TBI.

Results: Pre-war and war-related TBIs correlated with total health symptoms ( $r h o=0.19, p=.007 \&$ rho $=0.32, p=.000$, respectively). War-related TBIs also predicted total health symptoms $(\beta=0.67$, $p=.000)$. This relation was only significant in Veterans with a pre-war TBI history $(\beta=0.83, p=0.001)$, not in Veterans without a pre-war TBI history $(\beta=0.48, p=0.099)$.

Conclusions: War-related TBIs predict current health symptoms in Veterans with a pre-war TBI history, indicating repetitive TBI has a negative impact on health. However, findings also suggest a pre-war TBI history has an influence on current health, likely due to an unknown moderating factor present before the war.

Correspondence: Megan K. Yee, M.A., Environmental Health, Boston University, 715 Albany Street, T4W, Boston, MA 02118. E-mail: meganyee@bu.edu

N. HANTKE, J. KONG, T. SHENG, M. YUTSIS, J. CHENG, L. KINOSHITA, J.K. FAIRCHILD, J.W. ASHFORD, P. BAYLEY \& M.M. ADAMSON. Diagnosis of PTSD Predicts Worse Delayed Free Recall Performance in Veterans with Complex Medical Problems: Results from the War Related Illness and Injury Study Center.

Objective: Veterans diagnosed with post-traumatic stress disorder (PTSD) often report difficulties with learning and memory. Few studies have investigated the unique contribution of PTSD to memory performance in Veterans with complex medical problems. We examined the extent to which a diagnosis of PTSD, independent of other medical factors, can predict performance on a standard verbal memory task, the California Verbal Learning Test - II (CVLT-II).

Participants and Methods: Fifty-one Veterans were evaluated at WRIISC CA located at VA Palo Alto for complex medical problems, including PTSD and mild traumatic brain injury (mTBI). Each Veteran received a brief cognitive screening battery. Hierarchical multiple regression was conducted to examine unique and combined contributions of different medical factors to performance on the CVLT-II.

Results: A diagnosis of PTSD and age were significant predictors for CVLT-II long delay free recall (LDFR) performance ( $\mathrm{R} 2=.24, \mathrm{p}=.01)$, even after accounting for education and gender. A history of mTBI and number of chronic medical issues (e.g., pain, sleep problems) were not significant indicators of LDFR performance. Post hoc regression models showed that age and PTSD status were also significant predictors of short delay free recall and long delay cued recall performance, although these models only approached significance.

Conclusions: Veterans with a diagnosis of PTSD performed significantly worse on the CVLT-II as compared to those without PTSD. PTSD status and age predicted performance on LDFR. These findings suggest that a diagnosis of PTSD has a unique association to memory recall difficulties that according to previous studies, may be related to hippocampal dysfunction.

Correspondence: Nathan Hantke, MIRECC/WRIISC, VA Palo Alto HCS, 3801 Miranda Ave 151Y, Palo Alto, CA 94304.E-mail:nathan.hantke@ va.gov 
A.R. KAUP, A. GAZZALEY, C. PELTZ \& K. YAFFE. A Novel Video Game to Assess the Cognitive Impact of Traumatic Brain Injury in Older Adulthood: A Pilot Study.

Objective: Traumatic brain injury (TBI) is an important dementia risk factor; however, it remains unclear how TBI impacts specific cognitive abilities in aging. Using a novel video game assessment, we investigated executive control among older veterans with TBI.

Participants and Methods: Participants were 33 older veterans (ages $50-90,84.9 \%$ male, mean education $=14.7$ years), 18 with TBI and 15 controls. Participants were administered Project:Evolution (EVO), an individually-adaptive video game that assesses executive control by measuring performance cost between multitasking and single-task performance ( $\%$ multitasking cost).

Results: Groups were well-matched in demographics and global cognition $(p>.05)$. Both groups exhibited wide performance variability (multitasking cost range: Controls $=-1.8-58.7 \%, \mathrm{TBI}=-11.4-83.5 \%)$, with no overall group differences $(\mathrm{p}>.05)$. Results suggested trend associations by TBI history. Compared to individuals with single TBI, those with repetitive TBI tended to show worse multitasking cost $(p=.06, \eta 2$ $=.20)$; this trend attenuated slightly after demographic adjustment $(\mathrm{p}$ $=.13$, partial $-\eta 2=.17)$. Compared to individuals with mild TBI, those with moderate-severe TBI tended to show worse single-task performance $(\mathrm{p}=.07, \eta 2=.19)$; this trend remained after demographic adjustment $(\mathrm{p}=.07$, partial- $\eta 2=.23)$. Among controls, multitasking cost worsened with age $(p=.03$, partial- $\eta 2=.37$, adjusted for demographics $)$.

Conclusions: These pilot results suggest the impact of TBI on executive control in late-life may depend on injury number and severity. EVO may be a sensitive tool to further investigate these complexities.

Correspondence: Allison R. Kaup, Ph.D., Mental Illness Research, Education and Clinical Centers (MIRECC), San Francisco VA / University of California San Francisco, 4150 Clement Drive, Bldg 11, 1-04, San Francisco, CA 94121. E-mail: allison.kaup@va.gov

S.L. CZIPRI, L.H. LU, T. LOUISE-BENDER PAPE, B. SMITH, J. BABCOCK-PARZIALE, W.M. HIGH \&. C.T. EVANS. The Impact of Psychiatric Distress on Neuropsychological and Daily Functioning in a Veteran Population with Mild Traumatic Brain Injury.

Objective: Among veterans with a history of mild traumatic brain injury (mTBI), comorbid psychiatric diagnoses are highly prevalent and may confound clinical presentation and slow functional recovery. The purpose of this study was to examine the extent to which neurocognitive functioning and cumulative psychiatric distress predict functional disability among veterans with symptoms persisting for more than 3 months after mTBI exposure.

Participants and Methods: Analysis was conducted using retrospective data from Operation Enduring Freedom and Operation Iraqi Freedom veterans ( $\mathrm{n}=438$ ) who completed the VA's Comprehensive TBI Evaluation. Veterans included in this study $(n=148)$ had valid scores on measures of performance and symptom validy and at least one clinician-rated mTBI exposure. A four-stage hierarchical regression was conducted to determine the degree to which cumulative psychiatric distress and memory functioning predict functional disability after controlling for demographic variables, injury factors, and attention.

Results: Attentional abilities did not significantly predict functional disability, and memory performance was only a significant predictor of functional disability when psychiatric distress was excluded from the regression model. With all variables included in the analysis, cumulative psychiatric distress was the only significant predictor of variation in functional disability $(\mathrm{p}<.01)$.

Conclusions: Among a veteran population, the presence of functional disability more than 3 months following mTBI exposure was best predicted by cumulative psychiatric distress with minimal impact of neurocognitive functioning. These findings provide meaningful information regarding diagnosis, screening procedures, and treatment-planning for veterans with residual difficulties following $\mathrm{mTBI}$.
Correspondence: Sheena L. Czipri, MA, Department of Psychology, Roosevelt University, 3101 Seaway Ct., Apt. 205, Tampa, FL 33629. E-mail:SCZIPRI@GMAIL.COM

C. GREEN, T. BERGQUIST, A. BROWN, M. HOOGS \& A.M. BUTTS. Demographic and Clinical Characteristics of a Traumatic Brain Injury Sample upon Admission to Brain Rehabilitation Clinic: A Descriptive Study Utilizing the Mayo Classification System of Injury Severity.

Objective: The study aim was to describe differences in a sample of adults with traumatic brain injury (TBI) upon admission to a brain rehabilitation clinic, based upon injury severity.

Participants and Methods: Seventy-two patients receiving rehabilitation, who sustained TBI and without comorbid CNS diagnoses (mean age $=46.25$ years, $\mathrm{SD}=17.36 ; 50 \%$ female), were included in the study. Sixty percent were classified as Definite, $26 \%$ as Probable, and $14 \%$ as Possible. All patients completed the Participation Index from the Mayo-Portland Adaptability Index (M2PI), the Patient Health Questionnaire (PHQ-9), and Satisfaction with Life Scale (SWLS).

Results: No significant differences were identified between Probable and Possible TBI groups, so they were combined. Definite and Probable/ Possible groups differed with regard to gender, employment, M2PI, SWLS, and PHQ-9 at admission $(p<.05)$. While the Definite group had more males, higher unemployment, greater level of disability, and lower life dissatisfaction, the Probable/Possible group endorsed more emotional distress. There was no gender difference on the PHQ-9.

Conclusions: Using the MCS, injury severity is related to lower employment, participation, and life satisfaction. Patients with less severe injuries report higher levels of distress. Possible reasons for this finding include that patients in the Possible/Probable group seek treatment because of increased emotional distress, while patients in the Definite group may report less distress due to limited awareness.

Correspondence: Cassie Green, Mayo Clinic, 4847 10th Street NW, Rochester,MN 55901.E-mail: cassiemgreen@gmail.com

C. YORK \& T.A. HAMMEKE. Somatization and PTSD Symptoms Predict Persistent Postconcussive Symptoms in a Veteran Outpatient Polytrauma Clinic.

Objective: Research suggests that persistent postconcussive symptoms (PCSs) after mild TBI (mTBI) may not be specific to mTBI and are potentially related to psychogenic factors. This study examined whether somatization tendencies and PTSD symptoms are better predictors of persistent PCSs than mTBI history in a veteran population.

Participants and Methods: Participants were 50 veterans (mean age 31; $96 \%$ male; $82 \%$ White) who were assessed during an appointment in an outpatient VA Polytrauma/TBI clinic after screening positive on a TBI screener. Somatization was assessed using the Modified Somatic Perception Questionnaire (MSPQ) and PTSD symptoms were assessed using the PTSD Checklist (PCLC). Participants were grouped by self-reported duration of loss of consciousness and posttraumatic amnesia: no TBI versus at least one mTBI. PCSs (memory, concentration, irritability, headache, sleep problems/fatigue) were assessed using a neurobehavioral status questionnaire.

Results: Of the 50 participants, $60 \%$ met criteria for a mTBI sustained during military service. MSPQ ( $\mathrm{t}=-.910$; ns) and PCLC $(\mathrm{t}=-1.74$, ns) scores did not differ between the TBI groups. MSPQ scores were significantly associated with headache $(\beta=.074, \mathrm{p}<.01)$, and PCLC scores were significantly associated with concentration $(\beta=.038, p<.01)$ and memory problems $(\beta=.031, p<.05)$. MSPQ and PCLC scores were both associated with irritability $(\beta=.030, p=.08 ; \beta=.041, p<.001$; respectively $)$ and sleep problems/fatigue $(\beta=.047, p<.01 ; \beta=.036, p<.001$; respectively). TBI status was not significantly associated with any of these PCSs.

Conclusions: These results suggest that both PTSD symptoms and somatization, rather than mTBI history, accounts for certain PCSs that persist beyond the expected timeline of recovery for $\mathrm{mTBI}$. 
Correspondence: Catherine York, Ph.D., Mental Health, Zablocki VA Medical Center, 5000 W. National Ave., Milwaukee, W 53295. E-mail: katie8283@gmail.com

J. LARA-RUIZ, W.D. LOPEZ, J. BIRATH, B.M. SMITH, D. BUDDING, D.J. HARDY, P. VESPA, D. HOVDA, D. MCARTHUR, T. GLENN, J. FUSTER, E. WOO \& M.J. WRIGHT. Activity Memory and Subjective Workload Following Traumatic Brain Injury.

Objective: We conducted the current study to determine how activity memory is related to subjective cognitive workload following severe traumatic brain injury (TBI)

Participants and Methods: A TBI group (1+ years post-injury; $\mathbf{n = 1 1}$ ) and healthy comparison group $(\mathrm{n}=10)$ were administered a battery of standard neuropsychological tests. Participants rated their subjective workload after each test with the NASA Task Load Index. Following completion of the test battery, participants were assessed for their recall and recognition of each test. Test performances served as the to-be-remembered activities.

Results: Mann-Whitney U tests were used to examine group differences and Spearman's rho was used to evaluate associations. The threshold for significance was set at $p<.05$. The groups differed in age, but were similar in years of education. Age was not associated with activity memory. The comparison group demonstrated greater activity memory discriminability and fewer false positives for activities on recognition testing. No other activity memory differences were observed. The comparison group showed no associations between subjective cognitive workload and activity memory. The TBI group demonstrated a negative correlation between subjective frustration and recognition hits and a positive correlation between subjective performance and recognition false positives.

Conclusions: As expected, the TBI group demonstrated poorer incidental activity memory than the comparison group. While subjective cognitive workload was not related to activity memory in the comparison group, it was related to subjective frustration (negatively) and subjective performance (positively) in the TBI group. These data suggest that TBI participants are aware of their ability to remember performed activities. Correspondence: Matthew J. Wright, Ph.D., Psychiatry, Harbor-UCLA Medical Center, 1124 W. Carson St., B-4 South, Rm. 111 (Box 490), Torrance, CA 90502. E-mail: mwright@labiomed.org

W.D. LOPEZ, D. WONG, J. LARA-RUIZ, H. KAKAVAND, J. GIBSON, Y. CERVANTES, D.J. HARDY, P. VESPA, D. HOVDA, D. MCARTHUR, T. GLENN, J. FUSTER, E. WOO \& M.J. WRIGHT. Event-Based Prospective Memory and Subjective Cognitive Workload Following Traumatic Brain Injury.

Objective: Event-based prospective memory (E-PM) is the ability to remember and execute an intended action following a cue. Our objective was to evaluate the relationship between subjective workload and E-PM following traumatic brain injury (TBI).

Participants and Methods: A TBI group (1+ years post-injury; $n=11$ ) and healthy comparison group $(\mathrm{n}=6)$ were administered an E-PM task via computer. The E-PM trials required participants to press a key when a specific border appeared; the border changed with each trial. The NASA Task Load Index (NASA-TLX) was administered after each test in a battery of standard neuropsychological tests. NASA-TLX scores for each cognitive domain were averaged to provide overall indices of cognitive workload.

Results: Mann-Whitney U tests were used to examine group differences and Spearman's rho was used to evaluate associations. The threshold for significance was set at $p<.05$. The groups were matched in age, education, and sex. The TBI group showed a lower percentage of correct E-PM responses, but not reaction times (RTs). The comparison group's percentage of correct E-PM responses correlated with subjective physical workload and effort. No other associations were observed.

Conclusions: TBI participants evidenced deficits in E-PM. While subjective cognitive workload correlated with E-PM in the comparison group, it did not correlate with E-PM in the TBI group. This lack of association in the TBI group could be due to reduced awareness with regard to cognitive ability. If so, then interventions that improve awareness of cognitive ability might help persons with TBI better focus their efforts and improve their functional outcomes.

Correspondence: Matthew J. Wright, Ph.D., Psychiatry, Harbor-UCLA Medical Center, 1124 W. Carson St., B-4 South, Rm. 111 (Box 490), Torrance,CA 90502.E-mail: mwright@labiomed.org

D. WONG, M.J. WRIGHT, I. SIKORA, C. MANNINO, M. CORONA, M. QUEZADA, W.D. LOPEZ, C. MCELWEE, J. BIRATH, H. KAKAVAND, P. VESPA, D. HOVDA, D. MCARTHUR, T. GLENN, J. FUSTER, E. WOO \& D.J. HARDY. Functional Outcome and Subjective Cognitive Workload Following Traumatic Brain Injury. Objective: We conducted the current study to determine how functional outcomes were related to subjective cognitive workload following severe traumatic brain injury (TBI)

Participants and Methods: A TBI group (1+ years post-injury; $\mathrm{n}=11)$ and healthy comparison group $(\mathrm{n}=10)$ were administered the Mayo-Portland Adaptability Inventory-4 (MPAI-4) and the NASA Task Load Index (NASA-TLX), following each test in a battery of standard neuropsychological tests.

Results: Spearman's rho was used to evaluate associations between subjective cognitive workload and functional outcomes. The threshold for significance was set at $p<.05$. The comparison group demonstrated significant correlations between subjective cognitive workload and adjustment to- and participation in functional activities. Whereas, the TBI group showed correlations between subjective physical demands and functional abilities and total functional outcome as well as subjective temporal demands and total functional outcomes.

Conclusions: Both groups showed associations between subjective cognitive workload and functional outcomes. TBI participants evidenced relations between temporal and physical demands and functional outcomes. These data suggest that TBI survivor's feel that speeded and physical demands are most salient in terms of their functional outcomes. Correspondence: Matthew J. Wright, Ph.D., Psychiatry, Harbor-UCLA Medical Center, 1124 W. Carson St., B-4 South, Rm. 111 (Box 490), Torrance, CA 90502. E-mail: mwright@labiomed.org

C. MCELWEE, D. WONG, M. CORONA, J. LARA-RUIZ, S. AMAYA, E. ROMERO, C.J. DALY, D.J. HARDY, P. VESPA, D. HOVDA, D. MCARTHUR, T. GLENN, J. FUSTER, E. WOO \& M.J. WRIGHT. Functional Outcome and Symptoms of Depression and Anxiety following Traumatic Brain Injury.

Objective: We conducted the current study to evaluate the relationship between functional outcome and symptoms of depression and anxiety following severe traumatic brain injury (TBI).

Participants and Methods: A TBI group (1+ years post-injury; $\mathrm{n}=10)$ and healthy comparison group $(n=10)$ were administered the Hospital Anxiety and Depression Scale (HADS) and the Mayo-Portland Adaptability Inventory-4 (MPAI-4).

Results: Spearman's rho was used to evaluate associations. The threshold for significance was set at $\mathrm{p}<.05$. The comparison group and the TBI group showed significant correlations between symptoms of depression and the Abilities, Adjustment, Participation, and Total scores on the MPAI-4. With regard to symptoms of anxiety, the comparison demonstrated significant correlations with the Abilities, Adjustment, Participation, and Total scores on the MPAI-4. The TBI group only showed significant correlations between symptoms of anxiety and the Participation and Total scores on the MPAI-4.

Conclusions: While both groups showed similar correlations between symptoms of depression and functional outcomes, the TBI group demonstrated fewer correlations between symptoms of anxiety and outcome than comparison group. Also, the TBI group demonstrated fewer correlations between anxious symptoms and outcome in contrast to their associations between depressive symptoms and outcome. These data 
suggest that depressive symptoms may be more detrimental to functional outcomes following TBI than anxious symptoms.

Correspondence: Matthew J. Wright, Ph.D., Psychiatry, Harbor-UCLA Medical Center, 1124 W. Carson St., B-4 South, Rm. 111 (Box 490), Torrance,CA 90502.E-mail:mwright@labiomed.org

I. SIKORA, C. MANNINO, M. CORONA, S. AMAYA, E. ROMERO, D. VALDEZ, F. MARTINEZ, J. BIRATH, B.M. SMITH, D. BUDDING, D.J. HARDY, P. VESPA, D. HOVDA, D. MCARTHUR, T. GLENN, J. FUSTER, E. WOO \& M.J. WRIGHT. The Item Specific Deficit Approach to Memory Dysfunction and Subjective Workload Following Traumatic Brain Injury.

Objective: We conducted the current study to evaluate the relationship between the Item Specific Deficit Approach (ISDA) indices of memory dysfunction and subjective workload following severe traumatic brain injury (TBI)

Participants and Methods: A TBI group (1+ years post-injury; $n=11$ ) and healthy comparison group $(\mathrm{n}=10)$ were administered the California Verbal Learning Test-II (CVLT-II) in addition to the NASA Task Load Index (NASA-TLX), following the administration of the CVLT-II.

Results: Mann-Whitney U tests were used to examine group differences and Spearman's rho was used to evaluate associations. The threshold for significance was set at $p<.05$. The groups differed in age, but were similar in education and sex. Given the age difference, demographically corrected scores on the CVLT-II were used. The ISDA indices were not associated with age. The comparison group outperformed the TBI group with regard to common CVLT-II indices. The comparison group also showed better encoding and consolidation than the TBI group. With regard to subjective workload on the CVLT-II, ISDA consolidation deficits were negatively related to temporal demands and frustration in the control group. In the TBI group, subjective mental effort was negatively associated with retrieval difficulties assessed by the ISDA.

Conclusions: TBI participants evidenced memory difficulties on the CVLT-II and deficits on the encoding and consolidation indices of the ISDA. ISDA consolidation indices were negatively related to subjective temporal demands and frustration in the comparison group. While retrieval deficits were inversely related to mental demands in the TBI group. These data suggest that TBI participants do not find memory retrieval mentally demanding.

Correspondence: Matthew J. Wright, Ph.D., Psychiatry, Harbor-UCLA Medical Center, 1124 W. Carson St., B-4 South, Rm. 111 (Box 490), Torrance,CA 90502. E-mail: mwright@labiomed.org

\section{L.S. BRADFORD. Misconceptions about Traumatic Brain Injury} Among US Army Behavioral Health Professionals.

Objective: The aim of this study was to investigate misconceptions about TBI held by US Army healthcare professionals and provide data regarding the perceived adequacy of current US Army TBI training.

Participants and Methods: US Army health professionals ( $\mathrm{N}=181$ ) were surveyed on common misconceptions about TBI (Gouvier, Prestholdt, \& Warner, 1988). Eight additional items were added to assess misconceptions pertaining to concussion. The sample consisted of independently licensed psychologists $(37 \%)$, social workers $(26 \%)$, psychiatric nurses $(16 \%)$, psychiatrists $(10 \%)$, with $12 \%$ of the sample identifying as other healthcare professionals. $80 \%$ of the sample were serving on active duty, $51 \%$ had a doctoral degree, and $25 \%$ had $10+$ years of experience.

Results: Misconceptions were identified across content domains related to amnesia, unconsciousness, and recovery. The total percentage correct on the mTBI items was 51\%. 39\% erroneously endorsed that mTBI has a worse outcome and recovery than concussion (equivalent terms in the DoD), $60 \%$ erroneously endorsed $\mathrm{mTBI}$ as a result of blast injury has worse cognitive outcome and recovery than mTBI from blunt trauma alone, and $77 \%$ erroneously endorsed that delayed symptoms onset is common after mTBI. In terms of recovery rates, only $28 \%$ participants endorsed that individuals who experience a mTBI will achieve full recovery, and only $53 \%$ correctly identified one week to three months as an expected recovery period for most people after mTBI. Only $13 \%$ of providers endorsed that their current training was adequate.

Conclusions: These findings raise concern that healthcare professionals in the US Army do not possess accurate information about TBI and could be passing on inaccurate information about concussion recovery to their patients. The importance of these considerations is underscored by literature suggesting early and accurate education of patients is important in preventing and reducing the development of persistent symptoms (Mittenberg, DiGiulio, Perrin, \& Bass, 1992).

Correspondence: Lonnie S. Bradford, PsyD, Neuropsychology Service, Tripler Army Medical Center, 47-291 HUI IWA ST APT B, Kaneohe, HI96744.E-mail: bradfordxu@gmail.com

J. HERSHAW, B. GUISE, D. BARRY \& M. ETTENHOFER. Mild TBI and the Aging Brain: Eye Tracking Evidence from a Novel Neurocognitive Assessment Tool.

Objective: The purpose of this study was to investigate the effect of mild TBI on age-related decline in distinct visual attention networks.

Participants and Methods: The task, the BEAM, is a novel cued attention paradigm in which participants are induced to use several distinct attention networks, including alerting, orienting, reorienting, and attentional disengagement. Saccadic and manual reaction times (RTs) were collected from 27 participants with history of mild TBI and 54 healthy controls (age $\mathrm{M}=33.75, \mathrm{SD}=11.77$ years). Analyses were conducted using hierarchical regression.

Results: History of mild TBI moderated the relationship between age and a saccadic composite score of visual attention, $\mathrm{R} 2$ change $=.05$, $\mathrm{F}(3,77)=8.67 \mathrm{p}<.05$, indicating that saccadic markers of visual attention declined more rapidly among those with a history of mild TBI. Follow-up analyses of component attention networks revealed that this moderating effect was most prominent for attentional disengagement, $\mathrm{R} 2$ change $=.05, \mathrm{~F}(3,77)=3.00 \mathrm{p}<.05$ and intra-individual variability of alerting, $\mathrm{R} 2$ change $=.04, \mathrm{~F}(3,77)=5.10, \mathrm{p}<.05$. No interactions between age and mild TBI were present for manual RT.

Conclusions: These results suggest that individuals with a history of mild TBI may be susceptible to accelerated aging of visual attention. Notably, these findings were demonstrated using saccadic responses but not manual responses. When overall performance was decomposed into various attention networks, results suggested that the brain systems underlying alerting and attentional disengagement appeared to be particularly susceptible to decline after mild TBI. These results suggest that neuropsychological assessment of visual attention after mild TBI should incorporate evaluation of multiple attention networks and response modalities, including eve movements.

Correspondence: Jamie Hershaw, MA, Medical and Clinical Psychology, Uniformed Services University, 9713 Braddock Road, Silver Spring, MD 20903.E-mail:jamie.hershaw@usuhs.edu

T. O'BRIEN, T. BARR, V. ZIZAK, J. LAPIN \& D. HAN. Impact of psychoeducation on perceptions of injury severity and cognitive complaints in veterans with TBI.

Objective: Research has demonstrated that misperceptions of injury severity and cognitive complaints may occur among individuals with TBI and psychiatric distress. We investigated the effects of psychoeducation intervention upon perceptions of injury severity and cognitive complaints among U.S. Veterans.

Participants and Methods: Veterans $(\mathrm{N}=107)$ referred from a TBI Polytrauma Service at a VA Healthcare System were enrolled in a single 45-minute psychoeducation group. Participants' perceptions of TBI severity and cognitive complaints were assessed prior to, and following, the intervention. In secondary analyses, 50 Veterans were followed individually to obtain objective measures of cognitive performance (RBANS) and self-reported psychiatric symptoms. Multiple regression analyses examined the relationship between perception of cognitive complaints 
and objective cognitive performance when accounting for psychiatric distress.

Results: Prior to receiving psychoeducation, one-third ( $\mathrm{N}=37$ ) reported that they did not know how to classify their injury, with $16 \%$ describing their injury as Mild and 50\% as Moderate or Severe. Psychoeducation had a significant effect, with less participants (20\%) responding "Don't Know" ( $\mathrm{t}=2.537, \mathrm{p}=0.013)$. Further, $36 \%$ described their injury as Mild and $43 \%$ as Moderate or Severe. Regression models indicated that for objective memory abilities, psychiatric distress accounted for $27 \%$ of variance $(\mathrm{F}=8.495, \mathrm{p}<0.001)$, and after controlling for psychiatric distress, subjective memory complaints did not account for additional variance $\left(\Delta R^{2}=0.014, p>0.05\right)$. Similarly, for objective attention abilities, psychiatric distress accounted for $32 \%$ of variance. After considering psychiatric distress, subjective attention complaints was not significant $\left(\Delta \mathrm{R}^{2}=0.010, \mathrm{p}>0.05\right)$.

Conclusions: Results suggest that Veterans' perceptions of injury severity may improve with a single session of psychoeducation. Objective cognitive performance may be more related to overall psychiatric distress than self-reported cognitive functioning.

Correspondence: Timothy O'Brien, Ph.D., VA Long Beach Healthcare Sstem,5901 E. 7th Street,Long Beach, CA 90822.E-mail:timobrien77@ gmail.com

L.M. FRENCH, T.A. BRICKELL, A. GRAHAM, R. GARTNER, A. DRISCOLL, Z. LI, E. SCHMIDT, D. PRUITT, M. WRIGHT, J. SMITH, B. PIZZANO \& R. LANGE. Neuropsychological Outcome from Military-related Traumatic Brain Injury (TBI): Preliminary Analyses of the Role of Resilience, TBI Severity, and Blast Exposure.

Objective: The purpose of this study is to examine the unique contribution of resilience, brain injury severity, and blast exposure on neuropsychological outcome from TBI.

Participants and Methods: Participants were 60 U.S. service members (Age: $\mathrm{M}=32.4, \mathrm{SD}=12.3 ; 91.2 \%$ male) prospectively enrolled from Walter Reed National Military Medical Center who had sustained a mild-severe TBI. Participants completed a 6-hour neuropsychological test battery $(\mathrm{M}=17.8$ months post-injury $[\mathrm{SD}=25.2])$ that included 13 common neurocognitive and neurobehavioral measures (32 and 39 scores of interest respectively).

Results: When divided into TBI severity groups (uncomplicated MTBI vs complicated MTBI-severe TBI), there were medium effect sizes on $37.5 \%$ of neurocognitive $(\mathrm{d}=.30-.59)$ and $41.0 \%$ of neurobehavioral $(\mathrm{d}=.30-.57)$ measures. When divided into blast groups (blast vs nonblast), there were medium effect sizes on $34.4 \%$ of neurocognitive $(\mathrm{d}=.33-.68)$ and $25.6 \%$ of neurobehavioral $(\mathrm{d}=.39-.92)$ measures. When divided into resilience groups (Low vs High), there were medium to large effect sizes on $68.8 \%$ of neurocognitive measures $(\mathrm{d}=.30-.76)$, and medium to very large effect sizes on $89.7 \%$ of neurobehavioral measures $(\mathrm{d}=.32-3.19)$. Using a series of linear regression analyses, resilience was a significant predictor $(p<.05)$ for $53.8 \%$ of neurobehavioral measures (accounting for 10-63\% of variance), and $34.4 \%$ of neurocognitive measures ( $8-15 \%$ of variance). Blast and TBI severity were rarely significant predictors of all measures.

Conclusions: Resilience was strongly associated with neuropsychological outcome following TBI; however, blast and TBI severity were not. The role of resilience on outcome from TBI is poorly understood and requires further research as a potentially useful indicator for clinical care. Correspondence: Rael Lange, DVBIC, WRNMMC, Defense and Veterans Brain Injury Center, Walter Reed National Military Medical Center, Building 8, Room 2264, 8901 Wisconsin Avenue, Bethesda, MD 20814. E-mail: rael.t.lange@gmail.com
E. DE GUISE, M. DESJARDINS, M. OUELLET, F. BANVILLE, S. BEAULIEU-BONNEAU, Y. RICHARD, H. AUDRIT, S. GAGNIER \& B. SWAINE. Stakeholder Perceptions on the Roles Clinical Neuropsychologists Can Play in Rehabilitation Service Delivery for Victims of a Traumatic Brain Injury with Mental Health Disorders. Objective: The aftermath of a Traumatic Brain Injury (TBI) can be complicated with physical, cognitive, emotional and behavioural limitations. When psychological maladjustment and psychiatric problems develop, specialized care becomes necessary to optimize recovery. The objective is to explore stakeholder perceptions on the roles clinical neuropsychologists can play in the development and implementation of action plans aimed at improving services delivery for victims of a TBI complicated by mental health disorders.

Participants and Methods: Eighty stakeholders (healthcare professionals, managers and decision-makers) with expertise in TBI, mental health or both were consulted during a provincial workshop (Québec, Canada) to develop action plans to improve rehabilitation services offered to TBI patients. Qualitative data (concrete and practical solutions) emerging in real time during discussions organized along four themes (screening-evaluation, accessibility, partnership and training) were analysed using a logistic management software (DRAP). A week later, the participants were invited to evaluate the importance of each statement on a Likert scale $(0=$ not important; $9=$ very important $)$. All statements containing the words "neuropsychology" and "neuropsychologist" were extracted from the database for analysis.

Results: Of the 340 statements, 29 contained the targeted words "neuropsychology", "neuropsychologist (s)" or "neuropsychological". The three most important action plans concerning neuropsychologists were 1) benefit from the expertise of neuropsychologists on-site to assess mental health disorders (mean importance $=8.33$ SD: 1.03); 2 ) diagnose the most common mental disorders (mean importance $=8.14$ SD: 1.07), and 3) promote neuropsychological group interventions for mental health problems among persons with a TBI (mean importance=8.12 SD: 1.12). Conclusions: Neuropsychologists are perceived as playing significant roles for victims of a TBI presenting mental health problems in the course of their recovery.

Correspondence: Elaine de Guise, University of Montréal, Département de Psychologie Pavillon Marie-Victorin CP 6128 Succ. Centre-Ville, Montreal, QCH3C3J7, Canada.E-mail: elaine.de.guise@umontreal.ca

E. DE GUISE, S. BÉLANGER, S. TINAWI, K. ANDERSON, J. LEBLANC, J. LAMOUREUX, H. AUDRIT \& M. FEYZ. Usefulness of the Rivermead Post-Concussion Symptoms Questionnaire for Outcome Prediction in Patients with Mild Traumatic Brain Injury. Objective: The aim of this study was to target the best tool for outcome prediction following mild traumatic brain injury (mTBI).

Participants and Methods: The study included 47 patients with mTBI referred to an out-patient rehabilitation clinic. The Rivermead Post-Concussion Symptoms Questionnaire (RPQ) and a brief neuropsychological battery were performed in the first few days following the trauma. The Mayo-Portland Adaptability Inventory-4 (MPAI-4) was completed within the first three months.

Results: The only variable associated with results on the MPAI- 4 was the RPQ score $(\mathrm{p}<0.001)$. The predictive outcome model including age, education, results of the Trail Making Test A and the Trail Making Test B had a pseudo-R2 of 0.02 . When the RPQ score was added, the pseudo-R2 climbed to 0.19 . This model indicates that the RPQ score is useful in predicting moderate to severe limitations following mTBI. Moreover, with a cut-off of 35 or more on the RPQ, the sensitivity for identifying cases with moderate to severe limitations was $90 \%$ and the specificity was $60 \%$.

Conclusions: The RPQ provides clinicians with a brief and reliable tool for predicting outcome functioning and can help target the need for further intervention and rehabilitation following mTBI. 
Correspondence: Elaine de Guise, University of Montréal, Département de Psychologie Pavillon Marie-Victorin CP 6128 Succ. Centre-Ville, Montreal, QCH3C3J7, Canada.E-mail: elaine.de.guise@umontreal.ca

N.J. PASTOREK, R. MARTIN, B. MILLER, J. ROMESSER, J. LINCK \& A. SIM. Association of Family Distress to Health Outcomes in Veterans with a History of Combat-Related Mild Traumatic Brain Injury.

Objective: Family distress has been shown to moderate outcomes in persons with mental health and neurological conditions, including traumatic brain injury (TBI). The aim of this study was to determine the relation between health status indicators and family distress in Veterans with combat-related mild TBI.

Participants and Methods: Participants were drawn from a sample of 212 veterans referred for neuropsychological evaluation secondary to combat-related mild TBI in the Iraq or Afghanistan wars. Seventy-three veterans were excluded based on evidence of symptom invalidity as determined by Minnesota Multiphasic Personality Inventory-2 (MMPI-2) FBS scores. The final sample consisted of 139 participants (mean age $=30.6$ years; $96 \%$ male). The MMPI- 2 FAM subscale was utilized as an indicator of family distress. Health outcomes were defined as total scores on the Neurobehavioral Symptom Inventory, Satisfaction with Life Scale, and Mayo-Portland Participation Index. Regression models were used to determine the association of family distress to health outcomes above and beyond that of comorbidities commonly identified in veterans (i.e., pain, insomnia, and PTSD).

Results: Family distress accounted for variance in life satisfaction $(p<.005)$ and neurobehavioral symptoms $(p<.01)$ above and beyond that accounted for by ratings of pain and insomnia and PTSD diagnosis. Post hoc analysis showed that family distress was significantly associated with somatic and affective neurobehavioral symptom clusters, but not cognitive symptoms. Family distress was not related to community participation.

Conclusions: Consistent with prior research relating family distress with outcomes in persons with psychiatric and neurologic conditions, family distress was associated with multiple indicators of health status in this sample of veterans with a history of combat-related mild TBI, though a causal link could not be established with the cross-sectional data. Nevertheless, the provision of family support may help to bolster health outcomes in this population.

Correspondence: Nicholas J. Pastorek, Ph.D., Michael E. DeBakey VA Medical Center, 2002 Holcombe Blvd, RECL 117, Houston, TX 77030.

E-mail: nicholas.pastorek@gmail.com

\section{Q. KENNEDY, M.M. ADAMSON, J. HUSTON \& P. NESBITT. Can a Simple Wargame Provide an Unobtrusive Indicator of TBI? A Case Study.}

Objective: To test the efficiency of a wargame to indicate TBI among active duty officers.

Participants and Methods: A military version of the Iowa Gambling task, the convoy task, was created to measure reinforcement learning and military decision making. The goal of the convoy task was to maximize total damage score by minimizing friendly damage and maximizing enemy damage incurred. Over 200 trials, 34 officers (aged 29 - 45 yrs) tried to learn the best route to send convoys. After each decision, officers received immediate feedback regarding friendly, enemy, and total damage. In addition to latency response (LR), attention to feedback was also measured by eyetracking. LR was defined as the proportion of trials in which officer's decision immediately after receiving feedback of moderate to heavy friendly damage was greater than 2 sd above their baseline time. Seventeen officers also completed a self-report Traumatic Brain Injury (TBI) survey.

Results: One officer (age $=45$; deployment time $=28$ months) had moderate TBI and was used for this case study. The decision performance, response to negative feedback, and eye scan pattern in the officer with TBI differed dramatically from the overall sample. First, his total damage score was 650 ( sample mean $=2334.375(\mathrm{sd}=1601.13)$. Second, in the sample, LR was positively associated with total damage score $(r=38, p=.03)$. The LR rate for the officer with TBI was $0 \%$ $($ sample mean $=19.68 \%)$. Third, preliminary eyetracking results in the sample revealed that looking at enemy damage was not correlated with total damage score; this officer spent almost double the amount of time looking at enemy damage $(12 \%$ vs. mean $=6.37 \%)$. Note that the officer with TBI scored in the $90 \%$ on Trails A and B, and within normal range for digit span forwards and backwards.

Conclusions: This case study illustrates the utility and efficiency of a military-specific task that can be used to indicate TBI in a population of active duty officers.

Correspondence: Quinn Kennedy, Psychology, Operations Research, Naval Postgraduate School, 1411 Cunningham Rd, Naval Postgraduate School, Monterey, CA 93943-5219.E-mail:mqkenned@nps.edu

R.A. BERNIER, J. FRENCH, A. ROY \& F.G. HILLARY. Functional Status at Discharge Is Higher Among Alcohol-Positive Older Adults after TBI.

Objective: There is a longstanding link between excessive alcohol use and risk for traumatic brain injury (TBI). Little is known about whether differences in alcohol use and its effect on functional outcome differ as a function of variables such as gender, age, and mechanism of injury, which may have implications for policy and prevention.

Participants and Methods: This study examined data among individuals 45+ from the Pennsylvania Trauma Outcome Study (PTOS), a database containing information about individuals with TBI admitted to trauma services in Pennsylvania hospitals from 1992 to 2009. The resulting sample includes 5,715 cases of moderate and severe TBI defined by GCS scores ranging from 3 to 12 .

Results: Likelihood of being alcohol-positive at time of ED admission varied across mechanisms of injury, gender, and age. Alcohol-related TBI was stable over time and, men were more likely than women to be alcohol+ as were individuals in the 45-65 age range compared to individuals 65+. Mean functional status at discharge (FSD) differed between alcohol-positive/negative groups. Alcohol-positive individuals aged 45-65 had higher FSD in TBI due to motor vehicle accident (MVA), $\mathrm{t}(1859)=-7.069, \mathrm{p}<.001$, and assault, $\mathrm{t}(798)=-3.362, \mathrm{p}=.001$. Alcohol-positive individuals 65+ had higher FSD due to falls, $\mathrm{t}(659)=-$ 6.464, $\mathrm{p}<.001$, and MVA, $\mathrm{t}(650)=-2.812, \mathrm{p}=.005$.

Conclusions: Mean outcome scores were consistently higher in alcohol-positive groups, but significance of improvement in alcohol-positive groups compared to alcohol-negative groups differs across mechanism, gender, and age in older adults. This latest finding is controversial but not unique to this literature and possible explanations will be discussed. Correspondence: Rachel A. Bernier, B.A., Psychology, The Pennsylvania State University, 111 Bruce V. Moore Building, University Park, PA 16802. E-mail: rub221@psu.edu

M. KRENGEL, E. BAKER, A. HENDRICKS, K. IVERSON, T. POGODA, K. STOLZMANN \& M. METERKO. Examination of NSI-symptoms in OEF/OIF Veterans with Multiple Concussions. Objective: To assess the impact of multiple TBI-related events on Neurobehavioral Symptom Inventory (NSI) symptoms in OEF/OIF Veterans after a VA Comprehensive TBI Evaluation (CTBIE). To examine the interaction of injury Etiology (Blast versus non-Blast) with Number of Events on symptoms, controlling for PTSD. It was hypothesized that number of events and symptom averages would be associated.

Participants and Methods: Participants were Veterans with a CTBIE between 2008 and 2013, all male, reported a single deployment-related event (Blast or non-Blast), and responded to a question of post-deployment head injury (Y/N). Veterans self-reported presence and severity of health symptoms. Severity scores were computed for each of 4 factor analyzed domains (Affective, Somatosensory, Cognitive, Vestibular) as the average across relevant items. 
Results: A 4-way between groups MANCOVA was computed with Number of TBIs (1 vs 2), Etiology (Blast vs non-Blast), PTSD (Y/N), and persistence of TBI symptoms (Y/N) as predictors and age as a covariate. PTSD was the strongest predictor $(\mathrm{p}<.0001)$ of NSI severity. Number of events was significant $(p=.001)$ and Current Status was marginally significant $(p=.042)$. There was an interaction between PTSD and Current Status $(p=.008)$ on severity. The impact of a second event on symptom severity was greater for those with blast. The pattern of this interaction varied by PTSD and Current TBI status for each NSI dimension, especially Cognition.

Conclusions: NSI symptom severity differs depending on type of deployment-related event, mental health status, persistence of symptoms, and number of events. Differential cumulative effects of Blast etiology are seen subsequent to a second, post-deployment TBI event. These findings raise the concern of multiple TBI events in maintenance of neurobehavioral symptoms in OEF/OIF Veterans.

Correspondence: Maxine Krengel, Ph.D., Neurology, Boston University School of Public Health, 725 Albany St, Suite 7B, Boston, MA 02118. E-mail:mhk@bu.edu

R.B. FROST, J. LONG, R. CAMPBELL, J.A. COOK, C. MCGREW, J.H. KING, A.R. MAYER, J.D. LEWINE, D.T. PULSIPHER \& R.J. THOMA. Cross-sectional Analysis of Cognition, Time Since, and Number of Concussions in Division I Athletes.

Objective: Cognitive recovery in sports concussion may be incomplete after resolution of other symptoms. Results from neuroimaging studies suggest a possible dose-response relationship between number of concussions and lingering neuro-physiological dysfunction. The current study looked at the relationship between total number of concussions (Number), days since last concussion (Days), and current cognitive functioning.

Participants and Methods: Cognition was assessed in an NCAA Division I student-athlete population ( $\mathrm{N}=87,8$ female and 79 male), between the ages of 18 and $24(\mathrm{M}=20.43, \mathrm{SD}=1.39)$. Within this group, 60 athletes reported no history of concussion (Unaffected) and 27 reported a prior history of concussion(s) (Concussion). The Immediate Post-Concussion Assessment and Cognitive Testing (ImPACT) battery was used to measure current cognitive functioning. Utilizing a MANOVA, the five ImPACT Composite scores were dependent variables, with Group (Unaffected, Concussion) entered as an independent variable, and prior number of concussions and days since last concussion entered as covariates.

Results: Days since last concussion ranged from 80 to 2495 days and was associated with Visual Memory Composite scores ( $p=.001)$ and Reaction Time Composite scores (RTC: $p=.004)$, such that greater number of days since last concussion was associated with higher visual memory scores and faster reaction times. A significant two-way Days $x$ Number interaction also emerged $(p=.001)$, such that fewer number of concussions and more days since injury predicted better RTC performance.

Conclusions: Cognitive recovery following sports related concussion is a complex process that is affected by previous head injury and elapsed time since injury and aspects of the ImPACT appear to be sensitive to these variables.

Correspondence: Robert B. Frost, PhD, Psychiatry, University of New Mexico Health Sciences Center, 308 Morningside Dr SE, Albuquerque, NM 87108.E-mail: rbfrost@salud.unm.edu

\section{J. DENBOER. Utilizing Serial Neuropsychological Assessment to Evaluate Recovery from Traumatic Brain Injury: Acute to 2 Years Post-Injury.}

Objective: The purpose of this study was to evaluate the effectiveness of utilizing serial neuropsychological evaluations to help assess and inform patient's recovery from TBI.

It was hypothesized that the inclusion of neuropsychological evaluation at important time points in the patient's recovery (i.e., 10 days post-injury, 1 month, 3 months, 6 months, 1 year, and 2 years post-injury) would greatly aid clinical-decision making (particularly in the area of prognosis).

Participants and Methods: Participants were 264 patients with diagnosed TBI.

Patients were administered a fixed battery of standard neuropsychological test measures at the following uniform time points: 10 days post-injury, 1 month, 3 months, 6 months, 1 year, and 2 years post-injury. Recovery from TBI was measured using reliable change index (RCI) analysis.

Neuropsychological evaluation results were combined with neuroimaging results at multiple time points, whenever available.

Results: Researchers found that the use of neuropsychological assessment measures at important time points in TBI recovery greatly aided diagnostic decision making. Neuropsychological evaluation was a significant and positive factor in guiding treatment interventions (e.g., speech therapy).

Although a positive neuropsychological trajectory was found throughout the 2 year period for the majority of patients, the most significant clinical change was found to be between 10 days and 1 month and 1 month and 3 months.

Conclusions: The results of this study provide the first comprehensive depiction of assessment of longitudinal TBI recovery utilizing serial neuropsychological evaluation. Results underscore the utility of neuropsychological assessment during TBI recovery and emphasize the need for earlier neuropsychological intervention during recovery from TBI. Correspondence: John DenBoer, Ph.D., The Nicholls Group, 9965 N. 95th St., Ste. 101, Scottsdale, AZ 85258.E-mail:jdb@thenichollsgroup. com

D.R. SEICHEPINE, M.K. YEE, A. LEMONS, P.A. JANULEWICZ, K. SULLIVAN \& M. KRENGEL. Frequency of Traumatic Brain Injuries in a Cohort of 1990-1991 Gulf War Veterans.

Objective: Traumatic brain injuries (TBIs) are a common consequence of war. However, the extent to which veterans from the 1990-1991 Gulf War $(\mathrm{GW})$ experienced TBIs is unknown. The aim of the present study was to examine the frequency of self-reported TBIs occurring before, during and after the war in a cohort of these veterans.

Participants and Methods: Two hundred and thirteen male GW Veterans (mean age $=54.9 \pm 8.4$ ) from the Ft. Deven's Cohort self-reported frequency of mild TBIs (mTBI) before, during and after the war. A definition consistent with the American Academy of Neurology's current guideline was utilized

Results: Thirty-one percent of Veterans indicated sustaining at least one war-related $\mathrm{mTBI}$ (median=0.0, range $=0-30$ ). Of those who sustained a war-related mTBI, $49.5 \%$ reported multiple injuries (at least two mTBIs). $53.9 \%$ of Veterans reported having at least one pre-war mTBI (median=1.0, range $=0-40$ ). Of those reporting pre-war mTBIs, $72.2 \%$ reported multiple injuries (at least two mTBIs). Following war, $43.7 \%$ reported having a $\mathrm{mTBI}$ (median $=0.0$, range $=0-75$ ). Of the veterans reporting post-war mTBIs, $61.3 \%$ noted multiple TBIs.

Conclusions: Approximately, one-third of GW Veterans reported experiencing a least one mTBI during the war. Additionally, most Veterans reported experiencing multiple mTBIs during their lifetime. Multiple mTBIs has been associated with long-term cognitive problems in veterans from the more recent wars in Iraq and Afghanistan and based on the current findings, future studies are needed to examine this relation in $\mathrm{GW}$ veterans.

Correspondence: Daniel R. Seichepine, Boston VA Research Institute, 150 S Huntington Ave, Building \#9, Room 330, Boston, MA 02130. E-mail: daniel13@bu.edu

D. STEED \& S. MILLER. Predicting Development of Dementia: A Parallel Process Latent Growth Curve Analysis.

Objective: Changes in vascular functioning frequently lead to developing neuropsychiatric symptoms (NPS). Furthermore, NPS are highly associated with incipient MCI and dementia. The present study included 
impaired and non-impaired individuals and examined progression from non-impairment and MCI to dementia on an ordinal latent variable, examining the relationships between NPS, vascular risk factors, and dementia. Since cerebrovascular disease is highly associated with NPS, it was hypothesized that development of vascular problems over time would be associated with a greater chance of developing NPS, and consequently, dementia.

Participants and Methods: We studied a sample of 1,052 individuals drawn from a national longitudinal database (National Alzheimer's Coordinating Center, 2013). Participants (age M = 74.68, SD = 9.67; Men $=53 \%$; Women $=47 \%$ ) were evaluated annually for six years with the and the Neuropsychiatric Inventory and a medical workup, part of which included detailing current vascular risk factors. Qualified health practitioners diagnosed either no dementia, MCI, or dementia during each visit. We summed the number of vascular risk factors for each patient at each time point. We employed parallel process latent growth curve modeling to estimate the hypothesized mediation of changes in NPS between changes in vascular risk factors and changes in dementia. Results: Changes in NPS did not mediate changes between vascular functioning and dementia status, though the baselines between the variables were related, suggesting some relationship. That is, a clear relationship existed between the variables, but the direction of the relationships was unclear based on the non-significant finding of the slopes indirect effect.

Conclusions: Findings do support a correlational, but not causal relationship between vascular risk factors, NPS and dementia, with NPS related to, but not necessarily mediating the changes between the vascular risk factors and dementia. The NACC database is funded by NIA Grant U01 AG016976.

Correspondence: Drake Steed, M.A., Illinois School of Professional Psychology at Argosy, Chicago, 1618 Mayo, Dayton, OH 45409. E-mail: drakery4@gmail.com

\section{J. LENGENFELDER, Y. GOVEROVER, A. SMITH \& N.D. CHIARAVALLOTI. Apathy and Quality of Life in Traumatic Brain Injury.}

Objective: To examine the differences in quality of life in individuals with Traumatic Brain Injury (TBI) with and without apathy.

Participants and Methods: Fifty-one individuals with moderate-severe TBI were divided into apathy and non apathy groups based on their performance on the Apathy Evaluation Scale (AES). Quality of life was measured with the two component scores, the mental component summary (MCS) and the physical component summary (PCS), of the 36-item Short Form Health Survey (SF-36). Functional abilities were assessed with the Functional Behavioral Profile (FBP), self and significant other forms.

Results: Apathy was present in $41 \%$ of the individuals. There were no differences between the apathy and non apathy groups in age, education, gender, time since injury, or premorbid IQ. There was a significant difference between the groups in depression $(p=.001)$ on the CMDI. After controlling for depression, the apathy group had significantly lower scores on the MCS $(p=.000)$, FBP self total $(p=.000)$, FBP self task performance $(p=.008)$, FBP self social interaction $(p=.000)$, FBP self problem solving $(\mathrm{p}=.000), \mathrm{FBP}$ significant other social interaction $(p=.040)$, and FBP significant other problem solving $(p=.033)$.

Conclusions: Persons with TBI with and without apathy demonstrated significant differences in quality of life, particularly in regard to mental health. In addition, persons with apathy showed significantly more difficulties with functional behaviors, including social integration. It is thus likely that interventions for apathy could improve quality of life in individuals with TBI.

Correspondence: Jeannie Lengenfelder, Kessler Foundation Research Center, 300 Executive Drive, Suite 70, West Orange, NJ 07052. E-mail: jlengenfelder@kesslerfoundation.org
L. SEIBERT, R.T. LANGE, J. KENNEDY, J. DUCKWORTH, T.A. BRICKELL, L.M. FRENCH \& J.M. BAILIE. Effect of Body Orientation to Blast on Risk of Post Concussive Symptoms among Active Duty Service Members.

Objective: The risk of post concussive symptoms may be affected by body orientation to a blast wave. Animal models of blast exposure have demonstrated that front (head-on) or top-down blast exposure increases over-pressure through the brain compared to left, right, or bottom-up exposure. Greater intracranial pressure increases the likelihood of associated neuropathology and symptomatology following a TBI. This study investigated whether orientation to blast affects report of post-concussive symptoms among active duty service members after mild TBI.

Participants and Methods: To reduce potential confounding effects of multiple blast exposures, only participants without a prior history of blast exposure before their most recent mTBI were examined $(\mathrm{N}=119)$. Participants were all active duty service members between 1 and 24 months post injury. Symptoms were measured with the Neurobehavioral Symptom Inventory (NSI). Blast orientation was self-reported.

Results: A clear effect was found of body orientation to blast for NSI Total score as well as for each NSI symptom cluster score (i.e., Physical, Cognitive, Affective, and Sensory) ( $p$ 's $<.05$ ). Medium effect sizes (Cohen's d) were found for every comparison: NSI Total Score $=0.6$; Physical $=0.4 ;$ Cognitive $=0.6 ;$ Affective $=0.5 ;$ and Sensory $=0.6$.

Conclusions: For individuals with no prior history of blast exposure, a front or top orientation to a blast that resulted in mTBI was associated with increased neurobehavioral symptoms, relative to other blast orientations. This suggests body orientation to blast is relevant clinical information to collect, to help differentiate severities among blast-related mTBIs.

Correspondence: Laura Seibert, Ph.D., Camp Pendleton, Defense \& Veterans Brain Injury Center, Defense \& Veterans Brain Injury Center, 33 Area, Bldg 33305, Camp Pendleton, CA 92055.E-mail:drlseibert@ verizon.net

K. AGBAYANI. Cognitive profile and rate of impairment comparisons between cerebellar and frontal lobe strokes.

Objective: Focal damage to areas of the cerebellum lead to impairments in executive functioning and behavior regulation, which are abilities typically associated with frontal lobe lesions. To date, comparisons of neurocognitive profiles after frontal lobe and cerebellar damage have not been reported. We compared cognitive test performance between patients with cerebellar and frontal lobe strokes.

Participants and Methods: Archival data from 31 patients with cerebellar stroke (9 left, 17 right, and 5 bilateral lesions), and 28 with frontal lobe stroke (11 left, 12 right, and 5 bilateral lesions) were analyzed. A comprehensive neuropsychological battery was administered an average of one month after stroke. Fisher's exact test and ANOVA were conducted for between-group comparisons.

Results: Patients with cerebellar and frontal lobe strokes both showed high rates of impairment (clinical impairment defined as $\mathrm{z}$-score $<-1.5$ ) on tests of mental flexibility (60\% impaired with cerebellar damage, $54 \%$ with frontal), verbal fluency ( $45 \%$ cerebellar, $54 \%$ frontal for phonemic fluency), processing speed, and list learning (with no significant Fisher's exact test $\mathrm{p}$ values for all comparisons). However, based on ANOVA, frontal lobe strokes resulted in lower mean performance compared to cerebellar strokes on most tests with significant differences found for attentional focus, visual-spatial judgment and construction, confrontation naming, list learning (all p's $<0.05$ ), and semantic fluency $(\mathrm{p}<0.01)$.

Conclusions: Results were consistent with previous research indicating impairments in cognition after cerebellar or frontal lobe stroke, and expand on the literature by showing cerebellar strokes result in similar rates of cognitive performance (but smaller magnitude of impairment) compared to frontal lobe strokes. Such findings were consistent with the concept of a highly integrated circuitry, consisting of feedforward 
and feedback loops between the cerebellum and areas of the cerebral cortex, including the prefrontal cortex.

Correspondence: Kristina Agbayani, M.A., University of Houston, 5805 Wales Court, San Jose, CA 95138. E-mail:kaagbayani@gmail.com

\section{G. LEFEBVRE, E. CHAMARD, E. DE GUISE \& H. THEORET. Neuropsychological Impact of Repeated Sub-Concussive Blows to the Head in Male and Female Athletes.}

Objective: Contact sports are associated with a high risk of concussion, and recent studies have shown alterations in cognitive function following head injury in athletes. However, it is not clear if repetitive hits to the head at a subclinical level, notably in contact sports like rugby, soccer and football, can induce similar impairments. The aim of the present study was to verify if repetitive sub-concussive blows to the head induce cognitive impairment in athletes practicing contact sports.

Participants and Methods: Three groups were studied: 1) healthy non-athletes ( $\mathrm{N}=21 ; 9$ male): 2 ) athletes practicing a non-contact sport (swimming; $\mathrm{N}=20 ; 9$ male) and 3) athletes practicing a contact sport (rugby and soccer; $\mathrm{N}=17 ; 7$ male). Each group was equivalent with regards to education level and age. All participants had no history of concussion, and all athletes were part of a University of Montreal varsity sport team. Neuropsychological evaluation was conducted using the Ruff 2\&7, D2, Symbol Digit Modality Test (SDMT), Color Word Interference Test (D-KEFS), Color Trail A \& B, and the Rey Complex Figure Test (RCFT).

Results: The only significant effect was a main group effect in the recognition part of the RCFT where lower results were observed in the contact sport athletes compared to both control groups $(\mathrm{F}=4,342 ; \mathrm{p}<0,05)$. Conclusions: The results of the present study suggest that receiving repeated sub-concussive hits is associated with limited cognitive impairment.

Correspondence: Genevieve Lefebvre, Departement de psychologie, University of Montreal, CP 6128, Succ. Centre-Ville, Montreal, QC H3C 3J7, Canada.E-mail: genevieve.lefebvre.10@umontreal.ca

D. KAPlan, S. BUllaRD, A. PACIlEO \& C. YANTZ. Multidisciplinary Concussion Assessment: The Relationship Between Neuropsychological and Vestibular Physical Therapy Measures.

Objective: This study evaluates the relationship between standardized neuropsychological measures and vestibular physical therapy measures in a multidisciplinary concussion clinic setting. Specifically, we examined attention and visuospatial neuropsychological performances as related to vestibular physical therapy measures.

Participants and Methods: The data from outpatients who were administered both standardized physical therapy tools (King-Devick and Dynamic Gait Index) and a neuropsychological assessment (including RBANS, WAIS-III Digit Span. TOMM. and WTAR) in the concussion clinic setting were retrospectively analyzed. Two patients with low effort were excluded from this dataset, and thirty-two participants remained in the sample and had no missing data. Spearman rank-order correlations between the physical therapy measures and hypothesis-driven selected neuropsychological measures were performed, with a modified Bonferroni correction.

Results: The analysis demonstrated a significant relationship ( $\mathrm{r}=$ $0.486, p=0.005$ ) between performance on RBANS Line Orientation and the King Devick, a physical therapy measure.

Conclusions: This relationship helps to confirm the overlap between our assessments in this population, particular to the visuospatial domain. The data suggests that post-concussive syndrome (PCS) may involve visuospatial impairment observable in both neuropsychological and vestibular therapy treatment settings. Clinical implications include when to refer concussion patients from neuropsychological clinics to vestibular physical therapy treatment. Future research to coordinate care between related rehabilitation disciplines who manage PCS is recommended.
Correspondence: Danielle Kaplan, Gaylord Specialty Healthcare, 596 East Slope Drive, Orange, CT 06477.E-mail: daniellekaplan728@ yahoo.com

D. Wong gonzalez \& L. BUChanan. Prospective Memory Following Traumatic Brain Injury: A Meta-Analysis.

Objective: Individuals with traumatic brain injury (TBI) report frequent and significant deficits of prospective memory, defined as the ability to remember to do something in the future at the right time and place (Henry et al., 2007; Shum et al., 2011; Uttl, 2008). The current study aimed to critically review and report on the growing literature on prospective memory in TBI through meta-analyses. Specifically, we explored the influence of cognitive demands imposed by prospective memory tasks on performance.

Participants and Methods: Individual studies with a sample of adult patients with TBI and matched control group, and a continuous behavioral measure of prospective memory were included.

Results: Random effects meta-analyses $(k=16)$ revealed that the pooled standardized mean difference (SMD) for prospective memory performance between TBI and control groups was $0.81 \mathrm{SE}=0.10,95 \%$ $\mathrm{CI}=0.61-1.01)$ for low cognitively demanding tasks, and 1.20 (SE $=0.14,95 \% \mathrm{CI}=0.92-1.48)$ for high cognitively demanding tasks. Heterogeneity estimates were low and non-significant.

Conclusions: Both estimates are large and significantly different from zero $(p<.001)$, indicating that patients with TBI have significantly lower prospective memory performance than matched controls. The SMD was slightly higher for high as opposed to low cognitively demanding tasks; indicating that prospective memory task demands influence performance. Implications and future directions will be discussed within the context of the current results.

Correspondence: Daniela Wong Gonzalez, Clinical Neuropsychology, Psychology, University of Windsor, 705-166 University Ave W, Windsor, ON N9A5H9, Canada.E-mail:wonggon@uwindsor.ca

D.M. LOGAN, K.R. HILL \& M.J. LARSON. Cognitive Control of Conscious Error Awareness: Variable Task Performance in Moderate-to-Severe Traumatic Brain Injury.

Objective: Cognitive functioning in the area of cognitive control and specifically, deficits in awareness have been linked to poor recovery and rehabilitation outcomes following moderate-to-severe traumatic brain injury (M/S TBI). Both the error-related negativity (ERN) and the error positivity (Pe) components of the event-related potential (ERP) are linked to error awareness and cognitive control processes. We tested levels of error awareness in a M/S TBI sample using behavioral measures and ERPs, including the ERN and Pe, as indicators of error awareness. Participants and Methods: Participants included 30 neurologically-healthy controls and 24 individuals with M/S TBI who completed a brief neuropsychological battery and self-report measures of mood. To measure awareness, participants completed the error awareness task (EAT), a modified Stroop go/no-go task shown to be effective in generating errors. Participants signaled awareness of errors committed on the previous trial. Robust analyses of variance compared between-group ERN and Pe amplitudes, and first and second half no-go accuracy and error awareness performance on the EAT.

Results: The M/S TBI group decreased accuracy while improving or maintaining error awareness compared to controls over time. There were no significant between-group differences for ERN and Pe amplitudes. Conclusions: The M/S TBI group had varying levels of attention and awareness over time, evidenced by improving awareness of errors when they are happening, but an inability to regulate performance sufficiently to improve accuracy. Support is provided for further study of behavioral error awareness performance within a task and relative to task requirements.

Correspondence: Dustin M. Logan, MS, Psychology, Brigham Young. University, 6537 Lafayette St., Omaha, NE 68132. E-mail: logan. dustin@gmail.com 
S.J. BANKS, S. MEDNICOFF \& C. BERNICK. Verbal Fluency and Brain Health in Boxers and Mixed Martial Arts Fighters.

Objective: Negative cognitive outcomes are sometimes seen in boxers and other combat sports professionals, and this is often characterized by slurred speech and difficulty with language, as well as other cognitive impairments. In prior work we have demonstrated correlations between fight exposure and diminished volume of the hippocampi and thalami, as well as diminished processing speed. Here we investigate the relationship between fight exposure, verbal fluency and brain structure volume in fighters.

Participants and Methods: We were interested in semantic and phonemic fluency in boxers and mixed martial arts fighters $(n=190)$. Semantic (animals) and phonemic (f words) fluency were collected as part of a larger battery of tests given to active fighters in a large longitudinal study. Fighters also undergo MRI and brain structure volumes are assessed using automated software. Partial correlations were used to assess relationships, controlling for the age of the fighter.

Results: Negative correlations were seen between exposure and semantic fluency. Similarly, positive correlations between semantic fluency and hippocampal volumes, bilaterally, were apparent. Phonemic fluency showed no correlations with exposure, thalamic or hippocampal volumes.

Conclusions: These results point to a potential impact of repetitive traumatic brain injury on temporal lobe functioning and hippocampal volumes, and appears to be more specific than simply reduction in processing speed.

Correspondence: Sarah J. Banks, PhD, Lou Ruvo Center for Brain Health, Cleveland Clinic, 888 W Bonneville Ave, Las Vegas, NV 89106. E-mail:bankss2@ccf.org.

M.E. ONEIL, K. GLEITSMANN, M. MOTU'APUAKA, M. FREEMAN, K. KONDO, D. STORZBACH, D. KANSAGARA \& K. CARLSON. Visual Dysfunction in Patients with Traumatic Brain Injury: A Systematic Review.

Objective: Determine the prevalence of visual dysfunction in individuals with TBI history.

Participants and Methods: We used a previous systematic review on visual problems in TBI to identify studies published prior to 2009. We searched Medline, PsychINFO, Cochrane, SPORTDiscus, Rehabilitation \& Sports Medicine Source, and Rehabdata for studies published since 2009. We included studies reporting visual dysfunctions likely to be treated in eye care clinics in patients over 5 years of age with a history of TBI.

Results: Of 1299 abstracts, 118 were full-text reviewed. 15 studies met inclusion criteria. Evidence from a large retrospective cohort study of U.S. Service Members diagnosed with visual dysfunction and treated in military healthcare settings suggests that visual dysfunction is infrequently diagnosed in unscreened populations with TBI history, occurring in less than $1 \%$ in most cases. Disorders of accommodation and refraction are slightly more common, with a frequency of $7.3 \%$. Other studies focused on Veterans seen at the Department of Veterans Affairs Polytrauma Rehabilitation Centers and Polytrauma Network Sites. Visual dysfunction was much more frequent in these populations, with estimates of over $50 \%$ for many conditions such as accommodation and refraction disorders, convergence insufficiency or dysfunction, dry eye syndrome, photosensitivity, pursuit or saccadic dysfunction, and self-reported visual impairments.

Conclusions: Studies report a wide range of frequency estimates, likely due to differences in setting and population. While some studies report results regardless of current symptoms, many studies were conducted in clinics that only serve Veterans with current symptoms as well as other serious comorbidities. Overall, visual dysfunction diagnosed in U.S. Service Members treated in military healthcare settings is uncommon; however, Veterans with TBI history and current symptoms who are treated in TBI rehabilitation clinics report much higher frequencies of visual dysfunction.
Correspondence: Maya E. ONeil, PhD, Portland VAMC/OHSU, 3710 SW US Veterans Hospital Rd, Portland, OR 97239.E-mail: maya.oneil@ va.gov

M. YUTSIS, N. KARANDIKAR, O. HARRIS \& S.A. ROPACKI. Treatment Effect Versus Natural Recovery in Moderate to Severe Traumatic Brain Injury: Efficacy of Postacute Rehabilitation.

Objective: To evaluate functional improvement following a moderate to severe Traumatic Brain Injury (TBI) after postacute rehabilitation with an emphasis on time since injury in order to examine the impact of natural recovery on treatment effects.

Participants and Methods: A total of 91 (moderate TBI=26; severe $\mathrm{TBI}=65$, females $=4$ ) patients admitted for comprehensive treatment at a postacute rehabilitation facility in the Veterans hospital completed comprehensive evaluations on admission and discharge including a neurobehavioral functioning measure (Mayo Portland Adaptability Inventory $[\mathrm{MPAI}-4])$. The patients were grouped by the time since injury (0-6 months [n=69], 6-24 months [n=12], >24 months[n=9]) and by the severity of neurobehavioral impairment at admission (moderate to severe $[\mathrm{n}=16]$ and minimal to mild $[\mathrm{n}=75]$ on MPAI- 4 total score). Rate of progress on the MPAI was calculated by the difference between admission and discharge scores /by length of stay. Two separate $2 \times 2$ MANOVAs with rate of progress on the MPAI-4 Total and subscale scores as dependent variables and either time since injury with the TBI severity or the severity of functional impairment at admission with the TBI severity entered as between-subjects factors.

Results: $82 \%$ of all patients showed clinically meaningful gains on the MPAI total score from admission to discharge. Age at the time of injury was not correlated with either outcome measure. The time since injury had a significant impact on gains made in rehabilitation $(F(2,88)=5.3$, $\mathrm{p}=.01, \eta=.12$ ) with patients in $0-6$ months post injury group outperforming each other group $(p=.03)$ regardless of the TBI severity or level of functional impairment on admission $(\mathrm{F}(2,88)=3.4, \mathrm{p}=.04, \eta=.08)$. Conclusions: Findings support efficacy of postacute rehabilitation in patients with moderate to severe TBI based on functional gains seen in patients with TBI beyond what can be explained by spontaneous recovery trajectory alone.

Correspondence: Maya Yutsis, PhD, Polytrauma, VA Palo Alto HCS, 3804 Miranda Ave, Palo Alto, CA 94304.E-mail:maya.yutsis@va.gov

J.L. PONSFORD, G. SPITZ, M. DOWNING \& D. MCKENZIE. Mortality Following Traumatic Brain Injury and Rehabilitation. Objective: The current study examined the rate and causes of mortality following mild to severe traumatic brain injury (TBI) and rehabilitation. Participants and Methods: Rates of death and survival were examined 1-26 years post-injury for 3341 individuals with mild to severe TBI, admitted to a rehabilitation program. A multivariate model of mortality was developed.

Results: 313 deaths were observed, equating to a mortality rate of $9.4 \%$. The standardised mortality ratio (SMR) was $1.05(95 \% \mathrm{CI}=$ $1.05-1.06)$. A slight but significantly elevated SMR of $1.20(95 \%$ $\mathrm{CI}=1.06-1.37)$ was observed for males, and a non-significant SMR of $.99(95 \% \mathrm{CI}=.79-1.23)$ for females. Elevated risk of death from external causes were found for both males ( SMR $=2.32,95 \% \mathrm{CI}=2.32$ -3.82 ) and females ( $\mathrm{SMR}=4.48,95 \% \mathrm{CI}=1.05-1.06)$. Females also had a significantly elevated SMR of $5.02(95 \% \mathrm{CI}=1.36-12.80)$ for intentional self-harm. Younger individuals had a two-fold increase in mortality, compared to the general population. The multivariate model showed increased risk of mortality was associated with older age, being male, being unemployed prior to injury, history of stroke, alcohol use, mental health issues, and a back injury sustained in the accident.

Conclusions: Premorbid lifestyle factors exerted a greater influence on mortality following TBI, than injury related factors. This risk was especially prominent for younger individuals, who died primarily due to external causes. These findings highlight the need for interventions addressing premorbid substance abuse and mental health issues. 
Correspondence: Jennie L. Ponsford, PhD, Psychological Sciences, Monash Ujniversity, School of Psychological Sciences, Building 17, Wellington Road, Clayton, VIC 3800, Australia. E-mail: jennie. ponsford@monash.edu

\section{E. CHAMARD, G. LEFEBVRE, M. LASSONDE \& H. THEORET. White matter long-term abnormalities in the corpus callosum of female concussed athletes.}

Objective: Concussion is now recognized as an injury affecting annually millions of people with a portion of them suffering from long-term sequelae. Recent studies have demonstrated long-term abnormalities in white matter (WM) in male athletes, notably in the corpus callosum (CC) and the corticospinal tracts (CST). However, these aspects have never been investigated in female athletes despite a higher risk of suffering a concussion, and a higher severity of symptoms.

Participants and Methods: 8 healthy, unconcussed female athletes were compared to 10 female athletes six months post-concussion. Groups were equivalent with regards to age and level of education. Diffusion tensor imaging of the CC and CST was conducted in a 3T MRI scanner.

Results: Analysis in the CC was divided in 7 regions based on a previous study (Hofer et al., 2006). DTI analysis showed no significant effects in the CST but revealed differences between groups notably in the anterior part of the CC. The concussed group had lower mean diffusivity (CC1: $\mathrm{t}=2,60, \mathrm{p}<.05)$, lower radial diffusivity (CC1: $\mathrm{t}=1,05, \mathrm{p}<.05 ; \mathrm{CC} 2$ : $\mathrm{t}=2.94, \mathrm{p}<.05)$, lower axial diffusivity (CC6: $\mathrm{t}=2,23, \mathrm{p}<.05)$, and a lower volume of WM fibers (CC3: $\mathrm{t}=2,14, \mathrm{p}<.05)$.

Conclusions: Long-term alterations in the corpus callosum of female athletes appear to affect the anterior part of the CC projecting to the frontal and motor areas. Further studies are needed to determine whether these alterations are related to the higher risk of concussed athletes to sustain a subsequent injury.

Correspondence: Emilie Chamard, University of Montreal, 90 Vincent d'Indy, Montréal, QC H2V 2S9, Canada.E-mail: emilie.chamard@ umontreal.ca

C. HAYS, D.C. OLSEN, S. MA, N. WHITSEL, H.J. ORFF, A.J. JAK \& E.W. TWAMLEY. Predictors of Academic and Employment Status in Veterans with a History of Traumatic Brain Injury.

Objective: While most people who experience mild traumatic brain injury (TBI) recover rapidly and return to their everyday activities, some show cognitive impairment and persistent postconcussive symptoms, which can limit functional recovery, including return to work or school. We explored demographic, psychiatric, neuropsychological, and postconcussive factors as predictors of academic and employment status in Veterans with a history of TBI.

Participants and Methods: We reviewed the neuropsychological reports of $414 \mathrm{OEF} / \mathrm{OIF}$ Veterans referred for clinical assessment of possible TBI-related cognitive complaints and had valid neuropsychological testing. Their TBI severity ratings were $85 \%$ mild, $9 \%$ moderate, and $6 \%$ severe. All patients were administered a comprehensive neuropsychological battery and employment and academic information was collected. Pearson correlations were used to identify significant demographic, brain injury, psychiatric, postconcussive, and neuropsychological variables associated with work and school participation. Variables that were significantly associated $(p<.05)$ were then entered into logistic regressions predicting work and school status.

Results: Age was the only predictor of academic status, with younger Veterans more likely to report being in school $(p<.001)$. Male sex $(p=.018)$, higher level of education $(p=.013)$, better processing speed $(p=.005)$, and less severe somatic postconcussive symptoms $(p=.017)$ predicted current employment.

Conclusions: Neuropsychological performance (faster processing speed) and less severe postconcussive svmptoms are associated with higher rates of employment in Veterans with a history of TBI, whereas severity of brain injury was not associated with employment. Evidence-based interventions addressing cognitive functioning and postconcussive symptoms may lead to better employment outcomes in this population. Correspondence: Chelsea Hays, UC San Diego, 1813 reed ave \#3, San Diego, CA 92109. E-mail: chelseahays@gmail.com

H.M. GENOVA, C. CAGNA, N.D. CHIARAVALLOTI, G. WYLIE \& J. LENGENFELDER. Facial Affect Recognition in Traumatic Brain Injury: an fMRI study.

Objective: Individuals with Traumatic Brain Injury (TBI) experience a wide range of cognitive and psychological difficulties, due to the diffuse nature of traumatic axonal injury. Recent research has shown that individuals with TBI have significant impairments in emotional processing, and specifically facial affect recognition. In the current study, facial affect recognition in TBI was examined using functional magnetic resonance imaging (fMRI).

Participants and Methods: Patterns of cerebral activation during performance of facial affect recognition task for a group of individuals with moderate to severe TBI was compared to a group of matched healthy controls (HCs). The task used in the scanner was the Queen Square Face Discrimination Task, during which subjects were asked to identify emotions from a series of facial stimuli developed by Dr. Paul Ekman. Results: During performance of the facial affect recognition task, individuals with TBI showed several brain regions of increased activation compared to HCs including the fusiform gyrus, parahippocampal gyrus, anterior cingulate and caudate. Additionally, the TBI group showed reduced brain activation compared to HCis in the cuneus.

Conclusions: During a task of facial affect recognition, the brain activation patterns of the TBI group differed from the HCs in several important ways. Increased activation in visual areas was noted, including the fusiform gyrus, as well as attentional regions such as the anterior cingulate gyrus. This is the first study to our knowledge to examine the neural substrates underlying facial affect recognition in TBI using fMRI. Correspondence: Helen M. Genova, Ph.D., Kessler Foundation Research Center, 300 Executive Dr., Suite 70, West Orange, NJ 07052. E-mail: hgenova@kesslerfoundation.org.

M. AMICK, C.B. FORTIER, W.P. MILBERG \& R. MCGLINCHEY. Number and Severity of Mild TBIs and PTSD Symptoms Predict Neuropsychological Performance in OEF/OIF Veterans.

Objective: A persisting effect of mild TBI upon neuropsychological performance has not been consistently demonstrated. Inconsistent findings could be due to several factors including: the severity and number of mild TBIs, presence of posttraumatic stress disorder (PTSD), or the interaction of these comorbid conditions. The current study examined the independent effects of PTSD, number and severity of mild TBIs acquired across a lifetime, and the interaction of PTSD and lifetime mild TBI upon neuropsychological performance in OEF/OIF Veterans. Participants and Methods: Neuropsychological tests and semi-structured interviews assessing PTSD and lifetime TBIs were completed with $288 \mathrm{OEF} / \mathrm{OIF}$ Veterans. Participants were excluded if they failed effort measures. Factor analysis reduced the neuropsychological data into five domains: verbal memory, speeded information processing, executive functioning, set shifting, and visual memory. Lifetime number of very mild TBI (vmTBI, defined as AMS and/or PTA $<15$ minutes and no LOC) and mild TBIs (mTBI, defined as any LOC $<30$ minutes and/ or AMS / PTA >15 minutes but< 24 hours) was calculated for each participant.

Results: Multiple regression analyses revealed that increasing PTSD symptom severity predicted worse verbal memory factor scores (Beta=$0.23, p=0.002)$ and increasing lifetime number of mTBIs predicted poorer set shifting factor scores (Beta $=-0.14, p=0.03$ ). The interaction of PTSD symptom severity and number of lifetime mTBIs predicted speeded information processing factor scores (Beta=0.21, $\mathrm{p}=0.005$ ). Conclusions: Mild TBI acquired across a Veteran's lifespan predicts speeded information processing and set shifting performance when 
number and severity of mild TBI and its interaction with PTSD symptom severity are considered.

Correspondence: Melissa Amick, VA Boston Healthcare System, $150 \mathrm{~S}$ Huntington Street, Boston, MA 02130. E-mail:melissaamick@gmail. com

R.T. KIM, A.L. CLARK, S. SORG, A.J. JAK, K.L. HANSON, H.J. ORFF, L. DELANO-WOOD \& D.M. SCHIEHSER. Physical Fatigue and Vegetative Symptoms Best Predict Quality of Life (QoL) in Veterans with Mild to Moderate Traumatic Brain Injury. Objective: Fatigue, sleep disturbance, and depression have been found to be the most relevant post-concussive symptoms related to poor quality of life (QoL) in Veterans with mild-moderate traumatic brain injury (mmTBI). However, little is known about which aspects of these multi-factorial symptoms may be most associated with different facets of QoL in this population.

Participants and Methods: mmTBI Veterans $(n=50)$ were administered the World Health Organization Quality of Life (WHOQOL)-BREF as a measure of QoL as well as measures of fatigue (Modified Fatigue Impact Scale; MFIS), sleep (Pittsburgh Sleep Quality Index; PSQI), and depression (Beck Depression Inventory-II; BDI-II). Four WHOQOL-BREF subscales and factor-analytic-derived subscales of the MFIS, PSQI and BDI-II were examined.

Results: Stepwise regressions revealed that overall elevations in fatigue (MFIS) and sleep problems (PSQI) best predicted worse Physical QoL, while fatigue alone best predicted Psychological and Social QoL and depression (BDI-II) best predicted Environmental QoL (p's<.03). Of these significant predictors, Physical Fatigue, Sleep Quality, and Sleep Latency best predicted Physical QoL $(p$ 's<.02) and Physical Fatigue best predicted both Psychological and Social QoL ( $p$ 's<.001); while Vegetative Symptoms of the BDI-II alone predicted Environmental QoL $(\mathrm{p}<.001)$.

Conclusions: Physical fatigue and vegetative symptoms are the most significant symptoms associated with worse QoL related to physical health, psychological well-being, social relationships, and everyday activities within the environment. Sleep quality and latency are also associated, albeit to a lesser degree, to physical health QoL. These findings underscore the importance of neurovegetative/somatic symptoms in QoL and could provide potential treatment targets to improve specific areas of QoL in Veterans with mmTBI.

Correspondence: Russell T. Kim, B.S. - Psychology, Research, VA San Diego Healthcare System, Veterans Medical Research Foundation, 3350 La Jolla Village Dr., San Diego, CA 92161. E-mail: russelltkim@gmail. com

J. RAKIC, T. JANTZ, R. DAVIS, D. KRAWCZYK \& S. CHAPMAN. Pre and Post Daily Functioning Comparison in Mild and Moderate TBI.

Objective: The purpose of this abstract is to compare pre and post daily functioning data in moderate and mild TBI participants that took part in training programs.

Participants and Methods: Seventy-one participants with mild and moderate TBI ( 40 male, 31 female) in chronic stages of recovery ( $>6$ months post TBI), between 19 to 65 years of age $(\mathrm{M}=42.80, \mathrm{SD}=$ 14.31), and an IQ range of 73 to $141(\mathrm{M}=108.70, \mathrm{SD}=11.32)$. Moderate and mild TBI was categorized using the GOS-E score of either 4 or 5 and 6 or 7 respectively. All participants were tested at baseline and after the completion of their respective training programs. The participants were randomly assigned to two different trainings that focused either on learning strategies (SMART program), or brain health (BHW program). Results: A paired sample t-test analysis found significant change $(p \leq$ $.05)$ between pre and post daily functioning measures like FSE, BDI-II, and Awareness Questionnaire.

Conclusions: This study extends previous evidence that there is a significant change in daily functioning of TBI participants after they participate in a training program. Training programs involve group activities, discussions, home assignments, and a sense of responsibility. Thus, individuals feel that their daily functioning has improved because they are socializing, learning new concepts, and are surrounded in a positive atmosphere. An improved understanding of how to prevent social isolation in individuals with a TBI could potentially aid in developing beneficial training programs.

Correspondence: Jelena Rakic, Center for BrainHealth, University of Texas at Dallas, 2200 West Mockingbird Lane, Dallas, TX 75235. E-mail:j.rakic@utdallas.edu

T.P. COTHRAN \& E.B. LARSON. The Relationship Between Sleep Variability and Reaction Time Performance Across CPT-II Blocks in Traumatic Brain Injury Inpatients.

Objective: Sleep disturbance contributes to fatigue in people with traumatic brain injury (TBI) and is associated with impaired selective attention (Ponsford et al., 2012). For example, persons with obstructive sleep apnea and moderate TBI were administered a vigilance task that demonstrated a relationship between sleep disturbance and sustained attention (Wilde et al., 2007). Sleep variability is one form of sleep disturbance that was shown to be associated with cognitive impairment in other neurological populations (e.g., Westerberg et al., 2010). It is unknown if sleep variability in TBI is related to declines in performance across the duration of a sustained attention task.

Participants and Methods: Twenty-eight inpatients with TBI (Rancho Los Amigos Scale $=4$ or 5 ) were administered the Connors' Continuous Performance Test (CPT-II) as part of a larger treatment study. Over the five nights before CPT-II administration, nursing logged hourly observation of sleep. Sleep variability was operationalized as the within-subject sleep standard deviation across the five nights. Bivariate correlations were conducted comparing sleep variability and the slope of reaction times and standard errors across blocks.

Results: Sleep variability was associated with slope of raw reaction times and with slope of standard errors across blocks $(r=0.368, p=$ 0.054 and $\mathrm{r}=0.365, \mathrm{p}=0.056$, respectively).

Conclusions: Sleep variability appears to be positively associated with sustained attention scores in TBI, with greater variability evincing longer and less consistent reaction times. Quantifying variance in CPT-II block change data associated with sleep disturbance may be a means to index severity of fatigue in these patients.

Correspondence: Thomas P. Cothran, Illinois Institute of Chicago, 3456 North Hoyne Avenue, Chicago, IL 60618. E-mail: tcothran@hawk.iit. edu

K. Pagulayan, E. PETrie, M. Reiter, E.R. PESKIND \& N. KLEINHANS. Differential Effects of Repeated Blast-Related mTBI on Limbic and Higher Level Cognitive Systems: A Resting State fMRI Study.

Objective: Changes in cognitive and emotional functioning are common among Veterans with a history of repeated blast-related mTBI. Despite the significant functional impact of these symptoms, the underlying neuropathology remains unknown. This study evaluated functional connectivity from the amygdala and cerebellum, two brain regions that may be important to these ongoing symptoms.

Participants and Methods: Resting state fMRI data were analyzed on 37 OIF/OEF/OND Veterans, of which 22 had a history of repeated blast-related mTBI. Functional connectivity analyses were conducted from three cytoarchitectonically determined amygdala subregions: centromedial (CM), laterobasal (LB) and superficial (SF) and the right and left Crus 1 of the cerebellum.

Results: From the cerebellar seed regions, the mTBI group showed significantly decreased connectivity to the superior, middle, and inferior frontal gyri, the superior parietal lobes, and the middle temporal gyrus. Significantly increased connectivity was observed to limbic regions including the bilateral amygdala, bilateral insula, bilateral nucleus accumbens, and the hypothalamus. In the amygdala analyses, the mTBI group showed evidence of overconnectivity from the $\mathrm{CM}$ seed region to 
the pons, midbrain, and cerebellar vermis and underconnectivity to the bilateral temporal lobes. In contrast, the mTBI group showed evidence of underconnectivity from the LB seed region to the cingulate gyrus, medial prefrontal cortex, middle frontal gyrus, and the superior parietal lobes. Overconnectivity was seen between the LB subregion and the bilateral hippocampal gyri.

Conclusions: Our findings provide new evidence of distinct patterns of abnormal functional connectivity in Veterans with a history of repeated blast-related mTBI. Notably, in the mTBI group, we find evidence of overconnectivity within limbic and brainstem regions associated with emotional processing and autonomic arousal and underconnectivity to cortical regions associated with higher order cognitive processing. Correspondence: Kathleen Pagulayan, PhD, VA Puget Sound, $1660 \mathrm{~S}$ Columbian Way, S-116-MIRECC, Seattle,WA 98115.E-mail:farkat@u. washington.edu

M.G. JEFFREY, K. GRAJZEL \& B.E. GAVETT. Psychological Distress Has a Larger Effect on Baseline Concussion Test Performance than Brain Injury History.

Objective: The purpose of this study was to assess the effects of premorbid psychological distress and past brain injuries on baseline cognitive performance. Previous research has proposed that psychological distress, particularly distress related to depression and anxiety, decreases cognitive performance on Simple Reaction Time, Complex Reaction Time, and Processing Speed tasks measured by the Concussion Resolution Index (CRI; Bailey, Samples, Broshek, Freeman, \& Barth, 2010). Participants and Methods: The Brain Injury Screening Questionnaire (BISQ), which asked about past brain injuries, and the Personality Assessment Screener (PAS), which assessed psychological distress, were administered to 124 Division II collegiate athletes at the same time as the baseline administration of the Immediate Post-Concussive Assessment (ImPACT). Multiple linear regression was conducted to evaluate the effects of prior brain injury (number or self-reported brain injuries) and PAS scores on four different ImPACT variables (Visual Memory, Verbal Memory, Motor, and Reaction Time). PAS variables included scores for Negative Affect, Acting Out, Social Withdrawal, Alienation, and Anger. Results: Negative Affect had the largest effect on a standardized composite of ImPACT baselines scores, $b=-0.23$ (95\%CI [-0.36, -0.09]). On individual ImPACT variables, Negative Affect exerted a significant effect on Visual Memory, b = -3.60 (95\% CI [-5.35, -1.86]) and Verbal Memory performance, $\mathrm{b}=-2.56$ (95\% CI [-3.88, -1.23]), while Motor and Reaction Time were not affected by any of the predictor variables (overall model R2 $=0.04$ for both). Number of past head injuries produced small, non-significant effects on all baseline ImPACT test scores. Conclusions: Personal distress, such as depression and anxiety, contributed more to baseline ImPACT test scores than the number of past head injuries. These results highlight the importance of assessing mood and psychological distress along with cognitive test performance in baseline assessment of athletes.

Correspondence: Katalin Grajzel, Master's of Art, Psychology, University of Colorado Colorado Springs, 924 Descendant Dr., Fountain, CO 80817.E-mail: lini676@yahoo.com

K.L. HANSON, D.M. SCHIEHSER, R.T. KIM, N. LUC, A.L. CLARK, S. SORG \& L. DELANO-WOOD. Alcohol-Related Psychosocial Problems are Associated with TBI Injury Characteristics, Greater Post-Concussive Symptomatology, and Poorer Cognition in Veterans with a History of Mild TBI.

Objective: Since little is known about alcohol use patterns and associations with cognitive and psychiatric functioning among veterans with a history of mild traumatic brain injury (mTBI), we aimed to (1) characterize alcohol use among mTBI veterans, (2) examine its relationship to mTBI and psychiatric symptoms, and (3) determine associations with neuropsychological performance.

Participants and Methods: Thirty-seven veterans ( $\mathrm{n}=21 \mathrm{mTBI}$; $\mathrm{n}=16$ military controls [MCs]; mean age $=32.11 ; 22 \%$ women; $55 \%$
Caucasian) were assessed for problem alcohol use (modified Customary Drinking and Drug Use Record [CDDR]), psychiatric (depression, anxiety, and post-traumatic stress disorder [PTSD]) symptoms, and cognition. Participants reporting current DSM-IV alcohol or substance dependence were excluded.

Results: The mTBI group reported more alcohol-related psychosocial problems (e.g., fights, poor judgment, physical injuries, emotional problems) relative to MCs $(p<.03)$. Within mTBI, more lifetime alcohol-related psychosocial problems were associated with combat exposure, longer post-traumatic amnesia from blunt injury, and higher depression, anxiety, and PTSD symptoms, as well as neurobehavioral symptoms (rhos=.33 to .55 , ps<.05). Additionally, greater lifetime withdrawal symptoms were associated with poorer attention and visual learning (rhos=-.52 to $-.57, \mathrm{ps}<.03$ ), while recent alcohol-related psychosocial problems were associated with poorer executive functioning ( $r$ hos $=-.53$ to -.73 , ps<.03).

Conclusions: Our findings suggest that lifetime alcohol-related psychosocial or withdrawal symptoms may affect post-concussive symptomatology and cognitive functioning in some veterans with a history of mTBI. Veterans with mTBI who are combat-exposed or with a longer post-traumatic amnesia may be at higher risk for alcohol-related psychosocial problems. A thorough assessment of alcohol-related problems may be important for understanding the sequela of mTBI in veterans. Additional research on pre- and post-mTBI alcohol-use patterns is needed.

Correspondence: Karen L. Hanson, Ph.D., VA San Diego Healthcare System, 3350 La Jolla Village Drive, 151B, San Diego, CA 92161. E-mail:klhanson@ucsd.edu

H.K.RAU, K.PAGULAYAN,J.B.SHOFER, G. LEE, E.A.COLASURDO, R.S. HANSON, M.L. WERHANE, D.K. MORELLI, E.R. PESKIND \&. C.W. WILKINSON. Comorbid Pituitary Dysfunction and mTBI Characterized by Specific Weaknesses in Verbal Memory: Evidence from Combat Veterans with Repeated Blast-Related mTBI.

Objective: We have previously reported a high prevalence of chronic hypopituitarism (CHP) after blast-related mild traumatic brain injury (mTBI). Preliminary evidence suggests that the presence of CHP accentuates impairments in attention, learning, and memory in mTBI patients. The purpose of this study was to identify specific cognitive weaknesses characterizing comorbid mTBI and CHP.

Participants and Methods: Combat Veterans with $(\mathrm{n}=39)$ and without $(n=16)$ a history of blast-related mTBI were assessed for chronic pituitary dysfunction and administered the Wechsler Test of Adult Reading (WTAR), Trail Making Test (TMT), Letter-Number Sequencing (LNS), Symbol Digit Modalities Test (SDMT), and California Verbal Learning Test-Second Edition (CVLT-II). Group differences were evaluated using T-tests and ANOVA.

Results: CHP was identified in $32 \%(\mathrm{n}=12)$ of mTBI Veterans (mTBI+CHP). Groups with and without CHP did not differ across age, education, and measures of general intelligence (WTAR) and attention (TMT, LNS, SDMT). However, mTBI+CHP Veterans performed significantly worse $(\mathrm{p}<.05)$ than mTBI-CHP and deployed controls across CVLT-II trials, including learning (total correct trials 1-5), short-delay free and cued recall trials, and long-delay free and cued recall trials. Conclusions: Prevalence of CHP in mTBI Veterans was consistent with prevalence rates observed in other mTBI populations. Importantly, Veterans with comorbid mTBI and CHP exhibited global difficulties on verbal learning and memory despite intact performances in other domains. Future research is needed to determine whether verbal memory impairment is unique to CHP following mTBI, especially in younger mTBI populations with otherwise preserved cognitive functioning. CHP treatment is effective, and earlier detection could improve mTBI outcomes. Correspondence: Holly K. Rau, MS, MIRECC, VA Puget Sound, 6528 42nd Ave NE, Seattle, WA 98115. E-mail: hollyrau@gmail.com 
A.L. CLARK, D.M. SCHIEHSER, S. SORG, M.W. BONDI, N. LUC, R.T. KIM \& L. DELANO-WOOD. Mild Traumatic Brain Injury (mTBI) Moderates the Association Between White Matter Lesion Burden and Memory.

Objective: Accumulating evidence has shown that diffuse axonal injury (DAI) results in white matter lesion (WML) pathology after severe head trauma; however, little is known about WMLs in mild TBI (mTBI). Additionally, few studies have investigated the relationship between WMLs and cognitive dysfunction in individuals with head trauma. The current study therefore explored associations between WML pathology and cognition in Veterans with and without history of mild TBI.

Participants and Methods: 45 Veterans (mTBI=23, Military Controls $[\mathrm{MCs}]=22]$ ) underwent structural magnetic resonance scanning (MRI) and cognitive assessment. Fluid attenuated inversion recovery images were examined for deep white matter lesion (DWML) and periventricular lesion (PVL) volumes. Memory performance was assessed using the California Verbal Learning Test-II, and psychiatric symptoms were rated using the Beck Depression Inventory-II and the Posttraumatic Stress Checklist

Results: WML volumes were not associated with age or psychiatric symptomatology $(p ' s>.05)$. MC and mTBI groups did not differ in Total WML or WML subtype volumes (p's > .05). MANOVAs revealed a significant Group x PVL volume interaction on memory performance (Wilk's $\lambda=.743, \mathrm{p}=.04$ ), such that poor memory was uniquely associated with increased PVL volume in the mTBI group. There were no significant associations between DWML volume and cognition in either group ( $\mathrm{p}$ 's $>.05)$.

Conclusions: Results show that mTBI may not result in increased WML pathology. However, PVL, not DWML, volumes were associated with poor memory performance in participants with history of mTBI. Findings suggest that PVLs may exert a more deleterious effect on cognition in those with a history of mild neurotrauma.

Correspondence: Alexandra L. Clark, B.S., UCSD/SDSU Joint Doctoral Program, 3350 La Jolla Village Dr., San Diego, CA 92161. E-mail: alexandra.leigh.cl@gmail.com

A. VAS \& S. CHAPMAN. Expanding Measurement of Abstract Thinking in Adults with Traumatic Brian Injury.

Objective: Impaired abstract thinking is purportedly a common consequence of TBI. There is a void of metrics to characterize functionally relevant higher-order abstract thinking abilities. The current study compared abstract thinking ability between adults with moderate to severe TBI and healthy controls on standard abstraction measures (Similarities and Proverbs) and a functionally relevant skill of abstracting meaning from complex information (Gist reasoning).

Participants and Methods: The current study is a part of a larger study. Thirty adults with moderate to severe TBI (19-55yrs. M=38.53) in chronic stages of recovery (> 1-yr. post injury) and 40 healthy controls (19-50 yrs. Mean=37.67) with comparable IQ, education, and socioeconomic status participated in the study. Performance on Similarities, Proverbs, and Gist reasoning was compared between groups on Analysis of Variance.

Results: A distinctly divided spectrum of performance was found between the TBI and control groups. Performance was comparable ( $p>.05)$ between groups on the relatively simpler task of Proverb interpretation on a multiple-choice format. However, TBI group performance was significantly impaired on increasingly complex tasks of Proverbs-free inquiry format $(p=.06)$, Similarities $(p=.02)$, and Gist reasoning $(p<.001)$. Conclusions: A TBI can have lasting impact on abstraction abilities even in chronic stages of recovery. Specifically, higher-order abstraction involving synthesizing meanings from daily life relevant complex information (Gist reasoning) could be informative in objectively measuring higher-order abstraction abilities. Furthermore, Gist reasoning performance could guide rehabilitation regime and monitor training outcomes.
Correspondence: Asha Vas, PhD, Center for BrainHealth, University of Texas at Dallas, 3213 Wells Drive, Plano, TX 75093. E-mail: asha. vas@utdallas.edu

J. COHEN, S. VARDHAN, D. SANCHEZ DIAZ, A. LEOW \& M. LAMAR. Differential Predictors of Stroke and Cardiovascular Disease as They Relate to Brain Structure and Function: Implications for the Human Connectome.

Objective: Cardiovascular disease (CVD) and stroke affect over 42.2 million individuals over 60 years of age. Risk prediction scores exist to quantify the likelihood of developing CVD or having a stroke, taking into account various albeit slightly different vascular risk factors (VRFs). While these algorithms may predict differential medical outcomes, it is unclear whether they also predict differential cognitive or neuropathological outcomes.

Participants and Methods: 29 non-demented, non-depressed adults 60 years or older participated in a comprehensive assessment of vascular risk, cognition and brain imaging. The Framingham Study risk prediction scores for probability of CVD and stroke within 10-years were used to examine differences in vascular risk and their associations to cognitive outcomes including composite $\mathrm{z}$-scores of executive functioning (EF), language, learning/memory/recognition, and attention/ information processing. White matter hyperintensity (WMH) volumes were quantified using T2-FLAIR.

Results: A paired-sample t-test of risk prediction scores revealed greater CVD than stroke risk in our sample, $t(28)=-3.2 ; p=.003$. Given our small sample size, we focused on differences in resulting Pearsons correlational r-values between CVD and stroke risk as related to cognitive $\mathrm{z}$-scores and WMH volumes. Hotelling's t/Steiger's z revealed significant differences favoring stroke risk associations for language, $\mathrm{EF}$ and WMH volumes (all p-values<.05).

Conclusions: Within our population, CVD risk may be a more sensitive medical predictor of future adverse events while stroke risk may be a more sensitive measure of brain correlates. Our results, while preliminary, have implications for behavioral outcomes and we are currently exploring their relevance to the human connectome using multi-modal image analysis techniques.

Correspondence: Jamie Cohen, University of Illinois at Chicago, 1601 West Taylor Street, MC 912, Chicago, IL 60612.E-mail: jcohen@psych. uic.edu

B. BUDISIN, Y. KOSHIMORI, B. SHARMA, C. BRADBURY, N. MENEZES, D. MIKULIS \& R. GREEN. Preliminary Evaluation of the Diagnostic Utility of Diffusion Tensor Imaging (DTI) for Detecting Mild Traumatic Brain Injury (mTBI) in Spinal Cord Injury (SCI) Patients.

Objective: Diagnosis of mild traumatic brain injury (mTBI) in spinal cord injury (SCI) population is frequently missed due to insensitivity of the current diagnostic tools to the microscopic neuropathology associated with mTBI. Our ability to detect the enduring effects of $\mathrm{mTBI}$ is also challenging, and moreover controversial, with some studies questioning the existence of a post-concussion syndrome with an organic basis. Thus, the diagnosis of mTBI using objective and more sensitive assessment tools such as diffusion tensor imaging (DTI) is an important clinical gap. Participants and Methods: First, we conducted a review of all published studies of TBI-induced fractional anisotropy (FA) changes to identify the regions of interest (ROIs) that best discriminate mTBI patients from healthy controls. Second, healthy controls and SCI patients with concurrent mTBI based on neurological, neuroimaging, and neuropsychological data received DTI two- to six-months post-injury. The FA values in our sample of mTBI patients with concurrent SCI $(\mathbf{n}=9)$ were compared to those of age-matched, healthy controls $(n=14)$ for each identified ROI, allowing us to discern the regions most susceptible to microscopic axonal injury following mTBI.

Results: In study 1, 14 midline and bilateral structures that most reliably discriminate between mTBI patients and controls were identified. 
Two regions containing corticospinal tracts were excluded due to their vulnerability to transneuronal degeneration secondary to SCI. In study 2 , multivariate analysis of variance (MANOVA) was conducted. The mTBI group had significantly lower $(\mathrm{p}<0.05) \mathrm{FA}$ values as compared to the controls in the genu of corpus callosum as well as right and left anterior corona radiata.

Conclusions: These preliminary findings provide evidence for: (i) increased DTI diagnostic sensitivity for mTBI when assessing FA values in specific ROIs; and (ii) enduring organic changes that might be associated with the post-concussive symptoms. Further research is needed to confirm these findings.

Correspondence: Bojana Budisin, Ph.D., Cognitive Neurorehabilitation Sciences Lab, Toronto Rehabilitation Institute, 23 Sheppard Avenue East, Unit 307, Toronto, ON M2N 0C8, Canada. E-mail: bojana. budisin@uhn.ca

S.A. EVANS, A. AHN, J.J. TANNER \& C. PRICE. Evidence for a Central Disorder of Pain in PD and Its Relevance to PD Cognition. Objective: Pain in Parkinson's disease (PD) has been attributed to the musculoskeletal complications of the movement disorder. Lacking definition are: 1) the neurological contributions to pain intensity and pain related disability, and, 2) relationships between pain and PD cognitive profiles.

Participants and Methods: From a federally funded investigation studying the neuroanatomical contribution to cognitive profiles of medicated non-demented individuals with idiopathic Parkinson's disease (PD; $\mathrm{n}=40$; UPDRS-III =17.60(10.73): age $=67.80(5.40))$ and non-PD matched peers $(n=40$; UPDRS-III $=2.75(3.36)$, age $=68.20(4.60)$ ) we used the Brief Pain Inventory-Short Form (Cleeland, 1991) to examine hypotheses regarding 1) group differences in pain intensity versus pain disability and 2) associated neuroanatomical and cognitive associations. All individuals completed the same neuropsychological measures and structural brain magnetic resonance imaging protocol (3T, Siemens). Results: PD had higher levels of pain-related disability ( $P D=11.40$ \pm 12.66 ); non-PD=3.98 $\pm 8.94 ; \mathrm{p}<.01$ ) despite similar levels of pain intensity $(\mathrm{p}=.05)$. Only for PD, pain ratings were negatively associated with thalamic volume (interference $\mathrm{r}=-0.45, \mathrm{p}<.01$; intensity $\mathrm{r}=-0.39$, $\mathrm{p}=.01$ ), with the amygdala associated only with pain interference (interference $=-0.38, \mathrm{p}=.02$; intensity $\mathrm{r}=-0.28 ; \mathrm{p}=.08)$, and a trend for the putamen with intensity (interference $=-0.22, \mathrm{p}=.16$; intensity $\mathrm{r}=-0.31$, $\mathrm{p}=.05)$. Pain ratings were not associated with motor severity, but did increase with years of $\mathrm{PD}(\mathrm{r}=0.41, \mathrm{p}<.01)$. Only for $\mathrm{PD}$, increased pain intensity associated with reduced processing speed $(\mathrm{r}=-0.38, \mathrm{p}=.02)$ and inhibitory functions $(\mathrm{r}=-.36, \mathrm{p}=.03)$.

Conclusions: Data show evidence for a central disorder of pain regulation in PD whose mechanisms may be distinct from the specific pathophysiology of the movement disorder. Pain was uniquely contraindicative for cognition in PD.

Supported by NINDS K23NS060660 (CP); 1R01NS082386 (CP)

Correspondence: Samantha A. Evans, University of Florida, 10110

Woodsong Way, Tampa, FL FL. E-mail: sevans14@ufl.edu

\section{J.C. TOMASZCZYK, B. COLELLA, B.K. CHRISTENSEN \& R. GREEN. Recovery of Variability in Attention Task Performance after Moderate-Severe Traumatic Brain Injury.}

Objective: Increased variability in cognitive performance is a hallmark of moderate-severe traumatic brain injury (TBI), but few studies have examined recovery of variability. Given recent demonstrations of $\operatorname{cog}$ nitive and neural declines in chronic TBI, we longitudinally examined changes in variability from 2 months to $2+$ years post-injury to evaluate the trajectory of recovery of variability early post-injury, and the possibility of chronic stage decline.

Participants and Methods: Data were collected in 46 moderate-severe TBI patients $(71.7 \%$ men; mean age $=40.2$ years; mean education $=$ 14.7 years) who had completed the Sustained Attention to Response Task (SART) at 2, 5, 12, and 24+ months post-injury in a prospective, longitudinal study. The SART is a computerized, go/no-go reaction time task designed to measure sustained attention.

Results: To examine recovery of variability while accounting for RT performance, coefficient of variability scores (COV; amount of variability as a percent of RT) were calculated. COV decreased (i.e., improved) from 2 to 5 to 12 mos. post-injury, but significantly increased (i.e., worsened) from 12-24+ mos. RT improved significantly from 2 to 5 months post-injury, with a gradual asymptote in performance thereafter.

Conclusions: Longitudinal performance on the SART from 2 to 24+ months post-injury revealed significant early recovery of RT, with a gradual plateau by $24+$ months post-injury. Although variability of RT performance (COV) also showed early improvement, later decline from 12 to $24+$ months was observed, with significantly increased variability in responding in the late stages of injury. These findings suggest that 1) recovery trajectories of RTs and of variability in attention task performance are at least partially dissociable constructs in this population, and 2) greater RT variability on this attention task over time may be a behavioural correlate of chronic stage neurodegeneration that we and others have observed in moderate-severe TBI patients.

Correspondence: Jennifer C. Tomaszczyk, Research, Toronto Rehabilitation Institute, 550 University Avenue, Toronto, ON M5G2A2, Canada.E-mail: jennifer.tomaszczyk@uhn.ca

K. MEYERS, J.L. WOODARD, G. TRIFAN, D. FUERST, R. GATTU, E. HAACKE \& R.R. BENSON. Computer-Based Neuropsychological Performance in Retired Professional Football Players.

Objective: Athletes engaged in contact sports are highly susceptible to mild traumatic brain injury (mTBI). Possible residual effects of mTBI many years after retirement from the sport have not been well-investigated, particularly among professional athletes. This study investigated neuropsychological performance in a sample of retired National Football League (NFL) players.

Participants and Methods: Retired NFL players $(\mathrm{N}=26$; mean age $=43.3$ years, range $=29-75$ years) were recruited as part of a larger study examining structural brain changes associated with mTBI. Participants were previously listed on an NFL roster as an active player, and no mTBIs were incurred off the field. Plavers averaged 6.2 vears (range $=1-10$ years) played in the NFL. Participants completed the Neuropsychological Assessment Metrics (ANAM4) Sports Medicine Battery. Results: Predictors of throughput, median reaction time (RT) for correct responses, and accuracy for each of the ANAM4 subtests were investigated using regression analysis. Symptom severity and number of years played predicted median RT to correct stimuli for the mathematical processing subtest $(\mathrm{R} 2=.22, \mathrm{p}=.04$ and $\mathrm{R} 2=.22, \mathrm{p}=.04$, respectively). Number of years played did not predict performance on other ANAM4 subtests or performance variables. Symptom severity also predicted performance on simple reaction time. Age-adjusted scores revealed the same performance pattern.

Conclusions: Retired NFL players with longer playing careers and more self-reported symptoms on a concussion symptom inventory demonstrated slower complex processing speed. Currently reported concussion symptom severity also predicted slowed simple reaction time. Slowed complex processing speed appears to be related to length of playing career among retired professional football players.

Correspondence: Kortni Meyers, B.A., Wayne State University, 5057 Woodward Ave., Detroit, MI 48202. E-mail: Kortmeye@wayne.edu

R. MARTIN, N.J. PASTOREK, B. MILLER, J. ROMESSER, J. LINCK \&. A. SIM. The Influence of Headache Impact on Life Satisfaction and Community Reintegration in Veterans with Mild Traumatic Brain Injury.

Objective: Mild traumatic brain injury (mTBI) in Operation Enduring Freedom/Operation Iraqi Freedom (OEF/OIF) Veterans is associated with negative health outcomes. Headaches may be one of the few problems that are uniquely related to a history of mTBI after considering comorbidities such as Post-Traumatic Stress Disorder. This study aims 
to identify predictors of headache impact in Veterans with a history of mTBI and determine the influence headaches have on life satisfaction and productivity.

Participants and Methods: 216 consecutively-referred Veterans who screened positive for TBI were seen for neuropsychological assessment. After accounting for symptom over-reporting with the MMPI-2 FBS, 139 Veterans remained (134 males; mean years of education=13.1; mean age $=30.6$ ). Headache impact was measured with the Headache Impact Test (HIT-6). Predictive characteristics included time since most serious deployment-related mTBI, number of mTBIs during deployment, and whether any combat-related mTBI resulted in loss of consciousness (LOC). Outcomes included Satisfaction with Life Scale (SWLS), Mayo-Portland Participation Index (M2PI), and productivity level (full-time, part-time, not productive).

Results: Bivariate correlations showed number of injuries and time since injury were not significantly related to HIT-6 ( $p>$.05). A one-way ANOVA indicated LOC was associated with greater headache impact $(p=.032)$. Bivariate correlations with outcome variables showed that both SWLS $(\mathrm{r}=-.33, \mathrm{p}<.001)$ and M2PI $(\mathrm{r}=-.44, \mathrm{p}<.001)$ were significantly related to headache impact. An ANOVA indicated that participants not engaged in at least part-time work/school had higher HIT-6 ratings than those with full-time work/school.

Conclusions: The current study demonstrates the relation of injury characteristics (i.e. LOC) and broad indicators of health outcome (i.e., satisfaction with life, community reintegration, productivity) with current headache impact ratings. Interventions for headache in this population should be a priority for their treatment providers.

Correspondence: Rebecca Martin, Emory University, 922 Rock Springs Ct NE, Atlanta, GA GA. E-mail: rmartin29@gmail.com

C.C. BOSWORTH, K. KRISHNAN, S. ASLAN, J. SPENCE, D. KRAWCZYK, M. CULLUM \& C. MARQUEZ DE LA PLATA. Factors Associated with Default Mode Network Functional Connectivity in Traumatic Brain Injury.

Objective: Traumatic brain injury (TBI) is believed to result in alterations in functional connectivity in intrinsically connected networks. One such network is the default mode network (DMN), which displays increased activation in absence of an externally-imposed task and decreased activation during cognitively demanding tasks. Studying factors associated with post-TBI functional connectivity in the DMN may help further elucidate the role of this network, as well as the effects of TBI on functional connectivity in general.

Participants and Methods: Participants $(n=63)$ sustained a mild-to-moderate TBI at least six-months prior to participating in the study. Each participant completed a cognitive assessment battery consisting of measures of executive functions, language, nonverbal reasoning, memory, mood symptoms, functional status, and estimates of intelligence. Participants were scanned using resting-state fMRI and structural imaging. DMN resting-state functional connectivity was assessed using an accepted seed-based methodology. A stepwise multiple linear regression analysis was used to develop a predictive model for post-TBI DMN functional connectivity. Factors entered into the model included the aforementioned cognitive testing data, demographic and injury factors, functional outcomes, brain volumetrics, and depressive symptoms.

Results: A statistically significant model consisting of age at assessment, global white matter volume, ventricular cerebrospinal fluid volume, post-TBI functional status, and performance on specific measures of attention, immediate verbal memory, verbal reasoning, and cognitive flexibility accounted for $38 \%$ of the functional connectivity variance within the DMN.

Conclusions: These results indicate that DMN post-TBI resting-state functional connectivity is related to several different categories of factors. Though causality cannot be inferred, the same factors that impact functional connectivity may also have an effect on cognition, volumetrics, and functional status.
Correspondence: Christopher C. Bosworth, PhD, Pediatric Neuropsychology, St. Louis Children's Hospital, 8723 Delmar Blvd, 1W, St. Louis, MO 63124. E-mail: chrisbosworth.phd@gmail.com

\section{Acquired Brain Injury (TBI/ \\ Cerebrovascular Injury \& Disease - Child)}

B. ASKEN, A. SNYDER, J. ZAREMSKI, S. SMITH \& R.M. BAUER. Pre-morbid Characteristics of Adolescent Student-Athletes: a Sport-Related Concussion Perspective.

Objective: Research in the area of sport-related concussion has grown dramatically over the past decade as concussion awareness continually improves. Signs and symptoms of concussion are variable due to heterogeneous injury mechanisms, pre-injury characteristics, and other factors. This study examines this variability using aspects of the Sport-Concussion Assessment Tool (SCAT-3). We report baseline prevalence of unreported prior concussion, diagnosed psychiatric conditions, and prevalence of symptoms comprising the ICD-10 diagnosis of Post-Concussion Syndrome (PCS) at baseline.

Participants and Methods: 267 high school student-athletes (205 males, 62 females) aged 13-18 were administered the SCAT-3 during their pre-participation physical examinations or at pre-season baseline collection events organized through their athletic team.

Results: Data were analyzed using descriptive statistics and 2-way Chi-Square analyses. $14.7 \%$ of males and $2.5 \%$ of females stated they had a concussion that they had not previously reported to coaches or medical professionals. Participants of both genders reported a history of psychiatric diagnoses at a significantly lower rate than would be typical for the general population. Lastly, $17.1 \%$ of males and $16.1 \%$ of females had a symptom profile consistent with an ICD-10 diagnosis of Post-Concussion Syndrome at baseline.

Conclusions: (1) Males are more likely than females to not report their concussion. (2) High school student-athletes report a lower rate of psychiatric diagnoses than same-aged members of the general population. (3) A substantial number of adolescents, with or without concussion history, report a similar number of baseline symptoms that are consistent with a PCS diagnosis. The importance of considering baseline/pre-injury profiles in interpreting concussion-related changes will be discussed. Correspondence: Breton Asken, Clinical and Health Psychology, University of Florida, Dept. of Clinical and Health Psychology, P.O. Box 100165, Gainesville, FL 32610.E-mail: basken8@phhp.ufl.edu

\section{Assessment/Psychometrics/Methods (Child)}

J. UDERMAN, Y. CUKIER, B. RABINOVITZ \& S. SCHAFFER. Psychometric Assessment of Social Cognition in Pediatric Populations.

Objective: Social impairment is a core feature of nonverbal learning disability (NVLD), a controversial diagnosis. Although NVLD is conceptualized as a mild autism spectrum disorder (ASD), social deficits in NVLD may more closely resemble those of other developmental disorders, such as attention-deficit/hyperactivity disorder (ADHD). Social deficits in ADHD and NVLD are thought to stem from weaknesses in other cognitive domains (e.g., executive dysfunction), whereas youth with an ASD have primary social cognitive deficits. The aim of this study was to examine one aspect of social cognition, emotion recognition, between NLVD, ADHD, ASD, and Asperger's Syndrome (AS); we hypothesized that performances would be similar across ADHD and NVLD populations but different from ASD and AS.

Participants and Methods: Participants included youth (ages 8-18) with ADHD $(n=24)$ or NVLD ( $n=17)$. Emotion recognition was assessed via NEPSY-II, Affect Recognition. Norms provided through NEPSY-II on ASD and AS populations were utilized for comparison. Social 
functioning was further assessed through interview and the BASC-2, social skills subtest.

Results: Affect recognition was intact for ADHD (mean ss $=9.2$, $\mathrm{SD}=2.5$ ) and NVLD (mean ss=9.9, $\mathrm{SD}=2.4$ ) groups, and no significant differences between ADHD, NVLD, ASD, and AS populations were found using bonferroni corrected t tests. On interview, $63 \%$ and $88 \%$ of parents of ADHD and NVLD youth, respectively, reported social difficulties. BASC scores were not elevated across groups.

Conclusions: Results indicate that emotion recognition may not be representative of social deficits in these populations. Moreover, overall findings highlight the importance of clinician interviewing and the current limitations of psychometrics in capturing these social difficulties. Correspondence: Jodi Uderman, M.A., Neurology, North Shore Long Island Jewish, 79-25 150th St. Apt F8, APT. F8, Flushing, NY 11367. E-mail: jodi.uderman@gmail.com

\section{Autism Spectrum Disorders}

\section{Y. GRANADER, P.K. ISQUITH, K.M. DUDLEY \& L. KENWORTHY. Updated BRIEF Profiles in Children with Autism Spectrum Disorders.}

Objective: Children with autism spectrum disorders (ASD) exhibit significant executive functioning (EF) deficits. This study examined profiles in children with ASD versus typically developing children (TDC) on a revised Behavior Rating Inventory of Executive Function (BRIEF). Participants and Methods: 262 children (214 males, 5-18 years, age $\mathrm{M}=10.2, \mathrm{SD}=3.3$ ) without ID (IQ M=101.6, SD=17.5) who met both DSM-IV-TR and NICHD/NIDCD Collaborative Programs for Excellence in Autism criteria for ASD using gold standard diagnostic instruments were matched for sex and age with 262 TDC (214 males, 5-18 years, age $\mathrm{M}=10.2, \mathrm{SD}=3.3$ ) drawn from the revised BRIEF normative sample. Parent BRIEF ratings were compared via ANOVA. ROC curve was plotted for classification accuracy based on discriminant function analysis. Results: The ASD group had significantly higher $(p ' s<.001)$ mean T-scores on all nine scales of the BRIEF. Less than $10 \%$ of TDC, but 30 to $74 \%$ in the ASD group had clinically elevated scores ( $>65)$ on any scale. The Shift scale had the highest proportion of clinically elevated scores $(74 \%)$ and also the highest mean T-score in the ASD group (M=71.7, $\mathrm{SD}=11.6)$. In combination, the BRIEF scales showed sensitivity of .86 with a false positive rate of $10 \%$.

Conclusions: Similar to previous investigations of parent report on the original BRIEF, EF difficulties in children with ASD were seen across all scales of the revised BRIEF, with the greatest problems reported on the Shift scale. Classification analyses suggest that the profile is highly sensitive to the presence of ASD

Correspondence: Yael Granader, Children's National Medical Center, 2201 L Street NW, Apt 708, Washington, DC 20037. E-mail: yaelgranader@gmail.com

\section{Emotional Processes}

A.M. REVUELTAS, M. PALE \& M. RAMÍFEZ-FLORES. Analysis of Mexican Children's Recognition of Facial Expression of Emotions. Objective: To analyze the recognition of facial expression of emotions in Mexican children aged 6 to 12 years old in peers and the differences between the ages. It is expected that older children will recognize better than the young ones; also, happiness will be the most recognized emotion, and fear the least recognized.

Participants and Methods: 74 children from 6 to 12 years old participated. They were divided into 7 groups according to their age. All the participants answered a task of recognition of facial expression, which consists in watching 70 photographs of Mexican children displaying one of the 6 basic emotions (happiness, surprise, disgust, sadness, anger and fear) or a neutral expression.
Results: The statistical analysis shows differences across the ages $\mathrm{F}(6,67)=4.546, p<0.001$, specifically between $6[\mathrm{x}=33.50 \pm 5.9]$ and 12 years old $[x=43.13 \pm 4.32](p<0.001)$, and $7[x=35 \pm 5.7]$ vs. 12 years old $(p<0.05)$. A positive Pearson correlation between RFEE and age was observed $\left(\mathrm{r}=0.4982, \mathrm{r}^{\wedge} 2=0.2482, p<0.0001\right)$. Data showed differences in the recognition of surprise $(H: 26.38, p<0.001)$ between 6 and 12 years old, and disgust (H:14.81, $p<0.05)$ between 6 and 12 years old. A Friedman analysis showed significant differences between the emotions regardless of the age $(p<0.0001)$, with happiness as the most recognized emotion $(p<0.0001)$, followed in second by the neutral expression, and in third by anger, both of them more recognized than surprise $(p<0.001)$, sadness $(p<0.05)$, disgust $(p<0.001)$ and fear $(p<0.001)$.

Conclusions: The recognition of facial expression of emotion is an ability that develops and improves over age, although there is a window between the age 8 and 11 years old in which this ability may be developing, who show no significant differences between the ages. The recognition of happiness seems to have consolidated at the age of 6 , surprise and disgust recognition seems to be improving over age. Correspondence: „, E-mail:

L.R. WEISS, P. SPECHLER, A.J. ALFINI, T.J. SMITH, M. VERBER \&. J. SMITH. Changes in Blood Oxygen Level-Dependent (BOLD) Response to Affective Picture Viewing After Acute Exercise.

Objective: A single session of exercise is known to decrease negative affect and increase positive affect, but little is known regarding the brain systems underlying exercise-induced changes in affective responsiveness. We aimed to determine differences in brain activation during emotional picture viewing after an acute bout of moderate intensity exercise compared to seated rest.

Participants and Methods: Nine healthy young adults (ages 20-30) completed two conditions on different days; a 30-minute session of a) seated rest or b) moderate intensity exercise on a cycle ergometer. Following rest and exercise, fMRI data were acquired while participants viewed 90 arousing pleasant, unpleasant, and neutral pictures from the International Affective Picture System (IAPS).

Results: During pleasant picture viewing, exercise resulted in greater activation in two frontal and four parietal regions, and reduced activation in bilateral temporal lobe regions and posterior cingulate, compared to rest $(p<.01)$. During unpleasant images, brain activation was lower after exercise versus rest in the left cingulate gyrus, right parahippocampal gyrus, and right superior occipital gyrus $(\mathrm{p}<.01)$. During neutral pictures, less activation occurred after exercise in two right parietal regions, with greater activity in left middle temporal gyrus $(p<.01)$.

Conclusions: A 30-minute session of moderate-intensity cycling resulted in changes in neural responses to affective stimuli. The reduced temporal lobe and increased frontal and parietal lobe activation during pleasant pictures, paired with the consistently decreased brain activation in response to unpleasant images following exercise, suggests a neural basis for changes in emotional responsiveness and improved mood after exercise.

Correspondence: Lauren R. Weiss, Kinesiology, University of Maryland, University of Maryland Dept of Kinesiology, School of Public Health 1225, College Park, MD 20740. E-mail: lauren.rf.weiss@gmail.com

J.R. VANUK, S. DIVATIA, L.A. DEMERS, S. MARKOWSKI, A. ALKOZEI \& W.D. KILLGORE. Napping in Conjunction with Brief Internet-Based Training as a Means of Enhancing Emotional Intelligence.

Objective: The ability to perceive, understand, and manage emotions in oneself and others is known as Emotional Intelligence (EI). Greater EI is thought to be associated with better coping and resilience, successful interpersonal relationships and increased work performance. We developed a three-week online training program to enhance EI skills. Because considerable evidence suggests that sleep plays a crucial role in emotional functioning and memory consolidation, we hypothesized 
that regular nappers would show greater improvement from the training than non-nappers.

Participants and Methods: Sixty-two healthy 18-50 year olds (31 men) were randomized to receive either a 6-lesson on-line EI-training program over a 3 -week period, or a matched placebo training program with similar intellectual challenge and activities. Although napping was not controlled, twenty-eight participants (16 men) also reported voluntarily taking naps at least one or more times per week throughout the course of the training. As a measure of EI, participants completed the Mayer-Salovey-Caruso Emotional Intelligence Test (MSCEIT) at baseline and after completion of the program.

Results: A 2 x 2 ANOVA yielded a significant interaction between EI training and napping conditions with regard to improvement in EI scores $(p=.006)$. Whereas EI training was enhanced among nappers relative to non-nappers, the placebo condition was not effective at changing EI scores, regardless of napping.

Conclusions: The findings suggest that inclusion of napping for individuals in the online training program may provide an increase in the efficacy of EI training over the duration of the program. Napping may improve memory consolidation, emotional regulation, or cognitive performance during the training.

Correspondence: John R. Vanuk, Bachlors of Science, Psychiatry, University of Arizona, 733 S. Herbert Ave., Tucson, AZ 85701. E-mail: johnnyv@psychiatry:arizona.edu

J.R. VANUK, A. FRIDMAN, L.A. DEMERS, S. DIVATIA \& W.D. KILLGORE. Engaging in Meditation and Internet-Based Training as a Means of Enhancing Emotional Intelligence.

Objective: Emotional Intelligence (EI) refers to the ability to understand, perceive, and manage emotions in oneself and others. A threeweek online training regimen, composed of six lessons, was created in an attempt to improve EI faculties. Since past research has shown a relationship between the practice of meditation and increased internal awareness, we hypothesized that meditators would show greater EI increases than non-meditators during EI training.

Participants and Methods: Sixty-two healthy adults (31 men), ranging in age from 18-50 years, were randomly assigned to one of two forms of web-based cognitive training; one employing non-EI or "external awareness" and the other employing EI or "internal awareness" training. Training differed in content, but was balanced on intellectual challenge, activities, and time requirements. As a measure of EI, the Mayer-Salovey-Caruso Emotional Intelligence Test (MSCEIT) was administered at baseline and at completion of the program. Due to the importance of sleep in emotional functioning, sleep problems were also assessed.

Results: Individuals who practiced meditation reported higher occurrences of sleep problems than controls. After accounting for the variance associated with sleep problems, a $2 \times 2$ ANOVA showed a significant interaction between meditation group and improvement in EI scores ( $p$ $=.04)$, with meditators showing greater benefit than non-meditators. Conclusions: This relationship may be the result of individuals attempting to mitigate their sleep problems by engaging in meditation. Promoting introspective behaviors (i.e. engaging in meditation) may augment EI training in a manner that increases its effectiveness, regardless of whether individuals are experiencing the detrimental effects incurred by interruptions in sleep.

Correspondence: John R. Vanuk, Bachlors of Science, Psychiatry, University of Arizona, 733 S. Herbert Ave., Tucson, AZ 85701. E-mail: johnnyv@psychiatry.arizona.edu

A.A. HAZAMY, L.J. ALTMANN, M. TROCHE, W. COWLES \& D. BOWERS. Emotional Sentence Processing in Persons with Parkinson's Disease.

Objective: Emotional processing deficits are commonly reported in persons with Parkinson's disease (PD) within a number of domains including prosody and facial processing. However, the extent to which these processing changes affect language is relatively unknown. This study explored whether persons with PD differed in their processing of emotional sentences compared to health adults.

Participants and Methods: Forty cognitively-intact individuals aged 35-85 participated (18 persons with PD, 22 healthy adults). Participants were presented with sentences on a computer screen. Sentence frames were neutral with a stimulus word appearing at the end of the sentence. Stimuli varied on emotional valence (i.e., positive, negative, neutral) and concreteness (i.e., abstract, concrete). Participants rated the sentences on the emotional dimensions of pleasantness and arousal. Results: Persons with PD demonstrated a significantly reduced valence response range overall. Planned pairwise comparisons of valence ratings revealed persons with PD significantly rated negative concrete sentences as less negative than healthy adults. Furthermore, there was a trend toward persons with PD rating negative abstract sentences as less negative and positive abstract sentences as less positive than healthy adults. Regarding arousal ratings, persons with PD rated positive concrete sentences as less arousing than healthy adults.

Conclusions: Group differences in emotional ratings and the overall reduced range of valence responses in persons with PD compared to healthy adults are indicative of blunted processing of emotional sentence stimuli, which may impact their full appreciation of emotional content in everyday discourse. These findings are consistent with research on processing of emotional faces, prosody, and pictures in Parkinson's disease. Correspondence: Audrey A. Hazamy, University of Florida, 336 Dauer Hall, PO Box 117420, Gainesville, FL 32611.E-mail: ahazamy1@ufl. edu

J.T. TWAITE, A.D. BONO, H.A. BOKHARI, R. STAFFORD, E. ARONOVA, S.D. GOLDE, J.L. STORBECK \& J.C. BOROD. Musical Training is Associated With Lower Levels of Alexithymia and Greater Introspective Thought.

Objective: Alexithymia is characterized by deficits in affective awareness (i.e., difficulty identifying and describing emotions) and paucity of introspective thought, and is a risk factor for multiple psychiatric and medical conditions. It has been proposed that alexithymia reflects deficits in the cognitive processing of emotion. We hypothesized that individuals with musical training would report lower levels of alexithymia, based on prior findings that musical training alters the neural processing of emotion and enhances emotional competence.

Participants and Methods: Participants were 56 musically-trained individuals $(59 \%$ women; $\mathrm{M}$ age $=22.1 \mathrm{yrs}(4.0), \mathrm{M}$ onset of training $=8$ yrs (2.5), M duration of training $=13.5$ yrs (4.5) and 57 demographically matched healthy controls $(52 \%$ women, M age $=20.9$ (4.2). Participants were screened for psychiatric, medical, and neurological history and then completed the Toronto Alexithymia Scale-20 (TAS-20). The TAS20 yields a total score and 3 factor scores: (1) Difficulty Identifying Feelings, (2) Difficulty Describing Feelings, and (3) Externally Oriented Thinking.

Results: Musicians scored significantly lower than non-musicians on the total TAS score $(\mathrm{t}=-2.15, \mathrm{p}=0.03)$ and on Factor $3(\mathrm{t}=-2.47, \mathrm{p}=0.01)$. Factor 2 approached significance $(t=-1.69, \mathrm{p}=0.09)$. Male participants scored significantly higher than female participants on the total TAS score $(\beta=4.87, p=0.02)$, however, group differences between musicians and non-musicians remained significant when gender was controlled $(\beta=4.26, p=0.04)$

Conclusions: We found that musical training is associated with lower levels of alexithymia, particularly less externally oriented thinking. This study has implications for how musicians process emotion as compared to non-musicians and offers further evidence of positive relationships between musical training and socio-emotional functioning. Evaluation of causal mechanisms is needed to assess if musical training represents a possible intervention for socio-emotional deficits.

Correspondence: Jamie T. Twaite, M.A., Neuropsychology, CUNY Graduate Center, 102-40 67th Rd, Apt 5N, Forest Hills, NY 11375. E-mail: jtwaite@qc.cuny.edu 
W.D. KILLGORE, L.A. DEMERS, S. DIVATIA, M. KIPMAN, O. TKACHENKO, M. WEBER, L. PREER, H. GOGEL, E.A. OLSON, J.R. VANUK \& S.L. RAUCH. Enhancing Emotional Intelligence via Brief Internet-Based Training.

Objective: The capacity to understand emotions and use them to enhance cognition is known as Emotional Intelligence (EI). Evidence suggests that EI is important to success in many settings, and correlates with cortical volume and functional activation of the insula and ventromedial prefrontal cortex. Whether EI capacities are malleable and can be improved through focused training is currently a topic of debate. We developed and tested a brief, six-lesson (three week), on-line training course to enhance EI abilities based on established literature.

Participants and Methods: Sixty-two healthy adults (31 men), ranging in age from 18-50 years were randomly assigned to undergo one of two parallel online training programs, matched in terms of activities and intellectual challenge, but differing only in content (e.g.. EI or "internal awareness" training versus non-EI or "external awareness" training). Results: The EI training program significantly enhanced Total EI scores on the Mayer-Salovey-Caruso Emotional Intelligence Test (MSCEIT) relative to the placebo condition $(p=.04)$. Further analysis of the four MSCEIT subscales revealed that the preliminary training program was effective at improving scores on the Perceiving Emotions ( $p=.04)$ and Facilitating Thought $(\mathrm{p}=.007)$ branches of EI, but not at improving the Understanding Emotions and Managing Emotions branches relative to controls.

Conclusions: Findings suggest that at least some EI capacities are malleable and can be improved through a brief internet-based intervention. With further development, such a program could be used to enhance critical emotional skills in a variety of settings. Whether these changes are associated with concomitant changes in brain structure and function remains to be explored.

Correspondence: William D. Killgore, Ph.D., Psychiatry, University of Arizona, Brain Imaging Center, McLean Hospital, 115 Mill Street, Belmont,MA 02478.E-mail: killgore@mclean.harvard.edu

W.D. KILLGORE, E.A. OLSON, M. WEBER, S.L. RAUCH \& L.D. NICKERSON. Emotional Intelligence is Associated with Coordinated Resting State Activity Between Emotion Regulation and Interoceptive Experience Networks.

Objective: Emotional Intelligence (EI) reflects an individual's capacity to accurately perceive, understand, and regulate emotions, and to apply that information to facilitate thought and performance. Although EI has been shown to play an important role in mental health and success in academic, professional, and social realms, the neurocircuitry underlying this capacity remains poorly understood. We explored the relationship between regional functional connectivity and two alternative models of EI (i.e., Trait versus Ability models).

Participants and Methods: Fifty-four healthy, right-handed adults (28 women, 26 men), with an average age of 30.1 years $(\mathrm{SD}=7.5$ years) completed standardized validated measures of Trait and Ability EI followed by resting state functional magnetic resonance imaging (rsfMRI). FSL and FSL MELODIC were used to implement an independent components analysis (ICA) with dual regression to investigate brain circuits (resting state networks, RSNs), whose activity was thought to be associated with greater EI capacities. All results are reported at $\mathrm{p}<0.05$, FWE corrected.

Results: Higher Ability EI (as opposed to Trait EI) was associated with stronger inverse correlations of the spontaneous FMRI signals from RSNs involved in affective regulation (e.g., prefrontal) with those involved in emotional responses and experiences (e.g., insula), and also between FMRI signals from emotionally responsive networks (e.g., insula) and those involved in self-reflective cognition (e.g., medial frontal/posterior cingulate).

Conclusions: Importantly, these findings suggest that stronger inverse correlations between signals from key intrinsic emotional regulation and interoceptive experience networks serve as a marker of higher emotional intelligence skills and abilities, perhaps reflecting greater capacity to regulate emotional responses through executive control processes.

Correspondence: William D. Killgore, Ph.D., Psychiatry, University of Arizona, Brain Imaging Center, McLean Hospital, 115 Mill Street, Belmont, MA 02478. E-mail: killgore@mclean.harvard.edu

S.E. BRUCE. An fMRI investigation of visual cortex activity in response to fearful faces in participants with PTSD.

Objective: The current study examines the neural correlates underlying visual processing differences between women with post traumatic stress disorder (PTSD) compared with healthy controls. Prior research suggest that individuals with PTSD report visually vivid reexperiencing symptoms; symptoms that are associated with atypical visual processing. Structural and functional imaging studies have provided evidence of deficits in visual processing in individuals with PTSD. However, these studies have been inconsistent, with some reporting higher (or lower) visual cortex activity in PTSD patients in response to threat-related visual stimuli.

Participants and Methods: Fifty-two women with a history of interpersonal trauma, currently diagnosed with PTSD, as well as 21 healthy controls completed a baseline assessment and an fMRI scanning session. While in the scanner participants completed an emotion conflict task, allowing for an examination of attentional and emotional components of brain activity.

Results: Results indicate main effects in several areas of the visual cortex, including lower activity in PTSD participants in the left and right middle occipital gyrus, as well left fusiform gyrus. The fusiform gyrus, part of the visual stream, is thought to be involved with facial recognition. Additionally, in the "attend-fear" condition, PTSD participants had significantly lower activity in the left cuneus (an area that receives visual information from the retina), and the superior occipital gyrus, which has shown to be involved in facial recognition.

Conclusions: Our results suggest that PTSD in women is associated with significant sensory processing deficits, including disruptions in attending to arousing visual stimuli. Additional analyses examining dorsal and ventral visual stream differences as well as relationships between occipital lobe functioning and PTSD symptom clusters will be conducted. Implications of the findings will be discussed.

Correspondence: Steven E. Bruce, Ph.D., Psychological Sciences, University of Missouri-St. Louis, 7606 Maryland Ave, Clayton, MO 63105.E-mail:brucese@umsl.edu

A. ALKOZEI, Z. SCHWAB \& W.D. KILLGORE. Looking for Evil Intent: Emotional intelligence and the use of socially relevant facial cues during an emotional decision making task.

Objective: Emotional intelligence (EI) is defined as the ability to understand, perceive and manage emotions. However, there is little research investigating how EI influences decision-making during emotionally difficult situations. We hypothesized that higher EI would correlate with greater utilization of socially relevant facial cues in emotional decision-making.

Participants and Methods: Sixty-two 18-45 year olds completed a decision making task mimicking an airport security screening involving an established terrorist threat. Participants were presented with a series of facial photographs of white men and women and required to decide which ones to detain for further interrogation. The faces were previously rated for certain character traits (e.g., aggression) by independent judges. Participants also completed measures of Trait and Ability EI and cognitive intelligence (IQ).

Results: With higher Ability EI, participants were more likely to detain individuals based on higher negative traits (e.g., "aggression," $\mathrm{r}=.27$, "meanness" $\mathrm{r}=.31$ ) and lower positive traits (e.g., "trustworthy," $\mathrm{r}=-$ .31 , "emotionally stable," $\mathrm{r}=-.35$, all $\mathrm{p}$ 's $<.05)$. These associations were driven primarily by the Facilitating Branch of EI (i.e., the ability to generate and use emotion to facilitate decision making). On the other 
hand, no association between Trait EI or IQ and detained individuals character traits was found.

Conclusions: Individuals with greater Ability EI, in particular greater capacity to use emotions to facilitate cognitive processes, were more likely to utilize more of the limited social information (i.e., facial features independently rated as indicative of underlying dispositional traits) when completing an emotional decision making task. These findings have implications for real-life situations involving similarly difficult emotional decision-making processes.

Correspondence: Anna Alkozei, Ph.D., Department of Psychiatry, University of Arizona Medical Center, University of Arizona Medical Center, Department of Psychiatry, 1501 N Campbell Ave, Tucson, AZ 85724.E-mail:aalkozei@psychiatry.arizona.edu

D. SZELES, T.W. CONWAY \& K.M. HEILMAN. The Influence of Spatial Presentation on the Emotional Perception of Pictures.

Objective: Emotional concepts are often conceptualized vertically such that "good" is up and "bad" is down - and laterally - with right being good and left being sinister. To date, a neurobiological explanation has not been proposed. The left hemisphere may mediate positive emotions while the right hemisphere mediates negative emotions. Likewise, the ventral stream, which is allocentrically oriented and attends to upper space, may enhance positive emotions while the dorsal stream, which is egocentrically oriented and attends to lower space, may enhance negative emotions. This study examined how spatial attention might influence emotional perception.

Participants and Methods: Thirty-two neurologically healthy adults (ages 21-40) participated. Pictures from the International Affective Pictures System were presented in upper, lower, right, left, and middle space. Participants rated how positive or negative pictures were by marking points along a valence line. A three (valence) by three (space) repeated measures analysis of variance was performed with valence line label position (positive/negative) as the between subjects factor.

Results: Pictures in lower space were rated more positively than pictures in middle space; pictures in right space were rated more negatively compared to middle space.

Conclusions: Contrary to predictions, downward presentations yielded more positive ratings and rightward presentations yielded more negative ratings. The mechanism for these asymmetries is unknown; however, positive pictures in lower space and negative pictures in right space may have breached participants' expectations, driving more positive and negative ratings for pictures in lower and right space, overall. Further studies are warranted to explore the nature of these effects.

Correspondence: Dana Szeles, B.S., Clinical and Health Psychology, Malcom Randall VAMC/University of Florida, 999 SW 16th Ave Apt 36, Gainesville,FL 32601.E-mail: dmszeles@phhp.ufl.edu

\section{L.B. DEL PIERO, D. SAXBE \& G. MARGOLIN. Cognitive and Emotional Correlates of Family Aggression.}

Objective: The goal of this study is to examine the impact of family aggression on three interrelated, but distinct measures of cognitive and emotional functioning during adolescence - a novel functional neuroimaging measure of emotional flexibility, self-reported emotion regulation, and a measure of cognitive control (inhibition/switching).

Participants and Methods: Participants included 22 youth (10 female) recruited from a longitudinal family violence study. Family aggression was assessed when youth were in early adolescence, using the Conflict Tactics Scale. In mid-to-late adolescence, youth completed the Difficulties in Emotion Regulation Scale, the Color-Word Interference subtest from the D-KEFS, and a novel emotional flexibility fMRI task.

The emotional flexibility task involved five conditions: positive and negative congruent, positive and negative incongruent, and resting baseline. Each condition included 15 IAPS images selected based on valence ratings. Participants were shown each image for 4 seconds, and then rated "how the scene made them feel" on a 4-point scale. They were then shown the image again with a caption. In the congruent trials, the caption maintained the emotional valence of the image. In the incongruent trials, the caption was designed to change the emotional valence of the image - tapping into participants' emotional flexibility. Participants then rated the image a second time.

Results: Neither self-reported emotion regulation nor cognitive control were associated with family aggression. However, family aggression was linked with neural activation during the emotional flexibility task in brain regions associated with emotion regulation as well as behavioral responses to the task.

Conclusions: Family aggression appears to have an impact on emotional flexibility (i.e., cognitive control specifically in the context of emotion). This finding highlights a selective difficulty that may make it challenging for violence-exposed youth to control their emotions and behaviors in emotionally-arousing contexts.

Correspondence: Larissa B. Del Piero, M.A., Psychology, University of Southern California, 10914 Rose Ave, Apt. 3, Los Angeles, CA 90034. E-mail: larissa.b.delpiero@gmail.com

L.B. ANDERSON, L.K. PAUL \& W.S. BROWN. Emotional Intelligence Deficits in Agenesis of the Corpus Callosum.

Objective: The impact of agenesis of the corpus callosum (ACC) on emotional intelligence and problem-solving is as yet unclear. Using the Mayer-Salovey-Caruso Emotional Intelligence Test (MSCEIT; Mayer, Salovey, \& Caruso, 2002), this study investigated ability-based emotional intelligence (EI) in ACC.

Participants and Methods: Twenty participants with ACC (age 18-57; FSIQ 78-129) were compared to 20 control participants (age 20-41; FSIQ 87-112) on the MSCEIT. The ACC group was also compared to the standardized MSCEIT norms.

Results: Compared to controls, the ACC group showed a more marked decline from Experiential to Strategic EI areas of the MSCEIT ( $p=$ $.002, \eta p 2=.23$ ), and greater fall-off in performance across the four MSCEIT branch scores as the tasks increased in complexity $(p=.004$, $\eta p 2=.11)$. The ACC group scored in the normal range on Experiential EI (Perceiving Emotions and Using Emotions), but were significantly lower than the standardized MSCEIT norms on Strategic EI (Understanding Emotions and Managing Emotions), t $(19)=-4.09, \mathrm{p}<.001$. Conclusions: Deficits in ACG on the more complex domains of the MSCEIT (Strategic) suggests that callosal absence interferes with the ability to process and synthesize the emotional information. Better than expected performance of the ACC group on the lower-level tasks of the MSCEIT (Experiential) may be due to their ability to reason through simple emotional problem-solving tasks when not under the time constraints imposed by real-world processing.

Correspondence: Warren S. Brown, Ph.D., Travis Research Institute, Fuller Grad Sch of Psych, 180 N. Oakland Ave., Pasadena, CA 91101. E-mail:wsbrown@fuller.edu

K. GLAZEWSKI, B. ZUCCATO, E. KEATLEY \& C. ABEARE. The Role of Stress and Emotion Regulation in Post-Concussive Symptom Reporting in Healthy Adults.

Objective: Stress is known to influence symptom reporting and stress is known to influence emotion regulation, but little is known about how emotion regulation may influence the relationship between stress and symptom reporting. The purpose of the current study was to examine this relationship in the reporting of post-concussive symptoms among non-concussed university students.

Participants and Methods: Seventy-seven undergraduates (64 female, 13 male) from the University of Windsor completed questionnaires regarding perceived stress (Perceived Stress Scale; PSS), emotion regulation (Difficulties in Emotion Regulation Scale; DERS), and post-concussive symptoms (Post-Concussion Symptom Scale; PCSS).

Results: Univariate and hierarchical regression analyses were used to examine the relationships between stress, emotion regulation, and post-concussive symptoms. As expected, the PSS was associated with PCSS scores $(r=.66, p<.01)$, and PSS was associated with DERS $(r$ 
$=.69, \mathrm{p}<.01)$. Regression analysis demonstrated that DERS scores partially mediated the relationship between perceived stress and PCSS scores $(\beta=1.46, p<.01)$.

Conclusions: That emotion regulation mediates the relationship between stress and symptom reporting may have significant treatment implications for concussed athletes with persistent post-concussive symptoms if the current pattern of findings holds in this population. While reducing exposure to stressful events following a concussion is standard protocol, results suggest that improving emotion regulation may help to prevent and decrease symptom reporting following concussion. Correspondence: Christopher Abeare, Ph.D., University of Windsor, 401 Sunset Ave., Windsor, ON N9B 3P4, Canada.E-mail: cabeare@ uwindsor.ca

E.J. Kellogg, P.L. JOHnson, G. POTTS \& C. Cimino. Investigating the Relationship Between Emotional Dysregulation, Impulsivity, and Executive Functions In a Non-Clinical Sample. Objective: Emotional regulation is the ability to modulate emotions to act in a goal directed manner. Associations between emotional dysregulation and facets of impulsivity as measured by the UPPS-P have been shown in clinical populations with impulsivity. Additionally, researchers have found associations between executive functions, (e.g., inhibition, working memory) and emotional regulation. This study sought to elucidate the relationship between emotional dysregulation, impulsivity, and executive functions in a non-clinical sample of young adults.

Participants and Methods: Forty-nine female, undergraduates completed the Difficulty in Emotion Regulation Scale (DERS), UPPS-P, Stroop, and Letter-Number Sequencing (LNS) in the context of an emotional memory paradigm. Correlational analyses were used to investigate relationships between DERS Total and subscale scores, UPPS-P, Stroop Color-Word trial, and LNS total.

Results: DERS was highly correlated with the UPPS-P. Notably, DERS Total $(\mathrm{r}=0.52, \mathrm{p}<.001)$ and subscales Impulse $(\mathrm{r}=0.57, \mathrm{p}<.001)$ and Awareness $(r=0.42, p<0.05)$ were correlated with UPPS-P Negative Urgency. Similarly, DERS Total $(r=0.41, p<0.05)$, Impulse ( $r$ $=0.43, \mathrm{p}<0.05)$, and Awareness $(\mathrm{r}=0.44, \mathrm{p}<0.05)$ were correlated with UPPS-P Positive Urgency. DERS Total $(r=-0.29, \mathrm{p}<0.05)$ and Clarity $(\mathrm{r}=-0.29, \mathrm{p}<0.05)$ were associated with Stroop Color-Word. However, LNS was not associated with either DERS or UPPS-P.

Conclusions: Greater emotional dysregulation was related to greater impulsivity in a non-clinical population. In partial support of previous research, executive function inhibition was related to emotional dysregulation, but not working memory.

Correspondence: Emily J. Kellogg, BS, Psychology, University of South Florida, 4202 East Fowler Ave, PCD 4118G, Tampa, FL 33620-7200. E-mail: ejkellogg@mail.usf.edu

P.L. JOHnSON, E.J. KellogG, G. POTTS \& C. CiMino. The Impact of Self-Reported Difficulty in Emotion Regulation on Emotional Memory and Emotional Processing of Pictures.

Objective: Emotion processing (indexed by Event Related Potentials; ERPs) and memory can be altered using emotional regulation strategies, demonstrated in experimental paradigms. When directed to use reappraisal, ERP amplitude decreases but emotional memory remains intact. When directed to use suppression, ERPs are unaltered (i.e., evidence expected larger amplitude to emotional stimuli), with decreased emotional memory. Memory and ERP amplitude to emotional pictures has not been examined in relation to self-reported emotional regulation abilities. Those with poor self-reported regulation were predicted to have decreased emotional memory and increased ERP amplitude to emotional stimuli, similar to those using suppression techniques.

Participants and Methods: Forty-nine females viewed 150 affective pictures (positive, negative, neutral), rating each on valence and arousal. Recall and recognition was tested after a delay. The Difficulty in Emotion Regulation Scale (DERS) measured amount of emotion regulation dysfunction.
Results: Higher DER was tended to recall less emotional pictures ( $\mathrm{r}=-$ 0.27, $\mathrm{p}=0.63)$, especially high arousing pictures $(\mathrm{r}=-0.33, \mathrm{p}<0.05)$. Some subscales also related to better recognition of positive high arousing pictures $(p s<0.10)$. Increased difficulty accepting negative emotion showed a trend relationship for larger $\mathrm{N} 2$ amplitude to negative pictures $(\mathrm{r}=-0.26, \mathrm{p}=0.067)$ and larger LPP amplitude to negative $(\mathrm{r}=0.31$, $\mathrm{p}<0.05)$ and neutral pictures $(\mathrm{r}=0.30, \mathrm{p}<0.05)$.

Conclusions: Higher DERS demonstrated decreased recall for emotional pictures, particularly negative arousing pictures, and increased ERP amplitude for negative pictures. These findings indicate that self-reported emotional regulation abilities may impact emotional memory and processing, specifically when participants are asked to evaluate pictures. These results are consistent with directed suppression techniques findings, which may indicate poorer self-initiated emotional regulation abilities when presented with emotional material.

Correspondence: Patricia L. Johnson, MA, Psychology, University of South Florida, 115 112th Ave. NE, Apt 503, Tampa, FL 33716. E-mail: pjohnson2@mail.usf.edu

R.M. BAULDRY, E.S. KORNBLITH, R. GARCIA, S.W. ADAMS, C.W. WRIGHT, D.T. LOBDELL \& R.M. BOWLER. Generalized Anxiety and Major Depressive syndrome measured by the SCL90-R in Two Manganese (Mn) Exposed Ohio Towns.

Objective: Environmental exposure to manganese (Mn) may cause generalized anxiety (GA) and major depression (MD) in residents living in Mn-exposed areas. Marietta and East Liverpool are two Ohio towns identified as having elevated levels of Mn. The objective was to determine if levels of Mn exposure were associated with levels of GA and MD. Participants and Methods: 186 participants (Mean age: $55.0 \pm 10.80$ ) were examined. Levels of air-Mn were assessed over a period of ten years using U.S. EPA's AERMOD dispersion model. Average air-Mn exposure was $0.53 \mu \mathrm{g} / \mathrm{m} 3$ in the two towns. The GA syndrome was comprised of anxiety, obsessive-compulsive, and phobic scales from the Symptom Checklist (SCL-90-R). The MD syndrome was comprised of depression, anxiety, and psychoticism scales also from the SCL-90-R. Linear regression models were used to determine the relationship between Mn and GA, MD and the specific components of each.

Results: Elevated air-Mn was associated with GA $(\beta=0.240, p=0.002)$, and MD $(\beta=0.202, p=0.011)$. Air-Mn was associated with specific components of GA anxiety $(\beta=0.255, p=0.001)$, phobic anxiety $(\beta=0.159$, $p=0.046)$, and obsessive-compulsive $(\beta=0.197, p=0.013)$. Similarly, components of MD syndrome suggested an association as well: depression $(\beta=0.180, p=0.023)$, anxiety $(\beta=0.255, p=0.001)$, and psychot $\operatorname{icism}(\beta=0.188, p=0.018)$.

Conclusions: The results suggest that residents with elevated exposure to environmental Mn have elevated levels of GA and MD. Residents who reported symptoms of anxiety, such as feelings of nervousness, tension, and restlessness were found to have the strongest association with elevated Mn-air concentrations.

Disclaimer: This abstract does not necessarily reflect EPA policy. Correspondence: Rose M. Bauldry, Doctorate, Psychology, California School of Professional Psychology, 4016 Mission street, San Francisco, CA 94112.E-mail: rmbauldry@gmail.com

S.M. SHDO, B. ADHIMOOLAM, B. MILLER \& K.P. RANKIN. Specific Right-Temporal Contributions to Distinct Behavioral Subcomponents of Empathy in Neurodegenerative Disease.

Objective: Neurodegenerative patients often lose the ability to behave empathically, but for very different neurological reasons. Evidence shows right temporal structures support empathy, but contributions of temporal subregions to specific components of empathy (reading emotions, perspective taking, feeling with others) are not well-known. Participants and Methods: 262 patients ( 56 behavioral variant frontotemporal dementia[bvFTD], 15 right temporal FTD[rtFTD], 24 semantic variant primary progressive aphasia [svPPA], 20 nonfluent variant PPA [nfvPPa], 62 Alzheimer's disease [AD], 23 progressive 
supranuclear palsy [PSP]), 62 healthy older controls) underwent social testing with The Awareness of Social Inference Test Emotion Evaluation (TASIT-EET) (emotion naming), UCSF Cognitive Theory of Mind (cToM) (cognitive perspective taking), and Interpersonal Reactivity Index (empathic concern (EC); emotional perspective taking (PT)). Atrophy corresponding to each score was shown using voxel-based morphometry of structural MRI controlling for age, sex, disease severity (MMSE), and total intracranial volume (TIV).

Results: cToM predicted right posterior middle temporal gyrus volume ([x,y,z, T-score $]: 32,45,0 ; 5.41)$. PT predicted right anterior middle/inferior temporal gyrus $(44,0,-29 ; 5.68)$. EET predicted bilateral fusiform $(41,9,-32 ; 6.16)$, medial temporal regions $(41,9,-32 ; 5.60)$, while EC was unilateral. All emotional tasks correlated with right anterior insula (EC: 24,17, -17; 6.63)(PT:30,17,-12; 6.20)(EET: 36,12,-15; 5.75) (All results $\mathrm{pFWE}<0.05)$.

Conclusions: Right anterior insula damage is the common factor in loss of emotion-based empathic functions, but distinct additional temporal regions, affected divergently across neurodegenerative syndromes, support other functions. Right anterior lateral temporal regions support "thinking about feelings", while cognitive perspective taking is more posterior. Basic emotion reading involves medial temporal regions bilaterally, while higher-order empathic concern is right-lateralized.

Correspondence: Suzanne M. Shdo, MPH, Neurology, UCSF, 737 Post St. \#1013, San Francisco, CA 94109. E-mail: sshdo@memory.ucsf.edu

T.L. MCAULEY, C.M. CONSIDINE, K. ROBERTS, A. GARANT, A. SEGUIN, C. ABEARE, A. DHAR \& W. RAJKUMAR. Objective \& Subjective Sleep Quality Differentially Relate to Depressive, Cognitive, and Cognitive-Affective Processes in an Obstructive Sleep Apnea Referral.

Objective: Sleep quality is associated with mood, cognitive functioning, and a related domain- cognitive-affective processing. Subjective and objective indicators of sleep quality are often differentially related to these domains. This study investigated which sleep quality construct was more strongly related to measured sequelae of disturbed sleep.

Participants and Methods: The present study divided an obstructive sleep apnea referral sample ( $\mathrm{N}=55$; Mage $=53.35$, 26 women $)$ into 'good' and 'bad' sleepers for both subjective (i.e., Epiworth Sleepiness Scale, ESS) and objective (i.e., Polysomnogram-determined Sleep Efficiency, $\mathrm{SE})$ measures to determine group differences, and potential interactions, for depressed mood (Center for Epidemiologic Studies Depression Scale, CES-D) and on parallel tasks for cognitive processing (i.e., Stroop) and cognitive-affective processing (i.e., Emotional Stroop Test, EST). Results: In a 2 (high vs. low ESS) x 2 (high vs. low SE) ANOVA, a main effect of ESS was found for CES-D score, $F(1,48)=4.15, p=0.05$. In a similar analysis of Stroop performance, a main effect trend was found for $\mathrm{SE}, \mathrm{F}(1,46)=2.98, \mathrm{p}=0.09$, and there was a significant interaction between SE and ESS, $\mathrm{F}(1,46)=4.68, \mathrm{p}=0.04$. Finally, for the neutral EST trial, a main effect trend was again found for SE, $\mathrm{F}(1,46)=3.83$, $p=0.06$. For positive and negative EST performance, no significant effects were observed.

Conclusions: Subjective sleep quality is associated with depressed mood, while objective sleep quality is more strongly related to inhibitory processes; for the latter, an interaction between objective and subjective measurement methodology was observed. Objective indicators also appear related to affective-inhibition, but the findings were less consistent. Correspondence: Tara L. McAuley, Psychology, University of Windsor, 10325 Eastcourt Dr., Windsor, ON N8R 1E4, Canada. E-mail: mcauleyt@uwindsor.ca
R. GARCIA, M. MORIYASU, D.T. LOBDELL \& R.M. BOWLER. Psychological Symptoms and Quality of Life Among Residents Exposed to Long-Term, Low-Dose Environmental Manganese (Mn).

Objective: Elevated levels of air manganese (air-Mn) exposure have been associated with adverse health effects. This study examined the relationship of air-Mn concentrations with mood and quality of life.

Participants and Methods: 185 residents (age mean $(M)=55.13 \pm 10.88$; education yrs $\mathrm{M}=13.77 \pm 2.60$; residence yrs $\mathrm{M}=41.01 \pm 16.91) \mathrm{ex}-$ posed to long-term air-Mn from two Ohio towns, and 90 residents (age $\mathrm{M}=55.53 \pm 10.96$; education yrs $\mathrm{M}=15.18 \pm 3.04$; residence yrs $\mathrm{M}=33.59 \pm 17.25)$ from an unexposed Ohio town completed the Healthy Days Measures of the BRFSS, and the Symptom Checklist-90-Revised (SCL-90-R). In the SCL-90-R, "caseness" is defined as at least two symptom dimensions at or above 90th percentile of the normative population. Air-Mn concentrations were estimated over ten years using the U.S. EPA's AERMOD dispersion model. ANCOVA, chi-square and regression analyses were used with years of residence and education as covariates.

Results: The exposed towns had proportionally more residents with $\geq 2$ elevated SCL-90-R dimensions ("cases") than the unexposed town (chi-square $=3.602, \mathrm{p}=.058$ ). Air-Mn concentrations were associated with higher levels of Anxiety $(\beta=.162, p=.031)$ and higher Positive Symptom Distress $(\beta=.147, p=.048)$. Obsessive-compulsive $(\beta=.137$, $\mathrm{p}=.071)$ and Psychoticism $(\beta=.136, \mathrm{p}=.072)$ approached significance. Air-Mn concentrations were associated with poor mental health in the past 30 days $(\beta=.168, p=.026)$. Exposed "case" residents compared to exposed "non-case" residents had significantly more days of poor physical health $(\mathrm{M}=7.23 \pm 9.72$ vs. $3.61 \pm 8.37, \mathrm{p}=.013)$ and poor mental health $(\mathrm{M}=9.27 \pm 10.05$ vs. $2.24 \pm 4.84$, p<.001). Exposed "case" residents had more days of physical and mental health limiting daily activities $(\mathrm{M}=4.55 \pm 8.27$ vs. $0.76 \pm 3.07, \mathrm{p}<.001)$.

Conclusions: Results suggest that environmental Mn exposure may have adverse consequences on mood disturbance and quality of life. Disclaimer: This abstract does not necessarily reflect EPA policy.

Correspondence: Raisa Garcia, California School of Professional Psychology, 3123 White Terrace, San Jose, CA 95127. E-mail: rysa. kg@gmail.com

\section{THURSDAY MORNING, FEBRUARY 5, 2015}

\section{CE 7: \\ Pediatric Mild TBI: Who Gets Better, Who Doesn't, and What's Neuropsychology Got to Do with It}

\section{Presenter: Michael Kirkwood}

$$
\text { 7:20-8:50 a.m. }
$$

\section{KIRKWOOD. Pediatric Mild TBI: Who Gets Better, Who Doesn't, and What's Neuropsychology Got to Do with It.}

In recent years, few other medical conditions have received as much scientific or popular attention as concussion or mild TBI. Even so, pediatric mild TBI remains a frequent source of misunderstanding, confusion, and controversy. Methodologically rigorous studies indicate that most school-aged children recover quickly and well. This workshop will focus on the minority who display more persistent problems and the role of neuropsychology in understanding and clinically managing these children. The goals are to summarize the background science most relevant to the natural clinical history of pediatric mild TBI, as well as the known risk factors for persistent problems, and to provide an empirically-backed rationale for why neuropsychology should play a crucial role in clinical management in these cases. 
Correspondence: Michael Kirkwood, Children's Hospital Colorado, 13123 E. 16th Ave, Aurora, CO 80045. E-mail: michael.kirkwood@ childrenscolorado.org

\section{CE 8:}

\section{Cognitive Reserve, From Theory to Intervention}

\section{Presenter: Yaakov Stern}

$$
\text { 7:20-8:50 a.m. }
$$

\section{Y. STERN. Cognitive Reserve, From Theory to Intervention.}

The concept of reserve has been put forward to account for individual differences in susceptibility to age-related brain changes and pathologic changes such as those that occur in Alzheimer's disease. The concept of cognitive reserve suggests that the brain actively attempts to cope with brain damage by using pre-existing cognitive processing approaches or by enlisting compensatory approaches. Although much work has been done applying the concept of reserve to aging and dementia, it has also been applied to many other conditions. This course address the theory underlying the concept of reserve; epidemiologic evidence; the neural substrate of reserve; clinical implications; and interventions.

Correspondence: Yaakov Stern, Columbia University, New York, NY 10032.E-mail:ys11@cumc.columbia.edu

\section{Invited Address: \\ Connectomics and Cognition: A Tale of Many Regions}

\section{Presenter: Deanna M. Barch \\ 9:00-10:00 a.m.}

D.M. BARCH. Connectomics and Cognition: A Tale of Many Regions.

A growing body of research clearly indicates that both functional and structural connectivity within and between core brain systems is a critical determinant of cognitive function in both health and disease. This talk will first overview the current state of the art in terms of tools and methods for assessing human brain connectivity. Second, this talk will illustrate the current state of our knowledge of core human brain networks as derived from either or both structural or functional connectivity methods. Third, the talk will illustrate the ways in which variation in brain connectivity relates to variation in specific cognitive functions in healthy individuals, as well as how impairments in functional brain connectivity relate to impaired cognitive function associated with either or both neurological and psychiatric disorders.

Correspondence: Deanna M. Barch, PhD, Washington University, Psychology, Campus Box 1125, St. Louis, MO 63130.E-mail:dbarch@ artsci.wustl.edu

\section{INS Early Career Award Presentation: Brain, Behavior and Beyond: Tracing the Social Landscape of Pediatric TBI}

\section{INS Early Career Award Winner: Miriam H. Beauchamp}

\author{
10:15-11:15 a.m.
}
Paper Session 1: TBI - DTI

10:15-11:45 a.m.

N. PRESSON, S. BEERS, L. MORROW, J. PENDERVILLE, D. OKONKWO \& W. SCHNEIDER. Advanced White Matter Imaging with High Definition Fiber Tractography Predicts Neuropsychological Test Performance in Adults with Traumatic Brain Injury.

Objective: To predict neuropsychological test performance based on quantifications of white matter tract integrity using High Definition Fiber Tractography (HDFT) in adults with traumatic brain injury (TBI); to test preliminary TBI classification efficacy for HDFT metrics.

Participants and Methods: All participants (11 control; 20 TBI) completed HDFT diffusion imaging sequence and neuropsychological tests. The battery consisted of measures including Trail Making Test, California Verbal Learning Test (CVLT), WAIS-IV Processing Speed Index (PSI), and Controlled Oral Word Association Test (COWAT), as well as self-report scales (Glasgow Outcome Scale-Extended, Brief Symptom Inventory, Neurobehavioral Symptom Inventory). Quantitative metrics of tract integrity included generalized Fractional Anisotropy ( $\mathrm{gFA}$; diffusion spectrum imaging analogue to FA), tract symmetry, and tract spread.

Results: For TBI subjects, several tract measurements of gFA, symmetry, and spread were significantly correlated with neuropsychological test performance (correcting for False Discovery Rate, $\mathrm{p}<.05$ ). Notably, the spread of the corpus callosum and its components was predictive of performance on both short and long delay CVLT free recall as well as Trails A and B (all rs > .45); mean gFA, particularly in the left hemisphere, was correlated with PSI for 9 tracts $(\mathrm{r} .40-.64)$.

Conclusions: Quantification of tract integrity using HDFT is a cutting-edge tool for detecting white matter changes after TBI. These promising findings indicate that with increased sample size, the HDFT methodology may yield meaningful diagnostic information.

Correspondence: Nora Presson, PhD, Learning Research and Development Center, University of Pittsburgh, 3939 O'Hara Street, University of Pittsburgh, Pittsburgh, PA 15260.E-mail: presson@pitt. edu

K.L. MAIN, S. SOMAN, F. PESTILLI, A. FURST, A. NODA, J. KONG, J. CHENG, J.K. FAIRCHILD, L. KINOSHITA, J.L. TAYLOR, J. YESAVAGE, J.W. ASHFORD \& M.M. ADAMSON. DTI Metrics from the Right Inferior Longitudinal Fasciculus and Thalamic Tract Best Discriminate TBI in Patients and Controls.

Objective: To find a statistically significant optimal cutpoint for a white matter fiber tract which can be utilized clinically to discriminate Traumatic Brain Injury (TBI) in patients and controls.

Participants and Methods: MRI scans were performed on patients and controls (Overall n = 126; Age: $\mathrm{M}=44.8, \mathrm{SD}=10.8$; Gender: $81 \%$ male; $n=73$ TBI: $n=53$ non-TBI; $58 \%$ TBI). Diagnosis of mild and moderate TBI was based on neurological examination. Diffusion Tensor Imaging (DTI) data were processed (Vista Lab, Stanford University) to identify 20 fiber tracts and extract two clinically relevant metrics from each fiber tract (Fractional Anisotropy (FA) and Mean Diffusivity (MD)). We used two individual receiver operating characteristic (ROC) models 
to analyze these two measures. We searched for predictors of TBI until statistically significant cutpoints were revealed in $2 / 3$ of the sample. A confirmatory regression analysis was performed in the other 1/3.

Results: In the ROC model using the FA metric, the most statistically significant $($ Kappa $=0.328$, chi-square $=9.812, \mathrm{p}<.01)$ cutpoint identified the presence of TBI $(25 / 32=78.1 \%)$ in Right Inferior Longitudinal (RIL) Fasciculus at or below the FA cutpoint of 0.375 . (Above that cutpoint, $21 / 49(42.9 \%)$ had a Dx of TBI). The RIL-FA model was validated by a regression analysis. In the ROC model using the MD metric, a statistically significant $($ Kappa $=0.408$, chi-square $=14.386, \mathrm{p}$ $<.001)$ cutpoint identified the presence of TBI $(31 / 40=77.5 \%)$ in Right Anterior Thalamic (RAT) Tract at or above the MD cutpoint of 0.830 . (Below that cutpoint, 16/44 (36.4\%) had a Dx of TBI). The RAT-MD model was validated by a regression analysis.

Conclusions: Presence of TBI was best identified in clinical DTI as the FA value for RIL approaches the cutpoint of 0.375 , and MD value for RAT approaches 0.830 . This finding demonstrates how DTI metrics can be used to identify patients with TBI in a clinical setting.

Correspondence: Maheen M. Adamson, PhD, Psyhiatry/VA Aging Clinical Research Center, Stanford University/VA PAHCS, 3801 Miranda Ave, Palo Alto, CA 94304.E-mail: madamson@stanford.edu

B. BUDISIN, B. SHARMA, Y. KOSHIMORI, G. TURNER, D. MIKULIS, C. BRADBURY, A. CRAWLEY \& R. GREEN. Diagnostic Uncertainty and Speculations Regarding Mild Traumatic Brain Injury (mTBI): Diffusion Tensor Imaging (DTI) Versus Conventional Neuroimaging.

Objective: The ability to reliably diagnose mild traumatic brain injury (mTBI) is limited by the insensitivity of conventional measures. Diffusion tensor imaging (DTI), however, is sensitive to the microscopic neuropathology of mTBI. Current research on the diagnostic utility of DTI consists of cohort-based analyses, although discrimination at the single-case level is needed. Therefore, in a case-series, we explored the effectiveness of DTI relative to conventional imaging.

Participants and Methods: From a larger sample $(\mathrm{N}=92)$ of spinal cord injury (SCI) patients, a subset of patients $(n=25)$ received DTI two- to six-months post-injury. MTBI diagnosis was made in 9 patients using a comprehensive and reliable assessment approach that included neurological, neuroimaging, and neuropsychological measures. Absence of mTBI was documented in 10 patients. We controlled for potential confounds (e.g., learning disability, intubation, etc.). DTI was also administered to 14 age-matched, healthy controls.

Results: Twelve regions of interest (ROIs; most susceptible to axonal damage following TBI) were identified through a comprehensive literature review. To control for any possible effects of SCI on brain white matter, ROIs vulnerable to transneuronal degeneration were excluded. For each ROI, the mean FA value across controls was computed and compared to the FA values of individual mTBI-positive patients. Patients were diagnosed with $\mathrm{mTBI}$ if they had a Z-score decrement in FA values of 2.0 or 1.5 in one or two ROIs, respectively, relative to healthy controls. Using this conservative threshold, DTI accurately diagnosed $5 / 9(55.6 \%)$ mTBI-positive patients. In comparison, only one of these patients $(11.1 \%)$ had MRI findings positive for mTBI. No patients had a positive CT scan.

Conclusions: Moving from group level analyses to single-case diagnosis, DTI presents with a significantly higher sensitivity relative to conventional neuroimaging. These preliminary data provide support for the clinical potential of DTI to be validated in larger trials.

Correspondence: Bojana Budisin, Ph.D., Cognitive Neurorehabilitation Sciences Lab, Toronto Rehabilitation Institute, 23 Sheppard Avenue East, Unit 307, Toronto, ON M2N 0C8, Canada. E-mail: bojana. budisin@uhn.ca
K. KLIPFEL, B.A. MUELLER, L.E. KRACH, K.O. LIM \& J.R. WOZNIAK. Microstructural, Functional-Connectivity, and Neurocognitive Disruption in Pediatric Traumatic Brain Injury: A DTI and Resting-State fMRI Study.

Objective: We investigated corpus callosum (CC) microstructure and inter-hemispheric connectivity, and associated neurocognitive deficits, in children with recent traumatic brain injury.

Participants and Methods: Fifteen children (ages 10-18) with mild, moderate, or severe TBI within 6-18 months were studied along with 15 non-injured controls. Participants completed a neurocognitive battery examining processing speed (WISC-IV/WAIS-IV PSI) and motor skills (grooved pegboard). MRI data included resting-state fMRI and DTI. White matter microstructure was evaluated by examining fractional anisotropy (FA) and mean diffusivity (MD) in the anterior and posterior CC. Inter-hemispheric functional connectivity was derived from a 6-minute resting-state fMRI. Analyses focused on participants falling beyond 1.5 standard deviations (SD) from the control mean on FA, MD, and inter-hemispheric connectivity.

Results: MANOVAs showed low FA in anterior (not posterior) CC in TBI as compared to controls (Cohen's d=.75). High MD was seen in both CC regions in TBI (Cohen's d=.87 \& .89). Inter-hemispheric connectivity was disrupted in cortical regions sub-served by anterior (not posterior) CC in TBI (Cohen's d=.78). Pearson correlations (.40-.73) showed association between white matter microstructural integrity and inter-hemispheric functional connectivity.

Among the TBI group, those with abnormalities >1.5 SD on FA and MD showed motor deficits (not processing speed) relative to those with more normative FA and MD. Similarly, outliers on the functional connectivity measures also showed motor deficits.

Conclusions: Research MRI methods (DTI and resting-state functional connectivity) reveal subtle abnormalities in children with TBI, and these abnormalities are associated with practical neurocognitive deficits, especially in the motor domain.

Correspondence: Katherine Klipfel, Kent State University, 1414 Laurel Avenue \#L307, Minneapolis, MN 55403.E-mail:kklipfel@kent.edu

\section{Symposium 1: \\ Investigating Preclinical Alzheimer's Disease: The Wisconsin Registry for Alzheimer's Prevention Experience}

\section{Chair: Ozioma Okonkwo}

\section{0:15-11:45 a.m.}

O.C. OKONKWO, S. JOHNSON, B.B. BENDLIN, S.A. SCHULTZ, L. BRATZKE, L.R. CLARK \& O.C. OKONKWO. Investigating Preclinical Alzheimer's Disease: The Wisconsin Registry for Alzheimer's Prevention Experience.

Symposium Description: There is presently broad consensus that the pathophysiological processes that characterize Alzheimer's disease (AD) begin many years before the onset of clinical symptoms. This preclinical stage of $\mathrm{AD}$ has rightly become the subject of intense scientific interest given that it might represent a critical window of opportunity for optimally characterizing the nature and temporal evolution of AD-related changes, as well as for implementing interventions that might potentially alter the disease trajectory. Founded in 2001, the Wisconsin Registry for Alzheimer's Prevention (WRAP) is one of the largest cohorts worldwide dedicated to the study of preclinical AD. It currently consists of $>1500$ late-middle-aged adults with specific risk factors for $\mathrm{AD}$. This symposium will highlight a selection of the diverse yet integrated portfolio of scientific inquiries that have been undertaken within this unique cohort. Dr. Sterling Johnson will describe the longitudinal brain and biomarker changes that have been observed in the cohort. Dr. Barbara Bendlin's talk will discuss how neuroimaging of myelinated nerve fibers in the 
brain shed light on disease-related loss of structural connectivity. Ms. Stephanie Schultz will present data suggesting that higher cardiorespiratory fitness favorably alters age- and amyloid-associated changes in cognition. Dr. Lisa Bratzke will explore how distinct multimorbidity clusters might be differentially associated with decline in specific cognitive domains. Dr. Lindsay Clark's presentation will review recent developments in the field regarding improved reliability and sensitivity of the diagnosis of mild cognitive impairment, and how these approaches may be applied to relatively younger, risk-enriched cohorts like WRAP. Finally, Dr. Ozioma Okonkwo will lead a discussion on ways that these findings, and those from similar research groups around the globe, inform and support the national mandate to prevent and effectively treat AD by 2025.

Correspondence: Ozioma C. Okonkwo, PhD, University of WisconsinMadison, 600 Highland Avenue, CSC J5/156M, Madison, WI 53792.

E-mail:ozioma@medicine.wisc.edu

S. JOHnSON, B. CHRistian, C.M. Carlsson, A. RACINE, B.B. BENDLIN, O.C. OKONKWO, B.P. HERMANN \& M.A. SAGER. Amyloid imaging and CSF biomarkers in the Wisconsin Registry for Alzheimer's Prevention.

Objective: The longitudinal time course of biomarkers among late middle-age people at risk for AD has not been established previously. This presentation will describe the longitudinal multi-modal imaging and CSF changes that occur over a 2 year interval in the Wisconsin Registry for Alzheimer's Prevention.

Participants and Methods: 201 participants (mean age 61) received positron emission tomography (PET) [C-11]Pittsburgh compound B (PIB) imaging and most also had glucometabolic fluorodeoxyglucose (FDG) PET imaging, volumetric, perfusion, and diffusion MRI. Half the sample also contributed cerebrospinal fluid (CSF). All underwent serial cognitive assessment (2-4 time points) beginning prior to imaging (at mean age of 53) at intervals of 2-4 years using a battery that assesses multiple domains, with focus on episodic memory, from which indices of cognitive decline were derived.

Results: At a mean age of 61 approximately $22 \%$ of the sample was amyloid positive. Over two years $6 \%$ of the PIB negative subjects became positive. At the initial PIB scan, there were no significant associations with cognition and only subtle associations with volume. Persons with an AD-like CSF profile tended to exhibit more wide-spread PIB amyloid burden. Results of longitudinal change in cognition associated with amyloid burden and CSF profiles will be described.

Conclusions: Amyloid positivity is already present at a mean age of 61 in $22 \%$ of a cohort enriched for AD-risk. The effects on markers of atrophy, neural function and cognition are subtle at this age. This is consistent with a prevailing model of early amyloid burden that precedes other biomarkers.

Correspondence: Sterling.Johnson, PhD, Geriatrics, 2870 University Ave \#106, Madison,WI 53705.E-mail:scj@medicine.wisc.edu

B.B. BENDLIN, A. ALEXANDER, K. MELAH, S. JOHNSON, O.C. OKONKWO, C.M. CARLSSON, V. SINGH, N. ADLURU, W. KIM, H. ZETTERBERG, B.P. HERMANN \& M.A. SAGER. Connectivity loss in preclinical Alzheimer's disease: insights from diffusion tensor imaging.

Objective: Brain changes associated with Alzheimer's disease (AD) begin decades before disease diagnosis. While $\beta$-amyloid plaques and neurofibrillary tangles are defining features of $\mathrm{AD}$, neuronal loss and synaptic pathology are closely related to the cognitive dysfunction observed in AD. Brain imaging methods that are tuned to assess degeneration of myelinated nerve fibers in the brain (collectively called white matter) include diffusion tensor imaging (DTI) and related techniques, and are expected to shed light on disease-related loss of structural connectivity. Methods: Participants ( $\mathrm{N}=343$, ages 47-76 years) from the $\mathrm{W}$ isconsin Registry for Alzheimer's Prevention (WRAP) study underwent neuropsychological testing in addition to comprehensive MRI, including DTI, hybrid diffusion imaging, myelin water fraction mapping and T2FLAIRbased lesion mapping to determine the extent to which preclinical AD affects brain white matter. A sub-set underwent lumbar puncture for cerebrospinal fluid (CSF) analysis. The sample was enriched for AD risk (APOE 4 and parental family history of AD).

Results: Risk factors for AD and CSF markers of preclinical AD pathology were significantly associated with imaging measures obtained in limbic white matter. In addition to these findings, this session will discuss new developments in modeling and analysis, including graph theory methods for understanding connectivity, and issues of interpreting the DTI signal.

Conclusions: Findings from the WRAP study demonstrate that preclinical AD involves loss of structural connectivity. These results shed light on the early pathological features of $\mathrm{AD}$, in addition to suggesting that white matter imaging measures may have utility as both tools of early disease detection and outcome measures for clinical trials.

Correspondence: Barbara B. Bendlin, CSC J5/Mezz MC:2420, 600 Highland Ave., Madison, WI 53792.E-mail:bbb@medicine.wisc.edu

S.A. SCHULTZ, E.A. BOOTS, R.P. ALMEIDA, S. YU, D. COOK, D. EDWARDS, R. DOUGHERTY, J. STEIN, J. EINERSON, C. KORCARZ, C.M. CARLSSON, B.B. BENDLIN, S. ASTHANA, B.P. HERMANN, M.A. SAGER, S. JOHNSON \& O.C. OKONKWO. Cardiorespiratory capacity attenuates the influence of age and amyloid- $\beta$ on cognition.

Objective: To examine whether higher cardiorespiratory capacity might favorably alter age- and amyloid-associated changes in cognition in a cohort of late-middle-aged adults at risk for Alzheimer's disease (AD). Participants and Methods: Ninety enrollees in the Wisconsin Registry for Alzheimer's Prevention (age $=64 \pm 6,68 \%$ women, $46 \%$ APOE $4+$, and $73 \%$ with family history of $\mathrm{AD}$ ) participated in this study. They completed a comprehensive neuropsychological exam that assessed six cognitive domains and performed a graded treadmill exercise test using a modified Balke protocol. Expired gases and heart rate were measured continuously and the peak oxygen consumption (VO2peak) during the test was used as an index of cardiorespiratory capacity. Forty-five participants also underwent lumbar puncture for collection of cerebrospinal fluid (CSF) samples, from which amyloid- $\beta 42$ (A $\beta 42)$ was immunoassayed. Regression analyses, adjusted for age, sex, education, and body mass index were used to test whether the effect of age or $A \beta 42$ on cognition was modified by cardiorespiratory capacity.

Results: Mean VO2peak was $26 \pm 7 \mathrm{ml} / \mathrm{kg} / \mathrm{min}$. There was a significant age*VO2peak interaction on Immediate Memory ( $\mathrm{p}=.004)$, and significant $\mathrm{A} \beta 42 * \mathrm{VO} 2$ peak interactions on Verbal Ability $(\mathrm{p}=.034)$, Verbal Learning \& Memory $(p<.001)$, and Immediate Memory ( $p<.001)$, with a trend for Speed \& Flexibility $(p=.096)$. Specifically, with advancing age and lower CSF A $\beta 42$ - both of which confer increased risk for ADindividuals with higher cardiorespiratory capacity exhibited better cognition compared with individuals with lower cardiorespiratory capacity. Conclusions: In a late-middle-aged, at-risk cohort, higher cardiorespiratory capacity was associated with an abatement of age- and amyloid-related alterations in cognition. These findings suggest that exercise might play an important role in the prevention of AD.

Correspondence: Stephanie A. Schultz, CSC J5/Mezz MC:2420, 600 Highland Ave., Madison,WI 53792.E-mail:sschultz@medicine.wisc. edu

L. BRATZKE, R. BROWN \& S. JOHNSON. Multimorbidity and cognition: Latent class analysis within the WRAP cohort.

Objective: Multimorbidity is the occurrence of two or more chronic illnesses and is a growing concern in public health due to its high prevalence and poor outcomes. A growing body of literature has documented the relationship between cognitive decline and single chronic illnesses, such as diabetes, heart and kidney disease. Unfortunately, the effects of multimorbidity on cognition are relatively unknown. The purpose of this 
study is to identify and explore multimorbidity that is associated with cognitive decline among a large sample of community dwelling adults. Methods: Participants from the Wisconsin Registry for Alzheimer's Prevention (WRAP) study underwent neuropsychological testing and completed a medical review that documented the presence of several common chronic illnesses as well as other clinical and demographic variables. Latent class analysis was conducted to identify multimorbidity classes and to predict the likelihood of relationship between an individual class cognitive decline.

Results: Four classes of multimorbidity were identified from the analysis. Participants who experienced cognitive changes consistent with amnesic mild cognitive impairment (aMCI) were significantly more likely to be part of the cardiopulmonary illness class. Participants who experienced cognitive changes consistent with non-amnesic mild cognitive impairment (naMCI) were not significantly associated with any of the multimorbidity classes.

Conclusions: Our findings suggest that there may be a multimorbidity phenotype for aMCI. Further, the multimorbidity phenotype for naMCI may differ from aMCI. Future studies using latent class analysis and other novel statistical methods are needed to validate these findings. Correspondence: Lisa Bratzke, 600 Highland Ave, Madison, WI 53792. E-mail:bratzke@wisc.edu

L.R. CLARK, L. DELANO-WOOD, E.C. EDMONDS, R.L. KOSCIK, O.C. OKONKWO, B.B. BENDLIN, B.P. HERMANN, S. ASTHANA, M.A. SAGER \& S. JOHNSON. Psychometric definitions of mild cognitive impairment and applications to identifying early cognitive change in the Wisconsin Registry for Alzheimer's Prevention (WRAP) study.

Objectives: This presentation will review developments to improve reliability and sensitivity of the diagnosis of mild cognitive impairment (MCI) and discuss the application of these methods to the Wisconsin Registry for Alzheimer's Prevention (WRAP) sample.

Participants and Methods: We recently compared the efficacy of MCI diagnostic criteria using conventional and comprehensive neuropsychological methods in a sample of 134 older adults (Clark et al., 2013) and in a separate sample from the Alzheimer's Disease Neuroimaging Initiative ( $\mathrm{N}=846$; Bondi et al., 2014). The application of these diagnostic methods to 1500 middle-aged WRAP participants is currently ongoing. Study aims include: 1) determine prevalence of MCI in the WRAP sample comparing definitional schemes, 2) investigate cognitive trajectories over eight years using mixed effects regression models, and 3) examine factors that contribute to progression to MCI.

Results: Our recent studies revealed variation in MCI prevalence and characterization of MCI subtypes depending on diagnostic criteria used. Furthermore, application of comprehensive criteria improved prediction of progression to dementia, diagnostic stability, and associations with CSF AD biomarkers. Results of MCI prevalence and cognitive trajectories among the WRAP sample will be presented. Initial results indicate that $13 \%$ met MCI conventional criteria at baseline and onethird reverted to cognitively normal status at follow-up. Application of comprehensive criteria is expected to reduce false positive diagnostic errors and improve sensitivity to detect cognitive change.

Conclusions: Use of comprehensive neuropsychological criteria improves reliability and sensitivity of MCI diagnoses. Identifying subtle cognitive changes in early stages will be essential in treatment efforts attempting to delay the progression of AD neuropathology.

Correspondence: Lindsay R. Clark, M.S., 600 Highland Ave, Madison, WI 53792.E-mail: lrclark@medicine.wisc.edu

\section{Paper Session 2: \\ Pediatric Neuropsychology \& Neuroimaging}

10:15-11:45 a.m.

Y. CHEUNG, D. BHOJWANI, W. LIU, N.D. SABIN, T.M. BRINKMAN, C. KIMberg, D. SRIVASTAVA, M.M. HUdSON, C. PUI \& K.R. KRULL. Association between Acute Treatment-related Neurotoxicities and Executive Dysfunction in Long-term Survivors of Childhood Acute Lymphoblastic Leukemia.

Objective: To identify treatment-related neurotoxicities associated with executive dysfunction in long-term survivors of childhood acute lymphoblastic leukemia (ALL).

Participants and Methods: Eligible survivors included patients who were treated at St. Jude Children's Research Hospital on a chemotherapy-only protocol, without cranial radiation or preexisting neurologic morbidity. 210 (73.7\%) of 285 survivors ( $51.4 \%$ male) completed measures of cognitive flexibility and verbal fluency (Delis-Kaplan Executive Function Scale) at $\geq 5$ years post-diagnosis, with a mean[SD] age of 14.3[4.8] years and 7.7[1.7] years since diagnosis. Acute/sub-acute leukoencephalopathy was identified through MRI. Treatment-related neurotoxic events (eg. seizures, stroke-like syndrome etc.), post-highdose methotrexate serum homocysteine and post-dexamethasone serum cortisol levels were analyzed with general linear modeling as predictors of executive dysfunction.

Results: Overall, $22.4 \%$ of the survivors developed leukoencephalopathy and $9.1 \%$ experienced Grade 2 to 4 neurotoxic events during treatment. Survivors performed worse than the normative sample on cognitive flexibility (mean[SD] Z-score -0.52[1.19], $\mathrm{p}<0.0001$ ) and verbal fluency $(-0.38[0.98], \mathrm{p}<0.0001)$. Univariate analysis revealed that neurotoxic events were associated with poorer cognitive flexibility $(p=0.035)$ and verbal fluency $(p=0.024)$. After adjusting for demographic and other physiologic factors, a higher level of post-high-dose methotrexate serum homocysteine was associated with poorer cognitive flexibility (Est. -0.013; SE 0.006; p=0.031). Notably, the survivors who experienced a neurotoxic event at a younger age demonstrated lower verbal fluency (Est. -0.115; SE 0.058; $p=0.049$ ).

Conclusions: This study demonstrated that the occurrence of acute neurotoxic events and elevated homocysteine level during active treatment are associated with executive dysfunction in long-term survivors of ALL; such patients may benefit from early cognitive intervention. Correspondence: Yin Ting Cheung, Ph.D., Department of Epidemiology \& Cancer Control, St. Jude Children's Research Hospital, 262 Danny Thomas Place, Memphis, TN 38105.E-mail: VinTing.Cheung@Stjude. org

J.E. ROSENQVIST, P. LAHTI-NUUTTILA, C. URGESI, J. HOLDNACK, S. KEMP \& M. LAASONEN. Neurocognitive Development in 3- to 11-Year-Old Children: An International Comparison.

Objective: While previous studies have shown that neurocognitive functions develop with age, and that this development generally is especially rapid before age 9; similarities and differences in this development between countries and languages have rarely been studied. We aimed at investigating how performance in areas of attention/executive functioning, language, memory/learning, social perception, and visuospatial processing differed between children from the USA, Italy, and Finland. Participants and Methods: The 2228 participants were 3 - to 11-yearold children from the USA $(\mathrm{n}=900)$, Italy $(\mathrm{n}=637)$, and Finland $(\mathrm{n}=$ 691), who participated in the standardizations of the NEPSY-II in each country. The children's performance on the subtests from the NEPSY-II domains Attention/Executive Functioning, Language, Memory/Learning, Social Perception, and Visuospatial Processing was outlined and differences in the cross-sectionally assessed development between the countries were statistically investigated. 
Results: The development differed significantly between the three countries for subtests within all NEPSY-II domains. The differences were some subtests over all being easier in one country than the others, or the developmental curve spurting and decelerating differently between the countries.

Conclusions: The present study confirmed that neurocognitive functions in general increase with age. However, the differences between the countries that occurred in the present study indicate that culture and language may influence the development of neurocognitive performance. These results have implications for clinicians assessing children in international settings and emphasize the need for an international perspective in developmental studies.

Correspondence: Johanna E. Rosenqvist, Institute of Behavioural Sciences, University of Helsinki, P.O. Box 9 (Siltavuorenpenger 1), Helsinki FIN-00014, Finland. E-mail: johanna.rosenqvist@helsinki.fi

M.E. FOX, D.E. STODOLA, C.A. BURGHY, T.Z. KING, M.J. ESSEX \& R.J. DAVIDSON. Dorsal Anterior Cingulate-Based Functional Connectivity in Adolescents with Negative Attentional Bias.

Objective: The role of the dorsal anterior cingulate cortex (dACC) in emotion processing and related networks remains disputed, particularly in adolescents. This study examined the relationship between 18-yearolds' negative attentional bias (NAB) and task-derived fMRI functional connectivity $(\mathrm{FC})$ between the right $\mathrm{A} \mathrm{ACC}$ and prefrontal regions following negative compared to neutral images.

Participants and Methods: Participants were 109 (60 female) 18-year-olds. $\mathrm{NAB}$ was measured using negative compared to neutral errors of commission in CANTAB's affective go/no-go task. FC analyses were run separately for reactivity $(2 s-6 s)$ and recovery $(6 s-12 s)$ periods following negative or neutral image display in the scanner. Most data reduction steps were performed using AFNI, images were warped to MNI space using DARTEL in SPM, and regressions were done with SPSS. Results: Participants with greater NAB showed stronger connectivity in the dACC-right and dACC-left lateral PFC networks during the reactivity period $(\mathrm{r}=0.317, \mathrm{p}<0.001 ; \mathrm{r}=0.383, \mathrm{p}<0.001$, respectively). During the recovery period, NAB was positively correlated with right dACC-right dorsomedial PFC FC $(r=0.393, p<0.001)$ and right dACG-left premotor cortex FC $(r=0.309, p=0.001)$. The difference in $\mathrm{FC}$ between the two time periods for the dACG-left premotor cortex pair was significantly correlated with $\mathrm{NAB}(\mathrm{r}=0.342, \mathrm{p}<0.001)$.

Conclusions: Heightened functional connectivity between the dACC and lateral PFC during the reactivity period in participants with greater NAB suggests that this component of the rostral limbic system may contribute to overinterpretation of negative stimuli. The dACC-dmPFC and dACC-premotor networks that remained more strongly paired through the recovery period in adolescents with greater $\mathrm{NAB}$ may play roles in extended maintenance of negative affect.

This work was supported by US NIH grants P50-MH084051, R01-MH044340, and P50-MH052354.

Correspondence: Michelle E. Fox, Psychology, Georgia State University, 475 Buckhead Ave., \#3402, Atlanta, GA 30305. E-mail: mfox9@ student.gsu.edu

C. MRAKOTSKY, C.G. WATSON, K. MALETSKY, A. BOUSVAROS, R.J. GRAND, M.J. RIVKIN \& D. WABER. Reduced Cortical Thickness and Neuropsychological Function in Children with Crohn's Disease.

Objective: Regional cortical thickness has been found to be altered in adults with inflammatory conditions such as Crohn's disease (CD). Mechanisms remain poorly understood, but may include systemic inflammation and steroid therapy. Little is known for pediatric CD. We previously showed decreased white matter density and gray matter volume in children with CD compared to healthy controls, with impact of steroids and inflammation (Mrakotsky et al, 2012,2013). We also found inflammation associated with poorer memory and mood. We here study cortical thinning and functional behavior outcomes in pediatric CD longitudinally.

Participants and Methods: Thirty-two children 9-14 years (12 CD, 20 healthy) underwent structural MRI, neuropsychological assessment, and phlebotomy for inflammatory markers. Patients were scanned and tested during active disease and retested 6 months later. Cortical thickness was measured with Freesurfer. Analyses were adjusted for age and sex, and included regression and group comparisons. Groups were comparable on demographics and IQ.

Results: CD patients had reduced cortical thickness across several brain regions compared to controls [superior, middle frontal (<.01-.05); superior, middle temporal (<.0001-.01); inferior parietal, supramarginal (<.0001-.001)]. Steroids robustly predicted cortical thinning; however, decreased thickness was also associated with higher inflammation $(<.05)$, poorer memory, metacognitive and school function (<.05-.01). Cortical thinning during active disease predicted poorer nonverbal memory and mood 6 months later, particularly in superior temporal, middle temporal and supramarginal regions.

Conclusions: Results support our prior findings of white and gray matter changes in pediatric $\mathrm{CD}$, with effects of both inflammation and treatment on cortical thickness. Our findings further suggest developmental impact of cortical thinning long term. Larger neuroimaging studies are underway to confirm these initial findings, and to elucidate underlying mechanisms and functional outcomes.

Correspondence: Christine Mrakotsky, Ph.D., Psychiatry, Boston Children's Hospital, 300 Longwood Avenue, Boston, MA 02115. E-mail: christine.mrakotsky@childrens.harvard.edu

K.R. FITZER, J.B. HALE, C. LEBEL \& M. WALTON. Cognitive Hypothesis Testing for Targeted Reading Disability Subtype Interventions: Impact on Reading Competency and White Matter Connectivity.

Objective: The ecological and treatment validity of neuropsychological evaluation and treatment of reading disabilities (RD) can be supported by changes in cortical activation and connectivity following reading intervention. This proof-of-concept pilot study sought to examine changes in reading achievement and white matter pathways in response to targeted reading interventions for RD subtypes.

Participants and Methods: Children resistant to special education reading instruction were referred for cognitive hypothesis testing (CHT) neuropsychological evaluations to determine phoneme-grapheme correspondence (PGC) or rapid naming/ fluency (RNF) RD subtypes and inform subsequent 12-week interventions targeted to their individual needs. Single subject slope, standardized reading achievement, and diffusion tensor imaging (DTI) of five brain pathways were used to determine treatment efficacy.

Results: For both RD subtypes, single subject reading performance data showed linear response to the different targeted interventions, and both showed different pre-post improvements on standardized reading measures. The PGC subtype showed changes in phonological, orthographic, and word attack skills, with concomitant fractional anisotropy (FA) changes in the right superior longitudinal fasciculus, left and right inferior longitudinal fasciculi, right inferior fronto-occipital fasciculus, and left arcuate fasciculus. The RNF subtype showed increased rapid word naming and reading fluency, and FA changes in the right superior longitudinal fasciculus and left arcuate fasciculus.

Conclusions: Preliminary findings suggest CHT neuropsychological evaluations that identify specific deficits related to different RD causes can lead to targeted and effective reading interventions. Different white matter pathway changes observed in each subject may reflect observable treatment effects measured by standardized reading assessments and classroom performance. Implications for practice and future research will be addressed

Correspondence: Kim R. Fitzer, MSc, Educational Psychology, University of Calgary, 723 Demel Pl.,, Victoria, BC V9C 3L6, Canada. E-mail: krfitzer@shaw.ca 
V.J. WILLIAMS, J. JURANEK, K. STUEBING, P.T. CIRINO, M. DENNIS \& J.M. FLETCHER. Lateral Ventricular Volume, White Matter Integrity, and Intellectual Outcomes in Spina Bifida and Shunted Hydrocephalus.

Objective: The relations of white matter integrity, post-shunted ventricular volume, shunt revision, and intellectual outcome in medically stable children with spina bifida and hydrocephalus (SBH) have not been previously evaluated. In the case of treated hydrocephalus, we hypothesized reduced white matter integrity in children with enlarged ventricles, and in those a higher number of shunt revisions.

Participants and Methods: Structural MRI and IQ testing was acquired from 80 children with SBH (mean age 13.7 years). Probabilistic diffusion tractography was performed to calculate average metrics of WM integrity along frontal and parietal tectocortical pathways. Resultant DTI metrics were associated with voxel-based total lateral ventricular volume from T1 scans, a composite IQ measure, as well as total number of shunt revisions.

Results: Larger ventricular volume and multiple shunt revisions were associated with increased fractional anisotropy (FA) and reduced radial diffusivity (RD) along both frontal and parietal pathways. Those with no shunt revision history had greater lateral ventricle volume as well as higher FA and reduced RD along frontal and parietal pathways compared than those with multiple shunt revisions. Although mean DTI metrics along parietal pathways were predictive of IQ, intellectual ability had no relation to ventricular volume or the number of lifetime shunt revisions.

Conclusions: Significant changes in DTI metrics were observed as a function of ventricular volume. Increased lateral ventricle volume was associated with elevated FA and reduced RD, congruent with patterns noted in mechanical compression, and potentially suggestive of the presence of subclinical attenuated hydrocephalus. DTI metrics may provide a sensitive indicator of chronic mild hydrocephalus in the absence of overt clinical symptoms due to potential elevations in intracranial pressure. The presence of enlarged ventricles and the occurrence of multiple shunt revisions did not compromise intellectual outcomes in children with SBH.

Correspondence: Victoria J. Williams, University of Houston, $3350 \mathrm{La}$ Jolla Village Drive, 116B, San Diego, CA 92161.E-mail:tori85@gmail. com

\section{Symposium 2: \\ International Perspectives on Education and Training in Clinical Neuropsychology}

\author{
Chair: Christopher Grote
}

10:15-11:45 a.m.

C. GROTE, J.L. PONSFORD, L. HOKKANEN, D. BODIN \& A.M. BUTTS. International Perspectives on Education and Training in Clinical Neuropsychology.

Symposium Description: Training directors from Europe, Australia and North American will discuss typical education and training pathways required to obtain the skills, licensure or board certification needed to practice or be best recognized as an independent provider of neuropsychological services on those continents. While these models can vary significantly, particularly in terms of whether post-doctoral fellowships are available or required, commonalities exist in challenges related to funding, optimal methods for selection of students and trainees, and how to best define the appropriate criteria necessary for verification of skills and knowledge. Dr. Grote will review changes proposed by the American Psychological Association for accreditation of post-doctoral training in neuropsychology and other specialties, and moderate the panel discussion. Dr. Bodin will review the development and current status of methods used to match post-doctoral applicants with training programs in the United States and Canada, and what can be done to improve this process. Dr. Butts will review recent survey data of recent or current North American fellows in neuropsychology regarding their perception of matching techniques and how this system, and training and outcomes can be improved. Drs. Ponsford and Hokkanen will discuss training models and outcomes in Australia and Finland, respectively. Presenters will discuss whether some international standardization of training models is advisable or feasible at some point in the future, and what training directors in different parts of the world can learn from one another in the interim.

Correspondence: Christopher Grote, PhD, Behavioral Sciences, Rush University Medical Center, 1645 W. Jackson, Chicago, IL 60612. E-mail: christopherlgrote@yahoo.com

D. BODIN. A Review of the Hstory and Crrent Issues and Challenges in North American Postdoctoral Training in Clinical Neuropsychology.

Training guidelines for clinical neuropsychology in North America began to be developed in the 1970s and were refined during a national conference in the 1990s (i.e., the Houston Conference). The Houston Conference identified a two-year postdoctoral residency as a required part of training and the capstone experience for independent practice. In addition, a two year postdoctoral experience is required for recent graduates to be eligible for board certification. Despite the strong organizational structure of postdoctoral training in North American clinical neuropsychology, problems remain within the field. The objectives of this talk are to describe the history and status of postdoctoral training in clinical neuropsychology in North American, define problems facing the specialty, and outlines future areas to be addressed.

Correspondence: Douglas Bodin, Ph.D., 700 Children's Drive, Columbus, OH 43205. E-mail: Douglas.Bodin@nationwidechildrens.org

A.M. BUTTS. A Fellow's outlook on recruiting and matching for postdoctoral training in clinical neuropsychology in North America.

Navigating the application and decision making processes to neuropsychology postdoctoral training programs can be very difficult for students in training. Within the current North American postdoctoral training model, sizable challenges exist during this process relating to time, finances, and other resources during an already challenging predoctoral internship year. The disparity between match and non-match programs may exacerbate the uncertainty and challenges students face as they approach a market with limitedly available and desirable positions. The potential dilemma may be particularly salient for those students who apply to both match and non-match participating training programs. Results from a recent survey of applicants suggest the majority of trainees felt that all programs should participate in the match. Unfortunately, many students ultimately accept less desirable, yet certain, positions rather than participating in the match. Further statistics and trainee opinions from this recent survey will be discussed. Ongoing discussions toward consensus on match vs. non-match applications procedures are needed for neuropsychology postdoctoral training programs in the United States.

Correspondence: Alissa M. Butts, Ph.D, 62 Grandeville Rd SW, Unit 1521, Rochester, MN 55902. E-mail:Alissa.m.butts@gmail.com

\section{J.L. PONSFORD. Australian Models of Ttraining in Clinical} Neuropsychology.

Models of training in clinical neuropsychology vary considerably across countries. Whereas, many countries require a qualification in Clinical Psychology before undertaking training in Clinical Neuropsychology, in Australia, training in Clinical Neuropsychology may occur as an alternative to training in Clinical Psychology (with overlapping components), commencing after a four year honours degree in psychology. Training may be undertaken in Masters, Masters/PhD or Doctor of Psychology in Clinical Neuropsychology programs. Each incorporates intensive 
coursework over two years, a substantial research project, at doctoral standard for the doctoral degrees, and 1000 or 1500 hours of clinical placements for the Masters and Doctoral qualifications respectively. All courses must adhere to strictly prescribed accreditation standards, overseen by the Australian Health Practitioner Regulation Agency. These standards prescribe a comprehensive range of subjects and teaching hours on each topic, focused on building core competencies, staffing ratios including clinical neuropsychologists, and offerings of placement opportunities in a range of settings with supervision by appropriately qualified and experienced supervisors, who must comply with rigorous training standards. Following completion of the degree, graduates must practise under supervision for one or two years, depending on the level of their training. Universities face many challenges, including competition in selection of students, the high costs of training, growing demand from hospitals for payment for placements and government pressure to train more students at Masters level to meet workforce demands cost-effectively. This is at odds with the ever increasing knowledge base and scope of disorders with which Clinical Neuropsychology trainees must become competent and the scientist practitioner model of training provided within a doctorate.

Correspondence: Jennie L. Ponsford, PhD, School of Psychological Sciences, Building 17, Wellington Road, Clayton, VIC 3800, Australia. E-mail: jennie.ponsford@monash.edu

\section{HOKKANEN. Neuropsychology Training Models and Issues in Europe.}

The specialist training models in Europe are heterogeneous. Despite efforts to find a common European reference, such as The European Qualifications Framework (EQF), the accreditation and certification models differ. The university degree system in most EU countries follows the so called Bologna model, but the specialist degrees have not been fully integrated into the third cycle. Within psychology, the EuroPsy diploma acts as the qualification standard for master's level and some specialization areas. A common description of the specialist training within neuropsychology however is lacking.

The neuropsychology specialist training programs in some countries are tied to a university degree (doctoral or equivalent), but in some they are offered as a separate certificate / diploma with varied lengths of study. Additionally, there are pressures for example in Finland, presented by the Ministry of Education, to remove the currently existing degree status, presumably thereby making the training program shorter and more directly serving the needs of the health service sector. If neuropsychological training is seen as a scientist-practitioner, this poses a threat to the level of scientific and research education.

Currently in many programs in Scandinavia and elsewhere the required practical training, the internship, is included in the program in full, to be completed before the degree/diploma is issued. In Finland, the program is 4 years part time study, part time work. All students entering the program have a position within the health care sector where they practice neuropsychology under supervision. Once the degree is complete, independent practice is permitted. Transitory post-doctoral training periods would enhance mobility and collaboration between centers but finding funding for the residencies or internship fellowships has been problematic.

Correspondence: Laura Hokkanen, Ph.D., P.O. Box 9, Helsinki FIN-14, Finland. E-mail: laura.hokkanen@helsinki.fi

\section{Poster Symposium: Executive Function in Pediatric Medical Conditions}

\author{
Chair: Jacqueline Sanz \\ 10:15-11:45 a.m.
}

\section{Executive Functions/Frontal Lobes}

\section{K.S. WALSH, L. KRIVITZKY, J. SANZ \& K.K. HARDY. Executive Function in Pediatric Medical Conditions.}

Symposium Description: Executive Function (EF) encompasses several component cognitive skills important to the regulation of behavior and emotions and the orchestration of cognitive abilities toward an organized and efficient attainment of goals. EF challenges are common in childhood disorders. Though each medical disorder has unique cognitive phenotypes (Dennis, 2000) reflecting disease specific factors, there appear to be commonly affected domains. This symposium has three complimentary aims. The first is to compare and contrast profiles of EF across a set of pediatric medical disorders using the Behavior Rating Inventory of Executive Function (BRIEF). Dr. Walsh's discussion of EF profiles in a variety of medical conditions suggests that working memory is particularly vulnerable. Using pediatric stroke as a model, Dr. Krivitzky will discuss the challenges of examining EF profiles in a heterogeneous disorder and methods for investigating factors that predict increased likelihood of EF problems (e.g., age at injury, volume of injury, medical comorbidity). The second aim is to understand EF's contribution to quality of life. Dr. Sanz will discuss EF in children with congenital heart disease, and how EF at school age is a better predictor of quality of life than indicators of medical severity or complexity. The third aim of the study is to begin exploring treatment options for EF problems in childhood medical disorders. Given that working memory has been shown to be particularly vulnerable, Dr. Hardy will discuss her work with computer-based working memory interventions (Cogmed). Despite varying cognitive phenotypes, there are common elements of EF affected across pediatric medical disorders. Thus, a better understanding of the similarities across disorders, the real-life functional impact of EF problems, and development and validation of novel cognitive remediation strategies has the potential to benefit a wide range of medically affected children.

Correspondence: Jacqueline Sanz, Children's National, 111 Michigan Ave., NW, Washington, DC 20010.E-mail: jsanz@cnmc.org

J. SANZ, J. WANG, M. BERL, A.C. ARMOUR, Y. CHENG \& M.T. DONOFRIO. Executive Function as a Predictor of Quality of Life in School Age Children with Congenital Heart Disease.

Objective: Children with congenital heart disease (CHD) have a high rate of Executive Dysfunction (EdF). This study evaluates the relationship between EdF, medical factors, and quality of life (QoL) in children with CHD.

Participants and Methods: 82 Parents of children with CHD (mean age 8.9; 49 male) completed the Behavior Rating Scale of Executive Function (BRIEF), Parenting Stress Inventory (PSI) and PedsQL. Latent class analysis identified two groups of patients with different likelihoods of EdF. 33\% of patients fell in the latent class with high probability of EdF across subscales (0.65-0.84), the remaining 67\% had low probability of EdF (0-0.17). EdF group membership and medical characteristics (type of defect, gender, prematurity, neurological abnormalities) were entered as predictors of QoL (as measured by PSI and PedsQL) using structural equation modeling (SEM).

Results: SEM indicated a direct negative association between EdF and QoL $(\mathrm{p}<.001)$. Medical characteristics did not directly influence QoL. Prematurity $(p=.003)$ and male gender $(p=0.025)$ had indirect, negative associations (via EdF) on QoL. History of two-ventricle repair (e.g., less 
severe CHD) had a marginally negative indirect impact on QoL via EdF $(p=.0 .055)$. Neurological abnormalities did not impact QoL or EdF. Conclusions: EdF predicts QoL in children with CHD, more so than medical complexity. While some medical factors (prematurity and gender) can affect later QoL, they do so indirectly by impacting risk for $\mathrm{EdF}$. This model suggests that $\mathrm{EdF}$ is essential to understanding risk for poor QoL in children with CHD. Assessment and amelioration of EdF may be important to improving QoL for children with CHD. Correspondence: Jacqueline Sanz, 111 Michigan Ave., NW, Washington, DC20010.E-mail:jsanz@cnmc.org

\section{KRIVITZKY. The Relationship Between Executive functioning and Age/disease factors in Pediatric Stroke.}

Pediatric medical disorders are typically heterogeneous in their make-up and thus understanding predictors of neuropsychological outcome is often very challenging. We use pediatric stroke as a model of a "heterogeneous disorder" as children present with stroke for many different reasons, at different ages, and have highly variable outcomes. Despite all of these diverse factors, there are themes that emerge across the spectrum of pediatric stroke/vascular disorders, including a high incidence of ADHD and associated problems with executive functioning skills. The goal of this presentation is 1) briefly review the scope of what is known about executive functioning problems in children with a history of pediatric stroke, 2) present more focused data on the area of working memory, as this has been found to be a particularly vulnerable skill in other pediatric disorders 3) discuss the challenges of examining the relationship between these neuropsychological variables and other factors (e.g., age, disease, etc) in a heterogeneous disorder 4) discuss ongoing and future efforts in the field to better define/study risk factors for executive functioning problems in pediatric stroke. In particular, we will focus on how to better define the subtypes of pediatric stroke (e.g., perinatal vs. childhood, AIS vs. hemorrhagic) and methods for more clearly defining injury characteristics (e.g., lesion volume using modified ASPECTS score).

Data for this presentation will include both data from the stroke registry participants at the Children's Hospital of Philadelphia and review of prior studies examining executive functioning in children with a history of pediatric stroke/vascular disorders. Data on executive functioning skills will include both parent report questionnaires (BRIEF) and paper/ pencil measures of working memory and other executive skills.

Correspondence: Lauren Krivitzky, PhD, 34th and Civic Center Blvd, CSH-Room 021, Philadelphia,PA 19104.E-mail: cheeriodog@aol.com

K.K. HARDY, S.A. HOSTETTER \& M.T. ACOSTA. Computerized Cognitive Training for Children with Cancer and Neurofibromatosis type 1.

Background: Children with neurofibromatosis, type 1 (NF1) and survivors of CNS-impacting cancers are at increased risk for executive dysfunction, yet few interventions targeting this domain have been empirically evaluated. Several recent studies have examined the efficacy of computerized working memory training in these populations. We will present data on changes in working memory functioning following completion of such training programs by samples of children with NF1, acute lymphoblastic leukemia (ALL), and brain tumors (BT).

Methods: Data from four, small-sample trials will be presented. Two studies are prospective, single-arm trials with a pre-test, post-test design including children aged 8-16 with either NF1 (Study 1, n=25) or newly-diagnosed ALL or BT (Study 2, n=20). The other studies are randomized controlled designs with survivors of ALL or BT aged 8-16 (Study 3, n=20) or 4-7 (Study 4, n=20). Across studies, participants completed a brief battery of neuropsychological tests at baseline, and parents provided questionnaire ratings of their children's psychological and executive functioning. Children meeting eligibility criteria completed a computerized working memory training program, Cogmed, at home with phone-based coaching support. Afterwards, children returned for follow-up evaluation. Primary outcomes were attention and working memory tasks, using both traditional and computerized measures.

Results and Conclusion: A total of 85 participants have participated in the cognitive training program across trials (58.9\% male). Treatment completion rates range from $50 \%$ (Study 2 ) to $85 \%$ (Study 3 ) with no adverse events reported. Neuropsychological gains following training tend to be greatest for near-transfer tasks of short-term memory and working memory. There is less evidence of far-transfer effects on real-world functioning. Changes in neurocognitive outcomes at follow-up will be discussed in terms of clinical meaningfulness and generalizability. Correspondence: Kristina K. Hardy, PhD, 111 Michigan Ave, NW, Washington, DC 20010. E-mail: kkhardy@childrensnational.org

K.S. WALSH, L. KRIVITZKY \& M. BERL. Patterns of Executive Functioning in Pediatric Medical Disorders affecting the Central Nervous System.

Objectives: Deficits in executive functioning (EF) are known sequelae of many childhood CNS-impacting medical conditions or their treatments. Differences or similarities in the patterns of EF difficulties across disorders are under studied. We compare EF profiles across multiple pediatric medical conditions and examine the relationship to age and disease onset.

Participants and Methods: Five groups of children (ages 3-18, FSIQ > 70) including Partial Epilepsy ( $\mathrm{n}=97)$, Neurofibromatosis-Type $1(\mathrm{n}=55)$, Brain Tumors $(n=83)$, Leukemia $(n=62)$, and Urea Cycle Disorders $(n=49)$ were studied. EF profiles were compared between groups with mixed model MANOVA based on parent report BRIEF.

Results: A group effect for medical diagnosis $(\mathrm{F}=1.510, \mathrm{p}=.042)$ revealed higher levels of executive dysfunction in the NF-1 and UCD groups. Working memory and planning/organization were consistently rated as the most elevated subscales (peaks in the profiles) across diagnoses (mean T scores 57-62 and 53 -60, respectively), although group means did not reach clinically significant levels $(T \geq 65)$. A trend for an association between younger age at diagnosis/symptom onset (birth/ infancy) and more executive dysfunction was found ( $\mathrm{F}=1.566, \mathrm{p}=.081)$. However, this finding is confounded, as the NF-1 and UCD groups both have a high percentage of early onset subjects (under 2).

Conclusions: Our results suggest that children with medical conditions that impact the CNS have similar EF profiles with mild elevations for aspects of metacognitive skills. A risk factor for greater executive dysfunction may include specific medical conditions, particularly those with earlier age of symptom onset/diagnosis, or treatment.

Correspondence: Karin S. Walsh, PsyD, 111 Michigan Avenue, Suite 1200,Washington, DC 20010.E-mail:kwalsh@childrensnational.org

\section{Poster Session 2: EF/Frontal, Forensic, \& Malingering \\ 10:15-11:45 a.m.}

\section{Executive Functions/Frontal Lobes}

A.C.ZANINOTTO, P.A. RODRIGUES, I.S. NEVILLE, C.Y.HAYASHI, J.P. SABINO, S.A. EL HAJJ, B. SHIEH, S.M. LEONARDO, M. NAGUMO, V.P. GUIRADO, F. FELTRIN, R.L. AMORIM, M.S. DE LUCIA \& W.S. PAIVA. Neuropsychological Assessment in Traumatic Diffuse Axonial Injury Patient Before and After Repetitive Transcranial Magnetic Stimulation - A Pilot Study.

Objective: This is a pilot study from a repetitive Transcranial Magnetic Stimulation (rTMS) research, which aims to analyze through cortical excitability and neuropsychological evaluation the early and late effects of 10 TMS sessions on patients with Diffuse Axonal Injury (DAI).

Participants and Methods: The pilot study participant is a 26 year-old severe DAI patient (hospital admission GCS $=4$ points) who suffered 
trampling on public via in 2010. Neuropsychological assessment included: Trail Making Test forms A and B, Stroop Colored test Victoria version, Grooved Pegboard, Five Points, Symbol Digit oral version, COWAT (FAS) and animal fluency. The participant was assessed one month before rTMS sessions and one week after and at the same days were measured the cortical excitability.

Results: Analyzing the results before and after rTMS, it was observed oscillation results of executive functions and processing speed. The results showed worsening in performance after rTMS sessions on Stroop test and Five Point, however, it was observed improvement on COWAT (FAS) and animals fluency test, Symbol Digit test, Trail Making Test A and B, Grooved Pegboard test. Cortical excitability was changed in time. Conclusions: On this pilot study it was observed that rTMS has positive effects on DAI patient, improving executive functions and processing speed. This technique has shown great efficacy in the treatment of some psychiatric disorders, and it is expected to bring promising results for TBI patients.

Correspondence: , . E-mail:

Y. KWON, J. LEE \& S. CHOI. Neurobiological characteristics associated with impulse control impairment of Internet Addiction: A resting state EEG study.

Objective: Patient with Internet Addiction(IA) is considered as having greater impulsivity than healthy person. Frontal Beta EEG amplitude is known as an index of cortical arousal. Decreased beta activity which is believed as an electrical phenotype of frontal hypo-metabolism is associated with impulsivity. In the present study, we examined whether low frontal Beta power is a neurophysiological marker that associated with high impulsivity of IA.

Participants and Methods: Eight patients with IA(mean age 23.13, $\mathrm{sd}=1.89)$ and age, sex, IQ matched 8 healthy controls participated this study. Resting state EEG record and Go/No-go task response ware obtained. Resting state EEG was recorded on both eye opened and eye closed conditions. Also, participants completed BIS-11 to measure self-reported impulsivity.

Results: In comparison with control group, IA group shown significantly higher error rate in No-go condition of Go/No-go task. Also, frontal high beta amplitude and occipital low beta amplitude of IA group was significantly lower than control group.

Conclusions: The results suggest that high impulsivity of IA is closely related to decreased frontal beta amplitude which could be regarded as pathophysiological feature of IA. Also, the present study raised the possibility that impulsivity is associated with decreased beta power on occipital area.

Correspondence: , . E-mail:

W.D. LOPEZ, C. MCELWEE, G. EISMAN, M. COTTINGHAM, D.J. HARDY, M.J. WRIGHT \&. T.L. VICTOR. English as a Second Language and Performance on Tests of Executive Functioning.

Objective: In 2011, 44\% of Californians spoke a language other than English at home (Ryan, 2013). Research shows bilinguals outperform monolinguals on tasks of executive functioning (Banich, 2004; Bialystok, 2005). Switching between languages is thought to possibly strengthen cognitive flexibility (Bialystok, 2008; Garcia, 2012). Giving this, we hypothesized that individuals for whom English is a second language (ESL) would outperform individuals for whom English is a first language (EFL) on tests of executive functioning.

Participants and Methods: Neurologically and psychologically healthy college undergraduate students participated in one of two groups, ESL $(\mathrm{n}=42)$ and EFL $(\mathrm{n}=69)$. Participants completed a neuropsychological battery that included tests of executive ability (Trail Making Test, Stroop Color-Word Test, and the Controlled Oral Word Association Test - FAS).

Results: Groups did not differ significantly with respect to age, education or gender. An independent t-test revealed significant differences in COWAT test performance (ESL $<\mathrm{EFL}, \mathrm{p}=.006)$. An independent t-test revealed no significance differences in Stroop Color-Word Test performance $(p=.239)$ and Trail Making Test performance $(p=.09)$. Conclusions: Results were not in support of our hypothesis. In fact, COWAT performance was significantly different between groups in the unanticipated direction. Some have argued that bilinguals have lower levels of vocabulary than monolinguals by virtue of having to know two languages (Bialystok, et al., 2009; Luo, et al., 2010), which may help explain the difference observed here on a test of verbal fluency. Results of the study are discussed in light of methodological limitations and directions for future research.

Correspondence: Walter D. Lopez, Psychiatry, LA BioMed, 1357 Elm Ave 5, Long Beach, CA 90813. E-mail: wdaniel31@hotmail.com

\section{C.C. CRANSTON \& P.D. BLANTON. A Case for the Use of Action Fluency in Medial-Frontal Lesions.}

Objective: Verbal fluency tasks are long-standing and frequently used measures of executive and language function. The sensitivity of these tasks has been well established while the specificity has been more equivocal. Semantic fluency has largely been limited to categories of nouns (i.e., animals or fruits and vegetables). Action fluency (verbs) has been developed as a measure of frontal-basal ganglia loop pathophysiology, but its limited research has focused mainly on degenerating dementias and not with stroke patients.

Participants and Methods: A patient with a history of bilateral basal ganglia and left internal capsule infarcts underwent comprehensive neuropsychological evaluation including verbal fluency tasks (i.e., phonemic, semantic, and action).

Results: Both Phonemic and Action fluency were severely impaired (5/20 and 1/20, respectively). Scores for semantic fluency were mild-to-moderately impaired $(3 / 20)$. The profile was notable for significantly weaker performance on Action fluency.

Conclusions: Based on the findings in this case, there appears to be a reasonable degree of divergent validity between Action and Noun fluency as well as specificity with regard to the frontal-basal ganglia loop pathophysiology. Although these findings represent only a single case, they are meaningful in that data were gathered on a purely clinical outpatient basis without exclusion or recruitment and provide preliminary clinical utility for Action fluency in medial-frontal stroke patients. Furthermore, these findings necessitate a call for further research with combined imaging and neuropsychological data in patients with varying frontal lesions in order to examine the extent of pathophysiological specificity between fluency tasks.

Correspondence: Christopher C. Cranston, MA, Mental Health, VAMC Birmingham, 712 Raliegh CT Apt A, Homewood, AL 35209. E-mail: christopher-cranston@utulsa.edu

J.R. WIENER \& M.W. HAUT. The Neurobehavioral Exam: A Measure of Mental Status and Frontal Lobe Function.

Objective: Measures of general brain dysfunction often do not capture frontal lobe dysfunction in neuropsychiatric patients. We piloted a new measure, the Neurobehavioral Exam (NBE), designed to evaluate frontal lobe dysfunction, as well as general mental status.

Participants and Methods: We standardized our informal neurobehavioral procedures and retrospectively reviewed the neuropsychological evaluations of a consecutive clinical sample of medical, neurologic, and psychiatric outpatients ( $\mathrm{N}=82$, Mean age $=53.20)$. The following NBE scores were calculated: total correct, total frontal errors, and total score. We examined NBE performance using traditional neuropsychological measures of frontal lobe dysfunction and general mental status.

Results: NBE total correct, total errors, and total score each correlated strongly with performance on the Trail Making Test, parts A (respectively: $\mathrm{r}=-.796, \mathrm{p}<.001 ; \mathrm{r}=.506, \mathrm{p}<.001 ; \mathrm{r}=-.788, \mathrm{p}<.001)$ and $\mathrm{B}$ (respectively: $\mathrm{r}=-.807, \mathrm{p}<.001 ; \mathrm{r}=.581, \mathrm{p}<.001 ; \mathrm{r}=-.814, \mathrm{p}<.001)$. NBE total correct and total score correlated with measures of perseverative errors, perseverative responses, and longest string of perseverative responses on the $\mathrm{W}$ isconsin Card Sorting Test (all $\mathrm{p}<.05)$. NBE total 
correct and total score also correlated with age, education, WRAT-4 reading, and MMSE performance (all $\mathrm{p}<.05$ ).

Conclusions: The NBE correlates strongly with traditional neuropsychological measures of frontal lobe function as well as general mental status. Thus, this brief new measure may be used to assess general brain dysfunction and frontal lobe dysfunction in clinical populations. Future work should include collection of normal control data and examination of the NBE performance in individuals with known frontal lobe disease. Correspondence: Jennifer R. Wiener, Psy.D., Department of Neurology and Rehabilitation Medicine, Lutheran Medical Center, 15055 th Street, Station 3-31,Brooklyn, NY 11220.E-mail: jennifer.wiener@gmail.com

M. WELSH, P. GORMAN BARRY, L. COOPER \& L. BEDDES. Homeless Men in Transitional Housing Receiving the BrainWise Curriculum: Baseline Data on Executive Functions, Problem Solving and Coping Self-Efficacy.

Objective: Homeless single men living in transitional housing are being taught the BrainWise curriculum, designed to enhance their critical thinking skills. At baseline, we examined the reliability and intercorrelations among measures of self-reported executive functions, coping self-efficacy, and problem solving. Further, the associations between the thinking skills taught in Brain Wise and these important adaptive constructs were explored.

Participants and Methods: Two hundred and six males ( $\mathrm{M}$ age $=$ $41.93 \mathrm{yrs}, \mathrm{SD}=11.22 \mathrm{yrs}, 18-65 \mathrm{yrs}$ ) were administered a battery of tasks including: Behavior Rating Scale of Executive Functions (BRIEF) yielding a Behavior Regulation Index (BRI) and Metacognitive Index (MI), the Wasik Problem Solving Rating Scale (WPS), Coping Self-Efficacy (CSE), and the BrainWise Knowledge Survey (BKS), developed for this study.

Results: The reliability of each measure was adequate (BKS alpha =.71, WPS alphas $=.76)$ to strong (CSE alpha $=.90$ to BRIEF alpha $=.97)$. Age correlated only with the WPS score, $r=.14, p=.03$. Significant correlations were found between CSE and WPS. CSE and the BRIEF indices, and WPS and the BRIEF indices, in the expected direction. The BKS significantly correlated in the expected direction with the BRIEF indices, WPS, and CSE.

Conclusions: Our findings indicate that these measures are reliable (internally consistent) in this high-risk, vulnerable population. Self-reported executive function deficits are related to problems with coping self-efficacy and problem solving; whereas, better coping and problem solving skills covary. Importantly, the skills taught in the BrainWise curriculum are associated with the adaptive processes of executive functions, problem solving, and coping.

Correspondence: Marilyn Welsh, PhD, School of Psychological Sciences, University of Northern Colorado, Box 94, University of Northern Colorado,Greeley, CO 80639.E-mail: marilyn.welsh@unco.edu

E.C. QUINTIN, M. COCHRANE \& M.A. GARCIA-BARRERA. Association Between Executive Functions and Loneliness Across the Adult Lifespan.

Objective: Inhibition, shifting, and other executive functions (EF) are constantly exercised during social interactions. In fact, perceived loneliness has recently been suggested to be a risk factor of accelerated $\operatorname{cog}$ nitive decline, especially affecting inhibition. However, little is known regarding the vulnerability of other aspects of a multidimensional executive system to self-perceived loneliness, and across the adult lifespan. Participants and Methods: This study investigated the association between EF (i.e., inhibition, shifting, updating working memory, reward processing, and problem representation), age ( $N=60$; Group 1: 16 young adults ages 30-40, Group 2: 22 middle-aged adults ages 50-60, and Group 3: 22 older adults ages 70+), and loneliness (i.e., UCLA Loneliness Scale). More specifically, the moderated effect of loneliness on the relationship between age and EF was examined, predicting that the combined effect of increased age and loneliness scores would be more strongly associated with decreased EF
Results: While there was no significant moderating effect in the middle-age group, regression analyses revealed significant patterns in the other groups. In young adults, loneliness moderated the relationship between age and problem representation (i.e., the ability to initiate problem solving; $\mathrm{t}(15)=-2.325, \mathrm{p}=.038)$. In older adults, loneliness moderated the relationship between age and inhibition $(t(21)=2.141$. $p=.046)$. Examining separate levels of the moderator variable in older adults revealed the following results: loneliness significantly predicted performance for the reward processing task $(\mathrm{t}(21)=-2.182, \mathrm{p}=.041)$, and age for the shifting and updating working memory tasks $(\mathrm{t}(19)=$ $-2.683, p=.015$ and $\mathrm{t}(21)=-3.064, \mathrm{p}=.006$ respectively $)$.

Conclusions: Overall, our findings supported the hypothesis that the combined effect of self-perceived social isolation and age seems to vary across age groups and performance on different EF measures, further reinforcing the heterogeneous process of cognitive aging.

Correspondence: Emilie C. Quintin, MSc, Clinical Neuropsychology, University of Victoria, P. O. Box 3050, University of Victoria, Victoria, BCV8W 3P5, Canada.E-mail: emiliecq@uvic.ca

A. ANTOINE, D.M. WHITESIDE, C. HOWARD, S. TAHSIN, R. SHAH, L. RICE, M.R. BASSO \& B. ROPER. The Relationship Between Cognitive Reserve and Executive Functioning.

Objective: Cognitive reserve (CR) is the ability of cognitive functioning underlying a task to sustain disruption and still operate effectively (Stern, 2002). The objective of this study was to examine the relationship between executive functioning (EF) and CR in mild traumatic brain injury (mTBI) patients. For this study, cognitive reserve was operationally defined as the level of premorbid functioning as measured by the Wechsler Test of Adult Reading (WTAR) (Green, et. al., 2008). Participants and Methods: This study consisted of 42 consecutive referrals to an outpatient clinic who completed all study measures and were diagnosed with mTBI. The average age was $42.77(\mathrm{SD}=16.07)$ and the average level of education was 13.38 years $(\mathrm{SD}=2.52)$; all participants passed performance validity tests (PVTs), specifically the Test of Memory Malingering (TOMM, Morey 1996).

Results: A correlational analysis was conducted on WTAR and EF measures including Verbal Fluency (FAS version) and Wisconsin Card Sort Test number of trials to the first category (WCST-Trials) and number of errors (WCST-Errors). The WTAR was significantly correlated with FAS, $r=.35, p<.01$; with WSCT-Trials, $r=-.29, p<.05$; and WCST-Errors, $\mathrm{r}=-.38, \mathrm{p}<.01$. A multiple regression was then conducted with the three EF variables as predictors. Results indicated that the EF variables significantly predicted WTAR and accounted for a significant percent of variance $\mathrm{R} 2=.21, \mathrm{~F}(3,60)=5.57, \mathrm{P}<.05$.

Conclusions: These results indicated that executive functioning performance is significantly related to premorbid cognitive reserve in credible performance mTBI patients.

Correspondence: Douglas Whiteside, Ph.D., Clinical Psychology, Argosy University-Seatle, 200 Hawkins Drive, Iowa City, IA 52242. E-mail: douglas-whiteside@uiowa.edu

T. FRANCIS, A. TAN, K. STEWARD, L.E. DELGATY \& M. BUNNER. Gender Effects on Self-Awareness of Executive Functioning Deficits in Adolescents with ADHD.

Objective: Recent studies have demonstrated that children with Attention Deficit Hyperactivity Disorder (ADHD) often display an inflated view of their executive functioning (EF) abilities. This study examines whether there are gender differences in self-awareness of executive functioning.

Participants and Methods: Participants (free of comorbid psychological disorders) include 22 control (male $n=9)$ and 35 (male $n=26$ ) ADHD subjects, aged 11-16. Behavior Rating Inventory of Executive Functioning Self and Parent (BRIEF-SR/PR) forms were collected for each subject from an archival database at a private Neuropsychology Clinic. Discrepancy scores were calculated for each domain by subtracting the adolescent's T-score from the parent's T-score. Positive 
discrepancy scores indicated that the adolescent reported fewer difficulties than the parent.

Results: Interaction between ADHD and gender was assessed using an MANOVA. Discrepancy scores in Metacognition Index (MI) were significantly more positive for children with ADHD compared to controls $(p=0.04)$. Discrepancy scores for Behavior Regulation Index (BRI) were significantly more positive for males than females in both the ADHD and control groups $(p=0.026)$. There was no significant interaction between ADHD and gender on reported discrepancy scores for MI, BRI, or GEC. Conclusions: Within the MI domain of EF, adolescents with ADHD tend to endorse fewer difficulties when compared to parent reports. Within the BRI domain of EF, males tend to endorse fewer difficulties than parents. Lack of self-awareness in both genders may create barriers in treatment aimed at improving EF. This study suggests that males have less self-awareness of their behavioral regulation. Future research directions and clinical implications regarding this gender difference are discussed.

Correspondence: Taylor Francis, Psychology, B.S., Neuropsychology (Pediatrics and Adults), Austin Neuropsychology Clinic, 2929 E Martin Luther King.Jr Blvd, Austin, TX 78702.E-mail:taycfran@gmail.com

S. GUNTHER \& S. PARDO. Exploring the Pencil Tapping Task: Analyzing Receptive Vocabulary and Obedience as Correlates of Inhibitory Control.

Objective: The Pencil-Tapping Task (PTT) is an assessment of key neuropsychological components, including executive function. Prior research indicates connections between executive function, intelligence, and obedient behavior. This study tests three hypotheses: 1 . Scores from an early administration of an inhibitory control task will predict scores on later administrations. 2. Scores from an inhibitory control measure will correlate with scores from a measure of receptive vocabulary. 3. Scores from an inhibitory control measure will correlate with scores from a measure of obedience.

Participants and Methods: Participants were 3349 children selected from 486 Head Start Classrooms for the Family and Child Experiences Survey (FACES), 2009 cohort. Children were given the Pencil Tapping Task and Peabody Picture Vocabulary Test-4th Edition while parents and teachers gave reports of the frequency and type of their child's disobedient behaviors. Data was collected initially in fall, 2009, then at 3 later time points, ending in 2012.

Results: Linear regression was used to analyze the first hypothesis, while bivariate correlation was used for remaining hypotheses. For the first hypothesis, intital PTT performance consistently predicted later performance $\mathrm{B}=.548 \mathrm{p}<.001$. For the second hypothesis, PTT performance correlated with receptive vocabulary at all time points, with the weakest correlation at $r=.11, p<.05$. No significant findings were observed for the third hypothesis.

Conclusions: Inhibitory control may be linked to receptive vocabulary development. Surprisingly, connections between inhibitory control and disobedient behavior were not supported in the sample. Future research is needed to explore the possibility of connections between inhibitory control and disobedient behavior. Further studies may indicate a relationship which develops later in child development than this study was able to survey (Buss, Kiel, Morales \& Robinson, 2014).

Correspondence: Steven Gunther, California School of Professional Psychology at Alliant International University-San Francisco, 52 E 16th Street Apt 6, Antioch, CA 94509.E-mail:sgunther@alliant.edu

D.A. DENNEY, N. PATEL, R. DEWEY \& L.H. LACRITZ. Relationships Between the Questionnaire for ImpulsiveCompulsive Disorders in Parkinson Disease-Rating Scale and Measures of Executive Function.

Objective: Individuals with Parkinson disease (PD) are observed to more frequently have impulse control disorders (ICDs) than the general population. Investigation of this phenomenon has revealed disturbances in corticostriatal regions and suggests a dysregulation of dopaminergic mesolimbic pathways. This study examined the relationship between the Questionnaire for Impulsive-Compulsive Disorders in PD-Rating Scale (QUIP-RS) and measures of executive function in individuals with PD. Participants and Methods: 17 patients [M age=66.8(8.7); education=14.8(1.6)] with PD were administered a neuropsychological battery as part of a clinical assessment that included Trail Making Test (TMT), Wisconsin Card Sorting Test (WCST), and Phonemic Fluency (FAS) as well as the QUIP-RS. QUIP-RS Total ICD scores were correlated with TMT, WCST, and FAS scores using Spearman's rho correlation coefficient.

Results: QUIP-RS scores were sub-threshold (i.e., below score of 10) for ICDs (M QUTP-RS=8.4(6.1; range=0-19). Overall performance on TMTA $[\mathrm{T}=44(8.8)]$ and TMTB $[\mathrm{T}=40(9.5)]$ was low average, while FAS $[\mathrm{T}=48(11.3)]$ and WCST Perseverations $[\mathrm{T}=50(10.6)]$ were average. The Spearman's rho revealed a significant relationship between QUIP-RS ICD score and TMTA $[\mathrm{rs}(16)=.45, \mathrm{p}=.04]$ and TMTB $[\mathrm{rs}(15)=.56, \mathrm{p}=.01]$. QUIP-RS ICD score was not significantly associated with FAS or WCST.

Conclusions: Counter to expectation, better performance on TMTA and TMTB was associated with higher reports of ICD symptoms whereas other measures of executive function were not significantly associated with reported ICD symptoms. While based on a small sample, these findings suggest better psychomotor speed and set shifting among PD patients with ICD symptoms and warrant further investigation with a larger sample.

Correspondence: David A. Denney, M.S., Psychiatry, UT Southwestern Medical Center, 5323 Harry Hines Blvd, Dallas, TX 75390-8846. E-mail:david.denney@utsouthwestern.edu

K. VAN MOORLEGHEM, N. HANTKE, A. MACKAY-BRANDT, R.E. MORRIS \& S.A. BEAUDREAU. Anxiety Predicts Performance on Stroop Interference but not Stroop Switching.

Objective: Elevated anxiety has been associated with poorer cognitive control on Stroop interference, which requires goal maintenance. However, we recently found that the interaction between elevated anxiety and elevated worry facilitated Stroop performance (fewer self-corrected errors) relative to elevated anxiety and low worry in older adults (Beaudreau, Kraemer, Henderson, \& O'Hara, in preparation). Here, we examined whether the association of anxiety on cognitive control was moderated by worry during a more complex Stroop task requiring goal maintenance and switching between naming the ink color and reading the word if in a box.

Participants and Methods: Cognitively intact community dwelling older adults $(\mathrm{N}=121$; mean age $=72.2, \mathrm{SD}=7.0)$ completed a full battery, including a health rating, Beck Anxiety Inventory (BAI), Penn State Worry Questionnaire (PSWQ), Beck Depression Inventory (BDI-II), and Delis-Kaplan Executive Function System (DKEFS) Color-Word Interference Test (CWIT). Multiple linear regressions adjusted for age, education, sex, self-reported health and examined whether self-reported anxiety (BAI), worry (PSWQ), depression (BDI-II), or their interactions predicted errors on switching (CWIT condition 4).

Results: Anxiety alone or in interaction with worry or depression, did not significantly predict uncorrected or self-corrected errors on switching. Conclusions: Associations between anxiety and Stroop performance are potentially limited to the interference condition of Stroop tasks (e.g.. CWIT condition 3 ) and are not necessarily captured by conditions that tap other aspects of cognitive control, such as switching. Future studies examining these dissociations in the relationship between anxiety and cognitive control performance in older adults would be helpful for developing theories regarding mechanisms of this association

Correspondence: Katie Van Moorleghem, Palo Alto University, 260 Palm Valley Blvd, Apartment 304, San Jose, CA 95123. E-mail: kvanmoorleghem@paloaltou.edu 
D. GUARDINO, D. KOO, K. GARRIDO-NAG \& L. PICK. Verbal Fluency Performance among Deaf Readers.

Objective: English reading abilities of Deaf signers vary from developing to proficient, which may impact their performance on measures of verbal fluency. Research including hearing individuals indicate stronger English vocabulary and more efficient mental flexibility contributes to better verbal fluency performance (Luo, Luk, \& Bialystok, 2010). By contrast. there is limited information regarding the effects of reading ability on verbal fluency in Deaf people. This study compared the verbal fluency performances of proficient versus developing Deaf readers, as well as contributions of intelligence.

Participants and Methods: 48 Deaf undergraduate students were split into two groups according to their WRMT-III and ACT scores. Participants were administered the WASI-II PRI subtests to estimate intellectual functioning as Deaf people perform equally to their hearing peers on nonverbal intellectual measures. The D-KEFS Verbal Fluency Test - Letter Fluency Condition was administered via American Sign Language (ASL).

Results: Independent samples t-tests revealed developing readers to perform worse on the WRMT-III Reading Comprehension subtest and the ACT Reading Test compared to proficient readers $(p<0.01)$. No significant differences were identified between two groups for the total number of correct words produced on the D-KEFS or the individual letter conditions $(p>0.05)$. Multivariate analyses were not significant for verbal fluency performances across the two reading groups using intelligence as a covariate ( $p>0.05)$.

Conclusions: Differences in reading ability appear not to influence performances on verbal fluency measures in Deaf signers. This is surprising given the extant literature on Deaf and hearing readers, and studies suggesting that hearing bilinguals show reduced verbal fluency (Sandoval, Gollan, Ferreira, \& Salmon, 2010). Current findings will be discussed in relation to what is known about the development of language and executive functions in Deaf people.

Correspondence: Donna Guardino, Psychology, Gallaudet University, 800 Florida Avenue, NE, P.O. Box \#1056, Washington, DC 20002. E-mail: donna.guardino@gallaudet.edu

B.D. HOLCOMBE \& K.S. WALSH. Exploring Differences in Gender and Disease Risk on Executive Function in Pediatric Survivors of Acute Lymphoblastic Leukemia (ALL).

Objective: Survivors of pediatric ALL are at risk for late neurocognitive sequelae, including executive dysfunction. Research has shown deficits in attention and working memory (WM), with possible gender-specific deficits. We aim to examine gender and disease-risk specific executive function (EF) patterns.

Participants and Methods: Data from 45 participants (58\% male, evaluation age $\mathrm{M}=11.8$ vrs, $\mathrm{SD}=3.38$ ) was abstracted from a larger study of neuropsychological functioning in childhood cancer survivors. Participants were excluded if they were on treatment or off therapy less than 1 year. Diagnosis and treatment protocol was used to document disease risk category (standard $\mathrm{v}$. high risk). This study targeted real-world EF functions by using the parent BRIEF and DuPaul ADHD-RS.

Results: Statistical analysis indicated a significant relation between gender and higher ratings of hyperactivity $[\mathrm{F}(1,36)=9.975, \mathrm{p}=.003$, Eta2=.217], males $(M=51.5 \mathrm{SD}=9.35)$ greater than females $(M=43.6$, $\mathrm{SD}=3.48$ ). No gender differences with inattention, flexibility, inhibitory control, or WM were found, but group means for WM and inattention neared clinically significant levels. Mean scores on WM were significantly higher than Shift $(p=.049)$ and Inhibit $(p=.000)$, and $>50 \%$ of the sample had clinically significant WM impairments. Analysis of disease risk and EF was not significant.

Conclusions: Previous associations between gender and patterns of EF deficits in ALL survivors were not replicated in this study. While the presence of hyperactivity was associated with being male, as a group, these did not reach clinical significance. Overall, there appears to be uniformity in the effects of ALL and associated treatments for boys and girls, regardless of disease severity, with disruptions in attentional control and WM being the most prevalent cognitive sequelae.

Correspondence: Billy D. Holcombe, M.A., Department of Psychology, Howard University, 460 L St NW, 314, Washington, DC 20001. E-mail: billyholcombe1@gmail.com

B.A. HUNTBACH, A. EASTVOLD \& Y. SUCHY. Differences in SelfReported Executive Functioning between Pedophilic and NonPedophilic Sex Offenders.

Objective: Research has shown differences between pedophilic (PED) vs. non-pedophilic child molesters (N-PEDS) on performance measures of executive functioning (EF; Eastvold, Suchy \& Strassberg, 2011), such that PEDs exhibit relative strengths in abstraction and planning, and weaknesses in inhibition and initiation (with no differences in flexibility and working memory). The present study examined whether similar profiles would emerge on self-report of EF.

Participants and Methods: 58 adult males $(P E D s=29$, N-PEDs $=29$, ages 19 to $49(\mathrm{M}=33.05 \mathrm{SD}=8.04)$ with 8 to 17 years of education completed the behavioral rating inventory of executive function (BRIEF).

Results: Repeated measures ANOVA showed a main effect of group with PEDs reporting more EF problems than N-PEDs overall across all BRIEF subscales $[F(1,56)=4.04, p=.049]$. Additionally, univariate differences emerged on initiation $(t=2.17, p=.034)$, working memory $(\mathrm{t}=2.02, \mathrm{p}=.048)$ and planning/organizing $(\mathrm{t}=2.32, \mathrm{p}=.024)$.

Conclusions: In contrast to a profile of relative strengths and weaknesses in EF performances among PEDs and N-PEDS, PEDs report greater EF problems than N-PEDs across all BRIEF subscales, demonstrating dissociation between subjective and objective measures of EF. Correspondence: Bryce A. Huntbach, University of Utah, 934 E $260 \mathrm{~N}$, Orem,UT 84097.E-mail: brycehuntbach@gmail.com

S. KAUR, M. GONZALES, D. EAGAN, H. TANAKA \& A. HALEY. Serum Brain Derived Neurotrophic Factor mediates the relationship between Central Adiposity and Executive Function in middle age.

Objective: Excessive adipose tissue, especially with a central distribution, is associated with increased risk of dementia in older adults. However, the mechanisms behind this relationship are poorly understood. As increased adiposity is also associated with lower circulating levels of Brain Derived Neurotrophic Factor (BDNF), a key molecule modulating brain plasticity and neuronal regeneration, we hypothesized that the changes to cognition that occur as a result of excessive central adiposity would be driven by lower levels of circulating BDNF.

Participants and Methods: Fasting blood samples were obtained from 60 participants aged $40-60$ years $(M=52.28, \mathrm{~S} . \mathrm{D}=5.61)$, and BDNF levels were assessed with an enzyme linked immunosorbent assay (ELISA). Central adiposity was measured using a ratio of waist circumference to hip circumference (WHR). Particpants also completed a neuropsychological assessment battery to assess cognitive function. Statistical mediation was assessed using traditional causal steps and nonparametric bootstrapping.

Results: Higher WHR was significantly associated with poorer performance on the Controlled Oral Word Association (COWA) letter fluency test $(\beta=-0.362, p=0.01)$, and lower levels of circulating BDNF $(\beta$ $=-0.345, p=0.006)$. Linear regression and bootstrapping methods indicated that BDNF fully mediated the relationship between WHR and performance on the COWA $(\mathrm{r} 2=-1.52,95 \% \mathrm{CI}=-3.79--0.26)$. Conclusions: The relationship between higher WHR and verbal fluency was fully mediated by circulating BDNF levels. The BDNF pathway is thus a useful probable mechanism through which cognitive decline occurs in individuals with high central adiposity. Future studies should examine the effects of targeted interventions known to modulate BDNF levels, such as voluntary exercise or stress reduction.

Correspondence: Sonya Kaur, M.A, Psychology, Universtity of Texas Austin, 2529 Rio Grande, Apt \#12, Austin, TX 78705. E-mail: sonya. kaur21@gmail.com 
J. SPAT, M.T. MORAN, C. WILSON \& M.W. HAUT. The Nature of Perseverative Errors on the Auditory Consonant Trigram Test: Relationship to Other Measures of Frontal Lobe Dysfunction.

Objective: Perseverative errors are indicative of frontal cortex dysfunction. The Auditory Consonant Trigram Test (ACT) is a measure of working memory/attention, sensitive to frontal lobe deficits. Few studies have used perseverative errors on the ACT as a measure of frontal functioning. The current study developed new methods of scoring perseverative errors and examined relationships with measures of frontal lobe function.

Participants and Methods: Eighty-seven consecutive patients referred for an outpatient neuropsychological evaluation ( $M=42$ years $)$ were retrospectively analyzed. Measures of perseverative errors included: longest consecutive perseveration strings, total sum consecutive strings, and total set of perseverations. Neuropsychological measures included: Trail-making Test (TMT), Wisconsin Card Sorting Task (WCST), Luria's loops and ramparts, and the Wide-Range Achievement Test (WRAT-4).

Results: Pearson product moment correlations revealed a significant negative relationship between level of education/premorbid intellectual functioning and number and strings of perseverative errors on ACT $(\mathrm{p}<.05)$. There were positive associations between the measures of perseverative errors and measures of executive functioning $(p<.05)$. Finally, t-tests revealed that those with higher perseverative responses on the WCST had more perseverative errors $(t(85)=-2.36, p=.021)$, longest consecutive strings $(\mathrm{t}(85)=-2.63, \mathrm{p}=.010)$, and increased sums of perseverative errors $(\mathrm{t}(85)=-2.43, \mathrm{p}=.017)$.

Conclusions: There were significant associations between the different measures of perseverative errors and measures of frontal lobe dysfunction. However, a majority of the variance was unaccounted for suggesting the new measures of perseverative errors may assess another domain of executive function. Factor analysis to examine where the new perseverative measures may load is a next step.

Correspondence: Jessica Spat, MA, Yeshiva University, 1165 Morris Park Avenue, Bronx, NY 10461.E-mail: spatjl@gmail.com

G.G. MONCRIEF, B. SCOTT, D. STORZBACH, S. TUN \& M.E. ONEIL. Cognitive Reserve and Neuropsychological Test Performance in Blast Exposed Operation Enduring Freedom/ Operation Iraqi Freedom (OEF/OIF) Veterans.

Objective: Examine the influence of cognitive reserve on neuropsychological test performance in a sample of OEF/OIF Veterans with blast exposure.

Participants and Methods: Participants were 92 previously deployed OEF/OIF Veterans at the Portland VA divided into high and low cognitive reserve groups using a median split and grouped according to blast exposure history. Estimated cognitive reserve level was based on education and performance on the RIST and WTAR. We compared mean standardized scores on a comprehensive neuropsychological test battery for each group. We conducted a MANOVA comparing mean scores on cognitive tests for Veterans based on blast exposure and cognitive reserve.

Results: No significant results were found for learning, memory, attention, and spatial tests. Results indicated an interaction effect for blast exposure and cognitive reserve groups on tests of executive function: Veterans with high cognitive reserve who were exposed to blast performed better on the Mazes and Categories tests than those with low cognitive reserve; however, high cognitive reserve was not protective for those without blast exposure. We ran the same tests as a MANCOVA adjusting for PTSD scores, and found that there were no longer statistically significant differences between the groups once PTSD was included as a covariate.

Conclusions: Higher levels of cognitive reserve may serve as a protective factor for executive functioning in Veterans with a history of blast exposure, though PTSD symptoms may mediate this effect, supporting existing research on the impact of psychological factors on test performance.

Correspondence: Grant G. Moncrief, BS, Psychology, Pacific University, 9309 SE Taylor St., Portland, OR 97216. E-mail: grant.moncrief@ pacificu.edu

\section{K. KLIPFEL, J. GUNSTAD, J. DUNLOSKY, C. WAS \& M. VAN DULMEN. Executive Functions and Dating Aggression in Young Adults.}

Objective: The current study is the first study of which we are aware to investigate the association between executive functions and dating aggression perpetration and victimization. The current study extends previously presented outcomes (INS, 2013) to the full sample of 138 dating couples and controls for the impact of intelligence on this association. Participants and Methods: Heterosexual dating couples ( $N=138), 18-$ 29 years of-age, completed individual assessment batteries. The Conflict in Adolescent Dating Relationships Inventory (Wolfe et al., 2001) assessed emotional/verbal, physical, and sexual aggression perpetration and victimization, the Wisconsin Card Sorting Task (Heaton \& PAR staff, 2003) assessed executive functions (i.e., perseverative errors), and the Spot-the-Word, Second Edition (Baddeley \& Crawford, 2012) estimated overall intelligence. Only those participants without clinically significant impairments in executive functions ( $T$-score $>40$ ) were included in the analyses ( $n=125$ males; $n=131$ females).

Results: Partial correlations revealed that poorer executive functions were associated with greater physical aggression victimization for females, net of intelligence $(r=-.18, p=.03)$. There was also a trend toward poorer female executive functions in association with greater sexual aggression perpetration net of intelligence $(r=-.13, p=.08)$. Follow-up t-tests confirmed that females who experienced physical aggression victimization showed poorer executive functions $[\mathrm{t}=2.87(20.87), \mathrm{p}=.01$, Cohen's $d=1.26]$. However, these associations did not hold for males, nor did the impact of partner executive functions relate to outcomes of dating aggression.

Conclusions: The current study confirmed that poorer executive functions are associated with interpersonal aggression and extended these findings to a normative, young adult dating sample. The association was largely specific to females and to physical aggression.

Correspondence: Katherine Klipfel, Kent State University, 1414 Laurel Avenue \#L307, Minneapolis, MN 55403.E-mail:kklipfel@kent.edu

\section{J. RAAK \& R.L. SKEEL. The Impact of Working Memory Deficits} on In-group versus Out-group Helping Behavior.

Objective: Deficits in working memory have been implicated in a broad range of disorders, especially those focusing on externalized behaviors. Few studies have examined the relationship between working memory and social behavior. This was attempted utilizing a model focused on Cowan's system of working memory, including layers of awareness and manipulation of memory content. The study attempted to establish a link between working memory and socializing behavior by manipulating working memory load and in-group/out-group identification in helping behavior scenarios.

Participants and Methods: This was done through a dual task paradigm wherein 66 undergraduate participants were randomly assigned into a concurrent n-back task or no-task control condition to complete the primary task. All participants completed a helping behavior scenario task, featuring images of individuals altered with photo-editing software. During this task, participants were required to report their willingness to help the individual using a blinded 1-100 slider. Results were analyzed using ANCOVA.

Results: Overall, there was no main effect for working memory load, or in/out-group depiction. Significant interactions showed that working memory impacted scores differently at different levels of burden, and a similar trend existed between in-group/out-group depiction and burden. Exploratory analysis revealed that within the WM load group, WM task 
performance significantly correlated with differences in their willingness to help in-group vs. out-group individuals.

Conclusions: The current results may provide a starting point for the development of a new measure of explicit racism, as well as a direction for future research on the interaction between cognitive abilities and bias.

Correspondence: Jacob Raak, Central Michigan University, 1020 York Drive, Mount Pleasant, MI 48858. E-mail: raak1jd@cmich.edu

J. KAYLEGIAN, R. RAMOSKAITE, S. HUNTER, E.L. GUSTAFSON, D. SMITH \& N. KARNIK. Academic Achievement and Executive Functioning is Mediated by Parental Involvement in Homeless Urban Youth.

Objective: This study aimed to identify whether a relationship exists between executive functioning (EF), academic skill development, and early developmental history for a population of shelter living urban homeless youth. We predicted that youth who lived with their biological parents would show greater EF and academic skill versus those who grew up in foster care.

Participants and Methods: 148 youth were recruited from homeless agencies in Chicago and Los Angeles (53\% female; $76 \%$ African-American; ages 18-24). Participants completed a standardized interview assessing developmental and psychiatric history. EF was assessed using the BRIEF Self-Report form. Academic function was assessed with the WRAT-4.

Results: Comparison of participants across WRAT-4 subtests revealed that those who grew up with their parents were significantly stronger academically. Effect sizes were in the moderate range. Results included WRAT-4 Word Reading: $\mathrm{t}(101)=2.13, \mathrm{p}=.036, \mathrm{~d}=.42,95 \%$ CI $[0.326,9.402]$; Reading Comprehension: $\mathrm{t}(101), \mathrm{p}=.017, \mathrm{~d}=.48$, 95\% CI [0.953, 9.498]; Spelling: $\mathrm{t}(101)=2.05, \mathrm{p}=.043, \mathrm{~d}=.42,95 \%$ CI $[0.161,10.500]$; and Math: $\mathrm{t}(101)=2.33, \mathrm{p}=.022, \mathrm{~d}=47.95 \%$ CI $[0.932,11.552]$.

Participants who knew their parents were significantly stronger in EF development, across the BRIEF. Effect sizes were in the moderate to moderately high range. Results included: BRIEF BRI: $\mathrm{t}(44)=1.76, \mathrm{p}=$ $.085, \mathrm{~d}=.59,95 \%$ CI $[-1.089,16.235]$; BRIEF MI: $\mathrm{t}(44)=2.25, \mathrm{p}=$ $.029, \mathrm{~d}=.74,95 \% \mathrm{CI}[1.016,18.210]$; and BRIEF GEC: $\mathrm{t}(44)=2.09$, $\mathrm{p}=.043, \mathrm{~d}=.69,95 \%$ CI $[.314,18.628]$.

Conclusions: Academic achievement and EF are greatest for homeless youth who have a history of living with and being supported by their biological parents. Parental presence and involvement in the life of homeless youth influences both the level of skill developed and the capacity to regulate and meet daily challenges.

Correspondence: Jaeson Kaylegian, B.A., Pediatric Neuropsychology, University of Chicago Medical Center, 558 W Van Buren St, Unit 3, Chicago, IL 60607. E-mail: jkaylegian1@yoda.bsd.uchicago.edu

\section{N. SWAN, J. BERG, J. MILLER \& S.J. BANKS. DKEFS Color Word} Interference Switching and Learning Ability.

Objective: Executive functions such as cognitive set-shifting require flexible application of lower-level processes and are commonly measured by switching trials on DKEFS subtests (e.g., color-word interference (CWI)). An atypical pattern of performance has been observed on the CWI in a subset of individuals who perform faster, with equal or fewer errors, on the more difficult switching than the inhibition trial. This study seeks to explore the cognitive underpinnings of this atypical pattern.

Participants and Methods: Records from 239 clinical referrals (Age: $\mathrm{M}=68.09, \mathrm{SD}=10.62$; Education: $\mathrm{M}=14.87, \mathrm{SD}=2.73$ ) seen for a neuropsychological evaluation as part of routine care in an outpatient dementia and movement disorders clinic were sampled. The standard battery of tests included measures of attention, learning, executive functioning and working memory. ANOVAs were conducted to compare the cognitive performance of those with typical versus atypical CWI patterns.
Results: An atypical pattern of performance was confirmed in $23 \%$ of our sample. Analyses revealed a significant group difference in acquisition of information on both non-verbal (BVMT) $(\mathrm{F}(1,213)=16.61$, $\mathrm{p}<.001)$ and verbal (HVLT) learning tasks $(\mathrm{F}(1,181)=6.43, \mathrm{p}<$ $.01)$ with the atypical group performing better on each task. Effect sizes were larger for nonverbal (Cohen's $\mathrm{d}=0.66$ ) than verbal learning (Cohen's d = 0.47).

Conclusions: Individuals demonstrating an atypical pattern of performance on the CWI inhibition trial also demonstrated relative strengths in both verbal and nonverbal learning. These findings suggest that for some individuals, cognitive flexibility on the CWI may be underscored by learning ability.

Correspondence: Natasha Swan, Ph.D. Clinical Psychology, Neuropsychology, Cleveland Clinic Lou Ruvo Center for Brain Health, 888 West Bonneville Ave., Las Vegas, NV 89106. E-mail: natashamswan@gmail.com

J. MEISTER, D. CALVO, M. WRIGHT, J. GUNSTAD \& M. SPITZNAGEL. Obesity Confers Greater Risk for Cognitive Difficulties Associated with Poor Sleep Quality.

Objective: Poor sleep quality and obesity are both associated with diminished executive function, but have rarely been examined together. This study examined how relationships between sleep quality and inhibition differ by weight. We predicted that poor sleep quality would be related to worse performance on indices of inhibitory control, and that these relationships would be more robust in obese than lean individuals. Participants and Methods: 67 healthy college-aged individuals (33 lean, 34 obese) completed the Pittsburgh Sleep Quality Index (PSQI) and the ANAM Go/No-Go.

Results: Within the total sample, reduced sleep quality was related to fewer GNG hits $(\mathrm{r}=-.24, \mathrm{p}=.05)$, more omissions $(\mathrm{r}=.29, \mathrm{p}=$ $.02)$, and greater reaction time variability (RTV; $r=.28, p=.02)$, but was unrelated to reaction time, commission errors, or discriminability. Within lean individuals, sleep quality was unrelated to any GNG indices. Within obese individuals, poorer sleep quality was significantly related to greater RTV $(r=.42, p=.01)$, and trended toward significance for hits $(r=-.31, p=.08)$ and omissions $(r=.31, p=.07)$.

Conclusions: Consistent with expectations, poor subjective sleep quality was related to diminished performances on several indices of inhibitory control; findings were driven by performance in the obese group. Results suggest that for otherwise healthy young adults, obesity may confer a greater risk for poor inhibition associated with reduced sleep quality. Correspondence: Jonah Meister, Psychology, Kent State University, 11169 Aquilla Road, Chardon, OH 44024.E-mail: jmeister@kent.edu

A. LUKOSE, H.N. HALAHALLI, N. AMBEKAR, A. SASIDHARAN, D. KUMAR, A.K. GUPTA \& J.P. JOHN. Correlates and Predictors of Endogenous-Cue Prospective Memory in Schizophrenia.

Objective: In a recent fMRI study, we recently reported maximum activations of the right rostral prefrontal cortex (BA10) with additional activations of the left dorsal anterior insula during the PM condition of a novel 'endogenous-cue prospective memory' paradigm involving incremental updating of working memory (WM) (Halahalli et al 2014). Both BA10 and anterior insula have been reported to show characteristic abnormalities in schizophrenia. Therefore, we aimed to examine the performance of patients with schizophrenia on the above PM task in comparison to healthy control subjects.

Participants and Methods: Twenty eight patients with schizophrenia and thirty four healthy control subjects were administered the above prospective memory paradigm, comprising of PM, WM and ongoing tasks.

Results: The accuracy of performance on the 'endogenous- cue' prospective memory task in patients with schizophrenia was significantly inferior to that of healthy control ( $p<0.01$, domain-wise corrected). The accuracy scores of the ongoing as well as WM tasks in the prospective memory paradigm, immediate recall in Rey's Auditory Verbal Learning 
Test (RAVLT), total proportion of correct target in Continuous Performance Test (CPT) and categories completed in Wisconsin Card Sorting Test (WCST) were significantly lower in patients with schizophrenia when compared to healthy control subjects. Prospective memory task accuracy in both patients and controls correlated significantly with the Matrix reasoning score, total proportion of correct targets in CPT, WCST preservative errors, WCST categories completed as well as ongoing and WM task accuracies. In both groups, PM task accuracy was predicted by the proportion of correct targets in CPT and WM accuracy scores. In patients with schizophrenia, the PM task performance was additionally predicted by WAIS Matrix reasoning and ongoing task accuracy scores. Conclusions: The findings have implications in understanding the neurocognitive correlates of prospective memory and its dysfunction in schizophrenia.

Correspondence: Ammu Lukose, PhD, Psychiatry, National Institute of Mental Health and Neurosciences (NIMHANS), Multi-modal Brain Image Analysis Laboratory (MBIAL), Neurobiology Research Centre, National Institute of Mental Health and Neurosciences (NIMHANS), Bangalore 560029, India. E-mail: ammu18lukose@gmail.com

E.H. GERST, P.T. CIRINO, J.M. FLETCHER \& H. YOSHIDA. Prediction of Academic Performance with Rating Scale and Cognitive Measures of Executive Function.

Objective: Executive Functions (EF) contribute to both math (Bull \& Scerif, 2001) and reading (Christopher, et al., 2012). EFs are assessed via behavioral ratings and cognitive measures which have modest interrelationships and are infrequently examined together in predicting academic skill. We expected both types of EF measures across four domains (working memory, planning, inhibition, and shifting) to predict academic skill.

Participants and Methods: Participants were 4th and 5th grade students ( $\mathrm{n}=93)$. WJ-III Calculations and Gates MacGinitie Reading Comprehension test assessed academic skill. Measures from the D-KEFS, WMTB-C, the Tower of London, and corresponding BRIEF-Teacher rating scales assessed EFs. Covariates included phonemic awareness, oral comprehension and educational program status. Multiple regression analyses assessed relationships.

Results: For each EF domain, rating scale and cognitive measures contributed uniquely to both math (all $\mathrm{p}<.05)$ and reading (all $\mathrm{p}<.05)$. Inclusion of covariates diminished the unique prediction of cognitive measures for reading, and rating scale measures for math. When prediction was considered across $\mathrm{EF}$ domain (reading: $\mathrm{R} 2=0.646, \mathrm{p}<$ .0001 ; math $\mathrm{R} 2=0.581, \mathrm{p}<.0001)$, only rating scales were unique predictors of reading $(p=.0003)$, while only cognitive measures were unique predictors of math $(\mathrm{p}=.0199)$.

Conclusions: Both rating scale and cognitive measures of EF are related to academic skill across domain, even after accounting for covariates. However, there was divergence in the type of EF that was most contributory to academic skill. Unique predictors were also different, emphasizing rating scale measures of working memory for reading and cognitive shifting performance for math.

Correspondence: Elyssa H. Gerst, University of Houston, 8383 el Mundo St, Apt 411, Houston, TX 77054. E-mail: elyssa.gerst@gmail.com

E. DE GUISE, A. ALTURKI, M. LAGUE-BEAUVAIS, J. LEBLANC, M. CHAMPOUX, C. COUTURIER, K. ANDERSON, J. LAMOUREUX, J. MARCOUX, M. MALEKI, M. FEYZ \& J. FRASNELLI. Olfactory and Executive Dysfunctions Following Orbito-basal Lesions in Traumatic Brain Injury.

Objective: To study the acute relationship between olfactory function and traumatic brain injury (TBI), cognitive functions and outcome.

Participants and Methods: Sixty-two patients with TBI were evaluated within the first two weeks following TBI. The Sniffin'Sticks identification test was used to assess olfaction. A neuropsychological evaluation was carried out to assess attention, verbal fluency, naming, memory, problem-solving and mental flexibility. The extended Glasgow Outcome
Scale (GOSE) and the Disability Rating Scale (DRS) were rated at discharge from acute care.

Results: Traumatic lesions located in the basal frontal area resulted in odor identification scores that were significantly lower than when lesions were elsewhere $(p<0.001)$. A significant positive correlation was shown between odor identification scores and mental flexibility scores $(\mathrm{p}=0.004)$, and patients with hyposmia had worse performances on executive tests measuring problem-solving, verbal fluency and mental flexibility $(p<0.01)$. Moreover, the odor identification score and the DRS total score were related $(p=0.019)$.

Conclusions: These findings add information regarding acute olfactory status following TBI and provide evidence on the importance of assessing olfaction very early post TBI in order to plan intervention and determine what accident prevention advice will be required for home or work reintegration.

Correspondence: Elaine de Guise, University of Montréal, Département de Psychologie Pavillon Marie-Victorin CP 6128 Succ. Centre-Ville, Montreal, OCH3C3J7, Canada.E-mail: elaine.de.guise@umontreal.ca

W.R. MOORE, E.C. DUGGAN \& M.A. GARCIA-BARRERA. Examination of the bilingual advantage in young adults using two behavioral rating scale measures of executive function.

Objective: Following up from a previous study using the same sample (Moore, Duggan, \& Garcia-Barrera, 2014) in which we identified differences in executive functioning (i.e., bilingual advantage) using computerized cognitive measures, the current study investigated whether or not these advantages are detectable at a behavioral level using two self-rating scales of executive functioning (EF).

Participants and Methods: 100 young adults (Age $\mathrm{M}=20.55, \mathrm{SD}=1.82$ ) who completed the BRIEF-Adult version and BASC-2-College self-rating scales were included in the present study. Participants were split into three groups: (i) early bilinguals $(n=44)$; (ii) late bilinguals $(n=$ 19); (iii) monolinguals ( $n=22$ ). Twenty-five participants were excluded due to age cutoffs for inclusion in the early and late bilingual groups. The BRIEF is a well-established measure of EF. Executive behavior was further examined using a recently derived screener from the BASC that assesses four EF components: problem solving, behavioral control, attentional control, and emotional control (Duggan et al., 2014). Raw subscale scores were converted to T-scores and compared using oneway ANOVAs.

Results: Interestingly, no significant differences were found for any of the EF components in the between-group comparisons.

Conclusions: The bilingual advantage was previously demonstrated for this sample using computerized neuropsychological measures of EF; however, these advantages are not identified when assessed using behavioral rating scales. Possible explanations include: (i) behavioral rating scales may not be sensitive enough to pick up subtle cognitive processing differences between bilinguals and monolinguals; (ii) executive behavior in early adults may be similar between groups because the underlying EF processes are at their peak of development; or, (iii) the bilingual advantage may exist for lower-level and specific EF networks, such as those involved in inhibitory control, but this advantage may be diluted at the level of more general behavioral outcomes.

Correspondence: William R. Moore, University of Victoria, 206-240 Cook St, Victoria, BCV8V 3X3, Canada.E-mail:wrmoore@uvic.ca

S. HANNA, L.K. PAUL \& W.S. BROWN. Performance on the Tower Test in Agenesis of the Corpus Callosum.

Objective: Previous research examined the ability of individuals with agenesis of the corpus callosum (ACC) to problem solve, but research thus far has not tested spatial problem-solving and planning. Using the Delis-Kaplan Executive Functioning System (D-KEFS) Tower Test, thie present study examined this ability in individuals with ACC compared to match controls.

Participants and Methods: : Participants with ACC (23) were between 16 and 52 years of age with FSIQ between 78 and 129, 7 with partial 
ACC and 16 with complete ACC. Controls included 13 neurotypical individuals between 20 and 44 years old with FSIQ between 84 and 116. All participants were administered the full D-KEFS as well as the Wechsler Adult Intelligence Scale as part of a larger research test battery.

Results: Individuals with ACC tended to score lower in total achievement $(p=0.06)$, but scale scores for individuals with ACC $(M=8.57)$ and controls $(M=9.85)$ were within one standard deviation of the norm. No significant differences were found in first move time, number of moves, total item completion time, or rule violations.

Conclusions: Although only a trend, the lower average total achievement scale score for individuals with ACC is consistent with previous finding of deficient problem-solving associated with callosal agenesis. Participants with ACC and controls performed similarly on the measures of time and number of moves, which is not consistent with previous findings of slower cognitive processing time in ACC, suggesting that the lower average achievement score in ACC was influenced by the combination of time and number of moves.

Correspondence: Warren S. Brown, Ph.D., Travis Research Institute, Fuller Grad Sch of Psych, 180 N. Oakland Ave., Pasadena, CA 91101. E-mail: wsbrown@fuller.edu

M. WELSH \& C. ROHRBACHER. Executive Functions and Hypothetical Risk-Taking as Predictors of Developmental Tasks in Emerging Adulthood: Academic Self-Efficacy and Career Decision Making.

Objective: Executive functions aid in future-oriented, goal-directed behaviors and have been found to be related to self-reported hypothetical risk-taking in our lab. We examined the degree to which each of these constructs predicted two important developmental tasks of emerging adulthood: academic self-efficacy and career decision making.

Participants and Methods: Forty-one college students (16 male, 25 females, $\mathrm{M}$ age $=19.61 \mathrm{yrs}, \mathrm{SD}=1.41 \mathrm{yrs})$ took a battery of tasks including: Behavior Rating Scale of Executive Functions (BRIEF) yielding a Behavior Regulation Index (BRI) and Metacognitive Index (MI), the Career Decision Making Scale (CDM) yielding an Uncertainty score, the Academic Self-Efficacy Scale, and the Domain-Specific Risk-Taking Scale (DOSPERT), measuring both hypothetical risk-taking and risk-perception across a range of behavioral domains.

Results: The prediction of individual differences in both ASE and CDM uncertainty indicated: 1 . the effect of the BRI on both outcomes was completely mediated by the MI of the BRIEF; 2 . hypothetical risk-taking in the financial, health and safety, and ethical risk taking were most predictive of the two outcomes; 3 . both the MI and DOSPERT made independent (unmediated) contributions to the two dependent measures, with $52 \%$ of $\mathrm{ASE}$ and $36 \%$ of CDM uncertainty explained in the final models.

Conclusions: The metacognitive aspect of executive function (e.g., working memory, planning) and potential risk taking are predictive of both academic self-efficacy and career decision making, two milestone achievements of young college students. Executive function deficits and higher hypothetical risk taking in health and safety, finances, and ethical dilemmas predict greater career uncertainty and lower academic self-efficacy.

Correspondence: Marilyn Welsh, PhD, School of Psychological Sciences, University of Northern Colorado, Box 94, University of Northern Colorado,Greeley, CO 80639.E-mail: marilyn.welsh@unco.edu

E.S. KORNBLITH, R.M. BOWLER, S. CASEY \& D.T. LOBDELL. Tremor, Motor, and Executive Function Profiles in Adult Residents Environmentally Exposed to Manganese.

Objective: Exposure to elevated levels of manganese (Mn) may be associated with tremor and impairment in motor and executive function (EF), clinically resembling Parkinson's disease (PD). PD research has identified tremor-dominant (TD) and non-tremor dominant (NTD) profiles. NTD PD presents with bradykinesia, rigidity, and postural sway, and is often associated with EF impairment and lower quality of life (QoL). Presence and impact of tremor, motor, and EF profiles on health-related QoL and life satisfaction were examined in air-Mn exposed residents of two Ohio, USA towns.

Participants and Methods: From two Ohio towns exposed to air-Mn, 186 residents (76 males) aged $30-75$ years were administered measures of EF (Animal Naming, ACT, Rey-O Copy, Stroop Color-Word, and Trails B), motor and tremor symptoms (UPDRS), QoL (BRFSS), life satisfaction (SWLS), and positive symptom distress (SCL-90-R). Air-Mn exposure in both towns was modeled with 10 years of air-monitoring data. Cluster analyses detected the presence of symptom profiles by grouping together residents with similar scores on these measures.

Results: Overall, mean air-Mn concentration for the two towns was $0.53 \mu \mathrm{g} / \mathrm{m} 3$ ( $\mathrm{SD}=.92)$. Two-step cluster analyses identified TD and NTD symptom profiles. Most residents in the NTD group lacked EF impairment; EF impairment represented a separate profile. An unimpaired group also emerged. The NTD and EF impairment groups were qualitatively similar, with relatively lower QoL and life satisfaction, and higher positive symptom distress and Mn exposure compared to other groups.

Conclusions: NTD and EF symptoms in Mn-exposed residents should be a focus of clinical attention. Neuropsychological evaluations identified symptom profiles, aiding treatment planning.

Disclaimer: This abstract does not necessarily reflect EPA policy.

Correspondence: Erica S. Kornblith, MA, CSPP San Francisco, 42 Cortland Ave, San Francisco, CA 94110.E-mail: eschimbor@alliant.edu

\section{A. CHILD \& P.T. CIRINO. Executive Functions and ADHD} Symptomatology.

Objective: Executive functions ( $\mathrm{EF}_{\mathrm{s}}$ ) have been consistently associated with ADHD. However, relatively little is known about the relative and collective impact of working memory, inhibition, shifting, planning, and fluency on symptoms of behavioral inattention and hyperactivity/ impulsivity. We hypothesize that inhibition, WM, and planning will be more strongly related to inattention versus shifting and fluency while inhibition will be the most strongly related $\mathrm{EF}$ to overactivity relative to the other EFs.

Participants and Methods: 276 3rd-5th grade participants from a larger sample completed EF tasks of working memory (Listening Span), shifting (Trails), inhibition (Stop Signal), planning (Tower of London), and fluency (Verbal Fluency). The SWAN ADHD rating scale was also completed by teachers. Analyses used correlation and multiple regression analysis with the five EF measures as predictors and SWAN scales as outcome variables.

Results: Zero-order correlations of the five EF variables to ADHD symptomatology ranged from $|r|=0.004$ to 0.300 for inattention, and $|\vec{r}|=0.027$ to 0.203 for overactivity. In combination, EFs significantly predicted inattention, $F(6,269)=7.03, p<0.001, R^{2}=0.136$, in the multiple regression. Of these, working memory $(p=0.013)$ and inhibition $(p<0.001)$ were unique predictors. The five predictor EFs also significantly predicted overactivity, $F(6,269)=4.71, p<0.001, R^{2}=$ 0.095 , in the multiple regression analysis with fluency $(p=0.006)$ and inhibition $(p=0.007)$ as unique predictors.

Conclusions: The significant relationships between inhibition and both ADHD symptom subscales are consistent with previous research, as is the relationship between working memory and inattention (Willcutt, Doyle, Nigg, Faraone, \& Pennington, 2005). This study also found unique associations with fluency and overactivity. These results affirm and extend our understanding of the type and degree to which EFs are related to ADHD symptomatology.

Correspondence: Amanda Child, Psychology, University of Houston, TIMES Developmental Neuropsychology Lab, 4811 Calhoun Rd, Mailroom 365, Houston, TX 77204.E-mail: amandachild11@gmail. com 
L.I. THOMPSON, B.E. WELLS, J.T. PARSONS \& S.A. GOLUB. Profiles of Executive Function in the Prediction of Alcohol Use Among Emerging Adults in NYC.

Objective: Alcohol is the most common substance of abuse among young adults, and is associated with additional risk-taking and adverse outcomes (e.g., aggressive acts, unintended pregnancy). We examined the relationship between neurocognitive performance and alcohol use in a NYC sample, aged 18-29, to explore whether executive functions predict alcohol consumption.

Participants and Methods: 300 participants (35\% female) of mixed educational experience $(17.6 \%$ current students) and race $(51 \%$ nonwhite) were recruited as part of a NIH-funded research project. Participants were eligible if they reported using $\geq 3$ standard drinks (SDs) in the last 90 days. Measures of decision making (DM; Iowa Gambling Task, Bechara et al., 1994), behavioral inhibition (BI; D-KEFS Color-Word subtest), working memory (WM; WAIS-III Digits Backward subtest), and behavioral data were collected. Negative binomial regression was used to examine relationships between DM, WM, and BI performance in the prediction of reported number of SDs across 30 days. Results: Controlling for age, gender, race, and education, WM and BI interacted with $\mathrm{DM}$ performance $(\operatorname{Exp}(\mathrm{B})=1.006, \mathrm{p} \leq .001 ; \operatorname{Exp}(\mathrm{B})=$ $.995, \mathrm{p} \leq .05$, respectively) to predict number of reported SDs. Lower WM and BI performance predicted greater SDs, but only when DM performance was also low. There were no main effects of task performance on SDs, nor did WM and BI interact to predict SDs.

Conclusions: These results suggest that deficits in a single domain of executive function may not be sufficient to observe a connection with drinking behavior. Interventions targeting poor DM may be particularly important for those with low WM or poor BI.

Correspondence: Louisa I. Thompson, B.A., Psychology, Gradate Center of the City University of New York, 76 India Street, Apt. A16, Brooklyn, NY 11222. E-mail: Louisa.Isoud@gmail.com

\section{A. SCHWEHM, K.A. WALKER, A. TORSTRICK, A. RILEY, M. YAKOBSON, J. MARTIN-COOPER, K. KHURSHID, C. DABREO \& R. WELLINGTON. Components of Executive Functioning Differentially Relate to Estimates of Intelligence.}

Objective: Several recent studies have found that distinct components of executive functioning (EF) differentially relate to IQ in healthy adults. The current study aims to extend these findings by assessing the relationship between commonly used estimates of IQ and components of EF. Participants and Methods: The current study assessed 170 young adults using a battery of $13 \mathrm{EF}$ measures and two estimates of IQ: the Test of Premorbid Functioning (TOPF) and the Wechsler Abbreviated Scale of Intelligence (W ASI-II). EF components were derived using confirmatory factor analysis (CFA) and compared to IQ measures using structural equation modeling (SEM).

Results: CFA indicated that a four-factor EF model (Working Memory, Set-Shifting, Inhibition, Planning) provided the best fit to the data $(\chi 2$ $(50)=66.602, p=.290$, TLI $=.965, \mathrm{CFI}=.976$, RMSEA $=.023)$. Using CFA to calculate the relationship between EF components and TOPF, only Working Memory was found to be significantly related to TOPF $(\beta=.51, p<.05)$, whereas an association between Set-Shifting and WASI-II was found $(\beta=.49, \mathrm{p}<.05)$. Using SEM to examine how all four EFs simultaneously related to intelligence (i.e., considering EF intercorrelations), we found that only Working Memory emerged as a strong, albeit non-significant, predictor of both TOPF $(\beta=1.04, \mathrm{p}>$ $.05)$ and WASI-II performance $(\beta=.41, \mathrm{p}>.05)$.

Conclusions: The results extend previous findings that suggest working memory and updating are more strongly related to IQ than other components of EF by demonstrating that this trend is maintained when IQ is estimated using brief measures commonly used in clinical practice. Findings require replication in a larger sample.

Correspondence: Keenan A. Walker, M.A., Psychology, St. John's University, 101-01 67th Dr., APT 4J, Forest Hills, NY 11375. E-mail: kwalke26@gmail.com
M. SIEBENMORGEN, E. CAMPBELL, B. MITCHELSON, C. REUSSER \& K.L. NETSON. Streamlining Assessment of Inhibition: The Value of Multi-Method Assessment.

Objective: Executive functions in real-world settings can be difficult to capture in a structured assessment environment. Parent and teacher ratings from home and school environments have been a proven method to compensate. Disadvantages include rater bias and functionality differing across contexts. This pilot investigation aimed to assess the relationship between performance and behavior rating measures of inhibition to better streamline evaluations.

Participants and Methods: In this retrospective review of a clinical data repository, 117 consecutive clinical cases were reviewed and $\mathrm{n}=14$ met inclusion criteria (Age=10-15; Completed BASC-2, BRIEF, TEA-Ch Walk/Don't Walk, and DKEFS Color-Word Interference). Participants included $n=9$ males; $n=12$ Caucasian, $n=1$ African-American, n=1 Hispanic. Mean(SD) age was 12.02(1.02). Six children had diagnoses of ADHD. Other primary diagnoses included Traumatic Brain Injury ( $\mathrm{n}=3$ ) and Autism, Obsessive Compulsive Disorder, Dyslexia, Borderline Intellectual Function, and Adjustment Disorder ( $\mathrm{n}=1$ each). The sample was of low average IQ overall $(\operatorname{Mean}(\mathrm{SD})=86.86(10.61)$; Range=68-106).

Results: Parent ratings of hyperactivity (BASC-2) and disinhibition (BRIEF) correlated significantly with each other $(r=.755, p=.002)$. Teacher ratings from the same measures followed a similar pattern $(\mathrm{r}=.884, \mathrm{p}<.001)$. Parent and teacher ratings correlated only moderately across raters $(r=.412-.584)$. Child/Adolescent self-report ratings of hyperactivity correlated most strongly with neuropsychological measures of inhibition including Walk/Don't Walk ( $r=-.636, p=.015)$ and Color-Word Inhibition $(r=-.663, \mathrm{p}=.010)$.

Conclusions: Results suggest that the measures correlate moderately at best, and that children may have a better understanding of their weaknesses than previously presumed. Multiple questionnaires per rater may provide overlapping, rather than novel and clinically relevant, information.

Correspondence: Marsha Siebenmorgen, MA, University of Tulsa, 800 South Tucker Drive, Tulsa, OK 74104.E-mail:marsha-siebenmorgen@ utulsa.edu

S. MCCAULEY, D. MENEFEE, J. TRAN, J. WANNER, J. HICKEY, W. LEOPOULOS, C. PEDROZA, R. YALLAMPALLI \& E.A. WILDE. Neuropsychological Improvement Following a Novel Inpatient Treatment for OEF/OIF Veterans with PTSD and Multiple Comorbidities.

Objective: This study investigated the recovery of Veterans with PTSD and comorbidities including alcohol abuse (ETOH) and mild traumatic brain injury (mTBI) following a 4-week, inpatient treatment that is novel in the VA system.

Participants and Methods: Participants included OEF/OIF Veterans with PTSD+ETOH $(\mathrm{n}=14)$ and PTSD+ETOH+mTBI $(\mathrm{n}=12)$ and combat-exposed Veteran controls $(n=15)$ without these disorders. The groups did not differ by age $(p=.84)$, IQ $(p=.11)$, or length of stay $(p=.36)$. The treatment was an intensive, concurrent approach (group and individual settings) rather than the traditional sequential trauma-focused treatment. Measures of executive functioning, episodic memory, and processing speed were obtained.

Results: Data were analyzed using a $3 \times 2$ mixed ANOVA with the fixed effect variable Group (control, PTSD+EOTH, PTSD+ETOH+mTBI) and random effects variables of episodic memory, inhibition/impulsivity, and processing speed. Significant improvement was found in both treatment groups for nonverbal memory $(p<.01)$, processing speed $(p<.02)$, response inhibition (Stroop; $\mathrm{p}<.02$ ), and the Two-Choice Impulsivity Paradigm $(\mathrm{p}<.0005)$, but only the PTSD+ETOH group improved on measures of complex reaction time and impulsivity self-report (Barratt Impulsiveness Scale, total score). The control group did not demonstrate significant changes across the two occasions on any measure. 
Conclusions: These are the first data demonstrating the effectiveness of a novel, inpatient, concurrent treatment approach in the VA system in terms of improved neurocognitive functioning in domains affected by PTSD, alcohol abuse, and mTBI. This treatment approach may have advantages over the traditional sequential treatment paradigm for these comorbid disorders.

Correspondence: Stephen McCauley, PhD, Physical Medicine and Rehabilitation, Baylor College of Medicine, One Baylor Plaza, BCM 637,Houston, TX 77030.E-mail:mccauley@bcm.edu

\section{R. BRANSON, D.R. DAWSON \& A. CLARK. Examining an Alternate Version of the Baycrest Multiple Errands Test.}

Objective: Due to the connectivity of the frontal lobe with other brain areas, executive function deficits resulting from frontal lobe damage can severely impact activities of daily living. While standardized neuropsychological measures alone sometimes fail to fully capture impairment, naturalistic assessments like the Baycrest Multiple Errands Test (BMET, Dawson et al., 2009) have shown promise in identifying deficits associated with brain injury. However, using such methods as outcome measures requires multiple versions. The purpose of the present study was to validate an alternate version of the BMET.

Participants and Methods: Eight stroke participants, seven traumatic brain injury (TBI) participants and age and education matched controls completed a neuropsychological battery as well as two versions of the BMET. Both versions involved participants following rules, while completing tasks in a shopping mall environment. Version A and Version $\mathrm{B}$ differed in the tasks to be completed (i.e., purchasing a stamp versus a pen). Performance was scored based on task completions, omissions, rule breaks, inefficiencies, and errors.

Results: Regardless of version, brain injury participants made more errors $(p<.05)$ and were less efficient $(p<.03)$ than control participants. When considering correlations between the two versions, task completions, omissions, errors, and rule breaks were significantly correlated $(p<.05)$ for the stroke group. However, within the TBI group, only omissions and inefficiencies were significantly related.

Conclusions: Despite overlap in performance between the two versions of the BMET, they did not assess executive deficits identically. This highlights the importance of careful construction and validation of alternative versions of naturalistic assessments.

Correspondence: R. Christopher Branson, Psychology, University of Tennessee at Chattanooga, 1101 Hamilton Ave, Chattanooga, TN 37405.E-mail:Raylan-Branson@utc.edu

E. TURNER, A.K. HILDENBRAND, R. GAMBER, E. NICHOLLS, M.D. MCCURDY, S. SASSAMAN \& B. DALY. The Relationship between Hot and Cold Executive Functioning and Childhood Maltreatment in College Students.

Objective: Childhood maltreatment (e.g., physical, sexual, emotional abuse) has been linked to compromised executive functioning (EF). College students with impaired EF are at risk for negative educational outcomes, including academic adjustment and performance difficulties. This study examined differences in impairments of the hot and cold components of EF in college students with and without a history of childhood maltreatment.

Participants and Methods: One hundred twenty youth ages 18 to 23 years $(M=20.44)$ were administered the Childhood Trauma Questionnaire (CTQ) and The Behavior Rating Inventory of Executive Function-Adult Version (BRIEF-A). The Emotional Control and Inhibition subscales represent functioning of the hot system whereas the Working Memory and Planning/Organizing subscales represent cool EF.

Results: Of the 120 participants, $33(27.5 \%)$ reported a history of childhood maltreatment. An independent samples t-test indicated significant differences between the maltreatment and no maltreatment conditions for hot EF scores $\mathrm{t}(118)=-3.04, \mathrm{p}=0.003$, and for cold $\mathrm{EF}$ scores $\mathrm{t}(118)=-2.74, \mathrm{p}=0.007$. Specifically, the maltreatment group demonstrated higher hot $\mathrm{EF}(\mathrm{M}=16.10, \mathrm{SD}=4.03)$ and cold $\mathrm{EF}$ scores
$(\mathrm{M}=15.01, \mathrm{SD}=3.08)$ than those reporting no history of maltreatment. Neither maltreatment type nor history of polyvictimization significantly predicted hot or cold EF scores.

Conclusions: College students that are victims of childhood maltreatment report more deficits in both hot and cold components of EF. These results extend previous findings that maltreatment victims display disrupted neurocognitive problems in addition to more common emotional disturbances. The history of a particular trauma subtype or multiple subtypes may not uniquely contribute to the presence of hot or cold EF deficits.

Correspondence: Elise Turner, B.A., Psychology, Drexel University, 3141 Chestnut Street, Stratton Hall 119, Philadelphia, PA 19104. E-mail: eliseturner30@gmail.com

\section{D.C. BLINKOFF, G. POTTS \& C. CIMINO. Apathy and Working Memory in a Non-Clinical Population.}

Objective: The relationship between apathy and executive functioning has been demonstrated in several neuropsychiatric patient populations. Studies on apathy, however, have mainly focused on patient populations. The purpose of this study was to examine normal variations in apathy in a non-clinical population and to explore its relationship with executive functioning in comparison to depressive symptoms using behavioral and ERP data.

Participants and Methods: 28 undergraduates, 8 males/ 20 females, ages 18-26 with no history of neurologic or psychiatric disorders, were recruited from the University of South Florida's subject pool. Participants reported apathy (AES) and depressive symptoms (CES-D). Participants were separated into high/low apathy and depression groups using a split-half approach. EEG data was acquired during the administration of computerized N-back and control tasks. Accuracy and reaction time (RT) were recorded for the tasks and EEG data was processed to create ERPs.

Results: All AES scores were below cut-offs for clinical levels of apathy. N-Back performance (RT) was significantly correlated with AES scores (1-back, $r=.529, p<.01 ; 2$-back, $r=.532, p<.01)$ but not CES-D scores. Control task performance was unrelated to self-reported apathy or depressive symptoms. There was a trending difference in P300 amplitude between the high and low apathy groups during N-back performance, $\mathrm{t}(21)=1.85, \mathrm{p}=.078$. There was no difference in P300 amplitude between apathy groups for the control task. No difference in P300 amplitude was found between the depression groups for either task.

Conclusions: This study reveals that sub-clinical, normal variations in apathy can predict executive functioning performance and provides support that apathy is dissociable from depression.

Correspondence: Danielle C. Blinkoff, M.A., Clinical Psychology, University of South Florida, 13103 National Drive, Apt. D, Tampa, FL 33617. E-mail: dblinkoff@mail.usf.edu

P.T. CIRINO, J. MICIAK, E.H. GERST, A. CHILD, E.A. HUSTONWARREN \& M. EDWARDS. Executive Functions, Self-Regulated Learning, and Reading Intervention.

Objective: Executive functions (EF) and self-regulated learning (SRL) are complimentary in promoting active engagement of reading comprehension (RC), because both emphasize preparation/planning, ongoing monitoring, and evaluation of performance. The present study evaluates the effectiveness of adding such processes to a text-based RC intervention for at-risk students. We hypothesized that this intervention would outperform business-as-usual (BAU) controls, or an intervention focused on text but with less of an emphasis on EF/SRL.

Participants and Methods: Two hundred and fifty-four 4th grade students were screened on a common RC measure (Gates MacGinitie). 75 students with performance $<25$ th \%ile were randomized to BAU, to a text-based intervention (TB), or to a TB+EF intervention. Participants were predominantly African-American and Latino/Hispanic students at schools with high rates of free/reduced lunch. Both active interventions were equated for content (fourth grade social studies) 
and time (approximately 20 sessions over 4 weeks). Primary outcomes were taught text, near-transfer text (another social studies topic), and far-transfer text (science). Analyses included ANCOVA with pretest as covariate.

Results: At pretest the groups did not differ. After the intervention, there was a significant effect of treatment, $\mathrm{F}(2,68)=5.16, \mathrm{p}=<.001$, with both $\mathrm{TB}+\mathrm{EF}(\mathrm{d}=+.42)$ and $\mathrm{TB}(\mathrm{d}=+.72)$ outperforming controls; the active groups did not significantly differ. Background knowledge also improved for both active groups $(\mathrm{p}<.001 ; \mathrm{TB}+\mathrm{EF}$ vs control, $\mathrm{d}=$ +1.09 ; TB vs control, $\mathrm{d}=+.94)$. There were no significant differences among groups for near or far transfer texts $(p>.05)$.

Conclusions: The TB and TB+EF groups each performed better than the control group at post-test on intervention-related content, but with no differences on transfer tasks. Results speak to the difficult in separating $\mathrm{EF}$ and SRL processes from strong instruction more generally, as well as the need to intervene directly on skills targeted for improvement. Correspondence: Paul T. Cirino, PhD, Psychology, University of Houston, UH TMC Annex, 2151 W Holcombe Blvd, 224a, Houston, TX77204-5053.E-mail: pcirino@uh.edu

H.L. COMBS, M.E. RUSSELL \& S.C. SEGERSTROM. Higher Heart Rate Variability Predicts Better Performance on Executive Functioning Measures in Older Adults.

Objective: Heart rate variability (HRV), a vagally-mediated index of parasympathetic nervous system activity, has been proposed to reflect the strength of central networks linked to executive functions and self-regulation. Pharmacological deactivation of the prefrontal cortex during self-regulatory tasks leads to decreased HRV, while engagement of the prefrontal cortex increases HRV. Self-regulatory performance has also been linked to higher resting HRV in younger adults. To date, most studies of HRV and executive functions have used subjective, self-report questionnaires and younger adults; less is known about the relationship between HRV and objective neurocognitive measures in older adults. Participants and Methods: In the present study, the Trail Making Test A \& B (TMT A \& TMT B) and Rey Auditory Verbal Memory Test (RAVLT) were administered to 82 older adults (Mage=78). Resting HRV, operationalized as spectral power in the high-frequency (.12-.40 $\mathrm{Hz}$ ) range, was determined using a ten-minute ECG reading at the time of testing. A multiple regression analysis was conducted to predict neurocognitive performance from resting HRV, controlling for age, education, and estimated intelligence.

Results: Higher HRV significantly predicted faster performance on TMT A $(\mathrm{R}=-.352, \mathrm{~F}(1,80)=11.328, \mathrm{p}<.001, \mathrm{~B} 1=-3.141)$ and TMT $\mathrm{B}(\mathrm{R}=-.298, \mathrm{~F}(1,78)=7.610, \mathrm{p}=.007, \mathrm{~B} 1=-9.767)$. HRV was not related to RAVLT performance.

Conclusions: These findings provide confirmatory data that HRV is specifically related to objective executive function abilities, particularly scanning and mental flexibility. In older adults, HRV may reflect the integrity and capacity of prefrontal cortical function, and could be employed as a physiological marker for prefrontal impairment.

Correspondence: Hannah L. Combs, M.S., Psychology, University of Kentucky, 3900 Crosby Dr. Apt. 2507, Lexington, KY 40515. E-mail: hannahlanecombs@gmail.com

H.B. MORAIS, J.L. GESS, J.K. FAUSETT, N.K. MILLER \& G. JAMES. Network connectivity and Proverb test performance. Objective: Proverb test, a commonly used measure of executive functioning (EF), consists of eight orally interpreted nonliteral sayings and requires integration of concrete information into abstract concepts. Previous studies have modeled functional correlates of nonliteral interpretation; however, little is known regarding the relationship between this EF facet and non-task dependent connectivity. This study examined the relationship between neural connectivity at rest and Proverb test scores. Participants and Methods: Data were collected through the Cognitive Connectome project, which seeks to translate functional neuroimaging into personalized medicine by comprehensively mapping normative variability in cognition and brain function in a normative sample of healthy adults $(\mathrm{n}=38)$. Independent component analysis (ICA) identified 70 functionally unique neural networks. Robust linear regression related functional connectivity (the correlation between networks' activity timecourses) during wakeful rest to total scores on the D-KEFS Proverb test. Results: Greater Proverb test performance corresponded to decreased resting-state connectivity between medial prefrontal cortex (MPC) and anterior cingulate $(\mathrm{t}<-5.3), \mathrm{MPC}$ and subgenual cingulate $(\mathrm{t}<-4.2)$, and ventromedial prefrontal cortex (VMPFC) and dorsolateral prefrontal cortex (DLPFC) $(\mathrm{t}<-3.8)$.

Conclusions: These regions have been associated with sense of self (subgenual cingulate), social cognition (MPC and VMPFC), error processing (anterior cingulate), and higher-order cognition (DLPFC). Our findings suggest that greater independence among these regions at rest may facilitate appraisal of abstract concepts. These findings are useful in elucidating the relationship between network connectivity and neuropsychological functioning in a non-clinical sample. Future research should consider whether greater temporal synchrony of these regions may characterize impairments in abstraction among clinical populations.

Correspondence: Hugo B. Morais, Ph.D. Candidate, Psychiatry Research Institute, University of Arkansas for Medical Sciences, 4301 Markam St., Little Rock,AR 72207.E-mail: HBmorais@uams.edu

T.J. FARRER, A.K. SMITH WATTS, E.R. HARTMAN, S. CORREIA, S. VAN HUIDEN, S.P. SALLOWAY, P.F. MALLOY \& S. DEONI. Association between White Matter Hyperintensities and Alternate Scoring for Trail Making Test Part B.

Objective: Subcortical white matter hyperintensities (WMH) on MRI correlate with impairment on executive measures such as Trail Making Test Part B (TMT-B). Our group previously reported a TMT-B "efficiency score" (TMT-Be) that captures performance variability among examinees who fail to complete the task within the standard 300s time limit. The present study compared the correlations between WMH and TMT-B and TMT-Be scores in elderly individuals with cognitive impairment. Similar correlations would support concurrent validity for the TMT-Be score.

Participants and Methods: Forty-two participants (mean age $78 \pm 7.19$; $62 \%$ female) with either mild cognitive impairment or Alzheimer's dementia (Clinical Dementia Rating Scale $\geq 0.5$ ) completed a neuropsychological battery including TMT-B administered according to standard procedures.

Our efficiency score (TMT-Be) takes into account move-efficiency [ratio of correct moves $(\mathrm{Mc})$ to commission errors (Ec)], time efficiency [time (T) per correct move], and omission errors (Eo):

TMT-Be $=[(\mathrm{Mc} /(24-\mathrm{Ec})) *(\mathrm{~T} / \mathrm{Mc})]+\mathrm{Eo}$

where: $24 \geq \mathrm{Mc}>0,24 \leq \mathrm{T} \leq 300 \mathrm{~s}, 0 \leq \mathrm{E} 0 \leq 23$

Increased Ec or Eo or greater T/Mc all serve to increase the value of TMT-Be indicating poorer overall efficiency.

Results: Both TMT-Be and TMT-B correlated significantly with WMH after controlling for parenchymal volume $(\mathrm{rs}=.48 \& .52$, respectively, $\mathrm{p} \leq .007)$. The correlations were not statistically different (Fisher's $\mathrm{z}=$ $-.20, \mathrm{p}=.84)$.

Conclusions: WMH correlates equally with TMT-B and TMT-Be supporting concurrent validity of the TMT-Be score. This suggests that TMT-B and TMT-Be relate similarly to white matter integrity among individuals who fail to complete standard administration of the task. Correspondence: Thomas J. Farrer, M.S., Psychology, Brigham Young University, 1001 Kimball Tower, P.O. Box 25543, Provo, UT 846025543.E-mail: thomasfarrer@yahoo.com

E.C. DUGGAN, M.A. GARCIA-BARRERA \& U. MÜLLER. Examining Executive Behavior of Young Adults: Convergent Validity Analyses of an Executive Functions Screener.

Objective: Examining patterns of correlation coefficients is the most common approach to evaluating the convergent validity between 
executive function (EF) rating scales. Yet, correlated scores may reflect the extent to which scales covary on other factors. Thus, this study examined the convergent validity of a four-factor (Problem Solving, Attentional Control, Behavioral Control, Emotional Control) EF screener (Duggan et al., 2014) derived from the Behavior Assessment System for Children-2 College version (BASC) by evaluating it against the Behavior Rating Inventory of Executive Function-Adult version (BRIEF) at the latent mean level, rather than via direct scores.

Participants and Methods: 97 healthy undergraduates completed the BASC and the BRIEF. The BRIEF scales most similar to the BASC EF factors were isolated as "target" scales (i.e., Plan/Organize, Working Memory, Inhibit, and Emotional Control), with the remaining scales considered as "non-target." Each BASC EF factor was first evaluated against target and non-target scales from the BRIEF using Pearson and Spearman correlations. Structural equation modeling was then used to evaluate the latent mean correlations between the four-factor BASC model and a four-factor target BRIEF model, as well as a nine-factor target/non-target model.

Results: All BASC EF factors were significantly correlated with their target BRIEF scales using simple correlations $(\mathrm{r}=.489-.652, \rho=.650$ $.480, p=.000)$ and latent mean correlations $(r=.896-.768, p=.000)$. These correlations were stronger than those between the BASC and BRIEF non-target factors.

Conclusions: These results suggest that the BASC EF screener has strong convergent validity with the BRIEF when the effects of error are disattenuated. These findings further support the validity of the BASC EF screener. Moreover, the success of this approach suggests that a similar methodology may be helpful in addressing the challenges associated with establishing convergent validity for other executive functions rating scales.

Correspondence: Emily C. Duggan, BA, Psychology, University of Victoria, PO Box 1700, Dept. of Psychology, University of Victoria, Victoria,BCV8W2Y2, Canada.E-mail:eduggan@uvic.ca

R. GOLDSWORTHY, A. FEDER, J. RAAK, R. O'NEIL, S. MARTIN, A. NEALE \& R.L. SKEEL. Sex Differences in Impulsivity Factor Structures of Behavioral and Self-Report Measures.

Objective: Research on sex differences in impulsivity has provided mixed conclusions on the degree of differences in impulsivity between sexes. One confounding factor is the lack of both behavioral and self-report measures being administered within a single study allowing for direct comparison of different facets of the construct of impulsivity (Sharma et al. 2014). In the current study we sought to determine if the construct of impulsivity was equivalent across sex.

Participants and Methods: Undergraduate participants ( $N=141)$ completed the following measures of impulsivity: a weighted risk task (Stoplight Task), a time estimation task (TE), a sequential risk task (Angling Risk Task), a delayed discounting task and Barratt Impulsiveness Scale (BIS). In addition, participants completed a single word reading measure (AMNART), and a measure of numeracy to control for processes associated with impulsivity.

Results: MANOVA analysis revealed group differences in all impulsivity measures with the exception of the BIS. Separate analysis also revealed group differences on both numeracy and reading ability. Exploratory factor analyses yielded similar two factor solutions for both men and women with Factor 1 representing a Cognitive/Intelligence Component and Factor 2 a Self-Report Component. However, one measure, TE loaded on different factors for men and women.

Conclusions: Current results suggest that despite absolute differences in responses on behavioral and self-report measures, the underlying factor structure is largely equivalent across sexes. TE may represent a unique dimension of impulsivity.

Correspondence: Reid L. Skeel, Ph.D., Psychology, Central Michigan University, 136 Sloan Hall, Mt. Pleasant, MI 48859.E-mail: Skeel1rl@ cmich.edu
M. FEIGON, J. JANECEK, M. GOWHARI, R. MOLOKIE, L. PIPER, M. VERNALE, N. PLISKIN \& D. NYENHUIS. Executive Dysfunction in Adults with Sickle Cell Disease: Depressed versus Non-Depressed Groups.

Objective: Executive dysfunction is associated with both depression and vascular related cognitive impairment. Patients with sickle cell disease (SCD) have higher rates of depression compared to non-clinical populations and may demonstrate cognitive impairment even without evidence of stroke. The present study compared a sample of patients with SCD and moderate to severe depressive symptoms, to a sample of SCD patients with minimal depressive symptoms, and to healthy controls to determine if both SCD and depression related to greater executive dysfunction.

Participants and Methods: One hundred thirty-one African-American, community dwelling adults (mean age $=35.39, \mathrm{SD}=1.51$ ) completed the 60 Minute Vascular Cognitive Impairment Protocol and the CES-D (Hachinski et al., 2006; Radloff, 1977). Those with SCD and depression $(n=25$; CES-D score $>=16)$ were compared to SCD participants with low to no depressive symptoms $(\mathrm{n}=38)$ against controls without evidence of depression $(n=68)$ on the executive functioning domain. The effects of age and education were controlled for and differences in groups between number of strokes and premorbid intelligence were also compared in posthoc analyses.

Results: All three groups differed on levels of executive dysfunction ( $p$ $<.01$ ). The most severely depressed group had the highest level of executive dysfunction and both SCD groups had higher levels of executive dysfunction compared to controls.

Conclusions: Individuals with SCD and comorbid depression are at increased risk of executive dysfunction and require more careful monitoring and intervention. Additional studies are needed to examine potential causes for these differences and the effect of intervention.

Correspondence: Maia Feigon, M.A., Illinois Institute of Technology, 3540 N Hermitage Ave, Chicago, IL 60657.E-mail: mfeigon@psych. uic.edu

M. SULLAN, J. BAXTER, R. HAMMAN, R. PORTER \& J. GRIGSBY. Adiposity and Cognitive Functioning among Older Adults in the San Luis Valley Health and Aging Study (SLVHAS).

Objective: Obesity has significant impacts on cognition, with varying effects across the lifespan. Younger adults with a high Body Mass Index (BMI) are associated with decreased performance on certain cognitive measures. However, among older individuals, higher BMI has shown mixed effects. In this study we examined whether BMI was associated with higher scores on cognitive measures in older adults, and analyzed gender-based differences between groups.

Participants and Methods: In the San Luis Valley Health and Aging Study (SLVHAS), a population-based study of chronic illness and disability among persons age 60 and above in rural southern Colorado, we examined this relationship in a cross-sectional analysis of 1,251 participants. For cognition, we examined general mental status, executive functioning (EF), working memory and constructional praxis. Both BMI and Waist-Hip Ratio (WHR) were used as measures of adiposity. We calculated independent models for females and males using multiple regression, adjusting for age, education, ethnicity, and several comorbid conditions.

Results: For males, both BMI and WHR were positively associated with EF, but with no other measures of cognition. For females, WHR was inversely associated with EF, while BMI was positively associated with general mental status. Executive functioning appeared to be more susceptible to influence than other cognitive variables for both men and women, although among women, mental status declined with decreasing BMI.

Conclusions: The results suggest that frail older people may be at increased risk for cognitive decline, and that obesity has differential effects on cognition dependent on gender among older individuals. 
Correspondence: Molly Sullan, BS, Clinical and Health Psychology, University of Florida, 230 SW 2nd Ave, Apt 403, Gainesville, FL 32601. E-mail:mollysullan@gmail.com

O.A. SANTOS, S.E. PARK, E.M. GIESE, M.M. LANGENKAMP, O.L. HARMELINK, S.J. ZUPEK, J.J. ANDERSON \& D.C. OSMON. Comparison of Ex-Gaussian Analysis of Reaction Time on NonExecutive and Executive Elementary Cognitive Tasks in ADHD and Control Subjects Versus Schizotypal and Control Subjects.

Objective: Subjects with ADHD or with schizotypal personality traits (SPT) have shown more skewed reaction time (RT) distributions compared to controls, although normal distribution parameters are typically used despite distributions of RT having a well-known positive skew. Additionally, RT tasks favoring executive functions (EF) have not been typically used despite EF deficits in ADHD or subjects with SPT. We hypothesized that ex-Gaussian analyses of RT may provide a more specific description of the RT distributions in subjects with ADHD or with SPT compared to normal distribution parameters.

Participants and Methods: Four elementary cognitive tasks (ECTs) based on information theory and a RT paradigm were used: a Simple and a Choice non-EF ECTs and two EF ECTs using internal rule response selection criteria. Two independent studies were conducted using college students. The first study included 24 ADHD subjects and 53 controls, and the second study included 28 subjects with SPT and 63 controls.

Results: Multiple models of RT distributions for ADHD, SPT, and control groups were examined separately. When modeling the RT distributions using mixtures of 2 or 3 normal distributions, ADHD subjects had impairment in the skewed part of the distribution only for the Simple ECT compared to controls. Contrarily, subjects with SPT were faster on both the normal and skewed part of the Simple ECT distributions, but were slower on the Choice and EF ECTs compared to controls. Conventional RT analyses showed no differences between groups. Conclusions: ADHD appears to be associated with greater lapses of attention on the Simple RT task compared to controls, but not on the EF ECTs in contrast to the conventional wisdom that ADHD is associated with frontal-executive impairment. However, subjects with SPT presented the opposite pattern, showing greater RT on the Choice and EF ECTs compared to controls. Further work is necessary to explore why deficits are not present on EF ECTs in ADHD and replicate findings in subjects with SPF.

Correspondence: Octavio A. Santos, BA, Psychology, University of Wisconsin-Milwaukee, 2441 E Hartford Ave, Department of Psychology, GarlandHall,Room 224, Milwaukee,WI53211.E-mail: osantossolano@ gmail.com

J. BARRASH, D. STUSS, N. AKSAN, S.W. ANDERSON, R.D. JONES, K. MANZEL \& D. TRANEL. Types of Acquired Personality Disturbances Following Brain Damage: Exploration with Cluster Aanalysis.

Objective: Over many decades, differing types of acquired personality disturbances (APD) have been proposed based on clinical observations and hypotheses regarding distinct prefrontal systems. However, there has been little systematic investigation of patients with neuroanatomically diverse lesions.

Participants and Methods: 157 adults with stable, adult-onset focal brain lesions were studied. Collaterals rated 30 characteristics with the Iowa Scales of Personality Change. Subtypes of APD were investigated with two approaches to cluster analysis (CA): K-means CA to determine whether five hypothesized types (below) characterize actual patients; and Ward's method to identify empirically-derived groups according to hierarchical 2- through 6-group solutions.

Results: Hypothesis-driven K-means CA classified patients into the five expected types: Dysexecutive, Emotionally Reactive/Disturbed Social Behavior, Hypo-Emotional/Amotivated, Distressed, and Normal. The two-group solution of Ward's CA yielded Normal and Mild Generalized groups, and subarchical Ward's CAs with additional groups resulted in successive division of the disturbed group - until the 6-group solution, when the Normal group split in two. Compared to the hypothesis-driven CA, very similar clusters emerged from the 5-group solution using Ward's empirical approach, though the latter tended to be larger and with less distinct patterns of disturbance. The Hypo-Emotional/ Amotivated type had a particularly specific profile of disturbances that was consistent across $\mathrm{CA}$ procedures.

Conclusions: Ward's hierarchical CAs are informative regarding interrelationships between types, while the hypothesis-driven CA better illuminates the distinctive pattern of disturbances in APD subtypes. Information from the two approaches was complementary and indicated four types of APD, plus a residual normal group. Findings will inform further investigation of APD types.

Correspondence: Joe Barrash, PhD, Neurology, Uinversity of Iowa College of Medicine, 200 Hawkins Dr., Iowa City, IA 52246. E-mail: joseph-barrash@uiowa.edu

J. POMMY, S.G. RYMAN, K. WITKIEWITZ, R.J. THOMA \& R.A. YEO. Neuropsychological Subtypes of Executive Function in Schizophrenia and Healthy Controls Using A Community Detection Approach.

Objective: Executive functioning (EF) impairments observed in schizophrenia (SZ) occur prior to onset of psychosis and are predictive of functional outcomes. There is significant variability in the nature and severity of EF deficits, however, and a better understanding of this heterogeneity could provide insight into the neurodevelopmental processes underlying both SZ and EFs. Using an approach similar to Fair et al., 2012, the present analysis examined heterogeneity in EFs and attempted to identify EF subtypes within healthy controls $(\mathrm{HC})$ and individuals with SZ.

Participants and Methods: As part of a larger investigation of SZ, 285 participants (SZ: N=128; HC: N=157) were recruited from the community. EFs were assessed using the Trail Making Test, Verbal Fluency test, Tower of London, and Continuous Performance Test. A 4-factor model of EF (fluency, planning, shifting, attention) was tested in the sample using a Confirmatory Factor Analysis (CFA). The presence of EF subtypes was assessed separately in both groups using community detection (CD), an analytic technique based in graph theory that enables an unbiased analysis of community structure within complex networks. Results: Results from the CFA supported a 4-factor model of EF $[\chi 2(29)=27.895$, RMSEA $=0.000, \mathrm{CFI}=1.0, \mathrm{TLI}=1.003]$. The $\mathrm{CD}$ analyses indicated greater modularity in SZ; and upon initial inspection, identified $7 \mathrm{EF}$ subtypes in the SZ group that nested within $5 \mathrm{EF}$ subtypes in the HC group.

Conclusions: Consistent with findings reported by Fair and colleagues, results support the existence of similar cognitive subtypes in the context of both normal and aberrant neurodevelopment.

Correspondence: Jessica Pommy, UNM, 6004 Burgos Avenue NW, Albuquerque,NM 87114.E-mail:.jpommy@gmail.com

T. KIELY, A.M. STAFFARONI, R. GOMEZ, L. TRETTIN, B.H. SOLVASON, A.F. SCHATZBERG \& J. KELLER. The Effect of Depression Chronicity and Severity on Executive Function.

Objective: Little is known about how Depression (MD) chronicity (DC) and severity (DS) affect cognitive ability. DC is defined as the total duration one has carried a MD diagnosis in their lifetime. This study investigated the contributing effects of DC and DS on executive functions (EF) in a group of adults with MD.

Participants and Methods: MD participants $(\mathrm{N}=71)$ were recruited at Stanford University. Participants were administered the Structured Clinical Interview (SCID) to obtain chronicity data. The Hamilton Depression Rating Scale was used to assess DS. Participants were administered several executive measures including the Trail Making Test-Part B (TMT-B), Stroop Color Word test, and the Semantic Fluency (SF) subtest of the COWA. Stepwise linear regressions examined the relative 
contribution of DC and DS on executive function measures. Age and education were used as covariates and entered in Step 1; DC and DS were entered in the model at Step 2 and 3, respectively.

Results: On the Stroop, the regression model revealed trending significance explaining $9.1 \%$ of the variance, $F(2,58)=2.91$, p $=.06$. Age (Beta=-2.60, $\mathrm{p}<.05$ ), was found to be a predictor in this model. On TMT-B, the model significantly explained $11.3 \%$ of the variance, $\mathrm{F}(2,60)=3.81, \mathrm{p}=.03$. Again, age $($ Beta $=.322, \mathrm{p}<.05)$, was found to be a predictor in this model. On SF, the model significantly explained $11.0 \%$ of the variance, $\mathrm{F}(2,60)=3.72, \mathrm{p}=.03$. Age $($ Beta $=-2.60, \mathrm{p}<.05)$ and education (Beta=.269, $\mathrm{p}<.05)$ were found to be unique predictors in the model. However, DC and DS were not found to be significant predictors on executive measures in this regression model.

Conclusions: Although there was a trend in model significance, DC and DS were not found to be unique predictors for executive dysfunction in this MD sample ( $>30 \%$ Chronic MD). These findings might be due to an adaptive compensatory response in the chronically depressed as a means to improve overall functioning. Future investigations are needed to see if those with Chronic Depression experience a rebound in EF. Correspondence: Thomas Kiely, Ph.D., Clinical Psychology, Palo Alto University, 113 Crescent Ave, Burlingame, CA 94010.E-mail: tkiely@ paloaltou.edu

S.N. LIGHT, L. BIELIAUSKAS \& J. ZUBIETA. Anhedonia, Executive Function, and Endogenous Opioids in Lateral Prefrontal Cortex in Major Depressive Disorder (MDD): A PET Study.

Objective: The relationship between cognitive dysfunction (e.g. reduced concentration, executive function difficulties, etc.) and anhedonia (i.e. the reduced ability to experience positive emotion) in Major Depressive Disorder (MDD) is currently poorly understood. This question is of importance because these particular symptoms are the two most commonly reported and persistent symptoms of the syndrome. The elucidation of the neurochemical basis of these often co-occurring symptoms was the aim of the current study, with emphasis on ventrolateral prefrontal cortex (VLPFC) given prior findings relating activity in this region to anhedonia (Light et al., 2011).

Participants and Methods: 39 volunteers with MDD were characterized (pre-antidepressant treatment) with a structured clinical interview for DSM-IV diagnosis (SCID-IV), a personality questionnaire (NEO-PI-R), and positron emission tomography (PET) and functional magnetic resonance imaging (fMRI) as part of the study. Two different PET tracers, $[11 \mathrm{C}]$ carfentanil $(\mathrm{CFN})$ and $[11 \mathrm{C}]$ raclopride, were used to track opioid and dopamine neurotransmission in prefrontal cortex during PET scans, respectively. The fMRI sessions were used to characterize anatomy and allow co-registration of fMRI and PET images. Results: Greater positive emotionality, as measured by the Positive Emotion subscale of the NEO-PI-R, related to better performance on the Wisconsin Card Sorting Test (i.e. Categories Complete, Perseverative Responses, Failure to Maintain Mental Set; all ps<.05). Similarly, positive emotionality related negatively to $\mu$-opioid receptor binding in VLPFC $(p<.05)$. Finally, executive function also related negatively to $\mu$-opioid binding in the VLPFC $(p<.05)$. A mediation analysis was completed; VLPFC $\mu$-opioid binding remained significant and NEO Positive Emotion became a marginal predictor of executive function (overall model: R-square $=30 \%, p=.015$ ).

Conclusions: $\mu$-opioid receptor binding in VLPFC mediates the relationship between positive emotionality and executive function.

Correspondence: Sharee N. Light, PhD, Psychiatry, University of Michigan, 2101 Commonwealth Blvd, Ann Arbor, MI 48105. E-mail: lights@med.umich.edu

\section{Forensic Neuropsychology}

J. BIRATH, S. MANGASSRIAN, S. FATOORECHI, Y. CERVANTES, D. VALDEZ, K.B. BOONE, D.J. HARDY, P. VESPA, D. HOVDA, D. MCARTHUR, T. GLENN, J. FUSTER, E. WOO \& M.J. WRIGHT. Performance Validity and Workload in Healthy Adults and Adults with Traumatic Brain Injury.

Objective: We conducted the current study to determine how scores on measures of performance validity tests (PVTs) relate to subjective workload in healthy adults and survivors of severe traumatic brain injury (TBI).

Participants and Methods: A healthy adult group ( $\mathrm{n}=19)$ and an adult TBI group (1+ years post-injury; $n=6$ ) completed three PVTs, the Dot Counting Test (DCT), the Rey Fifteen Item Test (ReyFIT), and a newly devised recognition trial to the Symbol Digit Modalities Test (SDMT-R) in conjunction with The NASA Task Load Index (NASA-TLX). The NASA-TLX was administered following each PVT.

Results: Pearson product-moment correlations were used for the healthy adult group and Spearman's rho was used to evaluate associations in the TBI group. The threshold for significance was set at $p<.05$. All participants demonstrated valid performances across the three SVTs. The healthy adult group demonstrated negative correlations between the DCT E-score and subjective physical demands and the ReyFIT combination score and subjective physical demands and frustration. The TBI group showed positive correlations between the DCT E-score and subjective physical demands, performance, frustration, and overall workload. The TBI group also showed a negative association between the ReyFIT combination score and overall workload. No correlations were observed between subjective workload and the SDMT-R in either group. Conclusions: The current data indicate that healthy adults and adults with TBI perceive PVTs differently. Additionally, these data suggest that studies of perceived workload across PVTs may be beneficial for the detection of malingering in various populations.

Correspondence: Matthew J. Wright, Ph.D., Psychiatry, Harbor-UCLA Medical Center, 1124 W. Carson St., B-4 South, Rm. 111 (Box 490), Torrance,CA 90502.E-mail: mwright@labiomed.org

K.A. GORGENS, N.M. GLOVER, J. GAFFORD, B.F. MCMILLAN \& J. DETTMER. Identifying and Treating the Superfecta: TBI, Mental Illness, Substance Abuse in a County Jail.

Objective: This paper highlights a novel collaboration funded by the Health Resources and Service Administration (HRSA) and the Colorado Office of Behavioral Health. This partnership between the State of Colorado, several adult and youth correctional facilities, a problem-solving court and the University of Denver, aids in the identification of risk and promotion of harm reduction in the most vulnerable offenders.

Participants and Methods: This study uses traumatic brain injury and neuropsychological screening to detect cognitive vulnerabilities and identify at-risk persons as well as offer individualized feedback and interventions. The inmates invited to participate were housed in the Denver County Jail, Larimer County Jail, Boulder County Jail, and the Department of Youth Corrections and Denver Juvenile Probation Results: In this project to date, $96 \%$ of inmates endorse a history of TBI, $92 \%$ of inmates have comorbid substance abuse and mental illness and $83 \%$ of the inmates sample show a combination of mental illness, substance abuse, criminal history and traumatic brain injury history; a condition we have termed the 'superfecta' and one that warrants specialized intervention. In this case, self-advocacy and TBI Toolkit programming designed for Veterans is being adapted to correctional settings and program evaluation data will be presented.

Conclusions: Programs aimed at reducing recidivism in mentally ill offenders should incorporate an understanding of the relationship between criminality, TBI, mental illness and substance abuse. The present paper highlights a project with those aims and outlines plans for recidivism surveillance and long-term risk reduction. 
Correspondence: Nicole M. Glover, M.A., Clinical Psychology, University of Denver, 975 S Vine St., Denver, CO 80209. E-mail: nicole.marie. glover@gmail.com

J. ETHERTON, R. CLARK, T. BRAND, B. HUMMER \& C. O'SHEA. Controlled Oral Word Association Test Performance is Not Impaired by Induced Pain.

Objective: To examine the degree to which induced pain affects performance on the Controlled Oral Word Association Test in healthy volunteers.

Participants and Methods: Thirty-seven non-clinical undergraduate volunteers (22 female) were administered two different versions of the COWAT (either FAS or PRW as the stimulus letters). The first version was administered normally, while the second version was administered during either painless warm water immersion (Control group; $\mathbf{n}=19$ ) or during cold pressor-induced pain (Pain group; $n=18$ ). Participants were randomly assigned to groups, and order of COWAT version was counterbalanced.

Results: A mixed-design repeated-measures ANOVA was conducted, with the two COWAT administrations (Standard vs Water Immersion) as the repeated measure and group (Pain vs Control) as the between-groups variable. There was a main effect for order of administration, in that performance on the second COWAT administration improved $($ Version 1 mean $=32.16$, sd $=1.1 ;$ Version 2 mean $=34.25$, $\mathrm{sd}=1.39)$. There was no significant between-groups main effect, $\mathrm{F}(1$, $35)=0.0, p=0.99$, and no condition by administration interaction, $F(1,35)=1.97, p=0.17$, indicating that the Pain group did not show significant decrement in performance during pain induction.

Conclusions: Results indicate that pain does not reduce COWAT performance. Thus, verbal fluency decrements observed in chronic pain patients are not likely attributable to pain, but possibly to other variables including sedating pain medications, sleep deprivation, emotional disturbance, poor effort, or symptom exaggeration.

Correspondence: Joseph Etherton, Ph.D., Psychology, Texas State University, 1905 Ford St, Austin, TX 78704.E-mail: joe.etherton@ gmail.com

\section{F.L. COOLIDGE. Assessment of DSM-5 Neurocognitive Disorder in 3,090 Adult Prison Inmates.}

Objective: The present study attempted to assess the prevalence (as a function of severity) of the new DSM-5 Neurocognitive Disorder in a large sample of prison inmates.

Participants and Methods: Participants were 3,090 adult prison inmates who were given the 250-item Coolidge Correctional Inventory (CCI) upon routine admission to the Colorado Department of Corrections. Among other scales, the CCI contains a 31-item scale which assesses Neurocognitive Disorder according to various criteria in DSM-5. Results: When compared to norms based on normally functioning, community dwelling adults, approximately $10.4 \%$ of the inmates were identified as either purportedly having Mild or Major Neurocognitive Disorder, with 7.7\% as having Mild Neurocognitive Disorder and 2.7\% as having Major Neurocognitive Disorder.

Conclusions: As neurocognitive dysfunction can profoundly affect treatment programs, the present study emphasizes the importance of neuropsychological screening, even if by self-report, in correctional institutions.

Correspondence: Frederick L. Coolidge, PhD, Psychology, Univ of Colorado, Colorado Springs, 1420 Austin Bluffs Parkway, Psychology, Colorado Springs, CO 80918. E-mail:fcoolidg@uccs.edu

C.B. PADUla, P. TOOFANiAN-ROSS, S. NITCH \& D. KINNEY. Cognition and Trial Competency Restoration: Using the RBANS as a Treatment Indicator for Patients Deemed Incompetent to Stand Trial.

Objective: Intact cognitive ability is a fundamental component for someone to be deemed competent to stand trial. This study sought to ascertain whether a brief cognitive screening instrument, the Repeatable Battery for the Assessment of Neuropsychological Status (RBANS), was associated with the length of hospitalization required to restore trial competency.

Participants and Methods: A total of 288 forensic psychiatric inpatients undergoing competency restoration treatment were included in the study. Schizophrenia was the most common primary diagnosis in the sample. As part of the intake assessment, the RBANS was administered upon admission to the hospital. Length of stay was calculated based on admission and discharge dates. Individuals with a diagnosis of malingering were excluded from the study.

Results: All analyses were adjusted for age, race/ethnicity, gender, years of education, and neurocognitive disorder diagnoses including dementia. Linear regression analyses revealed that individuals who demonstrated poorer overall RBANS performance required longer hospitalizations to be restored to trial competence $(p<0.001)$. Age was the only significant covariate, such that older individuals also required longer lengths of stay $(\mathrm{p}<0.001)$. In addition, individuals deemed incompetent to stand trial scoring in the 51-60 range on the RBANS Total Scale Index were 2.63 times more likely to require hospitalizations beyond the average (7.2 months) length of stay ( $p=0.01)$.

Conclusions: Findings support the use of the RBANS to identify individuals at risk for requiring longer hospitalizations to restore trial competence due to cognitive ability. Identifying these individuals early in the treatment process and providing more intensive and personalized restoration treatment for trial competence may improve treatment outcomes.

Correspondence: Claudia B. Padula, PhD, Psychiatry, VA Palo Alto Health Care System, 3801 Miranda Ave, 151Y, Palo Alto, CA 94304. E-mail: claudiapadula@gmail.com

K.L. CURTIS, K. GREVE \& K. BIANCHINI. A Comparison of Mild Traumatic Brain Injury and Chronic Pain Patients on Symptom Self-Report, Objective Measures of Cognitive Functioning, and Psychological Factors.

Objective: Despite injury mechanism differences, there is considerable overlap in the symptoms reported by mild traumatic brain injury (mTBI) and chronic pain (CP) patients. The current study compared rates of subjective symptom report, performance on objective measures of working memory and processing speed, and psychological elevations in both samples.

Participants and Methods: Only patients with valid scores on performance (PDRT, TOMM, and RDS) and symptom (F, Fb, Fp, and FBS) validity measures were included in analyses to best elucidate patient group similarities and/or differences. The final sample consisted of 60 mTBI patients, $106 \mathrm{CP}$ patients, and 35 moderate-severe (M/S) TBI patients, serving as a comparison group. Group analyses examined rates of spontaneously-reported symptoms, demographically-adjusted T-scores for the Working Memory (WM) and Processing Speed (PS) Indexes of the WAIS-3, and psychological factors (Scales 1 [Hypochondriasis], 2 [Depression], 3 [Hysteria], and 7 [Psychasthenia]) from the MMPI-2. Results: Spontaneously-reported symptoms were not correlated with WM or PS T-scores. All three groups did not differ on WM or PS scores and all group averages were within one standard deviation from normal. Group means for Scales 1, 2, and 3 of the MMPI-2 were nearly identical for the mTBI and CP groups (e.g., Scale 1: $\operatorname{mTBI} M=75.2$; CP $M=75.8$ ). Both groups had significantly higher MMPI-2 scores than M/S patients.

Conclusions: The current study shows a lack of relationship between self-reported symptoms and objective measures of cognitive functioning. More importantly, the study demonstrates that mTBI and CP patients are essentially indistinguishable on psychological variables.

Correspondence: Kelly L. Curtis, Ph.D., Psychology, High Point University, 833 Montlieu Avenue, Drawer 37, High Point, NC 27262. E-mail: kcurtis0@highpoint.edu 
K.L. CURTIS, L. AGUERREVERE, K. BIANCHINI \& K. GREVE. The Influence of Exaggeration and Presence of Spinal Pathology on Select MMPI-2-RF RC Scales in a Chronic Spine-Related Pain Sample.

Objective: This study examined the contributions of spinal pathology and exaggeration on rates of psychopathology in patients with chronic spine-related pain (CP). Specifically, elevations rates were examined on the RCd, RC1, RC2, RC3, and RC7 scales from the MMPI-2-RF.

Participants and Methods: Chronic pain patients with $(n=147)$ and without $(n=235)$ evidence of spinal pathology were systematically categorized into "no/low" ( $n=126$; validity score composite $<2)$, "some" $(n=74$; validity score composite $=2$ or 3$)$, and "high" ( $n=182$; validity score composite $>3$ ) exaggeration groups using cognitive (PDRT, TOMM, and RDS), functional (FCE), and symptom (F-r, Fp-r, Fs-r, FBS-r, and RBS-r) validity indicators. The cutoffs used to determine cognitive validity were based on published cutoffs in TBI. Cutoffs for RF validity scales were from the MMPI-2-RF manual.

Results: A 2 (presence vs. absence of spinal pathology) by 3 (exaggeration group) MANOVA of RC scale scores showed a main effect for exaggeration only. Follow-up ANOVAs showed an apparent dose-response relationship between level of exaggeration and all RC scale scores. RC1 showed the highest level of scale elevations with $69 \%, 91.9 \%$, and $96.7 \%$ of the "no/low," "some," and "high" exaggeration groups scoring at a $\mathrm{T}$-score $>=65$, respectively.

Conclusions: This study indicated a strong relationship between the degree of exaggeration on validity indicators and RC scores. Further analysis examining MMPI-2-RF subscale scores as well as the contribution of additional psychosocial factors on rates of psychopathology in this population is warranted.

Correspondence: Kelly L. Curtis, Ph.D., Psychology, High Point University, 833 Montlieu Avenue, Drawer 37, High Point, NC 27262. E-mail:kcurtis0@highpoint.edu

V. KERCE, D. WHITESIDE, H. LUU, T. SAK, D. CARNS, L. RICE, M.R. BASSO \& B. ROPER. California Verbal Learning Test, Second Edition (CVLT-II) Recognition Measures as embedded Performance Validity Tests in a Mild Traumatic Brain Injury sample.

Objective: There is considerable research on Forced Choice (FC) Recognition subtest of the California Verbal Learning Test, Second Edition (CVLT-II) (Delis, Kramer, Kaplan, \& Ober, 2000) as embedded performance validity tests (PVT: Donders \& Strong, 2011) but relatively little on Total Hits (TH), Total False Positives (TFP), and Recognition Discriminability (RD). The current study examined the classification accuracy of these three CVLT-II measures for credible performance in a mild Traumatic Brain Injury (mTBI) sample. We hypothesized that TH, TFP and RD would have at least acceptable classification accuracy on Receiver Operator Characteristic (ROC) analysis.

Participants and Methods: The sample included 40 consecutive individuals age 18 and over who were diagnosed with mTBI (mean age $=48.2, \mathrm{SD}=14.6$; mean education $=13.3, \mathrm{SD}=2.8 ; 40 \%$ males, $92 \%$ Caucasian) referred for neuropsychological evaluation. The sample was divided into credible (participants who passed all PVTs, $\mathrm{N}=28$ ) versus non-credible performance (participants who failed at least 2 PVTs including the Test of Memory Malingering, $\mathrm{N}=12$ ) groups.

Results: TH and RD had excellent classification accuracy $(\mathrm{AUC}=.81$ and .83 , respectively) while TFP had acceptable classification accuracy $(\mathrm{AUC}=.70)$. At $90 \%$ sensitivity, the specificity for each CVLT-II measure was: TH $(50 \%)$, TFP $(30 \%)$, RD $(50 \%)$.

Conclusions: These results supported the classification accuracy of $\mathrm{TH}$ and RD, and to a lesser extent TFP, although TFP had relatively low sensitivity. Additionally, all three CVLT-II variables had cut-off values with appropriate specificity, indicating empirical support for multiple CVLT-II recognition measures as PVTs. Additional research in moderate/severe TBI and psychiatric samples would be beneficial in establishing the generalizability of these measures as embedded PVTs.
Correspondence: Douglas Whiteside, Ph.D., Clinical Psychology, Argosy University-Seatle, 200 Hawkins Drive, Iowa City, IA 52242. E-mail: douglas-whiteside@uiowa.edu

\section{Malingering/Effort Testing}

M.M. STIKA \& D.S. GOLDSTEIN. Relationship between Symptom and Performance Validity Test Performance in a Sample of Criminal Defendants.

Objective: Symptom validity tests (SVTs) and performance validity tests (PVTs) are utilized in neuropsychological evaluation in order to assess response bias, especially within the forensic setting. The purpose of this investigation was to examine the relationship between commonly used SVTs and PVTs in a sample of adult criminal defendants.

Participants and Methods: A large sample of felony defendants consecutively referred to a court clinic underwent comprehensive clinical interviews, effort, neurocognitive and personality assessment, and were classified into Optimal/Suboptimal cognitive effort groups and Exaggerated/Nonexaggerated psychological symptom endorsement groups. In order to examine the relationship between SVT and PVT performance, correlations were conducted between selected validity scales of the Minnesota Multiphasic Personality Inventory, 2nd edition (F, FB, FP, FBS) and Personality Assessment Inventory (INF, NIM) and a) stand-alone effort measures (Test of Memory Malingering. Trial 2; Word Memory Test, Delayed Recall; Victoria Symptom Validity Test, Hard Items; Rey 15-Item Test, Items); and b) embedded effort measures (California Verbal Learning Test, 2nd edition, Forced Choice; Reliable Digit Span). One-way ANOVAs were conducted, assessing 1) cognitive effort group differences on SVTs; and 2) psych group differences on PVTs, with follow-up univariate analyses when indicated.

Results: Descriptives for each of these groups is provided, including base rates of suboptimal cognitive effort and psychological response bias. Results from correlational and group analyses are presented.

Conclusions: Results contribute to the expanding literature on the relationship between SVTs and PVTs in samples with relatively high base rates of suboptimal effort, and increases our knowledge the PAI, a commonly used but less well-studied personality inventory.

Correspondence: Monica M. Stika, M.S., Psychology, Rosalind Franklin University of Medicine and Science, 3333 N. Green Bay Road, North Chicago,IL 60064.E-mail:monica.matthews@my.rfums.org

R. LEPPO, R. PLOTKIN, T. ZABEL \& J. REESMAN. Preliminary Examination of TOMM Performance in A Clinically-Referred Sample of Deaf and Hard of Hearing Children.

Objective: Despite the growth of research examining effort testing within various pediatric populations; no research has examined the performance of deaf children on various effort testing measures. This study examined the performance of clinically referred deaf and hard of hearing children (DHH) on the TOMM (Test of Memory and Malingering) to examine whether the TOMM may be useful as an effort testing measure with this population.

Participants and Methods: The present study was conducted via retrospective review of a de-identified clinical data set of children who were referred for clinical evaluation in a large neuropsychology department with a specialty clinic for children who are DHH. Only participants who were administered the TOMM and had a parent-reported history of hearing loss were included in the sample $(\mathrm{N}=25 ; 60 \%$ male, age range 5-18 years). Measures of adaptive functioning were available for 16 of the participants and Adaptive Behavior Assessment Scales (ABAS2) General Adaptive Composite scores ranged from 54 to 120. No other exclusionary criteria were applied.

Results: 5 of the 25 children $(20 \%)$ earned a score of 44 or below on Trial 1 of the TOMM. Of the children who were administered Trial 2; only $1(6.7 \%)$ scored below 45 on Trial 2 . This individual earned a raw 
score of 25 on Trial 1 and 26 on Trial 1 respectively, indicating concerns with consistent performance.

Conclusions: Preliminary results demonstrate that clinically referred children who are DHH perform similarly to clinical samples of children with typical hearing on the TOMM. Use of measures to assess consistency of effort in evaluations of DHH children is encouraged, and further investigation with the TOMM is encouraged based on preliminary results. Correspondence: Rachel Leppo, Educational Psychology, University of Texas at Austin, 520 Park Apt. 514, Baltimore, MD 21201. E-mail: rhtarantolo@gmail.com

\section{PIERCE \& A. STAROSTA. Failure Rates of Alternative TOMM} Indices and Cutoffs in a General Medical Population.

Objective: Evaluating performance validity is a critical component of neuropsychological testing as invalid performance undermines data quality and limits conclusions that can be drawn. One widely used performance validity test is the Test of Memory Malingering (TOMM). Recent research suggests that the traditional cut-off scores may lack sufficient sensitivity and specificity. We compared several alternative scoring methods and cutoff scores to determine the failure rates and degree of overlap between the techniques.

Participants and Methods: Participants were 281 general medical patients referred to a large urban medical center. Patients were administered the TOMM as part of a comprehensive neuropsychological evaluation, according to standardized instructions. Results from the TOMM were scored using the traditional cut-offs, and alternate scoring methods including the Albany Consistency Index (ACI), the Invalid Forgetting Frequency Index (IFFI), errors on the first 10 Items (e10), and recently reviewed stringent cutoff scores (i.e., $\leq 39$ correct on trial 1 and $<49$ correct on trial 2 or retention).

Results: The traditional scoring of the TOMM resulted in the lowest failure rate $(16 \%)$. Progressively greater failure rates were found in the trial 1 alternative scoring $(22 \%)$, IFFI (26\%), ACI (27\%), trial 2 and retention alternative scoring (31\%), and e10 (40\%). Substantial overlap was found between the various scoring methods.

Conclusions: In general medical patients, alternative scoring protocols and cutoffs produce a greater failure rate than the traditional scoring method for the TOMM. These methods are likely to produce greater sensitivity to invalid performance and enhance the utility of the TOMM as a performance validity measure.

Correspondence: Christopher Pierce, Ph.D., Psychiatry, Denver Health Medical Center, 667 Bannock St., MC 3450, Denver, CO 80204. E-mail: christopher.pierce@dhha.org.

\section{R. ZENISEK \& J.B. MILLER. Reliable Digit Span as a Measure of Effort in Dementia.}

Objective: The Reliable Digit Span (RDS) is a well-validated embedded indicator of performance validity. An RDS score of $\leq 7$ is commonly referenced as indicative of invalid performance; however, few studies have examined the classification accuracy of the RDS among individuals suspected for dementia. This study evaluated performance of the RDS in an outpatient memory disorders clinic when typical cutoffs were applied. Participants and Methods: Participants included 745 nonlitigating consecutive referrals suspected for dementia aged $19-93$ years (M $=70.5 ; \mathrm{SD}=10.4)$ with an average of 14.7 years of education $(\mathrm{SD}=$ 2.8 ; range $=3-22$ ). The sample was $52.1 \%$ male and predominantly Caucasian $(90.3 \%)$. The RDS was calculated for each participant in the context of a comprehensive neurpsychological assessment completed as part of routine clinical care. Score distributions were examined and one-way ANOVA was used to compare cognitive functioning of those falling below and above the RDS cutoff.

Results: RDS cutoff score of $\leq 7$ resulted in $27.8 \%$ of the sample falling below cutoff. One-way ANOVA revealed significantly lower MoCA total scores and poorer performance on all memory indices administered for those who fell below the RDS cutoff when a criterion of $\leq 7$ was applied.
Conclusions: These findings suggest that using the RDS cutoff score of $\leq 7$ results in an unacceptably high false-positive rate and increases risk of misinterpreting genuine cognitive impairments as invalid performance. Thus, the use of the RDS as a measure of performance validity in individuals suspected for dementia is ill-advised.

Correspondence: Justin B. Miller, Ph.D., Cleveland Clinic Foundation, 888 West Bonneville Ave, Las Vegas, NV 89106.E-mail: justin.b.miller@ gmail.com

L. LAU, M.R. BASSO, E. ESTEVIS, D. WHITESIDE, A.K. MILLER \& D.R. COMBS. Effects of Symptom- and Test-Coaching on the Detection of Feigned Neuropsychological Deficits.

Objective: Performance validity tests (PVTs) and symptom validity tests (SVTs) are often administered during neuropsychological evaluations. However, dishonest examinees impairment may be coached to simulate deficits and elude detection by PVTs and SVTs. Yet little research has evaluated the impact of such coaching. In this experiment, the effects of such coaching were evaluated in neurologically normal subjects. Participants endeavored to simulate mild head injury symptoms. Standalone and embedded PVT measures were administered in addition to SVTs.

Participants and Methods: Neurologically-normal college students participated. 20 received sophisticated information concerning head injury symptoms, 20 received superficial details, and 20 received detailed symptom information and strategies to evade detection by PVTs. All were asked to imagine they were seeking compensation for a mild head injury. 20 honestly-responding individuals participated, and were asked to give their best effort on all measures. All were administered the TOMM, WMT, CVLT-2, JLO, and MMPI-2-RF.

Results: The symptom-coached subjects were readily detected by the SVTs and PVTs. Nonetheless, a minority of those instructed in test-taking strategies elude detection by SVTs and PVTs.

Conclusions: Simulated symptoms of head injury are readily detected by the SVTs and PVTs. Embedded PVTs detected feigners, but their sensitivity was lower than the standalone measures. Most of those educated concerning test-taking strategies were detected. Yet, a minority successfully simulated mild head injury, and eluded detection by the SVTs and PVTs. This raises concerns about the validity of extant measures of response bias.

Correspondence: Lily Lau, PhD, Psychology, The University of Tulsa, 712 Raineer Ct., San Leandro, CA 94577. E-mail: lily-lau@utulsa.edu

M. BAR-HEN, G. DONIGER, M. GOLZAD \& A. SCHWEIGER. Validation in Patients of an Algorithm for Effort Assessment Embedded in a Neuropsychological Computerized Battery.

Objective: The validity of psychological assessments depends, among parameters, on the examinees exerting good effort on testing. Consequently, many tools were devised to assess effort on testing. However, many of these tools may be transparent, in the sense that they rely on simple memory tasks. In addition, validity may be compromised by prior guidance to examinees by attorneys in forensic contexts. The present study was designed to provide an algorithm for assessment of negative response bias (NRB) within the NeuroTrax, a standardized, computerized neuropsychological battery, thus making it very difficult to appreciate its purpose and thereby feign good effort.

Participants and Methods: Data were collected from the archived medical records of 75 outpatients with mild to moderate ?head injury, all being involved in litigation. Participants were classified into two groups: either low or high likelihood for NRB, ?based on their performance on a widely used effort test (Test Of Memory Malingering). Variables judged to be prone? to exaggeration and those showing large differences between the groups, were entered into a ?logistic regression analysis.

Results: The final, statistically significant, model classified correctly 94.7 percent of the patients and differentiated ?efficiently between the 
high and low NRB groups, with a high specificity $(98.0 \%)$ and sensitivity ?? (87.5\%).

Conclusions: It is concluded that the algorithm in the NeuroTrax battery may be a hard to detect tool for ? assessing effort of patients while being tested on a typical computerized battery designed to assess cognitive functioning.?

Correspondence: Moran Bar-Hen, MA, Psychology, Ben-Gurion University of the Negev, Israel, 1675 York Ave., 7D, New York 10128, USA Minor Outlying Islands. E-mail: moranbarhen@gmail.com

A. TEAGUE, J. GRETLER, Z. QUITTNER, A. ROSEN, L. SODOS \& R. MACHER. Debunking the "Amotivational Syndrome" in Chronic Cannabis Users With Objective Measures of Effort.

Objective: With the recent legalization of medical and recreational cannabis in several states and the rising use of marijuana, it is increasingly important to examine the effects of chronic cannabis use. A persistent stereotype of cannabis users is that they suffer from an "amotivational syndrome," which negatively affects their ability to complete tasks and positively contribute to society. Even recent publications reflect this popular perception of poor effort among cannabis users (U.S. Department of Health and Human Services, 2011). The present study utilized objective measures of effort to examine the accuracy of this stereotype. Participants and Methods: 62 chronic cannabis users and 48 non-users were administered a neuropsychological battery, which included both stand-alone and embedded measures of effort (Word Memory Test. Reliable Digit Span, and Trail Making Test B:A ratio). Published cutoff scores were used to identify participants who demonstrated suspect effort on each of the three measures. A Chi-square test for independence was used to compare rates of suspect effort between the user and nonuser groups.

Results: There were no significant differences in the rates of suspect effort between users and non-users. Consistent with previous studies, neuropsychological performance significantly correlated with an index of overall effort.

Conclusions: Results of this study suggest that users of cannabis put forth equivalent effort compared to non-users, as measured by objective symptom validity tests. These findings may help to refute the "amotivational syndrome" often associated with chronic cannabis use, which could have widespread implications for medical marijuana users and marijuana legislation. Further implications will be discussed.

Correspondence: Zoe Quittner, Palo Ato University, 1791 Arastradero Rd, Palo Alto, CA 94304.E-mail: zquittner@paloaltou.edu

M. IAMPIETRO, K. WASHINGTON, M.N. SCOTT \& S. HUNTER. Word Memory Test Findings in a Pediatric Mixed Clinical Sample. Objective: Green's Word Memory Test (WMT), designed to assess effort, has been reported to be highly sensitive in children with developmental disabilities, producing very few false positive results for poor effort. Few studies have investigated this claim in a large clinical sample of children with various developmental disorder diagnoses.

Participants and Methods: Participant diagnoses and WMT data were obtained during a clinical neuropsychological evaluation within the Pediatric Neuropsychology Clinic at The University of Chicago Medicine. Data from 100 children (Age range 7-23 years, Mage $=12.78, \mathrm{SD}=3.34$, $43 \%$ female) chosen at random were analyzed in a retrospective study. Results: Twenty-one percent of the total study sample failed at least one of three diagnostic subtests on the WMT, as did $26 \%$ of children with learning disorder diagnoses $(n=47)$. In the total study sample, lower full scale IQ scores (WISC-IV; MFSIQ=92.57, SD=19.37) were significantly associated with poorer performance on WMT Delayed Recall, $r(73)=$ $.32, \mathrm{p}<.01$ and WMT Consistency, $\mathrm{r}(71)=.35, \mathrm{p}<.01$.

Conclusions: Approximately 1 in 4 children in a mixed clinical population failed at least one diagnostic subtest on the WMT, and results indicate that performance is influenced by IQ. The WMT may be less sensitive in assessing effort in a clinical population of children with developmental disorders, and clinicians should be cautious of false positive results in this population.

Correspondence: Mary Iampietro, Psychology, Temple University, 1701 N. 13th St., Weiss Hall, Floor 6, Philadelphia, PA 19122. E-mail: mary. iampietro@temple.edu

M. VASSERMAN, G. WITKIN, B. KAHN, M. MAIMAN \& W.S. MACALLISTER. Utility of CVLT-C Recognition Discriminability as a Measure of Effort in Clinically Referred Children with Developmental Disorders.

Objective: Assessment of effort is essential in pediatric populations. While multiple standalone measures have been validated in various pediatric groups, fewer embedded measures have been explored. The California Verbal Learning Test-Children's Edition, Recognition Discriminability (CVLT-C(RD)) was found to be moderately sensitive in detecting poor effort in a pediatric mTBI sample. The current study aims to evaluate whether this measure is effective in identifying poor effort in clinically-referred children with and without ADHD.

Participants and Methods: The present study utilized data from 103 clinically-referred patients (ages 6-15; $\mathrm{M}=10.72 ; \mathrm{SD}=2.76 ; 63 \%$ males; ADHD n=77). The CVLT-C(RD), TOMM and Reliable Digit Span (RDS) were administered as part of a clinical battery. Established cutoffs were utilized for RDS and TOMM. A z-score of $\leq-1$ (Baker, 2014) was used for CVLT-C(RD). Failure of both RDS and TOMM was considered as poor effort.

Results: One participant failed both RDS and TOMM, but passed CVLT-C(RD). Fifteen failed RDS but passed the TOMM. While no group difference were found between ADHD and non-ADHD groups on RDS or TOMM scores, the groups differed significantly on CVLT-C(RD) $(\mathrm{t}=-2.43 ; \mathrm{p}=.017)$. Overall, $19 \%$ of the sample scored $\leq-1.0$ on RD. Of those who failed CVLT-C(RD), 81\% were diagnosed with ADHD and 81\% were male. CVLT-C(RD) scores correlated significantly with IQ, $\mathrm{r}(101)=.261, \mathrm{p}<.01$. Lastly, children who failed the CVLT-C(RD) or RDS tended to have lower IQ (RDS $\mathrm{t}(96)=2.28, \mathrm{p}<.025$; CVLT $-\mathrm{C}$ (RD) $\mathrm{t}(99)=2.34 ; \mathrm{p}<.021)$.

Conclusions: The CVLT-C(RD) cutoff score of $\mathrm{z}$-score $\leq-1.0$ produced an unacceptably high false positive rate in a sample of clinically referred children who did not demonstrate other indicators of poor effort. CVLT-C(RD) is strongly correlated with IQ and appears to be sensitive to deficits common in children with developmental disorders. RDS also resulted in a high number of false positives which is consistent with previous literature.

Correspondence: Marsha Vasserman, Psy.D., Child and Adolescent Psychiatry, NYU Langone Medical Center, 245 East 25th, 6E, New York, NY 10010.E-mail:marsha.vasserman@nyumc.org

D.E. TRAHAN. Specificity of the CVMT Symptom Validity Scale in Normal Adults.

Objective: The Continuous Visual Memory Test (CVMT) (Trahan \& Larrabee,1988) measures visual memory using complex designs and a recognition format. Larrabee (2008) developed a Symptom Validity Scale (SVS) for the CVMT to assist in detecting malingering or suboptimal performance in TBI litigants. The recommended cutoff was an SVS score below 14. This study provides additional support for use of the SVS by providing specificity data for neurologically normal adults. Participants and Methods: Participants were 203 normal adults (83 male, 120 female) ranging in age from 18-91. They were divided into four age groups: 18-29, 30-49, 50-69, and 70+. All were administered the CVMT as part of a larger standardization battery.

Results: Analysis of data revealed SVS specificity rates of $0.98,0.99$, 0.94 , and 1.00 for the four age groups and 0.98 for the combined sample. Analysis of variance for SVS scores revealed no significant differences among age groups, $\mathrm{F}(3,199)=1.85, \mathrm{p}=.14$. The mean numbers of SVS items correct for the groups were 17.45, 17.84, 17.25, and 17.45 , respectively. Correlations between SVS and CVMT Total Scores were not significant for any of the age groups. 
Conclusions: Results revealed that specificity rates for the CVMT SVS scale were very high for neurologically normal adults. Using the recommended cutoff score, the false positive rate for the entire sample was only 0.02 , substantially lower than the failure rate reported in litigating samples (Larrabee, 2008). These data provide additional support for use of the SVS scale in distinguishing between normal patterns of performance and possible malingering or suboptimal effort.

Correspondence: Donald E. Trahan, Center for Behavioral Studies, 3560 Delaware, Suite 105, Beaumont, TX 77706.E-mail:dtrahan@ swbell.net

D.T. PUlSIPHER, E. MONTAGUE, W. KEENER, E. LONG \& L.D. STANFORD. Effort Test Failure, Intervention, and Degree of Neuropsychological Impairment in Children/Adolescents with Concussion or Epilepsy.

Objective: Effort test failure in adults with concussion is associated with greater, but non-credible, neuropsychological impairment. Routine effort assessment with children is less common, and findings regarding the value of effort assessment in this population are less clear. The goal of this study was to determine if effort test failure is related to lower cognitive scores and if intervention following effort test failure improves subsequent performance.

Participants and Methods: 146 consecutive referrals ages 8-18 years with epilepsy or concussion were administered one effort test prior to any neuropsychological measures. Those who failed one effort measure spoke with a psychologist and were then administered a second effort measure. A single Global Deficit Score (GDS) was calculated for each individual based on 22 scores obtained from the same neuropsychological measures administered to all patients. The GDS of those that passed all effort tests was compared to those who failed the first effort test but passed the second.

Results: Twenty-four (16\%) individuals failed the first effort test. After discussion, all 24 passed a second effort test. There was no significant difference on GDS $(\mathrm{d}=0.02)$ between those who passed all effort tests and those who failed the first effort test.

Conclusions: Those who failed a standardized measure of effort but subsequently spoke to a neuropsychologist did not show evidence of greater impairment across a large battery of tests. These findings suggest that failure of a single effort measure does not necessarily indicate that low scores will occur on neuropsychological tests, and speaking frankly to children regarding their effort may improve subsequent performance. Correspondence: Dalin T. Pulsipher, Ph.D., NeuroDevelopmental Science Center, Akron Children's Hospital, 215 W. Bowery St., Suite 4500, Akron, OH 44308.E-mail: dalin.pulsipher@my.rfums.org.

\section{S. AMEDORO \& G. LOCASCIO. Exploratory Analysis of Mood/ Anxiety and Effort Following Pediatric Concussion.}

Objective: Use of the Test of Memory Malingering (TOMM), a visual recognition measure of effort, has revealed suboptimal effort in some pediatric patients following concussion (e.g., Kirkwood \&. Kirk, 2010). The current study examines the relationship between performance on the TOMM and presence of mood/anxiety symptoms before and after concussion.

Participants and Methods: Retrospective chart reviews were completed for 53 pediatric outpatients in a concussion clinic (aged 14.7 $\pm 2.17,64 \%$ female) who were administered the TOMM due to suspected symptom exaggeration or suboptimal effort on neuropsychological testing. Data were gathered from clinic notes and developmental questionnaires regarding presence of (yes/no) pre-injury, initial, and discharge mood/ anxiety symptoms as well as discharge disposition.

Results: Fourteen patients (aged 13.7 $\pm 3.0,71 \%$ female) failed the TOMM (Trial $2<45$ ) and 39 patients (aged 15.0ะ1.7, 62\% female) passed. Between these groups, gender was not significantly different $(\chi 21=0.438, p=0.51)$; however, age approached significance $[\mathrm{t}(51)=2.0$, $\mathrm{p}=0.052]$. While no significant differences were noted for presence of mood/anxiety symptoms at any time point, a significant difference was revealed regarding discharge disposition. Specifically, $87 \%$ of patients who passed the TOMM returned for scheduled follow-up visits and were ultimately discharged from clinic; however, only $43 \%$ of those who failed returned $(\chi 21=10.932, \mathrm{p}<0.001)$.

Conclusions: Patients who failed an effort measure did not have documentation of more mood/anxiety symptoms, but were significantly less likely to return to clinic. Small sample size was likely a factor. These findings have important clinical implications for pediatric concussion management, as these patients are likely in need of additional education and intervention.

Correspondence: Sarah Amedoro, Graduate Psychology, Immaculata University, 1611 Greenspring Dr, Lutherville, MD 21093. E-mail: sarah. amedoro@gmail.com

L. WARDIN, D. WHITESIDE, L. RICE \& L. NICHOLS. Classification Accuracy of the Wisconsin Card Sorting Test (WCST) in Detecting Noncredible Cognitive Performance in Neuropsychological Outpatients.

Objective: The current study examined the classification accuracy of the Wisconsin Card Sorting Test (WCST) as an embedded performance symptom validity test (PVT) among three different samples.

Participants and Methods: The sample of interest included 110 participants with mild traumatic brain injury (mTBI). For comparison, the study included 69 participants with moderate to severe traumatic brain injury (TBI) (STBI) and 155 non-neurological patients with mixed depression/anxiety psychiatric diagnoses (PSYCH). Furthermore, a logistically derived combination of Number Correct, Perseverative Responses, and Trials to First Category was created as an additional predictor variable named WCSTCOMB.

Results: Results indicated significant group differences between the credible performance (PASS) and non-credible performance (FAIL) groups for only the PSYCH and STBI samples. Receiver operating characteristic (ROC) analysis found that Perseverative Responses and WCSTCOMB had acceptable classification accuracy (Area under the Curve $>$.70) in the PSYCH group. In the STBI group, ROC analysis found that the following WCST variables had at least acceptable classification accuracy of at least .70: Number of Trials, Total Errors, Perseverative Errors, Perseverative Responses, Number of Categories Completed, and WCSTCOMB. Sensitivity, specificity, positive predictive power, negative predictive power, and recommended raw cutoff scores were calculated for WCST variables with acceptable classification accuracy. Acceptable classification accuracy was not found among any WCST variables in the mTBI sample.

Conclusions: Results do not provide support for the WCST as an embedded PVT with mTBI. However, results did provide support for the WCST as embedded PVTs with populations with moderate to severe TBI and psychiatric issues.

Correspondence: Lydia Wardin, Adler School of Professional Psychology, 411 E Hillside Ave, Barrington, IL 60010.E-mail: lwardin@my.adler. edu

L. WARDIN, D. WHITESIDE \& A. HAHN-KETTER. Trail Making Test (TMT) as a Performance Validity Test (PVT) in Neuropsychological Outpatients.

Objective: Embedded performance validity tests (PVTs) are often considered less vulnerable to coaching and are conveniently integrated into assessment batteries. This study examined the classification accuracy of potential embedded PVTs, the Trail-Making Test Part A (TMT-A) and Part B (TMT-B) as embedded PVT measures.

Participants and Methods: Participants were consecutive mixed neuropsychological outpatients. Participants were assigned to the noncredible performance (NP, N=59) group based upon failure on any TOMM trial (Tombaugh, 1996), which consisted of $59.3 \%$ males, $40.7 \%$ females, with an average age of $46.24(\mathrm{SD}=13.67)$ and 12.42 years of education $(\mathrm{SD}=2.71)$. Those participants who passed the TOMM were assigned to the credible performance $(\mathrm{CP}, \mathrm{N}=806)$ group, which 
consisted of $44.8 \%$ males, $55 \%$ females, with an average age of 44.38 $(\mathrm{SD}=17.73)$ and 13.3 years of education $(\mathrm{SD}=2.42)$.

Results: The groups significantly differed in gender and education. T-tests indicated both Trail-Making Test trials were significantly slower in the $\mathrm{NP}$ group compared to the $\mathrm{CP}$ group. $\mathrm{ROC}$ analysis indicated that only Trails B had acceptable classification accuracy ( $\mathrm{AUC}=.72$ ), while TMT-A did not achieve acceptable classification accuracy. Sensitivity, specificity, and positive and negative predictive power were calculated for Trails B. When specificity was set at $90 \%$, sensitivity was .24 . Conclusions: Results provide support for TMT-B as an embedded PVT when used with other free-standing and embedded PVTs, but lacks the sensitivity to be utilized alone.

Correspondence: Lydia Wardin, Adler School of Professional Psychology, 411 E Hillside Ave, Barrington, IL 60010.E-mail: lwardin@my.adler. edu

D. PLOETZ, H. DAYA, C. LALIBERTE', T. LOW \& B. BROOKS. Using the Automatized Sequences Task as a performance validity test in youth with neurological diagnoses.

Objective: Performance validity tests (PVTs) are typically developed in adult populations first and then data are extrapolated for use in pediatric populations. Recently, Kirkwood and colleagues (2013) developed a PVT for youth that uses an automatized sequences task (AST; e.g., counting) to help discern performance validity. The purpose of this study is to examine the AST in children with neurological diagnoses.

Participants and Methods: Youth between 5-17 years old ( $\mathrm{N}=162$; mean age $=13.0, \mathrm{SD}=3.9$; mean $\mathrm{FSIQ}=87.8, \mathrm{SD}=17.4)$ completed neuropsychological evaluations at a tertiary hospital including the AST and the Test of Memory Malingering (TOMM).

Results: Using the cut-off scores for the AST described by Kirkwood et al. (2013), 30\% of our sample was flagged as 'invalid' using the total score (e.g., Total Time $\geq 27$ seconds; ranging from $22-42 \%$ flagged on 1 of the 4 items). When using TOMM performance as the validity criterion for our sample, sensitivity was $43 \%$ and specificity was $70 \%$ based on total time $\geq 27$ seconds. However, a cut-off score of total time $\geq 40$ seconds obtained a more optimal $90 \%$ specificity with only $29 \%$ sensitivity. Conclusions: A cut-off score allowing more time to be taken may be warranted in a sample of children referred for a variety of neurological conditions, although sensitivity is low.

Correspondence: Danielle Ploetz, Ph.D., Neurosciences, Alberta Children's Hospital, 2113 Edenwold heights NW, Calgary, AB t3a3y2, Canada.E-mail: danielle.ploetz@gmail.com

D. PROTO, N.J. PASTOREK, B. MILLER, S.M. LIPPA, J. ROMESSER, A. SIM \& J. LINCK. Effect Sizes of Neuropsychological Performance Decrements as a Function of Sample-Derived PVT Hit Rate.

Objective: Previous research indicates that accuracy and hit rates of performance validity tests (PVTs) can vary significantly by measure and cut score, and that results from PVTs explain a large portion neuropsychological variance. Building on these concepts, the current study examined the neuropsychological effect sizes associated with failing PVTs with varying hit rates. The authors hypothesized an inverse relation between sample-derived hit rate and effect size (i.e., lower hit rates associated with larger effects).

Participants and Methods: $213 \mathrm{OEF} / \mathrm{OIF} / \mathrm{OND}$ veterans with history of $m$ TBI were seen for neuropsychological evaluation. The sample was $94 \%$ male with mean age of 32.5 (7.54) years and mean education of 13.2 (1.69) years. Evaluated cognitive domains were processing speed, lexical fluency, cognitive flexibility, sustained attention, and memory. PVTs included the Word Memory Test (WMT), TOMM, Reliable Digit Span, CVLT-II Forced Choice (FC), Wisconsin Card Sorting Test Failure to Maintain Set, and Rey-15 Test with recognition.

Results: Neuropsychological testing performances of those passing and failing the PVTs were significantly different in most instances, with effect sizes ranging from -.16 to 1.92. Steadily-increasing effect sizes were associated with smaller PVT hit rates across the WMT, TOMM, and FC on measures of processing speed and executive functioning. Memory findings were split by modality, with larger effects associated with memory-based PVTs.

Conclusions: The current study showed limited evidence of increasing effect sizes as PVT hit rate decreased and a PVT modality effect in memory, suggesting that failing PVTs with low hit rates or of a similar cognitive modality may be associated with greater neuropsychological "cost."

Correspondence: Daniel Proto, Ph.D., Michael E. DeBakey VA Medical Center, 790 Veterans Way, Pensacola, FL 32507.E-mail: dproto1@ alumni.lsu.edu

B.E. GAVETT, A. GURNANI, K. GRAJZEL, S.E. JOHN, N. JIVAN \& M. BERLIN. Do "Effort" Tests Measure Effort? A Call to Abandon this Misleading Term.

Objective: Performance validity tests (PVT) are commonly used to interpret cognitive test results. Failure of PVTs is often judged to reflect suboptimal effort exerted by the examinee and are described as providing a measurement of latent "effort." For instance, in a commonly referenced study, Green et al. (2001) claim, "effort has a greater effect on test scores than severe brain injury." In contrast, some authors have questioned the interpretation of PVTs as measures of "effort." We hypothesized that, if PVTs provide a valid measure of effort, their results should correlate with participants' subjective sense of effort.

Participants and Methods: Participants were 30 cognitively healthy undergraduates administered one PVT, the Word Memory Test (WMT), as part of a larger battery of tests. Participants were also administered a modified version of the Paced Auditory Serial Addition Test (PASAT), which required participants to continue the test as long as possible while giving their "best effort," regardless of performance accuracy. Pearson's correlation coefficient was used to estimate the magnitude of the relationship between WMT scores and participants' subjective effort. Results: All participants passed the WMT. Subjective effort on the PASAT correlated $\mathrm{r}=0.18$ with WMT Immediate Recognition and $\mathrm{r}=$ 0.29 with WMT Delayed Recognition and Consistency scores. At most, participants' subjective effort accounted for $8.6 \%$ of the variance in PVT scores.

Conclusions: The current results caution against the interpretation of PVT results as measures of "effort" because they are not strongly related to respondents' own sense of their effort level.

Correspondence: Brandon E. Gavett, Ph.D., Psychology, University of Colorado Colorado Springs, 1420 Austin Bluffs Parkway, Department of Psychology, Colorado Springs, CO 80918.E-mail:bgavett@uccs.edu

B.E. GAVETT, J.W. ADAMS, G. SILK-EGLIT, J. LYNCH \& R. MCCAFFREY. Linking Standalone Performance Validity Test Scores.

Objective: Performance validity tests (PVTs) are commonly used in neuropsychology as a method to aid interpretation of cognitive test scores by providing an indication of the validity of the examinee's responses. Differences in the design of PVTs has not allowed for a direct comparison of test scores. Although it has been reported that some PVTs are more sensitive and specific than others, these differences could reflect the fact that the cutoff scores used to identify invalid performance are not equivalent across tests. The goal of the current study was to use equipercentile linking methods to place the scores from three standalone PVTs on the same scale.

Participants and Methods: Archival data from 256 participants, including 153 cognitively healthy undergraduate volunteers and 103 litigants, were analyzed. Participants were administered the Test of Memory Malingering (TOMM), Victoria Symptom Validity Test (VSVT), and the Word Memory Test (WMT) as part of a larger battery of tests. The sample distributions from each test variable were used as the basis for linking test scores based on cumulative percentages.

Results: The rate of failure of at least one PVT was $23.9 \%$. The precision of linkings was generally good, with standard errors ranging from 
0.84 to 4.48; however, precision decreased with lower test scores and when Delayed Recognition trials (WMT and TOMM) were used. When all PVTs were scaled on the same metric as the TOMM, cutoff scores for Immediate Recognition trials (WMT, VSVT, and TOMM), Delayed Recognition trials (WMT and TOMM), and consistency scores (WMT and TOMM) differed considerably.

Conclusions: Linking the three PVTs supported the hypothesis that the manual cutoff scores are not equivalent, which may account for differences between the three tests that has been reported in the literature. For some examinees, what appears to be fluctuating effort may in fact represent psychometric differences between tests. Future research should extend these findings to include additional PVTs, both standalone and embedded.

Correspondence: Brandon E. Gavett, Ph.D., Psychology, University of Colorado Colorado Springs, 1420 Austin Bluffs Parkway, Department of Psychology, Colorado Springs, CO 80918.E-mail:bgavett@uccs.edu

W.S. MACALLISTER, V. DELBENE, M. MAIMAN, K. COULEHAN \&. M. VASSERMAN. Adventures in Pediatric and Adolescent Performance Validity Testing: A Case Series.

Objective: Performance validity tests (PVTs) are a critical component of neuropsychological evaluation in children and adolescents. Despite this, some clinicians remain skeptical of their utility in this population. By way of selected case examples, we demonstrate the utility of PVTs to identify symptom exaggeration in cases of implausible symptom presentations and contrast this with examples of youngsters with bonefide severe cognitive dysfunction who "pass' these measures.

Participants and Methods: Four clinically referred cases are presented; all were administered PVTs as part of neuropsychological workup. Case 1 is a 10 -year-old girl presenting with psychiatric symptoms and claims of sudden-onset-dyslexia status-post flu vaccination. Case 2 is a 14-year-old female with pseudoseizures. Case 3 is a 19-year old female status-post severe encephalitis that resulted in major impairment (FSIQ $=55$ ). Case 4, an 18-year-old male with epilepsy, was status-post right temporal lobectomy. Later imaging identified a hypothalamic hamartoma. Laser ablation surgery damaged the mamillary bodies, resulting in a profound amnestic syndrome.

Results: Cases 1 and 2 presented with implausible neuropsychological test results and failed multiple PVTs despite only mild cognitive difficulties in school. However, cases 3 and 4 , who present with severe cognitive deficits, passed the TOMM.

Conclusions: This case series demonstrates that PVTs are able to detect non-credible performances in youngsters feigning or exaggerating cognitive deficits whereas even youngsters with severe cognitive deficits are able to pass these measures, given adequate test engagement.

Correspondence: William S. MacAllister, PhD, Neurology, NYU, 223 East 34th St, NYU Epilepsy, New York, NY 10016. E-mail: william. macallister@nyumc.org

S.A. PARIKH, D. WHITESIDE, H. LUU, R. COSTELLO, L. RICE, B. ROPER \& M.R. BASSO. Using the Personality Assessment Inventory to Predict Non-Credible Cognitive Performance in Patients with Mild Traumatic Brain Injury.

Objective: This study evaluated whether the Personality Assessment Inventory (PAI) validity scales had adequate classification accuracy for non-credible cognitive performance in mild traumatic brain injury (mTBI) patients. The hypothesis was that PAI validity scales would predict non-credible performance.

Participants and Methods: Participants were placed into credible performance ( $n=143$ ) or non-credible performance $(n=24)$ groups based on PVT performance. Non-credible performance was defined as failing at least two free standing and/or embedded performance validity tests (PVTs), while credible performance was defined as passing all PVTs. The average age of participants was 43 with a standard deviation (SD) of 14.1. Participants had 12.7 years of education with a SD of $2.5 .84 \%$ of participants were Caucasian while $3.9 \%$ were Hispanic. $55 \%$ of the sample was female and $38 \%$ were male. Patients were administered a series of neuropsychological tests specific to mTBI.

Results: A logistic regression was used to calculate the combined variable consisting of the PAI validity scales (ICN, INF, PIM and NIM). Independent $t$-tests indicated that the combined variable and INF scales were significantly different between the credible and non-credible groups ( $p$ $<.05)$. Receiver operating characteristic (ROC) analysis indicated the combined PAI variable had acceptable classification accuracy, with Area Under the Curve $(\mathrm{AUC})=$.7.The PAI validity scales did not reach this threshold. Using an optimal cutoff score of 1.134 , sensitivity was 25 when specificity was set at $90 \%$.

Conclusions: The results suggested that the combined PAI validity scale variable might have some limited utility as an embedded PVT due to the specificity level.

Correspondence: Shravan A. Parikh, Clinical Neuropsychology, Adler School of Professional Psychology, 215 W Washington St, Apt 1501, Chicago, IL 60606.E-mail: sparikh@my.adler.edu

\section{J. ROBInson, R. COLlins, B. Miller \& V. PACHECO. Examination of the Severe Impairment Profile in Dementia Evaluations.}

Objective: Within the past decade, several studies have examined the PVT performance in older individuals with presumed mild cognitive impairment (MCI) and dementia. Certain PVT's such as the Test of Memory Malingering have shown failure rates of up to 27-76\% (Tombaugh, 1996; Teichner \& Wagner, 2004). In the current study, we sought to replicate and extend the findings of Green and colleagues (2011) to examine the genuine memory impairment profile of the Word Memory Test (WMT).

Participants and Methods: The study was a retrospective review of patients referred for an outpatient neuropsychological evaluation for memory loss. The final sample is comprised of 125 patients who underwent a full neuropsychological evaluation that included objective memory testing as well as the WMT.

Results: Following the evaluation, a comprehensive chart review was done to determine whether or not the patient had genuine memory impairment. This chart review included the evaluation in question, neuroimaging, and chart notes following the evaluation, up to two years post. Using this classification standard, the WMT showed $97.43 \%$ specificity and $8.33 \%$ sensitivity in the detection of poor effort as indicated by thorough chart review.

Conclusions: These results suggest that the context of the evaluation is important (e.g., secondary gain contexts) when evaluating the efficacy of PVT's. Additionally, it is important to consider data from multiple sources and time points to make this determination, although this level of information may not be available in all situations.

Correspondence: Jordan Robinson, Michael E. DeBakey VAMC, 3023 BroadmeadDr, Houston, TX 77025.E-mail:Jayking:robinson@gmail. com

R. COLLINS, B. MILLER, J. ROBINSON \& V. PACHECO. The Relation between the WMT and CVLT-2 in a Sample of Patients Evaluated for Dementia.

Objective: The Word Memory Test (WMT) is a widely utilized measure of performance validity (PVT). Across neurological populations (e.g., TBI \& epilepsy), PVTs account for a significant portion of variance in associated neuropsychological test scores (Green, 2007). Less is known, however, about the relation between patient PVT and cognitive test performance in patients being evaluated for dementia. The current study was conducted to assess how different levels of effort on the WMT are related to performance on a measure of list learning (CVLT-2 sub scores), mood, and caregiver report.

Participants and Methods: The study was a retrospective review of patients referred for an outpatient neuropsychological evaluation for memory loss. The final sample was comprised of 110 patients who underwent a full neuropsychological evaluation that included objective 
memory testing as well as the WMT. Patients were divided into 4 groups based on WMT easy subtest scores: high pass $(n=39$; all scores in the pass range), pass ( $n=13$, no scores in the fail range, at least one caution), fail ( $n=8$, no more than one score in the fail range), bad fail $(n=50$, at least 2 scores in the fail range). Given the non-normality of the data and unequal group sizes, Kruskal-Wallis (K-S) was used to determine between group differences.

Results: The overall K-S model was significant for 11/13 CVLT-2 sub scores $(p<.01)$, for both raw and standardized values, with most effect sizes ranging between 0.20 and 0.34 . Post-hoc analyses suggest the fail groups performed consistently worse than the pass groups, which were not significantly different from one another. The WMT was not significantly related to self-reported or caregiver ratings.

Conclusions: While poor WMT performance appears to be related to list learning, this relation does not appear to be as robust for patients passing the WMT. The relation between PVT and cognition in younger neurologic populations has been well established, however less is known about this in older patients with dementia.

Correspondence: Robert Collins, PhD, Neurology, MEDVAMC, 2002

Holcombe, Houston, TX 77030.E-mail: robert.collins3@va.gov

K. WYMAN-CHICK, S. CAZZANTI \& R.W. SCHROEDER. Performance Invalidity in Non-Clinical Undergraduate Research Participants: A Follow-Up Study.

Objective: Recent studies have found differing rates of performance invalidity among undergraduate volunteers recruited for neuropsychological research. One study found between $30.8-56.6 \%$ of participants failed at least one free-standing performance validity measure. Another study utilized free-standing and embedded performance validity measures among undergraduate volunteers and reported $2.26 \%$ of the sample failed one or more measures. The purpose of the current study was to examine rates of performance invalidity in a separate group of college students, given the wide discrepancy in the previously reported statistics. Participants and Methods: Archival data from 50 undergraduate students were included in this analysis. The students volunteered for a study on learning preferences, and each participant was compensated with a $\$ 5$ gift card for their time, regardless of their cognitive performances on testing. Each student completed an approximately 1.5 hour neuropsychological evaluation that included completing a learning preference questionnaire, intellectual testing, and memory testing. Four embedded memory-based validity measures were administered during the evaluations: California Verbal Learning Test - 2nd Edition Forced Choice, Logical Memory II Forced Choice, Rey Complex Figure with Recognition True Positive Recognition, and Brief Visuospatial Memory Test - Revised Recognition Hits.

Results: The results indicated that all participants passed all embedded memory-based validity measures. Moreover, the embedded measures did not indicate that any of the 50 students were providing an invalid performance during the study.

Conclusions: These findings are consistent with those reported in one of two previous studies indicating that the base rate of performance invalidity among undergraduate volunteers is low. Further details and implications are discussed.

Correspondence: Kathryn Wyman-Chick, M.A., Univeristy of Kansas School of Medicine, 7829 Rockhill, Ste 105-106, Wichita, KS 672063919. E-mail: kwyman@pacificu.edu

\section{J. LICHTENSTEIN, L. ERDODI \& M. HOLCOMB. Introducing a Forced Choice Recognition Task to the CVLT-C (FCR-Child): Preliminary Findings.}

Objective: Applying instruments developed for adults to assess performance validity in children has produced a growing body of evidence that PVTs can be extended to lower age ranges. We developed a measure for the CVLT-C modeled after the FCR task of the adult version.

Participants and Methods: Data from 43 children (28 males; M Age $=11.0$ years, $\mathrm{SD}$ Age $=2.6$; range $6-15)$ were used to examine the potential of this novel instrument to detect invalid response patterns in pediatric patients clinically referred for neuropsychological testing. Results: Using the cutoff of $\leq 14 / 15$, the FCR-Child had a low base rate of failure (5/43). Only $3 / 43$ scored $\leq 13 / 15$. Cross-validation analyses used a composite PVT (EI-3) as a reference, based on the RDS, DS-ACSS and CVLT-C d' z-score. At < 14, the FCR-Child achieved acceptable specificity (.90), but low sensitivity (.17) against the EI-3, with comparable performance against individual PVTs at published cutoffs (sensitivity .11-.20, specificity .88-.91).

Conclusions: This signal detection profile is largely consistent with the literature on the adult FCR. Due to the relatively small sample size and low failure rate on the FCR-Child, these parameter estimates might be unstable and need replication before they can be used clinically. However, given the ease of administration, the paucity of pediatric PVTs, the lengthy cost of administering free-standing PVTs in this population, and the good performance of the FCR-Child against established instruments, it has the potential to become a useful embedded indicator of cognitive performance validity.

Correspondence: Jonathan Lichtenstein, Psy.D., Psychiatry, Geisel School of Medicine at Dartmouth, HB 7550, Department of Psychiatry, DHMC, One Medical Center Drive, Lebanon, NH 03756. E-mail: jonathan.d.lichtenstein@dartmouth.edu

C. COPELAND, J.J. MAHONEY, C.K. BLOCK, N.J. PASTOREK, B. MILLER, J. ROMESSER, A. SIM \& J. LINCK. Relative Utility of Performance and Symptom Validity Tests for Assessing Cognitive Performance and Symptom Report.

Objective: The relevance of performance (PVT) and symptom (SVT) validity tests for cognitive performance and symptom report was examined. It was hypothesized that individuals who passed a PVT (PVTPass) would demonstrate better cognitive performance and decreased symptom report relative to individuals who failed a PVT (PVT-Fail), and that individuals who passed an SVT (SVT-Pass) would demonstrate decreased symptom report and no difference in cognitive performance relative to individuals who failed an SVT (SVT-Fail).

Participants and Methods: 181 military veterans with mTBI were administered the following respective PVT, SVT, cognitive performance, and symptom report measures: the Test of Memory Malingering, the Response Bias Scale of the Minnesota Multiphasic Personality Inventory, the California Verbal Learning Test, and the Neurobehavioral Symptom Inventory.

Results: Post-hoc analyses revealed that PVT-Pass, relative to the PVTFail, demonstrated significantly better cognitive performance, $F(1,178)$ $=26.40, p<.001, \eta^{2}=.13$ and decreased symptom report, $F(1,178)$ $=29.20, p<.001, \eta^{2}=.14$. Likewise, SVT-Pass, relative to the SVTFail, demonstrated significantly better cognitive performance, $F(1,179)$ $=7.56, p=.007, \eta^{2}=.04$ and decreased symptom report, $F(1,179)=$ $40.71, p<.001, \eta^{2}=.19$.

Conclusions: Despite similar patterns of statistical significance for the PVT and SVT, greater variance in cognitive performance was explained by the PVT (13\%) relative to the SVT $(4 \%)$, and greater variance in symptom report was explained by the SVT $(19 \%)$ relative to the PVT (14\%). Results tentatively support previous suggestions of differential utility for PVTs and SVTs (Van Dyke et al., 2013).

Correspondence: Christopher Copeland, Ph.D. Candidate, Psychiatry and Behavioral Sciences, Oklahoma University Health Sciences Center, 617 NW 5th St., Oklahoma City, OK 73102. E-mail: christophercopeland@ouhsc.edu

J. STENCLIK, G. SILK-EGLIT, J. LYNCH \& R. MCCAFFREY. Increasing Classification Accuracy of the TOMM: Comparison of the Albany Consistency Index and the Invalid Forgetting Frequency Index.

Objective: Prior research has examined the utility of consistency indices created from the Test of Memory Malingering (TOMM) in attempt to more accurately classify performance invalidity. Recently, the Albany 
Consistency Index was modified as to not penalize examinees for a "valid learning pattern", and thus, the Invalid Forgetting Frequency Index (IFFI) was created. This study sought to compare the diagnostic accuracy of the standard TOMM criteria, ACI, and IFFI.

Participants and Methods: An archival analysis was conducted on 48 medico-legal examinees with a diagnosis of a mild traumatic brain injury. Twenty-one were males and 45 were right-handed. Average age was 45.94 vears $(S D=10.55)$. education was 14.23 years $(S D=2.90)$ and Full-Scale IQ was 94.08 (SD=11.08). The criterion for performance invalidity was defined as failing two or more performance validity tests (PVTs). PVTs included the Word Memory Test, Victoria Symptom Validity Test, Rey-15 Item Test, and Reliable Digit Span.

Results: The standard TOMM criteria significantly predicted performance invalidity; however, they had the poorest overall classification accuracy and sensitivity (SN; $\mathrm{SN}=42 \%)$. The ACI (SN=71\%) and IFFI ( $\mathrm{SN}=52 \%$;) were also both significant predictors of performance invalidity, however, the ACI showed better SN and accounted for a larger amount of variance, while maintaining specificity (SP) above $90 \%$. Conclusions: Results indicated that the standard TOMM criteria were the least accurate predictors of performance invalidity. Although the IFFI did improve diagnostic accuracy of the TOMM and increased SN by $10 \%$, the ACI increased SN by almost $30 \%$ and was the most accurate measure of performance invalidity.

Correspondence: Jessica Stenclik, Ph.D., Neurology, University of Virginia, 4000 City Walk Wak, Apt 437, Charlottesville, VA 22902. E-mail: jhstenclik@gmail.com

T.A. BRICKELL, R. LANGE, D. TULSKY \& L.M. FRENCH. Influence of Symptom Validity Test Performance on the Traumatic Brain Injury Quality of Life (TBI-QOL) Scale in U.S. Military Service Members.

Objective: The purpose of this study was to examine the influence of Symptom Validity Test (SVT) performance on a new measure of Health Related Quality of Life in persons following traumatic brain injury (TBI); the Traumatic Brain Injury Quality of Life (TBI-QOL) scale. Participants and Methods: Participants were 80 U.S. military service members (Age: $\mathrm{M}=30.4, \mathrm{SD}=8.6 ; 92.5 \%$ male) who sustained a mild-severe TBI; prospectively enrolled from Walter Reed National Military Medical Center (71.3\% mild TBI). Participants were divided into two groups based on the validity scales of the Minnesota Multiphasic Personality Inventory-2-RF: (a) SVT-Fail $(n=27)$ and (b) SVT-Pass $(n=53)$. Participants were evaluated on average 19.3 months post-injury $(\mathrm{SD}=27.6)$.

Results: Participants in the SVT-Fail group had worse scores on all 14 TBI-QOL scales compared to the SVT-Pass group ( $p<.05 ; \mathrm{d}=.90$ to $\mathrm{d}=1.96$, very large effect sizes). The largest effect sizes were found on the Anger $(\mathrm{d}=1.96)$, Emotional and Behavioral Dyscontrol (d=1.91), Anxiety $(\mathrm{d}=1.90)$, and Depression $(\mathrm{d}=1.81)$ scales. When considering all 14 scales simultaneously, the SVT-Fail group consistently had a significantly $(p<.05)$ higher number of TBI-QOL scales that were classified as reflecting poor outcome (i.e., scores $>1$ SD from a normative sample) compared to the SVT-Pass group. For example, $70.4 \%$ of the SVT-Fail group had six or more scales classified as reflecting poor outcome, compared to $5.7 \%$ of the SVT-Pass group ( $p<.001$, Phi-coefficient; $\phi=.69$ ). Conclusions: It is common knowledge that the use of SVTs is a critical component for all neuropsychological evaluations following TBI. Patients who fail SVTs typically report inaccurate symptoms on other self-report measures. Not unexpectedly, the TBI-QOL is vulnerable to these effects and should be administered with well validated SVTs. Correspondence: Rael Lange, DVBIC, WRNMMC, Defense and Veterans Brain Injury Center, Walter Reed National Military Medical Center, Building 8, Room 2264, 8901 Wisconsin Avenue, Bethesda, MD 20814. E-mail: rael.t.lange@gmail.com
M.J. SHARLAND, S. WARING, A. TARAN, B. JOHNSON, J. PALCHER, A. PATTOCK \& T. RUSIN. Examination of Embedded Performance Validity Indicators for the Conners' Continuous Performance Test (CPT-II) and Brief Test of Attention (BTA) in a Large Clinical Sample.

Objective: Previous studies have examined the utility of embedded performance validity tests (PVTs) in both the Conners' Continuous Performance Test - 2nd edition and the BTA (Busse and Whiteside, 2012; Lange, et al, 2013; Ord, et al, 2010). The aims of the current study were to expand and apply the previous studies' findings to a large clinical outpatient hospital population.

Participants and Methods: Between 2010 and 2014, subjects completed comprehensive neuropsychological evaluation with multiple PVTs (TOMM, VSVT, DCT, WAIS-IV RDS, CVLT-II Forced Choice) as well as the BTA and CPT-II. 615 subjects between 18 to 64 years of age were included in the study. $47 \%$ of the population was female $(\mathrm{n}=286)$, and the overall sample had a mean education of 12.6 years. Subjects were classified as either showing biased or unbiased responding based on performance validity test scores. $444(72 \%)$ subjects passed all PVT variables. Subjects with failed PVT scores were subclassified into 3 groups: $9 \%$ who failed the TOMM Trial 2 or Retention, $28 \%$ who failed any 1 PVT, and $12 \%$ who failed 2 or more PVTs. ROC analysis with sensitivity, specificity, PPV, and NPV were compared on variables of the CPT-II and BTA between the sufficient effort group and the 3 failed PVT groups.

Results: Overall classification accuracy using ROC analyses yielded $\mathrm{AUC}$ values ranging from 0.66 to 0.86 . The $\mathrm{BTA}$ was most accurate $(\mathrm{AUC}=0.86)$ while the CPT-II Commission Errors was least accurate $(\mathrm{AUC}=0.66)$. With a base rate of $12 \%$, sensitivity was .55 and specificity was .94 for the BTA errors (PPP $=48 \%, \mathrm{NPV}=97 \%)$. For the CPT-II. sensitivity was between .50 and .53 with specificity between .85 to.87 $(\mathrm{PPP}=24-28 \%, \mathrm{NPV}=95 \%)$.

Conclusions: The current study replicated and extended Busse and Whiteside's (2012) previous study within a large outpatient hospital sample. Obtained results support use of CPT-II and Brief Test of Attention variables as embedded measures of performance validity with the BTA performing better as a PVT.

Correspondence: Michael J. Sharland, PhD, Neurology, Essentia Health, 400 East Third St, Duluth, MN 55805.E-mail: michael.sharland@ essentiahealth.org

A.C. PARKS \& J.D. GFELLER. Using the Structure Inventory for Malingered Symptomatology (SIMS) to Detect Feigned Postconcussional and Posttraumatic Stress Symptoms in Simulators.

Objective: The Structured Inventory of Malingered Symptomatology (SIMS; Widows \& Smith, 2005) is a self-report screening symptom validity test (SVT) designed to detect persons feigning psychological and neurological symptoms. While the utility of the SIMS has been explored in several simulation and clinical studies, its validity with populations susceptible to feigning symptoms of postconcussional disorder (PCD) and posttraumatic stress disorder (PTSD) is lesser known. The present study sought to examine the detection accuracy of the SIMS and its subscales with simulators instructed to feign symptoms of PCD and PTSD. Participants and Methods: One-hundred and four ( $\mathrm{N}=104)$ undergraduate students were assigned to one of four symptom simulation groups: (1) Feigned PCD, (2) Feigned PTSD, (3) Feigned PCD \& PTSD, (4) No symptoms. Participants were given a scenario involving an automobile accident with a pending lawsuit. Next, they were informed of a fictional monetary incentive if they showed psychological and neurological impairment. Adequate time was provided to study the symptom list. Lastly, a set of neuropsychological tests was administered, including the SIMS and other SVTs.

Results: Classification analyses revealed the SIMS Total score to be the most accurate validity test indicator, correctly classifying $86 \%$ of study participants. Additional analyses showed that the Affective Disorders 
subscale was the most sensitive across the simulator groups. Correlations between the SIMS scales and the other validity tests were moderately high $(r=.34-.70)$. An examination of the SIMS recommended cutoff scores was also performed.

Conclusions: This study provides evidence that the SIMS is an accurate screening SVT for use with persons suspected of feigning PCD and/or PTSD symptoms. It is recommended that only the Total Score be interpreted clinically, as several subscale scores lacked adequate specificity in this study. Additional research should continue examining the validity of the SIMS in both clinical and litigious populations.

Correspondence: Adam C. Parks, Clinical Psychology, Psychology, Saint Louis University, 920 Stanton L. Young Boulevard, Williams Pavilion Room 3059, Oklahoma City, OK 73104.E-mail:Adam-Parks@ouhsc. edu

R.D. SHURA, H.M. MISKEY, J.A. ROWLAND, R.E. YOASH-GANTZ \&. J.H. DENNING. Embedded Performance Validity Measures with Post-Deployment Veterans: Cross-Validation and Efficiency with Multiple Measures.

Objective: Embedded validity measures can provide a more comprehensive assessment of performance validity compared to any single measure in isolation. We evaluated the accuracy of individual embedded measures and then identified the least redundant combination that best predicted validity test failure.

Participants and Methods: The sample included 212 post-deployment Veterans from an ongoing research study (Average: age $=35$, education $=14$, male $=89 \%$, Caucasian $=72 \%$ ). Twenty-one percent of the sample failed Green's Word Memory Test (WMT). Thirty embedded measures were initially evaluated as predictors of WMT performance (derived from the CVLT-II, CPT-II, TMT, Stroop, WCST-64, WAIS-III: LNS, RCFT, BVMT-R, and FTT). Eight non-overlapping measures with the highest AUC values were entered into a logistic regression analysis. Embedded measure accuracy was also compared to previously reported cutoffs.

Results: Overall, previously developed cutoffs for individual measures showed relatively poor sensitivity (SN) in the current sample, though CPT-II Total Errors had the highest sensitivity using published cutoffs $(\mathrm{SN}=.41)$. CVLT-II (Total 1-5 raw score) showed the best overall accuracy $(\mathrm{AUC}=.80)$. After redundant measures were statistically eliminated from analysis, the model including RCFT (True Positives), CPT-II (Total Errors), and CVLT-II (Total 1-5 raw score) increased overall accuracy compared to the CVLT-II alone $(\mathrm{AUC}=.87)$.

Conclusions: Cutoffs in the existing literature showed relatively low sensitivity in this non-forensic, research Veteran sample. Despite starting with 30 embedded validity indices, a combination of 3 measures from the CPT-II, CVLT-II, and RCFT was the most accurate/efficient in predicting WMT failure.

Correspondence: Robert D. Shura, PsyD, Hefner VAMC, MA-MIRECC, 11M-2/MH\&BS, 1601 Brenner Ave., Salisbury, NC 28144. E-mail: shura@marshall.edu

M. SEMLA, D.M. WHITESIDE, L. BALABAN, T. SAK, L. RICE, M.R. BASSO \& B. ROPER. Classification Accuracy for Non-Credible Performance of the Personality Inventory (PAI) Psychosocial Scales in Traumatic Brain Injury.

Objective: Individuals with mild traumatic brain injury (MTBI) who fail performance validity tests (PVTs) often respond more pathologically on personality/emotional measures compared to individuals with severe traumatic brain injury (STBI) who pass PVTs (Lange et al., 2012). This study examined the classification accuracy for non-credible performance for several Personality Assessment Inventory (PAI) scales and as a logistically derived combined PAI variable. The PAI scales included Stress (STR), Nonsupport (NON), Treatment Rejection (RxR), Dominance (DOM), and Warmth (WRM). It was hypothesized that that the combined variable would have at least acceptable classification accuracy for credible performance based on Receiver Operating Characteristics (ROC) analysis within the MTBI/STBI samples.

Participants and Methods: The combined PAI variable was analyzed between MTBI participants $(\mathrm{n}=131)$ placed into credible $(\mathrm{n}=106)$ versus non-credible $(n=25)$ performance based on passing or failing PVTs such as the Test of Memory Malingering (TOMM). STBI participants ( $\mathrm{n}$ $=50)$ were likewise placed into credible $(\mathrm{n}=44)$ and non-credible $(\mathrm{n}$ =6) performance groups.

Results: None of the individual or combined PAI variable reached acceptable classification accuracy in the MTBI group $(\mathrm{AUC}=.59)$ but the combined PAI variable had excellent classification accuracy (AUC $=.85)$ in the STBI sample. This combined variable, using a cut-off score of 1.97 , with specificity set at $91 \%$, had sensitivity $=67 \%$ but the individual variables did not reach acceptable classification accuracy $(\mathrm{AUC}=.46-.60)$.

Conclusions: This study indicated that the combined PAI variable has potential as a PVT in STBI, but not MTBI, samples.

Correspondence: Douglas Whiteside, Ph.D., Clinical Psychology, Argosy University-Seatle, 200 Hawkins Drive, Iowa City, IA 52242. E-mail: douglas-whiteside@uiowa.edu

N. ROTHONG, J.M. CUNNINGHAM, S. KNOX, F. LOPEZ, M. WINGATE, J. NEUDECKER, M. MELINDER \& J. HOGG. Relationships between Word Memory Test Scores and Neuropsychological Performance in Patients with Epilepsy.

Objective: This study utilized the WMT General Memory Impairment Profile (GMIP) to identify and group false positives before investigating the relationship between WMT and neuropsychological test scores in an epilepsy population. We hypothesized that a significant number of patients who scored below WMT failure cutoff would have positive GMIPs, and that patients with WMT suboptimal or GMIP performance would have significantly lower neuropsychological test scores than patients with optimal WMT scores.

Participants and Methods: A retrospective chart review was conducted of patients referred to a Regional Epilepsy Center for neuropsychological evaluation. Participants included 81 patients ( $\mathrm{N}=56$ pre-surgical; $\mathrm{N}=25$ non-surgical) who were administered a battery of cognitive tests and the WMT. Patients were categorized into WMT optimal, suboptimal, and GMIP groups. Rohling's Interpretive Method (Miller \& Rohling, 2001) was used to interpret cognitive data. Multiple regressions were employed to examine the relationship between WMT performance and neuropsychological test scores.

Results: Forty-three percent $(\mathrm{N}=35)$ of patients were in the WMT optimal group, $36 \%(\mathrm{~N}=29)$ in the suboptimal group, and $21 \%(\mathrm{~N}=17)$ in the GMIP group. WMT performance accounted for $29 \%$ of the variance in overall neuropsychological performance $(p<.001)$ and from $17-40 \%$ of the variance across cognitive domains (all $p$ values $<.001$ ). Patients in suboptimal and GMIP groups typically obtained significantly lower neuropsychological scores than optimal group patients, though these differences were not consistently of practical significance.

Conclusions: Cognitive limitations, particularly on learning and memory tasks, may impact the ability of patients with epilepsy to perform above WMT failure cutoff. WMT performance did not impact all cognitive domains equally, suggesting that certain cognitive domains may be more sensitive to the impact of suboptimal performance. Overall, results support the use of the WMT and GMIP analysis in patients with epilepsy. Correspondence: Nichelle Rothong, PhD, Counseling Psychology, Marquette University, 616 N. 7th St., Unit 1105, St. Louis, MO 63101. E-mail:nichelle.rothong@gmail.com

G. SILK-EGLIT, J. STENCLIK, J. LYNCH \& R. MCCAFFREY. Use of the Impairment Index and Neuropsychological Deficit Scale as Indicators of Performance Validity.

Objective: Previous research has demonstrated that examinees exhibiting performance invalidity produce worse scores on summary measures from neuropsychological test batteries, including the overall test battery 
mean (OTBM) and the Impairment Index and Neuropsychological Deficit Scale from the Halstead-Reitan Neuropsychological Battery (HRNB). Recently, the OTBM has been used as an embedded performance validity measure. The current study investigated whether the Impairment Index and/or the Neuropsychological Deficit Scale might be used as a performance invalidity index as well.

Participants and Methods: Archival data on forty-eight examinees diagnosed with mild TBI seen for medico-legal purposes were examined. Examinees were classified as performance invalid (Mage $=45.48$, SDage $=10.28,58 \%$ male) if they failed $>=2$ free-standing PVTs and classified as performance valid (Mage $=42.78$, SDage $=12.01,45 \%$ male $)$ if they failed $<2$ free-standing PVTs.

Results: Performance invalid examinees produced greater Impairment Index, $\mathrm{t}(46)=4.00, \mathrm{p}=0.0003$, and Neuropsychological Deficit Scale, $\mathrm{t}(46)=4.66, \mathrm{p}=0.00002$, scores; however, these indexes were unable to achieve sufficient classification accuracy for clinical implementation. Conclusions: Despite large group differences, these indexes are not appropriate for detecting performance invalidity at the individual examinee level.

Correspondence: Grahan Silk-Eglit, M.A., Psychology, University at Albany, Social Sciences 399, 1400 Washington Ave, Albany, NY 12222. E-mail:grahammse@gmail.com

\section{G. SILK-EGLIT, J. STENCLIK, J. LYNCH \& R. MCCAFFREY. Use of Abnormal Score and Overall Test Battery Mean Intra-Individual Variability Scores as Measures of Performance Validity.}

Objective: Recent research demonstrated that performance invalid mild TBI examinees produce greater intra-individual variability (IIV) scores on the overall test battery mean (OTBM) than performance valid mild TBI examinees. The current study evaluated whether IIV scores can identify performance invalidity among mild TBI examinees. In addition, we also evaluated whether IIV on three levels of abnormal scores (1, 1.5 , and 2 standard deviations below the norm-referenced mean) can detect performance invalidity.

Participants and Methods: Archival data on 49 examinees diagnosed with mild TBI seen for medico-legal purposes were examined. Examinees were classified as performance invalid $($ Mage $=45.72$, SDage $=$ $10.29,60 \%$ male) if they failed $>=2$ free-standing PVTs; all other examinees were classified as performance valid (Mage $=42.92$, SDage $=11.75,58 \%$ male $)$.

Results: Performance invalid examinees did not produce greater IIV on the OTBM than performance valid examinees, but did demonstrate greater IIV at all three levels of abnormal scores. However, a scatterplot between IIV on abnormal scores and number of abnormal scores produced suggested a curvilinear association, which was confirmed by significant quadratic terms for these correlations at $1, \beta=0.002, \mathrm{t}=$ $49.82, \mathrm{p}<.001,1.5, \beta=-0.003, \mathrm{t}=14.97, \mathrm{p}<.001$ and $2, \beta=0.0004, \mathrm{t}$ $=-9.99, \mathrm{p}<.001$, standard deviations below the norm-referenced mean. Conclusions: The curvilinear association between IIV on abnormal scores and number of abnormal scores produced suggests that the number of abnormal scores most accurately classifies examinees, not IIV scores.

Correspondence: Grahan Silk-Eglit, M.A., Psychology, University at Albany, Social Sciences 399, 1400 Washington Ave, Albany, NY 12222. E-mail: grahammse@gmail.com

R. LANGE, T.A. BRICKELL, A. GRAHAM, R. GARTNER, A. DRISCOLL, Z. LI, E. SCHMIDT, D. PRUITT, M. WRIGHT, J. SMITH, B. PIZZANO \& L.M. FRENCH. Examination of the 'Mild Brain Injury Atypical Symptoms' and 'Validity-10' scales to Detect Symptom Exaggeration in U.S. Military Service Members.

Objective: The purpose of this study is to examine the clinical utility of two recently developed validity scales designed for use with the Neurobehavioral Symptom Inventory (NSI) and the PTSD Checklist (PCL); the Mild Brain Injury Atypical Symptoms Scale (mBIAS; Cooper et al. 2011) and Validity-10 scale (Vanderploeg et al., 2014).
Participants and Methods: Participants were 63 U.S. military service members (Age: $\mathrm{M}=31.9, \mathrm{SD}=12.5 ; 90.5 \%$ male) who sustained a MTBI; prospectively enrolled from Walter Reed National Military Medical Center. Participants were divided into two groups based on the validity scales of the Minnesota Multiphasic Personality Inventory-2-RF: (a) SVT-Fail (n=24) and (b) SVT-Pass (n=39). Participants were evaluated on average 19.4 months post-injury ( $\mathrm{SD}=27.6$ ).

Results: Participants in the SVT-Fail group had significantly higher scores $(\mathrm{p}<.05)$ on the mBIAS $(\mathrm{d}=.85)$, Validity-10 ( $\mathrm{d}=1.89)$, NSI $(\mathrm{d}=2.23)$, PCL-C $(\mathrm{d}=2.47)$, and the vast majority of the MMPI-2-RF scales $(\mathrm{d}=.69$ to $\mathrm{d}=2.47)$. Sensitivity, specificity, and predictive power values were calculated across the range of mBIAS and Validity-10 total scores to determine the optimal cut-off to detect symptom exaggeration. For the mBIAS, a cut-off score of $\geq 8$ was considered optimal, which resulted in low sensitivity (.17), high specificity (1.0) and positive predictive power (1.0), and moderate negative predictive power (.69). For the Validity-10 scale, a cut-off score of $\geq 13$ was considered optimal, which resulted in moderate-high sensitivity (.63), high specificity (1.0), and high positive (.93) and negative predictive power (.83).

Conclusions: These findings provide strong support for the use of the Validity-10 as a tool to screen for symptom exaggeration when administering the NSI and PCL-C. The mBIAS however was not a reliable tool for this purpose and failed to identify the vast majority of people who exaggerated symptoms.

Correspondence: Rael Lange, DVBIC, WRNMMC, Defense and Veterans Brain Injury Center, Walter Reed National Military Medical Center, Building 8, Room 2264, 8901 Wisconsin Avenue, Bethesda, MD 20814. E-mail: rael.t.lange@gmail.com

R. PATRICK \& M.D. HORNER. Psychological Characteristics of Individuals who put forth Inadequate Cognitive Effort.

Objective: Substantial research has been devoted to detecting inadequate effort on cognitive testing. However, little research has sought to identify psychological characteristics of individuals who exert inadequate effort after accounting for symptom over-reporting-the objective of the current study.

Participants and Methods: Fifty-two Veterans referred for clinical outpatient neuropsychological evaluation were divided into adequate $(n=29)$ and inadequate $(n=23)$ effort groups based on TOMM and/ or WMT, and specific behavioral indicators (e.g., Slick et al., 1999), Psychological functioning was characterized with the MMPI-2-RF. Influence of potential secondary gain was controlled by including only patients who had external financial incentive at the time of testing, though these were not forensic or disability evaluations. Variance attributable to symptom over-reporting was statistically controlled by covarying for F-r scores. Groups were compared using a series of multivariate analyses of variance.

Results: The inadequate effort group scored higher on all over-reporting scales (F-r, Fp-r, Fs, and FBS-r). After covarying for F-r, the inadequate effort group scored higher on Neurologic Complaints (NUC), and lower on Behavioral/Externalizing Dysfunction (BXD), Antisocial Behaviors (RC4), and Disconstraint (DISC-r).

Conclusions: Lower scores on BXD, RC4, and DISC-r could indicate higher behavioral constraint, which has been linked to strategic information processing motivated by desire to obtain high-value future rewards. Inadequate effort, therefore, may have reflected a decision-making strategy employed by patients with higher behavioral constraint aimed at securing external incentive (i.e., malingering). Alternatively, the findings could reflect a self-presentation strategy aimed at minimizing externalizing symptoms and antisocial behaviors in order to appear more psychologically healthy.

Correspondence: Regan Patrick, PhD, Neuropsychology, McLean Hospital, 20 A Lexington Terrace, Waltham, MA 02452. E-mail: regan. patrick11@gmail.com 
THURSDAY AFTERNOON, FEBRUARY 5, 2015

\section{The Birch Memorial Lecture: Optimal Outcome in Autism Spectrum Disorders}

\author{
Presenter: Deborah Fein
}

12:00-1:00 p.m.

\section{FEIN. Optimal Outcome in Autism Spectrum Disorders.}

A serious obstacle for progress in autism research, as with other psychiatric and neurodevelopmental disorders, is the existence of significant heterogeneity at all levels of analysis (genes, cells, behavior, etc.) and in all domains of functioning. Some theorists have even questioned the utility of the construct of autism itself. In addition, findings at different levels of analysis have not been found to correlate in any straightforward way. I will review some approaches to this variability, including that of the DSM-5. One domain of extreme heterogeneity is outcome: although varied outcome has been noted for many years, normalization of social communication and repetitive behaviors with loss of diagnosis has not generally been thought possible. I will report on a group of individuals who have reached this optimal outcome, and present data on cognitive functioning, psychiatric comorbidity, early development and intervention, and neuroimaging. Possible paths to this outcome, and implications for the heterogeneity problem, will be discussed.

Correspondence: Deborah Fein, University of Connecticut, 406 Babbidge Road, Unit 1020,Storrs, CT 06269. E-mail: deborah.fein@uconn.edu

\section{Invited Symposium: \\ Cannabis Effects in Vulnerable Populations}

\section{Chair: Deborah Yurgelon-Todd}

$$
\text { 1:30-3:00 p.m. }
$$

D. YURGELUN-TODD, D. YURGELUN-TODD, N. SOLOWIJ \& S. TAPERT. Cannabis Effects in Vulnerable Populations.

Symposium Description: Despite the widespread use and recent legalization of marijuana (MJ) in some US states, relatively little is known about the effects of cannabis on cognition, brain structure, or function in vulnerable populations. This symposium will examine the neurobiological effects of cannabis use in two vulnerable populations; (1) individuals at risk for psychotic disorders and (2) individuals undergoing adolescent brain maturation. Dr. Nadia Solowij will discuss alterations in neuroimaging results as they relate to attentional control and cannabis use. Specifically, the differential effects of cannabis use in individuals with psychosis compared to individuals with no psychiatric history will be presented. Dr. Deborah Yurgelun-Todd will review functional connectivity data in adolescents with heavy cannabis use indicating that MJ exposure impacts the developmental trajectories of brain circuits involved in cognition and mood

Dr. Susan Tapert will present functional and structural imaging data and neuropsychological measures in adolescents with and without a history of regular cannabis use who have been followed longitudinally. This work indicates that adolescents who use cannabis regularly perform more poorly at all time points and show changes in brain imaging measures compared with adolescents with a limited substance use histories. By examining the neurobiological effects of cannabis use in these vulnerable populations this symposia aims to provide insight into the effects of MJ which may impact future research and policy.

Correspondence: Deborah Yurgelun-Todd, PhD, Department of Psychiatry, University of Utah School of Medicine, 383 Colorow Drive Rm 323, Salt Lake City, UT 84108.E-mail:Deborah.Yurgelun-Todd@ hsc.utah.edu
D. YURGELUN-TODD. Abberant Orbitofrontal Connectivity in Adolescent Marijuana Smokers.

Objective: Orbitofrontal (OFC) circuits have been implicated in the pathophysiology of substance use disorders (SUD). We examined OFC functional connectivity and clinical variables in marijuana using adolescents (MJ) as compared to healthy controls (HC) to determine if difference in connectivity patterns could be seen in the adolescent MJ cohort. Participants and Methods: Functional MRI resting-state data was obtained on a 3 Tesla MRI scanner on 31 HC, and 43 adolescent participants who were heavy MJ smokers. Analyses controlling for both age and gender were performed between groups (MJ, HC) for the left and right OFC separately. Regression analyses between OFC functional connectivity and lifetime MJ use and age of first MJ use and clinical variables were also performed.

Results: We found increased OFC functional connectivity to frontal (cingulate and dorsolateral prefrontal cortex) and motor regions in adolescents with heavy MJ use as compared to HC. MJ users also had increased functional connectivity of the right $\mathrm{OFC}$ to motor regions with earlier age of first MJ use. In contrast, increasing lifetime MJ use was associated with increased OFC functional connectivity to several posterior brain regions. Measures of depression and impulsivity were also associated with connectivity.

Conclusions: The findings of atypical OFC functional connectivity patterns in adolescents with heavy MJ use in key attentional/executive, motor and reward networks, may be related to the suboptimal decision making capacities and increased impulsivity leading to substance use initiation, abuse and dependence. In addition, our study suggests that different OFC connectivity patterns may be present in adolescents with early onset of MJ use and high lifetime exposure to MJ. Additional studies examining the distinct biological risk factors for initiation of SUD and chronic use of substances on brain developmental trajectories is needed in individuals with SUD as these biological differences may impact prognosis and treatment.

Correspondence: Deborah Yurgelun-Todd, PhD, 383 Colorow Drive Rm 323, Salt Lake City, UT 84108. E-mail: Deborah.Yurgelun-Todd@hsc. utah.edu

N. SOLOWIJ. Cannabis Effects on the Brain in Schizophrenia and as a Vulnerability Toward Psychosis Phenotypes.

Objective: Long-term heavy cannabis use results in alterations to brain structure and function and the development of psychotic-like symptoms, all of which resemble aspects of schizophrenia. This talk will describe several studies that we have conducted to 1) determine whether there are additive effects of cannabis exposure to the pathophysiology of schizophrenia; 2) investigate vulnerability markers for psychosis-like phenotypes in otherwise healthy cannabis users; and 3) examine the effects of different compounds within cannabis that have been shown to have detrimental versus potentially neuroprotective effects on the brain. Participants and Methods: Patients with schizophrenia with and without comorbid cannabis use, and otherwise healthy cannabis users and non-user controls completed clinical and neuropsychological assessments and structural brain scans. Healthy cannabis users and controls also completed brain electrophysiological paradigms that investigated chronic and acute effects of cannabinoids (THC and CBD).

Results: Cannabis users without psychosis showed reduced hippocampal and amygdala volumes relative to healthy controls. Patients with schizophrenia and cannabis use showed the greatest alteration of hippocampal shape, followed by healthy cannabis users and then patients with schizophrenia without cannabis use. A similar hierarchy of alterations was observed for cerebellar white matter. Cannabis using patients performed better, however, than non-using patients on neuropsychological tests. Cannabis users show a signature of brain electrophysiology that differed in pattern to that observed in schizophrenia. Preliminary findings from acute administration of THC and CBD will be presented.

Conclusions: The implications of our findings for cannabis users and people with schizophrenia will be discussed in light of public policy 
debates around therapeutic cannabinoids and legalization for recreational use.

Correspondence: Nadia Solowij, PhD, School of Psychology and Illawarra Health and Medical Research Centre, Wollongong, NSW 2522, Australia.E-mail: nadia@uow.edu.au

\section{S. TAPERT. Neuropsychological and Neuroimaging Findings in Adolescent Marijuana Users: Longitudinal Results.}

Objective: The effect of adolescent marijuana use on brain development remains unclear despite relaxation of legal restrictions, decreasing perception of harm, and increasing use rates among youth. The aim of these prospective studies was to evaluate the long-term neurocognitive effects of adolescent marijuana use.

Method: Adolescent marijuana users $(M J, n=49)$ and control teens with limited substance use histories $(\mathrm{CON}, \mathrm{n}=59)$ were given neuropsychological and substance use assessments as well as a magnetic resonance imaging (MRI) session including diffusion tensor imaging, functional MRI, and structural MRI at project baseline, when they were ages 16-19. They were then re-assessed and re-scanned 18 and 36 months later. Results: MJ users performed significantly worse than controls, across time points, in the domains of complex attention, memory, processing speed, and visuospatial functioning $(p s<.05)$. Earlier age of marijuana use onset was associated with poorer processing speed and executive functioning by the 3 -year follow-up (ps $\leq .02$ ). Group differences in white matter quality, neural macrostructure, and neural activation to cognitive tasks were also observed.

Conclusions: Frequent marijuana use throughout adolescence and into young adulthood appeared linked to worsened cognitive performance. Earlier age of onset appears to be associated with poorer neurocognitive outcomes that emerge by young adulthood, providing further support for the notion that the brain may be uniquely sensitive to frequent marijuana exposure during the adolescent phase of neurodevelopment. Continued follow-up of adolescent marijuana users will determine the extent of neural recovery that may occur if use abates.

Correspondence: Susan Tapert, Ph.D., 9500 Gilman Drive, 0603V, San Diego,CA 92093-0603.E-mail: stapert@ucsd.edu

\section{Paper Session 3: Sleep}

$$
\text { 1:30-3:00 p.m. }
$$

J. KAMPER, R. NAKASE-RICHARDSON, D. SCHWARTZ, M. MCCARTHY, T. KRETZMER, J. GAROFANO, R. GECK \& W. ANDERSON. The Validity of Actigraphy as a Sleep Correlate in the TBI population.

Objective: Sleep disturbance is a common problem during both acute and chronic phases of TBI recovery, with potential for significant $\operatorname{cog}$ nitive and physical morbidity. Given the high cost and limited recording interval of the gold standard, polysomnography (PSG), alternative mechanisms for screening and monitoring sleep sequelae are needed. The purpose of this study was to examine the concordance of wrist-worn actigraphy (ACG) with PSG.

Participants and Methods: 42 participants with a history of TBI (median GCS=5). Participants were primarily male (89\%) and Caucasian $(79 \%)$, with a median age of 27 . Subjects were a median of 77 days (range $=28-1628)$ from initial injury. Participants underwent overnight polysomnography and concurrent actigraphy. Sleep indices were compared during the lights-off through lights-on period of PSG. PSG was administered and scored by certified sleep-technologists with review by board-certified pulmonologists in sleep medicine.

Results: ACG and PSG were found to be significantly correlated (Total Sleep Time $[r=.77 ; p<.001]$ and Sleep Efficiency $[r=.70 ; p<.001])$ across the entire cohort. Subgroup analyses revealed that ACG and PSG were significantly correlated for patients with and without obstructive sleep apnea (OSA), although sleep time r-values were higher for the OSA group ( $r=.84$ for OSA vs. $r=.64$ for non-OSA).

Conclusions: ACG measurements of sleep are comparable to PSG results in TBI patients. This association is stronger for but not contingent on the presence of a sleep disorder such as OSA. Given these data, ACG is a feasible first-line measure of sleep for detecting sleep disorders in TBI populations.

Correspondence: Joel Kamper, Ph.D., Neuropsychology, Tampa VAMC, 2406 Earlswood Ct, Brandon, FL 33510.E-mail: joelkamper@gmail. com

A.A. GARNER, M.M. MILLER, J. FIELD, Z. SMITH, O.B. NOE \& D.W. BEEBE. Impact of Experimentally Manipulated Sleep on Adolescent Simulated Driving.

Objective: We have previously shown that multi-night sleep restriction (SR) impairs adolescents' attention, which could contribute to driving problems. Here, we examine the impact of experimental SR on adolescent drivers, considering whether that impact is moderated by the driving environment (urban/suburban vs. rural) or individual differences in participants' parent-rated vulnerability to SR.

Participants and Methods: 17 healthy 16-18-year-olds with a valid driver's license completed two 5-night sleep conditions: SR $(6.5 \mathrm{hrs}$ in bed) vs. Healthy Sleep (HS; 10 hrs in bed) in counterbalanced order. Vulnerability to SR was computed as a change in parent ratings of adolescent inattention across the sleep conditions. Adolescents also completed simulated rural and urban/suburban drives in each condition. Change in standard deviation of speed, mean speed and standard deviation of lateral lane position (SDLP) was assessed via ANCOVAs (within-subject factors $=$ sleep and drive conditions, between subject factor $=$ vulnerability to SR, covariate $=$ years licensed $)$.

Results: Effects for speed mean and standard deviation were non-significant. For SDLP, the 3 -way interaction (sleep $\mathrm{X}$ drive $\mathrm{X}$ vulnerability) was significant. During the rural drive, teens had less consistent lateral vehicle control in SR than HS, regardless of vulnerability to SR. During the urban/suburban drive, SR worsened SDLP only among teens reported to be vulnerable to SR.

Conclusions: SR of a severity regularly experienced by millions of adolescents appears to be a significant, modifiable contributor to driving problems. This impact is widely present during boring rural drives, and in a subset of teens during engaging, urban/suburban drives.

Correspondence: Annie A. Garner, Ph.D., Behavioral Medicine/Clinical Psychology, Cincinnati Children's Hospital Medical Center, 3333 Burnet Avenue, ML 10006, Cincinnati, OH 45229. E-mail: annie. garner@cchmc.org

T.M. BRINKMAN, C. KIMBERG, Y. MZAYEK, C. PUI, M.M. HUDSON \& K.R. KRULL. Sleep disturbance contributes to attention and memory problems in adolescent and young adult survivors of childhood acute lymphoblastic leukemia (ALL).

Objective: Survivors of ALL are at risk for neurocognitive impairment. The contribution of sleep disturbance, bevond established treatment effects, to neurocognitive problems is not well understood. We examined associations between sleep disturbance and performance on tasks of attention and memory in long-term survivors of ALL.

Participants and Methods: Ninety-two survivors of ALL (mean [SD] current age $=14.3[4.7]$ years, time since diagnosis $=7.7[1.9]$ years) treated on a chemotherapy only protocol completed measures of attention (inattention, variability, detectability) and memory (verbal and nonverbal memory span; verbal and nonverbal working memory). Age-adjusted standardized scores were calculated using national normative data. Survivors and their caregivers completed questionnaires assessing sleep onset latency, total hours slept, nighttime awakenings, premature morning awakenings, and daytime sleepiness. Generalized linear models were used to examine associations between sleep and neurocognitive outcomes while adjusting for treatment exposures. 
Results: Fifty percent of survivors reported delayed sleep onset latency, nighttime awakenings, and daytime sleepiness; $37 \%$ reported premature morning awakenings. In multivariable models adjusted for sex, age at diagnosis, time since diagnosis, and treatment risk group (low vs. standard/high), survivors with delayed sleep onset latency performed significantly worse on indices of sustained attention (inattention: $\beta=10.14$, $p=0.002$; variability: $\beta=5.71, p=0.02$ ). Survivors with premature awakenings performed worse on sustained attention (inattention: $\beta=8.63$, $p=0.028$ ), verbal and nonverbal working memory (verbal: $\beta=1.39$, $p=0.046$; nonverbal: $\beta=1.27, p=0.059)$, and nonverbal memory span tasks $(\beta=1.5, p=0.009)$.

Conclusions: These findings suggest a specific contribution of sleep disturbance to reduced attention and memory performance in survivors of childhood ALL. Sleep interventions may improve neurocognitive function in these survivors.

Correspondence: Tara M. Brinkman, PhD, Epidemiology and Cancer Control, St. Jude Children's Research Hospital, 262 Danny Thomas Place, MS 735, Memphis,TN38105.E-mail:tara.brinkman@stjude.org

Y. MZAYEK, T.M. BRINKMAN, C. KIMBERG, A. KUNINBATSON, A. PANOSKALTSIS-MORTARI, C. PUI, M.M. HUDSON \& K.R. KRULL. Neurocognitive Function, Oxidative Stress and Sleep Disturbances in Long-Term Survivors of Childhood Acute Lymphoblastic Leukemia (ALL).

Objective: To investigate whether systemic inflammation and oxidative stress are associated with neurocognitive function and sleep disturbance in long-term survivors of childhood ALL.

Participants and Methods: Thirty-six survivors of childhood ALL (mean[SD] age $=12.1[2.8]$ years, time since diagnosis $=7.1[1.7]$ years) completed neuropsychological testing. Measures of memory span (Wechsler Digit Span) and executive function (Delis Kaplan Executive Function System) were administered and age-adjusted standard scores and impairment (standard score $>1$ SD below normative mean) were calculated. Sleep quality was assessed through parent report and survivor self-report. Serum collected on the day of testing was assayed for markers of inflammation (interleukin-6, tumor necrosis factor-alpha, C-reactive protein) and oxidative stress (oxidized low-density lipoprotein, myeloperoxidase, and malondialdehyde). Mann-Whitney U test was used to assess associations between biomarkers and neurocognitive function and sleep disturbances.

Results: Lower memory span scores were associated with increased levels of oxidative stress biomarkers: myeloperoxidase $(p<0.02)$ and malondialdehyde $(p<0.04)$. Survivors with impaired cognitive flexibility had higher oxidized low-density lipoprotein concentrations compared to those without impairment (mean, 175.4[49.9] vs. 119.4[49.3] ug/L, $\mathrm{p}<0.03)$. Survivors with nighttime awakenings had higher myeloperoxidase levels compared to those without $(p<0.003)$. Malondialdehyde levels were higher in survivors with either daytime sleepiness $(\mathrm{p}<0.05)$ or premature awakenings $(\mathrm{p}<0.007)$ compared to those without. Results were not associated with infection, fever or body mass index.

Conclusions: These findings suggest that oxidative stress is related to neurocognitive impairment and sleep disturbances in survivors of childhood ALL. Future research will assess whether the effects of sleep on neurocognitive function are mediated by oxidative stress.

Correspondence: Yasmin Mzayek, B.S. Neuroscience, Epidemiology and Cancer Control, St Jude Research Hospital, 1277 winter springs In, Cordova, TN 38016.E-mail: yasmin.mzayek@stjude.org.

\section{Symposium 3: \\ A New Look at Chemobrain: Conceptualizing and Measuring Cognition in Cancer Patients}

\author{
Chair: Todd Horowitz
}

1:30-3:00 p.m.

T. HOROWITZ, T. HOROWITZ, K.K. HARDY, J. COSMAN \& L.S. PADGETT. A new look at chemobrain: Conceptualizing and measuring cognition in cancer patients.

Symposium Description: Many cancer patients experience impaired cognition after treatment, a phenomenon dubbed "chemobrain" or "chemo fog". These impairments can last for months or years after the treatment is complete. There is a substantial neuropsychological literature on chemobrain. However, the scientific picture of chemobrain is blurry. There is no clear agreement on which cognitive faculties are impaired. Estimates of the degree and prevalence of impairment are contradictory. Perhaps most critically, neuropsychological estimates of the effect sizes often do not agree with patients' own subjective impression of their problems.

In this symposium, we will bring new perspectives to bear on this problem. Lynne Padgett will examine cognitive deficits in adults using the CHC (Cattell-Horn-Carroll) model of intelligence as a theoretical lens to guide assessment and intervention targets. Todd Horowitz will present an analysis of chemobrain data from the cognitive psychology point of view. Kristina Hardy will present a dual screening and monitoring approach to studying cognitive deficits in children undergoing treatment for cancer. Finally, Joshua Cosman will present a tablet-based testing battery developed from tasks used extensively in cognitive psychology. We hope that these new theoretical and measurement tools will spur more creative approaches to understanding and ameliorating chemobrain. Correspondence: Todd Horowitz, Ph.D., Basic Biobehavioral and Psychological Sciences, National Cancer Institute, 9609 Medical Center Drive, Rockville,MD 20850.E-mail:todd.horowitz@nih.gov

\section{L.S. PADGETT. Looking At Cancer Related Cognitive Impairment Through The Lens Of CHC Theory: Does It Improve Our Vision?}

Research examining cancer related cognitive impairment in adults, has established a broad consensus that executive function, memory, attention, speed of processing, as well as verbal and visual spatial abilities are affected areas. However, research has presented a confusing picture with results from both individual studies and meta-analyses conflicting each other.

I propose that the lack of a theoretical approach to provide a cohesive view of deficits has left the field with a large body of conflicting results often based on lack of concordance in assessment. The Cattell-Horn-Carroll (CHC) model of intelligence provides the strong research and theory base that facilitate an integrated view of cancer related cognitive impairment, leading to improved measurement of cognitive processes. When reviewing individual studies or meta-analyses, it becomes apparent that assessment instruments are often classified in different domains, thus complicating the ability to integrate data from multiple sources. CHC Theory, using concepts of broad and narrow abilities, provides a conceptual and statistically robust model of intelligence and cognitive processes as well as classification of assessments to more clearly delineate these narrow abilities. Research findings of verbal and visual spatial abilities will be re-examined using CHC theory to clarify incongruence and illustrate the importance of further research in this area advancing both our scientific understanding of impairment and rehabilitation.

Correspondence: Lynne S. Padgett, PhD, 9609 Medical Center Drive, 3E616, Rockville,MD 20850.E-mail: lynne.padgett@nih.gov 


\section{T. HOROWITZ. Attention And Chemobrain: The View From Cognitive Psychology.}

There is now substantial evidence that chemotherapy patients experience significant cognitive impairments ("chemobrain"). However, methodological problems make it difficult to determine which specific cognitive domains are impaired. As an illustrative example: across six meta-analytic reviews of the chemobrain literature, the major point of consensus was that attention was not impaired. However, I argue that most of the "attention" measures in this literature (e.g. memory span tests) have little or nothing to do with attention as cognitive scientists understand it. Conversely, tests with some validity as attention tests (e.g., letter cancellation, Stroop) are sometimes excluded from the attention category. Re-analyzing the existing datasets in light of contemporary theories of attention, I find evidence for impairments in visual search and response selection. However, given the limitations of the existing tests, this result should only be taken as a suggestive starting point for future research. If we are going to make progress in understanding the effects of chemotherapy on attention, we need measures that are designed to be sensitive to specific aspects of attention. More broadly, I argue that we need a strategic shift in the way we measure cognition in cancer patients. Much progress has been made recently in studying disorders such as autism and attention deficit disorder by testing patients in way that makes direct connections to contemporary theories of cognition and its neural underpinnings. A similar approach to chemobrain would be of great benefit.

Correspondence: Todd Horowitz, Ph.D., 9609 Medical Center Drive, Rockville, MD 20850.E-mail: todd.horowitz@nih.gov

\section{K.K. HARDY, L. EMBRY, R.D. ANNETT, S. SANDS, S. PATEL, K.S. WALSH \& R. NOLL. Neuropsychological Assessments within the Children's Oncology Group: A Model for Success.}

Background: The Children's Oncology Group is an international clinical trials consortium including over 250 sites. The Behavioral Science Committee has pursued the goal of completing neuropsychological assessments of children receiving treatment affecting the central nervous system as study end points and as part of standard clinical care. Our vision has resulted in the successful implementation of two strategies: (1) a standardized screening battery administered by a psychologist; and (2) a computerized monitoring battery administered by clinic staff. Methods: The 60-90 minute screening battery consists of both performance-based and questionnaire measures evaluating intellectual functioning, processing speed, working memory, verbal learning, behavior, and adaptive functioning. The 30 -minute monitoring battery consists of brief computer tasks that assess potentially vulnerable processes such as reaction time, visual attention, working memory, and executive functioning. The batteries can be used individually or combined to address research questions or monitor clinical functioning.

Results: The screening battery is currently used with six brain tumor trials and has accrued 500+ participants across 150 sites. The computerized monitoring battery is currently used with one high-risk acute lymphoblastic leukemia trial and two brain tumor trials across more than 120 institutions. Data collection rates exceed 90\% with both methodologies

Conclusions: Our approach has proven to be feasible in the cooperative group setting. Clinically, this approach has the potential to identify children with emergent problems before significant functional impairments develop using safe, tolerable, cost-effective, and low-burden methods. From a research perspective, this strategy resulted in strong accrual, and the ability to recruit a large, diverse sample with the same outcome measures.

Correspondence: Kristina K. Hardy, PhD, 111 Michigan Ave, NW, Washington, DC 20010.E-mail:kkhardy@childrensnational.org

\section{J. Cosman. Mobile Tests For Assessing Cognition In Aging And} Disease.

Basic cognitive tests are becoming increasingly important for assessing changes in neurological functioning due to aging, disease, or therapeutic interventions. Ideally, individuals would be able to obtain comprehensive cognitive testing on a regular basis in order to determine their "baseline" level of function, using this as a point of comparison to detect deviations if they arise. However, comprehensive assessment of cognitive function is currently expensive and time consuming, and tests often must be administered and scored by individuals trained in cognitive assessment (e.g.., clinical neuropsychologists). Further, given the heterogeneous cognitive symptomatology within and across diseases, it is critical that cognitive tests be precise enough to determine which aspects of cognition (e.g., memory, attention, executive function) are undergoing change. To this end, our group has developed a mobile tablet-based cognitive testing platform that is designed specifically for use by non-experts, providing a simple user interface and easy to understand multimedia instructions and feedback. The application consists of novel implementations of twenty extensively validated cognitive psychology and neuropsychological tests measuring aspects of attention, memory, executive function, motor control, and processing speed. In this talk. I will discuss how this application is currently being used to measure cognitive function in home, clinical, and research settings, and discuss how such an application could be used to assess cognition and improve patient monitoring and care in cancer in particular.

Correspondence: Joshua Cosman, PhD, 1700 state street, Nashville, TN 37203.E-mail: joshua.d.cosman@vanderbilt.edu

\section{Symposium 4: \\ Using Neuroimaging and Connectivity Modeling to Understand Network Plasticity After Brain Injury: Advancing Theory and Methods}

\author{
Chair: Frank G. Hillary
}

$$
\text { 1:30-3:15 p.m. }
$$

F.G. HILLARY, G. WYLIE, E.A. WILDE, V. CALHOUN \& E. BIGLER. Using neuroimaging and connectivity modeling to understand network plasticity after brain injury: advancing theory and methods.

Symposium Description: Connectivity modeling has received considerable attention in the systems neurosciences over the past decade, and increasingly, work is being conducted with the goal of integrating identifiable brain networks and behavioral states. The goals of this symposium are to: 1) examine the literature using functional brain imaging methods and connectivity modeling to understand the consequences of traumatic brain injury (Drs. Hillary, $\left.W_{y l i e}\right), 2$ ) to integrate structural and functional brain imaging methods to examine plasticity after TBI (Dr. Wilde), and 3) describe the very latest network modeling techniques including their application in brain injury and other neurological samples (Dr. Calhoun). This symposium will focus on theory and method with the goal of identifying consistencies in broad literatures examining traumatic brain injury and its long-term consequences including mild cognitive impairment and Alzheimer's disease. We explore developing methods for network modeling that permit previously unavailable examination of the systems plasticity and recovery in humans. Discussion following the speakers will be led by Dr. Erin Bigler, a renowned researcher in the area of imaging and TBI.

Correspondence: Frank G. Hillary, PhD, Psychology, Pennsylvania State University, 313 Moore Building, University Park, PA 16802. E-mail:fhillary@psu.edu 
F.G. HILLARY \& G. WYLIE. When physical disruption increases brain connectivity: the "hyperconnectivity hypothesis" after brain trauma.

The purpose of this talk is to outline the current findings in network neuroscience examining the consequences of traumatic brain injury (TBI). First, by summarizing over 100 studies focused on connectivity modeling, we propose that a common response to neurological disruption is network hyperconnectivity. This "hyperconnectivity hypothesis" helps to explain findings in TBI and provides a framework for bridging these findings to degenerative processes such as mild cognitive impairment and Alzheimer's disease. We then directly test the hyperconnectivity hypotheses in patients during the first year after injury and patients decades after injury to examine the trajectory of the hyperconnectivity response. This talk aims to describe the transient/situational demands associated with hyperconnectivity and the physical resource thresholds that permit its expression.

Correspondence: Frank G. Hillary, PhD, 313 Moore Building, University Park,PA 16802.E-mail: fhillary@psu.edu

F.G. HILLARY \& G. WYLIE. Investigation of information flow during a novel working memory task in individuals with traumatic brain injury.

We used a novel working memory paradigm, CapMan, to investigate effective connectivity associated with the capacity and manipulation processes of working memory in individuals with traumatic brain injury (TBI) relative to healthy controls (HCs). CapMan allows the independent investigation of brain regions associated with capacity and manipulation, while minimizing the influence of other working memory related sub-processes. Areas of the fronto-parietal working memory network, previously identified in healthy individuals as engaged in capacity and manipulation during CapMan, were analyzed with the Independent Multiple-sample Greedy Equivalence Search (IMaGES) method to investigate the differences between healthy individuals and individuals with TBI in information flow. In addition to differences in behavioral performance, we found that TBI participants evince more connectivity than HC participants, both during high capacity and high manipulation conditions. This pattern of increased connectivity (hyperconnectivity) was found despite the fact that the capacity and manipulation conditions rely on different networks. Because of the diffuse axonal damage that is common in TBI, this hyperconnectivity may reflect greater local connectivity in the underlying networks, and the clinical implications of this will be discussed

Correspondence: Glenn Wylie, 300 Executive Dr, Ste 70, West Orange, NJ 07052.E-mail: gwylie@kesslerfoundation.org

\section{E.A. WILDE. Integration of structural and functional imaging data in understanding connectivity.}

Diffusion tensor imaging (DTI) has demonstrated significant potential as an imaging modality which can be used to probe the structural properties of white matter tracts connecting distant components of distributed brain networks. Though functional and structural imaging techniques measure different phenomena and are focused upon different tissue types (white matter vs gray matter), data about the structural integrity of the physical "connections" between areas of a network may inform some of the functional consequences that are observed. This talk will review how structural imaging (DTI in particular) may enhance our understanding of disease states which alter neural connectivity. Using traumatic brain injury as an example, potential mechanisms underlying both transient and sustained alterations in connectivity will be reviewed. Strategies for the integration of structural and functional imaging data will be discussed.

Correspondence: Elisabeth A. Wilde, Ph.D., 1709 Dryden Rd., Ste 1200, Houston, TX 77025. E-mail: ewilde@bcm.edu
F.G. HILLARY \& V. CALHOUN. Functional network connectivity as a marker of brain injury.

The use of network-based approaches for capturing whole brain patterns of activity that are interacting and changing has become more common. The advantage of approaches that can evaluate both within and among network connectivity are many, not the least of which is they provide a whole brain perspective on functional connectivity. In this talk I will briefly review the use of independent component analysis for characterizing brain connectivity as well as approaches for addressing potential confounds such as motion. I will also discuss the use of classification approaches to make individual subject predictions from such data in the context of mild traumatic brain injury as well as other disorders. In addition, I will discuss advantages of more recent approaches that can capture time-varying changes in brain connectivity.

Correspondence: Vince Calhoun, 1101 Yale Blvd. NE, Albuquerque, NM 87106.E-mail:vcalhoun@mrn.org

\section{Poster Session 3: Aging \& Epilepsy 1:30-3:00 p.m.}

\section{Aging}

C. SUMIDA, E.J. VAN ETTEN, G.M. WAGNER, J.D. HILEMAN, H.M. HOLDEN \& P. GILBERT. Who, When, and Where: AgeRelated Differences on a Novel Episodic-Like Memory Task.

Objective: Age-related deficits in episodic memory have been documented using well-validated list learning tests such as the California Verbal Learning Test-II (CVLT-II). The present study examined age-related differences on a novel episodic-like memory test assessing memory for "who, when, and where" in addition to associations among these elements.

Participants and Methods: Young (ages 18-25) and older adults (ages $65+$ ) were administered the CVLT-II and a novel episodic-like memory test. Our test consisted of two trials during which the participant was asked to remember a sequence of pictures of different faces paired with different places. The participant then was asked to pair each face with the correct place and put the face-place pairs in the correct sequence. Results: Our test correlated significantly $(p<.05)$ with the CVLT-II, providing preliminary evidence for validity. Older adults remembered significantly fewer face-place pairs and correct pairs in sequence compared to young adults on both trials $(\mathrm{ps}<.05)$. Although older adults committed more face and place intrusion errors on the first trial ( $\mathrm{ps}<$ $.05)$, there were no significant group differences on the second trial.

Conclusions: Using a single test, we demonstrated that older adults are impaired in remembering associations between faces and places, as well as the temporal sequence in which face-place pairs were presented; both are critical for everyday episodic memory. Given that intrusion errors for the individual faces/places did not differ on the second trial, these age-related associative memory differences are not due solely to impaired memory for the individual items in the associations.

Correspondence: Catherine Sumida, San Diego State University, 17838 Aguamiel Rd., San Diego, CA 92127. E-mail: casumida@yahoo.com

C.S. MARTIN, B. KLUGER, D.B. CORBETT, D. GUNDERMANN, M. MARSISKE, I. BABU HENRY SAMUEL \& T.M. MANINI. Association of Subjective Fatigue Complaints with Objective Cognitive Performance in Older Adults.

Objective: Fatigue is a common complaint in older adults which is associated with both disability and increased mortality. Unfortunately, the cause(s) of fatigue in this population are poorly understood and there are no evidence based treatments. As aging is also associated with significant changes in cognition we hypothesized that cognitive 
factors may be related to fatigue complaints in this population. The objective of this case-control study was to determine whether cognitive performance over time differed between older adults with high and low fatigue complaints.

Participants and Methods: We recruited 21 older adults with high perceived fatigue and 27 older adults with low fatigue. Group status was determined using the Functional Assessment of Chronic Illness Therapy-Fatigue (FACIT-F) Scale with scores either 1 standard deviation above or below age-adjusted normative means. Cognitive performance was measured using a continuous 20-minute working memory task (N-back test), which was scored by response time (RT), accuracy, and non-response rate over four 5-minute blocks.

Results: Five subjects were excluded for high (> 50\%) non-response rates, 4 of whom were in the high fatigue group. While the remaining subjects had similar performances during the first block, the high fatigue subjects differed significantly from controls over time in terms of failure to learn (proportion correct improved for controls but was flat for cases); higher rates of no responses (suggesting lower effort); and increasing proportion of incorrect responses (suggesting increased cognitive fatigability).

Conclusions: Fatigue complaints are associated with significant differences in objective cognitive performance. Further studies are needed to determine whether fatigue affects cognition through perceptions of effort and/or heightened cognitive fatigability drives fatigue complaints. Correspondence: Christine S. Martin, BA, Neurology, University of Colorado, 4028 S. Malta St., Aurora, CA 80013. E-mail: christine. martin@ucdenver.edu

R.K. LIMKEMAN \& S. ROGERS. Ease Up, Study Up, or Forget About It: How Neuroticism Relates to Learning, Memory, and Cognitive Decline in Older Adults.

Objective: This study examined how older adults' verbal and visual learning and memory vary with their dispositional levels of neuroticism. Participants and Methods: A total of 79 older adults (59 women, 20 men) of mixed ethnicity with a mean age of 77.15 years $(\mathrm{SD}=$ $10.75)$ and mean education of 15.82 years $(\mathrm{SD}=2.55)$ completed a comprehensive neuropsychological battery. Verbal and visual memory measures included Logical Memory \& Visual Reproduction from the Wechsler Memory Scale, Third Edition (WMS-III); the California Verbal Learning Test, Second Edition (CVLT-II); the Rey Complex Figure Test (RCFT). The NEO-Five Factor Inventory was administered to assess levels of neuroticism.

Results: Canonical correlation analyses revealed a significant relationship between the trait of neuroticism and the set of memory measures, $\mathrm{p}<.02$. Bivariate correlation analyses showed significant negative relationships between the degree of older adults' neuroticism and Logical Memory I, p <.01, Logical Memory II, p <.01, CVLT-II Trials 1-5 Total, $\mathrm{p}<.04$, CVLT-II List B, $\mathrm{p}<.03$, CVLT-II Long Delay Free Recall, $\mathrm{p}<.04$, Visual Reproduction I, $\mathrm{p}<.001$, Rey-O 3', $\mathrm{p}<.01$, and Rey-O 30', $\mathrm{p}<.01$. The only measure in bivariate correlation analyses that was not related to neuroticism was Visual Reproduction II, $\mathrm{p}<.10$. Conclusions: Older adults with higher levels of neuroticism are performing significantly worse on almost all aspects of memory, including verbal and nonverbal memory, as well as learning and delayed recall. From these findings, it is clear that personality has neurological substrates and connections, not just in frontal regions, as previously thought, but also in temporal lobe functions. Although the direction of influence is uncertain, it is possible that assigning activities like brain games to strengthen memory may lower levels of neuroticism. It is equally possible that therapeutic interventions to help lower neuroticism may delay cognitive decline and strengthen memory.

Correspondence: Robert K. Limkeman, Westmont College, 955 La Paz Road, M.S. 1904, Santa Barbara, CA 93108. E-mail: rlimkeman@ westmont.edu
S. ROGERS \& B.L. ALLEN. Are Older Couples More Similar or Different in Cognition?

Objective: Longitudinal investigations have suggested that married couples share similarities on many variables, including intelligence. This study examined the degree of similarity or difference in the cognitive functioning of older adults who are part of a married couple.

Participants and Methods: Twenty-two, heterosexual, older adult couples with a mean age of 75.18 years $(S D=10.82)$ completed a comprehensive battery of neuropsychological measures. They were also classified into age-related diagnoses (e.g., normal aging, mild cognitive impairment, and dementia).

Results: Bivarate correlation analyses revealed significant positive relationships between husbands' and wives' scores on MMSE, $p<.01$. and the Boston Naming Test, $p<.04$. The level of depression between husbands and wives was also significantly correlated, $\mathrm{p}<.03$, but there were no other significant relationships between spouses' cognitive or emotional functioning. T-test analyses revealed that wives performed significantly better on FAS than their husbands, $\mathrm{p}<.03$, but there were no other significant differences in cognition. Moreover, chi-square analysis showed no significant differences in the age-related diagnoses of husbands and wives.

Conclusions: Older husbands and wives appear to share significant similarities in their gross cognitive abilities and mood, but these similarities disappear in specific cognitive abilities. With the exception of wives outperforming their husbands in phonemic fluency, older spouses are neither significantly similar nor different in their individual cognitive abilities. Older members of a married couple therefore seem to maintain independent cognitive abilities that neither resemble nor overtly compensate for each other. This has distinct implications for the way clinicians and researchers view older adults (e.g., as individuals rather than a couple), approach cognitive interventions (e.g., not relying on a compensatory role for spouses), and understand the differential impact of marriage on cognitive functioning.

Correspondence: Steve Rogers, Ph.D., Psychology, Westmont College, 955 La Paz Road, Santa Barbara, CA 93108. E-mail: sarogers@ westmont.edu

M. SOTO-AÑARI, G. CÁCERES-LUNA, R. RIVERA \& N. LOPEZ. Inhibitory Control and Response Suppression as Latent Variables in Older Illiterate Bilinguals.

Objective: Analyze inhibitory control and response suppression as latent variables of inhibition and estimate the effect of bilingualism over these factors. Our hypothesis predicts the existence of both factors and the influence of bilingualism on measures of inhibition.

Participants and Methods: We evaluated 52 healthy elderly illiterate subjects, 35 bilingual $(\mathrm{Me} .=70.26$, Ds $=5.86)$ and 17 monolingual $(\mathrm{Me}=73.94, \mathrm{Ds}=8.77)$. The inhibitory control was evaluated with NS and interference Stroop test scores (modified version for illiterates) and semantic fluency scores (animal category); for the response suppression factor, we used the subtest of conflicting instructions and control from go / no go task, also the scores of the Tower of Hanoi test were taken on account.

Results: Structural equation modelling shows that the inhibitory control reach an appropriate model fit, while the response suppression is unstable. Inhibitory control has a significant effect on the observed scores of NS, interference and fluency; it also received an important effect of bilingualism and gender. Bilingualism and gender does not affect response suppression, and this factor seems to have no effect on the observed measures.

Conclusions: These findings show that inhibition can be divided in inhibitory control and response suppression, and bilingualism appears to have an important effect on the inhibition variable. Bilinguals have to selectively attend to a relevant stimulus cue while ignoring a competing cue. These continuous practice will promote higher levels of inhibitory control. Results should be interpreted with caution given the sample size and the unreached goodness fit in the model, but show an interesting 
line of research on bilingualism as a measure of cognitive reserve for illiterate subjects.

Correspondence: Marcio Soto-Añari, M-Sc., Psychology, Universidad Católica San Pablo, Villa médica torre 2, dpto. 504, Arequipa 04001, Peru.E-mail:msoto@ucsp.edu.pe

P.E. MAY \& J.L. WOODARD. Engagement in Activities and Cognitive Functioning Among Older Adults in the Health and Retirement Study.

Objective: This analysis tested the "use it, or lose it" hypothesis, by examining how engagement in cognitive and social activities impacts verbal memory in a longitudinal sample of older adults. Two models were contrasted: 1) baseline activity engagement predicting initial level and longitudinal change in verbal memory, and 2) baseline verbal memory predicting change in activity engagement over time.

Participants and Methods: The sample included 3,397 respondents aged $\geq 60$ years from the Health and Retirement Study's 2008, 2010. and 2012 waves. Respondents completed a verbal memory test during each wave, as well as items ranking frequency of cognitive and social activity engagement in 2008 and 2012. Latent growth curve (LGC) and latent difference models were used to test the two hypothesized models. Results: Delayed recall decreased over time. A LGC model was specified using baseline cognitive and social activity engagement as predictors of intercept and slope for delayed recall over three time points. At baseline, frequent engagement in cognitive activities was associated with greater delayed recall. Conversely, frequent engagement in social activities was associated with lower delayed recall. Neither activity frequency predicted delayed recall slope. Latent difference models indicated that cognitive and social activity frequency did not significantly change over time.

Conclusions: Results lend support for the "use it, or lose it" hypothesis, as engagement in cognitive activities was positively associated with verbal memory at baseline. However, baseline cognitive and social activity engagement did not significantly impact verbal memory change over four years. Respondents' frequency of cognitive and social activity engagement remained stable over time.

Correspondence: Pamela E. May, M.A., Psychology, Wayne State University, 420 N Crooks Road, Apt 25, Clawson, MI 48017. E-mail: dy3110@wayne.edu

E.M. LANE, D. LIU, X. HAN, L. SAMUELS, E. BENSON, L. LOGAN, K. GIFFORD, T. HOHMAN, E.C. SUMNER, K. WISNIEWSKI, E. WIGGINS, M. GODFREY, F. RUBERG \& A.L. JEFFERSON. Abnormal Nocturnal Fluctuations in Ambulatory Blood Pressure Relate to Worse Cognitive Performance in Older Adults: The Vanderbilt Memory \& Aging Project.

Objective: Ambulatory blood pressure (ABP) measures diurnal fluctuations in blood pressure at pre-specified intervals. This study examines whether ABP variability in systolic blood pressure (SBP) and diastolic blood pressure (DBP) relates to poorer cognitive performance in older adults with normal cognition (NC) and mild cognitive impairment (MCI).

Participants and Methods: At enrollment, MCI ( $\mathrm{n}=81,73 \pm 7,41 \%$ female) and NC participants ( $\mathrm{n}=83$, age $=71 \pm 7,48 \%$ female) from the Vanderbilt Memory \& Aging Project completed 24-hour ABP capturing SBP and DBP every 30 minutes and a comprehensive neuropsychological protocol. Linear regressions, adjusted for age, sex, race, education, diabetes, and prevalent cardiovascular disease, cross-sectionally related SBP and DBP variables to neuropsychological performances for all participants. Secondary models tested ABP by diagnosis interactions.

Results: Compared to dipping (expected 10-19\% decrease in nocturnal $\mathrm{SBP})$, a nocturnal SBP increase $(>0 \%)$ related to worse performance on the Montreal Cognitive Assessment $(\beta=-1.27, p=0.015)$, Boston Naming Test $(\beta=-1.83, p=0.001)$, and Hooper Visual Organization Test (HVOT; $\beta=-1.32, p=0.047$ ). Similar results were observed between nocturnal DBP increase and these same neuropsychological tests (all p-values $<0.030)$. Extreme DBP dipping ( $\geq 20 \%$ decrease in nocturnal pressure) related to worse performance on the Tower Test $(\beta=-2.18$, $p=0.018)$, Letter Fluency $(\beta=-4.45, p=0.045)$, and HVOT $(\beta=-1.25$, $\mathrm{p}=0.038)$. NC vs. MCI diagnosis did not modify these associations.

Conclusions: Abnormal nocturnal SBP and DBP fluctuations relate to poorer global cognitive, naming, letter fluency, planning, and object recognition performances among older adults, regardless of cognitive status. Future work is warranted to assess the long-term impact of abnormal nocturnal BP fluctuations on cognition.

Funding: F32-AG046093, K24-AG046373, IIRG-08-88733, R01-AG034962, UL1-TR000445

Correspondence: Elizabeth M. Lane, PhD Psychology, Vanderbilt Memory \& Alzheimer's Center, Vanderbilt University Medical Center, 2525 West End Avenue Suite 1200, Nashville, TN 37203. E-mail: elizabeth.m.lane@vanderbilt.edu

P. BREWSTER, J. JONES, C. HUGENSCHMIDT, K. SMITH, O. MEYER, L.B. ZAHODNE, D. MUNGAS \& M. THOMAS. Influence of Physical and Mental Health on Long-Term Cognitive Training Intervention Outcomes.

Objective: Poor health is associated with greater cognitive decline in old age. Whether cognitive training attenuates adverse health effects on cognition or improves subsequent physical or mental health is unknown. We examined relationships between self-reported physical and mental health, training gains, and subsequent cognitive trajectories over ten years in the ACTIVE trial.

Participants and Methods: ACTIVE participants were a volunteer sample of 2,832 older adults. Cognitive interventions consisted of 10 training sessions for memory, reasoning or processing speed, along with booster sessions 11 and 35 months post-training in a subset of adherent participants. Participants completed cognitive tests and the SF-36, a self-report measure of physical and mental health, at baseline and five follow-up assessments over 10 years.

We examined mean composite scores reflecting physical and mental health (as assessed by the SF-36) and performance on tests of memory. reasoning and processing speed. Multiple-group latent growth curve models (MG-LGGM) evaluated whether: (1) baseline physical and mental health influenced cognitive training gains; (2) cognitive training ameliorated the influence of poor physical and mental health on cognitive decline; and (3) cognitive training affected the course of self-reported physical and mental health over time.

MG-LGCMs were estimated separately for each cognitive outcome and by each health variable.

Results: Results suggested: (1) training gains were independent of baseline health; (2) reasoning training slightly reduced the effects of poor physical health on cognitive decline; and (3) processing speed training reduced 10-year declines in mental health, and reasoning training reduced 10-year declines in physical health.

Conclusions: Our main finding was that training outcomes are broadly independent of physical and mental health at baseline. Cognitive training may further yield modest long-term physical and mental health benefits.

Correspondence: Paul Brewster, University of Victoria, 3800 Finnerty Rd, Victoria, BC 90101, Canada.E-mail: pbrew@uvic.ca

D. BARULLI \& Y. STERN. Cognitive Reserve is associated with Strategy Selection Independently of Executive Abilities.

Objective: The ability to select the best cognitive strategy for a given task has been previously demonstrated to decline with age. However. Cognitive Reserve (CR) moderates this association between age and diminished strategy selection ability. The present study attempted to investigate whether this moderation holds even after controlling for executive abilities and general arithmetic skill.

Participants and Methods: A computational estimation task with an explicit strategy component was administered to 32 younger adults $($ mean age $=30.75 ; 23-47)$ and 33 older adults (mean age $=64.75$; 
50-75), wherein participants were asked to estimate the product of two 2-digit numbers by using one of two explicitly delineated strategies. Participants also received several neuropsychological assessments aimed at approximating their CR, including WAIS Vocabulary score and the AMNART. They were given a standardized test of their arithmetical abilities, and underwent testing of a selection of tests from the NIH EXAMINER battery to assess executive function.

Results: The results revealed a significant interaction of age group with AMNART IQ in predicting strategy selection, and a near-significant interaction of age group with WAIS Vocabulary score. None of the other covariates were significant predictors.

Conclusions: CR is significantly associated with strategy selection ability in both young and old adults, even after controlling for executive functions and arithmetic skill. We speculate that strategy use may play a mechanistic role in CR.

Correspondence: Daniel Barulli, Columbia University, 435 West 119th St., Apt 9M, New York, NY 10027. E-mail: djb2168@columbia.edu

\section{S. MALLYA \& A.J. FIOCCO. The Association between Global Health Habits and Cognitive Function in Non-Demented Older Adults.}

Objective: Previous research has demonstrated that psychosocial and behavioural factors can differentiate between older adults who maintain cognitive function into late life and those who experience cognitive decline. However, many of these studies are limited by the use of brief cognitive batteries that cannot provide detail about the specific cognitive domains affected by these health habits.

Participants and Methods: We studied 60 non-demented, healthy older adults enrolled in a larger study investigating the relationship between perceived stress and cognitive function. We explored whether a number of global health habits (e.g., sleep, social engagement, diet) were associated with declarative memory and executive function, two areas that are known to be vulnerable to age-related decline. Cognitive function was measured using the California Verbal Learning Test-II (CVLT II), Trail Making Test A \& B (TMT A \& B), and the FAS verbal fluency task. Multivariate regression was used to identify the global health factors associated with performance on these tasks.

Results: Global health habits were significantly associated with performance on the TMT A, F (1,32)=1.84, $\mathrm{p}=.045$; TMT B, $\mathrm{F}(1,32)=2.19$, $\mathrm{p}=.015$; and facets of the CVLT and FAS.

Conclusions: Considering the projected rise in dementia cases in North America, this research points to the importance of maintaining healthy lifestyle habits in older age to preserve cognitive health.

Correspondence: Sasha Mallya, MA, Psychology, Ryerson University, Ryerson University, Psychology Department, 350 Victoria Street, Toronto, ONM5B 2K3, Canada. E-mail: sasha.mallya@psych.ryerson. ca

B.M. SCOTT, J.E. MAYE, J. JONES, K. THOMAS, E. TRIFILIO, C. HASS, M. MARSISKE \& D. BOWERS. Walking as a Stressor in Cognitively Normal Older Adults: Post-Exercise Pulse Pressure Better Predicts Executive Function than Pre-Exercise Pulse Pressure.

Objective: Exercise "stress tests" are widely used to access functional cardiovascular abnormalities. In line with the view of exercise as a stressor, the present study examined cardiovascular reactivity in a sample of healthy nondemented older adults both before and immediately after light physical exercise. Based on known relationships between hypertension, executive function and cerebral white matter changes, we hypothesized that greater post-exercise reactivity, indexed by blood pressure and heart rate (HR), would be more related to worse performance on frontal-executive tasks than pre-exercise physiologic measures.

Participants and Methods: Participants included 84 nondemented community-dwelling older adults ( $\mu$ age $=78.0, \mu$ education $=16.8$ ) who received neuropsychological testing as part of the Village Interactive
Training and Learning Study. All participants underwent a Six Minute Walk Test (6MWT), along with blood pressure and HR measures obtained immediately before and after the walk. Pulse pressure (PP) was derived from blood pressure as an indicator of vascular auto-regulation. An executive function composite was computed from Lexical Fluency, Stroop, and TMT-B.

Results: As predicted, worse executive function scores were significantly associated with greater post-exercise PP $(\beta=-0.242, p=0.02)$ but not pre-exercise PP or HR. Pre-walking PP was unrelated to demographic variables (e.g., age, body mass index), but was significantly predicted by use of antihypertensives $(\beta=0.229, p<0.05)$. There was no relationship between use of hypertensive medications and executive function scores. Conclusions: Results suggest that PP following system stress in the form of walking may be more reflective of the state of vascular integrity and associated executive dysfunction in older adults than baseline physiologic measures. These findings may relate to less than optimal modulation of hypertension and dysfunctional autoregulatory mechanisms that disrupt fronto-subcortical networks.

Supported by Mcknight Research Foundation award (DB, MM)

Correspondence: Bonnie M. Scott, M.S., Clinical and Health Psychology, University of Florida, $538 \mathrm{NW}$ 39th Road, Unit 305, Gainesville, FL 32607.E-mail: bonnie.m.scott@gmail.com

E. TRIFILIO, B.M. SCOTT, K.R. THOMAS, L.L. JORDAN, J. JONES, M. MARSISKE \& D. BOWERS. Age Related Changes in Apathy, but not Anticipatory Anhedonia in Cognitively Normal Older Adults. Objective: Previous research with Parkinson disease (PD) suggests that apathy, a motivational disorder, is associated with anticipatory, but not consummatory anhedonia. These constructs map onto neural circuits underlying 'wanting' and 'liking', each differentially associated with dopaminergic and accumbens networks. In light of age-related neurotransmitter changes, we tested the hypothesis that apathy and anticipatory anhedonia would be associated with increased age in older adults. Participants and Methods: A sample of 113 cognitively normal well-educated older adults, between ages 50 and $93(\mathrm{M}=75.9$ ), underwent cognitive screening and completed the following measures: Apathy Scale (AS), Temporal Experience of Pleasure Scale (TEPS), Behavioral Inhibition System/Behavioral Approach System (BIS/BAS), Beck Depression Inventory-II (BDI-II). Anticipatory and consummatory anhedonia indices were computed from the TEPS. Data were analyzed with multiple hierarchical regressions.

Results: Approximately $21 \%$ met criteria for apathy vs $4 \%$ for depression, based on cutoff scores from the AS and BDI-II. Similar to PD patients, apathy was significantly associated with anticipatory, but not consummatory anhedonia. Increased age significantly predicted worse scores on the AS, the BDI-II, and drive scale from the BIS-BAS. However, neither anticipatory nor consummatory anhedonia were associated with age. Instead, an overall health comborbidity index significantly predicted anticipatory anhedonia.

Conclusions: Findings highlight age-related differences in apathy, drive, and depression symptoms in a sample of cognitively normal older adults. We did not find that anticipation of pleasure was influenced by age, but instead was associated with worsened health. However, because we did not sample younger adults, we cannot rule out possibility of naturally occurring age-related changes in anhedonia. Findings will be discussed from the perspective of neural, biologic and psychological changes in aging.

Supported by Mcknight Research Foundation (DB,MM)

Correspondence: Erin Trifilio, BA, Clinical and Health Psychology, University of Florida, 1185 SW 9th Rd, Gainesville, FL 32601. E-mail: etrif07@ufl.edu 


\section{S. ROGERS \& A. KNEVELBAARD. The Counterintuitive Relationship between Sleep Quality and Neuropsychological Functioning in Older Adults.}

Objective: In light of recent research showing that quantity of sleep may not affect older adults' cognitive abilities, the current study examined how quality of sleep, particularly broken sleep, may relate to the cognitive functioning of older adults.

Participants and Methods: A total of 90 older adults (68 women, 22 men) of mixed ethnicity with a mean age of 78.72 years $(S D=9.88)$ and mean education of 16.11 years $(\mathrm{SD}=2.23)$ completed a comprehensive battery of neuropsychological measures. On an independent questionnaire, they also indicated if they struggled with insomnia, broken sleep, and other sleep disturbances.

Results: MANOVAs revealed significant differences in the set of attention and memory measures between those with and without broken sleep, $\mathrm{ps}<.04$. When divided into verbal and visual memory, the scores on the sets of both verbal, Wilks $\lambda, .81, \mathrm{p}<.02$, and visual, Wilks $\lambda=$ $.83, \mathrm{p}<.05$, memory measures were significantly higher among those with broken sleep relative to those not reporting broken sleep. Individual t-test analyses showed that those with broken sleep performed better on W AIS-III Digit Span, $p<.03$, WMS-III Logical Memory I, $p<.05$, WMSIII Logical Memory II, p <.02, CVLT-II Total Trials 1-5, p <.01, and WMS-III Visual Reproduction I, $p<.01$. There were no significant differences in the set of processing speed, language, visuospatial functions, and frontal-executive abilities.

Conclusions: Contrary to popular expectation, older adults who experience more broken sleep appear to exhibit better neuropsychological performance in select areas of attention and memory, particularly learning relative to delayed recall. Broken sleep may therefore be associated with enhanced attention and memory in older adults, or it may be that those who are generally more cognitively active are simply experiencing more broken sleep. This should alleviate some older adults' concern about sleep quality and provide new direction for understanding those factors associated with better learning, memory, and attention. Correspondence: Steve Rogers, Ph.D., Psychology, Westmont College, 955 La Paz Road, Santa Barbara, CA 93108.E-mail: sarogers@ westmont.edu

\section{B.E. SNITZ, B.J. SMALL, T. WANG, C.H. CHANG, T.F. HUGHES \& M. GANGULI. Temporal Dynamics of Subjective and Objective Memory Change in Aging.}

Objective: To investigate whether subjective memory complaints and objectively measured cognition in aging influence each other over time, and if so, which is the stronger pathway of change: objective to subjective, subjective to objective, or a dual pathway of reciprocal influence ? Participants and Methods: Participants were enrolled in the population-based Monongahela-Youghiogheny Healthy Aging Team study of $\mathrm{n}=1982$ older adults residing in small-town Southwestern Pennsylvania and initially screened for severe cognitive impairment. We applied bivariate latent change score modeling to 5 annual cycles of multi-domain neuropsychological assessment and subjective memory questionnaire data. Within domains of memory, language, and executive functions, respectively, we tested 4 alternative hypotheses: 1) no coupling between subjective memory and objective cognition over time; 2) subjective memory as the leading indicator of change in objective cognition; 3) objective cognition as the leading indicator of change in subjective memory; 4) dual coupling over time, with both subjective memory and objective cognition leading subsequent change in the other.

Results: The dual-coupling models best fit the data for all three objective cognitive domains. Results also suggest that subjective-objective memory temporal dynamics differ qualitatively compared to other domains and may reflect deficits in insight and self-awareness specific to memory impairment.

Conclusions: Subjective memory and objectively measured cognition reciprocally influence each other over time, over a broad spectrum of cognitive functioning in aging. Study results further our understanding of the nuanced temporal dynamics between subjective and objective cognition in aging, and may shed light on inconsistent relationships in other studies.

Correspondence: Beth E. Snitz, Ph.D., Neurology, University of Pittsburgh, 3501 Fifth Avenue, Suite 830, Pittsburgh, PA 15213. E-mail: snitzbe@upmc.edu

K. MCINERNEY, E. HAREN, A. PALKO, J. SCRUGGS, T. THIEMAN \&. J. SUHR. Neurocognitive Correlates of Hazard Perception in Normal Aging.

Objective: Hazard perception is the ability to detect and react to dangerous situations on the road. It is a skill that has been found to have particularly strong associations with accident involvement (Horswill \& McKenna, 2004). Unfortunately, data on hazard perception in older adults is scarce. Unlike other ways to examine driving (e.g., on-the-road tests, driving simulator tasks, UFOV performance) for which there is ample data on older adults, hazard perception has a much longer tie to the novice driver literature and has only recently been examined in older adult driving. The purpose of the study was to investigate the neurocognitive correlates of hazard perception in a non-demented older adult population.

Participants and Methods: 60 (21 male) participants between the ages of 61 and 88 (mean age $=70.72$ ) were screened for dementia using the RBANS. They were also administered a series of neuropsychological measures as well as a Hazard Perception Test (HPT).

Results: Better reaction time to touching the target hazards on the HPT was correlated with better performance on Trailmaking Test B $(r=.265$, $\mathrm{p}=.020)$, on Useful Field of View (UFOV) part $3(\mathrm{r}=.295, \mathrm{p}=.011)$, and on a list recognition task $(\mathrm{r}=-.383, \mathrm{p}=.001)$. A lower number of errors on the HTP was associated with better story recall $(r=-.215$, $p$ $=.050)$, better list learning $(\mathrm{r}=-.263, \mathrm{p}=.021)$, list recall $(\mathrm{r}=-.270$, $\mathrm{p}=.019)$ and list recognition $(\mathrm{r}=-.393, \mathrm{p}=.001)$, better performance on Trailmaking Test A $(\mathrm{r}=.221, \mathrm{p}=.045)$ Trailmaking Test $\mathrm{B}(\mathrm{r}=$ $.453, \mathrm{p}=.000)$, and UFOV part $3(\mathrm{r}=.333, \mathrm{p}=.005)$.

Conclusions: As expected hazard perception skills were associated with both memory and executive functioning skills in this older adult sample. Correspondence: Katalina McInerney, Clinical Psychology, Psychology, Ohio University, 200 Porter Hall, Ohio University, Athens, OH 45701. E-mail:km171107@ohio.edu

C.P. MOLDOVAN, M. MCDONNELL, C.H. NORSETH, Y. XU, E. LIMA, R. JONES, F.O. LEAF, A. ARÉCHIGA, S. RAJARAM \& J. SABATÉ. The Impact of Age, Education, and Spiritual WellBeing on Executive Functioning in Healthy Older Adults.

Objective: The progressive increases in life expectancy and dementia in the United Sates highlight the importance of preventative care and maintenance of physical and cognitive functions. Executive functioning (EF) represents the executive control center of the brain that affects behavior and cognition. EF impacts performance on all activities of everyday living such as walking, driving, and managing a household. Previous studies have reported a positive association between education and EF. Alternatively, prior research has shown that EF declines with age; thus, preserving EF is crucial in older adults. The impact of sociocultural factors, such as spirituality, on EF has not been examined in the current literature. This study tested the influence of age, education, and spiritual well-being on EF in healthy older adults.

Participants and Methods: Participants were 365 adults ages 62-80 years $(M=69.57, \mathrm{SD}=3.90)$ with a high level of education $(M=15.65$, $\mathrm{SD}=2.40$ ). The Trail Making Test, Part B measured EF and the Self-Efficacy and Life Schemes subscales of the Spirituality Index of Well-Being measured spiritual well-being. Regression analysis was conducted to examine age, education, and spiritual well-being as predictors of EF.

Results: The analysis revealed that $8.9 \%$ of the variance in EF was accounted for by age, education, and spiritual well-being, $\mathrm{F}(4,343)=$ $8.40, \mathrm{p}<.001$. EF was significantly predicted by age $(\beta=.124, \mathrm{t}=2.41$, 
$p<.05)$, education $(\beta=-.125, \mathrm{t}=-2.35, \mathrm{p}<.05)$, and self-efficacy $(\beta$ $=-.238, \mathrm{t}=-3.44, \mathrm{p}<.01)$.

Conclusions: Results revealed spiritual well-being as a new predictor of EF. Further, our findings support previously reported relationships between age, education, and EF. Outcomes from this study have major implications for older adults, suggesting that education and a sense of well-being and self-confidence may mitigate the effects of aging on cognitive functioning.

Correspondence: Christina P. Moldovan, Bachelor of Arts, Psychology, Loma Linda University, 11130 Anderson Street, Loma Linda, CA 92350.E-mail: cmoldovan@llu.edu

A. KARIMIAN, Z. MAHMOOD, A. STEINER, A. ARENTOFT, P. SAYEGH, N.S. THALER, S. ROYE, M.U. ELLIS \& A.D. THAMES. The Interactive Effects Of Age And Stress On Cognition And Functional Abilities Among HIV+ Individuals.

Objective: Research has explored the relationship between aging and stress on cognitive functioning. However, few studies have investigated this relationship in HIV+ populations, who often report high levels of psychosocial stress. This study explored how age and recent stress impact cognition and functional abilities in HIV+ individuals.

Participants and Methods: Eighty-two HIV+ participants were recruited from the community. Participants were grouped by age (younger $<50 ; n=26$ vs. older $>50 ; n=56)$. Participants completed a questionnaire that assessed recent stress and a comprehensive neuropsychological battery. The Barkley Functional Impairment Scale (BFIS) assessed functional impairment.

Results: Younger adults reported more recent stress than older adults, $F(1,81)=3.89, p=.05$. Older adults performed worse on neurocognitive testing, $\mathrm{F}(1,81)=15.13, p<.0001$, and reported greater functional impairment, $F(1,81)=4.46, p=.04$. Stress was associated poorer performance in cognitive domains of attention and processing speed ( $p$ 's $<.05)$. Stress was also associated with greater functional impairment, $\mathrm{r}(82)=.26, p=.02$. While there was a non-significant age $X$ stress interaction trend on global neurocognitive functioning, $F(1,82)=2.109$, $\mathrm{p}=.10$, there were significant interactions for attention, $\mathrm{F}(1,82)=6.73$, $\mathrm{p}=.01$, and processing speed, $\mathrm{F}(1,82)=4.28, \mathrm{p}=.04$. Older adults with high stress levels performed worse on these cognitive domains. There was no significant age $\mathrm{X}$ stress interaction on functional impairment. Conclusions: Findings suggest that while younger HIV+ individuals report more distress due to recent stressors, the impact of stress on cognition is greater among older HIV+ individuals. The impact of stressors for older adults should be considered despite reports of minimal distress. Correspondence: Ahoo Karimian, M.A., Psychiatry and Biobehavioral Sciences, University of California, Los Angeles, 760 Westwood Plaza C8-226, Los Angeles, CA 90095. E-mail: AKarimian@mednet.ucla.edu

J. GRIGSBY, M. SULLAN, A. SHARMA, J. BAXTER \& R. HAMMAN. Sensory Loss and Cognitive Decline among Older People: The San Luis Valley Health and Aging Study (SLVHAS).

Objective: Recent research suggests an association between hearing loss and dementia among older people. We tested whether hearing loss and visual impairment affect cognition, producing mild cognitive impairment rather than frank dementia. We examined the relationships between sensory loss (hearing and vision) and impairment of two aspects of cognition (mental status and executive functioning).

Participants and Methods: The San Luis Valley Health and Aging Study (SLVHAS) is a population-based epidemiologic study of chronic illness and disability among persons age 60 and above in rural southern Colorado. We conducted both cross-sectional and longitudinal analyses, adjusted for demographic and clinical variables. Follow-up interviews were conducted an average of of 22 months after baseline. Hearing impairment was measured by self-report and free field voice testing (FFVT), a brief hearing screening exam. Visual impairment was assessed by two self-report interview questions. Mental status was measured by the Mini Mental State Exam (MMSE), and executive functioning by the Behavioral Dyscontrol Scale (BDS).

Results: All analyses used multiple regression, adjusted for age, sex, education, ethnicity, and comorbid chronic illnesses. In cross-sectional analyses $(n=1,077)$, visual impairment and hearing loss independently contributed to deficient performance on both the MMSE and the BDS, with all $p$-values falling between $\alpha=0.026$ and $\alpha=0.001$. In longitudinal analyses $(n=1,297)$, change in BDS score after 22 months was predicted by both hearing (FFVT) and vision (self-report). Change in MMSE score was predicted by self-rating of visual impairment, but fell short of significance for hearing loss $(p=0.10)$.

Conclusions: The results suggest that among older people, both hearing loss and visual impairment are independently associated with impaired mental status and executive functioning, and with increased risk for cognitive decline.

Correspondence: Jim Grigsby, PhD, Psychology; Medicine, University of Colorado Denver, University of Colorado Denver, 1200 Larimer Street, Campus Box 173, Denver, CO 80217-3364.E-mail: jim.grigsby@ ucdenver.edu

J. EPPIG, L. CAMPBELL, E.C. EDMONDS, A.J. JAK, L. DELANOWOOD, M. SANDERSON-CIMINO \& M.W. BONDI. Superior Verbal Memory in SuperAgers: Generalizability to Learning, Visual Memory, Language, and Executive Functioning.

Objective: Research provides evidence for the existence of 'SuperAgers' - older adults with superior age-normed performance on verbal memory testing. We examined how learning contributes to superior delayed recall and potential generalization to other measures of memory, language, and executive functions.

Participants and Methods: 21 SuperAgers and 21 typically-aging older adults received a comprehensive neuropsychological assessment of memory, language, and executive functioning. Participants were $\geq 70$ years and did not meet Jak/Bondi criteria for MCI. SuperAgers were defined as performing $\geq 1.5 \mathrm{SD}$ above norms on long delay free recall (CVLT/CVLT-II) and $\geq-1.0$ SD above norms on tests of language and executive functions.

Results: There were no differences in demographics, depression, or global cognition between groups ( $p$ 's $>10)$. SuperAgers recalled significantly more words across CVLT immediate recall trials $(p<.012)$ than typical-agers. However, there were no differences in semantic clustering, serial clustering, or learning slope (all p's >.25). On Logical Memory, there was no difference between groups in immediate recall ( $p>.21)$; however, SuperAgers demonstrated significantly better recognition discriminability $(\mathrm{p}=.01)$ and a trend towards greater delayed recall $(p=.07)$. No differences were noted on visual learning or memory ( $p$ 's >.16). SuperAgers were significantly faster on Trails B than typical agers $(p=.048)$, though no other differences in language or executive functions were noted.

Conclusions: Superior verbal memory in SuperAgers appears to be related to greater immediate recall output, although verbal clustering strategies did not differentially aid in that learning. Superior verbal memory does not appear to generalize to visual memory or language abilities, but does appear to be related to better executive functioning. Future studies should explore how additional measures in SuperAging inclusion criteria affects the generalizability of superior memory performance to other cognitive domains.

Correspondence: Joel Eppig, B.A. in psychology, Clinical Psychology, SDSU/ UCSD, 1205 Colusa St, Apt \#3, San Diego, CA 92110. E-mail: joel.eppig@temple.edu

K. BEITZ, F. KELLER, A. SABARTINELLI, B. MARCHANT \& H.P. DAVIS. Performance on a Manual and Computerized Tower of London across the Life Span.

Objective: Shallice (1982) developed the Tower of London (TOL) to examine problem solving in patients with either anterior or posterior damage in the right or left cortex. The Drexel (TOL) is a manual version 
that has norms across the lifespan. We have developed a computerized version that allows the creation of any problem set with the three bead TOL. Performance on both versions of the TOL were examined across the life span to determine the suitability of the computer version for evaluation of problem solving.

Participants and Methods: Participants (N=154) were divided into five age groups, each encompassing $20 \%$ of the data: $7-18 ; 19-20$; 21-24; 25-38; and 39-89 years of age. Participants were administered the manual version of the Drexel Tower of London or a computerized version.

Results: Excess moves (SD) for the 5 age groups on the Drexel TOL from the youngest to oldest individuals were 36.8 (2.7), 28.1 (2.9), 28.5 (3.8), 28.3 (3.1), and 34.7 (3.1) and for the computer version were 33.7 (3.4), 22.3 (2.7), 25.4 (3.5), 23.8 (3.1), and 30.7 (3.6). An ANOVA with age as the between variable and excess moves as the within variable detected a significant effect of test type, $F(1,148)=5.39, p=.02$, MSE $=235.04$, age $\mathrm{F}(4,148)=3.12, \mathrm{p}=.02$, and no significant interaction. LSD comparisons between age groups revealed that the youngest and oldest age groups had the most difficulty on both tasks compared to young adults (aged 19-38). Pearson correlations for the age groups from youngest to oldest for excess moves were $.09, .21, .55, .28$, and 08 . Conclusions: Performance on the two test types were essentially parallel across the life span with fewer excess moves on the computerized version. The correlations indicated a weak relationship for performance on the two test types by the youngest and oldest age groups with a moderate relationship for the remaining age groups. The advantages of ease of administration, recording of each move, and millisecond timing provide support for use of the computerized TOL.

Correspondence: Hasker P. Davis, Ph.D., Psychology, University of Colorado Colorado Springs, Department of Psychology, 1420 Austin Bluffs Parkway, Colorado Springs, CO 80918.E-mail: hdavis@uccs.edu

Y. XU, A. HOLNESS, C.H. NORSETH, C.P. MOLDOVAN, I.A. PALMA, M. MCDONNELL, F.O. LEAF, R.M. WEISE, F. RIVERA, A. ARÉCHIGA, S. RAJARAM \& J. SABATÉ. Depression: The Effect of Mood on Cognitive Functioning in a Sample of Healthy Older Adults.

Objective: Previous studies have found that individuals with late life depression perform poorly in the areas of processing speed, attention, and executive functions. Depression has been found to be a prodromal symptom and risk factor for dementia. Researchers have not fully investigated how cognitive functioning is impacted by modest elevations of depressive symptoms, specifically in healthy older adults. The current study evaluated the effect of minimal depressive symptoms on executive functions, visuoperceptual processing, psychomotor speed, and memory retrieval.

Participants and Methods: The study consisted of 365 healthy older adults. The TMT-B was used to measure executive control and attention. Psychomotor speed and visuoperceptual processing were measured by the SDMT. The BNT was used to measure memory retrieval and confrontation naming. Depressive symptoms were measured using the Hamilton Depression Inventory.

Results: The results suggested that participants with a slight elevation in depressive symptoms performed significantly lower in executive control and working memory $(\mathrm{M}=116.87, \mathrm{SD}=65)$ as well as in visuoperceptual processing and psychomotor speed $(\mathrm{M}=48.35, \mathrm{SD}=9.11)$, compared to participants with no significant depressive symptoms, respectively $(\mathrm{M}=93.00, \mathrm{SD}=45.09),(\mathrm{M}=45.21, \mathrm{SD}=8.39), \mathrm{t}(41)=-2.2, \mathrm{p}<.05$. Individuals with no significant depressive symptoms showed better performance in memory retrieval and on a confrontation naming task $(\mathrm{M}=54.90, \mathrm{SD}=5.2)$, compared to those with minimal depressive symptoms $(\mathrm{M}=51.95, \mathrm{SD}=6.54), \mathrm{t}(43.98)=2.72, \mathrm{p}<.01$.

Conclusions: Healthy aging adults with minimal depressive symptoms may have a greater risk of experiencing additional cognitive decline. Thus, depressive symptoms should be monitored in order to prevent a decline in mental functions. If early assessments detect noticeable mood change, preventive interventions, such as cognitively stimulating activities or increased social support could be implemented to decrease the risk for cognitive impairment and dementia.

Correspondence: Yangmu Хи, M.A., Psychology, Loma Linda University, 901 Golden Springs Dr., Unit \#A10, Diamond Bar, CA CA. E-mail: jymqx224@gmail.com

\section{E.I. FRANCHOW \& Y. SUCHY. Complex Motor Planning: Sensitivity to Cognitive Concerns of Community-Dwelling Older Adults.}

Objective: Clinical experience suggests that many older adults self-reporting cognitive concerns perform within normal limits on traditional cognitive measures. However, previous work in our laboratory demonstrates that unusual fluctuations in planning time on a motor programming task are sensitive to subtle pre-clinical cognitive changes in community-dwelling elders (Suchy et al., 2011). The purpose of the current study was to determine whether older adults with cognitive concerns exhibit unusual fluctuations in motor planning, suggestive of subclinical cognitive changes.

Participants and Methods: 32 adults ( $M=68.5$ years old; $62.5 \%$ female; $\mathrm{M}=14.8$ years of education) completed 7 items assessing cognitive concerns in the recent past (Concern), a screening measure of global cognitive functioning (Dementia Rating Scale; DRS-2), and a computerized motor programming task (Push-Turn-Taptap Task; PTT) assessing planning time across 4 blocks of increasing complexity.

Results: Consistent with clinical experience, High and Low Concern groups (created via median split) did not differ on total age-corrected DRS-2 scores $(t(28)=-4.09, p=.686)$. Repeated measures analysis of variance with median planning times on PTT as the dependent variables, PTT Blocks as a within-subjects factor, and Concern as the between-subjects factor showed an interaction between PTT Block and Concern $(\mathrm{F}(3,26)=3.69, \mathrm{p}=.024)$, demonstrating a greater increase in planning time in the concerned group on the most complex block. Groups were comparable on age, sex, years of education, and an estimate of premorbid intelligence based on word reading.

Conclusions: These results suggest that motor planning may be sensitive to subtle preclinical changes and that such changes may underlie cognitive concerns in older adults.

Correspondence: Emilie I. Franchow, MS, Psychology, University of Utah, 220 E. 3300 S., Apt. 212, Salt Lake City, UT 84115. E-mail: emilie.franchow@psych.utah.edu

\section{A.R. MENATTI, K. MCINERNEY, P. DEMIREVA, B. MORGAN \& J. SUHR. Longitudinal Prediction of Driving Risk in an Older Adult Sample.}

Objective: Recent studies have suggested that dementia/memory concerns, visuospatial abilities, and executive functioning are related to driving risk in older adults, although many existing studies are cross sectional rather than longitudinal in nature. In the present study, we examined whether measures of memory and/or executive functioning would be related to driving risk over time.

Participants and Methods: 43 older adults who participated in two larger driving studies were examined to address the study's hypotheses. These 43 adults had participated in two studies and consented to have their data linked. Participants ranged in age from 61-89 (mean 71) and the time interval between the two studies was on average 26 months (range 15-34 months). Data at both time periods included neuropsychological screening and various experimental driving measures. Time 1 evaluation included the Repeatable Battery for the Assessment of Neuropsychological Status (RBANS) and the Trailmaking Test (TMT); each subtest was converted to "impaired" or "not impaired" (using a $<16$ th percentile cutoff). Time 2 evaluation included the Useful Field of View (UFOV) and a Hazard Perception (HP) driving test.

Results: Number of impaired subtests across all cognitive domains at Time 1 predicted Time 2 UFOV risk category $(.34, \mathrm{p}=.02)$ and Time 2 HP errors (.42, $p=.005)$ and was also related to experience of a driving 
accident in the past year $(\mathrm{p}=.03)$ and having a traffic violation in the past year $(p<.001)$. Number of Time 1 impaired memory tests was not associated with these variables, but number of Time 1 impaired executive measures was associated with driving risk (all p<.05). Neuropsychological performance was not related to self-reported restriction of driving (which was usually limitations on night driving).

Conclusions: Results suggest that it is impaired executive functioning, not impaired memory, that may be particularly predictive of driving risk in older adults.

Correspondence: Andrew R. Menatti, M.S., Psychology, Ohio University, 200 Porter Hall, Athens, OH 45701. E-mail: am855010@ohio.edu

A. COOLIN, E. ERDFELDER, D.M. BERNSTEIN, A.E. THORNTON \& W.L. THORNTON. Inhibitory Control Underlies Recollection and Reconstruction Processes in Older Adults' Hindsight Judgments.

Objective: Outcome knowledge influences recall of earlier predictions of an event. Older adults are particularly susceptible to this hindsight bias (HB) phenomenon. We recently extended the multinomial processing tree (MPT) model for HB (Coolin et al., 2014) to incorporate individual differences in cognitive abilities in the underlying recollection and reconstruction processes. This prior work revealed that older adults with poorer inhibitory control had lower recollection and higher reconstruction bias. In this study, we assessed whether experimentally increasing inhibition demands during the HB task affects the underlying HB processes, and whether the effects of the manipulation on these processes vary with cognitive ability.

Participants and Methods: We collected cognitive ability and HB data on 80 older adults (Mage $=71.40$, range $=65$ to 87). Using our extended MPT model, we directly incorporated individuals' cognitive ability data into parameter estimation using a logistic link function, and then compared individuals' parameter estimates across a standard memory judgment condition and a cognitive load condition.

Results: Cognitive load lowered recollection rates (additive manipulation effect $=-0.17, p=.02$ ). Furthermore, cognitive load tended to increase reconstruction bias in individuals with strong inhibitory control, resulting in these individuals performing more similarly to individuals with poor inhibitory control (interactive effect $=-0.41, \mathrm{p}=.059$ ).

Conclusions: Our findings provide empirical support for the role of inhibitory control in recollection and reconstruction processes underlying older adults' hindsight judgments, and suggest that the degree to which inhibitory control influences reconstruction bias varies with available processing resources and task demands.

Correspondence: Alisha Coolin, Simon Fraser Univeristy, 312-3575 Euclid Avenue, Vancouver, BCV5R6H5, Canada.E-mail: acc27@sfu.ca

A. TSAPANOU, N. SCARMEAS, Y. GU, D.M. O' SHEA, T. EICH \& Y. STERN. Relationship of Self-reported Sleep Problems to Cognition in Aging.

Objective: Changes in the pattern of sleep are a common feature of aging. Recent findings suggest that poor subjective sleep quality may be associated with cognitive impairments. We examined cross-sectionally the association between sleep problems and cognition in community-dwelling elderly adults.

Participants and Methods: Participants $(\mathrm{n}=2358)$ with a mean age of 79.58y (SD: 6.51), were selected from the Washington Heights-Inwood Community Aging Project (WHICAP). 417(17.7\%) had MCI while $237(10 \%)$ were demented. Sleep problems were assessed using the Sleep Scale from the RAND Medical Outcome Study. This is a self-report 12item questionnaire which yields 5 summary measures: sleep disturbance, snoring, sleep short of breath/with headache, sleep adequacy, and sleep somnolence. Cognitive functioning was assessed with a neuropsychological battery. Four cognitive composite scores were calculated: memory, language, speed of processing, and executive function.

Results: Generalized Linear Model analyses revealed that increased sleep somnolence (including; feeling drowsy/sleepy, having trouble staying awake, taking naps during the day) was associated with a decrease in language $(\beta=-.017, p=.000)$, speed of processing $(\beta=-.024$, $p=.009)$, and memory performance $(\beta=-.018, p=.003)$. No significant associations were revealed between other sleep measures and the $\operatorname{cog}$ nitive domains. After excluding the demented participants, results remained significant for language $(\beta=-.193, p=.005)$, speed $(\beta=-.317$, $\mathrm{p}=.012)$, and memory $(\beta=-.201, \mathrm{p}=.042)$.

Conclusions: Our results suggest that increased sleep somnolence is significantly associated with impaired cognition in the elderly population. A possible explanation could be that sleep problems affect concentration. which, results in decreased cognitive performance. According to the existing literature, a mental or organic pathologic condition could also affect both sleep and cognition.

Correspondence: Angeliki Tsapanou, Taub Institute, Columbia University Medical Center, 630 West 168th Street, New York, NY 10032. E-mail:at2859@cumc.columbia.edu

S. GARCIA, A. FEDOR \& J. GUNSTAD. Sleep as a Mediator for the Effects of an Exercise Regimen on Cognition in Older Adults. Objective: Increased life expectancy has led to a greater prevalence of age-associated cognitive impairment. Exercise has been shown to reverse some cognitive deficits; however, it does not fully explain these gains. Sleep might help account for the cognitive benefits of exercise, as it associated with cognition and exercise levels in older adults.

Participants and Methods: A total of 69 older adults were assigned to either a brief water exercise program $(\mathrm{n}=33$, age $63.1 \pm 7.56$ years, $78.6 \%$ female) or control group $(\mathrm{n}=36$, age $65.6 \pm 6.96$ years, $75.8 \%$ female). A brief neuropsychological test battery was conducted before and after the exercise program. Actiwatch devices were worn throughout the study to determine sleep efficiency.

Results: Repeated measures ANOVAs indicate that the exercise group improved in attention $(\mathrm{F}(1,25)=12.40, \mathrm{p}<0.01)$, executive function $(\mathrm{F}(1,25)=69.44, \mathrm{p}<0.01)$, memory $(\mathrm{F}(1,25)=13.27, \mathrm{p}<0.01)$, and language $(\mathrm{F}(1,26)=6.23, \mathrm{p}=0.02)$ whereas the control group remained stable. Sleep efficiency in the exercise group did not change and worsened in controls. Mediation analyses showed sleep efficiency did not mediate the relationship between exercise and attention $(\mathrm{CIb}=$ $-0.00,0.01)$, executive function $(\mathrm{CIb}=-0.09,0.05)$, memory $(\mathrm{CIb}=$ $-0.04,0.47)$, or language $(\mathrm{CIb}=-0.04,0.58)$.

Conclusions: Findings suggest that greater exercise is related to better cognitive performance in older adults, but sleep may not be affected and does not mediate this relationship. Further work is needed to clarify the mechanisms linking exercise and cognition in this population.

Correspondence: Sarah Garcia, M.A., Kent State University, 144 Kent Hall, Kent, OH 44240.E-mail: SGarcia6@kent.edu

D.S. BROWN, L.S. HYNAN, M.F. WEINER, L.H. LACRITZ \& A. MINHAJUDDIN. Relationship of Metabolic Syndrome to Rate of Cognitive Decline in Normal Controls, Mild Cognitive Impairment and Alzheimer's Disease.

Objective: To determine if metabolic syndrome (MetS) contributes to a higher rate of decline in cognitive abilities of participants in a longitudinal Alzheimer's disease (AD) research cohort.

Participants and Methods: 6,687 participants were followed for a median of 5 years as part of the National Alzheimer's Coordinating Center's longitudinal study cohort. At baseline, 3704 had no cognitive impairment, 1725 had mild cognitive impairment (MCI), and 1258 had dementia. Participants were administered a neuropsychological battery including Mini-Mental State Examination (MMSE), Trail Making Tests A \& B (TMT-A and TMT-B), Wechsler Adult Intelligence Scale-Revised Coding, and 30-item Boston Naming Test. At entry, 3139 (46.9\%) participants had MetS defined as having at least two of the following: a) BMI $>30$, b) hypertension c) diabetes, and d) hypercholesterolemia. The data were analyzed using hierarchical linear regression models to assess if participants with MetS have a higher rate of cognitive decline over time, adjusting for age, gender, education, and APOE status. 
Results: Participants with MetS had a higher rate of decline in MMSE and TMT-B performance, while no differences were seen for other cognitive measures.

Conclusions: These results support MetS as a risk factor for more rapid rate of cognitive decline in older adults and warrants further exploration. Correspondence: Daniel S. Brown, Bachelor of Music in Jazz Studies and Psychology, Division of Psychology, Department of Psychiatry, University of Texas Southwestern Medical Center, 3443 Mahanna St. \#3211, Dallas, TX 75209. E-mail: brown.daniel@gmail.com

A.L. NORMAN, J.L. WOODARD, K.A. NIELSON, M. SEIDENBERG, J. SMITH, S. DURGERIAN, M.A. SUGARMAN \& S.M. RAO. Baseline Structural MRI Volumes and Five-year Follow-up Neuropsychological Performance in Cognitively-Intact Older Adults.

Objective: Examination of how structural neuroimaging relates to longterm neuropsychological outcome in cognitively-intact older adults is somewhat limited. However, these studies may inform our understanding of pathological aging. We examined the relationship between structural MRI (sMRI) volumes at baseline in cognitively healthy older adults and their neuropsychological performance after five years.

Participants and Methods: Sixty-nine cognitively-intact older adults were administered a neuropsychological test battery and sMRI at baseline and after five years. A principal components analysis (PCA) using seven neuropsychological tests administered at the five-year visit identified three components explaining $66 \%$ of the total variance: Verbal Memory, Processing Speed, and Executive Functioning. Regression models used age (step 1) and individual baseline structural MRI volumes (step 2) as predictors of participants' five-year visit neuropsychological component scores. Structural volumes (intracranial volume-corrected) included total gray and white matter, hippocampal, cerebellar, and frontal gray matter regions.

Results: Baseline age was associated with regional brain volumes. Baseline right superior frontal gray matter volume predicted five-year verbal memory performance beyond age $(p<.01)$. Baseline hippocampal presubiculum and cerebellar volumes predicted five-year processing speed beyond age $(p<.01)$. Baseline right superior frontal gray matter and hippocampal presubiculum volumes predicted five-year executive functioning beyond age $(p=.02)$.

Conclusions: Baseline superior frontal gray matter predicted both verbal memory and executive function after five years. The right presubiculum correlated with future processing speed and executive function, suggesting a common attentional component, or perhaps representing relative efficiency of frontal-presubiculum white matter projections. Further, results are consistent with recent experimental findings that place greater emphasis on cerebellar involvement in processing speed. Correspondence: Andria L. Norman, M.A., Psychology, Wayne State University, 5057 Woodward Ave. 7th Floor, Detroit, MI 48201. E-mail: andria.norman@wayne.edu

A.C. GENESER, S.A. SPERLING, K. FUCHS, B.M. MIZUKI, S.V. FARLEY, I. WILLIAMS \& C.A. MANNING. Subjective Memory Complaints in Older African Americans.

Objective: Ten to fifty percent of older adults report subjective memory complaints (SMCs). Although the relationship between SMCs and cognitive dysfunction is unclear, SMCs have been associated with depression, anxiety, and increased risk of future cognitive decline. This study examined whether SMCs were associated with objective memory impairments, depression, or anxiety in older African Americans.

Participants and Methods: Ninety-six older African Americans without a diagnosis of mild cognitive impairment or dementia were recruited at their primary care visit, scheduled for reasons unrelated to cognitive complaints. Patients completed the Computer Assessment of Memory and Cognitive Impairment (CAMCI) that included questions regarding SMCs, depression, and anxiety. The memory subtests from the CAMCI (i.e., verbal word recall, nonverbal recall, functional recall, and incidental recall) were examined.

Results: Significantly more people with SMCs $(67 \%)$ had self-reported depression $(\mathrm{x} 2=18.68, \mathrm{p}<.0001)$ and anxiety $(\mathrm{x} 2=14.79, \mathrm{p}<.0001)$. When controlling for mood, age, and education, SMCs were not significantly correlated with objective measures of memory \{verbal memory $[\mathrm{F}(1)=.189, \mathrm{p}=.66]$, nonverbal memory $[\mathrm{F}(1)=.30, \mathrm{p}=.58]$, functional memory $[F(1)=2.58, p=.11]$, or incidental memory $[F(1)=.95, p=.33]$. Conclusions: These results indicate that African Americans with SMCs had higher rates of depression and anxiety. However, SMCs were not associated with actual memory impairment. These findings highlight the need for integrating neuropsychological testing in primary care and similar settings, as SMCs are unreliable indicators of objective memory impairment. Longitudinal studies are needed to determine if African Americans with SMC are at increased risk for future cognitive decline. Correspondence: Alexandra C. Geneser, PsyD, Neurology, University of Virginia, 423 North 1st St, Apt 3B, Charlottesville, VA 22902. E-mail: AG6BZ@virginia.edu

S. PORAT, K. HWANG, N. GOUKASIAN, E. WOO, T. ZANTO \& L. APOSTOLOVA. Personal Experience with Dance and Cortical Gray Matter Thickness in the Cognitively Normal and Mild Cognitive Impaired Elderly.

Objective: To investigate the effect of dancing experience on gray matter thickness and cognitive performance in elderly with and without mild cognitive impairment (MCI).

Participants and Methods: 39 cognitively normal and 48 MCI participants from the longitudinal ImaGene study at the Mary S. Easton Alzheimer's Disease Center at the University of California - Los Angeles, were mailed a questionnaire regarding their lifetime experience with music, dance, and song. Participants were self-identified as dancers or non-dancers. All participants received structural 1.5T MRI scans and detailed clinical and neuropsychological evaluations. An advanced 3D cortical mapping technique was then applied to calculate cortical thickness values.

Results: There were no significant differences between the groups in terms of age, gender, education, or MMSE, however, diagnosis was trending. Dancers had significantly lower cortical thickness in areas such as primary somatosensory cortex, motor cortex, and inferior frontal regions, when compared to non-dancers. The results remained significant after correcting for diagnosis. Despite having a thinner cortex (i.e. less gray matter), dancers preformed better in certain cognitive domain tasks involving learning and memory, such as the California Verbal Learning Test (CVLT) short delay free recall $(\mathrm{p}=0.004)$, CVLT long delay free recall $(p=0.003)$, and CVLT trials $1-5(p=0.001)$.

Conclusions: In agreement with previous studies dancers had thinner gray matter relative to non-dancers in cortical areas that are crucial to motor activity and coordination. Interestingly, dancers also showed better cognitive performance suggesting that dance as an art form may result in an enhancement of cognitive reserve.

Correspondence: Shai Porat, Bachelor's of Science, Neurology, University of California, Los Angeles, 10911 WEVBURN AVE, Suite 200, Los Angeles, CA 90024.E-mail: sporat@mednet.ucla.edu

C.C. STEWART, P. BOYLE, L. YU \& D.A. BENNETT. Diverse Resources Contribute to Decision Making in Non-demented Older Adults.

Objective: Aging and lower education adversely affect decision making in non-demented older adults. However, it is unclear if aging and education affect decision making directly or indirectly through cognition, contextual factors such as literacy, or personality-related factors such as risk aversion. This study aimed to determine if the relations of aging and education with decision making represent direct effects versus indirect effects due to cognition, literacy, or risk aversion.

Participants and Methods: Participants were 621 non-demented older adults. All underwent assessments of decision making, cognition, 
literacy, and risk aversion. We used path analysis to test a model linking age and education to decision making directly and indirectly through cognition, literacy, and risk aversion. The model also linked cognition to decision making directly and indirectly through literacy and risk aversion.

Results: Overall model fit was adequate $\left(\mathrm{X}^{2}=1.95\right.$ ( $\left.\mathrm{df}=1, p=.16\right)$, RM$\mathrm{SEA}=.04, \mathrm{CFI}=.99)$. The total standardized effect of age on decision making was $-0.30(p<.0001)$. Most of this represented indirect effects through cognition ( $47 \%$ of total effect) and literacy $(19 \%)$; the indirect effect through risk aversion was small $(2 \%)$. The total standardized effect of education on decision making was $0.33(p<.0001)$. Most of this reflected indirect effects through cognition (43\%) and literacy $(24 \%)$; the contribution through risk aversion was minimal $(3 \%)$. The total standardized effect of cognition on decision making was 0.48 ( $p<.0001)$. The majority of this was direct $(62 \%)$ or due to an indirect effect through literacy $(36 \%)$; the indirect effect through risk aversion was small $(2 \%)$. Conclusions: In non-demented older adults, the adverse effects of aging and lower education on decision making are largely due to lower cognition and literacy. Indirect effects on decision making through risk aversion were small. Findings are consistent with a prior study showing that higher literacy bolsters decision making in the context of aging and lower cognition.

Correspondence: Christopher C. Stewart, Ph.D., Behavioral Sciences, Rush University Medical Center, 1645 W. Jackson Blvd., Chicago, IL 60612.E-mail: christopher_stewart@rush.edu

\section{J.J. STEWART \&. C. GOLDEN. Verbal IQ as an Intervening Variable in Age-related Memory Decline.}

Objective: To examine the simple indirect effect of verbal IQ on the relationship between age and auditory and visual episodic memory, separately.

Participants and Methods: The present study randomly sampled 95 adults ages 16 and 70 years. The mean age was $35.63(\mathrm{SD}=16)$. Forty-eight were males. Mean level of education was 15.33 years $(\mathrm{SD}=$ 2.285 ). Individuals with a psychiatric or head injury history, medical illness and current pharmacological treatment were excluded. English was the primary language for all subjects. Non-age corrected composite variables were computed for verbal IQ, auditory memory and visual/ graphic memory using subtests from the WAIS-IV and WMS-IV

Results: To test the indirect effect, bootstrapping using 5,000 samples with replacement was employed (Preacher \& Hayes, 2004). Results were considered significant at the .01 level. Verbal IQ had a significant indirect effect on the relationship between age and both auditory (ab $=.0037 ; 99 \%$ CI $[.0005, .0088])$ and visual (ab $=.0053 ; 99 \%$ CI $[.0010$, .0137]) memory. However, rather than mediating the age-memory relationship, measured verbal IQ served as a suppressor variable.

Conclusions: Findings revealed verbal IQ to be a salient mechanism of age-related change in auditory and visual memory. Methodologically, omitting verbal IQ from mediation models that examine age-related memory decline possibly weakens the effect of age on auditory and visual memory and leads to erroneously finding full mediation when in fact there is a remaining effect being overlooked. Clinically, the results introduce verbal IQ, which facilitates better language processing and formation of verbal representations, as a potential compensatory factor of age-related decline in auditory and visual memory.

Correspondence: Jada J. Stewart, M.S., Center for Psychological Studies, Nova Southeastern University, 220 6th Ave N., Apt 7, St. Petersburg, FL33701.E-mail: js565@nova.edu

\section{L.C. BUTTERFIELD, J. JONES, K.R. THOMAS, M. MARSISKE \& D. BOWERS. Apathy and Fatigue are Better Predictors of Cognitive Performance than Other Mood Variables in a Sample of Healthy Older Adults.}

Objective: Apathy has been frequently linked to executive functioning, dementia risk, dopaminergic dysregulation, and frontal subcortical integrity. While most studies have evaluated these relationships in
Alzheimer's and related dementias, neurodegenerative diseases involving frontal subcortical changes, and schizophrenia, few have evaluated the associations between apathy and cognition in healthy older adults. Participants and Methods: Participants included 86 cognitively normal healthy older adults (mean age $=78.1$, edu $=16.7$ ) enrolled in the Village Interactive Training and Learning study (VITAL). Participants completed standard measures of depression (BDI-II), anxiety (STAI), apathy (AS), and fatigue (FSS) as well as a battery of cognitive tests. Stepwise regression analyses examined the differential influence of apathy, depression, anxiety, and fatigue, above that of age and education, on five broad cognitive domain composites: a) Immediate Memory, b) Delayed Memory, c) Attention/Working Memory, d) Executive, and e) Processing Speed.

Results: After controlling for age and education, apathy alone best predicted immediate and delayed memory performance while fatigue alone best predicted attention/working memory and processing speed performance.

Conclusions: The lack of association between executive performance and apathy was unexpected. It is possible that neural mechanisms underlying the relationship between apathy and cognition differ in healthy older adults relative to other populations, reflecting integrity of multiple neural systems. Novelty seeking, a key component of apathy, has been associated with both dopaminergic/frontal and amygdala/hippocampal systems. Additionally, apathy has been shown to be a strong predictor of amnestic MCI. Future studies should investigate possible mechanisms (novelty seeking, efficacy, cardiovascular risk factors) underlying the relationship between apathy and memory in healthy elderly and the impact of interventions.

Supported by McKnight Research Foundation (DB,MM)

Correspondence: London C. Butterfield, MA, Psychology, University of South Florida, 4202 E. Fowler Ave., PCD 4118G, Tampa, FL 33620.

E-mail: londonbutterfield@hotmail.com

E.Z. GROSS, J.L. WOODARD, K.A. NIELSON, M. SEIDENBERG, J. SMITH, S. DURGERIAN, A.L. NORMAN \& S.M. RAO. Detection of Age- and Alzheimer's Disease Risk-Related Compensatory Strategies UsingEx-Gaussian Response Time Parameter Estimates. Objective: Age-related differences in response times (RT) between healthy older adults might indicate successful compensation for ageor Alzheimer's disease risk-related cognitive changes. Ex-Gaussian parameter estimates $(\mu, \sigma$, and $\tau)$ may be more sensitive to $R T$ differences than mean and standard deviation (SD). $\mu, \sigma$, and $\tau$ reflect the mean and SD of the Gaussian component and variability in the right tail of the distribution, respectively. It was hypothesized that the ratio of $\tau / \mu$ would correlate with age.

Participants and Methods: Cognitively healthy older adults completed a two-choice semantic memory task at baseline $(\mathrm{n}=115)$ and18 months ( $\mathrm{n}=97$ ). Bootstrapped parameters were estimated by maximum likelihood. Correlations between $\tau / \mu$, sample statistics and age were compared. Significant correlations were examined by APOE $\varepsilon 4$ status. Results: Mean and SD were not significantly correlated with age. $\tau / \mu$ was significantly correlated with age at baseline and follow-up $(\mathrm{r} \tau / \mu 0$ Age $0=-0.22, \mathrm{p}=.02 ; \mathrm{r} \tau / \mu 1-$ Age $1=-0.21, \mathrm{p}=.04)$. When stratified by APOE $\varepsilon 4$ status, $\tau / \mu$ was significantly correlated with age at baseline only for APOE $\varepsilon 4$ carriers ( $\mathrm{r} \varepsilon 4 \tau / \mu 0$-Age $0=-0.35, p=.02$ ).

Conclusions: Negative correlations between age and $\tau / \mu$, but not between age and mean RT, suggest that healthy older adults compensate for age-related cognitive changes by adjusting RTs to maintain performance. Compensation strategies that might result in similar $\tau / \mu$ decreases could involve systematic alterations in decision threshold and RT. Cognitively healthy APOE $\varepsilon 4$ carriers at risk for AD may be adopting such compensatory strategies in response to preclinical deficits relative to non-carriers

Correspondence: Evan Z. Gross, B.A., Psychology, Wayne State University, 21546 Gregory Street, Dearborn, MI 48214. E-mail: evan. gross@wayne.edu 
R. VAN PATTEN, L.J. LARSON-PRIOR, D. BALOTA \& D.A. KAUFMAN. Effects of Context Maintenance on Cued-Stroop Performance in Healthy Aging.

Objective: The Stroop task is a widely-used measure for characterizing attentional control. Previous modifications of this task have highlighted the importance of context maintenance processes that are needed to override prepotent response tendencies in clinical populations. The current study used a cued-Stroop paradigm to investigate the effects of enhanced context maintenance demands on the attentional control resources of healthy older adults.

Participants and Methods: Ten healthy older adults completed a cued, trial-switching version of the Stroop task. Each trial began with a cue (the word "color" or the word "word"), followed by a 1-second or 4-second delay, which was then followed by either a congruent or incongruent Stroop stimulus. Participants responded to Stroop stimuli via a button press, depending on the instructional cue.

Results: Stroop task accuracy and reaction time (RT) were subjected to repeated measures ANOVAs. Context maintenance delay interacted with Stroop congruency for error rates such that accuracy was reduced for incongruent trials presenting a 4s delay, as compared to other trial types. Furthermore, main effects of RT demonstrated slower RTs in the $4 \mathrm{~s}$ versus the $1 \mathrm{~s}$ condition and in the incongruent versus the congruent condition.

Conclusions: Increased delay between the instructional cue and the Stroop stimulus taxed the attentional control resources of healthy older adults and led to a decrease in task performance. It appears that this increase in context maintenance demands improves the sensitivity of this classic task to detect subtle deficits in attentional control. Future research should address this question in pathological aging populations. Correspondence: Ryan Van Patten, B.S., Psychology, Saint Louis University, 1209 Covington Manor Lane, Apt 5, Saint Louis, MO 63125. E-mail: rvanpatt@slu.edu

\section{STEPHENS, B.M. BETTCHER \& J.H. KRAMER. Examining Interleukin-6 as a Predictor of Change in Processing Speed in Healthy Older Adults.}

Objective: The aim of the current study was to better understand the inflammatory mechanisms that contribute to the diversity in brain aging trajectories, particularly in its relationship to processing speed. We sought to examine biological predictors of change in processing speed using two time point data. Specifically, we investigated whether baseline interleukin- 6 correlated with changes in processing speed across two time points.

Participants and Methods: We studied 86 healthy, community dwelling older adults (age: 71.2; Education: 17.7) who were selected from the University of California, San Francisco Memory and Aging Center. Participants were evaluated at two time points, where participants underwent a series of 10 processing speed tasks (Hale \& Myerson, 1996), as a part of a comprehensive neuropsychological evaluation, in addition to a blood draw. Inclusion criteria included a Mini-Mental State Exam score of $\geq 25$, Clinical Dementia Rating score of 0 , and no report of cognitive decline during the previous year.

Results: Hypotheses were tested using a multiple regression with change in speed as the dependent measure and baseline interleukin- 6 as the predictor adjusting for baseline speed, age, and education. Results suggested that higher baseline interleukin- 6 predicted greater slowing on measures of processing speed $(\beta=.22 ; p<.05)$, even when vascular blood markers (hemoglobin A1C and Homa-IR) were included in the model. Conclusions: These findings offer some support that interleukin- 6 has an independent effect on longitudinal changes in processing speed. Correspondence: Melanie Stephens, PhD, Neurology, University of California, San Francsico, 21455 Millard Lane, Cupertino, CA 95014. E-mail:mstephens@memory:ucsf.edu
D.M. O' SHEA, B. HILL, D.J. BARULLI, Q. RAZLIGHI, D. LIU \& Y. STERN. The differential influence of extraversion on the association of age with cognition and the brain.

Objective: The present study investigated whether the personality trait 'extraversion', modified the association between age and cognitive performance as well as the association between age and global measures of brain volumes in a sample of healthy adults.

Participants and Methods: Data from a group of 140 healthy right handed adults (73 female, 67 male) was selected (mean age (standard deviation) 49.8 (17.27), range $=20-77$ ). Composite measures of two core domains; were calculated: memory and fluid reasoning. Three global neuroanatomic measures were selected; total cortical grey matter and white matter volumes, and total cortical thickness derived from T1-weighted magnetic resonance imaging using FreeSurfer. The personality factor 'Extraversion' were obtained, based on the Five Factor Model, using the 50-item IPIP.

Results: Individual multiple regression analysis predicting memory, fluid reasoning, and the three neuroanatomic measures each revealed significant interaction effects between age and extraversion. The pattern of results was similar across both the cognitive and neuroanatomical outcome measures. Higher extraversion was associated with better cognition and greater brain volumes with younger age but this association was reversed with older age.

Conclusions: Results suggest that the personality trait 'extraversion' may have a differential association with cognition and the brain across the lifespan. Being extraverted may reflect both a cognitive and neuroanatomical advantage in early life but serves as a disadvantage in later life. One possible explanation for these findings is that the measure of extraversion may be capturing similar but differing aspects of behavior in different age groups. In the young it may be measuring the prototypical concept of extraversion i.e., of having a positive affect and a tendency to seek out social situations, while in the older age groups it may be capturing more of a diminished social inhibition as a result of some biological and/or cognitive age-related decline.

Correspondence: Deirdre M. O'Shea, MS, The Taub Institute, Columbia University,Medical center, 630 West 168th street, P\&S box 16, New york, NY 10032.E-mail:dmo2123@cumc.columbia.edu

L.D. MEDINA, F. DE LEON, G. RODRIGUEZ, J. EVANS, M.J. WRIGHT, L. APOSTOLOVA, J. RINGMAN \& E. WOO. The Impact of Dysexecutive Symptoms on Prospective Memory in Healthy Aging, Mild Cognitive Impairment, and Dementia.

Objective: The executive skill of planning is integral to prospective memory (PM), the ability to execute an intended action in the future. It is known that PM is affected by the aging process. The present study investigated the impact of dysexecutive symptoms on PM in healthy older adults (OA), mild cognitive impairment (MCI), and individuals with dementia.

Participants and Methods: A total of 153 participants (66 OA, 67 MCI, 20 dementia) were administered the PM task, in which they were asked to remember to request a "pill" after completing each of eight cognitive tasks. Participants' study partners completed the Dysexecutive Questionnaire (DEX), on which they rated the frequency with which participants experienced difficulty with 20 symptoms of executive dysfunction.

Results: For each group, regression analyses were conducted to determine the impact of dysexecutive symptoms on PM. DEX total score and subscale scores were not significantly related with PM performance in any group. For individual dysexecutive symptoms, increased apathy predicted poorer PM performance in the MCI group. In the dementia group, euphoria, poor insight, poor response inhibition, and difficulty dissociating between "knowing" and "doing" were predictive of poorer PM performance. In OAs, dysexecutive symptoms did not predict PM performance.

Conclusions: Overall, symptoms of executive dysfunction predicted PM in individuals with dementia, but not in healthy aging. In MCI, 
apathy predicted PM. These findings demonstrate that apathy negatively impacts prospective memory when individuals begin to exhibit cognitive difficulties.

Correspondence: Ellen Woo, UCLA, 10911 Weyburn \#200, Los Angeles, CA90095.E-mail:ewoo@mednet.ucla.edu

J.S. MILLER, V. LLAMAS, K. BAERRESEN, E. HANSON, R. DYE, K.J. MILLER \& G.W. SMALL. The Appraisal of Subjective Cognitive Complaints to Detect Severities of Cognitive Decline.

Objective: Subjective Cognitive Impairment (SCI) is defined by the presence of cognitive complaints despite normal neuropsychological performance. Investigators in the field contemplate whether SCI represents a pre-clinical stage of Mild Cognitive Impairment (MCI). The current study examined diagnostic outcomes and severity of perceived memory difficulties.

Participants and Methods: A sample of 119 adults (age, mean=62.07 $\mathrm{SD}=11.13)$ with SCI $(\mathrm{n}=52)$, MCI $(\mathrm{n}=60)$ and dementia $(\mathrm{n}=7)$ were followed. SCI criteria was determined based on subjective memory complaints (assessed through the Memory Functioning Questionnaire or MFQ), Clinical Dementia Rating Scale $(<1)$, Hamilton Depression Inventory $(<6)$, and normal cognitive performance on a standard neuropsychological battery.

Results: Frequency analyses revealed $21 \%$ of SCI subjects at baseline converted to MCI in 3 years; the rest remained stable. Of those with MCI at baseline, $15 \%$ converted to dementia after 3.4 years. There were no significant differences between reassessment intervals after post-hoc adjustments $((\mathrm{F} 2,12.7)=2.80, \mathrm{p}>.05)$. ANOVA analyses revealed both the MCI and demented groups endorsed more severe complaints than SCI subjects at each time point, and demented subjects endorsed more severe complaints than those with MCI but only at reassessment $(p<.05)$; group differences occurred on circumscribed MFQ factors, with Factor 1 ("Frequency of Forgetting") being the most robust to detect differences. Conclusions: The MFQ may serve as a useful diagnostic screening tool in determining cognitive change among aging adults, particularly in identifying those who present with SCI. Further research may be valuable in investigating whether SCI may represent as a pre-clinical stage for MCI.

Correspondence: Justin S. Miller, MA, Graduate Psychology, Fuller Theological Seminary, 765 E California Blvd, Pasadena, CA 91106. E-mail:justin.s.miller@me.com

C. MOORE, M. GRANT, H. XIAN, M. LOGUE, M. PANIZZON, C. FRANZ, R. HAUGER, W. KREMEN \& M. LYONS. Erectile Dysfunction and Cognitive Change Over Time.

Objective: Given the negative impact that vascular disease has on cognition, the purpose of this study is to explore whether men with erectile dysfunction (ED), a harbinger of vascular disease, are at greater risk to experience cognitive decline compared to healthy peers. This is an extension of previous research that has documented that men with ED demonstrate significantly poorer cognition in attention-executive-psychomotor domains.

Participants and Methods: Participants were from the Vietnam Era Twin Study of Aging (VETSA). Sample consisted of 485 male twins (M age $=55.4 ; \mathrm{SE}=.1)$ free from $\mathrm{CVD}$ at baseline. Participants were given tests across 13 cognitive domains. Erectile function was assessed with the International Index of Erectile Function-15 (IIEF-15). Tests were re-administered five years later. The analyses were non-twin analyses, i.e., individuals, not twin pairs, were the unit of analysis. All analyses adjusted for the clustering of twins within pairs with mixed modeling in SPSS 19.

Results: Cross-sectionally, ED was associated with poorer cognitive performance on 7 of 13 neuropsychological domains. At follow-up, men with ED at baseline had poorer cognitive performance in 10 out of the 13 neuropsychological domains. By evaluating these statistics, it appears that ED's relationship with cognition strengthens over time. However, when looking at the change scores, baseline ED appears to only significantly predict cognitive change in aspects of executive function (set-shifting, verbal fluency), mechanical knowledge, and vocabulary. Conclusions: ED is an important variable to consider when evaluating a patient without cardiovascular disease. ED has long been documented to predict conversion from subclinical to clinical cardiovascular disease. These findings suggest that ED may help identify those at risk for vasculogenic cognitive change. Further, ED may be a more valuable warning sign than traditional vascular risk factors.

Correspondence: Caitlin Moore, Boston University, 506 Wisconson St, San Francisco, CA 94107. E-mail: caitlin.s.moore@gmail.com

\section{C.B. WOOLVERTON, M. CRAWFORD, M.D. GRILLI \& E. GLISKY.} Self-imagining Improves Memory in Older Adults.

Objective: Previous studies in older adults have demonstrated that encoding information in reference to the self provides positive benefits for memory - the self-reference effect (SRE). Recently, in a series of studies of individuals with traumatic brain injury, we showed that a form of self-referential processing called self-imagination, provided an even greater mnemonic benefit than the standard procedure. The present study extends that research to a group of normally-aging older adults. Participants and Methods: Thirty-five healthy older adults (ages 6592 ) and 31 younger adults (aged 18-22) encoded neutral and emotional sentences under three processing conditions: baseline, semantic, and self-imagining, followed by a yes/no recognition memory test.

Results: Results indicated a self-imagination effect (SIE): recognition memory was greatest in the self-imagination condition. There was also a main effect of emotion, which interacted with condition. Emotional sentences were recognized better than neutral sentences in the baseline and semantic conditions but not in the self-imagination condition. Young adults performed better than older adults, and age group did not interact with any of the other variables. A secondary analysis showed that older adults who were carriers of the APoE e4 allele also showed the SIE although they failed to show the emotion effect.

Conclusions: These results demonstrate that although older adults performed more poorly overall than young adults, self-imagination provided an equivalent memory benefit for both age groups. It also benefited both $\mathrm{APoE}$ e4 carriers and non-carriers equivalently. Interestingly, however, APoE e4 carriers did not show the same benefits of emotion as non-carriers.

Correspondence: Cindy B. Woolverton, Bachelor of Science, Psychology, University of Arizona, 1503 E University Blvd., P.O.Box 210068, Tucson,AZ 85721.E-mail: cindyw@email.arizona.edu

D. WALD, J. JONES, T. CUMMINGS, A. MIKOS, R. RODRIGUEZ, M.S. OKUN, C. HASS \& D. BOWERS. Is Exercise More Important for Cognitive and Mood Functioning among Parkinson's Disease Patients than Normal Elderly?

Objective: This study examined the relationship between reported aerobic exercise, cognition, and mood in individuals with Parkinson's disease (PD) versus normal elderly controls. We hypothesized that exercise would be significantly correlated with better cognition and mood in both PD and control groups.

Participants and Methods: Participants included 54 PD patients $(\mathrm{N}=54)$ and 31 healthy controls $(\mathrm{N}=31)$ who were part of an NIH-supported RCT for treatment of masked faces. Data from a self-report exercise measure was converted into metabolic equivalents (METS) following the guidelines of Strath et al. (2013). Cognitive tasks included a dementia screen (DRS-2), memory measures (CVLT-2, Logical memory), naming (BNT), and measures of mood (apathy, anxiety, depression). Multiple hierarchical regression analyses examined the influence of exercise (mets) on cognition and mood.

Results: The PD and Controls did not differ in demographic variables, DRS-2 scores, or reported exercise (METS). A significant group (PD, control) by exercise interaction was found for CVLT-II delayed recall. Specifically, increased participation in exercise related to better delayed recall scores for Controls but not for individuals with PD. Exercise did 
not relate to other measures of cognition or mood. Of note, PD patients performed normally on tasks involving self-appraisal of other behaviors. Conclusions: Contrary to our hypothesis, subjective report of aerobic exercise intensity may be less related to memory performance for PD patients, compared to Controls. Findings were not due to a general defect in behavioral appraisal by PD patients. Further research with objective measures of exercise intensity is needed to determine if high intensity aerobic exercise is a suitable intervention for cognition and mood in PD patients.

Supported by RO1-NSNS50633

Correspondence: Danielle Wald, Psy.D., University of Florida, $1316 \mathrm{NW}$ 102th Way, Gainesville, FL 32606. E-mail:d_wald593@hotmail.com

\section{A.H. COOK, A. MARTERSTECK, D. OHM, A. RADEMAKER, M. MESULAM, S. WEINTRAUB \& E. ROGALSKI. Reduced Cortical Atrophy in Cognitively Successful Elderly Adults.}

Objective: Northwestern University has identified a group of SuperAgers, individuals over age 80 who score at least in the average range for 50-65 year olds on a test of episodic memory and have at least average-for-age performance on the Boston Naming Test, categorical fluency, and Trail-Making Test. Previous findings suggest that SuperAgers display no cortical atrophy compared to cognitively average-for-age 50-65 year-olds at a single time point and have thicker cortices compared to their cognitively average-for-age, same age peers. This pilot study examined the maintenance of cortical integrity in SuperAgers over 18-months compared to same-age, cognitively average elderly adults who maintained their cognitive status over the 18-month interval.

Participants and Methods: Baseline and 18-month structural MR scans from 16 SuperAgers and 6 cognitively average-for-age elderly adults were compared using the longitudinal FreeSurfer pipeline. Paired t-tests were used to determine differences in rate of volumetric change over time for normalized whole brain cortical volume, cortical volume by hemisphere, and cortical volume in regions of interest (ROI) associated with age-related atrophy, including, among others, the cingulate, inferior and superior parietal gyri, and precuneus.

Results: SuperAgers demonstrated smaller rates of whole brain atrophy compared to cognitively average elderly $(p<0.05)$ as well as smaller rates of atrophy in three ROIs: right post-central gyrus, right superior temporal gyrus, and right precuneus ( $\mathrm{p}^{\prime} \mathrm{s}<0.05$, uncorrected).

Conclusions: On average, SuperAgers had larger cortical volumes and smaller rates of atrophy over time compared to their cognitively average same age peers suggesting that slower rates of cortical atrophy may contribute to preserved cognitive abilities in advanced age. A modest increase in sample size will provide adequate power to detect differences in aging-vulnerable brain regions.

Correspondence: Amanda H. Cook, MA, Northwestern University, 320 East Superior St, Searle Building, 11th Floor, Chicago, IL 60611. E-mail: amandacook2017@u.northwestern.edu

A.H. COOK, S. KIELB, K. WHITNEY, S. WEINTRAUB, M. MESULAM, R. LOGAN \& E. ROGALSKI. Psychological WellBeing in Cognitively Successful Elderly Adults.

Objective: The SuperAging program at Northwestern University has identified a cohort of individuals over age 80 who perform as well as individuals 20-30 years their junior on a test of episodic memory (Rey Auditory Verbal Learning Test, RAVLT) and maintain above-average performance for age on the Boston Naming Test, category fluency, and Trail-Making Test (SuperAgers). Psychosocial characteristics that contribute to such successful cognitive aging have not yet been explored. Previous research indicates that individuals with greater psychological well-being have better levels of cognitive performance. Thus, the present study investigates whether psychological well-being can distinguish cognitively successful from cognitively average-for-age elderly adults. Participants and Methods: 28 SuperAgers and 7 cognitively average-for-age elderly adults completed the Psychological Well-Being-42 questionnaire (PWB), a well-validated measure of psychological well-being in older adults. T-tests were used to compare the groups on the PWB's subscales: Autonomy, Environmental Mastery, Personal Growth, Positive Relations, Purpose in Life, and Self-Acceptance. Pearson correlations were used to examine the relationship between the RAVLT and PWB subscales.

Results: SuperAgers endorsed greater levels of Positive Relations compared to cognitively average-for-age elderly adults $(p<0.05)$. Further, RAVLT scores were positively correlated with Positive Relations $(\mathrm{r}=$ $.42)$, as well as Environmental Mastery $(r=.38)$ and Self-Acceptance $(\mathrm{r}=.38$; all p's $<0.05)$. Groups did not differ on other PWB subscales. Conclusions: Positive relationships with others distinguish between SuperAgers and cognitively average-for-age elderly adults. Further, this factor is positively correlated with episodic memory performance, suggesting that positive social relationships may contribute to highly successful cognitive aging.

Correspondence: Amanda H. Cook, MA, Northwestern University, 320 East Superior St, Searle Building, 11th Floor, Chicago, IL 60611. E-mail:amandacook2017@u.northwestern.edu

C. COOK, K. PATEL, A.R. MENATTI, A. KINZER, K. MCINERNEY \& J. SUHR. Hypertension and Driving Risk: Neuropsychological Ability as Mediator.

Objective: Hypertension (HTN) is associated with cognitive deficits in older adults, particularly in the domains of attention, learning/memory, visuospatial abilities, and executive functioning. Driving ability is also associated with many of these same cognitive areas. The purpose of the study was to examine the association of HTN status to driving variables and driving-related neuropsychological skills in older adults.

Participants and Methods: Participants were taken from a larger driving study; participants included only those who were not diagnosed with diabetes, other major cardiovascular illness, or other major neurological or medical disorders potentially impacting cognition $(\mathrm{N}=53$, age 61 88, mean age 71, 17 males). All individuals with HTN ( $\mathrm{N}=16)$ were receiving treatment for HTN. The two groups (HTN, no HTN) were not significantly different in age, education, or gender.

Results: The HTN group performed significantly worse on a predictor of driving risk (Useful Field of View, UFOV) and significantly more of them were judged to have at-risk driving, based on this measure $(p<.05)$. The HTN group also performed significantly worse on Block Design, Trailmaking Test part B, Stroop interference, and several subtests of the Repeatable Battery for the Assessment of Neuropsychological Status, including story learning, semantic fluency, digit span, and coding. The relation of HTN status to driving risk as measured by UFOV was mediated by neuropsychological performance.

Conclusions: Results suggest that HTN-related cognitive impairment is related to driving risk. Future research should examine whether this relationship holds among younger adults with HTN.

Correspondence: Carolyn Cook, B.A., Psychology, Ohio University, 8864 United Lane, Apt. 32, Athens, OH 45701.E-mail: cc541611@ohio.edu

A. FEDOR, S. GARCIA \& J. GUNSTAD. The Effects of a Brief, Water-Based Exercise Intervention on Cognitive Function in Older Adults.

Objective: Physical inactivity is a modifiable risk factor for cognitive decline and other adverse neurocognitive outcomes in older adults. Exercise interventions confer cognitive benefits, but long-term, land-based programs often suffer from poor adherence and high attrition rates. Water-based exercise has become increasingly popular as it provides the same physiological benefits with reduced risk of injury. The current study evaluated the effects of a short-term, water-based exercise intervention on cognition, and cardiovascular fitness in a group of older adults.

Participants and Methods: 60 older adults were assigned to either a brief water exercise program $(\mathrm{n}=27$, Mage $=63.26 \pm 7.64,78 \%$ female $)$ or a control group $(n=33$, Mage $=65.67 \pm 6.69,75 \%$ female $)$ for one 
week. Neuropsychological tests, and a measure of cardiovascular fitness were administered to both groups before and after the intervention. Results: Repeated measures analysis of covariance indicated the exercise group demonstrated significant improvements in cardiovascular fitness $(\mathrm{F}(1,26)=6.22, \mathrm{p}<.05)$, while the control group declined. The exercise group demonstrated improvements in attention $(\mathrm{F}(1,24)$ $=12.82, \mathrm{p}<.01)$, executive function $(\mathrm{F}(1,24)=58.46, \mathrm{p}<.001)$, and memory $(\mathrm{F}(1,24)=11.37, \mathrm{p}<.01)$, whereas the control group did not improve.

Conclusions: Findings suggest that a brief exercise program can provide benefits in fitness and cognitive function in older adults, despite being much shorter than existing programs (i.e. 3-12 months). Future work is needed to clarify the mechanisms for these gains and determine whether they persist over time.

Correspondence: Andrew Fedor, Kent State University, Kent Hall, Kent, OH 44242. E-mail: AFedor1@kent.edu

C.K. BLOCK, E. LOGUE, N.S. THALER, D.M. SCARISBRICK, J.J. MAHONEY, J. SCOTT \& K. DUFF. The Interaction Between Medical Burden and Anticholinergic Cognitive Burden on Neuropsychological Function in a Geriatric Primary Care Sample. Objective: To examine the potential neuropsychological burden imposed by multiple chronic medical conditions and by medications with anticholinergic effects.

Participants and Methods: Participants included 290 community-dwelling primary care patients ages 65 and older. Measures included a medical history form, self-report medication data, RBANS-Form A, and the Anticholinergic Cognitive Burden Scale. Our two predictor variables included the number of self-reported medical conditions (medical burden/MB) and the cognitive burden of participants' medication regimens (anticholinergic burden/AB). Moderated multiple regression analyses examined the main and interactive effects of our predictors on RBANS performance, with post hoc analyses performed on all RBANS indexes and at the subtest level.

Results: Low MB/low AB participants exhibited overall better RBANS performance than low $\mathrm{MB} /$ high $\mathrm{AB}$ participants. In contrast, individuals with greater $\mathrm{MB}$ did not display a significant sensitivity to increased $\mathrm{AB}$, but still exhibited comparatively poorer neuropsychological functioning overall. Post-hoc analyses indicated that high $\mathrm{AB}$ participants performed more poorly on the List Learning, Story Memory, Line Orientation, Coding, and Story Recall subtests. A significant AB main effect and $M B \times A B$ interaction effect was observed for the Digit Span subtest. List Recall and List Recognition subtests had significant MB x AB interaction effects.

Conclusions: To our knowledge, this is the first study to date to examine the complex relationship between $\mathrm{MB}, \mathrm{AB}$, and neuropsychological performance. Our results extend MB-neuropsychological findings by Duff et al. (2007) and AB-neuropsychological findings (Campbell et al., 2009; Boustani et al., 2008). Due to the advent of the ACA and associated increased catchment of older Americans into the U.S. healthcare system it will soon become more important than ever to delineate the burden imposed by multiple chronic medical conditions, and the medications designed to treat them.

Correspondence: Cady K. Block, Ph.D., Medical-Clinical Psychology, Department of Psychology, Division of Medical-Clinical Psychology, The University of Alabama at Birmingham, 1410 Avalon Pines Drive, Coram, NY11727.E-mail: cady.block@gmail.com

T. GIOVANNETTI, S.C. SELIGMAN, I. LAWRENCE, J. KURCZEWSKI, E. SCHELL, B. DECK \& G. MARTIN. Relations between Multiple Collateral Reports of Everyday Functioning and Performance-Based Assessment in MCI.

Objective: Functional assessment in MCI is crucial for diagnosis, estimating prognosis, and evaluating interventions. Typical collateral reports evaluate gross functional status and show weak relations to performance-based measures of functioning and cognition. This study examined whether more specific collateral reports might show stronger relations with performance measures.

Participants and Methods: Informants for 26 people with MCI completed 3 questionnaires: ECog - reported change in episodic memory or executive functions over 10 years; IADL- reported ability to perform tasks in the home; and Prediction- predicted how well participant might perform everyday tasks in the lab. MCI participants completed the MMSE, tests of episodic memory and executive function, and the Naturalistic Action Test (NAT), a performance-based measure of everyday functioning that includes three tasks (e.g., make a lunch). NAT recordings were coded for overt (task step never performed or performed inaccurately) and micro-errors (incorrect object touched but not subsequently used) to yield a NAT Error score.

Results: Correlations among collateral report variables were all significant ( $r>37$ ); exploratory factor analysis showed they comprised a single factor $(70 \%$ variance explained). Though relations between collateral reports and NAT Errors were all weak and non-significant $(\mathrm{r}<.25)$, relations between IADL/ECog and NAT Errors were consistently in the unexpected direction (i.e., collateral reports of better functioning associated with more NAT errors). NAT Errors were correlated with the MMSE $(r=-.36)$ and tests of episodic memory $(r=-.57)$.

Conclusions: Collaterals were consistent in their reports of participants' abilities, even when questions were framed quite differently (i.e., predictions vs. appraisals of current functioning). The weak relations between collateral reports and performance measures replicate and extend findings in the literature and suggest that collateral reports should be used cautiously in MCI clinics and research.

Correspondence: Tania Giovannetti, PhD, Psychology, Temple University, Weiss Hall, Psych Dept, 1701 N 13th St, Philadelphia, PA 19121.E-mail:tgio@temple.edu

M. DEKHTYAR, K.V. PAPP, R.E. AMARIGLIO, E.E. SHAW, M. CHUTE, T. MENEIDE, C. MUNRO, S. JAIMES, M. LAPOINT, S. WIGMAN, A. DAGLEY, A. SCHULTZ, K.A. JOHNSON, R.A. SPERLING \& D.M. RENTZ. Optimal Cognitive Aging in those $75+$ is Associated with Greater Brain Reserve (larger hippocampal volumes) and Reduced Genetic Risk (E4-).

Objective: Age-related declines in memory have been well-documented. However, some individuals reach their 8th -10th decade while maintaining strong memory performance. We were interested in which demographic and biomarker variables distinguished individuals aged $75+$ who score in the top 20th percentile for memory.

Participants and Methods: Our sample included 127 clinically normal individuals $(\mathrm{CDR}=0)$ aged 75-90 (mean education: 15.54 \pm 3.12 ; MMSE: $28.79 \pm 1.07$ ) participating in the Harvard Aging Brain Study. Memory was assessed using a composite derived from standardized and challenging tests: 6-Trial Selective Reminding Test, Memory Capacity Test, and Face-Name Associative Memory Exam. Participants were grouped into high performers, i.e., those who scored in the top 20th percentile on the memory composite $(n=25)$ vs. typical performers $(n=102)$. T-tests and $\chi 2$ tests were used to compare groups on APOE $4, \beta$-amyloid accumulation, ICV-adjusted hippocampal volume, and white matter hyperintensity volume (WMH).

Results: There were no group differences in age, sex, education, or estimated IQ. However, high performers had larger hippocampal volumes compared with typical performers $[\mathrm{t}(121)=-2.60, \mathrm{p}=0.011]$. There was a trend level difference in APOE 4 status with $36 \%$ of typical performers being E4+ compared with only $15 \%$ of the high performing group $[\chi 2(1,95)=3.223, p=0.073]$. There were no group differences in $\mathrm{A} \beta$ accumulation or WMH volume.

Conclusions: Underlying brain reserve (i.e., larger hippocampi) and reduced genetic risk (i.e. E4-) is associated with successful cognitive aging in those $75+$. Understanding brain reserve factors associated with optimal cognitive aging can help us better understand what factors contribute to the dementia trajectory. 
Correspondence: Maria Dekhtyar, Boston University, 221 Longwood Ave, BL-104, Boston, IL 02115. E-mail: mdekhtyar@partners.org

E.E. SHAW, A. SCHULTZ, R.A. SPERLING \& T. HEDDEN. Functional Connectivity Within and Across Cortical Networks is Associated With Cognition During Aging.

Objective: In this study we explored the relationship between functional connectivity and cognition in a group of cognitively healthy older adults. We examined four association cortex networks and three domains of cognition. We predicted that individuals with greater network integrity would exhibit higher cognitive scores.

Participants and Methods: Participants were 276 healthy, community dwelling older adults (ages 65-90) involved in the Harvard Aging Brain Study. Cognition was assessed with factor scores derived from a neuropsychological battery, representing processing speed, executive function, and episodic memory. Resting-state BOLD data were acquired in two six-minute acquisitions on a 3-Telsa scanner, screened for data quality metrics including motion, and processed using the Template-Based Rotation (TBR; Schultz et al., 2014) procedure to extract individual-level metrics of network integrity in multiple cortical networks.

Results: Integrity in multiple large-scale cortical networks was positively related to all cognitive domains. Controlling for the correlations between networks, only a positive relation between the fronto-parietal control network and executive function was significant, suggesting specificity in this relationship. An exploratory analysis of cluster-level relationships with cognition found that both within-network and across-network connections were related to cognition.

Conclusions: These results extend prior work (e.g., Andrews-Hanna et al., 2007), demonstrating that functional connectivity metrics in multiple cortical networks are associated with individual variation in cognition, and further suggest that TBR may be a useful tool for measuring relationships between reduced network integrity and cognition during aging.

Correspondence: Emily E. Shaw, B.S., Psychiatry \& Neuroscience, Massachusetts General Hospital, 149 13th Street, Office \#2651C, Charlestown, MA 02129.E-mail:EESHAW@mgh.harvard.edu

\section{R.F. ZEC, S. KOHLRUS, R. ROBBS \& T. ALA. Effects of Age and Education on MMSE Scores.}

Objective: The objective was to determine the effects of age and education on MMSE scores in a large sample of older "non-demented" adults. Participants and Methods: There were 946 "normal" elderly (age 50-99) who were administered the MMSE as part of a comprehensive cognitive battery in a study of normal aging. In the $50 \mathrm{~s}, 60 \mathrm{~s}, 70 \mathrm{~s}, 80 \mathrm{~s}$ and 90 s age group there were 167, 361, 313, 98 and 7 participants, respectively. Mean educational levels were 14.94, 14.41, 14.11, 14.08 and 12.29 for the aforementioned age groups. In addition to comparing MMSE scores across four age decades (50s - 80s), MMSE scores were stratified by both age decade and three educational levels $(<12,=12$, $>12$ years $)$.

Results: The mean (+/-SD) MMSE scores for the 50s, 60s, 70s, $80 \mathrm{~s}$ and 90s age decade group were 29.11 (1.21), 29.11 (1.08), 28.82 (1.33), $28.33(1.59)$, and $28.17(2.23)$, respectively (5th \%ile cutoffs $=25,27$, $27,25,24 ; 16$ th $\%$ ile cutoffs $=28,28,28,2724)$. The mean difference scores comparing the age extremes $(50$ s vs 80 s and 50 vs 90 s age groups) were less than 1 point on the MMSE indicating that age has a small effect on MMSE. The effect of years of education on the MMSE was appreciably larger than the age effects. In taking the difference between mean MMSE score between the $<12$ years group and the $>12$ year group, the difference scores for $50 \mathrm{~s}, 60 \mathrm{~s}, 70 \mathrm{~s}$, and $80 \mathrm{~s}$ age groups were $2.01,1.22,1.38$ and 0.17 points with most of the difference due to appreciably lower mean scores for the $<12$ years education group for the $50 \mathrm{~s}, 60 \mathrm{~s}$, and $70 \mathrm{~s}$ age group.

Conclusions: In this cross-sectional study of normal aging, the effects of age across the five age decades from the 50 s to the 90 s had a very small effect on mean MMSE ( $<1$ point). This indicates that the MMSE is largely insensitive to normal cognitive aging in older adults. Education had an appreciably larger effect on the MMSE due to lower mean MMSE scores in the $<12$ years education group compared to the $=12$ or $>12$ groups for the $50 \mathrm{~s}, 60 \mathrm{~s}$, and $70 \mathrm{~s}$ age groups.

Correspondence: Ronald F. Zec, Ph.D., Neurology, Southern Illinois University School of Medicine, P.O. Box 2832, Springfield, IL 62708. E-mail: rzec@siumed.edu

M. SANDERSON-CIMINO, L. DELANO-WOOD, A.L. CLARK, N. LUC, J. EPPIG, L. CAMPBELL, A.J. JAK \& M.W. BONDI. Age Moderates the Effect of Elevated Pulse Pressure on White Matter Lesion Burden in Older Adults.

Objective: Recent research has widely established the significance of vascular risk factors, including pulse pressure (PP), in the development of white matter lesions (WML). However, little is known about how the association between WML burden and PP differs with age. This study explores the relationship between age, PP, and WML in nondemented older adults.

Participants and Methods: 60 older adults underwent structural MRI and physiological health exams. Fluid-attenuated inversion recovery (FLAIR) images were examined for WMLs, which were manually traced and categorized as periventricular (PVL), or deep white matter lesions (DML). Subjects were separated by decade into three age groups: 60s (n $=21), 70 \mathrm{~s}(\mathrm{n}=21)$, and $80 \mathrm{~s}(\mathrm{n}=18)$. PP was reflected as the difference between systolic and diastolic blood pressure.

Results: After co-varying for gender, ANCOVAs revealed a significant Age Group x PP interaction in right hemispheric PVL $(p<.05)$, such that high PP in the 60s group was uniquely associated with increased WML volumes relative to the $70 \mathrm{~s}$ and 80 s groups. Though not significant, Age Group X PP interactions trended for left hemispheric PVL and total PVL ( $\mathrm{p}$ 's $<.10$ ), indicating the need for further examination. No significant associations were observed between PP and DWML for any age group.

Conclusions: High PP in midlife adults has a substantial effect on the development of WMLs, but this effect is minimized at later ages. As the deleterious effects of WMLs on cognition have been observed, findings suggest that targeted hypertensive interventions should also include those in midlife. Such interventions may reduce the negative consequences on WML burden on cognition and decrease risk for the later development of dementia in late life.

Correspondence: Mark Sanderson-Cimino, B.S. Psychology, VMRF Psychology, 4347 Hamilton street, San Diego, CA 92104 . E-mail: mesandci@gmail.com

J.E. MAYE, V. GOGOI, K. MCCOY, C. MCCRAE \& M. MARSISKE. Nightly Sleep and Cognitive Performance in Older Adults With and Without Amnestic Mild Cognitive Impairment.

Objective: Sleep is one predictor of cognitive functioning. Whether sleep plays a more important role in cognitively compromised individuals is unknown. The present study investigated associations between sleep and cognitive performance in older adults with and without amnestic mild cognitive impairment (aMCI).

Participants and Methods: 68 non-insomniac, community-dwelling, cognitively normal $(\mathrm{CN} ; \mathrm{n}=53)$ and aMCI $(\mathrm{n}=15)$ participants aged $\geq$ $65(\mathrm{M}=78.0+/-5.75)$ completed sleep diaries and daily cognitive assessments for 31 days. Sleep diaries yielded estimates of total sleep time and wake time after sleep onset. Level and rate of change in cognitive performance (attention, processing speed, working memory, immediate and delayed recall) were predicted by total sleep time, wakefulness, sleep variability, group (aMCI vs. CN), and interactions amongst these variables.

Results: aMCI participants displayed poorer average working memory and recall than CN. Groups did not differ in sleep time, wakefulness, or sleep variability. Across participants, less sleep was associated with worse cognitive performance on all tasks except delayed recall. More wakefulness was associated with worse attention. Immediate and 
delayed recall performance was more positively related to sleep time for MCI elders than CN elders. Wakefulness was more negatively associated with delayed recall for aMCI than $\mathrm{CN}$; unexpectedly, it was also more positively associated with attention and processing speed for aMCI. Improvement occurred in all cognitive domains over the 31 days, likely due to practice. Higher initial levels of attention and working memory predicted slower change. With few exceptions, rate of change did not differ by group status, sleep time, wakefulness, or sleep variability.

Conclusions: Poorer sleep was associated with poorer cognitive performance, disproportionately for the aMCI group, especially in memory. Results highlight sleep's importance for cognitive aging and suggest that sleep improvement could benefit memory performance of persons with aMCI.

Correspondence: Jacqueline E. Maye, M.S., Clinical and Health Psychology, University of Florida, PO Box 100165, Gainesville, FL 32610.E-mail:jmaye@phhp.ufl.edu

J.E. MAYE, B.M. SCOTT, M. MARSISKE \& D. BOWERS. Cardiovascular Disease Risk Factors and Cognitive Performance in Cognitively Normal VITAL Study Participants.

Objective: Cardiovascular disease imposes a greater risk of cognitive decline in older adults. Its risk factors (CRFs) include age, hypertension, high pulse pressure (PP; systolic - diastolic blood pressure), high cholesterol, diabetes, and family history of cardiovascular disease. The current study examined the relative influence of various CRFs on the cognitive performance of a group of cognitively normal (CN) elderly 'SuperAgers.' Participants and Methods: Participants ( $\mathrm{N}=87$; Age $78.1+/-7.5)$ enrolled in the Village Interactive Training and Learning study (VITAL) completed cognitive testing at baseline. Self-reported CRF information was extracted from health and family history questionnaires. PP was measured after a 6-minute walking task. Multiple regression analyses examined the relative association of various CRFs to performance in five separate neurocognitive domains.

Results: $54 \%$ of participants reported a parental history of stroke or heart attack, $53 \%$ had high pulse pressure $(>60)$, $42 \%$ had high cholesterol, $32 \%$ had hypertension, and $7 \%$ had diabetes. While analyses examined the influence of all possible CRFs, including age alone, only $\mathrm{PP}$, both as a continuous and dichotomous variable (normal/high), was related to executive functioning (EF: composite score of Trail Making Test B, Stroop Color-Word Test, and Controlled Oral Word Association Test); p<.001). Higher PP predicted poorer EF. No CRFs predicted performance in the domains of attention/working memory, delayed episodic memory, language, or processing speed.

Conclusions: Greater PP in this sample of CN elders appears to predict poorer EF. The sensitivity of PP as a CRF for EF differences within this particularly high-functioning group of elderly adults suggests its possible utility as an early identifier for subtle cognitive change and cerebrovascular disease assessment referrals.

Supported by McKnight Research Foundation award (DB,MM) Correspondence: Jacqueline E. Maye, M.S., Clinical and Health Psychology, University of Florida, PO Box 100165, Gainesville, FL 32610.E-mail:jmaye@phhp.ufl.edu

C.B. Padula, J. Weitlauf, A.C. ROSEN, G. REIber, M. NAUGHTON, M. RISSLING, J. HUNT, W. LI, B. COCHRANE, M. GOLDSTEIN, K. YAFFE \& M.A. ESPELAND. Longitudinal Cognitive Trajectories of Women Veterans from the Women's Health Initiative Memory Study.

Objective: Examination of age-related cognitive decline in older women Veterans is critical since this cohort has higher educational and occupational attainment, but greater vascular risk than non-Veteran peers. The aim of this study was to compare cognitive trajectories of women Veteran and non-Veteran participants from the Women's Health Initiative Memory Study (WHIMS).

Participants and Methods: Participants were 7330 women aged 65-79 at baseline who participated in the WHI hormone trial and were later enrolled in WHIMS, an ancillary study associated with WHI. Global cognitive functioning was assessed at study baseline, and annually thereafter for 8 years, in women Veterans $(\mathrm{n}=279)$ and non-Veterans $(\mathrm{n}=7051)$ using the Modified Mini-Mental State Examination (3MSE). Results: Relative to their non-Veteran peers, Veteran participants in WHIMS were more likely to be older, Caucasian, college graduates, have higher occupational attainment, and history of angina, stroke and smoking. ANCOVA adjusted for age, education and ethnicity revealed no baseline differences in 3MSE between groups $(p=0.72)$. Generalized linear models showed Veteran women experienced accelerated cognitive decline compared to non-Veterans over an eight-year follow-up $(p=0.002)$. Tests of 3 -way interactions revealed the accelerated decline was more marked in older women $(p=0.008)$, college graduates $(\mathrm{p}=0.001)$, and those with higher occupational attainment $(\mathrm{p}=0.03)$.

Conclusions: Veteran women demonstrated accelerated cognitive decline across an eight-year follow up period compared with non-Veteran participants despite similar baseline 3MSE, higher education and occupational attainment. Indirect evidence of greater vascular burden suggests that Veterans are able to compensate for vascular risk due to protective factors, but eventually decline more rapidly.

Correspondence: Claudia B. Padula, PhD, Psychiatry, VA Palo Alto Health Care System, 3801 Miranda Ave, 151Y, Palo Alto, CA 94304. E-mail: claudiapadula@gmail.com

S. SAPKOTA, G. MCFALL, L. BÄCKMAN \& R.A. DIXON. Genetic Risk for Executive Function Decline in Non-Demented Aging is Associated with APOE and Selectively Intensified by BDNF and COMT.

Objective: Single candidate gene association studies have produced encouraging but inconsistent results with neurocognitive phenotypes. From a genetic-risk intensification perspective, we examine independent and synergistic effects of three polymorphisms related to executive function (EF) performance and change in non-demented aging. The three single nucleotide polymorphisms are: Apolipoprotein E (APOE; rs7412; rs429358), Catechol-O-methyltransferase (COMT; rs4680), and Brain-derived neurotrophic factor (BDNF; rs6265). We test (a) independent roles of COMT (risk=G+), BDNF (risk=A+), and APOE (risk $=\varepsilon 4+$ ); (b) synergistic (additive and interactive) effects in the three polymorphism pairs; (c) effect modification by APOE.

Participants and Methods: We assembled a three-wave ( 9-year) longitudinal sample of non-demented older adults ( $\mathrm{N}=632$; age range $=53-95$ years) from the Victoria Longitudinal Study. First, confirmatory factor analysis established an EF latent variable (four standard indicators) and invariance. Second, latent growth curve modeling and path analysis tested (a) independent effects, (b) pairwise synergistic effects, and (c) COMT and BDNF effect modification by APOE.

Results: Significant results with EF factor scores include: (a) APOE risk carriers performed worse than their counterparts $(\beta=-0.194 ; \mathrm{SE}=0.097$; $\mathrm{p}=0.045$ ); (b) BDNF $\mathrm{x}$ APOE allelic risk group had the worst performance $(\beta=-0.446 ; \mathrm{SE}=0.165 ; \mathrm{p}=0.007)$ and more 9-year decline $(\beta=-0.019 ; \mathrm{SE}=0.009 ; \mathrm{p}=0.030) ;(\mathrm{c}) \mathrm{COMT}+\mathrm{BDNF}$ allelic risk showed an additive risk effect at age $75(\beta=-0.294 ; \mathrm{SE}=0.120 ; p=0.014)$ and borderline decline $(\beta=-0.012 ; \mathrm{SE}=0.006 ; \mathrm{p}=0.059)$ but only in the APOE risk group.

Conclusions: APOE $\varepsilon 4$ carriers in non-demented old age are at increased risk for EF decline, especially given vulnerability-inducing contributions marked by genetic risk from COMT and BDNF.

Correspondence: Shraddha Sapkota, University of Alberta, Department of Psychology, P-217 Biological Sciences Bldg, University of Alberta, Edmonton, AB T6G 2E9, Canada.E-mail:ssapkota@ualberta.ca 
L.B. ZAHODNE, J. MANLY, A.M. BRICKMAN, A. NARKHEDE, E. GRIFFITH, V.A. GUZMAN, N. SCHUPF \& Y. STERN. Longitudinal Application of a Novel Method for Quantifying Cognitive Reserve in Aging Based on the Decomposition of Episodic Memory Variance. Objective: The concept of cognitive reserve states that some individuals are more resilient to brain pathology than others. Study of reserve is limited by common proxy variables (e.g., education) that are static and imprecise. Using a large, randomly sampled and community-based cohort, we previously replicated a novel method for quantifying cognitive reserve as residual variance in memory performance after accounting for demographics and brain structure (Zahodne et al., 2013). The current study extended these results to a longitudinal framework

Participants and Methods: 303 older adult participants in the Washington Heights-Inwood Columbia Aging Project underwent neuropsychological and structural MRI on two occasions, separated by 4.6 $(\mathrm{SD}=1.0)$ years. Regression was used to compute the residual at both occasions. Latent difference scores were used to examine change over time. Results: On average, hippocampal and total gray matter volumes decreased, white matter hyperintensities increased, memory performance declined, and the residual remained stable. There were significant individual differences in all change parameters. Some participants' memory scores declined proportionately to their brain changes (stable residual), others' declined disproportionately to their brain changes (declining residual), and others' improved despite advancing brain structural changes (increasing residual). Multiple-group models revealed stronger associations between brain and memory changes among individuals with declining residuals. These individuals were also more likely to convert to dementia.

Conclusions: Computing the residual variable at one time-point may underestimate cognitive reserve in non-demented older adults because the residual does not reflect reserve prior to a neural challenge (e.g., brain structural changes). Changes in the residual variable may be a useful way to summarize individual cognitive responses to brain changes. Results provide evidence for the dynamic properties of this approach to quantifying reserve in older adults.

Correspondence: Laura B. Zahodne, PhD, Columbia University, 630 West 168th Street, P \& S Box 16, New York, NY 10032. E-mail: lbz2105@columbia.edu

J. GRAVANO, N. LUC, M. SANDERSON-CIMINO, K.J. BANGEN, W.M. PERLSTEIN, L. DELANO-WOOD, D.A. NATION, E.C. EDMONDS \& M.W. BONDI. White Matter Lesion Burden and Lateralized Recognition Memory Abilities in Old Age.

Objective: Cognitive dedifferentiation theory generally predicts increases in correlations of cognitive skills with age; however, circumscribed lesions or specific network degradation differentially affect cognitive processes. White matter lesion (WML) pathology is associated with advanced age, and likely interferes with connectivity of neural networks. Thus, in a sample of well-characterized non-demented older adults, we tested whether verbal and visual recognition memory abilities would increase in association with increased WML burden, or whether elevated WML would instead be associated with reduced association of these lateralized memory functions, consistent with the disconnection of distinct cortical processors.

Participants and Methods: Participants were 70 non-demented (DRS $>130$ ) community-dwelling older adults (mean age: 74.9, SD: 8.5) who underwent neuropsychological testing, and fluid-attenuated inversion recovery (FLAIR) images were evaluated for total WML in a semi-automated manner via voxel thresholding. Memory measures used were subtests of the WMS-R (Logical Memory and Verbal Reproduction).

Results: With respect to recognition memory, linear hierarchical regression showed that verbal memory scores predicted visual memory performance $(\mathrm{p}<.001)$. Total WML did not predict visual memory $(\mathrm{p}=.77)$; however, the interaction between total WML and verbal memory was significant $(p=.009)$, such that verbal memory performance was worse at predicting visual memory performance with increasing WML load, independent of age, education, handedness, and gender.

Conclusions: Results suggest that leukoaraiosis contributes to cortical disconnection rather than dedifferentiation in recognition memory, resulting in less dependence between lateralized memory functions. Future studies should attempt to further decompose this phenomenon, investigate the extent to which WML in specific regions mediate this process, and investigate longitudinal progression of cognitive dysfunction in the context of WML burden.

Correspondence: Jason Gravano, M.S., University of Florida, 3901 SW 20th Ave \# 608, Gainesville, FL 32607.E-mail:jtgravano@phhp.ufl.edu

L.J. ROTBLATT, C. SUMIDA, E.J. VAN ETTEN, J.D. HILEMAN, G.M. WAGNER, E. PIROGOVSKY TURK \& P. GILBERT. Temporal Order Memory Declines Across the Adult Lifespan: Insights into Middle and Old Age.

Objective: Memory for the temporal order of items or events in a sequence has been shown to be impaired in older adults compared to young adults. However, less is known about the efficiency of temporal order memory in middle age. The present study sought to examine the effects of temporal interference on memory for sequences of visuospatial stimuli across the adult lifespan.

Participants and Methods: Young adults (YA; 18-25 years; $\mathrm{n}=60$ ), middle aged adults (MA; 40-55 years; $n=43$ ), and older adults (OA; $65+$ years; $n=43)$ completed a visuospatial temporal order memory test (TOMT) involving varying levels of temporal interference.

Results: Results demonstrated that all three age groups improved as temporal interference decreased. However, significant differences were found among all age groups on the TOMT. The YA group significantly outperformed the MA and OA groups $(\mathrm{p}<.05)$. In addition, the MA group significantly outperformed the OA group $(p<.05)$. Age group was not found to interact with level of interference.

Conclusions: The data indicate that temporal order memory for sequences of stimuli may begin to decline as early as middle age and the decline may continue into old age. However, the effect of interference on temporal order memory may be similar across the adult lifespan. The findings also suggest that tests of temporal order memory may be useful in detecting cognitive change in middle age and older adulthood. Given the importance of temporal order memory in activities of daily living, the present findings may have important everyday implications. Correspondence: Lindsay J. Rotblatt, Master's (Candidate), Psychology, San Diego State University, 10033 Rio San Diego Drive, APT 229, San Diego, CA 92108.E-mail: lindsayrotblatt@gmail.com

\section{HUNT, Y.H. TSUI-CALDWELL \& M.J. LARSON. Performance} Monitoring in Older Adults: A Meta-Analytic Review.

Objective: Older adults (ages 55+) show decreased performance monitoring abilities relative to young adults. The error-related negativity (ERN) component of the event-related potential is an electrophysiological indicator of performance monitoring. Evidence suggests reduced ERN amplitudes in older adults relative to young adults indicating impaired performance monitoring processes. This meta-analysis was conducted to quantitatively assess the effect size of age on ERN amplitudes. Participants and Methods: We identified 750 relevant studies in a comprehensive literature search. 20 studies met all criteria for inclusion. Total participants included in the meta-analysis were 357 older adults and 357 young adults (total $n=714$ ). Cohen's $d$ was calculated for each study as a measure of effect size comparing the mean (+sd) of ERN amplitudes between old and young adults. An overall between-groups effect size was calculated using a random effects model.

Results: The between-groups effect size for age on ERN amplitudes was large and significant $(\mathrm{d}=0.95, \mathrm{p}<0.01)$. Tests of study heterogeneity were also significant $Q(\mathrm{df}=19)=78.6, \mathrm{p}<0.01$, indicating confounding methodological differences in the literature. Previous meta-analyses of ERN amplitudes have shown reduced cross-study heterogeneity in studies that used the same cognitive task (Flanker, Go/NoGo, Stroop. 
etc.) to elicit ERNs. In an analysis including only studies using a Flanker task $(k=7)$, the between-groups effect size for age on ERN amplitudes remained large and significant $(\mathrm{d}=0.99, \mathrm{p}<.01)$ with heterogeneity dropping to non-significance $Q(d f=6)=7.36, p=0.29$.

Conclusions: The current results support the consensus that performance monitoring is reduced in older adults. Much of the heterogeneity in the literature can be explained by cognitive task type.

Correspondence: Isaac Hunt, Brigham Young University, $4287 \mathrm{~W}$ Queens FerryDr, South Jordan, UT 84095.E-mail: isaacjameshunt@ gmail.com

S.W. LIEBEL, U.S. CLARK, X. XU, J.T. MCMAINS, H.H. RISKINJONES, B.E. HAWKSHEAD, N.F. SCHWARZ, D. LABBE, B.A. JERSKEY \& L.H. SWEET. Evaluating the Reliability and Construct Validity of an FMRI-Compatible Symbol Search Task. Objective: To determine whether a Symbol Search paradigm developed for FMRI is a reliable and valid predictor of cognitive processing speed (CPS) in healthy older adults. As all older adults are expected to experience cognitive declines due to aging, and CPS is one of the domains most affected by age, establishing a reliable and valid measure of CPS that can be administered inside an MR scanner may prove invaluable in future clinical and research settings.

Participants and Methods: We evaluated the reliability and construct validity of a newly developed FMRI Symbol Search task by comparing the performance of 45 healthy older adults $(\mathrm{M}$ age $=63.09$; $\mathrm{SD}=8.44$ ) in and outside of the scanner and to the widely used and standardized Svmbol Search subtest of the WAIS. A brief battery of neuropsychological measures was also administered to assess the convergent and discriminant validity of the FMRI Symbol Search task

Results: The FMRI Symbol Search task demonstrated high test-retest reliability when compared to performance on the same task administered outside the scanner $(r=0.791: p<.001)$. The criterion validity of the new task was supported, as it exhibited a significant positive correlation with the WAIS Symbol Search $(\mathrm{r}=.717 ; \mathrm{p}<.001)$. Predicted convergent and discriminant validity patterns of the FMRI Symbol Search task were also observed.

Conclusions: The FMRI Svmbol Search task is a reliable and valid measure of CPS in healthy older adults and exhibits the expected sensitivity to the effects of age on CPS performance.

Correspondence: Spencer W. Liebel, Psychology, University of Georgia, 144 Jolly Lane, Athens, GA 30606.E-mail: swliebel@uga.edu

L. SAlMinen, T. CONTURo, D. LAIDlaW, R. CABEen, E.M. LANE, J.M. HEAPS, J. BOLZENIUS, L. BAKER, S. COOLEY, S.E. SCOTT, S. PHILLIPS \& R. PAUL. Age-related Changes in Gray Matter and White Matter Diffusivity among Healthy Older Adults. Objective: Aging is associated with microstructural changes in brain tissue that can be visualized using diffusion tensor imaging (DTI). While previous studies have established age-related changes in white matter diffusion using DTI, the impact of age on gray matter diffusion remains unclear. The present study utilized DTI metrics of mean diffusivity (MD) to identify age differences in gray matter and white matter microstructure in a sample of healthy older adults.

Participants and Methods: Sixty participants (male $\mathrm{n}=22$, female $\mathrm{n}=38$ ) were divided into three age brackets (ages 50-59, 60-69, and $70+$ ) to examine differences in MD by decade. MD was examined bilaterally in the frontal, temporal, parietal, and occipital lobes of both gray matter and white matter tissue. Statistical analyses were completed using a series of multivariate analyses of variance. A Tukey HSD post hoc analysis was completed to determine the nature of significant differences between groups.

Results: Significantly higher MD was observed in bilateral gray matter of the temporal $(p<0.001)$ and parietal lobes $(p<0.01)$, and in right hemisphere white matter of the frontal $(p<0.01)$ and temporal lobes $(p<0.01)$ of older individuals. Across all analyses, the most robust differences in MD were between the 50-59 and 70+ age groups.
Conclusions: Results indicated that aging has a significant and differential impact on gray matter and white matter diffusion in this cohort of healthy older adults. Longitudinal studies are needed to determine the evolution of diffusion changes in gray matter and white matter regions during normal aging.

Correspondence: Lauren Salminen, B.A., Psychology, University of Missouri Saint Louis, 8001 Natural Bridge Road, St. Louis, MO 63121. E-mail: lespr4@umsl.edu

E. SULLIVAN, M. BARNETT, B. DUMAS, K. SLIGAR \& T.D. PARSONS. The Effect of Distractions on Geriatric and College-Aged Samples within a Virtual Apartment Stroop Task.

Objective: The impact of cognitive load in aging is a widely studied topic. The more solid generalizations about attentional performance across the lifespan suggest that as persons age distracting stimuli can hinder attention by increasing perceptual and cognitive load. The objective of this study was to compare the performance of two cohorts (college age and older age) on a distracting condition of the Virtual Apartment Stroop Task. We hypothesized that the older age sample would be more affected by distracting stimuli in the VR environment.

Participants and Methods: 31 aging participants (mean age $=74.4$; $\mathrm{SD}=8.74$ ) and 45 college-aged participants (mean age $=20.9: \mathrm{SD}=$ 4.84) completed the Virtual Apartment Stroop Task (VAST) as part of a larger neuropsychological battery. The VAST presents stroop stimuli on a display within a virtual apartment as colors are spoken aloud. Participants respond to congruent verbal and visual cues—replicating conditions of the traditional paper-and-pencil Stroop. Each section of the VAST is performed with and without visual, auditory, and visuo-auditory distractors.

Results: Repeated-measures ANOVAs were performed to examine the effects of distracting stimuli on each sample. The aging sample was significantly impacted by distractors, as evidenced by higher distraction condition response times for color-naming $(\mathrm{F}=11.492, \mathrm{p}<.005)$, word-reading $(\mathrm{F}=6.017, \mathrm{p}<.05)$, and interference $(\mathrm{F}=15.896, \mathrm{p}<$ .001) conditions. The college-aged sample was less affected by distractors, only showing higher distraction response times in the color-naming condition $(\mathrm{F}=8.506, \mathrm{p}<.01)$

Conclusions: When performing a virtual apartment Stroop task, geriatric individuals were more impacted than a college-aged sample by distractors. The potential for increased ecological validity using a virtual environment with real world distractors supports the notion that these results may generalize to the day-to-day lives of aging persons, indicating this cohort may have decreased attention when confronted by distractions.

Correspondence: Erin Sullivan, Clinical Psychology, University of North Texas, 2025 Lakepointe Drive, Apt 28D, Lewisville, TX 75057. E-mail: ea.sullivan@hotmail.com

J. MCNEELY, K. STEWARD, S. KAUR, C. CASSILL \& A. HALEY. Blood Glucose Mediates the Relationship between Cognitive Function and Sleep Quality in Middle-Aged Adults.

Objective: Previous work suggests that sleep disturbances and hyperglycemia independently contribute to impaired cognitive function. However, since fragmented sleep promotes alterations in glucose metabolism. it is possible that the negative impact of sleep problems on cognitive test performance is driven by disturbances in glucose metabolism. The purpose of the current study was to examine the relationship between sleep quality, blood glucose, and cognitive function in a healthy, middle-aged population.

Participants and Methods: One hundred twenty-three participants (mean age $=49.76,50 \%$ male), ages 40-60, completed the Medical Outcomes Sleep (MOS) questionnaire, a full neuropsychological assessment, and a general health assessment, including a fasting blood draw, to assess for glucose levels.

Results: Linear regression models adjusted for age, education level, and gender were used for the analyses. Results demonstrated that greater 
sleep problems positively correlated with higher glucose levels $(B=.27$, $\mathrm{p}=.002)$ and worse performance $(\mathrm{B}=.22, \mathrm{p}=.01)$ on Trails B. Likewise, higher glucose levels indicated worse performance $(B=.24, p=.007)$ on Trails B. A mediation analysis, using Baron \& Kenny's traditional causal steps approach, revealed that sleep no longer predicted worse performance on Trails $\mathrm{B}$ when adjusted for glucose levels $(\mathrm{B}=.15, \mathrm{p}=$ .089). Results of the Sobel test, used to test for indirect effect, were also significant $(p=.04)$.

Conclusions: These findings indicate that greater sleep problems correlate with worse performance on Trails B as a result of higher glucose levels in middle-aged adults. The findings provide novel insight into the relationship between blood glucose, cognition, and sleep disturbances. Correspondence: Jasmine McNeely, Psychology, University of Texas at Austin, University of Texas at Austin, Department of Psychology, 1 University Station A8000, Austin, TX 78712. E-mail: jasdmcneely@ gmail.com

N.E. NICODEMUS \& S.A. ROGERS. Does Anxiety Modulate the Effect of Education on Older Adults' Cognitive Abilities?

Objective: While research has indicated a relationship between anxiety and cognitive impairment, little attention has been given to how anxiety may modulate the effects of factors that seem to positively impact cognition, like degree of education. The current study explored the interaction between education and anxiety on older adults' memory and frontal-executive functioning.

Participants and Methods: A total of 222 older adults (M age $=78.1$ ) were recruited from the community and completed a comprehensive battery of memory and executive measures, as well as the Beck Anxiety Inventory.

Results: Hierarchical multiple regression analyses revealed that those with high levels of education showed stronger verbal list learning and recognition, nonverbal memory for simple visual material, phonemic fluency, and working memory when they had lower levels of state anxiety, but their performance in these areas was significantly worse when they had higher levels of state anxiety. In contrast, those with low levels of education performed better when they had higher levels of state anxiety and worse when they had less state anxiety. Those with high levels of education also show stronger delayed story recall, divided attention, and response inhibition when they had low levels of state anxiety, but the performance of low levels of education in each of these areas did not seem to vary as significantly with state anxiety. All ps $<.05$.

Conclusions: Greater state anxiety appears to impair the memory and frontal-executive functions of those with high levels of education, but it may aid the memory and frontal-executive functioning of those with low education. These findings call for an increasingly holistic approach to assessing, conceptualizing, and treating the cognitive functioning of older adults, particularly attending to the way anxiety and education modulate each other's effects.

Correspondence: Natalie E. Nicodemus, Psychology, Westmont College, 7210 Manzanita Street, Carlsbad, CA 92011.E-mail:nnicodemus@ westmont.edu

\section{A. SEELYE, N. MATTEK, D. HOWIESON, T. RILEY, K. WILD \&. J.A. KAYE. Unobtrusively Measured Sleep Disturbance and Sleep Variability Impact Neuropsychological Performance in Cognitively Intact Older Adults.}

Objective: In this study, we examined the impact of sleep disturbance, sleep duration, and sleep variability on cognitive performance in cognitively intact older adults using unobtrusive in-home sensor technologies installed in their homes. Specifically, we examined the impact of sleep the night prior, the week prior, and the month prior to a neuropsychological evaluation on cognitive performance.

Participants and Methods: Participants were 112 community dwelling cognitively intact older adults (mean age $=85.8 \pm 6.9$ years; 90 female) who were part of a longitudinal aging cohort study. Sleep data were collected using an unobtrusive activity assessment system consisting of motion and contact sensors in the home of each participant. Sleep variables were total movement in bed at night, times up at night, and total sleep time, which were collected on a nightly basis for 30 days before the neuropsychological evaluation.

Results: Results showed that more movement in bed the night prior to cognitive testing was predictive of reduced working memory $(p<.05)$. More movement in bed and more variable times up at night the week prior to cognitive testing was predictive of worse working memory and attention/processing speed $(\mathrm{p}<.05)$. Sleep duration was unrelated to cognition; however, it was related to higher levels of pain.

Conclusions: In-home, unobtrusive sensor monitoring technologies provide an ecologically valid method for objective, long-term assessment of sleep and other everyday activities that can contribute to variability or decreased cognitive performance in cognitively intact older adults. Correspondence: Adriana Seelye, Oregon Health \& Science University, 3181 SW Sam Jackson Park Road, Portland, OR 97239. E-mail: seelyea@ohsu.edu

L.N. CORREA, D.M. MOSQUERA, J.A. RODRIGUEZ, L. SALAZAR BEJARANO, D. LAMB, Z. SCHWARTZ, R.A. COHEN, K.M. HEILMAN \& A. FALCHOOK. On Target: Fitt's Law and Aging. Objective: Fitt's law describes an inverse relation between speed and accuracy. Cognitive and motor slowing are common with increasing age and may be related to changes in the frontal-subcortical networks. Fitt's law predicts that this slowing would be associated with increased accuracy. However, it has not been determined if with aging, motor slowing is associated with increased accuracy. Alternatively, the frontal-subcortical networks that change with aging may not only induce motor slowing, but these changes may also impair accuracy of ballistic aiming movements.

Participants and Methods: To test these contradictory hypotheses, we recruited 12 healthy younger adults and 12 healthy older adults. The task was to mark 16 circles distributed across a page positioned at an arm's length away from the participant while performance was timed. In between marking each circle, the participants were instructed to bring the pen back to mark a card centered directly in front of them. Participants were instructed to complete the task as quickly as possible while not sacrificing accuracy for speed. The sizes of the circles and hand used to complete the task varied on different trials.

Results: As the size of the target circles decreased, older adults performed the task relatively more slowly and more accurately than the younger adults.

Conclusions: These preliminary results demonstrate that during an aiming task, healthy older adults are less likely to sacrifice accuracy for speed than are the younger adults, but the reason for this difference remains to be determined and further research is needed to elucidate the possible brain mechanisms.

Correspondence: Lauren N. Correa, College of Medicine, University of Florida, 1220 SW 11 AVE D308, Gainesville, FL 32601. E-mail: lcorrea@ufl.edu

L. CAMPBELL, J. EPPIG, E.C. EDMONDS, L. DELANO-WOOD, A.J. JAK, M. SANDERSON-CIMINO \& M.W. BONDI. The Relationship between SuperAging and APOE Genotype, Vascular Risk and Brain Morphology.

Objective: Research provides evidence for the existence of 'SuperAgers' - older adults with superior age-normed performance on verbal memory testing. We determined the proportion of SuperAgers among a sample of healthy older adults and examined differences in MRI brain volumes, vascular and genetic risks, and subjective memory compared to typically-aging older adults.

Participants and Methods: 95 community-dwelling, nondemented older adults (age $\geq 70)$ underwent comprehensive neuropsychological assessment, including scales of subjective memory, depression, and vascular risk. Structural MRI and APOE genotyping were available on a subset of participants. Participants were excluded if they met Jak/ 
Bondi criteria for MCI. SuperAging was defined as performing $\geq 1.5 \mathrm{SD}$ above norms on long delay free recall (CVLT/CVLT-II) and $\geq-1.0 \mathrm{SD}$ above norms on tests of language and executive functioning. Individuals identified as SuperAgers were compared to a randomly sampled group $(\mathrm{n}=21)$ from the larger sample.

Results: 21 individuals (22\%) met criteria for SuperAging and did not differ from the typically-aging group on demographics, depression, or global cognition (all p's>.10), nor were differences noted between groups on vascular risk, APOE genotype, or subjective memory (all $\mathrm{p}$ 's $>0.14)$. In a subsample who had undergone neuroimaging, SuperAgers demonstrated larger right hippocampi $(p=.036)$ and whole brain volumes $(\mathrm{p}=.007)$.

Conclusions: SuperAgers do not present with fewer dementia risk factors and perceive their memory as equal to same-aged peers. Whole brain volume and hippocampal size may play a role in SuperAgers' resistance to normal age-related decline of verbal memory. Future research should examine longitudinal neurocognitive outcomes as well as generalization of superior performance to other cognitive domains. Correspondence: Laura Campbell, Veteran's Medical Research Foundation, 4755 Cather Ave., San Diego, CA 92122. E-mail: lauracampbell166@gmail.com

\section{Assessment/Psychometrics/Methods (Adult)}

E. LOGUE, D.M. SCARISBRICK, N.S. THALER, J.J. MAHONEY, C.K. BLOCK, J. HOFFMEISTER, R. ADAMS \& J. SCOTT. CriterionValidity of the WAIS-IV Cognitive Proficiency Index (CPI).

Objective: The WAIS-IV Cognitive Proficiency Index (CPI) is a combination of working memory and processing speed subtests that purports to represent a purer measure of cognitive processing. However, little research has validated the CPI in clinical samples. To address this, the current project examined the CPI's sensitivity to neurocognitive dysfunction in a mixed clinical sample.

Participants and Methods: Twenty-five individuals with MMSE scores of $<24$ (cognitively impaired group) and 43 individuals with MMSE scores of 30 (control group) completed the CPI and RBANS Attention Index. The impaired and control groups were compared on these indexes and effect sizes were examined. ROC analyses examined the sensitivity and specificity of each index in discriminating the groups. Stepwise DFA was also run to examine positive and negative predictive values. Results: Although both indexes had large effect sizes in differentiating the groups, the effect size for the CPI $\left(\eta^{2}=.506\right)$ was considerably larger than that of the RBANS Attention index $\left(\eta^{2}=.300\right)$. The CPI demonstrated better sensitivity (SE) than and equal specificity (SP) $(\mathrm{SE}=0.953 ; \mathrm{SP}=0.840)$ to the RBANS Attention index $(\mathrm{SE}=0.767$; $\mathrm{SP}=0.840)$. The stepwise DFA retained only the CPI in the model and correctly classified $91.2 \%$ of the cases.

Conclusions: These findings suggest good criterion-based validity for the CPI, and suggest that this index is more beneficial than the RBANS Attention index in detecting cognitive impairment.

Correspondence: Erin Logue, University of Oklahoma Health Sciences Center, 12901 N. MacArthur Blvd., Apt. \#38, Oklahoma City, OK 73142.E-mail:Erin-Logue@ouhsc.edu

\section{Dementia (Alzheimer's)}

D.M. SCARISBRICK, E. LOGUE, N.S. THALER, C.T. COPELAND, J. HOFFMEISTER, R. ADAMS \& J. SCOTT. The Relationship between Neurocognitive and Functional Status: The Utility of Neurocognitive Performance in Predicting Collateral Informant Symptom Ratings.

Objective: The current study examined the relationship between informant-reported and neurocognitive performances in a sample of individuals diagnosed with Alzheimer's dementia. We hypothesized that the memory and language indices of the RBANS would predict collateral reported functional level of impairment.

Participants and Methods: This study included 223 individuals diagnosed with dementia, Alzheimer's type who were selected from an archival database. Neurocognitive performance was measured by the Repeatable Battery for the Assessment of Neuropsychological Functioning (RBANS). Functional abilities were measured by the Functional Rating Scale for Symptoms of Dementia as reported by a collateral (spouse, family member). Hierarchical regressions examined the relationship between RBANS indices and factors extracted from the functional rating scale.

Results: Factor analysis extracted three factors that included Cognition, Activities of Daily Living, and Social/Emotional Functions. For the Cognition Factor, the RBANS Language Index displayed a significant predictive relationship, $\beta=-.338, \mathrm{p}<.001$. For the ADL factor, both Language, $\beta=-.195, p=.011$, and Visual/Constructional, $\beta=-.193, p$ $=.01$, indices predicted performance. Language was the only significant predictor of the Social/Emotional factor, $\beta=-.177, p=.026$.

Conclusions: These findings suggest that patients' language and visual/ constructional abilities play a role in the collateral ratings of patients' difficulties.

Correspondence: David M. Scarisbrick, Ph.D., Neuropsychology Lab, Keck School of Medicine of USC, 425 N. Oklahoma Ave., Apt 2117, Oklahoma City, OK 73104.E-mail: scarisdm@gmail.com

\section{Epilepsy/Seizures}

\section{IOVINO \& L. CHAPIESKI. Academic Performance and Attention in Pediatric Epilepsy.}

Objective: Students with epilepsy are at risk for poor academic performance, which may, in part, be related to problems with attention and impulse control. This study evaluated the impact of these factors on academic performance and the underlying seizure-related risk factors. Participants and Methods: Participants $(\mathrm{N}=76)$ ages 6-19 y/o with intractable seizures and IQ $>=70$ were included. Academic functioning was measured using the Academic Performance (SBS-AP) and Academic Habits Scales (SBS-AH) of the Student Behavior Survey, a teacher report measure and the School Scale from the Parent Child Behavior Checklist (CBCL). Aspects of attention were assessed with the errors of omission and commission, reaction time and reaction time variability from the Connors' Continuous Performance Test (CPT-II). Seizure variables included seizure frequency, number of AED's, seizure focus, age of onset and duration of disorder. Verbal intelligence was measured with an age-appropriate Wechsler test.

Results: Regression analyses, controlling for Verbal IQ, revealed that reaction time and commission errors significantly contributed to scores on the CBCL, $p<.01$, but CPT performance was not significantly correlated with the SBS-AP. SBS-AH was significantly correlated with reaction time only, $p<.05$. Seizure frequency was inversely correlated with reaction time, $\mathrm{p}<.01$. No other seizure variables were significantly associated with reaction time or errors of commission.

Conclusions: Academic functioning in children with epilepsy is strongly influenced by verbal intelligence but components of attention are important as well. Frequent seizures are a risk factor for inattention. Correspondence: Isabella Iovino, Ph.D., Pediatrics, Baylor College of Medicine (BCM),6701 Fannin St., Houston, TX77030.E-mail: iiovino@ bcm.edu

A. LEVAN, B. TUDOR, R.B. BURR \& S.D. GALE. Preliminary Investigation of the SSIS in Children with Epilepsy.

Objective: To compare self-report and caregiver ratings in children with epilepsy seen on an epilepsy monitoring unit with a less well-studied measure of social skills.

Participants and Methods: 17 children ( 7 males; average age $=12.1$, $\mathrm{SD}=2.77$ ) were recruited on the Epilepsy Monitoring Unit after being 
admitted for observation of seizure activity. All children were diagnosed with epilepsy via Video-EEG. Each child and their parent/caregiver completed the Social Skills Improvement System (SSIS).

Results: Significant group differences between parent and child ratings of social skills $(p=.05)$, problematic behaviors $(p=.04)$, cooperation $(p=.05)$, responsibility $(p=.03)$, and empathy $(p=.04)$ were found. Children rated themselves more positively on social skills, cooperation with others, sense of responsibility, and empathic behavior compared to parental/caregiver ratings. Both parent and child ratings were generally within the average range compared to normative data for healthy populations. However, parents/caregivers of children with epilepsy reported their child as having more problematic behaviors compared to healthy controls.

Conclusions: Preliminary findings demonstrate that social skills, cooperation, responsibility, and empathy, may be overrated by children with epilepsy when compared to reports from caregivers. Furthermore, children may be less likely to report problematic behaviors than their parents.

Correspondence: Ashley Levan, M.A., CRC, Clinical Psychology, Brigham Young University, 1423 S. 465 W., Orem, UT 84058. E-mail: Levanaj17@gmail.com

P. PRITCHARD, M. WAGNER, M. ABELL \& P. KERSEY. Episodic Misperception of Time Associated with Temporal Lobe Epilepsy. Objective: We report two cases of time distortion associated with complex partial epilepsy. We speculate about anatomical structures involved with the perception of time.

Participants and Methods: Cases come from a medical university teaching hospital, both followed for idiopathic treatment of refractory epilepsy beginning in midlife. Case 1 described "mind racers" during which time actions were felt to be occurring at a very rapid pace including speech and walking. Case 2 presented in the ED with a second seizure and asystole resulting in the placement of a pacemaker. Semiology was initially stereotypic visual, but evolved to the perception of speech as being very rapid and in high pitched voices during the preictal phase. Results: EEG localized seizure onset to the left temporal lobe in case 1 and to the right in case 2 . Both had frequent lateralized interictal spikes. Case 1 had fusion PET/MRI that was unremarkable. Case 2 had a pacemaker with serial unremarkable CT studies. Both subjects underwent neurocognitive evaluation and results failed to reveal any focal or lateralizing signs (see Tables and Figures). Case 2 suggested posterior temporal onset with a stereotyped visual scene and propagation to insular cortex supported by bradycardia and asystole.

Conclusions: We know of no reported cases of complex partial seizures causing temporal distortion. We hypothesized that these cases support the notion of the temporal lobe as integral part of timekeeping where transient functional disruption of the medial temporal structures disrupts a neural network mediating the perception of time.

Correspondence: Mark Wagner, Ph.D., Neurology, Medical University of South Carolina, 96 Jonathan Lucus St, Charleston, SC 29403. E-mail: wagnermt@musc.edu

C. LEVERONI, S. SPRICH, J. BURBRIDGE, M. GORMAN, S. SAFREN \& H. DUNCANSON. Case Series of CBT in Reducing Seizure Frequency.

Objective: Researchers have demonstrated strong correlations between stress levels, negative emotional states, and seizure frequency. However, data are mixed as to whether psychological treatment reduces seizure frequency. This is likely due to differing types of interventions. We developed a cognitive behavioral intervention focused on teaching organization, planning, problem-solving, and cognitive restructuring combined with mindfulness and relaxation training. We piloted the intervention in individuals with medically intractable seizures to determine efficacy.

Participants and Methods: Participants diagnosed with poorly controlled epilepsy for one year and reported at least mild stress over the past 6 months were included in the study. Nine participants passed screening. Two participants were disqualified due to previous psychotherapy treatment and one due to concurrent alcohol abuse. Three participants completed eight 50-minute sessions over a 12 week period. Measures of seizure frequency, perceived stress, and level of depression and anxiety were collected at baseline, 3 and 9 months post-treatment. Results: Preliminary results revealed that the protocol was feasible and acceptable to patients with epilepsy. Seizure frequency declined from baseline in all three subjects. In two subjects, depression and anxiety increased while stress remained stable. One subject showed declines in all measures.

Conclusions: Preliminary findings suggest that stress management techniques may affect seizure frequency independent of level of anxiety and depression. Additional variables likely account for the reduction in seizure frequency such as increased problem solving skills. The small sample size in the current study limits generalizability. Future studies should aim to recruit more subjects and quantify changes in executive functioning pre- and post-treatment

Correspondence: haley Duncanson, MA, psychology, Suffolk University, 253 center st, Randolph, MA 02368.E-mail: haleyduncanson28@gmail. com

E.H. GERST \& L. CHAPIESKI. The Impact of Anticonvulsant Medication on Academic Skills as Mediated Through Processing Speed and Working Memory.

Objective: Children with epilepsy are at risk for poor academic performance. This study examines the contribution of anticonvulsant drug (AED) treatment to academic performance. We hypothesized that the impact of AEDs on academic skills is mediated through working memory and processing speed.

Participants and Methods: Participants were 114 children with intractable seizures (6-19 years) taking AEDs $(\mathrm{M}=1.80, \mathrm{SD}=.76)$ with IQ's > 69. Verbal Comprehension Index (VCI), Perceptual Reasoning Index (PRI), Processing Speed Index (PSI) and Working Memory Index (WMI) from the Wechsler intelligence tests were administered. Math and reading skills were assessed using the Math and Reading Composite scores from the Kaufman Test of Educational Achievement. Correlations and multiple regression assessed relationships among variables.

Results: Number of AEDs was significantly correlated with math, but not reading scores $(p=.042)$. Number of AEDs was inversely correlated with PSI and WMI ( $\mathrm{p}=.014 ; \mathrm{p}<.0001$, respectively), but not VCI or PRI. When number of AEDs, PSI, and WMI were entered into a single stepwise multiple regression to predict math scores, only PSI and WMI remained in the model, explaining $39 \%$ of the variance $(p<.0001)$.

Conclusions: We found that a higher number of AEDs was associated with lower math scores, but the effect was mediated through sluggish cognitive tempo and poor working memory. Our findings indicate that high numbers of AEDs should be considered a risk factor for poor math skills in children with epilepsy.

Correspondence: Elyssa H. Gerst, University of Houston, 8383 el Mundo St, Apt 411, Houston, TX 77054. E-mail: elyssa.gerst@gmail.com

\section{J. SCHWARTZ \& J. TITUS. Executive Functioning and Behavioral Profiles in Childhood Absence Epilepsy and Juvenile Myoclonic Epilepsy.}

Objective: Childhood Absence Epilepsy (CAE) and Juvenile Myoclonic Epilepsy (JME) are idiopathic generalized epilepsies that are associated with executive dysfunction (e.g., Conant et al, 2010). Patients with both have higher internalizing psychopathology, but patients with JME have less stable externalizing behavior. The present study is a preliminary comparison of the executive functioning and behavioral profiles of these conditions in an attempt to better define their different psychological presentations.

Participants and Methods: This study included 11 children with CAE and 6 children with JME who presented for testing at a tertiary care medical center in Texas. CAE and JME diagnoses were confirmed by 
board certified pediatric neurologists. The mean age of the group was 12.75 years. The sample consisted of 8 males and 9 females, and 3 children were left handed. Two children were African American, 2 were Hispanic, and the remainder were Caucasian.

Results: The JME group had significantly lower Full Scale IQ scores than the CAE group $(p=.023)$. This appeared to be due to lower Verbal IQ $(p=.009)$ by the JME group. The JME group also performed lower in verbal fluency $(p=.015)$. Scores on the Wisconsin Card Sorting Test, Digit Span, and CVLT were similar. On the BASC-2, parents rated the CAE group significantly higher on the Depression scale $(p=.043)$. Additional scales assessing internalizing psychopathology approached statistical significance.

Conclusions: Verbal fluency was the only significant executive functioning difference between the groups. Children with JME perform lower on verbal IQ, while nonverbal IQ is similar. It is unclear whether the lower verbal IQ accounts for the difference in verbal fluency, or whether the verbal fluency contributes to lower verbal IQ. These results suggest a cognitive distinction between the groups based on verbal processing. Our study also reveals internalizing problems in the CAE group, but there is no evidence of greater externalizing problems in the JME group. Correspondence: Jeffrey Titus, Phd, Psychology, The University of Texas, 3828 Epperson Trail, Austin, TX 78732.E-mail: jbtitus@seton. org

\section{A. MIRSKY, M. LEVAV, D.A. GREENBERG, L.M. FRENCH \& C.C. DUNCAN. Gender Differences in Sustained Attention in Idiopathic Generalized Epilepsy.}

Objective: Females outnumber males with idiopathic generalized epilepsy (IGE), including juvenile myoclonic epilepsy (JME) and childhood absence epilepsy (CAE). Family studies yield ratios of 12.5:1 female to male (e.g., Pal et al., 2006). We summarize three studies of gender differences in sustained attention in IGE (Lansdell \& Mirsky, 1964; Levav, 1992; Mirsky \& Greenberg, 2006).

Participants and Methods: Subjects were 110 seizure cases (65 focal, 45 IGE) and 97 normal controls. The Lansdell and Mirsky (1964) diagnoses were: Focal disorders, 42 males, 23 females; IGE, 8 males, 11 females. Levav (1992) studied 6 IGE males and 8 females. Mirsky and Greenberg (2006) studied 12 IGE cases, adults and children. Other patient data are in the respective publications. Neuropsychological tests were given, including measures of sustained attention (i.e., the Continuous Performance Test, or CPT; Mirsky et al., 1991, 1995). CPT tasks were Visual $\mathrm{X}$ and $\mathrm{AX}$ and Auditory Tones. In each case, performance of male and female participants was compared.

Results: In two studies, male IGE patients, children and adults, scored higher than females on the CPT $(p<.05$ to $p<.001)$. Similar differences were also seen in probands' parents (Levav, 1992). In general, focal patients did not differ from controls (Lansdell and Mirsky, 1964). The results of the Mirsky and Greenberg (2006) study showed the same trend. Moreover, the data suggest differences in CPT impairment between CAE vs. JME cases.

Conclusions: Our findings provide additional insight into the differential sensitivity of females to IGE. A mouse model of JME offers very similar findings as well as male-female anatomical differences. This may ultimately assist in genetic and/or pathophysiological investigations of the origins of IGE. Our data may also contribute to the substantial information about the reverse gender sensitivity to certain attention-related disorders, in which males are more vulnerable than females, such as ADHD.

Correspondence: Allan Mirsky, Walter Reed National Military Medical Center,8901 Rockville Pike,Bethesda,MD 20889.E-mail: afmirsky@ gmail.com
J.E. BOOTH, N.M. WISDOM, J. ROBINSON, D. CHEN \& R. COLLINS. Chaining Likelihood Ratios to Detect Psychogenic Non-epileptic Events (PNEE).

Objective: The Health Attitude Survey (HAS) is an 8-item screener designed to assess for somatization in general medical clinics. Similarly, the Health History Checklist (HHC) is a brief checklist of physical symptoms that maps on to DSM-III diagnostic criteria for Somatization disorder. The purpose of this study was to calculate the diagnostic classification statistics for the HAS and HHC both alone and in combination to differentiate between patients with epilepsy and patients experiencing psychogenic non-epileptic events (PNEE).

Participants and Methods: The HAS and HHC were administered to veterans referred as part of a larger neuropsychological screening. All of the patients were referred by the neurology department at a large VA hospital and were undergoing week-long observation on an epilepsy monitoring unit. Group membership (epilepsy vs PNEE) was determined by a board-certified neurologist and director of the epilepsy program. 180 of the patients were successfully classified as either having epilepsy ( $\mathrm{n=82}$ ) or PNEE (n=98) using "gold standard" video EEG findings following induction.

Results: Using a cut-score $>19$, the HAS demonstrated good diagnostic classification statistics in patients diagnosed using vEEG ( $\mathrm{SE}=.54$, $\mathrm{SP}=.92 ; 68 \%$ post-test probability). Using a cut-score $>16$, the HHC was relatively poor at identifying PNEE ( $\mathrm{SE}=.24, \mathrm{SP}=.90 ; 45 \%$ posttest probability). However, chaining the likelihood ratios of the two measures resulted in a post-test probability of $84 \%$ when a positive finding occurred on both measures.

Conclusions: The HAS is moderately effective at identifying PNEE while the $\mathrm{HHC}$ was relatively poor. Importantly, however, combining the measures resulted in considerable more accuracy at identifying PNEE. These brief measures represent a cost-effective way of screening individuals for PNEE and might be effective for identifying other somatoform disorders. Limitations include not all patients being induced and a few patients with both PNEE and confirmed epilepsy.

Correspondence: Nick M. Wisdom, Ph.D., Michael E. DeBakey Veterans Affairs Medical Center, 2002 Holcombe Blvd, Houston, TX 77030. E-mail: nickandjen@gmail.com

\section{J.B. DYKSTRA \& K.A. MCNALLY. Utility of Routine Screening for Mental Health Problems in an Outpatient Pediatric Epilepsy Clinic.}

Objective: The primary objective of this study was to investigate the feasibility and utility of mental health screening for all children presenting to a pediatric epilepsy clinic.

Participants and Methods: Retrospective chart review was performed for 285 patients (ages 5-18) who were administered a screening measure of mental health problems (Strengths and Difficulties Questionnaire; SDQ) as part of a routine clinical care visit in a multidisciplinary outpatient epilepsy clinic. SDQ scores were regressed on age, sex, socioeconomic status (SES), epilepsy syndrome, duration of epilepsy, and seizure frequency.

Results: High rates of mental health problems were reported on the SDQ with $48.8 \%$ of the population scoring above established clinical cut-offs on at least one subscale. In contrast, only $26.9 \%$ had received previous mental health treatment. SES and seizure frequency emerged as the most significant individual predictors of elevations on the SDQ, ps $<.05$. Children identified as having seizures at least daily were at highest risk compared to other seizure frequency groups; $75 \%$ of children experiencing daily seizures had at least one clinical elevation on the SDQ. Conclusions: Findings highlight the feasibility and utility of routine mental health screening using the SDQ in children with epilepsy to identify previously unmet mental health needs. Children with daily seizures and also those from lower SES backgrounds may have the greatest mental health needs. 
Correspondence: Jana B. Dykstra, M.A., Psychology, Nationwide Children's Hospital, 101 1/2 E Frankfort Street, Columbus, OH 43206. E-mail: jana.dykstra@gmail.com

J.M. STINSON, J. GRABYAN, J. ROBINSON, D. PROTO, D. CHEN \& R. COLLINS. Psychometric Properties of the Patient Competency Rating Scale in a Seizure Disorder Population.

Objective: The Patient Competency Rating Scale (PCRS) was developed to measure the extent to which individuals are able to function in four domains: activities of daily living, emotional, interpersonal, and cognitive. The PCRS is widely used in traumatic brain injury populations and was recently validated on a stroke population, but has not yet been validated for use of other chronic medical populations, such as seizure disorders. Previous research has found that perceived competency to successfully accomplish tasks predicts long-term outcomes and quality of life in epilepsy; thus, having a valid instrument to track a patient's competency has important clinical implications. The current study sought to examine the underlying factor structure of the PCRS and, in particular, to validate the structure when used with a seizure disorder population.

Participants and Methods: Participants included 225 veterans who were referred to an inpatient epilepsy monitoring unit. In addition to completing the PCRS, participants completed neuropsychological tests, self-report symptom inventories, the MMPI-RF, and symptom validity measures.

Results: Given previous inconsistent research on the PCRS factor structure, an EFA was used. A PCA with a direct oblimin rotation was performed on the original 30 items. The final results indicated a three factor solution, explaining $59.59 \%$ of the variance $(\mathrm{KMO}=.94$, Bartlett's test of sphericity $[406,4459.21], p<.01)$. The three factors were: Activities of Daily Living ( 7 items, $\alpha=.91$ ), Emotional/Interpersonal (13 items, $\alpha=.92$ ), and Cognitive ( 9 items, $\alpha=.92$ ). One item was deleted due to lack of fit.

Conclusions: The underlying factor structure for the PCRS in a seizure disorder population appears to differ from the original conceptualization. In addition to reducing the structure to three factors, some items were a better fit within different factors than originally designed. This study highlights the importance of validating measures for unique populations.

Correspondence: Jennifer M. Stinson, PhD, Mental Health Care Line, Michael E. DeBakey VA Medical Center, 2002 Holcombe Blvd, Houston, TX 77030.E-mail:jenstinson@gmail.com

W.A. SCHRAEGLE \& N. NUSSBAUM. The Neuropsychologist's Users Guide for Language Lateralization Using Functional Imaging and Dichotic Listening: A case study.

Objective: The reliable and valid determination of language function by non-invasive techniques (e.g., fMRI and MEG) has become an essential part of the presurgical workup of children facing a neurosurgical intervention due to intractable epilepsy. As atypical language organization is most prevalent in children, language determination is often a complex process requiring corroborating evidence from several sources. For the neuropsychologist involved in localization of language functioning, an understanding of these imaging modalities and paradigms used to derive findings has become increasingly important as imaging findings do not always align and are not standardized across centers. Therefore, we offer a practical guide to the language lateralization process through case study.

Participants and Methods: Neuropsychological data of a 16 year old, left-handed male, with a history of tuberous sclerosis and intractable temporal lobe epilepsy are presented, along with functional language mapping findings derived from an auditory decision task (fMRI) and a word repetition task (MEG). Neuropsychological, functional imaging and structural MRI data were integrated for lesion localization and surgical planning.
Results: On neuropsychological examination, a strong left ear advantage was found on a dichotic listening task. fMRI language activation was found in left Broca's area and right Wernicke's area suggesting crossed dominance, while MEG reported right Broca's area and Wernicke's areas. Structural MRI showed multiple tubers especially in the left lateral posterior temporal/parietal lobe.

Conclusions: We discuss the discordance in imaging and dichotic listening results in the context of case history and paradigm selection. A broader discussion will also be provided on neuroanatomical localization of common fMRI and MEG language tasks. Additionally, we will provide an overview of atypical language organization and will discuss the contribution of neuropsychological assessment in defining the functional integrity of language systems.

Correspondence: William A. Schraegle, MA, The University of Texas at Austin, 1307Kinney Ave \#114, Austin, TX 78704.E-mail:waschraegle@ utexas.edu

S. THAMILAVEL, R. GALIOTO, A. BLUM \& G. TREMONT. SelfAwareness of Cognitive Deficits in Older Adults with Epilepsy and Mild Cognitive Impairment.

Objective: Older adults with epilepsy demonstrate cognitive impairment of similar severity compared to individuals with Mild Cognitive Impairment (MCI) without epilepsy. Little is known about awareness of cognitive deficit in epilepsy and how it compares to awareness in MCI. This study compared awareness in epilepsy and MCI and examined its relationship with neuropsychological performance.

Participants and Methods: Sixty-two older adults (31-epilepsy, 31MCI) completed neuropsychological testing and the attention and delayed memory subscales of the Cognitive Difficulties Scale (CDS), a self-report measure of everyday cognitive skills. Informants completed the CDS only. Cognitive domain scores were created. Awareness was defined as the difference between patient and informant CDS scores: greater informant complaints indicated limited awareness.

Results: Neuropsychological performance was similar between groups for all domains except delayed memory $(t(60)=2.49, p<.05)$, which was worse for MCI. CDS scores were similar between patient groups ( $p$ $>$.05). Epilepsy and MCI informant CDS scores were related to aspects of objective cognitive function. Limited awareness was found in $29.0 \%$ of epilepsy and $61.3 \%$ of MCI participants. Awareness was not related to cognition in epilepsy but was related to worse delayed memory $(r=$ $-.41, \mathrm{p}=.02)$ in MCI

Conclusions: Older adults with epilepsy and MCI had similar cognitive deficits, with the exception of delayed memory, but the MCI group had less awareness of deficit suggesting that delayed memory may be a critical factor for awareness of deficit. Future research should also examine the potential contribution of differences in informant perceptions of cognitive deficits between these two groups.

Correspondence: Rachel Galioto, MA, Warren Alpert Medical School of Brown University, 110 Village Grn N, Apt B, Riverside, RI 02915. E-mail: rachel_wiedemann@brown.edu

R. GALIOTO, S. THAMILAVEL, A. BLUM \& G. TREMONT. Subjective Cognitive Complaints vs. Objective Neuropsychological Performance in Older Adults with Epilepsy.

Objective: Memory complaints are common among epilepsy patients, though discrepancy between subjective complaints and objective performance often exists, suggesting the need for use of informant reports. This study examined the relationship between patient and informant ratings of everyday cognition and objective performance in older adults with epilepsy.

Participants and Methods: Thirty-one adults 55 or older with epilepsy completed a brief battery of neuropsychological tests, the Beck Depression Inventory, and the Cognitive Difficulties Scale (CDS), a self-report measure of everyday cognitive skills. Informants completed the CDS only. Cognitive domain scores (attention/executive function, immediate 
and delayed memory, language, visuospatial skills) were created. Higher CDS scores reflect greater cognitive complaints.

Results: Objective cognitive impairment (1.5 SD's below normative mean) was most common for immediate $(58.1 \%)$ and delayed $(67.7 \%)$ memory. Epilepsy patients reported greater cognitive complaints than their informatnts $(p<.01)$. Greater subjective cognitive complaints by epilepsy patients were related to lower immediate memory scores $(\mathrm{r}=-.40, \dot{\mathrm{p}}=.026)$. After controlling for patient depression, CDS and immediate memory were no longer significantly related. Informants' ratings were not associated with objective cognitive performance, but correlated significantly with patients' depression $(r=.48, p=.003)$. Conclusions: Subjective cognitive complaints in older adults with epilepsy have stronger relationships with depression than objective cognitive performance. Informants' ratings were not related to objective performance and were influenced by patient-reported depression. These results highlight the need for caution when using patient, and even informant, report as a proxy measure in this population.

Correspondence: Rachel Galioto, MA, Warren Alpert Medical School of Brown University, 110 Village Grn N, Apt B, Riverside, RI 02915. E-mail: rachel_wiedemann@brown.edu

\section{A. ALLEN, A.R. LOUGHAN, J. HERTZA \& M. COHEN. Varying Impairments in Children with Landau-Kleffner Syndrome: Short- term Auditory Memory and Attention.}

Objective: Landau-Kleffner Syndrome (LKS) is a rare epileptic syndrome with symptoms presenting typically in childhood. In addition to seizures, LKS is associated with language regression, as well as attention and behavioral difficulties. Previous research suggested that all children with LKS had impairments in short-term auditory memory and attention. This study sought to validate this earlier research.

Participants and Methods: Data was collected on 11 children (Mage $=$ 9; range 6-13) diagnosed with LKS who completed neuropsychological testing. Sixty-four percent were male. All children were Caucasian with overall low average IQ $(\mathrm{MIQ}=82$; range 59-101). Data was entered and analyzed via SPSS.

Results: During neuropsychological attention measures, 9/11 (81\%) performed below average in the CMS Attention Index. Five out of 9 $(56 \%)$ performed below average during WISC Digit Span. Attention deficits were supported on both parent and teacher questionnaires (BASC-2). Sixty-seven percent (6/9) of teachers and 50\% (5/10) of parents reported elevated inattention symptoms ( $\mathrm{T}>65)$; while $33 \%(3 / 9)$ of teachers and $30 \%(3 / 10)$ of parents endorsed impulsive symptomology. Regarding short-term auditory memory, $50 \%(5 / 10)$ performed in the below average range on the CMS Immediate Verbal Memory Index. Conclusions: Although many children performed in the below average range during auditory short-term memory and attention measures (approximately half or above), not all children were below average as expected given previous reports. This suggests that attention and auditory memory are areas of concern for some, but may not always be impaired in children with LKS.

Correspondence: Ashlee R. Loughan, Ph.D., NeuroBehavioral Associates, 1355 Independence Dr, Augusta, GA 30901.E-mail: aspicer88@yahoo. com

\section{A.L. WARE, J.D. SLATER, O.A. HOPE, G.P. KALAMANGALAM, N. TANDON \& J.I. BREIER. The Intracarotid Amobarbital Procedure Predicts Change in Verbal Memory in Patients With Good Pre-operative Memory Who Undergo Temporal Lobectomy in the Language Dominant Hemisphere.}

Objective: To address whether the intracarotid amobarbital procedure (IAP), whose utility is questioned in the literature, predicts change in verbal memory function after temporal lobectomy in the language dominant hemisphere (DTL).

Participants and Methods: 39 patients with intractable epilepsy (age $\mathrm{M}=36, \mathrm{SD}=11$; Males=32) underwent DTL. Variables included difference in delayed memory (Memdf) on the Buschke VSRT or CVLT-II across the operation (post-pre) and IAP memory performance. IAP memory testing consisted of presentation of 8 target objects during each injection, followed by post-injection recognition of targets among 16 foils. Total correct were adjusted for false positives. Hemispheric dominance was determined by IAP language testing.

Results: Memdf negatively correlated, $\mathrm{r}=-.53, \mathrm{p}<.0005$, with pre-operative memory, but not with IAP scores in either hemisphere. However, when patients with low IAP scores in the dominant hemisphere and good pre-operative memory (i.e., anomalous scores) were removed from analyses $(n=7)$, IAP scores in the dominant hemisphere and Memdf correlated, $r=-.50, p<.003$. Furthermore, pre-operative memory was a poor predictor of Memdf for those with average pre-operative memory $(\mathrm{n}=10), \mathrm{p}>.7$, while IAP scores in the dominant, $\mathrm{r}=-.62, \mathrm{p}<.05$ and non-dominant, $\mathrm{r}=.64, \mathrm{p}<.05$ hemispheres and total score in both hemispheres, $\mathrm{r}=-.79, \mathrm{p}<.01$, correlated with Memdf.

Conclusions: Anomalous IAP scores likely represent procedural confounds and should be questioned. Furthermore, the IAP has particular utility in participants with average pre-operative memory, where pre-operative memory was not valuable; higher total IAP scores suggested greater risk for memory decline. This may result from disrupted interhemispheric subcortical-cortical connections following DTL.

Correspondence: Ashley L. Ware, M.A., Psychology, University of Houston, 2003 Southgate Blvd, Apt 2, Houston, TX 77030. E-mail: aware2004@gmail.com

E. FARRELL, M. MAIMAN, L. VAURIO, W.S. MACALLISTER \& W. BARR. Use of the Repeatable Battery of Neuropsychological Status (RBANS) in Patients with Epilepsy.

Objective: The RBANS has been shown to be a useful brief screening measure in dementia and other clinical populations. The current study evaluated use of the RBANS in patients with epilepsy.

Participants and Methods: A total of 98 patients with a diagnosis of epilepsy or suspected seizures completed the RBANS as part of routine neuropsychological evaluations, conducted on either an inpatient or outpatient basis. The sample was well distributed with regard to gender, education, and type of epilepsy. Mean IQ was in the average range (96.01, SD=19.06).

Results: One-third of the subjects performed in the extremely-low range $(<70)$ on the RBANS Total Score Index. Mean scores on the Immediate Memory, Visuospatial, and Language indices fell within the low average range. Attention (mean=79.7, $\mathrm{SD}=19.8$ ) and Delayed Memory (mean=76.5, $\mathrm{SD}=21.9$ ) scores fell in the borderline impaired range. A total of $32.3 \%$ performed in the extremely low range on the RBANS Attention Index and $34.4 \%$ scored at that level on the Delayed Memory Index. Results from multivariate analyses (MANOVA) revealed significant differences among the index scores with the primary deficit observed on the Delayed Memory Index.

Conclusions: Overall, this study suggests that the RBANS has utility in evaluating relevant deficits in patients with epilepsy and is sensitive to finding specific deficits in attention and delayed memory, consistent with results from other studies on epilepsy using more extensive test batteries. The findings suggest that the RBANS provides a sensitive means of evaluating neuropsychological functions in patients with epilepsy. Correspondence: Eileen Farrell, NYU, 1330 Grand Street, Apt 506, Hoboken, NJ 07030.E-mail: eileen.farrell@gmail.com

D.D. HARgRAVE, J. PFALLER, B. BELL, B.P. HERMANN, J. JONES \& R.K. MAGANTI. Appropriateness of Rey 15-Item Test with recognition trial and Dot Counting Test for Assessing Performance Validity in Adults with Epilepsy.

Objective: Undetected invalid cognitive performances in adults with epilepsy could lead to inappropriate treatment plans, contributing to elevated morbidity. Some studies suggest significant variability in failure rates on performance validity tests in epilepsy samples, suggesting the potential need for adjustments to typical cutoffs. This study sought to determine the appropriateness of the Rey 15-Item Test with recognition 
trial (FIT) and the Dot Counting Test (DCT) for adults with documented epilepsy.

Participants and Methods: Individuals admitted to the epilepsy monitoring unit of a university epilepsy program were included if they had epilepsy (and no psychogenic non-epileptic seizures) diagnosed by a neurologist specializing in epilepsy, were administered the standard clinic battery including the FIT or DCT, and had an estimated WAIS-IV IQ > 69. After exclusions, 38 patients were selected (all 38 had the FIT and 37 had the DCT).

Results: Individuals ranged from 18 to 69 years of age $(M=40.00$, $\mathrm{SD}=15.52)$ and averaged greater than a high-school education $(\mathrm{M}=$ $13.08, \mathrm{SD}=2.15)$. WAIS-IV estimated IQ ranged from 71 to $119(\mathrm{M}=$ 93.89, $\mathrm{SD}=12.39)$. A majority of patients $(\mathrm{n}=33)$ were taking one or two antiepileptic drugs at the time of testing. Four individuals (10.81\%) failed the DCT (e-score $\geq 17 ; \mathrm{M}=11.46, \mathrm{SD}=3.09$ ) and four individuals $(10.52 \%$ ) failed the FIT (combination score $<20$; $\mathrm{M}=27.08$, SD $=4.28$ ). One of these patients failed both measures. This person and one other individual failed at least two validity indicators (including performances on embedded validity indicators) and were judged to have performed non-credibly. The remaining failures numbered three $(8.3 \%)$ for the DCT and two $(5.6 \%)$ for the FIT.

Conclusions: The failure rates found in this inpatient adult epilepsy sample approximate reported rates of invalid performance in general medical populations, suggesting that the FIT and DCT may be appropriate validity measures for this population.

Correspondence: David D. Hargrave, Psy.D., Neurology, University of Wisconsin-Madison, 600 Highland Ave, Madison, WI 53792. E-mail: david.hargrave@gmail.com

K.J. MCKITTRICK, K. SKILLICORN, D. KRIZ \& S.T. LI. The Impact of Illness-related, Psychopathological, and Demographic Variables on Families of Children with Epilepsy.

Objective: Few studies have examined the impact of epilepsy on family functioning. This study investigated the impact of epilepsy on families of children with this condition using a well-validated measure of family impact. The purpose of the study was to examine the relationship between illness-related, comorbid psychopathological, and demographic variables and the impact of epilepsy on families of pediatric patients. It was hypothesized that several factors including seizure severity, number of antiepileptic medications, internalizing and attention problems, and age would be associated with higher scores on the Impact on Family Scale (IOFS, Stein \& Jessop, 1985).

Participants and Methods: Preliminary analyses were conducted on an initial sample of 7 participants with epilepsy, ages 8 to 13, from an ongoing study. The subjects participated in a pediatric neuropsychological evaluation as part of their epilepsy care.

Results: The impact of seizure severity on families was assessed by examining the mean difference in IOFS scores for children with frequent ( $>31$ seizures/month; $\mathrm{n}=3$ ) and infrequent $(<31$ seizures/month; $\mathrm{n}=$ 4) seizures. This analysis indicated that families of children with more frequent seizures tended to report higher negative impact. The impact of age was also assessed by examining the mean difference on IOFS scores for older children (ages >10) and younger children (10 and below). This analysis suggested that families of older children with epilepsy also tended to report higher negative impact. Further, moderate positive correlations were found between IOFS scores and scores of internalizing and attention problems. The mean difference in IOFS scores reported by families of children taking 5 or more AED's and children taking less than 5 AED's was not qualitatively different.

Conclusions: Based on these analyses, preliminary data suggests support for seizure severity, attention problems, internalizing problems, and older age as being related to greater negative impact on families of children with epilepsy.

Correspondence: Kevin J. McKittrick, M.S., School of Professional Psychology, Pacific University, 2229 NW Everett st Apt 1, Portland, OR 97210.E-mail:mcki7796@pacificu.edu
M. MAIMAN, M. VASSERMAN, B. KAHN, H. MURPHY, H.A. BENDER, R. COHEN \& W.S. MACALLISTER. Sensitivity of the Wisconsin Card Sorting Test-64 versus the Tower of London for Detecting Executive Dysfunction in Children with Epilepsy.

Objective: It is well established that executive function deficits are prominent in children and adolescents with epilepsy. The Wisconsin Card Sorting Task (WCST) is often considered the 'gold standard' for executive function assessment, but the sensitivity of this instrument, particularly the short 64-card version (WCST-64), is insufficiently established in pediatric samples, including epilepsy. The goal of the present investigation is to assess the sensitivity of the WCST-64 in detecting executive function impairment in children with epilepsy in comparison to another established executive function measure, the Tower of London-Drexel Version (TOL).

Participants and Methods: 88 consecutively evaluated children and adolescents with epilepsy (age range 7-18) were administered both the WCST-64 and TOL as part of a comprehensive clinical neuropsychological evaluation. Using a criterion of standard score $<79$ as the threshold for impairment (i.e., 1.5 SD's below normative mean or greater), the sensitivity of WCST-64 and TOL variables were established. Correlational analyses assessed the relations between WCST and TOL variables with epilepsy severity factors including age of epilepsy onset, seizure frequency, and number of anti-epilepsy medications.

Results: The most sensitive of all WCST-64 variables detected executive function impairment in fewer than $20 \%$ of the population (perseverative responses, $19.3 \%$ impairment) versus TOL variables that detected impairment in about half (e.g., Rule Violations, $50 \%$ impaired, Total Moves $45.5 \%$ impaired). The relations between TOL variables and epilepsy severity variables were more commonly statistically significant and overall more robust than those seen between the WCST and epilepsy severity variables.

Conclusions: In comparison to the TOL, the WCST is less sensitive to executive function impairment in children and adolescents with epilepsy. Correspondence: William S. MacAllister, PhD, Neurology, NYU, 223 East 34th St, NYU Epilepsy, New York, NY 10016. E-mail: william. macallister@nyumc.org.

K.R. WOLFE, L. HAMIWKA \& K. MCNALLY. Executive Functions following Pediatric Epilepsy Surgery: Reliable Change Index Analysis.

Objective: Evaluate change in executive functions (EF) following pediatric epilepsy surgery compared to controls with complex partial seizures (CPS) who did not undergo surgery.

Participants and Methods: Retrospective clinical data were obtained from 35 youth ages 8-18 with intractable epilepsy who underwent neuropsychological evaluation pre- and post-epilepsy surgery $(\mathrm{M}=18.06$ months apart; range $=9.00-46.00$ months), as well as from a control group of 20 youth ages 8-18 with CPS who underwent repeat evaluations ( $\mathrm{M}=22.75$ months apart; range $=7.00-34.00$ months). Reliable change index (RCI) was utilized to assess the frequency and magnitude of change in EF (i.e., working memory, cognitive flexibility, perseveration, and concept formation) in the surgery group.

Results: Paired t-tests indicated that the surgery group improved across all $4 \mathrm{EF}$ domains ( $\mathrm{ps}<05$ ). The control group improved in cognitive flexibility $(p<.05)$. RCI analysis yielded wide confidence intervals secondary to high test-retest variability in controls. Despite this variability, $8-33 \%$ of participants in the surgery group improved in working memory, concept formation, and perseveration using RCI. Most participants in the surgery group showed no change outside of the RCI in EF. However, no participants in the surgery group showed RCI decline in EF

Conclusions: A subset of epilepsy surgery patients significantly improved in EF following surgery, which could be attributed to improved seizure control. Use of a clinical control sample illuminates the inherent variability in $\mathrm{EF}$ over time in pediatric epilepsy, highlighting the need for rigorous methods such as RCI when exploring post-surgical outcomes in this population. 
Correspondence: Kelly R. Wolfe, Nationwide Children's Hospital, 105 E. Frankfort St., Columbus, OH 43206.E-mail: kelly.ross.wolfe@gmail. com

K.D. SRNKA, C. KAY, A. HAMM, D. JACKSON, J. JONES, D. ALMANE, K. DABBS, B.P. HERMANN \& M. SEIDENBERG. Intra-Individual Variability in Children with Recent Onset Epilepsy.

Objective: Intra-individual variability (IIV), traditionally viewed as an index of error variance, has recently been re-considered as an index of cognitive and neural efficiency. Many children with childhood onset epilepsy (CWE) show significantly lower mean performance in intelligence quotient (IQ) and academic achievement. However, the potential use of IIV in characterizing their performance has not been investigated. Mean omission (OM) and commission (COM) errors on the Connors' Continuous Performance Test II (CPT-II) and variability in response speed consistency (IIV) was examined between CWE and healthy controls (HC) to determine the predictive utility of IIV in IQ and academic achievement.

Participants and Methods: Ninety HC and 143 CWE participants (Mage=12 years) were administered the CPT-II, Wechsler Abbreviated Scale of Intelligence-Second Edition (WASI-II), and the Wide Range Achievement Test-3 (WRAT-3). No group differences were evident for age or gender ( $p$ 's $>0.05)$. Analyses included pairwise t-tests; logistic regression analyses, and multiple regression analyses.

Results: CWE showed increased IIV compared to HC ( $\mathrm{p}<0.01 ; \mathrm{d}=0.66)$. IIV added to prediction of correct group classification for COM $(\beta=0.07$; Wald=17.95; $p<0.01)$. Additionally, higher IIV predicted lower performance IQ $(\beta=-0.25 ; \mathrm{t}=2.36, p=0.01)$, word recognition $(\beta=-0.19$; $\mathrm{t}=1.79 ; \mathrm{p}=0.08)$, spelling $(\beta=-0.19 ; \mathrm{t}=1.79 ; \mathrm{p}=0.08)$, and arithmetic $(\beta=-0.27 ; \mathrm{t}=2.62 ; \mathrm{p}=0.01)$.

Conclusions: IIV is a useful measurement construct in examining IQ and academic achievement in childhood epilepsy. Our future work will examine the neural basis for increased IIV in CWE, and its impact on prospective cognitive and behavioral adjustment.

Correspondence: Kyle D. Srnka, Psychology, Rosalind Franklin University of Medicine and Science, 3503 Green Bay Rd, \#307, North Chicago, IL 60064.E-mail: kyle.srnka@my.rfums.org

M.R. SCHOENBERG, K.E. EICHSTAEDT, A. TABAK, W.E. CLIFTON, S.R. BENBADIS, A.M. BOZORG, N.T. RODGERSNEAME, M.L. MATTINGLY \& F.L. VALE. Improved Surgical Treatment for Temporal Lobe Epilepsy? Neuropsychological Outcome Following the Inferior Temporal Gyrus Approach for Selective Amygdalohippocampectomy.

Objective: The study examines pre- to postoperative changes in neuropsychological status among a sample of localization related temporal lobe epilepsy (TLE) patients who had undergone a new selective amygdalohippocampectomy procedure using an inferior temporal gyrus approach.

Participants and Methods: Participants included nine left TLE (LTLE) and 13 right TLE (RTLE) patients who achieved Engel Class I surgical outcome. Measures included Wechsler Adult Intelligence Scale (WAIS-IV) full-scale intellectual quotient (FSIQ); Rey Auditory and Verbal Memory Test (RAVLT) immediate and long delay trials; and trials I and II of the following Wechsler Memory Scale (WMS-IV) subtests: Logical Memory, Paired Associates, Designs, and Visual Reproduction. Results: No significant demographic differences existed between the left and right TLE groups in terms of age, education, or gender, with $\mathrm{p}$ values equaling .71,.07, and .94, respectively. No changes in FSIQ were observed within either group, with LTLE and RTLE $p$ values of .62 and .80, respectively. Among the LTLE patients, postoperative verbal memory decline was evident, with significant declines in performance on WMS-IV Verbal Paired Associates II $(p=.02, \eta 2=.33)$, and RAVLT immediate delay $(\mathrm{p}<.01, \eta 2=.39)$. Among the RTLE patients, a trend approaching significance was noted regarding potential decline in visual memory on WMS-IV Designs II $(p=.09)$.

Conclusions: Initial findings suggest this selective amygdalohippocampectomy approach spares global intellectual functioning, but material specific verbal memory decline continuing to occur among patients with LTLE. Trend decline in a measure of visual memory observed for RTLE cases, but small sample size prevents determination of any meaningful decline in this measure of visual memory.

Correspondence: Katie E. Eichstaedt, MA, Florida School of Professional Psychology at Argosy University, Tampa, 5452 Baywater Drive, Tampa, FL 33615.E-mail:keichstaedt@stu.argosy.edu

\section{CHAPIESKI \& E.H. GERST. Do Memory Tests Provide any} Localizing Information in Pediatric Epilepsy?

Objective: Adults with focal temporal lobe seizures often show a pattern of lateralized, material specific memory impairments but this pattern has not been as commonly reported in pediatric cases. This study investigated the localizing value of memory tests in a pediatric sample while controlling for potentially confounding variables.

Participants and Methods: Participants included108 pediatric patients with focal seizures and IQ's > 69. Principal Component Analysis of memory tests revealed four factors, explaining $81 \%$ of the variance: Factor I (WRAML Story Memory subtests); Factor II (WRAML Verbal Learning subtests) Factor III TOMAL Visual Learning subtests); and Factor IV (TOMAL Facial Memory subtests). Scores from the Peabody Picture Vocabulary Test loaded highly on Factor I and scores from Judgement of Line Orientation loaded highly on Factor III. Participants were categorized by seizure focus-temporal lobe, frontal lobe or occiptial/pariental. Temporal lobe patients were also divided into left and right hemisphere and with and without mesial involvement. Age of onset, duration of disorder, seizure frequency, number of anticonvulsant drugs (AED) and age were evaluated as possible confounding variables. Results: Composite memory scores were created and group differences were evaluated with ANOVA. With one exception, there were no group differences on any of the memory test composites. The one exception was the lower scores on the Story Memory composite for the temporal lobe group with mesial involvement, irrespective of hemisphere, $\mathrm{p}<.01$. Inclusion of age and other seizure variables did not alter the findings. Seizure frequency was inversely related to scores on the Verbal Learning composite, $p<.01$ and number of AED's was inversely related to scores on all of the memory tests, $\mathrm{p}<.05$.

Conclusions: Performance on memory tests appears to be more dependent on the specific material to be recalled than seizure focus in young people with epilepsy. AED's have a uniformly dampening effect on memory test performance.

Correspondence: Lynn Chapieski, Ph.D., Baylor College of Medicine, 6621 Fannin St.-CC-1250, Houston, TX 77030.E-mail: mlchapie@ texaschildrens.org

A.K. STEFANATOS \& N. NUSSBAUM. Clinically Meaningful Change in Psychosocial Functioning Following Pediatric Epilepsy Surgery.

Objective: The goal of this study was to examine changes in clinical classification on a measure of psychosocial functioning in children with intractable epilepsy following a surgical intervention.

Participants and Methods: Participants included 21 children (ages 6 to 17) seen for pre- and post-surgical evaluation through a comprehensive epilepsy program. Parents completed the Achenbach Child Behavior Checklist at baseline and again at follow-up approximately 1 year after surgery. Scores on behavioral scales were classified as clinically or non-clinically significant, and the proportion of children who improved in clinical status between evaluations was examined.

Results: Results from paired-samples t-tests revealed a significant decrease in externalizing and internalizing behavior problems following resective surgery $(p=.035 \& .002$, respectively), with effect sizes in the range of .49-.78. An exact McNemar's test determined there was a 
statistically significant decrease in the proportion of scores falling in the clinically significant range ( $\mathrm{T}$-score $>60$ ) on the Internalizing Behavior scale $(p=.031)$ at follow-up. Additionally, further examination revealed that, irrespective of clinical classification, 7 children demonstrated clinically significant improvement (1 SD decrease) post-operatively on the Internalizing Behavior scale, while 6 children demonstrated clinically significant improvement on the Externalizing Behavior scale.

Conclusions: While a number of studies have reported decreased behavioral and emotional problems following epilepsy surgery, few studies have attempted to determine whether clinically meaningful change can be observed at an individual level. The implications, strengths and limitations of the current study will be discussed.

Correspondence: Arianna K. Stefanatos, MA, Psychology, The University of Texas at Austin, 108 East Dean Keenton St., Austin, TX 78712. E-mail:akstefanatos@utexas.edu

\section{A. GOODING \& M.J. HAMBERGER. The relationship between age, executive functioning, and verbal learning and memory in epilepsy.}

Objective: Memory and executive functioning are often reduced in individuals with epilepsy, and both of these functions decline with normal aging. However, relatively little is known about the potential interaction between aging and epilepsy related factors on cognition. We examined the relation between executive functioning and verbal learning and memory in younger and older adults with epilepsy.

Participants and Methods: Participants included 76 younger (18$40)$ and 15 older $(60+)$ adults with epilepsy who underwent neuropsychological evaluations. Correlations followed by regression analyses assessed the influence of executive functioning (i.e., DKEFS Color Word Interference, Trails B) on verbal learning and memory (i.e., CVLT-II). Results: In younger adults, inhibition (DKEFS) and set switching (Trails $\mathrm{B})$ correlated with learning $(\mathrm{r}=.38, \mathrm{p}<.01$ and $\mathrm{r}=.24, \mathrm{p}<.01)$ and delayed recall $(\mathrm{r}=.31, \mathrm{p}<.01$ and $\mathrm{r}=.21, \mathrm{p}<.01)$. In older adults, inhibition was correlated with learning $(r=.61, p<.05)$, however, set switching was not correlated. Hierarchical multiple regression analyses revealed that in younger adults, both education and inhibition independently predicted learning and delayed recall performance $(\mathrm{p}$ 's $<.05)$. In older adults, inhibition uniquely predicted verbal learning $(\mathrm{p}<.01)$, but was not a significant independent predictor of delayed recall; education had no significant contribution for older adults ( $p$ 's $>.05)$.

Conclusions: Cognitive inhibition influences verbal learning in both younger and older adults. However, inhibition appears to be a more salient factor, and more contributory than other executive functions in older adults with epilepsy. Greater reliance on inhibitory mechanisms with age might be related to epilepsy related memory decline, normal aging, or the combination of these factors.

Correspondence: Amanda Gooding, Neurology, Columbia University Medical Center, 38 Morton Street, \#4C, New York, NY 10014. E-mail: amandalgooding@gmail.com

A.M. CARSON \& L. CHAPIESKI. Social Functioning in Pediatric Patients with Intractable Epilepsy: Relative Contributions of Seizure-Related Variables, Cognitive Functions and Parental Anxiety about Epilepsy.

Objective: Individuals with epilepsy are at risk for social problems. This study evaluated the relative contributions of seizure-related variables, cognitive functions and parental anxiety about epilepsy to social functioning in young people with intractable seizures.

Participants and Methods: Participants were 111 pediatric patients with intractable epilepsy, ages $5-19$ years-old $(\mathrm{M}=12$; $\mathrm{SD}=3)$, presenting for pre-surgical evaluations. Social functioning was assessed with the Social Competence and Social Problems Scales from the Child Behavior Checklist (CBCL), a parent report measure, and the Social Skills and Social Problems Scales from the Student Behavior Survey (SBS), a teacher report measure. The Verbal Comprehension Scale from an age-appropriate Wechsler Intelligence Scale was administered and one parent was asked to complete the Parent Anxiety Questionnaire. The relative strength of these variables as risk factors for poor social functioning was compared to a number of seizure-related variables, including age of onset, duration of epilepsy, seizure frequency, seizure type and number of anticonvulsants, with stepwise multiple regression.

Results: Verbal IQ and parental anxiety about epilepsy explained $16 \%$ of the variance on CBCL Social Competence Scale, $\mathrm{p}<.001$ and the Social Problems Scale, $p<.001$ as well as $15 \%$ on the SBS Social Competence $\mathrm{p}<.01$. Verbal IQ explained $11 \%$ of SBS Social Problems, $\mathrm{p}<.02$. No seizure-related variables remained in any of the models.

Conclusions: This study revealed the relative importance of verbal intelligence and parental anxiety about epilepsy for social development in the pediatric population. The contribution of parental anxiety suggests an important area for intervention for children with seizures and poor social functioning.

Correspondence: Audrey M. Carson, PhD, Baylor College of Medicinel Texas Children's Hospital, 6701 Fannin St, Suite 1630, Houston, TX 77030.E-mail:amcarson@texaschildrens.org

E. CHIN, M. LOMAN, C. HINKLE \& J. KOOP. The Utility of a Lateralization Rating of Cognitive Dysfunction in Pediatric Epilepsy Presurgical Evaluations.

Objective: Neuropsychological evaluation is part of gold-standard presurgical epilepsy workup to gauge the potential cognitive risks of surgical resection. Most knowledge about cognitive risk is from adults with temporal lobe epilepsy, in which a lateralized cognitive pattern concordant with the side of epileptic focus was found. Examination of this in pediatric patients has been limited due to the variability of seizure type and localization and possible functional reorganization. The purpose of the study is to investigate the utility and accuracy of a lateralization rating based on standard neuropsychological battery among pediatric epilepsy patients.

Participants and Methods: Data from 225 patients referred for a presurgical evaluation were examined. 109 patients who were English speaking with IQ>55 and the ages of 4-18 years were included. EEG data was used to determine the lateralization of the epileptic focus $($ left $=34$, right $=36$, other $=9)$. Resective surgery was performed (rightsided=32, left-sided=30, other surgeries=47). A cognitive lateralization rating index (LRI) was calculated based on data derived from cognitive domains that have been attributed to dominant and nondominant hemispheres.

Results: LRI score was dichotomized to indicate dominant $(>1)$ or nondominant $(<-1)$ hemisphere dysfunction. Chi square analysis indicated significant difference between patients with right- and left-sided epileptic foci on LRI, $\chi 2(1, \mathrm{~N}=48)=5.37, \mathrm{p}=.020$. Overall correct classification rate was $67 \%$. The sensitivity $(71 \%)$ and specificity $(68 \%)$ of LRI were higher for patients with left-sided epileptic foci. 12 patients demonstrated non-lateralized profile ( $L R I=0)$ despite lateralized epileptic foci (left=6 and right $=6$ ), in which suspected functional reorganization was noted in 3 patients with left-sided epileptic foci.

Conclusions: The results of this study indicate that it is possible to identify a lateralized cognitive profile in pediatric patients being considered for epilepsy surgery despite variability in localization of seizure onset. Correspondence: Esther Chin, Medical College of Wisconsin, 800 Hinman Ave, Apt 612, Evanston, IL 60202.E-mail: echin@mcw.edu

C. VEGA, L. BRENNER, D. WABER, M. VALLE \& K. BOYER. Verbal Memory Decline After Left Temporal Lobe Epilepsy Surgery in a Pediatric Sample.

Objective: To assess verbal and non-verbal memory outcomes following surgery for treatment of left temporal lobe epilepsy (LTLE) in a pediatric sample. We hypothesize that LTLE will be associated with post-surgical decline in verbal but not non-verbal memory.

Participants and Methods: Patients were identified through archival record review. The final sample consisted of 21 patients ( $52 \%$ female). Mean age at surgery was 14.86 years $(\mathrm{SD}=3.80$; range $=8-21)$. Wada/ 
fMRI testing documented left-lateralized language in all patients except for one. All patients completed tests of story memory (Children's Memory Scale [CMS] or Wechsler Memory Scale [WMS]), learning a word list (the California Verbal Learning Test-Children's and Adult Versions) and memory for faces (CMS or WMS) prior to surgery and within the first year after surgery (mean=7.33 months, $\mathrm{SD}=2.46)$. Paired sample t-tests were conducted.

Results: All patients had been seizure free for six months at the time of the post-surgical assessment (Engel Class I). There was a significant decline in performance on immediate recall of stories, as well as on word list learning $(p<.01)$. Seven patients did not have positive findings on structural MRI prior to surgery and were more likely to experience a decline. There was no decline in memory for faces (Immediate, $p=.91$; Delayed, $\mathrm{p}=.96$ ).

Conclusions: Decline in verbal memory occurs after dominant, left temporal lobe surgery in pediatric patients, but memory for faces is preserved. This study has implications for pre-surgical decision-making in pediatric populations.

Correspondence: Clemente Vega, Psy.D., Neurology/Psychiatry, Boston Children's Hospital/Harvard Medical School, 300 Longwood Ave, Fegan 9, Boston, MA 02115. E-mail: clemente.vega@childrens.harvard.edu

K.E. EICHSTAEDT, J.R. SOBLE, S.R. BENBADIS, A.M. BOZORG, N.T. RODGERS-NEAME, M.L. MATTINGLY, F.L. VALE \& M.R. SCHOENBERG. Verbal Fluency Performance in Temporal Lobe Epilepsy: General Verbal Ability Accounts for Lateralizing Effect of Phonemic but Not Semantic Fluency.

Objective: A sizeable minority of studies have shown lateralizing effects of phonemic verbal fluency performances among temporal lobe epilepsy (TLE) patients, suggesting more temporal lobe involvement than typically assumed for this predominantly frontal-mediated function. This study examines whether lateralizing effects exist for TLE patients in phonemic fluency beyond what can be accounted for by differences in general verbal ability.

Participants and Methods: Participants included 40 left TLE (LTLE) and 36 right TLE (RTLE) patients seen in consultation for preoperative neuropsychological assessment. Measures included Wechsler Adult Intelligence Scale, Fourth Edition (WAIS-IV) Verbal Comprehension Index (VCI); Controlled Oral Word Association Test (COWAT), and Animal Fluency Test.

Results: No significant demographic differences existed between the left and right TLE groups in terms of age, education, gender, duration of epilepsy, full scale IQ, with all $\mathrm{p}$ values $\geq .15$. Groups differed in terms of WAIS-IV VCI ( $p=.02)$, COWAT (.03), and Animal Fluency $(\mathrm{p}=$ .005 ) performances, with the LTLE group performing worse on all three measures. However, a series of ANCOVAs found that, when WAIS-IV VCI was used as a covariate, groups only differed in terms of Animal Fluency $(p=.03, \eta 2=.07)$, but not COWAT $(p=.46)$ performances. Stepwise hierarchical logistic regression with WAIS-IV VCI entered as step one found that COWAT was not a significant predictor $(p=.43)$ while Animal Fluency $(p=.03, \operatorname{Exp}(B)=1.06)$ remained a significant predictor of side of seizure onset.

Conclusions: Findings support previous research suggesting Animal Fluency is an independent predictor of seizure laterality. In contrast, data refute that phonemic fluency predicts seizure laterality beyond the variance accounted for by between-group differences in general verbal ability. Results contribute to testing data interpretation in the context of confirming lateralization/localization in preoperative epilepsy evaluations.

Correspondence: Katie E. Eichstaedt, MA, Florida School of Professional Psychology at Argosy University, Tampa, 5452 Baywater Drive, Tampa, FL33615.E-mail:keichstaedt@stu.argosy.edu

\section{Invited Symposium: Refining Our Expectations and Understanding of Cognitive Aging}

\author{
Chair: Emily J. Rogalski \\ 3:15-4:45 p.m.
}

E. ROGALSKI, E. ROGALSKI, S. CHAPMAN \& W.J. JAGUST. Refining Our Expectations and Understanding of Cognitive Aging. Symposium Description: The parallel observations that memory complaints are widespread among the elderly and aging is a major risk factor for Alzheimer's disease (AD), leads to the impression that a gradual loss of intellectual ability, eventually culminating in dementia, may be a nearly universal consequence of getting old. This impression raises significant concern, since people age $80+$ constitute the fastest growing segment of the U.S. population and there is currently no cure for AD. This symposium will question this impression by demonstrating that exceptional memory performance over age 80 is possible (Rogalski) and that there is potential for improving cognitive function in normal aging persons (Chapman). In addition, Dr. Jagust will share data about how the aging brain may undergo compensatory or plastic alterations that help maintain cognitive performance even in the face of amyloid deposition. Results from these studies are important for separating age-related changes of cognition and brain that are inevitable from those that are not necessarily universal in order to promote strategies for optimizing cognitive health and quality of life in old age.

Correspondence: Emily Rogalski, PhD, CNADC, Northwestern University, 320 E Superior Street, Searle 11-569, Chicago, IL 60611. E-mail: e-rogalski@northwestern.edu

\section{E. ROGALSKI. Neurobiologic Features of Cognitive SuperAging.} Memory complaints are widespread among the elderly, leading to the belief that a gradual loss of intellectual ability is an inevitable part of "normal" aging. However, recent data from our cohort of "SuperAgers," individuals in their 80 s and 90 s suggest that it is possible to have memory test scores at or above the average range for healthy individuals at least 20 to 30 years their junior. This session will describe the psychosocial, cognitive, neuroimaging, neuropathologic and molecular features associated with unusually preserved episodic memory performance over age 80. Identification of neurobiologic factors that contribute to exceptionally successful cognitive aging may make it possible to help elderly avoid disease and disability and improve quality of life.

Correspondence: Emily Rogalski, PhD, 320 E Superior Street, Searle 11-569, Chicago, IL 60611.E-mail:e-rogalski@northwestern.edu

\section{S. CHAPMAN. Brain and Cognitive Enhancement in Aging through Complex Reasoning and Aerobic Training.}

Growing efforts are searching ways to harness residual neuroplasticity in healthy older adults to counter diminishing cognitive capacity. Indeed, $87 \%$ of the adult population are not predicted to develop Alzheimer's disease but nonetheless are showing relentless and insidious loss of cognitive capacity and degradation of brain function. I will share new data contrasting the significant benefits from two protocols: cognitive training versus an aerobic exercise program. We found both training programs enhanced cognition and brain functions but in distinct ways. The results strengthen prior findings of the robust plasticity retained in the fronto-temporal cortical regions - the areas of greatest vulnerability to age-related losses and that represent networks subserving cognitive control and memory mechanisms. The accumulating data suggest that otherwise healthy adults - the largest population at risk for cognitive decline even in the absence of a frank dementia- can significantly regain cognitive losses and enhance their cognitive brain health. The scientific impact of showing cognitive enhancement (in both complex reasoning and aerobic exercise) and training-induced improved neural plasticity (through an MRI-derived resting state CBF non-invasive scans) offers 
promise in two ways: (1) lifestyle changes can be adopted to strengthen cognitive performance and (2) MRI-CBF measures may offer one informative methodology to monitor improved brain health states. To date, validated and safe metrics of improved neural health are nonexistent yet critical to incentivize attention to our brain's health. The impact of identifying a neuromarker of brain health with short-term trainings will influence the fields of neuroscience, psychology, aging, education, and medicine.

Correspondence: Sandra Chapman, 2200 W. Mockingbird Lane, Dallas, TX75235.E-mail: schapman@utdallas.edu

\section{W.J. JAGUST. Factors Associated With Age-Related Cognitive Decline and Compensation.}

As we age, many older people note subjective alterations in cognition and some measures of cognitive performance may also decline. These changes are not uniform and many older people maintain normal cognitive function. Brain aging is, in fact, a heterogeneous process not just in terms of performance but also in terms of the underlying biological factors that affect performance. For example, many older people show evidence of deposition of $\beta$-amyloid $(A \beta)$ in the brain. Despite the widely held view that this is a deleterious process, direct links between this aggregated protein and impaired cognition are weak. This talk will explore alterations in neural function that occur in aging from the perspective of how different processes associated with age, including $A \beta$ deposition might affect the brain. For example, older people without $A \beta$ show evidence of functional change in comparison to younger people; this may help maintain cognition in the face of age-related biological change. Even those with evidence of $A \beta$ are able to function in a normal range and there is also evidence of compensatory neural processes in response to $A \beta$ that may underlie these adaptations. Together these data provide a window into factors associated with brain aging and about why some individuals may retain good cognitive function into late life. Correspondence: William J. Jagust, M.D., 132 Barker Hall MC\#3190, Berkeley,CA 94720.E-mail: jagust@berkeley.edu

\section{Symposium 5: \\ Biological Markers of Social and Emotional Impairment after Traumatic Brain Injury}

\section{Chair: Miriam H. Beauchamp}

$$
\text { 3:15-4:45 p.m. }
$$

M.H. BEAUСHAMP, M.H. BEAUCHAMP, K.E. ROBINSON, J.A. RUSHBY, S. MCDONALD \& V. ANDERSON. Biological Markers of Social and Emotional Impairment after Traumatic Brain Injury.

Symposium Description: Social and emotional dysfunction following traumatic brain injury (TBI) is both common and persistent, often representing the most debilitating of all sequelae and affecting patients' ability to adapt to home, school, work and the community. There is increasing evidence from neuropsychological and behavioural studies that socio-cognitive and affective problems are characterized by theory of mind deficits, executive dysfunction, and emotional dysregulation; however, little is known of the biological substrates of these impairments. The advent of social neuroscience techniques enables researchers to empirically measure biological markers of social and affective functioning, including structural and functional neural correlates and physiological activity. In this symposium, four speakers will introduce their work devoted to understanding social and affective functioning after TBI. The studies span the developmental period from infancy to adulthood and the full range of TBI severity. First, we explore the effects of early mild TBI on social and affective functioning, as measured by theory of mind and electrophysiology. Second, differences in neuroanatomical integrity in children with TBI are linked to executive skills, theory of mind, and social adjustment. Third, the impact of severe TBI on emotional arousal in adulthood is examined using evidence from electrophysiology and magnetic resonance imaging. Finally, psychophysiological methods are introduced as markers of social cognition and potential targets for biofeedback intervention after severe TBI in adulthood. The studies will be discussed within the broader context of outcome and intervention after TBI, as well as the applicability of social neuroscience methods to TBI research. Together, the findings will suggest that biological markers are useful in understanding the origins, nature and role of social impairments after TBI at all ages, and that they are potential loci for social and affective remediation.

Correspondence: Miriam H. Beauchamp, PhD, Psychology, University of Montreal, CP 6128 Succursale Centre-Ville, Montreal, QC H3C3J7, Canada.E-mail: miriam.beauchamp@umontreal.ca

F. D'HONDT, J. BELLEROSE, P. VANNASING, F. THEBAULTDAGHER, C. BEAUDOIN, J. GRAVEL, M. LASSONDE \& M.H. BEAUCHAMP. Theory of Mind and Emotional Face Processing after Early Mild Traumatic Brain Injury.

Objectives: Brain injuries under the age of five are common and can result in worse outcomes because of the fragility of the developing brain and vulnerability of emerging abilities. The appearance of appropriate social functions is a key milestone of this period, yet, little is known of the social and emotional consequences of early mTBI. The goal of this study was to identify early behavioural and biological markers of social functioning in preschool children with mTBI.

Participants and Methods: Preschool children (n=39, 18-60 months, $\mathrm{M}=42.59, \mathrm{SD}=11.18$ ) with $\mathrm{mTBI}$ were assessed 6 months post-injury on measures of theory of mind (ToM) and compared to 37 typically developing children $(\mathrm{M}=45.17, \mathrm{SD}=12.78$ months). Eighteen children with mTBI and 15 controls additionally completed an event-related potential protocol during which they looked at angry, neutral and happy faces. Results: Children with mTBI exhibited ToM deficits related to reasoning about the desires of others $(t(69)=.32, p=.01, d=0.64)$. ERP results revealed differences in arousal processing after mTBI compared to controls, as suggested by significant interactions at the level of P1 (shorter latencies for emotional faces than for neutral faces), N1 (lesser amplitudes for emotional faces) and LOP (lesser amplitudes for emotional faces).

Conclusion: The findings of this study suggest that even mild brain injuries sustained during the preschool years can lead to socio-cognitive difficulties, characterized by changes in ToM and alterations in the cerebral processing of emotional stimuli. These findings may have implications for broader social competence after early mTBI.

Correspondence: Miriam H. Beauchamp, PhD, CP 6128 Succursale Centre-Ville, Montreal, QC H3C3J7, Canada. E-mail: miriam. beauchamp@umontreal.ca

K.E. ROBINSON, E.D. BIGLER, M. DENNIS, H.G. TAYLOR, K. RUBIN, K. VANNATTA, C. GERHARDT, T. STANCIN \& K.O. YEATES. Executive Function and Theory of Mind as Predictors of Social Adjustment in Childhood TBI: Regional Brain Injury as a Moderator.

Objectives: Children with traumatic brain injury (TBI) are at risk for poor executive function and social cognition, and related adjustment difficulties. These difficulties may be a function of the impact of TBI on brain regions linked to social, behavioral, and emotional regulation. Participants \& Methods: A subset of 8- to 13- year old participants in a study on social outcomes following TBI underwent MRI following severe $(n=15)$, moderate $(n=6)$, or complicated-mild $(n=24)$ TBI. Children completed assessment of executive function (EF; TEA-Ch) and theory of mind (TOM). Parents rated children's social, behavioral, and emotional adjustment (BASC-2, ABAS-II). Children were divided into tertiles based on an adjustment composite score. We explored differences in brain integrity between the lowest $(n=15)$ and highest tertiles 
$(\mathrm{n}=15)$, and the impact of brain integrity on the association between $\mathrm{EF} / \mathrm{TOM}$ and adjustment.

Results: Children in the lowest adjustment tertile were more likely than children in the highest tertile to have a brain abnormality $(100 \% \mathrm{v}$ $60 \% ; \mathrm{X} 2=7.50, \mathrm{p}=.006)$ and temporal lobe abnormalities (33\% v 7\%; $\mathrm{X} 2=3.33, \mathrm{p}=.068)$. Although brain volumes in specific regions of interest were not related to adjustment, temporal lobe (TL) pathology moderated the association between EF/TOM and adjustment. For children without TL pathology, higher EF/TOM predicted better adjustment; the relationship was not significant for children with TL pathology.

Conclusions: MRI documentation of brain insult, particularly TL injury, increases children's risk for maladjustment, even when EF/TOM are intact. Broad and specific indicators of neuropathology should be considered, particularly when studying complex skills and with small/ heterogeneous samples.

Correspondence: Kristen E. Robinson, Ph.D., 700 Children's Drive, Columbus, OH 43205. E-mail: kristen.robinson@nationwidechildrens. org

J.A. RUSHBY, S. MCDONALD, A. FISHER, N. PARKS \& O. PIGUET. Diminished Arousal and Emotional Responsivity after Severe Traumatic Brain Injury.

Objective: The aim of this study was to investigate neurophysiological correlates of dysregulated emotional arousal after severe traumatic brain injury (TBI). We examined whether a relationship between arousal [indexed by EEG alpha power and skin conductance levels (SCL)] and brain atrophy (specifically in the insula and amygdala) mediates emotional responsivity after TBI.

Participants and Methods: Nineteen adults with severe TBI and 19 age-, education-, and gender-matched controls (all p's > 0.05) participated. Mean EEG alpha power and SCLs were recorded simultaneously across four conditions: eyes-closed baseline, view neutral face, happy face and angry face. Magnetic resonance imaging (MRI) scan established bilateral insula, amygdala and hippocampus volumes.

Results: Scalp-wide alpha suppression occurred from pre-task baseline to the face-viewing conditions $(\mathrm{p}<.001)$, but was diminished in TBI $(p=.04)$. TBI participants exhibited marginally significantly lower SCL $(p=.051)$, and elevated alpha power in the outer left/right hemisphere regions, contrasting with controls' midline dominance $(\mathrm{p}<$ $.01)$. Significant atrophy was observed in most structures in TBI participants ( $p$ 's $=.004-0.04)$. Larger left insula, left amygdala and right amygdala correlated positively with alpha power, alpha suppression and SCL, whereas reduced hippocampal volume did not relate to any of the arousal variables.

Conclusions: Findings suggest that alpha power provides a sensitive measure of dysregulated emotional arousal post-TBI. Atrophy in pertinent brain structures may contribute to these disturbances. These findings have implications for the assessment and remediation of TBI related arousal deficits, by directing more targeted remediation, and better assessing post-TBI recovery.

Correspondence: Jacqueline A. Rushby, PhD, PO Box 6355, UNSW, Sydney, NSW 1466, Australia.E-mail: j.rushby@unsw.edu.au

S. MCDONALD, F. HEATHER, A. FISHER \& J.A. RUSHBY. Heart Rate Variability as an Index of Emotional Processing Disturbance in People with Traumatic Brain Injury.

Objective: Heart rate variability (HRV) indexes autonomic regulation: balance between sympathetic and parasympathetic activity. HRV correlates with neural activity in the prefrontal cortex during emotional tasks and is disrupted in disorders of arousal such as depression, anxiety and pain. It has never been examined in people with traumatic brain injury (TBI). Learning to breathe at an optimal rate (biofeedback) normalises HRV in migraine, depression and anxiety and is associated with improved mood and cognitive function.
The following study aimed to determine whether HRV (1) is disrupted in people with TBI, (2) is associated with poor emotional functioning and (3) can be modified with biofeedback.

Participants and Method: 30 adults (23 males: mean age 46) with TBI (Mean PTA $=46$ days) and 30 control participants (23 males: mean age 47) were tested. HRV was monitored via electrodes on the wrists before and after a biofeedback session. Participants were also assessed for alexithymia (TAS-20), empathy (BAVQ, IRI), and social cognition (TASIT). Results: The TBI group was more variable on resting HRV than controls. There was no difference between groups at baseline. In the TBI group parameters of resting HRV, specifically, lower SDNN, rMSSD, LF and $\mathrm{HF}$ were associated with lower self-reported empathy and poorer social cognition. Lower SDNN and LF were correlated with greater alexithymia. HRV improved after biofeedback in both groups.

Conclusions: HRV indexes emotion processing disturbance in people with TBI. Brief biofeedback improves HRV in both people with TBI and controls and may have promise as a remediation approach.

Correspondence: Skye McDonald, PhD, UNSW, Sydney, NSW 2052, Australia.E-mail:s.mcdonald@unsw.edu.au

\section{Symposium 6: \\ Functional Mapping for Presurgical Planning Using dEEG Source Localization and Transcranial Stimulation}

\author{
Chair: Catherine Poulsen
}

3:15-4:45 p.m.

C. POULSEN, C. POULSEN, C. KUO \& P. LUU. Functional Mapping for Presurgical Planning Using dEEG Source Localization and Transcranial Stimulation.

Symposium Description: Functional brain mapping is an important component of presurgical planning, both for assessing the viability of a candidate for neurosurgery, and for guiding resection away from eloquent cortex. Current clinical practice for functional brain mapping can include the use of intracranial grids and strips for local recording and stimulation of cortical areas, administration of the Wada test for determining hemispheric lateralization of language, and intraoperative stimulation mapping. All of these techniques are invasive and pose additional patient risks. With the advent of noninvasive brain imaging, researchers have begun to develop safer protocols for functional mapping. Once validated, these noninvasive methods may serve to supplement and guide the more invasive techniques, and eventually even supplant them. This symposium outlines a strategy, and presents preliminary support, for a noninvasive functional mapping approach that combines electrical source imaging (ESI) of dense-array electroencephalography (dEEG) with transcranial stimulation, either transcranial magnetic stimulation (TMS) or transcranial electrical neuromodulation (TEN) using either direct or alternating current stimulation (tDCS or tACS). In this dEEG approach, ESI is first used to identify active cortical regions during domain task performance (e.g., language, motor, memory). TMS or TEN can then be applied to 1 ) facilitate or interrupt performance and thereby verify the role of identified regions, and 2) study network propagation dynamics. In this symposium, we present two examples of functional mapping with ESI in the language and motor domains. We then illustrate how transcranial stimulation can be applied to verify and probe functional regions and networks using a motor protocol.

Correspondence: Catherine Poulsen, Ph.D., Electrical Geodesics, Inc., 500 East 4th Ave., Suite 200, Eugene, OR 97401.E-mail: cpoulsen@ egi.com 


\section{POULSEN. Dense-array EEG Source Localization of Language Function.}

Objective: We seek to develop a dense-array electroencephalography (dEEG) protocol to reliably identify hemispheric dominance and functional localization of receptive and expressive language processes. Once validated, this protocol will offer a safer, non-invasive alternative to the Wada test and intracranial recording.

Participants and Methods: Eight healthy, right-handed adult volunteers (mean age $=22$ years; 3 male $)$ completed auditory word and picture versions of the Verb Generation Task (VGT) and Picture Naming Task while 256-channel EEG was recorded. Event-related potentials (ERPs) were computed and LORETA linear inverse source analysis was performed to localize the scalp-recorded activity to cortical source regions. Results: Scalp ERPs indicated significantly left-lateralized activity for all language tasks, greatest for the auditory VGT compared to either the picture VGT or naming task. Localized source activity was consistent with current language models (e.g., Hickok \& Poeppel, 2007) and fMRI research. Source activity for the auditory VGT showed clear left-lateralization in lateral inferior prefrontal, and temporal cortex regions associated with language processing. Similar regions were active for the picture VGT and picture naming task, but the activity was weaker and more bilateral.

Conclusions: Source-localized dEEG shows promise as an affordable and portable non-invasive language mapping method. Results were most robust with the auditory VGT protocol. Future research will extend this protocol to neurosurgical candidates cross-validated with fMRI, intracranial recording, and the Wada test. Finally, transcranial magnetic and/or electrical stimulation will be selectively applied to localized language regions to disrupt or facilitate performance.

Correspondence: Catherine Poulsen, Ph.D., 500 East 4th Ave., Suite 200,Eugene, OR 97401.E-mail: cpoulsen@egi.com

C. KUO. Dense-array EEG Source Localization of Motor Function. Objective: Increases and decreases in different electroencephalographic (EEG) frequency bands are associated with movement preparation and execution, with a dominant scalp distribution over the contralateral motor cortex. How these frequency specific changes relate to the primary motor and somatosensory cortices are not clear. Therefore, the goal of the present study is to estimate the cortical sources of these frequency specific changes to determine their relation to the underlying cortex. Participants and Methods: In the present study, parallel dense-array EEG (dEEG) and functional magnetic resonance imaging (fMRI) sessions with similar thumb movement tasks were performed in five participants. Movement-related changes in five frequency bands (theta, mu, beta, low gamma and high gamma) were localized using 256-channel dEEG recordings and high-resolution individual head models. Electrical source imaging (ESI) was performed on the dEEG data and source results were compared with localizations from fMRI data as well as transcranial magnetic stimulation (TMS) maps of primary motor cortex. Results: Event-related desynchronizations (power decreases) in the beta-band $(14-30 \mathrm{~Hz})$ were the most robust effects across all participants, consistently localized to the contralateral hand region of the primary motor cortex, and consistently aligned with fMRI and TMS localizations. Conclusions: Dense-array ESI of movement-related spectral changes demonstrated good localization accuracy for functional identification of the primary motor cortex. This information can be used to guide presurgical planning as well as information for cortical neuromodulation of motor cortex for rehabilitation.

Correspondence: Ching-Chang Kuo, 275 S.Garden Way Apt 114, Eugene, OR 97401.E-mail: bensonk@uoregon.edu

\section{P. LUU. Neuromodulation of Primary Motor Cortex with Transcranial Direct Current Stimulation.}

Objective: Transcranial direct current stimulation (tDCS) is a developing technology that permits "non-invasive" modulation of cortical function. Lasting changes can be observed in the function of the targeted brain region, reflecting the impact of the injected current on mechanisms of neuroplasticity. Dense-array electrode technology can be employed to improve tDCS targeting. The goal of the present study is to demonstrate that standard dense electrode arrays can be employed, along with high-resolution electrical head models, to target and modulate primary motor cortex function.

Participants and Methods: In six participants, localization of primary motor (M1) cortex was accomplished with dense-array EEG electrical source estimation (ESI) and transcranial magnetic stimulation (TMS). Using the same high-resolution electrical head model employed for ESI for each participant, tDCS plans were derived for targeting M1. tDCS was then applied and the amplitudes of TMS motor evoked potential (MEP), from M1, before and after tDCS were compared.

Results: MEP amplitudes increased or decreased, depending on the polarity of the injected current, after tDCS in all participants. There was also variability in the MEP responses that is consistent with existing literature.

Conclusion: Accurate targeting of underlying brain structure for neurostimulation can be achieved from a dense network of scalp EEG sensors. tDCS can facilitate or inhibit function of the targeted region. Therefore, it has great therapeutic potential to verify functional localization for presurgical planning. Additionally, tDCS can be used to facilitate recovery of function during rehabilitation or treatment of neurological (e.g., epilepsy) and psychiatric (e.g., depression) disorders.

Correspondence: Phan Luu, Ph.D., 500, Eugene, OR 97401. E-mail: pluu@egi.com

\section{Poster Symposium: Assessment of Physicians: From Prospective Screening to Rehabilitation}

\author{
Chair: Kelly D. Garrett
}

3:15-4:45 p.m.

\section{Forensic Neuropsychology}

K.D. GARRETT, L. KORINEK, K.D. GARRETT, W. PERRY, E. GRACE \& K.D. GARRETT. Assessment of Physicians: From Prospective Screening to Rehabilitation.

Symposium Description: Many physicians wish to continue professional practice beyond typical age-based milestones of retirement. Given the anticipated shortage of physicians in the next decade (HSS/HRSA, 2008) there are pressures to retain competent physicians of age. Increasingly, Medical Staff Offices and governing boards seek input from neuropsychologists to address the question: Is this physician able to meet the task demands of their practice?

Increased vigilance is indicated as baserates of cognitive decline and compromise of other skills related to medical practices increase with age. Additionally, there are potentially problematic issues with relying solely on peer and other performance evaluations.

Neuropsychologists are uniquely positioned to proffer opinions regarding Medical Staff and Governing Board policies and procedures for screening, evaluation, assessment, and developing and executing recommendations of physicians of age. Yet this process is wrought with challenges for the neuropsychologist: from validity of assessments to potentially sticky colleagial interactions and other professional/legal/ ethical/risk management issues.

This symposium will present the issues and highlight best practices drawn from screening, assessment, and improvement of physician performance from three organizations: UCSD's Physician Assessment and Clinical Education (PACE)/ Physician Enhancement Program (PEP); the Center for Personalized Education for Physicians (CPEP); and 
Intermountain Healthcare, who has an active prospective screening program for all Medical Staff of age.

Correspondence: Kelly D. Garrett, Ph.D., Geriatrics/Center on Aging, Intermountain Healthcare \& University of Utah, 8th Ave \& C St, LDSH Geriatrics, 2N, Salt Lake City, UT 84103.E-mail: kelly.garrett@imail. org

\section{KORINEK. Overview and Scope of Neuropsychological} Performance Among Physicians of Advanced Age.

Due to the growing demand for physicians in the workforce and the tendency of physicians to retire at a later age than other professionals, the average age of practicing physicians has increased over the last 30 years. According to the AMA, while the number of physicians has increased by approximately 200 percent, the number of physicians over 65 has risen by approximately 400 percent. As a greater number of physicians continue to practice into advanced ages, their neuropsychological functioning is more prone to the effects of normal cognitive aging as well as an increased likelihood of brain injury or insult. Over the past approximately two decades, research has demonstrated that physicians with competency concerns have a greater likelihood of cognitive impairment compared with same-age peers. As a result of these findings, physician competency programs have increasingly included neuropsychological assessment as one component of their evaluation processes for physicians whose competency is in question. More recently, hospitals and other agencies regulating or assessing physicians are beginning to develop programs that use neuropsychological assessment in their ongoing evaluation of the practices of all physicians as they age, regardless of specific competency concerns. As these trends continue, many questions have arisen among neuropsychologists and other physician evaluators regarding procedural issues, ecological validity, and ethical considerations.

Correspondence: Lauri Korinek, PHD, 9364 Teddy Lane, Unit 204, Lone Tree, CO 80124.E-mail: LIKorinek@gmail.com

\section{K.D. GARRETT. Systematic Prospective Cognitive Screening Programs for Medical Staff.}

Many Medical Staff Offices and Governing Boards responsible for credentialing health care professionals have self-imposed regulations requiring transition of physicians of age from active practice to emeritus status. Given the factors described in the previous presentation, Medical Staff Offices and Boards are increasingly faced with requests to grant exceptions to their bylaws to extend their mandatory retirement. Pulled between physician staffing shortages, and the reality that some physicians' competency to practice will wane with age and age related conditions, some chose to take a proactive approach to the renewal-vsretirement question. Some healthcare systems have chosen to conduct prospective screening as a part of their regular credentialing renewal for physicians of age. Specifically, favorable cognitive screening and physical examination, together with peer review, become new requirements for practice privileges to be renewed. We will review the elements programs should consider in developing a prospective screening process, tradeoffs of using various cognitive screening protocols, setting the criteria for "screen positive", and what to do when a physician's cognitive performance on screening falls in a zone of concern.

Correspondence: Kelly D. Garrett, Ph.D., 8th Ave \& C St, LDSH Geriatrics,2N, Salt Lake City, UT 84103.E-mail:kelly.garrett@imail. org

\section{W. PERRY. Neuropsychological Assessment of Late Career Physicians.}

Changes in neurocognitive function can influence the way older physicians reason through clinical problems. Normal aging involves sensory and neurocognitive changes. It has also been repeatedly shown that normal cognitive aging involves a decline in fluid intelligence, whereas crystallized intelligence tends to remain stable. Both crystallized and fluid intelligence are important when physicians engage in clinical decision making. In this talk, the implications of aging on professional performance will be presented, and integrated with discussion of planning and executing clinical neuropsychological assessment of a physician/patient. Case studies will be used to illustrate the value of neuropsychological testing in the context of fitness for duty decisions and consultations. Finally, challenges and considerations regarding modifications in practice will be discussed.

Correspondence: William Perry, PhD, 200 West Arbor Dr, San Diego, CA92013-8218.E-mail:wperry@ucsd.edu

L. KORINEK, K.D. GARRETT \& W. PERRY. Professional Issues and Ethics for Neuropsychologists in Screening, Evaluation, and Rehabilitation of Physicians.

Neuropsychologists working with physician/patients at any stage of the assessment process (program development, screening, assessment, rehabilitation, retirement) encounter unique circumstances. We will highlight the professional issues mired in this work, and identify best practices and pitfalls. As such, participants in this symposium will better appreciate the professional decision making process (ethics, risk management, clinical, legal) involved when neuropsychologists are involved in the program development, research, screening, clinical evaluation, and intervention with Medical Staff.

Correspondence: William Perry, PhD, 200 West Arbor Dr, San Diego, CA 92013-8218. E-mail: wperry@ucsd.edu

E.S. GRACE. Signed, Sealed, and Delivered: What Referring Organizations Need in Neuropsychological Screening and Testing Reports on Aging and Other Physicians.

Cognitive screening and neuropsychological testing can be instrumental in leading to the identification of issues that may contribute to professional difficulties and/or interfere with remediation. The physician tested and the referring organization must decide how to translate the findings and recommendations in the report into decisions about practice, with consideration of the safety of the physician's patients first and foremost. What do these organizations and individuals need from us to translate report findings and recommendations into practical workplace recommendations?

In this presentation, case studies from a physician evaluation and remediation center will be presented to highlight the challenges in the practical applications of cognitive screening and testing findings and recommendations.

Correspondence: Elizabeth S. Grace, MD, 720 S. Colorado Boulevard, Suite1100-N, Denver, CO 80246. E-mail: esgrace@cpepdoc.org

\section{Poster Session 4: Cross Cultural, Drugs, Genetics, HIV/AIDS, \& MS/ ALS}

$$
\text { 3:15-4:45 p.m. }
$$

\section{ADHD/Attentional Functions}

E.L. SUDIKOFF, A.L. AVIV, M.S. MILLS \& D.A. KAUFMAN. Medication Effects on ADHD as Revealed by a Novel Continuous Performance Test.

Objective: ADHD is one of the most common neurobehavioral disorders of childhood. The MOXO-CPT is a new computerized test that has been validated for the identification of ADHD symptoms. Although little research has examined medication effects on continuous performance tests, some results suggest that psychostimulants decrease reaction time and variability of responses. This study examined the effects of psychostimulant medication on MOXO-CPT performance in a sample of children with ADHD. 
Participants and Methods: Sixteen participants (ages 6-16) completed the MOXO-CPT task with and without medication as part of a neuropsychological evaluation. Performance scores for baseline and medication sessions were subjected to repeated measures ANOVA. MOXOCPT performance was divided into four subscales, which measured attention, reaction time variability, impulsiveness, and hyperactivity. All participants were taking one psychostimulant medication, with no other current prescriptions.

Results: A strong main effect of medication was seen in the data, with psychostimulant use resulting in better MOXO-CPT scores overall. More specifically, attention, reaction time variability, and hyperactivity scores all improved as a result of medication. Impulsiveness was not significantly affected by medication use.

Conclusions: The use of psychostimulant medication significantly improved MOXO-CPT scores, leading to better performance in attention, reaction time variability, and hyperactivity in a sample of children with ADHD. These findings help to illuminate the beneficial effects of psychostimulants on specific ADHD symptoms. Expanded understanding of medication efficacy in ADHD is critical to evaluate treatments and promote evidence-based practice for this clinical population.

Correspondence: David A. Kaufman, Ph.D., Psychology, Saint Louis University, 221 N Grand Blvd, Shannon Hall, Room 216, St. Louis, MO63103.E-mail: dkaufma3@slu.edu

A. HEITZER, B. PETERS, J. PIERCY \& S. RAZ. Hyperactivity and Disinhibition Among Preschool Age Children Born Prematurely. Objective: A large body of literature reveals that compared to children born at term, preterm children are at increased risk for difficulties with inhibitory control and hyperactivity. Less consistency exists, however, in the limited body of research exploring the contribution of early biological risk to behavioral disinhibition within the population of children born preterm. Therefore, our goal was to examine perinatal variables that may influence activity level and inhibitory control among preterm preschoolers.

Participants and Methods: Ninety-eight, nondisabled, preterm (23.4 - 33.9 weeks gestation) preschoolers (3-4 years) participated in the study. A direct measure of motor disinhibition (Statue subtest. NEPSY-II) as well as parental ratings of behavioral disinhibition on the Child Behavior Checklist (CBCL), Externalizing Problems scale, were used to evaluate behavior.

Results: We used simultaneous linear regression analyses with gestational age, perinatal complications, and antenatal growth z-score (birth weight standardized by gestational age and sex) as predictors of interest. Socioeconomic status, sex, multiple gestation, and age at testing were our "covariates." Unexpectedly, we found that within our preterm sample, total number of complications was inversely related to the CBCL Externalizing Problems scaled score $(\mathrm{F}[1,96]=4.98, \mathrm{p}=.028)$. Sex, but not perinatal medical status, was significantly related to performance on the NEPSY-II Statue subtest, with males displaying reduced motor inhibition $(\mathrm{F}[1,81]=4.45 ; \mathrm{p}=.038)$.

Conclusions: Preschoolers with a greater number of complications obtained lower Externalizing Problems scaled scores, suggesting a link between increased perinatal risk and reduced behavioral initiation. The reduced motor inhibition in boys, though consistent with the expected male outcome disadvantage documented in the prematurity literature, should be interpreted with caution due to the unavailability of normal controls.

Correspondence: Sarah Raz, Ph.D., Merrill Palmer Skillman Institute, Wayne State Univ, Merrill Palmer Skillman Institute, 71 E. Ferry St., Detroit, MI 48202.E-mail: sarahraz@wayne.edu

\section{Cognitive Intervention/Rehabilitation}

E. PAEK \& L. MURRAY. Intervention of Developmental Dyslexia through Working Memory Treatment.

Objective: Prior research has established that working memory (WM) components such as the phonological loop and/or central executive component play an important role in reading and writing skills (Amtmann, Abbott, \& Berninger, 2008; Berninger et al., 2010). Furthermore, deficits in reading and writing in children and adolescents are strongly associated with impaired WM capacity (Chiappe, Siegel, \& Hasher, 2000; Willcutt et al., 2001). In contrast, there has been nominal investigation of whether treatments that target WM can evoke improvements in adolescents with developmental reading or writing disorders; initial research involving adults with developmental dyslexia, however, suggests these adults' reading skills can benefit from WM training (Horowitz-Kraus \& Breznitz, 2009; Shiran \& Breznitz, 2011). Therefore, this study examined the benefits of WM training on developmental reading and writing difficulties using a single subject, multiple baseline treatment design across subjects.

Participants and Methods: Two male adolescent participants (16 and 17 years old, respectively) were provided weekly one-hour treatment for 13 weeks. Training consisted of practicing six verbal WM tasks (N-back, reconstitution of words from oral spelling, grammaticality and rhyming, odd-one out, updating, keeping track); all target treatment stimuli had a low probability of phoneme-to-grapheme conversion, and included functional items to foster the participants' motivation.

Results: With treatment, both participants showed improved performance of all trained WM tasks. Their probe data indicated significant positive changes, particularly on the WM probe task, which required monitoring the phonological buffer. On post-treatment testing, however, each participant demonstrated a different pattern of transfer effects in their spelling skills.

Conclusions: Adolescents with dyslexia were responsive to WM treatment. The implications of the results will be discussed in terms of both clinical recommendations and future research.

Correspondence: Eun Jin Paek, M.S., Speech and Hearing Sciences, Indiana University, 2810 Kellis Way Apt. D, Bloomington, IN 47403. E-mail: epaek@indiana.edu

A. WONG, N.E. CARLOZZI, D. TULSKY, R.K. HEATON \& A.W. HEINEMANN. Analyzing the Relationship of Disrupted Cognitive Function on Social Health and Participation after Neurological Disorders: a Structural Equation ModelingApproach. Objective: Neurological disorders due to trauma or disease can cause lasting cognitive impairments and lead to restrictions in social participation. The purpose of this study was to create and test a structural equation model of the complex relationship between cognitive functioning and participation among persons with traumatic brain injury (TBI), spinal cord injury (SCI), and stroke.

Participants and Methods: A total of 604 participants (TBI =184; $\mathrm{SCI}=209$, Stroke $=211 ;$ average age $=47.3$ years; $64.1 \%$ men; $58.6 \%$ white; $67.2 \%$ completed more than high school education) who were at least 1-year post-injury or disease, completed a series of neuropsychological tests and computer adaptive testing-based patient-reported measures. Participation outcomes were assessed using the Participation Enfranchisement Scale and Patient-Reported Outcomes Measurement Information System (PROMIS) - Social Health subtests. Cognitive functioning was measured by subtests of NIH Toolbox Cognitive Battery and a battery of traditional neuropsychological tests.

Results: The final model fit the data relatively well $(\mathrm{CFI}=0.918$; TLI= 0.884; RMSEA $=0.082$ ). Measures of fluid intelligence that included a psychomotor component (i.e., executive function, processing speed and working memory), and crystallized intelligence (i.e., reading and vocabulary) were related to social health. Measures of fluid intelligence that did not include a motor component were related to participation enfranchisement and social health. 
Conclusions: The findings demonstrate the complex interplay between cognitive functioning and participation. Results also suggest that efforts to improve the detection and treatment of cognitive deficits in neurological rehabilitation may also enhance social health and participation. Correspondence: Alex Wong, PhD, Department of Neurology and Program in Occupational Therapy, Washington University School of Medicine, 4444 Forest Park Ave, Campus Box 8505, St. Louis, MO 63108-0278.E-mail:wongal@wusm.wustl.edu

\section{Cognitive Neuroscience}

O. NITZAN-TAMAR, B. KRAMARSKI \& E. VAKIL. Eye-Tracking Patterns as a Tool to Identify and Classify Cognitive Styles.

Objective: Varied tools have been designed to classify the global-local dimension of the cognitive style, most of based on behavioral data which reveal little about how this dimension processing function. Therefore, the main goal of the current study is to validate a new tool for classifying the preferred cognitive style, by means of real time monitoring of eye movements (EM) of the participant while simultaneously taking the Extended Cognitive Styles Analysis - Wholistic-Analytic (E-CSA-WA) test. Participants and Methods: Seventy-seven students participated in this study. Participants answered the E-CSA-WA test while having their EM monitored. The test contains, 40 wholistic stimuli which require matching figures task, and 40 analytic stimuli which require embedded figures task.

Results: According to the nature of the test, when the task matched the preferred style (wholists on the matching task and analytics on the embedded task), the overall average of Reaction Time (RT) was significantly lower. However, the overall RT of the wholist group was lower on both tasks. Comparison of the EM pattern of the wholist and analytic groups on both tasks revealed a qualitative difference, beyond the quantitative differences between the groups, in the process of solving the task. Conclusions: The wholist group is characterized by less fixations and transitions as compared to the analytic group. These differences are interpreted as a reflection of the whole (gestale) and part (step by step) processing, respectively. The differences in the EM between the wholistic and the analytic groups might be interpreted as an influence of the impulsive-reflective style differences, respectively.

Correspondence: ,, . E-mail:

\section{Cross Cultural}

N.C. MOSS, S.J. ERICKSON, R.E. RIEGER, S. DUVALL, R.K. OHLS \& J.R. LOWE. The Relationship Between Primary Language, Socioeconomic Status, and Executive Function in Prechoolers Born Very Low Birthweight (VLBW).

Objective: Cultural differences and socioeconomic factors in parent-child interactions may help explain variability in developmental outcomes. We examined the relationship between language spoken, socioeconomic factors, and measures of verbal and performance abilities and executive functioning (EF) among preschoolers born very low birth weight VLBW ( $\leq 1500$ grams).

Participants and Methods: Preschoolers born VLBW (3-4.5 years) were assessed. Caregivers provided demographic information including language spoken (English: $n=66$; Spanish: $n=16$ ), number of people in home, and mother's education. Verbal abilities (VIQ), and performance abilities (PIQ) (WPPSI-III) and EF (Gift Delay, Bear/Dragon, BRIEF-P) scores were obtained.

Results: No significant differences were found between English and Spanish speaking VLBW groups on gestational age, birth weight, illness severity, test age, sex, or number of people in the home. English speaking participants reported higher household income $(p<.001)$ and maternal education $(p=.014)$. English speaking participants scored significantly higher on VIQ ( $p=.006)$, but there were no group differences on PIQ, Gift Delay, Bear/Dragon, or BRIEF-P scales.

Controlling for maternal education and income, a significant difference between English and Spanish speaking groups on VIQ (controlling for education: $\mathrm{p}=.014$; controlling for income: $\mathrm{p}=.044$ ) remained, with English speaking participants scoring significantly higher.

Conclusions: Spanish speaking preschoolers evidenced lower VIQ scores compared with English speaking preschoolers; and maternal education and income did not fully explain this difference. The two language groups did not differ on PIQ or any EF measures, suggesting outcome equivalence in non-verbal domains. VIQ results may be due to an instrumentation artifact rather than children's verbal ability, as the WPPSI is not normed on Spanish speaking children. Awareness of potential scoring discrepancies is required when verbally laden tests are used with Spanish speaking preschooolers.

Correspondence: Natalia C. Moss, M.A., Psychology, University of New Mexico, 301 Central Ave. NE \#300, Albquerque, NM 87102. E-mail: ncmoss@unm.edu

J.M. GALUSHA-GLASSCOCK, D.K. HORTON, M.F. WEINER, L.S. HYNAN, D. REAM, J. SHORE, J.G. HARRIS \& M. CULLUM. Comparison of ANAM4 with Common Neuropsychological Tests in a Racially Diverse Sample.

Objective: To compare scores from the Automated Neuropsychological Assessment Metrics-Version 4 (ANAM4) with standard neuropsychological tests of working and declarative memory, processing speed, and attention and explore convergent validity of ANAM4 in a racially diverse sample.

Participants and Methods: The ANAM4 was administered to a diverse group of 185 community-dwelling subjects (African American: $\mathrm{n}=63$, American Indian: $\mathrm{n}=60$, Hispanic: $\mathrm{n}=62$ ) with no neuropsychiatric history. Age ranged from 19 to $50(\mathrm{M}=32.78)$ and the mean educational level was 13.51 years. ANAM4 scores were compared with standard neuropsychological tests representing the domains of working memory, episodic memory, processing speed, and attention: WAIS-4 Letter-Number Sequencing, Hopkins Verbal Learning Test-Revised, WAIS-IV Coding, and Trails A and B. Pearson correlations compared ANAM4 Throughput scores with standard neuropsychological tests, using $\mathrm{p}=.001$ for all statistical comparisons.

Results: ANAM4 Procedural Reaction Time ( $r$ 's $=.29$ to .38 ) and Code Substitution Learning ( $r^{\prime} s=.28$ to .41$)$ were significantly correlated with most of the standard neuropsychological tests. Math Processing $\left(r^{\prime} s=.25\right.$ to.43) was significantly correlated with all standard tests. Matching to Sample was significantly correlated with WAIS-4 Letter Number Sequencing, WAIS- 4 Coding, and Trails B ( $r$ 's= .24 to .30). The only significant correlation involving Code Substitution Delay was with the Hopkins Verbal Learning Test ( $\mathrm{r}=.27)$. ANAM4 Simple Reaction Time was unrelated to any of the standard neuropsychological scores $(\mathrm{r}$ 's $=.07$ to .18$)$.

Conclusions: The ANAM4 shows moderate, significant correlations with standard neuropsychological tests in healthy diverse populations, with some predictable domain-specific relationships.

Correspondence: Jeanine M. Galusha-Glasscock, Master of Science, Department of Psychiatry, University of Texas Southwestern Medical Center, 323 Deaton Drive, Rockwall, TX T=75087. E-mail: jeanine. galusha@utsouthwestern.edu

C.K. Petranovich, N.C. Walz, M. StaAt, P. CHIU \& S.L. WADE. Differing Aspects of Social Information Processing and Parent-reported Social Competence in Internationally Adopted Girls with a History of Institutionalization.

Objective: Internationally adopted (IA) children often experience deficits in social functioning. Measures of social information processing (SIP) have been suggested to offer information about cognitive processes that underlie social competence, but have yet to be used in studies of international adoption. 
Participants and Methods: Participants included girls between the ages of 6-12 years old who were adopted from China $(n=32)$ or Eastern-Europe $(\mathrm{n}=25)$ and a comparison group of American-born, never-institutionalized girls $(\mathrm{n}=25)$. Participants completed the Dodge Video Vignettes (DVV), a measure of SIP. Parents completed the Home and Community Social Behavior Scale (HCSBS), a standardized measure of social competence in everyday behavior.

Results: Groups did not significantly differ on the DVV. Compared to the Chinese adoptees $(\mathrm{t}=2.25, \mathrm{p}=.02)$ and the controls $(\mathrm{t}=3.71$, $\mathrm{p}=.001)$, the Eastern European group evidenced significantly poorer parent-reported social competence (mean $\mathrm{T}=50.48, \mathrm{SD}=8.80)$. Controlling for group and family income, hostile $(\beta=-3.75, \mathrm{t}=-2.23, \mathrm{p}=$ $.02)$ and competent $(\beta=1.57, t=2.72, p=.008)$ responding on the DVV significantly predicted parent-reported social competence. Fewer hostile responses and more competent responses were associated with better social competence. Passive responding was non-significant.

Conclusions: These findings suggest that IA children with a history of institutionalization are vulnerable to negative social outcomes that may be influenced by hostile and competent responses tendencies. A better understanding of SIP in IA children may contribute to our understanding of the influence of early institutionalization on social development. Correspondence: Christine K. Petranovich, Dept. of Psychology, University of Cincinnati, 1 Edwards Center ML 0376, Cincinnati, OH 45221.E-mail:karvercl@mail.uc.edu

\section{ANDREOTTI \& K. HAWKINS. Role of Age, Health Status, and Education on RBANS Performance in an Older African American Community Sample.}

Objective: Previous research has documented cross-cultural discrepancies in performance on neuropsychological tests including the Repeatable Battery for the Assessment of Neuropsychological Status (RBANS). We explored sources of variance including age, common medical conditions, and education in order to provide clinicians with the opportunity to tailor their interpretation of test scores and further overall comprehension of the effects of sociocultural factors on RBANS performance in African Americans.

Participants and Methods: Three hundred fifty-five individuals participated in a study of cognition in normal older self-identified African Americans living independently in the community (210 females; mean age $=64.3$ years, $\mathrm{SD}=8.0$ years, range $=55-87$ years . All participants completed the RBANS, WRAT-3 Reading Test, as well as a comprehensive demographic and health history interview. A total of 184 participants $(63.0 \%)$ reported a diagnosis of hypertension, and $66(22.6 \%)$ reported a diagnosis of diabetes mellitus type 2 . Multivariate analysis of variance (MANOVA) was employed to determine whether diabetes or hypertension significantly affected RBANS performance. Additional linear regression analyses were then conducted to determine whether years of education, WRAT-3 reading score, and age each accounted for unique variance in the RBANS variables.

Results: Diabetes, but not hypertension, significantly predicted RBANS performance, Wilk's $\lambda=0.96, \mathrm{~F}(6,283)=2.24, \mathrm{p}=.04$, but both illness conditions were unrelated to overall RBANS performance once age and education were accounted for. Age, education, and WRAT-3 Reading score (a proxy for scholastic attainment) were independent predictors of RBANS performance ), $\mathrm{F}(3,279)=45.8, \mathrm{p}<.001$.

Conclusions: These results support established relations between education and neuropsychological test performance, especially in minority populations, underscoring the importance of African American population-specific norms based on education and other demographic covariates.

Correspondence: Charissa Andreotti, Ph.D., Psychiatry and Behavioral Sciences, Memorial Sloan Kettering Cancer Center, 641 Lexington Avenue, 7th Floor, New York, NY 10022.E-mail: andreotc@mskcc.org
K.R. BRYANT, P.M. DEAN, M.W. HAUT, J. SPAT, M.T. MORAN, C. WILSON \& J.R. WIENER. Education Attainment and Premorbid Ability Affects Boston Naming Test Performance in a Rural Sample. Objective: This study examined age, education, and premorbid ability (i.e., reading level) effects on Boston Naming Test (BNT) performance within a rural clinical sample.

Participants and Methods: A consecutive clinical sample of 176 adults referred for neuropsychological evaluation at a rural academic medical center as part of their clinical care was utilized. Pearson $\mathrm{r}$ correlations examined the hypothesis that education (range $=6-20$ years, mean $=$ 13.23 , SD $=2.75$ ) and premorbid ability, represented by a regression factor combining WRAT-4 performance and education level (Premorbid-Education Factor), would be more strongly related to a lack of semantic knowledge (Proportion Spontaneously Correct) relative to word retrieval deficits (Proportion Correct with Cues). Additionally, Chi-Square tests examined the effects of the Premorbid-Education Factor on individual items using a median split.

Results: Separately, educational attainment and WRAT-4 Reading, but not age were correlated with BNT performance in this sample. As hypothesized, the Premorbid-Education Factor was more strongly related to BNT Proportion Spontaneous correct $(r=.53, p=.000)$ relative to the BNT Cued Proportion correct $(\mathrm{r}=.45, \mathrm{p}=.000)$. The Education-Premorbid Factor significantly affected twenty-five percent of the items. Conclusions: It is helpful to consider premorbid factors when evaluating BNT performance, especially in rural areas where education quality and cultural experience vary considerably. Educational attainment and reading skill were correlated with BNT performance, but not age for this rural sample. Education and reading level impacted performance on specific items. Evaluation of cue effectiveness may help to differentiate semantic knowledge errors versus word retrieval deficits. Correcting for both educational attainment and reading level may be appropriate for rural communities.

Correspondence: Kirk R. Bryant, Ph.D., Psychiatry and Behavioral Medicine, West Virginia University, 7 Grapevine Village, Morgantown, WV 26505.E-mail:kb124300@gmail.com

G. RIDER, R.L. HICKS, B.A. WEISS, M.D. AHRONOVICH \& I. BARON. Cognitive Outcomes of Bilingual Preterm Children at Ages Three and Six.

Objective: Cognitive outcomes of bilingualism in children born preterm are rarely studied, with language deficit emphasized over other domains. We aimed to examine general cognitive functioning of monolingual and bilingual extremely low birth weight (ELBW; $<1,000 \mathrm{~g})$, late preterm (LPT; 34-36 weeks' gestation), and Term participants at ages 3 and 6. Participants and Methods: A retrospective cross-sectional study of 1,275 participants born 2004-2009: $232 \mathrm{ELBW}(n=160$ age $3 ; n=$ 72 age 6$) ; 709$ LPT ( $n=512$ age $3 ; n=197$ age 6$)$; or 334 Term $(n=$ 226 age $3 ; n=108$ age 6 ). $26.4 \%$ were bilingual. Differential Ability Scales-II general conceptual ability (GCA), special nonverbal composite (SNC), verbal, nonverbal reasoning, and spatial cluster scores were outcomes.

Results: Bilingual had significantly lower verbal performance than monolingual; all groups, both ages $(p s<.01)$. Large effect sizes at 3 for all groups (ELBW $d=0.63$; LPT $d=0.92$; Term $d=1.33)$; reduced effect sizes age 6 for LPT and Term only (ELBW $d=0.69$; LPT $d=0.27$; Term $d=0.27)$. Bilingual had significantly lower GCA than monolingual only for LPT and Term at age $3(p<.05 ; d=0.38 \& 0.52)$ but not age 6 $(p>05 ; d=0.08 \& 0.29)$. No significant differences between bilingual and monolingual were found for nonverbal, spatial, or SNC scores at any age for any group $(p>.05)$.

Conclusions: Only verbal differences were found for bilingual participants at 3 and 6. Nonverbal assessment (e.g., SNC) should provide better estimates of cognitive capacity into early school age. Decreased differences by 6 for LPT and Term, but not ELBW, suggest LPT and Term delay if bilingual, and ELBW deficit. 
Correspondence: G. Nicole Rider, Fairfax Neonatal Associates at Inova Children's Hospital, 201 Q Street NE APT 3451, Washington, DC 20002. E-mail: nicole.rider@gmail.com

A. LEON, C. CARRION \& N. PLISKIN. The Effect of Educational Attainment on Assessment of Adaptive Functioning in a Sample of Monolingual Spanish-speaking Hispanics.

Objective: The Instrumental Activities of Daily Living scale (IADL; Lawton \& Brody, 1969) is a questionnaire used to assess adaptive functioning in older adults, using self-report and caregiver-report. Since functional impairment is required for a dementia diagnosis, this scale provides valuable information that aids in differential diagnosis. However, low educational attainment is a strong predictor of functional disability in Hispanics, even after controlling for health risk status (Latham, 2012). The purpose of this investigation was to compare the effects of educational attainment, age, and diagnoses on adaptive functioning as assessed by the patient form and the caregiver form of the IADL.

Participants and Methods: Participants were 37 monolingual, Spanish-speakers [mean age $=65(14.9)$; mean years of education $=6.35(3.7)]$ referred for neuropsychological testing to address cognitive complaints. Fifteen participants were diagnosed with dementia or mild cognitive impairment (MCI). Twenty-two participants were diagnosed with non-neurodegenerative conditions.

Results: In the linear regression model, years of education was the strongest predictor of total IADL scores measured via self-report $(\beta=.45$; $p=.019)$; age $(\beta=.15)$ and diagnosis $(\beta=.19)$ did not significantly predict these scores. Similarly, when assessing the effects of these variables on caregiver reports, years of education was the strongest predictor $(\beta=.397 ; p=.01)$; age $(\beta=-.03)$ and diagnosis $(\beta=.23)$ did not significantly predict total IADL scores.

Conclusions: Findings suggest that monolingual Hispanics' educational attainment strongly influences assessment of adaptive functioning as measured by patients and their caregivers. Implications for assessment of adaptive functioning and its effect on dementia diagnosis in this population will be explored.

Correspondence: Adeline Leon, MSW, Psychology, Illinois Institute of Technology, 2635 N Spaulding Ave Apt 1E, Chicago, IL 60647. E-mail: aleon1@hawk.iit.edu

A. LEON, L. PIPER, C. CARRION, V. MONTGOMERY \& N. PLISKIN. Analyzing the Efficacy of the Spanish Version of the Frontal Systems Behavior Scale (FrSBe) in Assessing the Executive Functioning of a Sample of Monolingual Spanish-speaking Hispanics.

Objective: The Frontal Systems Behavior Scale (FrSBe; Grace \& Malloy, 2001) provides an estimate of behavioral dysfunction across three domains: apathy, disinhibition, and executive dysfunction. A Spanish version of the FrSBe was approved by its authors and includes a family and self-rating form, with high construct validity demonstrated in the former using Rasch modeling (Caracuel et al., 2012). However, studies have not focused on evaluating the Spanish FrSBe subscales' association with corresponding neuropsychological tests. The purpose of this investigation was to examine the executive dysfunction subscale of the Spanish FrSBe family-rating form by comparing it to objective measures of executive functioning (EF): (Color Trails-2 (CTT-2), Stroop Color-Word, and Wisconsin Card Sorting Test (WCST)).

Participants and Methods: Participants were 48 monolingual Hispanic, Spanish-speakers [mean age $=61.6(\mathrm{SD}=14.4)]$ referred for neuropsychological testing with cognitive complaints: twenty-five were diagnosed with dementia or mild cognitive impairment, and 23 were diagnosed with non-neurodegenerative conditions. A correlational analysis was conducted between the FrSBe family-rating of executive dysfunction [mean=73.4(16.3)], the CTT-2 [M = 185.8 (SD = 100.9)], the Stroop Color-Word [mean=22.8(9.1)], and the correct responses [M $=59.8(20.5)]$ and total number of errors $[\mathrm{M}=61.8(24.2)]$ of the WCST.
Results: The FrSBe was significantly correlated with WCST errors [ $\mathrm{r}=-$ $0.34(\mathrm{p}=.038)]$ and WCST correct responses $[\mathrm{r}=.37(\mathrm{p}=.03)]$; the CTT-2 and Stroop Color-Word were not significantly correlated with the FrSBe. Conclusions: Results suggest that the FrSBe executive dysfunction subscale likely measures behavioral aspects of executive functioning comparable to cognitive aspects measured by the WCST, but not other measures of EF. Utilizing a variety of cognitive and behavioral measures in neuropsychological evaluations may improve sensitivity when assessing executive disturbance.

Correspondence: Adeline Leon, MSW, Psychology, Illinois Institute of Technology, 2635 N Spaulding Ave Apt 1E, Chicago, IL 60647. E-mail: aleon1@hawk.iit.edu

I. FLORES, K.B. CASALETTO, M.J. MARQUINE, A. UMLAUF, D.J. MOORE, D. MUNGAS, R. GERSHON, J.L. BEAUMONT \& R.K. HEATON. Performance of Hispanics and Non-Hispanic Whites on the NIH Toolbox Cognition Battery: The Roles of Ethnicity and Language Backgrounds.

Objective: As the Hispanic/Latino population in the United States continues to grow, it has become imperative to understand how Hispanic ethnicity and speaking Spanish may impact performances on neuropsychological tests standardized in the U.S. We examined performances of Hispanics tested in English (Hispanic-English) or Spanish (Hispanic-Spanish) and Non-Hispanic White adults on the NIH Toolbox Cognition Battery (NIHTB-CB).

Participants and Methods: Participants included 93 Hispanic-English, 93 Hispanic-Spanish, and 93 Non-Hispanic White healthy adults matched on age, sex, and education who participated in the NIH Toolbox norming project. All participants completed the NIHTB-CB (Fluid and Crystallized Composites) and self-reported measures of culturally-relevant variables (e.g., language spoken, place of birth).

Results: Hispanic-Spanish individuals performed worse than both Hispanic-English $(\mathrm{p}=0.01)$ and Non-Hispanic White $(\mathrm{p}<.001)$ individuals on the uncorrected (for demographics) Fluid scores; the latter two groups did not differ from each other on Fluid scores. Crystallized scores showed no group differences. Among Hispanics $(\mathrm{n}=186)$, including those tested in English and Spanish, reported bilingualism was strongly associated with better performances on the Fluid composite regardless of the language the individual was tested in $(p=0.01)$, but was not associated with the Crystallized composite $(p=0.40)$. Within Hispanics tested in Spanish, those who spoke Spanish at home and who were born outside of the US were at risk for poorer Fluid performances $(\mathrm{ps}<0.05)$.

Conclusions: Hispanic-Spanish individuals performed the poorest of all groups and degree of acculturation (bilingualism, speaking Spanish at home) may be driving those differences. These findings highlight the importance of normative corrections that account for ethnic and language differences on neuropsychological test performances, as well as the need to better understand the factors that differentiate neuropsychological performances among the Hispanic population.

Correspondence: Ilse Flores, Psychology, San Diego State University, 330 W Plymouth St, Inglewood, CA 90302.E-mail: ilse.flores3@gmail. com

C. CARRION, A. LEON, V. MONTGOMERY, L. PIPER, T. KAEMMERER \& N. PLISKIN. Cognitive and Behavioral Predictors of Adaptive Functioning in a Monolingual SpanishSpeaking Hispanic Sample.

Objective: Cognitive assessment measures of executive functioning have shown strong positive correlations with adaptive functioning in Hispanics (Farias, Mungas, Hinton, \& Haan, 2011). However, cognitive measures do not comprehensively depict adaptive functioning. Investigators have found that combining cognitive and behavioral measures improves the ability to predict adaptive functioning (Karzmark et al., 2012); however, these findings might not generalize to Hispanics. The objective of this study is to determine whether the Wisconsin Card Sorting 
Test (WCST) and the Executive Dysfunction scale (E) of the Frontal Systems Behavior Scale (FrSBe; Grace \& Malloy, 2001) similarly predict adaptive functioning in a sample of monolingual Hispanics.

Participants and Methods: Using archival data, the files of 26 individuals with cognitive complaints were analyzed [mean age $=59.31$, $\mathrm{SD}=14.4$; mean education 7.23, $\mathrm{SD}=3.28]$. The FrSBe scale $\mathrm{E}$ and the WCST (correct and perseverative responses, failure to maintain set, total errors) served as predictors of adaptive functioning, measured by the Instrumental Activities of Daily Living scale (IADL; Lawton \& Brody, 1969). Hierarchical regression was employed to control for demographic variables in step 1 (age, gender, education, diagnosis) and predictor variables were entered in step 2 (WCST and FrSBe).

Results: Results revealed that demographic variables accounted for $11.1 \%$ (adjusted R2=.111) of the variance in IADL scores; adding cognitive and behavioral measures increased the variance explained by $37.3 \%$ (adjusted R2=.484). The FrSBe scale E score was the only statistically significant variable in the model $(\beta=-.462 ; p=.021)$.

Conclusions: Findings suggest that adding behavioral measures to standard neuropsychological assessments improves the prediction of adaptive functioning in Hispanics.

Correspondence: Carmen Carrion, MA, Psychology, Roosevelt University, 1938 W Thomas St, 2nd FL, Chicago, IL 60622. E-mail: carrionneuropsy@gmail.com

S. HUNTER, E.L. GUSTAFSON, J. KAYLEGIAN, D. SMITH \& N. KARNIK. Executive Functioning, Risk Behaviors, and Time Homeless: The Significance for LGBTQ Homeless Youth.

Objective: A significant proportion of US homeless youth are LGBTQ. These youth experience different challenges with homelessness than their heterosexual peers. We explored possible differences in the effect of homelessness on executive functioning (EF) and risk-taking behavior between LGBTQ and heterosexual youth. Duration and frequency of homelessness was examined as a potential mediator of EF and risk-taking.

Participants and Methods: 148 youth were recruited from homeless agencies in Chicago and Los Angeles (53\% female; 76\% African-American; ages 18-22). EF was assessed with the BRIEF, D-KEFS, and Iowa Gambling Task (IGT). Risk behaviors were assessed with the CDC National Youth Risk Behavior Survey (YRBS). Duration measures included age at first homelessness, number of homeless episodes, and length of homelessness, obtained through structured interviews. Classification of orientation came from homeless agencies' reports regarding LGBTQ youth in their shelters.

Results: Partnering homeless agencies reported $20-30 \%$ of youth are LGBTQ. Statistical modeling indicated better subjective EF was significantly related to lesser duration and frequency of homelessness. Similarly, less risk taking behavior was also related. This finding was observed across heterosexual and LGBTQ youth. Differences in EF skill across youth with varying frequencies of homelessness were found, but no differences seen for length of homelessness. Only modest relationships were found for objective EF measures (e.g., IGT).

Conclusions: LGBTQ youth are at a greater risk for homelessness. They also engage in a greater number of survival based risk behaviors than their heterosexual peers. Our data suggest that shelter-based LGBTQ youth show similar EF struggles as their non-LGBTQ homeless peers, given increased duration and frequency of homelessness. This highlights a need for a consistent model of intervention across homeless youth populations, taking individual experience into account.

Correspondence: Jaeson Kaylegian, B.A., Pediatric Neuropsychology, University of Chicago Medical Center, 558 W Van Buren St, Unit 3, Chicago, IL 60607. E-mail: jkaylegian1@yoda.bsd.uchicago.edu

\section{J. BENNETT \& S.P. VERNEY. Socio-cultural Factors Outweigh Language Factors in WRAT Reading Performance.}

Objective: The Wide Range Achievement Test (WRAT) Reading subtest is used in neuropsychology to measure academic achievement, estimate premorbid functioning and to gauge quality of education. The WRAT is widely used throughout the US with the majority culture and ethnic groups. The largest US ethnic group is the Hispanic population, many of whom are Spanish-English bilinguals. The purpose of this study is to investigate cultural and linguistic factors that affect bilingual Hispanic students' performance on the WRAT Reading subtest.

Participants and Methods: Monolingual European-American ( $\mathrm{n}=30$ ) and bilingual Hispanic $(\mathrm{n}=99)$ students at an English-speaking US Southwestern university completed the WRAT Reading subtest and an acculturation measure, the Scale of Ethnic Experience (SEE). Hispanic students, self-identified as fluent in English and Spanish, were recruited into three groups: Spanish dominant, English dominant, and balanced bilingual.

Results: A three-step linear regression resulted in language dominance accounting for $2.5 \%$ of the variance of WRAT reading score in step 1 $(p=0.08)$. In step 2, estimated socio-economic status (SES) accounted for an additional $14 \%$ of the variance of reading scores $(p<0.00)$. In step 3 , SEE cultural variables accounted for an additional $6 \%$ of variance in the reading scores $(\mathrm{p}<0.00)$.

Conclusions: SES and cultural factors, not linguistic considerations, significantly accounted for much of the variance in WRAT Reading scores $(\mathrm{R} 2=0.20)$. This may point to lower SES and Hispanic students receiving lower quality of education in the US and/or the need to consider cultural factors, such as level of acculturation, when assessing WRAT Reading scores for individuals from ethnic groups or from a lower SES.

Correspondence: Jennifer Bennett, PH.D. Psychology, Psychology, Univ New Mexico, 1 University of New Mexico, MSC03-2220, Albuquerque, NM 87131-1161.E-mail: jenben@unm.edu

J. AVILA, A.T. FLOWERS \& J. RAZANI. The Relationship Between Cultural Factors and Executive Functioning in English-speaking and Farsi-speaking Iranian individuals.

Objective: The purpose of this study was to evaluate the effects of education obtained in the US and level of acculturation on the neuropsychological test performance of English-speaking and Farsi-speaking Iranian individuals.

Participants and Methods: Sixty-four healthy Iranian adult participants composed two groups in this study: 28 English-Speaking Iranian, and 36 Farsi-Speaking Iranian. Participants' performance on the Wisconsin Card Sorting Test (WCST), Trail Making Test parts A and B (TMT), and Color Trails Test parts 1 and 2 (CTT) was assessed either in English or in Farsi (depending on their English fluency). Also, a measure assessing level of acculturation (ACC) and percent of education obtained in the US (EDUS) was obtained.

Results: Bivariate correlations revealed significant relationships between EDUS and performance on TMT-B and CTT-2, as well as, ACC and performance on TMT-A, TMT-B, CTT-2, and WCST set failure, conceptual level responses, and categories completed. Several hierarchical regressions, entering EDUS first, followed by ACC as the independent variables, were conducted with each neuropsychological variable as the dependent variable. Results revealed that EDUS alone accounted for significant variability in TMT-B and CTT-2 performance. The addition of ACC in the model accounted for additional variability in TMT-B and all WCST variables, but not in CTT-2.

Conclusions: It appears that for Iranians the proportion of education obtained within the U.S. as well as their level of acculturation to U.S. customs affects performance on the majority of executive neuropsychological tasks despite whether the test is given in their native language or in English.

Correspondence: Justina Avila, M.A., Psychology, University of New Mexico, 3003 Adams St. NE, Apt \# M74, Albuquerque, NM 87110. E-mail:jfavila@unm.edu 


\section{Drug/Toxin-Related Disorders (Including Alcoholism)}

D. SHAKED, J.E. KISSER, D.K. LEIBEL, S.R. WALDSTEIN, M.K. EVANS \& A.B. ZONDERMAN. The Influence of Substance Intake on Neuropsychological Performance.

Objective: Examine history of cocaine, opiate, marijuana, and cigarette use and neuropsychological performance.

Participants and Methods: Participants were 1,831 neurologically intact African-Americans (AAs) and Whites (Mage $=50$ years; $43 \%$ male, $42 \%$ White, $44 \%$ below poverty) from the Healthy Aging in Neighborhoods of Diversity across the Life Span (HANDLS) study. History of cocaine, opiate, marijuana, and cigarette use, each quantified as "ever/ never," was summed into a count variable. Participants completed the California Verbal Learning Test (CVLT), Benton Visual Retention Test, Digit Span, and Trail Making Test. Multivariable regression analyses, stratified by race and poverty status, examined interactive relations of substance use and sex with neuropsychological function adjusting age, education, literacy, depression, and biomedical risk factors.

Results: Substance use * sex interactions were noted as follows: CVLT learning slope $(\beta=.243, p=.010)$ and CVLT total score $(\beta=.177, p=$ $.035)$ in AAs below poverty; CVLT long delay $(\beta=.251, \mathrm{p}=.027)$ and Trails B $(\beta=-.252, \mathrm{p}=.020)$ in Whites below poverty; and Trails A $(\beta=-.188, p=.040)$ for Whites above poverty. With greater substance use, men performed better in all instances except for CVLT long delay on which women performed worse.

Conclusions: Substance use history was largely associated with better performance on select tests of learning and memory, perceptuo-motor speed, and executive function among men. These associations were not explained by biomedical risk factor profile, and may, in part, reflect relative activation with current use.

Correspondence: Danielle Shaked, BA, Taub Institute, Columbia University, 136 W. 87th St., Apt. \#2, New York, NY 10024. E-mail: danielle.shaked1@gmail.com

L. BURRELL, N. WHITEHEAD, V. BRYANT, A. DIGGINS \& R.A. COHEN. Effects of Cannabis Consumption on Verbal Fluencies.

Objective: Examine the effects of cannabis on general verbal fluency task performance and on a task of specific (verb) fluency.

Participants and Methods: Participants ( $\mathrm{N}=187$ ) completed a battery of neurocognitive measures as part of a larger NIH-funded study on HIV associated brain dysfunction. Verbal fluency was measured using the Action Fluency and Controlled Oral Word Association (COWA) tasks. Cannabis use was measured via self-report. A series of linear regressions, using standardized neurocognitive scores, were employed to test the hypothesis that current (past 6 months) cannabis use is associated with deficits in frontally mediated verbal fluency.

Results: Our results showed that individuals who reported consuming cannabis performed significantly worse than individuals who had not on a frontally mediated Action Fluency; R2 $=.020, \mathrm{~F}(1,144)=4.001 \mathrm{p}=$ $.047, \beta=-.164, p=.047$. However, there were no significant differences between individuals who had and had not consumed cannabis on COWA scores; $\mathrm{R} 2=-.006, \mathrm{~F}(1,143)=.123 \mathrm{p}=.726, \beta=.029, \mathrm{p}=.726$.

Conclusions: Our results suggest that individuals in this population may benefit from interventions enhancing the functions of frontal networks such as cognitive training. These results support previous investigations which have demonstrated cannabis to affect the verbal memory and executive functions. Future investigations in this area should further elucidate the role of cannabis in neurocognition. DTI and fMRI studies would be helpful in determining how specific brain regions are affected and interacting in individuals who use cannabis.

Correspondence: Larry Burrell, M.S., Clinical and Health Psychology, University of Florida, 716 SW 16th Ave., Apt. 304, Gainesville, FL 32601.E-mail: leburrell2@phhp.ufl.edu
B.E. HAWKSHEAD, N.F. SCHWARZ, S.J. BRYANT, B. DUDA, S.W. LIEBEL, J. MACKILLOP \& L.H. SWEET. Neurocognitive Markers of Relapse Risk Assessed Using FMRI.

Objective: Despite treatment, most smokers who attempt to quit are unsuccessful. Treatment may be improved by identifying neurocognitive markers of quit success. Previous studies suggest that working memory (WM) may be related to successful cessation, but research on the relationship between neural correlates of WM and treatment outcome is scarce. We hypothesized that performance and patterns of brain activity during a FMRI WM task may be markers for smoking cessation treatment outcome.

Participants and Methods: 17 treatment-seeking, community-recruited healthy smokers (Mcig/day=18.44) enrolled in a 9-week smoking cessation program. At treatment's end, $35.3 \%$ of participants were unsuccessful (i.e., never quitters and relapsers). Prior to treatment, neural correlates of verbal WM were assessed using an n-back paradigm with echoplanar BOLD-FMRI at 3T. The relationships between WM task performance, brain activity, and treatment outcome were investigated in 12 a priori ROIs selected from a meta-analysis on the n-back.

Results: WM performance (2-back accuracy) was correlated with WAIS-IV Letter Number Sequencing (LNS) $(r=.541, p=.013)$. As predicted, brain activation (2-back $>0$-back) was significant in the a priori ROIs, and significantly related to 2-back accuracy $(r=.604, p=.005)$ and LNS ( $\mathrm{r}=.629, \mathrm{p}=.003)$. Moreover, treatment success was related to 2-back accuracy $(\mathrm{r}=.440, \mathrm{p}=.039)$ and task-associated activation in the ROIs $(r=.398, p=.057)$.

Conclusions: Our results indicate that smoking cessation treatment outcome is strongly associated with WM task performance and is related to task-associated patterns of brain activity. This suggests that both WM-associated neural patterns and WM task performance are likely valuable markers for smoking cessation treatment outcome, providing novel methods to assess and test neurocognitive models of addiction and relapse in future research.

Correspondence: Brittany E. Hawkshead, Psychology, University of Georgia, Psychology Department, University of Georgia, Athens, GA 30602.E-mail:bhawks@uga.edu

M.R. EHRLER, E. MCGLADE, D. YURGELUN-TODD \& P. RENSHAW. Lifetime Methamphetamine and Cigarette Consumption Differentially Impact Executive Functioning in Men and Women.

Objective: Debate remains whether methamphetamine (MA) differentially affects cognitive functioning among men and women. Nicotine is thought to have neuroprotective properties when used in conjunction with MA. This investigation explored the relationship between sex, MA, and cigarette use on cognitive functioning.

Participants and Methods: Twelve male and sixteen female MA users were administered measures of cognitive functioning including Stroop, WCST, Verbal Fluency, CPT-II, and WMS-IV. Simple one-way ANOVA's were used to compare group differences. Linear regression was used to assess the relative contribution of total-lifetime MA and cigarette use on cognitive functioning for men and women.

Results: Men and women performed similarly on measures of cognitive functioning. Greater MA use among men predicted more trials needed on the WCST ( $p=.028)$, diminished concept formation $(p=.050)$, trending scores toward more total errors $(p=.066)$ and perseverative errors $(\mathrm{p}=.092)$ in-so-doing, and more failures in maintaining response set $(p=.032)$. Although cigarette use did not uniquely predict scores, more cigarette use corresponded with better cognitive performance and added to overall predictive value of the model. This was not true for women. MA use accounted for the majority of variance and cigarette use did not contribute to the model.

Conclusions: Negative relationships between higher quantities of MA use and poorer executive functioning for both men and women exist; however, greater cigarette use correlated with better performance for men, but did not appear to impact female performance. Nicotine use in 
conjunction with other demographic and neurobiologic variables should be further explored as a potential mediating agent in MA dependence. Correspondence: Marie R. Ehrler, PhD, Psychiatry, University of Utah Brain Institute, 7739 Rope Key Drive, \#R204, Midvale, UT 84047. E-mail:mrehrler@gmail.com

\section{AASE \& N. PLISKIN. Alcohol Use History is Associated with Social Perception Task Performance.}

Objective: Recent cognitive and functional neuroimaging studies suggest differences in social perception performance between alcohol users and control subjects that persist with abstinence and are associated with treatment outcomes (Charlet et al., 2013; Foisey et al., 2007, Maurage et al., 2013). Moreover, while some alcohol use history variables have been associated with functional neuroimaging performance, research exploring how these variables may be associated with more common neuropsychological measures is limited. The present study measured social perception performance in patients during early alcohol abstinence. We hypothesized that alcohol use history indicators would be negatively associated with performance on neuropsychological measures of social perception.

Participants and Methods: Subjects were 30 patients (10 women, 20 men) participating in an inpatient treatment program for alcohol use disorders. Each subject had a primary alcohol diagnosis, had completed detoxification, and had at least 2 weeks of sobriety. Subjects were interviewed and administered a brief neuropsychological battery.

Results: Overall social perception scores were significantly lower than age-corrected normative scores. Scores on the Prosody and Pairs subtests were negatively associated with number of lifetime alcohol treatment episodes. Moreover, scores on the Pairs subtest were negatively associated with a self-report indicator of alcohol use severity. Scores were not associated with self-reported years of alcohol use.

Conclusions: Findings are consistent with previous literature documenting social perception deficits among individuals with alcohol use disorders, and also suggest that specific deficits may be associated with indicators of alcohol use severity. Future directions and limitations of the study are discussed.

Correspondence: Darrin Aase, PhD, Governors State University, 1 University Parkway, G108,University Park,IL 60484.E-mail: daase@ govst.edu

R.M. BOWLER, C.L. BESELER, V.V. GOCHEVA, E.S. KORNBLITH, M. COLLEDGE, Y. KIM, M.D. LEZAK, C.W. WRIGHT \& D.T. LOBDELL. Manganese in Air: Associations in Residents with Tremor and Motor Function.

Objective: An environmental study examined air manganese (Mn) exposed residents of two towns in Ohio: Marietta (near a ferro-manganese smelter) and East Liverpool (EL)(adjacent to an open-storage ore packaging facility). Air Mn inhalation is associated with neuropsychological/neurological sequelae. Positive relationships between air Mn and neuropsychological/neurological dysfunction were hypothesized. Participants and Methods: AERMOD dispersion modeling and ten years of fixed-site outdoor air monitoring data were used to estimate air Mn inhalation exposures. Motor tests of Finger Tapping, Hand Dynamometer, Grooved Pegboard, and CATSYS Tremor were administered bilaterally to 186 adult Mn-exposed residents (Marietta: n=100; East Liverpool: $n=86$ ). Bayesian modeling was used to assess associations between tremor and motor function and air $\mathrm{Mn}$.

Results: Air Mn exposure was significantly correlated with CATSYS hand tremor intensity $(p=0.02)$, center frequency $(p<0.0001)$ and harmonic index $(\mathrm{p}<0.0001)$ in bivariate analyses. Center frequency $(\beta=1.04, p<0.0001)$ and harmonic index $(\beta=-0.08, p<0.0001)$ left hand showed strong and significant associations with air Mn in a Bayesian path analysis model. This model was significantly associated with lower Finger Tapping dominant $(r s=-0.26, p<0.001)$ and non-dominant $(r s=-$ $0.16, p=0.03)$ hand T scores. Exposure to other metals (Pd, Cd, Hg and
Fe) did not explain these results. Household income was significantly associated with motor dysfunction but not with tremor.

Conclusions: Tremor and motor function were negatively associated with higher exposure to airborne Mn. Tremor scores showed stronger associations in the Bayesian model than the motor function scores. This may be due to the higher prevalence of subtle effects of long-term Mn exposure.

Correspondence: Rosemarie M. Bowler, SFSU, 8371 Kent Dr, El Cerrito, CA94530.E-mail: rbowl@sfsu.edu

J. PETRIE, B.L. BROWN, D.E. FLEMING \& S. STEFFENSEN. Visual Attention, Object Recognition Processing, Cognition, and Physiological Differences in Males and Females with Substance Abuse and Opioid Addiction.

Objective: Electroencephalogram (EEG) event-related potential (ERP) components are an objective technique for studying neuronal information processes associated with normal and abnormal cognition including that found in long term drug use. However, sex differences in EEG ERPs are controversial. For example, Steffensen et al. (2008) reported sex differences in a visual object recognition (ObR) task with female P300 amplitudes significantly greater than males. The present study extends these previous findings to determine if sex differences are found in ERPs and reaction time (RT) for male and female substance abusers (i.e., opiate addicts) in using a relevant simple go-no-go visual ObR task and a basic cognitive task.

Participants and Methods: This was a between-subjects design of 30 participants diagnosed with opiate addiction (males $=16$, females $=14$; age range $=18-53$ years - recruited from a community-based, high intensity residential substance abuse and detoxification treatment program) and 46 age- and sex-matched controls (males $=24$, females $=22$ ). All were administered 3 timed basic neuropsychological tests including the Symbol Digits Modalities Test (SMDT) and 4 computer visual processing protocols.

Results: P300 amplitudes in male and female addicts were significantly lower than controls $(\mathrm{F}=11.8, \mathrm{p}<.001$ and $\mathrm{F}=5.2, \mathrm{p}<0.02)$. However, while male addicts had significantly longer P300 latencies than controls $(\mathrm{F}=39, \mathrm{p}<0.0001)$, female addicts was characterized by significantly shorter P300 latencies $(\mathrm{F}=98, \mathrm{p}<0.0001)$. Females had significantly slower RT compared to males ( $\mathrm{F}=10.3, \mathrm{p} \leq 0.003)$, with no significant RT differences between controls and addicts by gender. SDMT scores indicated significant cognitive differences between controls and addicts $(\mathrm{MC}=62.1, \mathrm{SD}=1.1 ; \mathrm{MA}=50.62, \mathrm{SD}=8.3)$ but no significant gender differences.

Conclusions: There appear to be important EEG sex differences in visual processing as identified in the ObR task and RT, while those with opiate addiction were also characterized by SDMT cognitive processing deficits.

Correspondence: JoAnn Petrie, PhD, Psychology, Brigham Young University, 1001 SWKT, Psychology Dept, Provo, UT 84602. E-mail: joann_petrie@byu.edu

S. ALI, K.A. KERNS \& B.P. MULLIGAN. Investigating Attention and Executive Functions Impact on Intraindividual Variability in Children with Fetal Alcohol Spectrum Disorder (FASD).

Objective: Research on intraindividual variability (IIV) in clinical and aging populations indicates that attention and executive function (EF) play a role in IIV. Previous research suggests improvements in attention decreases IIV in children with FASD. We examine the relationship between attention, EF and IIV in children with/without FASD.

Participants and Methods: The FASD group included 65 previously diagnosed children (8 to 16 years) and age-matched controls $(n=21)$ with no prenatal alcohol exposure. IIV was calculated via the IIV standard deviation (ISD) method on a go-no/go task. Attention and EF composites were used in a path analyses to assess the impact of attention and EF on IIV. 
Results: Findings indicate attention accounted for significant variation in IIV and EF in the full sample while EF failed to account for variation in IIV. This finding was based primarily on results of control participants, with no relation between these abilities and IIV found in the FASD participants.

Conclusions: Attention is known to play a role in performance on EF tasks, reinforced here. The differential role attention plays with IIV between the clinical and control group will be further discussed.

Correspondence: Sheliza Ali, Clinical Neuropsychology, Psychology, University of Voctoria, 141 Eberts Street, Victoria, BC V8S 3H8, Canada.E-mail:sheliza@uvic.ca

G. CÁCERES-LUNA, S. SHELACH, J.P. QUINTANILLA, M. MONTALVO, L. MIRANDA, A. MANRIQUE \& M. SOTOANARI. Analysis of predictive factors for psychoactive substances consumption among college students in Peru.

Objective: Analyze executive functions, impulsiveness and social-family factors that best predicts consumption of alcohol, tobacco and marijuana.

Participants and Methods: We evaluated 67 college students between 16 and 28 years $(\mathrm{Me} .=18.60, \mathrm{SD}=2.06)$. For executive functions we used a computerized version of the Tower of Hanoi, the Simon task and the Iowa Gambling Task; the BIS-11 scale for impulsivity and AUDIT test for alcohol consumption; finally we used a questionnaire for indicators of family integration. For test our objective we used a multivariate analysis.

Results: The results of linear and multinomial regression showed that scores on the Iowa Gambling Task, the scale of impulsivity and male gender significantly predict the consumption of tobacco and marijuana; in this latter case we also observed that family type is a predictor of consumption. Finally, alcohol is not explained significantly by any of the variables analyzed.

Conclusions: Factors associated with impulsivity, somatic marker, gender and some dimentions of family integration are the best predictors of marijuana and tobacco consumption, for the case of alcohol we could not found significant predictors. These findings support the idea that psychoactive substances are associated with difficulties in emotional regulation. We recommend a deeper analysis of these variables to have a more complex view of the phenomenon and to develop intervention strategies.

Correspondence: Marcio Soto-Añari, M-Sc., Psychology, Universidad Católica San Pablo, Villa médica torre 2, dpto. 504, Arequipa 04001, Peru.E-mail:msoto@ucsp.edu.pe

D.B. HAMMERS, K. DUFF, A.Y. WANG, K.R. SMITH \& N.L. FOSTER. Bad Air, Bad Cognition: The Effects of Wintertime Inversions on Executive Functioning Among Elderly Residents.

Objective: While limited research has indicated deleterious effects of air pollution on cognition in children and older adults, these studies have focused on areas of chronic air pollution. Less is known about the impact of acute and periodic air pollution on cognition that occurs during Utah wintertime 'inversions.

Participants and Methods: Neuropsychological data were taken from a clinical database collected between 2012 and 2014. Air quality measurements, defined by Particulate Matter 2.5 (PM2.5 in $\mu \mathrm{g} / \mathrm{m} 3$ ) concentrations, were obtained from the EPA. Elderly clinical patients $(n=304)$ assessed during the four worst air quality months and the four best air quality months were compared on multiple cognitive domains, including global cognition, memory, language, attention, and executive functioning, using a series of independent samples t-tests.

Results: Patients evaluated during the worst air quality months displayed significantly worse performance on select measures of executive functioning and attention (ranging from $\mathrm{p}<.05$ to $\mathrm{p}<.10$ ) than those assessed during good air quality. Group differences were not observed on measures of global cognition $(p=.26)$, memory $(p=.39)$, language $(p=.83)$, depression $(p=.50)$, or anxiety $(p=.71)$.
Conclusions: Acute bouts of reduced air quality, in this case during the worst months of Utah wintertime 'inversions,' appear to adversely affect executive functioning and attention in this elderly sample. Air quality may be an under-appreciated confounding factor in many clinical studies, particularly when pollution is episodic in nature. As pollution-related cardiac or respiratory compromise may indirectly contribute, further studies are necessary to better assess mechanisms underlying these findings.

Correspondence: Dustin B. Hammers, Ph.D., Neurology, University of Utah, 650 Komas Dr. 106-A, Salt Lake City, UT 84108. E-mail: dustin. hammers@hsc.utah.edu

M. HUCKANS, B.E. FULLER \& J.M. LOFTIS. The Association Between Plasma Inflammatory Markers and Depression, Anxiety, and Cognition in Adults with and without Methamphetamine Dependence.

Objective: The purpose of this study was to evaluate the role of plasma immune factor dysregulation in the expression of specific neuropsychiatric symptoms (i.e., depression, anxiety, memory complaints, and executive function) in non-dependent adults and adults with current or recent methamphetamine (MA) dependence.

Participants and Methods: Blood samples and measures of anxiety [Generalized Anxiety Disorder-7 Scale (GAD-7)], depression [Patient Health Questionnaire-9 (PHQ-9)], memory complaints [Prospective-Retrospective Memory Questionnaire (PRMQ)], and executive function (D-KEFS Verbal Fluency) were collected from demographically (i.e., age, gender, race/ethnicity, education) similar groups of active methamphetamine users (MA-ACT = 17), adults in early remission from methamphetamine dependence (MA-REM = 36), and non-dependent controls with no history of alcohol or drug dependence (CTLs $=31$ ). Multi-analyte profile (MAP) analysis was used to evaluate plasma levels of an array of 47 immune factors (e.g., brain derived neurotrophic factor, interleukin-4, eotaxin-1, matrix metalloproteinase-3) (Myriad Rules Based Medicine, Inc.'s [Austin, TX] Human InflammationMAP v1.0 panel).

Results: Compared with CTLs, the MA-ACT and MA-REM groups evidenced significantly $(p<0.050)$ higher levels of depression and anxiety, more memory complaints, and poorer performance on the executive function task. Regression analyses revealed that, regardless of substance abuse status, neuropsychiatric and cognitive problems were each significantly predicted by a subset of immune factors (i.e., a protein signature). Conclusions: Results demonstrate that altered expression of a network of plasma immune factors contributes to neuropsychiatric symptom severity and cognitive impairment in adults with and without methamphetamine dependence.

Correspondence: Marilyn Huckans, Ph.D., Mental Health and Behavioral Neurosciences, Portland VA Medical Center, Portland VA Medical Center, P3MHN, 3710 SW U.S. Veterans Hospital Road, Portland,OR 97239.E-mail: marilyn.huckans@va.gov

J.J. MAHONEY, A. KALECHSTEIN, S. BAJO, A. RENO, A. DE MARCO, D. BROSHEK, T. NEWTON \& R. DE LA GARZA. The Impact of Premorbid IQ on Cognitive Functioning in Individuals with a Cocaine Use Disorder.

Objective: To determine whether premorbid IQ mediates cognition in individuals diagnosed with a cocaine use disorder (CUD).

Participants and Methods: Recently abstinent cocaine users ( $N=113)$ were administered measures sensitive to the effects of cocaine on $\operatorname{cog}$ nition: Continuous Performance Task-II (CPT-II), N-Back working memory test (n-back), and Hopkins Verbal Learning Task-Revised (HVLT-R). Premorbid IQ was calculated using the Oklahoma Premorbid Intelligence Estimate, which integrates scores from the WAIS-III and demographic information. The sample was then divided into tertiles based on pre-morbid IQ and group comparisons were conducted using one-way ANOVA. 
Results: Participants were primarily African-American males, $\sim 44$ years of age, reported using $\sim 2$ grams of cocaine/day, $\sim 17$ days out of the last 30 days, and an average of $\sim 18$ years of use. Participants with a higher estimated premorbid IQ (109.6 \pm 0.8 ; mean \pm S.E.M. $)$ demonstrated better performance in comparison to individuals with a lower premorbid IQ $(85.1 \pm 1.1)(\mathrm{F}(1,82)=317.9, \mathrm{p}<0.0001)$ on the following indices: HVLT-R learning trials $(\mathrm{F}(1,82)=19.7, \mathrm{p}<0.0001)$ and delayed recall subtest $(F(1,82)=14.1, p<0.001)$, greater mean span $(F$ $(1,82)=17.3, p<0.001)$ and maximum $\operatorname{span}(\mathrm{F}(1,82)=13.5, \mathrm{p}<0.001)$ of working memory, and greater accuracy of responding to visual stimuli on the n-back $(\mathrm{F}(1,82)=15.0, \mathrm{p}<0.001)$.

Conclusions: These findings demonstrate that lower pre-morbid IQ is a risk factor for the onset of impaired episodic and working memory in individuals diagnosed with a CUD. Because these impairments are risk factors for poor treatment outcomes, it is important to consider the need to modify treatment programs to account for these issues during the course of treatment.

Correspondence: James J. Mahoney, University of Virginia, 816 Kent Road,Waynesboro,VA 22980.E-mail:jjm5ej@hscmail.mcc.virginia.edu

M.F. REGNER, N. SAENZ, K. MAHARAJH, J. TREGELLAS, D. YAMAMOTO, R.I. PERRY \& J. TANABE. Substance Dependence Demonstrates Altered Efficiency and Increased Bidirectional Network Causal Connectivity.

Objective: Complex changes in reward, learning, executive control, and interoceptive processing may underlie pathologic behavior in substance dependent individuals (SDI). Connectivity between networks associated with these constructs may be altered in SDI. We hypothesize that compared to controls, SDI demonstrate altered network efficiency and increased connectivity between default mode networks (DMN) and basal ganglia networks (BGN).

Participants and Methods: 50 SDI (28M/22F, 34 28 years) were compared to 50 age- and sex-similar controls $(25 \mathrm{M} / 25 \mathrm{~F}, 32 \pm 9$ years $)$. SDI met DSM-IV criteria for dependence on methamphetamine, cocaine, or both. GRE fMRI scans were acquired over five minutes during quiet rest with eyes closed. Group independent component analysis (ICA) was conducted on SDI and controls separately. Fourteen components were selected using template matching to standardized resting state networks. After adjusting for motion, CSF, and white matter signal fluctuations, the number, direction, and strength of connections between components was analyzed with Granger Causality. Within-group thresholds were $p<0.005$ using a bootstrap permutation (200 iterations) and between group comparisons were $p<0.05$, FDR-corrected for multiple comparisons

Results: SDI showed higher network density $(p<0.01)$, higher global efficiency $(p<0.01)$, and lower local efficiency $(p<0.05)$ than controls. SDI showed more bidirectional connectivity whereas controls showed more unidirectional connectivity. SDI showed significantly stronger connectivity from DMN to BGN.

Conclusions: Higher global and lower local efficiency in SDI suggests that connectivity is more random in SDI. Greater connectivity from DMN to BGN in SDI compared to controls suggests that interoception and internal processing (DMN) influence habit formation (BGN) to a greater extent in SDI than controls; pathological habit is associated with drug-seeking behavior in SDI. Increased bidirectional connectivity overall suggests less efficient functional organization in brains of drug users. Correspondence: Michael F. Regner, M.D., Department of Radiology, University of Colorado - Denver, 12700 E. 19th Avenue, Mailstop C278, Aurora,CO 80045.E-mail:michael.regner@ucdenver.edu

M. CHERNER, A. MiNASSIAN, R. Ellis, P.A. SUAREZ, R.K. HEATON, C. BOUSMAN \& I. GRANT. COMT Val158Met Val/ Val Genotype May Mitigate Methamphetamine-related Executive Dysfunction.

Objective: Catechol-O-methyltransferase (COMT) is responsible for dopamine (DA) clearance in prefrontal cortex. The COMT Val158Met genetic polymorphism has been linked to differences in executive function. COMT Val alleles confer a high rate of dopamine catabolism, whereas Met alleles result in slower clearance. Met/Met homozygotes typically show advantages on tests of executive function, perhaps resulting from greater bioavailability of DA. Methamphetamine (METH) use causes large releases of DA. This, in turn, is associated with injury to brain tissues. Heavy METH users show a number of cognitive deficits, including executive problems. We hypothesized that, contrary to its effect in non-METH-using populations, faster DA clearance conferred by the $\mathrm{Val} / \mathrm{Val}$ genotype will be associated with better executive function in METH users.

Participants and Methods: COMT Val158Met genotype was obtained for 85 methamphetamine dependent (METH+) and 62 healthy comparison (METH-) Caucasian men. Val/Met heterozygotes were excluded, resulting in $16 \mathrm{METH}$ - Met/Met, $18 \mathrm{METH}-\mathrm{Val} / \mathrm{Val}, 20 \mathrm{METH}+$ Met/ Met and 16 METH+ Val/Val. Within each METH group, genotype groups had comparable background characteristics. All received the Wisconsin Card Sorting Test 64-item-computerized version as part of a larger battery. Number of perseverative responses was transformed into a demographically adjusted T-score and used as the dependent variable in a multiple regression analysis with METH group, COMT genotype, and their interaction as predictors.

Results: There was a significant COMT $x$ METH interaction $(p=.0096)$. Among METH-, those with Met/Met genotype outperformed those with $\mathrm{Val} / \mathrm{Val}[\mathrm{T}-\mathrm{sc}(\mathrm{SD})=51(11)$ vs. 44(13)], while among METH+, $\mathrm{Val} / \mathrm{Val}$ outperformed Met/Met [46(12) vs. 39(8)] and performed comparably to METH-

Conclusions: Val/Val genotype may confer protection against METH-related executive dysfunction, possibly as a result of greater inactivation of DA in prefrontal cortex, suggesting genetically influenced differences in vulnerability to METH effects.

Correspondence: Mariana Cherner, PhD, Psychiatry, University of California San Diego, 9500 Gilman Drive, Mail Code 0847, La Jolla, CA 92093.E-mail: mcherner@ucsd.edu

T.M. SCOTT, M. RIVERA-MINDT, F. ARIAS, C. CUNNINGHAM, Z. MASSEY, A. MANGALONZO, P. OLSEN \& J. ARNSTEN. Neuropsychological Function is Improved among Opioid Dependent Drug Users Who Adhere to Opiate Agonist Treatment with Buprenorphine-Naloxone.

Objective: Among persons with substance use disorders (SUDs), neuropsychological (NP) dysfunction is associated with depression, active substance use, and poor substance abuse treatment retention, while improved NP function is associated with abstinence and opioid agonist treatment (OAT). However, no study to date has examined whether OAT adherence, depression, and SUD type are associated with NP change over time. We hypothesized that high OAT adherence and NP improvement are independently associated after adjustment for depression and SUD type.

Participants and Methods: Participants included 21 ethnically-diverse adults with opiate use disorder (OUD; M Age $=45$ years $[\mathrm{SD}=7.9] ; 71 \%$ male) receiving OAT (buprenorphine-naloxone) who completed a NP battery and self-reported OAT adherence (\% adherent) and psychiatric/ SUD (BDI-II, DSM-IV) measures at baseline and 6-months. Seven NP domains were assessed (learning, memory, attention/working memory, processing speed, verbal, executive, and motor). Demographically-corrected average $\mathrm{T}$-scores and $\mathrm{NP}$-change scores (Time 2-Time 1) were computed for Global NP and each domain. We divided SUD-type into two groups (OUD-Only and Polysubstance Use).

Results: OAT adherence was associated with Global NP, Learning, and Memory change scores (r's=.54-.67, $\mathrm{p}$ 's $\leq .01)$. BDI-II and SUD type were not related to adherence or NP change ( $p$ 's $>$.10). In multiple regression analyses including BDI-II and SUD type, adherence remained significantly associated with Memory change $(p<.01)$.

Conclusions: High adherence to OAT with buprenorphine-naloxone is associated with improved global NP, learning, and memory over time, 
and uniquely predicts improvement in memory, adjusting for depression and SUD type. Enhancing OAT adherence may be especially important in improving and/or preserving NP functioning in individuals treated for opioid dependence.

Correspondence: Travis M. Scott, BA, California State University, Northridge, 8752 Delco Ave, Los Angeles, CA 91306.E-mail: tscott11@ lion.lmu.edu

\section{Genetics/Genetic Disorders}

\section{A. ARENIVAS \& A.D. CANNON. Neuropsychological Profile of Pediatric Beta Thalassemia Major: A Case Study.}

Objective: Beta Thalassemia Major (B-Thal) is a rare, chronic, and genetically determined hematological disorder most commonly found in Mediterranean, African, or Asian countries. In the US, approximately 1000 individuals have B-Thal, which is characterized by erythropoiesis, hemolysis, and severe anemia. Treatments include blood transfusions (potentially leading to iron overload) or bone marrow transplantation. The cognitive and behavioral presentation of B-Thal is not well-established. Limited research in children suggests risk for attention problems, increased motor activity, and anxiety/withdrawal. This study aimed to contribute to improved understanding of the neuropsychological profile of pediatric B-Thal through presentation of child (Joe) diagnosed with B-Thal at age 1, who was assessed across several domains of cognition, including those not currently reported in the literature.

Participants and Methods: Joe is a 7-year-old right-handed male seen for a comprehensive evaluation in an outpatient pediatric neuropsychology clinic in an urban medical setting. Joe underwent matched sibling bone-marrow transplant at age 5 . Parent concerns included reading and fine-motor skills.

Results: Findings revealed average intellectual ability with average or higher basic auditory and visual attention and working memory, visual perception, receptive and expressive language, verbal fluency, memory, and academic skills in reading and math. Weakness included fine-motor dexterity, divided attention, aspects of EF, and adaptive behaviors. Anxiety was elevated on standardized rating-forms.

Conclusions: Aspects of Joe's presentation are consistent with research, although other difficulties were noted. Findings suggest that in order continue to aid clinical practice, ongoing research across multiple domains of cognition to establish a neuropsychological profile of this rare disease is warranted.

Correspondence: Ana Arenivas, PhD, MPH, Neuropsychology, Kennedy Krieger Institute/Johns Hopkins University School of Medicine, 1750 E. Fairmount Ave, Baltimore, MD 21231.E-mail: arenivas@ kennedykrieger.org.

\section{R. RAO \& J. MILLER. Neuropsychological Outcome in Russell- Silver Syndrome.}

Objective: Russell-Silver Syndrome (RSS) is a clinically and genetically heterogeneous congenital imprinting disorder in which genetic/epigenetic mutations can be found in up to $50 \%$ of cases. RSS is characterized by intrauterine and postnatal growth retardation and accompanying physical abnormalities including atypical facies and mild growth and digestive system abnormalities.

Participants and Methods: We present the case of an 11 year old female who was the result of a pregnancy complicated by preeclampsia and severe fetal heart decelerations, born at 26 weeks gestation and diagnosed with RSS at age two. Global developmental delay, short stature necessitating growth hormone therapy, fifth finger clinodactyly, and facial dysmorphism complicated her early history. Prior diagnoses included ADHD-C, dyscalculia, and anxiety disorder, and ongoing problems related to underdeveloped social skills were reported.

Results: Neuropsychological evaluation revealed average intellectual functioning and learning and memory skills that were contrasted by impairments in visual working memory, fine motor dexterity and coordination, and visual spatial estimation, and relative weakness in psychomotor processing speed and executive functioning including sustained attention and planning and organization skills. Significantly better-developed reading skills relative to math skills were also noted, as were parent and self-reported problems with attention and concentration, mild depression, and poor social proficiency marked by peer rejection.

Conclusions: Findings are consistent with previous case studies suggesting that the right hemisphere of the brain may be more susceptible to insult in cases of RSS. Results further support the utility of neuropsychological evaluation at school age to identify necessary psychosocial and academic supports

Correspondence: Julie Miller, Psy.D., Kettering Medical Center, 3533 Southern Blvd, Kettering, OH 45429.E-mail: julie.miller@khnetwork. org

E.B. LEAFFER, R.J. FEE \& V.J. HINTON. The impact of sleep quality on executive function deficits observed in children with dystrophinopathy.

Objective: To examine whether poor sleep quality, as measured by daytime sleepiness, contributes to reported executive function difficulties in children with dystrophinopathies. Dystrophinopathies are genetic disorders characterized by progressive muscular weakness and lack of the protein dystrophin in muscle and brain. Affected boys have higher rates of parent reported executive deficits. Children with dystrophinopathy often experience increased waking at night due to motor limitations; therefore, we postulated that these children would be more likely to report daytime sleepiness. Given that overall sleep quality impacts executive function, we hypothesized that reported sleep quality would contribute to the executive function deficits observed in children with dystrophinopathy.

Participants and Methods: 51 boys with dystrophinopathy, aged 6 to 18 were given the Epworth Sleepiness Scale (ESS) and parents completed the Behavior Rating Inventory of Executive Function (BRIEF). ESS score can range from 0-24, with $>10$ as "sleepy." BRIEF scores of $T \geq 67$ were set to reflect significant problems (found in $<4 \%$ of the general population). Descriptive statistics and chi-square tests $(\alpha=0.05)$ were run.

Results: Children with dystrophinopathy did not report daytime sleepiness on the ESS (mean $=4.0, \mathrm{sd}=3.0$, range $=0-16$ ), and only one participant had a score above 10 . We dichotomized the variable to those who scored above 8 (or slightly increased sleepiness, $n=6$ ) and those $<8$ $(n=45)$. As expected, parents reported executive function difficulties: $19.6 \%$ on Behavioral Regulation Index $(n=10), 2.0 \%$ on the Metacognition Index $(n=1)$, and $7.8 \%$ on the Global Executive Composite $(n=4)$. Chi-square analyses showed no significant association between higher sleepiness scores and significant executive problems.

Conclusions: Overall, our sample did not report poor sleep quality. Parent-reported executive function difficulties were present, however these problems do not appear to be attributed to sleep quality.

Correspondence: Emily B. Leaffer, MPH, Columbia University, 630 West 168th Street, PH 19, New York, NY 10019.E-mail: ebl2121@cumc. columbia.edu

\section{M.W. FONG \& M. LACY. Neuropsychological Profile in Hereditary Spastic Paraplegia (HSP): A Case Report.}

Objective: Hereditary spastic paraplegias (HSPs) are a group of rare genetic neurodegenerative disorders that are characterized by their physical features of lower limb spasticity and weakness. Accompanying cognitive decline is unclear (Murphy et al., 2009). Only scant research examining familial variants have been published often using screening tools. We provide a history and an in-depth cognitive assessment of a patient diagnosed with HSP.

Participants and Methods: We present data gleaned from an examination of a 58-year old Caucasian adopted female with a 1 year decline in mobility, with bilateral lower extremity weakness, and memory 
problems. Medical history included COPD and rheumatoid arthritis. Family history was notable for balance problems and monoclonal gammopathy. Neurological exam revealed hyperflexia, clonus, scissoring gait, and cortical thumbs.

Results: Results revealed a woman of average premorbid intellect who displayed a decline in intellectual ability. Assessment revealed significant executive dysfunction with severe problem solving deficits with milder processing speed, mental flexibility, spatial judgment, and planning deficits. Clear strengths were documented on verbal retrieval, naming, and speeded phonemic fluency tasks. Motorically, she displayed bilateral grip weakness with low average fine motor dexterity speed. Understandably, she also endorsed significant depression and anxiety. Conclusions: In the current case presentation we document a patient with prominent rapidly progressive lower extremity weakness along with prominent executive dysfunction. While her psychiatric state and history of pulmonary dysfunction may be playing a role, this is consistent with other case reports suggestive of an accompanying subcortical cognitive pattern of dysfunction (Webb et al., 1998). Clinicians should be aware of this potential condition in patients presenting with cognitive dysfunction with a rapid motor component.

Correspondence: Mandy W. Fong, Illinois Institute of Technology, 3105 South Dearborn, Chicago, IL 60616. E-mail:wfong1@hawk.ït.edu

\section{V.J. HINTON, R. FEE \& E.B. LEAFFER. Verbal Working Memory Selectively Contributes to Academic Achievement in Children with Dystrophinopathy.}

Objective: To examine the contributions of verbal working memory versus executive control on academic performance in children with dystrophinopathy. Dystrophinopathy is characterized by a genetic mutation and lack of dystrophin in muscle and brain. Affected boys have lowered verbal IQ and academic performance. We have argued there is an etiologically specific selective weakness in verbal working memory that underlies their cognitive profile, while others have suggested more generalized executive problems account for the findings. To test this, contributions of executive function and verbal working memory on academic performance were examined.

Participants and Methods: Participants: 24 boys with dystrophinopathy $(6-15$ years, mean $11.1+2.7)$. Measures: academics (WJ-III Academic Achievement), IQ estimate (Peabody Picture Vocabulary Test-4, Comprehensive Test of Nonverbal Intelligence), executive function (NIH Toolbox Flanker Inhibitory Control, Dimensional Change, BRIEF General Executive Composite), verbal working memory (WISC-IV Working Memory Index, NIH Toolbox List Sorting) and demographics (income, age, illness severity). Data analysis: Pearson correlations were run and variables significantly associated with academic outcome were entered into a regression analysis.

Results: Academic achievement was significantly associated with all cognitive variables and income $(\mathrm{p}<.01)$, but was not associated with BRIEF scores, age or illness severity. Linear regression with academic achievement as the dependent variable and other measures entered as independent variables was significant $(\mathrm{F}(6,15)=9.87, \mathrm{p}=.000)$, but only the Working Memory Index score contributed significantly ( $t=3.37$, $\mathrm{p}=.004)$ making up $64 \%$ of the variance in academic scores.

Conclusions: Verbal working memory contributes significantly to academic outcome in children with dystrophinopathy, whereas measures of general executive control do not. This is further evidence that there is a selective core deficit related to impaired verbal working memory. Correspondence: Veronica J. Hinton, PhD, Sergievsky Center \& Department of Neurology, Columbia University, P\&S Box 16, Columbia University, 630 West 168th Street, New York, NY 10025. E-mail: vjh9@ columbia.edu

R.J. FEE, E.B. LEAFFER \& V.J. HINTON. The Role of Socioeconomic Status in the Dystrophinopathies.

Objective: Dystrophinopathies (including Duchenne and Becker muscular dystrophy) are genetic diseases characterized by progressive muscular weakness and cognitive deficits associated with abnormal expression of the protein dystrophin in both muscle and brain. The cognitive profile is characterized by lowered verbal IQ, academic difficulties and selectively limited verbal working memory, but is not associated with disease severity. The dystrophinopathies are equally common across race and ethnic groups, but the role of socioeconomic status (SES) has not been explored in this population.

Participants and Methods: 38 boys with Dystrophinopathy from a range of socioeconomic backgrounds in $\mathrm{NYC}$ were included. Income group (low, middle, and high) was based on reported household income and number of individuals in the home. Measures: Estimated verbal IQ (Peabody Picture Vocabulary Test), nonverbal IQ (Comprehensive Test of Nonverbal Intelligence), working memory (WISC-IV Working Memory index), academic achievement (Woodcock-Johnson III Achievement Test), disease severity (Brooke and Vignos scale of physical functioning), behavior (Behavior Assessment System for Children-2). Chi-square tests were run to assess the relationship between family income and cognitive, physical and behavioral outcomes.

Results: There was a significant $(p<.05)$ relationship between SES and cognitive performance on: PPVT, $\mathrm{X}^{2}(4,38)=23$; WJ, $\mathrm{X}^{2}(4,36)=$ 11; WMI, $X^{2}(4,34)=12$. Lower SES was associated with lower verbal, academic and working memory performance. However, no significant relationships between SES and nonverbal intelligence, physical severity or child behavior were found.

Conclusions: SES is significantly related to cognitive performance in boys with Dystrophinopathy especially in areas of known vulnerability, but is not associated with disease severity or child behavior. Environmental influences such as low family income may make brain areas even less resilient

Correspondence: Robert J. Fee, Sergievsky Center, Columbia University/ Queens College, 630 West 168th St. PH19, New York, NY 10032. E-mail: rf237@columbia.edu

F.P. BERNIER, J.S. PARBOOSINGH, A. INNES, C. SMITH \& D. DEWEY. Complex Neurodevelopmental Profile, Reminiscent of Williams Syndrome, in a Child with EP300-related Syndromic Intellectual Disability.

Objective: This case study characterized the neurodevelopmental profile of a child with a pathogenic mutation in EP300, which has been linked to Rubinstein Taybi syndrome.

Participants and Methods: This child was initially assessed at 22 months of age due to global developmental delays, microcecephaly, short stature and minor dysmorphic features (i.e., brachycephaly, arched and thin eyebrows, down-slanting palpebral features, a depressed nasal tip, thin lips). At eight years of age, height, weight and head circumference were below the 3rd centile but she had normal interval growth velocities. She is healthy with no chronic illnesses. Whole exome sequencing identified a pathogenic mutation in EP300.

Results: She presented as a friendly, happy child who loved adult attention. Full Scale IQ was in the borderline range $(F S=71)$. Significant discrepancies were noted between performance on the Verbal Comprehension Index $(\mathrm{VC}=87)$, and the Perceptual Reasoning $(\mathrm{PR}=70)$, Working Memory (WM=74) and Processing Speed (PS = 70) indicies. On the NEPSY-II, deficits were noted in attention and executive function, and visual and social perception. She scored in the average range on measures of language, memory and sensorimotor functioning. Significant deficits were noted in fine and gross motor function. Parent report measures indicated difficulties in attention, inhibition, working memory, planning and organization and peer relationships. Academically, this child's functioning ranged from grade level (i.e., oral reading) to two years delayed (i.e., mathematics).

Conclusions: This child, with a mutation of EP300, displays a unique neurodevelopmental profile similar to that of Williams syndrome. Of note was the observation that her social behavior profile was consistent with Rubinstein Taybi syndrome. Clear documentation of neurodevelopmental profiles in individuals with genetic syndromes provides much 
needed information on the natural history of these syndromes for parents and professionals, but also helps developmental support services. Correspondence: Deborah Dewey, PhD, Paediatrics and Community Health Sciences, University of Calgary, Behavioural Research Unit, Alberta Children's Hospital, 2888 Shaganappi Trail NW, Calgary, AB T3B 6A8,Canada.E-mail: dmdewey@ucalgary.ca

A.G. GERTSBERG, R. KAPLAN, K.J. MANNING \& B.L. HENNIG. Neuropsychological Predictors of Driving Outcomes in Patients with Huntington's Disease.

Objective: Huntington's disease (HD) disrupts cognitive processes necessary for safe driving. However, limited evidence connects declines in cognitive abilities to actual on-road performance in HD, resulting in insufficient practice parameters concerning driving recommendations. The present aims were to validate proposed clinical guidelines for driving evaluation in HD and to elucidate the association between on-road driving capacity and cognition in this population.

Participants and Methods: Thirty-seven HD patients aged 23-73 $(\mathrm{M}=47.13, \mathrm{SD}=13.02)$ completed a neuropsychological test battery, including the RBANS, Trail Making Test, Stroop and the NAB Attention module as part of a routine clinical visit. Significant cognitive impairment resulted in a mandatory DMV referral for on-road assessment with three possible outcomes: Pass, Pass With Restrictions, and Fail. We then compared the cognitive performance of patients in these groups with patients who were not referred for the DMV driving assessment. Results: Completed DMV referrals resulted in either a decision of restriction $(n=5)$ or failure $(n=5)$. Six patients relinquished driving privileges before the road test. Patients not referred to the DMV performed significantly better than other groups on tests of attention, processing speed, and executive functioning (e.g., Stroop Test, F $(4,40)=11.41$, $\mathrm{p}<0.001)$. Of note, a trend analysis $(\mathrm{p}=0.07)$ suggested worse Stroop performance in patients who failed the road test $(\mathrm{M}=22.20, \mathrm{SD}=7.29)$ compared to those who passed with restrictions $(\mathrm{M}=35.40, \mathrm{SD}=8.99)$. Conclusions: These preliminary findings indicate that tests of executive functioning and processing speed may reliably distinguish patients who are likely to perform poorly on an on-road exam. Cognitive assessments may be clinically useful in making driving recommendations for patients with HD.

Correspondence: Anna G. Gertsberg, University of Connecticut Health Center, 263 Farmington Avenue, Farmington, CT CT. E-mail: anna. gertsberg@gmail.com

G. SCHWARZ, D.A. BENNETT, C.B. MERVIS \& B.P. KLEINTASMAN. Relations between Lab-Based and Parent-reported Executive Functioning in Children and Adolescents with Williams Syndrome.

Objective: Youth with Williams syndrome (WS), a neurodevelopmental disorder of genetic origin, show elevated rates of ADHD and related difficulties in executive functioning. The main objective was to examine relations between parental ratings of everyday executive functioning and performance on a lab-based executive function task.

Participants and Methods: Participants were 74 children with WS (40 girls, 34 boys), ages $8-15$ years $(M=11.27, S D=2.49)$ and their parents. Parents completed the Behavior Rating Inventory of Executive Functioning (BRIEF). Lab based executive functioning was assessed by the Dimensional Change Card Sort (DCCS) and cognitive functioning by the Kaufman Brief Intelligence Test - Second Edition (KBIT-2).

Results: Clear delays in EF were notable (e.g., only $50 \%$ of the $12-15$ year olds with WS passed the third level of the DCCS, which is the typical pass rate for 5-year-olds). Development of EF with age was evident $(\mathrm{r}=.42, \mathrm{p}<.001)$. A majority of parents reported child EF difficulties at clinical levels (T score 65+: General Executive Composite: 55.26\%, Behavioral Regulation: $44.74 \%$, Metacognition $68.42 \%$ ). DCCS performance was related to raw scores on all BRIEF indexes with medium effect sizes (r's: -.33|-.38, p-values .001-.004). A strong relation was found between DCCS performance and the KBIT-2 verbal ability raw score $(r=.68, p=<.001)$.

Conclusions: Consistent with the high comorbidity of WS and ADHD, a majority of participants show parent-reported problems in behaviors related to EF at clinical levels. Performance on the lab-based measure of EF relates to verbal abilities and to parental ratings of everyday EF behaviors in children with WS.

Correspondence: G. Nathanael Schwarz, B.A., Psychology, University of Wisconsin Milwaukee, 1010 E Hadley St., Milwaukee, WI 53212. E-mail: gschwarz@uwm.edu

G. MCFALL, S.A. WIEBE, K.J. ANSTEY \& R.A. DIXON. An Alzheimer's Genetic Risk Composite Interacts with Diabetes Status to Predict Neurocognitive Speed Level and Change in NonDemented Older Adults.

Objective: Risk factors associated with Alzheimer's disease (AD) may predict neurocognitive decline prior to clinical onset. Among these biomarkers are non-modifiable biological (e.g., genes) and modifiable health/environmental (e.g., diabetes; lifestyle) factors. We examine the cross-domain interactive effects of a new Alzheimer's Genetic Risk Composite (AGRC) and type 2 diabetes (T2D) on level and change of neurocognitive speed (NCS) in non-demented older adults.

Participants and Methods: This accelerated longitudinal design included non-demented older adults $(\mathrm{n}=600$, baseline $\mathrm{M}$ age $=70.4$, age range 53-95) followed over 9 years. Latent growth modeling tested independent and interactive AGRC $x$ T2D status effects on level (centering age $=75$ ) and change in a NCS latent variable (i.e., choice reaction, sentence verification, lexical decision). The AGRC was comprised of cumulative risk from APOE, CLU, CR1, and PICALM.

Results: First, T2D status independently predicted slower NCS performance at age $75(b=.465, p=.004)$ and more 9-year NCS decline $(\mathrm{b}=.021, \mathrm{p}=.032)$. Second, T2D and high AGRC risk predicted slower NCS performance at age $75(b=.456, p=.066)$ and more 9-year NCS slowing $(\mathrm{b}=.036, \mathrm{p}=.007)$ than the no-diabetes-high-AGRC (NDHGR) reference group. Third, T2D and high AGRC (minus CR1) exhibited significantly lower NCS performance at age $75(b=.686, p=.003)$ than the NDHGR group. Fourth, T2D $x$ high AGRC (minus PICALM) predicted more 9-year NCS slowing than the NDHGR group $(b=.044, p=.005)$.

Conclusions: Separately, these polymorphisms and diabetes are risk factors for AD. High AD-related genetic risk (indexed by AGRC) can intensify deleterious effects of diabetes on cognitive performance and change in non-demented aging.

Correspondence: G. Peggy McFall, University of Alberta, P-306 Biological Sciences Building, Edmonton, AB T6G 2E9, Canada. E-mail: gmcfall@ualberta.ca

C. MORSE, P.J. MOBERG, M. RICHMAN, V. KAMATH, B. TURETSKY, R. GUR \& R. GUR. Neurocognitive Functioning in Patients with 22q11.2 Deletion Syndrome: A Meta-Analytic Review.

Objective: The 22q11.2 Deletion Syndrome (22q11.2DS) is a known risk factor for development of schizophrenia and is characterized by a complex neuropsychological profile. To date, a quantitative meta-analysis examining cognitive functioning in 22q11.2DS has not been conducted.

Participants and Methods: A systematic review of cross-sectional studies comparing neuropsychological performance of individuals with 22q11.2DS with age-matched healthy comparison subjects was carried out. Potential moderators were analyzed.

Results: Analyses included 29 articles (158 effects) that met inclusion criteria. Large and heterogeneous effects were seen for global cognition $(\mathrm{d}=-0.89)$ and in specific neuropsychological domains (intellectual functioning, executive functioning, and motor; $\mathrm{d}$ range $=-0.76$ to -2.43 ). Moderator analysis revealed a significant role for demographic (age, sex) and clinical factors (externalizing behavior). Results revealed significant differences between pediatric and adult samples, with isolated analysis 
within the pediatric sample yielding large effects for global cognition $(\mathrm{d}=-0.99)$ and in several neuropsychological domains (intellectual functioning, motor, verbal memory; $d$ range $=-0.95$ to -2.88 ).

Conclusions: Large cognitive deficits in intellectual functioning and specific neuropsychological variables in individuals with 22q11.2DS represent a robust finding, but these deficits are influenced by several factors, including age, sex, and clinical status. These findings highlight the clinical relevance of characterizing cognitive functioning in $22 q 11.2 \mathrm{DS}$ and the importance of considering demographic and clinical moderators in future analyses.

Correspondence: Chelsea Morse, M.S., Drexel University, 3201 Chestnut Street, Philadelphia, PA 19130. E-mail: chelsealmorse@gmail.com

\section{HIV/AIDS/Infectious Disease}

N.S. THALER, P. SAYEGH, A. ARENTOFT, A. KARIMIAN, Z. MAHMOOD, E.J. SINGER \& A.D. THAMES. Behavioral dysregulation is associated with increased neurocognitive variability.

Objective: Elevated neurocognitive intra-individual variability (IIV) is an indicator of frontal lobe dysfunction. Frontal dysfunction can result in behavioral dysregulation and poor emotional control. However, there is limited research on the relationship between IIV and behavioral dysregulation. The current study used a convenience sample of HIV+ patients to examine this relationship.

Participants and Methods: This sample included 65 patients living with HIV. Neurocognitive IIV was calculated as the within-group standard deviation of test scores. Behavioral regulation was measured with the BRIEF Behavioral Regulation (BR) Index. Groups were split into no impairment and impairment based upon a t-score cutoff (i.e., $>65$ ). These groups were compared on measures of mood and distress, IIV, and mean neurocognitive performance.

Results: Those with BR impairment reported significantly higher levels of depression, $\mathrm{F}(1,64)=11.63, \mathrm{p}<.001$, than individuals without impairment. This group also had a trend for poorer emotional control $\mathrm{F}(1,64)=3.1, \mathrm{p}<.08$. Dispersion (i.e., IIV) significantly differed between BR groups, $\mathrm{F}(1,64)=6.34, \mathrm{p}=.014$, partial $\eta 2=.090$, such that the impaired group had higher IIV. Groups did not differ on overall test battery mean score $(p=.589)$ or executive functioning $(p=.285)$. Conclusions: Results suggest that behavioral dysregulation is associated with mood and increased IIV. We did not find group differences in neurocognition when using mean performance, suggesting that IIV might be more sensitive to dysfunction in behavioral regulation. Additional research is required to determine causal relationships between IIV and behavioral dysregulation.

Correspondence: Nicholas S. Thaler, Ph.D., Department of Psychiatry and Biobehavioral Sciences, UCLA Semel Institute, 760 Westwood Blvd, C8-849,Los Angeles, CA 90095.E-mail:Nthaler@mednet.ucla.edu

A. STEINER, J.S. MILLER, A. BENJI, R.A. ZALDIVAR, K. SMITH, D.J. HARDY \& E. LOPEZ. Working Memory Deficits in Spanishspeakers with HIV-Associated Neurocognitive Disorders.

Objective: HIV infection disproportionately impacts Spanish-speaking adults in the United States (CDC, 2013) yet there are limited culturally sensitive measures developed to detect working-memory impairment associated with HIV-associated neurocognitive disorders (HAND) in Spanish-speakers (Levine et al., 2011). Previous research suggests working-memory is compromised by HIV infection (Hinkin et al., 2002). This study investigated the potential deficits in working-memory (verbal and visual) between HAND and No-HAND groups. We hypothesized the No-HAND group would signifficantly outperform the HAND group on Digit-Span Backward (DSB) and Spatial-Span Backward (SSB).
Participants and Methods: Our study included $100 \mathrm{HIV}$-seropositive Spanish-speaking adults (47 HAND, 53 No-HAND). Individuals with neurological, psychiatric, or medical disorders were excluded.

Results: An ANCOVA controlling for age and education revealed the No-HAND group $(\mathrm{M}=5.06, \mathrm{SD}=1.93)$ performed significantly better than the HAND group $(\mathrm{M}=3.87, \mathrm{SD}=1.31)$ on DSB $[\mathrm{p}<.0001, \eta 2$ $=.167]$ and the No-HAND group $(\mathrm{M}=7.36, \mathrm{SD}=1.91)$ performed significantly better than the HAND group $(\mathrm{M}=5.32, \mathrm{SD}=1.76)$ on SSB $[p<.0001, \eta 2=.279]$. A Pearson's correlation found a strong relationship between DSB and SSB $(r=.555)$ suggesting convergent validity for Spanish-speakers.

Conclusions: Large effect sizes were observed on the visual and auditory working-memory tasks, implying that both types of instruments were sensitive to distinguishing between the HAND and the No-HAND group's level of impairment. Findings indicate that these measures are valid to assess working-memory deficits related to HAND and future research should continue investigating and developing culturally sound measures for Spanish-speakers.

Correspondence: Alexander Steiner, M.A., CSPP at Alliant International University, Los Angeles, 1816 Purdue Ave \#103, Los Angeles, CA 90025. E-mail: Alexsb812@aol.com

L. BAKER, U.S. CLARK, D.A. BYRD \& S. MORGELLO. Cognitive Predictors of the Medication Management Task-Revised in an HIV+ Racial/Ethnic Minority Cohort.

Objective: HIV is associated with cognitive and functional impairments. Rates of asymptomatic and mild cognitive impairment are increasing in the HIV+ population, resulting in greater efforts to utilize performance-based functional capacity measures as a more objective complement to self-report questionnaires when diagnosing HIV-associated neurocognitive disorders (HAND). We examined the relationship between a performance-based (Medication Management Task-Revised [MMT-R]) and self-report measure of functional capacity in an advanced-stage $\mathrm{HIV}+\mathrm{racial} / \mathrm{ethic}$ minority sample. We also explored the degree to which cognition predicted MMT-R scores.

Participants and Methods: Ninety-four HIV+ advanced-stage ethnic minority (African American; Hispanic) adults aged 36-69 years completed a performance-based functional capacity measure (MMT-R), a self-report functional capacity questionnaire (Activities of Daily Living [ADL] Scale), and a comprehensive neuropsychological battery. Spearman's correlations determined associations between ADL scores and MMT-R performance. Linear regression examined neuropsychological predictors of MMT-R covarying for education.

Results: Seventy-two percent of participants exhibited impairment on the MMT-R (cutoff: $\geq 5$ ), whereas $32 \%$ reported ADL impairment (cutoff: $\geq 2$ ). The correlation between MMT-R and ADL scores was non-significant $(r=-.19, p>.05)$. Neuropsychological performance significantly predicted MMT-R scores $(\mathrm{F}=4.9 ; \mathrm{p}<.001)$, with memory retrieval $(p<.05 ; \beta=.38)$ and abstraction $(p<.10 ; \beta=.30)$ domains driving this effect.

Conclusions: MMT-R performance was strongly related to cognitive measures, whereas little correlation was observed with self-reported ADL. Results are similar to previous studies examining MMT-R utilization in HAND diagnosis among less-advanced HIV+ patients. Findings highlight the contribution of cognitive abilities to MMT-R performance, and raise the question of whether the MMT-R is more closely aligned with cognitive than functional capacity.

Correspondence: Laurie Baker, M.A., University of Missouri- St.Louis, 300 N.4th Street, \#504, Saint Louis, MO 63102.E-mail:lauriebaker@ umsl.edu

A.R. AMBROZIAK \& S.E. KARPIAK. Does Depression Prevalence Contribute to Neurocognitive Disorder Overdiagnosis in HIV?

Objective: Due to effective HIV treatment more older adults are living with HIV. HIV associated neurocognitive disorder (HAND) is becoming an issue. Importantly, depression is one of the exclusion criteria for 
diagnosing HAND. This may be confounding the incidence of HAND since depression prevalence is often greater than $50 \%$. This study assesses the predictive value of demographic and illness characteristics on the prevalence of depression in an HIV-infected older adult population. Participants and Methods: Research on Older Adults with HIV (ROAH), conducted by ACRIA, collected health data on a sample of $\sim 1000$ New York City-based residents aged 50 and older. We analyzed a subsample of $710 \mathrm{ROAH}$ participants who completed the depression questionnaire CES-D (Center for Epidemiological Studies Depression Scale). The sample mean age was 55.36 with a range of 50-78.

Results: $76.06 \%$ of the sample showed significant depressive symptoms $(\mathrm{CES}-\mathrm{D} \geq 16)$. Depression decreased with age $(p=0.0044)$. Multivariate modeling showed that age, time since HIV diagnosis and employment status explained the prevalence of depression with high probability $(p=0.0010)$. Gender, race/ethnicity, and AIDS diagnosis were not related to depression symptom prevalence.

Conclusions: This data underscores the high prevalence of depression among older HIV-positive adults and suggests that HAND may be over-reported if individuals are not screened for depression. When diagnosing HAND, attention should be given to unemployed individuals closer to 50 years of age and with a long time since HIV-diagnosis. That group is at increased risk for being depressed.

Correspondence: , . E-mail:

P.L. FAZELI, M.J. MARQUINE, C.A. DUFOUR, B.L. HENRY, J. MONTOYA, B. GOUAUX, R.C. MOORE, S. LETENDRE, S. WOODS, I. GRANT, D.V. JESTE \& D.J. MOORE. Moderate Physical Activity is Associated with Better Neurocognitive and Everyday Functioning in Older Adults with HIV Disease.

Objective: The current study examined the association between physical activity (PA), neurocognitive impairment (NCI), and instrumental activities of daily living (IADLs) among older HIV+ persons.

Participants and Methods: One hundred older ( $\geq 50$ years) HIV+ adults completed the International Physical Activity Questionnaire (IPAQ) to assess frequency, duration, and intensity of PA over the previous week. Global and domain-specific NCI was determined with a comprehensive battery. Participants were classified as IADL Dependent or Independent using a modified Lawton and Brody ADL scale.

Results: Higher levels of moderate PA were associated with lower odds of global NCI ( $p=0.01)$, even when relevant covariates were modeled. Examination of domain-specific NCI revealed the association between moderate PA and global NCI was driven by executive function $(p=0.04)$. Higher levels of moderate PA were also associated with lower odds of IADL Dependence ( $p=0.03$ ), although this relationship fell to the trend level $(p=0.08)$ when including relevant covariates. Lastly, a follow-up analysis showed that those with both NCI and IADL Dependence had lower moderate PA than those with neither condition $(p=0.03)$.

Conclusions: These cross-sectional findings suggest PA is associated with better neurocognitive and everyday functioning among older HIV+ adults. Previous studies have shown that PA can promote neurogenesis and reduce NCI risk factors, such as metabolic and cardiovascular comorbidities among older HIV-uninfected adults. Longitudinal studies with older HIV+ persons utilizing objective PA methods are needed to more precisely evaluate: 1) the directionality of the association between PA, NCI, and everyday functioning, and 2) the underlying mechanisms of these associations.

Correspondence: Pariya L. Fazeli, PhD, Psychiatry, University of California San Diego \& HIV Neurobehavioral Research Center, 3720 8th Avenue, San Diego, CA 92103.E-mail:pfazeli@ucsd.edu

D.P. SHEPPARD, J.E. IUDICELLO, K.L. DOYLE, E.E. MORGAN, P. MASSMAN, P. GILBERT, M.W. BONDI \& S. WOODS. Gender Differences in the Risk of Mild Cognitive Impairment in HIV Disease.

Objective: With the increasing prevalence of adults in their 50's and 60 's living with HIV disease, concerns have emerged about possible increases in the risk of non-HIV-associated dementias. The current study examined the prevalence of MCI in older HIV+ adults, since MCI is an intermediate state between typical cognitive aging and dementia that is commonly observed in this age range.

Participants and Methods: Participants included $104 \mathrm{HIV}+$ adults aged 50 years and older and $85 \mathrm{HIV}$ - subjects who were demographically comparable, with the exception of a slightly higher prevalence of men in the HIV + group. Subjects with functional dependence, dementia, or severe psychiatric and neurological conditions were excluded. Participants completed a research neuropsychological evaluation, which was used to classify MCI according to the Jak et al. (2009) Comprehensive Criteria.

Results: A logistic regression showed an interaction between HIV serostatus and gender in predicting MCI diagnoses. Post-hoc analyses revealed significant HIV effects among the women, such that HIV+ women were 12 times more likely to have MCI than HIV - women $(95 \%$ confidence interval 1.3,112.4). No other significant pair-wise comparisons were observed (ps>.10). Among the HIV+ women, MCI was significantly associated with a shorter duration of HIV infection $(p<.01$, Hedges $g=2.12$ ).

Conclusions: Among older women, HIV infection is associated with a considerably elevated risk of MCI, which may increase their risk of developing non-HIV-associated dementias as this population ages. Future studies examining the course, neurobiological mechanisms, and functional implications of MCI in HIV+ women are warranted.

Correspondence: David P. Sheppard, San Diego State University, 2111 Holly Hall St. Apt \#3902, Houston, TX 92107. E-mail: dsheppard.uh@ gmail.com

C. WILLIAMS, N. DASHER \& P. VIK. Components of Executive Functioning and Visuospatial Memory in a Sample of HIV Positive Individuals with a History of Alcohol Abuse.

Objective: HIV and alcoholism have the potential to result in distinct neurocognitive complications, but their synergistic effects may be especially problematic. Studies have suggested that individuals with comorbid HIV infection and a history of alcohol abuse tend to have poorer episodic memory, verbal reasoning, and reaction time functioning than either individuals with a history of alcoholism or HIV infection, respectively (Fama et al., 2009; Green, Saveanu, \& Bornstein, 2004). This study sought to replicate as well as extend those findings to a relatively novel test of visuospatial and procedural memory, the Northwest Trails Test (formerly Idaho Trail Learning Test; NTT; Vik et al., 2013).

Participants and Methods: Twenty-four individuals (19 male; M age $=44.8(\mathrm{SD}=9.1))$ received a neuropsychological battery as part of their comprehensive HIV care. The test battery consisted of a selection of subtests from the Delis-Kaplan Executive Functioning System (D-KEFS) and the Wechsler Adult Intelligence Scale (WAIS-IV), as well as the Ruff $2 \& 7$ Selective Attention Test, which were then separated into Processing Speed, Flexibility, and Attentional composites. The NTT was treated as a separate test.

Results: After controlling for nadir CD4 count, a history of binge drinking significantly predicted poorer Processing Speed $(\mathrm{t}=-2.762, \mathrm{p}=.012)$ and Attention ( $\mathrm{t}=-2.517, \mathrm{p}=.020)$ composite scores, but not Flexibility Composite scores ( $\mathrm{t}=-1.753, \mathrm{p}=.09)$. Binge drinking and number of days of recent alcohol consumption significantly predicted NTT performance in several domains (e.g., free recall).

Conclusions: These results support previous findings despite limitations in how heavy alcohol abuse history was assessed. Implications for NTT are also discussed.

Correspondence: Catherine Williams, PhD, Psychology, Idaho State University, 2156 Marigold Street, Apt. 4, Pocatello, ID 83201. E-mail: willcath@isu.edu 
D.J. MOORE, P.L. FAZELI, K.B. CASALETTO, S. LETENDRE, C. SCOTT, S. WOODS, D.V.JESTE \& I. GRANT. Neuropsychological functioning is associated with multitasking in older HIV+ adults. Objective: The number of older adults with HIV disease $(\mathrm{HIV}+)$ is rising, and these older HIV+ adults are at increased risk for neurocognitive and everyday functioning deficits. Multitasking is an important aspect of everyday functioning that has not been examined in older HIV+ persons. Participants and Methods: One hundred older ( $\geq 50$ years) HIV+ adults completed a comprehensive, seven-domain, neuropsychological (NP) battery. The multitasking test (MT) is a performance-based measure that asks participants to complete, concurrently, several everyday tasks (i.e., medication management, financial management, cooking, telephone communication) (Scott et al., 2011). Participants are asked to prioritize the financial management and cooking portions of the task, and also to complete as many of the tasks as possible within a given time limit.

Results: Better NP test performance (global T) was associated with better overall MT performance ( $\mathrm{rho}=0.34 ; \mathrm{p}<0.001)$, whereas HIV disease severity and depressive symptoms were not (ps>.10). Average NP T-scores in the domains of verbal fluency, executive functions, speed of information processing, learning, working memory, and motor skills (but not delayed recall) were significantly correlated with total MT scores (all p's $<0.05$ ). Interestingly, persons who were currently employed scored better on the financial management portion of the MT (Cohen $\mathrm{d}=0.45 ; \mathrm{p}=0.04)$.

Conclusions: Consistent with findings among younger HIV+ persons, neuropsychological performance is strongly associated with a laboratory-based test of the ability to multitask activities of daily living. The association does not seem to be domain specific; that is, performance on the MT was associated with almost all neuropsychological domains (delayed recall being the exception). The association of MT performance with current employment status supports the ecological validity and broader utility of the multitasking test for older HIV+ persons.

Correspondence: David J. Moore, Ph.D., Psychiatry, University of California, San Diego, 220 Dickinson St., Suite B, Mail Code 8231, San Diego, CA 92103.E-mail: djmoore@ucsd.edu

M. KEUTMANN, R. GONZALEZ, J. VASSILEVA, P. MAKI, C. FRANCO, S. CHEN, L. LADD \& E. MARTIN. Sex Differences in Visuospatial Memory Impairment among HIV+ Drug Users.

Objective: To compare the effects of sex and HIV serostatus on visuospatial episodic memory performance by substance dependent individuals (SDIs). Previous studies of HIV-Associated Neurocognitive Disorder (HAND) have focused primarily on verbal memory and have not compared performance of HIV+ men and women directly, despite recent speculation that $\mathrm{HIV}+$ women might be more vulnerable to memory deficits.

Participants and Methods: A group of $120 \mathrm{HIV}+$ and 157 EIA-verified HIV - SDIs, 91\% African American and 44\% female, completed the Brief Visuospatial Memory Test as part of a larger study of neurocognitive effects of HIV and drugs of abuse. Groups were well-matched on demographic, substance use and comorbid characteristics and verified abstinent at testing.

Results: The HIV+ groups scored significantly more poorly compared with HIV - controls on BVMT immediate recall testing, $\mathrm{p}<.05$; on delayed recall, the HIV+ women scored significantly more poorly compared with the other three groups, $\mathrm{p}<.05$. Additionally, delayed recall was significantly poorer among HIV+ women with a history of cocaine dependence.

Conclusions: Visuospatial episodic memory deficits were evident among HIV + compared with HIV-SDIs but impairment was more prominent among $\mathrm{HIV}+$ women. These findings support the hypothesis that HIV+ women are more vulnerable to episodic memory deficits, and the impairment is not limited to verbal memory. A history of cocaine dependence was associated with greater memory impairment among the $\mathrm{HIV}+$ women; these data are consistent with recent neurocognitive and
fMRI findings from the Women's Interagency HIV Study. Supported by HHS R01 DA12828 to E.M. Martin

Correspondence: Eileen Martin, PhD, Psychiatry, Rush University Medical Center, 1645 W. Jackson Blvd. Suite 600, Chicago, IL 60612. E-mail: eileen_martin@rush.edu

K. VAN DYK, S.A. GOLUB, J.B. PORTER, K.J. ROBIN, W.J. KOWALCZYK, J.C. TOMASSILLI, J.J. LY, C. VILACASTELAR \& N.S. FOLDI. Covert Orienting in HIV: the Effects of Self-Reported Physical Health and Aging.

Objective: Covert orienting (Posner, 1980) is among the attentional abilities affected by HIV, where valid cues fail to facilitate subsequent speed of target location in symptomatic patients (Martin et al., 1992). The effects of aging and physical health status in this population could impact attention, but effects on covert orienting are unknown. We hypothesized that older age and worse physical health in persons with HIV would compromise the facilitative effect of orienting cues.

Participants and Methods: Forty-two non-demented HIV+ individuals participated. A $2 \times 2 \times 3$ design was used, with independent variables of age (young $(<50)$; old $(50+)$ ), self-reported physical health status (better and worse as determined by a median split of Physical Health Summary Scores on the Medical Outcomes Study HIV Health Survey) and cue type (valid = cue same location as target; invalid $=$ opposite location; neutral $=$ cues in both locations). Reaction time (RT) was recorded.

Results: There were no main effects of age or self-reported physical health status. A significant cue $x$ physical health interaction $(p<.05)$ indicated that those with worse self-reported health were slower following invalid cues, compared to those with better self-reported health. Other interactions were non-significant.

Conclusions: We found that self-reported physical health status in HIV impacted attention: those with worse self-reported health had greater difficulty re-orienting after invalid cues. Contrary to expectation, older age did not affect orienting either alone or interacting with health status. We suggest that poor self-reported physical health - regardless of age - should be considered as an important indicator of attentional decline in HIV.

Correspondence: Kathleen Van Dyk, PhD, Psychiatry, UCLA Semel Institute for Neuroscience and Human Behavior, 760 Westwood Plaza, 37-360B,Westwood, CA 90095.E-mail: kathleen.van.dyk@gmail.com

R. KAMAT, K.L. DOYLE, J.E. IUDICELLO, E.E. MORGAN, S. MORRIS, D. SMITH, S. LITTLE, I. GRANT, S. LETENDRE \& S. WOODS. Neurobehavioral changes in acute and early HIV infection.

Objective: Acute and early HIV infection (AEH) is accompanied by neuroinflammatory processes, as well as impairment in neurocognitive and everyday functions. However, little is known about the prevalence and correlates of neurobehavioral disturbance (e.g., apathy, disinhibition) during this period.

Participants and Methods: Thirty-four AEH (median duration of infection=2.05 months) and $39 \mathrm{HIV}$-seronegative participants completed neuromedical and neuropsychological assessments, a structured psychiatric interview, and the apathy, disinhibition, and executive dysfunction subscales of the Frontal Systems Behavioral Scale (FrSBe).

Results: AEH status, but not lifetime substance use disorder or mood disorder, was a significant, independent predictor of current apathy and executive dysfunction, but not disinhibition. Retrospective ratings of pre-seroconversion levels of apathy, executive dysfunction, and disinhibition were higher in the AEH group relative to HIV - participants $(p ' s<.01)$. Following seroconversion, the AEH cohort noted increases in current apathy and executive dysfunction ( $p$ 's<.05), but not disinhibition. In the AEH cohort, higher current total FrSBe score was significantly associated with lower nadir CD4 level $(\rho=.42, p=.01)$, slowed information processing speed $(\rho=-.37, p=.031)$, and greater everyday functioning problems $(\rho=.72, p<.001)$. 
Conclusions: Individuals who have recently acquired HIV may exhibit higher levels of neurobehavioral disturbance prior to seroconversion. Premorbid levels of apathy and executive dysfunction, but not disinhibition, are exacerbated during AEH, particularly in association with lower immune function. Of clinical relevance, global neurobehavioral disturbances were moderately associated with neurocognitive slowing and declines in everyday functioning and employment. These data may have implications for early initiation of antiretroviral therapies to reduce the risk of neurobehavioral complications in HIV.

Correspondence: Rujvi Kamat, Ph.D., UCSD, 220 Dickinson St, San Diego,CA 92103.E-mail: rkamat@ucsd.edu

K.B. CasaletTo, P.L. Fazeli, S. WOODS \& D.J. MOORE. Metacognition Partially Mediates the Relationship between Neurocognitive and Everyday Functioning among Older HIV+ Adults.

Objective: Although it is well established that neurocognitive impairment is associated with poorer everyday functioning outcomes (e.g., medication management), factors that mediate this relationship are not well understood. Poor metacognition (awareness of neurocognitive abilities) is associated with greater errors in everyday life and may represent one such factor. Our study assessed the role of metacognition as a mediator between neurocognitive impairment and everyday functioning among older adults with HIV disease.

Participants and Methods: 100 older ( $\geq 50$ years) HIV+ participants completed a performance-based task, which assessed four daily living skills (e.g., medication and financial management), alongside a comprehensive neuropsychological battery. Participants were also queried regarding their global metacognition of their daily task performance, which included 'metacognitive knowledge' (self- and task-knowledge) and 'online awareness' (task appraisal, self-monitoring, and self-evaluation).

Results: Using bootstrapping methods, global metacognition emerged as a partial mediator between neurocognition and everyday task performances (95\%CI: 0.01, 0.26). Specifically, metacognitive knowledge (95\%CI: 0.02, 0.22), but not online awareness (95\%CI: -0.02, 0.12) partially mediated the relationship between neurocognition and everyday functioning. Within metacognitive knowledge, self-knowledge (95\%CI: $0.12,0.17)$ appeared to be driving the mediation more than task-knowledge (95\%CI: $-0.01,0.11)$.

Conclusions: Among older HIV+ participants, insight into one's functioning is one mechanism by which neurocognitive impairment results in poorer daily functioning. In particular, self-knowledge, which included ratings of self-efficacy and self-predictions, emerged as an important pathway by which neurocognition influences successful everyday functioning. As such, developing targeted interventions to enhance metacognition among HIV+ older adults may help to remediate everyday functioning impairment in this vulnerable population.

Correspondence: Kaitlin B. Casaletto, MS, Psychiatry, SDSU/UCSD Joint Doctoral Program, 220 Dickinson St., Suite B, San Diego, CA 92103.E-mail:kaitlin.casaletto@gmail.com

P. SAYEGH, K.I. WILSON, D. SCHONFELD, N.S. THALER, A. ARENTOFT, Z. MAHMOOD, E.J. SINGER \& A.D. THAMES. The Interactive Effects of Body Mass Index and Depression on Neurocognitive Functioning Among HIV+ Adults.

Objective: Increased body mass index (BMI) has been associated with cognitive impairment and depressive symptoms. While depression is known to be common among HIV+ individuals, obesity is also becoming increasingly prevalent (Crum-Cianflone et al., 2010). We examined the concomitant effects of BMI and depression on neurocognition in HIV+ adults.

Participants and Methods: Participants included $68 \mathrm{HIV}+$ African-American and Caucasian adults (means: age [years] $=49.34$, $\mathrm{SD}=10.90 ; \mathrm{BMI}=26.11 \mathrm{~kg} / \mathrm{m} 2, \mathrm{SD}=4.81)$. We used linear regression to determine the interactive effects of BMI and depression on performance across neurocognitive domains.

Results: BMI was significantly correlated with depressive symptoms. $r(68)=.30, p=.01$ and negatively correlated with psychomotor processing speed, $r(68)=-.23, p=.05$. Depression was negatively correlated with psychomotor processing speed, $r(68)=-.26, p<.04$. Our overall model was significant, $\mathrm{F}(3,64)=3.88, \mathrm{p}=.01, \mathrm{R} 2=.15$, for psychomotor processing speed. There was a statistically significant BMI $\mathrm{x}$ depression interaction $(\beta=-.27, p=.04)$, with higher BMI and depression symptom levels predicting poorer psychomotor processing speed.

Conclusions: Higher BMI and depression symptom levels, which are becoming more common in HIV, resulted in poor performance on psychomotor processing speed. This result is consistent with neuroanatomical findings that have linked obesity with white matter disease and decreased grey matter volume in the right cerebellum, which are important for psychomotor function and processing speed. The combination of high BMI and depression appears to have a greater negative effect on psychomotor processing speed. Intervention efforts should simultaneously target depression and obesity among HIV+ individuals. Future research should strive to identify the mechanisms underlying these findings.

Correspondence: Philip Sayegh, PhD, MPH, Semel Institute for Neuroscience \& Human Behavior and Resnick Neuropsychiatric Hospital, University of California, Los Angeles, 760 Westwood Plaza, Los Angeles, CA 90095. E-mail: psayegh@gmail.com

\section{C.D. HINKLE, E. CHIN \& A. HEFFELFINGER. Neuropsychological Profile of Anti-NMDA Receptor Encephalitis.}

Objective: Anti-N-methyl-d-aspartate (NMDA) receptor encephalitis is a recently identified, life-threatening autoimmune disorder resulting in a neuropsychiatric syndrome characterized by seizures, dyskinesias, and cognitive impairment. The extent and specific tasks associated with cognitive dysfunction in anti-NMDA receptor encephalitis have not been fully investigated.

Participants and Methods: Neuropsychological assessments were conducted with two female patients (age 16 and 17) with confirmed anti-NMDA receptor encephalitis. Prior to disease onset, the patients were active, healthy teenagers and above average students. They were initially evaluated as inpatients during the acute phase of disease also received follow-up outpatient neuropsychological evaluations in the subacute phase 4-5 months following discharge.

Results: Both patients initially demonstrated confusion, irritability, agitation, perseverative speech, slowed interactions, and worsening expressive language. One patient also experienced a single seizure and right-hand motor complaints. During the initial evaluation, she was disoriented and exhibited flat affect, echolalic and tangential speech, and distractibility. The other patient exhibited paranoia and periods of elation. During her initial evaluation, cognitive status fluctuated. Significant improvements were observed at neuropsychological reassessment. While both patients exhibited average intellectual functioning and intact performance on visuomotor integration and sequencing tasks, both continued to exhibit impairment on tasks of working memory, inhibition, verbal memory, and confrontational naming.

Conclusions: Results suggest that cognitive deficits constitute a longterm morbidity of anti-NMDA receptor encephalitis. While the extant literature identifies executive functioning as an area of protracted impairment, our findings suggest that problems with memory and language may persist. The importance of early identification and treatment and repeat neuropsychological assessment will be discussed.

Correspondence: Clayton D. Hinkle, Ph.D., Neurology, Medical College of Wisconsin, MCW Department of Neuropsychology, $9200 \mathrm{~W}$. Wisconsin Ave., Milwaukee,WT 53226.E-mail: cyhinkle@mcw.edu 
M. ARCE RENTERIA, D.A. BYRD, C. MIRANDA, A. FUENTES \& M. RIVERA-MINDT. Reaction Time Variability in HIV+ Adults with and without Current Substance Use Disorders.

Objective: HIV and substance use disorders (SUD) independently affect frontostriatal circuits associated with neurocognitive (NC) and immunological dysfunction. Although findings of comorbid effects are equivocal, intra-individual variability (IIV) in NC performance is a promising area of investigation. IIV is associated with subtle changes in frontostriatal functioning and linked with $\mathrm{NC}$ and immunological dysfunction in HIV (e.g., lower CD4 count and higher HIV viral load). However, IIV is not well understood in HIV+ adults with and without current SUDs. It was hypothesized that greater IIV would be associated with SUD-status and $\mathrm{NC}$ and immunological dysfunction.

Participants and Methods: Fifty-five HIV+ adults (67\%male; mean age $=49$ yrs $[\mathrm{SD}=7.4]$; mean education $=13 \mathrm{yrs}[\mathrm{SD}=3]$; SUD $+\mathrm{n}=22$ ) completed comprehensive neuromedical (CD4 count, viral load) and NC evaluations; diagnostic interview; and the computerized Vigil Continuous Performance Test (VCPT). IIV was operationalized as reaction time variability across 4 VCPT trials (auditory, visual). SUDs were based on DSM-IV diagnoses.

Results: There were no differences in IIV between the SUD+ and SUDgroup and IIV was not associated with measures of immunological functioning ( $p$ 's $>10)$. However, IIV was negatively associated with processing speed $(r=-.61)$ in the SUD+ group, and verbal fluency $(r=-.35)$ in the SUD- group (p's<.05).

Conclusions: In this sample of HIV+ adults, current SUDs did not increase risk of greater IIV. However, greater IIV might place HIV+/ SUD+ adults at greater risk for processing speed dysfunction. In contrast to prior research, greater IIV was not related to worse immunological functioning. Unsupported findings could be related to methodological limitations related to the VCPT (i.e., limited trials, sensory modalities). Correspondence: Miguel Arce Renteria, B.A., Fordham University, 685 Academy St, Apt 27, New York, NY 10034.E-mail: marce3@fordham. edu

T. SEIDER, A. GONGVATANA, S. CORREIA, K. TASHIMA, B. NAVIA \& R.A. COHEN. Clinical Factors Affecting Cerebral White Matter Damage in HIV.

Objective: Human Immunodeficiency Virus-1 (HIV) infection is a risk for cerebral white matter damage, which can be quantified by measuring white matter hyperintensities (WMH) on magnetic resonance imaging (MRI). Fractional anisotropy (FA) measures white matter structural integrity. This study investigates the influence of $\mathrm{HIV}$-associated clinical factors on these two measures of white matter and how these measures relate to each other in the context of HIV.

Participants and Methods: 97 participants with HIV received MRIs and 84 had usable DTI data. WMH were quantified using a semi-automated process and FA was calculated using tract-based spatial statistics (TBSS). Pearson correlations compared WMH and FA. Forward selection stepwise regression predicted WMH and FA from Hepatitis $\mathrm{C}$ virus (HCV) coinfection, duration of HIV infection, current CD4 cell count, detectable/undetectable HIV RNA. history of AIDS, and combination antiretroviral therapy (cART) use.

Results: Greater WMH highly correlated with lower FA for 17 of the 18 white matter tracts. Greater WMH was significantly associated with HCV coinfection. Predictors of lower FA were HCV coinfection and lack of cART use.

Conclusions: HCV coinfection strongly predicts white matter damage in people with HIV. Medication use is associated with subtle white matter change, but not with outright damage. FA and WMH, while highly correlated, reflect different aspects of neural white matter and are both important to consider when studying the neurological effects of HIV. Use of HIV medications and treatment of comorbid conditions may protect from white matter changes.

Correspondence: Talia Seider, University of California, San Francisco, 2004 Mowry Rd., Gainesville, FL 32610.E-mail: tseider@phhp.ufl.edu
J. IUDiCELlo, E.E. MORgan, R. KAMAT, L.R. CLARK, M.W. BONDI \& S. WOODS. Does HIV Disease Accelerate Neurocognitive Aging?

Objective: Recent controversies have emerged regarding whether HIV "accelerates" the typical aging process in several organ systems, including brain structure and function. This study sought to examine the accelerated neurocognitive aging hypothesis by determining whether an average 55 vear-old with HIV disease demonstrates neurocognitive functioning similar to that of an average healthy seronegative adult in their 70s.

Participants and Methods: Participants included 40 older adults with HIV disease $(\mathrm{O}+$, mean age $=54.9)$ and 48 age-matched HIV seronegative comparison subjects $(\mathrm{O}-$, mean age $=55.9)$ between the ages of 50 and 65 years. A group of 40 healthy "older old" (i.e., age 65 years or older) HIV seronegative adults were also examined (OO-, mean age $=73.6)$. The study groups were comparable for gender, ethnicity, and education. Participants completed a brief battery of neuropsychological tests assessing auditory attention. information processing speed, verbal learning and recall, executive functions, and language. Group differences were explored using ANOVA, effect sizes, and equivalence testing. Results: An "accelerated aging" profile was observed on digit span and word list learning, whereby the $\mathrm{O}+$ and $\mathrm{OO}$ - groups performed significantly worse than the $\mathrm{O}$ - group $(\mathrm{ps}<0.05)$, but did not differ from one another ( $p s>0.10$ and confirmed by equivalence tests). Simple aging effects were observed on measures of information processing speed and executive functions, but no other significant effects were noted.

Conclusions: Older adults with HIV disease may experience a notable acceleration of neurocognitive aging. specifically within the domains of auditory attention and verbal learning, but not in other higher-order functions. Future research elucidating the underlying neurobiological mechanisms of this phenomenon (e.g.., telomere shortening, immunosenescence) is needed to better understand the interplay between HIV disease and aging on neurocognitive declines.

Correspondence: Jennifer Iudicello, Ph.D., Psychiatry, HIV Neurobehavioral Research Center; SDSU/UCSD, 220 Dickinson Street, Suite B, San Diego, CA 92103.E-mail: jüudicello@ucsd.edu

M.J. MARQUINE, D.J. MOORE, M. CHERNER, P.L. FAZELI, R. DEUTSCH, D. FRANKLIN, R. ELLIS, S. LETENDRE, S. MORGELLO, A. COLLIER, C. MARRA, D. CLIFFORD, B. GELMAN, J. MCARTHUR, D. SIMPSON, J.A. MCCUTCHAN, I. GRANT \& R.K. HEATON. Neurocognitive Decline in HIVinfected Hispanics: Rates and Predictors.

Objective: Hispanics are disproportionally affected by HIV and at increased risk for $\mathrm{HIV}$-associated neurocognitive (NC) impairment; yet, little is known about ethnic/racial differences in longitudinal NC decline among $\mathrm{HIV}$-infected persons. We compared rates and predictors of $\mathrm{NC}$ decline between HIV-infected adults who self-identified as either Hispanic or non-Hispanic White.

Participants and Methods: Participants were English-speaking. HIV-infected, adults assessed at six U.S. medical centers (57 Hispanics, 185 non-Hispanic Whites). Baseline characteristics for the combined sample were $(M[S D]$ or $\%)$ : Age $=43.9(8.8)$, Education=13.3(2.5). Male $=88 \%$, and AIDS=60\%. Participants completed comprehensive NC assessments semiannually (mean follow-up=34.6 months). Published, regression-based norms for $\mathrm{NC}$ change were used to generate overall change status (decline/no decline).

Results: Hispanics $(40 \%)$ were twice as likely to decline as were non-Hispanic Whites $(19 \% ; p<.01)$. These differences persisted $(O R=3.4, C I=1.5-7.8)$ when adjusting for covariates that differed across groups (age, education, current and nadir CD4, AIDS, antiretroviral therapy, detectable plasma RNA, lifetime major depression, non-HIV comorbidities, baseline NC impairment). Findings were also similar when the non-Hispanic White comparison group was restricted to participants who were demographically-matched to Hispanics. Within Hispanics, those of Puerto Rican origin/descent had higher rates of decline 
$(n=16 ; 63 \%)$ than those of Mexican origin/descent $(n=22 ; 27 \% ; p<.05)$. Separate multivariable models by ethnic group, showed that non-HIV comorbidity was the strongest predictor of $\mathrm{NC}$ decline for Hispanics $(p<.01)$, and current major depression for non-Hispanic Whites $(p=.04)$. Conclusions: HIV-infected Hispanics are at increased risk for $\mathrm{NC}$ decline compared to non-Hispanic Whites, and predictors of decline vary by ethnic group. Future studies should explore culturally-relevant risk factors for $\mathrm{NC}$ decline to help inform the development of targeted interventions.

Correspondence: Maria J. Marquine, PhD, University of California San Diego, 220 Dickinson Street, Suite B (MC 8231), San Diego, CA 92103. E-mail:mmarquine@ucsd.edu

R. PAUL, J. JOSKA, C. WOODS, S. SEEDAT, S. ENGELBRECHT, J. HOARE, J.M. HEAPS, V. VALCOUR, B. ANCES, L. BAKER, L. SALMINEN \& D. STEIN. Impact of the HIV Tat C30C31S dicysteine substitution on neuropsychological function in patients with clade $\mathrm{C}$ disease.

Objective: Previous animal studies have identified a C31S residue substitution in the C30C31 dicysteine motif of the Tat protein that is associated with reduced neurovirulence in clade C HIV. However, clinical studies of patients infected with clade C HIV have reported significant levels of cognitive impairment. To date no study has specifically examined cognitive function in clade C-infected patients as a function of the presence or absence of the Tat C31 substitution.

Participants and Methods: The present study investigated the impact of the Tat C30C31S genetic substitution among individuals residing in South Africa infected with clade C HIV that either exhibited the C30C31 motif ( $\mathrm{n}=128$ ) or the C31S motif $(\mathrm{n}=46)$. A control group of seronegative individuals were included to examine the overall impact of HIV on cognitive performance. All individuals completed a comprehensive neuropsychological battery consisting of tests sensitive to HIV

Results: Results revealed that clade C-infected individuals performed significantly worse across cognitive tests compared to seronegative controls. However, there were no significant differences in cognitive performances between individuals with the C.31S motif versus those without the C31S substitution. Proximal CD4 cell count and plasma viral load were unrelated to cognitive performances for either group.

Conclusions: These results confirm that the C31S dicysteine motif substitution of the Tat protein does not appreciably moderate neuropsychological outcomes in clade C. Further, these findings highlight the importance of clinical management of cognitive symptoms among individuals infected with this viral clade worldwide.

Correspondence: Robert Paul, PhD, Psychology, University of Missouri, St. Louis, 16735 Deveronne Cir, Chesterfield, MO 63005. E-mail: paulro@umsl.edu

D. EAGAN, S. KAUR, A.T. DEMARCO, W. HERTZING \& A. HALEY. Herpes Simplex-1 Infection is Associated with Reduced Right Hippocampal Volume Among Middle Aged Individuals with Genetic Risk for Alzheimer's Disease.

Objective: ApoE4 is a genetic risk factor for Alzheimer's disease (AD). Herpes Simplex-1 (HSV-1) is a common viral infection with higher incidence among ApoE 4 carriers, and is also linked to cognitive deficits. The current study sought to determine if hippocampal volumes differed among ApoE4 carriers and non-carriers who were also either positive or negative for HSV-1.

Participants and Methods: 111 individuals aged 40-60 were grouped according to risk for cognitive decline based on ApoE4 and HSV-1 status. Participants completed a general health assessment, including assay of HSV-1 seropositivity, and underwent structural neuroimaging. Hippocampal volumes were measured using Freesurfer software. Group differences were assessed with $2 \times 2$ ANOVA. Age, level of education, full scale IQ, systolic blood pressure, and total intracranial volume were included in the model as covariates.
Results: There was a significant main effect of HSV-1 on right hippocampal volume $\mathrm{F}(2,109)=1.50, \mathrm{p}=.01$, partial $\eta 2=.057$.

Conclusions: HSV-1 positive individuals had significantly smaller right hemisphere hippocampal volumes than HSV-1 negative individuals, regardless of ApoE4 status. Thus HSV-1 may be considered a risk factor for structural brain vulnerability in otherwise healthy middle-aged individuals. The identification of risk factors in mid-life allows time for intervention prior to the onset of cognitive changes. Further longitudinal research on the effects of HSV-1 suppression on brain health is warranted as a potential means of reducing risk for cognitive decline. Correspondence: Danielle Eagan, MA, Psychology, The University of Texas at Austin, 480 Oakdale Rd NE, Apt \#2, Atlanta, GA $3030 \%$. E-mail:deeagan@gmail.com

D. EAGAN, K. STEWARD, S. KAUR, W. HERTZING \& A. HALEY. Infection with Herpes Simplex-1 is Associated with Reductions in Executive Function and Full Scale IQ (FSIQ) Among Healthy Middle Aged Adults with Genetic Risk for Alzheimer's Disease.

Objective: ApoE4 is a genetic risk factor for Alzheimer's disease (AD). Herpes Simplex-1 (HSV-1) is a common viral infection, with higher incidence among ApoE 4 carriers, and is also linked to cognitive deficits. This study sought to determine if cognitive differences were present in healthy middle-aged ApoE4 carriers, who were either positive or negative for HSV -1 .

Participants and Methods: 115 individuals aged 40-60 were grouped according to risk for cognitive decline based on ApoE4 and HSV-1 status. Participants completed a health assessment and a neuropsychological battery assessing FSIQ, executive functioning, and verbal memory. Age, level of education, and systolic blood pressure were included in the model as covariates. Group differences were assessed with 2x2 ANOVA. Results: There was a significant interaction between ApoE 4 and HSV-1 on Executive Functioning domain score $(p=.01)$, as well as a significant main effects of HSV-1 ( $p=.015)$ and ApoE $4(p=.02)$ on FSIQ. No group differences were observed for verbal memory $(p=0.10)$.

Conclusions: HSV-1+ ApoE4 carriers performed significantly lower on tests of executive functioning than HSV-1- ApoE4 carriers. HSV-1 infection also correlated with significantly lower FSIQ, whereas ApoE4+ status was linked to higher FSIQ. Thus HSV-1 may be an additive risk factor for future cognitive vulnerability in individuals with genetic risk for AD. Further longitudinal research on the effects of HSV-1 suppression on cognition is warranted as a potential means of reducing risk for cognitive decline.

Correspondence: Danielle Eagan, MA, Psychology, The University of Texas at Austin, 480 Oakdale Rd NE, Apt \#2, Atlanta, GA 30307. E-mail:deeagan@gmail.com

M. SAKAMOTO, R. DEUTSCH, J. SEVERSON, J. COSMAN, T. HENDRIX, M. MERICKEL, I. GRANT \& T. MARCOTTE. Development of a brief iPad-based screening tool for detection of HIV-related neuropsychological impairment.

Objective: With the introduction of combination antiretroviral therapy, the severity of HIV-associated neurocognitive disorders (HAND) has decreased; however, mild forms of HAND remain prevalent. Existing screening batteries (e.g., HIV Dementia Scale, International HIV Dementia Scale) are insensitive to mild neuropsychological (NP) impairment. The current study aimed to develop a pilot iPad-based NP screening tool to quickly and accurately identify individuals with HAND. Participants and Methods: Twenty-two HIV-infected participants completed a seven-domain, "gold standard" NP battery and iPad-based NP screening. The iPad battery included seven tests assessing cognitive abilities previously shown to be sensitive to HAND in the cART era. All instructions were given both visually and auditorily. Global deficit scores were calculated based on the gold standard NP tests; nine participants (41\%) were considered NP impaired.

Results: Using stepwise logistic regression, four tests in three domains - working memory, processing speed, and executive function - were 
identified as predictors of NP impairment. Using the four tests in a discriminant analysis yielded $77 \%$ classification accuracy $(78 \%$ sensitivity and $77 \%$ specificity); however, small sample sizes prevented cross-validation.

Conclusions: This pilot study suggests that a brief iPad-based NP battery could be sensitive to detect HAND. It is important to validate this within larger cohorts and to examine its clinical utility/feasibility in HIV clinics. Given that the tool requires minimal staff involvement in administration, and that data could be automatically scored, this iPad-based screening battery could be beneficial for busy HIV clinicians. Correspondence: Maiko Sakamoto, PhD, Medicine, Saga University, 5-1-1, Nabeshima, Saga 849-0935, Japan.E-mail: maimaisakamoto@ gmail.com

K.N. DEVLIN, A. GONGVATANA, S. CORREIA, S. ROSS, B. NAVIA, K. TASHIMA, S. DE LA MONTE \& R.A. COHEN. IP-10 Mediates HIV-Associated Neuropsychological Dysfunction.

Objective: Chronic inflammation has been proposed as a mechanism for the persistence of HIV-associated neurocognitive disorders (HAND) in the era of combination antiretroviral therapy (cART). Elevated pro-inflammatory cytokines have been linked with both HIV infection and neuropsychological (NP) dysfunction. However, no clinical studies have directly tested the mediating role of inflammatory cytokines in the relation between HIV and NP dysfunction. Using a structural equation path model, we investigated whether IP-10, a marker of chronic inflammation, mediates HAND.

Participants and Methods: $117 \mathrm{HIV}+(84 \%$ cART-treated, $71 \%$ virally suppressed, mean HIV duration 13.45 years) and $73 \mathrm{HIV}$ - adults aged 23-79 were administered a NP assessment including measures of processing speed and verbal learning/memory with high predictive validity for HAND. Plasma IP-10 was measured using multiplexed bead array immunoassay.

Results: A path model was estimated using maximum likelihood estimation in MPlus 7. IP-10 was regressed on HIV, and NP function was regressed on IP-10 and HIV. The indirect effect of HIV on NP function via IP-10 was also tested. The analysis converged to an admissible solution with good model fit $[\chi 2 \mathrm{M}(6)=5.36, \mathrm{p}=.50$; RMSEA $=.00$; $\mathrm{CFI}=1.00 ;$ SRMR $=.02]$. HIV was associated with elevated IP-10 (unstandardized path coefficient $=0.41, \mathrm{p}<.01$ ), and elevated IP-10 was associated with poorer NP function $(-0.31, p<.01)$. The indirect effect of HIV on NP function via IP-10 was significant $(-0.13, \mathrm{p}=.02)$, as was the direct effect of HIV on NP function $(-0.28, p=.02)$.

Conclusions: Present findings suggest that IP-10 is among the factors that mediate the effect of HIV on NP dysfunction. Thus, chronic inflammatory processes may partly explain the persistence of HAND in the cART era. The present findings have important clinical implications, such as the potential utility of IP-10 as a biomarker of HAND, and the potential utility of anti-inflammatory agents in HAND treatment. Funding sources: NIH R01 MH074368, P30 AI042853.

Correspondence: Kathryn N. Devlin, Sc.B., Department of Psychology, Temple University, Weiss Hall 1701 North 13th Street, Philadelphia, PA 19122. E-mail:kathryn.devlin@temple.edu

\section{Imaging (Functional)}

\section{A. KUlKarni, C. MARQUeZ De la Plata, D. QUAlls \& P. PLENGER. Frontal Lobe Inefficiency After TBI Detected Using fNIRS During Stroop Task.}

Objective: To evaluate neural changes within the frontal lobes of individuals with TBI using fNIRS while performing a Stroop task.

Participants and Methods: Fourteen healthy controls and 15 patients with moderate to severe TBI were included. fNIRS data were collected using a Hitachi ETG-4000 and a 52 channel optode array. Data were acquired using a block design during a Stroop task (i.e., Condition A = Dot Color Naming, Condition B = Incongruent task). Visual stimuli were presented on a monitor. Oxygenated hemoglobin $(\mathrm{HbO})$ was recorded every $1 / 10$ of a second. One sample t-tests using SPM were conducted to determine $\mathrm{HbO}$ differences within groups of patients and controls during the tasks. A repeated measures t-test was conducted to examine signal intensity differences between conditions for each group.

Results: Behaviorally, number of errors were comparable between patients and controls for both conditions. Spatially, there were no differences in $\mathrm{HbO}$ between conditions $\mathrm{A}$ and $\mathrm{B}$ among patients; whereas controls show $\mathrm{HbO}$ increases in bilateral frontal lobes that are unique to condition $\mathrm{B}(\mathrm{p}<0.05)$. Furthermore, patients demonstrated greater involvement of bilateral dorsolateral prefrontal cortex (PFC), right ventrolateral PFC, and left motor cortex than controls during the simpler condition A.

Conclusions: While the simple dot color naming condition of the Stroop task did not result in more errors among patients than controls, neural activity was greater among patients suggesting a greater cognitive demand to maintain accuracy. Furthermore, the spatial distribution of increased activity during this relatively simple condition among TBI patients involved several bilateral areas of frontal cortex. Also, patients' degree of neural activity during the simple condition was comparable to that of their activity during the complex condition, suggesting relatively simple tasks may be neurally demanding on frontal lobes after TBI. Results suggest that fNIRS can identify frontal lobe inefficiency in TBI that is commonly observed with fMRI.

Correspondence: Carlos Marquez de la Plata, PhD, Pate Rehabilitation, 2655 Villa Creek Dr., Suite 140, Dallas, TX 75234.E-mail: cmarquez@ paterehab.com

\section{Imaging (Structural)}

D. UKUEBERUWA \& P. ARNETT. Diffusion Tensor Imaging Links Regional Brain Integrity to Fatigue Severity in MS.

Objective: While fatigue is one of the most commonly reported symptoms among people with multiple sclerosis (MS), and it greatly impacts quality of life, few studies have directly investigated the relationship between neuropathology and fatigue. Recent findings indicate that damage or reduced functioning of subcortical structures, especially the basal ganglia and thalamus, and prefrontal cortex may be associated with experience of fatigue in neurological disorders. This study used diffusion tensor imaging (DTI) to assess for a relationship between global or regional white matter structure and self-reported fatigue in MS.

Participants and Methods: 45 participants (31 female) with MS completed the Fatigue Severity Scale (FSS), a self-report measure of fatigue symptoms over the past week, as well as an MRI scan during which DTI data were acquired. DTI was then processed using FSL, and brain volume was calculated using SPM8 to account for any atrophy effects. Multiple linear regression analyzed DTI fractional anisotropy (FA) measures of white matter integrity as predictors of FSS scores, while controlling for brain volume.

Results: Regional FA values were statistically significant predictors of FSS at $p<.05$. Specifically, decreased FA in the anterior thalamic radiation $\left(R^{\wedge} 2=.14\right)$ and cingulate gyrus white matter $\left(R^{\wedge} 2=.10\right)$ were related to increased FSS scores. However, brain volume and mean whole brain FA were not significant predictors of FSS.

Conclusions: Consistent with predictions, these findings indicate that higher levels of fatigue were reported by people with MS who had decreased white matter integrity of the anterior thalamic radiation and the cingulate gyrus, structures that connect subcortical thalamic and limbic regions to frontal cortex. This study contributes evidence for a network of white matter structures whose integrity, when compromised, is related to elevated fatigue in MS.

Correspondence: Dede Ukueberuwa, M.S., Penn State University, 372 Moore Building, University Park, PA 16802. E-mail: dedemu@psu.edu 


\section{Medical/Neurological Disorders/Other (Child)}

D. POTVIN, K.K. HARDY, M.T. ACOSTA \& K.S. WALSH. The Effects of ADHD on the Cognitive Profiles of Children with NF1. Objective: While NF1 research has documented a downward shift in global intellect compared to healthy peers, research has not examined the unique contribution from each of the indices or the cognitive impact of comorbid ADHD. We examined WISC-IV profiles in children with NF1, NF1+ADHD, and neurodevelopmental ADHD to characterize the profile of children with NF1 and understand the impact of comorbid ADHD.

Participants and Methods: A retrospective file review captured 72 children (40 NF1, 12 NF1+ADHD, 20 ADHD), with FSIQ > 70.

Results: Examining NF1 and ADHD populations independently, both groups showed discrepancies within their WISC-IV profiles, with specific weaknesses on WMI and PSI: NF1 $(\mathrm{F}=7.325, \mathrm{p}<.01, \eta p 2=.126)$ and ADHD $(\mathrm{F}=3.678, \mathrm{p}=.017, \eta \mathrm{p} 2=.162)$. Post-hoc analysis showed that NF1+ADHD group performed lower than the NF1 group across all indices, although the pattern did not reach significance $(\mathrm{F}=2.774, \mathrm{p}=$ .102 , пр $2=.053)$. Further analysis of performance of all NF1 participants within the PRI revealed that Block Design was significantly weaker than other subtests in the domain $(\mathrm{F}=13.581, \mathrm{p}<.01, \eta \mathrm{p} 2=.241)$.

Conclusions: When examining aspects of intellectual functioning, we demonstrate similar vulnerabilities for working memory and cognitive efficiency weaknesses in children with NF1 and developmental ADHD. The challenges in overall nonverbal functioning for children with NF1 appear to be driven by particular deficits in spatial awareness and integration of visual-motor processes. There is some evidence to suggest added cognitive morbidity with comorbid ADHD in children with NF1. Correspondence: Deborah Potvin, PhD, Neuropsychology, Children's National Medical Center, 15245 Shady Grove Road, Suite 350, Rockville,MD 20850.E-mail:dpotvin@cnmc.org

\section{Multiple Sclerosis/ALS/Demyelinating Disorders}

S.M. LOPES COSTA, D. THIBODEAUX, H.M. GENOVA, L. STROBER, N.D. CHIARAVALLOTI \& T. L ALVAREZ. Saccadic Eye Movements and Cognition in Multiple Sclerosis: a Case Study. Objective: Both cognitive and visual impairments are common in persons with Multiple Sclerosis (MS). However, the interaction between cognition and saccadic eye movements is poorly understood. This study explores the inter-relationship between saccadic eye movements' latency (initiation of the movement) and cognition in MS patients.

Participants and Methods: Saccadic eye movements were recorded from three healthy controls (HC) and one MS patient. Three saccadic conditions were tested (1) gap (2) no-gap and (3) overlap, which stimulates a cognitive load ranging from low to high (gap $<$ no gap $<$ and overlap). Eye movements were recorded with an ISCAN Eye Movement Monitor (infrared source and camera, Woburn, MA, USA). Data were analyzed with custom MATLAB software.

Results: Results demonstrated that saccadic latency was greater for the MS participant for all conditions [gap mean $=0.28$ (standard deviation $(\mathrm{SD})=0.09)$, no gap mean=0.32 $(\mathrm{SD}=0.10)$; and overlap mean $=0.38$ $(\mathrm{SD}=0.11)$ ], compared to the HC group [gap mean=0.22 ( $\mathrm{SD}=0.07)$, no gap mean=0.25 $(\mathrm{SD}=0.07)$; overlap mean=0.29 $(\mathrm{SD}=0.10)]$. Given the larger discrepancies in saccadic eye movements between the different conditions in the persons with MS as compared with the HCs, it appears that cognitive load had a greater impact on saccadic latencies for the MS patient.

Conclusions: Data indicate that the MS patient required more time to initiate saccadic eye movements in contrast to the HC group, and this difference increased as a function of cognitive load. Preliminary data are promising and further research is needed to determine whether saccadic eye movement latency can differentiate persons with MS and HC. Correspondence: Silvana M. Lopes Costa, PhD, kessler Foundation/ Rutgers, the state university of New Jersey, 300 executive drive, suite 70, West Orange, NJ 07052-3390. E-mail: scosta@kesslerfoundation.org.

P.M. RIVERA, J. BARTON, P. GARCIA \& A. HOMEIDAN. Affective Disorders and Cognitive Performance in Multiple Sclerosis.

Objective: Individuals with Multiple Sclerosis (MS) acquire affective disorders that often coincide with their disease progression. Little is known about the neuropsychological connection between these disorders and disease progression in relation to cognitive performance. The following paper will discuss the literature pertaining to the relationship between the development of these disorders with cognitive performance in neuropsychological exams.

Participants and Methods: The authors conducted a qualitative analysis of the literature consisting of 10 peer-reviewed articles published from 2005 to 2013 using the keywords Multiple Sclerosis, affective disorders, cognition, cognitive performance, depression, and anxiety. Searched databases included EBSCOhost, ProQuest, Academic Search Premier, PsychINFO, PsychArticles, ScienceDirect, PubMed, and CoBimet. Initial searches revealed a total of 300 possibly applicable articles. Exclusion criteria included those that were not published within the time range, not published in English, case studies, and/or that were not peer-reviewed.

Results: The Literature review demonstrated associations between affective disorders and disease progression in relation to poor cognitive performance. Poor performance is present in areas related to processing speed, attention, and executive functioning. Imaging studies have also demonstrated a lack of functional connectivity in prefrontal areas.

Conclusions: The findings are relevant in understanding the relationship between affective disorders and disease progression conceptually. Accurately understanding this connection will assist clinicians in providing effective treatment and rehabilitation for individuals with MS and their caregivers.

Correspondence: Patricia M. Rivera, Carlos Albizu University, 8601 sw 21 st, Miami,FL 33155.E-mail: pattyrivera12@gmail.com

N.S. BANERJEE, S.R. DELGADO, M. FORTE, S.A. ANDERSON, C.I. BERMUDEZ, V. RIESGO, H.L. KATZEN, G. RODRIGUEZ, M.R. ORTEGA, K. RAMMOHAN \& B.E. LEVIN. Is Vitamin D Insufficiency Related to Neuropsychological Function in Multiple Sclerosis?

Objective: Insufficient vitamin D (vitD) levels are well documented in Multiple Sclerosis (MS). Recent research has also suggested that suboptimal vitD levels may be associated with cognitive function in select neurodegenerative diseases. Little is known about the relationship between vitD and cognition in MS. This study examined the association between vitD insufficiency and cognitive and emotional wellbeing in patients with MS.

Participants and Methods: Twelve vitD insufficient (serum 25(OH)D of $20-29 \mathrm{ng} / \mathrm{mL}$ ) and 19 age-matched vitD sufficient (serum 25(OH)D $>30 \mathrm{ng} / \mathrm{mL}$ ) MS patients completed the CVLT-II, SDMT, and BDI-II as part of a comprehensive neuropsychological battery. Mean Z-scores were compared between the vitD insufficient and vitD sufficient groups using an independent-samples t-test.

Results: No significant differences were observed between the vitD insufficient (mean vitD $=23.8 \mathrm{ng} / \mathrm{mL}$, sd $=2.6 \mathrm{ng} / \mathrm{mL}$ ) and vitD sufficient ( mean vitD $=46.8 \mathrm{ng} / \mathrm{mL}$, sd $=12.3 \mathrm{ng} / \mathrm{mL}$ ) MS groups for any of the CVLT-II subtests, SDMT or BDI.

Conclusions: While insufficient vitD levels may play a role in immunological dysregulation and disease progression in MS, the findings from this pilot study argue against its negative effect on memory, processing speed and self-rated depression. An important point is that this study focused on vitD insufficiency, and a more robust relationship may exist between cognition and vitD deficiency, defined by levels below those 
used in our study (serum $25(\mathrm{OH}) \mathrm{D}<20 \mathrm{ng} / \mathrm{mL}$ ). Limitations include a small cognitive battery and sample size. Future studies should focus on a broader range of vitD levels to increase power.

Correspondence: Nikhil S. Banerjee, Bachelor's, Psychology, University of Miami, 350 S Miami Ave, Apt. 1704, Miami, FL 33130. E-mail: nbanerjee@med.miami.edu

M. FORTE, M.R. ORTEGA, N. BANERJEE, S.A. ANDERSON, C.I. BERMUDEZ, V. RIESGO, H.L. KATZEN, G. RODRIGUEZ, S.R. DELGADO, K. RAMMOHAN \& B.E. LEVIN. Moderate Caffeine Intake and Verbal Memory in Multiple Sclerosis.

Objective: Cognitive changes are common in MS and exacerbated by complaints of fatigue and sluggishness. Caffeine may play a protective role and improve cognition via the attentional networks (Ritchie et al., 2007). This study explored whether caffeine intake positively affects memory, speed of processing and emotional well being in MS.

Participants and Methods: Sixty three patients (18M, 45F) with confirmed MS (44 RMMS, 4 SPMS, 6 PPMS, 9 unknown MS Type; EDSS range: 2.0-8.) were administered the MMSE, CVLT-II, SDMT, BDI as part of a screening battery for a larger clinical trial. The two groups were equivalent on MMSE. Daily caffeine intake (coffee+tea) amount was collected through self-report. Mann-Whitney $U$ test was used to compare MS patients consuming 0-1 caffeine units daily vs. those consuming 2 or more units daily.

Results: MS participants who consumed 2 or more units of caffeine/ day outperformed MS participants consuming 0-1 caffeine units on the CVLT-II- LDFR U $=676.5, p=.012$; CVLT-II-Total Learning $\mathrm{U}=715$, $\mathrm{p}=.002 ;$ CVLT-II-SDFR, $\mathrm{U}=675, \mathrm{p}=0.012$. No significant differences were found between groups on the SDMT and BDI.

Conclusions: Our data show that moderate caffeine intake may be linked to better verbal memory performance in MS. These data are consistent with previous studies showing better verbal memory among those consuming moderate amounts of caffeine daily (Ritchie et al., 2007). It also lends support to the view that caffeine acts on dopaminergic and cholinergic networks in the CNS linked to the attentional networks. Correspondence: Mayte Forte, B.A., Neurology, University of Miami Miller School of Medicine, 1120 NW 14th Street, Miami, FL 33136. E-mail:Mayte.Forte001@umb.edu

\section{L.STROBER,J.ARMSTRONG,J.DELUCA\& N.D. CHIARAVALLOTI. Should I Stay or Should I Go? Employment Concerns Among Individuals Diagnosed with Multiple Sclerosis (MS) Within the} Past Five Years.

Objective: Rates of unemployment are high in multiple sclerosis (MS), with reports that as many as $70 \%$ are unemployed within five years of diagnosis. These rates are striking given that individuals with a relapsing-remitting course may endure several years with the disease before experiencing significant disability. Other factors, such as personality and self-efficacy have been proposed as contributing to unemployment in MS. The present investigation included individuals diagnosed with MS within five years and compared individuals who felt they may need to cut back or leave work and those that felt they could remain in the workforce.

Participants and Methods: Forty-three individuals with MS were enrolled in the study. Of this, $14(33 \%)$ reported feeling that they may need to cut back/leave work. Participants completed questionnaires of disease symptoms, psychological functioning, person-specific factors, and coping.

Results: Individuals feeling they needed to change their employment status reported more fatigue $(\mathrm{p}=.014)$ and neuroticism $(\mathrm{p}=.025)$, and lower extraversion $(p=.013)$ and general and MS self-efficacy $(\mathrm{p}=.009, .030$, respectively $)$. Further, they reported using behavioral disengagement as a means of coping $(p=.010)$.

Conclusions: Early on the disease, individuals who reported feeling the need to change their work status experienced more fatigue as well as other person-specific factors that may warrant examination.
In particular, lower self-efficacy, higher neuroticism, and low extraversion. These latter two are consistent with the Type D personality, which is generally associated with poor outcomes in medical settings. Greater attention to these factors when making employment decisions seems imperative.

Correspondence: Lauren Strober, Ph.D, Kessler Foundation Research Center, 300 Executive Drive, Suite 70, West Orange, NJ 07052. E-mail: lstrober@kesslerfoundation.org

S. NEIDINGER, S. O'BRYAN, C. RAY, C. MAVIS, B. ROBERG, J. EHANA, A. BRUCE, D. CATLEY, L. STROBER, A. BRADLEYEWING, S. LYNCH, A. NESS, M. GLUSMAN, K. GOGGIN \& J. BRUCE. Conscientiousness and Depression are Related to the Ability to Delay Gratification in Multiple Sclerosis.

Objective: Delaying gratification is the ability forgo immediate reward in order to obtain a larger delayed reward. The ability to delay gratification has been linked to improved health outcomes. By discounting the immediate and focusing on future rewards, patients able to delay may be better suited to follow through with healthy lifestyle changes (e.g., quitting smoking and treatment recommendations). No researchers have addressed influences delayed gratification in multiple sclerosis (MS). The present investigation examined clinical and neuropsychiatric factors that influence MS patients' ability to delay gratification.

Participants and Methods: Non-adherent, relapsing-remitting MS patients ( $\mathrm{N}=61$ ) were recruited from several MS specialty clinics, internet advertisements, and a regional MS Society newsletter as part of a larger study that examined adherence to disease-modifying therapy. Participants completed a neurologic evaluation and a battery of neuropsychiatric tests that included the Delaying Gratification Inventory (DGI-35). Results: Pearson correlations revealed higher overall disability, fatigue, anxiety, depression, lower conscientiousness, and slowed information processing speed were associated with difficulty delaying gratification (all $\mathrm{p}<.05)$. The stepwise linear regression showed both conscientiousness $(\Delta \mathrm{r} 2=.40, \mathrm{p}<.001)$ and depression $(\Delta \mathrm{r} 2=.06, \mathrm{p}<.05)$ accounted for unique variance in the ability to delay gratification.

Conclusions: The ability to delay gratification was most significantly related to high levels of conscientiousness and euthymic mood. Interventions that address lack of conscientiousness and depressive mood may be effective in enhancing MS patient's ability to delay gratification to improve overall health outcomes. More research is needed to understand modifiable factors that contribute to improved healthcare decision-making in MS.

Correspondence: Sandra Neidinger, Undergraduate Student, Psychology, University of Missouri-Kansas City, 5030 Cherry Street, Rm.350, Kansas City, MO 64110.E-mail: sandyneidinger@gmail.com

M.R. BASSO, A.K. MILLER, P. CANDILIS, D.R. COMBS, D. WHITESIDE \& S. WOODS. Neuropsychological Correlates of Time-Based Versus Event-Based Prospective Memory in Multiple Sclerosis.

Objective: Prospective memory (PM) concerns memory to perform a future behavior. Initial research implies that people with multiple sclerosis (MS) show impaired prospective memory for activities of daily living (Rendell et al., 2007; 2012). Distinctions have been made between time-based and event-based PM cues. Specifically, time-based PM pertains to someone performing a behavior at a particular time, whereas event-based PM involves performing a behavior in response to a particular cue (Henry et al., 2004). Time-based cues presumably depend upon intact executive function, whereas event-based PM relies upon declarative memory.

Participants and Methods: Participants included 97 patients with MS (76 females and 21 males; age: $\mathrm{M}=45.29, \mathrm{SD}=10.61$; education: $\mathrm{M}=14.41, \mathrm{SD}=2.30)$ and 34 controls (22 females and 12 males; age: $\mathrm{M}=40.29, \mathrm{SD}=13.21$; education: $\mathrm{M}=14.35, \mathrm{SD}=2.21)$. All were administered the Memory for Intentions Screening Test (MIST), a validated 
measure of prospective memory, and a battery of neuropsychological tests.

Results: Correlations examined the relationship between time-based and event-based PM and performance on neuropsychological measures. Time-based PM correlated with working memory, executive function, and declarative memory, whereas event-based PM was associated only with declarative memory.

Conclusions: These results accord well with the multiprocess theory. Time-based PM generally places greater demands on strategic monitoring and cue detection processes, and is more sensitive to executive dysfunction (e.g.., planning and cognitive flexibility) commonly associated with pre-frontostriatal injury (e.g., Raskin et al., 2012). In contrast, event-based PM tends to be more automatic and more strongly associated with declarative memory linked to temporolimbic systems. Correspondence: Ashley K. Miller, PhD, Psychology, University of Tulsa, 333 S. Delaware Ave, Apt \# 736, Tulsa, OK 74104. E-mail: ashleymiller@utulsa.edu

\section{HANCOCK, H. WESTERVELT, D. AHERN \& J. BRUCE. A Case Series of Aging Multiple Sclerosis Patients Presenting with Dementia.}

Objective: Up to $65 \%$ of multiple sclerosis (MS) patients experience cognitive deficits associated with the disease regardless of their age. To date, few studies have attempted to describe the cognitive profiles of aging MS patients whose deficits are best characterized as dementia. Our aims were to 1 ) identify the neuropsychological characteristics of major neurocognitive disorder in MS and 2) propose suggested guidelines for distinguishing MS-related cognitive changes from MS-related dementia. Participants and Methods: Comprehensive neuropsychological battery data for a case series of 4 patients presenting with both clinically definite MS and dementia are outlined.

Results: Patients were relatively young $(52.2 \pm 5.3$ years $)$ and had been diagnosed with MS for more than ten years (13.25 \pm 6.4 years) at the time of evaluation. Patients scored in the impaired range on tasks of attention $(\mathrm{z}=-1.3)$, working memory $(\mathrm{z}=-1.7)$, processing speed $(\mathrm{z}=-$ 2.3 ), aspects of executive functioning (Trails $B: z=-2.9$, verbal fluency: $\mathrm{z}=-2.6)$, visuospatial skills $(\mathrm{z}=-2.4)$, verbal learning and memory $(\mathrm{z}=-$ 2.6 and $\mathrm{z}=-2.7$, respectively), and visual learning and memory $(\mathrm{z}=-3.1$ and $\mathrm{z}=-3.1$, respectively).

Conclusions: Despite the fact that mild neurocognitive changes are a common result of the disease, MS patients can also present with a progressive dementing illness. The neuropsychological profiles of these patients reveal deficits in 3 or more domains with scores more than 2 standard deviations below the mean of the normative sample. Recommendations and guidelines for distinguishing between MS-related mild neurocognitive disorder and MS-related major neurocognitive disorder are outlined. These distinctions help identify individuals at risk for developing major neurocognitive early so that preventative steps can be taken. Future research is needed to further identify and characterize the presentation of MS-related dementia.

Correspondence: Laura Hancock, Ph.D., Psychiatry and Human Behavior, Alpert Medical School of Brown University, Rhode Island Hospital, 593 Eddy Street, Physicians Office Building Suite 430, Providence, RI 02903. E-mail: laura_hancock@brown.edu

\section{K.D. EVANKOVICH, J. ELIAS \& T.E. LOTZE. Longitudinal Evaluation of Cognitive, Academic, and Adaptive Functioning in Pediatric Multiple Sclerosis.}

Objective: Initial studies suggested that children with pediatric MS accrue significant cognitive burden. Recent reports indicate early weaknesses in visual motor integration, processing speed, and attention that remain stable over short time frames. Studies assessing functional outcomes are limited. The present study assesses changes in cognitive, academic, and adaptive functioning in pediatric MS.

Participants and Methods: Eighteen patients underwent initial and follow-up neuropsychological evaluations including standardized measures of cognitive and emotional functioning, the KTEA-II, CBCL, and VABS. Disability was measured with the EDSS.

Patients were $72 \%$ female; 39\% Hispanic, $28 \%$ African American, and $33 \%$ Caucasian. Mean maternal education was 13.3 years. Mean age of onset was 12.6 years. All received DMT. Mean time from symptom onset was $1.36 \pm 1.2$ years and $3.2 \pm 1.24$ years at follow-up. Annualized relapse rate was 2.7 and 1.2 follow-up. Minimal disability was accrued (mean EDSS score $=.44$ initially and .35 at follow-up).

Results: Impairment was defined as $\geq 1$ SD below published normative means. Impairment rate was $33 \%$ initially and at follow-up. Paired sample t-tests indicated no significant changes in mean standardized score on cognitive, academic, or adaptive measures. Mean IQ and academic development remained in the Average range. Compared to normative data, impairments were noted in visual-motor integration ( $\mathrm{SS}=$ $80 \pm 16$, follow-up SS $=75 \pm 12$ ), and attention $(T=61 \pm 30$, follow-up $T$ $=62 \pm 17.6)$. Parents reported inattention $(T=62 \pm 8$, follow-up $\mathrm{T}=64 \pm$ 6.2 ) and lower adaptive functioning ( $\mathrm{SS}=82 \pm 10$, follow-up $\mathrm{SS}=81.9$ \pm 12.5 ). Multiple regression indicated $86 \%$ of variance in adaptive functioning was accounted for by self-report of anxiety/depression, fine motor skill, and attention $(\mathrm{F}=6.4 . \mathrm{p}=.04)$.

Conclusions: Cognitive ability, academic development, and adaptive functioning remained stable. Low adaptive functioning was accounted for by patients' emotional functioning and weaknesses in fine motor dexterity and attention.

Correspondence: Karen D. Evankovich, PhD, Pediatrics-Psychology, Baylor College of Medicine, 6621 Fannin, Suite 1250, Houston, TX 77030.E-mail:kdevanko@texaschildrens.org

A. CANAS, T. SCHREINER, K. BJORAKER \& C. HUTAFF-LEE. Cognitive Decline without Neuroimaging Evidence of Disease Progression in an Adolescent Patient with MS.

Objective: Pediatric multiple sclerosis (MS) is an autoimmune disease of the CNS that has been increasingly recognized. Although MRI plays a pivotal role in the diagnosis and management of MS, the suboptimal correlation between clinical symptomology and MRI results has led to the recognition of a "clinical-radiographic paradox". This paradox, however, has focused on the presence of physical disability and fatigue in the absence of positive disease-related neuroimaging activity. We present a case in which cognitive decline was evidenced on neuropsychological measures in the context of stable neuroimaging results.

Participants and Methods: A neuropsychological assessment was conducted on a 16-year-old male with a history of MS, who was referred by his neurologist to assess current levels of functioning. Recent MRI results were also reviewed.

Results: Results of the evaluation revealed decline in performance on measures of working memory, processing speed, verbal fluency, and vocabulary knowledge within the context of generally intact cognitive abilities. Weaknesses in aspects of academic fluency and executive functioning were also noted. Visual-motor integration, verbal learning and memory, and attention remained stable. MRI revealed stable T2 hyperintense lesions, without evidence of disease progression.

Conclusions: This case highlights the complex neurodegenerative aspects of MS and the limitations of imaging techniques. Commonly used T2-weighted sequences are insensitive to cortical demyelination and damage to gray matter structures, which may affect cognitive functioning in MS. Additionally, MRI does not capture quality of life factors that contribute to changes in cognitive functioning. For these reasons, routine neuropsychological assessments should be conducted to monitor functioning and inform treatment planning.

Correspondence: Angela Canas, Texas Woman's University, 3700 Orleans Ave, \#5235, New Orleans, LA 70119. E-mail: acanas@twu.edu 
J.M. GALUSHA-GLASSCOCK, D.S. BROWN, S. HUGHES, B. GREENBERG \& L.H. LACRITZ. Test-Retest Stability of a Novel Executive Function Measure in Patients with Multiple Sclerosis.

Objective: To compare scores from the Texas Card Sorting Task (TCST), a brief executive function measure, with standard neuropsychological tests and examine test-retest stability in a multiple sclerosis (MS) sample.

Participants and Methods: Seventy-two participants with MS $[$ Mage $=44.51$ (11.61), Medu=15.44(1.95)] were administered a cognitive battery at baseline and follow-up as part of a larger longitudinal study of cognition in MS. Tests included: verbal fluency (FAS and Animals); Stroop Color and Word Test; Oral Symbol Digit Modalities Test (SDMT); Brief Visuospatial Memory Test-Revised (BVMT-R); California Verbal Learning Test-II (CVLT-II); and TCST. Pearson $r$ correlations were performed between TCST total logical sorts (LS) and selected cognitive variables at baseline. Test-retest reliability coefficients were calculated for TCST LS over a short $(<9 \mathrm{mo} ; \mathrm{n}=40)$ and long interval (>12mo; $\mathrm{n}=32)$.

Results: TCST LS $[\mathrm{M}=6.07(1.25)]$ was moderately correlated $(\mathrm{p}<.01)$ with SDMT $(r=.40)$, Category fluency $(r=.36)$, Stroop Word $(r=.41)$, Color $(r=.33)$, and Color-Word $(r=.39)$; BVMT-R delayed recall $(r=.30)$, and CVLT-II total learning $(\mathrm{r}=.32)$, and mildly correlated $(\mathrm{p}<.05)$ with BVMT Total learning $(r=.29)$ and CVLT-II long delay $(r=.27)$. Test-retest reliability for TCST LS was $r=.66$ over the short and $r=.65$ over the long interval.

Conclusions: The TCST yielded moderate correlations with other neuropsychological tests commonly used in MS and was stable over time. TCST was more highly correlated with tests associated with frontosubcortical systems (i.e, attention and processing speed) than long term memory. This study provides further support for the validity and stability of the TCST for use in an MS population.

Correspondence: Jeanine M. Galusha-Glasscock, Master of Science, Department of Psychiatry, University of Texas Southwestern Medical Center, 323 Deaton Drive, Rockwall, TX T=75087. E-mail: jeanine. galusha@utsouthwestern.edu

D.S. BROWN, S. HUGHES, B. GREENBERG \& L.H. LACRITZ. The Impact of Fatigue, Depression, Sleep and Daytime Sleepiness on Cognition in Multiple Sclerosis.

Objective: To examine the impact of fatigue, depression, sleep, and daytime sleepiness on cognition in Multiple Sclerosis (MS).

Participants and Methods: Eighty-six participants [Mage $=44.67$ (11.06), range: $20-75$ years; Meduc $=15.38(2.19)$, range: $8-20$ years] diagnosed with MS were asked how many hours of sleep they received the prior night and administered the Modified Fatigue Impact Scale, Epworth Sleepiness Scale, Quick Inventory of Depressive Symptomology, Stroop Color and Word test, Verbal Fluency (FAS and Animals), Oral Symbol Digit Modalities Test (SDMT), Brief Visuospatial Memory Test-Revised (BVMT-R) and California Verbal Learning Test-II (CVLT-II). Data were collected as part of a broader IRB approved longitudinal study examining cognitive functioning in MS. Linear regressions were performed for each self-report measure and selected $\mathrm{T}$ scores from the neuropsychologal variables. Age, education, ethnicity and gender were included as covariates if they contributed at least marginally (alpha level=.15) to the respective regression models.

Results: Hours of sleep did not significantly predict any cognitive scores ( $p$ 's $>.05)$. The MFIS significantly predicted Stroop Color (adjusted R-squared=.07), SDMT (adjusted R-squareed=.12), and BVMT-R delayed recall (adjusted R-squared=.17; p's <.05). The ESS significantly predicted only CVLT-II long delay free recall (adjusted R-squared $=.20$, $\mathrm{p}=.01$ ). The QIDS significantly predicted only Stroop Color (adjusted R-squared $=.06, p=.02$ ).

Conclusions: Hours of sleep was not associated with neurocognitive performance in this sample. However, fatigue, and to a lesser degree daytime sleepiness and depression, significantly predicted cognitive test scores. These results offer additional evidence that cognition is sensitive to fatigue in individuals with MS, particularly for processing speed and aspects of memory.

Correspondence: Daniel S. Brown, Bachelor of Music in Jazz Studies and Psychology, Division of Psychology, Department of Psychiatry, University of Texas Southwestern Medical Center, 3443 Mahanna St. \#3211, Dallas, TX 75209. E-mail: brown.daniel@gmail.com

K.R. BRYANT, J.D. GFELLER, K. SANDS \& H. HUGHES. The Relationship between Depression and Cognitive Dysfunction in Individuals with Multiple Sclerosis.

Objective: The aim was to examine the relationship between depression and cognitive dysfunction in a fairly large sample of Multiple Sclerosis (MS) patients. Additionally, differences in both cognitive dysfunction and depression prevalence between Relapsing Remitting (RRMS) and Progressive (PMS) subtypes were explored.

Participants and Methods: The sample consisted of 205 persons diagnosed with MS who received an outpatient neuropsychological evaluation as part of their clinical care. Mean demographic data are as follows: age $=47.47$, education $=14.5$, and duration of diagnosis $=$ 9 years. A majority of the sample was female $(79.0 \%)$, right handed $(87.8 \%)$, Caucasian (93.6\%), and diagnosed with RRMS (61\%). Partial correlations and ANCOVAS controlling for demographic variables that significantly differed between the groups (e.g., age and disease duration) were utilized.

Results: Depressive symptoms were significantly and positively correlated with cognitive dysfunction in the areas of attention / processing speed, verbal fluency, and executive functioning. PMS subtypes evidenced significantly poorer performance across a majority of cognitive domains. No difference in depression was found between the groups.

Conclusions: Cognitive inefficiencies in processing speed, attention, and executive functioning, which are characteristic of both depression and MS, likely interact to produce increased deficits in these areas. Compared to persons with RRMS, persons with PMS demonstrated a greater degree of cognitive dysfunction, even after controlling for age and disease duration, suggesting that other factors are contributory. MS subtype is not strongly related to depressive symptoms.

Correspondence: Kirk R. Bryant, Ph.D., Psychiatry and Behavioral Medicine, West Virginia University, 7 Grapevine Village, Morgantown, WV 26505. E-mail:kb124300@gmail.com

M. CADDEN \& P. ARNETT. Beyond Binary: Exploring the Merits of Multiple Depression Groups.

Objective: Depression has been traditionally explored in the context of Multiple Sclerosis (MS) as a binary construct (depressed, not depressed). However, given the $50 \%$ lifetime prevalence rate of depression in MS, it may be useful to consider not only currently depressed versus non-depressed patients, but to evaluate groups that better characterize the complexity of MS depression. The objective of the current study was to evaluate daily hassles (a measure known to predict depression in MS) across three groups: currently depressed, depression in remission, and never been depressed.

Participants and Methods: Fifty-one individuals with MS were examined. Current depression status was measured using the Beck Depression Inventory-Fast Screen. Former depression was evaluated using the Structural Clinical Interview for DSM-IV and a semi-structured psychosocial interview. The Hassles and Uplifts Scale was used to measure participants' self-reported daily hassles.

Results: In agreement with existing literature, depressed MS patients had a significantly higher hassles score $(p=.01)$ compared with non-depressed patients. However, when participants were classified into three depression groups, post-hoc tests revealed only that the currently depressed group had a significantly higher hassles score $(p=.043)$ than the never depressed group. The depression in remission group's reported hassles did not significantly differ from either of the other two groups. Conclusions: Results suggest that individuals who have never been depressed may drive differences between depressed and not depressed MS 
groups, at least on self-reported daily hassles. Examining other variables among these groups may be fruitful in providing a more nuanced view of depression in MS.

Correspondence: Margaret Cadden, Psychology, The Pennsylvania State University, 372 Moore Building, University Park, State College, PA 16802.E-mail:mhc147@psu.edu

S.R. O'BRYAN, B. ROBERG, L. TANGEMAN, B. WILLIAMS, C. MAVIS, M. GLUSMAN, C. RAY, S. NEIDINGER \& J. BRUCE. Slowed Saccadic Eye Movements in Multiple Sclerosis.

Objective: Recent research has shown slower saccadic eye movements are related to poorer overall cognition in multiple sclerosis (MS). The present study compared saccadic eye movements between MS participants and healthy controls. The study also sought to replicate and extend earlier research suggesting a significant association between saccadic eye movements and cognition in MS.

Participants and Methods: MS patients $(n=60)$ and controls $(n=31)$ were recruited by word of mouth, flyers, and advertising through the National MS Society as part of a study investigating social cognition in MS. Participants were administered the vertical trial of the Speedy Eyes Test (V-SET), a brief measure of purposeful saccadic eye movements. Subjects also completed a neuropsychological battery that assessed memory, attention, executive functioning, and information processing speed. Disease severity was measured using the Guy's Neurological Disability Scale.

Results: There was a significant effect of group on V-SET scores, $F(1$, $89)=19.80, p<.001, \eta^{2}=.18$ with MS patients requiring more time in seconds to complete the test (mean $\pm S D=21.72 \pm 3.70)$ than controls $(18.09 \pm 3.69)$. Within the MS group, slower eye movements were associated with poorer performance in overall cognition $(r=-.39, p=.002)$, greater disability, $(r=.38)$ and longer disease duration $(r=.32)$.

Conclusions: MS patients had significantly slower saccadic eye movements on the V-SET than controls. Furthermore, slower eye movements were associated with greater disease burden and diminished cognitive test performance within the MS sample. Pending further research, a brief, nontechnical measure of saccadic eye movement may be useful clinically in detecting cognitive impairment and monitoring disease progression in MS.

Correspondence: Sean R. O'Bryan, BA, Psychology, University of Missouri - Kansas City, 5030 Cherry St, Cherry Hall Room 350, Kansas City, MO 64110.E-mail: sean.r.obryan@ttu.edu

B.L. ROBERG, J. BRUCE, L. TANGEMAN, B. WILLIAMS, C. MAVIS, C. RAY, S. NEIDINGER, S. O'BRYAN \& S. LYNCH. Differences in Theory-of-Mind Abilities between Multiple Sclerosis Subtypes.

Objective: Theory-of-mind (ToM) allows a person to make inferences about the mental and emotional states of others. Recent research indicates that people with multiple sclerosis (MS) perform more poorly on ToM tests than healthy controls. However, no study has compared ToM abilities between MS subtypes. The current study examined ToM performance between people with different subtypes of MS and controls. Participants and Methods: People with relapsing-remitting MS (RRMS, n=34), secondary-progressive MS (SPMS, n=32), and controls $(n=31)$ were recruited; MS diagnosis was confirmed by patients' neurologist. As part of a larger neuropsychological battery, participants were administered the Social Perceptions Subtests of the Advanced Clinical Solutions battery. The variable of interest was the Social Perceptions total score.

Results: Analysis of variance revealed a significant effect of group on ToM performance, $\mathrm{F}(2,94)=11.54, \mathrm{p}<.001$. These differences persisted when controlling for age and disease duration. Tukey's post-hoc tests showed SPMS patients performed significantly worse than both RRMS patients $(p<.001$, Cohen's $d=1.14)$ and controls $(p=.006, d=0.75)$. There were no significant differences between RRMS patients and controls.
Conclusions: This was the first study to compare ToM abilities between MS subtypes. People with SPMS performed significantly worse than people with RRMS and controls. This research suggests that people with SPMS may have difficulty deciphering the mental and emotional states of other people. More research is needed to understand if these findings have real-world implications impacting the social relationships of people with SPMS.

Correspondence: Brandon L. Roberg, Clinical Psychology PhD Program, Psychology, University of Missouri-Kansas City, 5030 Cherry Street, Suite 324, Kansas City, MO 64110. E-mail:blrp7c@mail.umkc.edu

C.A. ROMAN \& P. ARNETT. Performance on the Symbol Digit Modalities Test Predicts Decreased White Matter Integrity a Decade Later in Multiple Sclerosis.

Objective: Performance on the Symbol Digit Modalities Test (SDMT) has been shown to be a strong predictor of cognitive impairment in multiple sclerosis (MS). However, its ability to predict additional, more neuropathological consequences of MS, such as white matter damage, is unclear. The present study aims to examine whether performance on the SDMT predicts decreased white matter integrity 10 years later. Participants and Methods: Twenty-two individuals (4m, 18f) with MS were administered the SDMT at Time 1 and were scanned during a diffusion tensor imaging (DTI) protocol at Time 2 (an average of 10.68 years later). A mean fractional anisotropy (FA) map and skeleton were created using Tract-Based Spatial Statistics (TBSS). FA was calculated for 4 ROIs, including the cingulate gyrus (CG), corpus callosum (CC), forceps minor (FM), and superior longitudinal fasciculus (SLF). Standard scores for the SDMT were calculated using a sample-based control group.

Results: Multiple regression analyses were conducted, controlling for age, gender, and education. Poorer performance on the SDMT at Time 1 predicted lower global FA ( $p=.046)$ and FA in the $\mathrm{CG}(\mathrm{p}=.013), \mathrm{FM}$ $(\mathrm{p}=.008)$, and CC $(\mathrm{p}=.003)$ at Time 2. After correcting for multiple comparisons, poorer performance on the SDMT predicted lower FA in the FM and CC.

Conclusions: This is the first study to examine the ability of the SDMT to predict white matter damage in MS more than a decade later. These data not only point to the predictive power of the SDMT in MS, but they also present a possible avenue to predict neuropathological damage. Correspondence: Cristina A. Roman, B.S., Psychology, The Pennsylvania State University, 939 Southgate Drive, State College, PA 16801. E-mail: cristina.a.roman@gmail.com

C.A. ROMAN \& P. ARNETT. Cognitive Reserve Moderates the Effect of Depression on Working Memory Performance in Multiple Sclerosis.

Objective: Depression and cognitive impairment are highly prevalent in multiple sclerosis (MS). Numerous studies have linked depression to poorer performance on working memory tests. Cognitive reserve (CR) has been shown to reduce the impact of MS on cognitive functioning. Yet, the relationship between these variables is unclear, and will, therefore, be explored.

Participants and Methods: Forty-five individuals (14m, 31f) with MS were assessed for depression and working memory using the Beck Depression Inventory-Fast Screen (BDI-FS) and Paced Auditory Serial Addition Test-3s (PASAT-3), respectively. Scores on the BDI-FS revealed that 14 individuals were "currently" depressed. Standard scores for total items correct on the PASAT-3 were calculated using a sample-based control group. In addition, standard scores for education and premorbid intelligence (as measured by the Wechsler Test of Adult Reading (WTAR)) were averaged to create a CR composite.

Results: The total hierarchical multiple regression model predicting PASAT-3 performance was significant and accounted for $24 \%$ of the variance. There was a main effect for depression $(p=.022)$, indicating that depressed individuals performed worse on the PASAT-3 than nondepressed individuals. More importantly, there was a significant 
Depression $x$ CR interaction ( $p=.043)$. When CR was high, depressed and nondepressed patients did not differ on the PASAT-3; however, when CR was low, depressed patients performed significantly worse than the nondepressed group.

Conclusions: To our knowledge, this is the first study to examine CR as a moderator of the relationship between depression and working memory in MS. The data reveal important relationships among protective factors, cognitive impairment, and psychopathology.

Correspondence: Cristina A. Roman, B.S., Psychology, The Pennsylvania State University, 939 Southgate Drive, State College, PA 16801. E-mail: cristina.a.roman@gmail.com

L. OASAY, S.E. SUNDARAM, N. HUBBARD, A. OUYANG, H. HUANG \& B. RYPMA. The Effects of Aging on White Matter Integrity in Relapsing-Remitting Multiple Sclerosis: A DiffusionTensor Imaging Study.

Objective: Multiple sclerosis (MS) is a CNS disease associated with cognitive impairment due to white matter lesions, although increased age is also associated with decreased white matter integrity (WMI). Some studies have failed to identify age as a significant moderator of performance on neuropsychological measures with MS patients, suggesting that increased age may not significantly exacerbate impairments in cognition already present due to MS. No studies have used neuroimaging variables to test this hypothesis to date.

Participants and Methods: The current study examined whether increased age is significantly associated with decreased WMI between healthy controls (HC) and relapsing-remitting MS patients (RRMS). Participants included 53 adults, ages $21-66, \mathrm{M}(\mathrm{SD})=45.04(10.68)$, who were diagnosed with RRMS $(\mathrm{n}=28)$ or classified as HCs $(\mathrm{n}=25)$. Data collected included demographic variables and four composite variables of diffusion tensor imaging (DTI) to assess white matter integrity [fractional anisotropy (FA), axial diffusivity (AD), radial diffusivity (RD), and mean diffusivity (MD)].

Results: Independent samples t-tests identified significant differences between the RRMS and HC group on AD, $\mathrm{t}(51)=-2.50, \mathrm{p}=.016$, RD, $\mathrm{t}(51)=-2.44, \mathrm{p}=.015$, and $\mathrm{MD}, \mathrm{t}(51)=-2.61, \mathrm{p}=.010$, with the RRMS group having higher means compared to the HC group. Pearson $r$ correlations identified a moderate, negative, statistically significant relationship between age and $\mathrm{FA}$ for the $\mathrm{HC}$ group, $\mathrm{r}(23)=-0.44, \mathrm{p}=$ .026 , but not for the RRMS group, $r(26)=0.02, p=.907$. One-tailed t-tests identified a statistically significant difference between these correlations, $\mathrm{Z}=-1.711, \mathrm{p}=.044$. Disease duration was not associated with age or the DTI variables in the RRMS group.

Conclusions: The absence of associations between aging and WMI in MS patients suggests that the debilitation of the CNS intrinsic to the disease may undermine or obfuscate the effect of increased age on cognitive functioning.

Correspondence: Saranya E. Sundaram, Master in Rehabilitation Counseling, Center for Brain Health, University of Texas at Dallas, 5555 East Mockingbird Lane, \#2612, Dallas, TX 75206. E-mail: ssundaram1341@gmail.com

T. MARCOTTE, T. WOLFSON, R. MEYER, T. ROSENTHAL, H. BENTLEY, B. GOUAUX, C. SCOTT \& J. COREY-BLOOM. Driving Performance at Clinically Effective Cannabis Doses for the Treatment of MS Spasticity.

Objective: Cannabis may improve spasticity associated with multiple sclerosis (MS). Despite its psychoactive effects, little is known about the impact on driving abilities when smoked at doses yielding clinical benefits. The aim of this study was to determine the effects of such dosing levels on driving performance.

Participants and Methods: Seventeen drivers with complaints of spasticity and at least moderate increase in tone (Modified Ashworth Spasticity (MAS) Scale >=3) were recruited from a 2-week, 8-visit, randomized, double blind, placebo-controlled, crossover study of smoked cannabis for MS spasticity (Corey-Bloom et al., 2012). In the parent study, participants completed a baseline assessment, 3 days of treatment with $4 \%$ THC cannabis (CA) or placebo (PL), an 11-day washout period, and 3 days of CA or PL. The parent study demonstrated improved spasticity following treatment. Driving simulations assessing 1) car following and 2) lane tracking/divided attention (LT/DA) were completed at baseline, as well as $2 / 3$ hours post-treatment and the next morning at the end of each CA/PL week.

Results: There was no difference between CA or PL conditions in the ability to follow the lead car, nor reaction time to lead car speed changes, although on CA participants drove closer to the lead car and had a shorter time-to-collision. While on CA, participants demonstrated more in-lane variability, and were more likely to provide an incorrect or missed response to the DA stimulus. Most of these effects were absent the following morning. Higher $\mu \mathrm{g} / \mathrm{kg}$ of THC was associated with worse performance on the LT/DA simulations. A reduction in the MAS was associated with faster reaction to lead car changes and less lane variability. Conclusions: Treatment with cannabis at levels that improve MS-related spasticity results in acute (negative) effects on the most challenging driving tasks, but also better performance in select aspects of driving. Correspondence: Thomas Marcotte, PhD, University of California, San Diego, 220 Dickinson St., Suite B, San Diego, CA 92103. E-mail: tmarcotte@ucsd.edu

O. RILO CANO, N. IBARRETXE-BILBAO, J. PEÑA, A. CABRERA, A. ANTIGÜEDAD, M. MENDIBE BILBAO, A. GÓMEZ, I. GABILONDO-CUÉLLAR \& N. OJEDA. White matter injury and its relation with compensatory cortical activation in multiple sclerosis.

Objective: To investigate the relationship between white matter (WM) lesion burden and compensatory cortical activation during verbal memory paradigm in multiple sclerosis (MS).

Participants and Methods: Twenty-seven MS patients and 12 healthy controls (HC) were recruited. 3D-T1-weighted and functional magnetic resonance (fMRI) images were acquired using a Philips Achieva 3T scanner. fMRI paradigm design consisted of a 10-block learning and 10-block recognition tasks separated by a 20 min resting period, both alternating activation and control conditions ( 5 blocks each). In learning activation condition, patients had to indicate whether they liked or disliked the visually displayed words while in recognition activation condition had to recognize previously presented words. Patients within normal limits, scored above -1.5 S.D relative to the HC group mean in recognition condition, were included in the study $(n=25)$. WM lesions were manually segmented from 3D-T1 images (ITK-SNAP) and their volume was calculated (Sienax-FSL). Multiple regression analyses were performed to analyze the association between WM lesion volume and compensatory cortical activation distribution in learning and recognition tasks (SPM).

Results: Results showed that higher WM lesion volume was associated to an increased cortical activation during learning task in two clusters: right inferior frontal gyrus (IFG) (Brodmann area-BA 47) ( $\mathrm{pFWE}<.001$, $\mathrm{KE}=53)$ and left middle frontal gyrus (BA 10) (pFWE<.01, KE=22). Additionally, WM lesion burden was positively correlated to the activation of a cluster located in the right IFG $(\mathrm{pFWE}<.001, \mathrm{KE}=51)$ during the recognition task.

Conclusions: These results suggest that multi-focal WM injury in MS may induce a compensatory activation of the frontal cortex in tasks involving verbal memory.

Correspondence: Oiane Rilo Cano, Psychology, Department of Methods and Experimental Psychology, University of Deusto, Avenida de las Universidades,24., Bilbao 48007, Spain.E-mail: oiane.rilo@deusto.es

C. TILL \& B. HOSSEINI. Perceived Parental Support and Social Stress as Moderators of Cognitive Decline in Pediatric-Onset Multiple Sclerosis Patients.

Objective: Cognitive impairment affects 30 to 50 per cent of pediatric-onset multiple sclerosis (MS) patients. Little is known about the 
long-term impact of the disease on cognitive development and how psychosocial predictors may moderate cognitive outcomes. This study determined how perceived parental support and social stress impact the maturation of cognitive abilities in pediatric-onset MS patients. Patients who reported poorer relations with their parents and higher levels of social stress were hypothesized to show poorer cognitive trajectories over time.

Participants and Methods: Serial cognitive data were collected from 35 individuals with pediatric-onset MS. Of the 35 participants, 8 completed only one assessment, 4 completed two assessments, 16 completed three assessments, and 7 completed four assessments. Patients completed measures of vocabulary (Woodcock-Johnson Picture Naming), verbal learning (Word Selective Reminding test from the Test of Memory and Learning), working memory and processing speed (Trails Making Test-Part B and Symbol Digit Modalities). Growth curve modeling was used to assess cognitive trajectories over time and to examine how youth's ratings on the Relations with Parents and Social Stress scales on the Behaviour Assessment System for Children-2nd edition (BASC-2) may moderate rate of change.

Results: Pediatric-onset MS patients who endorsed lower parental support showed a slower rate of development on measures of processing speed, working memory and vocabulary $(\mathrm{p}<0.02)$. Patients who reported higher levels of perceived social stress showed poorer long-term trajectories on measures of verbal learning and vocabulary $(\mathrm{p}<0.05)$. Patient endorsement of social stress trended with poorer parental relations $(p<0.10)$.

Conclusions: Perception of higher levels of social stress and less availability of parental support increases the vulnerability for disrupted $\operatorname{cog}^{-}$ nitive development in youth with MS, suggesting an important role for family care in this population.

Correspondence: Christine Till, PhD, Psychology, York University, 4700 Keele St., Toronto, ON M3J 1P3, Canada.E-mail: ctill@yorku.ca

B. TYSON, M. CHAKRAVARTY, J. FORD, J. RANDOLPH, E. REILLY \& H. WISHART. Structural Changes in Thalamic Subnuclei Associated with Pain and Attention Performance in Multiple Sclerosis.

Objective: Recent research suggests that pain selectively affects attention processes in MS. Further, studies have demonstrated that both pain and attention are associated with structural changes in the thalamus. However, no previous studies have investigated the role of individual thalamic subnuclei in relation to pain and attention in MS.

Participants and Methods: We investigated the relationship of thalamic subnuclei to self-reported pain level and attention performance in 65 individuals with MS. Structural magnetic resonance images were acquired at $3 \mathrm{~T}$ and thalamic subnuclei were segmented using a multiatlas technique that relies on an automatically generated template library. Results: Hierarchical multiple regression analyses showed that whole thalamic volume was a significant predictor of attention performance, even after controlling for relevant demographic and disease variables. Whole thalamic volume was not predictive of self-reported pain. Investigation of individual thalamic subnuclei showed that estimated baseline intellect along with decreased volume of the anterior dorsal nuclear complex (anterior and lateral dorsal thalamic nuclei) were significant predictors of attention performance. No thalamic subnuclei volumes were predictive of self-reported pain. However, individuals who demonstrated both elevated pain and impaired attention performance showed increased white matter lesion volume and decreased volume of the central thalamic nuclei.

Conclusions: These results highlight the contribution of the anterior thalamic nuclei to attention performance in MS and suggest a shared region of pain and attention processing in the central thalamic nuclei. Correspondence: Brad Tyson, Psy.D., Dartmouth Hitchcock Medical Center, 1 Medical Center Drive, Lebonon, NH 03753. E-mail: b.radtyson@yahoo.com
K. ROMERO, B. PAVISIAN, R. STAINES \& A. FEINSTEIN. Effects of Cannabis Use on Gray Matter Volume and Cognition in Patients with Multiple Sclerosis.

Objective: A subset of patients with multiple sclerosis (MS) smoke cannabis to relieve symptoms including spasticity and anxiety. Recent evidence suggests that cannabis use negatively impacts cognitive functioning in MS patients and is linked to dysfunctional brain activation. To date, however, no study has investigated whether these cognitive deficits are associated with regional, structural brain changes. The present study addresses this gap in the literature.

Participants and Methods: Twenty patients with confirmed MS who smoke cannabis (MS-C) for symptom relief, and 19 matched non-cannabis-smoking MS patients (MS-noC) were administered the Brief Repeatable Neuropsychological Battery (Buschke Selective Reminding Test, 10/36 Spatial Recall Test, Verbal Fluency, Symbol Digit Modalities Test, \& Paced Auditory Serial Addition Test) followed by T1weighted and FLAIR MRIs. Images were segmented into gray matter (GM) and white matter (WM) using VBM8, and subsequently analyzed with Partial Least Squares (PLS), a data-driven multivariate technique used to ascertain brain-behaviour associations.

Results: The MS-C group was more impaired than the MS-noC group on the PASAT $(\mathrm{t}=2.41, \mathrm{p}=.02)$ and $10 / 36$ spatial reacall test $(\mathrm{t}=2.29$, $\mathrm{p}=.03$ ). In both groups, regional GM atrophy in the thalamus, basal ganglia, medial temporal, and medial prefrontal regions, and WM atrophy in the fornix, parietal, and lateral frontal regions correlated with cognitive deficits. Crucially, the analysis indicated that atrophy correlated significantly with indices of verbal and visual memory, executive function and information processing speed in the MS-C group, but only information processing speed in the MS-noC group.

Conclusions: Smoking cannabis in MS results in more extensive cognitive deficits, which are correlated with GM and WM atrophy in subcortical and prefrontal regions. These are the first findings demonstrating structural changes due to cannabis use in these patients, and suggest a network of vulnerable brain regions

Correspondence: Kristoffer Romero, PhD, Psychiatry, Sunnybrook Health Sciences Centre, 2075 Bayview Ave, Toronto, ON M4N 3M5, Canada.E-mail:kris.romero@utoronto.ca

\section{Invited Address: Tales from Both Sides of the Brain \\ Presenter: Michael S. Gazzaniga}

$$
\text { 5:00-6:00 p.m. }
$$

\section{M.S. GAZZANIGA. Tales from Both Sides of the Brain.}

My fifty-year exploration of split-brain patients has revealed secrets of human brain organization that remain challenging to our understanding of mind and brain. In this account both the major scientific milestones and many people involved in the lifelong project will be portrayed. The importance of this dwindling split-brain patient population will be discussed in the context of current neuroscience endeavors. Overall the scientific life is full of sociality, hard work and just plain fun. Taking the long view, it is easy to see how we are influenced by others and how we do the same to them. Indeed, a life in science is a social process. Correspondence: Michael S. Gazzaniga, PhD, University of California, Santa Barbara, Santa Barbara, CA 93106. E-mail: m.gazzaniga@ psych.ucsb.edu 
FRIDAY MORNING, FEBRUARY 6, 2015

\author{
CE 9: \\ Sleep: A Silent Contributor to Cognitive Problems \\ Presenter: Mark S. Aloia
}

7:20-8:50 a.m.

M. ALOIA. Sleep: A Silent Contributor to Cognitive Problems.

Sleep is something experienced everyday by all of us. If perturbed, sleep can affect cognitive function in predictable ways. Sleep can also herald the onset of certain cognitive disorders, making knowledge of sleep and its effects on cognition important to any clinician. An exhaustive review of sleep and its related disorders that affect both adults and children would take at least a full day to review. We will, in this shorter period, review some of the highlights from the recent literature including the effects of sleep deprivation, sleep apnea, and occult sleep disorders on cognitive functioning. Neuropsychological test results and neuroimaging will be included.

Correspondence: Mark Aloia, National Jewish Health, 1400 Jackson St, Room B116, Denver, CO 80206. E-mail: aloiam@njhealth.org

CE 10:

Learning from Your Mistakes? Errorless Learning in Amnesia and Dementia

\author{
Presenter: Roy P. Kessels \\ 7:20-8:50 a.m.
}

R.P. KESSELS. Learning from Your Mistakes? Errorless Learning in Amnesia and Dementia.

Patients with an amnesic syndrome or dementia have profound deficits in explicit anterograde memory, while implicit learning is relatively spared. In clinical practice though, it is complicated to assess implicit learning capacity and to apply it in setting up interventions aimed at the acquisition of new information or skills. Errorless learning is a concept that optimizes residual learning capacity in amnesic patients by reducing errors or interference during learning. This approach may increase the patients' independence and reduce their care burden. This workshop will introduce the fundamentals of this approach first. Second, studies that have applied errorless learning in amnesia or dementia will be discussed. Third, I will describe how the principle of errorless learning can be applied in clinical practice, providing examples from daily practice and advice on implementation, including sample scripts and step-by-step instructions based on a recently developed practical manual. The workshop is targeted at (clinical) neuropsychologists working with amnesia/dementia patients or those who supervise formal caregivers in e.g. memory clinics, general hospitals, psychiatric hospitals or rehabilitation centers.

Correspondence: Roy P. Kessels, PhD, Radboud University Nijmegen, PO Box 9104, Nijmegen 6500 HE, Netherlands. E-mail: r.kessels@ donders.ru.nl

\section{Invited Address: \\ Lifetime Trajectories of Cognition - From Birth Cohorts to Aging Studies}

\author{
Presenter: Laura Hokkanen
}

9:00-10:00 a.m.

\section{HOKKANEN. Lifetime Trajectories of Cognition - From Birth Cohorts to Aging Studies.}

Cognitive performance fluctuates during the life span as a result of aging and neurological damage. Individual responses vary, however, and the concept of cognitive reserve may explain the gap between symptoms and pathology in many brain disorders. Educational attainment is often used as a proxy, but can be affected by developmental learning disorders. This talk invites the question: what is the impact of abnormal cognitive development on cognitive reserve and aging?

Longitudinal research on cognitive markers of brain illness offers one perspective. Studies on factors influencing adult intelligence include epidemiological, prospective population-based and retrospective registry-based approaches. Clinically relevant information on abnormal development comes from birth cohorts with perinatal risks, such as prematurity, low birth weight, and neonatal encephalopathy. Longitudinal studies on developmental conditions, such as ADHD, examine the persistence of cognitive impairment, but have seldom been extended into adulthood. Furthermore, longitudinal aging studies typically begin after midlife, but can combine prospective and retrospective data to assess the impact of variables in early adulthood on the onset of aging disease decades later. This talk will present important insights from our prospective longitudinal birth risk study, now middle-aged, and how their illnesses will be studied into old age.

Correspondence: , . E-mail:

INS Mid-Career (Benton) Award Presentation:

A Glimpse Behind the Veil: Multimodal Assessment and Rehabilitation of Memory and Executive Functioning

\section{Benton Award Winner: Brian Levine}

$$
\text { 10:15-11:15 a.m. }
$$

\section{Paper Session 4: Alzheimer's Disease}

10:15-11:45 a.m.

J. CHOI, J. FISZDON, K. WILKINS, P. KIRWIN, C. VAN DYCK, D. DEVANAND \& M.D. BELL. Self-efficacy for cognitive rehabilitation in Alzheimer's disease.

Objective: While cognitive rehabilitation (CR) for Alzheimer's disease $(\mathrm{AD})$ is becoming more readily available, persons with dementia (PWD) may have difficulty adhering to these cognitive treatments due to denial of memory deficits, lack of motivation and apathy, or a sense of hopelessness, which may be primarily due to illness or secondary to geriatric depression. For this reason, CR treatments that incorporate motivational strategies to enhance treatment engagement and self efficacy may provide greater benefits than standard CR training.

Participants and Methods: 72 PWD were randomly assigned to 3 months of computer brain games (BG, active control), computer-based memory training (MT), or Cognitive Vitality Training (CVT)—-the same memory training but contextualized in a milieu specifically designed to increase adherence to treatment by reducing hopelessness, enhancing motivation, and empowering patients to be actively involved in 
treatment. A wait-listed group of 29 served as a control group. Assessments of cognitive function, QOL, neuropsychiatric symptoms, activities of daily living, treatment adherence, and self-efficacy were administered to all groups at baseline, 2, 4, 6 mo, and 1 year.

Results: CVT was associated with greater resistance to memory decline compared to MT and BG at 1 year. Patients in CVT and their caregivers reported less overall depression throughout the intervention and at 6 mo. Interestingly, baseline self-efficacy for treatment predicted cognitive outcome at $1 \mathrm{yr}$, even after accounting for variance attributable to group assignment, memory ability, symptoms, and depression.

Conclusions: While PWD (and their caregivers) experience a tremendous loss of control in their lives, CVT, and potentially other therapies that re-introduce elements of control and self-efficacy may offer greater benefits than standard CR both for PWD as well as their caregivers. Findings from a more preventive approach in the prodromal stages of dementia and add-on therapies directly targeting hopelessness will also be discussed.

Correspondence: Jimmy Choi, PsD, Mental Health Services \& Policy, Columbi University Medical Center, 1051 Riverside Dr, Mailbox 100, New York, NY 10032.E-mail: jc3110@columbia.edu

\section{B.M. HAMSPTEAD, A.Y. STRINGER \& K. SATHIAN. Evidence of Transfer following Mnemonic Strategy Training in Patients with Mild Cognitive Impairment.}

Objective: The current single-blind, randomized controlled trial examined whether 1) MCI patients successfully transferred mnemonic strategy training (MST) while learning novel stimuli and 2) MST engaged different neural networks relative to an active control condition, spaced retrieval training (SRT), matched to treatment parameters.

Participants and Methods: Fifty-eight MCI patients were randomly assigned to MST or SRT of object-location associations. During three training sessions, MST participants were taught to 1) identify a salient environmental feature, 2) develop verbally-based reason(s) linking the feature and object, 3) create a mental image. Participants underwent pre- and post-training fMRI while encoding object-location associations. To assess training transfer, participants completed a novel object-location memory test that quantified the magnitude of inaccuracy (distance from target location using a touch-screen monitor) during free-recall, cued-recall, and recognition. Different versions were used pre-training, post-training, and at 1-month.

Results: MST patients demonstrated significantly greater improvement in memory compared to the SRT patients for both cued-recall $(p=0.003)$ and recognition $(p=0.001)$. At 1 month, significant differences were evident during free recall $(p=0.006$; MST $>$ SRT). Although performance in the MST, but not the SRT, group remained significantly better than pre-training, the interaction effects fell short of significance for cued-recall and recognition. fMRI revealed significantly increased activation in the left-hemisphere lateral frontoparietal network that mediates cognitive control and the left hippocampus of the MST group. SRT patients demonstrated reduced activation consistent with repetition suppression effects.

Conclusions: MCI patients are capable of transferring MST to novel stimuli, with training being partially restorative as it (re)engages brain regions involved in "normal" memory encoding.

Correspondence: Benjamin M. Hamsptead, PhD, Emory University \& Atlanta VAMC RR\&D CoE, 1441 Clifton Rd NE, Room 170, Atlanta, GA 30322.E-mail:bhampst@emory.edu

D.A. NATION, E.C. EDMONDS, K.J. BANGEN, L. DELANO-WOOD, D.P. SALMON, D. GALASKO \& M.W. BONDI. Cognitive profiles of tau pathology and amyloidosis in prodromal AD.

Objective: To characterize the empirically-derived cognitive profiles associated with tau and amyloid pathologies in nondemented older adults from the Alzheimer's Disease Neuroimaging Initiative (ADNI).

Participants and Methods: Participants were 748 nondemented (MCI=601; $\mathrm{NC}=276$ ) older adults who underwent cognitive assessment, health exam, and lumbar puncture to assess cerebral spinal fluid (CSF) biomarkers of phosphorylated tau (P-tau) and amyloid-beta (Abeta). Cluster analysis determined empirically-derived neuropsychological profiles based on 6 tests across 3 domains (memory, language, executive functioning). Biomarker profiles were: Biomarker-negative (Abeta-Ptau-), Abeta-positive only (Abeta+Ptau-), P-tau-positive only (Abeta-Ptau+), and combined biomarker positivity (Abeta+Ptau+). Chi-square and ANCOVA with post-hoc LSD tests examined cognitive differences across biomarker profiles. Covariates were age, gender, education, vascular risk factors, body mass index, and apolioprotein-E4 carrier status.

Results: Relative to the Abeta-Ptau- group, the Abeta+Ptau+ group was more likely to exhibit an amnestic $(53.7 \%$ vs. $34.8 \%)$ or dysnomic profile $(20.2 \%$ vs. $9.6 \%)$, the Abeta+Ptau- group was more likely to exhibit an amnestic $(55.8 \%$ vs. $34.8 \%)$ or dysexecutive profile $(11.6 \%$ vs. $1.7 \%$ ), and the Abeta-Ptau+ group was more likely to display a dysexecutive profile $(9.2 \%$ vs. $1.7 \%)$. Additionally, the Abeta+Ptau+ group displayed worse performance on all neuropsychological measures relative to the Abeta-Ptau- group.

Conclusions: Elevation in both phosphorylated tau and amyloid-beta was associated with the greatest degree of cognitive impairment, which is consistent with an interactive model of both biomarkers in the context of prodromal AD. Cognitive differences between biomarker profiles characterized by isolated tau versus amyloid pathologies could suggest that the earliest stages of these disease processes may impact partially distinct neural pathways.

Correspondence: Daniel A. Nation, Ph.D., Psychiatry, VA San Diego Healthcare System / University of California San Diego, School of Medicine, 3350 La Jolla Village Dr., 151B, San Diego, CA 92161. E-mail:dnation@ucsd.edu

R.P. ALMEIDA, S.A. SCHULTZ, E.A. BOOTS, S. YU, H. ZETTERBERG, B.B. BENDLIN, B.P. HERMANN, M.A. SAGER, C.M. CARLSSON, S. JOHNSON, S. ASTHANA \& O.C. OKONKWO. Cognitive Reserve Modifies Age-Related Alterations in CSF Biomarkers of Alzheimer's Disease.

Objective: Advancing age is the strongest risk factor for both the development of symptomatic Alzheimer's disease (AD) and the accumulation of AD-related pathophysiological abnormalities. Recent studies have shown that there are individual differences in susceptibility to age-related alterations in AD biomarkers. Cognitive reserve has been posited as an explanation for this mismatch. Accordingly, we investigated whether cognitive reserve modifies age-related changes in cerebrospinal fluid (CSF) biomarkers of AD.

Participants and Methods: Two hundred and forty nine cognitively normal and 57 cognitively impaired individuals from the Wisconsin Registry for Alzheimer's Prevention and the Wisconsin Alzheimer's Disease Research Center participated in this study. They underwent lumbar puncture for collection of CSF samples, from which $A \beta 42, t-t a u$, and p-tau were immunoassayed. Additionally, we computed t-tau/A $\beta 42$ and $\mathrm{p}$-tau/A $\beta 42$ ratios. Cognitive reserve was indexed by years of education, with $\geq 16$ years taken to confer high reserve. Covariate-adjusted regression analyses were used to test whether the effect of age on CSF biomarkers was modified by cognitive reserve.

Results: There were significant age ${ }^{*} \operatorname{cognitive}$ reserve interactions for CSF t-tau ( $\mathrm{p}=.039), \mathrm{p}$-tau $(\mathrm{p}=.020)$, and $\mathrm{p}$-tau/A $\beta 42(\mathrm{p}=.017)$, with a trend for $\mathrm{t}$-tau/A $\beta 42(p=.062)$. Specifically, with advancing age, individuals with high cognitive reserve exhibited attenuated adverse alterations in these CSF biomarkers compared with individuals with low cognitive reserve.

Conclusions: In a sample comprised of both cognitively normal and cognitively impaired subjects, higher cognitive reserve was associated with a diminution of age-related alterations in CSF biomarkers of AD. This suggests one pathway through which cognitive reserve might favorably alter lifetime risk for symptomatic AD. 
Correspondence: Rodrigo P. Almeida, Geriatrics, University of Wisconsin, CSC J5/Mezz, 600 Highland Ave, Madison, WI 53792. E-mail: rodrigopina03@gmail.com

V.A. GUZMAN, G. TOSTO, M.E. ZIMMERMAN, Q. RAZLIGHI, Y. GU, A. NARKHEDE, B. LAST, S. JANICKI, M. ICHISE, Y. STERN, J. MANLY, N. SCHUPF, R. MAYEUX \& A.M. BRICKMAN. Regional White matter Hyperintensities and Fibrillar Amyloid Deposition. Objective: Alzheimer's disease (AD) is thought to be due to deposition of amyloid and tau pathology, but recent research suggests that small vessel cerebrovascular disease, visualized as white matter hyperintensities (WMH) on MRI scans, plays at least an additive role and possibly interacts mechanistically with $\mathrm{AD}$ pathology. We showed that WMH are elevated in individuals at risk for AD and predict progression to clinical $\mathrm{AD}$, particularly when they are distributed in parietal lobes. The purpose of this study was to determine whether WMH and $\mathrm{AD}$ pathology interact by examining the regional distribution of $\mathrm{WMH}$ in individuals with and without fibrillar forms of beta amyloid.

Participants and Methods: Participants came from an ongoing study of aging and dementia in northern Manhattan. The subset of 89 participants in the current study had complete amyloid PET data (Florbetaben) with concomitant T2-weighted MRI scans. Each subject's PET scan was rated to determine whether there was evidence of fibrillar amyloid deposition (amyloid positive) or not (amyloid negative). White matter hyperintensities were quantified regionally in each cerebral lobe. Using a general linear model controlling for age, we examined the difference in regional distribution of WMH between amyloid positive and negative individuals.

Results: Participants were, on average, 84-years-old and 29 (31.5\%) were amyloid positive. Five $(17 \%)$ of the amyloid positive individuals and none of the amyloid negative individuals had dementia $(\chi 2=11.48$, $p=0.001)$. Individuals who were amyloid positive had more severe WMH volume, particularly in temporal and parietal regions (Amyloid status X Region interaction, $\mathrm{F}(3,258)=2.99, \mathrm{p}=0.031)$.

Conclusions: The findings suggest that the regional distribution of WMH and presence of fibrillar forms of amyloid co-occur. Our previous observations linking parietal WMH to AD might reflect shared underlying pathology with amyloid or mechanistic interaction between small vessel cerebrovascular disease and AD pathology.

Correspondence: Adam M. Brickman, Ph.D., Department of Psychiatry, Mount Sinai School of Medicine, 630 West 168th Street, P\&S Box 16, New York, NY 10032.E-mail: amb2139@columbia.edu

N.T. BOTT, A. RADKE, T. SHANY-UR, P. POORZAND, B. ADHIMOOLAM, B. MILLER \& K.P. RANKIN. Altered Sense of Humor Comprehension in Neurodegenerative Disease: Neuroanatomical Correlates.

Objective: Humor comprehension involves detecting that something is surprising yet coheres with what precedes it. Previous studies show increased errors on humor tasks occur in healthy aging. We examined nonverbal humor comprehension in subjects with neurodegenerative disease and healthy older controls $(\mathrm{NC})$ to investigate the neuroanatomic basis for impaired humor comprehension.

Participants and Methods: One hundred fifty-five participants (36 Alzheimer's disease, 31 behavioral variant frontotemporal dementia, 14 progressive supranuclear Palsy, 7 semantic variant primary progressive aphasia, 5 nonfluent variant primary progressive aphasia, 4 corticobasal syndrome, and $58 \mathrm{NCs}$ ) selected one of four optional endings to incomplete cartoon strips representing scenes depicting correct funny (CF), straightforward (SF), humorous nonsequitur (HNS) and unrelated nonsequitur (UNS) conclusions. Group differences of the overall, logic, and surprise elements of humor comprehension were analyzed using GLMs, and scores were correlated with brain volume using DARTEL-based voxel-based morphometry analysis of structural MRI gray matter maps controlling for age, sex, MMSE, and total intracranial volume (TIV).
Results: Neurodegenerative disease patients chose fewer CF endings than NCs $(p<0.05)$. Atrophy specifically related to non-CF responses included bilateral basal ganglia $([\mathrm{x}, \mathrm{y}, \mathrm{z}, \mathrm{T}$-score $]$ : $-30,-8,9, \mathrm{~T}=5.0 ; 30$,$14,8, \mathrm{~T}=4.8 ;-6,8,-5, \mathrm{~T}=4.7 ; 9,15,-2, \mathrm{~T}=4.3)$, right insula $(45,11,-6$, $\mathrm{T}=4.9)$, right superior temporal pole $(54,8,0, \mathrm{~T}=5.0)$, and medial pregenual cingulate $(0,35,-6, T=4.6)(p<0.001 \mathrm{FWE})$, with distinct regions associated with failure to detect surprising versus logically continuous elements.

Conclusions: Our findings show that altered sense of humor in neurodegenerative disease can involve regions mediating affective salience and perspective taking, monitoring of expectation discrepancy, as well as indexing of social-cognitive information for emotion generation.

Correspondence: Nicholas T. Bott, Neurology, University of California San Francisco, 401 Quarry Rd., MC 5719, Palo Alto, CA 94305. E-mail: nbott@stanford.edu

\section{Symposium 7: \\ Developmental Motor Disorders: From Genes to Brains to Behavior}

\section{Chair: Deborah Dewey \\ 10:15-11:45 a.m.}

F.P. BERNIER, P.J. ANDERSON, S.K. THORNTON, K.D. TEN EYCKE, D. DEWEY, K.O. YEATES \& D. DEWEY. Developmental Motor Disorders: From Genes to Brains to Behavior.

Symposium Description: Motor impairments are commonly seen in children with neurodevelopmental disorders such as developmental coordination disorder (DCD) and attention deficit/hyperactivity disorder, as well as in children born very preterm. Failure to identify and address these motor problems at a young age can significantly affect educational outcomes and long-term physical and mental health. Research indicates that neurodevelopmental disorders are highly heritable with estimates as high as $70 \%$, suggesting a genetic component. Imaging studies suggest that children with motor impairments have altered brain structure and function compared to typically developing children. Further, children with motor deficits have been found to display impairments across a broader range of neurobehavioral functions (e.g., memory, visual perception). This symposium will present the findings of recent research that has investigated the genetic, neurobiological and neurobehavioral basis of developmental motor impairments in children with neurodevelopmental disorders and children born very preterm. Dr. Bernier will present the findings of a recent study that explored the underlying genetic basis of DCD. Dr. Anderson will discuss research that investigated the ability of brain magnetic resonance imaging (MRI) at term equivalent age to predict motor impairment in very preterm children. Ms. Thornton will present findings from a study that investigated differences in functional brain activation on a motor inhibition task among children with isolated or co-occurring DCD and ADHD. Drs. Ten Eycke and Dewey will discuss their research on the neuropsychological outcomes of children with DCD compared to children with other neurodevelopmental disorders. The findings of the studies presented at this symposium will provide insight into the relationship between genes, neural networks and neuropsychological functions in specific populations that display motor impairments.

Correspondence: Deborah Dewey, PhD, Paediatrics and Community Health Sciences, University of Calgary, Behavioural Research Unit, Alberta Children's Hospital, 2888 Shaganappi Trail NW, Calgary, AB T3B6A8,Canada.E-mail:dmdewey@ucalgary.ca 
F.P. BERNIER, S.J. MOSCA, L. LANGEVIN, A. INNES, A.C. LOINEL, C.C. MARSHALL, S.W. SCHERER, J.S. PARBOOSINGH \& D. DEWEY. Copy-number Variation in Canadian Children with Developmental Coordination Disorder Implicates Neurodevelopmental Genes.

Objective: Developmental coordination disorder (DCD) is a common neurodevelopmental disorder characterized by functional motor performance deficits that interfere with daily activities in children and adults. Copy number variants, both sub-microscopic deletions and duplications, are increasingly being identified in a subset of children with neurodevelopmental disorders including autism spectrum disorder and ADHD. DCD has similar heritability rate to other neurodecelopmental disorders and is also a common co-morbidity. Our studies there aim to explore the underlying genetic or genomic basis and DCD.

Participants and Methods: We performed copy number variant (CNV) analysis in 82 well-characterized Canadian children between the ages of 8 and 17 with DCD and 2,988 healthy European controls using identical 2,500,000 SNP microarrays and CNV calling algorithms.

Results: We detected a statistically significant increased rate of both rare CNVs $(p=0.018)$ and rare, genic CNVs $(p=0.009)$ between 500 and $1000 \mathrm{~Kb}$ in cases compared to controls. Within genic CNVs, there was an enrichment of duplications spanning brain-expressed gene $(p=0.039)$ and genes previously implicated in other neurodevelopmental disorders $(p=0.043)$. Genes and loci previously implication in other neurodevelopmental disorders were of particular interest and included: GAP43, RBFOX1, PTPRN2, SHANK3, 16p11.2, and distal 22q11.2.

Conclusions: Our findings provide compelling evidence supporting a genetic basis for DCD, and further implicate rare $\mathrm{CNV}_{\mathrm{s}}$ in the etiology of neurodevelopmental disorders. Our data also suggests that there might be shared susceptibility genes for DCD and other neurodevelopmental disorders.

Correspondence: Francois P. Bernier, 2500 University Dr NW, Calgary, ABT2N1N4,Canada.E-mail:francois.bernier@albertahealthservices. ca

P.J. ANDERSON, A. SPITTLE, D. THOMPSON, L.W. DOYLE, T.E. INDER \& J. CHEONG. Neonatal MRI Predicts Motor Impairment in Very Preterm School-aged Children.

Objective: The rate of motor impairment in preterm children is three to four times the rate in the general population. This study investigated the ability of brain magnetic resonance imaging (MRI) at term equivalent age to predict motor impairment in very preterm (VPT) children.

Participants and Methods: Participants were 224 children in a prospective longitudinal cohort born $<30$ weeks' gestation and/or $<1250 \mathrm{~g}$. Infants underwent MRI at term equivalent age. Structural scans were qualitatively assessed for white matter and grey matter pathology, and regional brain volumes were calculated. Diffusion parameters were calculated in 12 manually selected regions of interest. Motor functioning was assessed at 5 years of age with the Movement Assessment Battery for Children (MABC) and at 7 years of age with the MABC2.

Results: $50 \%$ of the VPT cohort had a motor impairment. Moderate to severe white matter pathology increased the odds of motor impairment at 5 years nine-fold (OR 9.4; 95\% CI 3.2-28.1). Motor impairment was also associated with smaller brain volumes, particularly in sensorimotor $(p<0.001)$, parieto-occipital $(p<0.001)$, cerebellum $(p=0.001)$, premotor $(p=0.006)$ and subcortical grey matter $(p=0.004)$ regions. Furthermore, decreased fractional anisotropy of the internal capsule was found in those with motor impairment. At age 7 years, motor impairment was predicted by white matter pathology, as well as cerebellar and subcortical grey matter abnormalities.

Conclusions: Brain MRI in the neonatal period is predictive of later motor impairment in VPT children, and could be helpful in detecting high-risk infants that warrant close monitoring and early intervention. Correspondence: Peter J. Anderson, 50 Flemington Road, Parkville, VIC 3052, Australia.E-mail: peter.anderson@mcri.edu.au
S.K. THORNTON, S. BRAY, L. LANGEVIN \& D. DEWEY. Functional Brain Activation during a Motor Inhibition Task in Children with Developmental Coordination Disorder and Attention Deficit/ Hyperactivity Disorder.

Objective: Developmental coordination disorder (DCD) and attention-deficit/hyperactivity disorder (ADHD) co-occur in up to $50 \%$ of cases. Previous studies have typically looked at these disorders in isolation. This study investigated whether children with isolated or co-occurring DCD and ADHD display different functional brain activation compared to controls on a motor inhibition task that demanded both motor and attention skills.

Participants and Methods: Functional magnetic resonance imaging (fMRI) was performed in children with DCD $(\mathrm{n}=11)$, ADHD $(\mathrm{n}=$ $22)$, co-occurring DCD/ADHD $(\mathrm{n}=18)$ and controls $(\mathrm{n}=19)$ during performance of a GO-NOGO task. Attention and executive function were assessed using standardized measures and correlated to fMRI measures. Results: Children with co-occurring DCD/ADHD showed decreased brain activation compared to controls in the bilateral inferior parietal lobules (IPL), superior parietal lobules (SPL), right angular gyrus (ANG), left precentral gyrus (preCG) and left inferior frontal gyrus (IFG) $(p<0.05 \mathrm{FEW}$ cluster corr.). No significant differences were found between children with isolated DCD, ADHD and controls. Functional activation in the IPL bilaterally, and left IFG, were positively correlated with measures of attention and executive function.

Conclusions: These results suggest that children with co-occurring DCD/ ADHD display significant alterations in brain function that may contribute to problems in motor inhibition. In contrast to previous work, we did not find that children with DCD or ADHD showed significant functional alterations on motor inhibition tasks. It is important to consider co-morbidity when developing treatments and predicting outcomes of children with developmental disorders.

Correspondence: Siobhan K. Thornton, 2500 University Dr NW, Calgary, AB T2N1N4, Canada.E-mail: skthornt@ucalgary.ca

K.D. TEN EYCKE \& D. DEWEY. Executive Function Deficits in Children with Developmental Coordination Disorder and Attention Deficit Hyperactivity Disorder: Same or Different.

Objective: Research investigating executive function (EF) in children with developmental coordination disorder (DCD) and attention deficit/ hyperactivity disorder (ADHD) has reported inconsistent results. Some studies suggest children with these disorders display deficits, whereas others do not. This inconsistency could be due to how EF is measured. This study investigated EF deficits in children with DCD, ADHD and co-occurring DCD+ADHD on performance-based versus parent-report measures of EF.

Participants \& Method: 315 children, $6-17$ years of age $(\mathrm{M}=10.1, \mathrm{SD}=$ 2.62) participated (53 DCD, 76 ADHD, 79 ADHD+DCD, 107 Controls). Performance-based EF was measured using an average of $6 \mathrm{EF}$ scores on the NEPSY-II (i.e., Animal Sorting, Auditory Attention, Response Set, Inhibition (Naming, Inhibition, Switching)). Parent-reported EF was measured using the BRIEF.

Results: On performance-based measures, the DCD and DCD+ADHD groups obtained a significantly lower NEPSY-II score than children with $\mathrm{ADHD}$ and Controls $[\mathrm{F}(3,308)=28.747, \mathrm{p}<.001 ; \mathrm{MDCD}=8.86$, $\mathrm{MADHD}=9.50, \mathrm{MDCD}+\mathrm{ADHD}=7.83, \mathrm{MNT}=10.49]$. In contrast, on the BRIEF, children in the ADHD and co-occurring DCD+ADHD had significantly lower BRIEF scores than children in the DCD and Control groups $[\mathrm{F}(3,281)=59.48, \mathrm{p}<.001 ; \mathrm{MDCD}=55.67, \mathrm{MADHD}=67.35$, $\mathrm{MDCD}+\mathrm{ADHD}=66.98, \mathrm{MNT}=48.47]$.

Conclusions: Children with DCD or ADHD displayed different outcomes on performance-based and parent-report measures of EF, suggesting that different EF constructs are assessed by these measures. Moreover, children with DCD+ADHD exhibited impairments on both performance-based and parent-reports indicating that these children display a broader range of deficits in EF. 
Correspondence: Kayla D. Ten Eycke, 2500 University Dr NW, Calgary, AB T2N1N4, Canada.E-mail: kteneyck@ucalgary.ca

D. DEWEY, L. LANGEVIN, S.G. CRAWFORD, K.M. BARLOW, J. LEMAY \& F.P. BERNIER. Developmental Brain Dysfunction: Co-occurrence is Associated with Impaired Neuropsychological Functioning.

Objective: Co-occurrence is the rule for virtually all neurodevelopmental disorders. This study examined the influence of comorbidity on neuropsychological functioning in children with developmental coordination disorder (DCD), attention deficit/hyperactivity disorder (ADHD) and reading disorder $(\mathrm{RD})$.

Participants \& Method: 412 children, 6-16 years of age were recruited through family physicians, pediatricians, schools and community organizations. They were assessed on standardized psychometric measures and classified as DCD, ADHD, RD, a comorbid condition or typically developing (TD). The main outcomes were neuropsychological functioning on the NEPSY-II.

Results: MANOVAs were used to investigate group differences. To control for multiple comparisons significance was set at $p<.01$. On measures of attention and executive function, children with DCD+ADHD+RD performed significantly poorer than TD children and children with only DCD or ADHD. Children with RD and comorbid disorders performed significantly poorer than TD children and children with only DCD or ADHD on a measure of phonological processing. On a test of visual memory, children with DCD and comorbid disorders displayed poorer performance than TD children, whereas on a test of verbal memory the $\mathrm{DCD}+\mathrm{ADHD}+\mathrm{RD}$ group performed significantly poorer than the TD, DCD and ADHD groups. Children with DCD+ADHD and DCD+ADHD+RD scored significantly lower than TD children and children with ADHD only on a measure of visual motor skills. On a measure of visual perception, children in the $\mathrm{DCD}, \mathrm{DCD}+\mathrm{ADHD}$ and $\mathrm{DCD}+\mathrm{ADHD}+\mathrm{RD}$ groups performed significantly worse than TD children.

Conclusion: Compared to TD children and children with only DCD and ADHD, children with co-occurring disorders were more likely to display impairments across a broader range of neuropsychological functions. To direct more efficacious treatment, children diagnosed with DCD, ADHD and RD need to be investigated for comorbid disorders.

Correspondence: Deborah Dewey, PhD, Behavioural Research Unit, Alberta Children's Hospital, 2888 Shaganappi Trail NW, Calgary, AB T3B 6A8, Canada.E-mail: dmdewey@ucalgary.ca

\section{Paper Session 5: Alcohol-Related Dysfunction}

\section{0:15-11:45 a.m.}

S. RASKIN, A. BENEDETTO \& E. AIKEN. Effect of Drinking Behavior on Cognitive Functions in College Students.

Objective: The objective of the study was to determine the effect of drinking behavior on cognitive functions in college students.

Participants and Methods: 1296 college students ages 18-21 were administered the Cogstate and JAva NEuropsychological Test (JANET) including One Back; Two Back; Groton Maze Learning and Recall; Visual Paired Associate Learning; Digit Symbol; Balloon Analogue Risk Task (BART); Penn Conditional Exclusion Test (PCET). Measures of impulsivity were Barrett's Impulsivity Scale and Behavioral Inhibition/ Activation Scales (BIS/BAS). Measures of mood were Beck Depression Inventory and State Trait Anxiety Inventory. Self-Rating Effects of Alcohol (SREA), Modified Timeline Follow-back (TFLB), and Alcohol Effects Questionnaire (AEQ) were used to divide participants into three categories. Non-drinkers had never consumed alcohol. Light drinkers (1) did not meet criteria for AUD and (2) drank $<50 \%$ of the weeks during the preceding 6 months. Heavy drinkers either (1) met criteria for current AUD or (2) drank $\geq 50 \%$ of the weeks in the preceding 6 months and typically binge drank ( $\geq 4$ drinks/occasion for females or $\geq 5$ drinks/occasion for males for more than half of the number of drinking incidents reported.

Results: One-way analysis of variance (ANOVA) demonstrated heavy drinkers performed significantly more poorly $(\mathrm{p}<.01)$ then the other groups on the PCET and Groton Maze recall $(p<.01)$ but light drinkers were not different from the other groups. Heavy drinkers scored significantly higher in impulsivity than the other groups $(p<.001)$ and light drinkers scored significantly more highly on measures of impulsivity than nondrinkers $(p<.01)$. No differences were found between groups on measures of mood.

Conclusions: These data suggest that college students who demonstrate heavy drinking both have poor impulse control and demonstrate reduced cognitive performance on measures of memory recall and executive functions. This study is remarkable for having a relatively large sample size and heterogeneous population.

Correspondence: Sarah Raskin, Ph.D., Psychology, Trinity College, 300 Summit Street, Hartford, CT 06119.E-mail: sarah.raskin@trincoll.edu

K.A. WALKER, A. TORSTRICK, Y. CUKIER, S. PAVlos, M. AMBROSE, G. DAMEWOOD, A. HAMLET, S. CONNELLY \& R. WELLINGTON. Executive Functioning Deficits in Offspring of Alcohol Dependent Probands Predate Alcohol Dependence.

Objective: Several studies have demonstrated executive functioning (EF) deficits in the offspring of alcohol dependent probands that predate heavy alcohol use. Some have proposed that these heritable deficits in EF may confer a vulnerability that places family history positive (FHP) individuals at an elevated risk for development of alcohol dependence. The current study aimed to extend these findings by examining specifically which aspects of EF were affected in adult offspring of alcohol dependent individuals.

Participants and Methods: A sample of young adults ( $\mathrm{n}=170)$ were assessed using a comprehensive battery of EF measures. Those with heavy alcohol use or dependence as defined by the CAGE criteria were excluded ( $\mathrm{n}=30)$. The FHP group $(\mathrm{n}=24)$ was compared to family history negative (FHN) group $(\mathrm{n}=116)$ on four EF component composite scores that were derived using confirmatory factor analysis.

Results: A series of hierarchical linear regression analyses compared FHP to FHN individuals on EF composite scores of Working Memory, Set-Shifting, Inhibition, and Planning while covarying for estimated IQ and current alcohol consumption. FHP status was associated with poorer Planning $(\beta=.27, p<.01)$, but was not related to poorer performance on other components of $\mathrm{EF}(\mathrm{p}>.05)$. Further analyses revealed that both maternal $(\beta=.22, \mathrm{p}=.01)$ and paternal $(\beta=.21, \mathrm{p}<.05)$ alcoholism were related to poorer Planning.

Conclusions: The findings indicate that planning deficits may predate heavy alcohol consumption in FHP adults, suggesting a possible cognitive vulnerability to alcohol dependence. Planning deficits should be further examined as a possible endophenotype for alcohol dependence. Correspondence: Keenan A. Walker, M.A., Psychology, St. John's University, 101-01 67th Dr., APT 4J, Forest Hills, NY 11375. E-mail: kwalke26@gmail.com

J.R. WOZNIAK, A. FUGLESTAD, J. ECKERLE, B. FINK, H. HOECKER, C.J. BOYS, J. RADKE, M. KROUPINA, N. MILLER, A. BREARLEY, S. ZEISEL \& M. GEORGIEFF. Choline supplementation improves memory performance in preschool children with fetal alcohol spectrum disorders: A randomized controlled trial.

Objective: There are few cognitive interventions and no biological treatments for Fetal Alcohol Spectrum Disorders (FASD), but animal models suggest that the micronutrient choline may treat cognitive deficits. Potential mechanisms for its effects include facilitation of cell-structure, enhancement of acetylcholine, and epigenetics. The primary goal of this study was to determine whether choline supplementation improves hippocampal-dependent memory performance in children with FASD. 
Participants and Methods: This was a double-blind, randomized, placebo-controlled trial in 60 children (ages 2.5 to 5 years) with FASD randomized to $500 \mathrm{mg}$ choline or placebo daily for 9 months. Primary outcome measures were an elicited imitation memory paradigm and the Mullen Scales of Early Learning.

Results: Choline was safe and well-tolerated - with participants receiving a dose on $88 \%$ of the days in the study. The only adverse event linked to the choline arm was fishy body odor. Choline supplementation was associated with improvement in elicited imitation memory performance for the youngest participants (2-3 year-olds), with an increase of 21-28 percentage points over nine months of treatment compared to smaller change in the placebo group and the older children (4-5 year-olds) receiving choline (range: $4-15$ percentage points). In the choline arm, there was an inverse relationship between choline dose adjusted for body weight $(\mathrm{mg} / \mathrm{kg})$ and memory improvement, suggesting that the universal dose of $500 \mathrm{mg}$ that was administered may have been higher than optimal for some participants.

Conclusions: Choline supplementation in children with FASD improved memory performance on an elicited imitation task in 2-3 yearold children, but not 4-5 year-olds. This is the first study to demonstrate the efficacy of choline supplementation in children with FASD. Choline's effects on hippocampal development may be long-lasting and may have application to other neurodevelopmental disorders.

Correspondence: Jeffrey R. Wozniak, Ph.D., Psychiatry, Univ. of Minnesota, F256/2B West, 2450 Riverside Ave., Minneapolis, MN 55454.E-mail:jwozniak@umn.edu

R. MIGLIORINI, E.M. MOORE, L. GLASS, M. INFANTE, S. TAPERT, K.L. JONES, S.N. MATTSON \& E.P. RILEY. Anterior Cingulate Cortex Structure Relates to Behavioral Inhibition in Children with Heavy Prenatal Alcohol Exposure.

Objective: Prenatal alcohol exposure is associated with behavioral disinhibition, yet the neural correlates have not been adequately explored. The anterior cingulate cortex (ACC) has been widely implicated in inhibitory control. We hypothesized that the size of the ACC would predict inhibition performance in alcohol-exposed children.

Participants and Methods: Children (12-17y) with histories of heavy prenatal alcohol exposure (AE,n=32) and controls $(\mathrm{CON}, \mathrm{n}=21)$ underwent structural neuroimaging. A subset $(n=41)$ completed the NEPSY-II Inhibition subtest. ACC structure (volume, surface area, thickness) was extracted from T1-weighted MRI (Freesurfer-v.5.3). Between group comparisons were made for ACC with MANOVA and inhibition variables (time, errors) with t-tests. Multiple linear regressions estimated the main effects of group and ACC and the group $x$ ACC interaction in predicting inhibition performance, accounting for age and sex.

Results: The AE group performed worse than controls on inhibition completion time $(p=.01)$ and had reduced surface area $(p=.02)$, driven primarily by the right caudal ACC. There were no group differences on inhibition errors, volume, or cortical thickness. Regressions revealed a significant group $\mathrm{x}$ right caudal ACC surface area interaction $(\mathrm{R} 2=.36, \mathrm{p}=.006)$. Reduction in ACC surface area predicted slower inhibition time for the AE, but faster inhibition time for the CON.

Conclusions: Accounting for age and sex, the nature of the relationship between ACC and inhibition depends on alcohol exposure history. Alcohol's teratogenic effect on the ACC may contribute to poorer inhibition, perhaps by impacting the speed at which children can process and respond to information requiring cognitive control. Understanding the neural underpinnings of inhibition deficits in alcohol-exposed children may lead to the development of better treatment strategies.

Research supported by NIAAA R01-AA019605, T32-AA013525, F31-AA022261 and NSF GRFP DGE-1247398.

Correspondence: Robyn Migliorini, Psychiatry, University of California, San Diego, 8939 Villa La Jolla Drive, Suite 230, La Jolla, CA 92037. E-mail: robyn.migliorini@gmail.com
L. GLASS, E.M. MOORE, R. MIGLIORINI, M. INFANTE, E.P. RILEY \& S.N. MATTSON. Academic Achievement Deficits in Children with Heavy Prenatal Alcohol Exposure: Presence, Prevalence, Neural Correlates.

Objective: Adverse effects of heavy prenatal alcohol exposure on cognition are well documented, however the presence, prevalence, and neural correlates of poor academic performance are not understood.

Participants and Methods: Children (8-16y) with heavy prenatal alcohol exposure (AE,n=67) and controls $(\mathrm{CON}, \mathrm{n}=61)$ were assessed for academic deficits using the WIAT-II. Impairment in spelling, reading, and math were determined by MANOVA and 2 -tailed $\mathrm{z}$-tests. Structural brain images were acquired for a subset of subjects $(13-16 y, n=44)$. FreeSurfer-v5.3 was used to examine relations between cortical surface area and performance, controlling for age and multiple comparisons. Results: The AE group performed worse than controls and had higher rates of impairment (1SD cutoff below control mean) across academic domains (ps<.001): Spelling (AE:37.3\%,CON:14.8\%), Reading (AE:40.3\%,CON:14.8\%), Math (AE:65.7\%,CON:16.4\%). Within the AE group, impairment was more frequent for math $(p<.001)$ than reading or spelling. The imaging analysis identified clusters in the parieto-temporal region, bilaterally that were differentially related to math performance across group and sex. The left cluster was related to math in alcohol-exposed males $(\mathrm{r}=0.54, \mathrm{p}=0.02)$, but not control males $(\mathrm{r}=-$ $0.30, \mathrm{p}=0.40$ ), whereas the right cluster was related to control females $(\mathrm{r}=0.86, \mathrm{p}=0.03)$, but not alcohol-exposed females $(\mathrm{r}=-0.24, \mathrm{p}=0.60)$. Reading and spelling were not related to cortical surface area.

Conclusions: Alcohol-exposed children have 2-4x higher rates of academic impairment with the highest prevalence rate in mathematics. Reduced parieto-temporal surface area may contribute to mathematical reasoning impairment in alcohol-exposed males. Reading and spelling, while impaired in alcohol-exposed children, do not appear to have cortical surface area correlates and may relate to broader neural circuitry dysfunction.

Research supported by NIAAA grants R01 AA019605, T32 AA013525, F31 AA022033, F31 AA022261

Correspondence: Sarah N. Mattson, Ph.D., Psychology, San Diego State University, 6330 Alvarado Court, Suite 100, San Diego, CA 92104.

E-mail:sarah.mattson@sdsu.edu

\section{Symposium 8: \\ Behavioral Genetics in Neuropsychology: Exploring New Frontiers in MS and mTBI}

\author{
Chair: Peter Arnett \\ 10:15-11:45 a.m.
}

P. ARNETT, V.C. MERRITT, J. MEYER, M. CADDEN \& D. UKUEBERUWA. Behavioral Genetics in Neuropsychology: Exploring New Frontiers in MS and mTBI.

Symposium Description: In recent years, methods for the acquisition of genetic data have been greatly simplified and are remarkably inexpensive. Integrating genetic data into neuropsychological research could be richly informative but is still relatively uncommon. Still, the $\varepsilon 4$ allele of the Apolipoprotein E (APOE) genotype has been examined in some studies as a risk for functional declines following brain injury or disease, and the short allele of the Serotonin Transporter (5-HTT) genotype has been examined as either a psychiatric risk factor or a cognitively protective factor in other studies. In this symposium, we present data from studies on mild Traumatic Brain Injury (mTBI) and multiple sclerosis (MS) that integrates genetics with neuropsychological data. Peter Arnett will begin by providing a brief overview of the issues covered in the symposium. Victoria Merritt will then show that the APOE $\varepsilon 4$ allele is a risk factor for increased post-concussion symptoms following mTBI in collegiate athletes. Next, Jessica Meyer will present data revealing that 
collegiate athletes who are homozygous for the short 5-HTT allele are much more likely to show negative affective biases post-concussion compared with athletes with all other 5-HTT allele combinations, something that could put them at risk for depression. Margaret Cadden will then review data demonstrating that MS patients with the APOE $\varepsilon 4$ allele show high levels of depression regardless of disability level, whereas MS patients without this allele only show high levels of depression when they also have high levels of disability. Finally, Dede Ukueberuwa will show that the presence of the short allele in MS patients' 5-HTT genotype may be protective against working memory decline and be indicative of greater cognitive reserve than the homozygous long genotype. A discussion moderated by Peter Arnett exploring the ethical, theoretical, and practical issues raised by these data will then ensue.

Correspondence: Peter Arnett, Ph.D., Psychology, Penn State University, 352 Moore Building, University Park,PA 16802.E-mail: paa6@psu.edu

\section{V.C. MERRITT \& P. ARNETT. Identifying the Role of the Apolipoprotein E (APOE) Gene in Concussion Outcome in Collegiate Athletes.}

Objective: In recent years, there has been an increased interest in exploring the relationship between genetic factors and outcome following brain injury. However, few studies within the sports-concussion literature have examined the influence of genetic factors on outcome following concussion. Therefore, the purpose of this study was to determine how a specific genetic variant-the $\varepsilon 4$ allele of the apolipoprotein $\mathrm{E}$ (APOE) genotype-influences symptom severity following sports-related concussion.

Participants and Methods: Participants included 42 collegiate athletes (83.3\% male) who completed neuropsychological testing within three months after sustaining a concussion. Athletes also provided a buccal sample that was analyzed to determine the make-up of their APOE genotype. The average age of athletes at the time of concussion was 20.05 years, with the majority being Caucasian $(71.4 \%)$.

Results: Dependent variables included the Post-Concussion Symptoms Scale (PCSS) total score, four PCSS symptom clusters (based on previous factor analytic work), and three PCSS global indices of distress. T-tests revealed significantly higher scores for athletes with the $\varepsilon 4$ allele compared to athletes without the $\varepsilon 4$ allele on the PCSS physical symptom cluster, $\mathrm{t}=2.50, \mathrm{p}<.05$, the PCSS global severity index, $\mathrm{t}=$ $-2.43, \mathrm{p}<.05$, and the PCSS positive symptom total, $\mathrm{t}=2.26, \mathrm{p}<.05$. Additionally, the PCSS cognitive symptom cluster approached significance, $\mathrm{t}=-2.00, \mathrm{p}=.052$.

Conclusions: These findings show that athletes with the $\varepsilon 4$ allele have a tendency to report a greater number of symptoms post-concussion than athletes without the $\varepsilon 4$ allele, suggesting that the APOE $\varepsilon 4$ genotype may confer risk for increased symptoms following concussion. Future studies examining the role of the $\varepsilon 4$ allele in concussion outcome are warranted. Correspondence: Victoria C. Merritt, M.S., 372 Moore Building, University Park,PA 16802.E-mail: vcabarnes@gmail.com

\section{J. MEYER \& P. ARNETT. Influence of Serotonin Transporter Genotype Status on Affective Bias Post-concussion.}

Objective: Response to concussion is markedly variable and can include emotional as well as cognitive sequelae. Some of the variability in concussion response may be attributed to genetic differences. Variation in the promoter region of the serotonin transporter genotype (5-HTT) has been found to contribute to stress sensitivity and subsequent depressive symptoms. The present study aimed to evaluate the contribution of 5-HTT variability on post-concussive depressive symptoms as measured by an affective bias test (Affective Word List).

Method: 38 athletes tested post-concussion were included from a university-based sports concussion program. The Affective Word List was administered and affective bias was calculated by subtracting the number of negative words recalled from the number of positive words recalled. Genetic samples were obtained through buccal swabs. A cutoff score of
1 SD from the mean bias score (bias score of -4) was used to determine a significant negative bias.

Results: Significantly more athletes who were homozygous for the 5-HTT short allele (5 of $8-63 \%$ ) showed a negative affective bias post-concussion compared with athletes who were homozygous for the long allele $(0$ of $18-0 \%$ ) or heterozygous for the short allele ( 0 of 12 $-0 \%)(\chi 2=21.59, p<.001)$.

Conclusion: Our findings suggest that 5-HTT status may moderate the risk for post-concussion depression via performance-based negative affective bias, as such a bias often predicts depression more generally. Predictors of post-concussion depression, such as genetic status, can help influence recovery protocols. Future work should focus on replicating this finding in larger samples.

Correspondence: Jessica Meyer, 373 Moore Building, University Park, PA 16801. E-mail: jem508@psu.edu

\section{CADDEN \& P. ARNETT. APOE 84 Allele Alters Depression Course in MS.}

Objective: Although depression has been found to be highly co-morbid with Multiple Sclerosis (MS), little research has been done investigating genetic factors that may influence this relationship.

Participants and Methods: Fifty-four individuals with MS participating in a neuropsychological research study of MS-related factors were examined. Depression was measured using the Beck Depression Inventory-Fast Screen (BDI-FS). In addition to collecting demographic (age, education, and sex) and disease (EDSS) related variables, participants underwent genetic testing (using buccal swabs) for APOE allele status. A regression on depression (BDI-FS) controlling for demographic variables and including EDSS, APOE status ( $\varepsilon 4+$ or $\varepsilon 4-)$, and their interaction was explored.

Results: The interaction between the EDSS and APOE status was significant, $\mathrm{t}(47)=-2.20, \mathrm{p}=.034$. Simple effect testing revealed that, after controlling for demographic variables, the EDSS significantly predicted depression in $\varepsilon 4$ - individuals $(\mathrm{t}(47)=3.33, \mathrm{p}=.002)$ but did not predict depression in $\varepsilon 4+$ individuals $t(47)=-.121, p=.904)$. At low levels of disability, $\varepsilon 4+$ individuals were marginally more depressed than $\varepsilon 4-$ individuals, $\mathrm{t}(47)=-1.96, p=.056$. At high levels of disability, there was no difference in depression between $\varepsilon 4+$ and $\varepsilon 4-$ individuals.

Conclusions: APOE $\varepsilon 4+$ patients showed high levels of depression regardless of disability level, whereas those with APOE $\varepsilon 4$ - only showed high levels of depression when they also had high levels of disability. The APOE $\varepsilon 4$ allele may be a risk factor for depression at low levels of disability and potentially unyokes the relationship between high disability and greater depression in MS.

Correspondence: Margaret Cadden, 372 Moore Building, University Park, State College, PA 16802.E-mail:mhc147@psu.edu

\section{UKUEBERUWA \& P. ARNETT. Cognitive Functioning in MS and Relationship to Serotonin Transporter Genotype.}

Objective: Cognitive problems are commonly reported by people with multiple sclerosis (MS), although the presence and severity of these problems varies among individuals. Examination of genetic factors may suggest a mechanism for this variation. In healthy individuals, presence of the short allele in serotonin transporter (5-HTT) genotype has been linked to better attentional control and working memory. This study examined the role of 5-HTT genotype in cognitive functioning in MS. Participants and Methods: 22 participants with MS (18 female) completed genetic testing and a neuropsychological test battery, including the Wechsler Test of Adult Reading (WTAR) and the Paced Auditory Serial Addition Test (PASAT) 3" version. Participants' 5-HTT genotype was determined and two groups were formed based on the presence of the short allele versus the homozygous long genotype (ss/sl vs. II). Cognitive test scores were compared between groups.

Results: Groups did not show a statistical difference in age, education, or EDSS. However, consistent with predictions, the short allele group 
scored higher on the WTAR $(p<.05)$ and the PASAT $(p<.05)$ than the homozygous long group.

Conclusion: In accordance with previous studies of healthy participants. this study of MS found better working memory performance among patients with the 5-HTT short allele compared to those with the homozygous long genotype. The short allele group also showed greater premorbid intelligence, which is thought to reflect cognitive reserve in people with neurological disorders. Thus, the 5-HTT short allele may be indicative of greater cognitive reserve and protect against working memory decline in people with MS.

Correspondence: Dede Ukueberuwa, M.S., 115C Moore Building, University Park, PA 16802. E-mail: dedemu@psu.edu

\section{Poster Session 5: Imaging (Structural \& Functional) \& Psychopathology/Neuropsychiatry}

\section{0:15-11:45 a.m.}

\section{Acquired Brain Injury (TBI/ Cerebrovascular Injury \& Disease - Child)}

L.S. BLACKWELL \& A.M. TAYLOR. Gender Differences in Children and Adolescents with Head Injury.

Objective: There is growing recognition that gender may be associated with quality, duration and severity of symptoms following concussion. The objective of the current study is to further investigate the effect of gender on self-reported symptoms and neurocognitive performance following concussion.

Participants and Methods: To this end, we examined patients admitted to a hospital for head injury acutely and at follow up in a cross-sectional sample. A total of 223 patients were seen for inpatient neuropsychological screening; a second cohort of 145 patients was seen in an outpatient setting. Mean patient age was $13.24(\mathrm{SD}=3.2), 30 \%$ female and $14.4(\mathrm{SD}=3.0), 43 \%$ female, respectively. We defined premorbid risk factors as history of concussion, headache, ADHD, learning disability or emotional symptoms. Patients completed measures of working memory (digit span) and speed of processing (sequencing task).

Results: Logistic regression analyses were conducted in order to obtain relative risk ratios. Results indicated that females were more likely to experience headaches immediately post injury $(p<.01)$, have a history of headaches $(p<.05)$, anxiety $(p<.05)$ and depression $(p<.05)$ when compared to their male counterparts. No significant differences were found on working memory and speed of processing tasks in males versus females. In patients seen for at an outpatient follow up visit, females experienced significantly more headache symptoms than males $(p<.01)$, but again were not different on tasks of working memory and speed of processing.

Conclusions: Overall, these findings continue to support past literature illustrating gender as a risk factor for acute symptoms and prolonged symptom duration following concussion.

Correspondence: Laura S. Blackwell, Ph.D., Psychiatry, Boston Children's Hospital, 758 Tremont Street, \#2, Boston, MA 02118. E-mail: laura.blackwell@childrens.harvard.edu

\section{Acquired Brain Injury (TBI/ Cerebrovascular Injury \& Disease - Adult)}

H.M. MISKEY, R.D. SHURA, R.E. YOASH-GANTZ \& J.A. ROWLAND. Personality Assessment Inventory Profiles of Post-Deployment Veterans: Differential Effects of Mild Traumatic Brain Injury and Psychopathology.

Objective: Neuropsychiatric complaints often accompany mild traumatic brain injury (mTBI), a common condition in post-deployed Veterans. Objective, multi-scale personality inventories may elucidate the pattern of psychiatric distress in this cohort. This study investigated valid Personality Assessment Inventory (PAI) profiles in post-deployed Veterans.

Participants and Methods: Measures of psychopathology (SCID-I. PAI) and TBI were examined in a sample of 144 post-deployed Veterans divided into the following groups: healthy controls $(n=40)$, mTBI only $(\mathrm{n}=31)$, mental health diagnoses only $(\mathrm{MH} ; \mathrm{n}=25)$, comorbid mTBI and Posttraumatic Stress Disorder (mTBI/PTSD; $\mathrm{n}=23$ ), and comorbid mTBI, PTSD, and other psychological diagnoses (mTBI/PTSD/MH; $n$ $=25)$.

Results: There were no significant differences between the mTBI and the control group on mean PAI subscale elevation, or number of subscale elevations above $60 \mathrm{~T}$ or $70 \mathrm{~T}$. The other three groups had significantly higher overall mean scores, and more elevations above 60T and 70T compared to both controls and mTBI only. The mTBI/PTSD/MH group showed the highest and most elevations. ANOVAs investigating potential moderation effects revealed no interaction effects between mTBI and presence of psychopathology. After entering demographics, mTBI, PTSD, and other psychological diagnoses into hierarchical regressions using the entire sample, PTSD and other diagnoses were significant predictors of overall mean score and number of elevations above 60T or 70T. MTBI was not a significant predictor of any outcome measure. Conclusions: This study suggests that mTBI alone is not uniquely related to psychiatric distress in Veterans, but that PTSD and mental health diagnoses account for self-reported symptom distress.

Correspondence: Holly M. Miskey, PhD, Mental Health and Behavioral Sciences, Hefner VA Medical Center, 1601 Brenner Avenue, 11M-2/ MH\&BS, Salisbury, NC 28144.E-mail: Holly.Miskey@va.gov

\section{Acquired Brain Injury (TBI/ Cerebrovascular Injury \& Disease - Child)}

J.M. SMITH, B. KUROWSKI, H.G. TAYLOR, K.O. YEATES, T. STANCIN, N. MINICH, P. CHIU \& S.L. WADE. Genetic and Environmental Influences on Executive Functioning after Pediatric Traumatic Brain Injury.

Objective: The primary aim of this study was to evaluate the association of the dopamine receptor D4 7 repeat (DRD4-7) allele, a gene linked to dysfunctional higher order cognitive processes, with measures of executive function $(\mathrm{EF})$ in children and to examine how these associations are moderated by pediatric traumatic brain injury (TBI) and home environment.

Participants and Methods: 174 children ages 3-7 years (87 TBI, 87 orthopedic controls [OI]) completed 6 month assessments post-injury. Of those, 122 participants $(53.3 \%$ male, $76.2 \%$ white $)$ provided DNA samples (67.2\% DRD4-7 absent, 32.8\% DRD4-7 present). The Verbal Fluency subtest of the NEPSY and Shape School were used to assess EF. The Home Observation for Measurement of the Environment (HOME) Inventory was used to assess environmental factors.

Results: Regression analyses found a significant interaction of gene by injury type for Verbal Fluency scores $(p=.001)$. An OI and absence of the DRD4-7 allele predicted significantly better scores $(\mathrm{M}=10.68)$ than the other three groups (M range: 8.40-8.79). A significant triple interaction of gene $\mathrm{x}$ injury type $\mathrm{x}$ HOME score was also found for Total Efficiency scores on the Switch subtest of the Shape School $(p=.02)$. 
The group with presence of the DRD4-7 allele, TBI and low HOME scores had the lowest mean score $(\mathrm{M}=0.36, \mathrm{SE}=.06)$, whereas the opposite group (absence of DRD4-7 allele, OI, high HOME scores) had the highest mean scores $(\mathrm{M}=0.56, \mathrm{SE}=.03)$.

Conclusions: Preliminary analyses suggest that a combination of genetic and environmental factors may influence recovery following a pediatric TBI.

Correspondence: Julia M. Smith, Clinical Psychology PhD, Psychology, University of Cincinnati, 5562 Verulam Ave, Apt \#17, Cincinnati, OH 45213.E-mail:smith8j2@mail.uc.edu

\section{Imaging (Functional)}

A.F. LIM, C. GONZALEZ, L. CARRIER \& R.J. YOUMANS. Neural Correlates for a New Cognitive Flexibility Measure Using Functional Near-Infrared Spectroscopy.

Objective: The current study was conducted using functional near-infrared spectroscopy (fNIR) to gather converging evidence of neural correlates within the PFC for a new cognitive flexibility (CF) puzzle-task measure (Gonzalez et al, 2013). CF is typically defined as one's ability to abandon one strategy in favor of a more optimal strategy. We predicted that higher activation of oxygenated hemoglobin (oxy- $\mathrm{Hb})$ in the PFC would occur as a function of more switch-moves required to complete the $\mathrm{CF}$ task, and also predicted that there would be a significant relationship between lower switch costs and higher levels of oxy-Hb across the PFC.

Participants and Methods: Twenty-two college students participated and were randomly assigned to one of three groups: random switch order $(n=6)$, low-to-high switch order $(n=10)$, and high-to-low $(n=6)$ switch order. Participants included both (males $=8$; females=14) and the average age ( $\mathrm{M}=24.2$ years). Participants wore the 16 -channel fNIR device on their forehead while completing a computerized CF puzzle-task which included seven trial blocks (2-14 switch moves per trial). Measures on the CF task includes switch costs (i.e., difference between switch and non-switch move times averaged across all trials), and neural activity was assessed by measuring absorption changes of oxygenated and deoxygenated-hemoglobin in the PFC.

Results: Bivariate correlations were conducted between switch costs and switch moves across all 16-channels to assess whether higher oxy-Hb was associated with lower switch costs (i.e., higher CF; faster performance). However, we did not find any support for our hypotheses to suggest any significant relationship between higher oxy-Hb and lower switch costs, and switch moves.

Conclusions: Despite finding no support for our hypotheses, we did however find trends in the direction we predicted. We consistently found that lower switch costs were negatively associated with higher levels of oxy-Hb within the PFC, although additional work would be needed to further investigate this.

Correspondence: Alex F. Lim, MA, Experimental Psychology, Psychology, California State University, Dominguez Hills, 1308 Greenwood Ave., Torrance,CA 90503.E-mail: alim@csudh.edu

D. MALFAIT, A. TUCHOLKA, J. LEROUX, L. CARMANT, M. SROUR, P. MAJOR \& S. LIPPÉ. Children with Benign Epilepsy with Centro-temporal Spikes: an fMRI, Cognitive and Behavioral Study.

Objective: Children with benign epilepsy with centro-temporal spikes tend to show cognitive impairments, especially in language. In this study, our aim was to understand brain correlates of reading comprehension abilities in Rolandic epilepsy (RE), and to draw an overall picture of these children.

Participants and Methods: 15 children with RE (11 y.o. \pm 4 m; 12 boys) and 18 healthy children ( 11 y.o. $8 \mathrm{~m} . \pm 20 \mathrm{~m}$.; 11 boys) performed an fMRI task in which they read a pair of sentences and decided whether the first sentence was true or false given the meaning of the previous one. In addition, a standard neuropsychological assessment was also conducted with each child and parents completed behavioral questionnaires. We performed analysis using SPSS and SPM. To study brain activity, we used an ROI approach for group comparison. We also applied correlations between neuropsychological variables and brain activity.

Results: In heatlhy children, reading task revealed significant brain activations in 5 different clusters ( $p<0.01 \mathrm{FWE}$ corr.): left inferior frontal gyrus, left middle temporal gyrus, left superior/middle temporal gyrus $(\mathrm{p}=0,000)$, left supramarginal and angular gyri, right superior/ middle temporal gyrus. Moreover, significant differences between groups were found in the left caudate and putamen ROI $(p=0,001)$. In addition, patients demonstrated impairments in language, working memory, attentionnal and executive domains. We also found some interesting correlations between cognitive scores and brain activity, especially between left putamen and verbal memory $(r=0,663 ; p=0,019)$.

Conclusions: We mentioned various cognitive impairments in epileptic patients and we created an fMRI task adapted for children, which activates significantly language specific regions. Even if patients showed in general a similar reading network compared to controls, we have demonstrated differences in left putamen and caudate. This constitutes an interesting finding given that some structural abnormalities have been found in striatum in patients with RE.

Correspondence: Domitille Malfait, Psychology, University of Montreal, 61 avenue Mont Royal Est, app.2, Montreal, QC H2T 1N6, Canada. E-mail:domitille.malfait@umontreal.ca

N.K. MILLER, J.K. FAUSETT, J.L. GESS, H.B. MORAIS \& G. JAMES. Neuroanatomical Contributions to Planning and the Relationship Between Resting State fMRI and DKEFS Tower Test. Objective: The Tower of London (ToL) assesses clinical deficits in planning and sustained attention. Functional neuroimaging studies have related these deficits to disruptions of frontoparietal and frontostriatal networks. The purpose of this study was to examine the relationship of DKEFS Tower performance to the brain's functional organization during wakeful rest in order to better understand functional neuroanatomical contributions to planning.

Participants and Methods: These data were collected through the Cognitive Connectome project, which seeks to map neural representations of cognition in a normative sample of healthy adults $(\mathrm{n}=37$; males and females aged 18-50). Independent component analysis (ICA) of resting-state fMRI data identified 70 functionally unique neural networks. Robust linear regression related functional connectivity (the correlation between networks' activity time courses) during wakeful rest to total score on the DKEFS Tower test.

Results: Tower performance corresponded to decreased resting-state connectivity between inferior occipital lobe and bilateral striatum $(\mathrm{t}<-$ $4.1)$, between right and left frontoparietal $(t<-3.6)$, and between primary visual area and bilateral superior temporal sulci $(\mathrm{t}<-3.3)$.

Conclusions: These regions have been associated with visual processing, planning, and working memory. While modest, our findings suggest that greater independence among these regions at rest (as indicated by weaker correlations) may facilitate visually-presented planning. Findings provide an initial step in understanding neuroanatomical contributions to visual planning within a normal sample. Future research is required to gain greater understanding of these networks to better understand abnormal neural representations within clinical populations. Correspondence: Nichole K. Miller, Psy.D, Clinical Psychology, The Chicago School of Professional Psychology, 1603 Rockwater Blvd, \#301, North Little Rock, AR 72114. E-mail:nkm5120@ego.thechicagoschool. edu 
R.S. SCHEIBEL, N.J. PASTOREK, M. TROYANSKAYA, J. KENNEDY, J.L. STEINBERG, M. NEWSOME, X. LIN \& H. LEVIN. Altered Brain Activation Associated with Posttraumatic Stress Symptoms.

Objective: Studies using cognitive paradigms have provided evidence for attenuated activation in association with posttraumatic stress symptoms (PTSS) within components of an executive control network, including prefrontal cortex. The present investigation examined the relationship between PTSS and activation during a cognitive control paradigm, the Arrows Task, in subjects who had been deployed to Afghanistan or Iraq. Participants and Methods: Functional magnetic resonance imaging was performed with seven subjects who reported current PTSS with scores of 39 or more on the Posttraumatic Stress Disorder Checklist - Civilian, but who had not sustained a traumatic brain injury or experienced blast exposure during deployment.

Results: During the Arrows Task these subjects exhibited large activation decreases (i.e., deactivation) and a regression analysis revealed decreasing activation in association with greater PTSS (i.e., negative correlation) within higher-order association areas of the frontal, temporal, and parietal cortex. Greater PTSS severity was also associated with heightened activation (i.e., positive correlation) within structures with basic sensory and motor functions, as well as some that are thought to have a role in monitoring and regulating internal bodily states (i.e., insula, right orbitofrontal cortex).

Conclusions: Deactivation extended beyond the executive control network and the pattern of positive and negative regression findings is consistent with widespread neural dysfunction, including changes similar to those thought to occur under conditions of acute stress and elevated noradrenergic activity.

Correspondence: Randall S. Scheibel, Ph.D., Physical Medicine and Rehabilitation, Baylor College of Medicine, 1709 Dryden Road, Suite 1200, Houston, TX 77030.E-mail: scheibel@bcm.tmc.edu

\section{J. THOMPSON, E.A. WILDE \& S.D. GALE. Social Outcome and Fractional Anisotropy of the Frontal Lobe in Children with Chronic Traumatic Brain Injury.}

Objective: To examine the association between fractional anisotropy (FA), social adjustment, and its related behaviors (i.e. attention, anxiety, and school problems) in children with a history of chronic traumatic brain injury (TBI).

Participants and Methods: Twenty-one children (57\% male; $\mathrm{M}$ age $=$ 12.8 years, $\mathrm{SD}=2.3$ years) at least 1 year post-injury were evaluated with the Child Behavior Checklist (CBCL), as completed by their caregivers, and underwent diffusion tensor imaging (DTI). Social outcome was evaluated using the Social Problems, Anxiety Problems, School Problems, and Attention Problems subscales from the CBCL. DTI data was analyzed using PRIDE software. Bivariate regression was used to determine whether there is a correlation between FA values in the frontal lobes and subscale scores from the CBCL.

Results: There was not a significant correlation between FA values in the left or right frontal lobes and the Social Problems subscale ( $p>.05)$. However, left frontal FA values significantly predicted School, Anxiety, and Attention Problems $(\mathrm{p}<.05)$. In contrast, right frontal FA values were not predictive of these behavioral outcome measures ( $p>.05)$.

Conclusions: FA values in children with chronic TBI were predictive of measures of attention, anxiety, and problems in school one year post-injury. Specifically, FA in the left, but not right, frontal lobe predicted performance even though there was no significant difference in FA values between hemispheres. Further study including replication is warranted to evaluate this potential hemispheric difference in the association between frontal lobe FA and behavioral outcome in children greater than one year post-injury.

Correspondence: Juliann Thompson, Clinical Psychology, Brigham Young University, 1190 North 900 East, Provo, UT 84604. E-mail: thompson.juliann@gmail.com
C. TAKEDA, M. NOTOYA, N. SUNAHARA, D. KIMURA, K. INOUE, T. FUJITA \& K. NAKATANI. Effects of order of task execution on Trail-Making Test performance.

Objective: The Trail-Making Test (TMT) is a measure of frontal lobe function comprising two tasks (Parts A and B). A previous study has indicated that the order of TMT task presentation is a factor affecting frontal lobe activity during the execution of Part B. Focusing on task order, the present study used near-infrared spectroscopy (NIRS) to re-investigate the effects of the order of TMT presentation on TMT execution. Participants and Methods: The participants were 48 healthy adults (mean age, 22.8 \pm 2.5 years). They were divided into two groups based on whether they completed Part A (Group-A) or Part B (Group-B) first. NIRS measurements were performed using an ETG-4000 optical topography system with 44 channels to measure changes in oxyhemoglobin $($ oxy-Hb) concentration in bilateral frontal lobes during TMT. The oxy-Hb value for Part A was subtracted from the oxy-Hb value for Part B (B-A oxy-Hb) to obtain an index for frontal lobe activity during Part B. Results: B-A oxy-Hb values were significantly greater in Group-B than in Group-A ( $\mathrm{t}$-test, $\mathrm{p}<0.05)$. Multiple regression analysis was conducted with the three factors (sex, knowledge of TMT, order of TMT) suggested in a previous study to affect TMT performance as independent variables and demonstrated that order of task presentation affected performance. Conclusions: The present study demonstrated greater oxy-Hb values for Part B when Part B was presented before rather than after Part A. The present findings suggest that consideration should be given to the order of task presentation when administering the TMT.

Correspondence: , , E-mail:

D. PUTCHA, R.S. ROSS, A. CRONIN-GOLOMB, A.C. JANES \& C.E. STERN. Altered Intrinsic Functional Coupling Between Core Neurocognitive Networks in Parkinson's Disease.

Objective: Three core neurocognitive networks, the default-mode network (DMN), the salience network (SN), and the central executive network (CEN), have been established as critical to high-level cognitive function. Striatal function may modulate the interaction between these core networks. Parkinson's disease (PD) pathophysiology is characterized by nigrostriatal dopamine depletion, which may affect the interaction between these large-scale neurocognitive networks.

Participants and Methods: 24 non-demented individuals with PD and 20 age-matched control participants (MC) were scanned during rest with fMRI. Inter-network coupling between the three core neurocognitive networks and the striatum were examined due to known striatal disruptions in PD.

Results: We establish that in MC, the CEN is positively coupled with the SN and negatively coupled with the DMN. In contrast, the PD group demonstrated less positive CEN coupling with the $\mathrm{SN}(\mathrm{t}=2.4, \mathrm{p}=0.02)$ and more positive coupling between the CEN and the DMN $(t=2.1$, $\mathrm{p}=0.04$ ), compared to MC. Additionally, increased disease severity in PD is related to reduced functional coupling between the striatum and $\mathrm{SN}$, suggesting that striatal dysfunction disrupts intrinsic SN activity $(\mathrm{r}=-0.45, \mathrm{p}=0.03)$.

Conclusions: These results illustrate that in individuals with PD intrinsic functional connectivity between the CEN and both the salience and default mode networks is disrupted, suggesting that the striatum may be critical to controlling the interaction between these core neurocognitive networks. Furthermore, we suggest that disrupted interactions between these three core neurocognitive networks likely underlie cognitive impairments associated with PD.

Correspondence: Deepti Putcha, M.A., Department of Psychological and Brain Sciences, Boston University, 2 Cummington Mall, Room 109, Boston, MA 02215. E-mail: dputcha@bu.edu

U.M. VENKATESAN \& F.G. HILLARY. Organization of Functional Network Representations within Posterolateral Parietal Cortex. Objective: Recent work in neural connectivity has identified densely interconnected "rich club" hub regions, which occupy important roles 
in functional brain networks. However, the posterolateral parietal cortex (PPC), comprising the inferior parietal lobule (IPL) and intraparietal sulcus (IPS), are not included in this definition of hubs despite their participation in diverse cognitive functions and networks. The current study examined resting-state functional connectivity (RSFC) of anatomic subregions of the IPL and IPS to further characterize PPC functional heterogeneity and its relation to large-scale networks.

Participants and Methods: Fifteen healthy participants completed resting-state fMRI. Ten IPL and IPS subregions were defined in group data using a probabilistic cytoarchitectonic atlas. Connectivity analyses included: 1) seed-voxel connectivity of each seed; 2 ) seed-seed connectivity within the IPL/IPS; and 3) quantification of IPL/IPS network structure using graph theory metrics.

Results: RSFC of anterior IPL, posterior IPL, and middle IPL and IPS regions was observed with traditional salience, default, and executive control regions, respectively. Posterior IPL seeds showed no connectivity with anterior IPL, but portions of both maintained connectivity to middle IPL (cytoarchitectonic area PFm) and IPS seeds. In graph analyses, betweenness centrality was highest for PFm and middle IPS (cyto. hIP2). IPS seeds also evidenced relatively high clustering coefficients and degrees.

Conclusions: Consistent with previous literature, the present study indicates functional heterogeneity of the PPC. Further, results suggest that network representations within this region converge on distinct anatomic subregions that serve as local hubs, or links between large-scale networks not readily evident in whole-brain analyses. Future research should work to clarify how "micro-networks" of the PPC relate to brain function more globally, as this may have implications for neurological conditions involving disrupted neural networks.

Correspondence: Umesh M. Venkatesan, Psychology, The Pennsylvania State University, 419 Moore Building, University Park, PA 16802. E-mail: umv5002@psu.edu

\section{A. ROY, R.A. BERNIER, J. FRENCH, D. FEGER \& F.G. HILLARY. A novel voxel-based approach to examine network plasticity during recovery from Traumatic Brain Injury.}

Objective: Several studies have confirmed that traumatic brain injury (TBI) leads to altered functional connectivity in the brain. This study aims to understand how functionally connected regional-pairs (FCRPs) in a resting-state network evolve during recovery from moderate to severe TBI. Using whole-brain analyses, we present a data-driven voxel-based approach to discover the FCRPs that distinguish the TBI-subjects from the controls.

Participants and Methods: Subjects included 13 individuals with moderate to severe TBI between the ages of 21 and 54, and 12 healthy adults with comparable age and education. Individuals with TBI were examined using fMRI at 3, 6, and 12 months following resolution of post traumatic amnesia. The controls were examined at 2 time points separated by 3 months to establish a stable functionally-connected (FC) neural-network. Desired FCRPs were found by first selecting the voxel-pairs for which the overlap between the control and the TBI FC strength (correlation-coefficient) distribution was minimal (ROC-value $>0.95$ ) and then grouping them by adapting a cluster-pairing approach developed by Zalesky, 2012.

Results: The number of FCRPs characterizing the TBIs at months 6 and 12 was significantly larger than at month 3 . At 3rd and 6th month most functional-connections for TBI subjects were relatively weaker than the controls. In contrast, after 12 months post-injury hyper-connectivity was observed.

Conclusions: A voxel-based approach provides sensitivity to network plasticity during the first year of recovery from TBI. The early results suggest the FC map of a TBI subject drifts away from the controls during the recovery period showing heightened connectivity at 1-year post injury.
Correspondence: Arnab Roy, Ph.D., Psychology, pennsylvania state university, Moore-419, University park, State college, PA 16801. E-mail: aroy2@binghamton.edu

J.L. WOODARD, M.A. SUGARMAN, K. MEYERS, E. GROSS, A.L. NORMAN, M. SEIDENBERG, K.A. NIELSON, J. SMITH, K. REITHER \&. S.M. RAO. A Novel Method for Assessing Behavioral and Neural Correlates of Levels of Semantic Knowledge.

Objective: Imaging studies of semantic memory typically use tasks that reflect basic familiarity with a presented stimulus, such as a famous name. Although these tasks may characterize broad aspects of semantic memory functioning, they are limited in their ability to evaluate higher-order nuances in semantic knowledge networks and organization. We developed a novel strategy for assessing higher-order category and attribute-level semantic knowledge. This task can be used in behavioral and neuroimaging studies to investigate the hierarchical structure of semantic memory.

Participants and Methods: Eleven cognitively intact, community-dwelling adults over age 65 (mean age $=69.5$ years) participated. During task-activated functional magnetic resonance imaging (fMRI), participants were asked to discriminate via button press: 1) famous and non-famous names (familiarity); 2) which of two occupational categories is most associated with a target name (category knowledge); 3) which of two specific attributes (e.g., song or movie title) is most associated with a target name (attribute knowledge).

Results: Accuracies for all tasks exceeded 88\%. Mean response times increased significantly in correspondence with the depth of semantic knowledge required for a correct response (familiarity $=1005 \mathrm{msec}$, category knowledge $=1619 \mathrm{msec}$, attribute knowledge $=1947 \mathrm{msec}$, $p$ 's<.001). We detected fMRI activation in regions consistent with the semantic memory and default mode networks, with increasingly greater extent and magnitude observed for tasks requiring greater semantic knowledge specificity.

Conclusions: Our newly developed semantic knowledge tasks show behavioral and imaging correlates of superordinate categorical and attribute levels of semantic knowledge. Varying the concept specificity of semantic probes may facilitate evaluation of the functional neuroanatomy associated with semantic knowledge storage and access.

Correspondence: John L. Woodard, Ph.D., Psychology, Wayne State University, 5057 Woodward Ave., 7th Floor, Detroit, MI 48202. E-mail: john.woodard@wayne.edu

B. DUDA, B.E. HAWKSHEAD, N.F. SCHWARZ, X. XU, U.S. CLARK, J. GUNSTAD, L. MILLER \& L.H. SWEET. Cognitive Reserve Predicts Bilateral Compensatory Brain Activation in Older Adults During a Working Memory Task.

Objective: Examine the influence of cognitive reserve (CR) on the relation between left and right middle frontal (LMF/RMF) regions (caudal and rostral middle frontral gyrii [MFG]) in older adults. Consistent with models of increased bilateralization in older adults and expected left lateralization of the verbal working memory paradigm (VWM), we hypothesized that CR would predict brain activity in the RMF regions over and above age, WM performance, and their LMF homologues.

Participants and Methods: 34 right-handed, healthy older adults (Mage $=64.35)$ underwent FMRI and a brief neuropsychological assessment. Formal years of education and a premorbid IQ estimate were combined into a composite proxy of CR. Neural correlates of VWM were assessed using an N-back paradigm with echoplanar BOLD-FMRI on a Siemens 3T system. FMRI response to the 2-Back compared to 0 -Back control task was found to be significant in all four frontal a priori ROIs selected from a meta-analysis on the N-back. We conducted two multiple regressions to test our hypotheses. We then conducted two moderation analyses to further investigate the influence of CR on the relationship of each RMF/LMF set.

Results: CR significantly predicted response to the 2 -Back in the caudal MFG ( $\mathrm{R} 2$ change $=.046, \mathrm{~B}=.219 ; \mathrm{p}=.019)$. CR predicted the right rostral 
MFG response ( $\mathrm{R} 2$ change $=.040, \mathrm{~B}=.202 ; \mathrm{p}=.050)$. The left rostral $\mathrm{MFG}^{*} \mathrm{CR}$ interaction term was not significant. The left caudal MFG activity ${ }^{*} \mathrm{CR}$ term explained a significant amount of variance in right caudal MFG activity ( $\mathrm{R} 2$ change $=.031, \mathrm{~B}=.-200 ; \mathrm{p}=.039$ ). Simple slopes revealed a significant association between left and right caudal MFG. Conclusions: Results suggest that CR may predict bilateral compensatory brain activation performed by older adults during a VWM task. Additionally, results suggest that individuals with lower levels of CR are more likely to exhibit bilateral compensatory activation. This finding is consistent with the Hemispheric-Assymtery Reduction in Older Adults (HAROLD) model.

Correspondence: Bryant Duda, University of Georgia, 1197 Cedar Shoals Drive, Apt 109, Athens, GA 30605.E-mail: bryant.duda@gmail. com

\section{J. YUAN, H. BLUMEN \& R. HOLTZER. Intrinsic Functional} Connectivity of Gait and Executive Functioning in Older Adults.

Objective: The relationship between cognition and gait is robust, particularly in aging. Dual-task methodology assesses a defined facet of executive functioning and has been used to demonstrate the causal role that attention and executive functioning have in the cortical control of gait under single- and dual-task conditions. Functional neuroimaging studies of gait, however, have been scarce due to methodological limitations of existing neuroimaging methods. Additionally, executive functioning is multifactorial. We utilized resting-state fMRI to identify shared and distinct intrinsic neural activity of gait (under single- and dual-task conditions) and commonly used neuropsychological measures of executive functioning in older adults.

Participants and Methods: 6 minutes of resting-state fMRI data were acquired in 30 cognitively-healthy older adults. We performed independent components analysis to separate resting-state fMRI data into 20 group-level statistically independent spatial components correlated with gait velocity under single- and dual-task conditions, as well as performance on a number of neuropsychological tests that assess executive functioning.

Results: Both single- and dual-task gait velocities were correlated with intrinsic functional connectivity in fronto-parietal regions. Dual-task gait velocity, however, was uniquely associated with increased connectivity in the prefrontal cortex. As expected, intrinsic functional connectivity in prefrontal regions was also associated with several measures of executive functioning.

Conclusions: Our results provide additional evidence that executive functioning and its underlying functional brain substrates are fundamental in supporting gait, particularly when cognitive demands are maximized. In addition, our findings encourage further investigation as to whether patterns of intrinsic functional connectivity follow the distinctions apparent in other neuroimaging studies between subcomponents of executive functioning.

Correspondence: Jennifer Yuan, Ferkauf Graduate School of Psychology, 205 E 95th St Apt 26B, New York, NY 10128.E-mail: jenniferdyuan@ gmail.com

J.J. STEWART, D. AMEN, K. WILLEUMIER \& C. GOLDEN. Diagnostic Accuracy of SPECT Scans: Examining Specific Brain Areas of Hypoperfusion at Baseline in Alzheimer's Disease.

Objective: To investigate the sensitivity and specificity of regional hypoperfusion, as measured by single photon emission computerized tomography (SPECT), in distinguishing Alzheimer's disease (AD) patients within an unmatched mixed clinical sample.

Participants and Methods: A randomized mixed clinical subsample of 118 adults, who participated in a larger SPECT study, were included. The mean age was 49 years and $62 \%$ of the sample was male. Clinical categories in the sample included Dementia of the Alzheimer's Type $(n=28)$, Vascular Dementia ( $n=5)$, PTSD $(n=9)$, unspecified Anxiety $(n=26)$, unspecified Depressions $(n=36)$, Bipolar $(n=3)$, brain trauma $(n=48)$, Schizophrenia/Psychosis $(n=3)$, Substance Use disorders $(\mathrm{n}=13)$ and normal aging adults. Individuals with comorbid diagnoses were excluded. Participants were administered baseline SPECT scans and self-report psychiatric and cognitive symptom questionnaires.

Results: Area under a ROC curve (AUC) was calculated for 128 specific regions of measured perfusion and the "memory problems" factor from the self-report questionnaire. Discrimination analysis showed the following areas under the ROC curves: right amygdala ( $\mathrm{AUC}=.750 ; 95 \%$ CI $[.650, .895])$; left $(\mathrm{AUC}=.762 ; 95 \%$ CI $[.621, .904])$ and right (AUC $=.736 ; 95 \%$ CI $[.591, .880])$ palladium; left $(\mathrm{AUC}=.742 ; 95 \%$ CI $[.594$, $.889])$ and right ( $\mathrm{AUC}=.694 ; 95 \% \mathrm{CI}[.538, .851])$ putamen. Cerebellar hypoperfusion also showed significant discrimination between Alzheimer's and non-Alzheimer's patients. The 7-item self-repot "memory problems" factor had less than chance sensitivity and specificity (AUC $=.433 ; 95 \%$ CI $[.295, .572])$.

Conclusions: Findings confirm that certain areas of the brain show reduced blood flow specific to Alzheimer's disease. Hypoperfusion to these areas have clinical utility for identifying individuals with Alzheimer's and parsing out those who meet the criteria for a differential diagnosis. As AUC values were <.8; SPECT data should be integrated with additional diagnostic information.

Correspondence: Jada J. Stewart, M.S., Center for Psychological Studies, Nova Southeastern University, 220 6th Ave N., Apt 7, St. Petersburg, FL33701.E-mail:js565@nova.edu

\section{K.M. ZIMMERMANN \& A. JANSEN. Connectivity of the bilateral FFA during eye gaze perception.}

Objective: The current model of face perception consists of two cortical systems. The "core system" is formed by the occipital face area (OFA), the fusiform face area (FFA) and the superior temporal sulcus (STS). The "extended system" is composed of variable cortical areas that contribute to the prevailing process according to context. In the context of eye gaze, brain regions associated with the spatial attention network seem to be involved. Although face perception is a right-lateralized process, each region of the "core system" has its homologue in the left brain halve. The present fMRI study aims to extend existing findings by investigating the connectivity of the right and the often neglected left FFA during the observation of eye gaze.

Participants and Methods: 20 male subjects (22-29 years) participated in the experiment. They observed videos showing a virtual face with moving eyes (DAZ Productions, UT) or rigid scrambled pictures. The eyes of the faces either moved to the left or right or were opened and closed. For the contrast eyes left/right against eyes open/close, we performed general psychophysiological interaction (gPPI) analyses using the left and right FFA as seed regions, respectively. FFA seeds were defined by the application of an independent face localizer task (faces against objects).

Results: The left FFA showed context-dependent functional coupling with the left superior orbitofrontal gyrus, the left temporoparietal junction and the left inferior occipital gyrus ( $p<.001$, uncorr.). The gPPI analysis employing the right $\mathrm{FFA}$ as seed region revealed functional coupling with the right temporoparietal junction $(\mathrm{p}<.001$, uncorr.). Conclusions: The gPPI analysis depicted functional coupling of the right and the left FFA with ipsilateral brain regions. The temporoparietal junction and the orbitofrontal gyrus are typically associated with spatial attentional processing and their connectivity with the FFA could possibly relate to subjects shifting their attentional focus in the opponents' direction.

Correspondence: Kristin M. Zimmermann, Brainimaging, University of Marburg, Rudolf-Bultmann-Str. 8, Marburg 35039, Germany. E-mail: Kristin.Zimmermann@med.uni-marburg.de

M. GONZALES, T. TARUMI, R. SKOCYPEC, H. TANAKA \& A. HALEY. Aerobic Fitness and the Brain at Midlife: Evidence for the Role of the Cerebellum.

Objective: Accumulating evidence indicates that aerobic fitness attenuates age-related cognitive decline. Understanding the mechanisms 
governing fitness-related cognitive enhancement, particularly within middle-aged adults, may yield important insights into neural flexibility in the adult brain. Accordingly, the aim of the current study was to explore the impact of cardiorespiratory fitness on cognition and its underlying neural substrates in middle-aged adults.

Participants and Methods: Thirty-two endurance-trained and twenty-four sedentary adults, aged 40-65 years, underwent general health assessment, neuropsychological testing, and functional magnetic resonance imaging (fMRI) during a 2-Back working memory task. Group differences in working memory-related blood oxygen level-dependent (BOLD) response were assessed with mixed effects analyses, thresholded by retaining clusters of $\mathrm{Z}>2.3$ and applying a familywise error corrected cluster significance $(\mathrm{p}<0.05)$.

Results: As compared with sedentary controls, the endurance-trained group displayed faster reaction time on the working memory task ( $t(54)$ $=-2.125, \mathrm{p}=0.038)$ and a modest trend towards enhanced executive function performance $(\mathrm{t}(54)=1.702, \mathrm{p}=0.095)$. fMRI analyses revealed that the endurance-trained group displayed higher BOLD response bilaterally in the cerebellum for the 1-Back $>0$-Back contrast (left cerebellum: Max Z-Stat $=4.33$, MNI Coordinates: $(-2,-80,-26)$; right cerebellum: Max Z-Stat $=3.42$, MNI Coordinates: $(34,-52,-34))$. Conclusions: The cerebellum plays a critical role in governing executive function performance through its diffuse connections with the prefrontal and parietal cortices. Thus, greater task-related cerebellar activation in the endurance-trained group may indicate enhanced neural efficiency that may serve to facilitate executive function performance and preserve cognitive function across the lifespan.

Correspondence: Mitzi Gonzales, Psychology, University of Texas Austin, 2908 Pearl St Unit D, Austin, TX 78705.E-mail: mitzi.m.gonzales@ gmail.com

J.S. PRICE, A.C. JANES, M. CHAKRAVARTY, S. LUKAS \& M. COPERSINO. Thalamo-cortico-thalamic Circuitry Differences in Emerging Adult Nicotine Users Seeking Treatment for Substance Dependence.

Objective: Substance dependence is characterized by a lack of behavioral control in the context of increased reward to drug stimuli; further, expanded connectivity patterns in the default mode network (DMN) has been demonstrated. Such difficulty in self-modulatory behavior is especially pertinent to youth engaged in substance use and for whom ongoing neurodevelopment is a consideration. The present study sought to examine anatomic and connectivity indices in a sample of emerging adult smokers with and without polydrug dependence.

Participants and Methods: Participants were 11 polydrug-dependent, emerging adult patients engaged in relapse prevention partial hospitalization treatment (8 males; ages 18-25) and 18 non-drug using healthy controls (8 males). Both groups were nicotine smokers.

Results: Subcortical volumes and surface areas were examined in the polydrug-using nicotine smokers and compared to a demographically similar group of non-substance using nicotine smokers. Gray matter volumes in the left thalamus, left striatum, and bilateral thalamic sub-regions were significantly greater in non-drug using controls than in substance use dependent patients; there were no between group differences in surface areas. MELODIC analysis of resting-state fMRI data yielded 32 independent components. There was an anti-correlation between demeaned days of drug use in the past 30 days and DMN connectivity (cluster corrected to $\mathrm{Z}=2.3, \mathrm{p}<.05,5,000$ permutation testing). As any drug use decreased over the past month, there was increased connectivity between the DMN and insula and the posterior cingulate cortex. Conclusions: Significant sub-cortical morphometric differences, particularly in thalamic sub-nuclei, were found between drug users and healthy controls. These findings support an association between thalamo-cortico-thalamic circuitry. Results of the analysis of resting-state fMRI data support significant associations in insula and posterior cingulate cortex functional connectivity and quantity of recent drug use in young adults.
Correspondence: Jenessa S. Price, Ph.D., Psychiatry, McLean Hospital - Harvard Medical School, 115 Mill St., Belmont, MA 02478. E-mail: jprice@mclean.harvard.edu

\section{P. HERHOLZ, V. SCHUSTER, S. FRÄSSLE \& A. JANSEN. Lateralization Paradigms in fMRI Studies - Robustness and Reliability.}

Objective: A variety of imaging studies used an even greater variety of paradigms to assess cognitive functions and their lateralization. For many of these paradigms however, neither reliability nor robustness has been formally tested so far. In the present study, we therefore aimed to assess the test-retest reliability of routinely used fMRI paradigms for language (LA) (a word generation task (WGT), and a semantic fluency taks (SFT)), visuospatial attention (VSA) (two version of the Landmark task (LT)) and face perception (FP) (a one-back task (FPo) and a passive presentation task (FPp)) at both, the group and single subject level. Participants and Methods: 20 subjects (10 men, age range 20-30 years) performed all paradigms twice, in a randomised order. The time interval between the sessions was one week. All data were acquired on a 3-Tesla TIM-Trio MR Scanner (Siemens medical systems) and analysed with SPM8. To assess the test-retest reliability we calculated for each voxel intra-class coefficients (ICCs) using the matlab-based ICC-toolbox (Caceres et al. 2009). Median ICCs were subsequently calculated for the whole brain, the activated network and task specific regions-of-interest (ROIs). Reliability was described by the median ICC at both the group level and in single subjects.

Results: All paradigms evoked the expected activation patterns. ICCs at the whole brain and within activated networks were mostly poor $($ ICC $>0.4)$ (with the exception of WGT and SFT), whereas ICCs of specific ROIs were good to excellent. While the results at group level were stable at any point, the results in single subjects showed a higher variance (especially for the LT).

Conclusions: LA, VSA and FP and their lateralization can be robustly detected by the investigated paradigms at group level but not necessarily in single subjects. Furthermore the results revealed a possible task dependency of the activated networks and their ICCs. Therefore future studies have to consider these findings and address it by improving existing paradigms.

Correspondence: Peer Herholz, Clinic for Psychiatry and Psychotherapy, Section of Brainimaging, University hospital Giessen and Marburg, Rudolf-Bultmann-Straße 8, Marburg 35033, Germany. E-mail: herholz@staff.uni-marburg.de

V. SCHUSTER, P. HERHOLZ, S. FRÄSSLE \& A. JANSEN. In Search of a Robust and Reliable Paradigm to Assess Visuospatial Functions: A Functional MRI Study.

Objective: In the present study, we aimed to optimize routinely used fMRI paradigms for visuospatial functions (VSF) to robustly and reliably assess hemispheric lateralization at the single subject level. In contrast to language functions, which can be detected robustly and reliably by existing paradigms, the applicability of previously used VSFtasks is debatable.

Participants and Methods: In a first step, 15 subjects ( 6 men; mean age 24.6 years) performed three VSF-fMRI paradigms, Dots-in-space (D), Mental Rotation (MR) and Landmark-task (LT). To assess the robustness of the paradigms, we developed a stepwise approach, with the following mandatory requirements:

1. Sufficiently strong activation $(p .001, k=20)$ in predefined frontal and parietal ROIs in more than $50 \%$ of participants

2. The Lateralization Index (LI) (bootstrap-approach) of group activation in these ROIs is $\leq 0.4$

3. The $\mathrm{LI}$ in these ROIs is $\leq 0.4$ in more than $40 \%$ of participants In a second step, another sample of 15 subjects (6 men; mean age: 24.9 years) were investigated with two further versions of the LT that varied in difficulty. All subjects were investigated twice in one week to 
assess the paradigms' test-retest-reliability. Reliability was described by calculating intra-class correlation coefficients (ICGs).

Results: Only the LT fulfilled the required constraints and revealed sufficiently strong right-lateralized activation patterns not only at the group level, but also in single subjects. Test-retest reliability of the LT revealed fair to good ICCs (0.5 to 0.8 ).

Conclusions: Lateralization of the VSF-network can be robustly detected at the group, but not necessarily at single subject level. Using a stepwise approach, including a broad spectrum of methods, we identified one of the optimised LT as the most suitable paradigm to reliably and robustly assess individual VSF activation patterns.

Correspondence: , . E-mail:

J.A. RAO, M. KASSEL, J.ZUBIETA, R. WELSH, S.A. LANGENECKER \&. S. WEISENBACH. Cognitive Control Network Disruption and its Relationship with Gray Matter Volume in Late Life Depression. Objective: Previous studies of patients with late life depression (LLD) have found increased activation during tasks involving cognitive control (CC) relative to healthy aging, despite no behavioral changes. One hypothesis is that individuals with LLD must utilize greater neural resources, or "compensate", in order to maintain performance. We sought to strengthen this hypothesis by directly comparing cerebral function (fMRI) during a task of sustained attention and inhibitory control and structure (grey matter concentration) in LLD.

Participants and Methods: Twenty-four individuals with LLD (M age $=66$ ) and 23 healthy controls (HCs; M age=68) completed a go/ no-go task while undergoing functional MRI. Behavioral performance measures found no differences between groups.

Results: Functional analyses results revealed 17 ROIs that were significantly more active in LLD than in HCs $(p<.001)$, and no regions where the HCs had greater activation than LLD. These 17 ROIs, largely in the $\mathrm{CC}$ and limbic networks, were then subjected to voxel based morphometry in order to determine their grey matter concentration. VBM analysis found that only the middle frontal gyrus differentiated groups, in that HCs had significantly greater grey matter concentration $(p=.02)$ than LLD. Additionally, when correlating grey matter concentration with fMRI activation, HCs had more significant positive relationships ( 7 of 17) than LLD (3 of 17), particularly in the bilateral caudate and right parahippocampal functional regions. In LLD, activation in the right cuneus demonstrated the most consistent positive correlations (5 of 17). Conclusions: These results are consistent with prior research that LLD requires greater activation in fronto-striatal regions in order to maintain performance during a CC-dependent task; however, the relationship between structure and function is weaker in LLD when compared to normal aging.

Correspondence: Julia A. Rao, Ph.D., Psychiatry, University of IllinoisChicago, 912 S Wood St.Rm 841, Chicago, IL 60612.E-mail: jrao@ psych.uic.edu

\section{A. ALKOZEI, D. PISNER \& W.D. KILLGORE. Emotional intelligence is differentially correlated with prefrontal cortical responses to backward masked fearful and angry faces.}

Objective: Emotional intelligence (EI) is the ability to understand and regulate emotions to facilitate problem solving. The neurobiological mechanisms behind this ability are unclear, however brain regions including the prefrontal cortex (PFC), amygdala, insula, and anterior cingulate cortex appear to be crucial. We hypothesized that in response to subtle social threat cues (masked fearful and angry faces), EI would correlate positively with activation within these brain regions.

Participants and Methods: Fifty-four 18-45 year olds underwent blood oxygen dependent (BOLD) functional magnetic resonance imagining (fMRI) while viewing images of fearful and angry faces, each presented for $20 \mathrm{~ms}$ and "masked" immediately by a neutral face for $100 \mathrm{~ms}$ to prevent conscious visual perception. Measures of Trait and Ability EI were individually regressed against BOLD activation in response to the masked face tasks in SPM8
Results: In response to masked fear, higher Ability EI correlated positively with bilateral activation in the PFC, and the left insula $(\mathrm{p}<.005$, uncorrected). In contrast, in response to masked angry faces, increases in Ability EI correlated negatively with bilateral activation in the PFC, and the insula. No associations with Trait EI were found.

Conclusions: The results suggest different adaptive responses to nonconscious fearful versus angry faces in those with higher EI. Increased activation within the PFC in response to fearful faces suggests motivation to localize the source of danger and possible coping strategies. Contrarily, PFC deactivation in response to angry faces might provide a survival advantage by circumventing relatively slow top down regulatory processes to allow more rapid and automatic defense systems to respond unencumbered.

Correspondence: Anna Alkozei, Ph.D., Department of Psychiatry, University of Arizona Medical Center, University of Arizona Medical Center, Department of Psychiatry, 1501 N Campbell Ave, Tucson, AZ 85724.E-mail:aalkozei@psychiatry.arizona.edu

N.A. CRANE, J.R. GOWINS, A.M. BARBA, S.R. DELDONNO, L.M. JENKINS, K. MEYERS, K.E. HAZLETT, D.T. HSU, B.J. MICKEY, J. ZUBIETA \& S.A. LANGENECKER. Independent Component Analysis of Cognitive Control as Treatment Predictors for Major Depressive Disorder.

Objective: Identifying biomarkers of treatment response in depression, such as cognitive control, is one potential option for prospectively identifying the best treatments. The present study examined whether independent component analysis (ICA) of fMRI during a cognitive control task might predict treatment response for Major Depressive Disorder (MDD). Participants and Methods: Thirty-four individuals with MDD, aged 18-55 completed the Parametric Go/No-Go Task (PGNG) during fMRI at baseline and then completed at least 8 weeks of treatment with duloxetine $(n=19)$ or venlafaxine $(n=15)$. The PGNG task measures cognitive control including commission and rejection trials. Treatment response was the percent change of Hamilton Depression Rating Scale (HDRS) scores from pre- to post-treatment. Using temporal ICA with behavioral regressors (in 13 MDD with all events), 28 components were found. Multiple linear regressions with the 10 components most strongly related to inhibition (commissions minus correct rejections) were used to predict depression symptom change.

Results: Two of 10 ICA components were related to treatment response. More BOLD activation during commissions (and relative to correct rejections) in the bilateral dorsal, anterior frontal lobe predicted poorer treatment response $(\beta=-0.56, p=.049)$. In contrast, less BOLD activation during correct rejections (and more during commissions) in the medial prefrontal cortex and posterior cingulate cortex predicted better treatment response ( $\beta=-0.53, p=.06$; trending).

Conclusions: Neural activation during inhibition predicted treatment response. Importantly, increased activation in areas important for error processing and also in the default mode network during failed inhibition were related to poorer treatment response.

Correspondence: Natania A. Crane, University of Illinois at Chicago, 1007 W Harrison Street (M/C 285), Chicago, IL 60607. E-mail: ncrane3@uic.edu

Z.Z. ZLATAR, C. HAYS, D. GALASKO, M.J. MELOY, J. KERR \& C.E. WIERENGA. Sedentary Time is Associated with Higher Tau Protein Load in Healthy Aging.

Objective: Sedentary behavior has emerged as a risk factor for negative health outcomes, independent of physical activity (PA), which confers health benefits. PA interventions in Alzheimer's disease (AD) risk are emerging. To elucidate the mechanisms of action, we examined the preliminary relationship between biomarkers of $\mathrm{AD}$, objectively-measured PA and sedentary time, and whole brain resting cerebral blood flow (WBrCBF) in cognitively normal older adults.

Participants and Methods: PA (1952 CPM) and sedentary time $(<100$ CPM) were measured for 7 days with a hip worn accelerometer in 13 
cognitively normal older adults (ages 67-79). AD risk biomarkers included: WBrCBF measured via arterial spin labeling MRI, and cerebrospinal fluid (CSF) Beta Amyloid 42 (A $\beta 42)$ and total tau levels collected via lumbar puncture.

Results: Spearman correlations indicated that those who accumulated on average more sedentary minutes had higher CSF levels of tau protein $(\rho=.69, p<.05)$. There were no significant relationships between the biomarkers and PA levels, although this might change as sample size increases. WBrCBF was significantly associated with $\mathrm{A} \beta 42(\rho=.83$, $\mathrm{p}<.01)$ and the ratio of tau $\mathrm{A} \beta 42(\rho=-.68, \mathrm{p}<.05)$, which is sensitive to cognitive decline.

Conclusions: There is a strong association between CSF-measured levels of tau protein and sedentary time, pointing to a possible important role of sedentary behavior as a risk factor for AD. The relationship observed between WBrCBF and $\mathrm{A} \beta 42$ is consistent with the two-hit vascular hypothesis of AD. Future studies should investigate if reductions in sedentary time affect CSF levels of tau and how they vary in relation to $\mathrm{CBF}$.

Correspondence: Zvinka Z. Zlatar, PhD, Psychiatry, University of California, San Diego, 9500 Gilman Dr MC 0664, La Jolla, CA 92093.

E-mail: zvinka@gmail.com

A.J. ALFINI, H. OH, L. WEISS, T.J. SMITH, B.P. LEITNER, S. SAHEB, R. TOLEDO, L.M. GUTH, R.Q. LANDERS-RAMOS, K. CORRIGAN, E.E. SPANGENBURG, J.M. HAGBERG \& J. SMITH. The Effects of Exercise Training Cessation on Cerebral Blood Flow: an Arterial Spin Labeling Analysis.

Objective: While exercise is known to improve cerebrovascular health, it is not known how a short-term cessation of regular exercise training may impact brain health. Our aim was to measure changes in resting cerebral blood flow (CBF) after a 10-day cessation of exercise training in healthy physically fit older adults.

Participants and Methods: Ten master athletes (men=7), defined as 50 years of age or older with a 15-year history of endurance exercise training, were recruited from local running clubs. Criteria for endurance exercise training included four training sessions, and at least four hours of high intensity exercise, per week. Before and immediately after the 10-day cessation of exercise training, CBF was measured using interleaved, multi-sliced, perfusion weighted magnetic resonance imaging. Paired T-tests were used to assess CBF changes over time.

Results: We found that a 10-day cessation of exercise training altered $\mathrm{CBF}$ in several brain areas with general decreases in the frontal, temporal, and parietal lobules $(\mathrm{p}<0.05)$. The strongest alterations were decreases in the bilateral precuneus and right cerebellar tonsil; and increases in the right insula and superior temporal gyrus $(p<0.01)$. Additional decreases also were found in the left thalamus, bilateral posterior cingulate, and parahippocampal gyri $(\mathrm{p}<0.05)$.

Conclusions: The cessation of exercise training for a period of 10days in healthy physically fit older adults resulted in decreased resting parenchymal blood flow in several brain regions. This suggests that the cerebrovascular system may be very responsive to the effects of exercise, and that even short-term decreases in exercise training in healthy older adults may reverse these effects.

Correspondence: Alfonso J. Alfini, M.S., Kinesiology, Cognitive Motor Neuroscience, University of Maryland, 3413 Tulane Drive, Apt. \#12, Hyattsville, MD 20783. E-mail: aalfini@umd.edu

K. KRISHNAN, S. ASLAN, J. SPENCE, W. RINGE, M. CULLUM, C. BOSWORTH \& C. MARQUEZ DE LA PLATA. Longitudinal Change in Resting-State Networks Over the Course of Recovery from TAI.

Objective: Little is known about neural network connectivity immediately after a traumatic axonal injury (TAI). This is the first longitudinal study in TAI to examine functional connectivity in the Default Mode Network (DMN) and Central Executive Network (CEN) within 48 hours after injury with repeat imaging 7 months later. Aims: (a) characterize connectivity in these networks at the sub-acute stage of injury, (b) evaluate longitudinal change in networks over time, and (c) explore how this change might be associated with structural connectivity and neurocognitive outcome.

Participants and Methods: Resting-state fMRI and diffusion tensor imaging (DTI) scans were acquired from 21 patients with moderate-severe axonal injuries compared with 8 non-injured controls. Functional connectivity data for DMN and CEN nodes was acquired for both injury time points and for controls. Metrics for white matter tracts connecting regions of these networks was identified. Neurocognitive outcome was assessed at 7 months post-injury.

Results: Results revealed lower resting-state DMN connectivity 48 hours after TAI compared to non-injured controls, and this persisted 7 months after injury $(\mathrm{p}<0.05)$. CEN connectivity was comparable between acutely injured patients and controls, though patients demonstrated increased CEN connectivity at 7 months $(p<0.05)$. These patterns of functional connectivity in patients were associated with alterations in structural connectivity, where areas of decreased functional connectivity were associated with decreased integrity of white matter tracts connecting those regions. However, some regions within these networks demonstrated increased functional connectivity despite presence of structural damage.

Conclusions: Results suggest disruptions in functional and structural connectivity are present as early as 48 hours after a TAI. Alterations in functional connectivity during the recovery period may be explained either by structural damage and could suggest the presence of neural compensation in functional connectivity.

Correspondence: Kamini Krishnan, PhD, Mayo Clinic, 151 Grande Woods Ave SW, Unit 3523, Rochester, MN 55902. E-mail: krishnan. kamini@mayo.edu

\section{Imaging (Structural)}

J.D. Medaglia, F. PasqualetTi, S. GU \& D. BassetT. Addressing Brain and Cognitive Reserve with Network Control Theory.

Objective: The term "reserve" describes the observation that there is an imperfect correlation between brain pathology and clinical sequelae in numerous brain disorders. While much research has been conducted on reserve in a number of neuropathological populations, models that address dynamical neural network mechanisms may produce an improved ability to understand brain resilience and dysfunction in neuropathological contexts. We introduce a novel extension to reserve theory with a recent innovation in dynamical systems analysis: network control theory. We apply analyses of network controllability in conjunction with advanced neuroimaging techniques (e.g., diffusion spectrum imaging) to predict network dynamics in a healthy sample to investigate its ability to explain network findings commonly reported in the literature

Participants and Methods: Eight healthy subjects underwent serial diffusion spectrum imaging scanning. Tractography was segmented using the Lausanne atlas family. A model of lagged neural signalling was used to compute the controllability Gramian of brain regions for the entire system to predict functional connectivity. Regions were classified as modal controllers, average controllers, and boundary controllers according to network control theoretical guidelines.

Results: Network controllability analyses identified that the modal controllability, average controllability, and boundary controllability of brain regions strongly predicted network functional connectivity for cognitive control, default mode, and attention systems across the entire brain.

Conclusions: Network control theory provides a critical mechanistic explanation for functional dynamic across the entire brain and is a theoretical innovation over previous approaches to brain network analysis. Applications for predicting neural dynamics in healthy and neuropathological populations are concretely introduced. A specific emphasis 
is placed on the theoretical implications of network control theory to questions within reserve research.

Correspondence: John D. Medaglia, M.S., Psychology, Pennsylvania State University, 347 Moore Building, University Park, State College, PA 16802.E-mail: johnmedaglia@gmail.com

\section{J. POMMY, P. BARBOSA, R.J. THOMA \& M. BOGENSCHUTZ. Intact Cerebellar Structure and Fine Motor Function with Religious Use of Ayahuasca.}

Objective: Ayahuasca is a hallucinogenic tea that is used within religious contexts. The brew contains N,N-Dimethyltryptamine and several monoamine oxidase inhibitors collectively referred to as harmala alkaloids. In high concentrations harmala alkaloids have the potential to induce cerebellar damage in some animal models via over-excitation of the Purkinje neurons. The impact of Ayahuasca on fine motor function and cerebellar structures in humans, however, is unknown. In the present analysis, cerebellar volumes and fine motor skills were examined in individuals from an Ayahuasca-using church (AUC) to assess the impact of Avahuasca on the cerebellum.

Participants and Methods: As part of a larger investigation on religious participation and neurocognitive function, 20 individuals from an AUC and 16 individuals from a matched control group (MC) were recruited from the community. Cerebellar structure was assessed using two neuroimaging techniques: (1) Voxel-Based Morphometry, an unbiased, voxel-wise assessment of gray matter volumes, and (2) Freesurfer, a semi-automated analysis of volumetric measures from specific brain regions. Fine motor skills were assessed using a pegboard test.

Results: Both volumetric analysis techniques revealed no significant differences between the AUC and MC groups ( $p<0.001)$. Similarly, no significant differences were found between groups on the pegboard task $(p<0.05)$. Finally, within the AUC sample, cerebellar structure was assessed relative to years of participation and results again showed no significant correlation between cerebellar volume and duration of Avahuasca use $(\mathrm{P}<0.05)$.

Conclusions: Overall, results suggest Ayahauasca, when used in a religious context, is not associated with smaller cerebellar volumes or impaired fine motor function.

Correspondence: Jessica Pommy, UNM, 6004 Burgos Avenue NW, Albuquerque, NM 87114.E-mail: jpommy@gmail.com

\section{E. MCINTOSH, A. JACOBSON, E. GREEN, N. KEMMOTSU \& C. MURPHY. Metabolic Syndrome and the Entorhinal Cortex: A Cortical Thickness Study.}

Objective: To examine cortical thickness differences in the entorhinal cortex in adults with metabolic syndrome.

Participants and Methods: Twenty-six adults with and without metabolic syndrome were included in the present study. Clinical data were acquired prior to the imaging session and included measurements of body mass index (BMI), waist circumference, and blood pressure. Additionally, participants reported their medical histories and medications. T1-weighted magnetic resonance images (MRI) with prospective motion correction were acquired in a 3.0T scanner. Structural images were processed using Freesurfer image analysis suite. Analyses of covariance were performed to investigate group differences in cortical thickness in the entorhinal cortex. Relationships between clinical data and cortical thickness were explored using partial correlations in further analyses. Results: Group analyses revealed differences in cortical thickness in the entorhinal cortex $(p=0.013)$. Partial correlation analyses showed significant associations between entorhinal cortical thickness and BMI $(p<0.001)$, waist circumference $(p=0.001)$, diabetes $(p=0.010)$, and hypertension $(\mathrm{p}=0.015)$

Conclusions: The findings in this study demonstrated that adults with metabolic syndrome have decreased cortical thickness in entorhinal cortex, relative to metabolically healthy adults. The medial temporal lobe, particularly the entorhinal cortex, is consistently implicated in early Alzheimer's disease pathology. Past studies show metabolic and cardiosvascular abnormalities in middle age and late life increase risk for cognitive impairment and dementia. The results of the present study may help us better understand the relationships between metabolic syndrome and increased risk for cognitive decline and dementia.

Correspondence: Elissa McIntosh, BS, Psychology, San Diego State University, 6363 Alvarado Court, Suite 101, San Diego, CA 92120. E-mail:emcintos@rohan.sdsu.edu

J.J. TANNER, A. CHOI, D. RAMON, N. SCHWAB \& C. PRICE. Comparison of Manual Versus FreeSurfer Calculated Intracranial Volumes in Older Adults.

Objective: Calculating total intracranial volume (TICV) from MRI is necessary for volumetric brain structure analyses particularly in relation to behavioral and cognitive data (Bigler \& Tate, 2001) but manual calculations are very time consuming. Automated methods exist but with varying reliability of TICV (Keihaninejad et al., 2010; Ridgway et al., 2011). FreeSurfer (Fischl, 2012) provides estimates of intracranial volume (eTIV; Buckner et al., 2004) but such estimates have limitations (Nordenskjöld et al., 2013). We hypothesized, however, that the brainmask volume from FreeSurfer would represent an accurate measurement of TICV as an alternative to the estimation created using Buckner et al.

Participants and Methods: Participants included 40 individuals with Parkinson's disease (age $=67.80(5.44)$ ) and 40 non-PD demographically-matched controls (age $=68.18(4.64))$. 3T brain MRI was acquired for all participants (Siemens Verio; T1: $1 \mathrm{~mm}^{\wedge} 3$ voxels). An expert rater manually created TICV masks for all participants including all regions within the inner surface of the skull above the foramen magnum. All MRIs were processed using FreeSurfer 5.3, producing brainmask.mgz (grav+white+CSF) and eTIV values.

Results: Brainmask volumes from FreeSurfer were larger than manual (brainmask mean: 1697cc; manual mean: $1665 \mathrm{cc}$ : $1.94 \%$ difference) and correlated with manual TICV $(\mathrm{r}=0.93$; ICC $=0.92 ; \mathrm{p}<0.01)$ with high spatial overlap (DSC $=0.95$ ). FreeSurfer's estimated intracranial volume (eTIV) underestimated TICV (mean: $1564 \mathrm{cc} ; 6.06 \%$ difference) and also correlated with manual masks $(\mathrm{r}=0.82 ; \mathrm{ICC}=0.68 ; \mathrm{p}<0.01)$.

Conclusions: Our results provide support for using FreeSurfer brainmask volume rather than eTIV as a measure of TICV. The brainmask volume could be utilized in place of time-intensive manual measurements in older adult populations.

Supported by NINDS R01 NS082386 (CP) and NINDS K23NS60660 (CP)

Correspondence: Jared J. Tanner, Ph.D., Clinical and Health Psychology, University of Florida, 4916 NW 80th RD, Gainesville, FL 32653.E-mail: jjtanner@phhp.ufl.edu

M.E. MCLAREN, S.M. SZYMKOWICZ, A. O'SHEA, A. WOODS, T.M. MANINI, S.D. ANTON \& V.M. DOTSON. Symptom Dimensions of Depression and Frontal Brain Volume in Older Adults.

Objective: Subthreshold depression is common in older adults and is linked to structural changes in frontal brain regions. Few studies have examined whether symptom clusters of depression correspond to unique structural changes in these regions. This study was conducted to examine the relationship between symptom dimensions of depression and structural brain changes. It was hypothesized depressed mood and lack of well-being would be more strongly linked to decreased brain volume than somatic symptoms, which may be more related to changes in paralimbic and subcortical structures.

Participants and Methods: This study included 47 healthy, community dwelling older adults (mean age $68.85 \pm 7.49$ years). Participants received a structural Magnetic Resonance Imaging scan and completed the Center for Epidemiologic Depression Scale (CES-D). Subscale scores for depressed mood, lack of well-being, and somatic symptoms were calculated. Volumetric data were based on Freesurfer Regions of Interest (ROIs). 
Results: We examined the association between symptom dimensions and ROIs using linear regression models controlling for gender, age, and total grey matter. Lack of well-being scores were negatively correlated with volumes in the left medial orbitofrontal cortex $(p<0.05)$, and the pars orbitalis region of the left inferior frontal gyrus $(\mathrm{p}<0.05)$. Depressed mood and somatic symptoms, however, were not correlated with frontal ROIs.

Conclusions: Results suggest decreased brain volumes in the left medial orbitofrontal and inferior frontal cortices are associated with lack of well-being symptoms. These results support previous findings of decreased frontal volumes in depression, and suggest this association may be driven by lack of well-being symptoms.

Correspondence: Molly E. McLaren, PhD, Clinical and Health Psychology, University of Florida, Department of Clinical and Health Psychology, P.O. Box 100165, Gainesville, FL 32610-0165. E-mail: memclaren@phhp.ufl.edu

K. REITER, K.A. NIELSON, T.J. SMITH, L. WEISS, A.J. ALFINI \& J. SMITH. Walking intervention increases VO2 peak and cortical thickness in MCI and healthy older adults.

Objective: Cortical atrophy is a well-known biomarker in Alzheimer's disease (AD), which contributes to clinical symptoms. This study sought to understand the influence of exercise on cortical thickness in healthy older adults (HOA) and those with Mild Cognitive Impairment (MCI), a group at high risk for $(\mathrm{AD})$.

Participants and Methods: Thirty-five older adults (ages 61-88, 17 MCI, $18 \mathrm{HOA}$ ) underwent MRI before and after participating in a 12 week walking intervention. Prior to intervention, participants exercised less than three days weekly. Fitness change was assessed using residualized scores of $\mathrm{VO} 2$ peak change (VO2PC) from pre to post intervention. Structural images were processed using FreeSurfer v5.1.0.

Results: Cortical thickness change showed robust positive correlations with VO2PC in bilateral insula, precentral gyri, precuneus, posterior cingulate, and inferior and superior frontal cortices in both MCI and HOA $(p<.01)$. To assess variables that influence VO2PC and thus cortical thickness, regression analysis were performed and showed that age, sex, education, and group together significantly predicted VO2PC $(\mathrm{F}(4,27)=3.09, \mathrm{p}=.032)$, though only age was independently significant $(\beta=-.51, p<.01)$.

Conclusions: A 12-week moderately intense walking intervention lead to greater fitness in both MCI and HOA. This was associated with robust cortical thickness increases in multiple brain regions, which was not differentiated by cognitive status group. Therefore, improved fitness results in increased cortical thickness in older adults regardless of cognitive status. Thus, regular exercise is an especially beneficial intervention to counteract cortical thinning, which may buffer against future cognitive decline in both HOA and older adults who have begun to experience cognitive decline.

Correspondence: Katherine Reiter, M.S. working toward Ph.D., Psychology, Marquette University, 3909 N. Murray Ave Apt 804, Milwaukee,WI 53211. E-mail: kereiter@gmail.com

\footnotetext{
A.K. SMITH WATTS, T.J. FARRER, L.A. WALKER, E.R. HARTMAN, S. NELSON SCHMITT, A. LEE, S. CORREIA, S.P. SALLOWAY, P.F. MALLOY \& S. DEONI. Pilot Test of the Utility of a Visual Rating System for Identifying Artifacts in Diffusion-Tensor Imaging (DTI) Data.

Objective: Motion, vibration, ghosting and other artifacts can lead to inaccurate DTI metrics and erroneous correlations with neuropsychological data. These artifacts are often best identified by visual inspection but few clear guidelines exist. We developed and pilot tested a Diffusion Visual Rating System (DiffVRS) consisting of visual exemplars to guide novice raters (NRs) to reliably identify such artifacts.

Participants and Methods: The DiffVRS was compiled from DTI datasets (3-Tesla, b=0, 1300; 64 directions) from an Alzheimer's disease (AD) study. An expert rater introduced two novice raters (NRs) to the
}

DiffVRS and supervised practice on 3 DTI datasets. The novice and expert raters then independently rated the same 10 randomly selected DTI datasets (640 Diffusion-Weighted Images; DWIs). Each DWI volume was rated as acceptable vs. unacceptable.

Results: After independent rating, the NRs had identified 31 problematic volumes in total (NR1 identified 25, NR2 identified 24). Of these, 18 volumes overlapped (58\%). The expert rater identified 32 problematic volumes. NR1 agreed with the expert on 18 volumes ( $56 \%$ sensitivity); NR2 agreed on 24 (75\% sensitivity). Compared to the expert rater, specificity was $\geq 99 \%$. Kappa for NRs vs. expert was acceptable (NR1: $\mathrm{k}=.578, \mathrm{p}<.001$, moderate; NR2: $\mathrm{k}=.776 ; \mathrm{p}<.001$, substantial). Raters were unanimous in identifying datasets too problematic to be included in further analysis.

Conclusions: Visual exemplars such as the DiffVRS facilitate novice raters in identifying problematic DTI data, but training on only 3 datasets appears insufficient for mastery.

Correspondence: Ashley K. Smith Watts, Brown University, 5 Squire Lane, Apt.B, Riverside, RI 02915.E-mail: ashley_smith_watts@brown. edu

J.L. BUCHHOLZ, L.A. DEMERS, R. FUKUNAGA \& I.M. ROSSO. Anxiety Sensitivity Correlates with Left Anterior Insula Volume in Posttraumatic Stress Disorder.

Objective: The Anxiety Sensitivity Index (ASI) measures a dispositional trait involving fear of anxiety-related sensations, a vulnerability factor for anxiety disorders including posttraumatic stress disorder (PTSD). Functional imaging studies have found ASI scores to be positively associated with activity in the anterior insula, a region known to play a critical role in conscious interoceptive awareness. Moreover, morphology findings specifically implicate anterior insula abnormalities among various anxiety disorders. Despite such reports, there is little research examining insula morphology as a potential neural correlate of anxiety sensitivity (AS) in PTSD. To better understand this relationship, we investigated whether ASI scores were correlated with insula volume among adults with PTSD and healthy participants.

Participants and Methods: Twenty-three right-handed adults with PTSD (8 male) and 26 healthy control subjects (11 male) ranging in age from 20-60 years completed structured clinical interviews and the ASI, and underwent 3T magnetic resonance imaging. Correlation and regression analyses examined ASI scores relative to anterior and posterior insular cortex volumes between and within subject groups.

Results: Groups did not differ significantly in ASI scores, intracranial volume, or insula volumes. Total ASI scores were significantly correlated with left anterior insula volume in the combined sample and the PTSD group specifically, but not in the control group.

Conclusions: These findings are consistent with a role of the anterior insula as a neural correlate of AS in PTSD. They add to evidence of transdiagnostic commonalities in the neurobiology of AS, and motivate future research exploring the relationship of $\mathrm{AS}$ with the anterior insula in anxiety- and stress-related disorders.

Correspondence: Jennifer L. Buchholz, McLean Hospital, 115 Mill St., Belmont,MA 02478.E-mail: jbuchholz@mclean.harvard.edu

L. HIZEL, S. LEININGER, J.J. TANNER, E. WARNER, N. SCHWAB, P. NGUYEN \& C. PRICE. Organizational and Neuroanatomical Contributions to the Rey-Osterrieth Complex Figure in Nondemented Older Adults with Parkinson's Disease.

Objective: Parkinson's disease (PD) is typically not associated with learning impairment. When it does occur, is often attributed to slow processing, reduced organizational strategy, and reduced frontal-subcortical integrity. We examined the organizational and neuroanatomical (gray and white) contributions to memory performance on the ROCF in PD and controls.

Participants and Methods: Non-demented individuals with idiopathic PD $(n=40) \&$ controls $(n=40)$ completed neuroimaging and neuropsychological testing including ROCF copy, immediate, and delay. 
Examiners scored organizational components using a flow, scored via Savage (1999) criteria. Neuroimaging included T1-weighted (caudate, entorhinal, and cortical volumes by lobe) and diffusion imaging metrics (mean lobar FA).

Results: Group by ROCF Condition (Copy, Immediate, Delay) repeated measures analysis had a significant interaction $[F(2,152)=5.81, p<.01$; $\mathrm{PD}<$ non-PD for immediate and delay; not copy condition]. On delay recall, 25\% PD scored one or more SDs below the mean. Behaviorally, better organization positively related to better delay recall (non-PD rho=.41, $\mathrm{p}=.01$; PD rho=.51, $\mathrm{p}<.01)$. PD had smaller entorhinal volumes $(p=0.02)$, a trend for thinner right temporal lobes $(p=0.8)$ but no frontal thinning. For non-PD only larger entorhinal volumes related to better delay $(r=.37, p<.02)$, while for PD there were no clear anatomical cortical gray, white, or subcortical gray associations. Delay recall impairment (<-1.0 SD cut off) for PD showed some evidence of reduced entorhinal volume relative to non-impaired PD peers $(t=-1.40, p=.17$; no pattern for other structures).

Conclusions: Individuals with PD have significant impairment in ROCF recall relative to controls. Although PD exhibited temporal loss relative to controls, the best correlation with recall performance was organization during the copy condition. Only for controls was entorhinal volume an additional significant association, leaving specific neuroanatomical contributions in PD unclear. NINDS R01 NS082386, K23NS60660 Correspondence: Loren Hizel, BA, Clinical and Health Psychology, University of Florida, $1810 \mathrm{NW} 23 \mathrm{rd}$ Blvd Unit 160, Gainesville, FL 32605.E-mail: Ihizel@phhp.ufl.edu

A. HOOD, E. WESONGA, T. HERSHEY, J. SHIMONY, R.C. MCKINSTRY, D.K. GRANGE, S. CHRIST \& D.A. WHITE. White Matter Integrity Mediates the Relationship Between Prolonged Exposure to High and Variable Phenylalanine Levels Over the Lifetime and Strategic Processing in Children with Phenylketonuria.

Objective: Phenylketonuria (PKU) is an autosomal recessive disorder characterized by disruption in the metabolism of the amino acid phenylalanine (Phe) that if untreated, typically leads to cognitive disability. Previous work has separately shown that children with PKU have reduced white matter integrity and impaired executive abilities. Further, high, and/or variable Phe levels are related to lower executive abilities and compromised white matter. However, the mechanisms by which Phe levels, white matter, and executive abilities are related not yet fully understood.

Participants and Methods: In this study, we tested the hypothesis that white matter integrity in three brain regions (centrum semiovale, prefrontal cortex, and posterior parietal-occipital cortex) of children with early- and continuously-treated PKU $(\mathrm{N}=36)$ mediated the relationship between prolonged exposure to Phe over the lifetime (mean exposure, standard deviation exposure) and strategic processing, which is an executive ability. Six separate mediation analyses were conducted. Results: Results showed that white matter integrity in the centrum semiovale negatively and statistically mediated the relationship between mean Phe exposure and strategic processing (95\% BCa CI -3.11 to -.11) and mediated SD Phe exposure and strategic processing (95\% BCa CI of -2.84 to -.01$)$. White matter integrity in the posterior parietal-occipital cortex negatively and statistically mediated the relationship between mean Phe exposure and strategic processing (95\% BCa CI of -3.57 to -.41), but did not significantly mediate the relationship between SD Phe exposure and strategic processing. Significant mediations had medium effect sizes. White matter integrity in the prefrontal cortex was not a significant mediator for either mean or SD exposure.

Conclusions: Findings suggest that, in children with PKU, compromised white matter integrity may help provide a mechanism to explain the relationship between exposure to elevations and variability in Phe and executive abilities.
Correspondence: Anna Hood, M.A., Psychology, Washington University in St Louis, One Brookings Drive, St Louis, MO 63130. E-mail: annahood@wustl.edu

J. BURCIAGA, D. TOSUN, D. BICKFORD, M. SUSANNE, P. INSEL, D. TRURAN-SACREY, C. NELSON, M. WEINER, J. ROTHLIND \& R. MACKIN. The Association of Cognitive Functioning and Cortical Atrophy in Late Life Depression.

Objective: Cognitive impairment is a frequently comorbid condition in Late Life Depression (LLD). However, the etiology of these impairments is not clear. An increasing body of literature has demonstrated a relationship between structural brain abnormalities (SBA) and LLD. While the majority of studies have linked LLD with subcortical white matter hyperintensities in the frontal lobe, growing evidence suggests cortical atrophy contributes to LLD. To date, only two studies have examined the association of SBA on cognitive functioning in LLD. The present study was conducted to examine the association between LLD, cognitive functioning and cortical thickness in a sample of older adults. We expected participants with LLD would exhibit lower performance on measures of executive function and exhibit bilateral cortical thinning of the frontal lobe when compared to controls. We also expected that reduced cortical thickness would predict lower performance on both cognitive measures.

Participants and Methods: In the Normal Control (NC) group, 59 had data for the Symbol Digit Modalities Test-Oral Version (SDMT) and 37 for the Stroop Color-Word condition (Stroop) and 20 had both neuroimaging and cognitive data. In the LLD group, 89 subjects had data for the SDMT and 87 for the Stroop, and 44 had both neuroimaging and cognitive data.

Results: LLD participants performed lower on both measures of cognitive functioning: SDMT F $(1,148)=10.88, p=.001$; Stroop F $(1,123)$ $=9.60, p=.002$. LLD participants also demonstrated thinner cortex in left frontal regions $\mathrm{F}(1,60)=8.98, \mathrm{p}=.004$. Yet, none of the regression models showed cortical thickness to be predictive of cognitive test performance.

Conclusions: This study demonstrated lower performance on cognitive measures and cortical thinning in left frontal regions in the LLD groups. Contrary to our expectations, there was no evidence of a significant relationship between cognitive performance and cortical thickness, suggesting something else may be driving cognitive differences.

Correspondence: Joaquin Burciaga, PhD, Psychology, San Francisco VA Medical Center, 707 Continental Circle, Apt 1713, Mountain View, CA 94040. E-mail: joaquinburciaga@gmail.com

M. LANCASTER, M. SEIDENBERG, S. DURGERIAN, K.A. NIELSON, J.L. WOODARD, J. SMITH, M.M. STIKA, A.M. BUTTS, N. HANTKE, C. KAY, C. KANDAH, K.E. HAZLETT, C. FIGUEROA, M.A. SUGARMAN \& S.M. RAO. Diffusion Tensor Imaging Predictors of Cognitive Decline in Healthy Older Adults. Objective: Several studies have demonstrated that certain structural and functional neuroimaging measures in the mesial temporal lobe are predictive of cognitive decline in older adults, but diffusion tensor imaging (DTI) has been understudied. Here we investigated the ability of DTI measurements in select white matter tracts to predict memory change over 3 years in cognitively intact older adults.

Participants and Methods: Fifty-one cognitively asymptomatic older adults (Mage $=73.3, \mathrm{SD}=4.9)$ underwent DTI scanning and administration of the Rey Auditory Verbal Learning Test (RAVLT), with repeat administration of the RAVLT (alternate form) after 3 years. Seventeen participants possessed at least one Apolipoprotein E epsilon 4 (APOE $\varepsilon 4)$ allele. DTI measures in the bilateral cingulate-hippocampal tract (CGH), the fornix/stria terminalis $(\mathrm{FxS})$, and the uncinate fasciculus (UNC) were calculated from regions derived by superimposing the ICBM DTI-81 WM atlas on a mean white matter skeleton. Residualized change scores were calculated for total RAVLT Delayed Recall. Hierarchical 
multiple regression models were created to predict Delayed Recall change after controlling for age and APOE status.

Results: Fractional anisotropy (FA) in the right $\mathrm{F}_{\mathrm{xS}}$, mean diffusivity in the left CGH and left UNC, axial diffusivity in the bilateral CGH and bilateral UNC, and radial diffusivity in the right CGH predicted Delayed Recall change. Lower FA and higher absolute diffusivity was related to decline in Delayed Recall.

Conclusions: DTI may serve as a useful biomarker of subsequent cognitive decline in healthy older adults. Future studies should compare the sensitivity of DTI in predicting decline with structural and functional neuroimaging measures.

Correspondence: Melissa Lancaster, M.S., Rosalind Franklin University of Medicine and Science, 3035 W. Jackson Blvd \# 2E, Chicago, IL 60612. E-mail: melissa.lancaster@my.rfums.org

\section{J.J. TANNER, T. MARECI, D.J. LIBON \& C. PRICE. Reduced Temporal White and Gray in Non-demented PD with Verbal Memory Deficits.}

Objective: Controversy exists regarding neuroanatomical contributions (i.e., frontal vs. temporal) to memory disturbance in individuals with Parkinson's disease (PD). We hypothesized that PD individuals with significant verbal memory deficits would have associated degradation of temporal lobe structures relative to their non-impaired PD peers. To test this hypothesis, we examined neuroanatomical regions within the left temporal lobe: entorhinal cortex, temporal cortex, temporal white matter fractional anisotropy (FA), and white matter connecting entorhinal and retrosplenial cortices (via temporal cingulum). Left frontal gray and white matter values served as dissociation variables.

Participants and Methods: Participants included 40 non-demented PD (age $>60$ ) with normative memory data derived from 40 age, education, and gender matched controls. All participants completed neurocognitive testing and neuroimaging. $20 \%$ were classified as having significant verbal memory deficits $(\mathrm{z}<-1.5$ relative to non-PD peers) using the WMS-III Logical Memory (LM) subtest and the Philadelphia (repeatable) Verbal Memory Test (PrVLT). An expert rater manually created volumes of left entorhinal cortex (ERC) and performed cingulum tracking with additional neuroanatomic variables derived through FreeSurfer and FSL.

Results: PD with verbal memory deficits, relative to PD peers, had smaller ERC $(p<0.04)$, thinner temporal cortex $(p<0.01)$, and a trend towards lower temporal FA ( $p=0.08$; Cohen's $d=0.76)$. Temporal cingulum connectivity, frontal thickness, frontal FA, disease duration, and disease severity did not differ between groups.

Conclusions: PD with significant memory impairment have smaller ERC and thinner left temporal lobes relative to non-impaired PD peers; this supports the idea that a subset of PD experience anterograde deficits and is suggestive of heterogeneity of pathology. PD with memory deficits might be at increased risk for later dementia.

Supported by NINDS R01 NS082386 (CP) and NINDS K23NS60660 (CP)

Correspondence: Jared J. Tanner, Ph.D., Clinical and Health Psychology, University of Florida, $4916 \mathrm{NW}$ 80th RD, Gainesville, FL 32653.E-mail: jjtanner@phhp.ufl.edu

\section{J.J. TANNER \& C. PRICE. Template Matters in Tract-Based Spatial Statistics.}

Objective: Tract-Based Spatial Statistics (TBSS; Smith, 2006) is a robust, well-established, and widely used method for quantifying diffusion tensor imaging (DTI) differences between groups. Researchers acknowledge that templates can change neuroimaging results (Keihaninejad et al., 2012). Previous research, however, has not investigated template effects in PD or quantified changes to TBSS results. We compared TBSS output based on three common template options as part of a larger NIHfunded study examining white matter in Parkinson's disease.

Participants and Methods: The imaging database included T1 and diffusion-weighted imaging (64 directions, $\mathrm{b}=1000)$ from 40 non-demented PD and 40 matched controls (age $>60$ ). Templates were chosen by: 1) aligning all fractional anisotropy (FA) images to each other with a script selecting a 'representative image' (RI; tbss_2_reg -n); 2) using the FSL-created FMRIB58_FA template (adults ages 2050); and 3) creating a study-specific template (SST) using Advanced Normalization Tools (Avants et al., 2010). TBSS was run with each template. Significant voxels where FA for PD was less than Controls were binarized and corrected for FA skeleton volume in order to compare output.

Results: Regardless of template, PD had reduced FA compared to controls in frontal and callosal regions. Two templates showed parietal FA differences. Volume and ratio to mean skeleton volume differed by template: 1) RI: $2720 \mathrm{~mm}^{\wedge} 3$; Ratio: .0228; 2) FMRIB58_FA: 6813 mm^3; ratio: .0595; 3) SST: $3033 \mathrm{~mm}^{\wedge} 3$; ratio: .0242.

Conclusions: Volume and anatomical regions differed by template. All templates demonstrated frontal and callosal FA differences; however, the FMRIB58_FA template resulted in more areas of reduced FA, including in the parietal lobes. The RI and SST produced similar results. Templates change TBSS results; therefore, careful consideration should be given to choice of template and template details should be included in manuscripts. Supported by: NINDS R01 NS082386 (CP) and NINDS K23NS60660 (CP)

Correspondence: Jared J. Tanner, Ph.D., Clinical and Health Psychology, University of Florida, $4916 \mathrm{NW}$ 80th RD, Gainesville, FL 32653.E-mail: jjtanner@phhp.ufl.edu

J.C. FLEMING, L. VER HOEF, R.C. MARTIN, H. DESHPANDE \& S. LEE. The Relationship between Hippocampal Dentation and Neuropsychological Memory Performance in Healthy Adults.

Objective: While the hippocampus has long been identified as the integral structure in memory functioning, the relationship between structure and function has yet to be fully explained. The current study investigates the neural substrates of memory beyond hippocampal volumetric analyses described in previous research (Petten, 2004; Molnar \& Keri, 2014). The inferior aspect of the healthy adult hippocampus has a dentated or "toothed" appearance that varies in prominence between individuals, yet this feature has not been explored in relation to memory. The current study examines the hypothesis that increased dentation is associated with better memory performance.

Participants and Methods: Twenty healthy adult participants were assessed for intelligence estimates (WASI; WRAT-III Reading; Boston Naming Test) and memory outcomes (CVLT-II; Rey Complex Figure Test), and were imaged with an ultra high-resolution 3 T structural MRI technique developed to show detailed hippocampal structure. Dentation was classified by a board-certified neurologist according to a 3-point visual rating scale.

Results: Regression analyses indicated a positive linear relationship between the primary verbal memory outcome, CVLT Trials 1-5 Total Score, and left hippocampal dentation $(p=.017)$. Secondary analyses, including the relationship between RCFT immediate recall and left dentation ( $p=.032)$ and RCFT delayed recall and left dentation ( $p=.039)$, were also significant.

Conclusions: Therefore, hippocampal dentation was positively associated with memory performance in a healthy adult population. Future studies will investigate this relationship in disease populations, particularly those affecting memory and the hippocampus, including temporal lobe epilepsy, as well as examine the relationship between hippocampal dentation and total volume.

Correspondence: Julia C. Fleming, B.S., Medical/Clinical Psychology, University of Alabama at Birmingham, 2105 English Village Lane, Birmingham, AL 35223.E-mail: flemingj@uab.edu 
B.B. TROTTER, C.B. FORTIER, M.E. ROBINSON, D.H. SALAT, W.P. MILBERG \& R. MCGLINCHEY. Effect of Adolescent Binge Drinking on Frontal White Matter Circuitry in Veterans of OEF/ OIF/OND.

Objective: Veterans of OEF/OIF/OND suffer from high rates of psychological problems, including alcohol abuse. This study examined whether Veterans who began binge drinking (BD) in early adolescence have decreased WM integrity (fractional anisotropy; FA) in a neural pathway essential for behavioral inhibitory control and good decision-making. Participants and Methods: Thirteen Veterans with early onset of BD (aged 12-14) were compared to fifteen with late (18-20) onset of BD for differences in FA in a pathway connecting the nucleus accumbens (NAcc) to the orbitofrontal cortex (OFC). Groups were matched demographically and for quantity of lifetime alcohol consumption. A structural pathway from the accumbens area to the medial OFC was defined using an anatomical seed within the accumbens to obtain whole brain seed-based functional connectivity maps in each individual and then probabilistic tractography procedures within the FSL data processing tools to structurally connect the accumbens area to the cortical region exhibiting functional connectivity.

Results: Tractography revealed a robust structural connection between the NAcc seed and OFC target bilaterally in all subjects. Analysis of mean FA in ROI defined by tractography revealed a significant difference between BD groups $(\mathrm{p}<0.05)$, with those reporting early onset of BD having a lower mean FA in this ROI than late onset.

Conclusions: The integrity of WM in a critical inhibitory control circuit is adversely affected by early BD. A pattern of BD during early adolescence may result in altered brain development that interferes with the normal maturation of executive functions and heightens risk for alcoholism.

Correspondence: Benjamin B. Trotter, Bachelor of Science, Research, VA Boston Healthcare System, 150 South Huntington Ave, Boston, MA 02130.E-mail: benjamin.trotter308@gmail.com

S.G. SHOLLENBARGER, K. MAPLE \& K. LISDAHL. Impact of Sleep Quality on Prefrontal Gyrification in Cannabis Using Emerging Adults.

Objective: Cannabis legislation changes highlight the need to determine its impact on populations with greater use, such as young adults (Johnston et al., 2014). We previously found reductions of prefrontal (PFC) gyrification in users compared to controls (Shollenbarger et al., in progress), and more use predicted poorer sleep quality (Maple et al., in progress). We hypothesize that poorer sleep quality will mediate the association between cannabis use and PFC gyrification.

Participants and Methods: Participants were 37 cannabis users (ages 18-25). Exclusions included major medical/neurologic/psychiatric disorders; or excessive other drug use. PFC gyrification was measured using FreeSurfer's local gyrification index (LGI: Schaer et al., 2008). Sleep quality was measured using the Pittsburgh Sleep Quality Inventory (Buysse et al., 1989). Cannabis and alcohol use were measured using a modified Time Line Follow Back (Sobell et al., 1979). Multiple regressions determined if past year cannabis use predicted LGI and if sleep mediated this relationship.

Results: After controlling for age, gender, and past year alcohol use, marginal relationships were observed between increased past year cannabis use and reduced right: ventral-medial (beta $=-.35, p=.1$ ), ventral-lateral (beta=-.30, $\mathrm{p}=.12$ ) and frontopol (beta=-.32, $\mathrm{p}=.09$ ) PFC gyrification; whereas, sleep did not mediate this relationship.

Conclusions: We found marginal dose-dependent relationships with right orbital frontal regions in cannabis users. Animal studies suggest that ventral aspects of the PFC may regulate the ability to foresee outcomes of one's behavior (Lucantonio et al., 2014), suggesting abnormalities may relate to poor insight. Although young users have demonstrated sleep problems (Cohen-Zion et al., 2009; Maple et al., in progress), this study demonstrates that sleep quality did not mediate the relationship between use and PFC gyrification. Thus, the aforementioned abnormal gyrification patterns observed in cannabis users cannot be explained by poor sleep quality.

Correspondence: Skyler G. Shollenbarger, M.S., Psychology, The University of Wisconsin-Milwaukee, 3324 S. Quincy Ave, Milwaukee, WI 53207.E-mail: skyler.shollenbarger@gmail.com

L.E. BRADSTREET, E. HECHT, D.L. ROBINS, J.A. TURNER \& T.Z. KING. The Examination of White Matter Microstructure, Autism Traits, and Social Cognitive Abilities in Neurotypical Adults.

Objective: Social cognition requires coordinated activity of multiple cortical and subcortical brain regions. White matter (WM) tracts, including uncinate fasciculus (UF) and inferior longitudinal fasciculus (ILF), may play an integral role in neural networks that underlie these processes. Structural differences in UF and ILF have been found in individuals with autism spectrum disorder (defined by disruptions in social behavior). The current study examines the relationships among mentalizing abilities, self-reported autism traits, UF, and ILF in neurotypical adults.

Participants and Methods: Twenty-four neurotypical adults (Mage $=21.92$ years, $\mathrm{SD}=4.72$ years; 15 women) completed the Autism Spectrum Quotient (AQ), the Dynamic Interactive Shapes Clips (DISC) task (mentalizing task), WASI-II, BASC-2, and diffusion tensor imaging scans. Tract-based spatial statistics was used to conduct voxelwise cross-participant comparisons of fractional anisotropy (FA) values in bilateral UF and ILF, with corticospinal tract (CST) as a control tract, as predicted by DISC scores and AQ total scores.

Results: FA values in left ILF were positively related to AQ total scores $(p<.05)$ and not related to DISC scores. The UF was not significantly related to either. DISC scores were not significantly associated with AQ total scores $(\mathrm{r}=.11, \mathrm{p}=.62)$.

Conclusions: The findings are consistent with prior research suggesting that higher levels of self-reported autism traits are associated with altered WM microstructure in the left ILF (see Iidaka et al., 2012). The ILF may play an important role in bringing sensory information to brain regions in the "social network." The results highlight the complex relationships among measures of WM microstructure and function. Correspondence: Lauren E. Bradstreet, Clinical Neuropsychology Ph.D., Psychology, Georgia State University, 855 Peachtree St. NE, Unit 2003, Atlanta, GA 30308.E-mail: lbradstreet1@gmail.com

S.M. SZYMKOWICZ, M.E. MCLAREN, A. O'SHEA, A. WOODS, T.M. MANINI, S.D. ANTON \& V.M. DOTSON. Subthreshold Depressive Symptoms are Associated with Age-related Structural Brain Changes.

Objective: Subthreshold depression, defined as clinically significant symptoms of depression that do not meet diagnostic criteria for major depression, is prevalent and associated with a range of negative health outcomes. Both frontal and limbic structures play a role in the development of depression. These same structures undergo atrophy with increasing age. While age-related structural brain changes in frontal and limbic regions appear to be associated with major depression, their role in subthreshold depression remains unclear. We hypothesized that increased depressive symptoms would be associated with age-related atrophy in frontal (anterior cingulate, orbitofrontal) and limbic (amygdala, hippocampus) structures.

Participants and Methods: Fifty-four adults (38 females; mean age $=$ $59.53 \pm 20.38$ years) completed the Center for Epidemiologic Studies Depression Scale and underwent structural magnetic resonance imaging scanning at 3T. Volumetric segmentation was performed used FreeSurfer and regional values were entered into hierarchical regression models in SPSS, controlling for sex and total gray matter volume.

Results: A depressive symptoms by age interaction was found for the left medial orbitofrontal cortex $(p=.04)$. Age differences in the left medial orbitofrontal cortex were greater at higher depressive symptoms, 
such that volumes were smaller in older adults with higher depressive symptoms.

Conclusions: Consistent with previous research, our findings suggest that structural changes in the left medial orbitofrontal cortex are associated with increasing depressive symptoms, particularly with advanced age. Further research is needed to better understand possible mechanisms through which subthreshold depressive symptoms affect specific brain regions during aging.

Correspondence: Sarah M. Szymkowicz, University of Florida, Dept of Clinical \& Health Psychology, PO Box 100165, Gainesville, FL 326100165. E-mail: smszymkowicz@phhp.ufl.edu

S. COOLEY, T. CONTURO, D. LAIDLAW, R. CABEEN, E.M. LANE, J.M. HEAPS, J. BOLZENIUS, L. SALMINEN, L. BAKER, S.E. SCOTT \& R. PAUL. Impact of Blood Pressure on White Matter Tracts in Healthy Older Adults.

Objective: Hypertension is a major contributor to age-related decline in white matter (WM) microstructural integrity. Previous studies have utilized diffusion tensor imaging (DTI) metrics fractional anisotropy (FA) and mean diffusivity (MD) to examine the relationship between hypertension and microstructural changes in several WM tracts demonstrating age-related decline. The impact of hypertension on other WM tracts similarly affected by aging remains unclear. The purpose of the present study is to identify the effect of hypertension on the inferior-fronto-occipital fasciculus (IFOF), inferior longitudinal fasciculus (ILF) and superior longitudinal fasciculus (SLF).

Participants and Methods: Sixty-six healthy older adult participants were divided into groups, normotensive $(\mathrm{n}=47)$ and hypertensive ( $\mathrm{n}$ =19), to examine differences in FA and $\mathrm{MD}$ of selected $\mathrm{WM}$ tracts. A secondary analysis included the grouping of "pre-hypertensive" (normotensive $\mathrm{n}=15$, pre-hypertensive $\mathrm{n}=32$, hypertensive $\mathrm{n}=19)$. Group differences were identified using multivariate analyses of variance (MANOVAs) with Tukey HSD post-hoc analysis.

Results: Hypertensives exhibited significantly decreased FA in bilateral IFOF and ILF, significantly decreased MD in the right IFOF, and significantly increased MD in bilateral SLF compared to normotensives. With three groups, significantly decreased FA was observed among hypertensives compared to pre-hypertensives in bilateral IFOF, and compared to normotensives in the right IFOF and left ILF. Hypertensives exhibited significantly decreased MD in the right IFOF and SLF compared to normotensives, and left SLF compared to pre-hypertensives.

Conclusions: Results from this study suggest that hypertension significantly impacts WM tracts in healthy older adults in a similar pattern to that of normal aging.

Correspondence: Sarah Cooley, Psychology, University of Missouri - St. Louis, 1 University Blvd., Stadler 442B, St. Louis, MO 63121. E-mail: sac232@mail.umsl.edu

K. BIRTCHER, N. GILES, A. DRESDEN, B. KIRWAN \& R. HOPKINS. Hippocampal, Medial Temporal Lobe, and Ventricle Volumes: Longitudinal follow up of Amnesic Subjects.

Objective: To assess longitudinal hippocampal, ventricle, and medial temporal lobe (MTL) volumes in amnesic subjects with hippocampal damage.

Participants and Methods: Longitudinal structural MRI scans were obtained from two male, amnesic patients (JRW and RS) who have selective hippocampal damage following anoxic injury. Structural MRI scans obtained in 2014 (time 2) were compared to initial MRI scans obtained 13 and 19 years ago (time 1). Age-matched controls' brain volumes were compared to subject volumes at time 2 .

We used Analysis of Functional NeuroImages (AFNI) to obtain volumetric measurements of hippocampus, MTL, and total intracranial volumes for subjects and controls. Inter-rater reliability (measured as Dice similarity coefficient) was good (mean DSC>.7). Lateral ventricle volumes and total intracranial volumes were obtained using FreeSurfer. Brain volumes were corrected for head size using total intracranial volumes so regions of interest could be compared across patients and controls.

Results: There was minimal change in hippocampal volume as a proportion of total brain volume over time (JRW time $1=.08 \%$, time 2 $=.06 \%$; RS time $1=.08 \%$, time $2=.07 \%$ ); however, there was an increase in total ventricle size in patient JRW $(+1.09 \%)$ and patient RS $(+1.06 \%)$. Patients had significantly reduced hippocampal volumes and increased ventricle volumes relative to controls at time $2(p ' s<.05)$. These volume differences appear to be due to anoxic injury. There was no significant difference in overall MTL volume ( $p$ 's >.1).

Conclusions: These longitudinal findings suggest that structural regions affected by anoxic brain injury are stable over time.

Correspondence: Kacie Birtcher, Psychology, Brigham Young University, 528 N. 720 E., 316, Provo,UT 84606.E-mail: kacie.birtcher@hotmail. com

L. COLON-PEREZ, S.J. CROWLEY, W. TRIPLETT, P. NGUYEN, C. PATTEN, A. FORD, M. CORTI, T. MARECI \& C. PRICE. A Majority Rule Approach for Segmenting the Corticospinal Tract from High Angular Resolution Diffusion Imaging.

Objective: Researchers often rely upon hand-drawn regions of interest to guide tractography. This method is vulnerable to rater bias, scan variability, and participant variability, however. Here, we report on a "majority rule" approach for ROI creation to guide tractography for an important white matter structure: the corticospinal tract (CST).

Participants and Methods: Diffusion imaging from one healthy participant was acquired across ten separate sessions over the course of one month (3T Siemens Verio). We calculated whole brain high angular resolution tractography with 64 seed points per voxel. Four raters identified ROIs within the left hemisphere (Cerebral Peduncle (CP); Internal Capsule (IC); Hand Bump (HB))using a group-established standard operating procedure. Each rater traced each ROI twice for every scan session. The overlap of these two ROIs was used to define a representative rater ROI. These ROIs were combined to create a majority rules ROI, in which each voxel had to be selected by at least three of four raters. Reproducibility for ROIs and CST segmentations were analyzed with the Dice Similarity Coefficient (DSC).

Results: Intra-rater reliability for each ROI was high (DSCs $\geq 0.83$ ). Inter-rater reliability was moderate to adequate (DSC range .54-.75; lowest for ICP). Using intersected ROI rater masks, the resulting CST showed improved overlap (DSC $=0.82$ ) across the ten intervals.

Conclusions: Despite high intra-rater reliability, there was low inter-rater reliability which is consistent with the expectation of rater bias by region. Employing a majority rules method resulted in improved overlap between scan sessions. The next step is to apply the majority rule approach to a dataset that assesses structure/function associations. Supported in part by NINDS and NINR.

Correspondence: Samuel J. Crowley, Clinical and Health Psychology, University of Florida, 2220 SW 34th Street Apt 207, Gainesville, FL 32608.E-mail:samjcrowley@ufl.edu

M. DÍEZ-CIRARDA, N. IBARRETXE-BILBAO, J. PEÑA, A. CABRERA, O. LUCAS-JIMÉNEZ \& N. OJEDA. Distinct Brain Volume, Diffusivity and Activation in Parkinson`s Disease MCI Subtypes.

Objective: To evaluate structural and functional cerebral differences in Parkinson`s disease (PD) patients with different MCI subtypes, focusing on the differences between patients with impairment in executive function (EF) and memory.

Participants and Methods: Thirty-three PD-patients underwent a neuropsychological battery and structural magnetic resonance images (MRI), diffusion tensor imaging (DTI) and AMRI were acquired while performing a memory task. Four groups were created based on patient's performance in EF or memory. Patients with impaired EF ( $\mathrm{n}=15)$ and memory ( $n=21)$ were compared with patients with preserved EF ( $n=18)$ and memory $(n=12)$ respectively. Cognitive function was impaired if 
patients scored below 1.5S.D regarding the mean of the matchedhealthy control group. Gray (GM) and white matter (WM) volumes, WM fractional anisotropy (FA) and brain activations were calculated and compared between groups.

Results: PD-patients with impaired EF showed significantly lower GM $(t=2.40 ; p<.022)$, WM volume $(t=2.40 ; p<.027)$, total brain volume $(\mathrm{t}=2.61 ; \mathrm{p}<.014)$, mean FA $(\mathrm{t}=2.28 ; \mathrm{p}<.030)$ and activation in the left $(\mathrm{t}=5.91 ; \mathrm{p}<.001)$ and right $(\mathrm{t}=.018 ; \mathrm{p}<.018)$ inferior orbitofrontal cortex (iOFC) compared with those with preserved EF. PD-patients with impaired memory showed lower activation in left $(\mathrm{t}=2.91 ; \mathrm{p}<.006)$ amygdala, left hippocampus $(\mathrm{t}=2.61 ; \mathrm{p}<.014)$, left $(\mathrm{t}=2.28 ; \mathrm{p}<.030)$ and right $(\mathrm{t}=3.35 ; \mathrm{p}<.002)$ caudate, left $(\mathrm{t}=2.60 ; \mathrm{p}<.014)$ and right $(\mathrm{t}=2.35 ; \mathrm{p}<.025)$ iOFC comparing with those with preserved memory. Conclusions: Lower brain activation in several limbic structures might be related to memory impairment in PD, while reduced activation in bilateral $\mathrm{OOFC}$ seems to be involved in both $\mathrm{EF}$ and memory impairment. Brain atrophy and reduced mean FA are related to EF impairment in PD. In conclusion, these results may suggest that different MCI subtypes in PD show different neural bases.

Correspondence: ,, . E-mail:

\section{K. STEWARD, J. MCNEELY, D. EAGAN, S. KAUR, P. PARKER \& A. HALEY. Peripheral Inflammation Does Not Relate to Cortical Thickness in Middle Aged Adults.}

Objective: High levels of C-reactive protein (CRP), a marker of systemic inflammation, are associated with impaired cognitive function in the elderly. Even in cognitively normal middle-aged adults, elevated CRP has related to disturbances in cerebral metabolism reminiscent of prodromal Alzheimer's disease. Since cortical thickness is a more proximal measure of brain integrity than cognitive test performance, the aim of the current study was to determine if CRP relates to cortical thickness in middle-aged adults.

Participants and Methods: One hundred and thirty adults (49\% male), aged 40-60, were included in this study. Participants completed a health assessment including a fasting blood draw to assess CRP levels, high-resolution structural magnetic resonance imaging (MRI) to assess cortical thickness in the frontal and temporal lobes, and a comprehensive neuropsychological battery to assess global intelligence, executive functioning, and verbal memory.

Results: After controlling for age, education, body mass index, and blood pressure in multiple linear regression models, CRP levels were not significantly related to cognitive performance, or to frontal or temporal cortical thickness (all p>.05). Interestingly, a trend between higher CRP levels and better memory performance was noted $(\mathrm{p}=.081)$.

Conclusions: Contrary to expectations, CRP elevation in middle age was not related to cortical thinning, despite being related to early disturbances in cerebral metabolism in prior studies. Thus, we concluded that markers of cerebral metabolism are potentially more sensitive indicators of brain integrity in midlife than cortical thickness. It is imperative that the relationship between inflammation and brain vulnerability be examined further in future longitudinal studies.

Correspondence: Kayla Steward, B.S., Medical/Clinical Psychology, University of Alabama at Birmingham, 1620 12th St. S., Birmingham, AL35205.E-mail: kasteward@comcast.net

A.D. THAMES, S.A. CASTELLON, E.J. SINGER, R. NAGARAJAN, M. SARMA, J. SMITH, N.S. THALER, J.H. TRUONG, D. SCHONFELD, A.M. THOMAS \& C. HINKIN. Using Multimodal Neuroimaging to Understand Neurocognitive Functioning and Fatigue in Patients With Hepatitis C.

Objective: The current study used multimodal neuroimaging methods (proton magnetic resonance spectroscopy [MRS] and diffusion tensor imaging $[\mathrm{DTI}]$ ) to understand neurocognitive performance and fatigue among a sample of Hepatitis-C infected adults (HCV+). We expected to observe structural brain abnormalities, poor neurocognitive performance, and fatigue among our HCV participants when compared to HCV-negative controls.

Participants and Methods: HCV+ individuals $(\mathrm{n}=76)$ and 20 controls were recruited to undergo neurocognitive and clinical assessments. A subset of HCV + individuals $(\mathrm{n}=29)$ and all controls $(\mathrm{n}=20)$ underwent MRI. DTI was acquired using a single-shot echo planar dual SE sequence with ramp sampling. Proton spectra were collected using the 2D MRSI technique with point-resolved spectroscopy (PRESS).

Results: HCV+ demonstrated greater fractional anisotropy (FA) in basal ganglia, thalamus, and striatum as well as greater mean diffusivity (MD) in the fronto-occiptal fasciculus, internal capsule, and external capsule than did HCV - controls. HCV+ participants also demonstrated lower levels of $\mathrm{N}$-acetylaspartate (NAA) in bilateral parietal white matter and elevations in myo-inisotal (MI) in bilateral frontal white matter compared to HCV - healthy controls (all p's $<.05)$. Cognitive dysfunction was also more prevalent among $\mathrm{HCV}+$ patients with particularly poor performance in processing speed, verbal fluency and executive functioning. HCV+ patients reported higher levels of fatigue than did controls and fatigue was significantly correlated with diffusivity in the superior fronto-occipital fasciculus, elevations in $\mathrm{mI}$ in frontal white matter, and overall cognitive performance.

Conclusions: Our results suggest that HCV disrupts frontal-striatal structures, which is associated with increased fatigue and cognitive deficits. The current study demonstrates how abnormalities in frontal/ parietal and subcortical structures have independent and overlapping relationships with cognitive performance and fatigue.

Correspondence: April D. Thames, Ph.D., Psychiatry, University of California Los Angeles, 740 Westwood Plaza C8-226, Los Angeles, CA 90095.E-mail:athames@mednet.ucla.edu

D. HAN, P. BOYLE, B. JAMES, L. YU, K. ARFANAKIS, D. FLEISCHMAN \& D. BENNETT. Grey Matter Correlates of Susceptibility to Scam in Community-Dwelling Older Adults.

Objective: Vulnerability to scams is a significant issue among older adults, and reports suggest even cognitively intact older adults may be susceptible. We investigated voxel-based grey matter associations with scam susceptibility in a large community-based cohort of nondemented older adults and tested the hypothesis that this would be associated with less grey matter in frontal and temporal regions.

Participants and Methods: Nondemented adults ( $\mathrm{N}=327$, mean age $=81.55$, mean education $=15.30,78.9 \%$ female and $94.2 \%$ white) from the Rush Memory and Aging Project completed a measure of scam susceptibility, 19 cognitive measures, and a $1.5 \mathrm{~T}$ MRI scan. Structural scans were processed using voxel-based morphometry (VBM). Regressions were used to examine the relationship between voxel-based estimates and susceptibility to scam. Multiple comparisons were corrected at the cluster level using random field theory (voxel threshold $p<0.001$ ). Results: Results indicated an inverse association between overall grey matter volume and susceptibility to scam $(\mathrm{r}=-0.133, \mathrm{p}<0.016)$. No significant associations were observed for white matter and CSF. Wholebrain models adjusted for age, education, and sex revealed seven clusters showing significantly lower grey matter in the right parahippocampal region $(t=4.72)$, the left middle temporal region $(t=3.93)$, the left inferior frontal region $(t=4.00)$, the right medial frontal region $(t=3.78)$, the right middle temporal region $(t=4.38)$, the right precuneus region $(\mathrm{t}=3.63)$, and the right dorsolateral prefrontal region $(\mathrm{t}=4.05)$. In wholebrain models further adjusting for global cognition, results revealed three significant clusters showing lower grey matter in the right parahippocampal region $(t=4.26)$, the right hippocampal region $(t=3.71)$, and the right middle temporal region $(\mathrm{t}=4.05)$.

Conclusions: These results suggest grey matter reductions in specific brain regions may be associated with susceptibility to scam, above and beyond the effects of cognition.

Correspondence: Duke Han, PhD, Behavioral Sciences, Rush Alzheimer's Disease Center, 600 S. Paulina St., 1022, Chicago, IL 60612. E-mail: Duke_Han@rush.edu 
T.M. HARRISON, A.M. PAIK, A. BURGGREN, G.W. SMALL \& S. BOOKHEIMER. The Relationship between Common Cognitive Reserve Measures and Brain Structure in Nondemented Older Adults.

Objective: Recently cognitive reserve has undergone a process of conceptual refinement. However, the way best to measure cognitive reserve during neuropsychological assessment remains unclear. In the present study, we use two common measures of cognitive reserve, years of education and the Wechsler Test of Adult Reading (WTAR), to examine relationships between each measure and brain structure.

Participants and Methods: To investigate the relationship between cognitive reserve and cortical thickness in older nondemented adults, 156 subjects were grouped into high and low education groups (EDU-hi, EDU-lo) using a median split. For the WTAR analyses, a subset of subjects with WTAR scores available were used ( $\mathrm{n}=125$; WTAR-hi, WTAR-lo). A general linear model was used to compare cortical thickness between groups across the whole brain. Statistical thresholding was based on Monte-Carlo simulations, with 10,000 iterations and a cluster p-value of 0.05 .

Results: In the EDU-hi/lo comparison, a significant cluster was identified in the frontal lobe, including parts of orbital frontal cortex and medial prefrontal cortex, where cortical thickness was greater in the EDU-hi group. In the WTAR-hi/lo analysis, a significant cluster where cortical thickness was greater for the WTAR-hi group was found. This cluster included portions of the temporal pole and inferior temporal lobe. Both significant clusters were registered to individual subjects' space and used as regions-of-interest (ROIs) for further analysis. Interestingly, thickness in the EDU-hi/lo cluster was not significantly correlated to any other cognitive measures, but thickness in the WTAR-hi/lo cluster had a significant $(\mathrm{p}<0.001)$ relationship with MMSE $(\mathrm{r}=0.36)$, MemCDR $(\mathrm{r}=-$ 0.37), LM Delay ( $r=0.38)$, VP Delay $(r=0.41)$ and Trails B $(r=-0.34)$. Conclusions: In the cluster identified by comparing high and low WTAR, cortical thickness correlated with several cognitive scores. Thus, WTAR may be a more useful measure of cognitive reserve for studies of brain structure.

Correspondence: Tessa M. Harrison, UCLA, 760 Westwood Plaza, Suite B8-169, Los Angeles, CA 90095. E-mail: theresamaria@gmail.com

J.M. GULLETT, A. BOHSALI, R.M. BAUER, T. MARECI \& D. FITZGERALD. The Gating Role of the ACC in PTSD ReExperiencing: A Connectivity Study.

Objective: Persons with Post-Traumatic Stress Disorder (PTSD) often experience intrusive symptoms, such as re-experiencing past life-threatening events. Several fMRI studies associate under-activation of the amygdala by the prefrontal cortex with PTSD symptoms, as well as the Anterior Cingulate Cortex (ACC) with state re-experiencing severity in PTSD. However. little is known about the role of white matter connectivity between the ACG, amygdala, and hippocampus as it relates to the self-report of re-experiencing symptoms in PTSD. We hypothesize that the integrity of white matter in the ACC-amygdala and ACC-hippocampus pathways in veterans with PTSD will be associated with reports of traumatic re-experiencing.

Participants and Methods: Participants were 32 male OIF/OEF veterans with reported symptoms of PTSD on the PCL-M and a past history of mild TBI. MRI data consisted of structural T1 scans used to determine automated segmentation of Regions of Interest (ROI's), and 64-direction DTI scans to infer white matter tract volume and integrity between ROI's.

Results: After removing variance due to depressive symptoms, white matter tract volume between the ACC and amygdala was negatively associated with re-experiencing symptom report $(p<.05)$. Similarly, fractional anisotropy of white matter connections between the ACC and hippocampus was negatively associated with re-experiencing symptoms report $(\mathrm{p}<.05)$.

Conclusions: White matter connectivity between the ACC and the amygdala is reduced in veterans with PTSD, as is the integrity of
ACG-hippocampal connections. These results provide structural evidence that is consistent with findings from previous fMRI work suggesting that changes in ACC-amygdala/hippocampal connectivity alters the ability of the ACC to exert its normal inhibitory/excitatory (gating) influence on these medial temporal structures, leading to pathological re-experiencing of traumatic episodic memories.

Correspondence: Joseph M. Gullett, B.S., Clinical and Health Psychology, University of Florida, Health Science Center, PO Box 100165, Gainesville,FL 32610.E-mail: gullettj@phhp.ufl.edu

M. SULLAN, A. BOHSALI, J.M. GULLETT, J. GOLDSTEIN, R.M. BAUER, T. MARECI \& D. FITZGERALD. Proof-of-concept for in vivo mapping of the human locus coeruleus using Fast Gray Matter Acquisition T1 Inversion Recovery (FGATIR) Imaging Data.

Objective: To establish a reliable protocol for in vivo localization of the human locus coeruleus (LC) using high-resolution MR imaging. This structure provides nearly all the noradrenergic innervation to the brain and plays key roles in sleep cycles, attention, arousal and other cognitive functions. However, its small size ( 1-2 mm in diameter) and location within the brainstem present many challenges for LC localization. The present study demonstrates a reliable protocol for LC localization using a novel MR imaging approach.

Participants and Methods: We collected structural MR data (T1weighted and FGATIR images) on 19 veterans and 19 healthy volunteers. The LC was first identified using a template developed by Keren et al. (2009) on T1-weighted scans, and then manually corrected using FGATIR image intensities. To establish reliability measures, LC masks of 10 randomly selected participants were re-traced using this protocol. To ensure reliable registrations between template spaces, and individual T1 and FGATIR images, we used the thalamus template from the Harvard-Oxford atlas to test registrations. The location and accuracy of the registration of the thalamic template was verified by a trained neuroanatomist $(\mathrm{AB})$

Results: The Dice similarity coefficient (DSC) was computed to assess the amount of overlap between the two sets of LC masks from the 10 randomly selected participants. The DSC for this structure ranged from .90 to .96 , exceeding the acceptability cutoff of 0.7 . Anatomic analysis revealed correct localization of the thalamic mask in all participants. Conclusions: This segmentation protocol provides a reliable method for $L C$ visualization in vivo.

Correspondence: Molly Sullan, BS, Clinical and Health Psychology, University of Florida, 230 SW 2nd Ave, Apt 403, Gainesville, FL 32601. E-mail:mollysullan@gmail.com

T.L. MERKLEY, E.A. WILDE, S. MCCAULEY, A. BARNES, X. LI, E.D. BIGLER \& H. LEVIN. Persistent Post-concussive Symptoms and Cortical Thinning in Mild Traumatic Brain Injury.

Objective: This study investigated how self-reported post-concussive symptoms (PCS) at 3 months post-injury relate to cortical thickness in mild traumatic brain injury (mTBI)

Participants and Methods: Participants included 58 individuals $(18 \mathrm{~F} / 40 \mathrm{M}$; mean age $=19.7 \pm 5.3)$ with a history of $\mathrm{mTBI}$ who underwent MRI scanning within 96 hours post-injury. A subset of 41 participants had scans at 3 months post-injury. At 3 months, residual cognitive, emotional, and somatic symptoms were rated on the Rivermead Post-concussion Symptoms Questionnaire. Those reporting a total score of $\geq 12$ were identified with persistent PCS. The MRI data were analyzed using the Freesurfer neuroimage analysis suite.

Results: A general linear model was fit at each vertex on the cortical surface for cortical thickness differences between high and low PCS groups, controlling for age at injury and gender. Within 96 hours post-injury, the high PCS group demonstrated reduced cortical thickness in left lateral orbital frontal $(p=0.003)$, right occipital $(p=0.002)$, and right fusiform gyrus $(p=0.002)$. At 3 months post-injury, the high PCS group demonstrated reduced cortical thickness in left lateral orbital 
frontal $(p=0.005)$, left temporal $(p=0.0001)$, and right anterior temporal $(\mathrm{p}=0.002)$.

Conclusions: Cortical reductions were observed within 96 hours in the high PCS group, which may reflect the interaction of premorbid vulnerability with reduced cortical thickness rather than pathological cortical thinning within this limited post-injury time interval. Larger regions of cortical reductions at 3 months post-injury may reflect an interaction between premorbid vulnerability and sequelae of injury.

Correspondence: Tricia L. Merkley, PhD, Barrow Neurological Institute, 745 N. Dobson Rd. \#156, Mesa, AZ 85201.E-mail: merkleytl@gmail. com

M.W. HAUT, D. COBIA, M. WISCHNEWSKI, G. KOWALSKI, X. HU \& C.M. BUETEFISCH. Primary Motor Cortex Thickness Correlates with the Ability for Motor Learning.

Objective: We examined variations in the thickness of the primary motor cortex (M1) in healthy subjects and their performance on a motor learning task.

Participants and Methods: Nine right-handed healthy individuals (6 females; mean age 61 years) participated. In training sessions, subjects manipulated a joystick pointing task with a parametric increase of difficulty (decreasing target size). Targets consisted of differently sized squares displayed at four locations on a monitor. After individual target feedback was provided about performance, the task continued if $<50 \%$. Each run consisted of 3 blocks of 21 pointing movements. Motor learning was expressed as training related increase in \% hits, normalized to the level of each subject's skillfulness at baseline and amount of training runs. High resolution 3T MPRAGE scans were processed with FreeSurfer. Surface-based cortical thickness of M1 was estimated and correlated with the standardized learning value for each hand in the contralateral hemisphere (FDR corrected).

Results: Thickness of the left M1 hand area correlated with right hand motor learning $(p<.05)$ and the thickness of the right M1 hand area correlated with left hand motor learning $(\mathrm{p}<.05)$. The extent and peak strength was greater for the left hand. There was no correlation between baseline motor skill and M1 cortical thickness.

Conclusions: We demonstrated a correlation between the ability to learn a visual motor task and corresponding M1 thickness. Considering that M1 plays a role in the early phase of motor learning, these results suggest that M1 structure contributes to the neuronal substrate for successful motor learning.

Correspondence: Marc W. Haut, PhD, Behavioral Medicine \& Psychiatry, WVU School of Medicine, 930 Chestnut Ridge Road, Morgantown, NY 26505.E-mail:mhaut@hsc.wvu.edu

C. WATSON, N. PATEL, M. WYNN, J.H. KRAMER \& B.M. BETTCHER. Perceived Stress and Corpus Callosum Integrity in Older Adults.

Objective: Chronic, perceived stress has been linked with a host of negative health outcomes, including effects on brain structure. Preliminary data associated self-reported stress levels with lower white matter volume in the corpus callosum in young adults. Limited information exists on its association with white matter integrity in older adults and whether this relationship is independent of depressed mood.

Participants and Methods: 87 normal older adults (Mean Age (SD) $=72.8(6.2)$; Males $=48 \%)$ underwent a 3.0T MRI diffusion tensor brain scan and completed self-report measures on depression (Geriatric Depression Scale) and perceived stress (Perceived Stress Scale, PSS). DTI corpus callosum (CC) regions of interest (ROI; genu, body, and splenium) were constructed from the JHU ICBM-DTI-81 white matter labels and mean fractional anisotropy (FA) was extracted using FSL. Generalized linear regression analyses were run with age, gender, ethnicity, and depression as covariates; perceived stress was the predictor of interest. CC ROIs served as dependent variables.

Results: Perceived stress was inversely correlated with FA in all regions of the CC, independent of demographics and self-reported depression scores (genu: Beta (SE) $=-.0019(.0009), p=.03$; body: Beta $=-.0017$ (.0008), $\mathrm{p}=.04$; splenium: Beta $=-.0012(.0005), \mathrm{p}=.03)$.

Conclusions: In conclusion, chronic, perceived stress is associated with poorer white matter microstructure in all regions of the corpus callosum in a cohort of community-dwelling older adults, independent of self-report depressive symptoms. Mediators of this relationship remain to be determined, but potentially include vascular and inflammatory factors. This work was supported by the Hillblom Foundation, and P50 AG023501 and 1R01AG032289 grants.

Correspondence: Christa Watson, Psy.D., Neurology, University of California, San Francisco, 675 Nelson Rising Lane, Suite 190, Memory and Aging Center, San Francisco, CA 94158.E-mail: christaleigh310@ gmail.com

A.E. BLANKEN, D. AVILA, N. GOUKASIAN, D. ELASHOFF, H. WILHALME, J. RINGMAN, E. WOO \& L. APOSTOLOVA. Cognitive Decline, Ventricular Enlargement, and Hippocampal Atrophy in Mild Cognitive Impairment.

Objective: To investigate the associations between hippocampal atrophy and ventricular enlargement with cross-sectional and longitudinal cognitive decline in cognitively normal $(\mathrm{NC})$ and mild cognitive impairment subjects (MCI).

Participants and Methods: 62 MCI and 49 NC subjects underwent structural MRI and detailed neuropsychological testing. Neuropsychological measures were combined in 5 cognitive domains - attention, executive, memory, visuospatial and language. Rates of longitudinal decline from baseline were calculated. MRIs were analysed using the radial distance technique followed by linear regression to detect in 3D associations between the cognitive performance, hippocampal atrophy and ventricular enlargement. We used permutation-based multiple comparison correction thresholded at $\mathrm{p}<0.01$.

Results: Initial univariate analyses revealed significant correlations between both baseline hippocampal atrophy and ventricular enlargement with impaired performance in all but the visuospatial domain. In multivariate models only the associations of memory and hippocampal atrophy remained significant.

Baseline hippocampal atrophy correlated with rate of decline in memory, attention, language and visuospatial function. Baseline ventricular enlargement showed a trend-significant association with rate of decline in the language and visuospatial domain on the left.

Conclusions: As expected both hippocampal atrophy and ventricular enlargement were significantly associated with poor cognitive performance at baseline. While both structural measures were predictive of decline in language and visuospatial function, hippocampal atrophy was also predictive of future worsening in memory and attention. These data confirm the value of structural imaging biomarkers for MCI prognosis. Correspondence: Anna E. Blanken, B.A., Neurology, UCLA, 13910 North West Passage, Apt 201, Marina del rey, CA 90292. E-mail: ablanken@mednet.ucla.edu

T.C. WU, T.L. MERKLEY, E.A. WILDE, A. BARNES, X. LI, B.C. SHIN, S. MCCAULEY, G. HANTEN \& H. LEVIN. Volumetric and DTI Analyses of Sports Concussion.

Objective: In recent years, the neurological, cognitive, and psychiatric sequelae of sports-related concussion (SC) have drawn increasing public attention. The current study utilized volumetric and diffusion tensor imaging (DTI) to study adolescents with SC.

Participants and Methods: Ten participants with SC, 12 with orthopedic injury (OI), and 12 typically-developing (TD) individuals aged 12-18 years were studied. All groups were demographically similar. SC participants had initial GCS scores between 13-15, LOC $<30$ minutes, PTA $<24$ hours, and negative intracranial CT. Participants underwent MRI scans within 72 hours and at 3 months post-injury. Volumetric and DTI tractography analyses utilized Freesurfer and Philips PRIDE software, respectively. DTI regions of interest included corpus callosum 
and its subdivisions, bilateral uncinate fasciculi, and bilateral cingulum bundles.

Results: DTI findings within 72 hours did not reveal significant differences between TBI vs. OI and TBI vs. TD for both fractional anisotropy (FA) and apparent diffusion coefficient (ADC). At 3 months post-injury, the SC group demonstrated significantly lower FA than TD group in CC (genu, splenium, and total), bilateral cingulum bundles, and right uncinate fasciculus. The SC group also had significantly higher ADC than TD group in the cingulum bundles bilaterally. No significant group differences for FA and ADC were observed between SC and OI groups. Volumetrically, there were no group differences in white matter volume of regions analogous to DTI pathways of interest at either 72 hours or 3 months post-injury.

Conclusions: Neuropathology resulting from SC may not be evident on neuroimaging immediately following injury, but may emerge with time. Further studies are needed.

Correspondence: Trevor C. Wu, PhD, Neuroscience, Mercy Health/ Baylor College of Medicine, 2329 Beatrice NE, Grand Rapids, MI 49505.E-mail:trevor:wu@mercyhealth.com

V. DELBENE, L.A. ROSS, M. WEITEKAMP, Y. WOO, G.A. ANDRADE, S. MOLHOLM, B.S. ABRAHAMS \& J.J. FOXE. Association Between Autism-Associated Polymorphism in CNTNAP2 and Bilateral Caudate Nucleus Volume Reductions in Healthy Adults.

Objective: Autism susceptibility genes have a direct role in neural metabolism and migration during development (Penagarikano \& Geschwind, 2008). In animal models, Cntnap2 knockout (KO) mice exhibit core features of autism, such as motor stereotypies (Penagarikano et al., 2011). In humans with autism, such repetitive behaviors have been linked with the caudate nucleus (CN; Wolff et al., 2013), with CN structural changes observed in this neurodevelopmental population (Cauda et al., 2011). We hypothesized that homozygous risk allele carriers will show $\mathrm{CN}$ volumetric reductions, relative to the heterozygous group, and both will be reduced compared to the homozygous non-risk allele carriers.

Participants and Methods: Healthy adults participated in a 3T MRI study, where T1-weighted images and genetic samples were acquired. The rs2710102 SNP from CNTNAP2 contains homozygous non-risk (TT; $\mathrm{N}=11$ ), heterozygous risk/non-risk ( $\mathrm{CT} ; \mathrm{N}=54$ ) and homozygous risk allele carriers (CC; $\mathrm{N}=20$ ). Volumetric differences were analyzed using ANCOVAs, with whole brain volume and gender as covariates.

Results: For SNP rs2710102, right CN volume was reduced for CC carriers $(\mathrm{M}=3760.05$, S.E.M. $=80.56)$ compared with $\mathrm{CT}(\mathrm{M}=3938.56$, S.E.M. $=48.72)$ and TT $(M=3900.74$, S.E.M. $=108.21), \mathrm{F}(4,80)=12.65$, $\mathrm{p}<0.001$. Left $\mathrm{CN}$ volume was reduced for CC carriers $(\mathrm{M}=3499.98$, S.E.M.=78.28), compared with CT $(M=3823.31$, S.E.M.=47.34 $)$ and TT $(\mathrm{M}=3656.58$, S.E.M. $=105.15), \mathrm{F}(4,80)=17.57, \mathrm{p}<0.001$.

Conclusions: Bilateral volumetric reduction of the $\mathrm{CN}$ in homozygous risk carriers of the CNTNAP2 SNP rs2710102 was observed. CN volume reductions may be reflective of reduced GABAergic interneuron density, as seen in Cntnap2 KO mice (Penagarikano et al., 2011).

Correspondence: Victor DelBene, MA, Ferkauf Graduate School of Psychology, Yeshiva University, 1225 Morris Park Avenue, Van Etten, 1st Floor, C-Wing, Bronx, NY 10461. E-mail: victor.delbene@gmail.com

J. CUI, O. TKACHENKO, H. GOGEL, M. KIPMAN, L. PREER, M. WEBER, S. DIVATIA, L.A. DEMERS, E.A. OLSON, J.L. BUCHHOLZ, J.S. BARK, I.M. ROSSO, S.L. RAUCH \& W.D. KILLGORE. Fractional Anisotropy of Frontoparietal Connections Predicts Individual Resistance to Sleep Deprivation. Objective: Sleep deprivation (SD) can degrade cognitive functioning. However, there are large individual differences in the vulnerability to this effect. Some evidence suggests that baseline differences in the responsiveness of a fronto-parietal attention system involved in working memory (WM) tasks may be associated with the ability to sustain vigilance during sleep deprivation. However, the neurocircuitry underlying this network remains virtually unexplored. Here, we combined functional magnetic resonance imaging (fMRI) and diffusion tensor imaging (DTI) to investigate the association between the microstructure of the axonal pathway connecting the frontal and parietal regions-i.e., the superior longitudinal fasciculus (SLF) — and individual resistance to SD. Participants and Methods: Thirty healthy participants (15 males) aged 20-43 years underwent fMRI and DTI at rested wakefulness prior to a 28-hour period of SD up to four days after scanning. Task-related fronto-parietal fMRI activation clusters during a Sternberg WM Task were localized and used as seed regions for probabilistic fiber tractography. DTI metrics including fractional anisotropy, mean diffusivity, axial and radial diffusivity were measured in the SLF. The Psychomotor Vigilance Test was used to evaluate resistance to SD.

Results: FMRI activation in the left inferior parietal lobule (IPL) and dorsolateral prefrontal cortex (DLPFC) positively correlated with resistance capacity. Higher fractional anisotropy of the left SLF comprising the primary axons connecting IPL and DLPFC was also associated with better resistance.

Conclusions: These findings suggest that individual differences in resistance to SD are associated with the functional responsiveness of a fronto-parietal attention system and the microstructural properties of the axonal interconnections between these regions.

Correspondence: William D. Killgore, Ph.D., Psychiatry, University of Arizona, Brain Imaging Center, McLean Hospital, 115 Mill Street, Belmont, MA 02478. E-mail: killgore@mclean.harvard.edu

T. KUHN, J. GULLETT, A. BOUTZOUKAS, A. FORD, P. CARNEY, D. FITZGERALD \& R.M. BAUER. Altered White Matter Connectivity in Adjacent Medial Temporal Circuits in Temporal Lobe Epilepsy.

Objective: Evidence for structural connectivity patterns within the medial temporal lobe derives primarily from post-mortem histological studies. In humans, the parahippocampal gyrus (PHg) is subdivided into parahippocampal (PHc) and perirhinal (PRc) cortices which receive input from distinct cortical networks and send distinct efferent projections to the entorhinal cortex (ERc). The PHc projects primarily to the medial ERc (M-ERc). The PRc projects primarily to the lateral portion of the ERc (L-ERc). Both M-ERc and L-ERc, via the perforant pathway, project to the dentate gyrus and hippocampal (HC) subfields. Until the recent advent of novel imaging techniques, these neural circuits could not be visualized in vivo.

Participants and Methods: Diffusion tensor imaging algorithms have been developed to segment grey matter structures based on probabilistic connectivity patterns. In this study, this segmentation procedure was used to classify ERc grey matter based on PRc, PHc, and HC connectivity patterns in 7 patients with temporal lobe epilepsy (TLE) without hippocampal sclerosis (mean age, $14.86+3.34$ ) and 7 healthy controls (mean age, $23.86+2.97)$.

Results: There was no significant between-group difference in surface area or volume of ERc connectivity-defined regions (CDR). In line with histology results, ERc CDR in the control group were well organized, uniform and segregated via PRc, PHc, and HC connections. In TLE, reduced spatial organization of ERc CDR was found. There was no clear delineation between M-ERc and L-ERc connectivity with PRc, PHc or HC in TLE.

Conclusions: Using current neuroimaging acquisition and analysis tools, adjacent temporal lobe circuits were successfully visualized in vivo. In the healthy group, segmentation revealed medial temporal connectivity patterns in agreement with extant histology literature. Conversely, the results suggest a breakdown of the spatial organization of $\mathrm{PHg}-\mathrm{ERc}-\mathrm{HC}$ connectivity in TLE.

Correspondence: Taylor Kuhn, M.S., University of Florida, 317 SW 54th Dr, Gainesville,FL 32607.E-mail: tkuhn@phhp.ufl.edu 


\section{Psychopathology/Neuropsychiatry (Including Schizophrenia)}

C. NOGGLE, J. THOMPSON, J. DAVIS \& A. LEVINE. Altered Neurocognitive Profiles on the WJ-III-Cognitive in a Middle-Aged Sample of Adult Depression: A Preliminary Analysis.

Objective: Neurocognitive deficits have commonly been associated with depression; however, this has predominately been noted in late adulthood. In comparison, fewer studies have focused on the middle adulthood population was raises substantial questions from a practice-based standpoint. This study sought to determine what if any neurocognitive deficits would arise in relationship to depression in middle adulthood and how such deficits would present on the WJ-III-Cognitive.

Participants and Methods: Middle-aged adults diagnosed with depression that had completed the WJ-III-Cognitive were extracted from an archival data set. Outcomes across the first seven subtests were compared to the normative sample. Individuals were selected based on them (1) having a primary diagnosis of Depression and no comorbid diagnosis, and (2) were between the ages of 35 to 55 (i.e. middle age). The resulting sample $(n=108)$ presented with a mean age of 44.06 (6.31) years, ranging from 35 to 55 years, and a mean of 13.39 (2.33) years of education.

Results: A very focal decline in an aspect of long-term memory and retrieval as evidenced by performance on the visual-auditory learning subtest of the WJ-III-Cognitive was noted $[\mathrm{t}(107)=-3.587, \mathrm{p}<.001]$, while sparing across other domains (i.e. verbal comprehension, spatial relations, sound blending, concept formations, visual matching, and numbers reversed was also found.

Conclusions: While findings contribute directly to the literature in terms of both deficits associated with depression in middle adulthood and specific deficits of this group on the WJ-III-Cognitive, they also carry implications for clinical practice and suggest multiple paths for future research endeavors.

Correspondence: Chad Noggle, Ph.D., ABN, Psychiatry, SIU School of Medicine, 315 W. Carpentedr St., Springfield, IL 62794. E-mail: cnoggle@siumed.edu

J. THOMPSON, C. NOGGLE, J. DAVIS \& A. LEVINE. Neurocognitive differences between Schizophrenia and Depression as measured by CNS Vital Signs.

Objective: Schizophrenia and depression are amongst the psychiatric presentations most commonly associated with neurocognitive problems. While some studies have compared the two, few have utilized computer-based assessment to determine differences between these clinical groups. The purpose of the present study was to determine if measureable differences in neurocognitive functioning not only exists between schizophrenia and depression, but also that computer-based assessment using CNS Vital Signs is sensitive to such differences.

Participants and Methods: The performances of 1,056 patients diagnosed with depression and 88 patients diagnosed with schizphrenia, both using the DSM-IV-TR criteria, were compared. Patients completed the core tests of the CNS Vital Signs computer-based assessment system. Group means and standard deviations were available for comparison. Results: Multiple independent sample t-tests were ran utilizing a Bonferroni correction to avoid inflation of family-wise error rate. Schizophrenia corresponded with significantly lower performance on verbal memory, visual memory, on the Stroop test, and in both reaction time and finger tapping. No significant difference was found between groups on a continuous performance test.

Conclusions: Overall, results both demonstrate the increased cognitive burden of schizophrenia compared to depression while also demonstrating the utility of CNS Vital Signs in making such comparisons. Correspondence: Chad Noggle, Ph.D., ABN, Psychiatry, SIU School of Medicine, $315 \mathrm{~W}$. Carpentedr St., Springfield, IL 62794. E-mail: cnoggle@siumed.edu
C. NOGGLE, J. THOMPSON, J. DAVIS \& A. LEVINE. Sensitivity of Computer-Based Assessment in the Identification of Neurocognitive Differences between Schizophrenia and Bipolar Disorder.

Objective: Psychiatric manifestations are commonly associated with cognitive problems. Both Schizophrenia and Bipolar Disorder have been linked with cognitive impairments. When compared, Schizophrenia more often than not is associated with greater cognitive burden compared to Bipolar disorder. However, few previous studies have investigated the sensitivity of computer-based assessments in identifying differences between these groups. The purpose of the present study was to determine if measureable differences in neurocognitive functioning exist between bipolar disorder and schizophrenia. In addition, we sought to evaluate the sensitivity of the CNS Vital Signs in identifying group differences, if any.

Participants and Methods: The performances of 447 patients diagnosed with bipolar disorder and 88 patients diagnosed with schizphrenia, both using the DSM-IV-TR criteria, were compared. Patients completed the core tests of CNS Vital Signs.

Results: Results revealed schizophrenia was associated with significantly lower performance in verbal memory and visual memory. In addition, individuals with schizophrenia performed significantly worse on the Stroop test, in reaction time, and in finger tapping. No significant difference was found between groups on a continuous performance test. Conclusions: Overall, results both demonstrate a greater impact on neurocognition in association with schizophrenia compared to bipolar disorder and support the sensitivity of CNS Vital Signs in identifying such deficits.

Correspondence: Chad Noggle, Ph.D., ABN, Psychiatry, SIU School of Medicine, $315 \mathrm{~W}$. Carpentedr St., Springfield, IL 62794. E-mail: cnoggle@siumed.edu

R.D. SHURA, L.L. DAVIDSON \& R.E. FEIERSTEIN. Assessment and Treatment of a Veteran with Conversion Myoclonus and PTSD. Objective: Conversion disorder refers to one or more symptoms of sensory or motor dysfunction that is not explained by neurological disease. A history of posttraumatic stress disorder (PTSD) was related to a 2.3 higher rate of conversion symptoms in a community sample (Andreski, Chilcoat, \& Breslau, 1998). However, little is known about the relationship between combat PTSD and conversion disorder.

Participants and Methods: A 66-year old Vietnam Veteran was referred for neuropsychological evaluation due to intense upper body myoclonus which began at age 62 . Since onset, the longest interval without a tic had been a few hours. His history was positive for combat PTSD, sleep apnea, tinnitus, HTN, and coronary artery disease. Multidisciplinary evaluations were completed.

Results: Brain imaging and neurological evaluation did not identify a cause for the myoclonus. Comprehensive neuropsychological evaluation results are presented. His MMPI-2 was consistent with PTSD (1-7-8; PK raw $=30)$. The myoclonus was conceptualized as a motoric conversion disorder in the context of PTSD. The Veteran later completed individual Prolonged Exposure. After treatment, he no longer met criteria for PTSD and his myoclonus resolved except during times of high stress. Conclusions: This case is unique in that it describes conversion disorder with a Veteran with combat PTSD and treatment of both disorders via evidence-based exposure therapy. A previous case study described the use of exposure therapy to resolve conversion mutism with a sexual assault survivor (Rothbaum \& Foa, 1991). Consequently, Prolonged Exposure may be a viable treatment for comorbid PTSD and conversion disorder.

Correspondence: Robert D. Shura, PsyD, Hefner VAMC, MA-MIRECC, 11M-2/MH\&BS, 1601 Brenner Ave., Salisbury, NC 28144. E-mail: shura@marshall.edu 
T. SAK, D.M. WHITESIDE, H. LUU, V. KERCE, L. RICE, K. NAYLON, D. CAIRNS, M.R. BASSO \& B. ROPER. The Relationship Between Executive Functioning and the Personality Assessment Inventory Validity and Clinical Scales in a Mixed Neuropsychological Sample. Objective: While previous meta-analyses reported depression was associated with executive functioning (EF) impairments (McDermott \& Ebmeier, 2009), there is limited literature examining EF in other psychiatric disorders (Snyder, 2013). The current study examined whether EF measures (including Verbal Fluency measures of FAS and Animal Fluency [AF], Trailmaking Test Part B [TMT-B], and Wisconsin Card Sorting Test-perseverative responses [WCST-PR]) could significantly predict scores on the Personality Assessment Inventory (PAI, Morey, 1996) validity scales, including NIM, PIM, ICN, and INF, and clinical scales including SOM, ANX, ARD, DEP, MAN, PAR, SCZ, BOR, ANT, ALC, DRG, AGG, and STR.

Participants and Methods: Consecutive neuropsychological evaluation referrals ages 18 and over who completed the study measures, passed cognitive performance validity tests (PVTs) and had interpretable PAI profiles $(\mathrm{NIM}<100)(\mathrm{N}=194$, mean age $=45.32[\mathrm{SD}=17.83]$, mean education $=13.48$ [2.42], 50.5\% female, 86\% Caucasian) were used in this analysis. The various PAI scales were the outcome variables, and the EF measures were the predictors in a series of multiple regression analyses. Results: Results indicated the EF measures could significantly predict $(\mathrm{p}<.05)$ the SOM (Somatic Concerns) scale $(\mathrm{R} 2=.06)$ and its subscales $(\mathrm{R} 2=.05$ to .06$)$, the AGG (Aggression) scale $(\mathrm{R} 2=.07)$ and the validity scales NIM $(\mathrm{R} 2=.09)$ and INF $(\mathrm{R} 2=.06)$, but not the other PAI scales. Conclusions: While the results of the study were only partially consistent with previous research, they provided evidence that EF is an important component of certain psychological issues, particularly somatization and aggression, as well as the PAI validity scales. Additional research exploring the relationship between executive functioning measures and psychiatric disorders is warranted.

Correspondence: Douglas Whiteside, Ph.D., Clinical Psychology, Argosy University-Seatle, 200 Hawkins Drive, Iowa City, IA 52242. E-mail: douglas-whiteside@uiowa.edu

I.N. MILLER, P. KEENAN, E. WEBER \& G.J. LAMBERTY. Utility of the MMPI-2-RF Fs Validity Scale in Determining Interpretability of Somatic/Cognitive Scales in a Veteran Sample.

Objective: The Infrequent Somatic Response (Fs) validity scale of the MMPI-2-RF is designed to identify overreporting of somatic complaints. Interpretive guidelines suggest that at the highest levels of somatic endorsement (Fs T score $\geq 100$ ), interpretation of Somatic/Cognitive problem scales is limited. The present study examines the utility of the Fs scale in distinguishing Somatic/Cognitive scale symptom endorsement in a sample of veterans referred for neuropsychological evaluation.

Participants and Methods: The current sample includes veterans who underwent neuropsychological assessment at a VA Polytrauma Rehabilitation Center ( $\mathrm{N}=336)$. Analysis of variance compared endorsements on the Somatic/Cognitive problem scales (Malaise (MLS), Gastrointestinal Complaints (GIC), Head Pain Complaints (HPC), Neurological Complaints (NUC), and Cognitive Complaints (COG)) for individuals with Fs endorsement in valid $(\mathrm{T}<80, \mathrm{~N}=215)$, overreporting $(\mathrm{T}=80-99$, $\mathrm{N}=83)$ and invalid $(\mathrm{T} \geq 100, \mathrm{~N}=38)$ ranges.

Results: Results revealed significant differences among Fs validity groups on all five Somatic/Cognitive scales (all ps<.001). While posthoc analyses revealed statistically significant differences between invalid and overreporting groups on GIC, HPC and NUC, endorsements on all somatic/cognitive scales for Fs overreporting and invalidity groups fell in clinically interpretable ranges $(T>65)$. Individuals in the Fs valid group endorsed clinically significant levels of symptoms on MLS, NUC and COG.

Conclusions: Results suggest that in a referred veteran sample, elevations on Fs and the Somatic/Cognitive scales are common. Distinguishing between Fs validity groups is not associated with clinically different elevations on these scales for this sample, particularly on scales that reflect common complaints related to neuropsychological referral. Correspondence: Ivy N. Miller, Ph.D., Neuropsychology, Minneapolis VA Medical Center, 1 Veterans Drive, Minneapolis, MN 55417. E-mail: ivy.miller2@va.gov

T. JERARD, K. RYAN, L. GABRIEL, C. NAGEL, J. HAYWOOD, S. WEISENBACH \& S.A. LANGENECKER. Personality Predictors of Cognitive Performance in Young Adults with Remitted Major Depressive Disorder.

Objective: Assessment of personality is often included in neuropsychological evaluation, as it may help explain cognitive performance. This study aimed to better understand the relationship between personality and cognitive performance in individuals with history of a major depressive episode.

Participants and Methods: Participants were recruited from the University of Michigan and University of Illinois at Chicago, ages 18-23, including 32 with remitted Major Depressive Disorder (rMDD), and 21 Healthy Controls (HC). The NEO-Personality Inventory was used to measure personality. Neuropsychological measures were divided into seven cognitive domains, consistent with previous literature (Langenecker et al., 2010).

Results: rMDD demonstrated stronger and a greater number of relationship between personality and cognitive performance than HC. In rMDD, Neuroticism negatively related to Verbal Fluency and Processing Speed (VFPS) and Processing Speed with Interference Resolution (PSIR); Extraversion positively related to VFPS and negatively related to Visual Memory (VM); Openness positively related to VFPS, Fine Motor Dexterity (FMD) and VM and negatively related to Conceptual Reasoning and Set Shifting (CRSS): Agreeableness positively related to CRSS; Conscientiousness positively related to VFPS, and PSIR (all ps<.05). In HC, Neuroticism positively related to PSIR; Openness positively related to VFPS, VM and Auditory Memory (AM); Agreeableness positively related to CRSS; and Conscientiousness positively related to PSIR and AM

Conclusions: Personality traits are more predictive of cognitive performance among individuals with history of depression than in their never-depressed peers. These findings underscore the importance of considering the contribution of personality to cognitive functioning among individuals with history of mood disorder, and might inform potential avenues for treatment.

Correspondence: Tracy Jerard, MS, MA, Neurology, UCSF, 600 North Kingsbury Street \#806, Chicago, IL 60654. E-mail: TracyJerard@ Gmail.com

A. HARLEY, S.K. GILL, J. NUNAN-SAAH, L. POSECION, L. COOPERSMITH, S. PAULRAJ, P. DUONG \& R. GOMEZ. Is ACTH or Cortisol a Better Predictor of Story Memory Deficits in Psychotic Major Depression?

Objective: Elevated cortisol levels are related to poor verbal memory and executive functioning in psychotic major depression (PMD; e.g. Gomez et al., 2006). However, there are limited studies on the relations between adrenocorticotropic hormone (ACTH) and memory in PMDs (Compono et al., 2011). This study investigated whether ACTH or cortisol was a better predictor of verbal contextual memory in PMD patients. Participants and Methods: Using archival data, 57 PMD patients completed several measures including the Logical Memory subtest.

Results: Two-tailed Pearson correlations indicated significant relations between ACTH and immediate and delayed logical memory ( $\mathrm{ps}<.05)$, but not with recognition. In contrast, cortisol was not related to any logical memory variables. Linear regressions revealed that ACTH $(\beta=$ $-0.335, p=.012)$ was a better predictor than cortisol $(\beta=0.035, p=$ .787) for immediate recall, explaining $11.2 \%$ of the variance. ACTH $(\beta=-0.307, p=.022)$ was also a better significant predictor of delaved recall compared to cortisol $(\beta=0.034, p=.795)$, explaining $9.3 \%$ of 
the variance. Lastly, these hormones accounted for only $1.1 \%$ of the variance of recognition memory, and neither were significant unique predictors.

Conclusions: Results indicate that elevated ACTH levels were related to poorer performance on immediate and delayed story memory in PMDs. Furthermore, ACTH is a better predictor of these deficits compared to cortisol. This suggests ACTH, a lesser-known neuroendocrine factor, may better account for logical memory deficits in PMD patients compared to the more well researched cortisol. Perhaps, treatments that affect ACTH levels may improve memory functioning.

Correspondence: Aikisha Harley, M.S., Palo Alto University, 505 Shell Parkway, \#1103, Redwood City, CA 94065. E-mail: aharley@paloaltou. edu

E.A. WILDE, D. MENEFEE, J. TRAN, J. WANNER, W. LEOPOULOS, J. HICKEY, C. PEDROZA, R. YALLAMPALLI \& S. MCCAULEY. Improvement in Psychiatric Status Following a Novel Inpatient Treatment for OEF/OIF Veterans with PTSD and Multiple Comorbidities.

Objective: This study investigated the recovery of Veterans with PTSD and comorbidities including alcohol abuse (ETOH) and mild traumatic brain injury (mTBI) following a 4-week, inpatient treatment that is novel in the VA system.

Participants and Methods: Participants included OEF/OIF Veterans with PTSD+ETOH $(n=14)$ and PTSD+ETOH+mTBI $(n=12)$ and combat-exposed Veteran controls $(\mathrm{n}=15)$ without these disorders. The groups did not differ by age $(p=.84)$, IQ $(p=.11)$, or length of stay $(p=.36)$. The treatment was an intensive, concurrent approach (group and individual settings) rather than the traditional sequential trauma-focused treatment. Measures of anxiety (PTSD-Checklist), depression (Beck Depression Inventory-II), and resilience (Connor-Davidson Resilience Scale) were obtained.

Results: Data were analyzed using a $3 \times 2$ mixed ANOVA with the fixed effect variable Group (control, PTSD+EOTH, PTSD+ETOH+mTBI) and random effects variables of anxiety, depression, and resilience. Significant improvement was found in both treatment groups for anxiety $(p<.0005)$, depression $(p<.0001)$, and resilience $(p<.01)$. The control group did not demonstrate significant changes across the two occasions on any measure.

Conclusions: These are some of the first data demonstrating the effectiveness of a novel, inpatient, concurrent treatment approach in the VA system in terms of improved psychiatric functioning in OEF/OIF Veterans with PTSD and comorbidities including alcohol abuse and mTBI. This treatment approach may have advantages over the traditional sequential treatment paradigm for Veterans with PTSD and these comorbid disorders.

Correspondence: Stephen McCauley, PhD, Physical Medicine and Rehabilitation, Baylor College of Medicine, One Baylor Plaza, BCM 637,Houston, TX 77030.E-mail:mccauley@bcm.edu

A. NORRIS-BRILLIANT, P. KLIMEK, D. AUSLANDER \& T. HILDEBRANDT. Neurocognitive Predictors of Eating Disorders. Objective: The objective of this study was to ascertain if neurocognitive functioning predicted severity of eating disorders.

Participants and Methods: The study included 25 participants who had all been previously diagnosed with an eating disorder. Their ages ranged from 15-42. They were given a brief neuropsychological assessment which consisted of a WASI, HVLT, BVMT, and selcted subtests of the DKEFS to assess for executive functioning, verbal, and visual memory.

Results: Hierarchical multiple regression analysis was used to test if measures of executive functioning, intelligence, learning and memory predict eating disorder severity. The predictors were the Trail Making $\mathrm{A}$ and B tests, the DKEFS Sorting Test (confirmed correct sorts), the DKEFS Tower Test (total achievement score), the WASI (Full Scale IQ), the HVLT (Total recall) and the BVMT (Total Recall, while the outcome variables were the EDE-Q Total score and Subscale scores. The results indicated that, after controlling for age, higher scores on the BVMT predicted higher levels of shape concern, $\beta=.049, \mathrm{t}(18)=$ $2.226, p<.05$, as well as higher levels of weight concern, $\beta=.063, \mathrm{t}(18)$ $=2.642, p<.05$. Levels of executive functioning did not significantly predict eating disorder symptoms.

Conclusions: Previous research has indicated possible executive dysfunction in subjects with bulimia and anorexia. This study did not find that difficulty with set shifting or planning predicted eating disorders, but rather that better visual memory predicted the severity of the illness. This indicates that subjects with body image disturbances process information in a more detailed way (e.g., perceiving and being preoccupied with distorted body parts). Further research into the neurocognitive functions of these subjects post-treatment is warranted.

Correspondence: Ami Norris-Brilliant, PsyD, Center for Attention and Learning Disorders, Lenox Hill Hospital, 210 East 64th Street, 4th Floor, New York, NY 10065.E-mail: Abrilliant@lenoxhill.net

L.S. CZEPIELEWSKI, L.A. SODRÉ, R. MASSUDA, J. BUCKER, A.L. SOUZA, K.P. BURKE, M. COSTANZI, R. RECKZIEGEL, A.A. GOLDANI \& C.S. GAMA. Improvement of Verbal Learning in Schizophrenia: Results from a Randomized, DoubleBlind, Placebo-Controlled Trial of Amantadine Adjunctive to Antipsychotics.

Objective: Amantadine (AMT), a memantine's derivate, is an antiviral substance with NMDA receptor antagonism properties that has been proposed as an add-on therapy for patients with schizophrenia (SZ). Several deficits in cognitive processes, such as in episodic memory and in verbal learning, have been shown in SZ. These cognitive impairments have also been associated to functioning in everyday life domains, especially in work performance. The aim of this study was to evaluate, as secondary outcome, the effect of AMT use on verbal learning of patients in recent onset of SZ.

Participants and Methods: 20 subjects with schizophrenia aged 2138 were enrolled in a randomized, double-blind, placebo-controlled, 12-weeks trial conducted at Hospital de Clinicas de Porto Alegre, Brazil to test the eficacy of amantadine $20 \mathrm{mg}$ /day adjunctive to antipsychotics for negative symptoms treatment. Inclusion criteria were diagnosis of schizophrenia confirmed by DSM-IV and less than 10 years of the disease. Clozapine use was exclusion criteria. Outcome measures were the Brief Psychiatric Rating Scale (BPRS) for symptomatology, Clinical Global Impression (CGI) and Functioning Assessment Short Test (FAST) for functionality, and Hopkins Verbal Learning Test - Revised (HVLT-R) for verbal learning and episodic memory.

Results: To analyze verbal learning, repeated measures general linear model was used for the 3 trials of immediate recall across both groups. At baseline, both placebo and AMT group showed similar verbal learning performances $(\mathrm{p}=.615, \mathrm{~F}=.501)$. After 12 -weeks, patients treated with AMT significantly improved their learning curve compared to placebo $(\mathrm{p}=.05, \mathrm{~F}=3.587)$.

Conclusions: The present study suggests that amantadine may be a potential adjunctive treatment strategy for improving cognition through learning in early phase of schizophrenia, even more so if combined with cognitive enhancement therapies. This is important once a better learning potential is associated with higher social functioning and quality of life.

Correspondence: Letícia S. Czepielewski, Universidade Federal do Rio Grande do Sul (UFRGS), Rua Ramiro Barcelos 2350 - Centro de Pesquisas, Porto Alegre 90035-903, Brazil.E-mail: czepielewski@gmail. com

\section{R. IP. Self-awareness of Patients on a Neuropsychiatric Unit.}

Objective: Neuropsychiatric patients often exhibit compromised self-awareness of their psychiatric and neurocognitive status. Impaired self-awareness (ISA) has been reported following many types of central nervous system dysfunction, such as dementia and acquired brain injury 
(ABI). Disruptions of the frontal subcortical system and inferior parietal lesions have been associated with ISA. The purpose of this study is twofold: 1) to investigate self-awareness of the neuropsychiatric patient subgroups by comparing their ratings of neuropsychiatric symptoms to those of the caregivers, and 2) to examine the association of their neurocognitive status and self-awareness.

Participants and Methods: The current study examined twenty inpatients on a neuropsychiatric unit at a mental health centre. They include ABI, geriatric, and psychiatric subgroups. Ratings of the Neuropsychiatric Inventory (NPI) were obtained from nursing staff and patients to compare/contrast their perceptions regarding frequency and severity of neuropsychiatric symptoms. Patients' demographics and diagnoses were examined in relation to perceptions of neuropsychiatric status. Their neurocognitive status was screened using the Repeatable Battery for the Assessment of Neuropsychological Status (RBANS), and associations between the NPI and severity of cognitive impairments were investigated. Results: Preliminary results indicate that neuropsychiatric patients underestimate the degree of their impairments when compared to caregivers. Clinical data and variables associated with these findings are discussed.

Conclusions: Self-awareness is an important factor that affects clinical outcome of the neuropsychiatric population. Patients with compromised self-awareness are often unable to recognize their impairments or set realistic goals. Their behaviours may be sometimes misinterpreted as willfulness or noncompliance by caregivers. Greater understanding of ISA and its manifestations will help to improve care for these patients. Correspondence: Rosa Ip, PhD, Psychology, Ontario Shores Centre for Mental Health Sciences, 700 Gordon Street, Whitby, ON L1N 5S9, Canada.E-mail:ipr@ontarioshores.ca

T. CARRATHERS, H. AMSBAUGH, J. ROSEBERRY, A.S. BODAPATI \& S. HILL. Examining Single Item Maintenance Impairment in Schizophrenia After Intact Updating.

Objective: Deficits in serial order processing information in working memory have been reported in schizophrenia under select conditions that require frequent updating when working memory load is three or more items. However, it is unclear whether this deficit involves a maintenance failure or a vulnerability of working memory stores to processes such as updating. The present study utilized a novel single item updating/maintenance task to limit working memory load and examine maintenance after updating in schizophrenia patients.

Participants and Methods: Schizophrenia patients $(n=16)$ and healthy controls $(n=20)$ were assessed for overall response accuracy on a novel updating/maintenance task. Participants were presented with a single item to maintain and were instructed to indicate whether the target item matched the spatial location of one to seven new items.

Results: Patients did not differ from controls on the initial response after updating, but demonstrated significantly lower accuracy for subsequent responses. For response lengths of $2[\mathrm{~F}(1,36)=5.52, \mathrm{p}<.05]$, $3 \quad[\mathrm{~F}(1,36)=7.74, \mathrm{p}<.01], \quad 4 \quad[\mathrm{~F}(1,36)=10.41, \mathrm{p}<.01], \quad 5$ $[\mathrm{F}(1,36)=5.47, \mathrm{p}<.05]$, and $7[\mathrm{~F}(1,36)=4.67, \mathrm{p}<.05]$ patients performed significantly worse.

Conclusions: The present study focused on updating and single item maintenance in working memory. Findings suggest that when patients are required to make multiple responses, performance drops significantly after the first response. Based on the results of the present study, updating appears intact in schizophrenia but maintenance appears vulnerable to rapid degradation once the maintained information has been accessed.

Correspondence: Tarra Carrathers, M.S., Rosalind Franklin University, 7305314th Ave, Salem,WI53168.E-mail:tarra.carrathers@my.rfums. org
E. LOJEK \& J. STANCZAK. Recovery from Depression: the Value of Executive Functions and Coping.

Objective: This study investigated the role of two individual resource factors - executive functioning and coping - which are believed to be important for recovery from neuropsychiatric disorders including depression.

Participants and Methods: Eighty seven patients with major depression and 87 controls took part in the investigation. The Questionnaire for the Measurement of Depression (QMD, Lojek, Stanczak, \& Wojcik, in press) was administered. It consists of four scales referring to depression and a scale measuring coping (Cope-S). The Color Trails Test (CTT) was used to evaluate cognitive functioning. Controls were tested once, the patients - before and after hospital treatment.

Results: Only the results of the Cope-S predicted level of depression after treatment, whereas CTT scores did not have significant influence on the QMD results on both assessments. Scores on the Cope-S negatively correlated with level of depression. Despite marked improvement after treatment, the patients were still significantly impaired on all measures compared with controls. After treatment, patients with the highest baseline scores on depression and the lowest baseline Cope-S scores improved the most, although their level of depression was still significantly higher than the other patients.

Conclusions: This study showed that coping may be a more valuable predictor of recovery from depression than executive functions. Severity of depression is related to the level of coping. The results may be important for the diagnosis and treatment of patients with depression. Correspondence: ,, . E-mail:

M. MATSUI, S. OTSUKA, T. HOSHINO, K. MIURA, Y. HIGUCHI $\&$ M. SUZUKI. Cognitive remediation therapy focusing upon the strategy coaching in schizophrenia.

Objective: Cognitive impairments in schizophrenia are found in a wide range of domains including attention, memory and executive functions. Although some cognitive remediation therapy (CRT) has been developed, there are only a few studies for Japanese patients. The aim of the present study was to examine the effectiveness and applicability of CRT focusing upon the strategy coaching for Japanese patients with schizophrenia.

Participants and Methods: Twenty-six participants diagnosed with schizophrenia were either assigned to CRT plus treatment as usual group or to treatment as usual alone group. The CRT intervention delivered in the study, over 12 weeks in weekly group sessions, was "compensatory cognitive training", focusing on four cognitive domains (prospective memory, attention, verbal memory and executive functions). Cognitive, functional and clinical symptom measures were implemented at pre-intervention (baseline), following the completion of CRT (post-intervention) and three months after CRT (follow-up).

Results: Effects of CRT on verbal memory, processing speed and social functioning at post-intervention were significant. Results also revealed that the effects on processing speed were maintained at follow-up. Effect sizes (Cohen's d) of CRT on cognitive measures at post-intervention were medium-to-large on verbal memory, large on processing speed and small-to-medium on executive functions, and these sizes were maintained at follow-up.

Conclusions: Our study suggests that CRT focusing upon the strategy coaching should have beneficial effects on cognitive functions with improvements on functional outcomes in Japanese patients with schizophrenia. Additionally, high degrees of attendance rate and level of satisfaction rated by the CRT participants ensure the fine applicability of this methodology to this population.

Correspondence: Mie Matsui, PhD, University of Toyama, 2630 Sugitani, Toyama 930-0194, Japan. E-mail:mmatsui@las.u-toyama.ac.jp 
S. ROGERS, D.A. LOWE \& E. ALBERT. How Does a History of Depression Influence the Current Neuropsychological Functioning of Older Adults?

Objective: Multiple studies have examined the relationship between cognition and older adults' current levels of depression, but this study examined how a history of depression influences the present neuropsychological functioning of older adults.

Participants and Methods: Ninety-nine older adults with a mean age of 76.66 years $(\mathrm{SD}=9.73)$ completed a comprehensive neuropsychological battery and indicated their history of depression on a background questionnaire.

Results: T-test analyses revealed that those with a history of depression performed significantly worse than those without a history of depression on WAIS-III PIQ, WAIS-III FSIQ, WAIS-III Digit Symbol, Trails A, DKEFS Color Naming, and Rey-Osterreith Complex Figure Copy, all ps $<.05$. There were also higher levels of current depressive symptomatology among those with a history of depression, $p<.02$. ANCOVA analyses controlling for this current level of depression showed that those with a history of depression continued to perform significantly worse than those without a history of depression in each of these cognitive areas. These findings also remained significant even when ANCOVA results covaried for the effects of age and education, all ps $<.05$.

Conclusions: Older adults with a history of depression appear to demonstrate worse nonverbal and overall intellectual abilities, visual processing speed, and figure copying skills than those spared of past depression. Although current levels of depression continue to be higher among older adults who have a history of depression, the impact of this history on their current neuropsychological functioning appears to be independent of their current symptomatology. This suggests that a prior history of depression may have a distinct neurological impact on nonverbal and overall intelligence, visual processing speed, and figure copying. This also supports right hemisphere involvement in depression and the importance of minimizing the risk of depression when younger. Correspondence: Steve Rogers, Ph.D., Psychology, Westmont College, 955 La Paz Road, Santa Barbara, CA 93108. E-mail: sarogers@ westmont.edu

R.B. FROST, J. LONG, V. CALHOUN, J. TURNER \& R.J. THOMA. The Relationship Between Symptom Severity and Performance on the MATRICS Consensus Battery in Symptomatic Schizophrenics Compared to Matched Controls.

Objective: Schizophrenia is associated with cognitive deficits in the domains of attention/concentration, processing speed, memory, and executive functioning. The MATRICS Consensus Cognitive Battery (MCCB) is a collection of neuropsychological measures that as a group have consistently been shown to be sensitive to cognitive dysfunction in schizophrenia. However, previous research has suggested that the MCCB is only modestly sensitive to symptom severity. The current study attempted to further investigate the relationship between performance on the MCCB and symptom severity in schizophrenics compared to matched controls.

Participants and Methods: Subjects consisted of 92 individuals diagnosed with schizophrenia (Mean age $=38.5 ; 72$ male) and 61 age, education, and sex matched controls (Mean age $=38 ; 61$ male). Subjects and Controls were administered the MCCB, and performances were compared using t-tests. Subjects only were administered the PANSS in order to measure symptom severity. Correlations between positive and negative symptom severity and performance on the MATRICS Overall Composite and index scores were calculated for subjects.

Results: No significant differences were noted for demographic variables. Student t-tests indicated significant differences between Subjects' and Controls' performance on the Overall Composite Index of the MATRICS ( $\mathrm{t}=-10.11, \mathrm{p}<.01)$, as well as on all subtests, with subjects performing on average 1.12 standard deviation units below controls. Negative symptom severity was negatively correlated with overall cognitive functioning $(\mathrm{r}=-.41, \mathrm{p}=<.01)$; however, positive symptoms were not.
Conclusions: In our sample the MATRICS was sensitive to the cognitive deficits seen in schizophrenia. In contrast with previous research, our data suggest that the MATRICS is at least moderately sensitive to the deleterious effects of negative symptoms in schizophrenia.

Correspondence: Robert B. Frost, PhD, Psychiatry, University of New Mexico Health Sciences Center, 308 Morningside Dr SE, Albuquerque, NM 87108.E-mail: rbfrost@salud.unm.edu

I. ZILBERFAYN, E.A. WILDE, J. TRAN, D. MENEFEE, R. SANYAL, J. HICKEY, W. LEOPOULOS, J. WANNER, E.D. BIGLER \& S. MCCAULEY. White Matter Integrity and its Relationship to PTSD Symptoms and Sleep Quality in OEF/OIF Veterans.

Objective: Studies utilizing diffusion tensor imaging (DTI) in Veterans with PTSD and sleep disturbances are limited, but previous reports have implicated decreased FA in the cingulum bundles and superior longitudinal fasciculus in PTSD. Extending our previous research on sleep disturbance in returning OIF/OEF Veterans with posttraumatic stress disorder (PTSD), this exploratory study examined PTSD symptoms and level of sleep disturbance in relation to white matter integrity.

Participants and Methods: Seventeen previously-deployed OEF/OIF Veterans aged 18-54 years with PTSD diagnosed via clinical diagnosis and verified using the Clinician-Administered PTSD Scale (CAPS) and without cormorbid mild traumatic brain injury (mTBI) or substance abuse underwent DTI. Sixteen Veterans of comparable age and sex and without PTSD, mTBI or substance abuse were used as a control group. Sleep quality was assessed with the Pittsburgh Sleep Quality Index (PSQI), and PTSD symptom level was assessed with the PTSD Checklist-Civilian Version (PCL-C).

Results: Group comparisons yielded significant differences in FA in the genu $(p=.054)$ and splenium of the corpus callosum $(p=.040)$ and left uncinate fasciculus $(p=.007)$, whereby the PTSD group demonstrated lower FA in all regions. There were no significant correlations between FA values in these regions and PCL-C, though significant negative correlations were observed between PSQI Total Sleep and right $(\mathrm{r}=-$ 0.736, $\mathrm{p}=0.003)$ and left $(\mathrm{r}=-.501, \mathrm{p}=0.030)$ centrum semiovale and left ventral striatum $(r=-0.570, p=0.042)$, indicating that higher levels of disrupted sleep were associated with lower FA in the participants with PTSD.

Conclusions: PTSD is known to be associated with significant sleep disturbance, particularly in Veteran populations. These problems with sleep may be associated with decreased white matter integrity and warrant further investigation and a need for treatment.

Correspondence: Irina Zilberfayn, MS, Clinical Psychology, Fielding Graduate University, 5534 Redstart, Houston, TX 77096. E-mail: izilber@yahoo.com

J.G. KEILP, G. WYATT, M. GORLYN, M. OQUENDO, A. BURKE \& J. MANN. Evidence Against Ventral Prefrontal Dysfunction in Suicidal Behavior.

Objective: Neuropsychological models of suicidal behavior suggest a role for ventral prefrontal dysfunction in this behavior, given the association of this brain region with impulse control and behavioral regulation. To test these models, we assessed individuals with past suicidal behavior on an alternation task, a measure sensitive to ventral prefrontal function adapted from primate studies.

Participants and Methods: 114 depressed patients with no past history of suicidal behavior, 64 with a history of low lethality attempts, 31 with a history of high lethality attempts, and 84 non-patients were administered a computerized object alternation task, similar to that used in recent imaging studies.

Results: Depressed non-attempters and low-lethality past attempters performed more poorly than healthy comparison subjects, but high-lethality past attempters, paradoxically, outperformed other depressed groups and were comparable to healthy comparison subjects. Additional analyses revealed that the vast majority of these high lethality past 
attempters used less violent methods in their attempts (e.g. overdose) with a high degree of planning, as opposed to violent, spontaneous methods.

Conclusions: There is a subgroup of individuals at risk for very serious suicidal behavior that do not exhibit the type of executive dysfunction associate with highly dysregulated behavior. While ventral prefrontal dysfunction may play a role in certain types of suicidal behavior, it cannot account for all types, especially those attempts that are more carefully planned and involve less violent means.

Correspondence: John G. Keilp, Ph.D., Psychiatry, NYSPI/Columbia University, Box 42, NYSPI, 1051 Riverside Drive, New York, NY 10032. E-mail:jgk13@columbia.edu

M. HUNTER, V.P. CLARK, V. CALHOUN, H. HE, B. YACKLEY \& T. LANE. Dynamic causal modeling of selective attention predicts relapse in patients recovering from addiction.

Objective: Relapse is a significant problem for patients recovering from drug dependence. Here we examined the hypothesis that effective connectivity obtained from fMRI data collected at an early stage of abstinence differentiates patients who later go on to relapse compared to those who will remain abstinent.

Participants and Methods: Three-stimulus oddball task performance was measured in 45 recently abstinent stimulant-dependent patients who were then monitored until 6 months of verified abstinence $(n=22)$ or stimulant relapse $(\mathrm{n}=23)$. Using an event-related functional MRI design, network-level timecourses were decomposed using high-dimensional ( $c=75)$ group independent component analysis (using the GIFT toolbox). Dynamic causal modeling (DCM) was then used to model both target and distractor effective connectivity among the posterior node of the default-mode network (DMN), the salience, and the task-specific oddball network.

Results: DGM showed that the effective connectivity from the DMN to the oddball network was significantly reduced in the relapse group compared to the abstinent group, independent of task modulation $(p=0.001)$. Furthermore, during distractor modulations, the DMN in the relapse group exerted more of an excitatory influence on the oddball network $(\mathrm{p}=0.04)$. Using classification algorithms on DCM parameter estimates and previously reported predictors of relapse (Clark et al., 2014), we were able to classify the 2 patient groups with $91 \%$ accuracy (with leave-one-out cross-validation).

Conclusions: These findings support the hypothesis for a dysregulation between the DMN and task-related networks in patients who relapse (Sutherland et al., 2012). These findings can also inform future treatment interventions that normalize these dynamics in order to prevent relapse.

Correspondence: Michael Hunter, M.S., Psychology, The University of New Me, 13208 Candelaria RD., Albuquerque, NM 87112. E-mail: mhunter1@unm.edu

\section{DALY \& D.J. WALDER. Characterization of Somatosensory Processing in Relation to Schizotypal Traits in a Sample of Nonclinical Young Adults.}

Objective: A core feature of schizophrenia spectrum disorders (SSDs) is a basic sensory processing disruption, yet few studies have examined somatosensation. This project examined differential patterns of associations of somatosensory abilities (tactile texture and spatial discrimination, letter recognition) with schizotypal traits (STs; positive, negative, disorganized) among individuals at varying degrees of psychometric risk for psychosis. The relative contributions of anxiety and depressive symptoms, and peripheral (morphologic features) and central (error types) contributions were also examined. We hypothesized that somatosensory performances would be differentially associated with ST dimensions and account for variability in STs beyond mood symptoms; and, central and peripheral contributions may contribute to, but not fully account for, associations among somatosensory abilities and STs.
Participants and Methods: Participants recruited from the City University of New York human subjects pool ( $\mathrm{n}=125 ; 37 \mathrm{M} / 88 \mathrm{~F}$; Mage $=20.55, \mathrm{SD}=3.27$ ), completed somatosensory tasks and mood and personality self-report measures. Fingerprints were obtained to assess morphologic features.

Results: Contrary to hypotheses, after accounting for the relative contributions of mood symptoms, better spatial discrimination and rough texture discrimination abilities were associated with more disorganized and negative STs, respectively ( $\mathrm{p} \leq 10$ ). Exploratory analyses demonstrated some differential contributions of dermatoglyphic features and letter recognition confusion errors to variability in STs. Specifically, more isomorphic errors were associated with fewer negative (and total) STs $(p \leq .05)$, and more minutiae were associated with more positive $\mathrm{ST}_{\mathrm{s}}(\mathrm{p} \leq .10)$.

Conclusions: Findings are discussed in the context of theories regarding neural substrates of somatosensory processing disruptions in SSDs. Implications for understanding differential patterns of sensory processing disruptions across the schizophrenia spectrum are posited.

Correspondence: Maureen Daly, Psychology, Psychiatry, Harvard Medical School, Partners Consortium, 16 Gilman Terrace, Somerville, MA 02145. E-mail: maureen.daly2@gmail.com

A.S. BODAPATI, E. HERBENER, E. OLSEN, O. BJORKQUIST, K.A. CHASE, C. ROSEN \& R. SHARMA. Cognitive Correlates of a Social Attentional Bias in Schizophrenia.

Objective: Deficits in social functioning are well-documented in schizophrenia. Furthermore, visual processing of social and affective stimuli has been studied in schizophrenia and deficits in emotion discrimination, gender identification, and discriminating facial stimuli have been found. A social attentional deficit may be contributing to these visual processing impairments. The authors hypothesized that individuals with schizophrenia would selectively attend to social stimuli less than healthy controls, and that various aspects of nonsocial cognition, particularly attention and processing speed, would be related to this social attentional bias.

Participants and Methods: The present study included individuals with schizophrenia (SZ) from the University of Illinois Medical District and age- and sex-matched healthy controls (HC). Study participants completed a novel 5-minute computerized dot probe task that measured their tendency to attend to social versus nonsocial visual stimuli. Participants also completed the MATRICS Consensus Cognitive Battery. Results: Groups differed in their attentional biases, $\mathrm{t}(28)=2.28, \mathrm{p}<.05$, such that HC attended toward social stimuli whereas SZ exhibited an attentional bias toward nonsocial stimuli. In HC, selectively attending toward social stimuli was significantly correlated with faster processing speed $[\mathrm{r}(22)=.41, \mathrm{p}<.05]$. In SZ, a social attentional bias was significantly correlated with better working memory $[\mathrm{r}(9)=.67, \mathrm{p}<.05]$ and visual memory $[\mathrm{r}(9)=.74, \mathrm{p}<.01]$, as well as processing speed $[\mathrm{r}(9)=.60$, $\mathrm{p}<.06]$ and attention $[\mathrm{r}(8)=.59, \mathrm{p}<.08]$, at trend.

Conclusions: These findings have implications for interventions. In particular, cognitive rehabilitation techniques that target domains strongly correlated with this social attentional bias may ultimately improve social functioning deficits in schizophrenia.

Correspondence: Anjuli S. Bodapati, MA, Psychology, University of Illinois at Chicago, 1007 W Harrison Ave, Chicago, IL 60607. E-mail: asingh31@uic.edu

W. POTHIER, M. ROY, S. TREMblay \& C. CELlard. Methodological Guidelines to Improve Research Designs on Cognition in Schizophrenia.

Objective: Research on cognition in schizophrenia has expanded over the last two decades. Clinical and methodological variables may influence the magnitude of cognitive deficits observed in schizophrenia. The main objective of this systematic review was to determine the effect of socio-demographic, clinical and methodological variables on cognition 
in schizophrenia in order to identify key variables to be considered in designing and interpreting clinical research.

Participants and Methods: A systematic review of meta-analyses was conducted in Pubmed database using keywords "schizophrenia", "meta-analysis", "cognition" and their related terms. Inclusion criteria were: 1) Group of patients with a diagnosis of schizophrenia and schizophrenia spectrum; 2) Comparative group (patients with schizophrenia or healthy control group); 3) Cognitive outcomes derived from standardised neuropsychological tests; 4) Moderators analysis computed on cognition. Results: Among the 361 records identified through Pubmed, 28 meta-analyses ( 11 with a healthy control group; 17 with a group of patients with schizophrenia) were included in this review. Socio-demographic variables such as age, gender and education influenced the most the cognitive deficits observed in schizophrenia. Clinical variables, namely the duration of illness, symptomatology (negative and disorganization symptoms), patient status and presence of a psychiatric comorbidity, showed an impact on cognition. Finally, methodological variables - publication year and sample size - should be considered in clinical research while interpreting the results from the literature.

Conclusions: High level of contradiction across meta-analyses was highlighted in this systematic review. Therefore, the limits identified may explain such contradictions and recommendations are provided to improve designs and validity.

Correspondence: William Pothier, Doctorate (Ph.D.), Psychology, Laval University, 148 rue de la Grand-Voile, Saint-Augustin-de-Desmaures, QC G3A 2H5, Canada. E-mail: william.pothier.1@ulaval.ca

C.E. RICHMOND, B.A. MARCOPULOS, B.C. ARREDONDO \& S. KOVACH. What Do Drawing Tasks Measure In Serious Mental Illness? A Preliminary Analysis Using The Boston Qualitative Scoring System For The Rey Complex Figure Copy.

Objective: Drawing tasks are included in neuropsychological assessments due to their sensitivity to brain dysfunction, and have a long history in psychiatric settings (Marcopulos \& Kurtz, 2012). We used the Boston Qualitative scoring system (BQSS) to explore drawing strategies (i.e., configurational versus piecemeal) and types of errors (e.g., omission, accuracy, confabulations, perseverations, fragmentation) on the Rey Complex Figure (RCFT) copy in a psychiatric hospital sample. We hypothesized that drawings reflect neurodevelopmental integrity/ maturity (Harrison \& Stiles, 2009) rather than the errors typical of acquired brain dysfunction.

Participants and Methods: Participants were psychiatric hospital patients referred for neuropsychological assessment $(\mathrm{N}=124)$. Average age was $37(\mathrm{SD}=13.3)$, duration of illness 13.9, $(\mathrm{SD}=10.3)$, education $12(\mathrm{SD}=2.6)$ years. Approximately half the sample had a diagnosis of schizophrenia spectrum illness with the remainder affective disorder, personality disorder or substance abuse.

Results: After controlling for familywise error using an alpha of .003571, preliminary correlational analyses revealed significant relationships between error types(omission, fragmentation, planning) and IQ as well as learning disability and special education. Error scores were not significantly correlated with patient demographic (e.g., age, education, gender), or clinical variables (e.g. length of illness, age of onset, diagnosis, history of neurological condition).

Conclusions: Our results are consistent with Seidman et al (2003) showing a fragmented approach to RCFT drawing. Qualitative errors on RCFT copy correlate with IQ and developmental history. We discuss what kind of information is obtained from the RCFT in psychiatric patients and whether drawing tasks add value to neuropsychological assessment in serious mental illness.

Correspondence: Bernice A. Marcopulos, PhD, Graduate Psychology, James Madison University, 70 Alumnae Drive, MSC 7401, Harrisonburg, VA 22807.E-mail: marcopba@jmu.edu
E. ESTEVIS, M.R. BASSO, R. PURDIE, P. CANDILIS, J. KAMPER, A.K. MILLER \& D.R. COMBS. Neuropsychological dysfunction and informed consent capacity among depressed inpatients.

Objective: Neuropsychological deficits occur commonly among people with depression, especially those who require inpatient treatment. In other clinical populations cognitive deficits correspond with compromised ability to make autonomous decisions regarding medical treatment. Initial research implies that memory impairment coincides with poor understanding of treatment options among inpatient depressives, but no previous study has investigated other facets of decisional capacity. We further evaluated whether cueing and repetition of treatment disclosures improved decisional capacity.

Participants and Methods: 18 unipolar depressed inpatients and 19 controls were administered a broad neuropsychological battery consistent with Marson's (1996) model of decisional capacity. To assess understanding, appreciation, reasoning, and choice concerning medical treatment options, the MacCarthur Competency instrument was administered. To assess performance effort, the Word Memory Test was given. Results: A mixed factor ANOVA was used to analyze the effect of depression on decisional capacity. Expressing a choice was not analyzed due to both groups obtaining perfect scores on this domain. The depressed group performed worse than the control group on the understanding, appreciation, and reasoning index. For both groups, repetition and cueing improved understanding and appreciation, but not for reasoning abilities. Multiple regression revealed that Stroop Interference predicted understanding and appreciation of medical decisions, whereas verbal concept formation predicted reasoning abilities.

Conclusions: Depressed inpatients have diminished medical decision-making capacity, especially regarding understanding, appreciation, and reasoning of treatment options. Executive function performance was the most salient predictor associated with decisional capacity. Indeed, patients that manifest impairments in this domain should be considered for an assessment of decisional capacity.

Correspondence: Eduardo Estevis, M.A., Clinical Psychology, James A. Haley VA Medical Center, 8801 Hunters Lake Dr. \#123, Tampa, FL 33647.E-mail: eduardo-estevis@utulsa.edu

E. ESTEVIS, M.R. BASSO, L. LAU, R. PURDIE, D. WHITESIDE, A.K. MILLER \& D.R. COMBS. Memory dysfunction in major depression: Not an artifact of poor effort.

Objective: Memory impairment has emerged as a frequent occurrence often among depressed individuals, especially in inpatients. However, memory impairment has been observed inconsistently across studies, as some investigations have found no evidence of deficit. Some assert that memory impairment reflects poor effort rather than a genuine deficit. Little research has directly assessed this issue. Towards this end, the present study examined CVLT-2 performance and performance effort in a group of depressed inpatients and healthy controls.

Participants and Methods: 42 unipolar depressed inpatients and 33 controls were administered a structured diagnostic interview, the CVLT2, and the Word Memory Test (WMT).

Results: Groups were compared using one-way ANOVAs to analyze the following CVLT-2 indices: Trial 1, Trial 5, Total Score, Short and Long Delayed Recall with and without cueing, Total Learning Slope, Total Recognition Hits, Across Trial Consistency, Primacy, Recency, Total Repetition, and Intrusion scores. In comparison to the control group, depressed inpatients had significant impairments $(\mathrm{p} \leq .01)$ across the majority of CVLT-2 indices except on Total Learning Slope, Primacy, Recency, Total Repetition, and Intrusion scores. These deficits occurred despite having displayed no evidence of poor effort on the WMT.

Conclusions: In contrast to previous findings, the current study revealed that depressed inpatients experience considerable memory impairment. This dysfunction reflects their psychiatric status rather than sub-optimal effort during testing. Indeed, depressed patients with valid performance on the WMT showed poor performance on the CVLT-2. These findings suggest that the cognitive dysfunction associated with 
major depressive disorder reflect genuine impairment, and is not an artifact of suboptimal effort.

Correspondence: Eduardo Estevis, M.A., Clinical Psychology, James A. Haley VA Medical Center, 8801 Hunters Lake Dr. \#123, Tampa, FL 33647.E-mail: eduardo-estevis@utulsa.edu

K.A. UMEKUBO \& S.L. DOLAN. Self-Reported Impulsivity Mediates the Relationship Between Schizotypy and Performance on the Wisconsin Card Sorting Test.

Objective: To better understand the etiology of psychotic disorders such as schizophrenia, prior research has searched for endophenotypic markers to help identify those at risk for developing psychosis. Previous research supports a negative association between schizotypal personality features and executive function performance on the Wisconsin Card Sorting Test (WCST), such that individuals reporting higher levels of schizotypy exhibit poorer performance on the WCST. The current study first aimed to replicate prior research on the relationship between schizotypy and executive function. Second, this study extended this research by investigating the effects of self-reported impulsivity on this association. We hypothesized that self-reported impulsivity would mediate the relationship between schizotypy and performance on the WCST.

Participants and Methods: Fifty-four undergraduates completed the Schizotypal Personality Questionnaire (SPQ), the Barratt Impulsiveness Scale (BIS-11), and the WCST. A mediation analysis was conducted between SPQ and WCST scores with BIS-11 scores as the mediator.

Results: Results revealed a significant negative correlation between unusual perceptual experiences and performance on the Learning to Learn category of the WCST $(p<.05)$. However, further investigation indicated that this relationship was mediated by the significant negative correlation between unusual perceptual experiences and motor impulsivity on the BIS-11 $(\mathrm{p}<.05)$.

Conclusions: Taken together, these results suggest that interpretations of the Learning to Learn category of the WCST for individuals with schizotypal personality features may be accounted for by motor impulsivity. When extended to the schizophrenia literature, these findings indicate that impulsive personality traits may help to explain some of the executive function deficits observed in individuals with schizophrenia. Correspondence: Kelsi A. Umekubo, Psy.D., Psychology and Neuroscience, Baylor University, 801 Washington Ave., Waco, TX 76701.E-mail:kelsi_umekubo@baylor.edu

A. R.-MERCIER, M. MASSON \& C. CELLARD. The effect of Maltreatment on Neuropsychological functioning of Children, Adolescents and Adults with Psychiatric disorders: Meta-analysis. Objective: The aims of this meta-analysis were to evaluate the impact of maltreatment on neuropsychological functioning in patients suffering from diverse psychiatric disorders across age span.

Participants and Methods: Twelve studies were identified according to the following criteria: 1) to have two groups recruited in psychiatric hospital or with psychiatric disorder, 2) to have a group who suffered from maltreatment during childhood or adolescence, 3 ) to report an assessment of neuropsychological functioning using standardized neuropsychological test, and 4 ) to have sufficient statistical data to compute effect sizes (Hedge's $g$ ).

Results: The results of this meta-analysis show a negative impact of maltreatment of small effect size on neuropsychological performance in the maltreated group with psychiatric disorders in comparison to the group with psychiatric disorders only $(g=-0.24)$. The most affected cognitive domains were working memory $(g=-0,967)$, attention $(g=-0.698)$ and visual memory $(\mathrm{g}=-0.500)$. Moreover, the type of maltreatment is a moderating variable as neglect shows a significant negative impact $(\mathrm{g}=-0.509)$ on neuropsychological functioning in the maltreated group with psychiatric disorders.

Conclusions: Results suggest that exposure to maltreatment and the presence of psychiatric disorders increase neuropsychological impairments in some cognitive domains. These targets should be considered while developing potential treatment strategies to improve cognitive and social functioning.

Correspondence: Alexandra R.-Mercier, 2 nd Ph.D., Psychology, Laval University, 792 rue rougemont App. 2A, Québec, QC G1X 2M2, Canada.

E-mail: alexandra.r-mercier.1@ulaval.ca

M. GORLYN, J.G. KEILP, A. BURKE, M. OQUENDO \& J. MANN. Semantic Fluency Deficit Is a Marker for High-Lethality Suicide Attempt Risk in Major Depression.

Objective: Major depression is characterized by deficits in language fluency, while suicidal behavior has been associated with associated with deficits in semantic, but not phonemic, fluency. Slowed processing speed may account for both sets of deficits.

Participants and Methods: Phonemic (FAS) and semantic (animal) fluency were compared in 283 unmedicated patients with current major depressive episode (145 with no suicide history, 83 with low-lethality attempts, 55 with high-lethality attempts) and 107 healthy volunteers. Scores were converted to T-scores using published norms adjusting for age, race and education. Psychomotor speed was assessed via the WAISIII Coding subtest.

Results: All patient groups performed more poorly on fluency tasks relative to healthy comparison subjects $(\mathrm{F}[3,386]=3.00, \mathrm{p}=.03)$, but these differences were eliminated after correction for psychomotor speed. High-lethality attempters, however, performed significantly more poorly on the semantic fluency task $(\mathrm{F}[3,366]=4.78, \mathrm{p}=.003)$ than all other groups, even after adjustment for psychomotor speed.

Conclusions: Verbal fluency deficits in depression appear to be a function of depressed patients' slowed processing speed, but deficits in semantic fluency in high-lethality suicide attempters are independent of this slowing. Risk for higher lethality suicide attempt appears related to a specific impairment in accessing semantic information. Patients at risk for more lethal suicidal behavior exhibit a particular vulnerability in the retrieval of connective and meaning-based information, which may play a role in generating alternatives and guiding goal-directed behavior. Semantic fluency deficits may serve as a marker for vulnerability to more medically severe suicidal behavior.

Correspondence: Marianne Gorlyn, PhD, NYSPI, 1051 Riverside Drive, Unit 42, New York, NY 10032.E-mail:mg643@columbia.edu

\section{W. PLANTE \& A.E. LANSING. Contributors of Executive Deficits in Incarcerated Youth.}

Objective: Elevated rates of psychiatric morbidity and executive deficits (ED) are documented in delinquent youth and associated with aggression and poor outcomes. To better inform executive functioning interventions, we aimed to evaluate gender and criminal engagement differences in predictors of self-reported ED in incarcerated youth.

Participants and Methods: Three groups of incarcerated youth were examined: Life course persistent delinquent (LCPD) boys $(n=50)$ and girls $(n=45$; ages 16-18), with at least one prior detention and disruptive behavior before age 10; and girls (Seeking Safety [SS]; n=49; aged 13-18) with evidence of substance abuse and complex trauma (using the Structured Clinical Interview Trauma and Loss Spectrum). Self-reported ED was measured with the Delis Rating of Executive Function, Executive Scale. Linear regression models were used to evaluate ED contributors in each group.

Results: Psychotic symptoms and early disruptive behavior were each individually associated with ED only in LCPD youth. Trauma symptoms, however, were important for both groups of girls, but not LCPD boys. Other psychiatric symptoms (especially attention and conduct problems) were related to ED in all groups. Attention problems, single-event PTSD avoidance symptoms, IQ and age were each independent contributors when controlling for emotional neglect in the best-fit model $(\mathrm{r} 2=0.65 ; \mathrm{F}(5,32)=11.85 \mathrm{p}<.001)$ for LCPD Girls. For LCPD bovs, attention problems, suspicious thoughts, race and early disruptive behavior were each independent contributors $(\mathrm{r} 2=0.60 ; \mathrm{F}(4,38)=14.18$ $\mathrm{p}<0.001)$. Lastly, the best-fit model $(\mathrm{r} 2=0.60 ; \mathrm{F}(4,40)=12.68 \mathrm{p}<0.001)$ 
for SS girls included independent contributions of attention problems, complex trauma symptoms and paranoid interpretations when controlling for physical abuse.

Conclusions: These results highlight that ED are affected by many psychiatric factors. Further, gender and delinquency subtypes must be taken into account when evaluating the importance of these factors and designing appropriate interventions.

Correspondence: Wendy Plante, Bachelor's of Science, Psychiatry, University of California, San Diego, 1780 Diamond St, San Diego, CA 92109.E-mail:wplante@ucsd.edu

D.D. LA, G. GOODALE, L. POSECION, A. NODA, L. LAZZERONI, B. HERNANDEZ, J. YESAVAGE \& L. KINOSHITA. Posttraumatic Stress Disorder and Obstructive Sleep Disorder Effects on Cognition in Older Veterans.

Objective: Veterans with posttraumatic stress disorder (PTSD) often report problems with sleep and cognition. Veterans may also be at higher risk for developing obstructive sleep apnea (OSA) compared to the general population. Both PTSD and OSA separately have been found to impact cognition. This study aims to investigate associations between PTSD, OSA, and cognition in older Veterans.

Participants and Methods: Participants include 176 older, male Veterans enrolled in a longitudinal PTSD study at the VA Palo Alto Health Care System (mean age $=63.5, \mathrm{SD}=5.8$ ). PTSD was measured using the Clinician Administered PTSD Scale (CAPS) current total score and OSA was measured by the apnea-hypopnea index (AHI) obtained from an overnight polysomnography. One task was used as a measure of attention, and principal component analyses (PCA) obtained separate factors for three additional cognitive domains: processing speed, auditory verbal memory, and executive functioning. Measures included in the PCA were obtained from the baseline neuropsychological evaluation. Regression was used to separately examine PTSD and sleep apnea effects for each cognitive domain.

Results: We found that greater severity in PTSD was significantly associated with decreased attention $(p=0.005)$ and processing speed $(p=0.001)$. No significant findings were noted regarding OSA and $\operatorname{cog}-$ nition or the interaction of PTSD and OSA on cognition.

Conclusions: In this population of older, male Veterans, PTSD was found to be associated with decreased attention and processing speed, which is consistent with the research literature. However, there were no significant findings associating OSA and cognition or the combined effects of PTSD and OSA on cognition.

Correspondence: Dawn D. La, M.S., Palo Alto University, 1851 Norseman Drive, San Jose, CA 95133. E-mail: DLa@paloaltou.edu

\section{K. JANG, S. MOON \& S. CHOI. Source localization of P300 in} college students with schizotypal traits.

Objective: We investigated the localization of cortical generators of P300 measured by an auditory oddball paradigm in individuals with schizotypal traits.

Participants and Methods: Based on the scores of Schizotypal Personality Questionnaire (SPQ) the schizotypal trait $(n=13, \geq 36$ scores on SPQ) and control ( $n=13,14-23$ scores on SPQ) groups were selected. Auditory oddball paradigm: Among a total of 200 tones, frequent standard tones $(1000 \mathrm{~Hz}, 80 \mathrm{~dB}$ SPL) were presented 160 times, and the rare target tones $(1500 \mathrm{~Hz}, 80 \mathrm{~dB}$ SPL) were presented 40 times. Participants were required to count the number of target stimuli presented and report this at the end of the experiment.

Electrophysiological recording procedure: EEG activities were recorded at 64 electrode sites. The EEG epochs $(1000 \mathrm{~ms}$ including a $100 \mathrm{~ms}$ pre-stimulus baseline) were averaged for standard and target tones, separately. The amplitudes and generators of P300 elicited by target stimuli were analyzed.

Estimation of current densities using sLORETA: The sLORETA analysis was conducted using Curry V6.0 after matching the electrode locations with the standard head model. For the spatial pre-processing of the current density image, we used the statistical parametric mapping toolbox (SPM8).

Results: The schizotypal trait group showed significantly smaller P300 amplitudes compared to the control group $(F(1,24)=60.08, p<.0001)$. In terms of source localization, the current densities in the left postcentral gyrus, precuneus, superior parietal lobule, right inferior parietal lobule were significantly reduced in the schizotypal trait group $(\mathrm{T}=2.80$, $\mathrm{p}=0.005)$ compared to control group.

Conclusions: These results indicate that P300 could be a biological marker of schizospectrum disorder and reduced P300 source activations observed in schizotypal trait group might be associated with dysfunctions of parietal area known as one of P300 generators.

Correspondence: Kyoung-Mi Jang, Sungshin Women's university, 249-1, Dongseon-dong 3-ga, Seoul 136-742, Korea (the Republic of). E-mail: cest20@gmail.com

Y.E. GALLEGOS RODRÍGUEZ, A. BOUCHARD, W.D. SPAULDING \&. E.L. GRANHOLM. Cognitive functioning outcomes in Cognitive Behavioral Social Skills Training (CBSST) for racial/ethnic minority individuals diagnosed with schizophrenia spectrum disorders.

Objective: The extent to which treatment outcomes vary as function of race/ethnicity in individuals with schizophrenia is not well understood. This study investigated whether individuals' racial/ethnic background is associated with improved cognition over the course of Cognitive Behavioral Social Skills Training (CBSST). We hypothesized that participants in CBSST would show better cognitive functioning outcomes compared to those in Supportive Contact (SC), regardless of racial/ ethnic background.

Participants and Methods: Forty-one White and 32 ethnic minority participants were randomized to CBSST, and 44 White and 32 ethnic minority participants were randomized to SC. Measures of memory, executive functioning, attention and processing speed were administered at baseline, mid-treatment, treatment end, and follow-up visits. Relationships among these variables were analyzed using multilevel models to more accurately represent unequal time between assessments, missing data, and dependency across time.

Results: CBSST was associated with gains in attention and memory, but no racial/ethnic differences were found during treatment in groups' rate of change on measures of executive functioning, attention, or processing speed. However, Whites and Latinos demonstrated a higher rate of change in memory when compared to Blacks. Relative to White participants, Latinos and Blacks did not retain gains in attention and memory during follow-up visits.

Conclusions: Analyses suggest CBSST is effective for non-white minorities in complex ways. Future research should be focused on development of culture-specific compensatory aids that promote CBSST skill practice and retention in racial/ethnic minority subgroups with SMI, particularly in light of results that attention and memory in these groups was not retained during follow-up visits.

Correspondence: Yuliana E. Gallegos Rodríguez, Ph.D., Psychiatry, VA San Diego Healthcare System, 3350 La Jolla Village dr., San Diego, CA 92161. E-mail: yulie1882@gmail.com

A. GONÇALVES, A. MENDES, N. VILA-CHÃ, I. MOREIRA, J. FERNANDES, J. DAMÁSIO \& S. CAVACO. Impulse Control Disorders and Apathy in Parkinson's Disease.

Objective: Objective: To explore the association between impulse control disorders and related behaviors (ICDRB) and apathy symptoms in Parkinson's disease (PD).

Participants and Methods: One hundred and forty-seven consecutive nondemented patients with PD Hoehn \& Yahr stage 1-3 (55\% men; mean age $=65 \pm 11$; mean education $=7$ years \pm 4 ; mean disease duration $=-$ 7 years \pm 5 ; levodopa equivalent dose $=717 \mathrm{mg} \pm 492 ; 52,4 \%$ with agonist medication) were assessed with the Apathy Evaluation Scale (AES), the Questionnaire for Impulsive-Compulsive Disorders in Parkinson's 
Disease (QUIP-Current-Short), the Hospital Anxiety and Depression Scale (HADS), and the Dementia Rating Scale-2 (DRS-2). Qui-square, Mann-Whitney Test, and Multiple Logistic Regression were used for data analyses.

Results: Twenty-eight patients (19\%) had ICDRB ( $>1$ positive answer on QUIP-Current-Short). These patients were younger $(p<0.001)$, were more educated $(p<0.001)$, had lower age at disease onset $(p<0.001)$, had higher levodopa equivalent dosages $(p=0.021)$, and had more dopamine agonist medication $(p=0.003)$. No significant association was found with sex $(p=0.132)$, HADS-anxiety $(p=0.199)$ and depression $(p=0.480)$ scores or DRS-2 total adjusted score $(p=0.084)$. ICDRB was not associated with AES-total $(p=0.819)$, Cognitive $(p=0.364)$ or Behavior $(p=0.855)$ scores. However, there was a tendency for patients with ICDRB to have higher AES-Emotional scores $(p=0.061)$. This tendency was clearer (adjusted $\mathrm{OR}=1.800 ; \mathrm{p}=0.007$ ), when relevant covariates (i.e., age and education) were taken into account. Both AES-Emotional items ( 7 and 14$)$ were significantly $(p<0.05)$ related to ICDRB.

Conclusions: ICDRB in PD was accompanied by emotional blunting, but not by other apathy symptoms or depressed mood. The reward-seeking behavior, characteristic of impulse control disorders in PD, appears to be related to diminished emotional responsiveness.

Correspondence: , . E-mail:

\section{C.Z. BURTON, P.D. HARVEY, T.L.PATTERSON \& E.W.TWAMLEY.} Neurocognitive Insight among Individuals with Schizophrenia.

Objective: Neurocognitive insight, or awareness of cognitive impairment, among individuals with schizophrenia is relevant to cognitive treatment adherence and outcome. Its characteristics and correlates, however, remain unclear. This study examined neurocognitive insight and its relationship to executive functioning, functional capacity, and psychiatric symptoms.

Participants and Methods: 214 outpatients with schizophrenia or schizoaffective disorder completed measures of cognition, functional capacity, psychiatric symptom severity, and self-reported frequency of cognitive problems. Based on cognitive performance and self-reported cognitive problems, latent profile analysis classified the participants with cognitive impairment into two groups: those with "intact" vs. "impaired" neurocognitive insight. The groups were then compared on cognitive, functional, and symptom variables.

Results: 168 participants $(78.5 \%)$ demonstrated objective cognitive impairment (global deficit score $\geq 0.50$ ). Self-reported frequency of cognitive problems was similar between those with and without objective cognitive impairment. Among the cognitively impaired participants, $54 \%$ showed "impaired" neurocognitive insight (i.e., reported few $\operatorname{cog}$ nitive problems despite objective cognitive impairment.) Participants with impaired vs. intact neurocognitive insight did not differ on measures of executive functioning, functional capacity, or negative symptom severity, but those with intact neurocognitive insight reported greater positive and depressive symptoms.

Conclusions: Self-reported frequency of cognitive problems does not differentiate those with and without objective cognitive dysfunction. Further, a substantial portion of individuals with objectively measured cognitive impairment appear unaware of their deficits. Patient self-report of cognitive problems, therefore, is not likely to reliably assess $\operatorname{cog}-$ nition. Difficulty self-identifying cognitive impairment may be unrelated to executive functioning, negative symptoms, and functional capacity. Correspondence: Cynthia Z. Burton, SDSU/UCSD Joint Doctoral Program in Clinical Psychology, 140 Arbor Drive, San Diego, CA 92103. E-mail:czburton@ucsd.edu
FRIDAY AFTERNOON, FEBRUARY 6, 2015

\author{
Invited Address: \\ Disconnection in the Connectome Era
}

Presenter: Marco Catani

12:00-1:00 p.m.

M. CATANI. Disconnection in the Connectome Era.

In a brain composed of specialized but connected areas, disconnection leads to dysfunction. This simple formulation not only underlay a range of classical neurological manifestations, referred to collectively as disconnection syndromes, but it has also recently been adopted to explain almost the entire spectrum of higher cognitive disorders in neurology and psychiatry. This paper will first trace the development of certain anatomical and physiological concepts at the origins of modern definitions of disconnection. Second, current developments of brain imaging methods will be discussed focusing on their application to the healthy and pathological brain. In particular modern tractography approaches based on diffusion imaging will be examined in detail with examples taken from disorders of language, visuospatial attention and praxis. Correspondence: Marco Catani, MD, King's College London, London WC2R 2LS, United Kingdom. E-mail: marco.1.catani@kcl.ac.uk

\section{Invited Symposium: \\ Norman Geschwind and the Lasting Influence of Disconnection}

\author{
Chair: Chris M. Filley
}

1:00-3:00 p.m.

C.M. FILLEY, K.M. HEILMAN, C.M. FILLEY, A. KERTESZ, M. DENCKLA \& R.A. YEO. Norman Geschwind and the Lasting Influence of Disconnection.

Symposium Description: Norman Geschwind (1926-1984), an American neurologist credited with introducing the term behavioral neurology, authored a seminal paper in 1965 entitled "Disconnexion Syndromes in Animals and Man." This Symposium commemorates the 50th anniversary of the publication of this article, the influence of which was immediately apparent and continues today. In this comprehensive work - 118 pages long and spread over two issues of Brain - Geschwind reviewed and synthesized a wide range of neurologic literature from 19th century Europe, and almost single-handedly re-established academic medical interest in brain-behavior relationships after a half century of neglect. His explanation of neurobehavioral syndromes in terms of lesions disconnecting brain regions from one another vigorously encouraged the renewed study of many neurologic disorders in light of altered cerebral connectivity, and set the stage for contemporary research on distributed neural networks. With no access to modern neuroimaging until late in his career, Geschwind significantly advanced behavioral neurology and neuropsychology through careful observation of patients and thoughtful interpretation of clinical data. To begin this Symposium, Dr. Filley will discuss progress in the understanding of white matter disconnection in behavioral neurology, particularly with respect to dementia. Dr. Heilman will then take up the syndrome of apraxia, which was crucial to Geschwind's thinking about disconnection. Dr. Kertesz will follow with a consideration of the aphasias, now being observed in neurodegenerative diseases as well as after focal lesions disconnecting language regions. Next, Dr. Denckla will review Geschwind's contributions to childhood disorders such as developmental dyslexia. Dr. Yeo will then conclude with a discussion of how Geschwind's influence is still widely evident in clinical and experimental neuropsychology. 
Correspondence: Christopher M. Filley, PhD, Department of Neurology, University of Colorado School of Medicine, Academic Office 1, Mail Stop B-185, 12631 East 17th Ave, Aurora, CO 80045. E-mail: christopher. filley@ucdenver.edu

\section{K.M. HEILMAN. Geschwind and Apraxia.}

I grew up in the Borough Park-Bensonhurst section of Brooklyn. New York, where Norman Geschwind, Edith Kaplan, and Arthur Benton also grew up, but I had not met them until many years later. My interest in apraxia started shortly after I met Geschwind, while I was a neurology resident. In the early part of the 20th century, Hugo Liepmann's reports induced a better understanding and enhanced interest in limb apraxia. However, after the First World War, interest in apraxia had waned. When Geschwind was working with Edith Kaplan at the Jamaica Plains VA, they evaluated a patient with a corpus callosum lesion who, when asked to pantomime transitive gestures, was impaired in his left but not his right hand. They reported this patient with a callosal disconnection in Neurology (1962). Based on this case report and review of the old German and French literature, Geschwind became aware of the importance of disconnection syndromes and wrote his classic 1965 Brain paper in which he summarized much of this research as well as posited explanations for many neurobehavioral syndromes. These papers helped to induce a renaissance of Neuropsychology and Behavioral Neurology, including much research that focused on the possible mechanisms of the different forms of apraxia which could be caused by disconnection or injury to action-movement representations. In this discussion, we will review many of Geschwind's contributions to the understanding of apraxia as well as update some of the concepts about apraxia that developed since Geschwind's classic disconnection paper.

Correspondence: Kenneth M. Heilman, MD, HSC Box 100236, Gainesville,FL 32610.E-mail: heilman@neurology.ufl.edu

\section{C.M. FILLEY. Disconnection and White Matter.}

The idea that white matter tracts interconnect brain regions and participate in neurobehavioral function seems self-evident today, but the contributions of white matter to the operations of cognition and emotion were slow to gain acceptance. Whereas the cerebral cortex has long enjoyed a well-deserved reputation as essential for the higher functions, Norman Geschwind's disconnection paper in 1965 expanded on this notion by emphasizing the importance of cerebral connectivity, in which white matter plays an indispensable role. Disconnection can produce such classic focal syndromes as aphasia, apraxia, and agnosia, and is being investigated in a variety of neuropsychiatric disorders; white matter lesions are also important in the pathogenesis of dementia, the main focus of my work. Modern neuroimaging, in particular magnetic resonance imaging, has been instrumental in this research, helping establish the basis for the syndrome of white matter dementia. The first and most compelling example of this syndrome was toluene leukoencephalopathy, a devastating dementia consequent to toxic white matter injury. Many other disorders feature a significant component of white matter pathology, and because the involvement is typically diffuse or multifocal, widespread disruption of distributed neural networks is typical. Cognitive slowing and executive dysfunction highlight the neuropsychological profile of white matter dementia, and a precursor syndrome of mild cognitive dysfunction has also been proposed. Recent studies have also disclosed that white matter damage is increasingly implicated in the pathogenesis of dementias such as Alzheimer's Disease and chronic traumatic encephalopathy. The investigation of white matter in the context of dementia has a host of intriguing implications, and owes a great deal to the synthetic vision of the brain and behavior offered by Geschwind in 1965.

Correspondence: Christopher M. Filley, PhD, Academic Office 1, Mail Stop B-185, 12631 East 17th Ave, Aurora, CO 80045. E-mail: christopher.filley@ucdenver.edu

\section{A. KERTESZ. Disconnexion Syndromes and Aphasia.}

Fixation and dissection of the brain by neuroanatomists led to the identification of most of the association tracts of the human brain. These tracts were recognized to connect widely dispersed brain regions, but their neurobehavioral significance was unclear. Beginning in the 19th century, clinicians adopted disconnection models for disorders of higher cognitive functions, such as conduction aphasia, visual agnosia, pure alexia, and apraxia, and these models increased the understanding of lesion-symptom relationships. Experimental psycholinguistic and quantitative approaches to the study of aphasia developed in parallel as psychologists began to think in terms of disconnection. Norman Geschwind's interpretation of the disconnexion syndromes in 1965 was highly influential, and has subsequently received further support from lesion studies, functional neuroimaging, and diffusion magnetic resonance imaging tractography. With regard to language and its disorders, Geschwind and his associates reinvigorated study of the syndromes of "Wernicke's," "Broca's," "conduction," "transcortical," and "anomic" aphasia, and clarified the dichotomy of fluency versus nonfluency. This work led to more accurate identification of selective linguistic impairment related to focal brain lesions, and in turn to a deeper understanding of the related functional anatomy of the brain. In this talk, examples of the disconnection syndromes will be presented and integrated with current evidence for network models derived from the study of degenerative disease, such as primary progressive aphasia.

Correspondence: Andrew Kertesz, MD, FRCPC, 268 Grosvenor Street, London, ON N6A4V2, Canada. E-mail: Andrew.Kertesz@sjhc.london. on.ca

\section{DENCKLA. Geschwind's Impact on Developmental Dyslexia and Related Disorders.}

I never planned to be a neurologist; but while rotating as a Beth Israel Internal Medicine resident at the Boston VA and meeting Norman Geschwind, I was "converted" and soon became one of the first fellows on the Aphasia Unit. I shared an office with Frank Benson, with whom I wrote my first publication, on dyscalculia after stroke. At my first faculty position at Columbia, I expected to start a Behavioral Neurology Section, but was instead assigned by Dr. Sidney Carter to attend Child Neurology Clinic to see every child whose chief complaint was either "can't talk yet" or "can't learn to read." While continuing to consult on Behavioral Neurology adult inpatients, I received research support for stroke-acquired dyslexia/dysgraphia/dyscalculia and their developmental counterparts. Then, I published my work on rapid automatized naming, based on hypotheses generated from the brain basis of acquired "alexia." Collaborating with the neuropsychologist Rita Rudel, I studied naming and its relation to reading, which remains fundamental to contemporary diagnosis, prognosis, and neuroimaging research on developmental dyslexia. In the days of the vague diagnosis "Minimal Brain Dysfunction," I investigated motor coordination in relation to dysgraphia and as a "neighborhood sign" relevant to the development of cognitive control. As in the naming/reading association, Geschwind's "proximity principle" proved fruitful in linking acquired lesions to developmental disorders. Before the term ADHD appeared in 1980, Rudel and I published on motor development in hyperactive boys. In the past 25 years, my group in Baltimore has been performing MRI, and lately TMS, to study motor control in ADHD. My journey from internal medicine to the neurodevelopmental field was entirely inspired by Geschwind. Poignantly, it was only in the last four years of his too short life that Norman joined me in memorably lively discussions of developmental dyslexia.

Correspondence: Martha Denckla, MD, 707 N Broadway, Baltimore, MD 21205.E-mail: denckla@kennedykrieger.org

\section{R.A. YEO. Geschwind's Impact on Clinical and Experimental Neuropsychology.}

In the 1970s and 1980s, the Boston VA Hospital was the place to be for anyone interested in neuropsychology, whether they were trainees or 
accomplished researchers. While the "Mecca" of expertise to be found within the Boston VA was certainly not the product of any one individual, no one defined the pervasive atmosphere of discovery, excitement, and opportunity better than Norman Geschwind. His enthusiasm for the "lively sciences" of behavioral neurology and neuropsychology was palpable and infectious. In neuropsychology, Geschwind's clinical impact was mediated by his long-term collaborator and friend, Edith Kaplan. The "process approach" that Edith taught to generations of clinicians, now incorporated into numerous neuropsychological tests, was clearly evident in the evaluation of Mr. K., the famous patient with left hand agraphia and apraxia she reported with Geschwind in 1962. Geschwind and Kaplan were keen observers of human behavior, and their emphasis on process over achievement brought clinical neuropsychology into basic, mainstream neurobehavioral science. Major domains of current neuropsychological research owe much to Geschwind's pioneering efforts. These include (1) the anatomic bases of functional asymmetry, (2) individual differences in brain organization associated with sex, age, and laterality, and (3) the importance of early neurodevelopmental variation for learning disabilities. But nowhere is Geschwind's impact more clearly seen than in contemporary research on neural networks, where his basic notions of centers and connections become elaborated into models capturing both local specialization and global integration. Correspondence: Ronald A. Yeo, PhD, Department of Psychology, University of New Mexico, Albuquerque, NM 87122. E-mail: ryeo@ unm.edu

\section{Symposium 9: \\ The Role of Physiological Factors and Novel Interventions in Mitigating Poor Neuropsychological Outcomes in Pediatric Brain Tumor Survivors}

\section{Chair: Donald Mabbott}

$$
\text { 1:30-3:00 p.m. }
$$

D. MABBOTT, L.M. JACOLA, H.M. CONKLIN \& K.K. HARDY. The Role of Physiological Factors and Novel Interventions in Mitigating Poor Neuropsychological Outcomes in Pediatric Brain Tumor Survivors.

Symposium Description: Curative therapies for pediatric brain tumors can have devastating and irreversible effects, including brain injury, neurovascular changes, epilepsy, endocrinopathy, hearing deficits and neuropsychological impairment. There is great interest in understanding particular physiological factors that may mitigate these deficits, and in developing individualized interventions to foster neuropsychological recovery. In this symposium we will focus on novel research using neuro-imaging (fMRI, MEG) and interventional designs to investigate the neural substrates of altered cognition and behavior in children with brain tumors, elucidate individual differences in specific physiological factors (i.e., sleepiness; fitness level) that may predict differences in neural activation and neuropsychological impairment, and evaluate the efficacy of specific interventions (exercise training and cognitive rehabilitation) for enhancing brain function and neuropsychological outcome. Our four talks will describe studies of: (a) the impact of daytime sleepiness on neural activation and neuropsychological outcome in patients with craniopharyngioma (Dr. Lisa Jacola), (b) how fitness level and motor proficiency relate to frontal activation and neuropsychological outcome in patients with craniopharyngioma (Dr. Heather Conklin), (c) resting state functional connectivity in children treated for posterior fossa tumors and the impact of exercise training in normalizing network connections (Dr. Donald Mabbott), and (d) the predictors of gains in neuropsychological functioning following computerized working memory training in survivors of pediatric brain tumors (Dr. Kristi Hardy). This symposium will describe emerging trends in innovative neuroimaging and intervention approaches in studies that seek to foster brain health and address neuropsychological dysfunction in survivors of pediatric brain tumors.

Correspondence: Donald Mabbott, Program in Neuroscience and Mental Health, The Hospital for Sick Children, 686 Bay Street, Toronto, ON L6M1C9, Canada.E-mail: donald.mabbott@sickkids.ca

L.M. JACOLA, E. DAVIS, M.A. SCOGGINS, R.J. OGG, V.M. CRABTREE, H.M. CONKLIN, J.M. ASHFORD, B.N. MANDRELL, M.S. WISE \& T.E. MERCHANT. The Impact of Excessive Daytime Sleepiness on Neurocognitive Performance and Brain Activation in Children Diagnosed with Craniopharyngioma. Introduction:Children treated for craniopharyngioma are at risk for increased sleepiness, sustained attention deficits, and behavior changes. In healthy adults, sleep restriction impacts neurocognitive performance and brain activation measured by fMRI. We examined the impact of excessive daytime sleepiness (EDS) on neurobehavioral functioning in children diagnosed with craniopharyngioma.

Methods:Patients ( $\mathrm{N}=38 ; 17$ males; $10.78 \pm 4.14$ years) completed sleep, cognitive, and fMRI ( $\mathrm{n}=23 ; 11$ males; $12.66 \pm 3.93$ years $)$ assessments after surgery and prior to proton beam radiation therapy (RT). Clinically significant EDS, defined as a mean sleep onset latency $\leq 10 \mathrm{~min}$ on the Multiple Sleep Latency Test, was present in $55 \%$ of patients. FMRI data from a sustained attention task (Continuous Performance Test;CPT) were analyzed with group comparisons (+EDS=13, -EDS=10).

Results:The +EDS group had elevated caregiver-reported symptoms of anxiety $(33 \%)$, depression $(33 \%)$, social withdrawal $(29 \%)$, and emotional dysregulation (30\%) compared to normative expectations (BASC-2 \& BRIEF: $p s<.05)$. CPT-II reaction time was slower for the + EDS group $(p=.06)$. Performance-related activation was seen in both groups in medial prefrontal cortex, inferior frontal gyrus (R>L), left inferior parietal cortex, and bilateral superior temporal and fusiform gyri $(p<.05$,corrected). Greater activation was seen in the +EDS group in inferior parietal cortex and in the -EDS group in fusiform and medial occipital regions, despite similar performance accuracy $(p=.83)$.

Conclusion: Prior to adjuvant RT, patients with craniopharyngioma present with EDS that impacts neurocognitive performance and behavior. FMRI results suggest compensatory neural activation during task performance in the +EDS group. Future analyses will examine the impact of patient factors (e.g. gender, endocrine status) on neural activation. Early sleep intervention is warranted in this population. Correspondence: Lisa M. Jacola, Ph.D., 262 Danny Thomas Place, Memphis, TN 38105.E-mail:Lisa.Jacola@STJUDE.ORG

H.M. CONKLIN, K.K. NESS, M.A. SCOGGINS, R.J. OGG, J.M. ASHFORD, F.A. BOOP \& T.E. MERCHANT. Aerobic Fitness in Relation to Cognitive Performance and Brain Function among Children Newly Diagnosed with Craniopharyngioma.

Craniopharyngioma survivors experience cognitive deficits that impact quality of life. Cognitive functioning is associated with aerobic fitness in typically developing children and physical exercise promotes recovery following brain injury. We investigated cognitive and neural correlates of fitness in a sample of craniopharyngioma patients. Methods: Patients treated for craniopharyngioma $[\mathrm{N}=57 ; 9.8 \pm 4.7$ years; $42 \%$ Male; $47 \%$ with diabetes insipidus (DI)] participated in fitness, cognitive and fMRI $(n=25)$ assessments following surgery but prior to proton beam radiation therapy. Results: Patients demonstrated impaired fitness [peak oxygen uptake (PKVO2) $=24.8 \pm 6.5 ; 43 \%$ impaired], motor proficiency [Bruininks-Oseretsky (BOT2 $)=38.6 \pm 8.2 ; 69 \%$ impaired] and sustained attention (e.g., CPTII Variability $=54.7 \pm 13.2 ; 30 \%$ below average). PKVO2 correlated with verbal fluency (WJII Retrieval Fluency $\mathrm{r}=.30$, $\mathrm{p}=.05$ ) and sustained attention (CPTII Variability $\mathrm{r}=-.28, \mathrm{p}=.03$ ). BOT2 correlated with verbal fluency (WJII Retrieval Fluency $\mathrm{r}=.35, \mathrm{p}=$ .02 ), sustained attention (e.g., CPTII Variability $\mathrm{r}=-.65, \mathrm{p}<.05)$, rapid naming (e.g., DKEFS- Colors $\mathrm{r}=.50, \mathrm{p}<.05$ ) and executive functions (BRIEF MI r= -.48, p<.01). Areas of neural activation during an n-back 
task included superior parietal lobule, ventral and dorsolateral PFC, middle and superior frontal gyri $(\mathrm{p}<.05$, corrected $)$. Higher activation in this network was associated with absence of DI, higher PKVO2, better BOT2, and slower, more accurate, performance during n-back $(p<.05$, corrected). Conclusions: Prior to adjuvant therapy, children with craniopharyngioma demonstrate impaired fitness, motor proficiency and sustained attention. Better fitness and motor proficiency are associated with better attention and executive functions, as well as greater activation of a well-established working memory network. These findings may explain differential risk for cognitive late effects and suggest targets for intervention.

Correspondence: Heather M. Conklin, Ph.D., 262 Danny Thomas Place, Mail Stop 740, Memphis, TN 38105-2794.E-mail: heather.conklin@ stjude.org

C. DOCKSTADER, S. GaUVREAU, E. BOUfFET, J. PISCIONE, S. LAUGHLIN, B. TIMMONS, S. DOESBURG \& D. MABBOTT. Exercise training alters functional connectivity in children treated for posterior fossa brain tumors.

BACKGROUND: The white matter damage that occurs in children treated for brain tumors of the posterior fossa (PF) likely disrupts typical neuronal communication, which in turn may underlie neuropsychological deficits. Exercise training has been reported to improve neuronal communication, as measured by functional connectivity. We investigated whether neuronal communication is altered in children treated for PF tumors and what effects exercise training may have on it. METHODS: We used Magnetoencephalography (MEG) to measure resting-state functional connectivity in 21 patients (8F; $12.18 \mathrm{yrs}+/$ 3.11SD) and 21 healthy matched controls (8F; 12.15yrs +/-3.81SD). Patients were scanned twice: prior to and following a three-month exercise program. Time-series were reconstructed from 116 coordinates of all cortical, subcortical and cerebellar sources in the AAL atlas, and filtered into frequency bandwidths. Weighted phase lag index values were computed to index functional connectivity. Connectivity strength for each region was calculated.

RESULTS: Prior to exercise, patients, relative to controls, showed increased local connectivity in high frequency bandwidths $(30-100 \mathrm{~Hz})$ in the cerebellum $(p<0.05)$ : a region with considerable white matter injury. Following exercise, patients' high-frequency hyperconnectivity, compared to controls, incorporated more long-range connections principally with anterior cingulate and inferior parietal regions $(p<0.05)$. CONCLUSIONS: Patients exhibit local high frequency hyperconnectivity in an area known to sustain considerable white matter damage. Exercise training appears to redistribute activity and facilitate longrange neuronal communication with regions that modulate attention and default-mode networks. Such redistribution may facilitate cognitive improvements associated with exercise training.

Correspondence: Donald Mabbott, 686 Bay Street, Toronto, ON L6M 1C9, Canada.E-mail:donald.mabbott@sickkids.ca

K.K. HARDY, B. CARLSON-GREEN \& S.A. HOSTETTER. Neuropsychological Functioning Following Computerized Working Memory Training with Survivors of Pediatric Brain Tumors.

BACKGROUND: Survivors of pediatric brain tumors (BT) are at high risk for neurocognitive late effects, including difficulties with working memory (WM). Home-based, computerized WM training has potential to improve functioning, but little is known about how medical, demographic, and cognitive factors affect which survivors may be best-suited for this intervention.

METHODS: We examined data from 46 BT survivors (aged 10.7+3.90, $52.2 \%$ female, $45.6 \%$ posterior fossa $[\mathrm{PF}]$ tumors) who completed a WM training program, Cogmed, across three trials. Following baseline evaluation, participants were asked to complete training at home and return for follow-up testing.
RESULTS: Nearly $85 \%$ of participants were adherent to the program. Compliance did not vary by gender, age, PF tumor location, age at diagnosis, or baseline WM abilities; however, those further from diagnosis were more likely to finish. Participants made significant gains in tasks of visual and verbal short-term memory and visual WM. Those with PF tumors were more likely to show improved verbal short-term memory following training. Evidence of transfer effects was variable; whereas there were no differences in parent ratings of WM, participants who completed tasks of applied math reasoning showed a trend towards improvement 6 months after training.

CONCLUSIONS: Computerized WM training appears feasible for a wide-range of survivors of pediatric BT, regardless of most medical or demographic characteristics. Near-transfer of WM gains was apparent in children who completed training, but far-transfer effects were less evident. Although promising, more research is needed to examine efficacy of this approach, particularly in combination with other treatment strategies.

Correspondence: Kristina K. Hardy, PhD, 111 Michigan Ave, NW, Washington, DC 20010.E-mail: kkhardy@childrensnational.org

\section{Symposium 10: \\ Stepping Out from Silence: Initiating Difficult Dialogues about Diversity in the Context of Neuropsychological Education, Training, and Leadership}

\section{Chair: April Thames}

\section{Discussant: Mariana Cherner}

$$
\text { 1:30-3:00 p.m. }
$$

A.D. THAMES, D.A. BYRD, X.E. CAGIGAS, P.A. SUAREZ, M.R. MADORE, A.L. FERNANDEZ \& M. CHERNER. Stepping Out from Silence: Initiating Difficult Dialogues about Diversity in the Context of Neuropsychological Education, Training, and Leadership.

Symposium Description: Difficult dialogues can occur among individuals with different beliefs, attitudes, beliefs, values, and perspectives about complex social issues and controversies, and often occur in the context of neuropsychological training across both domestic and international settings. Because difficult dialogues are often accompanied with powerful emotions, they are often avoided, which may inadvertently create tension. Although traditionally associated with discussions about race and ethnicity, difficult dialogues span a variety of areas that extend beyond race and include linguistic, religious, age, gender, and/or issues related to both domestic and international training.

This symposium will be led by a panel of national and international experts with the goal of discussing the types of difficult dialogues that are likely to occur in academic, clinical, and large organizational settings between supervisor-student patient/neuropsychologist, and among those serving leadership roles that interface with domestic and international policy and training. Guidelines will be provided for how to successfully initiate and resolve difficult dialogues. Special attention will be given to couching these dialogues in the context of training in neuropsychology and conducting of neuropsychological assessments. Our panel of neuropsychologists includes Drs. Desiree Byrd, Xavier Cagigas, Paola Suarez, Michelle Madore, and Alberto Fernandez (who will serve as our international representative). Together, the panel will discuss strategies for addressing and resolving difficult dialogues. Our Discussant, Dr. Mariana Cherner, will lead an open forum discussion/ dialogue about how we can use our knowledge and skills of initiating and resolving difficult dialogues in the context of international collaborations and training (e.g., different types of training "culture" around the world 
with regard to the level of formality between trainees and instructors, and perceptions of hierarchy).

Correspondence: April D. Thames, Ph.D., Psychiatry, University of California Los Angeles, 740 Westwood Plaza C8-226, Los Angeles, CA 90095.E-mail:athames@mednet.ucla.edu

D.A. BYRD. Demystifying the Process of Engaging in Difficult Dialogues at the Organizational Level of Neuropsychological Training and Practice.

The discussion of the sensitive aspects of diversity at the organizational level of neuropsychological training and practice is accompanied by challenges which can serve to discourage such conversations and lead to inaction. The goal of this presentation is to demystify the process and provide a guiding framework through the use of examples of successful and unsuccessful points of entry, management of emotions, and conflict resolution. This presentation will discuss how to foster an enriched environment whereby individuals in organizational and leadership roles can effectively manage difficult dialogues by increasing respectful organizational engagement with potentially contentious issues. The main objectives of the this presentation are to encourage self-reflection, conversation, and inquiry that penetrate boundaries created by difference; challenge those in leadership roles to recognize assumptions, attitudes, and behaviors that unintentionally perpetuate difficult dialogue, with consideration of alternative perspectives. Key issue that will be discussed include (1) Anticipating and identifying situations that result in difficult dialogue, (2) Reasons that difficult dialogues can derail organizational and training goals, (3) How to deal with emotions and other reactions that may emerge when under pressure, (4) Strategies to effectively stay in dialogue if the conversation starts to get off track. Participants will gain confidence in their ability to raise diversity concerns at the leadership level in a manner that encourages resolution and empowers those to move forward proactively to promote issues of diversity and inclusion. Correspondence: Desiree A. Byrd, 1 Gustave Levy Place, New York, NY 10029. E-mail: desiree.byrd@msm.edu

\section{X.E. CAGIGAS. Cross-Cultural Neuropsychology: A Difficult Dialogue.}

As much as two-thirds of the global population is multilingual, reflecting a linguistic diversity that has only recently begun to be felt within the United States. For example, the number of Spanish speakers alone is projected to rise through 2020 from 37 million to approximately 43 million (Ortman \& Shin, 2011). Although one approach in dealing with this demographic change is increasing recruitment and retention of Bilingual students to address the linguistic and cultural needs of this emerging majority-minority population, the magnitude of the census numbers suggests that ALL neuropsychologists, regardless of linguistic or cultural background, should be proficient in evidence-based procedures and best-practice guidelines for linguistically-discordant patient encounters (LDPE). In order for this change to occur, the field must disavow its lukewarm stance of simply acknowledging limitations in the reliability and validity of currently available instrumentation, and accept the challenge to actually respond to the complex needs of historically underrepresented and linguistically marginalized patients. This proactive stance potentially creates "difficult dialogues" by requiring neuropsychology move beyond the polarizing stance of its one-sizefits-all approach, or delegating LDPE to minority professionals or only those with an express interest in diversity. This presentation highlights preliminary steps to better serve the unique needs of LDPE by improving the generalizability of assessment practices through open expression of potentially opposing views in a constructive manner. For neuropsychology to remain relevant in the midst of these demographic changes, ALL neuropsychologists must be willing to engage in "difficult dialogues" throughout their years of training, practice, and academic discourse. This will ultimately lead to enhancing the overall health and well-being of every patient regardless of cultural or linguistic heritage.
Correspondence: Xavier E. Cagigas, Ph.D., 760 Westwood Plaza, C8746, Los Angeles, CA 90095-1759. E-mail: xcagigas@mednet.ucla.edu

\section{P.A. SUAREZ. Culturally Meaningful Feedback: A Difficult Dialogue in the Making.}

During neuropsychological feedback sessions, patients and their loved ones receive assessment results and recommendations that can optimize patients' daily functioning. When effective, feedback sessions are therapeutic encounters that motivate and empower patients to follow up with targeted recommendations thereby improving adherence and outcomes. Consideration of sociocultural factors and the examinee's worldview is critical to conceptualization AND effective communication of results in order to optimize patient outcomes. For effective communication to take place, neuropsychologists must communicate in language that is understandable and culturally relevant to all parties receiving the information. Demographically, the growth of minority populations underrepresented within our profession increases the likelihood of unfavorable language-discordant patient encounters (LDPE), and culturally-decontextualized feedback sessions. Rather than being therapeutic, such feedback sessions may resemble "difficult dialogues" wherein patients feel misunderstood and disenfranchised, thereby negatively impacting adherence [to recommendations], and outcomes. This session will provide specific examples under which "difficult dialogues" can arise in the context of variable levels of English proficiency, educational attainment, acculturation, and access to services between patients, loved ones, and neuropsychologists. We will conclude by providing some advice on how to successfully initiate, navigate, and resolve "difficult dialogues" that are common, but not unique, to the feedback setting.

Correspondence: Paola A. Suarez, 760 Westwood Plaza, Los Angeles, CA 90095.E-mail:psuare@mednet.ucla.edu

\section{M.R. MADORE. Unequal Positions of Power: Initiating Difficult} Dialogues in the Trainee and Supervisor Relationship.

Situations that spark difficult dialogues are common in the student-teacher or supervisee-supervisor relationship. In the neuropsychology training setting, differences in opinions often become most evident while working with interdisciplinary teams, handling patient care issues, and deciding upon the appropriate clinical diagnosis. Issues that surround diversity pose even greater potential for differences in perspectives and worldviews to emerge. Given the sensitive nature of conversations that surround issues of diversity, trainees may refrain from initiating in difficult dialogues about these issues. Furthermore, given the unequal positions of power, students are often hesitant to engage in such exchanges, which reinforces the idea that such topics and concerns are not appropriate for discussion and that silence is key for "getting by." Students are often concerned about how initiating a difficult dialogue may affect them in the future. Over time, this can greatly impact the quality of training. Thus, managing these kinds of conversations in the training environment is a skill, and critical to student development. The discussion will focus on providing strategies that students and trainees can use to successfully initiate and resolve difficult dialogues with their supervisors, teachers, and/or mentors. Strategies such as learning how to use curiosity as a guide to understanding different perspectives will be discussed. The presentation will also review circumstances in which it would be appropriate for trainees to seek outside mentorship as well as other mechanisms of support. The overall goal of the presentation is to provide a "toolkit" that students and trainees can use to effectively engage in difficult dialogues with supervisors that can occur in the context of neuropsychological training.

Correspondence: Michelle R. Madore, 4150 Clement St, San Francisco, CA 94121.E-mail:mador@gmail.com

A.L. FERNANDEZ. Translating measures for use across international settings: A critical analysis of current approaches. Neuropsychologists in international settings are accustomed to the various challenges with regard to the reliability and validity of available 
assessment measures. One common approach has been to simply translate measures to the language of the client. Students are often under the assumption that the use of translated measures will circumvent the issue of language differences, which will in turn allow accurate measurement of the intended construct (e.g., naming ability). However, the process of translation is confounded by language as well as adaptive versions that are not equivalent to the original. In training settings, the complexities involved in using translated measures create a difficult dialogue because one must understand and be transparent about the limitations regarding the generalizability of instruments and serious validity threats to the intended construct under measurement. Studies using international samples have shown that familiarity with test content has a significant impact on test performance. Rather than simply acknowledging these limitations, we need gain a more in-depth understanding of cultural influences on brain development and integrate this knowledge into neuropsychology training programs. The presentation will discuss the types of difficult dialogues that need to happen within international training settings in order to foster the types of critical thinking skills that are required for developing appropriate instruments and refining existing instruments.

Correspondence: Alberto L. Fernandez, PhD, Chile 279 PB, Cordoba 5000, Argentina. E-mail: neurorehab@onenet.com.ar

\section{Poster Symposium in Honor of Maureen Dennis}

$$
\text { 1:30-3:00 p.m. }
$$

\section{Acquired Brain Injury (TBI/ Cerebrovascular Injury \& Disease - Child)}

E. MAXWELL, P.T. CIRINO, C. SHARP \& L. EWING-COBBS. The Role of Anxiety, Family Burden, and Executive Functioning on Mathematical Performance in Children with Traumatic Brain Injury.

Objective: Mathematical performance is closely linked with anxiety and executive processes, which are both adversely impacted by traumatic brain injury (TBI) in children. The present study examined mathematical performance in children with TBI or orthopedic injuries (OI) at 2 months and 2 years post-injury, while considering the impact of anxiety, executive functioning, and family burden of injury.

Participants and Methods: Participants (ages 6 to 15 years) had been hospitalized for moderate or severe TBI $(n=51)$ or OI $(n=47)$ and were enrolled in a prospective longitudinal study. Children completed Woodcock-Johnson III Calculation and Applied Problems subtests. Parents completed the Family Burden of Injury Interview questionnaire, Child Behavior Checklist, and the Behavior Rating Inventory for Executive Function. Mediation and path analyses examined the contribution of the above variables to mathematical outcomes.

Results: Compared to children with OI, children with TBI had lower calculation and problem solving scores, elevated anxiety, higher family burden, and executive functioning impairment persisting 2 years post-injury $(p<0.05)$. Mediation analyses revealed that executive functioning was a significant predictor of calculation at 2 months (point estimate of $-1.37,95 \%$ bootstrap CI of -4.22 to -0.21 ) and of problem solving scores at 2 years post-injury (point estimate of $-0.91,95 \%$ bootstrap CI of -2.61 to -0.03 ), even after accounting for mathematical scores at 2 months post-injury. Neither anxiety nor family burden significantly impacted mathematical performance $(p>0.05)$.

Conclusions: Executive functioning difficulties at 2 months post-injury in children with moderate to severe TBI predict long-term functioning and may serve as a red flag regarding the need for interventions to improve educational and psychological health outcomes. This research was supported by NIH NS ROI 046308.
Correspondence: Emily Maxwell, University of Houston, 126 Heyne Building, Houston, TX 77204. E-mail: ecmaxwell@gmail.com

K. SINOPOLI \& M. DENNIS. "Is My Child Self-Aware?" Metacognitive Knowledge of Post-Injury Sequelae Following Childhood Traumatic Brain Injury.

Objective: Metacognitive knowledge, or awareness of one's own abilities and deficits, is disturbed in adults with a history of traumatic brain injury (TBI). The current study explored whether this deficit is also a consequence of childhood TBI.

Participants and Methods: Participants included 40 typically developing controls and 49 youth with TBI aged 7-17 years. Parents and teachers completed the Conners 3rd Edition questionnaires, and all participants over 8 years completed the self-report questionnaire. Metacognitive knowledge following TBI and the level of concordance between parent and teacher ratings was examined via analyses that focused on the level of agreement and consistency between raters using Cohen's Kappa statistic and intraclass correlations. Differences in ratings were explored between groups via analyses of variance (ANOVA).

Results: Although all raters rated youth with TBI as exhibiting a greater number of difficulties across subscales, no scores fell into the clinically significant range. However, parents and teachers indicated that children who met criteria for new-onset attention-deficit/hyperactivity disorder (i.e., secondary ADHD) exhibited greater difficulties at home and at school than those with TBI without ADHD. In comparison to parent ratings, youth with TBI seem moderately aware of their attention difficulties and learning needs, but less agreement was found for other externalizing behaviours (hyperactivity/impulsivity, aggression, oppositionality). There was no significant agreement between self-report and teacher ratings. Parents and teachers of youth with TBI displayed substantial agreement across hyperactivity, impulsivity, and executive functioning scales.

Conclusions: Pediatric TBI results in deficits in metacognitive knowledge of externalizing behaviours, but awareness seems intact for post-injury attention and learning difficulties.

Correspondence: Katia Sinopoli, PhD, Psychology, The Hospital for Sick Children, 555 University Ave., Toronto, ON M5G 1X8, Canada. E-mail: katia.sinopoli@sickkids.ca

\section{A. WILKINSON, N. SIMIC, M. DENNIS \& J. HUTCHISON. Predicting Attention Problems in the First Year Following Pediatric Traumatic Brain Injury (TBI).}

Objective: Attention problems are over-represented in children with TBI, both pre- and post-injury. These attention difficulties occur across all severities of TBI, suggesting severity of injury alone does not predict individual differences in attention outcomes. An exciting development in TBI research is the investigation of acute factors tested at the time of injury (such as serum biomarkers and neuroimaging) and the possible correlation between these factors and outcome after injury. The importance of biomarkers, as determinants of cognitive outcome in childhood TBI, has not been well described. Using multiple diagnostic investigations in the acute phase of an injury, and by assessing attention problems throughout the first year post-injury, we hope to gain a better understanding of not only the trajectory of attention problems, but why attention is so vulnerable to TBI.

Participants and Methods: 58 children/adolescents with mild to severe TBI were enrolled and followed (2009-2013) from PICUs at 5 Canadian children's hospitals. Institutional Review Boards provided approval of the study. Parents completed questionnaires assessing ADHD symptoms (Conners Rating Scale - 3rd Edition) at baseline, 3, 6, and 12 months post-injury. Brain MRIs, obtained within first 5 days post-injury, were classified using a novel clinical tool, developed at SickKids. ELISA test kits were used to analyze a selected group of serum biomarkers collected multiple times after injury. 
Results: Parent ratings of inattention and hyperactivity/impulsivity peak at 3 months post-injury, improve over time, but remain elevated in relation to baseline at 12 months.

Conclusions: Children with TBI are at risk for persisting attention difficulties at least 1 year post-injury.

Correspondence: Amy Wilkinson, MA, Psychology, The Hospital for Sick Children, 555 University Ave, The Hospital for Sick Children - 6th floor Psychology, Toronto ON, ON M5G1X8, Canada. E-mail: amy. wilkinson@sickkids.ca

\section{HARIK, C.P. JOHNSON \& L. EWING-COBBS. The Impact of Pediatric Traumatic Brain Injury on Written Expression: a Diffusion Tensor Imaging Study Utilizing Tract-Based Spatial Statistics.}

Objective: Written expression (WE), the ability to express oneself through writing, is negatively impacted by pediatric traumatic brain injury (TBI). This study explored the relation between TBI and WE using diffusion tensor imaging (DTI) in children.

Participants and Methods: Participants included children with TBI $(\mathrm{n}=35)$ and a comparison group of children with orthopedic injury (OI; $\mathrm{n}=28$ ) who were between $8-15$ years at time of injury. White matter integrity was assessed using fractional anisotropy (FA) values derived from tract based spatial statistics. General linear modeling predicted performance on WE (writing fluency, spelling and thematic maturity of a writing sample) measures one year post-injury from white matter integrity at three months post-injury. Mediation analyses explored contributions of specific white matter tracts to WE.

Results: Children with TBI showed reduced WE performance on writing fluency and spelling, and showed decreased white matter integrity in all pathways as compared to children with OI. Several tracts predicted WE performance and there was no interaction between group and DTI metrics. Mediation analyses revealed that white matter tracts mediate the relation between TBI and writing fluency when entered independently, but not when entered simultaneously.

Conclusions: White matter integrity predicted WE performance in both groups. The relation of TBI and writing fluency was mediated by FA values for all tracts but significant intercorrelation of white matter tracts prohibited any one tract from showing unique contribution. The results of this study are consistent with literature suggesting that white matter integrity facilitates cognitive and motoric fluency.NIH NS ROI 046308 Correspondence: Lindsey Harik, M.A., Department of Psychology, University of Florida, $3515 \mathrm{SW}$ 39th Blvd Apt 28B, Gainesville, FL 32608.E-mail:linharik@gmail.com

\section{A. TREBLE-BARNA, N. MINICH, H.G. TAYLOR, T. STANCIN, K.O. YEATES \& S.L. WADE. Classroom Functioning Following Traumatic Brain Injury in Young Children.}

Objective: Children sustaining traumatic brain injury (TBI) can demonstrate long-term problems with academic achievement. Studies of other student populations report associations of reduced levels of academic responding and higher rates of off-task behavior in the classroom with lower achievement. The objective of the present study was to examine the classroom behaviors of children with TBI utilizing ecobehavioral analysis.

Participants and Methods: Participants included children who sustained complicated mild to moderate $(n=22)$ and severe $(n=9)$ TBI or orthopedic injuries $(\mathrm{OI} ; \mathrm{n}=48)$ between ages $3-6$ years. At an average of 7 years post-injury, children were administered neuropsychological tests and were observed in their classrooms during a 1-hour instructional period using a time-sample method. The groups were compared on frequencies of individual teacher attention, competing or off-task behaviors, task management/preparation, and academic responding. Regression analysis examined associations of classroom behaviors with neuropsychological measures

Results: Children with severe TBI demonstrated significantly less academic responding $(p=.011)$ and trends for receiving more individual teacher attention $(p=.101)$ and demonstrating more task management/preparation $(p=.051)$ relative to OI children. Poorer classroom functioning was associated with poorer executive functioning and lower intelligence across groups.

Conclusions: Children with a history of severe TBI are less able to engage in instructional activities and may require more teacher support than their OI classmates long after their injuries. Associations of classroom functioning with performance on neuropsychological measures support the utility of neuropsychological testing in identifying children most in need of special educational interventions.

Correspondence: Amery Treble-Barna, PhD, Physical Medicine and Rehabilitation, Cincinnati Children's Hospital Medical Center, 3473 Brookline Ave, \#2, Cincinnati,OH 45220.E-mail: amery.treble-barna@ cchmc.org

A. TREBLE-BARNA, H. ZANG, N. ZHANG, H.G. TAYLOR, T. STANCIN, K.O. YEATES \& S.L. WADE. Parenting Practices as a Time-Varying Moderator of Executive Dysfunction Following Traumatic Brain Injury in Young Children.

Objective: Despite research documenting the influences of the family environment on neurobehavioral outcomes following traumatic brain injury (TBI) in children, it is unclear whether family characteristics have the potential to alter the developmental course of neurobehavioral recovery post-injury. The objective of the present study was to examine the relationship of parenting practices to changes in a behavioral measure of executive functioning (EF) over time following TBI in young children. Participants and Methods: Participants included children who sustained complicated mild to moderate $(\mathrm{n}=63)$ and severe $(\mathrm{n}=23)$ TBI or orthopedic injuries (OI; $n=117$ ) between ages $3-7$ years. The Parenting Practices Questionnaire and the Behavior Rating Inventory of Executive Functioning (BRIEF) were obtained at baseline ( $\mathrm{M}=40 \pm$ 20 days post-injury), 6 months, 12 months, 18 months, 3.5 years, and 7 years post-injury. Linear mixed models examined parenting practices as a time-varying moderator of changes in EF over time post-injury. Results: A significant GROUP $\mathrm{x}$ TIME $\mathrm{x}$ PARENTING interaction $(\mathrm{p}=$ .022 ) was found for authoritarian parenting. Specifically, the negative effects of mild to moderate TBI relative to OI became more pronounced over time for children exposed to higher levels of authoritarian parenting, with the most pronounced effects evident in children exposed to authoritarian parenting at 7 years post-injury.

Conclusions: Parenting practices are an important moderator of changes over time in the effects of complicated mild to moderate TBI on EF, with the negative effects of TBI exacerbated by less effective parenting practices. Thus, more effective parenting has the potential to buffer the effects of TBI on long-term EF in young children.

Correspondence: Amery Treble-Barna, PhD, Physical Medicine and Rehabilitation, Cincinnati Children's Hospital Medical Center, 3473 Brookline Ave, \#2, Cincinnati,OH 45220.E-mail: amery.treble-barna@ cchmc.org.

N. SIMIC, A. WILKINSON, M. DENNIS \& J. HUTCHISON. Predicting Executive Function Following Pediatric Traumatic Brain Injury (TBI).

Objective: Long-term consequences for TBI often include deficits in executive functions $(E F s)$. In the 1st year post-injury, there is often significant decline in EFs followed by partial recovery. "Partial" because many individuals continue to have persisting EF problems. Predicting who will go on to have EF problems is complicated by the heterogeneous nature of TBI and pattern of brain injury, and ongoing EF development through adolescence.

We assessed the pattern of EF changes over the first year post-injury. including what EFs are affected, whether and how they recover, and potential predictors of outcome 12-months post-injury.

Participants and Methods: 53 youth age 5 to 18 years who had sustained complicated mild to severe TBIs, recruited from five children's hospitals in Canada. Parents completed rating of EF symptoms 
(Behaviour Rating Inventory of Executive Function) at time of injury, at 3,6, and 12 months post-injury. Serum protein analysis and brain neuroimaging from the acute phase of injury provided potential biomarkers of TBI-related EF changes.

Results: Parent reports of EF changes are heterogeneous following pediatric TBI with some abilities reportedly minimally affected (inhibition, planning, organization), while others exhibit decline in the first 3 months (working memory and initiation) or 6 months (emotional control, flexibility, and monitoring behaviour). Many EFs fail to fully recover. Significant predictors of 12-month outcome included inflammatory marker Interleukin-8 (IL-8), astrocyte marker Glial Fibrillary Acidic Protein (GFAP), and cell adhesion markers Neural Cell Adhesion Molecule (NCAM) and Vascular Cell Adhesion Molecule (VCAM).

Conclusions: Serum biomarkers of brain injury may be important prognostic tools for predicting the course of EF changes following TBI, which is important for patients and their families because EFs are known to affect cognitive processes (memory and attention), academic achievement, and behaviour regulation.

Correspondence: Nevena Simic, PhD, Psychology, The Hospital for Sick Children, 50 Woodman Dr S, Hamilton, ON LSK 4E4, Canada. E-mail: nevena.simic@utoronto.ca

\section{Autism Spectrum Disorders}

J. HUBER, E. ROSENTHAL, T. JEGATHESAN, M. GOODMAN, S. CHEEMA \& E. YOUNG. Toward Social and Cultural Competence in Developmental Pediatrics: Parents' Perceptions of The Autism Diagnostic Observation Schedule.

Objective: Culture (thoughts, communications, actions, customs, beliefs, values, and institutions of racial, ethnic, religious, or social groups) and socioeconomic status are key determinants of health. The Autism Diagnostic Observation Schedule (ADOS) has materials that primarily represent Caucasian appearances (doll, figures, books, and pictures), activities typical of western societies (birthday party) and experiences common to families with resources (resort vacation picture). Given the diverse cultural, social and economic groups that utilize the ADOS, the tool's materials may not accurately reflect these populations. The study objective was to assess the social, economic and cultural relevance of the ADOS materials to an urban multi-cultural globalized developmental pediatric practice.

Participants and Methods: A cross-sectional study of 135 caregivers with children between 0-6 years old was conducted. Caregivers completed a questionnaire assessing perceptions of the ADOS. Demographics, immigration status, income, housing and ethnic background were collected. Fischer's exact t-test was used to identify differences between stratified groups.

Results: Of the 135 participants, 72(53\%) were immigrants. Compared to Canadian-born caregivers, a significant proportion of immigrants did not perceive the Caucasian doll $(52 \%, \mathrm{p}=0.0026)$, birthday party activity $(37 \%, \mathrm{p}=0.0020)$, or picture of the resort $(26 \%, \mathrm{p}=0.035)$ as relevant to their culture. Forty percent of low-income caregivers reported the ADOS would impact their opinion and relationship with the doctor compared to only $14 \%$ with higher incomes ( $p=0.0125$ )

Conclusions: Components of the ADOS may not be relevant to caregivers of immigrant and low economic status. Further research is needed to develop and validate materials that more broadly reflect multi-cultural, multi-economic populations.

Correspondence: Joelene Huber, Msc(A), PhD, MD, FRCPC, Pediatrics, St.Michael's Hospital, 61 Queen Street East, Toronto, ON (416) 8646060 Ext.8276, Canada.E-mail: huberjo@smh.ca

\section{Cancer}

K. EDELSTEIN, L. BERNSTEIN, R. SIMPSON, N. LAPERRIERE, C. CHUNG, B. MILLAR, C. MENARD \& W. MASON. Longitudinal Effects of Radiation on Neurocognitive Functions in Adults with Primary Brain Tumors.

Objective: Cranial radiation is associated with progressive declines in processing speed, attention, memory and executive functions, presumably due to deleterious effects on white matter growth in children and accelerated cognitive aging in older adults. Here we examine neurocognitive functions following radiation for a primary brain tumor in adults. Participants and Methods: Thirty-three primary brain tumor patients (18 male; mean age 45 years), underwent assessments within a year of radiation (baseline mean \pm SD: $0.11 \pm 0.28$ years), and $4.98 \pm 2.69$ years later. Patients who developed tumor progression prior to neurocognitive follow up were excluded. Neurocognitive test scores were converted to scaled scores, transformed to z-scores, and averaged to provide 5 cognitive domains: Speed, Attention, Immediate Memory, Delayed Memory, and Executive Functions.

Results: At baseline, Executive Functions were below average ( $t(32)$ $=-3.621, p=.001)$. All other scores were within normal limits at baseline and follow up (all p's >.05). Moreover, there was no difference in performance at baseline compared to follow-up across domains, nor was there an interaction between time and domain (time $\mathrm{x}$ domain repeated measures ANOVA, all $p>0.1$ ). There was also no difference in the number of tests that were impaired (i.e., z-score $<-1$ ) at baseline compared to follow up.

Conclusions: Adult brain tumor patients showed deficits in cognitive functions that did not appear to decline over time after radiation in the absence of tumor progression. Continued monitoring of neurocognitive functions following radiation treatment in a larger sample is warranted to identify factors contributing to neurocognitive decline across the lifespan in this population.

Correspondence: Kim Edelstein, PhD, Princess Margaret Hospital, 610 University Ave, Toronto, ON M5G2M9,Canada.E-mail:kim.edelstein@ uhn.ca

\section{Cognitive Neuroscience}

K.A. BRADLEY, J. JURANEK, A.M. ROMANOWSKA-PAWLICZEK, J. HANNAY, P.T. CIRINO, M. DENNIS \& J.M. FLETCHER. Corpus Callosum Microstructure and Auditory Interhemispheric Transfer in Spina Bifida Myelomeningocele.

Objective: The purpose of this study was to evaluate if early disruption in corpus callosum development due to spina bifida myelomeningocele (SBM) contributes to plastic reorganization of interhemispheric temporal lobe connections. Additionally, this study evaluated if diffusion tensor imaging (DTI) measures of integrity of the interhemispheric temporal tract specifically had functional relevance to a task that required auditory communication between hemispheres, dichotic listening.

Participants and Methods: Diffusion tensor images were acquired on 45 children and adults with SBM and 15 typically developing individuals, ages 8 to 36 . Probabilistic tractography was used to isolate the interhemispheric temporal tract connecting bilateral auditory processing regions. DTI measures were correlated with dichotic performance.

Results: Both hypoplasia and hypogenesis of the corpus callosum resulted in plastic re-wiring of interhemispheric temporal connections through alternate routes, particularly the anterior commissure. However, preservation of the normative pattern, a right ear advantage, on the dichotic listening task in people with SBM was associated with connections through the posterior corpus callosum and not the anterior commissure. Microstructural integrity of this tract was reduced in SBM as indicated by reduced fractional anisotropy (FA) and axial diffusivity $(\mathrm{AD})$, and increased radial diffusivity (RD). Changes in $\mathrm{AD}$ were associated with atypical left ear performance on the dichotic listening task. 
Conclusions: Macrostructural and microstructural evidence suggests that re-routed connections through the anterior commissure in SBM may not be compensatory but maladaptive, and reduced integrity of the interhemispheric temporal tract affected typical performance on the dichotic listening task. Given persistent hypotheses about the role of the anterior commissure and other potential compensatory connections, this study has important implications for understanding of the effects of early corpus callosum maldevelopment.

Correspondence: Kailyn A. Bradley, PhD, Department of Psychiatry, Mt. Sinai School of Medicine, 306 E 96TH ST, APT 2F, New York, NY 10128. E-mail: Kailyn.bradley@gmail.com

\section{Learning Disabilities/Academic Skills}

V. FERNANDEZ, J. JURANEK, A.M. ROMANOWSKA-PAWLICZEK, V. WILLIAMS \& J.M. FLETCHER. Cortico-cerebellar connectivity in reading impaired children: A probabilistic tractography study. Objective: Very little is known about cortico-cerebellar connectivity as it relates to individuals with dyslexia. Building on previous findings of decreased volume in the anterior lobe of the cerebellum, we examined tracts that originate in the anterior lobe of the cerebellum and project to cortical regions typically associated with reading: the temporoparietal region (TP), occipitotemporal region (OT), and inferior frontal region (IF).

Participants and Methods: We utilized novel cerebellar segmentation procedures and probabilistic tractography in 29 children with impaired single-word reading skills and 27 typical readers.

Results: We found greater FA for the poor readers in the tracts terminating in the TP and IF regions. In the OT region, differential FA trajectories were identified such that FA was greater for the older poor readers. The observed differences in FA were driven by reduced RD suggesting that increased myelination may be the microstructural mechanism of change.

Conclusions: This study provides evidence for discrete, regionally-bound functions of the cerebellum and suggests that the projections from the anterior cerebellum appear to have a regulatory effect on cortical functions important for reading.

Correspondence: Vindia Fernandez, M.A./Ph.D. Student, Department of Psychology, University of Houston, UCLA Semel Institute for Neuroscience and Human Behavior, 760 Westwood Plaza, Los Angeles, CA 90095. E-mail: vindia_f@yahoo.com

\section{Medical/Neurological Disorders/Other} (Child)

\section{N. SIMIC, M. DENNIS, P.T. CIRINO, J. JURANEK, P. TAYLOR \& J.M. FLETCHER. White and Grey Matter Relations to Simple, Choice, and Cognitive Reaction Time in Spina Bifida.}

Objective: Elevated reaction time (RT) is common in brain disorders. We studied RT in a neurodevelopmental disorder, spina bifida myelomeningocele (SBM), and typically developing (TD) individuals aged 8 to 48 years in order to establish the nature of lifespan-relations of RT and the brain.

Participants and Methods: Participants: 251 children and adolescents $(\mathrm{n}=120)$ or adults $(\mathrm{n}=131)$ including SBM $(\mathrm{n}=166)$ and TD $(\mathrm{n}=85)$. RT Measures: computerized tasks required speeded responses to the presentation of visual stimuli. In Simple RT, participants pressed a single button at the presentation of a centralized downward pointing arrow. In Choice RT, they pressed a left or right button for a downward arrow presented on the corresponding side of the screen. In Cognitive RT, a left or right button was pressed for a centralized upward or downward arrow. Accuracy and RT (speed and variability) were recorded.

MRI Analyses: Volumes of cortical white and grey matter in large networks.
Results: Accuracy and RT speed and variability were all impaired in SBM relative to TD, but the most important effects of SBM on RT are seen on tasks that require a cognitive decision rule. SBM showed smaller corrected corpus callosum proportions, larger corrected cerebellar white matter proportions, and larger corrected proportions for grey matter in the Central Executive and Salience networks. There were clear negative relations between RT measures and corpus callosum, Central Executive, and Default Mode networks in SBM; relations were not observed in TD. Statistical mediation analyses indicated that corpus callosum and Central Executive Network were important mediators.

Conclusions: While RT is known to rely heavily on white matter under conditions of typical development and in individuals with adult-onset brain injury, we add the new information that additional involvement of grey matter may be important for a key neuropsychological function in a common neurodevelopmental disorder.

Correspondence: Nevena Simic, PhD, Psychology, The Hospital for Sick Children, 50 Woodman Dr S, Hamilton, ON LSK 4E4, Canada. E-mail: nevena.simic@utoronto.ca

C. ARRINGTON, A.L. WARE, Y. AHMED, S. AFRAZ, P.A. KULESZ, J.M. FLETCHER \& M. DENNIS. The Effects of Shunt Revisions on Intelligence in Congenital Hydrocephalus: A Meta-Analysis.

Objective: Surgical shunt intervention is common in patients with congenital hydrocephalus. While deleterious effects of hydrocephalus on intelligence (IQ) are established, a dearth of literature has determined whether shunt-revisions further alter IQ in these populations and findings across studies are mixed. The current study utilized a meta-analytic approach to investigate whether shunt revisions affected IQ in congenital hydrocephalus.

Participants and Methods: Forty-three articles were reviewed to include studies which: (a) were empirical, (b) included target variables, and (c) included congenital hydrocephalus patients with history of shunting. Included articles were coded systematically, with five studies and 14 comparisons yielding means for meta-analysis. A random-effects model was used because of variations across studies.

Results: Mean effect size across studies was small $(g=0.25)$ and had a variance of 0.01 with standard error of 0.10 . Significance of the effect size was evaluated by computing $95 \%$ confidence intervals $[0.06,0.45]$, which did not include zero. Rejection of heterogeneity of effect size [Q $(14)=14.29, p>0.05]$ indicated generalizability across studies. The $\mathrm{I}^{2}$ statistic indicated little variance $(2 \%)$ attributable to heterogeneity among studies, despite demographic differences.

Conclusions: Results indicated that IQ differences between low revision and high revision groups significantly differ such that increased shunt revisions are related to lower IQ scores, but the effect is small. The effect sizes may be larger for adult than child cohorts, implying a lifetime effect. Findings highlight a need for longitudinal research in this population with a focus on intra-individual differences following hydrocephalus and shunt treatment outcomes.

Correspondence: C Nikki Arrington, Psychology, University of Houston, 126 Heyne, Houston, TX 77204. E-mail: cnarring@central.uh.edu

P.T. CIRINO, M. BARNES, A.L. WARE, J.M. FLETCHER \& M. DENNIS. Predictors of Academic Fluency in Spina Bifida Myelomeningocele.

Objective: Children and adults with spina bifida myelomeningocele (SBM) have difficulties within and across cognitive domains, including academics, slowed reaction time (RT), and other motor tasks. Proficient academic skill requires both accuracy and fluency, so understanding both is important. This study examined academic fluency in SBM and controls. For reading and writing fluency, single-word reading, cognitive RT, and vocabulary (and fine motor for writing) were expected predictors. For math fluency, effects of cognitive RT, vocabulary, and fine motor were expected; computations were also considered.

Participants and Methods: 182 children and adults with SBM and 83 controls were included. The sample was $52 \%$ children, $51 \%$ female, 
and of diverse ethnicity. SBM participants had hydrocephalus, 74\% had lower spinal lesions, and 94\% had Chiari II. All academic measures were from the WJ-III. Cognitive RT involved a rapid response by applying a decision rule; Purdue Pegboard was the motor predictor. Age and socioeconomic status were covariates. Analyses used regression for group differences and prediction.

Results: Controls outperformed SBM on all measures of academic fluency $(p<.001)$. For reading fluency $(p<.001, R 2=.68)$, vocabulary, word reading, and cognitive RT were unique predictors, as was group (all $\mathrm{p}<.05)$. For writing fluency $(\mathrm{p}<.001, \mathrm{R} 2=.62)$ results were similar, though fine motor but not cognitive RT, were significant. For math fluency $(p<.001, R 2=.47)$, vocabulary, fine motor skill, cognitive RT, and group were predictors (all $\mathrm{p}<.05$ ), but adding computations diminished the role of vocabulary, and modified the role of group.

Conclusions: Academic fluency is reduced and influenced cognitively in SBM, even when other academics and covariates are included. Predictors operate in similar fashion across group for reading and writing but diverge for math. where the relation of accurate and fluent mathematical skills was stronger for individuals with SBM than for controls.

Correspondence: Paul T. Cirino, PhD, Psychology, University of Houston, UH TMC Annex, 2151 W Holcombe Blvd, 224a, Houston, TX77204-5053.E-mail: pcirino@uh.edu

P.A. KULESZ, A. TREBLE-BARNA, V. WILLIAMS, J. JURANEK, P.T. CIRINO, M. DENNIS \& J.M. FLETCHER. Attention in Spina Bifida Myelomeningocele: Relations with Brain Volume and Integrity.

Objective: The recently identified associations between tectal malformations and alterations in white matter connectivity of both frontal and parietal cortical regions suggest that a more detailed exploration of both the orienting and executive control attention networks in relation to the brain abnormalities of spina bifida myelomeningocele (SBM) is timely. This study investigated the relations of tectal volume and superior parietal cortex, as well as alterations in tectocortical white matter connectivity, with the orienting and executive control attention networks in individuals with SBM.

Participants and Methods: Probabilistic diffusion tractography and quantification of tectal and superior parietal cortical volume was performed on 74 individuals aged 8 to 29 with SBM and a history of hydrocephalus with assessments of posterior (covert orienting) and anterior (conflict resolution, attentional control) attention network functions. The Pearson, percentage bend and skipped correlations were used to examine attention-brain relations.

Results: Lower tectal volume was associated with slower covert orienting; lower volume of the superior parietal cortex was associated with slower conflict resolution; and lower white matter integrity along both frontal and parietal tectocortical pathways was associated with poorer attentional control.

Conclusions: The findings suggest that the components of both the orienting and executive control attention networks are impaired in SBM. Neuroanatomical disruption to the orienting network appears more robust and a direct consequence of characteristic midbrain dysmorphology; whereas, executive control difficulties may emerge from parietal cortical anomalies and reduced frontal and parietal cortical-subcortical connectivity related to the pathophysiological effects of congenital hydrocephalus.

Correspondence: Paulina A. Kulesz, TIMES, University of Houston, 4811 Calhoun Road, Houston, TX 77204.E-mail: Paulina.Kulesz@ times.uh.edu

\section{Poster Session 6: \\ Assessment-Child, Dementia (AD), \& Medical/ Neurological Disorders-Child}

\author{
1:30-3:00 p.m.
}

\section{Assessment/Psychometrics/Methods (Child)}

G. DE GYVES \& M. RAMÍREZ-FLORES. Exploring decisionmaking in children from 6 to 9 years old using a risky choice task.

Objective: In decision-making one must make a choice between two or more options, requiring an analysis of costs and benefits, and the estimation of consequences in short, median and long term. Ventromedial prefrontal cortex is involved in adult decision-making, its immaturity in children may explain why children have a preference for immediate rewards and with few benefits. The objective of the present study was to analize decision-making and risky choice in children

Participants and Methods: 139 children from 6 to 9 years from public and private schools were assessed, using the computerized version of the "Hungry Donkey Task" which is used for evaluating risky choices. Results: Results don't show significant statistical differences between age groups in task performance $(\mathrm{p}=0.484)$ although six-year-olds have better scores. Gender differences neither are shown $(t=1.461, p=0.82)$. Conclusions: As described in other studies there are no differences between the age groups and in gender other studies report differences between boys and girls, without consistency between them. Hungry Donkey Task is a useful screening tool for assessing children's decision making.

Correspondence: ,, . E-mail:

J.K. RAI, J.E. CASEY \& L. FLARO. The ecological validity of neuropsychological tests of executive function in children with Fetal Alcohol Spectrum Disorder (FASD).

Objective: Although performance-based tests are used widely to evaluate executive functions (EFs), studies comparing scores from performance-based and behavioural measures of EFs suggest that the former have little, if any, ecological validity in various clinical populations (e.g.. Vriezen \& Pigott, 2002). This study investigated the ecological validity of performance-based EF tests in a new clinical sample: children with Fetal Alcohol Spectrum Disorder (FASD).

Participants and Methods: Retrospective data were analyzed from 96 children with FASD, aged nine to 16 years, who were assessed at the private practice of a child neuropsychologist. Using bivariate correlations, multiple regression, and canonical correlation analysis, both the extent and nature of the relationship between the participants' scores on three performance-based EF tests (Wisconsin Card Sorting Test (WCST), Trail Making Test (TMT), Controlled Oral Word Association Test (COWAT)) and the Behavior Rating Inventory of Executive Function (BRIEF) were examined.

Results: The majority of bivariate correlations and the canonical correlation analysis were not statistically significant, although more impaired scores on BRIEF Organization of Materials were weakly associated with fewer perseverative errors on the WCST $(r=.21)$. Performance-based test variables also did not meaningfully predict scores on the BRIEF Metacognition and Behavior Regulation Indexes.

Conclusions: These results add to the growing literature demonstrating a weak relationship between performance-based and behavioural measures of EF. It appears that performance-based and behavioural measures assess either different aspects of EF or different constructs altogether. There is a need for the development of new performance-based $\mathrm{EF}$ tests that follow from a strong theoretical conceptualization of EF. Correspondence: Jaspreet K. Rai, M.A., Psychology, University of Windsor, \#203, 40 Adams Lane, LaSalle, ON N9J3R4, Canada. E-mail: raii@uwindsor.ca 


\section{SOUBBOTINA, J.E. CASEY \& V. PETRAUSKAS. Clinical Utility of the BDEFS-CA ADHD-EF Index in Classifying Children with ADHD.}

Objective: The Barkley Deficit of Executive Functioning Scale - Children and Adolescents (BDEFS-CA; 2012) is a new parent-completed behaviour rating scale intended for clinical evaluation of Executive Functions (EF). At present there is no independent evaluation of the instrument. In addition to the five EF factor scales, the BDEFS-CA includes a screening scale, ADHD-EF Index, devised to identify children at risk for ADHD. The present study examined the ability of ADHD-EF Index to identify ADHD in a sample of children with a known ADHD diagnosis and a neurotypical group.

Participants and Methods: Sample comprised 66 children $(\mathrm{ADHD}=38$, Control=29), aged 8-15 years. Children referred to the study were assigned to the ADHD group if they were diagnosed with ADHD by a physician or psychologist in the community and had a T-score on the Conners 3P DSM-IV-TR Inattentive and/or DSM-IV-TR Hyperactive subscale(s) that was greater than or equal to 70. The accuracy of the ADHD-EF Index scale in classifying children in the ADHD group and in the neurotypical group was conducted by receiver-operating characteristic (ROC) curve analysis and an examination of utility estimates. Results: Classification accuracy using the ADHD-EF Index score was excellent (AUC=0.977; p<.001, SE +/- .014). Using the Youden Index, the maximum combined sensitivity and specificity was $95 \%$ and $89 \%$, respectively, corresponding to an ADHD-EF Index percentile rank of 76.5

Conclusions: Results provide preliminary evidence of the clinical utility of ADHD-EF Index in identifying children with ADHD. Replication comparing children with ADHD to clinical samples is necessary to extend generalizability.

Correspondence: Joseph E. Casey, Psychology, University of Windsor, 401 Sunset Avenue, Windsor, ON N9B 3P4, Canada. E-mail: jecasey@ uwindsor.ca

L. FERENC, A.E. PRITCHARD, H. SCHNEIDER \& M. MAHONE. Construct Validity of the BRIEF-P for Teacher-Rated Executive Functions.

Objective: The BRIEF-P Teacher version shows good internal consistency and convergent validity among typically developing children (Gioia et al., 2003). We examined the measure's construct validity among preschoolers with ADHD, as it is often used in clinical assessment of these children.

Participants and Methods: Children 3-5 years ( $64 \%$ male) were assigned as ADHD $(n=70)$ or control $(n=63)$ based on diagnostic interview and parent ratings. The multitrait-multimethod matrix was used to assess convergent and discriminant validity of BRIEF-P Teacher Inhibit subscale scores in comparison to: Conners' Teacher Rating Scale-Revised: DSM-IV Hyperactive/Impulsive (MonoTrait-MonoMethod; MT-MM), BASC-2-Preschool Teacher Internalizing Composite (HeteroTrait-MonoMethod; HT-MM), CANTAB Stop Signal (MonoTrait-HeteroMethod; MT-HM), and Peabody Picture Vocabulary Test-IV (HeteroTrait-HeteroMethod; HT-HM) scores.

Results: Within the ADHD group, MT-MM (r=.94, p<.0001) and MT-HM ( $\mathrm{r}=.48, \mathrm{p}=.008)$ correlations were significant; HT-MM $(\mathrm{r}=.28$. $\mathrm{p}=.084)$ and HT-HM $(\mathrm{r}=-.16, \mathrm{p}=.320)$ correlations were non-significant. MT-MM correlation was significantly higher than HT-MM $(\mathrm{z}=6.33, \mathrm{p}<.001)$ and HT-HM $(\mathrm{z}=8.36, \mathrm{p}<.001)$. MT-HM correlation was significantly higher than HT-HM $(z=2.67, p=.008)$, but not different from HT-MM $(\mathrm{z}=0.09, \mathrm{p}=.36)$.

Among controls, MT-MM correlation $(r=.84, p<.001)$ was significant, but MT-HM was not $(\mathrm{r}=.46, \mathrm{p}=.133)$. HT-MM $(\mathrm{r}=.31, \mathrm{p}=.131)$ and HT-HM $(r=.12, p=.536)$ correlations were small and non-significant. MT-MM correlation was significantly higher than both HT-MM $(\mathrm{z}=3.34$, $\mathrm{p}<.001)$ and HT-HM $(\mathrm{z}=4.20, \mathrm{p}<.001)$. MT-HM correlation was not significantly different than either the HT-HM $(\mathrm{z}=0.98, \mathrm{p}=.327)$ or HT-MM $(\mathrm{z}=0.44, \mathrm{p}=.66)$.
Conclusions: Results suggest good convergent and discriminant validity of the Inhibit subscale of BRIEF-P Teacher version among the ADHD group, with moderate convergent but good discriminant validity among controls.

Correspondence: Lisa Ferenc, MA, BS, AA, Neuropsychology, Kennedy Krieger Institute, 1750 E. Fairmount Ave, 3rd floor, Baltimore, MD 21231. E-mail:ferenc@kennedykrieger.org

N. HERTING, T. ERTELT, T. PETROS \& C. YEAGER. Neuropsychological, Academic Achievement, and Behavioral Differences Between the CBCL Pediatric Bipolar Profile, ADHD, and Emotionally Normal Controls.

Objective: The diagnosis of bipolar disorders in children and adolescents has increased substantially in recent years. The goal of the present study was to identify differences in neuropsychological functioning, as well academic achievement and emotional/behavior functioning, in children identified as having a bipolar disorder or attention-deficit/ hyperactivity disorder (ADHD) and emotionally normal children using the CBCL. The present study hypothesized that children with bipolar disorders would experience impaired performance on executive functioning tasks compared to the other groups and have higher reports of problematic behaviors.

Participants and Methods: A profile on the Child Behavior Checklist (CBCL), called the CBCL Pediatric Bipolar Disorder (CBCL-PBD) profile, has been identified as an efficient proxy method of screening children for bipolar disorders, and strong diagnostic accuracy has been reported when using the CBCL-PBD profile. The present study examined children between six and twelve years old who were positive for the CBCL-PBD and positive for a diagnosis of ADHD (CBCL-PBD + ADHD group, $n=17$ ), negative for the CBCL-PBD and positive for a diagnosis of ADHD (ADHD group, $n=44$ ), and negative for both the CBCL-PBD and an ADHD diagnosis (control group, $n=10$ ) on measures of problem solving, set shifting, sustained attention, alternating attention, verbal memory, visuospatial memory, academic achievement, and emotional/ behavioral functioning.

Results: No significant differences were observed between groups on measures of neuropsychological functioning; however, notable differences in academic achievement and emotional/behavioral functioning were observed.

Conclusions: The lack of significant neuropsychological differences for the CBCL-PBD group suggests the profile may not identify full-threshold cases of bipolar disorders. The importance of considering neuropsychological functioning in diagnosis is addressed.

Correspondence: Nicola A Herting, Ph.D. Clinical Psychology Graduate Student, Psychology, University of North Dakota, 5000 Curran Court Apt 308, Grand Forks, ND 58201.E-mail: nicola.rodwell@my.und.edu

S. SHIH, L. SEPETA, P.K. ISQUITH \& M. BERL. Everyday Executive functioning in Focal Pediatric Epilepsy on the Revised BRIEF.

Objective: We examine parallel-test reliability of the Behavior Rating Inventory of Executive Function (BRIEF) with a concise, revised version. Metacognitive aspects of executive functioning $(\mathrm{EF})$ on the BRIEF have been the most impaired but also included children with $\mathrm{IQ}<70$ or generalized epilepsy (MacAllister et. al, 2012; Parrish et. al., 2007; Slick et. al., 2006). We compare children who have had focal epilepsy (EPI) and IQ>70 to typically developing (TD) controls on both versions of the BRIEF.

Participants and Methods: Parent ratings on the two BRIEF versions were compared in a pediatric epilepsy group ( $\mathrm{n}=88$; mean age: 9.4 ; 48 male; mean FSIQ=104; mean duration 3.9 years) using a repeated measures MANOVA. Scores were categorized and compared via Cohen's kappa coefficient. Patients and TD controls (n=85; age: 9.6; 49 male) were compared with MANOVA.

Results: The two BRIEF versions had small (within 3 T-score points) but reliable $(\mathrm{p}<.05)$ differences for 3 (Inhibit, Working Memorv, Global 
Executive Composite (GEC)) of 11 scores. Agreement of clinically elevated T-scores $(\geq 65)$ ranged from kappa of .64 to .90. Patients were significantly higher on all scales, except for Inhibit and Organization of Materials. Working Memory demonstrated the largest effect (partial eta squared=.13). Approximately $30 \%$ of patients had clinically elevated Working Memory, Shift, and Task-Monitor scores versus $<6 \%$ of controls.

Conclusions: Scores and rates of clinical elevation in pediatric epilepsy were similar between the concise, revised BRIEF and the original BRIEF, encouraging future utilization. Excluding low IQ and generalized epilepsy patients, we found less inhibitory control difficulties, but working memory remains as the most problematic as rated by parents. Correspondence: Madison Berl, PhD, Children's National Medical Center, 111 Michigan Avenue NW, Washington, DC 20010. E-mail: mberl@childrensnational.org.

R.E. RIEGER, S.J. ERICKSON, N.C. MOSS, P.C. MACLEAN, R.K. OHLS \& J.R. LOWE. Associations among Maternal Depressive Symptoms, Interactive Behaviors, and Child Development in Very Low Birth Weight and Normal Birth Weight Preschoolers.

Objective: Sensitive and responsive parenting predicts better executive functioning (EF) in children. Risk factors such as low parental education and depression have been shown to predict child adjustment. All children are sensitive to family environment, but those born very low birth weight $(V L B W)$ are impacted more by quality of parenting. We examined relationships between maternal depressive symptoms and interactive behaviors with IQ and EF in VLBW and normal birth weight (NBW) preschoolers.

Participants and Methods: 54 VLBW ( $\leq 1500$ grams) and 33 NBW 3 to 4.5 years were evaluated using the WPPSI-III (PIQ and VIQ). EF was measured using Gift Delay and Bear Dragon. Videotaped play was coded for maternal intrusiveness and affect and child task persistence and emotion regulation with the Caregiver-Child Affect, Responsiveness and Engagement Scale (C-CARES). Mothers were assessed for depression (BDI) and education.

Results: The NBW group had significantly higher maternal education level $(p<.001)$, VIQ ( $p<.001)$, PIQ ( $p<.001)$, Gift Delay $(p=.002)$, and Bear Dragon $(\mathrm{p}<.001)$. No differences were found between groups for BDI or C-CARES.

For VLBW group: after controlling for illness severity, VIQ was correlated with Bear Dragon $(\mathrm{r}=.279, \mathrm{p}=.047)$ and child task persistence $(\mathrm{r}=.299, \mathrm{p}=.033)$, controlling for maternal education, child test age, and illness severity; there was a significant relationship between BDI and Gift Delay ( $\mathrm{r}=-.352, \mathrm{p}=.011)$; and negative affect was correlated with child emotion regulation $(\mathrm{r}=-.523, \mathrm{p}=.001)$ and child task persistence $(\mathrm{r}=-.513, \mathrm{p}=.001)$. Maternal intrusiveness correlated with VIQ $(\mathrm{r}=-.323, \mathrm{p}=.021)$.

For NBW group: maternal negative affect was correlated with child emotion regulation $(\mathrm{r}=-.425, \mathrm{p}=.015)$.

Conclusions: Maternal depression and interactive style correlated with lower IQ and EF scores in VLBW children. The association between maternal negative affect and child emotion regulation also supports this relationship. Future directions include investigating meditational models to explain relationships.

Correspondence: Rebecca E. Rieger, PhD, Psychology, University of New Mexico, 1510 A Gold Ave SE, Albuquerque, NM 87106. E-mail: rerieger@unm.edu

K.T. ERICKSON, J. WHITNEY, R. LIBOVE, K. CHANG, A. HARDAN \& J. PHILLIPS. A Comparison of Social Cognitive Deficits in Children with Autism Spectrum Disorder and At-Risk for Pediatric Bipolar Disorder.

Objective: Autism Spectrum Disorder (ASD) and Pediatric Bipolar Disorder (PBD) are both severely functionally impairing and chronic conditions for which early detection and treatment is correlated with more positive outcomes. The domain of social cognition has begun to garner attention for both populations, yet no studies to date have compared the two groups. We compared children with ASD and at-risk for PBD to healthy controls and siblings of children with ASD, to assess for differences in social cognition.

Participants and Methods: 136 children aged 8-12 with ASD ( $n=33)$, at-risk for PBD (n=27), siblings of children with ASD $(n=27)$ and healthy controls $(n=49)$ were administered the Social Perception subtests of the NEPSY-II (i.e. Theory of Mind [TOM] \& Affect Recognition [AR]), and their parents filled out the Social Responsiveness Scale (SRS). Data were analyzed using ANOVA for each outcome measure. Post-hoc analyses were performed for each outcome measure using Tukey HSD.

Results: For both AR and TOM, analysis revealed that the groups differed significantly with respect to their score (AR: $p=.001$; TOM: $\mathrm{p}=.031$ ). Post-hoc analyses revealed that the ASD group performed significantly worse on these measures than all other groups. On the SRS, significant group differences were found $(p<.001)$, with post-hoc analysis revealing that parents of children with ASD and PBD rated them significantly worse than siblings and healthy controls.

Conclusions: Results indicate children with ASD perform significantly worse than all other groups on objective tasks, providing evidence that the NEPSY-II is a good measure for differentiating between ASD and PBD. However, parents of children with ASD and PBD rated them lower than other groups on their social skills, highlighting the difference between social cognition and social interaction. Results also emphasize the need for better measures of social cognition across the lifespan.

Correspondence: Kalen T. Erickson, Master of Science, Psychology, PGSP-Stanford Psy.D. Consortium, 15 Jacinto Lane, South San Francisco, CA 94080.E-mail: kerickson@paloaltou.edu

B. BUCHHOLZ, S. AYLWARD, T. CARRATHERS, A.A. GRETENCORD \& J. DORFLINGER. Verbal Memory Evaluation by the Immediate Post-Concussion Assessment and Cognitive Testing (ImPACT) and the California Verbal Learning Test Children's Version (CVLT-C) in Patients Presenting With Concussion.

Objective: Brief computerized neurocognitive testing is frequently used for assessment of sport-related concussions in youth. Effective evaluation techniques are imperative for ensuring players' safety and informing return-to-play recommendations. The Immediate Post-Concussion Assessment and Cognitive Testing (ImPACT), has become widely used by schools, coaches, and clinicians. The purpose of this study was to examine the validity of the ImPACT Verbal Memory Composite (ImPACT $\mathrm{VM}$ ), a frequently used measure of verbal memory in concussed patients. This was accomplished by investigating the relationship between scores on the ImPACT VM to a widely accepted clinical neuropsychological measure, the California Verbal Learning Test (CVLT-C).

Participants and Methods: Pediatric patients $(n=51)$, aged 9 to 17-years-old $(\mathrm{M}=12.37, \mathrm{SD}=1.56)$ underwent neuropsychological evaluation after sustaining a concussion. Bivariate correlations were conducted to determine whether or not a relationship exists between verbal memory as measured by the CVLT-C and by the ImPACT VM composite, as they are intended to measure the same neuropsychological construct.

Results: No relationship was observed between the ImPACT VM index and the CVLT-C Total Score $[\mathrm{r}(49)=-.158, \mathrm{p}=.270]$. However, significant relationships were observed between ImPACT VM and CVLT-C Recognition scores, $[\mathrm{r}(47)=.427, \mathrm{p}=.002]$, and the ImPACT VM and CVLT-C Discrimination scores, $[\mathrm{r}(48)=.380, \mathrm{p}=.006]$.

Conclusions: CVLT-C Total Score is widely used by clinicians to assess verbal memory. No relationship was observed between the ImPACT VM composite and the CVLT-C Total Score. Moderate correlations were observed between ImPACT VM and CVLT-C recognition and discrimination scores, which may result from the format of the ImPACT VM composite more closely resembling recognition rather than spontaneous word-retrieval tasks. Caution is recommended when interpreting ImPACT Verbal Memory scores. 
Correspondence: Blythe Buchholz, B.A., Aleixan Neurosciences Institute, 1555 Barrington Road, Chicago, IL 60169. E-mail: blythebuchholz@ gmail.com

K.S. WALSH, K.K. HARDY \& P.K. ISQUITH. Executive Function Profiles in Children with Neurofibromatosis Type 1 Compared with Healthy Children on the Behavior Rating Inventory of Executive Functions-2.

Objective: NF1 is a disease with well-documented neuropsychological morbidity, including a high prevalence of ADHD and executive dysfunction (EF). We aim to examine the psychometrics of the parent rated BRIEF-2 specific to NF1, and group differences in EF profiles.

Participants and Methods: Data includes children with NF1 (age $\mathrm{M}=10, \mathrm{SD}=3.10 ; 53 \%$ male) age/gender matched healthy children (HC; $\mathrm{N}=96$ total). Groups were compared on the Parent BRIEF-2 via repeated measures MANOVA. Stepwise Discriminant analysis was used to analyze EF predictors of group membership and provide information on the specificity and sensitivity of this updated measure. Chi square analysis was used to examine the differences in rates of clinical elevation between groups.

Results: The BRIEF-2 correctly classified $77 \%$ of the sample to group (NF1 v HC). Discriminant analysis showed that the Plan/Organize scale predicted group membership $[\mathrm{F}(1,92)=32.54, \mathrm{p}=.000]$. There was a significant group effect $[\mathrm{F}(1,92)=30.46, \mathrm{p}=.000, \mathrm{Eta} 2=.249)$ and group by $E F$ interaction $[F=2.63, p=.020$, Eta2=.028], with significant differences across all domains. The NF1 group showed a significantly higher prevalence (19-45\%) of clinically elevated EF deficits than HC across nearly all domains $(\mathrm{p}<.03)$.

Conclusions: The updated parent-rated BRIEF-2 demonstrates good sensitivity and specificity (validity) in children with NF1. Greater deficits with strategic planning and organizational abilities on this measure appear to differentiate children with NF1 from healthy peers. Consistent with prior research, children with NF1 exhibit significantly greater executive dysfunction across most areas of EF compared to peers, and there is a notably higher prevalence of functionally impairing deficits for children with NF1, especially in the areas of cognitive self-monitoring and working memory.

Correspondence: Karin S. Walsh, PsyD, Neuropsychology, Children's National Health System, 111 Michigan Avenue NW, Washington, DC 20010.E-mail:kwalsh@cnmc.org

K.S. WALSH, K.K. HARDY \& P.K. ISQUITH. Executive Function Profiles in Pediatric Cancer Survivors Compared with Healthy Children on the Behavior Rating Inventory of Executive Functions-2.

Objective: Neuropsychological morbidity in pediatric cancer survivors is a well-documented consequence of the disease and its treatments, with the development of executive functions (EF) being highly vulnerable to disruption. We aim to examine the psychometrics of the BRIEF-2 in cancer survivors, and group differences in EF profiles.

Participants and Methods: Data includes survivors of brain tumor (BT, n=52) and leukemia (ALL, n=46) age/gender matched to healthy children (HC; $n=98$ ). Overall mean age is 11 years, with $57 \%$ males. Groups were compared on the Parent BRIEF-2 via repeated measures MANOVA. Discriminant analysis was used to analyze EF predictors of group membership and the specificity and sensitivity of this updated measure. Chi-square analysis was used to examine the differences in rates of clinical elevation between groups.

Results: The BRIEF-2 correctly classified $>80 \%$ and between $61-76 \%$ of the sample on parent and teacher forms, respectively. Discriminant analysis showed that the Working Memory, Inhibit, and Emotional Control predicted group membership $[p=.000]$. There was a significant group effect $[\mathrm{F}(2,193)=6.97, \mathrm{p}=.001, \mathrm{Eta} 2=.067)$ and a group by $\mathrm{EF}$ interaction $[\mathrm{F}=7.76, \mathrm{p}=.000$, Eta2 $=.074]$, with significant differences across all domains. The BT and ALL groups showed a significantly higher prevalence of EF deficits than HC across nearly all domains by both raters $(\mathrm{p}<.04)$.

Conclusions: The BRIEF-2 demonstrates good sensitivity and specificity in cancer survivors. Greater working memory and emotional regulation deficits, and better inhibitory control appear to differentiate survivors from HC. Survivors exhibit significantly greater executive dysfunction across most areas of EF compared to peers, and there is a notably higher prevalence of functionally impairing deficits for survivors, primarily in metacognitive domains.

Correspondence: Karin S. Walsh, PsyD, Neuropsychology, Children's National Health System, 111 Michigan Avenue NW, Washington, DC 20010.E-mail:kwalsh@cnmc.org

\section{MESSER. Development and Reliability of a New Academic Achievement Battery.}

Objective: The development and reliability of a new academic achievement battery will be discussed.

Participants and Methods: The Academic Achievement Battery (AAB) is a newly developed academic achievement battery that covers a wide range of achievement domains that are aligned with the Individuals with Disabilities Education Act (IDEA, 2004). The AAB Comprehensive Form assesses these constructs across 15 subtests, each of which addresses a specific function within a broader area of achievement, while the AAB Screening Form measure four specific academic construction: Letter/Word Reading, Spelling, Written Composition, and Mathematical Calculation. The conceptualization of each subtest, a review of the pilot $(\mathrm{n}=133)$, refinement $(\mathrm{n}=280)$, and standardization $(\mathrm{n}=1,274$ age-based, $n=1,447$ grade-based) studies, and a review of the reliability data including internal consistency, standard error of measurement, test-retest reliability, inter-scorer reliability, will be discussed.

Results: The reliability results provide strong evidence that the $A A B$ Comprehensive Form and Screening Form are reliable instruments. Internal consistency reliability ranged from .77 to .98 for the Comprehensive Form and .89 to .98 for the Screening Form across the subtests and composite scores. The test retest $(\mathrm{n}=147)$ reliabilities were found to range from .74-.95 for the Comprehensive Form and .83-.95 for the Screening Form across over a two week period.

Conclusions: A thorough development processes resulted in the development of a highly reliable comprehensive and screening assessment of academic achievement.

Correspondence: Melissa Messer, MHS, Psychological Assessment Resources, 16204 N. Florida Ave, Lutz,FL 33549.E-mail: mmesser@ parinc.com

M. MESSER, J. GREENE, P.K. ISQUITH \& G.A. GIOIA. Development and Reliability of a Revised Behavior Rating Inventory of Executive Function (BRIEF).

Objective: We describe the development and reliability of a revised BRIEF.

Participants and Methods: Factor analysis, item-total correlations, and item behavior were examined in over 5000 normative and clinical BRIEF forms. Weaker items were removed; no new clinical items were added, and a third validity scale was developed. Reliabilities were examined in new standardization samples ( $\mathrm{N}=1,400$ Parent Version; $\mathrm{N}=1,400$ Teacher Version; N=803 Self Report Version). Test-retest reliabilities were examined in a subset ( $\mathrm{n}=163$ Parent; $\mathrm{n}=173$ Teacher; $\mathrm{n}=190$ Self). Results: The iterative development process yielded parallel 63-item Parent and Teacher forms and a 55-item Self-Report form with three factors: Behavior Regulation comprised of Inhibit and Self-Monitor; Emotion Regulation including Shift and Emotional Control; and Cognitive Regulation with Initiate, Working Memory, Plan/Organize, Task Monitor and Organization of Materials scales. The Initiate and Organization of Materials scales were replaced by a Task Completion scale on the Self-Report. Internal consistency reliabilities ranged from .80 to .98 across forms, comparable to original BRIEF reliabilities. The median 
test-retest index score reliabilities across the three forms ranged from .77 to .83 over a two week period, again comparable to the original BRIEF. Conclusions: A thorough development processes resulted in a more concise revised BRIEF with increased parallelism, three embedded validity scales, and a consistent three factor structure while maintaining good internal consistency and temporal stability based on a large stratified standardization sample.

Correspondence: Peter K. Isquith, Ph.D., Psychiatry, Geisel School of Medicine at Dartmouth, 221 US Route 5S, Unit 15, Norwich, VT 05055.

E-mail: peter.isquith@gmail.com

A.R. SNYDER, B. ASKEN, J. ZAREMSKI, S. SMITH \& R.M. BAUER. Normative Values and the Impact of Demographics at Baseline for the Sport Concussion Assessment Tool (SCAT3) in Teenagers.

Objective: Baseline concussion assessment provides an efficient method for obtaining important demographic and clinical variables that can be used by physicians to tailor post-injury diagnostic and management decisions. In the event that an individual baseline test is unavailable, normative values become important as a means for comparison to expected values, allowing the identification of impaired scores after injury. The Sport Concussion Assessment Tool 2nd Edition (SCAT2) was recently revised and updated to the Sport Concussion Assessment Tool, Third Edition (SCAT3). It includes an expanded demographic section accompanied by modifications to the overall scoring scheme, but updated norms and information regarding the influence of added demographic variables are not yet available. The purpose of this study is to present normative values for the SCAT3, and also to provide an analysis of the impact of relevant demographic variables on baseline SCAT3 performance.

Participants and Methods: A community-based approach was implemented to collect cross-sectional baseline data on the SCAT3 from 335 youth athletes ages 13-18.

Results: Statistical analyses included parametric and non-parametric t-tests and ANOVAs comparing age, sex, and other demographic variables on component scores of the SCAT3 (self-reported symptom score and severity, cognition, and postural stability). Results demonstrated that there was no effect of age on any of the component scores. Females scored significantly better than males on the cognitive component only. Other demographic variables, such as past history of concussion, had no effect on baseline performance across SCAT3 components with the exception of psychiatric history, which was associated with higher ratings on symptom severity.

Conclusions: Similar to previous findings in the same age group, this study presents the SCAT3 as a useful tool for characterizing baseline performance in teenagers, but emphasizes that certain demographic variables need consideration when interpreting results.

Correspondence: Aliyah R. Snyder, B.S., Clinical and Health Psychology, University of Florida, 1225 Center Drive, Room 3151, Gainesville, FL 32611. E-mail: aliyahsnyder@phhp.ufl.edu

\section{Dementia (Alzheimer's)}

D. WALD \& R.M. BAUER. Spatial Disorientation in Mixed Alzheimer's Disease and Vascular Dementia.

Objective: To outline an interesting neurocognitive profile of mixed Alzheimer's disease (AD) and vascular dementia. We present a case of a 73-year-old, Caucasian male with a one year history of disorientation, memory problems, poor spatial awareness, and problems multi-tasking. Symptoms began with veering off the road when driving and misplacing items, but progressed to a point in which he frequently gets lost in his home. Patient experienced a sudden decline in spatial processing and gait disturbance. Ophthalmological evaluation revealed left homonymous hemianopia. The patient's wife provides assistance with all activities of daily living. CT study showed extensive periventricular subcortical white matter demyelination often seen in the setting of hypertension, diabetes, or hyperlipidemia. CT was also notable for an enhancing lesion in the right temporal lobe, likely vascular.

Participants and Methods: A comprehensive neuropsychological examination was administered.

Results: Estimated premorbid abilities were average. Results demonstrated impairment across all measures of spatial cognition requiring sustained visual attention. The patient had deficits in episodic memory for tasks requiring planning and organization and also in episodic memory for information with little executive demand. Visual memory was more impaired than verbal memory. Patient had difficulty on tasks of executive functioning and was remarkably perseverative. Mild difficulty was observed in working memory and confrontation naming.

Conclusions: This pattern of testing coupled with significant cardiovascular risk factors (cerebral artery occlusion with cerebral infarction, atrial fibrillation, heart disease) is most consistent with a diagnosis of mixed AD and vascular dementia. The case will be discussed in the context of a differential diagnosis involving syndromes of spatial disorientation, posterior cortical atrophy, and other dementia syndromes affecting spatial cognition.

Correspondence: Danielle Wald, Psy.D., University of Florida, $1316 \mathrm{NW}$ 102thWay, Gainesville,FL 32606.E-mail:d_wald593@hotmail.com

Y. TSUI-CaldWell, Z. CHRIstensen, C.E. PERry, T. SCHMIDT, S. LIN, T. JOHNSON, E.D. BIGLER, J.T. TSCHANZ \&. M.C. NORTON. Schelten et al. Ratings for Mesial Temporal Lobe Atrophy in the Cache County Study on Memory Health and Aging. Objective: The Cache County study on memory and aging (CCSMA) is an ongoing population based study. Because MRI clinical ratings require no special image analysis tools, are quick and economical to perform, they remain important in behavioral neurology and clinical neuropsychology. The Schelten et al. (1992) rating method for identifying mesial temporal lobe atrophy (MTA) has been widely used, but not previously in the CCMSA. The present study summarizes MTA in the CCMA sample in relation to general cognitive ability [Mini-Mental State Examination (MMSE)] and list-learning memory from the CERAD neuropsychological test battery.

Participants and Methods: Cognitive assessment and MRI studies were performed on 414 individuals $\geq 65$ years old with possible dementia as well as a 22 healthy controls. Using Schelten et al. criteria, three regions of interest (ROIs) were examined: (1) temporal horn (TH) width, (2) choroid fissure (CF) and (3) hippocampal (Hipp) height. Head and body size differences were adjusted for by an intracranial width (IW) measurement.

Results: Controls and those with possible dementia differed significantly on all measures. Significant negative CF correlations ( $r=-.32$, $\mathrm{p}<.05$ ) with MMSE scores were observed. Hipp height was positively related to CERAD List Learning $(\mathrm{r}=.25, \mathrm{p}<.05)$ and MMSE performance $(\mathrm{r}=.26, \mathrm{p}<.05)$ as was wider IW $(\mathrm{r}=.11, \mathrm{p}<.05)$. TH did not significantly relate to MMSE or list-learning memory.

Conclusions: Atrophy within mesial temporal lobe ROIs can be readily identified using the Schelten et al. rating scales, with results showing the expected relations between level of mesial temporal lobe atrophy and cognition in the CCMA sample.

Correspondence: Yoko H. Tsui-Caldwell, Ph.D, Clinical Psychology, Brigham Young University, 500N 541E Apt 23, Provo, UT 84606. E-mail: stsuiyoko@gmail.com

\section{J.M. GRABYAN, P. MASSMAN \& R. DOODY. Enhancing Prediction of Longitudinal Decline in Instrumental Activities of Daily Living in Alzheimer's Patients Using Functional and Cognitive Pre-} progression Estimates.

Objective: Appropriate planning for the future functional decline of patients with Alzheimer's disease (AD) is very important. An easy-toutilize, reliable method for predicting progression of deficits in instrumental activities of daily living (IADL) would have great clinical utility. Previous work demonstrated that an estimate of IADL decline prior to 
the patient's first assessment shows promising ability to predict subsequent IADL decline. It was hypothesized that adding a similar estimate of prior cognitive decline would enhance the model's predictive ability. Participants and Methods: Subjects were 785 patients with a probable $\mathrm{AD}$ diagnosis enrolled in the Baylor College of Medicine Alzheimer's Disease and Memory Disorders research database with a baseline visit and at least one annual follow-up (up to 13 total). Lawton's IADL scale scores were obtained based on informant report. Estimations of prior illness duration were made using standardized methods. Rates of pre-progression IADL and MMSE decline were calculated and used to predict IADL change over time (IADL at baseline - expected 'normal' IADL / estimated duration; MMSE at baseline - 30 / estimated duration).

Results: Mixed effects regression analyses revealed that the IADL pre-progression estimate significantly predicted longitudinal decline of IADL scores ( $p<0.001)$, even when other significant covariates available at baseline (age, gender, baseline MMSE scores) were included in the predictive model. The addition of the MMSE pre-progression estimate $(p<0.001)$ greatly enhanced model fit.

Conclusions: Accurately predicting the rate of decline in functional abilities can be of great utility for clinicians, patients, and their family members as it would allow them to better prepare for the changes to come and the timing of accommodations. The present method incorporating both IADL and MMSE pre-progression estimates appears to be a valid tool in the pursuit of this goal.

Correspondence: Jonathan M. Grabyan, M.A., University of Houston, 7490 Brompton, Apt 279, Houston, TX 77025. E-mail: jgrabyan@ gmail.com

T.J. SMITH, H. OH, L.R. WEISS, A.J. ALFINI, K. REITER, K.A. NIELSON \& J. SMITH. Effects of a 12-week Exercise Intervention on Default Mode Network Connectivity in MCI and Healthy Elders.

Objective: It is important to identify effective treatments to improve brain function in Mild Cognitive Impairment (MCI), an early stage of Alzheimer's Disease (AD). Cortical regions of the brain's Default Mode Network (DMN) are preferentially vulnerable to amyloid plaque deposition in MCI, which may hasten $\mathrm{AD}$ pathogenesis and disrupt functional connectivity. We investigated the effects of a 12 -week treadmill walking intervention on DMN functional connectivity in MCI and healthy elders. Participants and Methods: Sixteen MCI and 16 healthy controls, ages 60-88, engaged in a supervised 12 -week treadmill walking exercise intervention. Functional MRI (fMRI) was acquired at rest; functional connectivity analysis used the left precuneus as a seed.

Results: A 2(Group)x2(Time) analysis revealed significant interactions $(\mathrm{p}<0.01)$. The MCI group showed increased connectivity in right posterior cingulate and decreased connectivity in bilateral insula, and left frontal and middle gyri. Controls demonstrated decreased connectivity in right superior frontal and angular gyri, and right posterior cingulate. At baseline, the MCI group had greater connectivity in bilateral insula, right cingulate, and left inferior parietal lobule. The only group difference post-intervention was greater connectivity in the MCI group in the left cingulate.

Conclusions: These findings suggest 12 -weeks of exercise resulted in DMN functional connectivity pattern changes in both groups. The fact that the MCI group connectivity patterns were more similar to the healthy controls after the exercise intervention lends support to the use of exercise as a therapeutic intervention in MCI.

Correspondence: Theresa J. Smith, Kinesiology, University of Maryland, Department of Kinesiology, School of Public Health, The University of Maryland, College Park,MD 20742.E-mail:tsmith27@umd.edu

C.L. MARREIRO, S. TOMASZEWSKI FARIAS, D. MUNGAS \& B. REED. The Effect of Depression and Behavioral Disturbance on Cognitive Change in Diverse Older Persons.

Objective: The purpose of this study was to evaluate how self-reported depression and informant-reported behavioral disturbance relate to longitudinal trajectories of cognitive decline and how these relationships might vary depending on initial age and diagnostic status.

Participants and Methods: Data are from 288 diverse older adults (mean age 78) enrolled in a longitudinal study of cognition (mean number of annual visits $=5.5$ ). The sample was $25 \%$ Black, $25 \%$ Hispanic, 50\% non-Hispanic White; 48\% cognitively normal at baseline, $36 \%$ MCI, and $16 \%$ demented. Self-report of depressive symptoms was measured with the 15-item Geriatric Depression Scale (GDS) and informant-report of behavioral disturbance was measured through the Neuropsychiatric Inventory (NPI). Mixed - effects regression analysis was used to measure baseline status and longitudinal change in psychometrically matched measures of Episodic Memory, Executive functioning, and Semantic Memory.

Results: In models that controlled for demographic factors, diagnostic status (normal vs. MCI or demented) and the APOE 4 allele, presence of depression was associated with lower baseline Semantic Memory. The presence of behavior disturbance on the NPI was associated with lower baseline Executive functioning. Main effects for depression and behavior disturbance on longitudinal change were not significant. However, a set of significant interaction terms suggest that relationships between GDS and rate of decline is dependent on diagnosis and age for both executive functions and semantic memory. Similarly, interaction terms suggest that the association between NPI and rate of decline is dependent on age for episodic memory, executive functions, and semantic memory. Conclusions: The findings indicate that impact of depression and behavioral disturbance on rate of cognitive decline may vary depending on age and diagnostic status.

Correspondence: Catherine L. Marreiro, Ph.D., Neurology, UC Davis, 3306 Partridge Ave, Oakland, CA 94605.E-mail: clmarreiro@ucdavis. edu

A. GURNANI \& B.E. GAVETT. The Differential Effects of Alzheimer's Disease on Cognitive Performance: A Meta Analysis. Objective: Previously, we have presented results on the effect of definite Alzheimer's disease (AD) pathology on individual cognitive tests. AD pathology caused substantial decrements in performance on tests of verbal category fluency and list recall at immediate and delayed intervals, relative to controls. The present study expanded on previous research by examining how $\mathrm{AD}$ pathology affects cognitive performance within larger domains of ability.

Participants and Methods: A comprehensive literature search yielded 6 studies that met the inclusion criteria for the present meta-analysis; these studies together comprised 222 decedents with $\mathrm{AD}$ and 434 normal controls. Weighted Cohen's d effect size estimates and confidence intervals were calculated for the cognitive domains of memory, language, visuospatial skills, executive functioning, and mental status. Weighted least squares multiple regression was used to examine the effects of moderators (age, education, and interval from last assessment to death) on effect size estimates.

Results: Results revealed that the effect of having neuropathologically confirmed $\mathrm{AD}$ versus normal aging significantly lowered performance on the memory, $\mathrm{d}=-0.64,95 \%$ CI [-0.96, -0.31$]$, language, $\mathrm{d}=-0.64$, $95 \%$ CI $[-1.09,-0.20]$, visuospatial, $d=-0.52,95 \%$ CI $[-0.99,-0.04]$, and executive functioning domains, $\mathrm{d}=-0.66,95 \%$ CI $[-0.95,-0.37]$. The effect of $\mathrm{AD}$ on mental status was large but non-significant due to imprecision of the estimate, $\mathrm{d}=-0.94,95 \%$ CI $[-2.06,0.18]$. With the exception of mental status, education was a significant moderator of cognitive performance in all domains $(\mathrm{p} \leq .05)$.

Conclusions: Thus, the pathological features of AD eventually result in synaptic degeneration, neuronal loss, and overall brain atrophy causing changes in all domains of cognitive functioning. The moderating effects of education on performance in all domains indicate that cognitive reserve possibly mediates the start and rate of decline of cognitive functioning in $\mathrm{AD}$. 
Correspondence: Ashita Gurnani, University of Colorado at Colorado Springs, 1510 Dublin Blvd, Apt 160, Colorado Springs, CO 80918. E-mail:agurnani@uccs.edu

C.L. SANDERS, H. WENGREEN, S. SCHWARTZ, S. BEHRENS, C. CORCORAN, C.G. LYKETSOS \& J.T. TSCHANZ. Nutritional Status and Neuropsychological Functioning in Persons with Dementia: The Cache County Dementia Progression Study.

Objective: Previous studies have found nutritional status to predict better functional and cognitive ability in dementia. The purpose of the current study is to investigate the relationship between nutritional status and progression of neuropsychological impairment in a US sample of persons with dementia.

Participants and Methods: 243 persons with dementia (71.6\% Alzheimer's Disease, $52.3 \%$ female) were studied for up to 6 years in the population-based Cache County, UT Study. Mean (sd) age and dementia duration at baseline was 85.6 (5.4) and 3.4 (1.9) years, respectively. CERAD neuropsychological test battery (Word List Memory, praxis drawing and recognition, verbal fluency, and Boston Naming Test (30item)) was administered annually. Nutritional status was assessed using the Mini Nutritional Assessment (mMNA-revised) and scored (range 0-22). Linear mixed effects models examined the association between mMNA (time-varying) and each neuropsychological measure and rate of decline over time. Covariates tested included dementia type, gender, age of dementia onset and duration, education and neuropsychiatric symptoms.

Results: With mean (sd) baseline scores of 16.57 (2.94), mMNA scores decreased .4 pts/year ( $<$.001), suggesting increasing risk for malnutrition. Higher mMNA scores were associated with better neuropsychological test scores across time in praxis $(\beta=.09)$, verbal fluency $(\beta=.32)$ and naming $(\beta=.27)$, all $p<.003$, with the inclusion of covariates. mMNA scores were not associated with Word List Memory, praxis recognition, nor with rate of decline on any measure.

Conclusions: Nutritional status is positively associated with neuropsychological overall domains of executive functioning, verbal expression, and visuospatial abilities but not with memory.

Correspondence: Chelsea L. Sanders, Bachelors, Psychology, Utah State University, 650 South 100 East \#E305, Logan, UT 84321. E-mail: chels. laurae@gmail.com

K. HEGDE \& S. JEFFERSON. Rethinking the File Drawer Problem of Null Findings: Vascular Burden Did Not Predict Dementia in a Nationally Representative Sample of Older Adults.

Objective: The association between vascular burden and dementia has been found throughout the extant literature. The current study compared three different methods for determining vascular burden to identify which best predicted dementia in a nationally representative sample of 548 older adults who did not have dementia at baseline.

Participants and Methods: Data were derived from the Aging, Demographics, and Memory Study. Participants were assessed for dementia four times over a six-year period. A 3-4 hour in-home assessment of each participant was conducted by a nurse and a neuropsychological technician. Vascular burden was assessed via the Mast approach, the National Institute of Health's Adult Treatment Panel III (NIH-ATP III) approach, and the Framingham Risk Score (FRS) approach. A consensus expert panel of neurologists, neuropsychologists, geriatric psychiatrists, and internists reviewed the data and assigned dementia diagnoses.

Results: Separate multivariate logistic regression analyses were conducted to predict dementia using each of the three proposed vascular burden scoring systems. Counter to expectations, neither the Mast approach $(\mathrm{eB}=1.18)$, the NIH-ATP III approach $(\mathrm{eB}=.90)$, nor the FRS approach $(\mathrm{eB}=1.03)$ significantly predicted dementia in this sample. As a result, a comparison of the relative predictive utility of each of these methods is not possible.

Conclusions: The current null findings suggest that a more complex relationship likely exists between vascular disease and dementia than previous findings have suggested, particularly in population-based samples. These findings also intimate that this census-derived sample may be more resilient than previous studies indicated.

Correspondence: Krupa Hegde, M.A., Psychology, Eastern Michigan University, 501 SE 2nd Street, Apt 1240, Ft. Lauderdale, FL 33301. E-mail:khegde@emich.edu

S.V. FARLEY, B.M. MIZUKI, A.C. GENESER, S.A. SPERLING, K.L. FUCHS \& C.A. MANNING. RBANS Total and Memory Scores Differentiate AD from MCI and Other Dementia Subtypes.

Objective: Comprehensive neuropsychological assessment improves diagnostic accuracy when differentiating Alzheimer's Disease (AD) from Mild Cognitive Impairment (MCI) and other dementia subtypes. However, few studies have assessed the accuracy of cognitive screening measures in predicting differential dementia diagnosis. This study investigated the diagnostic utility of the Total, Immediate and Delayed Memory, and other index scores from the Repeatable Battery for the Assessment of Neuropsychological Status (RBANS).

Participants and Methods: Following neuropsychological and neurological evaluation at a memory disorders clinic, 273 patients (age: $M$ $=75.16 ; S D=7.07$ ) were diagnosed accordingly: $84 \mathrm{AD}, 80 \mathrm{MCI}, 18$ Vascular Dementia, 55 Dementia with Mixed Alzheimer's and Vascular Etiology, 26 Parkinson's and Lewy Body Dementias, and 10 Frontotemporal Dementias. Patients' comprehensive assessments included administration of the RBANS and the Geriatric Depression Scale (GDS), among other measures.

Results: Based on logistic regression, the RBANS Total score $(89 \%$ accuracy, $p<.01)$, as well as the Immediate and Delayed Memory scores $(90 \%$ accuracy, $p<.01)$, successfully differentiated AD and MCI patients. Furthermore, the Delayed Memory score (65-100\% accuracy, $p<.01)$ was diagnostically informative when differentiating $\mathrm{AD}$ from the other dementia subtypes (e.g., AD vs. Vascular); the Total score and other index scores were not significant. Gender, age, education, and depression did not significantly moderate the results.

Conclusions: The RBANS Total and both Memory scores are useful in distinguishing AD from MCI. In addition, the Delayed Memory score is sensitive to differences between $\mathrm{AD}$ and other dementia subtypes. The results support the clinical use of multi-domain cognitive screening measures such as the RBANS for early differential diagnosis of dementia and subsequent treatment planning.

Correspondence: Stephanie V. Farley, PhD, Neurology, University of Virginia, 115-B Longwood Drive, Charlottesville, VA 22903. E-mail: scv3q@virginia.edu

A.T. FLOWERS, J. AVILA, P. LU \& J. RAZANI. Effects of Demographic and Cognitive Factors on an Observation-Based Daily Functional Test.

Objective: The Direct Assessment of Functional Status (DAFS) is an observation-based activities of daily living task (ADL) that has been shown to characterize aspects of daily functional abilities in Alzheimer's Disease (AD). The purpose of the present study was to assess how demographic and cognitive factors affect and account for DAFS test performance.

Participants and Methods: A total of 54 patients meeting NINCDSADRDA criteria for AD were recruited from medical and Veteran's Administration centers in greater Los Angeles. Participants were administered the DAFS tests which is an observation-based ADL test with subscales assessing orientation, as well as the ability to shop, manage finance, communicate, and use transportation.

Results: A series of multiple regression analyses were performed using gender, education and the Mini Mental State Exam (MMSE) as the independent variables and the 5 DAFS subscales as the dependent variable. The results revealed that all five regression models were statistically significant, with the independent variables accounting for $34 \%$ to $61 \%$ of the variability in the DAFS performance of the AD patients. On closer examination, the MMSE was the best predictor of the 3 independent variables of all 5 DAFS subscales. However, gender was also found to 
account for significant variability in DAFS transportation and financial abilities, with males outperforming females on these tasks.

Conclusions: These findings indicate that as cognitive performance worsens in $\mathrm{AD}$, so do most areas of daily functional abilities. Additionally, gender must be taken into account when interpreting DAFS results as it seems to affect circumscribed daily skills.

Correspondence: Amina T. Flowers, B.A., Psychology, California State University, Northridge, 5454 Brynhurst Avenue, Los Angeles, CA 90043. E-mail: flowersamina@gmail.com

\section{CLEM, S. PANDYA, L.H. LACRITZ \& F.L. WOON. Baseline Neuropsychiatric Symptoms and Stability of Mild Cognitive Impairment.}

Objective: We examined the relationship between severity of baseline neuropsychiatric symptoms and MCI stability at 3-years.

Participants and Methods: Data from the National Alzheimer's Coordinating Center Uniform Data Set were examined for 1,059 subjects $($ males $=556$, Mage $=74.73 \pm 8.73$, Meducation $=14.93 \pm 3.46$ years $)$ diagnosed with MCI (amnestic=893, non-amnestic=166) at initial visit who subsequently had 3 annual visits. Subjects were classified as stable MCI if they were diagnosed at every follow-up visit as MCI, or as progressors if they were diagnosed at both 2-year and 3-year follow-up as dementia. Baseline Neuropsychiatric Inventory Questionnaire data was used to assess symptom severity ( $0=$ None, $1=$ Mild, $2=$ Moderate, $3=$ Severe). Stepwise logistic regression analyses were used to predict group membership (stable versus progressor) and baseline symptom severity scores for seven neuropsychiatric variables were entered as predictors, along with gender, age, education, MCI subtype and number of ApoE4 alleles entered as covariates. Follow-up stepwise logistic regression analyses by MCI subtype (amnestic and non-amnestic) were also conducted.

Results: At 3 -years, 596 subjects (56\%) remained MCI and 463 (44\%) progressed to dementia. Lower scores for apathy $(\mathrm{x} 2=18.35, \mathrm{OR}=1.57$, $\mathrm{p}<.01)$, anxiety $(\mathrm{x} 2=4.96, \mathrm{OR}=1.29, \mathrm{p}<.05)$ and motor disturbances $(\mathrm{x} 2=4.20, \mathrm{OR}=1.78, \mathrm{p}<.05)$ were predictive of stable MCI. In amnestic MCI, lower apathy $(\mathrm{x} 2=17.28, \mathrm{OR}=1.70, \mathrm{p}<.01)$ and anxiety $(\mathrm{x} 2=4.94$, $\mathrm{OR}=1.34, \mathrm{p}<.05)$ scores predicted stability, while in non-amnestic MCI only lower irritability $(\mathrm{x} 2=6.3, \mathrm{OR}=2.03, \mathrm{p}<.05)$ scores were predictive. Conclusions: MCI patients with less severe symptoms of apathy, anxiety, and irritability are likely to remain diagnostically stable at 3-years. This project was supported by the Friends of the UT Southwestern Alzheimer's Disease Center and by the UT Southwestern Alzheimer's Disease Center (NIH P30-AG12300).

Correspondence: Matthew Clem, MEd, Psychiatry, UT Southwestern Medical Center, 5323 Harry Hines Blvd, Dallas, TX 75235. E-mail: matthew.clem@utsouthwestern.edu

K.J. JENNETTE. The Association of Cognitive Endophenotypes and Risky Single Nucleotide Polymorphisms of Alzheimer's Disease within the Alzheimer's Disease Neuroimaging Initiative (ADNI) Database.

Objective: The purpose of this study is to assess the influence of three single nucleotide polymorphisms previously associated with Alzheimer's disease on specific domains of cognition, when controlling for APOE, in a sample of individuals with and without Alzheimer's disease.

Participants and Methods: The data were drawn from the Alzheimer's Disease Neuroimaging Initiative database, a comprehensive, longitudinal database of controls, subjects with mild cognitive impairment, and subjects with mild Alzheimer's disease. Each subject has a full neuropsychological assessment, neuroimaging, genetic sequencing, and physical evaluation. For the purposes of this study individuals were filtered based on the presence of the three SNPs of interest: CR1 (rs3818361),CLU (rs11136000), PICALM (rs3851179). Each SNP was then measured against the available tests of the ADNI neuropsychological battery that measured immediate and long delay memory, semantic fluency, and confrontation naming.
Results: Only the CR1 SNP (rs3818361_T) had significant findings. CR1 negatively associated with performance on logical memory recall total score, AVLT immediate recall trials 2 and 4, AVLT delayed recall, and confrontation naming in the 12-month control group. Logical memory and AVLT delayed recall were also negatively associated with CR1 in the 12-month AD case group

Conclusions: These results support the negative influence presence of the risky CR1 SNP in individuals without diagnosis of AD, and to a lesser extent, those with $\mathrm{AD}$, has on memory endophenotypes. These data also indicate a possible protective effect the presence of CLU has on measures of memory, albeit modestly and with marginal significance in this sample. Such findings can aid in the earlier detection of Alzheimer's disease, risk for domain specific cognitive impairment, and novel targets for personalized pharmacotherapy.

Correspondence: Kyle J. Jennette, Ph.D. Candidate, Department of Psychology and Neuroscience, University of Wisconsin Milwaukee, 2717 E. Hartford Ave, Milwaukee, WI 53211.E-mail:kjtommasi@gmail.com

C. VILA-CASTELAR, J.J. LY, L. KAPLAN, K. VAN DYK, J.T. BERGER, L.O. MACINA \& N.S. FOLDI. Short-Term Efficacy of Cholinesterase Inhibitors in Alzheimer's Disease Measured by Sensitive Tasks of Attention.

Objective: Documenting efficacy of cholinesterase inhibitors (AChE-I) in patients with Alzheimer's disease (AD) has depended on large sample comparisons and 4-6 months treatment minimum, with global outcome measures aggregating multiple cognitive domains. As acetylcholine (ACh) has a direct effect on attentional function (Bruno \& Sarter, 1997), we hypothesized that sensitive attention tasks could demonstrate drug efficacy, even after a short treatment period.

Participants and Methods: 23 newly diagnosed patients with AD participated in a longitudinal randomized double-blind placebo-controlled trial. All participants were tested at baseline (T1), randomized into Drug ( $\mathrm{N}=12$; Donepezil 5mg) and Placebo ( $\mathrm{N}=11$ ) groups, and retested after 6 weeks (T2). The attention tasks used were: 1) Foreperiod Task (FT), measured response time (RT) and RT variability comparing high versus low load; 2) Covert Orienting (COV) measured RT of valid, neutral, and invalid cues across 5 trial blocks; 3 ) Attentional Blink (AB) measured accuracy of top-down, guided search of an alphanumeric character under increasing temporal load. Non-parametric Wilcoxon signed-rank tests were applied.

Results: FT: The Drug group was significantly less variable under high load (rapid stimulus presentations) at T2 (Median (Mdn) $=106.75 \mathrm{msec})$ than T1 (Mdn=129.71ms), $\mathrm{z}=-2.04, \mathrm{p}=.041$. COV: The Placebo group significantly slowed across blocks at T2 (Block 5: Mdn= 487.33msBlock 1: $\mathrm{Mdn}=399.00 \mathrm{~ms}), \mathrm{z}=-2.84, \mathrm{p}=.004$. AB: Accuracy under high temporal load significantly worsened for the Placebo group at T2 (Mdn= 62\% accuracy) compared to T1 $(\mathrm{Mdn}=37 \%), \mathrm{z}=-2.20, \mathrm{p}=.028$. Conclusions: The Drug, compared to the Placebo group, was less variable (FT), less fatigued (COV), and showed more accuracy identifying an alphanumeric target presented at high load (AB). Therefore, confirming our prediction, these three attention measures were sensitive to subtle changes of AChE-I treatment after only 6 weeks.

Correspondence: Clara Vila-Castelar, Ph.D. in Clinical Psychology, Psychology, Queens College and The Graduate Center, CUNY, 6530 Kissena Boulevard, Psychology Department, Queens, NY 11367. E-mail: clara.vilacastelar@qc.cuny.edu

\section{J.N. TRAVIS SEIDL \& P. MASSMAN. Cognitive and Functional Correlates of Neuropsychiatric Syndromes in Mild Alzheimer's Disease.}

Objective: The Neuropsychiatric Inventory- Questionnaire (NPI-Q) is used to identify behavioral and psychiatric symptoms in patients with dementia. The present study sought to identify the factor structure of the NPI-Q in a sample of mildly demented Alzheimer's disease (AD) patients and to determine the cognitive and functional correlates of these factors. 
Participants and Methods: Data was utilized from 256 participants with mild AD (Clinical Dementia Rating of 0.5 or 1 ) who were enrolled in the Baylor College of Medicine Alzheimer's Disease and Memory Disorders Center. Exploratory Factor Analysis (EFA) was conducted to determine the factor structure of the NPI-Q in this sample. Regression analyses were conducted to identify associations between NPI-Q factors and cognitive and functional performance.

Results: Results of the EFA indicated that there were two factors: Negative/Oppositional (consisting of agitation, irritability, apathy, depression, disinhibition, and delusions) and Anxiety/Restlessness (consisting of nighttime behavior, anxiety, hallucinations, and appetite). Higher total NPI-Q score was associated with poorer functioning on Activities of Daily Living (ADLs) and Instrumental Activities of Daily Living (IADLs). Increased severity of Anxiety/Restlessness symptoms were associated with poorer performance on VR I and Block Design, and poorer performance on basic and instrumental ADLs.

Conclusions: Higher overall level of neuropsychiatric symptoms was related to functioning on basic and instrumental ADLs. The two identified factors were associated with different patterns of cognitive and functional performance. Negative/Oppositional symptoms were not related to performance on any neuropsychological measures, while symptoms of Anxiety/Restlessness were associated with visuospatial skills and functional performance.

Correspondence: Jennifer N. Travis Seidl, M.A., Psychology, University of Houston, 126 Heyne Building, Houston, TX 77204. E-mail: jennifer.t.seidl@gmail.com

J. DURANT, G.C. LEGER, S.J. BANKS \& J.B. MILLER. Relationship between the Activities of Daily Living Questionnaire and the Montreal Cognitive Assessment.

Objective: The Activities of Daily Living Questionnaire (ADLQ) is an informant report measure assessing functional impairment in daily living skills. Previous research has demonstrated correlations between the ADLQ and cognitive screening measures used in the assessment of dementia (e.g., Mini-Mental State Examination; Clinical Dementia Rating Scale). The following study examined the relationship between the ADLQ and the Montreal Cognitive Assessment (MoCA), an increasingly common cognitive screening measure.

Participants and Methods: Participants included 629 individuals referred for assessment at an outpatient neurology clinic specializing in neurodegenerative disease. The ADLQ and MoCA were administered as part of a comprehensive neurological and neuropsychological assessment conducted in the context of routine clinical care. The Pearson correlation coefficient was calculated to determine the relationship between ADLQ total score and MoCA total score and one-way ANOVA compared ADLQ scores of individuals whose MoCA scores fell within the impaired range.

Results: Pearson correlation indicated a significant negative correlation between MoCA total score and ADLQ total score $(r=-.33 ; \mathrm{p}<.001)$ such that lower MoCA scores were associated with higher levels of functional impairment as rated on the ADLQ. One-way ANOVA revealed significantly higher total ADLQ scores for individuals whose total MoCA score was within the impaired range $(\mathrm{F}(1,628)=15.60 ; \mathrm{p}<.001)$.

Conclusions: Understanding the relationship between ADLQ and MoCA may aid clinicians in estimating or predicting anticipated levels of functional impairment based on cognitive screening results. The present findings suggest that individuals who obtain MoCA scores within the impaired range are more likely to also have functional impairments. Correspondence: January Durant, Cleveland Clinic Lou Ruvo Centre for Brain Health, 888 W. Bonneville Ave., Las Vegas, NV 89106. E-mail: durantj@ccf.org
D. HOWIESON, N. MATTEK, H.H. DODGE, D. ERTEN-LYONS, T. ZITZELBERGER \& J.A. KAYE. Stability and Prognostic Value of Memory Complaints in Older Adults.

Objective: Interest in subjective memory complaints as a possible indicator of impending dementia has escalated in recent years as research focus has shifted toward identifying people who will develop Alzheimer's disease at the earliest possible stage. The stability and prognostic value of subjective memory complaints was studied in cognitively intact community-dwelling older adults.

Participants and Methods: The 156 participants with a mean age of 83 years were assessed for subjective memory complaints, cognitive performance, functional status, and mood at annual evaluations with a mean follow-up of 4.5 years.

Results: Subjective memory complaint at entry $(n=24)$ was not associated with impaired memory performance on standard neuropsychological tests and did not predict memory decline or progression to incipient dementia over time. Memory complaints were inconsistent across examinations for $62 \%$ of participants who reported memory problems. Conclusions: Memory complaint's value as a research criterion for selecting older adults at risk for dementia is weak among octogenarians. Age, length of follow-up, and other population characteristics may affect the implication of self-reported memory problems.

Correspondence: Diane Howieson, PhD, Neurology, Oregon Health \& Science University, Layton Alzheimer Center CR 131, 3181 SW Sam Jackson Park Rd, Portland, OR 97239-3098.E-mail:howiesod@ohsu. edu

B.M. MIZUKI, S.V. FARLEY, A.C. GENESER, S.A. SPERLING \& C.A. MANNING. DRS-2 Construction and Memory Scores Differentiate Between Alzheimer's Disease and Parkinsonian Dementias.

Objective: Screening measures are a useful and common tool in neuropsychological assessment batteries. While these measures can help accurately identify dementia, they are less successful at differential diagnosis of dementia. This study assessed the ability of the Mattis Dementia Rating Scale-2 (DRS-2) to differentiate between patients with Alzheimer's disease (AD) and Parkinsonian dementias, including Parkinson's disease dementia (PDD), Lewy Body Dementia (DLB), and Corticobasal Degeneration (CBD).

Participants and Methods: Seventy-five patients (age: $\mathrm{M}=76$; $\mathrm{SD}=$ 7.12) from a memory disorders clinic received comprehensive neurological and neuropsychological assessment that included the DRS-2, the Mini-Mental State Exam (MMSE), and the Geriatric Depression Scale (GDS). Based on their evaluations, 23 participants were diagnosed with Parkinsonian dementias (13 PDD, 8 DLB, 2 CBD) and 52 with probable Alzheimer's disease (AD).

Results: Binomial logistic regression was conducted using the DRS-2 Total and subscale scores, while accounting for variance explained by age, gender, education, mental status, and depression score. Results revealed that reduced visuospatial abilities (i.e., DRS-2 Construction) predicted classification into the Parkinsonian dementia group $(77 \%$ accuracy, $\mathrm{p}<.05$ ). Memory dysfunction (i.e., DRS-2 Memory) was more predictive of $\mathrm{AD}(77 \%$ accuracy, $\mathrm{p}<.05)$. There were no significant predictive values for the DRS-2 Total score or other subscales.

Conclusions: The results demonstrate the utility of the Construction and Memory subscales of the DRS-2 for quickly and accurately detecting differences between dementia etiologies. The findings are consistent with pathological consequences of the disease and our understanding of disease impact on neuropsychological functioning.

Correspondence: Brian M. Mizuki, Psy.D., Neurology, University of Virginia, 1258 Swan Lake Dr., Apt 308, Charlottesville, VA 22902. E-mail:brian.mizuki@gmail.com 
C.E. PERRY, D.W. HEDGES, S.D. GALE, E. WILSON, J.S. KAUWE \& L. ERICKSON. Association between cognition and latent toxoplasmosis in healthy older adults and in Alzheimer's disease. Objective: The intraneuronal protozoal parasite Toxoplasma gondii (T. gondii) has been associated with cognitive changes in rodents and humans. We investigated the association between latent toxoplasmosis and cognition in older healthy adults and in Alzheimer's disease (AD). Participants and Methods: From Washington University's ADRC project, we obtained serum and cognitive data from 133 healthy controls and 105 patients with AD. We evaluated latent toxoplasmosis status with micro-enzyme-linked immunosorbent assay (ELISA) analysis and cognition with standardized cognitive tests. We used multiple regressions to determine if latent toxoplasmosis predicted scores on cognitive measures while controlling for age and education.

Results: In healthy male controls, latent toxoplasmosis and/or higher antibody titers predicted better performance on the WMS letter number sequencing, associate learning, digit span backward, and mental control and on WAIS Information, word fluency, and a selective reminding test. In female healthy controls, latent toxoplasmosis predicted worse scores for WMS logical memory, WAIS block design, and the Trailmaking B test. In males with $\mathrm{AD}$, higher antibody titers predicted worse scores on the Boston naming test and a selective reminding test and females with $\mathrm{AD}$ and latent toxoplasmosis performed worse on the Trailmaking A test. Conclusions: Latent toxoplasmosis may be protective or enhancing for cognition in older males without AD but may be detrimental for males with AD. Additionally, latent toxoplasmosis may negatively affect cognition in older women without AD. These preliminary results suggest sex- and dementia-state dependent associations between latent toxoplasmosis and cognitive function in older adults.

Correspondence: Cynthia E. Perry, BS, Psychology Department, Brigham Young University, 260 Dorchester Dr., Salt Lake City, UT 84103.E-mail: cindeeperry@gmail.com

C.E. PERRY, S.D. GALE, D.W. HEDGES, E. WILSON, J.S. KAUWE \& L. ERICKSON. Association between latent toxoplasmosis and Alzheimer's disease.

Objective: While felines are the definitive host of the protozoal parasite Toxoplasma gondii (T. gondii), humans and a variety of other animals can be intermediate hosts. T. gondii has an affinity for the central nervous system, and latent toxoplasmosis has been both positively and negatively associated with cognitive and behavioral changes in rodents and humans. Specifically, one study found higher prevalence of latent toxoplasmosis in humans with Alzheimer's disease (AD). However, in a rat model of AD, another study found increased cognitive capacities for rats with latent toxoplasmosis, suggesting that latent toxoplasmosis may protect against $\mathrm{AD}$. We sought to determine if there is an association between latent toxoplasmosis as assessed by anti-toxoplasmosis IgG and $\mathrm{AD}$ in a large, well defined human sample.

Participants and Methods: From Washington University's ADRC project, we obtained serum and demographic variables from 133 healthy controls and 105 patients with AD. We evaluated latent toxoplasmosis status with quantitative immunoenzymatic determination of $\mathrm{T}$. gondii IgG-class antibodies using micro-enzyme-linked immunosorbent assay (ELISA) analysis.

Results: We found no significant differences in the prevalence of latent toxoplasmosis between the control and AD groups in the full sample ( $64 \%$ and $59 \%$ seronegative, $35 \%$ and $40 \%$ seropositive, respectively). Additionally, when using logistic regression to predict the diagnosis of AD based on latent toxoplasmosis with a fully controlled model, latent toxoplasmosis did not predict AD either in the whole group or in females and males separately.

Conclusions: In contrast to previous studies, we found no association between latent toxoplasmosis and $\mathrm{AD}$ in the largest study to date. However, cognitive function in AD may be affected by latent toxoplasmosis, rather than just an outcome of $\mathrm{AD}$, warranting further research.
Correspondence: Cynthia E. Perry, BS, Psychology Department, Brigham Young University, 260 Dorchester Dr., Salt Lake City, UT 84103.E-mail: cindeeperry@gmail.com

J. HASSENSTAB, A. ASCHENBRENNER, D. BALOTA, M. STORANDT, J.C. MORRIS \& R.J. BATEMAN. Cognitive Performance in the Dominantly Inherited Alzheimer Network (DIAN).

Objective: DIAN is an international registry study of individuals from families with known Autosomal Dominant Alzheimer Disease (ADAD) mutations. Participants undergo extensive neuroimaging, cerebrospinal fluid collection, and cognitive assessment every 1-3 years. We present cognitive data from the expanded baseline sample and a preliminary look at longitudinal cognitive data.

Participants and Methods: Participants included 331 individuals aged 39.4 (10.42) years. Groups were defined by mutation status and severity of symptoms using the Clinical Dementia Rating Scale (CDR). Non-mutation carriers (NCs, $n=118$ ) were used as the reference group in statistical analyses. Asymptomatic Mutation Carriers (CDR 0 MCs, $\mathrm{n}=130$ ), Mutation Carriers with very mild symptoms (CDR 0.5 MCs, n $=54$ ), and Mutation Carriers with mild symptoms (CDR 1 MCs, $n=29$ ) were contrasted on performance on a battery of 13 standard cognitive tests and 5 novel computerized measures of executive functioning. Linear mixed models were used to contrast groups on cognitive functioning at baseline and up to 5 years of follow-up. Tests were grouped into four z-score composites derived from exploratory factor analyses.

Results: At baseline, symptomatic MCs exhibited widespread cognitive impairment in multiple domains including memory, visuospatial, executive, and language functioning when compared to NCs (all p's <.01). In Asymptomatic MCs, performance was worse than NCs on a global cognitive composite $(p<.01)$ and showed trends for worse performance on the executive composite $(p<.10)$. Slopes of longitudinal cognition among symptomatic MCs largely reflected baseline results, with the exception that language functions declined more rapidly. In Asymptomatic MCs, a slight difference in cognitive trajectories was observed on episodic memory $(p<.10)$.

Conclusions: Individuals with genetic forms of AD show patterns of cognitive decline similar to sporadic $\mathrm{AD}$, even in the preclinical stage, further establishing that ADAD cohorts may be ideal for secondary prevention trials.

Correspondence: Jason Hassenstab, PhD, Neurology, Washington University in St. Louis, 4488 Forest Park Ave, Suite 130, St. Louis, MO 63130.E-mail: hassenstabj@abraxas.wustl.edu

\section{FARRELL, Y. STERN, J. PISCITELLO \& S. COSENTINO. Windows of Awareness in Alzheimer's disease: Insight into Word- finding Difficulty Brings Memory Deficits to Light.}

Objective: The domain-specificity of cognitive awareness in Alzheimer's disease (AD) is not well understood. Frequent endorsement of word-finding, but not memory difficulty, hints at selective preservation of language awareness. However, it is unclear whether word-finding complaints are mediated by accurate self-assessment or generic age-related concerns. The present research aimed to characterize the frequency, accuracy, and interdependence of two domains of cognitive awareness in AD: memory and word finding.

Participants and Methods: Participants included 164 individuals with mild AD $(\mathrm{CDR}=1)$. Awareness within the two domains was assessed using 2 items from a cognitive difficulties questionnaire, also completed by informants. Awareness of memory and word-finding difficulty was defined dichotomously, $(0=$ No Difficulty, $1=$ Difficulty $)$. Accuracy of self-reports was assessed via participant-informant discrepancy scores, and correlation between participant report and objective performance on domain-specific tests.

Results: Participants endorsed word-finding difficulty more frequently than memory difficulty $(51.6 \%$ v. $37.8 \%, p=.02)$. Conversely, $47.7 \%$ of informants endorsed word-finding difficulty in the participant, while 
$77 \%$ reported memory difficulty. Participant-informant discrepancy scores were significant for memory but not word-finding. Although there was no association between memory reports and memory performance, word-finding complaints were associated with worse performance on objective word-finding tasks.

Conclusions: Word-finding complaints are endorsed more readily than memory complaints in mild AD, and seem to reflect more accurate self-appraisal. However, domains of awareness were not completely dissociable; presence of a word-finding complaint was associated with increased odds of having a memory complaint (OR 1.8). Hence, it appears that insight into word-finding deficits may serve as a gateway into cognitive awareness more broadly.

Correspondence: Meagan Farrell, ProPhase, 587 Fort Washington Ave, New York, NY 10033.E-mail: mfarrell@prophase.com

D.A. NATION, E.C. EDMONDS, K.J. BANGEN, L. DELANOWOOD, B.K. SCANLON, D. HAN, S.D. EDLAND, D.P. SALMON, D. GALASKO \& M.W. BONDI. Elevated pulse pressure is associated with tau-mediated neurodegeneration, cerebral amyloidosis, and progression to dementia in the very-old.

Objective: (1) Determine the relationship between pulse pressure and cerebral spinal fluid (CSF) biomarker profiles and progression to dementia; (2) Investigate whether observed relationships are stronger among those with more advanced arterial age (very-old $\geq 80$ years).

Participants and Methods: Nondemented Alzheimer's Disease Neuroimaging Initiative (ADNI) study participants (ages 55-91) underwent baseline health assessment, including blood pressure assessment and lumbar puncture to assess CSF phosphorylated tau (P-tau) and beta amyloid (A $\beta 1-42)$ ( $\mathrm{N}=877)$. ANCOVA examined relationships between pulse pressure and CSF biomarker profiles, and Cox regression examined progression to dementia. Pulse pressure $x$ age-group (very-old vs. young-old) interactions were investigated. Covariates were age, gender, apolipoprotein E genotype, body mass index, vascular risk, and antihypertensive medication use.

Results: P-tau positive cases exhibited elevated pulse pressure, regardless of age. Among the very-old, a further increase in pulse pressure was observed in those exhibiting both $\mathrm{P}$-tau elevation and $\mathrm{A} \beta 1-42$ reduction, and those with higher baseline pulse pressure progressed to dementia more rapidly. Systolic pressure exhibited similar relationships among the very-old, but not the young-old. Diastolic pressure was reduced in young-old participants with isolated P-tau elevation.

Conclusions: Pulse pressure, an index of vascular aging, was associated with neurodegeneration prior to the onset of dementia across a broad age range. Among those with more advanced age, higher pulse pressure was also associated with amyloidosis in the presence of neurodegeneration, and more rapid progression to dementia. Diastolic contributions to these associations were limited to the young-old whereas systolic contributions were found only in the very-old.

Correspondence: Daniel A. Nation, Ph.D., Psychiatry, VA San Diego Healthcare System / University of California San Diego, School of Medicine, 3350 La Jolla Village Dr., 151B, San Diego, CA 92161. E-mail:dnation@ucsd.edu

A.M. BUTTS, M.M. MACHULDA, J.L. WHITWELL, E.A. STRAND, J.R. DUFFY, S. PRZYBELSKI, R.C. PETERSEN, R.J. IVNIK, V. LOWE \& K.A. JOSEPHS. Amyloid PET confirmed Alzheimer's disease: Neurocognitive differences in Logopenic Progressive Aphasia vs. Dementia of the Alzheimer's Type.

Objective: The logopenic variant of primary progressive aphasia (lvPPA) presents with language disruption while memory impairment is the cardinal feature of dementia of the Alzheimer's type (DAT). Despite similar pathology, few studies have compared neurocognition between these groups. We hypothesize that lvPPA will perform better on memory measures than DAT.

Participants and Methods: Fifty DAT subjects were matched to 50 lvPPA subjects on age, disease duration, and gender. All subjects showed positive beta-amyloid deposition on Pittsburgh Compound B (PiB) PET, defined using a standardized uptake value ratio (SUVR) of $\geq 1.5$. Subjects completed the Auditory Verbal Learning Test, Logical Memory, Visual Reproduction, Trail Making Test (TMT), Boston Naming Test (BNT), MMSE, the Clinical Dementia Rating Scale-Sum of Boxes (CDR), and APOE genetic testing.

Results: The groups did not differ on MMSE, PiB SUVR, or CDR. The DAT group had a higher frequency of the apolipoprotein $\varepsilon 4$ allele. Although both groups were impaired relative to normal controls, the DAT group performed more poorly than the lvPPA group on all delayed memory measures. Conversely, the lvPPA group performed more poorly than the DAT group on the BNT. The groups did not differ on TMT A or B. Conclusions: The pattern of neurocognitive deficits reflect greater language disturbance, but relatively less memory impairment, in lvPPA compared to DAT. Poorer memory in DAT may relate to $\varepsilon 4$ status. Results suggest that despite similar underlying pathology, PiB SUVR ratio, performance on a brief cognitive screen and a functional assessment, neuropsychological testing may be a useful tool for differentiating lvPPA from DAT.

Correspondence: Alissa M. Butts, Ph.D, Psychiatry and Psychology, Mayo Clinic, 62 Grandeville Rd SW, Unit 1521, Rochester, MN 55902.

E-mail: Alissa.m.butts@gmail.com

R.J. MELROSE, M. DAIANU, E. JIMENEZ, P. THOMPSON \& M.F. MENDEZ. Distinct Patterns of Structural and Functional Connectivity in Amnestic and Non-amnestic Early Onset Alzheimer's Disease.

Objective: Early onset Alzheimer's Disease (EAOD) presents clinically before age 65 and includes amnestic (A-EOAD) and non-amnestic (NA-EOAD) subtypes. We determined differences in the structural connectome of EOAD subtypes and their correspondence with functional connectivity.

Participants and Methods: NA-EOAD $(n=9)$ and A-EOAD $(n=10)$ were diagnosed following neurobehavior exam. Using 1.5-T diffusion-weighted images we computed whole-brain tractography to map connections between cortical regions (ROIs). We evaluated differences in nodal degree (sum of connections passing through an ROI) between subtypes $(\mathrm{p}<.05)$. Exploratory analyses assessed functional connectivity of ROIs showing differences in the structural connectome in a subset that completed resting state fMRI (NA-EOAD n=7, A-EOAD n=5; seed-based analysis in SPM8, p<.005).

Results: The structural connectome in NA-EOAD showed a lower nodal degree (fewer connections between pairs of ROIs) in left inferior frontal gyrus (IFG), pars opercularis, left supramarginal gyrus, and right cingulate. In contrast, A-EOAD showed reduced nodal degree in frontal regions (including left middle frontal gyrus- MFG) and right temporal cortex. Similarly, rsfMRI showed reduced functional connectivity between left IFG and bilateral middle/superior temporal gyri in NA-EOAD relative to A-EOAD. Regarding left MFG, NA-EOAD showed reduced connectivity with bilateral insula and temporal regions, while A-OEAD showed reduced connectivity with default mode network (DMN).

Conclusions: Preliminary findings suggest NA-EOAD and A-EOAD show distinct connectivity patterns, supported by both structural and functional analyses. NA-EOAD show reduced structural and functional connectivity between regions involved in language. A-EOAD show reduced structural connectivity in frontal regions and corresponding attenuation of frontal-DMN functional connectivity. Connectivity analyses reveal separate phenotypes and may advance understanding of the pathophysiology of atypical dementias.

Correspondence: Rebecca J. Melrose, PhD, Brain Behavior and Aging Research Center, VA Greater Los Angeles Healthcare System, 11301 Wilshire Blvd., 116AE, Los Angeles, CA 9007.E-mail: rjmelrose@ucla. edu 
M.A. TRIVEDI, T.P. COTHRAN, D.V. MERKITCH, V.T. DINH, G.T. STEBBINS, T.B. PARRISH, R.C. SHAH, D. BENNETT \& D.A. EVANS. The relationship between oral versus written discrepancy scores on the symbol digit modalities test (SDMT) and fMRI activation during SDMT performance and white matter integrity in middle aged adults with a parental history of Alzheimer's disease (AD): Influence of APOE genotype.

Objective: Normal aging is associated with increased risk for Alzheimer's disease $(\mathrm{AD})$ and declines in cognitive efficiency/processing speed. In middle-aged, adult children of individuals diagnosed with $\mathrm{AD}$, we examined the influence of APOE genotype on the relationship between cognitive efficiency/processing speed (discrepancy between oral-written symbol digit modalities test, SDMT, performance) and functional MRI (fMRI) activation during SDMT performance and diffusion tensor imaging (DTI) of white matter integrity.

Participants and Methods: We used an fMRI-modified version of the SDMT and DTI to examine the relationships between these neuroimaging measures and SDMT discrepancy scores. All participants were 40-65 years of age. There were 54 (31 APOE e4+, 23 e4-) and 52 (26 e4+, 26 e4-) participants in the fMRI and DTI analyses, respectively. Regression analyses were used to examine the relationship of SDMT discrepancy score with fMRI activation during SDMT performance, white matter integrity, and the influence of APOE genotype on these relationships. Results: Greater oral-written SDMT discrepancy scores were associated with greater fMRI activation (cluster FDRp=.05) in left inferior parietal, bilateral superior frontal, left posterior cingulate, and right anterior medial temporal cortices. Only clusters in the left inferior parietal cortex and bilateral superior frontal cortices remained significant after adjusting for APOE genotype. Greater SDMT discrepancy scores were also associated with greater white matter integrity in bilateral splenium of the corpus callous/cingulum bundle (FDRp=.05). After adjusting for APOE genotype, a more extensive relationship emerged with white matter regions in the right precuneus also being statistically significant. Conclusions: We found that SDMT oral-written discrepancy scores are associated with fMRI activation and white matter integrity in brain regions that have been implicated in aspects of SDMT performance. These relationships are partially modulated by APOE genotype. Correspondence: Mehul A. Trivedi, PhD, Neurology, Medical College of Wisconsin, 1827A N. Warren Ave, Milwaukee, WI 53202. E-mail: jazzymagic@gmail.com

E.A. BOOTS, S.A. SCHULTZ, R.P. ALMEIDA, S. YU, J.M. OH, R.L. KOSCIK, M.N. DOWLING, C.L. GALLAGHER, C.M. CARLSSON, H.A. ROWLEY, B.B. BENDLIN, A. LARUE, S. ASTHANA, B.P. HERMANN, S. JOHNSON \& O.C. OKONKWO. Occupational Complexity and Cognitive Reserve in Middle-aged Adults at Risk for Alzheimer's Disease.

Objective: Higher occupational attainment is reported to be associated with greater brain pathology and preserved cognitive function in Alzheimer's disease (AD), indicating that occupation could provide cognitive reserve. The objective of this study was to assess whether occupational complexity (OCC) corresponds with better memory test performance and decreased hippocampal volume in a middle-aged cohort at risk for $\mathrm{AD}$.

Participants and Methods: 330 cognitively healthy adults (age $=60.18 \pm 6.21$ years) enrolled in the $\mathrm{W}$ isconsin Registry for Alzheimer's Prevention underwent structural MRI scanning, cognitive assessment, and completed a work history questionnaire. Three complexity ratings (work with data, people, things) were coded using $\mathrm{O}$ *NET classifications, averaged across up to three reported jobs, weighted by years on each job, and summed to create a composite OCC rating. FreeSurfer was used to derive hippocampal volume.

Results: OCC was positively correlated with several AVLT components, including total score $(p=.024)$, long delay $(p=.035)$, and a composite AVLT score (total score+short delay+long delay+recognition, $p=.022$ ). Linear regression, adjusting for age, sex, APOE, family history, cognitive
testing-MRI scan time interval, education, and the composite AVLT score revealed a negative association between OCC and hippocampal volume $(p=.034)$. Negative associations between OCC and hippocampal volume were also seen while individually adjusting for AVLT components, including total score $(\mathrm{p}=.037)$, short delay $(\mathrm{p}=.048)$, long delay, $(\mathrm{p}=.038)$, and recognition $(\mathrm{p}=.032)$.

Conclusions: OCC is associated with better memory performance, and with decreased hippocampal volume given same educational attainment and memory performance. This suggests that, in people at risk for AD, OCC may provide cognitive reserve by preserving memory performance as brain pathology progresses.

Correspondence: Elizabeth A. Boots, B.S., Geriatrics, University of Wisconsin School of Medicine and Public Health, J5/Mezz Clinical Science Center (MC:2420), 600 Highland Avenue, Madison, W 53792. E-mail: eaboots@medicine.wisc.edu

W.A. ALVERSON, P. MASSMAN \&. R. DOODY. Characteristics Associated with Cognitive Asymmetry in a Large Sample of Alzheimer's Disease Patients.

Objective: Previous studies of cognitive profiles in Alzheimer's Disease have been limited by relatively small sample sizes. The present study aimed to define features of asymmetric cognitive profiles in a much larger clinical sample. More robust descriptions of associated demographic features might be used to provide evidence for asymmetric profiles as unique clinical subtypes of $\mathrm{AD}$.

Participants and Methods: Archival data was obtained from the Baylor College of Medicine Alzheimer's Disease and Memory Disorders Center. This study used neuropsychological test results to calculate asymmetry indices for a large number $(\mathrm{N}=788)$ of probable AD patients. Baseline scores from Rey-Osterrieth Complex Figure Copy, WAIS-R/WAIS-III Block Design, and WMS-R Visual Reproduction I were used to create a visuospatial composite score. Boston Naming Test, Animal Fluency, WAIS-R/WAIS-III Similarities, and WMS-R Logical Memory I were used to create a verbal composite. Composite scores were used to assign patients to a cognitive profile group.

Results: At baseline, $82.11 \%$ of patients were considered to have a symmetric, or globally impaired cognitive profile, versus $10.41 \%$ and $7.49 \%$ for low verbal and low visuo-spatial groups, respectively. Oneway ANOVA tests revealed significant differences between cognitive profile groups in current age $(\mathrm{F}=10.02, \mathrm{p}<0.01)$ and estimated age at onset $(\mathrm{F}=9.62, \mathrm{p}<0.01$; low visuo-spatial group significantly younger $)$, MMSE score $(\mathrm{F}=6.54, \mathrm{p}=0.002)$, and AMNART estimated IQ $(\mathrm{F}=$ $3.86, \mathrm{p}=0.026)$. Chi-square analysis did not yield significant associations between cognitive profile group and sex or number of ApoE e4 alleles.

Conclusions: This study provided an expanded and more generalizable description of certain demographic and cognitive features of unique cognitive profile groups. Relative proportions of patients in each profile group were similar to some previously published studies, although likely lower due to the inclusion of memory measures sensitive to Alzheimer's Disease in both composite scores.

Correspondence: William A. Alverson, B.S., Psychology, University of Houston, 2496 Bering Dr, Houston, TX 77057. E-mail:waalverson@ gmail.com

K. GIFFORD, D. LIU, L. SAMUELS, X. HAN, E. BENSON, L. LOGAN, T. HOHMAN, E.M. LANE, E.C. SUMNER, K. WISNIEWSKI, E. WIGGINS, M. GODFREY, F. RUBERG \& A.L. JEFFERSON. Ambulatory blood pressure variations relate to greater subjective cognitive decline in older adults: The Vanderbilt Memory \& Aging Project.

Objective: Subjective cognitive decline (SCD) may be a marker of prodromal Alzheimer's disease (AD) but its relation to vascular disease is poorly understood. Ambulatory blood pressure monitoring (ABPM) measures blood pressure intermittently over several hours, capturing normal and abnormal diurnal fluctuations, which can indicate vascular 
health. We examined whether variability in blood pressure (BP) relates to SCD in older adults with normal cognition (NC) and mild cognitive impairment (MCI).

Participants and Methods: MCI $(\mathrm{n}=81,73 \pm 7,41 \%$ female $)$ and NC participants ( $\mathrm{n}=83$, age $=71 \pm 7,48 \%$ female) from the Vanderbilt Memory \& Aging Project completed 24-hour ABPM capturing BP every 30 minutes and self-report SCD questionnaires. Linear regressions, adjusting for age, sex, race, education, diabetes, and cardiovascular disease, cross-sectionally related systolic (SBP) and diastolic blood pressure (SBP) to SCD for all participants with follow-up models testing ABPM $\mathrm{x}$ diagnosis (MCI, NC) interactions.

Results: Greater report of SCD was related to higher mean nocturnal $\operatorname{DBP}(\beta=0.37, p=0.03 ; \beta=0.35, p=0.02)$. Compared to normal nocturnal diastolic dipping (i.e., an expected $10-19 \%$ nocturnal decrease), individuals with no nocturnal diastolic dipping had greater SCD $(\beta=10.6$, $\mathrm{p}=0.03)$. In follow-up models, MCI participants showed stronger positive associations between greater SCD and increased nocturnal SBP $(\beta=2.8, p=0.02)$ and nocturnal non- $\operatorname{dipping}(\beta=106.3, p=0.005)$ than NC participants.

Conclusions: Compromised vascular health, including abnormal variations in nocturnal BP, related to greater subjective reports of cognitive decline. These associations were stronger in MCI than NC. SCD may have implications in brain aging prior to $\mathrm{AD}$ onset and may represent an appropriate window for intervention.

Correspondence: Katherine Gifford, PsyD, Vanderbilt University, 2525 West End Ave, 12th Floor, Suite 1200, Nashville, TN 37203. E-mail: katie.gifford@vanderbilt.edu

G. WEISSBERGER, T.H. GOLLAN, M.W. BONDI, R. MIZRAHI, S.M. JURICK \& D.P. SALMON. Longitudinal Semantic Fluency Performance in Hispanic Older Adults in the Early Stages of Alzheimer's Disease.

Objective: Semantic memory is vulnerable to early effects of Alzheimer's disease (AD). However, bilingualism may influence the robustness of semantic memory and its vulnerability to AD. The ability to rapidly generate words from specified semantic categories was assessed across 4 annual test sessions in bilingual Hispanics and non-Hispanics in the preclinical stages of $\mathrm{AD}$.

Participants and Methods: Participants were selected from the UCSD Shiley-Marcos AD Research Center's longitudinal study. We compared category fluency performance over 4 consecutive years in 9 Hispanics and 11 non-Hispanics who were cognitively normal at their initial evaluation but eventually developed AD ("decliners"). Groups were matched on age, education, and Dementia Rating Scale scores. Decliner groups were also compared to age and education matched controls $(\mathrm{n}=10$ Hispanics; $n=11$ non-Hispanics). Groups were compared on total correct words, summed across "animals", "fruits", and "vegetable" categories, across 4 time points. We also examined consistency with which specific semantic exemplars were lost over time in each group.

Results: Category fluency scores were lower in Hispanic than non-Hispanic controls, but higher in Hispanic than non-Hispanic decliners. This GroupXEthnicity interaction was significant at each annual evaluation (all pss.04). Hispanic and non-Hispanic decliners declined in their generation of words across all 4 years, but rate of decline did not differ between the groups $(p=.82)$. Overall, decliners showed significantly greater consistency of semantic exemplar loss than controls ( $p$ $=.03)$, but this did not differ in Hispanic and non-Hispanic decliners (GroupXEthnicity interaction not significant; $\mathrm{p}=.6$ ).

Conclusions: Findings suggest that early AD leads to semantic degradation in Hispanics, as has been reported in non-Hispanics (e.g., Salmon et al., 1999), but the impact of AD on semantic fluency may be less in bilingual Hispanics. Findings highlight the possible protective effects of bilingualism during development of $\mathrm{AD}$.
Correspondence: Gali Weissberger, M.S., Clinical Psychology, San Diego State University/University of California, San Diego Joint Doctoral Program, 811 West Nutmeg St. Apt 401, San Diego, CA 92103. E-mail: gweissbe@gmail.com

C.F. ELIASSEN. Hippocampal subfield atrophy in multi-domain, but not in amnestic mild cognitive impairment.

Objective: To investigate hippocampus (HP) subfield and HP sub-structure differences in amnestic mild cognitive impairment (aMCI), multi-domain amnestic MCI (mdMCI) and controls.

Participants and Methods: Employing a comprehensive neuropsychological classification approach, 19 patients characterized as aMCI were compared with 24 patients characterized as mdMCI and 31 controls by means of an automatic HP segmentation procedure.

Results: Using ANCOVA adjusting for age, gender and intracranial volume (ICV) we found significant atrophy of the perirhinal cortex (PRC) and entorhinal cortex (ERC) in aMCI relative to controls, whereas a more severe pattern of atrophy in most subfields, including total HP volume were evident in the mdMCI group. The mdMCI group also showed significant CA4-DG, subiculum and total HP atrophy relative to aMCI. Conclusions: The aMCI group showed atrophy in PRC and ERC, whereas significantly more severe affection of HP subfields was evident in mdMCI. The mdMCI group may thus represent clinical progression relative to aMCI, corresponding to separate patterns of HP subfield affection.

Correspondence: , . E-mail:

E.C. EDMONDS, L. DELANO-WOOD, D. GALASKO, D.P. SALMON \& M.W. BONDI. Characterizing Subtle Cognitive Decline and Biomarker Staging in Preclinical Alzheimer's Disease.

Objective: The criteria for "preclinical" Alzheimer's disease (AD) propose a staging method in which AD biomarkers follow an invariable temporal sequence in accordance with the amyloid cascade hypothesis, although recent findings do not align with the proposed temporal sequence. In addition, "subtle cognitive decline" may occur earlier than suggested in preclinical AD but has not yet been definitively operationalized. We aimed to define subtle cognitive decline using sensitive and reliable neuropsychological tests, and to examine the number and sequence of biomarker abnormalities in the Alzheimer's Disease Neuroimaging Initiative (ADNI).

Participants and Methods: 570 ADNI participants who were cognitively normal at baseline were classified based on the proposed staging model and separately based on the number of abnormal biomarkers associated with preclinical AD that each individual possessed. CSF was used to determine the presence of cerebral amyloidosis and tau-related neurodegeneration. Subtle cognitive decline was determined by actuarial neuropsychological criteria.

Results: The majority of participants did not follow the proposed temporal order of biomarker abnormalities. For those who demonstrated only one abnormal biomarker at baseline and later progressed to a diagnosis of MCI/AD, it was most common to have neurodegeneration alone and nearly equally common to have amyloidosis or subtle cognitive decline alone as the first sign of preclinical AD.

Conclusions: We provide an operational definition of subtle cognitive decline that that is based on individual test scores and captures both cognitive and functional decline. Additionally, we offer a new approach for staging preclinical AD based on number of abnormal biomarkers, without regard to their temporal order of occurrence. This method of characterizing preclinical AD is more parsimonious than the current staging system and does not presume that all patients follow a singular invariant expression of the disease.

Correspondence: Emily C. Edmonds, Ph.D., University of California, San Diego, 4635 Torrey Circle Apt. N104, San Diego, CA 92130. E-mail: ecedmonds@ucsd.edu 
S. KIELB, E. ROGALSKI \& S. WEINTRAUB. Subjective Cognitive Complaints and Early Cognitive Features in Preclinical Alzheimer's Disease.

Objective: Subjective cognitive complaints (SCCs) among cognitively normal older adults have been associated with the presence of in vivo Alzheimer's disease (AD) biomarkers and post-mortem AD neuropathology. This study investigated whether the presence of SCCs (SCC+) was associated with a higher frequency of autopsy-confirmed AD neuropathology and lower baseline cognitive scores compared to the absence of SCCs (SCC-).

Participants and Methods: Data were analyzed from 168 National Alzheimer' Coordinating Center (NACC) participants with normal cognition at their baseline assessment $(\mathrm{CDR}=0)$ who were followed annually to death and received brain autopsy. First, the frequency of AD neuropathology in SCC+ and SCC- groups was compared. In a second analysis, all participants with post-mortem $\mathrm{AD}$ neuropathology $(\mathrm{N}=71)$ who were classified as SCC+ or SCC- at their baseline assessment were compared on baseline scores from the Uniform Data Set neuropsychological tests. Results: More SCC+ than SCG- participants had evidence of AD neuropathology $(87 \%$ vs $37 \%$; $<0.05)$. Of the participants with AD neuropathology, the SCC+ group performed significantly worse than the SCG- group on a semantic fluency test $(\mathrm{p}<0.05)$. On average, SCC+ performance was also worse on tests of processing speed (Trail Making Test Parts A and B, WAIS Coding), but the group differences were not statistically significant.

Conclusions: Results are consistent with prior research suggesting that SCCs may be an early indicator of AD neuropathology. Among individuals with AD neuropathology, those with SCCs tended to show poorer preclinical cognitive scores than those without SCCs on average. More sensitive neuropsychological instruments may be helpful for detecting preclinical AD at the individual level.

Correspondence: Stephanie Kielb, Northwestern University, 320 E. Superior, Chicago, IL 60611. E-mail: stephaniekielb2012@u. northwestern.edu

C. HAYS, Z.Z. ZLATAR, M.J. MELOY, A. KELLER, K. BARON, N. WHITSEL, D. GALASKO, D. SHIN \& C.E. WIERENGA. Resting Cerebral Blood Flow Measured with Arterial Spin Labeling MRI is Associated with Cerebrospinal Fluid Biomarkers of Alzheimer's Disease.

Objective: There is growing evidence implicating cerebral perfusion in the pathogenesis of Alzheimer's disease (AD), yet it is unclear how perfusion relates to AD neuropathology. Studies using PET amyloid imaging indicate that lower cerebral blood flow $(\mathrm{CBF})$ and larger age-related change in $\mathrm{CBF}$ is associated with greater amyloid- $\beta(\mathrm{A} \beta)$ deposition. To extend previous findings and determine the utility of whole brain resting $\mathrm{CBF}$ (WBrCBF) as a potential candidate biomarker for $\mathrm{AD}$ risk, we examined the relationship between gray matter WBrCBF and cerebrospinal fluid (CSF) levels of $\mathrm{A} \beta 42$ and total tau in a group of cognitively normal older adults.

Participants and Methods: Fourteen cognitively normal older adults between the ages of 67-79 participated. WBrCBF was collected via pseudocontinuous arterial spin labeling (PCASL) MRI. A $\beta 42$ and total tau levels were collected from CSF via lumbar puncture. Spearman correlations were carried out between the measures of interest.

Results: Lower WBrCBF in gray matter was significantly associated with higher ratio of CSF tau/A $\beta 42(\rho=-.56, p<.05)$ and with lower levels of $\mathrm{A} \beta 42(\rho=.64, p<.05)$. There was no association between WBrCBF and tau levels.

Conclusions: The association between ASL MRI-measured WBrCBF and tau/A $\beta 42$ implicates a vascular contribution to the pathogenesis of $\mathrm{AD}$. Consistent with the two-hit vascular hypothesis of AD, A $\beta 42$ seems to drive this relationship. These novel preliminary results suggest that global measures of brain perfusion correlate highly with global levels of CSF biomarkers of neuropathology in healthy aging and implicate ASL MRI-measured CBF as a useful biomarker in AD.
Correspondence: Chelsea Hays, UC San Diego, 1813 reed ave \#3, San Diego, CA 92109. E-mail: chelseahays@gmail.com

B.M. BETTCHER, R. FITCH, Z.A. MILLER, M. WYNN, A. BOXER, L. MITIC, K. KOSIK, J.H. KRAMER \& A. KAO. Proinflammatory Chemokines Selectively and Negatively Associate with Episodic Memory in Alzheimer's Disease Phenotypes.

Objective: Recent literature demonstrates an association between inflammation and cognition in older adults, but potential mechanisms underlying these relationships and the specificity of affected cognitive domains remains unclear. MCP- 1 and Eotaxin are joint members of the C-C chemokine subfamily, whose genes have been localized on chromosome 17 . When elevated, they may play a pathogenic role in hippocampal functioning and reduce neurogenesis. We hypothesized that MCP-1 and Eotaxin would selectively and negatively associate with episodic memory in individuals with Alzheimer's disease (AD) phenotypes.

Participants and Methods: Plasma specimens were assayed for MCP1, Eotaxin, and control chemokines (located on chromosomes 4 and 16) in 170 participants at risk for or showing evidence of $\mathrm{AD}$ (CDR 0-2), of varying phenotypes (e.g. memory, executive, language, and spatial predominant phenotypes). Sixty individuals had available amyloid PET scans and all were positive. Memory measures consisted of CVLT-II short form and Benson figure delayed recall, and were analyzed as a composite factor.

Results: Higher MCP-1 was related to worse memory function in AD phenotypes, controlling for clinical severity, diagnosis and demographics (Unstandardized Beta $[\mathrm{B}]=-.00577,95 \% \mathrm{CI}=-.01058$ to $-.0009 ; \mathrm{p}=.02$ ). An interaction was noted between MCP-1 and Eotaxin, such that deleterious associations with memory were primarily seen when both chemokines were elevated $(\mathrm{B}=-.00012 ; 95 \% \mathrm{CI}=-.00012$ to $-.00001 ; \mathrm{p}=.016)$. These associations remained significant after adding APOE genotype and control chemokines into the model. Finally, MCP-1 and Eotaxin were not related to other cognitive domains assessed

Conclusions: Results suggest a potentially selective role for MCP-1 and Eotaxin in episodic memory dysfunction in the context of a wide array of Alzheimer's disease phenotypes.

Correspondence: Brianne M. Bettcher, PhD, Neurology, Memory and Aging Center, UCSF, UCSF Memory and Aging Center- Box 1207, 675 Nelson Rising Lane, Suite 190, San Francisco, CA 94158. E-mail: bbettcher@memory.ucsf.edu

M.L. WERHANE, J.J. HIMALI, K.J. BANGEN, D.A. NATION, R. AU, A. BEISER, A.C. MCKEE, T. STEIN, S. SESHADRI, S.H. AUERBACH, P.A. WOLF \& L. DELANO-WOOD. Dissociation of Alzheimer's Disease Neuropathological Burden and Vascular Pathology on Cognition by CDR Stage: Results from the Framingham Brain Donation Program.

Objective: To examine the antemortem cognitive correlates of postmortem cerebrovascular (CVD) and Alzheimer's disease (AD) pathologies across the Clinical Dementia Rating (CDR) continuum in participants from the community-based Framingham Heart Study (FHS).

Participants and Methods: Participants included 66 stroke-free older adults (mean age at cognitive testing $=85$; CDR: $0-3.0$ ) who came to autopsy and completed cognitive testing within 5 years of death. Secondary analyses were performed separately for individuals with CDR score of $0-0.5(n=39$, mean age $=83)$ and 1 or greater $(n=27$, mean age $=$ 87). The Ischemic Injury Score (IIS) was used as a composite measure of CVD burden. Neurofibrillary tangles (NFT), diffuse plaque (DP). and neuritic plaque (NP) densities were rated semi-quantitatively in 8 regions. Age at testing, time between testing and death, sex, and WRAT reading score were adjusted for in all analyses.

Results: Collapsed across group, IIS was not related to cognition; however, AD pathology significantly predicted poorer scores on executive functioning, verbal memory, and visual memory. Among individuals with CDR scores of 1 or greater, poorer verbal memory, visual memory, and executive functioning were associated with greater neuropathological 
burden. For CDR scores of 0 or 0.5 , only poorer executive function performance was associated with greater $\mathrm{AD}$ neuropathological burden. Conclusions: Across the aging-MCI-dementia continuum, subclinical CVD was not associated with cognition. Additionally, while AD pathology was strongly related to impaired performance across multiple cognitive domains, it was associated with only executive dysfunction among the nondemented subgroup.

Correspondence: Madeleine L. Werhane, PhD, SDSU/UC San Diego Joint Doctoral Program in Clinical Psychology, 9152 Regents Road, Apt I, La Jolla, CA 92037.E-mail: mlwerhane@gmail.com

\section{A. KINZER \& J. SUHR. Dementia Worry is Related to (In)accuracy of Self-reported Memory Complaints.}

Objective: Recent studies have suggested that subjective memory complaints can accurately detect the earliest stages of dementia and should be used in identifying individuals for early pharmacological treatments. However, existing data on subjective memory complaints suggests that, while memory complaints can be relatively accurate in non-selected (i.e., epidemiological) samples, they are not accurate, and instead more likely related to psychological concerns, in treatment-or assessment-seeking individuals who present in a clinical setting. Another factor potentially related to inaccuracy of subjective memory complaints is dementia worry. In the present study, we examined the relationship of dementia worry to the accuracy of self-reported memory complaints.

Participants and Methods: Participants were 87 older adults with no signs of dementia or mild cognitive impairment who participated in a larger study examining the psychometric properties of a new dementia worry scale and in an aging and driving study, from which neuropsychological data were drawn. Dementia worry was assessed using the new scale, and participants were divided into high and low worry based on mean split. Objective memory impairment was defined as scoring at least 1 SD below the mean on one of the memory subscales of the Repeatable Battery for the Assessment of Neuropsychological Status. Memory complaints were assessed using a subscale of the Memory Controllability Inventory.

Results: A 2 (worry level) by 2 (objective memory impairment) ANOVA, with worry as the dependent variable, showed a significant main effect for worry $(p<.05)$; there was no main effect for objective memory impairment and no interaction between worry and objective memory impairment.

Conclusions: Results showed that it is dementia worry that is related to subjective memory complaints, not objective impairment, raising concerns about use of subjective report (in the absence of objective testing) as a means to identify early dementia.

Correspondence: Adrianna Kinzer, Ohio University, 363 Richland Ave, Apt. 281, Athens, OH 45701. E-mail: ak143911@ohio.edu

\section{Language and Speech Functions/Aphasia}

R.K. PEACH. Treatment for Aphasia Associated with Attentional Impairment: A Comparative Effectiveness Study.

Objective: Recent approaches to aphasia rehabilitation have targeted attention processing though with weak results. It may be that, for attentional treatments to be effective for aphasia, they should target language under conditions that compete for attentional resources. The purpose of this research was to test the comparative effectiveness of an attention processing treatment and a language-specific skill-based treatment for the remediation of aphasia associated with attentional impairment.

Participants and Methods: Participants were four right-handed individuals with stroke-induced aphasia. A crossover, single-subject design was used to compare Attention Process Training-3 (APT-3) to Language-Specific Attentional Treatment (L-SAT), an approach that exploits increasingly complex linguistic devices known to focus attention. Probes consisting of a grammaticality judgment dual-task and the PASAT, results from comprehensive neuropsychological testing, and patient-reported outcomes were used to assess the results of these interventions.

Results: Participants showed greater attention allocation on grammaticality judgment, one significantly so, following APT-3. Two participants showed increased processing speeds on PASAT following APT-3 while one did so following L-SAT. Two participants showed improvements on a composite of language scores, one following APT-3, the other following L-SAT. Greater change was shown on a composite of attention scores following APT-3 with one participant showing positive results but two showing negative results. Three participants reported greater changes in communication and attentional abilities following L-SAT.

Conclusions: APT-3 resulted in larger improvements in attentional allocation and processing speed while L-SAT produced better patient-reported outcomes in communication and attention. Both approaches resulted in improvements in language scores while APT-3 generally produced declines in attention scores.

Correspondence: Richard K. Peach, Ph.D., Communication Disorders \&. Sciences, Rush University Medical Center, 1018B Armour Academic Center, 600 South Paulina Street, Chicago, IL 60612. E-mail: richard_k_peach@rush.edu

\section{Medical/Neurological Disorders/Other} (Child)

\section{A. AGOSTON, A.C. GROSS \& C.J. BOYS. Executive Functioning Predicts Interpretation of Social Intents in Children with Fetal Alcohol Spectrum Disorder.}

Objective: Children with FASD have social deficits and frequently misinterpret intentions of others. Although a study found executive functions (EF) contributed to social difficulties in FASD, no control group was used and comparable findings exist in non-FASD populations (Schonfeld et al., 2006). The current study investigated contributions of EF-specifically, concept formation and problem-solving - to interpreting intents (II) in children with/without FASD.

Participants and Methods: To minimize effects of multiple cognitive deficits on social functioning, only children with FASD: Alcohol-Related Neurodevelopmental Disorder (ARND) $(\mathrm{n}=82 ; \mathrm{M}$ age $=12.54, \mathrm{SD}=2.68)$ were selected. This group was comparable to a sample not meeting FASD criteria but referred for suspicion of prenatal alcohol exposure $(\mathrm{n}=80 ; \mathrm{M}$ age=12.29,SD=2.81). An FSIQ cutoff of 85 was used so groups did not differ on IQ (t=1.61, ns). EF was assessed using the Delis-Kaplan Executive Function System Sorting Test, composed of Free-Sortingconsisting of Correct Sorts (CS) and Sorting Description (SD)-and Sort Recognition (SR) conditions. A composite measure of II assessing social inference-making and understanding of metaphoric expressions was used from The Test of Language Competence-Expanded Edition. Results: Hierarchical multiple regression analyses were used to predict II from EF. FS predicted higher II in ARND (CS: $\beta=.50, p=.01, \mathrm{~S}$ $\mathrm{D}: \beta=.40, \mathrm{p}=.05$ ) but not in controls (CS: $\beta=.01, \mathrm{p}=.94, \mathrm{SD}: \beta=-.02, \mathrm{p}=.92)$. SR predicted II in both ARND $(\beta=.47, \mathrm{p}=.02)$ and controls $(\beta=.35, \mathrm{p}=.04)$. Conclusions: FS only predicted II in children with FASD. Whereas children without FASD may rely on automatic or intuitive perspective-taking to understand social situations/statements, children with FASD may utilize concept formation and problem-solving skills to compensate for social deficits. While SR predicted higher II in both groups, recognizing sorts may require better perspective-taking and predict II regardless of diagnosis. Future social skills interventions may build EF in children with FASD.

Correspondence: Anna Monica Agoston, University of Minnesota, 2512 South 7th Street, Minneapolis, MN 55454.E-mail: agost008@umn.edu 
M.P. FISCHER, R. PIERPONT, R.S. ZIEGLER \& J. EISENGART. Differential Neuropsychological Dysfunction Associated with Age of Treatment Onset in Three Relatives with Congenital Hypothyroidism.

Objective: We present an unusual case series involving three Somali relatives (a sibling pair and their uncle) with congenital hypothyroidism, who were deprived of standard treatment due to their refugee status. Treatment commenced in October 2007 after the family immigrated to the United States. We examine the degree of impairment as it relates to age of treatment onset.

Participants and Methods: Patient A (male, age 21) began receiving treatment for congenital hypothyroidism at age 14 years. Patient $\mathrm{B}$ (female, age 14) received treatment starting at age 7 years, and her sibling (Patient $\mathrm{C}$, male, age 7) received treatment starting at age 6 months. All three patients were assessed using standardized neuropsychological assessments of neurocognitive and adaptive functioning appropriate for their respective developmental levels.

Results: All three family members exhibited varying degrees of neurocognitive and adaptive impairments. Patient A, whose treatment was initiated the latest in life, demonstrated the greatest impairment, whereas Patient C, whose treatment was initiated the earliest, demonstrated the mildest impairment. Patient B whose age of treatment onset was in between showed a comparatively moderate level of impairment. She also met diagnostic criteria for selective mutism.

Conclusions: This familial case series provides evidence that an earlier age of treatment onset can reduce the severity of neurocognitive sequelae associated with congenital hypothyroidism. In this family, differential cognitive and adaptive outcomes were associated with striking variation in the duration of treatment deprivation. Cultural and communication challenges in neuropsychological assessment and interventions with a refugee family are also highlighted.

Correspondence: Melissa P. Fischer, Doctorate of Psychology in Clinical Psychology (PsyD), Pediatrics, University of Minnesota, 6413 Pleasant Ave. S. \#7, Richfield, MN 55423. E-mail: fisch326@umn.edu

T. ANTONINI, F. SAAVEDRA, W. DREYER \& S. CAUDLE. Neurocognitive Functioning in Infants Undergoing Cardiac Transplantation.

Objective: Children undergoing cardiac transplantation are at risk for neurodevelopmental impairment. A variety of factors can potentially impact their neurocognitive functioning, including neurological anomolies, preoperative hemodynamic instability, intraoperative hypoperfusion, embolic events, total circulatory arrest, transplant rejection, and immunosuppressant medication. Most studies examining neurocognitive functioning in this patient population have focused on post-transplant functioning, have included wide age ranges, and/or were conducted over a decade ago when less sophisticated medical intervention was available than is today. Examining functioning prior to transplantation may provide additional information as to who is at most risk for difficulties over time. This study provides updated information regarding neurocognitive performance in infants with complex congenital heart conditions who are being evaluated for cardiac transplantation.

Participants and Methods: Fourteen infants between the ages of 2 and 18 months completed the Bayley Scales of Infant and Toddler Development - Third Edition prior to cardiac transplantation. Mean cognitive, language, and motor scores were calculated and compared with normative population mean scores.

Results: Language $($ mean $=82.83, \mathrm{SD}=15.99, \mathrm{t}(11)=-3.72, \mathrm{p}=.003)$ and motor $($ mean $=71.85, \mathrm{SD}=19.07, \mathrm{t}(12)=-5.32, \mathrm{p}<.0001)$ scores both fell significantly below the normative population. The difference between patient and normative cognitive scores (mean $=87.50$, SD $=23.18)$ approached statistical significance $\mathrm{t}(13)=-2.02, \mathrm{p}=.065$. Conclusions: Results suggest that prior to cardiac transplantation, some infants with congenital heart conditions show impairments in neurocognitive functioning. Thus, identifying and tracking neurodevelopment over time in these patients will be vital for providing necessary interventions

Correspondence: Tanya Antonini, Texas Children's Hospital, 4055 South Braeswood Blvd., Apt 212, Houston, TX 77025. E-mail: tanya. antonini@gmail.com

E. SALONEN, H. NYMAN, I. KIZLING, J. AF GEIJERSTAM, O. FLODMARK, P. ASPELIN \& M. KAIJSER. Can a Computed Tomography Examination of the Head Affect Later Cognitive Functions? Follow-up of a Randomized Controlled Trial.

Objective: The aim was to examine if a computed tomography (CT) examination of the head in childhood can affect later cognitive functions. Participants and Methods: 147 participants from a randomized controlled trial on mild traumatic brain injury patient management, CT examination or inhospital observation, took part in the follow-up. The 67 female and 80 male participants' age at head injury was 6-16 years (median of 11.3) and age at assessment was $11-24$ years (mean of 17.8 \pm 3.0 ). Computerized measures of short and long-term nonverbal memory tests from the Wechsler Memory Scale III, as well as computerized tests for short and long term verbal memory, executive functions and sketchpad memory, motor speed and coordination, reaction time, selective attention, and visuospatial ability from the neurocognitive test battery EuroCog were used for the assessment. The Chi-square Test, Student's T-Test, and Wilcoxon Two Sample Test were used to analyze the descriptive data and the psychometric test results. Multivariate analyses with Factorial ANOVAs and Factorial Logistic Regressions were performed to control for the variables of sex, age at head injury, and age at assessment.

Results: The CT examination and inhospital observation groups did not differ in any of the cognitive measures and all the average means and medians were within norm values for both groups. Exposure status remained insignificant when sex, age at head injury, and age at assessment were included into multivariate analyzes.

Conclusions: A CT examination of the head at the age of 6-16 vears does not affect later cognitive functions

Correspondence: , . E-mail:

J. COHEN, M. BRINKER, J. SHARRETTE, S. SOARES, K. NEWMAN $\&$ T.A. HALL. A Comparison of the Intellectual and Adaptive Behavior Functioning of Children with Prenatal Exposure to Methamphetamine with Children with ADHD.

Objective: There is limited research literature concerning the neurocognitive and neurobehavioral effects of prenatal methamphetamine exposure (PME) on child development. The current study explores the developmental impact of PME on children's intellectual and adaptive behavior functioning.

Participants and Methods: Archival neuropsychological test data for 84 children with caregiver-reported PME and 84 children of similar ages with ADHD were used as a clinical and comparison sample, respectively. T-tests were used to compare the scores on neuropsychological and behavioral measures between the two clinical groups at three different age points in order to look for developmental trends. Participants were compared on an overall IQ score from the WISC-IV. WPPSI-III, or SB-5 and on adaptive functioning scores based on parent ratings using the ABAS-II.

Results: In the 4-7 year old age group there were no statistically significant differences between the groups in their WISC-IV/WPPSI-III/SB-5 overall IQ; the PME children obtained lower ABAS-II Social Composite scores (effect size $=.58, p=<.03$ ). In the 8-11 year old age group. children with PME achieved lower overall IQ scores (effect size $=.85$, $p=<.001$ ). Parent ratings on the ABAS-II resulted in lower scores for the PME group on the General Adaptive (effect size $=.90 ; p=<$ .003 ), Conceptual (effect size $=.80, p=<.006$ ), and Practical (effect size $=.93, p=<.002)$ Composites. In the $12-15$ vears old age group. children similarly showed lower overall IQ scores (effect size $=.97, p=$ $<.002)$ and ABAS-II General Adaptive (effect size $=1.14 ; p=<.003$ ). 
Conceptual $($ effect size $=1.13, \mathrm{p}=<.003)$, and Practical $($ effect size $=$ $.95, p=<.01)$ Composites.

Conclusions: PME may result in lowered neurocognitive and adaptive behavior functioning throughout the childhood/adolescent period that becomes more apparent with age.

Correspondence: Jodie Cohen, PhD, Neuropsychology, Northwest Neurobehavioral Health, 406 W. Ada St., Boise, ID 83702. E-mail: jodiescohen@gmail.com

S.E. ELLEFSON, D.L. COOL, T. KAEMMERER, M. LACY \& D. FRIM. Intellect, Spatial Planning and Executive Functioning in Children with Chiari Malformation Type I.

Objective: Chiari Malformation Type I (CMI) has been described as caudal displacement of the cerebellar tonsils below the plane of the forman magnum (Yassari \&. Frim, 2004). Recent research has suggested possible associated executive deficits (Allen et al, 2014). We previously identified constructional deficits in children with Chiari Malformation (Lacy et al, 2008). We currently investigate the possible executive dysfunction as a potential underlying mechanism.

Participants and Methods: Following consent, twenty-one children presenting for routine neurosurgical appointments completed the Cognitive Assessment System (CAS), Wechsler Abbreviated Scale of Intelligence- Second Edition (WASI-II), Wide Range Achievement Test 4 (WRAT4), Rey Complex Figure Test: Copy (RCFT-C), and Conner's Continuous Performance Test- Second Edition (CPT-II). Means and personal correlations were calculated between measures.

Results: The cohort had a mean age of 11.35 (SD=3.05) with $76.2 \%$ Caucasian. Data documented average premorbid and current intellectual functioning [WRAT-4 Reading SS $=106.00(\mathrm{SD}=12.54)$ and FSIQ $=107.86(\mathrm{SD}=14.29)]$. CMI children displayed mild constructional planning deficits [RCFT-C Z $=-1.63$ ] which were correlated with intellect [WASI-II: SI r=. 447, $\mathrm{p}=0.042$ ] and vigilance abilities (CPT-II: Omissions $r=-0.44, p=0.05$ ), while other executive measures did not [(CAS:EA r $=0.06, p=0.79$; PC $r=0.328, p=0.15$; and CPT-II: Commissions $\mathrm{r}=0.21, \mathrm{p}=0.36)$ ].

Conclusions: Children with CMI demonstrate mild construction deficits, which correlated with generalized intellectual functioning and selective executive measures. This is consistent with prior studies suggesting links between executive functioning and construction skills in children (Ogino et al., 2009) cerebellar mediated spatial planning and the diversity of executive skills (Testa et al., 2012).

Correspondence: Sarah E. Ellefson, M.S., Psychology, Illinois Institute of Technology, 5419 S. Harper Ave \#213, Chicago, IL 60615. E-mail: sellefso@hawk.iit.edu

R.B. TANGEN, E. LUNGREN, J. BUCHBINDER \& D. BOTHE. Visual and Verbal Memory Performance in Children wtih Fetal Alcohol Spectrum Disorders.

Objective: Research on memory problems in children with Fetal Alcohol Spectrum Disorders (FASD) has not been consistent. Studies have shown poor verbal memory in FASD, but variable visual memory. Also, it remains unclear how memory problems may differ among children with different FASD diagnoses. The present study was designed to investigate the hypothesis that children with FASD would have greater verbal than visual memory problems. A second hypothesis was that the severity of memory problems would depend on the FASD diagnosis, with the greatest memory problems in children with Fetal Alcohol Syndrome (FAS) or Partial FAS (Group 1), followed by Static Encephalopathy (Group 2), and then Neurobehavioral Disorder (Group 3).

Participants and Methods: Participants were 35 children assessed for FASD with the 4 Digit Diagnostic Code (Astley, 2004) as part of a multi-disciplinary FASD clinic at a regional medical center. Children were $40 \%$ female (mean age $=9.1$ years) and $82 \%$ were adopted/foster care. Children were divided into three groups based on diagnosis: FAS/ partial FAS ( $n=10)$, Static Encephalopathy ( $\mathrm{n}=14)$, Neurobehavioral Disorder $(\mathrm{n}=11)$. Children were administered a comprehensive neuropsychological battery including the Children's Memory Scale, which was analyzed with a series of mixed model ANOVAs.

Results: Contrary to hypothesis, results did not reveal significant within subject differences on verbal and visual memory indices. However, there were significant between group differences on verbal memory but not visual memory indices. Group 2 performed worse than Groups 1 and 3 on both immediate $(\mathrm{F}(1,28)=9.29, \mathrm{p}<.001)$ and delayed verbal memory indices $(\mathrm{F}(1,28)=5.27, \mathrm{p}<.01)$.

Conclusions: Results indicate that children with FASD have deficits in verbal and visual memory, but verbal memory performance differentiates groups better than visual memory. In addition, deficits in memory are present even in children with fetal alcohol exposure who do not meet full diagnostic criteria for FAS.

Correspondence: Rachel B. Tangen, Ph.D., Division of Developmental Pediatrics \&. Psychology, Rainbow Babies \& Children's Hospital, 10524 Euclid Ave., W.O. Walker Building, Suite 3150, Cleveland, OH 44106. E-mail: Rachel.Tangen@UHHospitals.org

M.A. PHOONG, S. CHESTER \& S. PEERY. Case Study: Neuropsychological Functioning in a 12-Year Old With Unilateral Right Cerebellar Hypoplasia.

Objective: Cerebellar hypoplasia is a rare malformation associated with atypical development of cognition, affect, and motor functioning (Schmahmann \& Sherman, 1998). Previous studies of cerebellar malformations suggest an asymmetrical cognitive profile with verbal abilities outpacing nonverbal abilities (Steinlin, Styger, LicPhil \& Boltshauser, 1999). This case study presents evidence of atypical development in an adolescent with unilateral cerebellar hypoplasia.

Participants and Methods: Subject is a 12-year old male with right unilateral cerebellar hypoplasia, referred for neuropsychological testing in an outpatient setting. A comprehensive battery measuring social, emotional, attention, psychomotor, language, visuospatial, learning, memory, and executive functioning was administered.

Results: Neuropsychological testing revealed deficits in visuospatial construction, integration, reasoning, and reduced efficiency in psychomotor processing. Measures of dual-tasking, affect recognition, executive functions, comprehension of multi-step instructions, and phonemic fluency were impaired, whereas verbal learning, memory, naming, semantic fluency, abstraction, and problem solving were in the average range.

Conclusions: In a 12-year old male diagnosed with unilateral right cerebellar hypoplasia, neuropsychological testing revealed a cognitive profile consistent with previous findings demonstrating a range in cognitive deficits. However, additional measures of social and affective functioning also indicated deficits in social cognition which may contribute to elevated levels of social anxiety. A split was evident between verbal and nonverbal measures of learning, memory, and executive functions, consistent with the limited extant literature. This study illuminates the preservation of language mediated abilities and impaired affective processing in one case study of unilateral cerebellar hypoplasia, contributing to the limited literature regarding the neuropsychological effects of this condition.

Correspondence: Malay A. Phoong, Clinical Psychology, Palo Alto University, 1072 Freestone Ave, Sunnyvale, CA 94087. E-mail: mphoong@paloaltou.edu

C.H. SALAMA, B. SLOMINE, S. SUSKAUER \& C.F. SALORIO. Functional Outcomes of Children with Brain Tumor following Inpatient Rehabilitation.

Objective: Children with brain tumors experience cognitive and functional sequelae due to disease and treatment. Understanding functional outcomes during rehabilitation may help with counseling regarding long-term prognosis. This study aimed to evaluate functional recovery during inpatient rehabilitation for children with brain tumors.

Participants and Methods: Ninety-five children with brain tumors of varying pathology presented to a single pediatric inpatient rehabilitation 
facility between 1994 and 2014. Patients were admitted due to functional decline associated with, resection, complication (e.g., hydrocephalus, stroke), or treatment effects (e.g., toxicity from chemotherapy). Functional status was evaluated at admission and discharge using the Functional Independence Measure for Children (WeeFIM) Self-care, Mobility, Cognitive, and Total Developmental Functional Quotient (DFQ) scores.

Results: Mean age was 10 years (range 1-21 years) with $55 \%$ males. Average length of stay was 44 days (range $3-149$ days). Mean Total DFQ at admission was 37 (range: 13-93) and 54 at discharge (range: 14-96). Average change in WeeFIM Total DFQ from admission to discharge was 16.8 (range: -8-58; $\mathrm{p}<.01$ ). Across the cohort, all WeeFIM domain scores improved between admission and discharge $(p<.001)$. Eightyeight children demonstrated significant impairments in functional skills at discharge, as defined by total DFQ $\leq 85$.

Conclusions: Results suggest that children with heterogeneous presentation of brain tumor demonstrate improvement in function during rehabilitation. Evaluation of the impact of type/location of tumor, reason for admission, time since diagnosis, and tumor-related treatments on recovery merit exploration. Further research is necessary to determine which children with brain tumors are expected to experience the most recovery during inpatient rehabilitation.

Correspondence: Christina H. Salama, M.A., Georgia State University, 707 N. Broadway, Department of Behavioral Psychology, Baltimore, MD 21205.E-mail: salama@kennedykrieger.org

J. BEAN JAWORSKI, A.R. CASSIDY, PH.D., J.W. NEWBURGER, MD, MPH \& D.C. BELLINGER, PHD, MSC. Differential Impact of Visuospatial Integration on Academic Skill Development in Children and Adolescents with Critical Cyanotic Congenital Heart Disease.

Objective: Visuospatial deficits are common in children with critical cyanotic congenital heart disease (CCCHD); however, their relevance for predicting functional outcomes remains unclear. Clinically, one's approach to managing visuospatial tasks (i.e., detail-oriented vs. integrated/configural) is thought to represent a more general cognitive style that may differentially impact academic skills development.

Participants and Methods: Rey-Osterrieth Complex Figure (ROCF; Developmental Scoring System) Copy Trial Style ratings were used to examine visuospatial integration in three groups of children/adolescents with CCCHD [d-transposition of the great arteries (TGA, $\mathrm{n}=139$ ), tetralogy of Fallot (TOF, $\mathrm{n}=65$ ), single-ventricle/Fontan recipients (SVF, $n=144)$ ], and healthy controls $(\mathrm{n}=110)$. MANCOVAs examined the predictive validity of Style for academic outcomes (covariates: age, SES, ROCF Basal Level).

Results: Part-orientation was more prevalent among SVF (49\%) than control $(31 \%)$ and TGA $(25 \%)$ participants $(\chi 2(9, \mathrm{n}=436)=22.27$, $\mathrm{p}=.008)$. Style $\mathrm{x}$ CCCHD interactions were not significant. Main effect of Style was significant for assembled (WIAT-II Reading Comprehension, Applied Problems, $\mathrm{p}=.03$ ), but not discrete/associative academic skills (Word Reading, Pseudoword Decoding, Math Calculations, p = .34). Part-orientation was associated with worse performance than more integrated approaches. Main effect of CCCHD group was significant for both assembled and discrete/associative skills (ps < .03), with controls generally outperforming CCCHD groups. Specific group comparisons will be reported.

Conclusions: Children with CCCHD are at risk for visuospatial and academic struggles. Children who showed a detail-oriented visuospatial approach were at increased risk for academic difficulties on tasks requiring synthesis/inferential reasoning, though the impact of integrative weaknesses did not vary as a function of CCCHD subtype. Developmental implications will be discussed.

Correspondence: Jessica Bean Jaworski, Ph.D., Psychiatry, Boston Children's Hospital, 75 Kilsyth Rd, Apt 10, Brighton, MA 02135. E-mail: jessica.bean07@gmail.com
J. NEUMANN, A. FAYAD, D.K. HORTON, S. HUGHES, A. AYRES, B. GREENBERG \& L. HARDER. Neuropsychological Functioning, Fatigue, and Educational Support in Pediatric Transverse Myelitis. Objective: Transverse myelitis (TM) is a rare CNS inflammatory disease that targets the spinal cord resulting in motor weakness and loss of sensation. Although classically defined as a spinal cord condition, recent literature shows cognitive problems in pediatric TM, suggesting that this disease may also impact the brain. Based on previous findings, it was hypothesized that TM participants would exhibit deficits in attention, memory, verbal fluency, and fine-motor skills. They would also be more likely to report fatigue, psychosocial difficulties, and receive educational support services.

Participants and Methods: Twenty participants, ages 6 to 18 years (mean=10.5), were consented for study participation during their attendance at a TM family camp. Participants completed neuropsychological measures, and questionnaires related to fatigue and psychosocial functioning. Caregivers completed an educational history questionnaire. Results: One-sample t-test revealed statistically significant differences from the mean $(\mathrm{p}<.05)$ for bilateral fine-motor skills and self-reported general and cognitive fatigue. No significant differences from the mean were found for attention, memory, or verbal fluency; however, $30 \%$ showed impairment in auditory attention, $25 \%$ in working memory and short-term memory, and $20 \%$ in verbal fluency. Seventy-five percent of the sample was enrolled in formal educational support services. One-sample t-test revealed statistically significant differences from the mean on BASC-2 Self-Report favoring lower rates of clinical symptom endorsement.

Conclusions: Findings suggest that pediatric TM patients are at risk for significant fine-motor impairment as well as physical and cognitive fatigue, often requiring formal educational support services. Although cognitive impairments were not significantly different from the mean, frequencies of impairment were above base rate expectations. Findings support the need for a multi-disciplinary approach, including surveillance by neuropsychology, in the care of pediatric TM patients. Correspondence: Joy Neumann, PsyD, Neuropsychology, Children's Medical Center, 6300 Harry Hines, Chase Bank Building 9th Fl, Dallas, TX 75235.E-mail: joy.neumann@childrens.com

S. WISE, K. OLSON, S.J. HARDY \& K.K. HARDY. Sluggish Cognitive Tempo and Executive Functions in Children with Sickle Cell Disease.

Objective: Sickle cell disease (SCD) is associated with deficits in executive functions including working memory; however, little is known about the role of sluggish cognitive tempo (SCT) in these problems. Our objective was to describe SCT and its effect on working memory and broad executive functioning in a sample of youth with SCD.

Participants and Methods: Youth ( $\mathrm{n}=21$, ages 7-16) with SCD completed a pre-intervention neuropsychological assessment before starting a computerized working memory training program (Cogmed). A primary caregiver completed the Behavior Rating Inventory of Executive Function (BRIEF) and Child Behavior Checklist (CBCL) while youth completed the 4-subtest short form of the Alloway Working Memory Assessment (AWMA) and Wechsler Abbreviated Scale of Intelligence. Results: Our sample was $52 \%$ female with a mean age of 11.38 years (SD=2.8). The mean T-score on the CBCL's SCT subscale was 56.76 $(\mathrm{SD}=7.69)$, and $23 \%$ of scores fell in the clinical range. A considerable portion of the sample also demonstrated clinical elevations on the BRIEF Metacognition Index (MI; 33\%; M=60.33, SD=10.50) and Working Memory (WM) subscale (43\%; M=64.19, SD=12.69). Participants scored in the Average range on all four AWMA subtests but demonstrated the greatest difficulty with nonverbal WM tasks (Dot Matrix M=90.9, SD=21.0; Spatial Recall $\mathrm{M}=93.4, \mathrm{SD}=10.6)$. SCT was significantly correlated with BRIEF WM ( $\mathrm{r}=.59)$ and MI $(\mathrm{r}=.67)$ scores, as well as the AWMA Spatial Recall task $(r=-.62)$. After controlling for age, gender, and intelligence, SCT predicted BRIEF WM $(\beta=.74, p<.01)$, BRIEF MI $(\beta=.81, p<.01)$, and AWMA spatial recall $(\beta=-.71, p<.01)$. 
Conclusions: A considerable number of youth in our sample exhibited elevated SCT symptoms. Caregiver-reported SCT was associated with working memory and executive function problems and may partially explain the relationship between SCD and deficits in executive functioning. Correspondence: Shane Wise, B.S., Hematology/Oncology, Children's National Medical Center, 111 Michigan Ave., Washington, DC 20010. E-mail: swise@childrensnational.org.

\section{HARRELL, A.R. LOUGHAN, J. HERTZA \& M. COHEN. Childhood Narcolepsy and ADHD Comorbidity.}

Objective: Narcolepsy is a sleep disorder defined as poor regulation of wake/sleep cycles. It has been shown to affect activities of daily living and negatively impact mood and cognition. Although research suggests a relationship between narcolepsy and ADHD, data examining specific deficits in childhood inattention, impulsivity, and executive functioning has been variable. This study examined the profile of children diagnosed with narcolepsy, symptoms of ADHD, parent/teacher reports, and measured attention deficits.

Participants and Methods: Participants included 5 children $($ Mage $=$ 8 , range $6-11 ; 80 \%$ boys; $80 \%$ Caucasian; $\mathrm{MFSIQ}=74$, range $53-105$ ) who were referred for neuropsychological evaluations. Sixty percent had a comorbid diagnosis of ADHD. All children completed the following tasks: Digit Span, Coding, Symbol Search and the CLOX. Data were entered and analyzed via SPSS

Results: When examining the dual diagnosis group, parents reported clinical hyperactivity $(67 \%)$ and inattention $(100 \%)$. Teachers reported clinical hyperactivity $(67 \%)$, yet denied inattention. Cognitive profiles revealed $40 \%$ performed below average on Digit Span, $67 \%$ performed below average on both Coding and Symbol Search, while 100\% demonstrated impaired performance on the CLOX. Individual attention profile scores will be included in the poster.

Conclusions: Children with narcolepsy display elevated symptoms of hyperactivity and inattention both at home and at school. Although some research suggests that ADHD rating scales may be more indicative of narcolepsy symptomology instead of developmental ADHD, this study supports true cognitive deficits of inattention, slow processing, and executive dysfunction. Given the small sample size, further research investigating the relationship between narcolepsy and ADHD is needed. Correspondence: Ashlee R. Loughan, Ph.D., NeuroBehavioral Associates, 1355 Independence Dr, Augusta, GA 30901.E-mail: aspicer88@yahoo. com

\section{A. ALLEN, A.R. LOUGHAN, J. HERTZA \& M. COHEN. Language Deficits in Children; An Investigation into Left Hemisphere Cortical Dysplasia.}

Objective: Cortical dysplasia (CD) is a congenital disorder most often diagnosed in childhood which results in lesions forming on the cerebral cortex. Fifty percent of children with $\mathrm{CD}$ are also commonly diagnosed with epilepsy. Research indicates that these children have normal language development until seizure onset, when language regression begins. Limited research is available regarding the association between language regression and lesion hemisphere placement. In addition, no studies have investigated specific expressive versus receptive language deficits.

Participants and Methods: Data was collected on 8 children (Mage = 12: range 7-15) diagnosed with left-hemisphere $\mathrm{CD}$ who completed neuropsychological testing. Sixty-three percent were male and $63 \%$ were Caucasian, with IQs ranging from 46-95 (MFSIQ = 78). All children had a history of seizures. Data were entered and analyzed via SPSS.

Results: Specific focus was placed on neuropsychological expressive and receptive language measures. Profile analysis revealed object naming as a significant deficit for all children. Seven of the 8 children had deficits in both receptive and expressive language measures. Individual language profile scores will be included in the poster.

Conclusions: All children with CD performed poorly during a measure of object naming, and all children had difficulty with at least one language measure. Language deficits in both expressive and receptive measures were found in 7 of the 8 children investigated; suggesting global language deficits for left-hemisphere children with CD. Correspondence: Ashlee R. Loughan, Ph.D., NeuroBehavioral Associates, 1355 Independence Dr, Augusta, GA 30901.E-mail: aspicer88@yahoo. com

J.C. PIERCY, A. HEITZER, B. PETERS, J. NEWMAN, A. DEBASTOS \& S. RAZ. Infertility Treatment and Neuropsychological Functioning in Preterm-Born Preschoolers.

Objective: Assisted conception involves the use of methods such as ovulation induction (clomiphene citrate) and in vitro fertilization (IVF). Infertility treatments have been reported to be linked to multiple births and associated prematurity or low birth weight. Therefore, in the current study we wished to determine whether such treatments explained a unique portion of neuropsychological outcome variance within the population of preterm-born preschoolers.

Participants and Methods: We used data from 231 nondisabled preterm-born (<34 weeks gestation) preschoolers (129 males), graduates of the William Beaumont Hospital Neonatal Intensive Care Unit, Royal Oak, MI. IVF had reportedly been used in 30 cases and clomiphene in 10 cases in our sample. The Wechsler Preschool and Primary Scale of Intelligence - Revised (WPPSI-R), the Preschool Language Scale-Third Edition (PLS-3), and the Peabody Developmental Motor Scales 2nd Edition (PDMS-2) were used to measure cognitive, language, and motor skills, respectively.

Results: Infertility treatment accounted for a significant portion of the variance in the Full Scale IQ (FSIQ), over and above the variance explained by socioeconomic status, sex, gestational age, maternal age at birth, multiple gestation, adequacy of antenatal growth, and ventilation days $(\mathrm{F}[2,221]=3.20 ; \mathrm{p}<.043)$. Though still within the Average range, the adjusted mean FSIQ in the clomiphene group fell approximately a standard deviation lower $(91.64 \pm 4.8)$ than the means for the IVF $(104.80 \pm 3.24)$ and natural reproduction $(103.23 \pm 1.11)$ groups. Conclusions: In this study of preterm preschoolers, we found a link between clomiphene use and global intelligence, but not language or motor skills. Chlomiphene effects through accumulation over consecutive therapy cycles are believed to be biologically plausible. However, the findings should be interpreted cautiously because of the small number of cases and due to the fact that the effects of clomiphene could not be assessed separately from the effects of subfertility.

Correspondence: Sarah Raz, Ph.D., Merrill Palmer Skillman Institute, Wayne State Univ, Merrill Palmer Skillman Institute, 71 E. Ferry St., Detroit,MI 48202.E-mail:sarahraz@wayne.edu

M.E. DIQUATTRO, A. CONNER \& J. KIEFEL. A Case Study: Neurocognitive Trajectory of Adolescent with a Complex Medical History.

Objective: A complex medical history can impact neuropsychological functioning. The present case study examines the neuropsychological impact of an adolescent female (age 13) with multiple medical diagnoses, including mitochondrial disorder, epilepsy, very long-chain acy-Coa dehydrogenase deficiency (VLCAD), and dysautonomia. Mitochondria disorder presents with variable developmental, cognitive, and medical concerns. Impacted body systems are highly individualized; however, difficulties with cognitive functioning, visuospatial abilities, memory, and executive functioning skills are often reported. Epilepsy, which is common among individuals with mitochondrial disorder, also presents with a variable neurocognitive profile, with difficulties with attention/ executive functioning, memory, processing speed, visuoconstruction/ visuospatial skills, and academic/behavior functioning. VLCAD may impact fatigue, and one's ability to sustain performance in cognitive, academic, and adaptive tasks. Finally, research suggests that individuals diagnosed with dysautonomia demonstrate average to above average verbal skills; yet visual reasoning abilities are more impacted. Previous studies have evaluated the individual neuropsychological profile of each 
disorder; however, limited research is available regarding the comorbid presentation.

Participants and Methods: The patient was evaluated at three time points (ages 6:11, 9:9, and 13:11).

Results: Results revealed improved functioning in receptive language and aspects of visual-spatial skills. However, difficulties with executive functioning, verbal memory for discrete pieces of information, and visual-motor skills persist. Working memory, processing speed, vocabulary, aspects of academic functioning, visual-spatial skills, and mood presentation appear to be more significantly impacted overtime.

Conclusions: When compared to the neuropsychological profile previously reported with each individual disorder, this patient presented with more substantial cognitive and behavioral/emotional impact.

Correspondence: Megan E. DiQuattro, PsyD, Neuropsychology, Children's Healthcare of Atlanta, 371 North Ridge Drive, Senoia, GA 30276.E-mail: megan.diquattro@choa.org

D.M. GRAHAM, L. GLASS \& S.N. MATTSON. Deficits in Encoding but not Retention of Facial Stimuli in Children with Heavy Prenatal Alcohol Exposure.

Objective: Facial memory deficits in studies of children with prenatal alcohol exposure have been found inconsistently. As discrepancies may relate to small samples or varied definitions of memory, we examined immediate and delayed recall of facial stimuli in a large sample.

Participants and Methods: Children with heavy prenatal alcohol exposure $(\mathrm{AE} ; \mathrm{n}=60)$ and controls $(\mathrm{CON} ; \mathrm{n}=52)$ completed immediate and delayed facial memory tasks from the NEPSY-II. Data were analyzed using a 2(Group: AE/CON) x 2(Condition: Immediate/Delayed) repeated-measures ANCOVA. Visuospatial processing was assessed using NEPSY-II Design Copying. The contribution of global and local visuospatial skills to facial memory performance was tested via hierarchical regression analyses.

Results: The AE group was impaired on all four NEPSY-II scores. While immediate and delayed recall of facial stimuli were both impaired, retention was intact. Further, in the AE group only, global but not local visuospatial processing contributed significantly to immediate recall of facial stimuli.

Conclusions: Impairments in recall of facial stimuli in alcohol-exposed children were related to encoding rather than retention of information. This pattern supports some but not all previous studies of visual memory and suggests that previous facial memory deficits were likely due to encoding deficits. Alterations in encoding facial stimuli may relate to other impairments in this population such as those in social problem solving. Further, global visuospatial processing contributed to performance in the AE group only, suggesting that interventions targeting global processing may improve encoding of facial information and ultimately improve social skills.

Research supported by NIAAA grants R01 AA019605 and F31 AA022261.

Correspondence: Sarah N. Mattson, Ph.D., Psychology, San Diego State University, 6330 Alvarado Court, Suite 100, San Diego, CA 92104. E-mail:sarah.mattson@sdsu.edu

A.M. COLBERT, R. LAJINESS-0'NEILL, F. HOODIN, R. KENTOR, K. HEINRICH \& J. CONNELLY. Effects of Processing Speed and Memory on Academic Outcomes in Children Following Allogeneic Hematopoietic Stem Cell Transplantation.

Objective: Allogeneic Hematopoietic Stem Cell Transplantation (HCT) is a treatment for many severe pediatric diseases. Unfortunately, the prevalence of late effects is high $-79 \%$ of HCT recipients experience chronic medical conditions. The few extant studies of cognitive late effects have focused on global measures of intelligence and are equivocal about potential HCT neurotoxicity.

Participants and Methods: Our archival study of 30 children (mean transplant age $=6$ years, mean time since HCT $=5$ years), aimed to characterize neuropsychological predictors of academic outcomes, focusing on processing speed, attention and memory.

Results: Mean intellectual and academic abilities were grossly average, but evidenced extreme variability. Similarly, attention and memory measures evidenced substantial variability, about $25 \%$ of the sample achieved borderline impaired performance or lower. In regression models exploring predictors of academic outcomes, processing speed and memory accounted for $69 \%$ and $61 \%$ of the variance in mathematics and reading, respectively. Exploration of medical and demographic predictors of outcome revealed longer intervals between HCT and testing were related to decrements in IQ, attention and memory. Non-cord blood recipients had lower visual-constructional skills; participants with busulfan conditioning regimens had poorer reaction time and visual memory; participants with chronic graft-versus-host disease (GVHD) had poorer visual-spatial and visual-motor functioning; those with acute GVHD and those who underwent steroid treatment paradoxically demonstrated better memory.

Conclusions: Our findings reveal processing speed and memory may strongly influence academic outcomes following HCT, and illuminate a potential resilience or neurotrophic factor for memory that requires replication and exploration.

Correspondence: Alison M. Colbert, Psychology, Eastern Michigan University, 810 1/2 N. Congress, Ypsilanti, MI 48197. E-mail: alison. mh.colbert@gmail.com

A. LUCCHETTI, T. DROSSOS, E. FITZSIMMONS-CRAFT, K. ANDERSON, E. ACCURSO, C. STILES-SHIELS, C. SKELLY \&. M. SZTAINER. Psychosocial Profiles in Pediatric Patients with Median Arcuate Ligament Syndrome.

Objective: Median Arcuate Ligament Syndrome (MALS) is a poorly understood vascular compression syndrome involving the celiac artery (Harjola, 1963). Less than 1\% of individuals with MALS experience painful gastrointestinal symptoms (Horton et al., 2005). MALS has overlapping physical symptoms with chronic abdominal pain (CAP; Mak et al., 2013); however, the psychosocial profile is relatively unknown. Children with CAP have increased risk for psychiatric and academic problems (Ramchandani et al., 2007). This study investigates the psychosocial profiles of pediatric patients with MALS.

Participants and Methods: Thirty participants (ages 8-17, 87\% female) completed a preoperative psychological evaluation, including a semi-structured interview (MINI, Sheehan et al., 2010), Children's Depression Inventory (CDI; Kovacs, 1985), Multidimensional Anxiety Scale for Children (MASC; March et al., 1997), and Pediatric Quality of Life Inventory (PEDsQL; Varni et al., 2001).

Results: Forty three percent of patients met criteria for a psychological disorder, with anxiety disorder being the most prevalent (23\%). Thirty seven percent had utilized therapy services and $30 \%$ took psvchotropic medication. Scores on the CDI $(M=8.1, S D=6.3)$ and MASC $(M=47.1$, $\mathrm{SD}=11.9$ ) did not indicate clinical elevations. An independent samples t-test revealed MALS patients had significantly poorer health-related quality of life on the PEDsQL when compared to patients with irritable bowel disease $(t=5.06, p>.01)$.

Conclusions: This study is the first to provide the preoperative psychosocial profiles of pediatric patients with MALS. Results indicate elevated risk for psychological distress consistent with findings in patients with CAP. A multi-disciplinary approach to treat these patients is imperative. Correspondence: Amanda Lucchetti, MA, Psychiatry and Behavioral Sciences, Children's Hospital Colorado, 5100 Leetsdale Drive, Unit 401, Denver, CO 80246.E-mail: amandalucchetti@gmail.com

S. RAU, L.A. JACOBSON, A.E. PRITCHARD \& T. ZABEL. An Examination of Factors Contributing to Sluggish Cognitive Tempo (SCT) in Spina Bifida (SB) and ADHD, Inattentive Type (ADHD-I). Objective: Individuals with SB often have brain dysmorphology that contributes to inattention and executive dysfunction, specifically low initiation. ADHD-I is also commonly diagnosed in individuals with SB. 
As low initiation is an aspect of the SCT phenotype, the present study had two aims: 1) Examine whether individuals with SB or ADHD-I have greater symptoms of SCT relative to a community sample and 2) delineate differences in SCT symptom presentation between the two clinical samples

Participants and Methods: Parent report data on ADHD and SCT symptoms were obtained for a clinically referred sample of patients with SB ( $\mathrm{N}=27,63 \%$ female) and age and verbal IQ-matched ADHD-I sample ( $\mathrm{N}=27,59 \%$ female). Analyses included one-sample t-test and independent samples t-test.

Results: Mean SCT scores for the clinical samples were significantly higher than the community sample mean (SB:t $(26)=5.09, \mathrm{p}<.05$; ADHD-I:t $(26)=9.35, \mathrm{p}<.05)$. Examination of between group differences on SCT symptoms using indepdent samples t-test revealed the ADHD-I sample had significantly higher ratings on $2 / 3$ factors of SCT. However, when inattention symptoms were residualized, differences between the SB and ADHD-I groups were markedly attenuated (i.e.,all p>.10). Conclusions: Individuals with SB and ADHD-I exhibit significantly more symptoms of SCT relative to a community sample. However, differences in symptoms of SCT between SB and ADHD-I are driven by symptoms of inattention suggesting SCT may not be a meaningful construct upon which to differentiate groups with SB from ADHD-I. Correspondence: Srishti Rau, M.A., Neuropsychology, Kennedy Krieger Institute, 5647 Columbia Rd, Apt 103, Columbia, MD 21044. E-mail: 56seth@cardinalmail.cua.edu

D. BADALY, E.K. HODGES, C. GOLDBERG \& K.P. HEINRICH. Cognitive, Behavioral, and Socioemotional Functioning in Children with Congenital Heart Disease Compared to Children Diagnosed with Attention-Deficit/Hyperactivity Disorder.

Objective: Similar to their peers diagnosed with attention-deficit/hyperactivity disorder (ADHD), children with congenital heart disease (CHD) tend to display inattention, impulsivity, and executive dysfunction, and are at risk for socioemotional maladjustment (Marino et al., 2012). Yet, little research has directly compared the functioning of these two groups (Hansen et al., 2012). We examined cognitive, behavioral, and socioemotional outcomes in children with CHD compared to children with ADHD.

Participants and Methods: Participants were 15 children with CHD (10 male; $M$ age $=9.28$ years) and 20 children with ADHD (16 male; $M$ age $=10.02)$ consecutively referred to an outpatient neuropsychology clinic. Cognitive measures included attention, impulsivity, and executive functioning (WISC-IV Working Memory, CPT Commissions, Trails B). The Conners-3 provided parent ratings of related behaviors. Measures of socioemotional functioning included parent ratings (Conners-3 Peer Relations) and self-report ratings (CDI-2, MASC).

Results: T-tests showed that children with CHD and ADHD did not differ on cognitive measures of attention, impulsivity, or executive functioning $(t s<1.98, p s>.05)$. However, parents rated children with ADHD as displaying more problematic behaviors in terms of inattention, hyperactivity/impulsivity, and executive functioning than those with CHD $(t \mathrm{~s}>2.17, p \mathrm{~s}<.05)$. Children with ADHD were also rated as having poorer peer relationships $(t=2.60, p=.01)$ and reported greater anxiety $(t=2.84, p=.01)$ than those with CHD. Across groups, behavioral ratings but not cognitive measures correlated with socioemotional outcomes.

Conclusions: Our findings suggest that, although children with CHD display fewer behaviors indicative of inattention, impulsivity, and executive dysfunction than peers with ADHD, these behaviors may similarly place them at risk for socioemotional maladjustment. As such, our results underscore the need to assess and treat such behaviors among children with CHD.

Correspondence: Daryaneh Badaly, PhD, Psychiatry, University of Michigan, 1311 Natalie Ln Apt 301, Ann Arbor, MI 48105. E-mail: d.badaly@gmail.com
D.L. COOL, V. MONTGOMERY, T. JERARD, F.T. CHARBEL \& J. JANECEK. Pre- and Post-Cerebral Bypass Neuropsychological Functioning of a 17-year-old Female with Moyamoya Disease and Atypical Cerebral Organization.

Objective: Moyamoya disesase is a rare, chronic, and progressive occlusive cerebrovascular condition that typically affects the internal carotid arteries and their proximal branches (Williams et al., 2011). The current case report sought to integrate the neuropsychological literature pertaining to moyamoya disease and atypical cerebral organization. The literature was then compared with a case of a 17-year-old left-handed female with sickle cell anemia, moyamoya disease, and a history of stroke at age 3 who underwent left-sided STA-MCA bypass with EDAS. Participants and Methods: The patient underwent a comprehensive neuropsychological examination, including medical chart review, clinical interview, and neurocognitive testing at 6 weeks pre-cerebral revascularization and again at 6 months post-cerebral revascularization. Standardized test scores were compared pre- and post-revascularization with cerebral bypass.

Results: Results from the comparison showed significant improvements $(\geq 1 \mathrm{SD})$ in the areas of perceptual reasoning (WAIS-IV Matrix Reasoning subtest), visuoconstruction (Rey Complex Figure Copy trial; WMS-IV Visual Reproduction Copy), visual learning (WMS-IV Visual Reproduction I), verbal memory (WMS-IV Logical Memory II), visual attention (Gordon Diagnostic System Vigilance and Distractibility trials), phonemic fluency (D-KEFS Letter Fluency trial), and non-dominant manual speed (Finger Tapping).

Conclusions: Atypical cerebral organization has been associated with shifts in manual dominance, language dominance, and re-organization of visuospatial cognitive functions (Satz, 1972). For this patient, it appears that increased left cerebral hemisphere perfusion was associated with improvements in a number of domains typically considered to be mediated by the right hemisphere, including perceptual reasoning, visual memory, visuoconstruction, and visual attention.

Correspondence: Danielle L. Cool, M.A., Psychology, Roosevelt University, 675 Lake St., Apt 314, Oak Park, IL 60301.E-mail: dcool@ mail.roosevelt.edu

S.J. HARDY, A. THOMPSON, S.A. HOSTETTER, S. WISE, K. OLSON \& K.K. HARDY. Neurocognitive Functioning in Pediatric Sickle Cell Disease: A Cumulative Risk Perspective.

Objective: Disease-related neurocognitive deficits are common in pediatric sickle cell disease (SCD). Youth with SCD also often present with psychosocial and socioeconomic factors known to affect cognitive outcomes in the general population. Using a cumulative risk model, we explored the influence of psychosocial/socioeconomic factors on neurocognitive and academic performance in SCD.

Participants and Methods: Youth with SCD (n=20) ages 7-16 completed a brief neuropsychological assessment; caregivers completed questionnaires. A Cumulative Risk Index (CRI) was calculated by summing dichotomized variables representing absence/presence of psychosocial, socioeconomic, and disease-related risk factors (e.g., family income, parent education, SCD severity). Outcomes included the WASI, WJ-III fluency subtests, Alloway Working Memory Assessment-Short Form (AWMA-S), and BRIEF.

Results: Sample was $55 \%$ female with mean age of 11.5 years (SD=2.8). Mean WASI scores were in the low average range (FSIQ M=87.48, $\mathrm{SD}=9.23$; VCI $\mathrm{M}=87.00, \mathrm{SD}=11.40$; $\mathrm{PRI} \mathrm{M}=90.38$, $\mathrm{SD}=10.52)$. Academic fluency was also below average, with lowest scores observed in Math Fluency $(M=83.30, \mathrm{SD}=14.55)$. AWMA scores fell in the average range, with greatest difficulty observed on Dot Matrix $(M=90.90$, $\mathrm{SD}=21.04)$. BRIEF scores were generally in the normal range. The CRI, ranging from 0-9, was significantly correlated with math fluency, nonverbal working memory, and the BRIEF Metacognition Index ( $\mathrm{r}=-.51$, $-.45, .46$, respectively).

Conclusions: Our sample of youth with SCD demonstrated low average cognitive abilities and academic fluency and mild deficits in executive 
functioning. Possessing multiple psychosocial, socioeconomic, and disease-related risks was associated with poorer math fluency and executive function. A multidimensional perspective on risk may lead to a more comprehensive understanding of neurocognitive functioning in pediatric SCD. This project was supported by Award Number UL1TR000075 from the NIH National Center for Advancing Translational Sciences. Correspondence: Steven J. Hardy, PhD, Hematology and Oncology, Children's National Health System, 111 Michigan Ave, NW, Washington, DC 20010.E-mail:sjhardy@childrensnational.org

\section{CASNAR, K. RIVERA, M. HELMS \& B.P. KLEIN-TASMAN. Parent and Teacher Perspectives on BASC-II Content Scales in Young Children with NF1.}

Objective: Children with NF1 are at increased risk for attention problems, executive functioning difficulties, and social and emotional challenges. In this study, parent and teacher report of social and emotional functioning in everyday contexts was examined for young children using the Behavior Assessment System for Children - Second Edition (BASC-II) content scales.

Participants and Methods: Participants were 62 children (37 boys) with NF1 ages 3 through $8(M=4.95, \mathrm{SD}=1.62)$. Teacher report was available for a subset of children (33 children, \# boys) ages 3 through $8(\mathrm{M}=5.16, \mathrm{SD}=1.59)$.

Results: Overall, all ratings of social and emotional functioning were in the normal range. Children with NF1 were rated significantly higher than the normative mean by their parents on the Developmental Social Disorders (DSD) scale $(\mathrm{t}(\dot{61})=2.88, \mathrm{p}=.005)$. Executive Functioning $(\mathrm{EF})$ and Negative Emotionality (NE) were not significantly elevated in comparison to the normative mean, but trends towards elevation were observed $(\mathrm{t}(61)=1.80, \mathrm{p}=.077 ; \mathrm{t}(61)=1.96, \mathrm{p}=.055)$. Teachers rated children with NF1 significantly higher than the normative mean on the EF scale $(t(32)=2.11, p=.04)$. A significant correlation between parent and teacher ratings of Bullying $(\mathrm{r}=.52, \mathrm{p}=.002)$, DSD ( $\mathrm{r}=.47, \mathrm{p}=.006)$ and Resiliency ( $r=0.434, p=.012$ ) was found, with no other significant correlations between raters. Relations to cognitive abilities, age and gender will also be examined.

Conclusions: This study demonstrates that the BASC-II content scales may be useful to measure problem and adaptive behaviors in young children with NF-1. Identification of early difficulties in psychosocial functioning may help children with $\mathrm{NF}-1$ receive the interventions they need.

Correspondence: Christy Casnar, M.S., University of Wisconsin Milwaukee, P.O. Box 413, Garland Hall, Rm 325, Milwaukee, WI 53201.E-mail: clcasnar@uwm.edu

\section{S. Whiting, C.W. KARLSON \&. E. MANNING. Cognitive and Psychosocial Functioning in Children with Sickle Cell Disease versus ADHD.}

Objective: Children with SCD are at risk for frontal-lobe strokes, which are related to impairments in intelligence, attention, visual-motor skills, and academic achievement. Even children with SCD who do not have a known cerebral infarct show similar reduced cognitive abilities. Children with Attention-Deficit/Hyperactivity Disorder (ADHD) also show reduced academic achievement, attention, and language performance. However, the similarities between these populations have not been determined. It was hypothesized that children with SCD will demonstrate cognitive deficits similar to those with ADHD.

Participants and Methods: Participants were 39 children with SCD $(\mathrm{M}=11.3 ; \mathrm{SD}=3.9$; African American $=97 \%)$ and 46 with ADHD $(\mathrm{M}$ $=10.6 ; \mathrm{SD}=3.7$; African American = 43\%). Children completed neurocognitive testing at pediatric hematology or neuropsychology clinics as part of clinical care. IRB approval was obtained for chart review. Test batteries include the WISC-IV, WAIS-III, or WASI (intelligence), WIAT-II (academic achievement), CVLT-C (verbal memory), CPT-II (attention), and CBCL (behaviors).
Results: One-way ANOVA revealed lower scores in the SCD group on academic achievement $(\mathrm{p}$ 's $<0.05)$ and false positive verbal memory recognition $(p<0.05)$. There was a trend for lower total verbal memory scores in the SCD group $(p=.07)$. Children with SCD had greater Somatic Complaints $(\mathrm{p}=.01)$ and higher Thought Problems $(\mathrm{p}=.04)$ scores. No other significant differences were found.

Conclusions: Children with SCD had similar cognitive and behavioral functioning as children with ADHD but showed lower academic achievement and verbal memory. Children with SCD may be at particular risk for academic difficulties. Academic assessment and support services, similar to those for ADHD, should be made readily available for children with SCD.

Correspondence: Sara Whiting, MA, Department of Psychiatry and Human Behavior, University of Mississippi Medical Center, $2500 \mathrm{~N}$. State Street, Jackson, MS 39216.E-mail: rwhiting@umc.edu

\section{K. OLSON, S.J. HARDY, S. WISE \& K.K. HARDY. Predictive Validity of Parent-Reported Working Memory in Pediatric Sickle Cell Disease.}

Objective: Youth with sickle cell disease (SCD) are at risk for neurocognitive deficits, particularly in the domain of working memory (WM). Our objective is to better understand WM abilities in a pediatric sample with SCD through analysis of parent-reported and performance-based measures of visual and verbal WM.

Participants and Methods: Children and adolescents (n=20) ages 7-16 with sickle cell disease enrolled in a home-based, computerized cognitive training program (Cogmed). All participants completed a pre-intervention neuropsychological evaluation that included the Behavior Rating Inventory of Executive Function (BRIEF) and the Alloway Working Memory Assessment (AWMA).

Results: The sample was $55 \%$ female $(n=11)$ with mean age of 11.5 years $(\mathrm{SD}=2.8)$. WM was the most elevated subscale on the BRIEF $(\mathrm{M}=63.70, \mathrm{SD}=12.81) ; 50 \%$ of the sample $(\mathrm{n}=10)$ had clinically significant WM scores. BRIEF WM score significantly predicted AWMA simple visual WM scores, $\beta=-.500, p=.025$, R2Adjusted $=.21$, and AWMA complex visual WM scores, $\beta=-.596, p=.006$, R2Adjusted $=.32$. After controlling for variance accounted for by age, sex, and intelligence, BRIEF WM score predicted a significant portion of unique variance in simple visual WM (R2change $=.28, \mathrm{p}<.05)$, complex visual $\mathrm{WM}(\mathrm{R} 2$ change $=.37$, $\mathrm{p}<.01)$, and complex verbal WM (R2change $=.20, \mathrm{p}<.05)$.

Conclusions: Decrease in parent-reported problems with WM predicted increase in performance-based visual WM abilities. The BRIEF WM subscale demonstrated unique predictive validity for simple and complex visual and complex verbal WM in children with SCD. Repeated administration of the BRIEF may allow for earlier detection of subtle changes in WM without concern for practice effects associated with performance-based measures.

Correspondence: Katie Olson, PsyD, Hematology \& Oncology, Children's National Medical Center, 111 Michigan Ave., Washington, DC 20010. E-mail:kaolson@childrensnational.org

T. KENNEDY, A.C. ARMOUR, V. TERWILLIGER, M. BERL, M.T. DONOFRIO \& J. SANZ. Emotional and Behavioral Outcomes in School Age Children with Congenital Heart Disease.

Objective: Children with congenital heart disease (CHD) have high rates of neurodevelopmental problems. We evaluated the prevalence and pattern of parent reported behavioral/emotional concerns in school aged children with CHD.

Participants and Methods: Parents of children with complex CHD completed questionnaires by mail (Child Behavior Checklist (CBCL), demographic survey). A control group was selected with equivalent mean age and gender distribution. MANOVA was used to evaluate subscale T-scores and Chi-Square analyses/odds ratios were used to evaluate frequency of clinical elevations in relation to type of CHD, neurological abnormalities, gender, and prematurity. 
Results: 172 children with mean age $9.0+/-2.7$ years; 104 male, were included. The 86 children with CHD had worse scores and higher rates of clinical elevations across domains (all $\mathrm{p}<0.01)$. Males with CHD had worse scores, were 6.67 times more likely to report aggressive behavior and 11.20 times more likely to report ADHD symptoms $(\mathrm{p}<0.01)$. Children with CHD born prematurely had worse scores, and were 3-6 times more likely to report inattention, somatic complaints, affective symptoms, oppositional defiance, conduct problems $(p<0.01)$, aggressive behavior, thought problems, and ADHD $(p<0.05)$. Type of CHD or neurological abnormalities did not impact emotional/behavioral outcome.

Conclusions: School age children with CHD have high rates of behavioral/emotional problems. Male gender and prematurity are associated with increased risk of inattention and disruptive behavior. Type of CHD and neurological findings did not relate to behavioral/emotional outcomes; thus, future studies comparing children born premature to children with CHD can help determine relative influence of CHD versus other risk factors such as prematurity/male gender.

Correspondence: Jacqueline Sanz, Children's National, 111 Michigan Ave., NW, Washington, DC 20010.E-mail: jsanz@cnmc.org

A.C. ARMOUR, T. KENNEDY, L. KENWORTHY, M.T. DONOFRIO \&. J. SANZ. Emotional and Behavioral Outcomes in School Aged Children with Congenital Heart Disease (CHD) and Autism.

Objective: Previous work shows high rates of behavioral/emotional concerns in children with CHD relative to controls, and similarities in executive function profiles in some children with CHD to autism. We compared behavioral/emotional concerns in CHD and autism.

Participants and Methods: 172 children with mean age $=9.21$ ( $\mathrm{SD}=2.64), 104$ males were included. Parents of children with CHD completed the Child Behavior Checklist (CBCL) by mail. Children with autism with equivalent mean age and gender distribution were selected from a database. Participants were divided into four groups by gender and diagnosis (males/CHD, females/CHD, males/autism, females/autism), and T-scores for DSM scales were evaluated with MANOVA and independent sample t-tests for post-hoc analyses.

Results: All main effects and interactions were significant $(p<.01)$, suggesting that the four groups have distinct profiles. Post-hoc tests suggest males with CHD have more elevations than females with CHD $(p=.03)$, but similar elevations to males/females with autism ( $p>.97)$. Females with CHD generally have fewer concerns than other groups $(p<.06)$. Post-hoc t-tests suggest male children with CHD do not differ significantly from male children with autism, but differ from female children with autism for somatic problems $(p<0.05)$.

Conclusions: Consistent with prior findings, male children with CHD are at greater risk for emotional/behavioral problems than female children with CHD. The emotional/behavioral profile of male children with CHD is similar to that of children with autism for mood, anxiety, and attention. Future work should examine prevalence of DSM diagnoses in children with CHD by gender, which may represent a risk factor for CHD psychosocial outcomes.

Correspondence: Jacqueline Sanz, Children's National, 111 Michigan Ave., NW,Washington,DC20010.E-mail: jsanz@cnmc.org

E. WESONGA, A. HOOD, J.S. SHIMONY, R.C. MCKINSTRY, D.K. GRANGE \& D.A. WHITE. Difference in the Relationship Between Age and Mean Diffusivity of White Matter in Children with Phenylketonuria.

Objective: Phenylketonuria (PKU) is a metabolic disorder associated with cognitive impairment and neurological abnormalities. Advances in diffusion tensor imaging (DTI) have allowed us to detect age-related declines in the microstructural integrity of white matter in children with PKU (White et al., 2010). However, decreases in diffusivity of white matter have also been reported in healthy children (Lebel et al., 2008). The present study examined whether or not the relationship between age and mean diffusivity (MD) is significantly different between children with PKU and healthy children.

Participants and Methods: Children with PKU ( $\mathrm{n}=31, \mathrm{M}=12.2$ ) were recruited through metabolic clinics and compared with healthy control children ( $n=54, M=12.1$ ). Ten ROIs were established prior to MRI. Several measures of diffusivity (e.g. MD) were acquired during DTI analyses of the structural images. Within-group Pearson correlations were obtained between age and MD for each ROI. Fisher's Z transformations were conducted on each correlation, and then significance tests were conducted on the difference between the correlations for each group.

Results: The relationship between age and MD was significantly different between the PKU and control groups for the optic radiation, hippocampus, and genu of the corpus callosum $(\mathrm{z}<-1.96)$. All ROIs demonstrated an inverse relationship between age and MD for both groups, such that older children had lower levels of MD. This relationship was significant for nine total ROIs within the PKU group, as compared with only five total ROIs within the control group.

Conclusions: We observed a stronger age-related decline in the mean diffusivity of three ROIs for children with PKU than for healthy children. Given the cognitive deficits typically observed in PKU, it is surprising that additional ROIs did not demonstrate significant differences between correlations of age and MD. Further examination of longitudinal data is needed to increase our understanding of trends of white matter development in PKU.

Correspondence: Erika Wesonga, Psychology, Washington University in St.Louis, St.Louis, St.Louis, MO 63108.E-mail: ewesonga@wustl.edu

\section{K.L. DINH \& K.L. NETSON. Neuropsychological Sequelae of} Opsoclonus-Myoclonus Syndrome: A Case Series.

Objective: Opsoclonus-Myoclonus Syndrome (OMS) is a rare movement disorder which typically presents in infancy. Clinical features of the disorder include erratic eye movements and generalized myoclonus.

Participants and Methods: The first case study involves a 3 year-old Caucasian boy diagnosed with OMS at 23 months of age. His treatment included chemotherapy, IVIG infusions, and ACTH, and he was asymptomatic at the time of neuropsychological evaluation.

The second case study involves a 6 year-old Caucasian boy diagnosed with OMS at 13 months of age. He had experienced multiple relapses despite treatment with chemotherapy and monthly IVIG infusions. Re-evaluation was also conducted following a dexamethasone treatment regimen.

Results: Case 1:

Intellectual functioning was in the borderline range (DAS-II GCA=79), while pre-academic skills and adaptive functions were at age-appropriate levels. Speech articulation was a weakness. No emotional or behavioral problems were present.

Case 2:

Intellectual functioning was in the borderline range (WISC-IV FSIQ=74). Executive functioning, attention, and motor dexterity were impaired; verbal memory, expressive vocabulary, and visuospatial skills showed mild weaknesses. Difficulties with behavioral regulation and speech articulation were also noted. At post-treatment re-evaluation four months later, FSIQ=83. Improvements were also noted in verbal memory and motor dexterity.

Conclusions: These neuropsychological presentations are largely consistent with other published case series on OMS. In general, OMS results in reduced global intellect, problems with speech and language, and behavioral dysregulation. Cognitive prognosis is heavily dependent upon management of OMS-related symptoms. Given the rarity of OMS, greater awareness of the disorder and its clinical manifestations is needed.

Correspondence: Kara L. Dinh, Psy.D., Neuropsychology, University of Kansas School of Medicine - Wichita, 9911 E. 21st St. N, Apt. 308, Wichita, KS 67206.E-mail: kshaneyfelt@gmail.com 
S.M. GREIF, E.M. MELLOTT, K.A. SWITALSKI, M. UDHNANI, A.L. FRIAS, S.C. HEATON \& P.A. JOHNS. Cognitive, Emotional, and Behavioral Features as Predictors of Adaptive Functioning in a Clinical Pediatric Population.

Objective: Adaptive functioning (AF) is a multidimensional construct measuring the effectiveness with which an individual is able to navigate everyday tasks. Impairment of adaptive abilities is commonly seen in clinical pediatric populations, and has been linked long-term to both decreased quality of life and lifetime achievement. Our study aims to address specific cognitive and behavioral features which may serve as predictors of overall levels of adaptive functioning via analyses of the Wechsler Intelligence Scale for Children, Fourth Edition (WISC-IV) and the Behavior Assessment System for Children, Second Edition, Parent Rating Scale (BASC-2-PRS).

Participants and Methods: A mixed pediatric clinical sample, comprised largely of medical/neurological patients, of 436 patients aged 6 -16 underwent neuropsychological evaluation including WISC-IV and BASC-2PRS administration. Statistical analysis included Exploratory Factor Analyses (EFA) of specific subscales of the BASC-2PRS and Composite Indices of the WISC-IV. These resulting factors were then entered into path analysis using The Analysis of Moment Structures, Version 5 (AMOS-5).

Results: Increased endorsement of problem behavior on all three factors of the BASC-2PRS predicted a decrease in AF, increased Cognitive Function positively predicted AF. Across the entire sample, and within sub-groups the Hyperactivity/Inattention $(p=0.001)$ construct consistently held the greatest relative magnitude as a predictor of adaptive functioning.

Conclusions: These data strongly suggest that formal evaluation of adaptive functioning may be relevant in children exhibiting internalizing symptoms, externalizing symptoms, and especially symptoms of inattention/hyperactivity, in addition to the classic consideration of a child with suspected low IQ. Relative magnitude of predictors may further aid physicians in treatment planning, and increasing accuracy of prognosis. Correspondence: Sarah M. Greif, BS, Clinical and Health Psychology, University of Florida, 507 NW 39th Rd, APT 202, Gainesville, FL 32607. E-mail:sgreif1021@phhp.ufl.edu

\section{Invited Symposium: \\ The Young Damaged Brain: A Symposium in Honor of Maureen Dennis}

\section{Organizers: Jack M. Fletcher, Brenda Spiegler}

\author{
Discussant: Erin D. Bigler
}

$$
\text { 3:15-4:45 p.m. }
$$

J.M. FLETCHER, E.D. BIGLER, L. TURKSTRA, R.J. SCHACHAR, J. JURANEK \& H.G. TAYLOR. The Young Damaged Brain: A Symposium in Honor of Maureen Dennis.

Symposium Description: This symposium involves some of the themes prominent in Maureen Dennis's many contributions to child neuropsychology. Throughout her career, Dr. Dennis applied concepts and methods from cognitive neuroscience, cognitive and experimental psychology and developmental psychology to atypical development, focusing on the effects of brain injury on the organization of function in children with acquired and developmental brain disorders. Among her more prominent contributions were multiple considerations of how language is organized after brain injury in children, most recently in relation to social cognition. She had an enduring interest in endophenotypes and cross-disorder comparisons, exemplified by her work in both language and attention. Dr. Dennis had a fascination with the cerebellum and disorders associated with damage "under the tent," with theoretical and methodological contributions to the role of the cerebellum in attention, cognition and motor function. The enduring theme tying together all of her work was an abiding interest in neural plasticity, which represented her earliest contributions and was a theme she returned to at the end of her career. Metaphorical and allegorical, Maureen Dennis was a major figure in child neuropsychology; her ideas will endure through her writings and her influence on her many students and colleagues

Correspondence: Jack M. Fletcher, PhD, Department of Psychology, University of Houston, 126 Heyne, Houston, TX 77204. E-mail: jack. fletcher@times.uh.edu

\section{TURKSTRA. Different Routes to Pragmatic Communication} Impairment in Adolescence.

Impairments in pragmatic communication characterize a variety of developmental and acquired disorders in adolescence, when social language problems may surpass limitations in formal language as a predictor of everyday success. Maureen Dennis was interested in the possibility of cross-disorder endophenotypes. Pragmatic communication phenotypes may overlap across disorders, and interventions designed for one disorder often are used with phenotypically similar others. Despite phenotypic similarities, substantial differences in underlying mechanisms across disorder groups mean that intervention designed for one group might be ineffective for another. To illustrate the importance of considering underlying mechanisms in clinical decision making, this talk summarizes the unique cognitive contributors to pragmatic language impairment in four groups with overlapping pragmatic communication phenotypes: Autism Spectrum Disorder, Specific Language Impairment, fragile $\mathrm{X}$ syndrome, and traumatic brain injury.

Correspondence: Lyn Turkstra, PhD, 474 Goodnight Hall, 1975 Willow Dr., Madison,WI 53706.E-mail: lsturkstra@wisc.edu

R.J. SCHACHAR. Etiology of Attention Deficit Hyperactivity Disorder (ADHD): Lessons from Cognitive Function and Traumatic Brain Injury.

ADHD is a complex, some would say "messy" phenotype that lacks precision and undermines efforts to dissect its pathophysiology. Cognitive deficits are common among individuals with ADHD and can be more rigorously defined than the behavioral phenotype thereby facilitating precision in research. Maureen Dennis focused on these types of phenotypic distinctions in many disorders characterized by attentional impairment, including deficient response inhibition, which is thought to be a signature deficit in ADHD. Measures of response inhibition and their psychometric properties are described. Evidence for deficits in various components of response inhibition is critically reviewed and evidence is presented to show that response inhibition is a stable, heritable and biologically informative trait that can leverage the understanding of the genetic and neural mechanisms of ADHD. Studies conducted with Maureen Dennis of response inhibition and ADHD secondary to traumatic brain injury afford insight into the differences between primary and secondary ADHD. Genetic studies in the general population and functional neuroimaging studies informed by an understanding of response inhibition are reviewed. These studies point to genetic and neural risk factors in ADHD and identify candidate functional pathways for future research.

Correspondence: Russell J. Schachar, MD, FRCPC, The Hospital for Sick Children, 555 University Avenue, Toronto, ON M5G 1X8, Canada. E-mail: russell.schachar@sickkids.ca

J. JURANEK. The Cerebellum in Neurodevelopmental Disorders. Despite our tendencies to be cerebrocentric in our investigations of human brain and behavior, recent advances in neuroimaging methods have demonstrated that looking under the tentorium is "not so boring" after all. In severe cerebellar malformations including Dandy-Walker, Joubert syndrome, and pontocerebellar hypoplasia, the orchestrated processes underlying hindbrain development in utero are either disrupted or poorly-timed. As Maureen Dennis pointed out in a major theoretical paper and many studies, the orchestration of cerebellar development in Chiari 
II is quite complex, involving a combination of regional volumetric reduction and enlargement. How these structural anomalies influence measures of behavioral performance is a key area of research interest. Children with spina bifida are most commonly known for motor deficits in lower limb function. However, a growing body of literature has described relative assets in other motor functions, particularly in motor learning. Across different muscle groups and with different forms of movement, children with SBM have consistently demonstrated a somewhat surprising ability to learn new motor skills, despite pronounced quantitative structural differences in both the cerebellum and striatum. Thus, anomalous structural development is not necessarily synonymous with behavioral deficits. Instead, some anomalies somehow afford "better than expected" behavioral performance. Such findings challenge our old fashioned views of brain organization and inspire us to look for clues about reorganization of function and neural plasticity.

Correspondence: Jenifer Juranek, UT Health Science Center at Houston, 6655 Travis, Houston, TX 77030-1312.E-mail: Jenifer.Juranek@uth. tmc.edu

H.G. TAYLOR. Plasticity of Function After Childhood Brain Injury. Maureen Dennis's historical analysis of Kennard's early work and of more recent findings on outcomes of pediatric brain injuries reveals several principles that are useful in summarizing current knowledge on plasticity of function. The aims of this presentation are to: 1) briefly summarize the foundational discoveries by Kennard, 2) consider the multiple factors related to longitudinal change after early brain insults, including the effects of age, time since injury, and other medical and non-medical risk factors, and 3) cite examples of plasticity from research on outcomes of childhood brain disorders. Evidence for plasticity will be evaluated in terms of the extent and nature of developmental sparing following early brain insults, the need to evaluate different types of outcomes, and the possibilities and limitations of neural reorganization. Consistent with Dr. Dennis's many intellectual and research contributions to the field, emphasis will be placed on the need for a nuanced conceptualization of the effects of childhood brain injury-one that emphasizes variations in developmental change after insult, moderating influences on these changes, and longer-term consequences. Discussion will include the implications of this nuanced view of plasticity for research, clinical assessment, and intervention.

Correspondence: H. G. Taylor, PhD, 10524 Euclid Ave Ste 3150, Cleveland, OH 44106.E-mail: hgt2@case.edu

\section{Paper Session 6: \\ TBI - Functional Imaging}

\section{3:15-4:45 p.m.}

T.A. HAMMEKE, M. MCCREA, G. CHEN, S.M. COATS \& S. LI. Recovery of Functional Brain Networks Following Sport-Related Concussion.

Objective: To better understand the natural recovery course and biomarkers of potential window of cerebral vulnerability following sport-related concussion (SRC).

Participants and Methods: Seventeen high school football athletes who sustained either a AAN Grade 2 or Grade 3 concussion were given measures of cognitive, postconcussion symptom, postural steadiness and resting state fMRI (rs-fMRI) at 13 hours and 7 weeks following injury. Uninjured teammates individually matched on demographic and preseason screening measures to injured athletes completed concurrent studies. Rs-fMRI data sets were acquired on a $1.5 \mathrm{~T}$ GE scanner from 116 predefined regions of interest and cross-correlated. Modular functional networks were identified for each subject using the spectral algorithm of Newman.

Results: Relative to controls, injured athletes reported significantly more concussion-related symptoms $(p=.003)$ and had poorer steadiness $(\mathrm{p}<.001)$, and showed a trend to poorer memory and choice RT at 13 hour after injury. These behavioral group differences resolved by 7 weeks after injury. Rs-fMRI revealed 7 functional networks in the controls that remained stable across imaging sessions. Relative to controls, injured athletes at 13 hours after injury showed fragmented functional networks that coalesced into more organized networks by 7 weeks after injury but mild differences from controls persisted.

Conclusions: Concussed athletes acutely showed fragmented functional brain networks that persisted beyond the resolution of postconcussion symptoms and neurocognitive deficits, implying that recovery from concussion persists beyond resolution of clinical symptoms. These findings suggest that cerebral vulnerability following SRC may persist well beyond resolution of postconcussion symptoms.

Correspondence: Thomas A. Hammeke, PhD, Neuropsychology, Milwaukee VAMC \& Med Coll Wisconsin, 1660 N Prospect Ave \#2305, Milwaukee, WI 53202.E-mail: thomas.hammeke@gmail.com

S. JIVANI, T.J. FARRER, E.D. BIGLER, T.J. ABILDSKOV, M. DENNIS, H.G. TAYLOR, K. RUBIN, K. VANNATTA, C. GERHARDT, T. STANCIN \& K.O. YEATES. Effects of Injury Severity on Default Mode Network Volume in Pediatric Traumatic Brain Injury and the Relationship to Attention.

Objective: Deficits in attention and executive function (EF) are common sequelae following pediatric traumatic brain injury (TBI). Damage to the default mode network (DMN) following TBI may be particularly influential on attention and EF. The DMN can be quantified volumetrically using FreeSurfer (FS; see Dennis et al. 2013). The Test of Everyday Attention for Children (TEA-Ch) provides indices of attention and EF and was examined in a pediatric sample with complicated mild to severe TBI compared to those with orthopedic injury (OI).

Participants and Methods: From the original sample ( $N=143$ (82=TBI, 66=OI); 52 Female, 91 Male; age at injury: $\mathrm{M}=7.8, \mathrm{SD}=1.9)$, 109 participants $(59=\mathrm{TBI})$ had FS data, for analysis and 104 completed the TEA-Ch. Overall injury severity for the TBI group, as measured by the Glasgow Coma Scale (GCS) score was in the moderate range $(\dot{M}=10.8, \mathrm{SD}=4.9)$.

Results: Combining TBI and OI groups and examining DMN volume with TEA-Ch composite scores, volume was positively related but did not reach significance $(\mathrm{rs}(96)=.191, \mathrm{p}=.059)$. Separately examining the TBI and OI groups showed that DMN volume significantly related to TEA-Ch composite in the OI $(\mathrm{rs}(45)=.338, \mathrm{p}=.02)$ but not in the TBI $(\mathrm{rs}(49)=.085, \mathrm{p}>.05)$ group. Injury severity was significantly correlated with DMN volume in the TBI group $(\mathrm{rs}(57)=.361, \mathrm{p}<.01)$.

Conclusions: Differential size-function relations of the DMN were found between OI and TBI children. Although injury severity related to reduced DMN volume, DMN volume in children with TBI did not systematically relate to TEA-Ch performance. Implications for using TEA-Ch findings in pediatric TBI will be discussed.

Correspondence: Sanam Jivani, Clinical Psychology, Brigham Young. University, 14925 Ox Cart Lane, Draper, UT 84020.E-mail: sanam47@ gmail.com

L. ZHAVORONKOVA, A. ZHARIKOVA, E. KUSHNIR, T. SHEVTSOVA, S. KUPZOVA \& O. MAKSAKOVA. Brain Functional Connectivity in Traumatic Brain Injury Patients and Healthy Persons.

Objective: Consequences of traumatic brain injury (TBI) are often associated with deficits in different spheres. Advances in diffusion tensor imaging (DTI) have demonstrated decrease of brain connectivity in TBI patients. Brain-behavioral correlation is mostly implemented by EEG coherence as a tool for study in brain connectivity. The aim of our study was to investigate functional brain connections involved in concurrent executions during dual tasks in TBI patients.

Participants and Methods: In the study was examined 47 TBI patients compared to 25 healthy volunteers. EEG and DTI studies were used for 
estimation of brain functional-structural connectivity. Dual (motor and cognitive) tasks were used as tool for modeling of everyday life Results: Clinical scales showed motor and cognitive deficits in TBI patients. According to DTI study was shown significant decrease of white matter integrity. The poor dual task performance was observed and these behavioral alterations accompanied by decrease of EEG coherence for distant diagonal pairs between associative areas as well as an increase ones for local pairs. While in healthy volunteers EEG-marker of successful dual task performance was an increase of theta-alpha EEG coherence for distant pairs as well as the decrease of EEG coherence for local networks

Conclusions: Alterations of structural-functional connectivity were observed in TBI patients. Functional EEG connectivity between distant associative brain areas shown in healthy volunteers during dual task performance may be marker of successful human functioning under information loads. In TBI patients the increase of the functional connectivity only in short networks may be marker of compensator brain mechanisms

Correspondence: ,, . E-mail:

M. ELLIS, C. YEATMAN, S. MARION, C. GIZA, C. KERNAN, T. BABIKIAN, A. MARTINEZ \& R. ASARNOW. Differential Outcomes in Neural and Cognitive Functioning in Children with Moderate-to-Severe Traumatic Brain Injuries: The UCLA RAPBI Study of Event-Related Potentials.

Objective: Traumatic brain injury (TBI) results in diffuse axonal injury and white matter damage. The corpus callosum is particularly vulnerable to injury following TBI, and damage to this white matter bundle has been associated with impaired neurocognitive functioning in children with TBI. Event-related potentials can identify stimulus-locked neural activity with high temporal resolution and may represent a physiological biomarker for network injury.

Participants and Methods: Event-Related Potentials (ERPs) were used in this study to measure interhemispheric transfer time (IHTT), an indicator of corpus callosum integrity. ERPs were conducted in 39 children with moderate-to-severe TBI at 3-5 months post-injury and compared to 28 healthy control children. Neurocognitive performance differences were also examined in these groups.

Results: Nearly half $(n=18)$ of the children with TBI had ERP-measured IHTTs that were more than 1.5 SDs worse than the control group children. This subgroup of children also performed significantly more poorly than healthy controls on neurocognitive measures of working memory $(\mathrm{F}(2,65)=6.25, \mathrm{p}<.01)$, executive functioning $(\mathrm{F}(2,63)=$ $4.56, \mathrm{p}<.05)$, and processing speed $(\mathrm{F}(2,64)=4.18, \mathrm{p}<.05)$, even after correction for pre-morbid intellectual functioning.

Conclusions: This study demonstrated that children with both moderate to severe TBIs and slow IHTT have worse neurocognitive functioning compared to healthy controls in the post-acute (i.e., 3-5 months) phase after injury. We discuss alternative models for the relationship between IHTT and neurocognitive function following TBI. Future studies should explore whether Event-related potential measures of IHTT can predict long-term neurocognitive functional outcomes for children with TBI. Correspondence: Monica Ellis, BA, Culture and Trauma Research Lab attn: Dr. Bryant-Davis, Pepperdine University Graduate School of Educationa and Psychology, 135 North Oakland Avenue, Fuller School of Psychology c/o Dr. Jeff Bjorck, Pasadena, CA 91182. E-mail: monicaellis@fuller.edu

\section{Symposium 11: \\ The Psychometric Assessment of Dementia and Related Conditions using the Latent Variable " $\delta$ "}

\author{
Chair: Donald Royall \\ 3:15-4:45 p.m.
}

D.R. ROYALL, R.F. PALMER, B.E. GAVETT \& A. KOPPARA. The Psychometric Assessment of Dementia and Related Conditions using the Latent Variable " $\delta$ "

Symposium Description: Using a novel Confirmatory Factor Analysis (CFA) in a Structural Equation Model (SEM) framework, we have created a latent dementia phenotype (i.e., " $\delta$ " for "dementia"). $\delta$ has the potential to revolutionize dementia's diagnosis and assessment. $\delta$ can be output as a composite " $\mathrm{d}$ score", which rank orders individuals along a continuous dimension of dementia severity. Cross-sectionally and longitudinally, the $\delta$ score achieves very high Areas Under the Receiver Operating Curve (AUC /ROC) for the diagnosis of dementia, across etiologies. $\delta$ is derived from Spearman's General Intelligence factor " $\mathrm{g}$ " and shares many of g's properties, notably g's "indifference to its indicators". Thus, $\delta$ can be derived post hoc or prospectively from almost any convenient cognitive battery, including item-level data from a single scale. As a latent variable, $\delta$ is relatively free of measurement error. $\delta$ scores can be exported across samples, languages or cultures without loss of its psychometric properties. As a continuous measure, $\delta$ can be easily interrogated for its biomarkers. $\delta$ has been associated with the Default Mode Network (DMN) structure and several serum protein cytokines.

This symposium will introduce $\delta$ to the participants and extend our work into new datasets:

"Welcome Back to Your Future: $\delta$ 's Rationale and Very Ancient History." Donald R. Royall, MD (Symposium Chair) University of Texas Health Science Center at San Antonio

"Future Dementia Status is Almost Entirely Explained by the Latent Variable $\delta$ 's Intercept and Slope." Raymond F. Palmer, PhD University of Texas Health Science Center at San Antonio

"Neuropathology Mediates Age, Race, and APOE effects on $\delta$ in Pathologically Confirmed AD" Brandon Gavett, PhD University of Colorado at Colorado Springs

" $\delta$ 's Replication in a European Sample: Serum Protein and Neuroimaging Biomarkers" Alexander Koppara, PhD Universitätsklinikum Bonn, GERMANY

"The 'd' Evolution of Cognitive Assessment.' Donald R. Royall, MD (Discussant)

Correspondence: Donald R. Royall, MD, Psychiatry, UTHSCSA, 7703 Floyd Curl Dr., San Antonio, TX 78229-3900. E-mail: royall@uthscsa. edu

D.R. ROYALL. Welcome Back to Your Future: $\delta$ 's Rationale and Very Ancient History.

Using a novel Confirmatory Factor Analysis (CFA) in a Structural Equation Model (SEM) framework, we have created a latent dementia phenotype (i.e., " $\delta$ " for "dementia"). $\delta$ is derived from Spearman's General Intelligence factor "g", which was first described in 1904. Our Method parses $g$ into two oblique factors: $\delta$ (i.e., the "cognitive correlates of functional status") and "g" (i.e., $\delta$ 's residual in Spearman's $g$, and the fraction of $g$ 's variance that is NOT related to functional outcomes. It is an empirical question whether dementia severity is associated with $\mathrm{g}, \mathrm{g}^{\prime}, \delta$ or domain specific cognitive performance (e.g., "Memory", "Executive Function", etc.). Our work to date suggests that dementia severity is entirely attributable to $\delta$ and not to any other compartment of variance.

This talk will serve as an introduction to $\delta$ and it's validation to date. $\delta$ rank orders individuals along a continuous dimension of dementia severity. $\delta$ scores achieve very high Areas Under the Receiver Operating Curve (AUC /ROC) for the diagnosis of dementia, across etiologies. $\delta$ appears 
to exhibit g's "indifference to its indicators". Thus, $\delta$ can be derived post hoc or prospectively from almost any convenient cognitive battery, including item-level data from a single scale. $\delta$ scores can be exported across samples, languages or cultures without loss of its psychometric properties. $\delta$ has been associated with the Default Mode Network (DMN) structure and several serum protein cytokines.

Correspondence: Donald R. Royall, MD, 7703 Floyd Curl Dr., San Antonio, TX 78229-3900.E-mail: royall@uthscsa.edu

R.F. PALMER. Future Dementia Status is Almost Entirely Explained by the Latent Variable $\delta$ 's Intercept and Slope.

Objective: To distinguish dementia-relevant variance from a longitudinal battery of cognitive performances tasks using a structural modeling framework. The resulting latent construct " $\delta$ " provides an "error free" continuously varying dementia-specific phenotype. We estimate $\delta$ 's rate of change over time.

Participants and Methods: Data from n $=2191$ participants from the Texas Alzheimer's Research and Care Consortium were used to construct a latent growth curve model (LGC) of longitudinal change over four years using five cognitive measures and one measure of physical function. The six resulting intercepts were then used as indicators of latent baseline $\delta$ (i.e., " $\mathrm{d}$ ") and residual non-dementing cognitive variance (i.e., "g"') in a confirmatory factor analysis (CFA), as previously described (Royall, Palmer \& O'Bryant, 2012). Similarly, the six slopes were combined into homologous latent factors addressing change in $\mathrm{d}$ and $g^{\prime}$ (i.e., $\Delta d$ and $\Delta g^{\prime}$ respectively). The four final latent factors: $g^{\prime}$, $\Delta \mathrm{g}^{\prime}, \mathrm{d}$ and $\Delta \mathrm{d}$, were simultaneously entered as predictors of wave 4 dementia severity using the Clinical Dementia Rating Scale (CDR). All observed baseline variables were adjusted for age, gender, ethnicity, and education.

Results: All observed measures exhibited significant change $[\mathrm{x} 2=1152$ $(\mathrm{df}=229)$; $\mathrm{CFI}=0.968 ;$ RMSEA $=0.043]$. The final model demonstrated excellent fit to the data $[\times 2=543(\mathrm{df}=245)$; CFI = 0.991; RMSEA $=0.023]$. All latent indicator loadings were significant, yielding four distinct factors. After covariate adjustment including baseline CDR scores, $\mathrm{d}$ and $\Delta \mathrm{d}$ were significantly independently associated with CDR4, explaining $25 \%$ and $49 \%$ of its variance, respectively. The latent variable g' significantly explained $3 \%$ of CDR4 variance independently of $\mathrm{d}$ and $\Delta \mathrm{d}$. $\Delta \mathrm{g}$ ' was not significantly associated with CDR4. Baseline CDR explained $16 \%$ of CDR 4 variance.

Conclusions: Future dementia severity is almost entirely explained by the latent construct $\delta$ 's intercept and slope.

Correspondence: Raymond F. Palmer, Ph.D., 7703 Floyd Curl Drive, San Antonio, TX 78212.E-mail: palmerr@uthscsa.edu

\section{B.E. GAVETT. Neuropathology Mediates Age, Race, and APOE effects on $\delta$ in Pathologically Confirmed AD.}

Objective. Latent dementia severity can be modeled as the construct $\delta$, representing the "cognitive correlates of functional status." We recently validated a model for estimating $\delta$ factor scores in the National Alzheimer's Coordinating Center's Uniform Data Set (U01 AG016976). Participants and Methods. Here, we used data from 684 decedents evaluated at Alzheimer's Disease (AD) Centers nationwide. Participants spoke English, had no genetic abnormalities, and were pathologically diagnosed with $\mathrm{AD}$ as a primary or contributing etiology. Clinical data from participants' last visit prior to death were used to estimate dementia severity ( $\delta$ factor scores). Results. A structural equation model using age, education, race, and APOE genotype (number of $\varepsilon 2$ and $\varepsilon 4$ alleles) as predictors and latent $\mathrm{AD}$ and cerebrovascular disease (CVD) pathology as mediators fit the data well (RMSEA=0.033; $\mathrm{CFI}=.951)$. AD pathology mediated the effects of age and APOE genotype on dementia severity. An older age at death was associated with less AD pathology and, in turn, less severe dementia. More $\varepsilon 4$ alleles were associated with more pathology and more severe dementia. In contrast, more $\varepsilon 2$ alleles were associated with less pathology and less severe dementia. Although age and race contributed to differences in CVD pathology in this sample of decedents with pathologically confirmed AD, CVD pathology was not related to dementia severity. Conclusions. Using $\delta$ as an estimate of dementia severity fits well within a structural model in which AD and CVD directly affect dementia severity and mediate the relationship between age and APOE genotype on dementia severity.

Correspondence: Brandon E. Gavett, Ph.D., 1420 Austin Bluffs Parkway, Department of Psychology, Colorado Springs, CO 80918. E-mail:bgavett@uccs.edu

A. KOPPARA, S. WOLFSGRUBER, L. KLEINEIDAM, J. KORNHUBER, W. MAIER, F. JESSEN \& M. WAGNER. Validation of the latent dementia phenotype $\delta$ in a German Sample with clinical data, CSF and neuroimaging biomarkers.

1. Objective: To replicate and validate the latent dementia phenotype " $\delta$ " (for dementia). $\delta$ is constructed from cognitive and functional measures, as proposed by Royall and Palmer, 2012. As a latent variable, it is free of measurement error, indifferent to its indicators and achieves high diagnostic accuracy for the diagnosis of dementia. In a large memory clinic sample of patients with MCI and AD dementia, individual d-scores were derived and subsequently associated with CDR-SoB, APOE-4 status, cerebrospinal fluid (CSF) biomarkers and hippocampal volume. A comparison with traditional neuropsychological tests was performed. 2. Participants and Methods: Subjects from a German multicenter memory clinic cohort ( $\mathrm{n}=1775), 1046 \mathrm{MCI}$ and 729 dementia (83\%AD cases) were used for the generation of the $\delta$ parameter, which was modelled in a structural equation modelling framework based on the ADAS-cog and IADL. ADAS-cog sum score and CERAD total score were compared to the $\delta$ parameter. From 658 cases (376 MCI, 282 dementia cases) additional biomarker information was available. Associations with CDR-SoB, A-beta 42, tau, p-tau, a-beta $42 /$ tau ratio and hippocampus volume were calculated.

3. Results: The $\delta$-parameter had the strongest association with CDRSoB, when compared to ADAS-cog total sum score and CERAD Total score. In addition, $\delta$ was significantly associated with ApoE4, right hippocampal volume and CSF biomarkers A-beta42, tau and the a-beta/ tau ratio. These biomarker associations were equal or more pronounced than the association of the ADAS-Cog and the CERAD.

4. Conclusions: The $\delta$ parameter was replicated in a German sample and associations with CDR-SoB and biomarkers show that it is a valid representation of the dementia process. The $\delta$ variable may constitute a useful single measure of outcome in clinical trials and provide also a risk marker for dementia.

Correspondence: Alexander Koppara, Dipl.Psych. PhD, Department of Psychiatry, University of Bonn, Bonn, Germany, German Center for Neurodegenerative Diseases, Bonn, Germany, Sigmund Freud Str. 25, Bonn 53105, Germany. E-mail: alexander.koppara@ukb.uni-bonn.de

\section{D.R. ROYALL. The 'd' Evolution of Cognitive Assessment.}

This symposium has described the latent dementia phenotype " $\delta$ ", and extended it to new datasets and applications. This presentation will address $\delta$ 's potential to revolutionize dementia's diagnosis and assessment. By placing cases on a continuous measure of dementia severity, $\delta$ has the potential to obviate the need for categorical discriminations, including "Mild Cognitive Impairment (MCI)". Because of its high diagnostic accuracy and indifference to its indicators, $\delta$ has the potential to obviate the need for expert psychometric evaluation or adjudication of dementia status. $\delta$ 's derivation from Spearman's g constrains the potential mechanisms of dementia to those that can conceivably affect ALL psychometrics, regardless of their content. In fact, it may be possible to extract $g$, and therefore $\delta$, from behavioral or physiological data. If so, then dementia assessment may be entering a new phase in which dementia severity may be estimable without direct clinician input. Finally, because our approach is modular, the psychometric assessment of depression, psychosis or age-related cognitive changes may soon benefit from the same advances. 
Correspondence: Donald R. Royall, MD, 7703 Floyd Curl Dr., San Antonio, TX 78229-3900. E-mail: royall@uthscsa.edu

\section{Symposium 12: \\ How Your Network Shapes Your Science and Vice Versa: New Ways to Think about Advancing Your Research Career and Obtaining Funding}

\author{
Chair: Lynne Padgett
}

$$
\text { 3:15-4:45 p.m. }
$$

L.S. PADGETT, T. HOROWITZ \& T. AHLES. How Your Network Shapes Your Science and Vice Versa: New Ways to Think about Advancing Your Research Career and Obtaining Funding. Symposium Description: This program brings together National Cancer Institute (NCI) program officers as well as current and former extramural investigators to present a variety of perspectives on pursuing NCI-funded research in cancer and cognition. These presentations go beyond discussing grant mechanisms and focus on the importance of integrating your scientific networks to drive innovative science. Dr. Lynne Padgett will highlight results of a portfolio analysis of grant proposals in cancer and cognition, highlight research gaps, and discuss the role of program officers in the grants process. Dr. Todd Horowitz will discuss his unique role as a former extramural investigator and current project officer at NCI. Lastly, Dr. Tim Ahles will present his perspective as a senior investigator as an example of how to build a grant funded career. Learn from three different perspectives how to leverage your interests, networks, and serendipitous opportunities to shape science and advance your career.

Correspondence: Lynne S. Padgett, PhD, Behavior Research Program, National Cancer Institute, 9609 Medical Center Drive, 3E616, Rockville, MD 20850.E-mail: lynne.padgett@nih.gov

L.S. PADGETT. Portfolios and Project Officers: Learning about Grant Funding at the National Cancer Institute.

As the phenomenon of "chemo brain" has entered into the public discourse, researchers have sought to better understand the mechanisms and outcomes. The purpose of this presentation is to describe currently funded topics of research examining the effects of cancer and chemotherapy on cognition and to highlight important gap areas as proposed topics for future research. Emphasis on utilizing project officer's knowledge of funding trends and priorities will also be discussed.

Participants and Methods: Two sets of analyses were undertaken. First, an examination of funded grants across NIH in the past ten years was conducted to classify proposals examining the effects of chemotherapy on cognitive function in non-CNS tumors. Based on these results, a subset of recent proposals focused on cognitive interventions in the past two years was also analyzed.

Results: Results indicated increasing numbers of applications over the past ten years, with larger growth in recent years. Animal models and neuroimaging studies are well represented in funded grants. Significant gap areas include measurement challenges, research in populations beyond breast cancer, cognitive rehabilitation, and developmental sequelae of exposure to in utero cancer treatment.

Conclusions: The results of the analyses illustrate understudied populations based on both disease and age, a lack of data on in utero exposure to cancer treatment, and interventions focused on cognitive rehabilitation. In addition, the role of the program officer in facilitating understanding of the gaps in the field and negotiating the grants process will be discussed.

Correspondence: Lynne S. Padgett, PhD, 9609 Medical Center Drive, 3E616, Rockville, MD 20850.E-mail:lynne.padgett@nih.gov
T. HOROWITZ. I Went from Principal Investigator to Program Officer, and You'll Never Guess What I Learned about the Granting Process!

You have a great research idea, but you need money to make it happen. You need to write a grant. But where can you apply to get money for neuropsychological research? What do you need to know before you write a grant? How does the granting process work? Writing grants to support your research is as critical to a scientific career as data analysis and scientific writing. After 12 years as a PI on soft money at Brigham and Women's Hospital, I jumped to the other side of the grants game to become a Program Director at the National Cancer Institute. In this talk, I will present insights into the grantsmanship process from both perspectives.

Correspondence: Todd Horowitz, Ph.D., 9609 Medical Center Drive, Rockville,MD 20850.E-mail: todd.horowitz@nih.gov

T. AHLES. Obtaining Grant Funding: Taking Advantage and Creating Opportunities.

Objective: The primary objective of this presentation is to use my $20+$ year history of grant funding as an example of how to build a grantfunded research career. Points of emphasis will include: 1) relationship (or lack thereof) between training and career / research focus; 2) the nonlinear path that a research career can take; 3) taking advantage of the strengths of your institution / environment to develop innovative research, particularly as a junior researcher; 4) leveraging findings from other research areas to develop multidisciplinary partnerships designed to foster translational research within your area of study; and 5) collaborating with NIH Program Directors in the development of grant ideas and understanding of trends for areas of funding priority. I will start with a brief overview of training and early research in pain and symptom management in cancer and non-cancer populations. The bulk of the presentation will use the development of a focus on cognitive changes associated with cancer and cancer treatments to illustrate integration of multiple assessment methods (self-report, neuropsychological assessment, imaging) and multiple disciplines (genetics, biomarkers, animal models) to develop transdisciplinary research, culminating in the development of the Neurocognitive Research Laboratory at Memorial Sloan Kettering Cancer Center. Special emphasis will be placed on identification and recruitment of investigators from other areas (basic science, animal model researchers) to become involved in your area of research. Correspondence: Tim Ahles, PhD, 641 Lexington Ave, New York, NY 10022.E-mail:ahlest@mskcc.org

\section{Poster Symposium: Anterograde Memory Disorder As Disconnection Syndrome}

\author{
Chair: J. Michael Williams \\ 3:15-4:45 p.m.
}

\section{Memory Functions}

\section{J. WILLIAMS, J. WILLIAMS, A. TART-ZELVIN, K. MCWILLIAMS, K. PATRICK \& K. OSIPOWICZ. Anterograde Memory Disorder As Disconnection Syndrome.}

Symposium Description: This symposium will include five presentations that examined the thesis that the hippocampus represents an interface between sensory systems in the cortex and the limbic systems of emotion generation and expression. The primary function of the hippocampus is to encode the emotional tone of memories so that memories have emotional valence when recalled in the future. Following this encoding, information is then stored in corresponding areas of the cortex. The hippocampus is a "pass through" system, in which 
sensory information from the cortex passes through the hippocampus, acquires encoded emotional information from the limbic system and is then conveyed to storage areas in the cortex. Lesion of the hippocampus interrupts this communication system and sensory information is not stored. The syndrome of anterograde memory disorder represents a disconnection of sensory information perceived in the primary and secondary areas of the cortex from storage sites in other parts of the cortex. We explored this model using fMRI, Resting State Functional Connectivity and Diffusion Tensor Imaging studies of the hippocampus, memory and emotion. The first presentation will cover a general theoretical model of hippocampal structure and function derived from the anatomy of the hippocampus. The second presentation will review the fMRI, Resting State Connectivity and DTI methods that were used in all the analyses. The third presentation will cover results of the fMRI studies, and the fourth presentation will cover results of Resting State Connectivity studies. The final presentation will present results of the DTI studies.

Correspondence: J. Michael Williams, PhD, Psychology, Drexel University, 3141 Chestnut St, Philadelphia, PA 19104.E-mail:jw37@ drexel.edu

\section{J. WILLIAMS. The Hippocampus As a Cortical Interface Derived From An Analysis of Its Structural Anatomy and Function.}

This presentation will describe the model of hippocampal function that underlies the subsequent analyses included in the symposium. The fundamental hypothesis is that the hippocampus represents an interface between sensory systems in the cortex and the limbic system. Information passes from primary and association sensory cortices to the hippocampus. The emotional valence associated with the sensory experience is then encoded by interaction with the hippocampus, amygdala and other parts of the limbic system. The emotion-encoded sensory information is then stored in sites widely spread across the cortex. The model is unique in the stress on the encoding of emotion and the flow of information from sensory association areas to hippocampus then back to cortical areas for storage. A simple expression of this model is that the hippocampus can be conceptualized as a sensory association area for emotion. One prediction derived from the model is that lesion of the hippocampus will interrupt the flow of information from sensory perception to memory consolidation and storage. The syndrome of anterograde amnesia, long associated with $\mathrm{H}$. M. and other amnesic cases, is then regarded as a type of disconnection syndrome. This introductory presentation will review the anatomy of the hippocampus with a focus on its extrinsic connections and the patterns of connectivity with the cortex and limbic system we tested using fMRI, resting state connectivity and Diffusion Tensor Imaging. With an emphasis on memory encoding and consolidation, many previous studies have understandably focused on intrinsic connections and possible memory consolidation mechanisms, such as reverberating circuits and long-term potentiation. The extrinsic connections of the hippocampus with cortical systems are still being discovered. Our objective is to map these functional connections using a variety of current neuroimaging methods.

Correspondence: J. Michael Williams, PhD, 3141 Chestnut St, Philadelphia,PA 19104.E-mail: jw37@drexel.edu

\section{K. OSIPOWICZ. Neuroimaging Methods Used to Examine the Hippocampus, Memory and Emotion.}

This section will review the methodology involved in the analysis of each of the subsequent presentations. Twenty healthy, neurologically normal participants underwent MRI scans in a Seimens trio 3t clinical scanner. We obtained fMRI, resting state fMRI (rsfMRI) and diffusion weighted imaging (DTI). SPM8 was utilized for processing all images. fMRI Preprocessing included conventional slice timing correction, coregistration and normalization with the NMI305 template and smoothing. Contrasts were made using the general linear model built into SPM8.

The Resting State fMRI data was processed by coregistering to MNI305 each subject's high resolution T1 image: this data was further segmented into grey matter, white matter, CSF, and other. Following rsfMRI processing, first level bivariate correlation between all ROIs as defined by the AAL atlas, and all voxels were computed, which were then modeled on the second level with a performance regressor modeled. These were then analyzed to explore functional connectivity matrices between all brain nodes. Lastly, analyses of functional connectivity within the hippocampal formations were conducted. The fMRI data was similarly modeled in a connectivity analysis with the effect of the experimental conditions removed, thus allowing the modeling of intrinsic network differences between emotional and neutral.

Diffusion Tensor Imaging was done using DTIStudio. The same standard gradient table was used throughout the study. The Automated Image Registration (AIR) realignment procedure was performed prior to tensor calculation. ROIs were imported from fMRI and rsfMRI analyses using ROIeditor, and fibers were tracked using the union operation between functional ROIs. Extracted measures of tract integrity (e.x. mean FA, number of fibers) were then entered into a multiple regression with performance modeled as the outcome variable.

Correspondence: Karol Osipowicz, PhD, 1221 West Porter St., Philadelphia,PA 19148.E-mail:kzo22@drexel.edu

K. MCWILLIAMS, K. OSIPOWICZ \& J. WILLIAMS. Results of Resting State Functional Connectivity Studies of the Hippocampus. The objective of this study was to investigate the whole brain functional connectivity of the hippocampal formation in the context of emotional and neutral stimuli. Twenty neurologically healthy adults between the ages of 18 and 25 underwent scanning. A seed based correlation approach was used to examine whole brain resting state functional connectivity patterns of the hippocampi. For each participant, Pearson correlation coefficients between the average time series of each seed region and that of each individual voxel were computed. A voxel-wise random effect one-sample $t$ test was used to identify brain regions that showed significant correlations with the seed regions. Multiple comparisons were corrected for using family wise error with a threshold of $\mathrm{p}<.05$. We observed hippocampal resting state functional connectivity with several brain regions involved in the processing and regulation of emotionally salient events including the amygdala. regions of the default mode network and the medio-dorsal attentional network. Additionally, we observe decreases in connectivity are associated with poorer memory performance. We interpret our findings as supporting functional connectivity differences between emotional and neutral stimuli. Correspondence: Kellie McWilliams, BA, 1708 Melon st, Philadlephia, PA 19130.E-mail:kam392@gmail.com

\section{K. PATRICK, J. WILLIAMS \& K. OSIPOWICZ. Results of Diffusion} Tensor Imaging studies of the Hippocampus and Emotion.

Twenty participants were examined using the DTI protocol described previously. DTI tractography was carried out between the following regions: bilateral hippocampi, areas modulated by performance in the resting state functional MRI (rsfMRI) data, and areas modulated by the experimental task on fMRI. Extracted measures, including Fractional Anisotrophy (FA), Mean Diffusivity (MD) and number of tracts were then modeled in a multiple regression predicting memory performance. We found posterior fibers emanating from to hippocampi toward the fornix, splenium of the corpus callosum, the posterior commissure; and anterior fibers entering the stria terminalis and the uncinate fasciculus. We also found a trend suggesting that lower levels of anatomical connectivity are associated with poorer performance. Lastly, we describe anatomical connectivity differences between emotional and neutral networks. The DTI analyses replicated the known pathways crossing the hippocampal formations. Additionally, we suggest that anatomical connectivity between the hippocampi and secondary, cortical nodes is at least partially responsible for memory performance at long term follow-up. Lastly, we describe network differences between the processing of neutral and emotional stimuli. 
Correspondence: Kristina Patrick, M.S., 506 S 41st St 2M, Philadelphia, PA 19104.E-mail: kristina.e.patrick@gmail.com

\section{A. TART-ZELVIN, J. WILLIAMS \& K. OSIPOWICZ. Results of fMRI Studies of the Hippocampus and Emotion.}

Patterns of hippocampal activations were examined using a conventional on-off boxcar design in which emotional and neutral pictures. words ("Kill" vs "Flower" and environmental sounds (e.g. glass breaking vs baby laughter) were alternated during a single scanning session. Memory for the stimuli was also assessed following the scanning session. As expected, participants had the best memory recall for pictures. Emotional stimuli of all types were better remembered than neutral stimuli. fMRI analyses using SPM revealed a robust effect for hippocampal activation associated with picture stimuli. The right hippocampus was more active than the left. There were no apparent hippocampal activations associated with the words or sounds. There was no effect associated with the emotional tone of the stimuli and no effect for gender. Consistent with many other studies, the results suggest that fMRI methods detect the dominant influence of the visual system on hippocampal activation. More sensitive methods, such as resting state connectivity and Diffusion Tensor Imaging studies of the hippocampus may be useful in testing the model of the hippocampus and emotion memory.

Correspondence: Ariana Tart-Zelvin, M.S., 584 Pheasant Ridge Drive, Apt.A, Chubbuck, ID 83202.E-mail: atartz@gmail.com

\section{Poster Session 7: \\ Behavioral Neurology, Dementia (Subcortical), \& Medical/Neurological Disorders-Adult}

$$
\text { 3:15-4:45 p.m. }
$$

\section{Assessment/Psychometrics/Methods (Adult)}

K.M. HARRELL, S. HORNING, M.K. CONNOR, S.S. WILKINS \& J. CHODOSH. Clinical Video Telehealth Compared to Face-to-Face Assessment Using the Montreal Cognitive Assessment: Is Clinical Video Telehealth Reliable?

Objective: Clinical Video Telehealth (CVT) is an emerging means for providing clinical services for those who otherwise have limited access to care. While objective cognitive testing via CVT is feasible and has good patient acceptance, further investigation is required to determine if results obtained via CVT can be equated to face-to-face (F2F) results. This study compares performance on the Montreal Cognitive Assessment (MoCA) under CVT versus F2F testing conditions.

Participants and Methods: Participants $(\mathrm{N}=28)$ were recruited from an outpatient geriatric medicine clinic (Mage $=85.64, \mathrm{SD}=6.11$, range: $70-97)$. The average years of education was $14.5(\mathrm{SD}=3.35$, range: 7-20). The majority of participants were Caucasian $(n=16)$ and African American $(\mathbf{n}=9)$. All participants with capacity to consent were included in the study, irrespective of clinical diagnosis, and were randomly assigned to condition (F2F or CVT), administrator (1 or 2), and MoCA Form (1 or 2 ) for their first testing. A counterbalanced design was employed for the second administration, conducted immediately after the first administration. Participants also completed surveys of their use and familiarity with computers and video-call technologies. A Pearson's $r$ was computed to analyze the relationship between the F2F and CVT performance.

Results: Seventy one percent of participants had used a computer and $11 \%$ had used some form of video-call technology at least once. The F2F performance $(\mathrm{M}=20.14, \mathrm{SD}=5.10)$ was strongly and significantly correlated with CVT $(\mathrm{M}=20.39, \mathrm{SD}=5.18)$ performance $(\mathrm{r}=.89$, df $=26, \mathrm{p}<.0001)$.

Conclusions: Performance on the MoCA via CVT is highly related to $\mathrm{F} 2 \mathrm{~F}$ performance in geriatric patients with a diverse set of demographic and clinical characteristics and suggests that there is adequate reliability to equate performance to that in the F2F setting. This preliminary data supports using the MoCA via CVT as a valid form of clinical care. Correspondence: Kathryn M. Harrell, Ph.D., Psychology, West Los Angeles VA Medical Center, 11301 Wilshire Blvd., Los Angeles, CA 90073. E-mail: kathryn.harrell@va.gov

R. GOLDSWORTHY \& R.L. SKEEL. Are Assumed Differences in Delay Discounting and Delayed Gratification Due to Procedural Variance?

Objective: Delay discounting refers to the subjective evaluation of reward values: a reward's value will subjectively decrease as the delay becomes longer (Bickel \& Marsch, 2001). Delayed gratification is the inclination to refrain from immediate satisfaction for the promise of a forthcoming larger reward (Hoerger et al., 2011; Mischel, et al., 1972). Although both sound conceptually similar, the literature has taken divergent paths in regards to what the two concepts are measuring, and whether the two represent the same underlying construct. The current study sought to make direct comparisons between these procedures using two novel operant procedures.

Participants and Methods: Eighty-five participants were randomly assigned to one of three conditions: operant delay discounting, operant delayed gratification, or hypothetical delay discounting. In the two operant procedures, participants chose between watching mediocre-rated videos immediately, or highly-rated videos after variable delays. The hypothetical condition was a questionnaire version of the operant procedure. In addition, all participants completed the a hypothetical monetary reward questionnaire, the Barratt Impulsivity Scale, and the Delayed Gratification Inventory.

Results: Both of the operant conditions suggested the novel task was valid: the effect of the delay significantly affected participants' responses. Area under the curve analyses indicated that the choices made in the two operant procedures were not significantly different from one another.

Conclusions: The current study supports models of delay discounting and delayed gratification that treat them as similar or identical processes. Limitations of the current study are also discussed.

Correspondence: Rachael Goldsworthy, Psychology, CMU, $600 \mathrm{~S}$. Washington Street, APT 19, Mt. Pleasant, MI 48858.E-mail: rgoldswo@ gmail.com

\section{Behavioral Neurology/Cerebral Lateralization/Callosal Studies}

V. HARMS, N. CZEMERES \& L. ELIAS. Put Your Best Side Forward: The Effect of Facial Attractiveness Asymmetry on Posing Biases.

Objective: When examining the posing orientation of subjects in portraits, a bias for the sitter to pose with their left cheek forward is typically observed. Further, it was found that participants were more likely to pose with the more expressive left cheek forward when asked to pose for a loving family portrait and with the less expressive right cheek forward when asked to pose for a stoic scientific portrait. This suggests an awareness of the asymmetry in emotional expressivity that influences posing behaviour. Further research has provided evidence that the right side of the face is perceived as more attractive compared to the left side. We examined posing bias in online modeling headshots to assess whether this attractiveness asymmetry influences posing direction in portraits specifically highlighting the attractiveness of the subject.

Participants and Methods: A total of 493 modeling headshots were sampled from professional photography and modeling websites. All images were coded for posing direction, sex of the portrait subject, and the amount of the body visible in the photograph.

Results: We observed an overall leftward posing bias for the modeling headshots. Males and females did not differ in posing bias, and the 
amount of body visible in the headshot photograph was not found to influence posing direction.

Conclusions: Consistent with the typical left-cheek bias observed in portraiture, this observed leftward bias suggests that either the rightward bias for facial attractiveness does not influence posing behaviour, or it is less dominant than other factors such as emotional expressivity. Correspondence: Victoria Harms, Psychology, University of Saskatchewan, Department of Psychology, 9 Campus Drive, Saskatoon, SKS7N 5A5, Canada.E-mail:v.harms@usask.ca

S.W. ADAMS, Y. KIM, C.W. WRIGHT, D.T. LOBDELL \& R.M. BOWLER. Movement Disorder Symptoms Associated with Unified Parkinson's Disease Rating Scale (UPDRS) in Two Manganese (Mn)-Exposed Communities.

Objective: The UPDRS is a commonly used neurological measurement to assess the presence and severity of parkinsonian symptoms. It has also been used to assess symptoms associated with Mn exposure. Objectives: to determine 1) if movement disorder symptoms were associated with UPDRS: Activities of Daily Living (ADL) and Motor abnormalities; and 2) which symptoms were most related to increased abnormalities on these UPDRS subscales.

Participants and Methods: Correlations between self-reported movement disorder symptoms from a health questionnaire and scores obtained on UPDRS: ADL and Motor subscales, and the Bradykinesia domain of the Motor subscale, were assessed during a medical examination among 185 Mn-exposed participants from two Ohio towns. Partial correlations were used for statistical analyses, controlling for age, sex, education and a history of musculoskeletal disease.

Results: The presence of movement disorder symptoms was positively associated with ADL $(\mathrm{pr}=0.647, \mathrm{p}=<0.001)$, Motor $(\mathrm{pr}=0.449, \mathrm{p}=$ $<0.001)$, and Bradykinesia $(\mathrm{pr}=0.418, \mathrm{p}=<0.001)$ domains on the UPDRS. Specific movement disorder symptoms most strongly associated with increased ADL and Motor scores included having difficulty getting out of chairs ( $\mathrm{pr}=0.458, \mathrm{p}=<0.001)$, writing $(\mathrm{pr}=0.481, \mathrm{p}=<0.001)$, skilled movements ( $\mathrm{pr}=0.478, \mathrm{p}=<0.001$ ), loss of coordination/balance $(\mathrm{pr}=0.457, \mathrm{p}=<0.001)$, changes in walking $(\mathrm{pr}=0.412, \mathrm{p}=$ $<0.001)$ and slowness of movement $(\mathrm{pr}=0.539, \mathrm{p}=<0.001)$.

Conclusions: Participants in the low, but long-term Mn-exposed communities reported movement disorder symptoms associated with abnormalities on the UPDRS: ADL and Motor subscales, especially with symptoms of Bradykinesia and difficulty with skilled movements. Correspondence: Shane W. Adams, SFSU, 37 Reed Blvd \# 7 , Mill Valley, CA 94941.E-mail: swadams2@gmail.com

J. UPSHAW, D. LEITNER, L. OHLHAUSER, M. REYNOLDS, J.C. PIERCY, E. CONCEPCION \& H. MILLER. Connecting the Disconnected: Importance of an Integrated Approach to Neuropsychological Evaluation in a Patient with ACC.

Objective: Corpus callosum agenesis (ACC) is a congenital brain malformation in which there is a failure to develop the large bundle of fibres that connect the cerebral hemispheres. Throughout existing literature, complete ACC has been associated with a number of readily testable cognitive, emotional, and social sequelae. This study presents the case of young man with complete ACC who is symptomatic in daily living, but presents as unusually competent in most tested areas.

Participants and Methods: This case study investigated a 27-year-old male with complete ACC, diagnosed at age 17. Existing CT scans of the head were obtained to confirm ACC, and a comprehensive neuropsychological evaluation and interview were conducted. Evaluations included tests to address cognitive, emotional, personality, and social issues, as well as day-to-day functioning.

Results: The patient reported relatively significant cognitive, emotional, and social issues in daily living. Formal assessment, however, was not congruent with these complaints. Aside from a mild-to-moderately depressed score on an assessment of learning, the patient performed in the average to superior range on all other tests administered. Though still within normal limits, a wide discrepancy between scores on verbal and performance-based tasks was also noted.

Conclusions: Comprehensive testing using standardized materials proved largely insensitive to the complaints of the patient and was not reflective of his actual level of daily functioning. These findings have implications for the importance of an integrated approach to neuropsychological evaluation in which testing is augmented with extensive clinical interview and measures of daily functioning, and is seen as only one part of a multi-dimensional process.

Correspondence: Jennifer Upshaw, Psychology, University of British Columbia Okanagan, 952 Cawston Avenue, Kelowna, BC V1Y6Y2, Canada. E-mail: jnupshaw@gmail.com

C.S. GHILAIN, S.A. ANDERSON, N. SUN-SUSLOW, C.I. BERMUDEZ, M. FORTE, I. BABAKHANYAN, B. GALLO, C. LUCA, J. JAGID, C. SINGER \& B.E. LEVIN. Assessing DBS Candidacy in Idiopathic PD: Do Emotional Factors Influence Outcome?

Objective: Deep Brain Stimulation (DBS) has been shown to offer significant symptom relief in select patients with Parkinson's disease (PD). However, there is no consensus to date how to best screen for DBS eligibility. Depression and anxiety symptoms in PD are pervasive, yet it is unknown whether depressed or anxious affect prior to surgery will impact post-surgical cognitive performance. This study examined whether emotional factors predating DBS surgery influence post-surgical outcomes.

Participants and Methods: 37 PD patients $($ Mage $=63.48, \mathrm{sd}=7.85$ ), underwent pre- and post-DBS neuropsychological evaluations. Patients were grouped based on severity of emotional symptoms, 13 depressed (BDI-II $>13$ ) and 25 anxious (BAI>9). Groups did not differ by gender, race, ethnicity, age, language of evaluation, span between pre/ post evaluation or disease duration. Years of education were significantly different in the depression $(\mathrm{t}(34)=3.23, \mathrm{p}=.007)$ and anxiety $(\mathrm{t}(34)=2.117, \mathrm{p}=.042)$ groups, respectively. ANCOVAs were used to determine differences in post-surgical outcomes by group, controlling for pre-surgical performance and years of education.

Results: Depressed and non-depressed groups did not differ significantly post-DBS on MMSE, digit span, confrontation naming, phonemic and semantic fluency, mental object assembly or judgment of line orientation, after controlling for both pre-surgical scores and years of education. Clinically anxious and non-anxious groups showed the same performance pattern.

Conclusions: In this small sample, PD patients who meet criteria for clinical depression or anxiety did not exhibit more impaired neuropsychological performance post-DBS than their non-depressed/non-anxious counterparts. Given that depression and anxiety are so common in PD, these data highlight the need to further elucidate what role emotional factors should play when assessing DBS candidacy.

Correspondence: Christine S. Ghilain, M.S., Department of Psychology, University of Miami, 5665 Ponce De Leon Blvd, Coral Gables, FL 33146. E-mail: csghilain@gmail.com

J. BAYNARD, D. GOODELL, S. ORCUTT \& S. BLAND. The Effect of Social Isolation on Aggressive Behavior and Cannabinoid Receptors in the Amygdala.

Objective: Social support systems are a major contributor to the healthy development of young children. Roughly $80 \%$ of individuals diagnosed with a psychiatric disorder are young adults that have encountered neglect or abuse at an early age. However, the behavioral and neuronal impact of aberrant social support systems is not fully understood. Previous research in the Bland lab showed male rats reared in social isolation (isolates) had an increase in aggressive behaviors and a decrease in markers of cell activation in the prefrontal cortex. Another study found that decreases in cannabinoid 1 (CB1) receptors are associated with aggressive behaviors in CB1 knockout mice. This study assessed 
whether an increase in aggressive behaviors in isolates is associated with a reduction of CB1 receptors in the amygdala.

Participants and Methods: In Sprague-Dawley rats, we manipulated housing conditions to assess the effects of the social support systems. For four weeks, rats were either housed in isolation or housed in groups of 3-4 per cage. On week five, the social behavior testing was completed to assess aggressive behaviors. Animals were later sacrificed and immunohistochemistry was performed to assess CB1 receptors in the amygdala. Results: The data showed that isolates displayed more aggressive behaviors than their group reared cohort. Additionally, we found a trend that isolates had more CB1 receptors than their group reared cohort. Conclusions: The trend in the expression of CB1 receptors in the isolates was contradictory to the hypothesis of the study. The trend may be due to an up-regulation of the CB1 receptors due to a decrease in endogenous endocannabinoids. Future research could assess the precursor proteins and enzymes of those endogenous endocannabinoids. This would add further clarity to the differences seen in the CB1 receptor levels of the two groups.

Correspondence: Jessica Baynard, B.A., Psychology, University of Colorado, Denver, 6583 Benton Circle, Arvada, CO 80003. E-mail: jessica.baynard@ucdenver.edu

R.D. ELLISON, K. BRYANT, C. WILSON \& J. GONZALEZ. LateOnset Rasmussen's Encephalitis: A Neuropsychological Case Study.

Objective: Rasmussen's encephalitis (RE) is an uncommon, idiopathic inflammatory disease that typically affects the brain unilaterally. RE is characterized by medically intractable seizures, declivity of cognitive and motor functions dependent on the effected hemisphere, and progressive atrophy. RE is most prevalent in children with an adult onset accounting for roughly $10 \%$ of all cases. As neuropsychological case reports involving $\mathrm{RE}$ are extremely rare, especially with adults, we describe the neuropsychological presentation of a case of adult-onset RE. Participants and Methods: A 25-year-old, left handed, female, with 18 years of education underwent a comprehensive neuropsychological evaluation to assess her cognitive functioning 7 months post RE diagnosis. She was diagnosed following hospitalization with prolonged status epilepticus. Brain MRIs with and without contrast were performed, which demonstrated cytotoxic edema and prominent decreases in neural tissue density of the left hemispherical neocortex and deep grey matter structures.

Results: The patient demonstrated deficits that were highly consistent with left hemisphere impairment. Specifically, on the WAIS-III, her Verbal Comprehension Index ( VCI = 76) was significantly lower than her Perceptual Reasoning Index (PRI = 96). Additionally, she had impairments in right-handed motor/sensory functioning (i.e., dexterity, speed, and sensation), aspects of language (e.g., reading, fluency, and confrontation naming), and mild right hemi-neglect. Memory was intact with no evidence of lateralization.

Conclusions: The neuropsychological findings were consistent with the unilateral, left hemispherical disease demonstrated by MRIs. This case study provides a rare glimpse into the neuropsychological functioning and lateralized deficits of an individual with late-onset RE 7 months post-onset.

Correspondence: Ryan D. Ellison, West Virginia University, 18 Hayeslett Lane,Washington,WV 26181.E-mail: relliso1@mix.wvu.edu

S.A. ANDERSON, C.I. BERMUDEZ, C.S. GHILAIN, N. SUNSUSLOW, M. FORTE, I. BABAKHANYAN, B. GALLO, C. LUCA, J. JAGID, C. SINGER \& B.E. LEVIN. Assessing DBS Candidacy in Idiopathic Parkinson's Disease: The roles of education and ethnicity.

Objective: Determining DBS candidacy in PD is challenging, especially among non-native English speakers and ethnic minorities. Educational attainment is likely related to DBS cognitive outcome and an important factor in candidate selection. The study aim was to understand the role of education for Hispanics $(\mathrm{H})$ and Non-Hispanics (NH) undergoing DBS evaluation.

Participants and Methods: Thirty-six individuals with PD, $20 \mathrm{H}$ and $16 \mathrm{NH}$, underwent pre- and post-DBS neuropsychological evaluation. Education ( $\mathrm{M}=12.47)$, months since DBS $(\mathrm{M}=24.12)$, and disease duration (M=13.52 years) were comparable between groups. Independent and paired sample t-tests were used to compare pre- and post-surgical test scores. Multiple regression was conducted to examine the relationship between years of education and post-surgical scores, after controlling for age and baseline (pre-surgical) performance.

Results: Pre-surgically, the H group showed significantly lower scores on digit span and confrontation naming compared to the NH group $(p<.05)$. Following DBS, the $\mathrm{H}$ group exhibited a more pervasive pattern of cognitive decline than NH, with significantly lower scores on MMSE, Similarities, BNT, COWAT, Immediate and Delayed Recall CVLT-II, and Hooper VOT $(\mathrm{p}<.05)$. The relationship between education and post-surgical performance was unique, where years of education was a significant predictor of outcome, but for different measures in $\mathrm{H}$ and NH groups.

Conclusions: These findings show that ethnicity is an important factor in understanding the role of education as a predictor of DBS cognitive outcome. These data argue against using a uniform approach to evaluating DBS candidacy among different ethnic groups. Further research is needed to disentangle the impact of ethnicity and language on DBS outcome.

Correspondence: Sharlet A. Anderson, Ph.D., Neurology, University of Miami Miller School of Medicine, 1120 NW 14 street, CRB 1369, Miami, FL33136.E-mail: s.anderson@med.miami.edu

\section{Cancer}

K.E. ROBINSON, M.M. PEARSON, C.J. CANNISTRACI, A.W. ANDERSON, J.F. KUTTESCH \& B.E. COMPAS. Psychosocial Functioning Following Pediatric Brain Tumor: Contribution of Executive Function, Brain Activation, and Coping to Predicting Outcome.

Objective: Neurocognitive and psychosocial late effects are well-documented following pediatric brain tumor (PBT). Although neurocognitive deficits may directly impact psychosocial functioning, this impact may occur via effects on survivors' ability to cope.

Participants and Methods: We assessed neurocognitive and psychosocial functioning of 17 survivors of PBT ( 6 girls) and 15 healthy children (9 girls). Children completed a working memory task (Nback) during functional magnetic resonance imaging (fMRI), a neuropsychological assessment of executive function (WISC-IV, D-KEFS), and ratings of psychosocial functioning (Revised Class Play (RCP), CBCL, YSR) and coping (Responses to Stress Questionnaire) were obtained from children and parents.

Results: Relative to healthy children, survivors performed more poorly on measures of executive function $(p<.001)$; groups did not differ in coping style ( $p s>.05)$. Survivors showed reduced Nback-related BOLD signal in superior frontal regions (BA 6,8) and increased signal in the anterior cingulate (BA 32) during fMRI. Survivors rated themselves as lower in popularity/leadership (RCP; $=.03$ ) and as having marginally elevated social problems (YSR; $p=.09$ ). Parents rated survivors as more sensitive/isolated, less popular, and more victimized (RCP; ps <.05) and as having significant social problems (CBCL; $\mathrm{p}<.01)$. Multiple mediator models using ordinary least squares path analysis showed that coping consistently predicted better social functioning across models. Brain activation during fMRI also predicted sensitivity/isolation by parent report, and executive function predicted sensitivity/isolation and social problems by child self-report.

Conclusions: Although neurocognitive deficits are well-documented following PBT, survivors' coping has received less attention, but mav more directly contribute to social deficits. These findings help clarify 
the nature of late effects in survivors, and suggest potential avenues for intervention.

Correspondence: Kristen E. Robinson, Ph.D., Pediatric Psychology \&. Neuropsychology, Nationwide Children's Hospital, 700 Children's Drive, Columbus, OH 43205.E-mail: kristen.robinson@nationwidechildrens. org

\section{Dementia (Subcortical, Specific Disorders, MCI, etc.)}

\section{N. DEYOUNG \& B. SHENAL. Classifying patients with and without dementia using cardiovascular risk factors.}

Objective: The relationships between cardiovascular risk factors (CVRs) and dementia can help providers identify patients at high risk for dementia. CVRs are often studied in isolation making it difficult to understand the dynamic relationships. Determining which CVRs are the strongest predictors of dementia can help determine which CVRs suggests a need for additional dementia evaluation.

Participants and Methods: 130 veterans were referred and evaluated in a memory assessment clinic (aged $\mathrm{M}(\mathrm{SD})=74.8(10.61)$, range $=44$ to $96,127 \mathrm{M} / 3 \mathrm{~F}$ ). The prevalence of dementia and CVRs was compared to the overall medical center population of 32,246 individuals. Discriminant function analysis created a predictive model for dementia based on linear combinations of CVRs.

Results: Memory assessment patients had significantly higher rates of dementia, cardiovascular disease, hypertension, ischemic heart disease, obesity, and peripheral vascular disease (all ps<0.001). The discriminant function analysis showed a strong association between group and predictors $\mathrm{F}(3,126)=5.78, \mathrm{p}=0.001, \mathrm{R}^{\wedge} 2=0.35$. The function was able to correctly classify $68 \%$ of the patients with dementia. Previous cardiovascular accidents, and ischemic heart disease had the best discriminative ability.

Conclusions: This study shows that evaluating the presence of CVRFs in patients with memory complaints can help inform care. A history of strokes and heart disease appear particularly powerful at detecting dementia. Evaluating CVRFs may help improve the sensitivity and specificity of dementia diagnosis and lead to improved clinical outcomes. Additional studies may be helpful to determine if CVRFs are predictive of mild neurocognitive impairment versus other types of dementia. Correspondence: Nathaniel DeYoung, Ph.D., MHSL, Salem Veterans Medical Center, 1970 Roanoke Boulevard, Salem, VA 24153. E-mail: nathaniel.deyoung@va.gov

\section{C.H. FONG, D.M. SCHIEHSER \& V. FILOTEO. Procedural-Based learning in Parkinson's Disease Motor Subtypes.}

Objective: Previous research has indicated that Parkinson's disease (PD) patients with a Tremor Dominant (TD) motor presentation tend to be less cognitively impaired than Postural Instability/Gait Difficulty (PIGD) patients. No study, however, has examined cognitive tasks thought to measure processes that emphasize the basal ganglia (BG). The present study sought to evaluate differences in procedural learning between TD-PD and PIGD-PD subtypes using a second-order Serial Reaction Time (SRT) task.

Participants and Methods: Patients were classified into subtypes using Movement Disorders Society criteria. Nineteen TD-PD, 18 PIGD-PD, and 14 controls were administered the SRT task, which required rapidly pressing one of four computer keys based on the appearance of an asterisk in a corresponding screen location over 6, 48 trial blocks. Each block contained a second-order repeating sequence, except block 5 , which consisted of a random sequence. Learning scores were computed by subtracting the median reaction times (RT) of blocks 4 and 6 from the random, non-sequenced block 5 .

Results: All three groups demonstrated procedural learning in that their RT decreased across blocks 1-4 and their learning scores were significantly different than zero. There were no group differences in learning scores among the three groups, however, indicating that procedural learning performances did not differ between the TD-PD and PIGD-PD subgroups.

Conclusions: This study did not identify any difference between TD-PD and PICD-PD groups on a classical measure of procedural learning. These findings suggest the BG structures responsible for procedural learning are not necessarily involved in the motor symptoms that define TD-PD and PIGD-PD subtypes.

Correspondence: Christopher H. Fong, BS, Research, VA San Diego Healthcare System, 3350 La Jolla Village Dr 116B, San Diego, CA 92161.E-mail:c4fong@ucsd.edu

\section{MURRAY, E. PAEK \& S. NEWMAN. Behavioral and Neural} Correlates of Word Retrieval Treatment for Dementia.

Objective: Whereas word retrieval in individuals with dementia may be improved or at least maintained via behavioral treatment (e.g., Jokel et al., 2014), questions remain regarding: (a) efficient treatments that evoke immediate gains and long-term maintenance of those gains (longterm outcomes are infrequently reported), (b) whether noun and verb retrieval respond to behavioral treatment (few prior studies have examined verb retrieval), (c) the neural and behavioral mechanisms that support treatment-induced linguistic recovery (neural mechanisms have been infrequently reported, particularly using DTI), and (d) prognostic variables (e.g., cognitive status; language profile) to guide clinicians in prescribing the type and amount of behavioral treatment needed to achieve functional change.

Participants and Methods: A multiple baseline, single-subject across subjects and behaviors design was used to examine both immediate and long-term effects of anomia treatment on the language skills of 2 participants with frontotemporal dementia (1 with progressive nonfluent aphasia; 1 with semantic dementia), and identify neural changes that accompany behavioral treatment effects. Participants completed pre- and post-treatment cognitive-linguistic assessment and MRI scanning sessions (including a 3 month post-treatment follow-up) and 16 60-min treatment sessions to target spoken naming of nouns and verbs. A variety of treatment tasks (e.g., semantic feature analysis; speeded confrontation naming) were included to foster transfer of treatment effects (Ehlardt et al., 2008).

Results: Both participants demonstrated improvements, but different patterns of: response to nouns vs. verbs, extent and loci of neural activation changes, and degree and types of language generalization and maintenance.

Conclusions: Individuals with progressive dementing diseases respond positively to naming treatment and at least some are able to maintain behavioral and neural activation gains. Discussion will focus on implications for future treatment research.

Correspondence: Laura Murray, Ph.D., Speech and Hearing Sciences, Indiana University, 200 S. Jordan Ave, Bloomington, IN 47405. E-mail: lmurray@indiana.edu

A.S. ORD, R. KINTZING, J. PHILLIPS, K. BHATTACHARJEE, S. SLOGAR, A. STITT \& S. SAUTTER. Adaptive Behavior, Practical Judgment, and Cognitive Functioning in Older Adults.

Objective: The goal of the present study is to investigate the correlations among practical judgment (as measured by the Test of Practical Judgment [TOP-J]), basic cognitive functioning (as measured by the Dementia Rating Scale-2 [DRS-2]), and adaptive behavior (as measured by the Texas Functional Living Scale [TFLS]), in order to better understand the impact of cognitive decline on daily living.

Participants and Methods: The participants were 278 older adults aged 56 to 95 who were seen for a neuropsychological evaluation at an outpatient neuropsychological clinic in Virginia. The mean age of patients was 74 years $(\mathrm{SD}=10.01)$. The mean DRS-2 total score was $124(\mathrm{SD}=14.61)$, the mean TFLS score was $38(\mathrm{SD}=9.97)$, and the mean TOP-J score was $20.3(\mathrm{SD}=5.38)$. Bivariate correlations were conducted to explore relationships among these variables. 
Results: DRS-2 total scores had a strong positive correlation with TFLS scores, $\mathrm{r}(278)=.79, \mathrm{p}<.001$, and with TOP-J scores, $\mathrm{r}(278)=.51, \mathrm{p}$ $<.001$. TOP-J scores were also positively correlated with TFLS scores, $\mathrm{r}(278)=.53, \mathrm{p}<.001$.

Conclusions: Results of this study indicate that there is a strong relationship between measures of adaptive behavior, practical judgment, and cognitive function. Further research of the relationship among these variables is recommended in order to determine whether measures of basic cognitive function may be used to predict patient's level of adaptive behavior and practical judgment. Clinical implications of these findings will be further discussed within this presentation.

Correspondence: Anna S. Ord, MBA, MA, PsyD Candidate, School of Psychology \& Counseling, Regent University, 3304 Eamon Court, Apt. 103,Virginia Beach,VA 23452.E-mail: annashi@mail.regent.edu

\section{A.S. ORD, J. PHILLIPS, R. KINTZING, K. BHATTACHARJEE, D. HOWERIN, A. STITT \& S. SAUTTER. A Factor Analysis Cross- Validation of the Dementia Rating Scale-2.}

Objective: The goal of the present study was to conduct a cross-validation of the Dementia Rating Scale-2 (DRS-2). Previous factor analyses of the DRS-2 have questioned the validity of its original five subscales, raising questions regarding their interpretations and clinical applications. The present study examined all 36 items of the DRS-2 to explore the underlying factor structure behind this measure.

Participants and Methods: The participants were 692 older adults aged 56 to 95 who were seen for a neuropsychological evaluation at an outpatient neuropsychological clinic in Virginia. The mean age of patients was 74 years $(\mathrm{SD}=10.01)$. The mean DRS-2 total score was $124(\mathrm{SD}=14)$.

Results: A principal components factor analysis was performed, and the best fitting model identified that five factors should be retained. This is consistent with the original five subscales identified within the DRS-2. However, factor loading of most items was not consistent with the conceptual classification of these items into subscales. In fact, most items had heavy loading on one factor with eigenvalue of 7.5 , and the rest of the factors had significantly lower eigenvalues (in the range of 1.5-2.9). Conclusions: In summary, the present study supports the findings of variability in DRS-2 factor composition reported in previous studies. Results also suggest that while factor structure in some samples may be multifactorial, DRS-2 scores in a large sample of older adults (who are primarily referred for evaluation due to memory concerns) may reflect a single factor (e.g., general dementia).

Correspondence: Anna S. Ord, MBA, MA, PsyD Candidate, School of Psychology \& Counseling, Regent University, 3304 Eamon Court, Apt. 103, Virginia Beach, VA 23452.E-mail: annashi@mail.regent.edu

\section{R. PABÓN, D. LOCKE, S. HANNA \& G. SMITH. Stability of MCI} Patient Centered-Outcomes Following Intervention.

Objective: Patient-centered care involves understanding which problems associated with Mild Cognitive Impairment are most concerning to individuals so affected. HABIT is a multi-component program for individuals with MCI and caregivers intended to impact daily function, mood, self-efficacy and quality of life. We are interested in knowing whether participating in the program itself shifts the way participants rank the importance of some aspects of their lives.

Participants and Methods: 36 participants (18 patients and 18 caregivers) completed a rank ordering of the 12 HABIT outcomes at the beginning and the end of the 10-day program prioritized from $1=$ most important to $12=$ least important.

Results: Wilcoxon signed-rank test showed that patients did not significantly change their prioritization of the assessed life aspects after HABIT. Changes were observed in the way caregivers ranked the anxiety of their loved ones $(\mathrm{Z}=-1.965, \mathrm{p}=0.049)$. Caregivers' median rank for Participant Anxiety was 7 at beginning and 9.5 at end of program.

Conclusions: MCI patients and caregivers appear to remain stable in their prioritization of outcomes even after intensive intervention, though small sample limits our ability to detect modest effects. Quality of life and self-efficacy remain paramount to patients and caregivers, suggesting these are robust targets for own interventions in MCI.

Correspondence: Ricardo Pabón, Ph.D., Clinical Psychology, Ponce School of Medicine and Health Sciences, Estancias del Rio, 26 Tallaboa, Aguas Buenas 00703. E-mail: ricardogonzalezpabon@hotmail.com

E. HESSEN, L. BLOMSø, C. HOLMEIDE, I. REINVANG, C.F. ELIASSEN, T. FLADBY \& D. AARSLAND. Cognitive profiles in MCI and newly diagnosed and unmedicated Parkinson disease. Objective: While typical features of cognitive impairment are well described in traditional MCI, characteristics of early cognitive impairment in Parkinson disease (PD) are not yet established. The purpose of this study was to compare cognitive profiles in newly diagnosed and unmedicated PD with age and education matched healthy controls and patients with non-PD MCI. We hypothesized that 1 . Than non-PD MCI would be associated with deficits both in memory and other cognitive domains compared to healthy controls, 2. that early PD would score poorer than controls on executive measures and 3. that non-PD MCI would be characterized by poorer memory than early PD.

Participants and Methods: A total 163 persons took part in the study. 75 were diagnosed with non-PD MCI, 38 had early PD and 50 were healthy controls.

Results: According to our hypothesis patients with non-PD MCI scored poorer than controls on all measures of memory and executive functions employed while patents with early PD were poorer than controls on 2 of 4 executive measures (COWA and TMT-B), but similar to the controls with regard to memory. In comparison between non-PD MCI and early PD, non-PD MCI scored significantly poorer on verbal and visual memory measures as well as on a measure of working memory. Conclusions: Newly diagnosed and unmedicated PD show deficits in some executive measures that require speeded cognitive processing, whereas patients with an established non-PD MCI diagnosis show deficits in multiple cognitive domains, including memory and executive functions.

Correspondence: Erik Hessen, PhD, University of Oslo, Krags vei 13, Oslo 0783, Norway. E-mail: erik.hessen@nevropsykologi.no

D. MECHANIC-HAMILTON, S. NEGASH, R. HAMILTON \& S. ARNOLD. Executive Function, Memory and Partner Report of Everyday Functioning in Healthy Controls, MCI and AD.

Objective: The relationship between cognitive domains and the ability to carry out everyday tasks is an important area of investigation and affects how we counsel patients on the impact of cognitive impairment. This study aimed to investigate how executive function drives the relationship between memory impairment and care-partner functional report.

Participants and Methods: Subjects were recruited from the Penn Memory Center and the Clinical Core of the University of Pennsylvania's Alzheimer's Disease Center. Study groups included normal controls $(\mathrm{N}=288)$ and patients with Mild Cognitive Impairment $(\mathrm{N}=347)$ and Alzheimer's Disease ( $\mathrm{N}=689)$. Subjects completed a battery of neuropsychological tests and a study partner completed a measure of functional status.

Results: There were significant correlations between executive function measures and functional status in all groups $(p<0.05)$. Memory measures were also correlated with functional rating for patient groups $(p<0.05)$. In controls, the relationship between memory and everyday function was modified by performance on tests of executive function $(\mathrm{p}<0.05)$.

Conclusions: Executive functioning may play a role in the relationship between areas of cognitive deficit and everyday functioning. Additionally, the strength of these relationships can be explored to determine how cognitive interventions targeted to a specific domain may impact on measures of everyday function. 
Correspondence: Dawn Mechanic-Hamilton, PhD, Department of Psychiatry, University of Pennsylvania, 1012 Fitzwater Street, Philadelphia,PA 19147.E-mail: dawn.mechanic@uphs.upenn.edu

D. STEED \& S. MILLER. Differential Predictive Influence on the Development of Dementia: Depression and Neuropsychiatric Symptoms.

Objective: The aim of this study was to investigate whether depression more specifically, or neuropsychiatric symptoms (NPS) more generally, were better predictors of the development of dementia over time.

Participants and Methods: We studied a sample of 1,052 individuals drawn from a national longitudinal database (National Alzheimer's Coordinating Center, 2013). Participants (age $\mathrm{M}=74.68, \mathrm{SD}=9.67$; Men $=53 \%$; Women $=47 \%$ ) were evaluated annually for six years with the Geriatric Depression Scale and the Neuropsychiatric Inventory. Qualified health practitioners diagnosed either no dementia, MCI, or dementia during each visit. Latent growth curve modeling techniques estimated the relationships between depression and dementia and NPS and dementia, respectively. Algebraic equality constraints were placed on the predicting parameters (slope and intercept) from neuropsychiatric and depression symptoms to dementia changes, with the understanding that if these constraints significantly worsen model fit, then depression and NPS would have differential predictive power.

Results: The initial level and changes in depression predicted the initial level of, but not the development of, dementia, while changes in NPS did in fact predict the development of dementia over time as well as its initial level. Thus, differential impact of changes was not estimated algebraically. However when the initial levels of NPS and depression were algebraically equal at estimating dementia changes, the model did worsen significantly, suggesting that the initial level of NPS is a more significant predictor in developing dementia over time than the initial level of depression.

Conclusions: Results extend earlier findings that, in addition to apathy, neuropsychiatric symptoms in general, are stronger predictors of dementia over time than depression. The NACG database is funded by NIA Grant U01 AG016976.

Correspondence: Drake Steed, M.A., Illinois School of Professional Psychology at Argosy, Chicago, 1618 Mayo, Dayton, OH 45409. E-mail: drakery4@gmail.com

S.C. SELIGMAN, T. GIOVANNETTI, G. MARTIN, J. KURCZEWSKI \& D.J. LIBON. Differences in Subtle and Overt Everyday Action Error Patterns in Healthy Aging, Mild Cognitive Impairment, and Alzheimer's Disease.

Objective: There is growing evidence that mild cognitive impairment (MCI) impacts everyday functioning. On performance-based measures of everyday action, individuals with Alzheimer's disease (AD) omit more task steps than healthy older adults, but action error patterns have not been compared across healthy older adults and individuals with MCI and AD. We predicted that subtle action errors (i.e., micro-errors) would differ between healthy older adults and those with MCI.

Participants and Methods: 15 healthy older adults, 25 individuals with MCI, and 19 individuals with $\mathrm{AD}$ were videotaped performing the Naturalistic Action Test, a performance-based test of everyday action. Videos were scored for overt errors (i.e., omissions - steps never performed; commissions - steps performed incorrectly) and micro-errors (i.e., subtle, non-overt errors deemed inefficient to task completion). A measure of global cognition (MMSE) was administered.

Results: ANOVAs were conducted to examine differences in error profiles, and correlations were conducted between MMSE scores and error proportions. Proportions of omissions $(\mathrm{F}=43.24$; $\mathrm{p}<0.001)$ and micro-errors $(\mathrm{F}=36.89 ; \mathrm{p}<0.001)$ differed across groups. As expected, omission proportion was greater in the $\mathrm{AD}$ group than both healthy adults $(p<0.001)$ and MCI $(p<0.001)$ but did not differentiate healthy adults and MCI $(p>0.05)$. Micro-error proportion was higher in MCI than healthy adults $(p<0.01)$ and $\mathrm{AD}(\mathrm{p}<0.001)$ and was higher in healthy adults than AD $(\mathrm{p}<0.001)$. MMSE score was positively associated with micro-error proportion $(\mathrm{r}=0.52 ; \mathrm{p}<0.001)$ and negatively associated with omission proportion $(\mathrm{r}=-0.61 ; \mathrm{p}<0.001)$.

Conclusions: Micro-errors are sensitive to subtle cognitive decline associated with MCI; however, overt omission errors best characterize the functional deficits associated with AD. Micro-errors may thus be useful in identifying those at risk for $\mathrm{AD}$, and strategies that preclude omissions may be most effective in preventing or delaying functional disability in AD.

Correspondence: Sarah C. Seligman, Psychology, Temple University, 1701 N. 13th Street, 6th Floor Weiss Hall, Philadelphia, PA 19122. E-mail:sarah.seligman@temple.edu

S.R. THORGUSEN, Y. SUCHY, G.J. CHELUNE \& B.R. BAUCOM. Examining a Theoretical Model of Practice Effects and Cognitive Decline: Contributions of Learning and Response to Task Novelty. Objective: The present study examined a theoretical model of the interrelationships among cognitive decline, learning, novelty effect and PEs in older adults. The model, proposed by Suchy et al. (2011), posits that learning and cognitive effects of task novelty differentially contribute to $\mathrm{PE}$ at different points along a trajectory of abnormal (or pathological) cognitive decline associated with mild cognitive impairment and dementia.

Participants and Methods: Sixty-six adults (ages 65-89) with normal to moderately impaired cognitive status completed a brief cognitive battery including measures of general cognitive functioning (DRS-2), list learning (RAVLT), response to task novelty (change in response efficiency between first and second trials of a motor programming task), and PEs (difference between two trials of the WAIS-IV Coding and Symbol Search subtests administered at 30-minute intervals).

Results: Results indicated a quadratic effect of cognitive decline on PEs for WAIS-IV Symbol Search $(b=-.119, t=-2.532, p=.014)$, such that largest PEs were observed at mild levels of decline. Novelty meditated this relationship for below-average cognitive status. Cognitive decline showed a positive linear relationship with PEs for WAIS-IV Coding ( $b=.640, t=3.028, p=.004)$; list learning did not mediate this relationship.

Conclusions: Results partially supported our model of PE. Explicit learning and novelty effect had unique effects on two different measures of $\mathrm{PE}$ and results suggest that these contributing variables may differentially influence PE at various levels of general cognitive functioning. While the results provide preliminary insights into cognitive processes underlying PEs, further research is needed to explicate the component cognitive processes related to changes in PEs with cognitive decline.

Correspondence: Sommer R. Thorgusen, PhD, Mental Health Service, VA Puget Sound Healthcare System, 5941 Suwannee Cir, Murray, UT 84123.E-mail:somrae@gmail.com

C.A. FYOCK \& B.M. HAMSPTEAD. Comparing the Relationship between the Memory Assessment Clinic Memory Scales, Objective Memory Performance, and Medial Temporal Lobe Volumes in MCI patients.

Objective: Recent literature has focused on the utility of subjective memory complaints for predicting subsequent conversion to mild cognitive impairment (MCI) or Alzheimer's disease. The Memory Assessment Clinic Self-Report Memory Scale (MAC-S) and the MAC-F (Family-Report) assess the patient's functioning in five memory scales: Remote, Numeric, Everyday, Semantic, and Spatial. The frequency of memory failures is measured in five additional memory scales: Semantic, Concentration, Everyday, Forgetfulness, and Facial. The current study examined the relationship between these subjective measures of memory complaint, as reported by both the MAC-s and MAC-F, objective memory test performance, medial temporal lobe (MTL) volumes.

Participants and Methods: Fifty-eight MCI participants completed the MAC-S and Repeatable Battery for the Assessment of Neuropsychological Status (RBANS), 51 informants completed the MAC-F, and 
40 patients completed structural magnetic resonance imaging (MRI). RBANS immediate (IMI) and delayed memory indices (DMI) were used as objective memory measures. Neuroquant ${ }^{\circledR}$ was used to obtain MTL volumes.

Results: None of the MAC-S scales were related to objective memory test performance; however, semantic ability and the frequency of forgetfulness were related to amygdala volumes. MAC-F semantic, spatial, and total memory ability were related to RBANS-DMI. Further, numeric memory ability was related to hippocampal volume. Remote, numeric ability, everyday ability, spatial ability, and total ability were all related to amygdala volume as were semantic frequency and facial frequency. Conclusions: Findings suggest that self-report of memory impairment is of minimal diagnostic value in MCI, likely due to anosagnosia, while informant report is comparatively more reflective of memory performance and MTL volumes in MCI patients.

Correspondence: Courtney A. Fyock, Emory University, 2397 Paul Ave NW, Atlanta, GA 30318. E-mail: cfyock22@gmail.com

L.A. SCHAEFER, D. HUREMOVIC, M. LEHMANN, A. ANAND \& A. ISAACSON. Imaging-Diagnosed Normal Pressure Hydrocephalus in a Patient Initially Presenting with Psychiatric Symptoms.

Objective: Normal pressure hydrocephalus (NPH) is a blockage of the flow of cerebrospinal fluid, without an increase in intracranial pressure. On neuroimaging, it appears as ventriculomegaly that is out of proportion to sulcal atrophy. NPH is traditionally characterized by dementia, gait dysfunction, and urinary incontinence. Psychiatric and behavioral manifestations are less common, which may hinder its diagnosis.

Participants and Methods: A 65-year-old male was initially seen by Psychiatry for delusional behavior. According to the chart, he had traveled from Ireland, reportedly to ask a "friend" to marry him. They called police, who brought him to the hospital. The patient reported not having seen a physician in over 20 years. Physical examination showed no focal neurological deficits. Gait instability was noted, however he was not incontinent. He was referred for cognitive testing and imaging. Results: Neuropsychological assessment revealed deficits in immediate and delayed memory, psychomotor speed, semantic fluency, and set-shifting. Auditory attention, visuospatial functioning, and naming were intact. The patient was oriented to date and had a pleasant demeanor and personality, but was disinhibited and demonstrated effortless and profuse confabulations. He was also delusional, believing he was at a train station. Magnetic resonance imaging (MRI) of the brain was performed, which showed marked dilatation of the lateral and third ventricles out of proportion with mild sulcal prominence, consistent with chronic NPH. There was no noticeable hippocampal atrophy.

Conclusions: This case highlights the potential for NPH to present psychiatrically, without the classic "triad". Given the aging of the population, recognition of delusions as a possible initial symptom is important. Neuroimaging in these cases could help increase detection of this potentially treatable form of dementia.

Correspondence: Lynn A. Schaefer, Ph.D., Physical Medicine and Rehabilitation, Nassau University Medical Center, 2201 Hempstead Turnpike, Box 31, East Meadow, NY 11554.E-mail: lschaefe@numc.edu

\section{R.K. BISS, K.J. MURPHY, G. ROWE \& L. HASHER. A Novel Method to Improve Face-Name Memory in Older Adults with Age-Normal Memory and aMCI.}

Objective: Recent research indicates that older adults use presentations of useful distraction to implicitly rehearse words from a memory list. This pilot study explored whether this distraction-as-rehearsal method can also boost memory for face-name associations in older adults with age-normal memory and those with amnestic-mild cognitive impairment (aMCI). Implicit processes are preserved and perhaps even enhanced as explicit memory declines in aMCI. Therefore, we predicted that implicit rehearsal opportunities might produce a particular memory boost among individuals with aMCI, for whom forgetting names is a primarily memory concern.

Participants and Methods: Five older adults with aMCI and 10 older controls studied 24 face-name pairs and were tested with an immediate cued recall test. During a 30-min retention interval, 10 of the face-name pairs re-occurred as distraction in an ostensibly unrelated face-judgment task, providing an opportunity to implicitly rehearse these pairs. Memory for pairs repeated during the retention interval was compared to unrepeated pairs using delayed cued recall and forced-choice recognition. Results: Overall, participants showed improved cued recall of repeated face-name pairs compared to unrepeated pairs, $\mathrm{t}(14)=2.32$, $\mathrm{p}=.04$, with a similar trend on recognition, $\mathrm{t}(14)=1.99, \mathrm{p}=.07$. This effect tended to be larger in the aMCI group $(\mathrm{d}=1.01)$ compared to older controls $(d=0.40)$, suggesting that individuals with reduced explicit memory disproportionately benefit from implicit rehearsals.

Conclusions: These findings indicate that distraction-as-rehearsal produces a robust benefit for face-name memory in older adults with and without aMCI. Future work will confirm this result with a larger sample and explore its application as a memory intervention strategy.

Correspondence: Renée K. Biss, Neuropsychology \& Cognitive Health, Baycrest Health Sciences, 3560 Bathurst St., Toronto, ON M6A 2E1, Canada.E-mail:r.biss@baycrest.org.

K.J. BANGEN, D.A. NATION, E.C. EDMONDS, L. DELANO-WOOD, M.L. WERHANE \& M.W. BONDI. Impact of cerebrovascular risk on brain amyloid- $\beta$, cerebral blood flow, and neuropsychological functioning in normal aging and mild cognitive impairment.

Objective: It is increasingly clear that underlying cerebrovascular disease is highly prevalent in mild cognitive impairment (MCI), a clinical entity thought to represent a prodromal stage of dementia such as Alzheimer's disease (AD). We therefore examined the influence of vascular risk burden on brain amyloid- $\beta$, cerebral blood flow (CBF), and cognition within the well-characterized nondemented Alzheimer's Disease Neuroimaging Initiative (ADNI) cohort.

Participants and Methods: 151 ADNI participants were classified into demographically-comparable groups according to comprehensive neuropsychological criteria (MCI=31; normal controls $[\mathrm{NC}]=120$ ) and underwent anatomical and arterial spin labeling MRI scans and florbetapir positron emission tomography. Vascular risk was quantified with the Framingham Stroke Risk Profile.

Results: MCI participants demonstrated significantly greater vascular risk burden relative to NC.s. Across all participants, greater vascular risk was associated with increased amyloid- $\beta$ burden and poorer cognition (memory, language, and executive dysfunction). Among NCs, elevated vascular risk was associated with increased CBF in the posterior cingulate, hippocampus, and frontal regions. In contrast, among individuals with MCI, greater vascular risk burden was related to trends toward decreased CBF in several regions.

Conclusions: Greater vascular risk burden was associated with amyloid- $\beta$ accumulation, alterations in CBF, and poorer cognitive function. Increased $\mathrm{CBF}$ in $\mathrm{NC}$ with elevated vascular risk burden may reflect cellular and vascular compensatory response to pathologic changes in preclinical dementia. Findings suggest that vascular risk assessment may have implications for detection of those at risk for progression to $\mathrm{AD}$ as well as treatment selection given that vascular risk factors may represent useful targets for preventing or slowing decline.

Correspondence: Katherine J. Bangen, University of California, San Diego, 9500 Gilman Drive, mail code 151B, La Jolla, CA 92093. E-mail: kbangen@ucsd.edu

C. LOBUE, L.H. LACRITZ, H.C. ROSSETTI, J. HART, K. WOMACK \& M. CULLUM. Self-reported History of Head Injury and Age of Diagnosis in Dementia.

Objective: To retrospectively examine whether history of traumatic brain injury (TBI) leads to earlier age of diagnosis of Alzheimer disease 
(AD), behavioral variant frontotemporal dementia (bvFTD), or Lewy Body dementia (LBD) within a large national cohort.

Participants and Methods: Subjects with a clinical diagnosis of AD, bvFTD, or LBD were obtained from the National Alzheimer's Coordinating Center Uniform Data Set. Subjects were categorized by group based on lifetime self-reported TBI with loss of consciousness but no chronic deficit (TBI+ nAD $=594 ; \mathrm{nbvFTD}=76 ; \mathrm{nLBD}=42)$ and compared to subjects without a history of TBI (TBI- $\mathrm{nAD}=7346$; $\mathrm{nbvFTD}=624$; $\mathrm{nLBD}=496)$ using ANOVA.

Results: Across diagnoses, the TBI+ and TBI- groups were similar in education (M EDUC=14.5), ethnicity, and vascular risk, although the TBI+ groups contained more males than the TBI- groups. TBI+ subjects were diagnosed with $\mathrm{AD}(\mathrm{M}$ Age $=73.6, \mathrm{SD}=10.5) 2.5$ years earlier $(\mathrm{p}=0.002)$ than TBI- subjects $(\mathrm{M}$ Age $=76.1, \mathrm{SD}=9.6)$. TBI+ subjects were diagnosed with bvFTD (M Age=61.5, $\mathrm{SD}=8.0$ ) 3.1 years earlier $(p<0.0001)$ than TBI- subjects $(\mathrm{M}$ Age $=64.6, \mathrm{SD}=8.9)$. Average age of diagnosis of LBD was not significantly different $(p=0.72)$ between TBI+ (M Age=74.4, SD=8.9) and TBI- groups (M Age=74.3, SD=7.7). Conclusions: History of self-reported TBI was associated with an earlier diagnosis of $\mathrm{AD}$ and bvFTD compared to those without TBI, but showed no relation to age of diagnosis in LBD. These findings provide preliminary evidence to suggest that TBI may be a risk factor for earlier expression of certain types of dementias later in life.

Correspondence: Christian LoBue, UT Southwestern Medical Center, 5953 Lofland Drive, Frisco, TX 75033. E-mail: christian.lobue@ utsouthwestern.edu

M.R. TIMPANO SPORTIELLO, D.M. CAMMISULI, E. CASTRO, S. TOCCHINI, M. GNOFFO \& M. BARONCINI. Italian Network Study Group on Parkinson's Disease-Mild Cognitive Impairment (INPM): Preliminary Results.

Objective: Our study aims at defining the neuropsychological profile of subjects with Parkinson's Disease-Mild Cognitive Impairment (PD-MCI) and evaluating the proportion of each PD-MCI subtypes.

Participants and Methods: 54 PD-MCI (M:F=60:40\%; Age: 72,4 47,5 ; Education: 6,8 $\pm 3,5)$ were strictly recruited by following the Movement Disorder Society guidelines (2012) for the assessment at Level II, including: Milan Overall Dementia Assessment (MODA); ADL and IADL; Digit Span, Corsi Span, Pairs Associates Learning, Memory of Prose, Corsi Suvra-span; Visual Search, Stroop Test; Street's Completion Test; Constructive Apraxia Test; Category Fluency Test, Boston Naming Test; Frontal Assessment Battery Subtest Go-no-Go, Brixton Test, Tower of London, Neuropsychiatric Inventory. A non-parametric Wilcoxon Signed-Rank Test was used to compare performances on neuropsychological measures.

Results: Orientation and autonomy were preserved, as expected. MODA total score was at the superior limits of the borderline area $(89 \pm 9)$. The subjects performed more poorly on long-term memory than short-term memory tests, both for verbal and non-verbal stimuli $(p<.05)$. Sensitivity to interference was more damaged than selective attention $(p<$ $.05)$. No significant differences were found by comparing instrumental extra-memory abilities. Among frontal domains, planning abilities were the most fragile sub-domain $(\mathrm{p}<.05)$. The capacity to produce word by using an unusual procedure was less compromised than the set-shifting/ rule detection $(\mathrm{p}<.05)$. Apathy $(56 \%)$ and depression/dysphoria $(45 \%)$ were the most relevant psychiatric symptoms. The majority of the sample was represented by amnesiac multiple domain (63\%), followed by non-amnesiac multiple domain $(20 \%)$, executive single domain $(11 \%)$ and attentive single domain $(6 \%)$.

Conclusions: Future research should clarify if the executive functions and visual-space episodic memory impairment may represent the main risk factors for PD-MCI conversion into Parkinson's Disease Dementia. Correspondence: Davide M. Cammisuli, Ph.D, Surgery, Medical, Molecular, and Critical Area Pathology, Pisa University School of Medicine, 6, Via Bonanno Pisano, Pisa 56126, Italy. E-mail: d.cammisuli@med.unipi.it
A.M. WEAKLEY, J. WILLIAMS, M. SCHMITTER-EDGECOMBE \& D. COOK. Automated Classification of Mild Cognitive Impairment and Dementia.

Objective: Accurate detection of cognitive impairment can be challenging, particularly when data is missing. Automated machine learning algorithms offer an alternative diagnostic pathway to traditional methods that yield valuable insights. This research explores the use of neuropsychological and demographic data to predict Clinical Dementia Rating scores (CDR) and clinical diagnoses through the implementation of two machine learning algorithms (i.e., naïve Bayes, C4.5 decision tree). Participants and Methods: Participants classified using clinical diagnosis criteria were 52 individuals with dementia, 97 with mild cognitive impairment, and 161 cognitively healthy older adults. Participants classified using $\mathrm{CDR}$ were 25 individuals with $\mathrm{CDR}=1,93$ with $\mathrm{CDR}=0.5$, and 154 with $\mathrm{CDR}=0$. Twenty-seven variables including age, gender, education, global cognitive status, memory, working memory, attention, visuospatial ability, executive functioning, language, and functional ability measures were used to predict diagnostic status.

Results: The clinical diagnosis dataset resulted in higher classification accuracies (68.4-79.7\%) than the CDR dataset (66.2-76.1\%). Diagnostic accuracy was slightly improved with the naïve Bayes model (73.2-79.7\%) compared to the decision tree (66.2-72.9\%). Accuracy, sensitivity and specificity were generally within acceptable ranges. Furthermore, Naïve Bayes resulted in higher accuracy levels when missing data was present. Therefore, naïve Bayes may be of particular benefit as it is not uncommon to have missing data in clinical settings.

Conclusions: Results from this study suggest that machine learning algorithms can reliably detect cognitive status using neuropsychological and demographic data. Therefore, machine learning algorithms have the potential to aid in diagnostic decision making and may be of increasing value when data is incomplete.

Correspondence: Alyssa M. Weakley, M.S., Psychology, Washington State University, $415 \mathrm{NW}$ Maryland st, Pullman, WA 99163. E-mail: alyweakley@gmail.com

\section{A.M. WEAKLEY, J. WILLIAMS, M. SCHMITTER-EDGECOMBE \& D. COOK. Neuropsychological Measures Essential for Cognitive Impairment Classification Using Machine Learning.}

Objective: Diagnosis of cognitive impairment is traditionally performed by one or more clinical experts using numerous sources of information. To maximize diagnostic accuracy with the smallest amount of data a variable selection method was applied to demographic and neuropsychological variables.

Participants and Methods: Analyses were performed on participants classified using clinical diagnostic criteria and clinical dementia rating (CDR). Participants classified using clinical diagnosis criteria were 52 individuals with dementia, 97 with mild cognitive impairment (MCI), and 161 cognitively healthy older adults. Participants classified using CDR were 25 individuals with $\mathrm{CDR}=1.0,93$ with $\mathrm{CDR}=0.5$, and 154 with $\mathrm{CDR}=0$. Twenty-seven variables including age, gender, education, global cognitive status, memory, working memory, attention, visuospatial ability, executive functioning, language, and functional ability measures were used in the analyses. A wrapper-based variable selection approach was applied to two machine learning algorithms (i.e., naïve Bayes, C4.5 decision tree). This approach evaluates combinations of variables and balances accuracy with number of tests required.

Results: Participant groups were reliably distinguished between with 78.7-80.6\% accuracy with 4-8 variables. Selected variables included age, executive functioning, language, attention, memory, and functional status measures. Memory was found to be more important for distinguishing between MCI/CDR=0.5 and cognitively healthy older adults/ $\mathrm{CDR}=0$. Yet, domains beyond memory, including language, executive functioning, and global cognitive status, were central to delineating between MCI/CDR=0.5 and dementia/CDR=1.

Conclusions: Cognitive domains essential for accurate diagnosis and stage of impairment were identified using machine learning techniques. 
Determining the fewest number of variables required for diagnosis translates to reduced assessment time and cost.

Correspondence: Alyssa M. Weakley, M.S., Psychology, Washington State University, 415 NW Maryland st, Pullman, WA 99163. E-mail: alyweakley@gmail.com

M. SEIDENBERG, C. KAY, M. LANCASTER, C. KANDAH, E. HOIDA, D.A. KELLY, S. DURGERIAN, J.L. WOODARD, K.A. NIELSON, J. SMITH \& S.M. RAO. Longitudinal Investigation of Recent and Remote Famous Names in MCI and Healthy Participants.

Objective: Alzheimer's Dementia (AD) is typically characterized by better remote memory than recent memory. Few studies have investigated this phenomenon with Mild Cognitive Impairment (MCI). We compared healthy controls and MCI groups for accuracy and response times (RT) recognizing remote and recent names at baseline and after 18 months. Participants and Methods: 31 cognitively intact participants (mean age 72), and 15 MCI participants (mean age 75 ) were shown 30 non-famous names and 30 famous names. Famous names included 10 recent famous, 10 famous 30 years ago, but not currently well-known (transitory), 10 famous 30 years ago and currently well known (enduring). Participants responded famous or non-famous by button press.

Results: MCI group demonstrated lower recognition baseline accuracy and greater decline than controls after 18 months $(p=.02)$. MCI group also showed greater decline for recent and transitory names, but not for enduring names $(p=.05)$. Controls demonstrated faster RT than MCI group $(p=.03)$. Enduring names were recognized faster than transitory names which were faster than recent names $(p=.001)$. Controls showed better discrimination between famous and non-famous names than MCI patients at baseline, and the difference was more pronounced at follow-up $(\mathrm{p}=.02)$.

Conclusions: Frequency of exposure rather than just memory age may determine the better recall of remote memories compared to recent memories. Enduring names have presumably been more frequently encountered than transitory names, and both have likely been more frequently encountered than recent names. These re-exposures update and re-consolidate previously acquired memories and may produce a stronger multimodal long-term representation.

Correspondence: Dana A. Kelly, Psychology, Rosalind Franklin University of Medicine and Science, 3507 Green Bay Rd, Apt 312, North Chicago, IL 60064.E-mail: dana.kelly@my.rfums.org

D.A. KELLY, E. HOIDA, C. KANDAH, C. KAY, M.M. STIKA, M. LANCASTER, M.A. SUGARMAN, J. SMITH, J.L. WOODARD, K.A. NIELSON, S.M. RAO, S. DURGERIAN, W. SONG, N. PLISKIN \& M. SEIDENBERG. Semantic Specificity and Memory Age of Famous Names Reflect Progression of Alzheimer's Disease.

Objective: Person identity is impaired in Alzheimer's Disease (AD) and is considered an early indicator of semantic memory decline. Typically, individuals with $\mathrm{AD}$ lose specific semantic knowledge before losing superordinate information and they provide less semantic knowledge for recent than remote famous names. We examined the effect of memory age and semantic specificity of famous names in healthy controls (HC), persons with Mild Cognitive Impairment (MCI), and persons with AD. Participants and Methods: 73 participants (ages 65-85) were categorized into three groups: HC $(\mathrm{n}=44)$, MCI $(\mathrm{n}=8)$, and $\mathrm{AD}(\mathrm{n}=21)$ and were administered a famous name semantic knowledge task, which assessed accuracy and response time to category, associative, attribute levels of semantic specificity across recent and remote time epochs.

Results: Mixed-design ANOVAs demonstrated a significant 3-way (group*level*epoch) interaction $(\mathrm{p}=.001)$. MCI and HC performed faster and more accurately than $\mathrm{AD}$ participants across all semantic levels and epochs. MCI were more impaired at the attribute level than the associative $(p=.003 ; d=0.68)$ and categorical $(p<.001 ; d=0.91)$ levels for recent names. MCI also performed more poorly than HC at the recent attribute level $(p=.009 ; d=0.80)$. All groups responded faster for categorical knowledge than for associative $(\mathrm{p}<.001)$ and attribute $(\mathrm{p}<.001)$ levels across epochs.

Conclusions: Results suggest that semantic memory deficits at the most specific semantic level are specific to persons with MCI. The semantic specificity of this task may have particular value in examining early indicators of decline.

Correspondence: Dana A. Kelly, Psychology, Rosalind Franklin University of Medicine and Science, 3507 Green Bay Rd, Apt 312, North Chicago, IL 60064.E-mail: dana.kelly@my.rfums.org

K. KAUZOR, A.T. FLOWERS, J. AVILA, G. CASTILLO, E. WOO, J. RINGMAN, P. LU, L. APOSTOLOVA \& J. RAZANI. Memory Performance in MCI Using Memory and ADL Tasks.

Objective: Mild cognitive impairment (MCI) is a mental state that is characterized by cognitive deficits, which lie in a prodromal state in between normal aging and dementia. The present study sought to determine if performance on an observation-based activities of daily living shopping task is similar to that of a commonly used neuropsychological memory test in MCI individuals.

Participants and Methods: A total of 95 individuals composed two groups: 63 normal controls, and 32 individuals diagnosed with MCI. All participants were administered the California Verbal Learning Test-II (CVLT) and the Direct Assessment of Functional Status (DAFS), as apart of a larger test battery.

Results: A series of multivariate analyses of covariance (MANCOVAs) with education as the covariate indicated worse performance by MCI individuals on the CVLT brief delay, free delay, cued recall, and total intrusions subtests relative to controls. Similarly, separate MANCOVA analyses indicated that MCI participants performed worse on the DAFS overall shopping test, as well as on the subscales of free-recall, and recognition shopping relative to controls. Results of correlation analyses performed on the MCI individuals revealed positive relationships between the CVLT brief delay and DAFS shopping recognition task $(\mathrm{r}=.736)$, CVLT free delay and DAFS shopping recognition ( $\mathrm{r}=.640)$, CVTL cued recall and DAFS shopping free recall $(r=.603)$ and CVLT cued recall and DAFS shopping recognition tasks $(r=.790)$.

Conclusions: These results support the notion that memory deficits may contribute to an inability to properly perform the complex activity of daily living task of shopping.

Correspondence: Amina T. Flowers, B.A., Psychology, California State University, Northridge, 5454 Brynhurst Avenue, Los Angeles, CA 90043. E-mail:flowersamina@gmail.com

E. PIROGOVSKY TURK, V. FILOTEO \& D. HARRINGTON. Volumetric Correlates of Episodic Memory in Nondemented Parkinson's Disease.

Objective: To examine the specific relationships among different components of verbal memory and brain volumes in frontostriatal (FS) and medial temporal lobe (MTL) systems in non-demented patients with Parkinson's disease (PD).

Participants and Methods: PD patients $(\mathrm{n}=56)$ were administered the California Verbal Memory Test II-Short Form (CVLT). Several key indices were assessed including learning (total words recalled on learning trials 1-4), retention (Trial 4 - Long Delay Free Recall (LDFR)), cued retrieval (Cued recall discriminability - LDFR), and recognition retrieval (Recognition memory D' Prime - LDFR). Structural MRI was used to measure brain volumes in a priori defined regions that encompassed structures within the FS and MTL systems. Partial correlations (adjusted for age) were conducted between CVLT variables and regions of interest volumes.

Results: Poorer learning correlated with smaller volumes in all MTL regions. No clear relationship emerged between brain volumes and retention. Improved memory with recognition testing (recognition retrieval index) correlated with reduced volume in one MTL region. Improved memory with cues (cued retrieval index) correlated with FS and MTL volumes. 
Conclusions: These findings suggest that various aspects of memory have different neural substrates in non-demented PD. These results could have implications for the identification of early cognitive deficits in PD.

Correspondence: Eva Pirogovsky Turk, UC San Diego, 6714 avenida andorra, La jolla,CA 92037.E-mail: evapturk@gmail.com

B.L. DECK, T. GIOVANNETTI, S.C. SELIGMAN \& I. LAWRENCE. Everyday Functioning in Mild Cognitive Impairment: Understanding Impairment Through Analysis of Action and Language Errors.

Objective: The functional difficulties associated with mild cognitive impairment (MCI) are difficult to assess and poorly understood. Efficient and accurate assessments would facilitate diagnostic decisions and improve clinical trials. Based on the premise that domain general control processes (i.e. a single cognitive system controlling multiple, different cognitive operations) govern planning/execution in action and language domains, we examined relations between action and language variables in MCI to understand everyday action and explore whether language tasks are useful for assessing functional abilities.

Participants and Methods: 28 individuals with MCI completed the Naturalistic Action Test- Complex Version (NAT-CV) and the Script Generation Test (SGT). Performance errors were similarly coded for both tasks: Commission (inaccuracies such as sequence errors or object/lexical substitutions), Omission (step not performed/reported), and Micro-errors (object was touched or moved but not used/ pauses and filler words within/between a sentence). Participants also completed the MMSE and tests of executive function (Digits BW), language (FAS), and episodic memory $(\mathrm{P}[\mathrm{r}] \mathrm{VLT})$.

Results: Errors from the SGT were weakly related to each other ( $r$ 's $<.30)$ and were each associated with unique cognitive measures (SGT Omissions x PVLT, r=.39; SGT Micro-errors x FAS, $r=-.45)$. Correlations between SGT and NAT-CV errors showed a significant relation between SGT Commissions and both NAT-CV Commissions ( $\mathrm{r}=.46$ ), NAT-CV Micro-errors ( $\mathrm{r}=.44)$; all other relations were nonsignificant $(r$ 's $<-.31)$.

Conclusions: Verbal script generation is a multidimensional task that requires verbal fluency/ episodic memory ability. Accuracy of verbal scripts was associated with accuracy on functional tasks, suggesting a "domain-general" process may explain task inaccuracy but not other forms of error (i.e. omissions). Thus, verbal tasks may be helpful in assessing specific functional abilities but they should not be used in lieu of performance-based tasks in MCI.

Correspondence: Benjamin L. Deck, Bachelor's of Science, Neuroscience, Temple University, 341 Swope Road, Bethel, PA 19507. E-mail: bdeck8317@comcast.net

C.L. LASSEN-GREENE, O.C. OKONKWO, M. CROWE, K. BALL, D.C. MARSON \& V.G. WADLEY. Mild Cognitive Impairment and Everyday Function: Longitudinal Changes in Speed versus Performance.

Objective: Cross-sectional research has demonstrated that speed in performing instrumental activities of daily living (IADLs) is a more sensitive and perhaps earlier marker of amnestic Mild Cognitive Impairment (MCI) than level of task performance. The present study investigated whether longitudinal changes in speed of IADL performance differed between persons with MCI and cognitively normal peers.

Participants and Methods: Sixty-two (62) persons with MCI and 63 cognitively normal older adults underwent clinical and research evaluations at baseline and at 1 to 4 annual follow-up visits. IADL speed and performance accuracy were assessed each year with selected domains of the Timed IADL assessment, the Observed Tasks of Daily Living, the Financial Capacity Instrument, and driving-related assessments (Useful Field of View, Road Sign Test). We used mixed-model, repeated measures MANCOVA to examine group differences in slopes of change in speed and performance on measures of telephone use, grocery shopping, medication management, financial management, and driving risk, controlling for group differences in age, depressive symptoms, visual acuity, and contrast sensitivity.

Results: Relative to controls, MCI participants had significantly steeper slopes of decline in speed, or smaller rates of speed improvement, in telephone use, financial management, and driving tasks $p$-values $<.05$ ) (grocery shopping $p=.055$ ). The groups did not differ on rates of change in performance in any IADL domain.

Conclusions: Everyday tasks that require sustained attention and information processing speed take longer for persons with MCI to accomplish successfully, relative to cognitively normal controls, and this disparity in task completion time increases over time.

Correspondence: Caroline L. Lassen-Greene, MA, Psychology, UAB, Holley Mears Building, 924 19th Street South, Birmingham, AL 35233. E-mail: cgreene@uab.edu

M.L. CHAN, L. KRITIKOS, B. MILLER \& J.H. KRAMER. The Clinical Utility of a Behavior Rating Scale in Distinguishing Alzheimer Disease (AD) and Behavioral Variant Frontotemporal Dementia (bvFTD).

Objective: bvFTD is a neurodegenerative disease characterized by early personality and behavioral changes. Given the salience of behavior, clinician-rating of patient behavior is potentially a powerful tool for differential diagnosis, but the clinical utility of this method has not been examined. This study assesses whether behaviors directly observed during a neuropsychological evaluation can reliably differentiate bvFTD from AD.

Participants and Methods: We studied 256 patients with probable $\mathrm{AD}$ and 69 with probable bvFTD with MMSE $\geq 15$. After the completion of neuropsychological testing, the examiner, who was blind to patient diagnosis, rated 10 behaviors (agitation, stimulus-boundedness, perseverations, decreased initiation, motor stereotypies, distractibility, social disengagement, impulsivity, social inappropriateness, and impaired alertness) by level of severity (none, mild, moderate, or severe) based solely on observed behaviors during testing.

Results: A generalized linear model revealed that bvFTD patients were rated significantly more severe on stimulus-boundedness, perseverations, decreased initiation, motor stereotypies, distractibility, social disengagement, impulsivity, and social inappropriateness than $\mathrm{AD}$ patients ( $p$ 's<.005). A discriminant function using these variables correctly classified $96.5 \%$ of the AD patients but only $58 \%$ of the bvFTD patients. Social disengagement, social inappropriateness, impulsivity, perseverations, and distractibility were the strongest predictors.

Conclusions: bvFTD patients exhibit more behavioral symptoms during cognitive testing than $\mathrm{AD}$ patients, specifically stimulus-boundedness, perseverations, decreased initiation, motor stereotypies, distractibility, social disengagement, impulsivity, and social inappropriateness. These behaviors are rarely exhibited by $\mathrm{AD}$ patients, indicating that a behavior rating scale offers diagnostic specificity. Sensitivity is only modest, but impressive given that the ratings are based on observations during a brief, structured evaluation.

Correspondence: Michelle L. Chan, University of California, San Francisco, 675 Nelson Rising Lane, Suite 190, San Francisco, CA 94158. E-mail:mchan@paloaltou.edu

P. EBERT, E. BISHOP, J. FISK \& J. INGLES. Proactive and Retroactive Memory Interference in Adults with Amnestic Mild Cognitive Impairment and Typically Aging Adults.

Objective: Discrimination between normal memory decline and pathological memory decline is essential for early detection of memory disorders that commonly precede Alzheimer's disease, such as amnestic mild cognitive impairment (aMCI). This study investigated differences in proactive and retroactive memory interference between adults with MCI and adults with no cognitive impairment (NCI).

Participants and Methods: Thirty-two MCI and 103 NCI cases were drawn from a population-based study. Memory interference was 
measured by correct recall and intrusion errors on a commonly used clinical memory test, the California Verbal Learning Test- II (CVLT-II). Memory interference was analyzed by six planned $2 \times 2 \times 2$ Mixed Model ANCOVAs composed of one between group variable (MCI vs. NCI) and two within group variables: List recalled and Semantic Category (Shared vs. Non-shared semantic category) on measures of immediate and delayed recall. Recognition data was analyzed by a 2 x 2 Mixed Model ANCOVA composed of the variables: Group and Semantic Category. Significant results were further analyzed with a set of a priori t-tests. Results: While no group differences were detected for proactive interference, the MCI group demonstrated increased vulnerability to retroactive interference on delayed recall and recognition tasks compared to the NCI group.

Conclusions: These results suggest that memory interference may be a potential mechanism underlying memory decline in adults with MCI. Retroactive memory interference may be used to help distinguish pathological aging from normal aging.

Correspondence: Patricia Ebert, Ph.D., Seniors Health, Alberta Health Services, Rockyview General Hospital, 7007 14th Street SW, Calgary, ABT2V1P9, Canada.E-mail: patricia.ebert@albertahealthservices.ca

R. GARRETT, A. LIEBERMAN, R. DHALL, F. PONCE, N. SALINS \& A.I. TRÖSTER. Relationship Between Specific Cognitive Domains and Quality of Life in Parkinson's Disease with Mild Cognitive Impairment (PD-MCI).

Objective: Prior research conflicts whether mild cognitive problems impact quality of life (QOL) in Parkinson's disease (PD). A prior study showed mild cognitive impairment impacts only select domains of QOL (Reginold et al., 2013), but this study did not address 1) which cognitive domains impact QOL and 2) the extent to which such relationships might be mediated by depressive symptoms. The present study aims to answer these questions.

Participants and Methods: The sample was comprised of 63 males and females that were diagnosed with PD-MCI per The Movement Disorder Society's proposed diagnostic criteria. Patients did not have a diagnosis for Major Depressive Disorder. Methods included correlation and multiple regression analyses.

Results: The highest bivariate correlations were between Beck Depression Inventory-II (BDI-II) scores and PDQ-39 subscales (emotional well-being, cognition, and communication). Regression analyses showed that the BDI-II score is the most important predictor of all three PDQ-39 subscales. Cognitive composite scores in visuospatial skills and language contributed to the PDQ-39 emotional well-being subscale, but were not significant contributors to PDQ-39 communication and cognition subscales after controlling for depression.

Conclusions: The current study confirms prior findings noting the importance of depressive symptoms and their impact on quality of life in PD-MCI. We need to consider depressive symptoms in patient care as they may be more important than cognitive symptoms in determining QOL in PD-MCI. Our discussion addresses issues regarding PDQ-39 item content for these subscales.

Correspondence: Robin Garrett, Psy.D., Clinical Neuropsychology, Barrow Neurological Institute, 222 W. Thomas Rd, Ste 315, Phoenix, AZ 85013. E-mail: Robin.Garrett@DignityHealth.org

H.M. HOLDEN, S. TIERNEY, A. HERNDON, S. WOODS, D.C. DELIS, J. COREY-BLOOM \& P.E. GILBERT. Verbal Learning and Memory in Premanifest and Manifest Huntington's Disease: Evidence from the California Verbal Learning Test-II.

Objective: The California Verbal Learning Test-II (CVLT-II) is a well-validated clinical test of word list learning and memory that is sensitive to subtle difficulties. The purpose of the current study was to utilize the CVLT-II to characterize learning and memory performance in individuals in premanifest and manifest Huntington's disease (HD). Participants and Methods: Participants included 78 individuals diagnosed with HD, 48 premanifest gene carriers for HD (Pre-HD), and
73 healthy adults who completed the CVLT-II using standardized procedures as part of a research neuropsychological assessment battery. A total of 11 CVLT-II age- and gender-corrected standard scores were examined, including all recall measures, recognition discriminability, several learning characteristics, and errors.

Results: The HD group performed significantly worse $(\mathrm{ps}<.05)$ than the healthy adults on all indices, with the exception of repetition errors. The Pre-HD group demonstrated significantly lower performance than the healthy adults ( $\mathrm{ps}<.05$ ) on all recall and recognition measures, but performed similarly on learning slope, semantic and serial clustering, and errors.

Conclusions: The current results extend prior literature by characterizing learning and memory deficits in HD using the CVLT-II. Importantly, the current study supports the utility of the CVLT-II in detecting subtle episodic memory changes in premanifest HD. The pattern of CVLT-II performance in premanifest and manifest HD is suggestive of difficulties in initial acquisition and subsequent retrieval. Other aspects of learning and memory, including learning rate and strategic aspects of encoding, were not affected in Pre-HD, suggesting that these processes may deteriorate later in the disease process.

Correspondence: Heather M. Holden, MA, SDSU/UCSD Joint Doctoral Program in Clinical Psychology, 6363 Alvarado Court, Suite 103, San Diego, CA 92120.E-mail: hholden365@gmail.com

A.M. STAFFARONI, J.H. KRAMER, J. KORNAK, R. BINNEY, S. ATTYGALLE, A. CAPLAN \& H. ROSEN. Longitudinal Assessment of Cognition in Semantic Variant Primary Progressive Aphasia.

Objective: The semantic variant of primary progressive aphasia (svPPA) is characterized by focal impairment in semantic memory. We know little about the trajectory of cognitive decline, however, and whether different facets of cognition change differently over time. The present study addresses this gap in the literature by assessing cognition in svPPA longitudinally over 3-4 visits.

Participants and Methods: 39 patients who met the 2011 international consensus criteria for svPPA received neuropsychological testing at 3 (n $=39 ; 23$ male) or 4 visits $(\mathrm{n}=26 ; 13$ male). Mean duration between visits was 0.9 years. Mean baseline age was $63.3(\mathrm{SD}=6.4)$. Based on previous work, four cognitive composites (semantic memory, episodic memory, executive functions and visuospatial) were created. Linear mixed-effects modeling was used to assess longitudinal cognitive changes.

Results: svPPA patients declined significantly on measures of semantic memory (0.33 z-score/year; 95\% CI 0.26-0.41, p<.001), episodic memory $(0.35 \mathrm{z}$-score/year; $95 \%$ CI $0.26-0.45, \mathrm{p}<.001)$ and executive functions ( $0.28 \mathrm{z}$-score/year; $95 \%$ CI $0.18-0.38$, p<.001), but not visuospatial functioning $(\mathrm{p}=.943)$.

Conclusions: svPPA patients not only decline in semantic memory, as expected, but also showed similar rates of decline in episodic memory and executive functions. In contrast, svPPA patients showed remarkable stability in visuospatial functioning across four visits. This is one of the first longitudinal studies of cognition in svPPA to include more than two visits. Our behavioral findings are consistent with neuroimaging studies, which have suggested a pattern of progressive atrophy and white matter disruption involving the anterior temporal lobes, hippocampus and ventromedial frontal areas, with relative sparing of the parietal and occipital regions responsible for visual perception.

Correspondence: Adam M. Staffaroni, M.S., Palo Alto University, 707 Continental Cir., Apt 636, Mountain View, CA 94040. E-mail: astaffaroni@paloaltou.edu

F. DE LEON, G. RODRIGUEZ, J. EVANS, I. HANULIK, K. TINGUS, J. Ringman, L. APOSTOLOVA, M.J. WRIGHT \& E. WOO. Prospective Memory and Functional Abilities in Healthy Aging and Mild Cognitive Impairment.

Objective: Prospective memory, the ability to execute an intended action in the future, is affected by the healthy aging process and also in mild cognitive impairment (MCI). In the present study, we investigated 
the role of prospective memory in the ability to perform cognitively mediated functional abilities in healthy older adults and individuals with MCI.

Participants and Methods: Participants included 60 healthy older adults and 51 persons with MCI. All participants were administered the prospective memory task, in which they were asked to remember to request a "pill" after completing each of 8 cognitive tasks. To evaluate functional abilities, participants' study partners completed the Everyday Cognition (ECog) Scales. On the ECog, study partners rated changes in the participants' ability to perform everyday tasks that are mediated by the following cognitive skills: memory, language, visual-spatial abilities, and three aspects of executive functioning (planning, organization and divided attention).

Results: For each group, regression analyses were conducted to determine the impact of prospective memory on functional abilities. For the MCI group, poorer prospective memory was predictive of declines in functional abilities that are mediated by memory, language, and planning. For the healthy older adults, prospective memory did not predict changes in functional abilities.

Conclusions: Overall, prospective memory predicted changes in functional abilities in MCI but not in healthy aging. Further, prospective memory was particularly important for abilities that were mediated by specific cognitive skills (memory, language, and planning). These findings demonstrate the role of prospective memory in specific aspects of everyday functioning when individuals begin to exhibit cognitive difficulties.

Correspondence: Ellen Woo, UCLA, 10911 Weyburn \#200, Los Angeles, CA 90095.E-mail:ewoo@mednet.ucla.edu

F. DE LEON, M.J. WRIGHT, G. RODRIGUEZ, J. EVANS, A. HE, L. APOSTOLOVA, J. RINGMAN \& E. WOO. Contingency-Based Prospective Memory in Mild Cognitive Impairment and Dementia. Objective: Prospective memory (PM) involves remembering to execute an intended action and is typically measured by asking participants to perform a simple action when signaled by an event. However, in everyday life, intentions are often based upon specific contingencies (e.g., taking a medication only after a meal). In this study, we examined contingency-based PM in healthy aging, MCI, and dementia.

Participants and Methods: Healthy older adults $(n=67)$ and participants with MCI $(n=67)$ or dementia $(n=20)$ were administered a contingency-based PM task, in which they were asked to remember to request a "pill" after completing each of 8 cognitive tasks. To examine contingencies, they were also asked to request "one pill" if the task just completed involved memorization and "two pills" if the task did not involve memorization.

Results: ANCOVAs were conducted to determine the impact of diagnosis on PM, with education as the covariate. For PM involving a simple action (requesting the "pill"), we found that the healthy older adults outperformed the MCI group, who outperformed the dementia group. For PM based on the contingency (requesting the correct number of pills based on the type of test just performed), the same performance pattern was observed.

Conclusions: Consistent with previous research, we found that individuals with MCI performed at an intermediate level compared to controls and persons with dementia for prospective memory for simple actions. Importantly, we found the same performance pattern when examining prospective memory based on contingencies, which more closely mirrors the actions performed in everyday life.

Correspondence: Ellen Woo, UCLA, 10911 Weyburn \#200, Los Angeles, CA 90095.E-mail:ewoo@mednet.ucla.edu
S. TIERNEY, H.M. HOLDEN, E. PIROGOVSKY TURK, S. WOODS, V. FILOTEO, J. COREY-BLOOM \& P.E. GILBERT. Memory for Intentions Screening Test Reveals Similar Prospective Memory Performance in Huntington's and Parkinson's Disease.

Objective: Prospective memory (PM) is dependent on executive and episodic processes adversely affected by subcortical neurodegenerative diseases such as Parkinson's disease (PD) and Huntington's disease (HD). The current study compared PM performance in HD and PD using the Memory for Intentions Screening Test (MIST).

Participants and Methods: Participants included 26 individuals diagnosed with PD and 20 individuals diagnosed with HD. All participants completed the MIST, which includes both time- (TB) and event-based (EB) PM subscales.

Results: Mann-Whitney tests reveal no significant differences between the PD and HD groups on MIST summary, TB and EB subscales, loss of content and loss of time errors, and recognition scores (all ps > .05). However, the HD group committed significantly more no response errors, while the PD group committed significantly more task substitution errors ( $p s<.05)$. Both groups performed significantly worse ( $p s<.05)$ on TB trails compared to EB trials. Individuals in the HD group were 1.8 times more likely to fail the 24-hour semi-naturalistic PM task, 95\% CI $[0.52,6.77]$. Findings were not better explained by demographic variables.

Conclusions: The findings reveal overall levels of PM performance on the MIST in PD and HD. Error analyses suggest that PM deficits in the HD group stemmed from difficulty with cue monitoring, whereas the PD group experienced increased online retrospective memory failures. While laboratory PM performance was comparable between the groups, worse performance in the HD group on the semi-naturalistic PM task offers preliminary evidence that individuals with HD may experience difficulty compensating for PM deficits in daily life.

Correspondence: Savanna Tierney, Psychology, San Diego State University, 1640 Maple Dr. \#55, Chula Vista, CA 91911. E-mail: Savanna386@gmail.com

J.C. SHERMAN, S. FLYNN, C. HENDERSON, J. GAIR, L. SHABO, K. CAPELLI, J. BEHRENDT \& B. LUST. Pronoun Problems in MCI: New Research Begins to Reveal the Source of Difficulty.

Objective: Current research is beginning to reveal linguistic deficits as indicators of prodromal $\mathrm{AD}$, though challenges remain regarding explanation. While syntactic knowledge is often argued to be retained in early AD, disordered pronoun use has long been observed. Paradoxically, AD speech includes higher pronoun frequency than normal speech, but with difficulty with pronoun comprehension. We report results of a study that tested syntactic and semantic factors in pronoun knowledge in MCI. We hypothesize that pronoun difficulties are due to ambiguity of pronoun reference and not a syntactic deficit.

Participants and Methods: Through a cross-institutional study with standardized testing and scoring methods, 21 MCI subjects were compared to 10 Healthy Aging on elicited imitation of sentences with pronoun subjects which varied semantic plausibility, structural direction of clause embedding and pronoun direction. Subjects' performances on these sentences were compared to a matched set of sentences with null subjects.

Results: MCI performance was significantly worse than that of HA on sentences with pronouns (t 2.01, p.04; mean correct for MCI .54 compared to .75 HA), but their deficit is significant on sentences with pronoun ambiguity and not on those where co-reference is grammatically blocked. MCI subjects reveal sensitivity to both the syntactic and semantic factors varied. The MCI production deficit is not simply due to the pronoun as it also occurs with null subjects involving ambiguity of reference.

Conclusions: Although our results confirm a pronoun deficit in MCI, they do not support a deficit in syntactic knowledge. We argue that pronouns, because essentially ambiguous require a resolution of reference in the outside world, as well an integration of this reference with the 
syntax of the sentence. It is this integration of syntax and semantics, a toggling between internal and external knowledge, which underlies the observed pronoun deficit.

Correspondence: Janet C. Sherman, Ph.D., Psychiatry, Mass General Hospital, 1 Bowdoin Square, 7th Floor, Boston, MA 02114. E-mail: jsherman@partners.org

A. RADKE, N.T. BOTT, T. SHANY-UR, P. POORZAND, L. NGUYEN, B. ADHIMOOLAM, K. POSSIN, D. MARIN, D. ZACKEY, B. MILLER \& K.P. RANKIN. Classification Accuracy of the Dynamic Affect Recognition Test (DART) in Neurodegenerative Disease.

Objective: The Dynamic Affect Recognition Test (DART) is a novel freely available tablet-based video emotion naming test designed for use with dementia patients. We evaluated the diagnostic precision of the DART for discriminating across major dementia syndromes, compared to an existing non-tablet, commercially-available video emotion naming test.

Participants and Methods: A total of 174 participants [26 behavioral variant FTD (bvFTD), 11 semantic variant primary progressive aphasia (svPPA), 9 right-temporal FTD (rtFTD), 12 progressive nonfluent aphasia (nfvPPA), 12 progressive supranuclear palsy (PSP), $41 \mathrm{Alz}$ heimer's disease (AD), 63 healthy older controls (NC)] took the DART and the Emotion Evaluation subtest (EET) of The Awareness of Social Inference Test (TASIT). Group differences were analyzed using GLMs, and scores were correlated with brain volume using DARTEL-based voxel-based morphometry (VBM) analysis of structural MRI gray matter maps, controlling for age, sex, MMSE, and total intracranial volume (TIV). Area under the curve (AUC) derived from the receiver-operating characteristic (ROC) analysis was used to test the classification accuracy of the DART in bvFTD vs. NC and other dementia groups.

Results: Only bvFTDs, rtFTDs, and svPPAs scored significantly worse than NCs $(p<0.05)$. A DART cutoff of $9 / 12$ yielded $90 \%$ sensitivity $/ 75 \%$ specificity for bvFTD vs. NCs (AUC: DART=.84; EET=.87), and $89 \%$ sens $/ 77 \%$ spec for any dementia vs. NC (AUC: DART=.82, EET=.82). Cutoffs of $8 / 12$ had $98 \%$ sensitivity for both. VBM showed atrophy in bilateral medial temporal, temporal pole, anterior insula, basal ganglia, and ventromedial frontal regions $(\mathrm{pFWE}<0.05)$.

Conclusions: We identified the cutoffs at which the DART discriminates bvFTD and other dementias from controls. DART score corresponds to brain regions associated with emotion naming, and performs similarly to an already validated but longer emotion reading test. Tablet administration and free availability provide additional advantages to using the DART with dementia patients.

Correspondence: Anneliese Radke, PGSP-Stanford Psy.D. Consortium, 4298 Wilkie Way, Apt. D, Palo Alto, CA 94306. E-mail: aradke@ stanford.edu

\section{S. PANDYA, M. CLEM, D.K. HORTON, L.H. LACRITZ \& F.L. WOON. Predictors of Reversion from Mild Cognitive Impairment to Normal Cognition.}

Objective: To examine baseline predictors of reversion from mild $\operatorname{cog}$ nitive impairment to normal cognition.

Participants and Methods: Data from the National Alzheimer's Coordination Center included 1778 MCI subjects (50.60\% male; Mage $=72.47 \pm 9.80)$, who were seen at baseline and for 3 subsequent annual visits. Of these, $176(9.9 \%)$ reverted to normal cognition and 423 $(23.8 \%)$ progressed to dementia at 2-years, with sustained diagnoses at 3 -years, and were used for this study. These subjects were classified at baseline as either Amnestic ( $n=507 ; 84.6 \%)$ or Non-amnestic $(n=92$; $15.4 \%)$ MCI. Baseline factors were categorized into demographic, cognitive, global functioning, medical health, and neuropsychiatric clusters. Logistic regression models were used to identify significant predictors of MCI reversion for each cluster, which were then entered into a final model to find the best overall predictor(s).

Results: Analyses yielded significant overall predictors of: younger age $(\mathrm{OR}=1.09 ; \mathrm{p}<.01 ; 95 \% \mathrm{CI}=1.04-1.14)$, absence of APOE 4 allele
(OR=3.29; $\mathrm{p}<.01 ; 95 \% \mathrm{CI}=1.53-7.07)$, lower Clinical Dementia Rating Sum-of-Boxes scores (OR=4.11; $\mathrm{p}<.01 ; 95 \% \mathrm{CI}=2.11-8.00)$, greater frequency of metabolic symptoms (e.g., hypertension, hypercholesterolemia) $(\mathrm{OR}=0.69 ; \mathrm{p}<.05 ; 95 \% \mathrm{CI}=0.48-0.98)$, and higher cognitive test scores [Vegetable Fluency ( $\mathrm{OR}=0.58 ; \mathrm{p}<.05 ; 95 \% \mathrm{CI}=0.36-0.95)$, Digit Symbol (OR=0.64; $\mathrm{p}<.05 ; 95 \% \mathrm{CI}=0.42-0.98)$, Logical Memory (Story A, Delay) ( $\mathrm{OR}=0.39 ; \mathrm{p}<.01 ; 95 \% \mathrm{CI}=0.28-0.55)$, Boston Naming (OR=0.57; $<<.01 ; 95 \% \mathrm{CI}=0.39-0.82)]$.

Conclusions: The strongest predictors of MCI reversion were absence of APOE 4 allele and higher global functioning, followed by younger age, more metabolic symptoms, and higher cognitive functioning. MCI individuals with these characteristics at baseline were more likely to revert to normal cognition than progress to dementia at 3-years.

This project was supported by the Friends of the UT Southwestern Alzheimer's Disease Center and by the UT Southwestern Alzheimer's Disease Center (NIH P30-AG12300).

Correspondence: Seema Pandya, Clinical Psychology, University of Texas Southwestern Medical Center, 3220 Maple Avenue, \#242, Dallas, TX 75201. E-mail: seema.pandya@utsouthwestern.edu

M.M. BURKE, E. PIROGOVSKY TURK, C.H. FONG, L. NGUYEN, I. LITVAN, S.L. LESSIG, D. SONG, V. FILOTEO \& D.M. SCHIEHSER. Detrimental Impact of Traumatic Brain Injury on Visuospatial Processing and Executive Function in Parkinson's disease.

Objective: Visuospatial processing speed and executive function deficits are common in Parkinson's disease (PD), yet the role of traumatic brain injury (TBI) in these areas of cognition in PD is unknown. We hypothesized that PD patients with a history of TBI (PD+TBI) would perform significantly worse on measures of processing speed and executive function compared to those without a history of TBI (PD-TBI).

Participants and Methods: PD+TBI $(\mathrm{n}=33)$ and PD-TBI $(\mathrm{n}=33)$ participants, who were matched on age, education, gender, disease and motor symptom severity, and overall cognition (Mattis Dementia Rating Scale), were administered the D-KEFS Color-Word Interference Test as a measure of visuospatial processing speed (color naming/word reading composite score) and executive function (inhibition and inhibition/ switching subtests).

Results: PD+TBI participants scored significantly worse on the color/ word reading composite score and inhibition and inhibition/switching subtests $(\mathrm{p}$ 's <.02). Frequency of impairment (defined as a scaled score of $<7$ ) was significantly higher in PD+TBI compared to the PD-TBI participants on inhibition/switching $(33 \%$ vs. $0 \% ; \mathrm{p}<.001)$, inhibition $(15 \%$ vs. $0 \% ; p<.05)$ and the color/word composite score $(24 \%$ vs. $3 \% ; \mathrm{p}<.03)$.

Conclusions: PD patients with a history of TBI evidence greater impairment relative to those without a history of TBI on measures of processing speed and executive function, with a greater number of individuals showing impairment on tests requiring a higher level of executive function (i.e., inhibition/switching). This study supports the notion that TBI may result in a detrimental effect on cognition in individuals with PD. Correspondence: Mathes M. Burke, VASDHS, 3350 La Jolla Village Dr. 116B, San Diego, CA 92161.E-mail:maburke@ucsd.edu

M. REYNOLDS, J.C. PIERCY, H. MILLER, D. LEITNER, L. OHLHAUSER, J. UPSHAW \& E. CONCEPCION. Frontotemporal Dementia: Two Case Studies.

Objective: We describe the progress of two patients with frontotemporal dementia (FTD). FTD, a group of cognitive disorders involving the degeneration of cells in the frontal and/or temporal lobes of the brain, is relatively rare with a prevalence rate of $10-15 \%$ of all dementia cases. We compared the neuropsychological, biological, social support, and affective experience of two patients with FTD.

Participants and Methods: Patient A and Patient B received comprehensive serial neuropsychological evaluations. Patient A is male and was followed for two years between the ages of 49 and 51. Patient B is female and was followed for two years from age 48 . 
Results: Presenting complaints for Patient A revolved around impaired expressive language, while Patient B presented with difficulties related to cognitive and behavioral disturbances with some expressive language disturbance. Both individuals demonstrated decline in neuropsychological performance and corresponding deterioration in functioning. Both individuals displayed aggressive behaviours and social inappropriateness. Patient B maintained some degree of insight related to FTD with subsequent affective response, while Patient A lacked insight and appeared indifferent and unaware of his situation. Differences in social support and behavioural disruption afforded Patient B the opportunity to remain living at home with a caregiver while Patient A now resides in an extended care facility.

Conclusions: We followed the progression of FTD in two individuals. These individuals initially presented with differing symptoms but showed a similar decline in neuropsychological performance and concurrent functioning. This study emphasizes the importance of social support, and yields beneficial clinical information on FTD and its management overtime.

Correspondence: Meredith Reynolds, University of Montana, 301 Kiwanis Street, Apartment 9, Missoula, MT 59802. E-mail: meredith. reynolds@umontana.edu

L. NGUYEN, E. PIROGOVSKY TURK, M.M. BURKE, C.H. FONG, I. LITVAN, S. LESSIG, D. SONG, V. FILOTEO \& D.M. SCHIEHSER. Predictors of Health-Related Quality of Life Decline in Parkinson's Disease.

Objective: Non-motor and motor symptoms have been found to play an important role in health-related quality of life (HRQoL) in individuals with Parkinson's disease (PD), yet it is virtually unknown which symptoms may predict decrements in HRQoL that are evident in this population. Therefore, the present study aimed to identify specific cognitive, motor, and psychiatric predictors of HRQoL change over time in PD. Participants and Methods: PD participants $(\mathrm{n}=70)$ were administered the Parkinson's Disease Questionnaire 39-item version (PDQ-39) as a measure of HRQoL at baseline and after a 2-year interval. Participants were also administered measures of cognition (Mattis Dementia Rating Scale), motor symptom and disease severity (Unified Parkinson's Disease Rating Scale), anxiety (State-Trait Anxiety Inventory), and depression (Hamilton Depression Rating Scale) at the baseline and follow-up evaluations.

Results: Average PDQ-39 summary score significantly declined from baseline to follow-up in this sample. PDQ-39 decline was significantly associated with lower memory and executive function scores, elevated anxiety, and increased disease severity at baseline. Baseline demographic characteristics, attention, visuospatial ability, motor symptom severity, and depression failed to correlate with PDQ-39 decline. A stepwise regression analysis revealed that baseline trait anxiety was the best predictor of decline on the PDQ-39, with poorer baseline memory also predicting a significant, albeit smaller, amount of variance.

Conclusions: The results of this study suggest that baseline trait anxiety and memory impairment best predict future decrements in HRQoL in PD. These findings have implications for potential treatment targets to improve quality of life in PD.

Correspondence: Lannee Nguyen, Bachelor of Science, Psychology, University of California, San Diego, 4926 W. Crystal Lane, Santa Ana, CA 92704.E-mail:lanneeek@gmail.com

S. KARANTZOUlis, B. CERBONE, M.o. SHEIKH, J. XU \& J.E. GALVIN. Sensitivity of the RBANS in Parkinson's disease with and without cognitive impairment.

Objective: We sought to compare how well the Repeatable Battery for the Assessment of Neuropsychological Status (RBANS), a brief measure of global neurocognitive functioning, and (2) the Neuropsychological Assessment Battery (NAB)-Executive Functions module (NAB-EF), a brief battery designed to measure an individual's judgment, conceptualization, cognitive response set, mental flexibility, verbal fluency contributes to diagnostic accuracy of cognitive impairment in Parkinson's disease. Neither the RBANS nor the NAB has been fully examined in PD. The RBANS has been criticized for its lack of executive function tasks mediated by disruption of fronto-striatal pathways, affected by PD.

Participants and Methods: Participants were 32 Healthy Controls $($ HC: $\mathrm{M}$ age $=70 \mathrm{yrs}, \mathrm{M}$ educ $=17 \mathrm{yrs}), 24$ idiopathic Parkinson's disease participants without cognitive impairment (PD: M age $=66 \mathrm{yrs}$, M educ $=18 \mathrm{yrs}$ ), $21 \mathrm{PD}$ participants with cognitive impairment (PDI: $\mathrm{M}$ age $=70 \mathrm{yrs}, \mathrm{M}$ educ $=18 \mathrm{yrs}), 12$ participants with mild cognitive impairment due to Alzheimer's disease (MCI: M age $=69 \mathrm{yrs}, \mathrm{M}$ educ $=$ 16 yrs). Regression analyses were used to determine which test(s) best predicted group membership.

Results: The RBANS Total Score was a strong predictor of group membership (adjusted R-square 26.5\%, p<.001). With RBANS Total Score in the model, the addition of the NAB-EF increases the adjusted $\mathrm{R}$-square to $34.5 \%$, but did not reach significance $(p=0.34)$. Further addition of the Controlled Oral Word Association Test (total score), Trail Making Test B (Time in Sec), and the Stroop Color Word (\# correct) minimally increased the adjusted R-square to $37.5 \%$ ( $\mathrm{p}=0.87$ ).

Conclusions: Despite evidence of early disruptions of fronto-striatal pathways in PD, the RBANS is a stronger predictor of PD-related cognitive deficits than measures designed specifically to address executive functions. These data suggest that the RBANS alone is sufficient to detect cognitive changes associated with PD and could be considered for use as an outcome in PD clinical trials.

Correspondence: Stella Karantzoulis, NYU Langone Medical Center, 2nd Floor, New York, NY 10016.E-mail: stella.karantzoulis@nyumc.org

L.M. NICCOLAI, K.L. TRIEBEL, T.O. MCPHERSON, M. FALOLA, G.R. CUTTER, R.C. MARTIN \& D.C. MARSON. Neuropsychological Predictors of Declining Financial Capacity in Persons with Mild Cognitive Impairment Due to Alzheimer's Disease.

Objective: To identify key neuropsychological predictors of declining financial capacity in individuals with mild cognitive impairment due to Alzheimer's disease (MCI-AD).

Participants and Methods: Participants were 49 older adults with a consensus conference diagnosis of MCI-AD who completed neuropsychological testing and a performance based financial capacity measure (Financial Capacity Instrument; FCI) at baseline and two-year follow-up visits. We calculated two-year change scores for the neuropsychological tests and FCI total score. Bivariate correlations between demographic, neuropsychological, and FCI variables were examined. The top 5 correlates were entered into a stepwise linear regression analysis to identify multivariable predictors of FCI decline in MCI-AD.

Results: The MCI-AD group showed significant decline over two years on FCI total score and most cognitive variables. Education was the only significant demographic correlate of FCI total score $(r=0.36, p=0.006)$. The top four neuropsychological correlates of FCI change scores were written arithmetic (WRAT-3 Arithmetic, $r=0.61$ ), visual attention/ processing speed (Trails A, $r=-0.40$ ), confrontation naming (Boston Naming Test, $r=0.47$ ), and immediate visual memory (WMS-III Visual Reproduction I, $r=0.45$ ) (all $p ' s<0.01$ ). In the multivariable regression model, written arithmetic, immediate visual memory, and visual attention/processing speed were predictors of longitudinal decline in FCI score. The model's overall adjusted $R^{2}$ was $0.47(p<0.001)$.

Conclusions: Declining abilities in written arithmetic, immediate visual memory, and visual attention/processing speed predicted twoyear declines in financial capacity in persons with MCI-AD. The results extended prior cross-sectional findings and demonstrated the critical importance of semantically based arithmetic knowledge, and also visual attention and visual memory to financial skills in prodromal AD.

Correspondence: Lindsay M. Niccolai, University of Alabama at Birmingham, 1530 3rd Avenue South, Campbell Hall 415, Birmingham, AL 35294.E-mail: niccolai@uab.edu 
M.L. COHEN, S. AITA, Z. MARI \& J. BRANDT. The Unique and Combined Effects of Apathy and Depression on Cognition in Patients with Parkinson's Disease.

Objective: Apathy and depression are associated with poor cognition in patients with Parkinson's disease (PD). However, the specific cognitive signature of each syndrome is not well understood. The cognitive consequences of having apathy or depression, versus apathy and depression, are also unclear. This study investigated the unique and combined effects of apathy and depression on cognition in patients with PD.

Participants and Methods: PD patients were identified from a clinical research database as having self-reported apathy $(\mathrm{AP}+\mathrm{DEP}-; \mathrm{n}=21)$, depression $(\mathrm{AP}-\mathrm{DEP}+; \mathrm{n}=11)$, or both $(\mathrm{AP}+\mathrm{DEP}+; \mathrm{n}=43)$. The $\mathrm{AP}+\mathrm{DEP}+$ group was no more apathetic than the AP+DEP-group, and no more depressed than the AP-DEP+ group. PD patients without apathy or depression served as the control group (AP-DEP-; $n=49)$. The groups were of similar age, education, disease severity, age of symptom onset, and medication status. A multivariate analysis of variance (MANOVA) compared the groups on 17 neuropsychological test scores in the domains of attention, motor and psychomotor speed, construction, language, episodic memory, and executive functioning.

Results: There was a significant overall effect of group: $\mathrm{F}(18,276)$ $=2.12, p=.006$, partial eta squared $=.12$. Univariate analyses and planned contrasts revealed medium-sized effects distinguishing only the AP-DEP- and AP+DEP+ groups (partial eta squared = 0.06-0.09) on measures of manual speed, psychomotor speed, and verbal fluency. Conclusions: PD patients with apathy alone or depression alone did not perform worse than PD controls on neuropsychological tests. Patients with apathy and depression performed worse than PD controls, but only on speed-based measures.

Correspondence: Matthew L. Cohen, Ph.D., Psychiatry and Behavioral Sciences, Johns Hopkins University School of Medicine, 20 Owens Landing Ct., Apt. A, Perryville, MD 21903.E-mail: matthew.l.cohen@ outlook.com

B. CERBONE, S. KARANTZOULIS, M.O. SHEIKH, J. XU \& J.E. GALVIN. The Relationship between MOCA Scores and Performance on the RBANS and NAB in Parkinson's Disease with and without Cognitive Impairment.

Objective: The relationship between the subdomains of the Montreal Cognitive Assessment (MOCA) and "gold-standard" neuropsychological measures in different neurological disorders is unclear. We sought to determine whether MOCA subdomains are significant predictors of performance on the Repeatable Battery for the Assessment of Neuropsychological Status (RBANS) and Neuropsychological Assessment Battery-Executive Functions (NAB-EF) Module among individuals with Parkinson's disease (PD) with and without cognitive impairment.

Participants and Methods: Participants were 32 Healthy Controls (HC: $\mathrm{M}$ age $=70 \mathrm{yrs}, \mathrm{M}$ educ $=17 \mathrm{yrs}$ ), 24 idiopathic $\mathrm{PD}$ participants without cognitive impairment (PD: M age $=66 \mathrm{yrs}, \mathrm{M}$ educ $=18 \mathrm{yrs}$ ), and $21 \mathrm{PD}$ participants with cognitive impairment (PDI: M Age $=70, \mathrm{M}$ educ $=18)$. Multiple regression models were conducted relating MOCA subdomains with RBANS Indices and NAB-EF, controlling for age, education, and total UPDRS scores.

Results: Performance on the MOCA executive functioning sub-tests predicted performance on the NAB-EF $(p<.01)$. Performance on the MOCA attention $(\mathrm{p}=.01)$, immediate and delayed memory (both p's $<.001$ ) sub-tests were significant predictors of performance on the corresponding RBANS indices. However, the language subtests of the MOCA (naming, letter fluency, and repetition) were not strong predictors of performance on the RBANS language index $(p>05)$.

Conclusions: With the exception of the language items on the MOCA, the executive functions, language, immediate and delayed memory items are strong predictors of "gold-standard" neuropsychological measures like the NAB and RBANS. Items on the MOCA may be useful for identifying cognitive-related impairments among individuals with PD and
PDI when more comprehensive assessments are not feasible and could be used for recruitment into clinical trials.

Correspondence: Brittany Cerbone, NYU Langone Medical Center, 145 East 32nd Street, 2nd Floor, New York, NY 10016. E-mail: brittany. cerbone@nyumc.org.

\section{Medical/Neurological Disorders/Other} (Adult)

J.W. KIRTON \& V.M. DOTSON. Moderating effects of age and education on the relationship between body mass and memory function in community adults.

Objective: Increased body mass index (BMI) can be detrimental for cognitive aging. Nonetheless, there are nuances, as research suggests that this relationship may be more complicated than more weight is worse. For example, some studies have found obesity in late life is not related to cognitive decline, whereas obesity in midlife increases risk for dementia. We examined the moderating effects of education and age on the association between BMI and memory.

Participants and Methods: Self-reported height and weight were used to calculate BMI for 56 healthy, dementia-free, community-dwelling older (mean age $65.72 \pm 7.40$ ) and younger (mean age 21.10 \pm 2.37 ) adults. Participants completed a neuropsychological battery that included The Hopkins Verbal Learning Test and the Brief Visual Memory Test-R. Composite scores for verbal, visual, immediate, delayed and discrimination memory were calculated. Mixed effects models were used to evaluate interactive effects of age, education, and BMI on memory composite scores.

Results: In younger adults, verbal and delayed memory decreased as BMI increased, which was worse in individuals with lower education. In older adults, higher BMI and lower education was associated with better verbal memory, whereas higher BMI and higher education was detrimental. Additionally, delayed memory performance improved in older adults as BMI increased, especially in those with higher education. Conclusions: In younger adults, higher BMI is detrimental to memory, but higher education may attenuate this effect. In older adults, the effect of higher BMI on memory function is less clear, and may be better at higher BMI in some instances.

Correspondence: Joshua W. Kirton, M.S., Clinical and Health Psychology, University of Florida, P.O. Box 100165, Gainesville, FL 32610.E-mail: joshuakirton@phhp.ufl.edu

A.D. BONO, J.T. TWAITE, R. STAFFORD, E.M. MURRAY, K. ALTERESCU, M. LUBOMSKI, K. SCORPIO, J.L. SPIELMAN, L.O. RAMIG \& J.C. BOROD. Demographic and Clinical Predictors of Facial Expressivity Improvement for Individuals with Parkinson's Disease (PD) Receiving the Lee Silverman Voice Treatment (LSVT).

Objective: PD is associated with impairments in facial mobility and emotional expressivity, which can impair communication and affect quality of life. LSVTC LOUD (Ramig et al., 2001) increases facial expressivity in individuals with PD. This study evaluated whether demographic (gender, age, \& education) and clinical factors (global cognitive status, depressive symptoms, illness duration, disease stage, \& side of initial motor symptoms) can predict likelihood of improvement from LSVT.

Participants and Methods: Participants were 62 individuals with idiopathic PD and 18 demographically-matched healthy controls $(61.1 \%$ male; M age=63.7). There were three groups of individuals with PD: 21 participants $(71.4 \%$ male; M age $=67.6)$ received LSVT, $19(73.7 \%$ male; $\mathrm{M}$ age $=67.9)$ received a control voice treatment, focusing on articulation (Spielman et al., 2012), and $22(66.7 \%$ male; M age=64.2) received no treatment. Facial expression was elicited through monologue tasks from the New York Emotion Battery (Borod et al., 1992) at baseline and one month post treatment. Facial emotional intensity was rated 
by 6 naïve healthy adult raters on a 7-point Likert scale. Demographic and clinical data were collected at baseline.

Results: Multiple regression analyses were performed for each group. Predictor variables were demographic and clinical variables, and the dependent variable was the pre-post testing change score for emotional intensity. For the LSVT group, change was predicted by age, gender, education, and disease stage in one monologue condition (talking about an everyday event; $\mathrm{R} 2=.784, \mathrm{p}=.014)$.

Conclusions: Results suggest that demographic and disease factors contributed to LSVT treatment responsiveness for one condition. However, for other conditions, these factors did not have an effect, suggesting that LSVT is relatively robust to individual differences. This study contributes to the rehabilitation literature regarding factors that may influence response to treatment.

Correspondence: Amanda D. Bono, Ph.D. Clinical Psychology, Psychology, CUNY Graduate Center, 152-72 Melbourne Ave. Apt. 1P, Flushing, NY 11367. E-mail: adbono922@gmail.com

\section{PERSAD, C. HERRST \& E. PRINCE. Relationship between depression and apathy post Deep Brain Stimulation Surgery in patients with Parkinson's disease.}

Objective: Changes in symptoms of depression and apathy following DBS surgery in patients with Parkinson's disease have been reported in the literature, however little research has examined the relationship between depression and apathy in this population. Although there is some overlap between these two constructs, and are often confused, the mechanisms behind depression and apathy are thought to be distinct. Understanding the changes that occur with DBS can be important for treatment.

Participants and Methods: 14 patients with Parkinson's disease undergoing STN-DBS surgery (Mean=61yrs, S.D=8.07) for treatment of motor symptoms were assessed before and on average 6-9 months after surgery. Patients completed the Geriatric Depression Scale-Short Form and the Marin Apathy Scale, while caregivers of the patients completed the Caregiver version of the Marin Apathy Scale.

Results: Paired Sample t-tests showed increased symptoms of apathy reported by both the patient and the caregiver after surgery. There was also a trend for higher symptoms of depression reported by the patients after surgery. Using cutoff scores, 2 patients could be classified as having some depression at baseline, while after surgery 5 reached criteria. Correlation analyses showed no association between patient and caregiver reports of apathy at baseline, or patient's report of apathy and depressive symptoms. Post-surgery, significant correlations were seen between self-reported depressive symptoms and apathy as well as caregiver and patient report of apathy symptoms. A similar pattern was noted when using change scores.

Conclusions: Changes in apathy, although mild, that can follow DBS surgery, appear to be related to an increase in depressive symptoms rather than an increase in apathy symptoms per se. This study needs to be replicated with a larger sample size and with the inclusion of a matched control group of Parkinson's patients who are not undergoing DBS.

Correspondence: Carol Persad, Ph.D., Psychiatry, Univerisity of Michigan, 2101 Commonwealth, Suite C, Ann Arbor, MI 48105. E-mail: cpersad@umich.edu

N. SCHWAB, H. HUANG, J.J. TANNER, H. PARVATENENI, M. RICE, I. SCHMALFUSS, M. DING \& C. PRICE. Preliminary Data: White Matter Burden and Processing Speed Relative to Acute Pre to Post Surgery Decreases in Default Mode Network.

Objective: Cognitive changes can occur for older adults after major surgery. Based on theories of cerebral reserve and preliminary findings suggesting that there are neuroanatomical predictors for type of cognitive changes after elective major surgery, we prospectively examined the relevance of baseline white matter burden (leukoaraiosis load; LA) and processing speed on pre to post operative reductions in the default mode network (DMN) for older adults electing total knee replacement surgery. Participants and Methods: As part of an on-going federally funded investigation studying neuroimaging predictors of post-operative $\operatorname{cog}$ nitive decline, twenty non-demented older adults $(69.25 \pm 6.70$ years old) completed a baseline neuropsychology assessment and a pre- and post-surgery (within 48 hours) structural and functional magnetic resonance (MR) scans. All participants had surgery completed by the same surgeon. Pre-surgery brain MR white matter disease burden (LA) was manually quantified by an expert rater and analyzed as a percentage of total white matter volume, corrected for total intracranial volume. Applying independent component analysis, the DMN was identified and it's components selected as regions of interest (ROIs). Zero-lag cross correlation (CC) and total interdependence (TI) were applied to assess changes in functional connectivity pre- and post-surgery.

Results: LA load positively associated with pre-post functional connectivity difference $(\mathbf{r}(20)=0.53 ; \mathrm{p}=0.03)$, such that high LA had greater reductions. LA load also negatively related to baseline processing speed $(r$ 's $=-0.46,-0.62, p<0.05$; Trail Making Text A; Digit Symbol respectively) in our sample. Processing speed showed trends in the appropriate direction for predicting declines in functional connectivity ( $r$ 's -0.32 and $-0.24)$ but were not significant.

Conclusions: Data support the rationale for continuing to examine white matter burden and cognitive function on acute brain network changes following elective major orthopedic surgery.

Correspondence: Nadine Schwab, M. S., Clinical and Health Psychology, University of Florida, PO Box 100165, Gainesville, FL 32601. E-mail: schwabnadine@phhp.ufl.edu

M. DEMIAN, R. SHAPIRO \& W.L. THORNTON. Health Literacy and Cognition in Kidney Transplant Recipients.

Objective: Poorer health literacy (HL) is linked to worse disease outcomes in various medical populations. HL has been shown to be dependent upon core neuropsychological (NP) abilities, however, the association between HL and everyday cognition has not been examined. Given that kidney transplant (TX) recipients experience difficulties in cognition, and the potential negative impact this may have on HL, we sought to further clarify the relationship between HL and cognition in this population.

Participants and Methods: 96 stable kidney TX recipients (M=53 years \pm 12.56$)$ participated. HL was comprehensively assessed by the REALM-T, NVS, and HLQ. Participants completed measures of verbal intelligence (VIQ) and traditional NP abilities (verbal memory, processing speed, working memory, and executive functions). A NP composite was created to sum the NP variables. Everyday cognition was measured by the Everyday Problem Solving (EPS) test. Correlational analyses were used to examine associations between HL and cognition.

Results: Amongst the three HL measures only the REALM-T and NVS were associated with one another $(\mathrm{r}=.47, \mathrm{p}<.01)$. The REALM-T and NVS demonstrated significant correlations with verbal intelligence $(\mathrm{r}=.74$ and $.72, \mathrm{p}<.01$ respectively) and EPS ( $\mathrm{r}=.40$ and $.33, \mathrm{p}<.01$ respectively). In contrast, the HLQ demonstrated much weaker correlations with verbal intelligence $(\mathrm{r}=.23, \mathrm{p}<.05)$ and EPS $(\mathrm{r}=.22, \mathrm{p}<$ $.05)$. None of the HL measures were associated with NP functioning. Conclusions: These results suggest that HL may specifically rely on VIQ and EPS abilities, as opposed to traditional NP abilities. The findings also demonstrate that the REALM-T and NVS are more similar to one another than to the HLQ, which is in line with its development as a tool to assess the social, in addition to cognitive, abilities necessary for HL. The findings have implications for the improvement of medication adherence in kidney TX recipients. The role of HL, verbal intelligence, and EPS in medication adherence remains to be examined.

Correspondence: Maryam Demian, HBSc, Psychology, Simon Fraser University, 8888 University Drive, Department of Psychology, Burnaby, BCV5A1S6, Canada.E-mail: demian@sfu.ca 
L. MANDERINO, M. SPITZNAGEL, G. STRAIN, M. DEVLIN, R.A. COHEN, R.D. CROSBY, J.E. MITCHELL \& J. GUNSTAD. Traditional Cognitive Deficiencies Predict Impaired Performance on an Emotion Recognition Task in Bariatric Surgery Candidates. Objective: Deficits in traditional cognitive domains (e.g. attention, executive function, memory, etc.) are prevalent in obese persons, but it is still unclear if such deficits can extend to social cognition. The present study examined whether impairment in such traditional domains of cognition correlated with a deficit in social cognition, as measured by an emotion recognition task, in bariatric surgery candidates.

Participants and Methods: 116 bariatric surgery candidates (avg age $=43.62 \pm 11.03 ; 81 \%$ female) completed the computerized Integneuro neuropsychological test battery. In addition to assessing function in traditional cognitive domains, the Integneuro also includes a measure of emotion recognition. This task presents 48 faces (8 different individuals depicting neutral, happiness, fear, sadness, anger, and disgust) and participants must choose the correct verbal label from the six expression options. Number of correct responses and average reaction time for correct responses served as primary dependent variables.

Results: Stepwise multiple regression analyses revealed that age, Maze Errors, and hypertension predicted accuracy in emotion recognition (Adjusted R2 $=.22, \mathrm{~F}(3,111)=11.86, \mathrm{p}<.001)$. Stepwise multiple regression analyses revealed that Switching of Attention-Digits, Long Delay Recall, and age predicted speed of responses (Adjusted R2 $=.26$, $\mathrm{F}(3,111)=13.00, \mathrm{p}<.001)$.

Conclusions: Results show several aspects of traditional cognition that predicted performance on an emotion recognition task, suggesting a link between traditional cognitive impairment and social cognitive impairment in obese persons. The mechanism(s) of this relationship is still unclear and requires further study, as social functioning may relate to outcomes in bariatric surgery candidates.

Correspondence: Lisa Manderino, Kent State University, 2004 Hidden Lake Dr, Apt F, Stow, OH 44224.E-mail: lmanderi@kent.edu

C. NOGGLE, J. THOMPSON, A. LEVINE \& J. DAVIS. Additive Effects of Comorbid Psychiatric Disorders Post-TBI on Aspects of Cognition.

Objective: Though changes in cognition, language, intelligence, and personality are most often discussed in relation to TBI, the manifestation of psychiatric disorders is also frequently reported. The current sought to evaluate not only the potential additive effect of psychiatric features on neurocognition, but compare the differential effects of specific types of psychiatric features.

Participants and Methods: An archival data set was used for group comparisons. Participants $(\mathrm{n}=141)$ were diagnosed with a moderate TBI. Participants presented with either no comorbid psychiatric presentation (Group 1), comorbid depression (Group 2), comorbid anxiety (Group 3), or comorbid psychosis (Group 4). Participants were compared on numbers reversed, auditory working memory, and decision speed subtests.

Results: Findings demonstrated that individuals with a history of TBI who were diagnosed with comorbid psychiatric disorders exhibited greater deleterious effects on cognitive functioning than individuals with a TBI alone. When specific psychiatric entities were compared (i.e. comorbid psychosis, depression, or anxiety), individuals that were post-TBI with comorbid psychosis had significantly lower scores in all testing domains in comparison to individuals post-TBI with either comorbid anxiety or comorbid depression. At the same time, individuals who were post-TBI that presented with comorbid anxiety or depression demonstrated more cognitive decline then those who were post-TBI and demonstrated no comorbid psychiatric presentation.

Conclusions: Outcomes demonstrate that TBI with psychiatric comorbidities has worse outcome in cognition than TBI alone. This speaks to an increased functional burden (i.e. additive effect) of comorbid psychiatric presentations in post-TBI populations thus emphasizing the need for comprehensive psychiatric care.
Correspondence: Chad Noggle, Ph.D., ABN, Psychiatry, SIU School of Medicine, $315 \mathrm{~W}$. Carpentedr St., Springfield, IL 62794. E-mail: cnoggle@siumed.edu

T.S. PATERSON, R. SHAPIRO \& W.L. THORNTON. Ill-Structured EPS Ability Predicts Medication Adherence Above and Beyond Well-Structured EPS and Traditional Measures of Intelligence in Renal Transplant Patients.

Objective: We have previously shown that ill-structured everyday problem-solving (EPS) ability predicts medication adherence post successful renal transplantation, such that greater ability to solve problems requiring generation of spontaneous, safe and effective solutions is associated with better adherence (Gelb et al., 2010). To date, we have not assessed the relative utility of well-structured EPS (problems dealing with everyday subject matter, requiring specific, discrete answers), or traditionally measured general intelligence (IQ), for predicting medication adherence in this group, compared to ill-structured EPS.

Participants and Methods: Participants were renal transplant recipients $(n=173)$. Ill-structured EPS was determined by the number of safe/effective solutions generated for real-world scenarios (EPS Test). Well-structured EPS was measured via a multiple-choice test covering everyday issues (ECB-KQ). IQ was measured using the KBIT-II.

Results: Regression analyses revealed that beyond age, gender, education, and English as a second language (ESL) status, ill-structured EPS $(\Delta R 2=.039, \mathrm{~F}=6.19, \mathrm{p}<.01)$, but not well-structured EPS or IQ, was predictive of reported medication adherence. Furthermore, controlling for the effects of well-structured EPS and IQ, ill-structured EPS remained a significant predictor of adherence $(\Delta \mathrm{R} 2=.022, \mathrm{~F}=$ $4.39, \mathrm{p}<.05)$.

Conclusions: Results indicate that performance on IQ and well-structured EPS measures do not provide the same potential utility in assessing likelihood of non-adherence as ill-structured EPS. Even when the effects of measured intelligence and problem solving requiring distinct responses are controlled for, the ability to solve problems requiring generation of spontaneous, safe, effective solutions continues to significantly predict medication adherence in this group.

Correspondence: Theone S. Paterson, M.A., Psychology, Simon Fraser University, 580 Oxford St. East, London, ON N5Y 3J1, Canada. E-mail: theonep@sfu.ca

\section{WAGNER \& M. PRIEUR. Too quiet: A case of auditory Charles Bonnet Syndrome.}

Objective: To present a case study of auditory variant Charles Bonnet Syndrome.

Participants and Methods: A 79 year old widowed female presented in an outpatient neurological clinic accompanied by her son with a chief complaint of persistent auditory hallucinations during the past 6 months. Past medical history was significant for profound hearing loss. premature ventricular contraction and carpal tunnel syndrome. Notably there was no psychiatric history and the only stressor was the premature death of an institutionalized daughter one year prior to the onset of the auditory hallucinations.

Results: Basic neurological exam was positive only for profound bilateral sensorineural hearings loss. Neuropsychological investigation revealed normal age associated memory loss and no evidence of psychopathology. Pertinent data included MMSE 27/30, Orientation 13/14. Logical Memory I SS=9, Logical Memory $2 \mathrm{SS}=11$, Trails B $\mathrm{t}=40$, Vocabulary $\mathrm{SS}=10$, Information $\mathrm{SS}=11$, Block Design $\mathrm{SS}=9$ and negative PAI. A prior CT was negative. MRI showed mild age related atrophy and minimal white matter change (see Table 1 for psychometric data and Figure 1 of MRI).

Conclusions: This case was discussed in the context of an auditory variant of Charles Bonnet Syndrome. The etiology of auditory hallucinations is diverse, but this case is classified as hypacusis variant. The pathophysiological mechanism is thought to be auditory deafferentiation in the context of neuropsychological vulnerability. 
Correspondence: Mark Wagner, Ph.D., Neurology, Medical University of South Carolina, 96 Jonathan Lucus St, Charleston, SC 29403. E-mail: wagnermt@musc.edu

K. YEN, A. TAN, J. MCNEELY, C. CASSILl, M. GONZALES, C. BEEVERS \& A. HALEY. Effects of Glucose Levels on Information Processing in College-Aged Adults.

Objective: Although recent studies have shown relationships between elevated glucose levels and cognitive deficits in older populations, research has been lacking on the effects of elevated glucose levels on specific cognitive abilities in college-aged adults. The current study examines participants with a range of glucose levels, both healthy and elevated $(>100 \mathrm{mg} / \mathrm{dL})$, in order to investigate specific deficits in information processing.

Participants and Methods: Data was collected from a university lab. The sample consisted of participants aged 18-24 $(\mathrm{n}=61$, mean $=20.1$, $\mathrm{SD}=1.6$ ) with glucose levels ranging from $68-134 \mathrm{mg} / \mathrm{dL}$ ( mean = 88.6, $\mathrm{SD}=11.6$ ). Glucose levels were obtained using finger stick and glucometers. To evaluate information processing, the $\mathrm{d} 2$ Test of Attention and COWAT were administered to each subject to measure for processing speed and rule compliance.

Results: The relationship between glucose levels and information processing was assessed using a linear regression analysis. Although higher glucose levels was not a significant predictor of processing speed on either test, it was a significant predictor of total errors on the $\mathrm{d} 2(\mathrm{p}=.015)$ and the COWAT $(\mathrm{p}=.009)$.

Conclusions: The results from this study suggest a significant relationship between elevated glucose levels and information processing errors in college-aged adults. This relationship may be an important consideration for young adults with unhealthy or even subclinical elevations of glucose levels given the information processing requirements in academic and occupational contexts. Other clinical implications and future research directions are discussed.

Correspondence: Kyle Yen, University of Texas at Austin, 2300 Nueces St, Apt \#427, Austin, TX 78705. E-mail: kyleyen1992@gmail.com

A. TAN, J. MCNEELY, K. YEN, C. CASSILl, M. GONZALES, C. BEEVERS \& A. HALEY. Effects of High Blood Pressure on Executive Function in College-Aged Adults.

Objective: Although studies have shown relationships between hypertension and cognitive deficits, research is lacking on the effects of higher blood pressure on higher-order cognitive functioning during young adulthood. The current study examines college-aged participants with a range of blood pressure levels, both healthy and elevated (>139/89 $\mathrm{mmHg}$ ), in order to investigate executive dysfunction.

Participants and Methods: Data was collected from a university lab. The sample consisted of participants aged 18-24 $(\mathrm{n}=66$, mean $=$ $19.85, \mathrm{SD}=1.60)$ with varying diastolic $($ mean $=69.73 \mathrm{mmHg}, \mathrm{SD}=$ $9.71 \mathrm{mmHg}$ ) and systolic (mean $=106.12 \mathrm{mmHg}, \mathrm{SD}=12.73 \mathrm{mmHg}$ ) blood pressure levels collected using a blood pressure cuff. To evaluate executive functioning, each participant was administered the Stroop, WAIS-III Digit Symbol, and Trail Making tests as measures of attentional control, processing speed, and cognitive flexibility, respectively. Results: The relationship between blood pressure level and executive function was assessed using a linear regression analysis with age as a covariate. Blood pressure was not a significant predictor of Trail Making $B$ raw scores. Higher diastolic blood pressure was a significant predictor of Stroop Word-Color raw scores $(\mathrm{p}<.05)$, and higher diastolic/ systolic blood pressures were significant predictors of Digit Symbol ( $p$ $<.01)$ raw scores.

Conclusions: Results suggest that higher blood pressure levels predict lower performance in the attentional control and processing speed in college-aged adults. This relationship may be an important consideration for young adults with even sub-clinical blood pressure levels given executive function requirements in academic and occupational contexts.
Correspondence: Alexander Tan, Austin Neuropsychology PLLC, 7205 Hart Ln \#3035, Austin, TX 78731. E-mail: a.tan518@gmail.com

J. MICHALOWSKI, M. HARCIAREK, J.B. WILLIAMSON, B. BIEDUNKIEWICZ, A. DEBSKA-SLIZIEN \& B. RUTKOWSKI. What do the event-related potentials tell us about the anterior attentional system in dialyzed patients with end-stage renal disease?

Objective: Patients with end-stage renal disease receiving dialysis have been shown to present with impaired attention and executive dysfunction. However, the cerebral mechanism(s) that may account for these cognitive problems remain unknown. Thus, by using event-related brain potentials (ERPs), the present study was designed to explore the temporal dynamics of attentional/executive processing in dialyzed patients with end-stage renal disease.

Participants and Methods: Fifteen non-demented hemodialyzed patients and 20 demographically matched healthy controls participated in this study. The given tasks were the simple reaction time test (RT) and two forced choice reaction time tests (i.e. Choice RT and Prepare RT), all derived from the ROtman-Baycrest Battery to Investigate Attention (ROBBIA). In the Prepare RT, a warning cue was presented 1 or 3 seconds prior to a target, whereas there was no warning cue in the Choice RT. While performing these tests, the EEG signal was recorded from all participants using a 128-channel EGI system.

Results: Across all forced choice tasks, targets evoked smaller N2 (but not P1 and P3) amplitudes in patients than in controls. Also, a marked increase in the $\mathrm{N} 2$ amplitude from the 1 to $3 \mathrm{~s}$ warning condition in the Prepare RT observed in healthy controls was not present in dialyzed patients. Moreover, patients had lower P1 and P3 amplitudes during processing of a warning cue, regardless whether this cue was presented 1 or 3 seconds before the target onset.

Conclusions: These data suggest that dialyzed patients may present with a selective inability to initiate attention-related cerebral activation as well as problems in sustaining achieved activation, probably owing to a dysfunction affecting superior medial frontal regions of the anterior attentional system.

Correspondence: , , E-mail:

M. HARCIAREK, J. MICHALOWSKI, J.B. WILLIAMSON, B. BIEDUNKIEWICZ, A. DEBSKA-SLIZIEN \& B. RUTKOWSKI. Anterior attentional/executive system in adequately hemodialyzed patients with end-stage renal disease. Evidence from the ROBBIA. Objective: Dialyzed patients have been repeatedly shown to present with cognitive changes, attentional/executive dysfunction in particular. Nonetheless, although our previous studies have suggested the attentional/executive deficits in this population may be relatively selective, likely owing to a dysfunction of fronto-subcortical systems, no research has yet systematically investigated these problems in dialyzed individuals. Thus, this study aimed at addressing the question of the potential selectivity of the attentional/executive dysfunction in adequately dialyzed patients with end-stage renal disease.

Participants and Methods: Fifteen non-demented hemodialyzed patients and 20 demographically matched healthy controls were tested using four reaction time (RT) subtests from the ROtman-Baycrest Battery to Investigate Attention (ROBBIA): 1) Simple RT, 2) Choice RT, 3), Prepare RT, and 4) Concentration.

Results: Both groups exhibited a strong foreperiod effect for unwarned stimuli, namely a gradual decrease in reaction time with increasing interstimulus intervals. Dialyzed patients were significantly slower on the Prepare RT (but only when a warning stimulus was presented 3 seconds before the onset of the target/letter) as well as on the Concentration. There was no group difference in the number of errors in each test. However, patients exhibited a different effect of the warning stimulus compared to controls, with the increase in RT from 1s warning condition to $3 \mathrm{~s}$ warning condition being significantly greater $(87 \mathrm{~ms}, 15 \%)$ than the control group's increase (11ms, $2.1 \%)$. 
Conclusions: This study indicates that dialyzed patients present with a selective inability to sustain attention, likely resulting from impaired energization (i.e. defective initiation and constant activation of the strength of the selected response mode). The results are discussed in light of the theory of the multiple frontal systems controlling response speed. Correspondence: ,. . E-mail:

J.N. IKANGA \& H.M. ELIZABETH. The Association Between the years of Essential Hypertension and Memory.

Objective: This study examines whether there is any association between the years of clinical hypertension (HTN) and memory performance while controlling for age.

Participants and Methods: Participants were150 individuals (82 male $/ 68$ female, mean years of education $=18.97, \mathrm{SD}=4.09, \mathrm{FSIQ}=$ 115.89, SD $=14.28)$, over 45 years of age $($ mean $=72.5, \mathrm{SD}=11.41)$, 58 normotensive/ 90 hypertensive, and with a mean of $13.66, \mathrm{SD}=14.41$ years of HTN duration. Outpatient neuropsychological evaluations were performed by the principal investigator and trained technicians. Medical histories and blood pressure (BP) readings were taken by registered nurses. Analysis of covariance (ANCOVA) controlling for age and the interaction of BP and age and general linear regression were conducted to determine whether there is any association between the years of clinical HTN and memory performance.

Results: There was a significant difference in memory performance between normotensive and hypertensive participants after controlling for age and the interaction between blood pressure and age, BP accounted for $3 \%, F(1,146)=3.92, p<.04$, the complete model accounted for $42 \%, \mathrm{~F}(3,146)=33.92, \mathrm{p}<.0001$.

Conclusions: In this study, poor performance in visual and verbal memory was associated with clinical hypertension. These findings are consistent with previous studies, which have underlined deleterious impact of HTN on the brain and the importance of treating and controlling HTN. Longitudinal and experimental studies which utilize JNC 8 (2014) guidelines of HTN and neuroimaging are required before these findings should be applied in clinical setting in the treatment of HTN.

Correspondence: Jean N. Ikanga, M.A, Rehabilitation Medicine, Emory University, $636 \mathrm{~W}$ Ponce de Leon Ave, Decatur, GA 30030. E-mail: jeanikanga@yahoo.com

D. FLODEN, R.M. BUSCH, S.E. COOPER, C.S. KUBU \& A.G. MACHADO. Mattis Dementia Rating Scale-2 Does Not Predict Quality Of Life After Subthalamic Nucleus Deep Brain Stimulation. Objective: To examine the recent claim that borderline scores on the Mattis Dementia Rating Scale - 2nd Edition (DRS-2) predict poor quality of life (QoL) outcomes after subthalamic nucleus deep brain stimulation (STN DBS) in patients with Parkinson disease.

Participants and Methods: Retrospective sample of 110 patients diagnosed with idiopathic Parkinson's disease who underwent STN DBS implantation at Cleveland Clinic between 2006 and 2013. Both before and after surgery, patients completed full neurological and neuropsychological evaluations (including the DRS-2), and rated QoL using the Parkinson Disease Questionnaire-39 (PDQ-39). Correlations and nonparametric analysis of variance tests examined the relationship between preoperative DRS-2 scores and postoperative PDQ-39 ratings and motor outcomes. Reliable change index scores were used to examine individual differences in QoL change after surgery.

Results: DRS-2 performance and QoL or motor outcomes were not significantly correlated. RCI analysis indicated that patients with borderline to impaired range preoperative DRS-2 scores demonstrated similar rates of improved, stable, or worsened QoL relative to patients who scored within normal limits.

Conclusions: Dementia screening with the DRS-2 is not sufficient to determine whether a Parkinson patient will experience improved QoL after STN DBS. Our data serves as a cautionary note for clinicians who might otherwise place undue emphasis on screening measures in ascertaining surgical candidacy.
Correspondence: Darlene Floden, Cleveland Clinic Foundation, 9500 Euclid Ave, Desk P57, Cleveland, OH 44195.E-mail: flodend@ccf.org

S.E. COOK, S.M. MCCLINTOCK, J. FELD, S.D. EVEREST, D. BOLAND \& M. STACY. Characterizing the Neurocognitive Profile of Parkinson Disease with Freezing of Gait.

Objective: Parkinson disease (PD) is a common neurodegenerative disorder associated with declining neurocognitive function. Freezing of gait is a specific phenotype of PD (PD+FOG) that may have a unique neurocognitive profile. The purpose of this study was to characterize the neurocognitive profile of patients with PD+FOG.

Participants and Methods: We enrolled 28 community-dwelling participants diagnosed with PD+FOG from an academic movement disorders center. Participants completed a comprehensive neurocognitive battery. Descriptive statistics were computed to create the neurocognitive profile.

Results: The sample had a mean age of $66.8(\mathrm{SD}=7.1)$ and estimated IQ of 105.0 (SD=7.0), $54 \%$ were male and $79 \%$ were Caucasian. Mean UPDRS score was 39.9 (SD=13.9). Mean standardized scores on neurocognitive measures fell within the average range on tasks of processing speed (Trails A SS=9.5, SD=3.9), working memory (Digit Span $\mathrm{SS}=10.3, \mathrm{SD}=2.6$, Spatial Span $\mathrm{SS}=9.8, \mathrm{SD}=2.9$ ), executive functioning (Trails B SS=9.3, SD=4.2, DKEFS Category Switching Accuracy $\mathrm{SS}=10.3, \mathrm{SD}=2.8$, DKEFS Correct Sorts $\mathrm{SS}=11.5, \mathrm{SD}=2.2$ ), verbal fluency (DKEFS Letter Fluency $\mathrm{SS}=10.8, \mathrm{SD}=3.7$, DKEFS Category Fluency $\mathrm{SS}=9.8, \mathrm{SD}=3.2$ ), and naming (BNT-SF z=0.3, $\mathrm{SD}=0.6)$. Mean overall learning was low average across measures, but there was no evidence of memory impairment (HVLT-R Learning T=41.1, $\mathrm{SD}=11.2$, HVLT-R Delay T=42.3, SD=11.3, HVLT-R Discrimination $\mathrm{T}=44.0$, $\mathrm{SD}=9.0, \mathrm{BVMT}-\mathrm{R}$ Learning $\mathrm{T}=40.0, \mathrm{SD}=10.5$, BVMT-R Delay $\mathrm{T}=44.5$, $\mathrm{SD}=12.4$, BVMT-R Discrimination Raw=5.3, $\mathrm{SD}=1.7$ ).

Conclusions: At group level, individuals with PD+FOG demonstrated mild weakness with learning new information, but otherwise had intact cognitive abilities. However, individual neurocognitive profiles varied widely. Further exploration in a larger PD+FOG cohort is necessary to confirm the neurocognitive profile.

Correspondence: Sarah E. Cook, PhD, Clinical Neuropsychology Service, Duke University, Duke Health Center at Morreene Road, 932 Morreene Rd., Durham, NC27705. E-mail:sarah.cook@duke.edu

L. BRENNAN, A. PANTELYAT, J.E. DUDA, J. MORLEY, D. WEINTRAUB, J. WILKINSON \& P.J. MOBERG. Effects of Memantine on Cognition in Parkinson's Disease Dementia and Dementia with Lewy Bodies: A Meta-Analytic Review.

Objective: To utilize meta-analysis in examining the effects of memantine on neuropsychological functioning in patients with Parkinson's disease dementia (PDD) and dementia with Lewy bodies (DLB).

Participants and Methods: Studies to be included in the meta-analysis were reviewed and followed these criteria: 1) inclusion of measures of cognition, 2) had a comparison group of participants not taking memantine (placebo-controlled trials and open-label trials), and 3) provided data allowing for the calculation of effect size. We examined effect sizes across global cognition and six neuropsychological domains . Clinical moderator variables examined included: 1) study diagnostic cohort (i.e., PDD only, DLB only, or mixed PDD-DLB cohort), and 2) study design (i.e., open label or placebo-controlled).

Results: Review of the literature resulted in 6 publications meeting our inclusion criteria totaling 57 effects for analysis. Homogeneity analysis across cognitive domains revealed a small overall effect size $(d=0.26)$ that was significantly heterogeneous $(p<.001)$. Specific neuropsychological domains assessed did not account for variability in effect size. Moderator analysis across the three diagnostic cohorts revealed significant heterogeneity among effects $(p=.011)$. Effect sizes were small to moderate in the mixed cohort of DLB and PDD patients $(\mathrm{d}=0.42)$, but small in DLB $(d=0.12)$ and PDD cohorts $(d=0.23)$. With regard to 
study design, open-label trials had significantly $(p<.001)$ larger effect sizes $(d=1.32)$ than placebo-controlled trials $(d=0.12)$.

Conclusions: The present study demonstrates that heterogeneity across studies of memantine in DLB and PDD patients is moderated by diagnostic make-up of the cohort and study design.

Correspondence: Laura Brennan, M.S., Psychology, Drexel University, 1505 Race Street, Philadelphia, PA 19102. E-mail: laura.brennan29@ gmail.com

H.M. MISKEY \& P.L. GROSS. Neuropsychological Assessment of a Veteran with a Large Arachnoid Cyst.

Objective: Arachnoid cysts comprise approximately 1\% of intracranial masses (Basaldella, 2007), and mostly occur in the temporal fossa (Wester, 2008). Although often silent, they can result in impaired verbal learning, visual perception, processing speed, memory, cognitive flexibility, and constructional skills (Wester, 2008).

Participants and Methods: A 65 year-old, left-handed, male Vietnam Veteran was referred for a neuropsychological examination following two years of progressive memory decline. Complaints included headache, difficulty recalling names, decreased focus, forgetting instructions at work, and difficulty multi-tasking. Relevant medical and psychological history included hypertension, hypothyroidism, hyperlipidemia, atrial fibrillation, untreated severe sleep apnea, PTSD, depression, and Agent Orange exposure.

Results: A head CT scan revealed a $16.4 \mathrm{~cm}$ x $7.7 \mathrm{~cm}$ arachnoid cyst spanning the left anterior and middle cranial fossa, mass effect on the left frontal and temporal lobes with midline shift, mild scalloping of the adjacent calvarium, and chronic microvascular ischemic changes. Premorbid functioning was estimated as average. Visuospatial construction, visual learning and memory, processing speed, set switching, and ability to maintain set were mildly impaired. Visuospatial impairments were notable in that the Veteran previously taught map reading. Nonverbal and verbal reasoning, vocabulary, simple auditory attention and working memory, verbal learning and memory, problem-solving, and conceptualization were average. Results were consistent with Mild Neurocognitive Disorder. Screening instruments indicated severe levels of depression and anxiety.

Conclusions: Despite the significant size of the arachnoid cyst and accompanying midline shift, this Veteran reported a history of excellent functioning and showed only mild deficits on testing. Results suggested vascular and psychological contributions to cognitive changes.

Correspondence: Holly M. Miskey, PhD, Mental Health and Behavioral Sciences, Hefner VA Medical Center, 1601 Brenner Avenue, 11M-2/ MH\&BS, Salisbury, NC 28144.E-mail:Holly.Miskey@va.gov

L.J. ALTMANN, E. STEGEMÖLLER, D. BOWERS, A.A. HAZAMY, M.S. OKUN \& C.J. HASS. Effects of an Aerobic Exercise Intervention in Parkinson's Disease.

Objective: Aerobic exercise can improve mood and cognition in older adults and persons with mild cognitive impairment, Alzheimer's disease, and developmental disabilities. Studies of the effects of exercise in Parkinson's disease (PD) are rare and show limited improvement. The current study examines the effects of aerobic exercise on disease severity, mood, sleep and cognition.

Participants and Methods: Twenty-two non-demented people with PD were randomly assigned to either a treadmill exercise or a no-contact control group. Both groups completed a range of mood, sleep, and cognitive tasks, and the UPDRS at intake and after the 16-week intervention. The treadmill group ( $\mathrm{N}=11)$ performed supervised, moderate intensity treadmill walking for an hour three times a week over 16 weeks, while the no contact control group $(\mathrm{N}=11)$ continued their normal activities. Results: The aerobic group improved significantly relative to the control group in depressive symptoms, sleep, and overall UPDRS scores, especially for motor symptoms. Performance on the Stroop color-word task diverged between groups. The control group responded faster and made more errors post intervention, while the aerobic group responded more slowly and more accurately post intervention, resulting in significant group differences in accuracy and RTs. Other speeded measures showed similar but non-significant trends.

Conclusions: Aerobic exercise led to important mood and performance gains for this small group of people with PD. We interpret the increased accuracy and slower responses on the Stroop as reflecting improvements in inhibitory capacity. The lack of pervasive improvements in cognition, though, are consistent with previous studies.

Correspondence: Lori J. Altmann, PhD, Speech, Language \& Hearing Sciences, University of Florida, 336 Dauer Hall, Box 117420, Gainesville,FL32611-7420.E-mail: laltmann@ufl.edu

E.E. KRAUSKOPF \& R. HOPKINS. The Impact of Cerebral Microemboli on Cognitive Function Following Bubble-Contrast Transcranial Doppler for Evaluation of Cardiac Shunt.

Objective: Paradoxical embolization via a patent foramen ovale (PFO), or right-to-left cardiac shunt (RLS), is a risk factor for stroke. The gold standard for diagnosis of PFO, Power M-mode Transcranial Doppler (TCD) evaluation, produces brain microembolization (bubbles) to determine shunt size and severity. We evaluated cognitive outcomes in patients who underwent routine TCD for RLS.

Participants and Methods: One hundred and four participants (mean age $36.8 \pm 11.3$ and education level $14.2 \pm 2.3$ in years; 77 women and 27 men) referred to Sorensen Cardiovascular Group were evaluated preand immediately post-TCD for changes in cognitive functioning using the processing speed (Detection) attention (Identification), and working memory (Two-Back) subtests of the CogState battery. Pre- post cognitive function were compared using repeated measures ANOVAs and the no shunt vs. moderate to severe shunt groups did not significantly differ in age $(p=.326)$, education $(p=.150)$, or gender $\left(\chi^{2}=1.78\right)$.

Results: Adjusting for practice effects, age, and education, the main effects for shunt group and shunt group by time interactions were not significant for processing speed $(p=0.17 ; p=0.87)$, attention $(p=$ $0.18 ; p=0.34)$, or working memory $(p=0.77 ; p=0.19)$, respectively. Conclusions: Results suggest that TCD-induced microemboli do not result in immediate cognitive changes in patients with moderate to severe RLS. However, findings are restricted to young $(30 \mathrm{~s})$, medically healthy, predominately Caucasian individuals assessed immediately following TCD. Studies are needed to assess the effects of TCD in other cognitive domains, on other neuropsychological tests, and the development of cognitive impairment at later time periods.

Correspondence: Erin E. Krauskopf, Doctorate, Neuropsychology, Brigham Young University, 231 East 400 South, Suite 335, Salt Lake City, UT 84111. E-mail: eekrauskopf@gmail.com

\section{J. JONES, M. MARSISKE, M.S. OKUN \& D. BOWERS. Latent Growth Curve Analysis Reveals Worsening Parkinson's Disease Quality of Life is Driven by Depression.}

Objective: Parkinson's disease is a neurodegenerative disorder resulting in a wide variety of motor and non-motor symptoms. The current study examined the influence of apathy, depression and motor symptoms on quality of life in Parkinson's disease patients. Information was drawn from an 18 month period.

Participants and Methods: Participants (N=397) were assessed for apathy (Apathy Scale), depression (Beck Depression Inventory-II), motor severity (Unified Parkinson's Disease Rating Scale, part III) and quality of life (Parkinson's Disease Questionnaire-39) at three time points: an initial clinical evaluation (baseline), a 6-month follow-up and an 18-month follow-up. Latent growth curve models were used to determine the influence of apathy, depression and motor symptoms on quality of life trajectories.

Results: Greater difficulties with quality of life at baseline showed the strongest relationship to more severe depression, followed by more severe motor symptoms, younger age and less education (all $\mathrm{p}$ values<.001). Worsening of quality of life over the 18 -month period was only predicted by a worsening (i.e. becoming more severe) of depression 
$(p=.003)$. Apathy was not related to quality of life either within- or between-subjects.

Conclusions: Overall, findings from the current study suggest that self-reported QoL among PD patients is primarily related to depression. Future efforts to improving clinical care of PD patients may benefit by focusing on improving psychosocial-adjustment or treatments targeting depression.

Correspondence: Jacob Jones, MS, CHP, University of Florida, 3901 SW20th ave apt 206, Gainesville, FL 32607.E-mail:jacob.jones126@ ufl.edu

B.N. RENN, A. FERNAU, A.L. SMITH, A.M. LALIK, N. BAUMANN, S. JARRELL \& L. FELICIANO. Screening for Cognitive Impairment in a Diabetic Primary Care Sample.

Objective: Cognitive impairment (CI) is often comorbid with and may be a sequela of diabetes. CI may have negative effects on medical treatment adherence. We investigated the cognitive profile and risk factors associated with CI in adult primary care patients with diabetes.

Participants and Methods: The present study utilized a cross-sectional examination of 217 patients $(75 \%$ female, Mage $=53.64 \pm 10.59$, range 22-82 years) recruited from health education classes for diabetes management at a federally qualified health center. The sample was diverse with regard to education (Meduc $=12.92 \pm 2.19$, range 6-18 years) and ethnicity $(37.8 \%$ non-White). The presence of CI was assessed using the St. Louis University Mental Status Examination (SLUMS). CI was dichotomized into presence (mild or severe) or absence (normal cognition) for subsequent chi-square analyses.

Results: Mean SLUMS score in this sample was 23.76 \pm 4.31 (mild impairment). The majority of patients $(64.1 \%)$ screened positive for CI; $42.4 \%(n=92)$ scoring within the mild neurocognitive impairment range and $21.7 \%(n=47)$ within the severe impairment/dementia range. Prevalence of CI varied by ethnic/racial minority status with $73.2 \%$ of non-White patients performing in the impaired range, compared to $58.0 \%$ of White patients $(\chi 2(1, \mathrm{n}=213)=4.38, \mathrm{p}=.036)$. CI status was not associated with age, gender, or depression.

Conclusions: CI was alarmingly high in this sample of primary care patients referred for diabetes management. Patients of ethnic minority status were at increased risk for CI. Implications for neuropsychological assessment and diabetes treatment adherence are discussed.

Correspondence: Brenna N. Renn, MA, Psychology, University of Colorado, Colorado Springs, 1420 Austin Bluff Pkwy, Dept of Psychology, Colorado Springs, CO 80918.E-mail: brenn@uccs.edu

\section{A. DODDS, C.I. HIGGINSON \& E.A. DISBROW. The Relation} Between Apathy and Quality of Life in Parkinson's Disease.

Objective: Research suggests that both apathy and depression are related to quality of life (QOL) in Parkinson's disease (PD). However, since apathy is a component of the syndrome of depression, it is unclear whether the relation between depression and QOL is due to apathy. The purpose of the current study was to address this question.

Participants and Methods: Fifty nondemented individuals with PD (26 female) were administered the Frontal Systems Behavior Scale (FrSBe), Geriatric Depression Scale (GDS), and Parkinson's Disease Questionnaire (PDQ-39) amongst a battery of neuropsychological measures. Mean (SD) age, education, and UPDRS motor scores (in "on" state) were 67.16 (5.76) years, 16.50 (2.67) years, and 19.98 (13.14), respectively.

Results: Both the FrSBe apathy subscale and the GDS significantly correlated with the PDQ-39, $r=.57, p<.001 \& r=.76, p<.001$, respectively. When entered into a hierarchical regression the FrSBe apathy subscale predicted $33 \%$ of the variance in PDQ-39 scores on the first step, $\mathrm{F}(1,48)=23.24, \mathrm{p}<.001$. On the second step the GDS predicted an additional $28 \%$ of the variance, $F(1,47)=32.93, p<.001$. Beta weights on the second step were marginal for the FrSBe apathy subscale, $\mathrm{t}=1.95, \mathrm{p}=.057$, but significant for the GDS, $\mathrm{t}=5.74, \mathrm{p}<.001$.
Conclusions: These results suggest that symptoms of depression other than apathy predominantly account for the relation between depression and QOL in PD.

Correspondence: Alicia Dodds, Psychology, Loyola University Maryland, 205 E.Melrose Ave., Baltimore,MD 21212.E-mail: alicia.dodds@gmail. com

M. FUIMARA, T. QUINN, J. MORTON, J. CREEK, S. CUEVAS, L. ZETTEL-WATSON \& B.J. CHERRY. Cognitive Clusters in Fibromyalgia, A Chronic Pain Condition.

Objective: Cluster analysis was performed to determine whether individuals with fibromyalgia (FM), a chronic pain condition, would perform similarly to the cognitive clusters found in Libon et al. (JINS, 2010). In the Libon study, participants with complex regional pain syndrome formed three groups: "normal" cognition, "dysexecutive" impairment, and "global" impairment based on assessments of executive function as well as semantic and declarative memory.

Participants and Methods: Sixty-eight individuals with FM (Mage $=59.5$ years; $92.6 \%$ women) were assessed on Digit Span Backward, animal fluency, and delayed recall on a 10-item word list (from the Consortium to Establish a Registry for Alzheimer's disease [CERAD]). A two-step cluster analysis was conducted to determine if subgroups were found within FM based on cognitive scores. Hierarchical algorithms determined the cluster distance (log-likelihood) and criterion (Schwarz's Criterion Measure) between the pre-clusters.

Results: Participants in Cluster 1 ( $\mathrm{n}=30$ [44\%] "normal") scored highest on all measures (DSB, AF, CERAD delayed recall). Participants in Cluster 2 ( $\mathrm{n}=18$, [27\%] "memory decline") showed normal executive function (DSB, AF), but lower scores on CERAD delayed recall. Participants in Cluster $3(\mathrm{n}=20$ [29\%], "global decline") performed lowest on all tests. MANOVA analysis revealed that all clusters were significantly different from each other, Pillai's Trace $=1.19, \mathrm{~F}(1,66)$ $=31.25, \mathrm{p}=.05$.

Conclusions: Cognitive clusters were different for FM than complex regional pain syndrome; however, both studies suggest that cognitive decline may be comorbid with chronic pain.

Correspondence: Barbara J. Cherry, PhD, Psychology, CSUF, $800 \mathrm{~N}$ State College Blvd, Fullerton, CA 92834.E-mail: bcherry@fullerton.edu

J.S. MILLER, A. CHAN, A. GOTTUSO \& J.C. WERTHEIMER. A Clinical Profile of Dysautonomia in Parkinson's Disease for Individuals with and without Deep Brain Stimulation.

Objective: Dysautonomia is common in Parkinson's disease (PD) and may precede the onset of motor symptoms. Although deep brain stimulation (DBS) is an effective treatment for motor symptoms, the effects of DBS on dysautonomia remain unclear. This study investigated the prevalence of dysautonomia in a PD population with and without DBS. Participants and Methods: A cross sectional research design was used. A total of 1489 individuals with PD participated in the study, including 413 with DBS and 1076 without DBS. Participants completed the Scale for Outcomes for Autonomic Symptoms (SCOPA-AUT) and the Hospital Anxiety and Depression Scale (HAD).

Results: Dysautonomia was highly prevalent (up to $90 \%$ of the sample), regardless of age and disease duration. Analyses revealed older adults and advanced disease duration yielded higher SCOPA-AUT Total Scores $(p=.035$ and $p=.0003$, respectively). There was no significant difference between the SCOPA-AUT Total Score and the majority of indices between DBS and Non-DBS groups, with exception to significantly greater difficulties with swallowing and less cardiovascular symptoms for the DBS group $(p<.0001$ and $p=.003$, respectively). Moderate correlations between dysautonomia and mood (HAD) were observed $(r=.49)$ and $57 \%$ of participants reported dysautonomia adversely impacts engagement in social activities.

Conclusions: Dysautonomia is significantly elevated in PD. Prevalence and severity of symptoms are associated with increased age and advanced disease duration. DBS therapy can impact autonomic functions. 
such as adversely affecting swallowing, while auspiciously impacting cardiovascular symptoms. Implications for neuropsychologists relating to non-motor clinical profiles, psychosocial variables, and recommendations (DBS therapy and rehabilitation intervention) are discussed. Correspondence: Justin S. Miller, MA, Graduate Psychology, Fuller Theological Seminary, 765 E California Blvd, Pasadena, CA 91106. E-mail: justin.s.miller@me.com

D. CALVO, J. GUNSTAD \& M. SPITZNAGEL. Obesity does not influence effects of fasting on attention and executive function.

Objective: Obesity is a risk factor for reduced attention/executive function, and is also linked to breakfast skipping. Poor glucoregulation may underlie cognitive deficits associated with skipping breakfast, suggesting this behavior could have greater cognitive consequences for obese individuals. We expected poorer attention/executive test performance in obese than lean persons with extended fasting, mediated by glucoregulation.

Participants and Methods: 34 lean and 33 obese participants fasted overnight $(8 \mathrm{hrs})$ and then completed assessments at baseline and 90 minute follow-up. Each assessment was comprised of glucometer reading of blood glucose and cognitive measures including ANAM-4 Go/ No-Go (GNG) and Continuous Performance Task (CPT).

Results: Obese individuals showed higher fasting glucose than lean at baseline and 90 minutes $(\mathrm{p}<.01)$, and glucose declined across both groups over the 90 minute period $(p=<.001)$. Repeated measures MANOVAs showed no main effects of lean versus obese on cognition. In the total sample, extended fasting resulted in faster GNG reaction time $(\mathrm{p}<.05)$, but slower CPT reaction time $(\mathrm{p}<.01)$ with more omission errors $(\mathrm{p}<.05)$ at 90 minutes.

Conclusions: Although glucoregulation was poorer in obese than lean individuals, this did not lead to greater attention or executive function deficit in this healthy sample. Extended fasting produced poorer vigilance and faster inhibitory control across groups; though simple sustained attention worsened, practice effects or rising to the occasion may have occurred for the inhibition task. Skipping breakfast may confer no greater cognitive risk for obese than lean individuals; however, given that our weight groups showed no cognitive differences at all, our healthy sample may not have had poor enough glucoregulation to impact cognition. Future studies that include participants with impaired glucoregulation may better capture any fasting cognitive differences between lean and obese individuals.

Correspondence: Dayana Calvo, Kent State Psychology, 3877 Lake Run Blvd, Stow, OH 33777. E-mail: dcalvo1@kent.edu

\section{K. WYMAN-CHICK. Effects of bilateral deep brain stimulation on verbal fluency in patients with Parkinson's disease: A meta- analysis.}

Objective: Previous meta-analyses suggest patients with Parkinson's disease experience significant decline in verbal fluency compared to non-clinical groups. Deep brain stimulation for Parkinson's disease is also associated with impairment in verbal fluency. The purpose of this study is to determine if bilateral deep brain stimulation in patients with Parkinson's disease is associated with greater impairment in verbal fluency when compared to a clinical control group managed by medication. Participants and Methods: Though a literature search yielded over 140 articles, 11 articles met inclusion criteria. Specifically, those studies that were of high methodological quality, from peer-reviewed journals, included bilateral stimulation with a comparison group of non-surgical patients with Parkinson's disease were included. A total of 462 patients who underwent deep brain surgery and 420 non-surgical controls were included. Cohen's d was calculated as a measure of effect size comparing the means (+SD) for verbal fluency performance.

Results: Tests of heterogeneity were non-significant and there were no outliers. The random effects model showed a medium effect size for phonemic fluency $(d=-0.49)$ and a small effect size for semantic fluency $(\mathrm{d}=-0.29)$. Moderators such as time elapsed since surgery and disease duration were not significant. Disease severity (Hoehn \& Yahr stage at baseline) was a significant moderator for phonemic fluency and age was a significant moderator for semantic fluency.

Conclusions: The results indicate patients with Parkinson's disease who have undergone bilateral deep brain stimulation have significant impairment in verbal fluency compared to patients with Parkinson's treated with medication.

Correspondence: Kathryn Wyman-Chick, M.A., Univeristy of Kansas School of Medicine, 7829 Rockhill, Ste 105-106, Wichita, KS 672063919. E-mail:kwyman@pacificu.edu

J.N. COPELAND \& A.I. TRÖSTER. Accuracy of Patient And Care Partner Reports Regarding Specific Cognitive Functions: Implications for Diagnosis of Mild Cognitive Impairment in Parkinson's Disease.

Objective: Recent criteria for Parkinson's disease-Mild Cognitive Impairment (PD-MCI) require a caregiver or patient complaint or clinician observation of cognitive decline (Litvan et. al, 2012). The present study examined agreement among patients' and care partners' reports of specific cognitive difficulties and objective performance on tests of attention, memory, language, visuoperceptual skills, and executive functioning.

Participants and Methods: A cross-sectional sample of 42 patients who met criteria for PD-MCI and their care partners subjectively reported absence or presence of deficits in each cognitive domain during clinical interview. Presence or absence of objective deficits in each domain was determined if the patient's performance was $\geq-1.5 \mathrm{SD}$ below the mean on selected cognitive tests.

Results: Objective deficits were observed least frequently in the language domain and most frequently in the memory domain. Patients' and care partners' accuracy in observing objectively identified deficits was poor ( $\leq 60 \%$ agreement; Kappa $\leq .07, \mathrm{~ns})$ in all 5 domains. Patient and care partners showed a moderate level of agreement between their subjective reports $(\geq 74 \%$ agreement; Kappa $\geq .43, \mathrm{p}<.01)$, in all domains except attention. Patients with objective executive deficits (and their care partners) endorsed these less than other domains. Objective testing detected subjective memory and executive difficulties best, whereas patient and care partner endorsement of language and attention difficulties was not as well detected.

Conclusions: PD-MCI patients and their care partners may not be accurate in their endorsement of cognitive deficits, which could potentially affect (and delay) diagnosis and treatment of PD-MCI in a clinical setting. Inaccurate reports may influence diagnostic rates of MCI, accuracy of epidemiologic data, and future research regarding treatment. Correspondence: Jacquelynn N. Copeland, Ph.D., Clinical Neuropsychology, Barrow Neurological Institute, $222 \mathrm{~W}$. Thomas Road, Suite 315, Phoenix, AZ 85013.E-mail: jacquelynn.copeland@ dignityhealth.org

\section{N.A. KIEWEL, E.N. ANDRESEN \& D. FLODEN. Can We Evaluate Visual Memory in Parkinson's Disease?}

Objective: Assessing nonverbal memory in Parkinson's disease (PD) can be difficult as most of the commonly used measures require motor control sufficient for drawing. Doors is a nonverbal memory measure which does not require a motor response (Baddeley, Emslie, \& Nimmo-Smith, 1994). The aims of the present study were to (a) compare performance of a PD sample to normative data and (b) examine the relationship between Doors performance and other cognitive abilities that are commonly affected by PD.

Participants and Methods: Participants included 49 PD patients (18 females, 31 males) who were evaluated through the Cleveland Clinic's Center for Neurological Restoration. Average age was 65.84 (SD = 7.18), years of education was $13.79(\mathrm{SD}=3.39)$, duration of $\mathrm{PD}$ in years was $8.79(\mathrm{SD}=4.94)$, and motor symptom severity (UPDRS-III) was 25.17 ( $\mathrm{SD}=12.40)$. Doors was administered as part of a comprehensive 
neuropsychological evaluation. Exploratory descriptive and correlational analyses were conducted.

Results: $51 \%$ of the PD sample performed in the impaired range on Part A. On Part B, 45\% had scores in the impaired range. Both parts were associated with different markers of disease severity, as well as processing speed and an index of global cognitive function.

Conclusions: The Doors test is a useful indicator of cognitive impairment in PD patients and appears to be sensitive to PD severity. The lack of motor demands on this task may make this task a valuable measure of nonverbal memory for a population that has been challenging to evaluate.

Correspondence: Nicole A. Kiewel, MS, Psychology, University of Houston, 126 Heyne Building, University of Houston, Houston, TX 77204-5022.E-mail:nicole.kiewel@gmail.com

\section{A.D. ROCHETTE, M. SPITZNAGEL, R.A. COHEN, L.H. SWEET, R. JOSEPHSON, J. HUGHES \& J. GUNSTAD. Gender is Associated With Memory Task Performance in Older Adults With Heart Failure.}

Objective: Neurocognitive impairment is found in up to $80 \%$ of heart failure (HF) patients and are associated with poor outcomes and diminished health-related quality-of-life. Though it is known that women perform better than men on memory tasks in the general population, this has not been explored under the context of HF and could have significant clinical implications. The current study examined the relationship between gender and cognition in a sample of older adults with HF.

Participants and Methods: A total of 189 older adults with HF (36.5\% women; avg age 68 years) completed a neuropsychological test battery as part of a larger project. Measures were chosen to assess functioning in attention/executive function (Trail Making Test A and B, Frontal Assessment Battery, Stroop Color Word Test) and memory (California Verbal Learning Tests Learning, Short Recall, Delayed Recall, Recognition). Results: After controlling for demographic (i.e. education) and medical factors (i.e. ejection fraction, hypertension status, and fitness levels), MANCOVA revealed women had better memory performance $[\lambda=0.9$, $\mathrm{F}(4,180)=5.00, \mathrm{p}=0.01]$. Posthoc tests revealed better performance on CVLT Learning, Short Recall, and Delayed Recall ( $p<.01$ for all). Men and women did not differ on tests of attention/executive function $[\lambda=0.96, F(5,172)=1.34, p=0.25]$.

Conclusions: Results suggest that HF is associated with greater memory impairment in men than women, though no differences emerged in attention/executive function. Future studies are needed to determine the mechanisms for this pattern and examine whether gender-specific clinical management of HF may improve outcomes in this high risk population.

Correspondence: Amber D. Rochette, BA, Psychology, Kent State University, 3494 Bent Tree Lane, \#213, Stow, OH 44224. E-mail: amber.rochette88@gmail.com

\section{A.B. RYAN \& J. BENGE. Influence of Depression on the Different Aspects of Quality of Life in Parkinson's.}

Objective: Health related quality of life (QOL) is a multidimensional construct in Parkinson's disease. While it is well established that depression is related to overall QOL, little research has evaluated how it impacts specific dimensions of QOL. The current study used the Parkinson's Disease Questionnaire - 39 (PDQ-39), a commonly used self-report measure, to examine the impact of depression on multiple dimensions of QOL in patients with Parkinson's disease.

Participants and Methods: 325 patients with Parkinson's disease were administered the PDQ-39. Patients were grouped by scores on the depression item of the PDQ-39 into none (score 0), mild depression (score 1), and moderate to severe depression (score $>2$ ). Mean scores for seven dimensions of QOL measured by the PDQ-39 were compared using non-parametric techniques.

Results: There were significant differences between groups across dimensions of QOL, and the differences remained significant after
Bonferonni corrections: mobility $\chi^{2}(2, \mathrm{~N}=325)=70.2, \mathrm{p}<.0001$, activities of daily living $\chi^{2}(2, \mathrm{~N}=325)=66.1, \mathrm{p}<.0001$, stigma $\chi^{2}(2, \mathrm{~N}=325)=32.4$, $\mathrm{p}<.0001$, social support $\chi^{2}(2, \mathrm{~N}=325)=38.2, \mathrm{p}<.0001$, cognition $\chi^{2}(2, \mathrm{~N}=325)=61.5, \mathrm{p}<.0001$, communication $\chi^{2}(2, \mathrm{~N}=325)=37.5$, $\mathrm{p}<.0001$, bodily discomfort $\chi^{2}(2, \mathrm{~N}=325)=56.4, \mathrm{p}<.0001$. Post hoc analyses indicated increasing severity of depression was associated with declines in each domain of QOL assessed.

Conclusions: Depression has a pervasive influence across dimensions of QOL, even when it is mild. As such, the influence of depression on QOL should be considered when QOL is used as an outcome variable in clinical trials. It should also be taken into account when designing interventions that address QOL.

Correspondence: Abigail B. Ryan, Ph.D., Neuropsychology \&. Concussion Management Associates, 220 Union Street, Rockport, ME 04856. E-mail: aabernett@gmail.com

M. Alosco, M.S. PENN, M. SPITZNAGEL, B.R. OTT, M. CLEVELAND \& J. GUNSTAD. Preliminary Evidence for the Adverse Impact of Depressive Symptoms on Driving Fitness in Older Adults with Heart Failure.

Objective: Heart failure (HF) patients perform poorly on driving simulation tasks, suggesting this population may be at risk for unsafe driving. Impaired driving in HF is believed to stem from cognitive dysfunction, although recent work suggests clinical modifiers of cognition also play a key role. Depression can be found in $>70 \%$ of HF patients, is linked with cognitive dysfunction, and contributes to impaired driving in non-HF samples. Yet, no study has examined the associations among depression, cognition, and driving in HF.

Participants and Methods: $18 \mathrm{HF}$ patients completed a validated simulated driving scenario, the Beck Depression Inventory-II (BDI-II), and a cognitive battery. Partial correlations controlling for age, ejection fraction, and diabetes examined the associations among BDI-II, cognition, and driving simulation indices.

Results: The average BDI-II was 3.00 (range $=0$ to 15 ). Higher BDI-II score correlated with greater number of collisions $(r=0.55, p=0.03)$, centerline crossings $(\mathrm{r}=0.66, \mathrm{p}=0.01)$, and $\%$ time out of lane $(\mathrm{r}=$ $0.70, p<0.01)$. Increased depressive symptoms predicted lower attention/executive function $(\mathrm{r}=-0.62, \mathrm{p}=0.02)$ and reduced performance in this domain correlated with greater number of collisions, centerline crossing, and \% time out of lane $(r=-0.50$ to -0.72$)$.

Conclusions: Depressive symptoms predict poorer driving simulation performance in HF, possibly via cognitive dysfunction. Prospective studies that use on-road testing are needed to confirm our findings and examine crash statistics, elucidate non-cognitive mechanisms by which depression influences driving, and examine whether depression treatment improves driving abilities.

Correspondence: Michael Alosco, MA, Kent State University, 4710 Waterford Circle, Stow,OH 44224.E-mail: malosco@kent.edu

\section{PIPER, E. LEE, M. FEIGON, M. VERNALE, N. PLISKIN \& D. NYENHUIS. Neuropsychological Profiles in Adults with Sickle Cell Disease.}

Objective: Cognitive impairment is documented in patients with sickle cell disease (SCD). Studies investigating cognitive impairment in this population have primarily examined group differences in neuropsychological performance, which may have overlooked the heterogeneity of cognitive functioning. To date, no study has examined individual differences in cognitive functioning that may categorize patients with SCD into subgroups. The objectives of this study were to determine whether distinct cognitive profiles occur in patients with SCD and, if so, to examine potential differences in demographic, clinical, and psychosocial characteristics.

Participants and Methods: Participants with SCD $(\mathrm{n}=73)$ and similarly matched controls $(n=82)$, completed a brief neuropsychological protocol (Hachinski et al., 2006) and self-report measures of pain and mood-related symptoms. Cluster analysis was used to identify groups 
of participants based on their cumulative scores across the domains of executive functioning, language, memory, and visuospatial skills. MANOVA was used to compare the cluster groups across the four cognitive domains. ANOVA and chi-square tests were used to compare cluster groups on demographic, clinical, and psychosocial characteristics.

Results: Results indicated three distinct cognitive subtypes: (1) executive and memory impaired ( $56 \%$ of participants); (2) globally impaired $(14 \%)$; and (3) cognitively intact $(30 \%)$. The three cluster groups did not differ on most demographic factors, stroke history, or pain severity, but differed on level of education and current mood-related distress.

Conclusions: Results demonstrated the presence of distinct cognitive profiles in adults with SCD, with a proportion of cognitively intact patients. Future studies should further explore differences between subtypes and their implications for treatment planning.

Correspondence: Lauren Piper, Illinois Institute of Technology, 1844 S. Blue Island Ave, Apt 3R, Chicago, IL 60608. E-mail: Ipiper129@ gmail.com

L.C. BUTTERFIELD, C. CIMINO, R. SALAZAR, C. LEE, W. HALEY, J. SANCHEZ-RAMOS, D. BOWERS \& M.S. OKUN. The Parkinson's Active Living (PAL) Program: A Behavioral Intervention Targeting Apathy in Parkinson's Disease.

Objective: Apathy, reflecting motivation and self-initiation impairment, is common in Parkinson's disease (PD) and associated with a host of negative associates. The purpose of this study was three-fold: (1) develop protocol materials to facilitate treatment delivery, (2) determine ease of training interventionists, (3) assess feasibility, acceptability, and estimated effect of a 6-week, primarily telephone-based activity scheduling/monitoring intervention on reducing apathy in non-demented, apathetic PD patients. To our knowledge, this is the first behavioral intervention program designed to target apathy in PD.

Participants and Methods: 34 non-demented, apathetic PD patients participated. 1. An interventionist manual outlining treatment protocol and a patient workbook were created. 2 . Interventionists were trained and evaluated for protocol adherence. 3. A one-arm uncontrolled trial was conducted to estimate treatment effect on self-rated apathy, depression, ADLs, QoL, and caregiver burden. Intervention consisted of one in-person Planning Session to identify and schedule tailored goals, and 6 weekly phone sessions. Outcomes were assessed at baseline, post-test, and 1-month follow-up. Paired t-tests examined differences in outcomes by time point.

Results: Treatment delivery, feasibility, and acceptability data reflected strong interventionist adherence, expected attrition, and high patient satisfaction. Apathy, depression, and QoL significantly improved post-treatment with medium to large effects and clinically meaningful changes. Improvements in apathy and depression were maintained at follow-up. Response to treatment was associated with higher baseline executive function, novelty seeking, and self-awareness.

Conclusions: A promising manualized intervention designed to target self-generational deficits common in PD is presented. Preliminary results showed improved motivation, mood, and QoL in PD through a telehealth intervention. A randomized controlled trial is needed to further investigate the efficacy of the PAL program.

Correspondence: London C. Butterfield, MA, Psychology, University of South Florida, 4202 E. Fowler Ave., PCD 4118G, Tampa, FL 33620. E-mail: londonbutterfield@hotmail.com

\section{FISCHER, T. SUlLIVAN \& P. SHEAR. Profound Memory} Impairment as Rare Complication Following Bariatric Surgery.

Objective: There is an extensive literature on alcoholic Wernicke-Korsakoff Syndrome (WKS); however, less is known about nonalcoholic origins of this syndrome, which may have a differing presentation. We describe a patient who developed profound memory loss and motor dysfunction secondary to an acute nutritional deficiency.

Participants and Methods: This single subject case study is based on a comprehensive clinical neuropsychological examination of a premorbidly high functioning 30-year old woman, referred for evaluation of cognitive deficits incurred following bariatric surgery for obesity. Post-surgically, she experienced an inability to retain oral nutritional supplementation, resulting in a thiamine deficiency that went untreated. Subsequent MRI results indicated bilateral thalamic hyperintensities and subtle hyperintensity in the periaqueductal gray.

Results: Neuropsychological testing revealed extensive anterograde and retrograde memory impairment that is comparable to what is observed in patients with WKS of an alcoholic origin, as well as marked motor dysfunction. She displayed relatively spared visuospatial and working memory functioning and, less expectedly, severe reduction in verbal abilities.

Conclusions: This patient demonstrated memory dysfunction comparable to previous reports in alcoholic WKS, although she has no history of excessive drinking. Her profound fine and gross motor difficulties are atypical for alcoholic WKS but have been reported as a rare adverse consequence of bariatric surgery. These data provide further evidence of the potential Wernicke-Korsakoff like symptoms as well as neuropathy in bariatric surgery cases with improper nutritional supplementation and highlight the critical importance of monitoring thiamine levels following this type of surgical procedure.

Correspondence: Mark Fischer, Psychology, University of Cincinnati, 2828CORTELYOU PL, Cincinnati, OH 45213-1202.E-mail: fischemk@ mail.uc.edu

J. SORDAHL, D. GONZALEZ, J. HIGHSMITH, J. MARCEAUX, K. MCCOY \& J. O'ROURKE. The Relationship Between Body Mass Index and Processing Speed.

Objective: Body mass index (BMI) is a common metric used for estimating an individual's body fat based on height and weight. Research has associated higher BMI with vascular risk factors as well as cognitive decline (e.g., lower MMSE scores). The purpose of the current research was to assess the relationship between BMI and processing in a veteran population.

Participants and Methods: Participants included a mixed-clinical sample of veterans ( $\mathrm{N}=258$ ) referred for neuropsychological evaluation $($ Mage $=61.74$, SDage $=10.3 ;$ Meducation=12.9, SDeducation=3). Bivariate correlations evaluated the relationship between BMI, several health risk factors, and processing speed index (PSI) from the WAIS-IV. Hierarchical regression evaluated BMI as a unique predictor of PSI after accounting for education, vascular risk factors, obstructive sleep apnea, type-II diabetes, and depression. ANOVA was used to compare mean PSI between healthy, overweight, and obese patients categorized according to BMI.

Results: The mean BMI for the group was 30.1 ( $\mathrm{SD}=5.2$; obese range), and PSI was 84 (SD=14.0; low average range). Neither bivariate correlations nor six-stage linear hierarchical regression demonstrated a significant relationship $(p<.05)$ between BMI and PSI. ANOVA also did not yield any BMI group differences in PSI. BMI was associated with diabetes and obstructive sleep apnea.

Conclusions: BMI was not a significant independent predictor of processing speed in a clinical sample of veterans. These findings differ from previous research using cognitive screening measures that found a relationship between BMI and global cognitive functioning, indicating that the relationship between BMI and neuropsychological functioning may differ by domain and population.

Correspondence: Jeffrey Sordahl, PsyD, Clinical Psychology, George Fox University, 1007 Caprese Ln, San Antonio, TX 78253. E-mail: jeffrey. sordahl@gmail.com

A.K. HILDENBRAND, R. AGGARWAL, R. JETHVA \& M. GRANT. Examining the Efficacy of Brief Adherence Interventions for Individuals with Phenylketonuria.

Objective: Despite the success of medical treatment in preventing cognitive and neurological sequelae of Phenylketonuria (PKU), an inherited metabolic disorder, 50-70\% of adolescents and adults display 
suboptimal adherence to treatment. We explored the efficacy of supplementing standard treatment with brief, clinic-based educational and behavioral interventions to address nonadherence.

Participants and Methods: This study used a retrospective longitudinal design. Participants included 53 individuals with PKU (high Phe intervention group: $\mathrm{n}=28$; high Phe control group: $\mathrm{n}=14$; low Phe control group: $\mathrm{n}=11$ ). For the intervention group, standard clinical care visits were supplemented with psychoeducation, assessment of barriers to adherence, and/or behavioral adherence strategies. Adherence was assessed by Phenylalanine (Phe) averaged over 12 months pre- and post-baseline. Intellectual outcomes were obtained from WISC-IV/III or WAIS-R.

Results: Growth curve analyses indicated that the effect of group on the cubic term significantly improved model fit $(\chi 2(4)=9.90 ; p=.04)$, indicating that groups differed in change in Phe over time. Model comparisons revealed a stable, linear trend in Phe for both control groups, whereas the intervention group displayed increasing Phe pre-intervention and decreasing Phe (better adherence) post-intervention. For the overall sample, FSIQ and Phe were negatively correlated $(p<.001)$, and change in Phe from 6 months pre- to post-baseline visit was positively associated with working memory $(p=.03)$ and processing speed $(\mathrm{p}=.04)$.

Conclusions: Findings are promising and suggest that brief educational and behavioral interventions delivered in a busy, multidisciplinary clinic may positively impact outcomes in nonadherent PKU patients. Future research should consider standardizing and comparing interventions and examining predictors of response to intervention.

Correspondence: Aimee K. Hildenbrand, M.S., Psychology, Drexel University, 333 S. 12th St., Apt 4F, Philadelphia, PA 19107. E-mail: akh38@drexel.edu

G.O. REYNOLDS, S. NEARGARDER \& A. CRONIN-GOLOMB. Gender Differences in Verbal Learning in Parkinson's Disease.

Objective: In Parkinson's disease (PD), corticostriatal dysfunction leads to cognitive compromise. Cognitive deficits are highly prevalent, often occur in the early stages of the disease, and significantly reduce quality of life. Because about twice as many men as women are affected by PD, men with this disorder may experience particular difficulties on cognitive tasks that are known to elicit gender effects (female advantage) in healthy adults. We hypothesized that on a standard test of verbal learning, men with PD would be significantly impaired relative to women with PD and relative to healthy men and women.

Participants and Methods: We administered the Rey Auditory Verbal Learning Test (RAVLT) to 44 non-demented individuals with mild-moderate idiopathic PD (21M, 23F; mean age 63.1, range 5073 ) and 42 matched normal control adults (NC; 17M, 25F; mean age 64.0, range 45-82).

Results: There were significant main effects of gender and disease status on the RAVLT (Total) but no interaction. NC consistently outperformed PD, regardless of gender, and women consistently outperformed men, regardless of disease status. Men with PD had the lowest mean performance of any group.

Conclusions: The results indicate that men with PD may be at a greater risk than women with PD for cognitive impairments related to verbal learning, which is of clinical importance. Future research on cognitive dysfunction in PD should more closely examine gender differences in order to better characterize and manage cognitive symptoms in mild to moderate stages of the disease, potentially leading to the development of subgroup-tailored treatment approaches.

Correspondence: Gretchen O. Reynolds, Psychological and Brain Sciences, Boston University, 648 Beacon Street, 2nd Floor, Boston, MA 02215.E-mail:gor@bu.edu
K.F. HOTH, M. ALOIA, K. MESCHEDE, J.D. CRAPO, B.J. MAKE, R.P. BOWLER, K.L. MOREAU, E. KOZORA, K.E. HOLM, D.J. MOSER, H.D. WEINBERGER \& F.S. WAMBOLDT. Nocturnal Oxygen Desaturation and Cognitive Performance in COPD.

Objective: Cognitive impairment is common in COPD. To date the association between nocturnal oxygen desaturation and cognition in COPD has not been well examined. We explored the association between the oxygen desaturation index (ODI) and cognition among patients with COPD.

Participants and Methods: Sixty former smokers with COPD (mean age $=70.3$ ) completed spirometry, neuropsychological testing, and 2 nights of nocturnal oximetry using a wrist device with continuous recording. Neuropsychological tasks were grouped into domains of immediate memory, delayed memory, executive functioning-processing speed, visuospatial skills, and language. ODI was calculated as the average number of decreases in Sp02 of 4 or more during each hour of valid recording time averaged over 2 nights. Five regression models were calculated including these predictors: ODI, age, sex, pack-years, premorbid intellectual functioning (WRAT4-Reading), airflow obstruction (post bronchodilator FEV1\% predicted), and average nocturnal Sp02. Results: The regression models predicting immediate memory $(\mathrm{F}=2.54$, $\mathrm{p}=0.03)$ and delayed memory $(\mathrm{F}=2.94, \mathrm{p}=0.01)$ were significant. More frequent oxygen desaturation events (immediate memory $\beta=-23.91$, $p=0.01$, delayed memory $\beta=-25.00, p<0.01)$ and lower estimated premorbid intellectual functioning (immediate memory $\beta=0.46, p<0.01$, delayed memory $\beta=0.44, p<0.01$ ) were associated with worse memory. Conclusions: More episodes of nocturnal oxygen desaturation were associated with reduced memory performance after adjusting for age, smoking history and severity of airflow limitation in patients with COPD. Replication studies examining nocturnal desaturation using complete sleep studies will be essential to confirm the association between nocturnal desaturation and cognition and determine if reduced memory is, in part, driven by co-occurring COPD and obstructive sleep apnea. Correspondence: Karin F. Hoth, Ph.D., University of Iowa, University of Iowa- Psychiatry, 200 Hawkins Drive, W278GH, Iowa City, IA 52242. E-mail:karin-hoth@uiowa.edu

D. ZIEGLER, P. ASHOURIAN, J.S. WONDERLICK, A.K. SAROKHAN, D. PRELEC, C.R. SCHERZER \& S. CORKIN. DRD2 Polymorphisms Confer Increased Risk of Behavioral Impulsivity in Parkinson Disease.

Objective: Parkinson disease (PD) is an age-related degenerative disease of the brain, characterized by motor, cognitive, and psychiatric abnormalities. Several symptoms of the disease, including impulse control behaviors, may stem from the dopaminergic medications used to control the motor aspects of PD. Converging evidence from animals and humans suggests that individual differences in the genes that affect the dopamine system influence the response of PD patients to dopaminergic medication. We hypothesized that patients taking dopamine replacement therapy who carry candidate alleles that increase dopamine signaling exhibit greater amounts of behavioral impulsivity. Our protocol examined the relation between DRD2 C957T and COMT Val158Met polymorphisms and two measures of behavioral impulsivity (delay of gratification and reflection impulsivity) in PD patients.

Participants and Methods: We recruited 130 patients with idiopathic PD. Inclusion criteria included: H\&Y stages I-III; taking dopamine replacement therapy; MMSE $\geq 26$; $\geq 12$ years of schooling; no history of other brain disorder; no use of psychoactive drugs; and BDI $<19$. Patients performed the Delay of Gratification Task and the Information Sampling Task. An ANCOVÄ compared each variable among DRD2 and COMT genotype, with age, sex, levodopa equivalent daily dose, disease duration, and $H \& Y$ stage as covariates.

Results: Individuals with the T/T and C/T genotypes of DRD2 had reduced ability to delay gratification compared to $\mathrm{C} / \mathrm{C}$ carriers. Patients with the DRD2 T/T genotype showed more reflection impulsivity than $\mathrm{C} / \mathrm{C}$ carriers. We found no significant effects of the COMT genotype. 
Conclusions: Our results support the hypothesis that the T allele of DRD2, associated with higher dopamine signaling, confers an increased risk for behavioral impulsivity in PD patients taking dopamine replacement therapy. We propose that patients with the lowest amounts of baseline dopamine are less likely than other groups to exhibit impulsive behavior while taking dopamine replacement therapy.

Correspondence: David Ziegler, UCSF, 675 Nelson Rising Lane, MC 0444, San Francisco, CA 94158. E-mail: david@gazzaleylab.ucsf.edu

\section{INS Presidential Address: Networks, Neural Connectivity and Neuropsychology}

INS President: Erin D. Bigler

$$
\text { 5:00-6:00 p.m. }
$$

E.D. BIGLER. Networks, Neural Connectivity and Neuropsychology.

For most of the 20th Century, neuropsychology could only infer what the potential underlying pathology may be in any given disorder, until post-mortem studies were performed. For 21st Century neuropsychology, the field has a myriad of in vivo neuroimaging methods that not only identify a variety of pathological conditions but also permit the study of brain morphology and connectivity in healthy, typically developing individuals. In this digital era, how neuropsychology utilizes available neuroimaging techniques may define the future of the field. This lecture will review a variety of magnetic resonance imaging (MRI) methods including structural imaging combined with diffusion tensor imaging (DTI), resting state functional connectivity mapping (rs-fcMRI) and functional MRI and their utility in neuropsychological investigations. How to integrate these methods with neuropsychological assessment in the study of neural networks in the living individual with age-typical development, or some neurodevelopmental, neurological and/or neuropsychiatric disorder will be reviewed. A systems-level approach to neural connectivity and functional neural networks provides a much improved perspective for using neuroimaging findings in the neuropsychological examination of cognition and behavior. Examples from studies of traumatic brain injury and autism will be presented demonstrating the utility of this approach.

Correspondence: Erin D. Bigler, Ph.D., Psychology and Neuroscience, Brigham Young University, 1001 SWKT, Provo, UT 84602. E-mail: erin_bigler@byu.edu

\section{SATURDAY MORNING, FEBRUARY 7, 2015}

CE 11:

\section{How Neurons Enable Language and Cognition}

\author{
Presenter: Stephen E. Nadeau \\ 7:20-8:50 a.m.
}

S.E. NADEAU. How Neurons Enable Language and Cognition. One of our foremost challenges in understanding cognition is to relate it to the function of neural tissue: neurons, cortical micro-columns, synapses, and electrochemical physiology. Key to meeting this challenge is the recognition that neural representations corresponding to cognitive entities are population encoded: they reflect the pattern of activity of millions of neurons firing simultaneously. Parallel distributed processing (PDP) research (the science of population encoding) has been extensively validated empirically in studies of both normal and damaged brains and it has informed us of the constraints that the neurobiology of neural networks places upon cognitive function and its breakdown. In this seminar, language, the single cognitive function about which we know the most, is considered from a PDP perspective, supplemented by knowledge of neuroanatomy, neural systems, neurophysiology, and data from the fields of cognitive psychology, cognitive neuropsychology, psycholinguistics, and multilingual aphasia studies. The neural basis for phonology, noun and verb semantics, lexical semantics, grammatic morphology, phrase structure rules, and syntax are reviewed from this perspective.

Correspondence: Stephen E. Nadeau, MD, University of Florida College of Medicine, HSC Box 100236, Gainesville, FL 32610. E-mail: stephen. nadeau@neurology.ufl.edu

CE 12:

Neurobiology of Socioemotional Behavior in Health and Neurologic Disease

\author{
Presenter: Katherine P. Rankin \\ 7:20-8:50 a.m.
}

\section{K.P. RANKIN. Neurobiology of Socioemotional Behavior in Health and Neurologic Disease.}

Technological innovation during the past decade has enabled significant advances in social cognitive neuroscience. Our understanding of the neural circuits underpinning socioemotional behaviors such as empathy, self-awareness, warmth, and reading others' intentions is more precise than ever before. Faculty will provide an overview of how specific neural networks normally function to support social behavior, and will show how this new information can improve our ability to understand the kinds of neurologically based behavioral dysfunction that result from disease and injury. Disturbances of socioemotional behavior in clinical neuropsychology will be discussed using data from patients with diseases that particularly impair social functions, such as frontotemporal dementia, autism, ADHD, sociopathy, and Williams syndrome. Specific neuropsychological tests and questionnaires that effectively measure these socioemotional factors will be reviewed, along with their correlation with brain structure and function.

Correspondence: Katherine P. Rankin, PhD, Department of Neurology, University of Californi, San Francisco, 675 Nelson Rising Lane, Suite 190, San Francisco, CA 94158.E-mail: krankin@memory.ucsf.edu

\section{Invited Symposium: \\ Exploring the Function and Dysfunction of the Brain's Default Network}

\section{Chair: Jessica Andrews-Hanna}

9:00-10:30 a.m.

\section{J. ANDREWS-HANNA, J. ANDREWS-HANNA, C. GRADY, M. IRISH \& S. GABRIELI. Exploring the Function and Dysfunction of the Brain' Default Network.}

Symposium Description: The brain's default network (DN) is a largescale brain system whose anatomical organization and adaptive functions have remained elusive until recent years. Insight into the functional-anatomic properties of the default network is critical because network alterations are a key source of cognitive impairment and/or mental health dysfunction across numerous populations. To clarify gaps in our understanding of the DN, this symposium synthesizes research across the lifespan and in clinical populations, drawing on methods spanning neuropsychology, functional and structural neuroimaging, and pharmacological and behavioral interventions. Jessica Andrews-Hanna will 
provide a brief introduction to the symposium and discuss the role of the DN in self-generated cognition in healthy young adults. Next, Cheryl Grady will explore how DN activity and connectivity (both within the DN and with other brain systems) changes with age, providing further insight into the DN. Muireann Irish will reveal how DN atrophy in neurodegenerative diseases can lead to impairments in goal-directed and spontaneous forms of self-generated cognition. Finally, Susan Whitfield-Gabrieli will tackle the mental health implications of DN dysfunction by considering a variety of psychiatric populations and disorders of consciousness, highlighting recent research suggesting that self-generated cognition can be improved with pharmacological and behavioral interventions that modulate the DN.

Correspondence: Jessica Andrews-Hanna, PhD, University of Colorado Boulder, Center for Innovation \& Creativity Office 182C, UCB 954, Boulder, CO 80309.E-mail: jessica.andrews-hanna@colorado.edu

\section{GRADY. Age Differences in the Functional Connectivity of the Default Network.}

Recently there has been much interest in understanding the fluctuating activity within brain networks, i.e., groups of regions that are functionally correlated at rest and during cognitive tasks. There has been particular focus on the default mode network (DMN) which is involved in multiple cognitive processes and is vulnerable to aging. Older adults show a number of interesting differences in the DMN relative to younger adults: 1) weaker functional connectivity within the DMN at rest; 2) less modulation of DMN activity during cognitive tasks; 3) differences in how resting functional connectivity is related to activity during memory tasks; and 4) alterations in how the DMN interacts with other brain networks. In addition, many life experiences, such as bilingualism and exercise, can influence functional connectivity in the DMN in older adults. Because the connections within the DMN, and between it and other brain networks, are related closely to multiple domains of cognitive function, this has important implications for understanding cognitive aging.

Correspondence: Cheryl Grady, Rotman Research Institute at Baycrest, 3560 Bathurst Street, 1064, Toronto, ON M6A 2E1, Canada. E-mail: cgrady@research.baycrest.org.

M. IRISH. The Wandering Mind Standing Still - Exploring the Functional Properties of the Default Network in the Dementias. Neurodegenerative disorders offer a unique window into the cognitive architecture of the brain. Alzheimer's disease (AD) and frontotemporal dementia (FTD) are two dementia syndromes characterized by the deterioration of dissociable large-scale neural systems. Clinical features of these syndromes include the disruption of cognitive functions posited to rely upon the integrity of the default network. Our work has shown that memory-based construction/simulation processes, such as remembering the past and imagining the future, are impaired irrespective of dementia type. These changes, however, reflect the degeneration of divergent brain regions, with posteromedial and medial temporal structures implicated in $\mathrm{AD}$, versus the involvement of predominantly anterior regions in FTD. Introspection about the mental state of others, via theory of mind, is exclusively impaired in FTD, attributable to the breakdown of a largely anterior frontoinsular brain network. The characteristic regional atrophy patterns of $\mathrm{AD}$, however, do not seem to disrupt this capacity to infer the mental states of others. Finally, our recent work reveals significant alterations in spontaneous cognition in the dementias during periods of low cognitive demand. Taken together, our findings point to multiple brain regions, within and beyond the default network, that must be functional to support complex constructive/simulation and introspective functions.

Correspondence: Muireann Irish, NeuRA PO Box 1165, Randwick, NSW 2031, Australia.E-mail:m.irish@neura.edu.au
S. GABRIELI. The Default Mode Network and Psychopathology. The default mode network (DMN) is an identified neural system that is associated with mind wandering and is associated with one's self: one's reminiscence of the past, one's introspection of present thoughts and feelings, and one's plans for the future. Neuropsychiatric diseases strike at these most important aspects of human experience, and correspondingly such diseases alter, or are altered by, DMN function and organization. Neuroimaging allows for a systematic study of how alteration in DMN function relates to specific aspects of diseases, such as paranoia in schizophrenia or rumination in depression. I will discuss how the functional pathology of the DMN relates to psychopathology and executive function in patients with a variety of psychiatric disorders including schizophrenia, bipolar disorder, ADHD and DOC (disorders of consciousness). I will also discuss how pharmacological and behavioral interventions can modulate (or normalize) DMN pathology. The findings that DMN functions are plastic and can be changed by interventions offer hope that effective treatments may help patients mitigate their symptoms and potentially augment their cognitive function.

Correspondence: Susan Gabrieli, 43 Vassar Street,Building 46 - 4033 (4th Floor), Cambridge, MA 02139.E-mail:swg@mit.edu

\section{Paper Session 7: Veteran Populations}

\section{9:00-10:30 a.m.}

K. Pagulayan, e. Petrie, B.A. Parmenter, h. RaU, M. REITER, E. PESKIND \& N. KLEINHANS. The Impact of Repeated Blast-Related mTBI on Brain Activation During a fMRI Working Memory Task.

Objective: Reports of persistent working memory difficulties are common among Veterans with a history of repeated blast-related mTBI. However, the neural substrates of these symptoms are not clear. This study evaluated patterns of brain activation during a working memory task in Veterans with and without a history of repeated blast-related mTBI.

Participants and Methods: 13 Operation Iraqi Freedom (OIF)/ Operation Enduring Freedom (OEF)/ Operation New Dawn (OND) Veterans with a history of repeated blast-related mTBI and 10 deployed controls completed a n-back visual working memory task during fMRI. Contrasts of interest were 2-back > 0-back, 3-back > 0-back, and a linear trend analysis. Data pre-processing, first-level analyses, and tests for between-group differences were conducted with FSL.

Results: No between group differences were observed in the 2-back condition. In the 3-back condition, the mTBI group exhibited decreased activation in the cerebellar vermis and areas V and VI, the pons, midbrain, and the lateral occipital cortex. Increased activation was present in the medial prefrontal cortex $(\mathrm{p}<.05$, whole brain corrected). Linear trend analysis yielded a similar pattern in the cerebellum (decreased activity in the mTBI group) and medial prefrontal cortex (increased in mTBI group). Further investigation of the medial prefrontal cortex finding revealed that the control group had a significant negative relationship between activation in that area and increased task difficulty; this pattern was considerably weaker in the mTBI group.

Conclusions: Repeated blast-related mTBI is associated with reduced activation in the cerebellum, midbrain and pons and atypically increased activation in the medial prefrontal cortex. Abnormal medial prefrontal cortex activation may indicate insufficient suppression of the default network when engaging in a complex working memory task. These results may be associated with the persisting cognitive difficulties frequently reported in this population.

Correspondence: Kathleen Pagulayan, PhD, VA Puget Sound, $1660 \mathrm{~S}$ Columbian Way, S-116-MIRECC, Seattle,WA 98115.E-mail:farkat@u. washington.edu 
S. SORG, N. LUC, A.L. CLARK, R.T. KIM, M.W. BONDI, D.M. SCHIEHSER \& L. DELANO-WOOD. Frontothalamic Structural Connectivity in Veterans with Mild Traumatic Brain Injury: Associations with Executive Functions.

Objective: White matter pathology is common in traumatic brain injury and is evident even in mild cases (mTBI). This damage may cause a disconnection syndrome via disruption of functional networks that mediate cognitive processes. The thalamus is a key relay station for many neural networks, and damage within frontothalamic white matter pathways may contribute to executive dysfunction following mTBI. In this study we computed measures of frontothalamic structural connectivity (FTSC) and examined its association with mTBI and measures of executive functions (EF) in a sample of US military Veterans.

Participants and Methods: Thirty-nine Veterans (mTBI=21, Military Controls $[\mathrm{MCs}]=18]$ ) completed cognitive testing, the Frontal Systems of Behavior Scale (FrSBe), and structural and DTI MRI scanning. Seed and target regions were identified via FreeSurfer and frontothalamic pathways were produced via probabilistic tractography. Measures of FTSC (i.e., proportion of streamlines from the thalamus reaching the frontal lobes) were extracted from the resultant tracks.

Results: Compared to MCs, the mTBI group had higher FrSBe scores across all subscales $(\mathrm{p}<.01)$ and performed worse on an EF composite score $(p<.01)$. MC and mTBI groups did not differ in measures of FTSC ( $\mathrm{p}$ 's $>.05$ ), however, FTSC was associated with loss of consciousness in the mTBI group $(p<.05)$. Within the mTBI group, right FTSC correlated with the $\mathrm{EF}$ composite $(\mathrm{r}=.57, \mathrm{p}=.02)$, while left FTSC was correlated with higher scores on FrSBe disinhibition $(\mathrm{r}=.57, \mathrm{p}=.01)$ and executive dysfunction $(\mathrm{r}=.57, \mathrm{p}=.01)$ scales. Such associations were not found in MCs.

Conclusions: Results show that altered structural connectivity of frontothalamic white matter pathways may disrupt processes vital to executive functions in Veterans with mTBI. While group differences in FTSC were not found, FTSC was associated with objective and subjective measures of executive functions and may be related to mTBI severity. Correspondence: Scott Sorg, VA Puget Sound Health Care System, 1660 S Columbian Way, Seattle,WA 98108.E-mail: scottsorg03@gmail.com

A.J. JAK, A. GREGORY, H.J. ORFF, C. COLON, N. STEELE, D.M. SCHIEHSER, L. DELANO-WOOD, S.M. JURICK \& E.W. TWAMLEY. Neuropsychological performance in treatment seeking OEF/OIF/OND Veterans with a history of mild TBI.

Objective: This study sought to better characterize widely variable clinical neuropsychological presentations of a large sample of OEF/OIF/ OND Veterans seeking treatment for cognitive concerns in the post-acute phase following mTBI.

Participants and Methods: Retrospective chart reviews of neuropsychological testing and mental health symptoms were conducted on 411 clinically referred OEF/OIF/OND Veterans with a history of mTBI.

Results: $29.9 \%$ of the sample performed below normative expectations on at least one performance validity test (PVT) and $47 \%$ performed below normative expectations ( $>1$ SD below norms) on no more than one measure (15\% failed PVTs but were still within normal limits on other cognitive measures), Therefore, only 38.1 percent showed valid deficits on two or more measures. In Veterans passing PVTs, $60 \%$ performed below normative expectations on no more than one measure (33.7\% performed within normal limits on all measures). Veterans in the Fail PVT group were significantly more likely to carry diagnoses of PTSD and Depression than those in the Pass PVT group. Mood and neurobehavioral symptoms were significantly elevated (all p's<.001) in Veterans in the Fail PVT group. In those with objective deficits, memory was the most likely domain to be impaired.

Conclusions: This is one of the largest examinations of the clinical cognitive presentation of OEF/OIF/OND Veterans with a history of non-acute mild TBI that provides detailed comprehensive neuropsychological information in the context of neuropsychiatric symptomatology. The data suggest that some OEF/OIF/OND Veterans with persistent cognitive complaints following mild TBI have objective impairments, but over half do not and a third demonstrated invalid neuropsychological assessments. Our results demonstrate high rates of psychiatric comorbidities in this population. Results also highlight the importance of objective neuropsychological testing and feedback regarding objective cognitive data to avoid misattribution of symptoms.

Correspondence: Amy J. Jak, Ph.D., VA Healthcare System, San Diego/ UCSD, 3350 La Jolla Village Dr., 151B, San Diego, CA 92161. E-mail: ajak@ucsd.edu

L.J. GRANDE, C.B. FORTIER, M.E. ROBINSON, D.H. SALAT, W.P. MILBERG \& R. MCGLINCHEY. Impaired Verbal Memory Associated with Close Blast Exposure in OEF/OIF Veterans.

Objective: TBI is described as the signature wound of the $\mathrm{OEF} / \mathrm{OIF}$ conflict, with exposure to blast munitions as a common cause. Many returning veterans report cognitive complaints following blast TBI and blast-exposure without TBI. Currently, there is no comprehensive understanding of the impact of blast exposure on brain function. Our laboratory has reported altered resting state brain activity related to close blast exposure $(\mathrm{CBE}<10 \mathrm{~m})$ for those with and without blast TBI (Robinson et al, under review, Hum Brain Map). The present study investigated CBE and cognitive functioning.

Participants and Methods: 124 Veterans enrolled in the TRACTS TBI Center of Excellence completed a battery of cognitive measures of executive function (i.e., DKEFs Trailmaking) and memory (i.e., CVLT and BVMT). Veterans were excluded for neurological diagnosis other than TBI and suboptimal effort. Veterans were classified into two groups based on proximity to one or more blasts: CBE $(<10 \mathrm{~m})$ and non-close (>10m or none).

Results: Multiple regression analyses revealed CBE significantly predicted performance on CVLT memory measures (Short Delay Free Recall: $\mathrm{B}=-1.326, \mathrm{t}(116)=-2.043, \mathrm{p}<.05$ and Long Delay Free Recall: $\mathrm{B}=$ $-1.431, \mathrm{t}(116)=-2.155, \mathrm{p}<.05)$ while controlling for PTSD, sleep, pain and age. Similar associations were demonstrated for short and delayed cued recall. When comparing CBE subgroups, worse delayed recall was noted for those with a blast TBI compared those without TBI, Further, comparison of CBE without TBI to a nonblast control group revealed an association between blast exposure and short and delayed memory Conclusions: Findings indicate decreased memory performance associated with close exposure to blast, with deficits iobserved even when blast does not result in a TBI. These findings have implication for understanding the impact of blast exposure on brain function and Veterans health. Translational Research Ctr for Traumatic Brain Injury \& Stress Disorders, RR\&D TBI Center of Excellence, VA Boston Healthcare System. Correspondence: Laura J. Grande, Ph.D., Psychology, VA Boston Healthcare System, Psychology Svc (116B), 150 South Huntington Ave, Boston, MA02130.E-mail: lgrande@heartbrain.com

J.R. SADEK, J.E. KELLER, D.T. CASTILLO \& K.Y. HAALAND. Changes in Executive Functioning with Successful PTSD Treatment.

Objective: To determine whether executive abilities would improve after psychotherapy for post-traumatic stress disorder (PTSD). Hypotheses included that PTSD treatment would improve inhibition/switching abilities relative to other neurocognitive abilities; lower baseline neurocognitive functioning would be associated with poorer response to treatment; and better treatment response would be associated with greater improvement in inhibition/switching.

Participants and Methods: 42 female veterans diagnosed with PTSD and 48 non-PTSD controls matched for age, education, and ethnicity were participating in a randomized controlled PTSD treatment trial and who were screened for confounding psychiatric or neurological disorders. Neuropsychological testing was completed before and after the 16 week treatment interval. Treatment sessions included 5 of exposure therapy, 5 of cognitive processing therapy, 4 of skill training, and 1 each introduction and conclusion. Standardized neuropsychological 
tests were used to comprise the ability domains of simple attention, working memory, verbal learning, retention, and inhibition/switching. Results: Mixed model regression showed no specific improvement in inhibition/switching in the PTSD treatment group. Baseline neurocognitive abilities did not predict treatment outcome. Clinical improvement was defined as Clinician Administered PTSD Scale (CAPS) improvement $>=10$ points (treatment responders) or post-treatment CAPS of $<45$ (loss of PTSD diagnosis). PTSD symptom improvement was associated with improvement in only inhibition/switching: Responder partial $\eta^{2}=.24$ (medium/large), and loss of diagnosis partial $\eta^{2}=.13$ (medium). There was no difference on baseline inhibition/switching.

Conclusions: Female veterans with PTSD who show a positive treatment response also show improvement in inhibition/switching only, consistent with the theory that subtle executive deficits maintain PTSD symptoms (Aupperle et al, 2012). PTSD treatment success may be mechanistically related to executive functioning.

Correspondence: Joseph R. Sadek, Ph.D., Behavioral Health, New Mexico VA Health Care System, 1501 San Pedro SE, BHCL 116, Albuquerque, NM 87108.E-mail: jsadek@unm.edu

N.M. WISDOM, J.E. BOOTH, R. TANG, B. MILLER, R. COLLINS \& N.J. PASTOREK. An Examination of Cognitive Deficits after Controlling for Respondent Validity in Veterans with Chronic PTSD.

Objective: A large volume of studies have reported an association between PTSD and cognitive deficits. A recent study (Wisdom et al., 2014), however, failed to find any deficits associated with reported PTSD symptomatology after excluding participants failing a performance validity test (PVT). The authors suggested the lack of findings might reflect the relatively young participants $(\mathrm{OEF} / \mathrm{OIF} / \mathrm{OND}$ veterans) and recommended the study be replicated in veterans with longstanding signs and symptoms of PTSD as chronic activation might result in cognitive deficits. The purpose of this study was to identify possible cognitive impairments in veterans with chronic PTSD after excluding those failing a well-validated PVT.

Participants and Methods: Veterans included 295 men and 18 women referred for a routine clinical neuropsychological evaluation by mental health $(59.7 \%)$, primary care $(32.3 \%)$, and other specialized clinics $(8 \%)$. Medical records were used to identify veterans with chronic PTSD and the Word Memory Test (WMT) was used to establish invalid performance. The final groups included 147 clinical controls (no PTSD, pass WMT), 68 honest responders (+PTSD, pass WMT), and 98 failing a PVT (+PTSD, fail WMT). One-way ANOVA and post-hoc analyses were used to identify any deficits between groups across a large array of cognitive measures.

Results: As hypothesized, those failing the WMT performed significantly worse on nearly every cognitive test administered; however, unlike Wisdom et al. (2014), the honest responders also performed significantly worse than clinical controls on a measure of long delay free recall (CVLT-II; $p=.047)$ and retention percentage $(p=.026)$.

Conclusions: In aggregate form, the veterans with chronic PTSD demonstrated impairment in free recall and retention of a word list albeit with very small effect sizes and qualitatively still in the average range. These findings continue to highlight the importance of assessing respondent validity in future research examining cognitive functioning in psychiatric illness.

Correspondence: Nick M. Wisdom, Ph.D., Michael E. DeBakey Veterans Affairs Medical Center, 2002 Holcombe Blvd, Houston, TX 77030. E-mail:nickandjen@gmail.com

\section{Symposium 13: \\ Using the NIH Toolbox for Neuropsychological and Behavioral Functioning in Individuals who have Disabilities}

\author{
Chair: David Tulsky
}

9:00-10:30 a.m.

D.S. TULSKY, R. GERSHON, D. TULSKY, N.E. CARLOZZI, K.B. CASALETTO, R.K. HEATON \& A.W. HEINEMANN. Using the NIH Toolbox for Neuropsychological and Behavioral Functioning in Individuals who have Disabilities.

Symposium Description: The NIH Toolbox was designed to be a leading assessment instrument and common data element in NIH funded research. The Toolbox consists of 4 broad domains (Cognitive, Emotional, Motor, and Sensory) of functioning, each of which takes approximately a half hour to complete. The Toolbox was developed and normed on a large standardization sample of adults and children (ages 3 - 85) with both English and Spanish speaking individuals who did not have disabilities. A recent study was conducted to test the Toolbox in a large sample of individuals $(\mathbf{N}=604)$ who have Traumatic Brain Injury, Stroke, or Spinal Cord Injuries. This marks the initial time that the NIH Toolbox has been tested extensively in clinical populations and the data have great relevance for utilizing the NIH Toolbox in clinical practice. This symposium will focus on the clinical utility of the NIH Toolbox and review important considerations when testing the Toolbox in such populations. The symposium will start with an initial overview and introduction to the NIH Toolbox. Following will be a presentation discussing the sampling and testing methodology of the study and present evidence of construct validity for using the Toolbox in clinical groups. Results are demonstrating that there is clinical utility across all 4 domains of the toolbox and a presentation will focus on the utility of the motor, sensory and emotional domains that could expand the neuropsychologists' clinical assessment battery. The Toolbox contains several permutations of scores including demographically corrected, age corrected, and uncorrected scores. One presentation will focus on the clinical utility of these different scores. Finally, for this study, the Toolbox was administered in the context of a much larger assessment session which included several measures of participation, social support, physical functioning, and environmental concerns. The final talk will discuss the Toolbox scores in relation to these other constructs.

Correspondence: David S. Tulsky, Ph.D., Physical Medicine and Rehabilitation, University of Michigan, North Campus Research Complex, 2800 Plymouth Road, Ann Arbor, MI 48109. E-mail: dtulsky@ gmail.com

R. GERSHON. Overview and update on what is new with Toolbox. Since the release of The NIH Toolbox for the Assessment of Neurological \& Behavioral Function by the NIH Neuroscience Blueprint in 2012, more than 350 studies have initiated the use of these instruments. Some investigators have opted to give all 4 batteries that cover the full range of function for 3-85 year olds, across the domains of Cognition, Emotion, Motor and Sensory function. Others choose to use a specialty instrument from a single domain. Advanced computer algorithms enable many cognition domains to be assessed in just a few minutes, with real-time scoring and norming lookups. Through the use of Computer Adaptive testing algorithms developed using data provided by tens of thousands of respondents, some of the Emotional Health measurement are now able to reach clinical significance in as little as 1 minute of assessment time for most respondents. And while less than 24 months have elapsed since the public release of the NIH Toolbox, more than 50 peer-reviewed articles have already been published describing it's validation, norming and research use in journals like Lancet Neurology, Neurology, Journal of the International Neuropsychological Society and Monographs of the Society for Research Child Development, as well as journals more 
familiar to motor, sensory and emotional health experts. The NIH Toolbox has now been validated for use with general population samples, as well as for research use in a growing list of disease populations including Parkinson's, Stroke and TBI. And the National Children's Study has selected numerous NIH Toolbox instruments in their Vanguard Study of 5,500 children and their parents in order to maximize reliability while at the same time minimizing participant burden. We should note that the NIH has recently committed to provide support for the NIH Toolbox for 4 more years. We will also announce and demonstrate the new iPad version of the NIH Toolbox that will be available in 2015 for qualified researchers and clinicians and

Correspondence: Richard Gershon, 625 N. Michigan Ave, Chicago, IL 60611.E-mail: gershon@northwestern.edu

D.S. TULSKY, N.E. CARLOZZI, C. BAUM, A. GOLDSMITH \& A.W. HEINEMANN. Using the NIH Toolbox Cognitive Battery (NIHTB-CB) in individuals who have had Traumatic Brain Injury (TBI).

The NINDS has been funding the development and selection of measures that would be common data elements across their funded grants to allow cross-study comparisons over time. The NIH Toolbox (NIHTB) for the Assessment of Neurological and Behavioral Function is one such measure and was developed and normed in individuals from the general population. Recently, the Toolbox was systematically administered to a large sample of individuals who have cognitive or physical disabilities. This paper examines the validity of the NIH Toolbox in individuals who have had a TBI and provides initial insight in how to use the measure in a clinical population. Our sample included adults with TBI $(n=184)$ who completed the NIHTB Cognitive Battery as well as a neuropsychological tests. TBI severity was classified using the lowest Glascow Coma Score within the first 24 hours of injury. Individuals with severe injury ( $\mathrm{n}=99$ ) had scores of $<$ or $=8$ and individuals with Moderate or Complicated Mild ( $\mathrm{N}=83)$ had scores of $9-12$ or $13-15$ with CT scan confirmation. Additionally, a sample of healthy individuals that was demographically-matched provided a comparison group. In general, moderate to high correlations were found between the Toolbox tasks and neuropsychological tests that measure the same construct (approximately in the 0.5 and 0.6 ranges). When compared to matched controls, individuals with severe TBI perform significantly lower on Fluid Reasoning and the majority of tests that comprise the Fluid scores. Differences between crystallized tasks (Reading and Picture Vocabulary) are not significantly different between the groups. For the Moderate/complicated Mild group, when compared with Control participants, scores are significantly lower on Fluid tasks, but at a less significant P-value, indicating more mitigated differences. The results provide the initial evidence of support for the construct validity of the NIH Toolbox in Individuals with TBI. Correspondence: David S. Tulsky, Ph.D., North Campus Research Complex, 2800 Plymouth Road, Ann Arbor, MI 48109. E-mail: dtulsky@ gmail.com

N.E. CARLOZZI, D.S. TULSKY, S. GOODNIGHT \& A.W. HEINEMANN. An Examination of the NIH Toolbox Motor, Sensory and Emotion Batteries in Individuals with Disabilities. Objective: The NIH Toolbox for the Assessment of Neurological Behavior and Function (NIHTB) includes a brief assessment of cognition, emotion, sensation and motor functioning. Since individuals with stroke, traumatic brain injury, and spinal cord injury not only experience deficits on traditional cognitive tests but also show significant deficits in sensory functioning, motor impairment, and lowered satisfaction with life, we examined their performance on the motor, sensory and emotion batteries of the NIHTB.

Participants and Methods: Participants with traumatic brain injury $(\mathrm{n}=184)$, stroke $(\mathrm{n}=211)$, and spinal cord injury $(\mathrm{n}=209)$ completed the NIHTB. Multivariate and univariate analyses were conducted to profile NIHTB performance for each of the three rehabilitation groups on the motor, sensory and emotion batteries.
Results: Results indicated significant group differences on almost all of the NIH Toolbox sensory (multivariate analyses) and motor tests (univariate analyses); all groups scored a standard deviation (or more) below the mean. For the NIHTB emotion battery (multivariate analyses), the only group difference was for General Life Satisfaction, where individuals with SCI indicated less satisfaction than individuals with TBI; regardless, all groups were within +/- 1SD of the mean for all of the measures of emotion. Normative data for each rehabilitation group is also available in order to aid clinical interpretation of findings.

Conclusions: Although neuropsychologists will gravitate to the NIHTB Cognition Battery (NIHTB-CB), the NIH Toolbox Sensory, Motor, and Emotional Domains offer a unique opportunity to assess other domains of functioning that are not traditionally assessed by the Neuropsychologist and provide clinically meaningful information.

Correspondence: Noelle E. Carlozzi, 2800 Plymouth Road, Ann Arbor, MI 48109.E-mail: carlozzi@med.umich.edu

K.B. CASALETTO, D.S. TULSKY \& R.K. HEATON. Uncorrected versus Demographically-Adjusted Scores on the NIH Toolbox Cognition Battery: What is the Difference?

Objective: Impacts of demographics on neuropsychological test performances are well established, but clinicians and researchers may have difficulty choosing and interpreting uncorrected versus demographically-corrected (DC) scores. Our study compared these two types of scores for clinical and nonclinical groups on the NIH Toolbox Cognition Battery (NIHTB-CB), and examined associations with demographic and clinical variables.

Methods: Our samples were adults with traumatic brain injury (TBI; $\mathrm{n}=181$ ) or stroke (CVA; $\mathrm{n}=209)$, and demographically-matched groups of healthy adults (TBI-matched n=184; CVA-matched n=210). NIHTB-CB Fluid and Crystallized Composites were analyzed, and DC scores controlled for age, education, gender, and race/ethnicity.

Results: Compared to matched controls, both the TBI and CVA cohorts demonstrated larger differences on corrected than uncorrected Fluid Composites (uncorrected to corrected effect sizes: $\mathrm{TBI} d=0.60$ to 0.77 ; CVA d $=0.88$ to 1.01). However, on Crystallized scores, differences between clinical groups and their matched controls became slightly smaller with DCs (uncorrected to corrected effect sizes: TBI $\mathrm{d}=0.23$ to 0.21 ; CVA $d=0.40$ to 0.17 ). In the clinical sample, demographic factors accounted for $29-36 \%$ of the variance in uncorrected scores ( $p s<0.001)$, but only $0-4 \%$ of the variance in corrected scores (ps>0.04). Clinical ratings of injury increasing severity were associated with greater impairment on the corrected Fluid scores within the TBI and CVA cohorts ( $p s<0.01)$; uncorrected Fluid scores were only significantly associated with injury severity within the CVA group.

Conclusions: DC scores resulted in higher rates of impairment in the clinical groups, and larger differences versus matched controls. Importantly, the DC scores were no longer associated with demographic factors and demonstrated good construct validity with a proxy for brain injury severity.

Correspondence: Kaitlin B. Casaletto, MS, 220 Dickinson St., Suite B, San Diego, CA 92103.E-mail: kaitlin.casaletto@gmail.com

A.W. HEINEMANN, A. WONG, D.S. TULSKY \& R. GERSHON. Relationships between NIH Toolbox Cognition and Emotional Measures and Indicators of Participation for CommunityDwelling Persons with Stroke, Spinal Cord Injury and Traumatic Brain Injury.

Aims: Impaired cognition may limit independent performance of activities and restrict participation in community activities. Neuropsychologists focus on assessments of cognition but often do not consider the consequences of impaired cognition on societal participation. Little is known about the relative influence of cognitive and emotional functioning on participation in community activities. This study examined the relationship between cognitive and emotional functioning as measured 
by the NIH Toolbox, several widely used neuropsychological measures, and patient-reported measures of participation.

Methods: We recruited a sample of 604 community-dwelling adults with traumatic brain injury, stroke, and spinal cord injury. We administered the NIH Toolbox, neuropsychological tests, the PROMIS - Social Health item bank using computer adaptive testing, and the Participation Enfranchisement instrument. We used structural equation modeling to evaluate the relative importance of cognitive and emotional functioning on participation.

Results: Toolbox and neuropsychological measures of fluid intelligence that required a psychomotor component $(p=0.02)$ and crystalized intelligence $(p=0.01)$ were related to social health, whereas measures of fluid intelligence that did not require a motor component were related to participation enfranchisement $(p=0.01)$ and social health $(p=0.01)$ $(\mathrm{CFI}=0.92 ; \mathrm{TLI}=0.88 ;$ RMSEA $=0.082)$. Toolbox measures of emotions were related to participation enfranchisement and social health (all ps $<0.001)(\mathrm{CFI}=0.94 ; \mathrm{TLI}=0.89$; RMSEA $=0.094)$.

Conclusions: Cognitive and emotional functioning was associated with participation in community activities. The results provide insights on how ameliorating cognitive deficits may allow improved participation outcomes for these populations. The NIH Toolbox, PROMIS, and participation measures can help the neuropsychologist measure a full range of functioning so that the person can be treated for multiple decrements. Correspondence: Allen W. Heinemann, Rehabilitation Institute of Chicago, 345 E Superior Street, Chicago, IL 60611. E-mail: a-heinemann@northwestern.edu

\section{Paper Session 8: Aging}

\section{9:00-10:30 a.m.}

M. JOANNETTE, H. CHERTKOW, C. BOCTI, B. MASSE, J. NIKELSKI, R. PILON, S. SOLOMON, P. SÉVIGNY-DUPONT, R. LANGLOIS, C. RAINVILLE, C. MCHENRY \& S. JOUBERT. Neuropsychological Performance of Normal Elderly with Significant $\beta$-amyloid Deposition: a PiB-PET Imaging Study.

Objective: It is unclear to what extent "normal cognitive aging" is dependant on the presence of quantifiable brain pathology. We wished to assess the effect of $\beta$-amyloid $(\mathrm{A} \beta$ ) deposition, a key signature of $\mathrm{Alz}$ heimer's disease (AD), on cognitive function in normal aging. Positron emission tomography (PET) with the amyloid tracer [11C] Pittsburgh compound $\mathrm{B}(\mathrm{PiB})$, provides a quantifiable measure of $\mathrm{A} \beta$, allowing us to examine in vivo the relationship between $A \beta$ burden and cognition in normal aging. Cognitively healthy individuals with high $A \beta$ may be on a path to $\mathrm{AD}$, or may remain normal for many years.

Participants and Methods: In order to explore the association between $A \beta$ deposition and different domains of cognition in normal aging, a group of 47 healthy older adults (mean age 73 years) completed a detailed battery of neuropsychological tests and had an anatomical MRI acquisition (3T). To measure A $\beta$ burden, participants underwent PiBPET imaging.

Results: Using a 1.23 SUVR cutoff, $36 \%$ of the subjects showed high $\mathrm{PiB}$ retention $(\mathrm{PiB}+)$. T-tests and non-parametric tests were carried out for neuropsychological performance. Overall, no differences in executive functions (Wisonsin Card Sorting Test and Stroop subtest of the D-KEFS) and in denomination (Test of Famous Persons) were found. $\mathrm{PiB}$ - participants had better performance than $\mathrm{PiB}+$ participants on the copy of the Rey-Osterrieth Complex Figure Test $(p<0.05)$ and for immediate and delayed recall $(\mathrm{p}<0.01)$. PiB- also had higher performance in the Rey Auditory Verbal Learning Test $(p<0.05)$, semantic fluency $(p<0.05)$, digit span $(p<0.01)$ and the Hit SE by ISI measure of the Conners' Continuous Performance Test $(p<0.05)$.

Conclusions: Preliminary results indicate that $\mathrm{PiB}+$ participants have poorer cognitive performance than $\mathrm{PiB}$ - participants on verbal and visual episodic memory, semantic memory, working memory, attention and visuoconstructional abilities. Brain amyloid burden measured with PiB-PET imaging correlates with poorer cognitive performance among clinically normal individuals.

Correspondence: Maude Joannette, PhD, Psychologie, Université de Montréal, 4545 chemin Queen-Mary, Montreal, QC H3W 1W4, Canada. E-mail: maude.joannette@umontreal.ca

K.J. MANNING, M.T. SCHULTHEIS, J. GALLO, S. MOELTER, D. CHUTE \& A.J. SAYKIN. Navigation Driving and Vehicle Control in Healthy Older Adults and Subjective Cognitive Impairment.

Objective: Navigation requires the selection, implementation, and monitoring of a travel route while simultaneously maintaining vehicle control. Navigation may result in driving errors not encountered in standard driving assessments where the examiner directs the examinee where to drive (i.e., guided-direction). We hypothesized that the challenging nature of navigation may be especially useful in the driving assessment of older adults with subtle cognitive difficulties. We therefore contrasted vehicle control in older drivers with subjective cognitive impairment (SCI) and healthy older adults (HC) under two conditions: guided-direction and self-guided navigation.

Participants and Methods: Participants completed a neuropsychological evaluation and underwent virtual reality driving simulation (VRDS). The sample included 8 drivers with SCI, who performed in the average range on formal neuropsychological examination and did not meet criteria for mild cognitive impairment (MCI), and $10 \mathrm{HC}$. Vehicle control was defined as the approach speed ( $\mathrm{mph}$ ) in the 100 feet prior to a stop sign measured under both guided-direction and navigation conditions. Results: Linear modeling revealed effects for group $(F(1,20)=6.35$, $\mathrm{p}<.05)$ and group by distance traveled $(\mathrm{F}(1,15)=15.66 \mathrm{p}<.05)$. Compared to HC, SCI participants engaged in behavior associated with a higher probability of accidents; they entered the 100 feet prior to the stop sign at higher speeds and engaged in more sudden braking. Effect of driving condition was not statistically significant $(p=.06)$.

Conclusions: Subtle cognitive changes in older adults are associated with worse performance on driving simulator maneuvers associated with successful vehicle control. Driving simulation may enhance driving evaluations by measuring alternative maneuvers to those assessed on-road. Correspondence: Kevin J. Manning, Ph.D., Psychiatry, University of Connecticut Health Center, 263 Farmington Avenue, Farmington, CT 06030.E-mail:manning@uchc.edu

D.M. RENTZ, K.V. PAPP, E.C. MORMINO, R.E. AMARIGLIO, K.A. JOHNSON \& R.A. SPERLING. Cognition and biomarker abnormalities in clinically normal older adults.

Objective: To determine if specific cognitive changes are associated with biomarker abnormalities in clinically normal (CN) older adults.

Participants and Methods: 158 CN subjects (CDR=0; MMSE $>25$; LMIIa > 7) enrolled in the Harvard Aging Brain Study underwent neuropsychological testing, APOE genotyping, positron emission tomography (PiB-PET and FDG-PET) and magnetic resonance imaging. Amyloid status was determined with PiB-PET. Neurodegeneration was measured using two a priori imaging markers: MRI hippocampal volume and FDGPET metabolism extracted from AD-vulnerable regions. Subjects were classified into 4 groups: 1$)$ biomarker negative $(n=76$, age $=71.0 \pm 4.8$; $14.5 \%$ APOE 4$) ; 2$ ) amyloidosis alone ( $\mathrm{n}=20$, age $=73.8 \pm 5.3 ; 50.0 \%$ APOE 4); 3) amyloidosis plus neurodegeneration ( $\mathrm{n}=26$, age $=76.5 \pm 6.3$; $42.9 \%$ APOE 4$)$ and 4$)$ neurodegeneration alone $(n=36$, age $=76.9 \pm 6.2$; $12.9 \%$ APOE 4 ). A Multivariate General Linear Model controlling for age and APOE 4 status explored cognitive performances between groups. Results: Compared to biomarker negative subjects, performance on a memory composite was significantly worse in both amyloidosis alone $(p$ $=0.032)$ and those with amyloidosis plus neurodegeneration $(\mathrm{p}=0.007)$. Post hoc analyses revealed that measures of immediate recall were associated with amyloidosis alone, while measures of immediate and cued recall were associated with amyloidosis plus neurodegeneration. 
Compared to biomarker negative subjects, performance on an executive composite was significantly worse in those with neurodegeneration alone $(p=0.047)$ but not in the other amyloid positive groups.

Conclusions: These findings suggest that subtle cognitive changes are associated with different biomarker abnormalities in CN adults. Understanding these cognitive phenotypes may be a more effective method for dissociating normal aging from those individuals at-risk for neurodegeneration prior to expensive imaging or biomarker procedures. Correspondence: Dorene M. Rentz, PsyD, Neurology, Harvard Medical School, 221 Longwood Avenue, Boston, MA 02115.E-mail:DRENTZ@ PARTNERS.ORG

C.E. HARRISON \& S.A. ROGERS. How Do Exercise and Leisure Differentially Relate to Frontal Functions of Older and Younger Adults?

Objective: The present study examined the way participation in personal wellness activities relates to the frontal-executive functions of both older adults and college students. Hopefully this will proffer a better understanding of age-associated effects of personal wellness on frontal functions.

Participants and Methods: Eighty-nine college-aged participants and 59 older adults $(\mathrm{M}$ age $=76.28)$ completed tests of frontal-executive functioning, including personality and mood. Participants indicated how many exercise and leisure activities they engaged in weekly.

Results: College-aged participants engaged in exercise 5.09 hours and leisure activities 9.75 times, per week. Older adult participants engaged in exercise 4.52 and averaged 5.36 leisure activities, per week. The number of times college students engaged in exercise was positively associated with extroversion and negatively correlated with neuroticism, ps <. 04. Their number of leisure hours was positively associated with anxiety and extroversion, ps <. 05 . For older adults, exercise was positively correlated with conscientiousness, and leisure activities were negatively correlated with depression, ps <. 04. The number of times college-aged participants engaged in leisure activities was positively associated with Trails A \& B and DKEFS Word Reading, ps <. 05. The number of times older adults engaged in exercise per week was positively correlated with DKEFS Inhibition and negatively correlated with errors on DKEFS Inhibition, ps <. 05. Amount of older adults' leisure activity was positively associated with WAIS-III Similarities, $\mathrm{p}<.004$.

Conclusions: Results show significant relationship between personal wellness and various aspects of frontal-executive functioning, including cognitive abilities, personality, and mood. These relationships decisively vary with age. Clinicians may find it helpful to integrate these findings into customized wellness programs, appreciating how age may influence impact of exercise and leisure on abilities associated with frontal functioning.

Correspondence: Cory E. Harrison, PhD, Neuropsychology, Pacific Neuroscience Medical Group, 515 W Foothill Blvd, Unit A, Monrovia, CA 91016.E-mail: cburns12@apu.edu

\section{Poster Session 8: \\ ABI-Child, Autism, Cognitive Neuroscience, \& Electrophysiology/EEG}

9:00-10:30 a.m.

\author{
Acquired Brain Injury (TBI/ \\ Cerebrovascular Injury \& Disease - Adult)
}

M. ALOSCO, M. SPITZNAGEL, R.A. COHEN, L.H. SWEET, S.M. HAYES, R. JOSEPHSON, J. HUGHES \& J. GUNSTAD. Reductions in Physical Activity Predict Cognitive Decline in Older Adults with Heart Failure.

Objective: High levels of physical inactivity likely contribute to cognitive decline and increased risk of dementia in patients with heart failure (HF). Yet, the longitudinal relationship between physical activity (PA) and cognition in HF is poorly understood due to limitations of past work. including single time assessments of PA. This is the first study to examine changes in objectively measured PA and cognition over time in HF. Participants and Methods: At baseline and 12-weeks later, $57 \mathrm{HF}$ patients (mean age $=69.67$ years, $\mathrm{SD}=10.34)$ completed psychosocial self-report measures, were administered a neuropsychological battery, and wore an accelerometer for seven days. Regression analyses controlling for medical (i.e., ejection fraction, diabetes), psychological (i.e., depressive symptoms), and baseline PA and cognitive function examined the association between 12-week PA and cognition.

Results: At baseline, sedentary behaviors were high (597.83 (SD = 75.91) minutes per day) and many participants exhibited impairments (i.e., $>1.5 \mathrm{SD}$ below the normative mean) in attention/executive function and memory. Steps per day declined from baseline to the 12 -week follow-up, $\mathrm{F}(1,56)=8.02, \mathrm{p}=0.01$. Decreases in moderate lifestyle PA predicted declines on an attention/executive function composite score over the 12 -week period $(\beta=.31, p=.045)$. No such pattern emerged for any other actigraphy measures or cognitive domains.

Conclusions: Reductions in moderate PA is associated with acute cognitive decline in HF. Exercise is a key treatment recommendation in $\mathrm{HF}$ that may attenuate cognitive decline and prospective studies are much needed to test this possibility and elucidate mechanisms.

Correspondence: Michael Alosco, MA, Kent State University, 4710 Waterford Circle, Stow,OH 44224.E-mail:malosco@kent.edu

\section{Acquired Brain Injury (TBI/ Cerebrovascular Injury \& Disease - Child)}

T. CARRATHERS, S. AYLWARD, B. BUCHHOLZ, A.A. GRETENCORD, J. LACE \& J. DORFLINGER. Impact of Age on Neuropsychological Measures Among Youth with Acute Concussions.

Objective: Research of neuropsychological performance following a concussion for children younger than high school is limited; the present study aims to directly compare pre-adolescent and high school students following a concussion.

Participants and Methods: High school students (n=96) were compared to pre-adolescent students $(n=43)$ who sustained a concussion in the past 30 days. Multivariate analysis of variance (MANOVA) was conducted to evaluate the impact of age (pre-adolescents v. adolescents) on neuropsychological measures.

Results: For the Auditory Consonant Trigrams, 9 second delay (ACT9) there was a significant effect of age $[\mathrm{F}(1,137)=5.196, \mathrm{p}<.05]$. There was also an effect of age for Woodcock Johnson Reading Fluency (WJreading) and Woodcock Johnson Math Fluency (WJmath), $[\mathrm{F}(1,137)=6.791, \mathrm{p}=.01]$ and $[\mathrm{F}(1,137)=7.937, \mathrm{p}<.01]$ respectively. For all measures, pre-adolescent students performed better than adolescent students with acute concussions. 
Conclusions: The current findings are contrary to previous research suggesting that younger age is associated with greater post-concussive impairment. Adolescence may represent a time during which concussed athletes are at-risk for increased post-concussive problems. Current findings suggest that there is not a linear relationship of age and concussion outcomes, or adolescence is a more vulnerable time for brain injury. There may be other factors, such as motivation to continue play or emotional variables, which may be influential (e.g. our previous research indicates that emotional factors are more salient for non-sportrelated concussions and older students report more emotional difficulties than younger students).

Correspondence: Tarra Carrathers, M.S., Rosalind Franklin University, 7305314th Ave, Salem,W 53168.E-mail:tarra.carrathers@my.rfums. org

\section{R. DIDIANO, M. DILUNA \& M. BEST. Gender Differences in BASC-2 Self and Parent Rating Scales after Concussion.}

Objective: Literature suggests the presence of emotional and social problems after sustaining a brain injury. However, many of the studies have been conducted using individual symptom report measures. The purpose of the current study was to investigate gender differences using a standardized assessment tool (BASC-II).

Participants and Methods: Fifty-eight patients aged 7-20 (M=15.31 years, $\mathrm{SD}=2.7$ ) with a history of one or more concussions were given the BASC-II Self-Report. Parents were asked to fill out the BASC-II Parent Report. Parent and patient responses were analyzed in order to determine gender differences in symptoms after injury.

Results: According to parent rating, concussed females reported higher incidence of anxiety symptoms after injury $\mathrm{t}(69)=1.100, \mathrm{p}=0.012$. Both female $(\mathrm{M}=54.77 ; \mathrm{SD}=13.63)$ and male $(\mathrm{M}=51.78 ; \mathrm{SD}=8.87)$ mean scores were in the normal range. Parents also reported concussed females to express more aggressive and depressive symptoms $(p<0.05)$. On both parent and self-ratings of somatization symptoms, females scored higher than males but neither score was found to be significant. For parent Somatization ratings, mean scores for females were in the "at risk" range $(\mathrm{M}=60, \mathrm{SD}=12.40)$. Males reported higher scores on a locus of control measure $\mathrm{t}(49)=-2.230 ; \mathrm{p}=0.000$.

Conclusions: Females reported higher incidence of anxiety, aggression, and depression symptoms after sustaining concussions. Males reported a higher locus of control score, indicating they feel less control over life events. Further research should examine effects of multiple concussions on BASC rating scales between genders.

Correspondence: Mary Best, Ph.D, Child Study Center, Vale University, 40 Temple Street, Suite7D, New Haven,CT06510.E-mail:mary.best@ yale.edu

\section{R. DIDIANO, M. DILUNA \& M. BEST. Gender Differences in} Naming Tasks Following Concussion.

Objective: Research indicates that gender differences in language-related tasks can arise after sustaining one or more concussions. However, few studies have investigated the direct effects. The aim of this study was to examine gender differences in word retrieval after one or more concussions.

Participants and Methods: Fifty-eight patients aged 7-20 (M=15.31 years, $\mathrm{SD}=2.7$ ) with a history of one or more concussions were given the Boston Naming and Auditory Naming Tests as part of a clinical neuropsychological evaluation at the Yale Pediatric Neuropsychology Clinic between 2012 and 2014. Auditory Naming measures included Total Correct and "Tip-of-The-Tongue" Responses (responses occurring after 2 seconds). Boston Naming Test score included Total Correct. Full-scale IQ was also analyzed.

Results: Overall, there were no significant gender differences on Full Scale IQ measures. indicating equivalent cognitive skills between the groups. Concussed females performed significantly better on the Total Correct measure of the Auditory Naming Test; $\mathrm{t}(38)=-2.026 ; \mathrm{p}=0.000$ (but not on the Tip of the Tongue measure). Visual naming was not found to be significant despite a difference in female $(M=100.67$; $\mathrm{SD}=18.48)$ vs. male $(\mathrm{M}=87.48 ; \mathrm{SD}=87.48)$ performance on the Boston Naming Task.

Conclusions: The findings indicate that Auditory Naming is more affected in males after concussion. Mean differences in the Boston Naming Task might suggest a possible trend in a similar direction. Further research to determine effects of concussion demographics on language, including number, severity, and length of time since is warranted.

Correspondence: Mary Best, Ph.D, Child Study Center, Yale University, 40 Temple Street, Suite7D, New Haven, CT 06510.E-mail:mary.best@ yale.edu

M. STUDER, B. GOEGGEL SIMONETTI, A. JOERIS, M. STEINLIN $\&$ T. HEINKS. Sleep is related with post-acute socio-behavioral outcome after pediatric mild traumatic brain injury.

Objective: Although there is evidence that sleep may be affected after pediatric mild traumatic brain injuries (mTBI), there is little research investigating the effects of sleep on neurocognitive and behavioral outcome. The aim of this prospective short-term longitudinal study was to explore the post-acute impact of sleep on neurocognitive and behavioral outcome.

Participants and Methods: We included children (age 6-16 years) after mTBI $(n=40)$ and orthopedic injuries (OI, n=38). Parents rated sleep via items of the Postconcussion Symptom Inventory (sleeping more, being tired, being drowsy) at the injury as well as post-acute at one (T1) and four months (T2) after the injury. Neurocognitive and socio-behavioral outcome was measured with standardized testing and questionnaires at $\mathrm{T} 1$ and $\mathrm{T} 2$.

Results: Our results revealed that there were no cross-sectional group differences concerning the amount of sleep problems or regarding neurocognitive and socio-behavioral outcome. While there was no association between sleep and neurocognitive outcome, we found significant and moderate sized correlations between sleep and behavioral outcome at $\mathrm{T} 1$ and T2 in both groups. Furthermore, sleep at T2 significantly predicted emotional problems at T2 $(\beta=.49, \mathrm{p}<.001, \mathrm{~F}(6,64)=4.61, \mathrm{p}<.01$, $\mathrm{R} 2=.32$ ), while injury group or pre-injury postconcussive symptoms were not significant predictors.

Conclusions: These results imply that parents of children after mTBI rate the need of sleep and symptoms of tiredness similar compared to parents of children after OI. Ongoing sleep symptoms seem not to be related with the performance in neurocognitive assessment, but with socio-behavioral outcome. Thus, sleep may affect socio-behavioral functioning in everyday life in children after mTBI and OI, indicating that sleep should be assessed more thoroughly in the post-acute phase after an injury to prevent sleep problems at an early stage.

Correspondence: Martina Studer, University Children's Hospital Bern, Inselspital,Bern 3010, Switzerland.E-mail:martina.studer@gmail.com

G.L. IVERSON, M. WOJTOWICZ, B. BROOKS, B. MAXWELL, J. ATKINS, R. ZAFONTE \& P. BERKNER. Concussion History in High School Athletes with Attention Deficit Hyperactivity Disorder.

Objective: Little is known about the lifetime history of concussions in high school athletes with attention-deficit hyperactivity disorder (ADHD). We hypothesized that high school athletes with ADHD would report a greater history of concussion than students without ADHD.

Participants and Methods: A large cohort of student athletes ( $\mathrm{N}=22$, 248), between the ages of 13 and 18, completed baseline, preseason testing with ImPACT® between 2011-2013. As part of the program, students were asked to self-report whether they have had "problems with ADD/hyperactivity" and to indicate the number of diagnosed concussions they have experienced.

Results: In the total sample, $6.5 \%(\mathrm{n}=1,452)$ self-reported a diagnosis of ADHD, representing $8.5 \%$ of the boys and $4.3 \%$ of the girls. A greater proportion of students with self-reported ADHD reported a history of one or more (ADHD: $24.2 \%$, no ADHD: $15.8 \%$; p<.0001), two or more 
(ADHD: $8.8 \%$, no ADHD: 4.3\%; p<.0001), three or more (ADHD: $4.2 \%$, no ADHD: $1.4 \%$; $<.0001$ ), and four or more prior concussions (ADHD: $2.1 \%$, no ADHD: $0.5 \% ; \mathrm{p}<.0001$ ) compared to those without ADHD. Stratified by gender, a greater proportion of boys with ADHD reported a history of one or more (ADHD: $25.6 \%$, no ADHD: $18.2 \%$; p<.0001), two or more (ADHD: 9.7\%, no ADHD: 5.5\%; p<.0001), three or more (ADHD: $4.6 \%$, no ADHD: $2.0 \%$; $\mathrm{p}<.0001$ ), and four or more concussions (ADHD: $2.4 \%$, no ADHD: $0.7 \%$; $<<.0001$ ) compared to boys without ADHD. Similarly, a greater proportion of girls with ADHD reported a history of one or more (ADHD: $21.2 \%$, no ADHD: $13.3 \%$; $\mathrm{p}<.0001$ ), two or more (ADHD: 6.9\%, no ADHD: $3.0 \%$; $<<.0001$ ), three or more (ADHD: $3.3 \%$; no ADHD: $0.9 \%$; $<<.0001$ ), and four or more concussions (ADHD: 1.3\%, no ADHD: 0.3\%; p<.0001) compared to boys without ADHD.

Conclusions: In this large-scale, retrospective survey study, boys and girls with ADHD were significantly more likely to report a history of concussion. Additional research is needed to determine if students with $\mathrm{ADHD}$ are more susceptible to injuryor have different recovery trajectories.

Correspondence: Magdalena Wojtowicz, PhD, Psychiatry; Physical Medicine and Rehabilitation, Harvard Medical School, 60 Alban St, Boston, MA 02124.E-mail: mwojtowicz@mgh.harvard.edu

\section{B. BIEKMAN, E.A. WILDE, A. SCHMIDT, X. LI, Z. CHU, H. LEVIN \& G. HANTEN. Resilience and Brain Imaging in Pediatric Traumatic Brain Injury.}

Objective: Adequate recovery of social functioning and reintegration into one's social world following traumatic brain injury (TBI) is an area of significant interest. However, the concept of resilience as a factor in recovery has been incompletely explored. We examined diffusion tensor imaging (DTI) metrics and their relation to a measure of resilience one year following pediatric TBI.

Participants and Methods: DTI and a cross-culturally valid measure of factors related to resilience (Child and Youth Resilience Measure; CYRM) were obtained 12 months after complicated-mild to severe TBI in children ages 10-18 years who sustained complicated-mild to severe TBI $(n=38)$ and a cmparison group $(n=28)$. Quantitative tractography was used to measure fractional anisotropy (FA) in the right and left uncinate fasciculus (UF), a white matter tract that connects the frontal and temporal poles. These regions are particularly vulnerable to injury in moderate to severe TBI.

Results: Children with TBI showed significantly lower scores than the control group on measures of resilience (all $p<0.05$ for total and subscales). Group comparison also revealed significantly lower FA in the right $(p=0.006)$ and left $(p<0.0001)$ UF FA in the children with TBI. In this group, there were no correlations with the CYRM total score or subscales and the right UF. However, FA of the left UF correlated with the total score $(\mathrm{r}=0.508)$ and the indiviudal $(\mathrm{r}=0.329)$, relationships with primary caregiver $(\mathrm{r}=0.563)$ and contextual factors $(\mathrm{r}=0.494)$ subscales, such that higher FA in this region was associated with greater resilience. Conclusions: Results underscore the need to attend to psychosocial factors in children sustaining a TBI as these may influence cognitive and potentially neurobiological recovery. They also indicate that children sustaining a TBI may require careful assessment of environmental and psychosocial factors and support in these areas in order to promote optimal brain plasticity and recovery from the long-term impact of pediatric TBI.

Correspondence: Elisabeth A. Wilde, Ph.D., Physical Medicine and Rehabilitation, Neurology and Radiology, Baylor College of Medicine, 1709 Dryden Rd., Ste 1200, Houston, TX 77025.E-mail: ewilde@bcm. edu

J.A. SOTOMAYOR, C. VAUGHAN, J. MOLLO \& G.A. GIOIA. Variability of Recovery in a Pediatric Concussion Sample.

Objective: Age, gender, and injury characteristics such as amnesia have been found to be related to length of recovery from concussion. The current study explores the relation between these demographic and injury variables and length of recovery.

Participants and Methods: The sample consisted of 237 children and adolescents (Mean age $=13.3, \mathrm{SD}=2.7$, range 5-19, 64\% male) with diagnosed concussion ( $54 \%$ sport/ $46 \%$ non-sport). Eighty-five percent reported full recovery at the time of this study; length of recovery ranged from 6 to 182 days $($ Mean $=56.47, \mathrm{SD}=37.09)$. Demographic (age, gender) and early injury (loss of consciousness, anterograde and retrograde amnesia) characteristics were examined to understand variability in length of recovery.

Results: Correlational analyses indicated no significant associations between age, gender, and amnesia (either retrograde or anterograde amnesia) with length of recovery $(p>.05)$. For those children who had not yet recovered, $71 \%$ of parents described persisting post-concussion symptoms, while the remaining group reported issues associated with impaired school functioning and lack of physical activity.

Conclusions: Recovery from concussion varies widely in children. Although age and gender have both been reported to be associated with length of recovery, we did not find this relationship in our clinic sample. Similarly, potential markers of injury severity were also not associated with length of recovery. Persisting problems in a subsample were associated with impaired school functioning and lack of physical activity. A selection bias in referral to a specialty clinic may explain the different outcome in this study. Large sample prospective studies are needed to better understand this course of concussion recovery in children and adolescents.

Correspondence: Jason A. Sotomayor, BA, Pediatric Neuropsychology, Children's National Health System, 4540 MacArthur Blvd, Washington, DC20007.E-mail: jasonasotomayor@gmail.com

J.J. MIETCHEN, S.D. GALE, E.A. WILDE, B. BIEKMAN, G. HANTEN \& H. LEVIN. Fractional Anisotropy of the Ventral Striatum Following Moderate to Severe Traumatic Brain Injury in Children.

Objective: Compare fractional anisotropy (FA) of the ventral striatum between children with chronic traumatic brain injury (TBI) to an orthopedic injury (OI) control group.

Participants and Methods: Participants were thirteen children with moderate to severe TBI (age $=15.9 \pm 2.5$ years; $30.8 \%$ female; $\mathrm{GCS}=8.7 \pm 5.4$ ) and $12 \mathrm{OI}$ controls (age $=13.1 \pm 1.8$ years; $41.7 \%$ female; $\mathrm{GCS}=15$ ). Diffusion tensor imaging (DTI) was acquired at one year post injury. Mean FA in the left and right ventral striatum was calculated using PRIDE software. In addition to group comparisons of FA, Glasgow Coma Scale (GCS) score and BRIEF scores were correlated with FA values.

Results: The TBI group was slightly older $(\mathrm{p}<.01)$ but comparable for gender ( $p>05)$ as shown by a chi-square analysis. FA in the left ventral striatum was significantly lower $(p<.05)$ in the TBI group, demonstrating a large effect size $(\mathrm{d}=0.94)$ though no difference was found in the right ventral striatum $(p>.05 ; d=0.30)$. In the TBI group, GCS did not correlate with the left or right ventral striatum $(p>.05)$ although significant correlations were found between the right ventral striatum and the Global Executive Composite $(\mathrm{p}<.05)$ and the Inhibit subscale $(p<.05)$ from the BRIEF.

Conclusions: Children with a history of TBI at one year post injury had significantly lower FA in the left, but not right, ventral striatum compared to orthopedic controls. Results support prior reports of persistent changes in FA as a result of TBI in children. FA in the right ventral striatum was also associated with a measure of executive function in children with TBI.

Correspondence: Jonathan J. Mietchen, BS Psychology, Department of Psychology, Brigham Young University, 1001 SWKT, Provo, UT $84602-$ 5543. E-mail: jonathan.mietchen@gmail.com 
J. WISE, T.Z. KING \& J. HAARBAUER-KRUPA. Word Fluency and Reading Comprehension in Young Children with Orthopedic and Traumatic Brain Injury.

Objective: Early childhood traumatic brain injuries (TBI) can negatively impact language development and executive functioning. The purpose of this study was to examine the relationships among executive functioning and reading comprehension between two groups of children who experienced an early TBI or orthopedic injury (OI).

Participants and Methods: Sixty-three children between the ages of 6 and 10 years old $(\mathrm{M}=8.17$ years $(1.26), 51 \%$ female, $65 \%$ Caucasian) who sustained a TBI $(n=33)$ or OI $(n=30)$ before age 6 (TBI M age of injury $=2.50(1.50)$ and OI Mean=4.32 (1.36)) were administered the Reading Comprehension subtest of the Woodcock Reading Mastery Test and Word Fluency subtest of the Pediatric Test of Brain Injury.

Results: A mediation model was tested using the simple mediation with bootstrapping script for SPSS (Preacher \& Hayes, 2004). Injury type (OI vs. TBI) significantly predicted reading comprehension performance. The children with a TBI had worse reading comprehension compared to children with an OI $(\beta=-7.21, \mathrm{t}(61)=-3.64, p<.001)$. Further, the indirect effect of word fluency skills was significant $(\beta=-2.86,95 \mathrm{CI}$ : $-5.53,-0.19)$. However, the relationship between injury type and reading comprehension performance remained significant when word fluency skills was added to the model $(\beta=-4.35, \mathrm{t}(61)=-2.74, \mathrm{p}=.008)$. Conclusions: Results indicated that word fluency skills partially mediated the relationship between injury group and reading comprehension skills. Results suggest that an early TBI affects later development of both reading comprehension and the speed of word generation, and that word fluency skill contributes to reading comprehension performance differentially across injury groups.

Correspondence: Justin Wise, Oglethorpe University, 4484 Peachtree Road NE, Atlanta, GA 300319.E-mail: jwise@oglethorpe.edu

H.L. COMBS, L.H. MASON, A.J. ANDERSON \& D. HAN. Irritability and Aggression as Indicators of Persistent Post-Concussive Symptoms in Adolescent Athletes.

Objective: Prevalence of sport-related concussions ranges between 300,000-3.8 million annually (Halstead et al., 2010; Langlois et al., 2006). Post-concussive symptoms vary, but typically include physical, emotional, and/or cognitive difficulties. Although research suggests most symptoms resolve within one week, younger athletes are frequently symptomatic 7-10 days longer (Field, Collins, Lovell, \& Maroon, 2003; McCrea et al., 2009). This extended recovery time for adolescents highlights the importance of identifying markers that suggest and predict such atypical recovery.

Participants and Methods: Thus, we examined 46 adolescent athletes (Mage $=14.39)$ with persistent concussion symptoms. Clinical interview and Beck Youth Inventory Second Edition (BYI-II) were completed as part of a larger neurocognitive battery. Self-reported irritability/ aggression was common and easily identified. Therefore, athletes were split into two groups: Those endorsing irritability/aggression $(\mathrm{n}=23)$ and those denying this symptom $(\mathbf{n}=21)$.

Results: Adolescents endorsing irritability/aggression subjectively described more fatigue $(p=.002)$, disordered sleep $(p=.001)$, anxiety $(p$ $=.028)$, depression $(p=.003)$, and mood lability $(p<.001)$ during interview. Adolescents reporting more irritability also had elevated scores on related psychometric scales, including the BYI-II Anxiety $(\mathrm{t}(41)=$ -2.916, $\mathrm{p}=.006)$, Depression $(\mathrm{t}(41)=-3.551, \mathrm{p}=.001)$, and Anger Scales $(\mathrm{t}(41)=-2.958, \mathrm{p}=.005)$.

Conclusions: These findings suggest that irritability/aggression may provide an easily identifiable marker of atypical recovery post-concussion, with utility for both clinical professionals and associated laymen. It may also predict other maladaptive symptoms, including mood instability. The causal relationships among these symptoms remain a focus of ongoing investigation.
Correspondence: Hannah L. Combs, M.S., Psychology, University of Kentucky, 3900 Crosby Dr. Apt. 2507, Lexington, KY 40515. E-mail: hannahlanecombs@gmail.com

J. BERNSTEIN, S.L. WADE, J. HUGENTOBLER, J. TAYLOR, A. CASSEDY, P. GUBANICH \& B. KUROWSKI. Benefits of Aerobic Training for Concussion in Adolescents: a Preliminary Report of a Pilot Randomized Clinical Trial.

Objective: To assess the benefits of aerobic exercise on post-concussive symptoms (PCS) and cognitive functioning in adolescents.

Participants and Methods: Participants included 13 adolescents (6 males; 7 females) with a mean age of 15.2 vears who demonstrated PCS for $>4$ weeks ( $M=53.5$ days). Participants were randomized to an aerobic training program or generalized stretching program. The aerobic intervention consisted of progressive, sub-symptom exacerbation aerobic training using a stationary bicycle. The stretching intervention involved a whole-body stretching program. Both interventions included a home program to be completed 5-6 days per week and weekly visits to review program implementation. The primary outcomes were parent and self-report, post-concussion symptom inventory (PCSI) ratings. Secondary outcomes included the NIH tool box cognitive battery assessments (crystalized and fluid cognition, processing speed, and executive function). Assessments were completed pre- and post-treatment. Paired t-tests were used to evaluate recovery within groups and general linear models, controlling for baseline scores, were used to compare post-intervention outcomes between groups.

Results: Within the aerobic group, participants showed improvement in self-reported PCSI ratings ( $p=.003)$, crystalized cognition $(p=.016)$, fluid cognition $(p=.011)$, and processing speed $(p=.02)$. Non-significant improvements were noted on parent PCSI ratings $(p=.14)$ and executive function measures $(p=.07)$. When compared to stretching, aerobic training was not significantly associated with outcomes ( $p$-range $=.06-.62$ ). Conclusions: These findings suggest that aerobic training is not harmful and may be beneficial for adolescents with persistent PCS. Larger comparative studies need to be performed to better elucidate efficacy and determine who will benefit.

Correspondence: John Bernstein, University of Rochester, 10 Ingleside Road, Lexington, MA 02420.E-mail:jberns16@u.rochester.edu

C. GAGNER, C. LANDRY-ROY, J. BELLEROSE, C. BEAUDOIN, A. BERNIER, J. GRAVEL \& M.H. BEAUCHAMP. Are Behavioural Problems Following Mild TBI in Preschoolers Associated with Theory of Mind?

Objective: Traumatic brain injury (TBI) can lead to theory of mind (ToM) deficits, as well as behavioural and emotional problems ( $\mathrm{Li} \&$ Liu, 2012; Walz, 2010). Hugues and colleagues (2005) have shown that individual differences in behavioural problems show a strong association with ToM in typically developing children (TDC). The aim of the current study was to examine the role of ToM impairments in behavioural problems following mild TBI in preschoolers.

Participants and Methods: Forty-six preschoolers (25 - 64 months, $\mathrm{M}=44.6, \mathrm{SD}=10.5)$ who sustained mild TBI were assessed 6 months after injury. Their parents completed the Child Behavior Checklist to provide an indication of the presence of internalizing and externalizing behavioural problems. In addition, the Can't Sleep Task (Moerbeek, 1994), a measure of false belief understanding was used to assess ToM. The results of the mild TBI group were compared to those of thirty-three typically developing children (24-66 months, $\mathrm{M}=45.9, \mathrm{SD}=12.5)$.

Results: An analysis of covariance using the false belief score as a covariate revealed significant group differences on the CBCL total problems scale $(\mathrm{F}(1,76)=7.89, \mathrm{p}=.01)$, suggesting that behavioural problems are not fully explained by the presence of ToM differences. Conclusions: In sum, the TBI group had more parent-reported behavioural problems than typically developing children even when controlling for ToM. Further studies should be conducted to assess whether 
other cognitive variables, such as executive functioning, may underlie behavioural problems after preschool mild TBI.

Correspondence: Charlotte Gagner, Doctoral, Psychology, University of Montreal, 6235, Molson street, apt.20, Montreal, QC H1Y 3C2, Canada. E-mail: charlotte.gagner@hotmail.com

C. LANDRY-ROY, C. GAGNER, C. BEAUDOIN, A. BERNIER, J. GRAVEL \& M.H. BEAUCHAMP. The Relationship between Emerging Behavioral Problems and Parental Distress following Mild Traumatic Brain Injury in Early Childhood.

Objective: Research suggests that paediatric traumatic brain injury (TBI) significantly increases the risk of developing behavioural and emotional problems (Li \& Liu, 2012). In addition, TBI can have a negative impact on families more globally, and parents of children who have sustained TBI report elevated levels of distress (Hawley et al., 2003). The current study aimed to examine the relationship between child behavioural problems and parental distress in preschoolers who sustain mild TBI.

Participants and Methods: Forty-seven children aged 16 to 58 months $(\mathrm{M}=38, \mathrm{SD}=11$ month at injury $)$ with mild accidental TBI were recruited at an urban paediatric tertiary care Emergency Department. Their parents completed the Child Behaviour Checklist (CBCL), the Parenting Stress Index and the Family Assessment Device (General Functioning Scale) acutely $(\mathrm{M}=53, \mathrm{SD}=103$ days $)$ and 6 months post-injury.

Results: There was a significant increase in behavioural problems between the acute and the 6 months post-injury assessments (Total CBCL Problems, $\mathrm{t}(46)=-1.89$, one-tailed, $\mathrm{p}=0.03)$. Increased behavioural problems predicted higher parental distress at 6 months post-injury, when controlling for premorbid family functioning. Premorbid family functioning accounted for $29 \%$ of variation in parental distress at 6 months post-injury, and the increase in behavioural problems explained an additional $6 \%(\mathrm{p}=0.05)$ of variation in the model.

Conclusions: The present study suggests that emerging behavioural problems following mild TBI are distressing to parents. Rapid identifying of potential behavioural problems is imperative to guiding TBI management and rehabilitation, and may alleviate parental distress. Correspondence: Catherine Landry-Roy, Psychology, Université de Montréal, 340 Sainte-Madeleine app 3, Montreal, QC H3K 0A5, Canada.E-mail: catherine.landry.roy@gmail.com

K.A. MCNALLY, K.E. ROBINSON, A. TREBLE-BARNA, J. DYKSTRA, C. HAJEK \& K.R. WOLFE. Effectiveness of a Brief Cognitive Behavioral Intervention for Children and Adolescents with Prolonged Post-Concussive Symptoms.

Objective: To examine the impact of a cognitive-behavioral treatment intervention for children and adolescents with prolonged post-concussive symptoms (PCS).

Participants and Methods: Participants were 30 children and adolescents (ages 10-18, M $=15.3, \mathrm{SD}=2.15$ ) who were referred for psychological treatment to address prolonged symptoms following mild traumatic brain injury. Initial appointments occurred on average 183 days post-injury $(\mathrm{SD}=170) .43 \%$ of injuries were sustained while playing sports; $27 \%$ motor vehicle collisions; $20 \%$ falls; $10 \%$ other. $83 \%$ of participants did not lose consciousness. Treatment involved 1-7 sessions (total sessions completed $\mathrm{M}=4.4 ., \mathrm{SD}=1.8$ ) of cognitive behavioral therapy. Treatment sessions included psychoeducation, activity scheduling, relaxation training/biofeedback, and cognitive restructuring. Paired t-tests were conducted to compare pre- to post-treatment PCS ratings and quality of life (Pediatric Quality of Life Inventory, PedsQL).

Results: For both parent and child ratings, PCS significantly declined following treatment (Pre-treatment parent PCS M = 25.7, $\mathrm{SD}=8.8$; post-treatment parent $\mathrm{PCS} \mathrm{M}=16.07, \mathrm{SD}=7.7, \mathrm{t}=4.98, \mathrm{p}<.001$; Pre-treatment child PSC M $=24.42, \mathrm{SD}=9.3$; post-treatment child PCS M $=15.6, \mathrm{SD}=7.2, \mathrm{t}=5.96, \mathrm{p}<.001)$. Similarly, parent-reported quality of life significantly improved with treatment (Pre-treatment
PedsQL M $=57.7, \mathrm{SD}=16.7$, post-treatment PedsQL M = 69.6, $\mathrm{SD}$ $=18.4, \mathrm{t}=-2.45, \mathrm{p}<.05)$ with the most significant change evident in the emotional domain.

Conclusions: Brief cognitive-behavioral treatment may be a promising intervention for children and adolescents who are experiencing prolonged PCS.

Correspondence: Kelly A. McNally, Ph.D., Psychology, Nationwide Childrens Hospital, 700 Children's Drive, Columbus, OH 43205. E-mail:kelly.mcnally@gmail.com

R.L. PETERSON, A.K. CONNERY, D.A. BAKER \& M. KIRKWOOD. Pre-Injury Anxiety and Postconcussive Problems in a Pediatric Sample.

Objective: Both injury-related and non-injury-related factors are known to influence recovery from mild traumatic brain injury (TBI). The goal of the current study was to characterize preinjury emotional-behavioral functioning in a sample of pediatric patients referred clinically because of concerns about lingering problems following mild TBI.

Participants and Methods: This clinical case series design included 387 patients aged 8-17 years old seen for neuropsychological evaluation following mild TBI and their parents. Parents retrospectively rated children's preinjury emotional-behavioral functioning on a broadband questionnaire (Behavior Assessment System for Children-Second Edition). Rates of clinically significant problems were compared to national norms.

Results: The rate of clinically significant preinjury anxiety was significantly elevated compared to national norms. Rates of other pre-injury emotional-behavioral difficulties were similar to the norming sample. Conclusions: Premorbid anxiety may play an important role in the establishment or maintenance of lingering symptoms after pediatric mild TBI, similar to what has been reported in adults. Anxious children appear more likely to report persistent postconcussive difficulties, perhaps because of hypervigilance to somatic symptoms. Providers should carefully consider preinjury emotional functioning when evaluating and managing children with persistent problems following mild TBI. Results also highlight the need for pediatric mild TBI outcome studies that rigorously account for preinjury functioning.

Correspondence: Robin L. Peterson, Ph.D., Rehabilitation Medicine, Children's Hospital Colorado, 13123 E. 16th Avenue, B285, Aurora, CO 80045.E-mail: robin.peterson@childrenscolorado.org

K. PEARCE, A. SUFRINKO, A. KONTOS \& L. HENRY. Near Point Convergence and Concussion: Demographic and Neurocognitive Findings.

Objective: Roughly $60 \%$ of the brain is involved with visual processing. Consequently, it is not surprising that visual dysfunction secondary to concussion is found in a significant number of cases. One type of binocular vision dysfunction, convergence insufficiency (CI), can result in a variety of functional impairments. CI has been observed in upwards of $46 \%$ of individuals following traumatic brain injury; however, few studies have evaluated the effects of CI on neurocognitive performance and symptoms secondary to concussion.

Participants and Methods: Participants included 107 individuals formally diagnosed with a concussion. Participants completed the ImPACT computerized neurocognitive test battery and underwent a vestibular and ocular-motor screening evaluation to measure posttraumatic changes in near point convergence (NPC) that can result in CI.

Results: NPC did not differ by age, $\mathrm{F}(6,107)=.621, \mathrm{p}=.713$, or gender, $F(1,107)=.331, p=.567$. Four hierarchical regressions were run to evaluate the predictive utility of NPC/CI on neurocognitive data following cerebral concussion. After controlling for age and total symptoms, NPC significantly predicted performance on visual motor speed $(\beta$ $=-.520, \mathrm{p}=.004)$, and reaction time $(\beta=.008, \mathrm{p}=.034)$.

Conclusions: Recent research suggests that concussion is a highly individualized injury. Consequently, screening for post-traumatic vision changes following concussion may better the clinician's understanding 
of the individualized nature of this injury and further guide treatment planning. Routine evaluation of NPC during concussion evaluations may be an effective tool used to gain insight into potential sources of concussion symptoms.

Correspondence: Kelly Pearce, PhD, Orthopaedics, University of Pittsburgh Medical Center, 3200 S Water St., Pittsburgh, PA 15203. E-mail: pearcekl@upmc.edu

T.J. FARRER, S. JIVANI, E.D. BIGLER, M. DENNIS, H.G. TAYLOR, K. RUBIN, K. VANNATTA, C. GERHARDT, T. STANCIN \& K.O. YEATES. Chronic MRI Findings, Ventromedial Prefrontal Cortex (vmPFC) Volume, and Behavioral Functioning in a Pediatric Sample with Complicated Mild Traumatic Brain Injury. Objective: In mild traumatic brain injury (TBI) positive day-of-injury (DOI) CT findings define complicated mild (cm) TBI or cmTBI. However, some DOI abnormalities are not identifiable after the acute stage. In a pediatric cmTBI sample, participants were divided into those with positive (MRI+) or negative MRI findings (MRI-) based on chronic imaging typically performed $>2$ years post-injury. Although both MRI+ and MRI- cmTBI children only met criteria for mild injury, and did not differ in GCS, we hypothesized that greater brain volume loss would occur within the ventromedial prefrontal cortex (vmPFC), because of its vulnerability to injury, in the MRI+ group compared to MRI- or orthopedically injured (OI) groups. The relation of vmPFC volume with behavioral outcome measured by the Behavioral Assessment System for Children (BASC) was also explored.

Participants and Methods: Of the 36 children that comprised the cmTBI group (age at injury: $\mathrm{M}=7.9, \mathrm{SD}=1.8$; time post injury: $\mathrm{M}=2.7$ years, $\mathrm{SD}=1.1), 22$ were in the MRI+ group. The OI group was comprised of 48 participants. vmPFC volume was based on FreeSurfer analyses.

Results: vmPFC did not significantly differ across groups. In the MRI+ group, vmPFC volume was significantly related to Aggression ( $\mathrm{rs}=$ $-.63, \mathrm{p}=.02)$ and Depression ( $\mathrm{rs}=-.58, \mathrm{p}=.03$ ). However, none of the select BASC subscales were related to vmPFC volume in the MRI- or OI groups. For OI controls, the correlation between vmPFC volume and Aggression and Depression was -.06 $(\mathrm{p}=.71)$ and -.05 $(\mathrm{p}=.72)$, respectively . For MRI- group, the correlation between vmPFC volume and Aggression and Depression was -.56 ( $\mathrm{p}=.07)$ and -.21 ( $\mathrm{p}=.52)$, respectively. Only the correlation for Aggression was significantly different between the MRI+ and Control groups (Fisher's $\mathrm{z}=-1.91, \mathrm{p}=$ $.05)$. No other correlations were significantly different between groups. Conclusions: Reduced vmPFC volume in children with mTBI and positive MRI findings in the chronic phase of injury relates to depression and aggression indices on the BASC.

Correspondence: Thomas J. Farrer, M.S., Psychology, Brigham Young University, 1001 Kimball Tower, P.O. Box 25543, Provo, UT 846025543. E-mail: thomasfarrer@yahoo.com

\section{R.Z. BLAHA, R.L. PETERSON, M. KIRKWOOD, H.G. TAYLOR, T. STANCIN, K.O. YEATES \& S.L. WADE. PTSD Symptoms After Complicated Mild to Severe TBI in Adolescence: What Role does Memory of Injury Play?}

Objective: Moderate to severe pediatric traumatic brain injury (TBI) increases the risk for post traumatic stress disorder (PTSD) symptomatology. Some have argued that impaired memory of injury (MOI), which is common following TBI, might preclude traumatization. However, we previously found that among adolescents who had sustained a serious TBI, little to no memory for the traumatic event was associated with higher levels of PTSD symptoms (Blaha et al., 2014). We aimed to replicate these findings in a different sample and to further elucidate the relationship between PTSD symptomatology and MOI following TBI in adolescence.

Participants and Methods: Participants included 143 adolescents ( $69 \%$ male) aged 11 through 18 years old $(M=14.97$ at time of injury) who had sustained a complicated mild to severe TBI within the previous
18 months. PTSD symptomatology and MOI were assessed using items from the UCLA PTSD Index (Adolescent Version).

Results: Adolescents who endorsed little to no memory for the event had higher levels of PTSD symptoms than those who endorsed some memory for the event $(t(138)=2.57, p=.011)$. This effect was disproportionately seen in females. Injury severity also played a role, with more severe injuries associated with higher PTSD symptomatology $(\mathrm{t}(59)=2.01, \mathrm{p}=.049)$.

Conclusions: The results confirmed our previous findings. Lack of memory for injury following adolescent TBI may increase the risk for PTSD symptomatology, particularly in females. Further investigation is needed to understand the mechanisms for this effect but poor MOI may capture a different aspect of injury severity than more traditional indices such as Glasgow Coma Scale.

Correspondence: Robert Z. Blaha, Master of Arts, Rehabilitation, Children's Hospital Colorado, 13123 E. 16th Ave. B285, Aurora, CO 80045. E-mail: robert.blaha@childrenscolorado.org

W. KEENER, E. MONTAGUE, E. LONG, L.D. STANFORD \& D.T. PULSIPHER. The Relationship Between Post-Concussion Symptoms and Psychological Status in Children and Adolescents With Prolonged Recovery Following Mild Traumatic Brain Injury. Objective: Post-concussive symptoms (PCS) resolve within 7-90 days of mTBI for most children and adolescents. However, a subset of youth continues to experience PCS even longer. Non-injury related factors (e.g. psychological factors or pre-morbid conditions) are believed to contribute to prolonged recovery. Our investigation aimed to explore the relationship between PCS and current psychological status in children and adolescents with extended recovery time following mTBI.

Participants and Methods: 101 consecutively referred individuals ages 8-18 years with mTBI completed the Post-Concussion Symptom Questionnaire (PCSQ; total number of symptoms and severity markers intensity, frequency, duration) and other self-report measures, including the Youth Self-Report [YSR] and anxiety and depression measures. Parents completed the Child Behavior Checklist (CBCL). Correlational analyses were used to determine relationships among variables.

Results: Total number of symptoms, as well as frequency, intensity, and duration of PCS, were correlated with endorsement of somatic complaints, anxious/depressed symptoms, withdrawn/depressed feelings, and thought problems $(r$ 's $>0.26)$. Total number of PCS was most strongly correlated with endorsement of depression and anxiety ( $r$ 's > 0.62). PCSQ symptom total, frequency, intensity, and duration were correlated with parent-reported internalizing problems and total problems on the CBCL (r's > 0.21); externalizing problems was associated with only PCSQ total symptoms $(r=0.24)$.

Conclusions: Children and adolescents with an extended recovery course following mTBI report high PCS rates of moderate severity and rate themselves higher on scales of somatic, anxiety, and depressive symptomatology, further supporting previous findings that psychological factors strongly contribute to prolonged recovery time in these youth. Correspondence: Whitney Keener, M.A. Clinical Psychology, NeuroDevelopmental Science Center, Akron Children's Hospital, 1946 Wiltshire Rd, Akron,OH 44313. E-mail: jkeener@chmca.org

D. RANSOM, M.D. SADY, C. MCGILL, C. VAUGHAN \& G.A. GIOIA. Evidence-Based Assessment of Cognitive Exertion and Academic Problems during Concussion Recovery.

Objective: The effects of cognitive overexertion (i.e., increasing symptoms in response to cognitive activities) are commonly reported during concussion recovery but have yet to be empirically explored in relation to school activities. This study examined the predictive utility of exertional effects to identify students at risk for post-injury academic problems. Participants and Methods: Participants included 221 students diagnosed with concussion (Mean age $=12.54 \pm 2.11,61 \%$ male) within four weeks of injury (Mdn=16 days). Demographics (gender, level of schooling, pre-injury diagnosis of $\mathrm{ADHD}$ and LD), time since injury, 
concussion history, and the Exertional Effects Index (EEI: in-vivo rating of increased symptoms with cognitive activity) were examined as predictors of self-reported post-injury academic outcomes $(3+$ school problems or $2+$ interfering symptoms or $2+$ impaired functional academic skills). Results: Classification statistics with receiver operating characteristic (ROC) analyses provided robust support for cognitive overexertion in identifying students with post-injury academic problems. Prediction was optimized with an EEI cut score of $+/-3$ and demographics were included in the model $(\mathrm{Sens}=.80, \mathrm{Spec}=.61, \mathrm{LR}+=2.04$, odds ratio=6.09, $\mathrm{AUC}=.77$ ). Sex (female) was a significant predictor of symptom-based problems (Sens=.80, Spec=.61, $\mathrm{LR}+=2.04$, odds ratio=6.09, $\mathrm{AUC}=.73$ ). Higher levels of schooling (high school) and pre-injury diagnosis of ADHD also significantly predicted skill-based problems (Sens=.60, Spec=.71, $\mathrm{LR}+=2.09$, odds ratio=3.75, $\mathrm{AUC}=.67$ ).

Conclusions: Students reporting symptoms of cognitive overexertion during concussion recovery are at greater risk for post-injury academic problems. Assessment of exertional effects can improve predictions of school problems, enhance clinical decision making, and target academic interventions for clinicians and administrators working with students recovering from concussion.

Correspondence: Danielle Ransom, PsyD, Neuropsychology, Children's National Health System, 15245 Shady Grove Road, Suite 350, Rockville, MD 20850.E-mail:DRansom@cnmc.org

D. RANSOM, M. ZAYAT, M. FURDA, A. GOKHALE, R. KROMASH, C. MCGILL, C. VAUGHAN \& G.A. GIOIA. Modeling Academic Effects of Concussion with Evidence-Based Assessment of Executive Functions.

Objective: Evidence-based assessment (EBA) offers an important perspective in the management of concussion, applying traditional strengths of neuropsychological assessment to individual clinical decision-making (Youngstrom, 2013). This study examined the predictive utility of everyday executive functions to aid in the identification of students at risk for academic problems.

Participants and Methods: Participants included 221 students diagnosed with concussion (Mean age $=12.54 \pm 2.11,61 \%$ male) within four weeks of injury ( $M d n=16$ days). Demographics (gender, level of schooling, pre-injury diagnosis of ADHD and LD), time since injury, concussion history, and a self-report measure of executive functions (BRIEF-SR monitoring version) were examined as predictors of self-reported academic problems (i.e., $3+$ school problems).

Results: Classification statistics with receiver operating characteristic (ROC) analyses revealed significant discriminative validity for reports of pre-injury retrospective baseline (RBL) and post-injury changes in executive functions for students with and without post-injury academic problems. While pre-injury RBL BRIEF ratings classified students significantly better than chance (Sens $=0.70$, Spec $=0.54, \mathrm{LR}+=1.55$, odds ratio=2.85; $\mathrm{AUC}=0.67, \mathrm{p}<.001)$, post-injury changes provided significant incremental validity $(z=6.14, p<.001$, Sens $=0.82$, Spec $=0.74$, $\mathrm{LR}+=3.18$, odds ratio=12.84, $\mathrm{AUC}=0.85, \mathrm{p}<.001)$. Prediction was optimized with a comprehensive model of assessment (i.e., demographics, injury-related variables, and pre/post executive functions) (Sens=.87, Spec=.77, LR+=3.85, odds ratio=21.56, AUC+=.89, p<.001).

Conclusions: Many students recovering from concussion are faced with impaired executive skills that impede their academic performance. Assessment of pre- and post-concussion executive dysfunction improves identification of students at risk for academic problems. EBA combines traditional psychometric methodology with individual risk factors to support clinical decision-making in the evaluation of concussion. Correspondence: Danielle Ransom, PsyD, Neuropsychology, Children's National Health System, 15245 Shady Grove Road, Suite 350, Rockville, MD 20850.E-mail:DRansom@cnmc.org
M. ZAYAT, C. MCGILL, J. SOTOMAYOR, M. LANCASTER, D. SRIRAM, C. VAUGHAN \& G.A. GIOIA. The Effectiveness of Concussion Education in Youth: A Pilot Study.

Objective: Concussion education is an important component of risk reduction (Tator, 2012), yet empirical research regarding mechanism and effectiveness of knowledge transfer is needed. This study aims to examine the initial knowledge about concussion and the impact of a brief concussion education session for youth.

Participants and Methods: Participants were 102 uninjured children (Mean age $=12.92 \pm 2.16,62 \%$ male, $86 \%$ Caucasian) who received their educational session individually, or in a small (2-4 children) or large (4+ children) group. The education addressed signs/symptoms of a concussion, what to do if you think you have a concussion, risks of ignoring a concussion, and concussion laws. A 10-question, multiple-choice quiz assessing concussion knowledge was completed before the education and then 30 minutes later. The effect of gender, age group (7-12 years, 13-18 years), and group size on knowledge gained was examined.

Results: A significant within-subjects increase in overall concussion knowledge was found $(\mathrm{F}(1,100)=153.99, \mathrm{p}<.001)$ from pre-to-posttest. The adolescent group demonstrated greater knowledge acquisition than the younger age group $(F(1,99)=10.47, p=.002)$, and there were no differences due to gender or group size. The greatest improvement in knowledge acquisition pertained to the concussion definition ( $46 \%$ increase), identifying emotional symptoms ( $42 \%$ increase), and the number of symptoms necessary for diagnosis ( $29 \%$ increase).

Conclusions: Knowledge of concussion is an important component of youth sport safety. This study demonstrated the effectiveness of live, brief education sessions in increasing concussion knowledge. Future research should examine the impact of knowledge acquisition on subsequent behavior change.

Tator, C. H. (2012). Sport concussion education and prevention. Journal of Clinical Sport Psychology, 6(3), 293-301.

Correspondence: Maya Zayat, Psy.D., Neuropsychology, Children's National Health System, 15245 Shady Grove Road, Suite 350, Rockville, MD 20850.E-mail:mzayat@childrensnational.org

G. VARGAS, J. CROWLEY \& H. SCHOFIELD. Correlates of Prolonged Recovery After Pediatric Concussion.

Objective: The nature of pediatric concussion remains elusive. Symptom report is considered as sensitive to concussion effects as neurocognitive or balance testing. Using symptom report as an indicator of concussion recovery, the influence of demographic, pre-injury and injury characteristics were investigated as to their effect on concussion recovery.

Participants and Methods: Participants were a consecutive cohort presenting to a multidisciplinary Concussion Clinic during one calendar year $(\mathrm{N}=278)$. They ranged from 10 to 19 years old and 38\% were female. Fifty-two percent of concussions were sports-related and $34 \%$ of children had a pre-existing exceptionality (e.g., ADHD). Length of recovery was determined by self and parent symptom report over time. Results: Length of recovery ranged from 0 to 48 weeks (average=5.7). Sixty-seven percent of children reported recoveries longer than 2 weeks and $22 \%$ longer than 8 weeks. Children whose recovery took longer than 8 weeks reported more severe symptoms at first evaluation $(\mathrm{t}=-$ $5.5, \mathrm{p}<.001)$ and had longer time between injury and first evaluation $(\mathrm{t}=-5.8, \mathrm{p}<.001)$. Girls reported longer recoveries $(\mathrm{t}=-2.04, \mathrm{p}<.05)$. Participants with exceptionalities did not have longer recovery times. Age and injury-related factors were not correlated with length of recovery. Conclusions: In this group, risk factors for protracted recovery included increased symptom endorsement at first evaluation, longer time before first evaluation, and female gender. Interestingly, children with pre-existing exceptionalities such as ADHD did not show longer recovery times. Further group and intraindividual analyses will lead to a better understanding of symptom recovery following pediatric concussion. 
Correspondence: Gray Vargas, Ph.D., Behavioral Health/Rehabilitation Medicine, Nemours/A.I. Dupont Hospital for Children, Nemours/A.I. Dupont Hospital for Children, 1600 Rockland Rd, Wilmington, DE 19803.E-mail: Gray.Vargas@nemours.org

M. NARAD, N. MINICH, H.G. TAYLOR, M. KIRKWOOD, T. BROWN, T. STANCIN \& S.L. WADE. Changes in Parent-Teen Interactions and Family Functioning During the Initial 24 Months Post Traumatic Brain Injury.

Objective: To examine whether Counselor Assisted Problem Solving (CAPS), an online version of Family Problem Solving Therapy (FPST), improves parent-adolescent interactions and family functioning over time relative to an Internet Resource Comparison (IRC) group in children with TBI

Participants and Methods: Participants were 132 teens with complicated mild to severe TBI $(65.3 \%$ male, $19.7 \%$ nonwhite, and $38.6 \%$ with severe TBIs; 65 treated with CAPS and 67 in the IRC condition). Measures of effective communication, problem solving, and conflict based on observed parent-child interactions and of ratings of family functioning were completed at baseline soon after injury and at 6-, 12-, and 18-months after baseline. Injury severity, SES, and child grade were examined as moderators.

Results: Mixed models analysis revealed that CAPS was superior to IRC in reducing severity of parent-adolescent conflict over time for older but not younger adolescents $(p=.04)$. CAPS was also protective of a decline over time in effective communication, but only for teens with moderate injuries $(p=.01)$.

Conclusions: Online FPST introduced shortly after injury appears to have some beneficial effects on parent-teen interactions after adolescent TBI, supporting the efficacy of this treatment approach in promoting more positive outcomes for families.

Correspondence: Megan Narad, PhD, Behavioral Medicine Clinical Psychology/Physical Medicine \& Rehab, Cincinnati Children's Hospital Medical Center, 7521 Glenover Drive, Cincinnati, OH 45236. E-mail: megan.narad@cchmc.org

A.A. GRETENCORD, S. AYLWARD, J. DORFLINGER \& J. HOPKINS. Gender Differences in Postconcussive Symptoms of Sport-related Concussions in Children and Adolescents.

Objective: Current research on concussions indicates that both younger age and female gender are associated with a greater number of symptoms and a lengthier postconcussive recovery time. The aim of this research was to examine postconcussive symptoms (PCS) resulting from a sports-related concussion in both male and female children/ adolescents. It was predicted that younger age and being female would be associated with worse performance on neuropsychological measures and more reported PCS.

Participants and Methods: Participants included 132 children/adolescents (10-18 years) who had sustained a sports-related concussion. Data was collected using neuropsychology measures (Auditory Consonant Trigrams Test, Conners' Continuous Performance Test-2nd edition, Immediate Post-Concussion Assessment and Cognitive Testing, Woodcock Johnson Tests of Achievement-Third Edition, and Behavior Assessment System for Children-2nd edition) and a neurological evaluation.

Results: Hierarchical regression analyses were conducted for each of the dependent measures. Results indicated evidence of subtle, but clinically significant, impairments in executive functioning. This was particularly true for those with a premorbid attention, learning, and/or mood disorder. In addition, a history of previous concussions was associated with a higher number of reported cognitive PCS. As predicted, female gender was associated with increased executive dysfunction and a higher report of cognitive PCS. Contrary to hypotheses, younger age was associated with less executive dysfunction and fewer reported cognitive PCS.

Conclusions: First, the majority of participants reported high levels of PCS. Second, executive functioning was the system most impacted. Third, these results emphasize the individualized nature of postconcussive recovery. A premorbid disorder, history of previous concussions, age, and gender were each identified as factors that impact the recovery process. Thus, appropriate postconcussive management must be adapted to the individual.

Correspondence: Ashley A. Gretencord, M.S., Clinical Psychology, Illinois Institute of Technology, 2451 S. Timberline Rd., Apt 5-107, Fort Collins, CO 80525. E-mail: agretencord@gmail.com

G. LALONDE, A. BERNIER, C. BEAUDOIN, J. GRAVEL \& M.H. BEAUCHAMP. The Quality of Mother-Child Interactions Six Months Post-TBI in Preschool Children.

Objective: Childhood traumatic brain injury (TBI) can have a detrimental effect on family functioning (Josie et al., 2008; Stancin et al., 2008), but little is known about the specific impact on the quality of parent-child interactions. This study assessed the quality of mother-child interactions after early TBI. It was expected that the quality of interactions between mothers and children with TBI would be lower when compared to dyads of mothers and non-injured children.

Participants and Methods: Forty preschoolers (18 to 60 months) who sustained accidental mild, moderate or severe TBI $(73 \%$ male, $\mathrm{M}=$ 42.95 months, $\mathrm{SD}=12.45$ months) were compared to 23 non-injured children ( $39 \%$ male, $\mathrm{M}=44.08$ months, $\mathrm{SD}=12.16$ months). The quality of mother-child interactions was assessed six-months post-injury during a free play activity using three components of the "Mutual Responsive Orientation' Scale (MRO; Aksan et al., 2006): Harmonious Communication, Mutual Cooperation, and Emotional Ambiance.

Results: The quality of interactions in TBI mother-child dyads was poorer than that of control dyads on the MRO Global $(\mathrm{t}(27)=-2.07$, $\mathrm{p}=.04)$, Emotional Ambiance $(\mathrm{t}(63)=-2.16, \mathrm{p}=.04)$, and Mutual Cooperation $(\mathrm{t}(63)=-3.01, \mathrm{p}=.004)$ scales. TBI mother-child dyads were less receptive to one another, not as proficient in reading each other's cues, appeared more disconnected and resistant, and had a greater tendency toward escalating conflicts.

Conclusions: These findings indicate that TBI during the preschool years may alter the quality of parent-child interactions and highlight the importance of monitoring social development after early brain injuries. Correspondence: Gabrielle Lalonde, University of Montreal, 2900 Boulevard Edouard-Montpetit, Montreal, QC H3T 1J4, Canada. E-mail: gabrielle.lalonde@hotmail.com

A. SUFRINKO, E. JOHNSON, K. PEARCE \& L. HENRY. The Influence of Sleep Duration and Sleep-related Symptoms on Baseline Neurocognitive Performance among Male and Female High School Athletes.

Objective: Due to the widespread use of computerized neurocognitive testing among athletes and their strong influence on return to play (RTP) decisions, it is vital to understand extraneous factors that influence baseline performance prior to injury. While sleep duration appears to influence baseline performance to a minimal degree, we hypothesized that examining sleep duration and sleep-related symptoms concurrently would influence baseline testing performance to a greater magnitude. Participants and Methods: A sleep problems group (i.e., 1+ sleep-related symptom(s) on the PCSS AND sleep duration $<5$ hours) and control group (i.e., 0 sleep-related symptoms and $>9$ hours sleep duration) were derived from a sample of high school athletes, ages 14-17 $(\mathrm{M}=15.26, \mathrm{SD}=1.09)$, who completed baseline Immediate Post-Concussion Assessment and Cognitive Testing (ImPACT). A 2x2 MANOVA with grouping factors of gender and sleep group (sleep problems $(n=78)$ and control group $(n=100)$ ) was employed to examine differences on neurocognitive composite scores/PCSS.

Results: A significant gender $\mathrm{x}$ group interaction (Wilk's $\lambda=.90, \mathrm{~F} 5,170$ $=3.96, \mathrm{p}=.002, \eta 2=.10)$ for verbal memory $(\mathrm{F} 1,174=5.13, \mathrm{p}=.025$, $\eta 2=.03)$, visual memory $(\mathrm{F} 1,174=7.26, \mathrm{p}=.008, \eta 2=.04)$, and visual motor speed $(F 1,174=4.09, p=.045, \eta 2=.02)$ revealed female athletes in the sleep problems group performed worse across these three 
neurocognitive composites, while female athletes in the control group performed best.

Conclusions: Clinicians should be wary of artificially deflated baseline performance among athletes with reduced sleep duration and sleep-symptoms, particularly adolescent girls, which could adversely influence return to play decisions following a concussion.

Correspondence: Alicia Sufrinko, PhD, Sports Medicine - Concussion Program, University of Pittsburgh Medical Center, 3200 S. Water St., Pittsburgh, PA 15203.E-mail: sufrinkoam@upmc.edu

\section{R.M. ROBERTS, J.L. MATHIAS \& S.E. ROSE. What White Matter Tracts are Most Affected by Pediatric TBI? A Meta-analysis of Diffusion Tensor Imaging (DTI) Research.}

Objective: Diffusion Tensor Imaging (DTI) has been used to assess microstructural changes to white matter following pediatric TBI. Many brain regions have been examined, at varying post-injury intervals, but the findings have not been consolidated. The current study meta-analyzed research that used DTI with pediatric TBI and control groups in order to identify the white matter regions that are most affected by pediatric TBI, both in the short- and medium-long term.

Participants and Methods: Fractional anisotropy (FA; measuring uniformity of diffusion) and apparent diffusion coefficient (ADC; measuring rate of diffusion) data were extracted from 20 independent studies ( $N=491$ TBI, $N=465$ controls). Cohen's d effect sizes, 95\% confidence intervals, and fail-safe $\mathrm{N}$ statistics were calculated for each region-of-interest.

Results: In the early stages after pediatric TBI (1-4 weeks), there are moderate-to-large increases in $\mathrm{FA}$, together with equivalent decreases in the ADC, in most white matter regions. In contrast, there are moderateto-very-large decreases in FA and increases in $\mathrm{ADC}$ in the longer term (3-45 months). FA values for the whole brain (short-term), cerebellum (medium-long term) and corpus callosum (medium-long term) appear to hold the greatest diagnostic potential because they appear to be most affected.

Conclusions: Many white matter regions are affected by pediatric TBI, albeit not equally, and the nature of these changes alters over time. The impact of injury severity and age/developmental stage on these metrics, and whether they accurately predict functional outcomes, remains to be determined.

Correspondence: Rachel M. Roberts, PhD, Psychology, University of Adelaide, School of Psychology, University of Adelaide, North Tce, Adelaide,SA 5005,Australia.E-mail:rachel.roberts@adelaide.edu.au

M. AMAYA-HODGES, C. MCGILL, J. SOTOMAYOR, M.D. SADY, P.K. ISQUITH \& G.A. GIOIA. Factor Structure of BRIEF Parent and Self-Report Forms for Monitoring Recovery in Children with Concussion.

Objective: To examine the factor structure of abbreviated BRIEF parent and self-report monitoring forms in youth with concussion.

Participants and Methods: Ratings of pre-injury and current executive functioning were collected from 712 youth ( $62 \%$ male; $70 \%$ white) evaluated within 30 days of concussion and their parents. Selected items from the BRIEF Working Memory, Plan/Organize, Task Completion/ Initiation, Inhibition, and Emotional Control scales were rated on an expanded 5-point dimensional scale. Parent- (ages 5-18) and self-report (ages 11-18) factor structures were first examined in post-injury reports using hierarchical confirmatory factor analysis of a previous three-factor model (Gioia et al., 2002) and were then applied to pre-injury reports. Results: On parent report, the three-factor model (i.e., Cognitive, Behavioral, and Emotional Regulation) generally fit within desired ranges for both post- $\left(\mathrm{CFI}=.91\right.$, SRMR=.05, RMSEA $90 \% \mathrm{CI}=.07$ to $.08 ; \chi^{2}$ / $\mathrm{df}=4.5)$ and pre-injury $(\mathrm{CFI}=.91$, SRMR $=.05$, RMSEA $90 \% \mathrm{CI}=.07$ to $.08 ; \chi 2 / \mathrm{df}=4.7)$ item responses. Two items were dropped due to weak factor loadings, resulting in a 29 -item parent measure. A similar three-factor, 28-item self-report form also met acceptable fit criteria for both post- $(\mathrm{CFI}=.90$; SRMR=.05, RMSEA $90 \% \mathrm{CI}=.07$ to $.08, \chi 2$ / $\mathrm{df}=3.9)$ and pre-injury (CFI=.88, SRMR=.06, RMSEA 90\% CI $=.07$ to $.08, \chi 2 / \mathrm{df}=3.9)$ item ratings. All final items exhibited strong factor loadings on each form, with moderate to large correlations between the three factors (parent $\mathrm{r}=.43$ to .56 ; self-report $\mathrm{r}=.36$ to .56 ).

Conclusions: Confirmatory factor analysis of BRIEF parent- and self-report monitoring forms supported a three-factor model in children with concussion. These findings provide preliminary support for their use in monitoring changes in executive functioning post-concussion. Correspondence: Meredith Amaya-Hodges, PhD, Memorial Hospital of RI - Brown University, 300 Front St. Unit 501, Pawtucket, RI 02860. E-mail: meredith.amaya@gmail.com

M. AMAYA-HODGES, D. SRIRAM, J. GREINER, S. ROBERTS, S. KARRAY \& G.A. GIOIA. Relations between Adolescents' Anxiety and Post-Concussion Symptom Reports.

Objective: To examine the relations between adolescents' anxiety and post-concussion symptomatology.

Participants and Methods: Adolescents ages 13-18 years ( $\mathrm{N}=74$; $53 \%$ male; $84 \%$ white) first treated within 30 days of concussion $(\mathrm{M}=12.0 \pm 7.2)$ underwent standard clinical assessment. Current and retrospective pre-injury ratings were obtained from youth and their parents on the Post-Concussion Symptom Inventory (PCSI) and an abbreviated Revised Children's Anxiety and Depression Scale (RCADS). Results: Parent and self-report ratings of generalized anxiety on the RCADS were correlated at a low level (pre-injury $r=.24, p=.04$; post-injury $r=.29, p=.01)$. Symptoms of generalized anxiety significantly increased following a concussion [parent $\mathrm{t}(73)=-4.4$, $\mathrm{p}<.001$; child $\mathrm{t}(73)=-$ $2.4, p=.02]$. When adjusting for pre-injury symptomatology, youth who experienced a larger increase in generalized anxiety symptoms following concussion were also significantly more likely to experience an increase in total post-concussion symptoms (parent $\mathrm{r}=.31, \mathrm{p}=.007$; child $\mathrm{r}=.26$, $\mathrm{p}=.03)$. While pre-injury generalized anxiety was moderately correlated with both pre- (parent $\mathrm{r}=.37, \mathrm{p}=.001$; child $\mathrm{r}=.42, \mathrm{p}<.001)$ and post-injury (parent $r=.39, p=.001$; child $r=.40, p=.001$ ) PCSI total, it did not predict a greater increase in PCSI symptoms post-concussion when controlling for pre-injury symptom levels.

Conclusions: Concussions were associated with an increase in adolescents' symptoms of generalized anxiety. Those who experienced a greater increase in anxiety following injury also tended to report more post-concussion symptoms overall. Retrospective reports of pre-injury anxiety were associated with higher pre- and post-injury scores on a concussion symptom measure. The relation between anxiety and overall post-injury symptomatology may be reciprocal, with implications for clinical assessment and management. Further exploration of this relation is warranted.

Correspondence: Meredith Amaya-Hodges, PhD, Memorial Hospital of RI - Brown University, 300 Front St. Unit 501, Pawtucket, RI 02860. E-mail:meredith.amaya@gmail.com

K. ARES, N. HARTLEY, D. SMITH, N. KARNIK \& S. HUNTER. The Mediating Role of Traumatic Brain Injury on the Relationship between Temperament and Psychopathology in Urban Homeless Youth.

Objective: Homelessness affects youths' decision making, emotion regulation, and coping. Maladaptive temperamental traits also develop, that further impact youths' ability to adjust to adverse environmental and social challenges. TBI is not an uncommon outcome of such challenges for some homeless youth. This study examined the relationship between temperamental negative affectivity and effortful control, psychopathology, and TBI.

Participants and Methods: 93 young adults, aged 18-22, with an acknowledged head injury history, were recruited as part of a larger study of homeless shelter-residing youth in Chicago, IL and Los Angeles, CA. Participants completed the Adult Temperament Questionnaire (ATQ) and the Mini International Neuropsychiatric Interview (MINI) to assess current and past psychopathology 
Results: Analyses revealed significant correlations between negative affectivity and depression $(\mathrm{r}=.050, \mathrm{p}<.05)$ and anxiety $(\mathrm{r}=.018, \mathrm{p}<.05)$. There were no significant relationships between effortful control and psychopathology. TBI was significantly correlated with anxiety $(\mathrm{p}<.05$, phi=.283). A significant interaction was found between negative affectivity and TBI when predicting anxiety disorder occurrence ( $p=.002$. $\mathrm{B}=1.015)$.

Conclusions: Findings suggest that negative temperamental traits in homeless youth increase their likelihood of developing internalizing disorders, with head injury playing a mediating role in regard to anxiety. These findings may help to better guide treatment recommendations, particularly regarding the maladaptive impact of head injury on internalizing behaviors in homeless youth.

Correspondence: Kathleen Ares, M.A., The Chicago School of Professional Psychology, 1360 North Lake Shore Drive, apt 415, Chicago, IL 60614. E-mail:kathleenares@gmail.com

G.A. GIOIA, P.K. ISQUITH, A. BURNS \& J. SOTOMAYOR. EvidenceBased Approach to Detecting Concussion in Children: Combining Symptom Reports and Cognitive Performance.

Objective: To examine an evidence-based, multidimensional approach to evaluating concussion in children, exploring detection rates using standardized symptom ratings and cognitive performance separately and in combination.

Participants and Methods: 85 children (84\% male; 8-18 years) with documented concussions seen within 14 days of injury were compared with age- and sex-matched controls. The Tasks of Executive Control (TEC), an N-back working memory and go/no-go task, was administered while the child's parent completed symptom ratings on the Post-Concussion Symptom Inventory (PCSI).

Results: Initial MANOVA's reveal parent PCSI ratings explained 38\% of variance while TEC Response Time (RT) and variability (RTSD) each explained $6 \%$ and $7 \%$, respectively. Discriminant functions showed a model with Parent PCSI and TEC RT, RTSD, working memory accuracy and response to increased working memory demand accurately ruled out 99\% of controls while detecting 68\% of injured children (Positive Likelihood Ratio $=58$, Odds Ratio=180, Classification Accuracy $=84 \%)$. ROC analysis showed a $6 \%$ false positive rate at $80 \%$ sensitivity ( $\mathrm{AUC}=.93$ ). The TEC alone identified $65 \%$ of injured children but ruled out only $60 \%$ of controls correctly, while the PCSI ruled out nearly all non-injured cases but identified only $50 \%$ of injuries.

Conclusions: An evidence-based multidimensional assessment approach combined parent symptom ratings with children's working memory performance offers substantial advantages over either one approach alone. Parent PCSI and children's TEC performance showed good specificity and sensitivity with a very strong odds ratio in differentiating injured from matched controls. Children with symptoms who perform slowly, more variably and with difficulty keeping pace with increasing demands on the TEC are substantially more likely to have concussion effects.

Correspondence: Gerard A. Gioia, PhD, Neuropsychology, Children's National Health System, 15245 Shady Grove Road Suite 350, Rockville, MD 20850.E-mail: ggioia@cnmc.org

\section{Autism Spectrum Disorders}

S. ADACHI, Y. IKEDA, I. FUJITA, K. HASHIMOTO \& M. NOTOYA. Language Development in School-aged Children with Pervasive Developmental Disorders: Examining Relationships among Syntactic, Vocabulary, and Speech Abilities.

Objective: [Objective] Language development in children with pervasive developmental disorders (PDD) has rarely been discussed based on the evaluation of language, examining each phase of language functions from comprehensive perspectives. This study aimed to clarify relationships among the development of syntactic, vocabulary, and speech abilities.

Participants and Methods: [Methods] Seventeen male school-aged children with PDD, aged 6 years and 2 months to 9 years and 0 months (mean: 7 years and 2 months) were studied, all of whom had been diagnosed with PDD by doctors during infancy. They underwent the following tests: the Syntax Test of Aphasia (STA), Picture Vocabulary Test (PVT), a verb test as part of the Test of Lexical Processing in Aphasia, Test of Question-Answer Interaction Development (Q\&A) based on the S-S method, Raven's Colored Progressive Matrices (RCPM), and Auditory

Memory Span as part of the WISC-III (AMS). Following instruction in the procedures, speech-language-hearing therapists individually conducted these tests.

Results: [Results] 1) Decreases in scores were observed in all tests, except for the intelligence test; 2) STA auditory comprehension was associated with sentence production, PVT, verb comprehension, Q\&A, and AMS, while STA sentence production was associated with auditory comprehension, verb comprehension and expression, PCPM, Q\&A, and AMS; and 3) the syntactic comprehension level was: I(word-meaning) in $8, \mathrm{II}$ (word-order) in 7 , and III (particle)in 2; none were defined in level IV (particle-complement sentences).

Conclusions: Based on these results, speech impairment in children with PDD may be associated with difficulty in appropriately acquiring vocabulary and syntactic abilities.

Correspondence: , . E-mail:

M.T. ACOSTA. Autism Spectrum Disorders (ASD) in Neurofibromatosis Type 1 (NF1): Clinical and Neurobiological correlations.

Objective: 1 . Review current evidence that demonstrate a high incidence of ASD symptoms and diagnoses in NF1 patients. 2. Present neurobiological evidence supporting the hypothesis that alterations in the NF1 gene impacting brain development are responsible for the ASD symptomatology.

Participants and Methods: Several international groups have reported a high incidence of ASD symptoms in NF1 populations. Current neurobiological knowledge from mice models in NF1, aim to explain the developmental impact of NF1 gene. Comparison of clinical and neurobiological findings in NF1 with typical ASD studies aim to propose a hypothesis for this association. A review of these results and methodologies will be presented.

Results: ASD incidence in NF1 is higher than expected, with a frequency of ASD symptoms in up to $40 \%$ of the population, and full criteria diagnosis of around $20 \%$. Clinically, patients with NF1 and ASD patients share significant deficits in executive function, cerebellar function, social cognition, and others. Genetic studies in large samples of ASD populations have demonstrated that several genes part of the RAS Pathway, including the NF1 gene; have been found to be associated with ASD. Further identification of behavioral, neurobiological, and neuroimaging data in patients and mice models of NF1 may be useful to explain the neurobiology of ASD.

Conclusions: Incidence of ASD is higher than previously described in children with NF1. Neurobiological and clinical correlations in NF1 and ASD may advance our understanding of this association. The high association of ASD symptoms in a single gene defect as NF1 may open the opportunity to explore neurobiological and genetic imprints in ASD. New available technologies may facilitate testing of potential pharmacological options to treat ASD.

Correspondence: Maria T. Acosta, MD, Neurology, Children's National Health System - The George Washington University, 111 Michigan Ave, NW,Washington,DC20010.E-mail:macosta@childrensnational.org 
B. CASTElluCCiO, M. STEVENS, I. EigSTi, R. SCHULTZ, L. NAIGLES, E. KELLEY \& D. FEIN. Characterizing the White Matter Tract Integrity of Youth with a History of Autism Spectrum Disorder Who Have Achieved Optimal Outcome.

Objective: A minority of persons with an autism spectrum disorder (ASD) lose the diagnosis, a phenomenon referred to as "optimal outcome" (OO). Ongoing research is revealing clinical differences between $\mathrm{OO}$ and ASD youth, but the neural basis of the phenomenon is currently unknown. Prominent among hypothesized neuroanatomical substrates of ASD are abnormalities in the brain's white matter connections. This study evaluated white matter tract integrity in $\mathrm{OO}$ youth to determine whether it differed from those with ASD

Participants and Methods: The three age- and IQ-matched study groups comprised 14 typically developing (TD), 22 high functioning ASD, and 11 youths with a well-established early childhood history of an ASD who no longer meet diagnostic criteria (OO). Participants underwent magnetic resonance diffusion tensor imaging (DTI). Anatomical prior-guided probabilistic tractography (TRACULA) localized 18 major white mater tracts. Mean tract fractional anisotropy (FA) values were extracted for each subject, and one-way ANOVA compared groups.

Results: Compared to youths with high functioning ASD, OO youths had reduced FA in left and right hemisphere cingulum bundles and the right inferior longitudinal fasciculus. Compared to TD youths, OO youths had lower FA in the right cingulum bundle and both left and right inferior longitudinal fasciculi. No differences were identified between youths with ASD and TD youths.

Conclusions: The presence of anatomical connectivity differences in $\mathrm{OO}$ raises the possibility that they contribute to achievement of OO. Provided they can be replicated, these findings can guide future research seeking to understand the relationship of white matter integrity to OO. Correspondence: Brian Castelluccio, Psychology, University of Connecticut, 406 Babbidge Road, Unit 1020, Storrs, CT 06269-1020. E-mail:brian.castelluccio@uconn.edu

K. STEPHENSON, M. MAISEL \& M. SOUTH. Alexithymia as a Predictor of Dimensional Scales of Autism Symptoms.

Objective: Alexithymia is characterized by difficulties identifying, expressing, and feeling emotional states. In addition to core clinical symptoms of autism spectrum disorders (ASD), researchers have found high rates of comorbid anxiety, depression, and alexithymia symptoms. However, there is little research investigating the association between these constructs and how they relate to dimensional measures of ASD symptoms. The goal of this study was to clarify this relationship.

Participants and Methods: Participants were 23 adults diagnosed with ASD as well as 20 neurotypical controls. All participants completed a battery of questionnaires including the Toronto Alexithymia Scale (TAS-20), the Autism Spectrum Quotient (AQ), the Social Responsiveness Scale (SRS-2), the State-Trait Anxiety Inventory (STAI), and the Beck Depression Inventory (BDI). We used multiple regression analyses to predict AQ and SRS-2 scores using alexithymia, anxiety, depression, and diagnosis as predictors.

Results: Multiple regression analyses indicated that both alexithymia and diagnosis were significant predictors of both AQ and SRS-2 scores when controlling for anxiety and depression. Furthermore, alexithymia emerged as a better predictor of dimensional ASD symptoms than categorical diagnosis. Additional Pearson correlations showed differing patterns between the study variables among the two diagnostic groups. In particular there appears to be different relationships between subscales of the TAS-20 and the SRS-2 in individuals with ASD.

Conclusions: Overall, alexithymia appears to be a robust predictor of dimensional measures of autism symptoms, even when controlling for related anxiety and depression symptoms. This evidence emphasizes the connection between autism and alexithymia and suggests the importance of an assessment of alexithymia in both clinical and research settings.
Correspondence: Kevin Stephenson, Psychology, Brigham Young University, 245 TLRB, Provo, UT 84602.E-mail:kevin.g.stephenson@ gmail.com

\section{A. HEFFELFINGER, J. KOOP, J. SCHUH \& L. LESCHKE. Factors} Influencing Age of Evaluation for Autism.

Objective: Ideal outcomes for children with autism spectrum disorder (ASD) are maximized by early diagnosis and treatment. The goal is for all children to be screened at 24 months, yet most do not receive formal diagnosis until 4 years (Lord et al., 2006). Many factors influence age of diagnostic evaluation.It was hypothesized that $\mathrm{A}$ ) children with medical/ neurological disorders (NEURO) were evaluated later than children without medical/neurological disorders; and B) socioeconomic factors influence age of first evaluation.

Participants and Methods: In a retrospective chart review of 67 preschool age children (mean age $=43.8+/-15.6$ months) seen for a neuropsychological evaluation with concerns for symptoms of ASD, 34 received a diagnosis of ASD.

Results: In this clinic, there was no difference in age between those in the NEURO group and those without $(42.93+/-16.5,44.6+-15.2$, respectively; $p>05)$. Of the 27 children with medical or neurological disorders, 10 were diagnosed with ASD (37\%). Of the 39 children without a medical/neurological disorder, $23(59 \%)$ met criteria for a diagnosis of ASD. These results demonstrated the need to evaluate children with medical/neurological disorders as well as children without. Race/ethnicity had a trend towards having differing age of evaluation $(\mathrm{F}=2.69, \mathrm{p}=$ 0.054 ), with children identified as Hispanic being seen at the youngest age (36.57+/-13.88 mo), Caucasian at $m=42.15+/-14.25 \mathrm{mo}$, and African American at the oldest age at 52.00+/-17.94 mo. SES as assessed through parent education did not differ.

Conclusions: In this community there is an active group of pediatricians that screen for ASD in their Hispanic patients, resulting in referrals at a younger age. PMDs need to be educated and encouraged to conduct the AAP recommended screening consistently at 24 months, especially within the African American population in Southeastern Wisconsin. These results suggest that there are several factors that likely influence age of ASD evaluations, particularly race.

Correspondence: Amy Heffelfinger, Medical College of Wisconsin, 9200 W.Wisconsin, Milwaukee, WI, WI 53212.E-mail: aheffelfinger@mcw. edu

M. MAISEL, M. SOUTH \& K. STEPHENSON. Dispositional Mindfulness Predicts Anxiety in People Diagnosed with Autism Spectrum Disorder.

Objective: Converging findings show that individuals diagnosed with autism spectrum disorders (ASD) often experience severe comorbid anxiety. Other research suggests that mindfulness skills (paying attention to the present moment in a non-judgmental way), are associated in neurotypical people with less anxiety and stress. Studies of potential moderators in ASD, such as mindfulness, can serve to fill the gap in current research of effective treatments for the comorbid anxiety often seen in ASD. This is the first study examining how dispositional mindfulness moderates anxiety in ASD.

Participants and Methods: Participants included 21 adults who have been diagnosed with ASD and a control group (CON group) consisting of 20 participants who reported no history of psychological diagnoses. Participants completed a brief intelligence test (WASI-II) and also a demographics form, the State Trait Anxiety Inventory, the Five Facet Mindfulness Questionnaire, and the Social Responsiveness Scale.

Results: Multiple regression showed that for both the ASD and CON group specific facets of mindfulness significantly predicted anxiety, while controlling for the severity of core autism symptoms. Mindfulness was shown to affect the two groups differently: the mindfulness facets of "observe" and "non-reactivity" significantly predicted anxiety in the ASD group, and the facet of "non-judging" significantly predicted anxiety in the CON group. 
Conclusions: These findings suggest that the current trend of incorporating mindfulness in psychological interventions may have utility in ASD populations. Moreover, as seen in this study, mindfulness may moderate anxiety differently in ASD people from neurotypical people. These findings may be helpful in tailoring interventions specifically for the comorbidity seen in ASD populations.

Correspondence: Max Maisel, Clinical Psychology, Brigham Young University, 165 North 1650 West \#i-104, Pleasant Grove, UT 84062. E-mail:max.e.maisel@gmail.com

\section{MACMULLEN FREEMAN, E. ROTHERAM-FULLER, J. LOCKE \& D. MANDELL. The Association between Executive Functions and Social Skills in Children with Autism Spectrum Disorder.}

Objective: Executive deficits in children with autism spectrum disorder (ASD) have been widely noted in the literature; however, few studies, have investigated the underlying neuropsychological components associated with these difficulties. This study examined the association between executive functions and social skills in children with ASD to understand the nature of these deficits and inform the development of effective interventions.

Participants and Methods: Participants included 23 children (19 males) with ASD who were fully included in first through fifth grade general education classrooms (ages 5-12). The mean age was 8.6 years $(\mathrm{SD}=1.8)$. Participants were administered the DAS-II and the NEPSY-II to measure shifting, inhibition, and working memory skills. Teachers completed The Behavior Rating of Inventory of Executive Function (BRIEF) questionnaire. Two social outcomes were measured: 1) playground engagement using direct observations; and 2) social network inclusion using peer nominations.

Results: Several BRIEF scales were associated with social functioning. Initiation was positively correlated with solitary play $(r=.52)$ and Working Memory was negatively correlated with time spent jointly engaged $(\mathrm{r}=-.44)$. Poor Working Memory $(\mathrm{r}=.54)$, Planning and Organization $(\mathrm{r}=.48)$, and Organization of Materials $(\mathrm{r}=.62)$ were all associated with an increase in peer rejection. No significant associations emerged between executive functions and overall social connectedness in the classroom

Conclusions: Executive skills (measured by teacher-report) may strongly affect peer interaction on the playground and peer rejection. Thus, social interventions may need to directly target these skills in an effort to increase social engagement and acceptance.

Correspondence: Laura MacMullen Freeman, Temple University, 1200 14th St Apt 2A, Miami Beach, FL 33139.E-mail: lauramacfreeman@ gmail.com

A.R. CARLEW \& T.D. PARSONS. Title: The Virtual Reality Classroom for the Assessment of Autism Spectrum Disorders.

Objective: Although the specific pattern of executive function deficits in Autism Spectrum Disorder (ASD) is not yet known, the more solid generalizations about executive functioning in ASD reflect a disturbance of "social executive" functioning that involves frontal motivation, self-monitoring, and volitional attention regulation. In this study, we aimed to compare performance of individuals with ASD to typically developing individuals using the VR Classroom Stroop Task.

Participants and Methods: Eight individuals diagnosed with ASD and eight neurotypical university students were administered both traditional versions of the Stroop task (paper-and-pencil and computerized) and the VR Classroom Stroop task. Within the VR Classroom, participants are asked to click a mouse button if the color of the stimulus presented matches the color spoken by the "virtual teacher." Ecologically valid auditory, visual, and audio-visual distractors are presented around the environment in the distraction condition.

Results: The Classroom succeeded in eliciting an interference effect from both the ASD group $\mathrm{F}(1,7)=15.832, \mathrm{p}<.001$, and the neurotypical group, $\mathrm{F}(1,7)=19.33, \mathrm{p}<.001$. However, significant differences in performance between the two groups were not observed.
Conclusions: The VR Classroom successfully elicited an interference effect using Stroop stimuli in both populations, suggesting it may be used as a measure of interference control. These results indicated individuals with ASD do not differ significantly from TD individuals in their ability to inhibit irrelevant information.

Correspondence: Anne R. Carlew, Psychology, Univerisity of North Texas, 940 West Round Grove Rd, 1425, Lewisville, TX 75067. E-mail: anne.carlew@gmail.com

M.J. PAGE \& A. CAMODECA. Executive Functioning Profiles in a Clinical Sample of Youth With and Without Autism.

Objective: Compared to their typically developing peers, children with Autism Spectrum Disorder (ASD) demonstrate executive functioning (EF) deficits (Rosenthal et al., 2013; Kretschmer, et al., 2014). Research has additionally suggested that, even in non-clinical populations, those with higher levels of ASD symptoms report more severe and more global weaknesses regarding EF (Camodeca et al.. 2011). While research attests to EF weaknesses as a secondary symptom of ASD (Geurts, et al., 2014), it may be that children with ASD demonstrate more global or severe EF weaknesses, even when compared to other clinical groups. The purpose of the present study is to compare parent-reported deficits in EF among youth with ASD with deficits among other clinical. non-ASD youth

Participants and Methods: Parents of 268 youth aged 6-18 who were referred for a comprehensive neuropsychological evaluation completed the Behavior Rating Inventory of Executive Function (Gioia et al., 2000). The sample was comprised of 69 youth diagnosed with ASD (82.6\% male) and 169 youth who did not receive an ASD diagnosis $(69.2 \%$ male $)$

Results: Group averages fell within the clinically significant range (T-score $\geq 65$ ) on 7 of the 8 subscales in the ASD sample; Organization of Materials fell within normal limits. For the non-ASD sample, only the Working Memory subscale fell within the clinically significant range. Independent samples T-tests indicated significant $(p<.01)$ between-group differences on all subscales except Organization of Materials $(p=.063)$. The largest mean differences were observed for the Shift (16 points) and Emotional Control (13 points) subscales.

Conclusions: These results provide support for weaknesses in cognitive flexibility and emotion regulation observed in youth with ASD. These results also highlight the severity of EF deficits when compared to other clinical populations

Correspondence: Matthew J. Page, PhD, Psychiatry, Allegheny General Hospital, 707 Duncan Ave, Apt 415, Pittsburgh, PA 15237. E-mail: matthewjlpage@gmail.com

\section{K. BRADBURY, T. DUMONT-MATHIEU, M. BARTON \& D. FEIN. A Comparison of High- and Low-Risk Children with Autism Spectrum Disorder.}

Objective: Due to elevated recurrence rates in high-risk younger siblings of children with ASD, the population is frequently used to examine early ASD symptomatology. However, the representative nature of this population compared to the general population of children with ASD is unknown. Previous research suggests that parents of children with ASD raise concerns about the development of subsequent children earlier than first-time parents or parents of typically developing children. Whether an actual discrepancy exists in the attainment of milestones in these populations has not yet been assessed. The current study proposes to compare high- and low-risk children with ASD with respect to behavioral differences and milestone attainment.

Participants and Methods: A high-risk sample of 23 children with ASD who had an older affected sibling was compared to a matching sample of 23 children with ASD drawn from a low-risk group. Samples were matched on age, gender, and maternal education. T-tests were utilized to detect differences between groups on ASD symptomatology, cognitive ability, adaptive functioning, and milestone attainment. 
Results: No group differences on ASD symptom severity were observed, although high-risk children had fewer communication-related symptoms than low-risk children and significantly higher cognitive scores. Adaptive functioning and milestone attainment appeared similar across groups.

Conclusions: High-risk children may be higher functioning than children drawn from the low-risk sample in their communicative skills and cognitive abilities. This may be partially related to high-risk parents' increased sensitivity to early signs of language and cognitive delay and may have implications for the generalizability of results from high-risk samples.

Correspondence: Kathryn Bradbury, University of Connecticut, 406 Babbidge Rd, Unit 1020, Storrs, CT 06269-1020. E-mail: kathryn. bradbury@uconn.edu

\section{E. MOULTON, M. BARTON \& D. FEIN. Developmental Trajectories in Toddlers with ASD.}

Objective: In order to best inform parents and clinicians, we must enhance of our understanding of the developmental trajectories of children with ASD.Emerging literature indicates that a subset of children with a documented ASD lose their diagnosis in toddlerhood and function in the average range of cognition and behavior (Optimal Progress, OP). The present study seeks to characterize and compare the developmental trajectories of these children and children who remain on the spectrum (ASD-ASD).

Participants and Methods: Participants include children who were evaluated at ages 2(T1) and 4(T2) following positive ASD-screening. Children (OP, ASD-ASD, N=19 each) were matched on gender, age, diagnosis and symptom severity at T1.The Autism Diagnostic Observation Schedule, Mullen Scales of Early Learning, Vineland Adaptive Behavior Scales (VABS-II), and Childhood Autism Rating Scale were completed at T1 and T2.

Results: At T1, the two groups demonstrated comparable adaptive and cognitive abilities ( $p>.05$ for each domain of the VABS and Mullen). Between T1 and T2, in each domain, the OP group demonstrated a steeper pattern of growth $(p<.05$ for each). On average, the OP group demonstrated an increase of 1.31 SD in VABS total scores, whereas the ASD-ASD group demonstrated an average decrease of 0.25 SD.The OP group demonstrated an average increase of 2.47SD in Mullen composite scores, whereas the ASD-ASD group demonstrated an average increase of .75SD

Conclusions: A range of outcomes are possible for children diagnosed with ASD.Children in the OP group demonstrated significant increases in cognitive and adaptive skills over 2 years.Children in the ASD-ASD group showed mild improvements in cognitive abilities, with less growth in adaptive skills.Importantly, the OP group demonstrated a steeper growth trajectory even when controlling for age- 2 symptom severity, indicating that differences in trajectory may be related to additional factors including subtle differences in adaptive skills, or other child and intervention factors.

Correspondence: Emily Moulton, Ph.D., Psychology, University of Connecticut, 406 Babbidge Road, Storrs, CT 06269. E-mail: emily. moulton@uconn.edu

\section{LEE \& J. BO. Visuomotor adaptation in children with Autism Spectrum Disorders.}

Objective: Children with Autism Spectrum Disorders (ASDs) often demonstrate significant motor dysfunctions and impaired motor learning; however, previous studies using prism and force field adaptation tasks have reported intact ability in motor learning (Gidley Larson, et al, 2008; Haswell, et al, 2009). Motor impairment may be overlooked due to great variability within (e.g., inconsistent performance) and between individuals (e.g., heterogeneity of ASDs). Furthermore, participants with ASDs may rely more on proprioception than vision when updating internal mapping. In light of the limitations, the current study employed a computerized visuomotor task to examine adaptation in children with ASDs.

Participants and Methods: Thirteen children with ASDs, age 8 to 12 , and thirteen age-, gender-, and IQ-matched controls completed a center-out pointing task with the visual feedback of the hand movement rotated $30^{\circ}$ counter-clockwise. The adaptability was measured by the after-effect on variables of motor planning (directional error [DE], the maximum directional deviation of the actual from the ideal movement) and motor control (root mean square error [RMSE], the average pointto-point spatial deviation of the actual from the ideal movement).

Results: Results revealed that children with ASDs demonstrated significant improvement in motor control (RMSE, p<.01) with minimal gain in motor planning (DE, $\mathrm{p}=.067)$, whereas controls demonstrated significant learning in both motor control and planning (all $\mathrm{p}<.005)$.

Conclusions: Children with ASDs showed less adaptability than controls in both motor planning and control, suggesting impaired visuomotor adaptation when learning heavily relies on visual inputs. Future study is needed to verify the findings and to uncover the underlying deficits of motor learning in children with ASDs.

Correspondence: Chi-Mei Lee, Master, Psychology, Eastern Michigan University, 1687 Broadway St., Apt. 402, Ann Arbor, MI 48105. E-mail: marg323.tw@gmail.com

K. PATRICK, F. AGATE, D.D. BOSENBARK, F. HUREWITZ \& M.T. SCHULTHEIS. Driving Behaviors of Young Adults with Autism Spectrum Disorders.

Objective: Although many young adults with autism spectrum disorders (ASD) are interested in driving, their families are often reluctant to seek licensure. Literature on ASD and driving is scarce and primarily based on questionnaires. We examined realistic driving behaviors using a virtual reality driving simulator.

Participants and Methods: As part of an ongoing study, 10 non-licensed young adults aged $18-24(\mathrm{M}=20.2, \mathrm{SD}=1.93 ; 80 \%$ Male $)$ either diagnosed with ASD $(n=5)$ or demonstrating typical development completed a driving simulator protocol. Participants first drove a rural route with no distractions. Participants then drove the same route with the addition of distractor tasks including changing the radio station several times, orally listing words beginning with "S," and maintaining conversation with the examiner. Speed and lane position were recorded and averaged over each quarter mile section.

Results: Mixed effects modeling revealed significant diagnosis by drive interaction effects ( $p s<.01)$. Specifically, during distractor tasks, young adults with ASD reduced their speed to nearly $5 \mathrm{mph}$ below the speed limit while controls maintained speeds near the speed limit. In addition, distractor tasks led to high variability in lane position for ASD participants but did not affect lane variability for controls.

Conclusions: In a preliminary sample, non-licensed young adults with ASD had significantly more difficulty maintaining appropriate speed and lane position while driving than controls when presented with realistic distractions. Findings indicate the need for continued research to determine whether specialized driving instruction programs may benefit individuals with ASD.

Correspondence: Kristina Patrick, M.S., Drexel, 506 S 41st St 2M, Philadelphia, PA 19104.E-mail: kristina.e.patrick@gmail.com

\section{A.E. WAGNER \& G. ALLEN. Neurocognitive Subtypes of ASD.}

Objective: Previous research has emphasized the importance of defining a cognitive profile of strengths and weakness in ASD, yet capturing the heterogeneity in one model may be unrealistic. This exploratory study aims to determine whether distinct neurocognitive profiles/subtypes exist among a small group of individuals with high functioning ASD by examining performance across a comprehensive neuropsychological test battery.

Participants and Methods: Participants were 20 young adult males (ages 18 to 24) with high functioning ASD. An initial hierarchical cluster 
analysis was followed up with a k-means cluster analysis. Initial cluster centers were randomly generated with up to 20 iterations allowed. Results: The 3-cluster solution was selected as providing the best separation between clusters. C1 denoted subjects with strengths in general intellectual ability (FSIQ), reasoning, and receptive and expressive language; and a weakness in fine-motor skills. C2 included subjects with high FSIQ scores and low scores on verbal learning/memory, visual learning/memory, and fine-motor skills. C3 was comprised of 2 subjects with low performance on tests of reasoning, cognitive flexibility, working memory, verbal learning/memory, expressive and receptive language, and visuospatial processing.

Conclusions: This study highlights the well-documented heterogeneity across ASD and suggests a method for parsing a sample of ASD subjects into smaller and more meaningful homogeneous groups using neuropsychological tests. Diagnostic clarification in the form of a "neurocognitive subtype" could give a client and their family useful information about cognitive strengths and weaknesses and provide service providers with direction for treatment and intervention planning. Variations in cognitive ability among the three clusters may be related to differences in underlying brain functioning and/or connectivity, thus future directions should include comparisons of functional imaging (fMRI) and brain connectivity (DTI, fcMRI) among cognitive subtypes of ASD. Correspondence: Amanda E. Wagner, School Psychology, Educational Psychology, University of Texas at Austin, 1310 Cloverleaf Drive, Austin, TX 78723. E-mail: amandaelizabethwagner@gmail.com

\section{A.E. RICHARD \& R. LAJINESS-0'NEILL. Visual Attention Shifting in Autism Spectrum Disorders.}

Objective: Abnormal visual attention is consistently observed in autism spectrum disorders (ASD). An understanding of specific aspects of visual attention that are abnormal in ASD may inform an understanding of aberrant neural networks in this disorder. This study investigated a deficit in attention shifting in ASD compared to neurotypicals (NT).

Participants and Methods: Participants were 20 ASD and 20 ageand gender-matched NT ages 7-14, who completed CogState Detection, Identification, and Set Shifting, as well as visual search (VS) and Navon tasks with attention shifting demands. VS consisted of targets defined in one of two dimensions; Navon required identifying a global or local target letter. Reaction time (RT) for attention shifting trials was compared to RT for non-shift trials.

Results: ASD and NT displayed similar shifting effects (i.e., slower RT on shift than non-shift trials) on VS and Navon. For NT, a correlation between Navon and VS RT difference trended toward significance $(r$ $=.39, p=.051)$; for ASD, Navon RT difference was correlated with Set Shifting $(r=-.59, p<.01)$. Interactions were observed between Identification and VS trial type, and between Detection and Navon trial type (i.e., larger shifting effects associated with slower processing/ psychomotor speed).

Conclusions: Results showed a typical shifting response rather than a deficit in attention shifting in ASD. Relationships between measures of shifting suggest ASD may use higher-order processing for attention shifting, while NT may use lower-level processing. Relationships between attention shifting and psychomotor and processing speed may be related to efficiency of short- and long-range neural networks.

Correspondence: Annette E. Richard, B.A., Psychology, Eastern Michigan University, EMU Psychology Clinic, 611 W. Cross St., Ipsilanti, MI 48197.E-mail: annette.e.richard@gmail.com

E.R. SEPPÄ, A. PESONEN, K. HEINONEN, R. PYHÄLÄ, P. HOVI, S. ANDERSSON, E. KAJANTIE \& K. RÄIKKÖNEN. The Association between Autism Spectrum Traits and Visual Processing in Young Adults with Very Low Birth Weight: The Helsinki Study of Very Low Birth Weight Adults.

Objective: There is growing evidence of autism spectrum disorders (ASD) being associated with atypicalities in visual processing. Preterm birth is a risk factor for psychological (e.g. autism spectrum) and neurocognitive (e.g. visual processing) disorders. We examined whether young adults born preterm at very low birth weight (VLBW; $<1500 \mathrm{~g}$ ) show an association between autism spectrum traits and visual processing skills, and whether the effects of AGA/SGA status moderate any of the associations.

Participants and Methods: 105 VLBW young adults of the Helsinki Study of Very Low Birth Weight Adults completed the Autism-Spectrum Quotient $(\mathrm{AQ})$ yielding two sum scores. They also completed the Rey-Osterrieth Complex Figure Test (ROCF) yielding three time scores, raw scores and residual memory scores. The effect of AQ scores on ROCF test scores was examined using linear regression analysis and necessary adjustments were made.

Results: The more autistic traits the VLBW group reported on the AQ Total sum score and Social interaction sum score, the slower they performed on all ROCF tasks ( $\mathrm{P}$-values $<0.05)$. The AGA/SGA status did not moderate the associations ( $\mathrm{P}$-values $>0.06)$.

Conclusions: Among adults born preterm at VLBW, the more autism spectrum traits they report, the slower they perform in visual tasks. Being born AGA or SGA does not modify the effects of autism spectrum traits on visual processing. These findings could indicate an underlying ability to process social situations visually affecting both the AQ scores and ROCF performance among adults born preterm at VLBW.

Correspondence: , . E-mail:

\section{T. DUFFIELD, H. TRONTEL \& E.D. BIGLER. Trails Performance in ASD.}

Objective: Salthouse (2011) has identified that processing speed and fluid cognitive abilities both contribute to successful trail making performance (i.e., A and B). However, it was also found using a contextual analysis that the simple difference $(\mathrm{B}-\mathrm{A})$ primarily reflects speed and that a ratio (B/A) does not completely eliminate the influence of speed, but it does so to a much greater extent than the simple difference. The aim of the current investigation was to apply the Salthouse (2011) contrasts (i.e., B - A and B/A) to the trail making task in an ASD sample as the literature is mixed concerning basic examination of Trails A and B performance in this population.

Participants and Methods: The Trail Making Test (TMT) was given to participants as part of a larger battery. Test administration was adhered to as outlined by Reitan (1958). T-tests were run to assess group differences on Trails A and B. Next, a logistic regression was run to examine group membership using speed (B - A) and fluid cognitive abilities (B/A) as predictors (Salthouse, 2011). Age was also included in the model. Results: Age and errors were not significantly different between the groups. ASD demonstrated significantly poorer performance on Trails A $\mathrm{t}(135)=1.60, \mathrm{p}=.02$. Trails B $\mathrm{t}(135)=1.96, \mathrm{p}=.05$, and FSIQ $\mathrm{t}(135)$ $=4.80, \mathrm{p}<.001$. Only FSIQ remained significant after controlling for family-wise error rate. The full model containing the three predictors (speed, fluid cognitive abilities, age) was statistically significant, $\chi 2$ (3, $\mathrm{N}=137)=12.63, \mathrm{p}=.006$, indicating the model was able to distinguish between TDC and ASD.

Conclusions: The findings indicated that both speed and fluid cognitive abilities, as derived from trail making contrasts, are reduced for those on the autism spectrum compared to typically developing individuals. Explanation of FSIQ group differences using the Salthouse (2011) contrasts are discussed.

Correspondence: Tyler Duffield, M.A., Brigham Young University, 1210 Harrison Ave, Salt Lake City, UT 84105. E-mail: tduffield2009@yahoo. com

L.D. HAISLEY, L. HERLIHY, E. TROYB, K. KNOCH, M. BARTON \& D. FEIN. Executive Functioning Deficits Associated with Parenting Stress: Parents of Children with ASD.

Objective: Studies investigating parenting stress in parents of children with autism spectrum disorder (ASD) show links to symptom severity, externalizing behaviors, and deficits in adaptive skills. Although neuropsychologists have documented the presence of executive function (EF) 
deficits in children with ASD, no studies have looked at the relationship between EFs and parenting stress. As parenting stress has direct implications for intervention gains, this is an important area of inquiry. Participants and Methods: Participants ( $\mathrm{N}=40$ ) were diagnosed with an $\mathrm{ASD}$ at 2 years $(\mathrm{M}=26.23$ months, $\mathrm{SD}=3.76)$ as part of a larger longitudinal study. Participants' diagnoses were confirmed at 4 (M=51 months, $\mathrm{SD}=5.28)$. At the final evaluation $(\mathrm{M}=9.87$ years, $\mathrm{SD}=0.81)$, 22 children were given direct EF measures (NEPSY-II: Inhibition, Animal Sorting, Word Generation; NEPSY: Tower), and 35 parents were given a questionnaire assessing the child's EF skills (BRIEF). ASD symptoms and cognitive skills were assessed (Vineland-II, CARS, ADOS and DAS-II/Stanford Binet). Parenting stress was measured with the Parenting Stress Index-Short Form (PSI).

Results: Spearman's correlations showed the PSI was related to Word Generation and Inhibition $(\rho=-.52$ and -.61). There was a significant relationship between the PSI and the Behavioral Regulation Index subscales (range: $\rho=.46-.59$ ) and most Metacognition Index subscales (range: $\rho=.41-.51$ ). A regression model using significant associations showed that BRIEF Index scores and Word Generation predicted PSI totals above ASD symptom severity or cognitive skills and accounted for an additional $39 \%$ of the PSI variance.

Conclusions: Parent reported EFs, and measured fluency predicted parenting stress over deficits in adaptive skills, ASD symptoms and cognitive skills. Neuropsychologists are in an important role to address these concerns with parents as they are frequently the providers who measure executive function and make recommendations for treatment and family support.

Correspondence: Lauren D. Haisley, B.A., University of Connecticut, 12 Stone Pond RD, Tolland, CT 06084.E-mail: lauren.haisley@uconn.edu

D.N. ABRAMS, D.L. ROBINS, L.B. ADAMSON \& D. FEIN. Early Child Characteristics Predict Transition from ASD to Non-ASD.

Objective: Research indicates that $10-20 \%$ of toddlers diagnosed with Autism Spectrum Disorder (ASD) no longer meet ASD criteria when they are older. The present study aims to identify predictors of no longer meeting ASD criteria by age 4 .

Participants and Methods: Toddlers ( $\mathrm{n}=261 ; 79 \%$ male) diagnosed with ASD at age 2 (T1, M=26.63 months, $\mathrm{SD}=4.49)$ were re-evaluated at age 4 (T2, M=49.56 months, $\mathrm{SD}=6.86) ; 50(19 \%)$ no longer met ASD criteria. The initial evaluation occurred after screening or surveillance indicated ASD risk. Evaluations included Autism Diagnostic Observation Schedule (ADOS), Mullen Scales of Early Learning (MSEL), and parent-report measures.

Results: Backwards model selection for logistic regression resulted in the inclusion of ADOS Calibrated Severity Score (CSS), presence (66\%) or absence $(34 \%)$ of non-ASD-specific intervention, and MSEL receptive language in the final model. Lower ADOS CSS $(\mathrm{OR}=.79, \mathrm{p}=.006)$ and higher MSEL receptive language $(\mathrm{OR}=1.02, \mathrm{p}=.029)$ were associated with greater odds of losing ASD diagnosis. Surprisingly, participation in non-ASD-specific intervention (e.g., speech, occupational therapy) was associated with decreased odds of diagnostic change (OR=.45, $\mathrm{p}=.027)$. Presence of ASD-specific intervention $(26 \%)$ was not a significant predictor.

Conclusions: Early child characteristics, including ASD symptom severity and receptive language ability, predict diagnostic change from ASD to non-ASD two years later. Children of parents who sought nonASD-specific intervention were less likely to change, perhaps due to factors that led them to seek intervention, such as increased severity. Future research should explore additional factors that impact intervention-seeking and loss of ASD diagnosis.

Correspondence: Danielle N. Abrams, B.A., Psychology, Georgia State University, 230 E Ponce de Leon Ave, Unit 223, Decatur, GA 30030. E-mail: dabrams1@student.gsu.edu
L. KENWORTHY, K.M. DUDLEY, C. PUGLIESE, Y. GRANADER, C. LUONG-TRAN, J. STRANG \& L.G. ANTHONY. The Executive Function Challenge Task (EFCT): A Lab-based Observational Measure of Flexibility and Planning in Typically Developing Children and Those with Autism Spectrum Disorder.

Objective: We have previously described a treatment sensitive observational measure, the EFCT, that challenges children to be flexible and planful (Kenworthy, Anthony et al., 2014). Here, we explore the internal consistency and discriminant and construct validity of the EFCT.

Participants and Methods: 30 typically developing children (TDC; age $\mathrm{M}=10.2$ years, $\mathrm{SD}=1.7$; FSIQ $\mathrm{M}=111.9, \mathrm{SD}=11.3)$ and 58 children with autism spectrum disorder (ASD; age $\mathrm{M}=9.5$ years, $\mathrm{SD}=1.1$; FSIQ $\mathrm{M}=110.8, \mathrm{SD}=16.7$ ) were given the EFCT, three standardized activities on which behavior is coded for flexibility and planning. Examiners achieved inter-rater agreement $>90 \%$. Parents completed the Behavior Rating Inventory of Executive Function (BRIEF).

Results: Chronbach's alpha statistics indicate good internal consistency for the EFCT Flexibility (FS; 0.78) and Planning (PS; 0.70) scores and item total correlations are all in the acceptable range. The FS and PS clearly distinguish individuals with ASD from the TDC group (t's $>6.2, p$ 's<.001), and are not significantly correlated with FSIQ in either ASD or TDC. Convergent validity was demonstrated between the FS and PS and the Parent reported BRIEF Shift and Plan/Org scores (r's $>0.30)$ for the combined TDC/ASD data.

Conclusions: In addition to showing sensitivity to a cognitive behavioral EF treatment, the EFCT: can be coded reliably; has good internal consistency; distinguishes children with ASD, who are know to have flexibility and planning deficits, from TDCs; and is moderately correlated with parent report of flexibility and planning problems. The EFCT has the potential to capture a unique set of EF behaviors that are important in characterization and treatment research efforts.

Correspondence: Lauren Kenworthy, Children's National Medical Center, 111 Michigan Ave, NW, Washington, DC 20010. E-mail: lkenwort@cnmc.org

\section{Cognitive Neuroscience}

\section{S. BROOKS HOLLIDAY, C.A. LOCKWOOD, S. NAMAZI, C. HANSEN \& M. REINHARD. Specialized Training in Neuropsychology: A Review of Doctoral-Level Programs.}

Objective: In 1997, the Houston Conference Guidelines established standards of neuropsychology training. Since then, the field of clinical neuropsychology has evolved quickly, as evidenced by the increasing number of training programs. This study evaluates the current status of specialized neuropsychological training at the doctoral level.

Participants and Methods: To identify programs for inclusion, a list of programs maintained by APA Division 40 was reviewed. Additional programs were identified using search terms such as "neuropsychology doctoral program" and "neuropsychology emphasis clinical psychology." Non-APA accredited programs were excluded. The final sample included 45 programs. Program websites and handbooks were reviewed to gather information regarding curriculum and other aspects of training. Results: Thirty-four programs described a formal neuropsychological concentration, while 11 programs appeared to offer specialized coursework or research opportunities. Approximately $47 \%$ of programs identified as members of the Association for Doctoral Education in Clinical Neuropsychology. Among programs with formal neuropsychology concentrations, $62 \%$ offered basic coursework in neuropsychology; $71 \%$ in neuropsychological assessment; and $50 \%$ in behavioral or functional neuroanatomy. Further, 38\% of programs offered child-specific courses, and $27 \%$ offered coursework in rehabilitation/intervention. Several programs offered elective classes in a variety of areas, including the neuropsychology of aging, forensic neuropsychology, and developmental disabilities. Consistent with the Houston Guidelines, many 
programs described practicum and research requirements related to neuropsychology.

Conclusions: Graduate programs offering specialized neuropsychology training are largely consistent with the Houston Guidelines. In addition, many programs offer elective coursework that provides an advanced understanding of areas of neuropsychology that are becoming increasingly salient (e.g., aging).

Correspondence: Stephanie Brooks Holliday, PhD, War Related Illness and Injury Study Center, Washington DC VA Medical Center, 50 Irving St, NW, Washington,DC 20422.E-mail: stephanie.holliday@va.gov

\section{L.E. SABBAH \& E. MOES. Distinction Between Dopamine and Norepinephrine Related Tasks.}

Objective: Dopamine (DA) and norepinephrine (NE) are two neurotransmitters essential for motor, sensory and cognitive functioning. The primary aim of this exploratory study was to use indirect behavioral measures of DA and $\mathrm{NE}$ to determine whether performance on DA proxy tasks correlate more closely with each other than with NE-dependent tasks and vice versa.

Participants and Methods: A total of 84 college students were recruited (63 women, 21 men, $\mathrm{M}=19.26$ years, age range: $18-23$ years). Participants were given a screening vision test as well as a demographics questionnaire and 7 measures (FTT: Finger Tapping Test, CS: MARS Contrast Sensitivity, WCST: Wisconsin Card Sorting Test (id-shift and ed-shift), ANT: Attention Network Test, SST: Stop Signal Test, AT: Anagram test, and RAT: Remote Associate test) during a 2 hour testing session.

Results: An exploratory factor analysis with oblique rotation produced a two factor solution, which explained $45 \%$ of the overall variance in task performance. The first factor explained $28.41 \%$ of the overall variance in task performance. Based on the literature, this factor was labeled as the "Dopamine" factor and was comprised of the FTT, the ANT executive condition, the WCST id-shift and the SST. The second factor explained $16.61 \%$ of the overall variance in task performance and was labeled the "Norepinephrine" factor which was comprised of the WCST ed shift, the Anagram test and the Remote Associate test. Conclusions: This research is useful in enabling clinicians to better understand disorders of the DA and NE systems, which also has important implications for treatment.

Correspondence: Liorah E. Sabbah, M.A., Psychology, Suffolk University, 75 Saint alphonsus street, apt 708, Boston, MA 02120. E-mail: liorah. sabbah@gmail.com

\section{A.L. SMITH, B.N. RENN, L. FELICIANO \& H.P. DAVIS. Cognitive Dysfunction among Adults with Hyperglycemia.}

Objective: There is increasing concern regarding cognitive decline or impairment in individuals with diabetes mellitus (DM). We examined cognitive impairment (CI) in adults with DM or hyperglycemia across multiple cognitive domains including fluid intelligence, executive function (inhibition and task switching), and memory (declarative and nondeclarative). We hypothesized poorer cognitive performance across domains for those with DM relative to controls because of the vascular and metabolic changes associated with DM.

Participants and Methods: Participants were selected from an ongoing study examining cognition across the lifespan. The diabetic group $(\mathrm{DG} ; \mathrm{n}=32$ ) was $59.5 \%$ female, Mage $=58.83 \pm 19.10$ (range $21-87$ ). Meducation $=14.93 \pm 1.93$ (range 12-20) $35.7 \%$ non- White. A series of one-way ANOVAs analyzed group differences on the St. Louis University Mental Status Examination (SLUMS), Trail Making Test-B (TMT-B), Stroop, WASI Matrix Reasoning (WASI-MR), Rey Auditory Verbal Learning Test (RAVLT) trials 1 and 5, and the Word Stem Completion Task (WSC).

Results: DG participants exhibited poorer SLUMS performance $(23.85+3.43$ vs. $25.49+3.19, p=.01)$ relative to control group $(C G)$. Executive function was impaired in DG relative to $C G$ as evidenced by slowed TMT-B $(p=.01)$ and Stroop $(p<.001)$ performance. DG also demonstrated poorer fluid intelligence (WASI-MR; $p<.001$ ), verbal memory (RAVLT; immediate, $\mathrm{p}<.001$; delayed, $\mathrm{p}=.01$ ), and nondeclarative memory (WSC; $\mathrm{p}=.01$ ).

Conclusions: Conclusions: Cognitive dysfunction was greater in DG than in the CG for all aspects of global cognition evaluated. Further examination is needed to understand the prevalence of CI and effects of DM on cognition.

Correspondence: Alison L. Smith, Bachelo of Arts, Psychology, University of Colorado Colorado Springs, 13275 Spring Wagon Rd, Peyton, CO 80831.E-mail:asmith@uccs.edu

Q. KENNEDY, J.L. TAYLOR, A. NODA, J. YESAVAGE \& L. LAZZERONI. The STEP Model: Characterizing Simultaneous Time Effects on Practice for Flight Simulator Performance Among Middle-Aged and Older Pilots.

Objective: Understanding possible practice and test-retest interval effects among middle aged and older adults in real world tasks, or whether domain relevant expertise impacts practice and test-retest interval effects has important implications for training interventions. We modeled the simultaneous effects of practice and test-retest interval on a real-world outcome-flight simulator performance. Because the participants varied in age and level of specialized training, we also determined if age, flight expertise, or cognitive ability moderated practice and test-retest interval effects.

Participants and Methods: Flight simulator performance data across five sessions from 263 middle-aged and older pilots who varied in flight expertise was used to create the STEP (Simultaneous Time Effects on Practice) model. The STEP model implemented a mixed effects model with unstructured covariance thatcontains both a Practice effect and a Test-Retest Interval effect.

Results: The STEP model demonstrated consistent positive effects of practice and negative effects of test-retest interval. Among this sample of high functioning middle-aged and older adults, neither age nor flight expertise interacted with these two effects. However, for particular measures of flight performance, cognitive processing speed and intra-individual variability in processing speed moderated (i.e. interacted with) the benefits of practice and/or the negative influence of test-retest interval.

Conclusions: Practice and test-retest interval effects occur in real world tasks. However, certain age-related cognitive abilities may moderate these effects more so than chronological age. Our results have implications for the design and assessment of training interventions targeted at middle-aged and older adults for complex real world tasks.

Correspondence: Quinn Kennedy, Psychology, Operations Research, Naval Postgraduate School, 1411 Cunningham Rd, Naval Postgraduate School, Monterey, CA 93943-5219.E-mail:mqkenned@nps.edu

\section{K.M. TOPPING \& B. BECKER. Gender Role Beliefs Mediate} Priming Effects of Gender-Congruent Verbal Fluency Task.

Objective: Previous research has demonstrated the effects of priming on information retrieval and cognitive performance (Davies et al., 2005). In this study, we hypothesized that differences would be observed among participants when given a gender-congruent versus gender-incongruent verbal fluency task. We were also interested in understanding the degree to which this relationship is mediated by traditional gender role beliefs. Participants and Methods: Sixty-eight college-aged participants were asked to provide boys' and girls' names within one-minute. Verbal fluency tasks were counter-balanced. Gender role beliefs were assessed using the Gender Roles Belief Scale (GRBS; Kerr \& Holden, 1996).

Results: Results indicated a gender $\mathrm{x}$ task interaction effect, $\mathrm{F}(1,66)$ $=6.66, p=.01$. As predicted, men and women performed better on their respective gender-congruent verbal fluency task compared to their performance on the gender-incongruent task. When adding GRBS as a covariate, the interaction was no longer significant, but GRBS was independently associated with differences across tasks suggesting that gender role beliefs mediated the relationship between gender and differences 
in performance across verbal fluency tasks. In follow-up analyses, we found that men with higher traditional gender roles beliefs were most impacted by the priming effects of this task.

Conclusions: These findings suggest that gender-relevant information influences performance, particularly among males with more traditional gender role beliefs and suggests the importance of considering the impact of gender-salient information when selecting a test battery.

Correspondence: Kimm M. Topping, Lesley University, 1 Marion Drive, Tewksbury, MA 01876. E-mail: kimmtopping@gmail.com

H.V. ECHLIN, S. PETER, C.M. CONSIDINE, K. ROBERTS, A. GARANT, A. SEGUIN, C. ABEARE, A. DHAR \& W. RAJKUMAR. The Influence of Sleep Quality and Health Behaviours on Mood and Attention.

Objective: Sleep quality and health behaviors have been shown to be related, and have demonstrated associations with cognition and mood. Obstructive sleep apnea (OSA) patients represent a clinical population at risk for neuropsychological sequelae related to sleep quality, health behavior, and therefore represent a population in which examination of these relationships may be particularly useful.

Participants and Methods: Patients referred for OSA evaluation $(\mathrm{N}=55$, Mage=53.35), who underwent polysomnography (PSG), completed a brief neuropsychological evaluation (including Trail Making Test - B; TMTB), the Epworth Sleepiness Scale, (ESS), questions related to unhealthy behavior (UB), and completed the Center for Epidemiological Studies - Depression Scale (CES-D), as part of a larger study. Results: A $2(\operatorname{good} v s$. bad UB) $x 2$ (high vs. low ESS) ANOVA on TMTB showed a main effect for $\mathrm{ESS}, \mathrm{F}(1,49)=4.26$, $\mathrm{p}=0.04$. A similar ANOVA on CES-D scores showed a main effect for UB, $F(1,51)=4.68$, $\mathrm{p}=0.04$. A $2(\operatorname{good} v s$. bad UB) $\times 2$ (high vs. low $\mathrm{O} 2$ blood saturation) ANOVA on CES-D controlling for age showed a main effect for $\mathrm{O} 2 \mathrm{blood}$ saturation $F(1,14)=2.48$, $p=0.02$, but not for UB.

Conclusions: The findings suggest that subjective sleep quality is associated with cognitive performance (TMTB), whereas objective sleep quality (O2 blood saturation) was not associated with attentional processing performance. In terms of mood, unhealthy behaviour and physiological sleep quality are associated with indicators of depression. Health behavior and sleep quality appear to independently affect both mood and cognition without any additive effects.

Correspondence: Holly V. Echlin, University of Windsor, 1950 College Avenue, Apt.408, Windsor, ON N9B3T6, Canada.E-mail:hollyechlin@ hotmail.com

K. ROBERTS, C.M. CONSIDINE, A. GARANT, A. SEgUIN, C. ABEARE, A. DHAR \& W. RAJKUMAR. Objective and Subjective Sleep Quality Determinants of Cognitive and Negative Affective Processing Performance in Good vs. Bad Sleepers.

Objective: Poor sleep quality is associated with decrements in cognitive performance, mood, and more recently researched cognitive-affective processing (CAP). Subjective sleep quality reports and objective polysomnogram are often differentially related to these neuropsychological constructs. This study investigated whether cognitive performance, negative affect, and CAP performance differed significantly using clinical cut-offs for "good" and "bad" sleepers.

Participants and Methods: Fifty-five (Nfemale=26 Mage=53.35) potential sleep apnea patients referred for polysomnography completed sleep quality and mood questionnaires (Epworth Sleepiness Scale, ESS; PANAS-X Negative Affect, NA), and neuropsychological evaluation including cognitive (Digit Vigilance Test, DVT) and CAP measures (Emotion Vigilance Test, EVT).

Results: Polysomnogram-determined Sleep Efficiency (SE) negatively correlated with NA, r $(50)=-0.27, \mathrm{p}=0.03$, and time on EVT sad and neutral sub-measures, $\mathrm{r}(48)=-0.26, \mathrm{p}=0.036$ and $\mathrm{r}(48)=-0.28$, $\mathrm{p}=0.026$. Polysomnogram-determined apnea severity $(\mathrm{AHI})$ was positively correlated with DVT and EVT total time, $\mathrm{r}(52)=0.36, \mathrm{p}=0.004$ and $\mathrm{r}(50)=0.37, \mathrm{p}=0.003$. ESS score positively correlated with NA, $\mathrm{r}(53)=0.44, \mathrm{p}<0.000$. A 2 (high vs. low ESS) $\mathrm{x} 2$ (high vs. low SE) ANOVA for NA found an ESS main effect, $F(1,48)=10.99, p=0.002$, and near-significant SE main effect, $\mathrm{F}(1,48)=3.86$, $\mathrm{p}=0.055$, with no interaction found. For DVT, AHI main effect neared significance, F(1, $50)=3.85, p=0.055$. No other results were significant.

Conclusions: In this sample, objective and subjective sleep quality was associated with negative affect. Objective measures were better predictors of dysfunction in sustained attention and in attention for negatively-valenced faces. This suggests differential effects of objective sleep quality versus subjective reported sleepiness on affect, attention, and potentially biased vigilance for negatively-valenced faces.

Correspondence: Katelyn Roberts, Psychology, University of Windsor, 135 Sacred Heart Drive, Windsor, ON N9J1S6, Canada. E-mail: robertsi@uwindsor.ca

L.M. JENKINS, S.R. DELDONNO, K. MEYERS, J. ZUBIETA, M.N. STARKMAN \& S.A. LANGENECKER. Effects of Music on Pleasure and Cognition During Functional Brain Imaging.

Objective: Music can elicit highly positive emotional states and changes in cerebral blood flow, particularly in the reward network. Music may also affect cognition via stimulating these brain regions.

Participants and Methods: Nine males aged 18-32 participated. Exclusion criteria were: history of head injury or neurological disorder, psychiatric disorder (assessed using the SCID), and MRI contraindications. Participants rated eight 90 second classical music segments (e.g. Mozart) on 5 point Likert-scales for pleasure/enjoyment. Their top 4 clips were identified.

On day two, participants completed two cognitive tasks during fMRI: a facial emotion perception task and a Monetary Incentive Delay (MID) task allowing examination of the neural circuitry involved in anticipation and attainment of rewards. Between cognitive trials, participants listened to music or noise (control condition) through pneumatic MRI compatible earphones.

Results: Music minus noise contrasts revealed activation in a variety of areas involved in emotion and reward, including caudate head, subgenual anterior cingulate, bilateral anterior insula, as well as bilateral dorsolateral prefrontal cortex (PFC).

A separate regression model examined music minus noise during the MID only, with accuracy following music minus accuracy following noise as a predictor. Regions that were more active during music that predicted worse performance included the right caudate head, insula, middle frontal gyrus and bilateral thalamus.

Conclusions: Our hypothesis that listening to music would activate areas of the reward network was supported, however, music did not increase reward-based performance. Future investigations include the capture of music relationships in major depression using this design. Correspondence: Lisanne M. Jenkins, PhD, Psychiatry, The University of Illinois at Chicago, $1601 \mathrm{~W}$ Taylor St, Chicago, IL 60612. E-mail: ljenkins@psych.uic.edu

F. SAAVEDRA, K. RAGHUBAR, J. OGHALAI, R. WILLIAMSON \&. S. CAUDLE. Verbal Cognition as a Predictor of Adaptive Behavior in Children with Cochlear Implants.

Objective: Children with cochlear implants have been shown to demonstrate weakness in verbal intellectual functioning. Although nonverbal intelligence has been shown to predict adaptive functioning in these children, it is unclear as to whether verbal outcomes predict performance over and above those of nonverbal intelligence. As such, the current study investigated aspects of verbal intelligence and its relation to adaptive functioning in children with cochlear implants.

Participants and Methods: Participants included 32 children with a history of bilateral hearing loss, treated with cochlear implantation (59\% unilateral implantation), ranging in age from 6 to 17 years of age at the time of the assessment. The average length of implant use 
was 5.67 year. Scores were available for nonverbal intelligence, verbal reasoning, vocabulary knowledge, and parent ratings of adaptive functioning.

Results: Participants performed better on a measure of verbal reasoning than a measure of vocabulary knowledge. Verbal reasoning but not vocabulary knowledge was significantly correlated with aspects of adaptive functioning, including communication, self-care, and leisure. Moreover, verbal reasoning accounted for a significant portion of unique variance after accounting for gender, age at test, maternal education, and nonverbal intelligence on parent ratings of communication, social, and leisure activities.

Conclusions: Overall, children with cochlear implants demonstrated an uneven pattern of development of verbal cognition. Verbal reasoning was an important predictor of adaptive skills even after accounting for nonverbal problem solving. Implications for intervention strategies and functional outcomes are discussed.

Correspondence: Fiorella Saavedra, BS, Pediatrics, Baylor College of Medicine, 6701 Fannin St, Houston, TX 77030. E-mail: fiorella. saavedra@bcm.edu

\section{MOON, K. MELAH, S. JOHNSON \& L. BRATZKE. Sleep Apnea and Grey Matter Volume in Individuals with Heart Failure: A Voxel-Based Morphometric Analysis.}

Objective: Nocturnal hypoxia from sleep apnea (SA) has been shown to reduce the grey matter volume and cognitive status in non-heart failure (HF) samples. The aim of the current study was to compare grey matter volumes and cognitive status between HF patients with and without untreated SA.

Participants and Methods: Among 24 individuals with HF (mean age $=70.1, \mathrm{SD}=5.2$, male $=70 \%$, we compared $9 \mathrm{HF}$ patients (Mean age $=70.9, \mathrm{SD}=4.622$ ) with $\mathrm{SA}$ and $7 \mathrm{HF}$ patients (Mean age $=69.1$, $\mathrm{SD}=6.2$ ) without $\mathrm{SA}$. SA was evaluated using a portable sleep apnea screening device. Grey matter volume differences were evaluated using voxel-based morphometry of grey matter segments derived from T1 weighted images acquired with a 3 T MRI. Analyses were conducted using a generalized linear model controlled for age, sex, and left ventricular ejection fraction (LVEF). Cognitive function was evaluated using comprehensive neuropsychological testing, and multiple regression was used to evaluate group differences after adjusting for age, sex, education, and LVEF

Results: There were no significant grey matter differences between HF patients with and without untreated sleep apnea controlling for covariates. Cognitive scores were not significantly different between HF patients with and without SA.

Conclusions: SA was not associated with reduction in grey matter volume or cognitive function. Further studies are warranted to better understand the association between SA, brain structure and cognition among HF patients. As our understanding increases, researchers and clinicians will be better able to identify phenotypes of cognitive impairment in HF and develop potential intervention strategies.

Correspondence: Chooza Moon, MSN, School of Nursing, University of Wisconsin-Madison, 701 Highland Ave, \#3156, Madison, WI 53705. E-mail: cmoon4@wisc.edu

\section{B. SHANE, A. ALKOZEI \& W.D. KILLGORE. The Contribution of General Intelligence and Emotional Intelligence to the Ability to Appreciate Humor.}

Objective: The ability to appreciate humor is an extraordinarily complex capacity that requires numerous cognitive processes, rapid perceptual-cognitive switches, intelligence, and creativity. It remains unclear the extent to which capacities including traditional intellectual capacity (e.g., cognitive intelligence (IQ) contribute to this ability, relative to emotional problem solving capacities such as emotional intelligence (EI)). The current study examined the relative contribution of IQ and EI on the ability to accurately identify humor.
Participants and Methods: Sixty-one 18-45 year olds completed the Humor Appreciation Test (HAT), which presents a series of pairs of nearly identical fictional newspaper headlines or nonverbal cartoons. Participants were asked to select the more humorous item from each pair. Participants also completed measures of IQ, and ability and trait EI. Stepwise multiple linear regression analyses were used to predict total HAT accuracy, picture HAT accuracy, and headline HAT accuracy using IQ and EI as predictors.

Results: IQ significantly predicted HAT total accuracy (R2=0.35, $\mathrm{p}<0.001)$, headline accuracy $(\mathrm{R} 2=0.45, \mathrm{p}<0.001)$ and picture accuracy $(\mathrm{R} 2=0.11, \mathrm{p}<0.010)$. However, neither trait nor ability EI explained additional variance in any of the models.

Conclusions: The ability to appreciate humor is strongly related to general IQ. However, once cognitive intelligence is accounted for, EI does not make any unique additional contribution to humor appreciation. Thus, the ability to appreciate humor appears to rely predominantly on basic intellectual capacities rather than on the attunement of emotional skills or affective status.

Correspondence: Bradley Shane, Neuroscience and Cognitive Science, College of Medicine, Psychiatry, The University of Arizona, 1444 E. Drachman St., Tucson, AZ 85719. E-mail: bshane@email.arizona.edu

K. CARBINE, A. CLAWSON, J.E. FAIR, P.E. CLAYSON, I. HUNT, S.A. BALDWIN \& M.J. LARSON. A Comparison of Neuropsychological Functioning in Major Depressive Disorder, Generalized Anxiety Disorder, Obsessive Compulsive Disorder, and Healthy Controls.

Objective: Individuals with major depressive disorder (MDD) and obsessive-compulsive disorder (OCD) exhibit known deficits in cognitive functioning, while cognitive functioning in generalized anxiety disorder (GAD) is less understood. Particularly, how individuals with similar psychopathologies differ from each other in cognitive functioning is unclear, and few studies have compared cognitive functioning across multiple psychiatric groups. We hypothesized that individuals with MDD and OCD would have greater cognitive deficits than individuals with GAD and healthy controls.

Participants and Methods: Participants included 38 individuals with MDD (Mean age $=22.2 ; \mathrm{SD}=3.7), 25$ with $\mathrm{GAD}$ (Mean age $=21.9$; $\mathrm{SD}=4.8$ ), 10 with OCD (Mean age=24.6; $\mathrm{SD}=10.2$ ), and 24 psychiatrically healthy controls (Mean age $=23.2 ; \mathrm{SD}=2.8$ ). All participants were free from psychiatric comorbidities, head injuries, or neurologic diagnoses. Symptom measures included the Beck Depression Inventory (BDI-II), State-Trait Anxiety Inventory (STAI), and Penn State Worry Questionnaire (PSWQ). Neuropsychological measures included the Rey Auditory Verbal Learning Test (AVLT), digits span, controlled oral word association test (COWAT), and Trail Making Test (TMT) parts $\mathrm{A}$ and $\mathrm{B}$.

Results: Statistical analyses consisted of one-way ANOVAs with posthoc Tukey's HSD. Groups differed on BDI, STAI, and PSWQ ( $\mathrm{ps}<.001)$, with healthy controls significantly differing on BDI and STAI and all groups differing on PSWQ, besides individuals with MDD and controls (all ps<.03). Groups did not differ on all neuropsychological measures (ps>.11).

Conclusions: Contrary to predictions, individuals with MDD, GAD, OCD, and controls did not significantly differ on measures of cognitive functioning. However, there were a limited number of individuals with OCD. Alternative measures of cognitive functioning, such as event-related potentials, could be considered in order to differentiate between the cognitive functioning of psychopathologies.

Correspondence: Kaylie Carbine, Psychology, Brigham Young University, 6605 Amber Lane, Pleasanton, CA 94566. E-mail: kaylie. carbine@gmail.com 
D. ZIEGLER, J. JANOWICH \& A. GAZZALEY. Dynamic Interplay Between Distractions and Internally- and Externally-directed Attention.

Objective: Attention can be oriented externally or internally and can be derailed by irrelevant information originating from either external (e.g., distracting sights or sounds) and internal sources (e.g., distracting or intrusive thoughts), but few studies have explored the nature and underlying mechanisms of internal distraction in the context of differing attentional orientation. In this study, we investigated whether internal distractibility is affected by the presence of external distractions, how modulation of internal distractibility in the presence of external distraction relates to task performance, and whether these dynamics are affected by healthy aging.

Participants and Methods: Healthy young and older adults performed an externally-oriented visual target detection task and an internally-oriented mental rotation task. Each task was performed with and without auditory noise delivered through headphones. After each trial, participants reported if they were distracted, and if so by internal thoughts or by an external factor.

Results: We found that on the visual discrimination task, auditory noise induced a significant decrease in performance in both young and older adults, and this was accompanied by a shift in the type of distractions reported (i.e., from internal to external). Further, those participants who exhibited the most reduced internal distraction in the noise condition had the highest task accuracy. In contrast, on the internal task, noise only affected accuracy in older adults and performance did not correlate with changes in internal distraction reports.

Conclusions: These results suggest that the impact of task-irrelevant noise differentially impacts performance on tasks with internal vs external goal orientations and this effect appears to be mediated by differences in the ability to self-regulate suppression of internal distraction. These results might help explain real-world behaviors, such as the propensity to seek out noisy vs. quiet work environments.

Correspondence: David Ziegler, UCSF, 675 Nelson Rising Lane, MC 0444, San Francisco, CA 94158. E-mail: david@gazzaleylab.ucsf.edu

T. KAEMMERER, M. TUNNEY, S.L. CZIPRI, K. ASH, C. HOWARD \&. L.H. LU. Age-Related Differences in Implicit Motor Sequence Learning: Exploring the Influence of Embedded Associative Structure.

Objective: Little is known about age-related differences in implicit motor sequence learning. Some evidence suggests age-related deficits in the implicit learning of sequences containing higher-order associations (two or more units predict subsequent unit) as opposed to simple associations (one unit predicts subsequent unit). We examined implicit motor sequence learning differences between healthy youths and older adults using a serial response time task (SRTT), controlling for the effects of associative structure (i.e., sequence predictability).

Participants and Methods: Healthy right-handed youths $(\mathrm{n}=22$, mean age $=11.95, \mathrm{SD}=5.18)$ and older adults $(\mathrm{n}=15$, mean age $=$ 65.47, SD = 5.66) completed two SRTTs. One sequence was comprised predominantly of first-order associations and the other of higher-order associations. For each task, the 7-stage target sequence was presented for four learning blocks followed by a random sequence in block 5 . Learning was defined as the difference in response time between the random block and the fourth learning block.

Results: Repeated measures ANOVA revealed no significant group $\mathrm{x}$ predictability interaction in learning, $\mathrm{F}(1,35)=2.19$, $\mathrm{p}=.15$. Learning was significantly greater for the sequence comprised mainly of first-order associations, $\mathrm{F}(1,35)=4.52, \mathrm{p}=.04$. Older adults responded more quickly than youths, $\mathrm{F}(1,35)=5.69, \mathrm{p}=.02$. Interestingly, older adults showed greater learning than youths in the task with greater associative complexity, $\mathrm{t}(35)=2.34, \mathrm{p}=.03$, but not in the simpler associative task, $\mathrm{t}(35)=0.63, \mathrm{p}=.54$.

Conclusions: Associative structure appears to impact implicit motor sequence learning rate regardless of age. In contrast with previous literature, healthy older adults responded more quickly than youths and showed greater learning than youths under conditions of increased associative complexity. In fact, healthy youths exhibited difficulty with the implicit learning of higher-order associations. These preliminary findings warrant further investigation.

Correspondence: Tobias Kaemmerer, University of Chicago, $2331 \mathrm{~N}$ Harding Ave, Chicago, IL 60647.E-mail: botias108@gmail.com

\section{T. KARPOUZIAN, H. BREITER \& J. REILLY. Neural Correlates of Working Memory Capacity.}

Objective: Working memory (WM) is the cognitive ability that allows for the temporary mental maintenance and manipulation of information to support higher order cognitive. The neural circuitry supporting WM is well established; however, it is not clear which regions underlie variability in capacity and stability of stored information over time. To elucidate the neural basis of variation in WM, we conducted an fMRI study in which we parametrically manipulated the amount of information (capacity) and length of time (stability) over which information is held in WM.

Participants and Methods: Twenty-one healthy adults completed a WM task while undergoing fMRI scanning. Subjects were briefly presented with a stimulus array consisting of 3,5 , or 7 items. After a variable delay period, a probe item was presented and the subject decided if it appeared in the same location as one of the array items. D' (proportion of hits to false alarms) was calculated to characterize task accuracy. Event-related fMRI analyses were conducted to identify regions of activation during correctly performed trials, and how activation changed with increased amounts of information stored in WM.

Results: A mixed-effects analysis revealed that WM accuracy parametrically declined with increasing load $(\mathrm{F}(2,60)=5.075, \mathrm{p}=.009)$ whereas performance did not change with increasing delay interval $(\mathrm{F}(2$, $60)=1.878, p=.162)$. Event-related fMRI analyses demonstrated activation in bilateral dorsolateral prefrontal, insular, and posterior parietal cortices, anterior cingulate, and bilateral thalamus. There was a linear increase in activation within this network as WM load increased.

Conclusions: Results confirmed that increased activation of a widespread cortical-thalamic network underlies successful WM performance as capacity levels increase. Consideration of the neural underpinnings of variation in this core cognitive ability is important as WM processes are altered in several neuropsychiatric disorders.

Correspondence: Tatiana Karpouzian, Northwestern University, 710 N Lake Shore Drive, Chicago, IL 60611.E-mail: t-karpouzian@ northwestern.edu

D. PISNER, A. ALKOZEI \& W.D. KILLGORE. Visuospatial reasoning mediates the relationship between emotion recognition and emotional intelligence.

Objective: A major component of emotional intelligence (EI) is the ability to understand emotions, a skill that goes beyond mere emotion recognition, and includes knowledge of how emotions form an interrelated symbol set. We propose that greater visuospatial reasoning may allow individuals to better analyze how emotions interrelate, which may in turn explain higher EI. Therefore, we hypothesize that visuospatial reasoning ability mediates the relationship between emotion recognition ability and EI

Participants and Methods: Sixty-one 18-45 year olds (males=31, mean age $=30)$ completed: 1$)$ the Ekman 60 Faces Task, which assesses emotion recognition accuracy in response to a series of emotional faces, and 2) the Design Organization test (DOT), a rapid visuospatial screening instrument similar to the ubiquitous 'Block Design' task, and 3) an ability based EI assessment.

Results: In a standard mediation analysis, we first used linear regression to confirm that: 1) emotion recognition significantly predicts EI ( $\mathrm{R}$ square=.22, $\mathrm{p}<.001) ; 2$ ) emotion recognition significantly predicts visuospatial reasoning $(\mathrm{R}$ square $=.22, \mathrm{p}<.001)$; and 3 ) visuospatial reasoning, controlling for emotion recognition, significantly predicts EI 
( $\mathrm{R}$ square=.28, $\mathrm{p}<.001)$. Finally, using a Sobel Test, we confirmed that visuospatial reasoning serves as a partial mediator between emotional recognition and EI, with a standardized indirect effect of .29 at a marginal level of significance $\mathrm{p}=.052$.

Conclusions: The results imply that Emotional Intelligence involves more than just recognizing emotions-it also involves visuospatial reasoning. Future work may explore whether enhancing visuospatial ability might improve EI, as well as investigate fMRI activity of highly visuospatial individuals during EI tasks.

Correspondence: Derek Pisner, Psychiatry, University of Arizona, 202 E12th st, 816, Tucson, AZ 85701.E-mail: dpisner@email.arizona.edu

J.E. LETZEN, L.S. SEVEL, J.G. CRAGGS \& M.E. ROBINSON. Temporal Differences in the Relationship Between Dorsal and Ventral Attention Networks Based on Pain Intensity.

Objective: Neurocognitive models of pain have suggested that bottom-up and top-down attentional processes are essential for creating the pain experience. Associated with these processes are the ventral and dorsal frontoparietal attention networks ( $\mathrm{vFPAN}$ and dFPAN), respectively. This study examined how differences in pain perception related to the temporal relationship of these neural networks.

Participants and Methods: Thirty-three healthy participants completed an fMRI paradigm that used thermal stimuli to induce pain. Participants rated pain intensity after each stimulus. A cluster analysis classified individuals as experiencing "high" or "low" pain. An independent component analysis was conducted on preprocessed fMRI data. Resulting components were spatially sorted to identify the vFPAN and dFPAN. Functional network connectivity analyses were then conducted to assess the temporal relationship of these components.

Results: "High" and "low" pain groups $(\mathrm{n}=20,13$; mean pain $=$ $51.40,28.20$, respectively) significantly differed in pain ratings $[\mathrm{t}(31)$ $=7.87, \mathrm{p}<.001]$. Both groups showed a significant temporal relationship between FPANs, with vFPAN activity preceding dFPAN activity $(p<.001)$. Additionally, the temporal lag between these networks was significantly slower in the low pain group $(p \leq .05)$.

Conclusions: As expected, the bottom-up vFPAN was engaged prior to the top-down dFPAN in both groups. Interestingly, higher pain ratings were associated with stronger temporal coupling of the networks. This finding suggests that the temporal relationship of the FPANs may serve as an index of pain-related vigilance and somatic focus. Future work can examine whether these networks can be selectively engaged to modulate pain perception, especially for clinical populations.

Correspondence: Janelle E. Letzen, M.S., Clinical and Health Psychology, University of Florida, 3461 SW 2nd Avenue, \#428, Gainesville, FL 32607.E-mail:jletzen@phhp.ufl.edu

\section{M.D. GRILLI \& M. VERFAELLIE. The Contribution of Memory to the Self-Concept in Amnesia.}

Objective: Neuropsychological research has demonstrated that individuals with amnesia can maintain a self-concept (i.e. personal identity). Although there is evidence to suggest that episodic memory may be used to support the self-concept in healthy adults, how, or even if, individuals with amnesia turn to memory to support the self-concept remains unclear.

Participants and Methods: 8 medial temporal lobe amnesic patients and 12 healthy adults matched on age, education, and verbal IQ participated. They completed eight "I Am" self-statements. For each of the four highest ranked self-statements, they then provided supporting information indicating why the statement was considered self-descriptive. Finally, they attempted to retrieve six episodic memories for each of these self-statements. Responses were recorded and scored as episodic, personal semantic (i.e. personal knowledge), and general semantic memories.

Results: Patients were less likely than controls to identify traits among their top four self-statements. Patients generated fewer personal semantic memories than controls to support trait self-statements, but not role self-statements. There were no differences across groups in the use of episodic or general semantic memories, but this was likely due to the infrequent use of such memories in either group. Patients demonstrated a pervasive impairment in self-defining episodic memory retrieval relative to controls.

Conclusions: These results reveal that traits are less central to the self-concept than roles in amnesic patients. This may reflect the fact that amnesic patients have difficulty accessing trait-supporting memories. We hypothesize that role-supporting memories may be more accessible to amnesic patients, because they are more likely to be linked to significant life experiences.

Correspondence: Matthew D. Grilli, Ph.D., VA Boston Healthcare System, 150 South Huntington Ave, Jamaica Plain, Boston, MA 02130. E-mail:matthew.grilli@va.gov

S. MARKOWSKI, W.D. KILLGORE \& A. ALKOZEI. Sleep Onset Latency and Duration are Associated with Self-Perceived Invincibility.

Objective: Insufficient sleep alters risk-taking propensity, but the direction and magnitude of the effect appear to differ according to a number of factors that remain poorly understood. One potential modifying influence on risk-taking is the extent to which a person believes that they will not be affected by the consequences of risky behavior-i.e., "invincibility." The present study explored the association between self-reported sleep parameter and self reported invincibility.

Participants and Methods: Sixty-one healthy individuals (Males = $31, \mathrm{M}$ age $=30$, range $=18-45)$ completed a series of self-report measures including a brief questionnaire about typical sleep habits, and the Invincibility Beliefs Index (IBI). A bivariate correlational analysis was used to examine the relationships between sleep onset latency (SOL), sleep debt, and scores on the IBI.

Results: Shorter SOL on weeknights was associated with higher self-perceived Total Invincibility scores ( $r=-.292, p=.023)$, and the Audacity subscale $(r=-.355, p=.003)$. Furthermore, participants who typically slept less than their optimum preferred amount (i.e., typical sleep hours - hours of sleep necessary to feel best) tended to show lower scores on Total Invincibility $(r=-0.248, p=.045)$ and Audacity $(r=-.355$, $\mathrm{p}=.005)$ of the IBI.

Conclusions: Individuals who typically fall asleep faster and obtain more sleep tend to report greater self-perceptions of invincibility than those who receive less sleep. These findings are consistent with evidence that sleep loss reduces motivation and self-confidence. Thus, it is unlikely that prior findings of increased risk-taking during sleep loss stem from increased perceptions of invincibility and may be due more to altered decision-making or reduced alertness.

Correspondence: Sarah Markowski, Psychiatry, University of Arizona, 5901 N. Oracle Rd Apt 3, Tucson, AZ 85704.E-mail:smarkows@ psychiatry.arizona.edu

\section{A.C. ROSEN, A. MATHUR, M. LAM \& S. HANGAL. Effect Of Phonemic Cuing On Recall Of Personally Relevant Names Derived From Email.}

Objective: Email is a lifelog that can be mined to study cognition in an ecologically valid context. We developed a system that automatically creates stimuli from participants' emails to probe memory for proper names in an autobiographical memory context (MUSE: Hangal, Lam, and Heer, 2011). In this web based study, participants view email messages they previously sent and inserted the proper name that was omitted. The task thus involves recall of a proper name and source memory when the participant matches it to the appropriate email context. Accuracy of recalling these proper names declines over the course of a year (Hangal,Rosen, Mathur and Lam, 2014). The objective of this current analysis is to test whether a letter cue is helpful for recall and whether the benefit of this phonemic cuing decreases with increasing delays over the course of the year. 
Participants and Methods: Forty eight people (average age $=30.2[19$ 60]) were paid $\$ 10$ for participation. Email archives were analyzed on a secure server at the Stanford Department of Computer Science and 40 sentences were derived as stimuli using a method previously described (Hangal et al., 2011). The task was to type the name that should fill in where the proper name was missing. Items were cued with blank lines substituted for the letters. After 20 seconds with no response, participants saw the first letter of the correct name.

Results: Average accuracy was $77.8 \%(\mathrm{SD}=10.86 \%)$. An ANOVA was performed on accuracy collected over the course of a year divided into 3 month intervals. There was a decline in accuracy over time $(\mathrm{F}(3,141)=7.83, \mathrm{p}=.00007$. Retrieval was more effortful over the year as evidence by increased use of the phonemic cues $(F(3,141)=4.03$, $\mathrm{p}=.0087$ ). Successful retrieval based on a phonemic cue decreased from $70 \%$ accuracy in the most recent quarter of the year $43 \%$ in the last quarter of the year $(\mathrm{F}(3,87)=4.39, \mathrm{p}=.006)$.

Conclusions: Results thus demonstrated that the effectiveness of phonemic cues in facilitating recall of proper names declines over time.

Correspondence: Allyson C. Rosen, Ph.D., ABPP-Cn, Psychiatry, Stanford University, Dept. of Psychiatry, C305, 401 Quarry Road, Stanford,CA 94305.E-mail: rosenally@gmail.com

M. LAMAR, O. AJILORE, J. COHEN, R. CHARLTON, J. GADELKARIM, A. LEOW, R. SWENSON, D.J. LIBON \& A. KUMAR. Cognitive and Neural Efficiency: What Planning and Organization Behaviors Reveal About Brain Connectivity and the Human Connectome.

Objective: Aging studies have long investigated cognitive efficiency of planning and organization. Innovative neuroimaging analysis techniques have created opportunities for investigating neural efficiency of networks and connectomes. Combining cognitive and neural information, we explore the interplay between functional and structural efficiency in aging.

Participants and Methods: 72 non-demented/non-depressed adults $>55$ years old completed the Clock Drawing Test (CDT). We determined cognitive efficiency by participants' use of anchor digits (placing 12,3,6,9 before other digits during CDT command condition). We compared participants that 'anchored' (Anchorers; $n=37$ ) to Non-Anchorers $(\mathrm{n}=35)$ on composite $\mathrm{z}$-scores of cognition and connectome-based graph theory analyses of network efficiency derived from grey matter volumes of T1-weighted MRI and white matter tract indices of DTI.

Results: Anchorers outperformed Non-Anchorers on executive functioning ( $p=.007)$ and learning/memory/recognition ( $p=.008)$ z-scores. Anchorers also excelled at verbal and visuospatial abstract reasoning ( $p$-values<.04). Connectome analyses revealed edge differences favoring Anchorers in an interhemispheric subnetwork involving the left putamen and right insula and significantly higher nodal efficiency for Anchorers versus Non-Anchorers in the left medial OFC, right rostral ACC and right superior frontal gyrus. Path length associated community estimates revealed nodal-level community structure differences between groups. Conclusions: Anchorers demonstrated superior cognitive and neural efficiency. Furthermore, Anchorers displayed distinct structural brain networks suggesting a significant interplay between cognitive skills and neural networks involved in planning and organization. Subtle measures of functional and structural efficiency may prove useful in monitoring change over time in high-functioning older adults.

Correspondence: Melissa Lamar, Ph.D., Psychiatry, University of Illinois at Chicago, 1601 W Taylor Street, MC912, Chicago, IL 60612. E-mail: mlamar@psych.uic.edu

A. DE LA VEGA, A. REINEBERG \& M. BANICH. Meta-analytic Function of Brain Regions Predicts Resting-state Connectivity.

Objective: Functional connectivity measures at rest have been shown reflect how likely regions are to coactivate in cognitive tasks. We were interested in making a direct link between resting-state functional coactivation and the similarity of regions' functions across a large-scale meta-analytic database of functional neuroimaging studies.

Participants and Methods: We used NeuroSynth, a database of over 9000 fMRI studies, to characterize a set of 200 a priori defined regions. First, we determined how often regions activated together across studies in the database by generating a coactivation correlation matrix. Next, we used multivariate ridge regression to determine which cognitive functions best predict activity in each region, and then generated a correlation matrix of regions' meta-analytically derived function. Then, using data from 100 participants that underwent rs-fMRI scans, we generated an average functional connectivity matrix using the same regions.

Results: We found regions with high resting-state functional connectivity were more likely to coactivate across the studies in the database, suggesting that resting-state connectivity reflects task-based activation (pearson's $\mathrm{r}=0.7, \mathrm{p}<0.001$ ). Regions with high functional connectivity were not only more likely to activate together, but also showed more similar meta-analytic functional profiles, suggesting they have more similar function (pearson's $\mathrm{r}=.6, \mathrm{p}<0.001$ ).

Conclusions: We found the first direct link between resting-state functional connectivity and meta-analytically derived function showing that functional connectivity at rest not only reflects similar dynamics, but indexes regions' similarity of function.

Correspondence: Andrew Reineberg, University of Colorado Boulder, 1406 Pine St., Apt.2, Boulder, CO 80302.E-mail: andrew.reineberg@ colorado.edu

R. SALAZAR, X. REN, O. BARTHELEMY \& A. CRONIN-GOLOMB. Effects of Cognitive Deficits on Gait in Parkinson's Disease.

Objective: Parkinson's disease (PD) is associated with abnormalities in cortico-striato-thalamocortical circuits, leading to deficits in cognition. Though it is known that cognitive load may exacerbate walking impairments, less is understood about the effects of specific cognitive demands on gait. Because PD is associated with dysfunction of prefrontal cortex early in the disease course, we hypothesized that frontal-associated cognitive deficiencies would relate to gait impairment.

Participants and Methods: We assessed multiple aspects of gait during overground walking (OptoTrak/3020 System) in 18 non-demented individuals with mild-moderate PD and 10 matched normal control adults (NC). Five baseline walking trials were followed by 6 dual-tasking trials: walking and performing Serial 7's (3 trials) or an auditory version of Trail Making Test B (3 trials). Single-task Serial 7's and TMT B were assessed in a separate session.

Results: Cognitive performance significantly declined (i.e., fewer subtractions, switches) under dual-tasking relative to single-tasking for PD (each test $\mathrm{p}<.01)$ and, to a lesser extent, for NC ( $\mathrm{p}=.022$ for TMT B; $\mathrm{p}=.085$ for Serial 7's). For PD and NC, increased cognitive load was related to reduced stride speed and stride length $(\mathrm{p}<.01)$ and, in PD only, also stride frequency $(p<.01)$. For both groups, more cognitive mistakes under dual-tasking correlated with reduced stride frequency $(\mathrm{p}<.01)$.

Conclusions: The results suggest that gait may be vulnerable to frontal-related cognitive load in PD. Future research should examine additional aspects of cognition. Targeting cognitive function may prove useful in mitigating gait impairment in PD and may bolster the effects of existing physical therapy interventions.

Correspondence: Robert Salazar, M.A., Boston University, 1 Fairland St, Roxbury, MA 02119.E-mail: rdsalaz@bu.edu

\section{L.Y. TARHAN, C.E. WATSON \& L.J. BUXBAUM. Action Understanding and Production: Common and Distinct Neural Substrates.}

Objective: Theories of motor simulation suggest that action understanding and action production share common neural and cognitive substrates. However, both associations and dissociations between the tasks have been reported. The current study aims to clarify this relationship by using voxel-based lesion-symptom mapping (VLSM) to directly compare the tasks' neural substrates in the same participants. 
Participants and Methods: 131 chronic left hemisphere stroke patients completed tests of meaningful tool-related Gesture Production (GP) and Gesture Recognition (GR). Patients' lesions were hand-drawn and warped to a common template. VLSM was used to assess the impact of lesions on behavioral scores at each voxel.

Results: Behaviorally, there was a significant correlation between GP and GR tasks, controlling for lesion volume $(p<.001)$. VLSM analyses revealed overlapping regions critical to both tasks in the posterior temporal lobe (pTL) and middle frontal gyrus (MFG). Controlling for the common variance between tasks revealed greater involvement of anterior inferior parietal lobe (IPL), motor cortex (M1), and inferior frontal gyrus (IFG) in GP, and posterior middle temporal gyrus (pMTG) in GR. Conclusions: These data are consistent with both common and distinct neural substrates for GP and GR, elucidating previously-reported associations and dissociations between the tasks. We suggest that simulation mechanisms requiring pTL and MFG integrity are active in both the planning and perception of action, whereas additional temporal, parietal, and frontal regions are differentially critical for one or the other task. Thus, patients whose lesions spare pTL and MFG may drive previously reported dissociations between GR and GP tasks.

Correspondence: Leyla Y. Tarhan, Moss Rehabilitation Research Institute, 50 Township Line Rd., Elkins Park, PA 19027. E-mail: lytarhan@gmail.com

J. BURKAUSKAS, J. BROZAITIENE, A. BUNEVICIUS \& R. BUNEVICIUS. Cardioselective Beta-blockers Effect Cognitive Functioning in Coronary Artery Disease Patients.

Objective: The impact of treatment with beta-blockers on risk for dementia remains controversial as specific cognitive functions affected by selective beta- 1 blockers use have been less studied. The aim of the study was to investigate the effect of selective beta- 1 blockers on cognitive functions in non-demented coronary artery disease (CAD) patients admitted for cardiac rehabilitation program.

Participants and Methods: A 3 year study included 723 CAD patients two weeks after acute myocardial infarction or unstable angina; 529 $(73 \%)$ men and $194(27 \%)$ women; mean age of 58 years (SD=9). Patients were evaluated for socio-demographic characteristics, medication plan, left ventricular ejection fraction (LVEF), for New York Heart Association (NYHA) class and the presence of arterial hypertension. Digit Span Test, Digit Symbol Test, Trail Making Test A and B were used to assess auditory attention, mental flexibility, psycho-motor performance, incidental learning, perceptual speed and task switching. Participants were considered impaired in the specific cognitive performance if their scores fell below the 25th percentile of the study population. Multiple logistic regression models were used to evaluate effects of treatment with selective beta- 1 blockers on impairment in each of the cognitive tests. Results: Controlling for potential confounders such as age, gender, education, NYHA class, LVEF, and arterial hypertension treatment with selective beta- 1 blockers was associated with a 2.65 -fold increase (95\% confidence, 1.309 to $5.381, p=.007)$ in risk for impairment in incidental learning as measured by Digit Symbol Test. There was no significant effect of beta-blockers to other measured cognitive functions. Conclusions: In CAD patients two weeks after acute cardiac events treatment with beta-blockers is associated with a selective impairment in incidental learning.

This research was funded by the European Social Fund under the Global Grant measure, Grant VP1-3.1-SMM-07-K-02-060.

Correspondence: ,, . E-mail:

\section{Electrophysiology/EEG/ERP}

G. STEFANATOS, B. EID, H. ZOBEL \& L. SCHUMACHER. Disassociation of steady-state and transient evoked response findings in Word Deafness.

Objective: Neuropsychological studies have attributed the characteristic impairment of auditory recognition in word deafness to temporal processing deficits. Few reports have utilized electrophysiological measures to help specify the precise nature and stage at which processing breaks down. We therefore investigated a case of word deafness utilizing a typical "oddball" evoked potential paradigm and a new steady-state adaptation of this procedure.

Participants and Methods: In our typical oddball paradigm, a complex tone $(250 \mathrm{msec})$ was presented repeatedly at irregular intervals (1-2.2 seconds). GL (59yrs) was instructed to respond whenever he heard an infrequent interspersed tone that differed in pitch (oddball). In the steady-state paradigm, GL listened to a continuous frequency-modulated (FM) tone comprised of repetitive up-going FM pulses (standard) presented four times a second. He was asked to respond to infrequent events lasting 1 second when the direction of the FM pulses was reversed. Results: The N100 and P200 components derived from the typical oddball paradigm were broadly within normal limits. The P300 response was visible, but modestly reduced in amplitude. In the steady-state paradigm, GL did not exhibit steady-state auditory evoked potentials to either the standard or the oddball pulsed FM sequences.

Conclusions: Results from the typical oddball paradigm suggested that the timing of information processing between stimulus and neural response was broadly within normal limits. By contrast, the steady-state paradigm revealed substantial abnormalities in neural processing of rapidly changing auditory events (both standard and oddball). These results suggest that the steady-state paradigm used here may disclose abnormalities in word deafness that can be missed using traditional paradigms.

Correspondence: Gerry Stefanatos, D.Phil., Communication Sciences \& Disorders, Temple University, 1701 N. 13th St., Philadelphia, PA 19122.E-mail:stefang@temple.edu

L.A. KAIS, C. LEE, S.M. SASS, J.L. STEWART, W. HELLER, G.A. MILLER \& R.L. SILTON. Anxiety Influences Neural Correlates of Inhibitory Control.

Objective: Anxiety has been shown to modulate anterior cingulate cortex (ACC) activity, but little is known regarding how these changes in ACC activity are related to impairments in top-down attentional control. The primary aim of this study was to evaluate the influence of anxiety on the neural correlates of inhibitory control. The frontocentral P300 ERP has been previously shown to index inhibitory processes. We predicted that the frontocentral P300 component of the event-related brain potential would be modulated by anxiety, indicating impairments in inhibitory control.

Participants and Methods: Participants (35 undergraduates, aged $18-21$ ) were identified as either healthy controls or having an anxiety disorder diagnosis based on DSM-IV criteria ( $\mathrm{n}=16$ participants with anxiety, $n=19$ healthy controls). All participants were right-handed with no known history of neurological disorders. To assess inhibitory function, participants completed a color-word Stroop task during EEG (electroencephalography). P300 mean amplitude was calculated for all participants at the Cz scalp site.

Results: P300 mean amplitude was larger for participants with anxiety than for healthy controls $(p=.02)$. Faster response time $(R T)$ was related to increased P300 mean amplitude $(r=-.42, p=.01)$. Group differences in RT were not significant $(p=.11)$.

Conclusions: Increased P300 amplitude was observed for individuals with anxiety, suggesting that more neural resources were recruited for inhibitory function. These findings are consistent with previous research that has shown that anxiety is related to increased ACC activity during inhibitory control tasks. Extra processing resources recruited during 
inhibitory control may be necessary in order to override the internally distracting nature of ruminative thoughts associated with anxiety. Developing a better understanding of abnormal neural correlates of inhibitory control may help inform the development of more effective treatments for individuals with anxiety.

Correspondence: Lorri A. Kais, B.A., Psychology, Loyola University Chicago, 1543 WWalton St., \#3, Chicago, IL 60626.E-mail:lorrikais@ gmail.com

P. MANGAL, J.A. LAFO, M.S. OKUN, M. BRADLEY \& D. BOWERS. Emotional Memory, the Late Positive Potential, and Parkinson's Disease.

Objective: Previous research with non-demented Parkinson individuals has found decreased episodic emotional memory that was not accounted for by a general amnestic difficulty. We tested the hypothesis that emotional memory deficits in PD patients were due to decreased arousal at the time of initial encoding. To test this hypothesis, participants viewed emotional pictures while an index of arousal (late positive potential; LPP) was obtained and then associated with subsequent memory.

Participants and Methods: Thirty-six individuals with idiopathic Parkinson disease or age- and education-matched healthy controls viewed a standardized set (IAPS) of high and low arousing pictures, while EEG was recorded from an EGI 64-electrode net. The LPP amplitude was computed over centro-parietal electrodes $500-800 \mathrm{~ms}$ post stimulus onset. Free recall of the viewed pictures was tested after a 30 -minute delay. Data were analyzed using mixed models ANOVA.

Results: At time of encoding, controls showed significantly higher LPP responses to high vs. low arousing pictures, whereas the PD group did not. Despite these differences, the PD group recalled significantly more high vs. low arousing emotional pictures, similar to controls. In terms of the subsequent memory effect, controls showed significantly higher LPP responses to recalled vs. forgotten high arousal pictures $(\mathrm{p}=.029)$, whereas this comparison was at trend level in individuals with PD $(p=.071)$. There were no differences in recalled vs. forgotten low arousing pictures for either group.

Conclusions: In contrast to our hypothesis, PD patients showed an enhanced emotional memory effect like controls. This occurred even though the PD patients had attenuated electrocortical reactivity at the time of encoding. These findings highlight a dissociation between abnormal LPP responses and normal memory indices of emotional processing in non-demented Parkinson patients. Findings will be discussed in terms of potential mechanisms that may underlie altered emotion processing. Supported by R21-NS079767.

Correspondence: Paul Mangal, B.S., Clinical Health Psychology, University of Florida, P.O. Box 100165, Gainesville, FL 32610-0165. E-mail: pmangal@phhp.ufl.edu

M. NIERMEYER, M. EULER, H. SCHRYVER, D. CHON, V. REDDY, E. VACCARIELLO \& Y. SUCHY. Effects of Task Novelty on Cognitive Contributions to Planning Times and EEG Activity During Complex Motor Sequencing.

Objective: Movement-related cortical potentials (MRCPs) are thought to reflect planning of motor output. Motor planning times in turn are related to both psychomotor speed (PS) and executive functioning (EF), but are also known to be affected by specific task demands, such as task novelty. This study sought to examine the unique contributions of EF vs. PS to planning times and MRCPs, under different novel vs. familiar conditions.

Participants and Methods: Forty healthy undergraduates completed a series of measures of PS and EF, followed by a motor sequencing task during EEG recording. Contextual and sequence novelty were manipulated pseudo-randomly on 20 and $10 \%$ of trials, respectively, with the remainder of the trials being familiar. MRCPs were measured at electrode $\mathrm{FCz}$, time-locked to participants' first movement on correct trials. Results: Hierarchical linear regressions using planning time as the dependent variable showed that PS contributed between $13 \%$ and $19 \%$ of variance in all conditions (novel context vs. novel sequence vs. familiar), all $\mathrm{p}$ values $<.022$. EF contributed $10 \%$ of unique variance (above PS) to planning times of novel (both context and sequence) trials, both $\mathrm{p}$ values<.04. When using MRCP amplitude as the dependent variable, EF contributed (above PS) 14\% of variance on novel context trials $(p=.016)$ and showed a trend for $8 \%$ to $9 \%$ of variance on familiar trials $(p<.077)$. PS was generally not a significant contributor. Neither PS nor EF contributed during novel sequences.

Conclusions: While planning times appear to be largely determined by PS, EF does contribute to planning under novel conditions. In contrast, MRCPs appear to be primarily related to EF (without much relationship to PS), particularly when context is novel, suggesting they may play a role in matching familiar motor output with novel contexts. However, MRCPs during novel sequences are unrelated to EF or PS, possibly reflecting a unique cognitive process.

Correspondence: Madison Niermeyer, BA in Psychology, Clinical Psychology Department, University of Utah, 9928 Roseboro Road, Sandy, UT 84092. E-mail: madison.niermeyer@psych.utah.edu

P.E. ClaYSON, J. HOSKIN, M. PRIMOSCH, S.W. BERGIN, A. CLAWSON, S. STEFFENSEN, M. LEYTON \& M.J. LARSON. Effects of Dopamine Precursor Depletion on Feedback-Related Performance Monitoring.

Objective: Studies using dopaminergic medications implicate dopamine in feedback-related performance monitoring. However, these findings might be confounded by medication side effects. To circumvent these potential confounding effects, we administered an amino acid mixture deficient in the dopamine precursors tyrosine (Tyr) and phenylalanine (Phe) to acutely and preferentially decrease dopamine synthesis in order to examine its effects on the feedback-related negativity (FRN), an electrophysiological index of feedback monitoring.

Participants and Methods: High-density event-related potential (ERP) data were acquired while 14 males completed a trial-and-error learning task four hours following mixture ingestion to target peak dopamine depletion. During the learning task participants were presented with arbitrary stimuli and were required to learn the correct stimulus-response pairings based on feedback. Using a double-blind within-subjects design, participants were administered, on two separate days, a nutritionally balanced amino acid mixture (placebo) and a mixture deficient in Tyr and Phe (depletion). Order of administration was randomly assigned. 2-Condition (depletion, placebo) x 2-Accuracy (correct, error) robust ANOVAs were conducted on FRN amplitude.

Results: FRN amplitude was more negative for error trials than correct trials $(\mathrm{TWJ} / \mathrm{c}=19.46, \mathrm{p}=.002)$; however, similar FRN amplitude was observed between depletion and placebo conditions $\left(\mathrm{T}_{\mathrm{s}}<1.53\right.$, $\left.\mathrm{ps}>.23\right)$. Conclusions: The findings contradict previous research that indicates decreased neural indices of feedback monitoring upon administration of dopamine agonist medications and demonstrate that FRN amplitude is not closely related to acute changes in dopamine. These results suggest that other factors may influence the relationship between certain medications and feedback monitoring. Implications of these findings for models of psychiatric illness are discussed.

Correspondence: Peter E. Clayson, MA, University of California-Los Angeles, 1285 Franz Hall, Box 951563, Los Angeles, CA 90095. E-mail: pclayson@ucla.edu

A. Clawson, B. AnDERSON, K. CARbine, M. SOUTH \& M.J. LARSON. Reversal Learning in Autism: Neural and Behavioral Performance Monitoring.

Objective: Individuals with autism spectrum disorder (ASD) show reduced flexibility in response to feedback. We used the feedback-related negativity (FRN), an event-related potential component that indexes reward sensitivity and prediction, to examine whether individuals with ASD utilized performance feedback to learn rules and improve performance. We hypothesized that individuals with ASD would display reduced FRN amplitudes following reversal and exploration negative 
feedback and increased perseverative and exploration errors relative to controls.

Participants and Methods: High-density electroencephalogram recordings were collected from 13 individuals with ASD and 16 control participants during a four-choice reversal-learning task. FRN amplitudes were analyzed using a Group x Feedback (reversal negative, first and second exploration negative, first positive) ANOVA. Errors were analyzed using a Group x Error (exploration, perseverative, reversal, spontaneous) ANOVA and response times (RTs) were analyzed using a Group x Response (first, second, third switch; correct) ANOVA.

Results: Analyses revealed a significant main effect of feedback but no significant main effects or interactions of FRN amplitude based on group $\left(F_{s}<1.8, p s>0.17\right)$. However, individuals with ASD displayed significantly less negative FRN amplitudes relative to controls during reversal negative feedback $(\mathrm{t}=-2.51, \mathrm{p}=0.02)$. For error rates and $\mathrm{RT}$, main effects and interactions with group were nonsignificant $\left(F_{s}<2.86\right.$, ps $>0.10)$.

Conclusions: Individuals with ASD displayed reduced neural activation following an unexpected rule change but did not differ from controls for any other feedback type. Behavioral performance did not differ between groups. Results are consistent with previous findings of differential neural activation in ASD despite similar behavioral performance possibly indicating altered neural network activation during feedback presentation.

Correspondence: Ann Clawson, B.S., Brigham Young University, 6575 S. 2475 E., Salt Lake City, UT 84121. E-mail: clawson.ann@gmail.com

M.B. MITCHELL, S.D. SHIRK, D.G. MCLAREN, J.S. DODD \& A. ATRI. ERP Age Effects during Face-Name Recognition: Relation to Neuropsychological Test Performance.

Objective: Classic event related potential (ERP) effects seen in recognition memory paradigms implicate frontal and parietal brain networks in sustaining memory and executive functions. In this study we aimed to examine age effects in frontal and parietal ERP activity during performance of a face-name recognition task in relation to clinical measures of executive function.

Participants and Methods: Thirty-three participants (16 young adults aged 18-39 and 17 older adults aged 55-69) studied 72 face-name pairs, of which 36 were presented four times $(4 R)$ and 36 were only presented once (1R). Participants made old/new judgments for 80 facename pairs, of which 40 were old (20 1R and $204 R$ ) and 40 were novel (N). ERP activity during correct identification of faces was analyzed using analysis of variance methods applied to a priori regions of interest (ROI). Participants additionally completed a battery of neuropsychological tests heavily focused on memory and executive function.

Results: Planned contrasts showed a significant difference between age groups in early frontal ROI activation (300-500 ms) for N vs. 1R ( $<<$ $.05)$. A significant difference between the two age groups was also observed in parietal ROI activation $(500-800 \mathrm{~ms})$ for $4 \mathrm{R}$ vs. $1 \mathrm{R}(\mathrm{p}<.05)$. Contrast scores indexing these two ERP effects were then correlated with measures of executive function. The parietal effect, as indicated by the difference between ERP activation in the Right Posterior Superior ROI for $1 \mathrm{R}>\mathrm{N}$ was correlated with a measure of cognitive set shifting (Trails $\mathrm{B}$ performance; $\mathrm{r}=-.38, \mathrm{p}<.05)$.

Conclusions: Findings complement robust spatial resolution fMRI signals of activity in the medial temporal lobes with temporal resolution frontal and parietal ERP signals that show classic old/new effects. These results suggest that multimodal assessment of associative memory processes using a combination of task-related fMRI and ERP data may provide complementary information regarding the dynamics of memory function and cognitive aging.

Correspondence: Meghan B. Mitchell, Ph.D., GRECC, Bedford VA Medical Center, 26 Main Street, Pepperell, MA 01463. E-mail: meghanbmitchell@gmail.com

\section{Paper Session 9: Cognitive Neuroscience}

\author{
10:45 a.m.-12:15 p.m.
}

M.J. VAN ZANDVOORT, E. DALMAIJER \& P. ROBE. Stimulation of Supplementary Eye Fields induces temporary neglect during awake neurosurgery.

Objective: Humans continuously interact with changing environments, relying primarily on vision by guiding the highest acuity of their visual field towards elements of interest (foveating). Before such an eye movement can be made, the most interesting elements must be resolved from the context to select the most relevant. One of the areas thought to be involved and associated with cognitive control, is the supplementary eye field (SEF). In this case study we found support for the role of SEF in humans.

Participants and Methods: Here we present our case, a 54-year old man who underwent surgery to (partially) resect a frontal brain tumour. During neurosurgery, the patient remained awake to test his behavioural responses to electrical stimulation $(3 \mathrm{~mA}, 50 \mathrm{~Hz})$. While electrical impulses temporarily disrupted neural activity, neuropsychological functioning was assessed using standard neuropsychological tests i.e. Stroop task, qualitative description of a scene, reading, and object naming.

Results: Our observations indicate $\mathrm{AB}$ suffered from a severe attentional bias towards the right side of his visual field during stimulation that disrupted SEF functioning. On the Stroop: the number of errors was not the same before, during, and after stimulation [, $\chi 2=10.0, \mathrm{p}=.007]$, with $\mathrm{A}$ omitting more words from the left half $(\mathrm{M}=4.8, \mathrm{SD}=1.5)$ of the display during SEF stimulation than both immediately pre and post. Conclusions: We presented the case of $\mathrm{AB}$, who demonstrated a severe rightward bias of spatial attention while his SEF functioning was temporarily electrically disrupted during awake neurosurgery. Our results fit in well with the idea that frontal areas, now including the SEF, play a role in the allocation of spatial attention. Additionally, our results support the idea that the SEF is not only involved in context monitoring and response selection, but is also involved in the preparation and execution of responses.

Correspondence: „, E-mail:

G. IARIA, A. ARNOLD, F. BURLES, I. LIU, E. SLONE, S. BARCLAY, T. BECH-HANSEN \& R. LEVY. Developmental Topographical Disorientation is associated with Decreased Functional Connectivity between the Hippocampus and Prefrontal Cortex.

Objective: The lifelong history of getting lost in familiar surroundings despite any brain injury or neurological condition is known as Developmental Topographical Disorientation (DTD). The objective of the this study is to investigate the neural correlates of this newly discovered cognitive disorder.

Participants and Methods: We asked a group of individuals with DTD and a group of control subjects to undergo a resting-state functional MRI (rsfMRI) scan. In addition, both groups performed a task-based fMRI study while forming a cognitive map of a virtual environment. Finally, we performed Voxel Based Morphometry to exclude structural differences between DTD and control groups.

Results: We found a decreased functional connectivity between the right hippocampus and the prefrontal cortex (PFC) in individuals with DTD. In addition, the fMRI study revealed decreased signal change in DTD individuals within the insular cortex, and the temporal and parahippocampal gyri in the right hemisphere while forming a cognitive map. No structural differences were revealed between groups.

Conclusions: Our findings provide the first group based neural evidence of DTD and suggest that ineffective functional connectivity between hippocampus and PFC may affect the monitoring and processing of spatial information while moving within an environment, resulting in the selective inability to form cognitive maps that are critical for orienting in the environment. 
Correspondence: Giuseppe Iaria, Psychology, University of Calgary, 2500 University Drive NW, Calgary, AB T2N 1N4, Canada. E-mail: giaria@ucalgary.ca

S.G. RYMAN, A.A. VAKHTIN, R.A. YEO, M. VAN DEN HEUVEL, M. DE REUS, R.A. FLORES, C. WERTZ \& R. JUNG. Structural Network Hub Connectivity Predicts Intelligence.

Objective: Intelligence is associated with efficiency of a widespread neural network within parietal and frontal regions (Jung \& Haier, 2007; van den Heuvel et al., 2009). Network analysis of the human brain has identified key brain regions, referred to as the rich club, which are highly connected, and responsible for integration between functional networks of the brain (van den Heuvel et al., 2012). The current study hypothesizes that due to its role in integrating communication across distant brain regions, the connectivity of the rich club will predict intelligence. Participants and Methods: We obtained behavioral and neuroimaging data from 250 healthy participants ( 167 male, age $\mathrm{M}=22+/-3.96$; FSIQ M=111.77+/-12.02, WASI-II). The network was mathematically described as a graph $\mathrm{G}=(\mathrm{V}, \mathrm{E})$, with cortical regions serving as nodes $(\mathrm{V})$, and reconstructed white matter fiber tracts serving as edges $(\mathrm{E})$. The rich club nodes of the brain were identified and connectivity measures corresponding to rich club connections, feeder connections, and local connections for each subject were extracted. Multiple regressions were used to determine which types of connections predicted FSIQ accounting for age and sex, examining connectivity, cost and length of the connections. Results: The rich club $(k=20)$ was comprised of a set of widely distributed brain regions. Higher intelligence scores were associated with the connectivity strength of the feeder and local connections to the rich club, but not the local and rich club connections. Furthermore, network cost and measures of network efficiency predicted intelligence.

Conclusions: The rich club provides a focal network through which the brain's long distance connections pass. This study found the paths related to individual differences in intelligence link the rich club regions to the rest of the brain. Examinations of the network's cost and efficiency suggest individuals with high intelligence have connections that facilitate efficient information transfer with a greater physical cost. Correspondence: Sephira G. Ryman, M.S., Psychology, University of New Mexico, 800 Madison St. NE, Albuquerque, NM 87110. E-mail: sephira.ryman@gmail.com

G. DOUCET, R. RIDER, N. TAYLOR, P. BARNETT, C. SKIDMORE, M. SPERLING, A. SHARAN \& J.I. TRACY. Pre-surgery restingstate graph-theory measures predict neurocognitive outcomes after brain surgery in temporal lobe epilepsy.

Objective: This study investigated the ability of resting-state functional connectivity (rsFC) graph-theory measures to predict neurocognitive status in temporal lobe epilepsy (TLE) patients who underwent an anterior temporal lobectomy (ATL).

Participants and Methods: Thirty-two TLE patients (16 left/16 right) that underwent a ATL realized a 5-min fMRI resting-state task, pre-surgery. Patients were administered standardized neuropsychological testing in order to evaluate neurocognitive change post-surgery in major domains: verbal and non-verbal episodic memory, language, working memory, and attention. We investigated three graph-theory properties (local efficiency, Eloc; distance, Dist; participation, P), measuring segregation, integration and centrality, respectively. These measures were computed for regions with functional relevance to both the cognitive domain of interest and the ictal pathology. Linear regression analyses were computed to predict the change in each neurocognitive domain, using the graph-theory measures as the independent variables.

Results: We found that the integration measure (Dist) was the most predictive of outcome in the left TLE. For the right TLE, the Dist and P measures appeared equally predictive. For both groups, the Eloc measure was the least predictive. For both TLE groups, the contralateral healthy hippocampus was one of the most predictive regions. Regardless of TLE group, the outcomes of verbal-related domains were best predicted by left-sided regions. Working memory outcome was predicted solely by right-sided regions.

Conclusions: This study brings evidence that pre-surgery rsFC measures help to determine neurocognitive outcomes in the major cognitive domains in TLE. The results have implications for refining our understanding of compensatory reorganization and predicting cognitive outcome after ATL using graph-theory measures. The results are encouraging with regard to the clinical relevance of using such measures in pre-surgical algorithms and decision making in the setting of TLE. Correspondence: Joseph I. Tracy, PHD, Neurology, Thomas Jefferson University/Jefferson Medical College, Health Sciences Bldg., Ste. 407, 901 Walnut Street, Philadelphia, PA 19107.E-mail: joseph.tracy@ jefferson.edu

E. JAKOBSEN, J. BOETTGER, P. BELLEC, S. GEYER, M. PETRIDES \& D.S. MARGULIES. Individual In Vivo Sub-parcellation of Broca's Region Using Functional Connectivity Glyphs.

Objective: Broca's region is functionally involved in language production and is comprised of two adjacent cytoarchitectonic areas, 44 and 45. These regions have distinct connectivity to superior temporal and inferior parietal regions in both macaque monkeys and humans. We made use of prior knowledge of sulcal anatomy and resting-state functional connectivity, together with a novel visualization technique, to manually parcellate areas 44 and 45 in individual brains in vivo.

Participants and Methods: 103 ICA-FIX denoised rfMRI datasets from healthy individuals, provided by the Human Connectome Project, were used. Left-hemisphere grayordinate correlation matrices were computed for every subject, and then visualized in brainGL. The full unthresholded connectivity profile is presented at each node of the surface based rendering in a small visual summary (glyph), representing the distribution of connections from the node to the rest of the cortical surface. By manipulating various visualization parameters of the glyphs, transitions between cortical areas can be observed. Using this technique, individual brains were manually parcellated, and then compared at the group-level. Additionally, the manual labeling approach was compared to a variety of pre-existing automatic clustering techniques.

Results: Group-level probability maps of the two areas show significant spatial consistency across individuals, while still demonstrating individual variability in cortical topography. Automatic parcellation techniques produced clusters with varying degrees of spatial overlap with the manual labels, indicating the need for further investigation and validation of machine learning cortical segmentation approaches. Conclusions: We could clearly delineate areas 44 and 45 from each other and neighboring regions in all subjects. These results are consistent with previous studies showing distinct connectivity patterns of areas 44 and 45 , and validate the usefulness of glyph visualization for individual-level manual cortical segmentation.

Correspondence: Estrid Jakobsen, Max Planck Research Group for Neuroanatomy and Connectivity, Max Planck Institute for Human Cognitive and Brain Sciences, Max Planck Institute for Human Cognitive and Brain Sciences, Stephanstrasse 1A, Leipzig 04103, Germany.E-mail: ejakobsen@cbs.mpg.de

R.A. YEO, S.G. RYMAN, M. VAN DEN HEUVEL, M. DE REUS, A.R. MAYER, R. JUNG \& J. POMMY. Rich Club connectivity and executive function in healthy controls and individuals with schizophrenia.

Objective: Graph theory can provide quantitative descriptions of networks from structural imaging data. A key network is the Rich Club (RC), a set of nodes that interact extensively with each other. We hypothesize that greater RC connectivity predicts better executive function $(\mathrm{EF})$.

Participants and Methods: We examined the importance of the RC connectivity for individual variation in executive function in healthy controls $(\mathrm{N}=113)$ and individuals with schizophrenia $(\mathrm{N}=82)$, based on diffusion-weighted imaging. Participants were recruited from four 
sites and network metrics were adjusted for site differences. Three executive skills (Planning, Fluency, Inhibition) were identified through principal components analysis of ten variables from six tests, and an overall composite (EF) was created by averaging them. The network was mathematically described as a graph $\mathrm{G}=(\mathrm{V}, \mathrm{E})$, with cortical regions serving as nodes $(V)$, and reconstructed white matter fiber tracts serving as edges $(\mathrm{E})$.

Results: Patients scored lower on each cognitive measure. They also had lower overall connectivity among the RC Nodes, as well as lower connectivity of local and feeder connections with RC nodes. Across groups, all three connectivity measures predicted EF, though effect sizes varied across specific components. In multivariate analyses, connections among $\mathrm{RC}$ nodes emerged as the most important network metric. Follow-up analyses revealed variation in the importance of specific RC nodes. Conclusions: RC connectivity predicts individual variation in EF and group differences in RC connectivity contribute to group differences in diverse EF skills. These results also demonstrate the importance of distributed brain regions for $\mathrm{EF}$.

Correspondence: Ronald A. Yeo, PhD, Psychology, University of New Mexico, Department of Psychology, University of New Mexico, Albuquerque, NM 87122.E-mail: ryeo@unm.edu

\section{Symposium 14: \\ The Many Faces of Memory Disorders: Video Case Examples and Neuroanatomical Correlations}

\section{Organizers: Kathleen Y. Haaland, Russell M. Bauer}

\section{Discussant: Michael Kopelman}

\section{0:45 a.m.-12:15 p.m.}

K.Y. HAALAND, K.Y. HAALAND, M. VERFAELLIE, M.G. O'CONNOR, J. DELUCA, R.M. BAUER \& M. KOPELMAN. The Many Faces of Memory Disorders: Video Case Examples and Neuroanatomical Correlations.

Symposium Description: Memory disorders and amnesia can result from damage to a distributed memory system that includes the medial temporal lobe, the diencephalon, and the basal forebrain. An inability to utilize memory in the service of cognition may additionally result from cortical lesions. The neuropsychological study of these disorders has been largely driven by information processing accounts that identify patterns of performance associated with encoding, consolidation, or retrieval deficits, or that attempt to account for performance dissociations on tasks of recollection or familiarity. This symposium will provide an overview of human memory disorders by utilizing video case examples of specific syndromes and discussing key cognitive and anatomical features of each form of amnesia presented. Mieke Verfaellie will introduce the symposium with emphasis on the heterogeneity of cognitive/information-processing deficits seen in the human amnesic syndrome. Kathleen Haaland will discuss findings from a patient who sustained an anoxic episode after cardiac arrest, displaying many of the features of temporal lobe amnesia. Margaret O'Connor will present a patient with alcoholic Korsakoff syndrome related to diencephalic and frontal lobe dysfunction. John DeLuca will report on case examples of basal forebrain amnesia featuring patients with anterior communicating artery aneurysms. Russell Bauer will present a young patient with Pick's disease who shows a striking inability to use recent or working memory to direct complex cognitive or motor performance due to frontal lobe involvement. Michael Kopelman will serve as a discussant emphasizing the importance of careful cognitive analysis coupled with attempts to localize the deficit within the human memory system both functionally and anatomically.
Correspondence: Kathleen Y. Haaland, PhD, Psychiatry and Neurology, University of New Mexico, MSC 095030,1 University of New Mexico, Albuquerque, NM 87131.E-mail: khaaland@unm.edu

M. VERFAELLIE. The Many Faces of Memory Disorders in the Context of Contemporary Memory Theory.

Qualitative differences in the nature of memory impairment among patients with distinct lesions have long been recognized. However, recent advances in understanding the functional properties of different types of memory and the neural substrates mediating them shed new light on various profiles of memory impairment. In the introduction to this symposium, I will highlight how advances in memory research can guide assessment and theoretical understanding of memory disorders associated with distinct etiologies.

Correspondence: Mieke Verfaellie, PhD, 150 S Huntington Ave (151A), Boston, MA 02130. E-mail: verf@bu.edu

\section{K.Y. HAALAND. Medial Temporal Lobe Syndrome: Amnesia in an} Anoxic Patient after Cardiac Arrest.

The amnesia of patients with medial temporal lobe (MTL) damage is characterized by relatively intact encoding but significantly impaired storage of new information, which is commonly supported by impaired delayed recall that cannot be explained by retrieval deficits. The neurobehavioral, neuroimaging, neurosurgical and/or neuropathological analysis of such patients has significantly influenced our views of the cognitive and neuroanatomical characteristics of MTL amnesia. For example, HM, Milner's bilateral temporal lobectomy patient, represents the bulwark of traditional conceptions of MTL amnesia. Zola Morgan and Squire's case was even more specific as they linked this form of amnesia in an anoxic patient after cardiac arrest to damage to the CA1 sector of the hippocampus. While we don't have verification of temporal lobe damage in the anoxic case presented here, the behavioral presentation is consistent with the traditional picture of MTL amnesia characterized by relatively intact encoding but severely impaired storage of new information and significant retroactive interference. MTL amnesia will be discussed in the context of traditional and more recent conceptions of memory.

Correspondence: Kathleen Y. Haaland, PhD, MSC 095030, 1 University of New Mexico, Albuquerque, NM 87131.E-mail: khaaland@unm.edu

\section{M.G. O'CONNOR. Wernicke Korsakoff's Syndrome: Contributions to Models of Memory.}

Patients with Wernicke Korsakoff's Syndrome (WKS) develop amnesia as a result of chronic alcohol abuse and thiamine deficiency. The cognitive phenotype often associated with WKS emphasizes encoding deficits, confabulation and temporally graded retrograde amnesia which may be due to the convergent effects of diencephalic damage and frontal systems dysfunction. Other confounding variables, including chronic alcohol abuse, prior head injuries, low baseline achievement, and psychiatric co-morbidity, affect the functional outcome of the individual with WKS. In this presentation, neuropsychological studies of WKS amnesics will be reviewed and the neuropsychological profiles of several patients will be presented. Findings will be discussed in relation to cognitive models of amnesia and the neural substrates of memory.

Correspondence: Margaret G. O'Connor, PhD, BIDMC/Neurology/330 Brookline Ave, Boston, MA 02215. E-mail:moconnor@bidmc.harvard. edu

\section{J. DELUCA. Profound Amnesia and Confabulation following Anterior Communication Artery Aneurysm.}

Patients who suffer an aneurysm of the anterior communicating artery $(\mathrm{ACoA})$ frequently suffer from severe amnesia, confabulation and personality change (referred to as the "ACoA syndrome"). This presentation will display video clips of the profound amnesia and confabulation often observed in these patients. The discussion will center around our 
understanding that the amnesia is a result of basal forebrain damage and how the basal forebrain modulates hippocampal functioning. The confabulation is a consequence of the interaction between basal forebrain damage and frontal lobe dysfunction. With cognitive rehabilitation, significant improvement was observed in frontal executive functions in this patient, although the profound amnesia remained. With treatment, despite the remaining amnesia, the patient was able to return to a high level of functional activity in everyday life.

Correspondence: John DeLuca, PhD, 1199 pleasant valley way, West Orange, NJ 07052.E-mail: jdeluca@kesslerfoundation.org.

\section{R.M. BAUER. When Working Memory Isn't Working: Profound Retrieval Deficits in a Case of Early-Onset Pick's Disease.}

Successful memory performance depends not only on a distributed memory system involving temporal, diencephalic, and basal forebrain structures, but also on interaction between frontal-intentional systems and memory-related structures. This presentation focuses on clinical and neuroimaging analysis of a 29-year-old with pathologically verified Pick's disease. The clinical examination, depicted in video segments, reveals profound impairment in working memory and a dramatic impairment in memory retrieval. The qualitative features of this case will be described and contrasted with other patients with memory disorders presented by other symposiasts. Despite these profound deficits, the patient retains certain motor skills and other capacities that do not depend on intentional remembering and retrieval; these examples of spared skills stand in stark contrast to the impairments in working memory and recall performance.

Correspondence: Russell M. Bauer, Ph.D., PO Box 100165 HSC, Gainesville, FL 32610.E-mail: rbauer@phhp.ufl.edu

\section{KOPELMAN. Discussion.}

Professor Kopelman will discuss the cases presented with respect to their clinical, neuropsychological, and theoretical implications.

Correspondence: Michael D Kopelman, Kings College London, St Thomas's Hospital, London SE1 7EH, United Kingdom. E-mail: kopelman.pa@kcl.ac.uk

\section{Poster Session 9: \\ Assessment-Adult, Cognitive Intervention/Rehab, \& Visuospatial/Neglect}

\section{0:45 a.m.-12:15 p.m.}

\section{Assessment/Psychometrics/Methods (Adult)}

\section{J.A. TAYLOR, A. BOETTCHER \& B.D. HILL. A Psychometric Examination of Pain Scales Commonly Used in the Management of Chronic Pain.}

Objective: Chronic pain is common condition that significantly impairs patients' lives and may result in disability. Pain scales are commonly used in presurgical work-ups for chronic pain patients. Despite their wide spread use, the psychometric properties of these scales is understudied. The purpose of this study was to examine which scales and specific items best measure latent pain constructs. It was hypothesized that all pain scales would index the same pain construct.

Participants and Methods: This study examined the psychometric properties of three common pain scales. Records were reviewed from 147 patients who had recently had either a presurgical behavioral medicine evaluation or simply a behavioral medicine evaluation ( $\mathrm{M}$ age $=51.1$, $\mathrm{SD}=13.578 ; 60 \%$ female; Age range $=22-88)$. Using principal axis factor analysis, two primary factors emerged related to disability and somatization, respectively.

Results: All items of the PCS and PDI were significantly correlated with disability factor scores. Correlations ranged from $r=.2-.8$. Cronbach's alpha was .933 for PCS and .858 for PDI. MSPQ items were not strongly related to disability factor scores $(\mathrm{r}=.2-.3$ range) but were more related to somatization factor scores $(\mathrm{r}=.4-.7$ range). Individuals who failed effort measures were removed from the dataset $(n=53)$. Scale content was examined with exploratory factor analysis using principal axis factoring and varimax rotation. Coefficient alpha was calculated to examine psychometric properties of the tools. Bivariate correlations were used to examine the association between the scales and latent pain constructs. Data were analyzed with SPSS.

Conclusions: Two primary factors emerged when all scales were analyzed that were interpreted as indexing disability (Factor 1, 29\% of variance) and somatization (Factor 2, 9\% of variance). Our hypothesis was not supported. The PCS and PDI primarily index aspects of pain related to endorsement of disability and the MSPQ was more related to endorsement of somatization.

Correspondence: Joshua A. Taylor, Psychology, University of South Alabama, 1875 Woodcrest Ct., Semmes, AL 36575.E-mail:jtaylor_02@ yahoo.com

J. CAVANAUGH. Examining the Differential Effects of Natural and Synthetic Aromas of Lavender and Peppermint on Cognition, Mood, and Subjective Workload.

Objective: There is reliable, empirical evidence that various inhaled aromas can significantly affect mood, cognition, physiology and behavior (Herz, 2009). However, the literature often does not document whether natural or synthetic fragrances were used when evaluating these factors. Also, no studies to date have compared natural and synthetic versions of the same odors.

Participants and Methods: The current study attempted to determine whether the natural aromas of lavender and peppermint were superior to their synthetic counterparts in terms of measurable differences in cognition, mood, and subjective workload among 180 college students. Specific Aim 1 compared the inhalation of natural fragrances to the inhalation of synthetic fragrances on measures of cognitive function. Specific Aim 2 addressed whether the inhalation of natural fragrances, as opposed to synthetic fragrances, was associated with greater group differences on a measure of self-reported mood. Finally, Specific Aim 3 compared the inhalation of natural fragrances to synthetic fragrances with respect to their ability to decrease subjective workload and increase subjective performance. This Aim also assessed whether subjective workload was a mediator of cognition and mood.

Results: The initial results from this study reinforced the assertion that fragrances are capable of altering cognition, with further evidence revealing that the natural aroma of peppermint is superior to its synthetic counterpart in bringing about these changes. However, after performing a statistical correction for conducting multiple comparisons, these results largely do not remain significant.

Conclusions: Combined with small effect sizes, this study was unable to provide conclusive evidence to support the statement that natural aromas are superior to their synthetic counterparts in terms of their ability to alter cognition, mood, and subjective workload.

Correspondence: Jamie Cavanaugh, MA, Psychology, University of Colorado Denver, 1140 Josephine St, Denver, CO 80206. E-mail: jamie. cavanaugh@ucdenver.edu

B.A. HUNTBACH \& Y. SUCHY. Predicting Performance on Sequencing and Backward Span Tasks Using a Verbal and NonVerbal n-Back Task.

Objective: Performance of the digit-span backward (DS-B) and letter number-sequencing (LNS) is thought to require spatial working memory (WM) because of the need to mentally visualize the stimuli (Sattler \& Ryan, 2012; Costa, 1975). However, this hypothesis has not been formally tested (Sattler \& Ryan, 2009). The purpose of this study was to examine the contribution of verbal and spatial WM to DS-B and LNS, as well as to spatial span backward (SS-B) tasks. 
Participants and Methods: 90 adult males, ages 19 to 49 (M=32.34, $\mathrm{SD}=7.67$ ) with 8 to 17 years of education completed the DS-B, LNS, SS-B, and verbal and spatial n-back tasks.

Results: Linear regressions using DS-B, LNS, and SS-B as criterion variables and verbal and spatial $n$-backs as predictors showed that both verbal and spatial n-backs were significant predictors of both LNS $[b=.746, t(87)=2.358, p<.021 ; b=.576, t(87)=2.201, p<.030$, respectively] and SS-B $(b=.429, t(87)=2.309, p<.023 ; b=.438, t(87)=2.855$, $\mathrm{p}<.005$, respectively]. However, only verbal $\mathrm{n}$-back predicted DS-B $(b=.735, t(87)=2.693, p<.008)$, with no contribution from spatial n-back $(\mathrm{p}=.668)$.

Conclusions: Contrary to expectation, backward digit span does not require spatial working memory, whereas both LNS and SS-B have both verbal and spatial WM contributions.

Correspondence: Bryce A. Huntbach, University of Utah, 934 E $260 \mathrm{~N}$, Orem,UT 84097.E-mail: brycehuntbach@gmail.com

L. OHLHAUSER, D. LEITNER, J. UPSHAW, M. REYNOLDS, J.C. PIERCY, E. CONCEPCION \& H. MILLER. Comparability of the Full and Short Forms of the Personality Assessment Inventory in a Stroke Population.

Objective: The Personality Assessment Inventory (PAI) is a self-report measure of psychopathology, interpersonal style, and factors that impact treatment. The Personality Assessment Inventory - Short Form (PAI-SF) contains the first 160 items of the full 344-item PAI. This study aims to determine the validity of using the PAI-SF in place of the full PAI in a stroke population.

Participants and Methods: Participants $(\mathrm{N}=43)$ were hospital inpatients (mean age $=67.69, \mathrm{SD}=12.98$ ) who underwent neuropsychological evaluation a mean of 8.61 days $(\mathrm{SD}=6.82)$ following stroke. Participants completed the full PAI, from which both PAI and PAI-SF scores were generated. Form comparability was determined by examining differences in mean $\mathrm{T}$-scores of the clinical scales, calculating the intra-class correlations, and determining agreement of clinically significant scores between the two forms.

Results: Mean difference scores fell within the standard error of the mean except for the Paranoia scale. The mean clinical scale intra-class correlations were between .88 and .97. Agreement of clinically significant scores between the PAI and PAI-SF ranged from $90.7 \%$ to $100 \%$. When the two forms disagreed on clinically significant scores, it was more often the PAI-SF which exceeded a T-score of 70, while the PAI remained below that threshold.

Conclusions: The PAI-SF provides a reasonable approximation of the full PAI in a stroke population. Though the PAI-SF useful for research purposes and psychopathological screening in the stroke population, the PAI includes additional validity measures and clinical subscales, and is recommended when making critical decisions about patient care. Correspondence: Lisa Ohlhauser, Bachelor of Science in Psychology with Honours, Psychology, University of British Columbia - Okanagan, 771 Royal Pine Drive, Kelowna, BCV119G2, Canada.E-mail: lismiroh@ gmail.com

\section{NOGGLE, J. THOMPSON, A. LEVINE \& J. DAVIS. Grooved Pegboard and RBANS Performance: Links between Motor and Cognitive Functioning.}

Objective: The grooved pegboard (GP) is a measure commonly included in neuropsychological assessments as a means of assessing manual dexterity, motor processing, and sensory-motor integration. While studies have demonstrated links between GP performance and general cognitive slowing, more expansive studies looking at the relationship between GP performance and broader neurocognitive status have not been done. The present study as a result sought to evaluate the relationship between outcomes on GP and the RBANS.

Participants and Methods: An archival data set was utilized for the current study. Participants $(n=669)$ included were part of a mixed clinical sample previously evaluated as either inpatients or outpatients at a large Midwestern hospital. All participants completed the Grooved Pegboard and The RBANS as part of their clinical workup.

Results: At a domain level, significant correlations were observed between GP Dominant-hand performance and outcomes in Immediate Memory $[-.454(<.001)]$, Visuospatial/Constructional $[-.425(<.001)]$, Attention [-.294 (.007)], Delayed Memory [-.404 (<.001)], and the Total Score $[-.460(<.001)]$. No significant correlation was found between GP-Dominant hand performance and outcomes in Language [-.165 (.139)]. Significant correlations were also found between GP Non-dominant hand performance Immediate Memory [-.327 (.007)], Visuospatial/Constructional [-.332 (.003)], Language [-.270 (.016)], Attention [-.364 (.001)], Delayed Memory [-.415 (<.001)], and the Total Score $[-.452(<.001)]$.

Conclusions: Findings demonstrate that GP performance does, in fact, provide some insight into overall neurocognitive functioning. Outcomes may suggest the grooved pegboards capability in providing a snapshot of general neurological functioning while more broadly supporting the concept of shared variance of sensory-motor functioning and neurocognition.

Correspondence: Chad Noggle, Ph.D., ABN, Psychiatry, SIU School of Medicine, $315 \mathrm{~W}$. Carpentedr St., Springfield, IL 62794. E-mail: cnoggle@siumed.edu

\section{J. THOMPSON, C. NOGGLE, A. LEVINE \& J. DAVIS. Relationship of Finger Tapping to General Neurocognitive Functioning.}

Objective: Finger tapping tests are commonly included as part of broader neuropsychological assessments. Prior research has demonstrated finger tapping tests can distinguish between individuals with brain dysfunction and those without. Other studies have even suggested a link between finger tapping performance and processing speed. The present study evaluated whether a linear relationship existed between finger tapping performance and a broader array of neurocognitive functioning.

Participants and Methods: An archival data set was utilized for the current study. Participants ( $\mathrm{n}=37$ ) included were part of a mixed clinical sample previously evaluated as either inpatients or outpatients at a large Midwestern hospital. All participants completed the RBANS as part of their clinical workup along with a finger tapping test.

Results: At a domain level, significant correlations were observed between dominant-hand finger tapping and Visuospatial/Constructional [.353 (.032)], Attention [.414 (.011)], Delayed Memory [.392 (.016)], and the Total Score $[.388(.018)]$. No significant correlation was found between dominant-hand finger tapping performance and outcomes in Immediate Memory [.259 (.122)] and Language [.018 (.916)]. Significant correlations were also found between non-dominant finger tapping and performance in Immediate Memory [.351 (.033)], Visuospatial/ Constructional [.356 (.031)], Attention [.492 (.002)], Delayed Memory $[.425(.009)]$, and the Total Score $[.454(.005)]$. No significant correlation was found between non-dominant finger tapping performance and outcomes in Language [.141 (.405)].

Conclusions: Findings demonstrate that finger tapping likely goes beyond simply demonstrating brain dysfunction per se, rather, it provides insight into overall neurocognitive functioning.

Correspondence: Chad Noggle, Ph.D., ABN, Psychiatry, SIU School of Medicine, $315 \mathrm{~W}$. Carpentedr St., Springfield, IL 62794. E-mail: cnoggle@siumed.edu

\section{J.C. TOMASZCZYK, B. SHARMA, J. MOK, B.K. CHRISTENSEN \& R. GREEN. Development of a Scale to Measure Patient Acceptability of Cognitive Therapy.}

Objective: Cognitive assessment and intervention for patients with acquired brain injury (ABI) carry burden. Currently, there are no metrics for measuring this burden, leaving open the question of how a given intensity and duration of a cognitive session impacts a patient, and how this impact may, in turn, result in benefits or consequences for 
recovery. We report on the development of an "acceptability scale" to measure this impact.

Participants and Methods: To construct the scale prototype, a multi-disciplinary team of clinical and scientific experts in neurorehabilitation (from neuropsychology, occupational therapy, psychology, social work, speech language therapy, and rehabilitative therapy) were recruited to refine the construct measured by the scale and to generate scale items. The first version of the scale was also piloted in a recent feasibility study.

Results: The panel reached consensus on a scale that would measure patient perception of the burden of therapy. The tool would be value-neutral regarding the impact of patient perception on clinical outcomes. The prototype generated contained 21 items that focused on potential cognitive, emotional, and somatic symptoms that patients may experience after a cognitive session and a four point scale that asks patients to rate each of these symptoms ("Less", "Same", "More" or "Much More") as compared to prior to the session. In the pilot study, moderate-severe ABI patients understood instructions, completed all items of the scale, reported no difficulty understanding test items and were able to self-complete. Preliminary clinician response was positive, with users reporting the tool easy and rapid to administer.

Conclusions: We have developed an acceptability scale for measuring patient perception of cognitive assessment and intervention burden. The tool was well-received by clinicians/therapists and patients in pilot testing. Further research into the validity and reliability of the tool is underway.

Correspondence: Jennifer C. Tomaszczyk, Research, Toronto Rehabilitation Institute, 550 University Avenue, Toronto, ON M5G2A2, Canada.E-mail: jennifer.tomaszczyk@uhn.ca

\section{A. WARD \& D. TRANEL. A Critical Evaluation of the Validity and Clinical Implications of Episodic Future Thinking.}

Objective: The aim of this critical narrative review is to assess the validity and clinical implications of episodic future thinking, an important aspect of cognition that may not be well measured in typical neuropsychological assessments.

Participants and Methods: A systematic review of episodic future thinking literature was conducted. This review included the critical evaluation of 26 reviews and 75 empirical journal articles to determine the construct and predictive validity as well as the clinical advantages and disadvantages of episodic future thinking tasks.

Results: The review concludes that evidence regarding the validity of episodic future thinking is lacking, since this construct has not been directly compared to related constructs in terms of its overlap and predictive validity. The clinical advantage of an episodic future thinking task is that it may capture an area of cognition that is not well measured within neuropsychological assessment. Episodic future thinking is critical for higher cognitive abilities and adaptive behavior, which in turn have implications for neurological populations. A primary limitation of episodic future thinking tasks is that they are too lengthy in terms of administration and scoring for a clinical setting.

Conclusions: The clinical utility of episodic future thinking is currently unknown due to the lack of research investigating incremental validity. Future research is needed to address outstanding questions before incorporating episodic future thinking into a clinical setting. The validity of episodic future thinking should be investigated to determine whether episodic future thinking predicts the ability to engage in adaptive functioning over and above similar constructs. An episodic future thinking task that is economical and efficient in terms of its administration and scoring is needed. Future work will help determine whether assessment of a key higher cognitive function is missing in clinical neuropsychological assessment.

Correspondence: Amanda Ward, Bachelor of Arts, University of Iowa, 1146 Hotz Avenue, Iowa City, IA 52245.E-mail: amanda-m-ward@ uiowa.edu
Y. GOVEROVER \& J. DELUCA. Actual Reality: Using the Internet to Assess Everyday Functioning after Traumatic Brain Injury.

Objective: to examine and compare the performance of actual everyday life activities in persons with Traumatic Brain Injury (TBI) and healthy controls (HC) using a new assessment tool called actual reality (AR).

Participants and Methods: A between-subjects design was utilized to compare 10 individuals with $\mathrm{TBI}$ and $10 \mathrm{HC}$ performing an AR task. Participants were asked to access the Internet to purchase a decorative arrangement of cookies and were administered a battery of neuropsychological tests and questionnaires to assess quality of life, functional status, affect symptomatology and prior Internet experience.

Results: Participants with TBI had significantly more difficulties than HC participants in accurately and independently completing the AR task-primarily due to cognitive impairment. In addition, self-reports of quality of life and everyday functional abilities were not correlated with $A R$ performance.

Conclusions: The results provide initial evidence supporting the use of the AR approach in assessing persons with TBI performing everyday life activities. The utilization and interpretation of the AR approach is a significant step forward in increasing the sensitivity, accessibility, and relevancy of functional assessments in people with cognitive impairments. Actual reality will be discussed in terms of accessibility and directions for assessment and treatment.

Correspondence: Yael Goverover, New York University, 35 W. 4 th street, 11th floor, new York, NY 10012. E-mail:yg243@nyu.edu

J.T. TWAITE, A.D. BONO, H.A. BOKHARI, E. ARONOVA, R. STAFFORD, S.D. GOLDE, J.L. STORBECK \& J.C. BOROD. Intellectual and Cognitive Performance of Musicians and Healthy Controls: Differences on the Montreal Cognitive Assessment (MoCA) and Other Measures.

Objective: Musical training and performance involves the simultaneous recruitment and integration of a wide variety of auditory, motor. and cognitive processes. Significant functional and neuroanatomical differences have been found between musicians and non-musicians. These findings have generated interest in possible cognitive differences between musicians and non-musicians, but research has, to date, been largely inconclusive. The present study compared the performance of musicians and non-musicians on several measures of intellectual and cognitive ability.

Participants and Methods: Participants were 56 musicians (59\% women; $\mathrm{M}$ age $=22.1$ years $(4.0), \mathrm{M}$ onset of training $=8$ years $(2.5), \mathrm{M}$ duration of training $=13.5$ years $(4.5)$ and 57 demographically matched controls $(52 \%$ women, $\mathrm{M}$ age $=20.9(4.2)$. Participants were screened for psychiatric, medical, and neurological history and then administered the MoCA, Raven's Advanced Progressive Matrices (APM), and the Wechsler Test of Adult Reading (WTAR).

Results: Musicians scored significantly higher than non-musicians on the WTAR ( $\mathrm{t}=4.63, \mathrm{p}=0.00)$, MoCA overall language domain $(\mathrm{t}=2.01$, $p=0.046$ ), and MoCA verbal fluency (total words generated: $t=2.79$, $\mathrm{p}=0.01$; MoCA score: $\chi 2=3.84, \mathrm{p}=0.04)$. These relationships remained after controlling for native language status and education. There were no significant group differences in overall MoCA scores, other MoCA domains, or APM scores.

Conclusions: Musicians outperformed non-musicians on measures of verbal ability. These findings are consistent with prior research showing that musical training is associated with superior phonological awareness. Prior studies have reported that children who took music lessons scored higher on measures of fluid intelligence as compared to untrained children. However, no relationship between musical training and APM scores was found in the present study. This discrepancy may reflect differences between individuals who receive musical training versus those who go on to become professional musicians.

Correspondence: Jamie T. Twaite, M.A., Neuropsychology, CUNY Graduate Center, 102-40 67th Rd, Apt 5N, Forest Hills, NY 11375. E-mail: jtwaite@qc.cuny.edu 
L. RUBINFELD, F. KINTZEL, S. JONES, B. BUTLER, R. KLEIN \& G. ESKES. Validity and Reliability of the Dalhousie Computerized Attention Battery in Healthy Older Adults.

Objective: Attention deficits are common in many psychiatric and neurological patient populations and are related to poorer functional outcomes. For this reason, the Dalhousie Computerized Attention Batttery (DalCAB) was developed with the purpose of accurately identifying attention difficulties using tests validated from cognitive neuroscience research in order to provide theory-driven assessment and targeted treatments for patients.

Participants and Methods: The current study gathered initial normative data and evaluated the validity and reliability of eight reaction time (RT) tests with repeated testing over a 24-hour interval with 50 healthy older adults $(\mathrm{M}(\mathrm{SD})$ age $=70$ (7.4) yrs, $\mathrm{M}(\mathrm{SD})$ education $=15.2$ (2.8) yrs). The battery included tests of vigilance and sustained attention (simple RT, choice RT), orienting and selective attention (visual search) and executive attention (go/no-go, flanker task, and memory span tasks).

Results: In terms of validity, expected task patterns were confirmed in relation to previous literature. Test-retest reliabilities (ICCs) were variable, ranging from good $(0.72-0.94)$ for mean RTs to as low as .45 for derived scores, such as memory scanning speed. Accuracy was overall very high.

Conclusions: Results suggest RT tasks derived from cognitive neuroscience theory can be useful for attention assessment in clinical and research settings in older adults, but interpretation of some attentional processes must be made with caution.

Correspondence: Lindsay Rubinfeld, PhD Clinical Psychology, Psychology and Neuroscience, Dalhousie University, 1459 Oxford St., PO Box 15000, Halifax, NS B3H 4R2, Canada. E-mail: lindsay. rubinfeld@dal.ca

B.M. SCOTT, A.M. STRUTT, P. LUNDBERG-LOVE, A.L. SCHMITT, E. TRIFILIO \& D. BOWERS. Comparison of Psychogenic Movement Disorder Patients with Non-epileptic Seizures vs Other Hyperkinetic Motor Manifestations: An Integrated Model of Psychosocial and Neuropsychological Functioning.

Objective: The present study compared the neurocognitive performance and psychological profiles of two psychogenic movement disorder (PMD) subtypes: non-epileptic seizures (PNES) and other hyperkinetic motor manifestations (PMD-H). It was hypothesized that frontally mediated functions (e.g., attention/working memory, executive function) would be among the most prominent in the current sample, and that patients with more dramatic motor manifestations (i.e., PNES vs PMD-H) would demonstrate greater neurocognitive impairment and more severe psychopathology.

Participants and Methods: Participants included 16 PNES (video-EEG verified) and 16 PMD-H (Fahn \& Williams criteria) patients. All participants underwent an abbreviated multi-domain neuropsychological battery and completed a series of self-report psychological measures.

Results: For combined groups, education and posttraumatic symptoms were significant predictors of general cognitive status $[\mathrm{F}(2,23)=14.8$, $\mathrm{p}<0.001]$. PMD-H participants outperformed PNES subjects across cognitive measures, with statistically significant differences in attention, lexical fluency, and set maintenance (WSCT). There were minimal differences on psychological measures. Binary logistic regression analyses identified daily functional status (i.e., employment, relationships) and set maintenance as significant predictors of group membership [omnibus $\chi 2(2)=20.8, p<0.001]$. This model accounted for $47.8-63.7 \%$ of the variance in group membership, with $75.0 \%$ of both groups correctly classified.

Conclusions: Results suggest that PMD patients with different hyperkinetic motor manifestations may be characterized by subtle differences in cognitive functions mediated by frontal lobe systems. While posttraumatic symptoms aided the prediction of general cognitive function for the total sample, between-group differences in cognitive function were not accounted for by differences in psychopathology. Implications for treatment and etiological conceptualizations will be discussed.

Correspondence: Bonnie M. Scott, M.S., Clinical and Health Psychology, University of Florida, $538 \mathrm{NW}$ 39th Road, Unit 305, Gainesville, FL 32607. E-mail: bonnie.m.scott@gmail.com

A.R. STABLER \& M. LACY. Cross-Cultural Sensitivity of the MiniMental State Exam (MMSE) as a Screener for Memory Impairment. Objective: Memory decline is one the first and most common symptoms of emergent mild cognitive impairment and dementia. Screening for this memory impairment is integral to providing early effective interventions and family planning. The Mini-Mental State Exam (MMSE) continues to be the most widely utilized, first-line screening measure for dementia and cognitive deterioration within clinical settings. However, multiple researchers have documented problems with the MMSE (Lacy et al., 2014), specifically pertaining to its sensitivity across racial groups (Fillenbaum et al., 1990; Parker \& Philp, 2004). In the current study, the sensitivity of the MMSE for detecting memory impairment was examined across racial groups.

Participants and Methods: The performance of 295 consecutive referrals as a function of racial group (Whites: $n=90$ ) (African Americans: $\mathrm{n}=205$ ) at a university-based outpatient memory clinic was examined on the Standardized MMSE (SMMSE) and the Hopkins Verbal Learning Test-Revised (HVLT-R).

Results: On average, Whites and African Americans scored comparably on the SMMSE. Among patients scoring $\geq 26$ on the SMMSE, $50 \%$ of Whites and $60 \%$ of African Americans demonstrated at least mild impairment on delayed recall $(Z=\leq-1.5)$, with significantly greater $(p=.05)$ percentage of African Americans at the level of moderate impairment $(\mathrm{Z}=\mathbf{s}-2.0)$.

Conclusions: The SMMSE appears to be an insensitive screening measure for memory impairment across race, with slightly poorer sensitivity seen in the African American patients presenting for dementia assessment. This insensitivity, particularly for African Americans, may represent a function of etiology, access to healthcare, testing bias, or other variables that will be presented.

Correspondence: Anthony R. Stabler, Doctoral, Roosevelt University, 1445 W Augusta Blvd., Chicago, IL 60642.E-mail: astabler@mail. roosevelt.edu

C.R. PENNINGTON, A. SCHMIDT, B.E. RIDGE, K.E. MCCALLUM, C.N. BRYSON, K.K. MARSHALL \& R.T. CHEIFFETZ. Personality Traits Influence Processing Speed Performance in a Neurologically Intact Population.

Objective: Previous research indicates that personality characteristics are associated with cognitive abilities including executive functions; however, little research has examined how personality factors may influence more fundamental cognitive operations such as processing speed. The current study addressed this question in a typically developing college population.

Participants and Methods: Participants included 22 undergraduate students at a midsized university. The sample group included 10 males and 12 females with a mean age of 22.4 ( $\mathrm{SD}=3.2$ ). Participants were ethnically diverse with $36.4 \%$ identifying as Caucasian, $31.8 \%$ identifying as African American, 22.7\% identifying as Hispanic, and 9\% identifying as biracial or other. All participants completed the NEO-PI-3, and tests from the Delis-Kaplan Executive Functioning System (Trails and Color-Word Interference) as part of a larger battery of personality, intelligence, and executive functioning measures.

Results: Agreeableness $(\alpha=-.47, p=.03)$ and Conscientiousness $(\alpha$ $=-.47, p=.03)$ were positively related to Color-Word Interference completion times. Conversely, Neuroticism was negatively related to Trails completion times $(\alpha=.48, p=.03)$ and trended towards being negatively related to Color-Word Interference completion times $(\alpha=$ $.42, \mathrm{p}=.06)$. 
Conclusions: The present findings suggest that, within a neurologically intact population, aspects of personality can systematically influence performance on measures of processing speed. If replicated in larger samples and generalized to clinical populations, the current findings may have implications for the interpretation of neuropsychological test data. Present results emphasize the utility of including measures of normal range personality functioning into standardized neuropsychological protocols.

Correspondence: Charlotte R. Pennington, Psychology, Sam Houston State University, 2451 Lake Road \#604, Huntsville, TX 77340. E-mail: crp007@shsu.edu

A. COSKUNPINAR, J. NOVITSKI, T. SUSMARAS, L. HENKLE, J. FLANNERY \& S. MUSIL. MMPI-2 Profiles in an Academic Medical Center: The Defended and the Defenseless.

Objective: In our evaluations of patients for whom there may be concern for somatization, we often see 2 distinctly different MMPI- 2 profiles: the "conversion- $\mathrm{V}$ " (CV) and the so-called "cry for help profile" (CFH), which is characterized by high $\mathrm{F}$, low $\mathrm{K}$, and multiple clinical scale elevations. Previous research has tended to focus on a particular group of interest (e.g., psychogenic nonepileptic seizures (PNES) patients) and examine mean MMPI-2 profiles. In this study, we took the reverse approach and grouped patients by MMPI-2 profile. The goal is to describe the characteristics of patients producing each profile and examine the diagnostic utility of each with regard to somatization risk.

Participants and Methods: 122 MMPI- 2 profiles were obtained from clinically referred patients (including 27 patients with PNES based on video EEG monitoring). CV and CFH profiles were identified by both visual inspection and quantitative procedures. CV profile was defined by $\mathrm{T}$-scores $>65$ on scales 1 and 3 , with both scales being at least 10 points higher than scale 2 . CFH profiles were defined as 6 or 7 scores of $\mathrm{T}>65$ on scales 1, 2, 3, 4, 6, 7, and 8. Data were analyzed using Chi Square analysis in SPSS.

Results: The CV profile was seen in $16 \%$ of patients whereas the CFH profile was seen in $20 \%$. There were no differences between groups with regard to age, race, or education. However, while CV was seen more commonly in women $(70 \%)$, the CFH profile was equally common in men and women. About half of patients with each profile had a history of abuse. Of 27 PNES patients, $22 \%$ had a CV profile while $37 \%$ had CFH. Conclusions: These results suggest that neither profile is specific to a particular diagnosis such as PNES, and that both are common. This has implications for treatment as individuals with different MMPI-2 profiles may respond to different treatment approaches. As such, treatment may be best guided by the MMPI-2 profile rather than a specific diagnosis, such as PNES.

Correspondence: Suzanne Musil, PhD, Behavioral Sciences, Rush University Medical Center, 1645 W. Jackson Blvd, Ste. 400, Chicago,

IL 60612.E-mail: suzanne_musil@rush.edu

M. LEGARRETA, E. BUELER, J. DIMUZIO, E. MCGLADE \& D. YURGELUN-TODD. Neuropsychological Performance in Veterans with Chronic Pain.

Objective: Chronic pain (CP) and cognitive impairment are prevalent and disabling in Veterans; however, their relationship has not been rigorously examined. We have completed a preliminary study of the association between pain and cognitive performance in a sample of Veterans with CP.

Participants and Methods: A total of 17 Veterans (mean age 35.5 years) completed neuropsychological (NP) testing including the Continuous Performance Task, the Stroop Task, and Vocabulary (from the WASI-II). Pain intensity was measured by McGill Pain Questionnaire. The Pain Catastrophizing Scale and the Pain Disability Index were utilized in assessment of functioning related to pain.

Results: On the Stroop interference subtest, increased time was related to high pain magnification thoughts $(r=0.627, p=0.009)$, and more errors were correlated with increased pain helplessness $(\mathrm{r}=0.517$, $\mathrm{p}=0.024)$. Lower Vocabulary scores on the WASI were associated with increases in catastrophizing ratings $(\mathrm{r}=-0.550, \mathrm{p}=0.034)$, with helplessness catastrophizing being especially relevant $(r=-0.557, p=0.024)$. Furthermore, attentional capacity was assessed with the CPT and findings revealed that individuals with higher pain catastrophizing ratings $(\mathrm{r}=0.610, \mathrm{p}=0.020)$, and helplessness catastrophizing ratings $(\mathrm{r}=0.646$, $\mathrm{p}=0.013$ ) produced more errors of omission.

Conclusions: These preliminary findings demonstrate an association between pain ratings and NP performance and suggest that pain catastrophizing is especially relevant when discussing reduced cognitive capacity in Veterans with CP. Interestingly, pain intensity and pain disability did not seem to impact NP performance. Additional research is needed to examine which cognitive functions are altered by pain, and what factors may be driving the relationship.

Correspondence: Margaret Legarreta, PhD, MIRECC, VA Salt Lake City Health Care System, 500 Foothill Drive, Mail Code - 116M, Salt Lake City, UT 84148. E-mail: mlegarreta@gmail.com

M. CAIRnCROSS, A. PETERSON, A. LAZOSKY, C. WEIJER \& A.M. OWEN. Neuropsychological Assessment of Decision-Making Capacity in Patients With Barriers to Communication.

Objective: Patient autonomy is contingent on the ability to demonstrate decision-making capacity (DMC). Standard capacity assessments are reliant on both the ability to make an adequately informed decision, and the ability to express that decision verbally. Thus, barriers to verbal communication, such as aphasia, present difficulties for assessing DMC, and create a risk for falsely concluding that a patient lacks DMC and thereby wrongly denying their autonomy. Due to barriers in communication, current capacity assessment tools (i.e. semi-structured/structured interviews) are inappropriate for this patient group.

Participants and Methods: In this paper, we review current DMC assessment tools in order to highlight the relevant challenges individuals with communication barriers may face (e.g., no means for patients to communicate non-verbally). We further review potential alternative strategies to accompany and enhance standard assessment tools for use in patients with communication barriers.

Results: Key to developing alternative strategies, we argue, is identifying the constitutive cognitive faculties that may be necessary for responsible decision-making, such as auditory comprehension, reading comprehension, memory, executive functions, reasoning, and judgment. A neuropsychological test battery adapted to this patient groups unique communication deficiencies that measures such faculties is proposed.

Conclusions: We will discuss how such modified techniques may eventually be used for the assessment of capacity in patients with severe barriers to communication. Doing so, we argue, ensures that efforts to respect patient autonomy are upheld regardless of the communication barriers any condition may pose to avoid the harm of wrongly denying autonomy to those with barriers to communication.

Correspondence: Molly Cairncross, MA, Psychology, University of Windsor, Psychology Department, University of Windsor, 401 Sunset Avenue, Windsor, ON N9B3P4, Canada.E-mail: cairncrm@uwindsor. ca

H. DUNCANSON, A. HOLLIS \& M.G. O'CONNOR. Error Analysis of the TMT: Relevance to Driving Safety.

Objective: Many studies have demonstrated the efficacy of the Trail Making Test (TMT) in the prediction of driving safety. Most focus on TMT-A and TMT-B completion time as the variable of interest. There is scant information regarding TMT errors in relation to driving. The goal of this study is to provide normative data on error analysis of the TMT using road test performance as the criterion for safe driving.

Participants and Methods: A total of 587 participants (351 male, 236 female) underwent a comprehensive driving evaluation including cognitive screening and a road test. Thirty-four participants were excluded due to incomplete data, loss to follow up, or cancelled appointments. Drivers averaged 72.97 years of age and 15.45 years of education. 
Underlying medical conditions varied across drivers including those with and without cognitive deficits.

Results: There was no effect of education on driving outcome. As expected, age was associated with road test performance: individuals who passed the road test were significantly younger $(M=68.21)$ than those who failed $(\mathrm{M}=76.40)$. Trails $\mathrm{A} \& \mathrm{~B}$ completion times of those who passed were faster (Trails A: $M=48.78$; Trails $B: M=120.17$ ) than those who failed (Trails A: $\mathrm{M}=67.88$; Trails B: $\mathrm{M}=143.88$ ). Error data were analyzed using the number of correctly completed items as the variable of interest. Number of completed items for the TMT-B was lower in those who failed the road test (Passed: $\mathrm{M}=22.84$; Failed: $\mathrm{M}=$ 19.29) but number of completed items on Trails A was not associated with road test outcome.

Conclusions: To our knowledge, this is the first study to examine a large cohort of at risk drivers in order to provide normative data on the TMT with regard to two different metrics from the TMT- processing speed and number of items completed. The normative information provided from this large cohort will assist clinicians in deciding whether a formal driving evaluation is warranted.

Correspondence: haley Duncanson, MA, psychology, Suffolk University, 253 center st, Randolph, MA 02368.E-mail: haleyduncanson28@gmail. com

J.C. PIERCY, D. LEITNER, M. REYNOLDS, L. OHLHAUSER, J. KLUFTINGER, E. CONCEPCION \& H. MILLER. Comparison of the Buschke Selective Reminding Test and the California Verbal Learning Test - Second Edition in a Stroke Population.

Objective: The Buschke Selective Reminding Test (SRT) and the California Verbal Learning Test - Second Edition (CVLT-II) are neuropsychological measures designed to assess verbal learning and memory. Studies with heterogeneous acquired brain injury (Leitner, et al., 2012) and TBI populations (Leitner, et al., 2014) suggest differences in memory and learning classification between the two tests. This study compared these two tests in a stroke population.

Participants and Methods: Forty-six stroke inpatients (mean age $=$ $70, \mathrm{SD}=11.2$; mean years education $=12, \mathrm{SD}=2.0$ ), 26 male and 20 female, participated in the study. Each participant received both the SRT and CVLT-II in their neuropsychological assessment.

Results: Participants scored lower on the SRT indices of learning, long delay free recall (LDFR), total recall, short-delay cued recall (SDCR), and recognition. These differences were consistent between sex, with exception of SDCR. Further, hemorrhagic or left hemisphere strokes scored lower on SRT learning and LDFR than those with ischemic or right hemisphere strokes respectively. This difference was not apparent on the CLVT-II. No differences were noted between anterior-posterior type strokes.

Conclusions: The SRT classified stroke patients as impaired more often than the CVLT-II. Further, the SRT learning and LDFR indices may be more sensitive to stroke etiology and location. These findings suggest that the SRT and CVLT-II are not interchangeable tests of learning and memory in patients who have experienced a stroke. Conclusions about learning and memory function and/or treatment implications may differ depending on which test is employed in assessment.

Correspondence: Damian Leitner, BSc. (Hons) Psychology, Kelowna General Hospital, 2268 Pandosy Street, Kelowna, BC V1Y 1T2, Canada.E-mail:Canadian_02@hotmail.com

C.B. DUNN, J. CELLAR, J.J. LAH, A.I. LEVEY \& F.C. GOLDSTEIN. Sensitivity and Specificity of the Benson Figure as a Cognitive Screen in Older Adults.

Objective: The Benson Figure is clinically attractive as a test of visuospatial functioning, planning/organization, and visual memory because of its relatively reduced stimulus complexity and shorter administration time and scoring demands than the Rey Osterreith Complex Figure. The Benson Figure includes a copy condition, and delayed recall is obtained after 10-15 minutes. The current study assessed the sensitivity and specificity of the Benson Figure among older adults diagnosed with normal cognition, mild cognitive impairment (MCI), or dementia.

Participants and Methods: Participants were evaluated at the Emory University Alzheimer's Disease Research Center between October 2012 and July 2014. They received a cognitive battery of memory, language, attention, and executive functioning tests as well as an assessment of their independent activities of daily living. Diagnoses were determined during consensus conferences, and were then re-evaluated to ensure they were still appropriate independent of performance on the Benson Figure. The final sample included 52 individuals with normal cognition, 40 with MCI, and 19 with dementia.

Results: There were significant differences among the three groups in the Benson Figure recall scores (normal > MCI > dementia). At an age and education corrected cut score of $\mathrm{z}=-0.5$, the sensitivity to distinguish MCI from normal cognition was .73 while the specificity was .60 . At a cut score of $\mathrm{z}=-1.5$, the sensitivity to distinguish dementia from normal cognition was .88 while the specificity was .79 .

Conclusions: The Benson Figure is a useful clinical tool to aid in distinguishing between normal cognition and dementia. However, the simplicity of the stimuli may hinder its ability to detect MCI.

Correspondence: Callie B. Dunn, MS, Department of Clinical and Health Psychology, University of Florida, PO Box 100165, Gainesville, FL 32610-0165. E-mail: cbeck@phhp.ufl.edu

C.A. LOCKWOOD, S. BROOKS HOLLIDAY, S. NAMAZI, C. HANSEN \& M. REINHARD. Effect of Pain, Emotional Distress, and Cognition on Social Role Outcome Measures.

Objective: The ability to participate in social roles and daily social activities may be affected by multiple variables, including pain, emotional distress, and cognitive abilities. This study examined the impact of pain, mental health, and cognition on social role performance in a sample of veterans with chronic pain.

Participants and Methods: Participants were recruited from a comprehensive complementary and alternative medicine clinic at a large VA hospital. The sample included 19 veterans (12 male, 7 female), mean age 48.05. Participants were administered self-report measures of pain, emotional distress, and cognition, as well as a measure of social role performance.

Results: Mean total score analyses revealed the sample endorsed mild to moderate difficulties with social roles $(M=27.74$; SD = 6.94). An analysis of individual items revealed that participants consistently endorsed mild to moderate difficulties. Scores on the Social Roles and Activities measure were significantly correlated with self-report of depression $(\mathrm{r}$ $=-.71, \mathrm{p}=.001)$, PTSD $(\mathrm{r}=-.62, \mathrm{p}=.006)$, and pain $(\mathrm{r}=-.49, \mathrm{p}=$ $.033)$. Self-reported general cognitive difficulties $(r=.69, p=.001)$ and executive difficulties $(r=.71, p=.001)$ were associated with decreased satisfaction in social roles and activities.

Conclusions: These findings suggest that both emotional distress and perceived cognitive abilities are associated with lower satisfaction in social roles and activities. This highlights the need for a better understanding of factors which may impact functional outcome measures, such as social role and participation in activities and satisfaction in pain samples.

Correspondence: Courtney A. Lockwood, Ph.D., WRIISC, Washington DC VA Medical Center, 50 Irving St, NW, Washington, DC 20422. E-mail: courtneylockwood@gmail.com

S.K. GILL, J. MOSES \& E. SHAPIRO. Visual Naming, Age, Educational Level and WAIS-III Factorial Components Mediate Level and Pattern of Performance on the Judgment of Line Orientation Test.

Objective: This study was designed to develop an interpretive model for analysis of the the effects of verbal mediation in areas of cognitive functioning.

Participants and Methods: Analyses were completed using archival data of 138 veterans from the VA Palo Alto Health Care System. We 
developed a three-component factor solution for the Boston Naming Test (BNT) in previous research. Factor scales were computed for three orthogonal components: BNT1 (items $35-60)$, BNT2 $(6-45)$ and BNT3 (items 1 - 10). Factor variable BNT1, which includes specialized vocabulary, loaded uniquely with the Judgment of Line Orientation (JLO) total score, indicating a verbally mediated component of visuospatial processing. Factorial variables BNT2 and BNT3 loaded uniquely on two separate factors. Second order factor scales were computed for these factorial components. The second order factors were refactored with age and educational level. Age loaded uniquely on the BNT2 factor and educational level loaded uniquely on the BNT1 incorporating the total score from the JLO. Factor scales were computed for each of these factorial components. These factor scale components were factored again with the four factorial components of the Wechsler Adult Intelligence Scale-III (WAIS-III).

Results: The factor involving BNT1, JLO total score and education loaded uniquely on the Verbal Comprehension (VC) component. The factor involving BNT2 with age loaded positively with Perceptual Organization (PO) and negatively with BNT3. The BNT3 factor loaded on the VC, PO, and Processing Speed (PS) components.

Conclusions: Current findings highlight education and verbal mediation as important components of visuospatial processing. Results show the need to analyze complex multivariate factorial components of visual naming and demographic variables on JLO performance in both empirical and clinical analysis. These findings highlight the usefulness of examining patterns of performance in learning strategies to support clinical applications in assessment and treatment settings.

Correspondence: Supria K. Gill, M.S., Clinical Psychology, Palo Alto University, 4010 Martis Street, West Sacramento, CA 95691. E-mail: sgill@paloaltou.edu

A. JONES, N.L. DENBURG \& J. BARRASH. Iowa Scales of Personality Change: Normative Data From Healthy Older Adults. Objective: Acquired personality disturbances (APDs) associated with various clinical conditions have been characterized using the Iowa Scales of Personality Change (ISPC), with demonstrated sensitivity and specificity to the pathological changes. Normative data will enhance the scientific and clinical value of the ISPC.

Participants and Methods: Fifty-nine healthy, independent older adults (ages 59-90, mean: 74.3 years) were studied. Using the ISPC, collaterals rated 30 characteristics on a 7-point scale for two epochs: middle age (until age 50), and currently (the last year). Descriptive analyses and independent samples t-tests were performed. "Acquired disturbance" required both (a) some disturbance in a specific trait and (b) an increase from the earlier level.

Results: ISPC ratings indicated little change, on average, across the 30 traits (mean negative change: 0.14 , positive: 0.18 ). Significant change $(p<.0017)$ was seen only for increased Lack of Stamina $(+0.89)$, and decreased Type A Behavior (-0.58). Regarding acquired disturbances in individual participants, a small number developed Lack of Stamina (8), Moodiness (7), and Inflexibility (6); otherwise, acquired disturbances were rare. Sex differences were slight, including higher longstanding levels of Insensitivity, Apathy and Social Withdrawal among men, and higher current levels of Poor Judgment among women (at lenient uncorrected .05 alpha).

Conclusions: Normative data indicate healthy adults generally exhibit stable personality into their later decades, as measured by the ISPC, unlike patients with clinical conditions manifesting characteristic APDs. Furthermore, ratings indicate that findings of specific acquired personality disturbances in patients with certain neurological diseases are not due to normal aging, and indicate good specificity of ISPC ratings. Correspondence: Joe Barrash, PhD, Neurology, Uinversity of Iowa College of Medicine, 200 Hawkins Dr., Iowa City, IA 52246. E-mail: joseph-barrash@uiowa.edu
N. DEYOUNG, S. BELKONEN, K. LUCI, M. SLATER, T. QUIGLEY $\&$ B. SHENAL. The reliability and accuracy of administering The Montreal Cognitive Assessment over telehealth.

Objective: Telehealth neuropsychological services are helpful for individuals in rural areas who face barriers to accessing care. The reliability and validity of neuropsychological assessment using telehealth needs to be further established. The Montreal Cognitive Assessment (MoCA), a screener for general neurocognitive dysfunction, can be given over telehealth with minimal adaptation. This study investigates the inter-rater reliability of the MoCA when administered remotely using telehealth technologies.

Participants and Methods: Seventeen veterans (Age $\mathrm{M}(\mathrm{SD})=62.8(14.50), 15 \mathrm{M} / 2 \mathrm{~F})$ referred to an outpatient neuropsychology clinic for various cognitive complaints participated in this project. Participants were administered the MoCa either in-person or over telehealth by a clinician. A second clinician observed the administration in-person or by telehealth and independently scored the test. The inter-rater reliabilities across conditions were compared to assess for differences between in-person and telehealth administrations.

Results: The inter-rater reliability of the MoCa across the three conditions of interest were acceptably high and ranged from $r=0.88$ to $r=0.98$. Reliability correlations were compared using Fisher r-to-z transformations, and no significant differences among the conditions were observed ( $p$ 's $>0.10)$. Beyond reliability, univariate comparison of the absolute mean differences in clinician scores showed no significant differences among the actual raw scores of the three conditions tested indicating accuracy $(\mathrm{F}(14)=0.01, \mathrm{p}=0.56)$.

Conclusions: The inter-rater reliabilities of MoCa scores across conditions were all acceptably high, and administration of the MoCa using telehealth technology did not significantly alter the total MoCa scores. Overall, the lack of significant differences suggests that administering the MoCa by telehealth is reliable, accurate, and well received by participants.

Correspondence: Nathaniel DeYoung, Ph.D., MHSL, Salem Veterans Medical Center, 1970 Roanoke Boulevard, Salem, VA 24153. E-mail: nathaniel.deyoung@va.gov

A. VINCENT, H. TYLER \& T. ROEBUCK-SPENCER. Test-Retest Reliability, Practice Effects, and Base Rates of Change for the ANAM-GNS Battery.

Objective: Computer-based cognitive assessment is rapidly expanding. However, computerized tests have been criticized as having low reliability and lacking key psychometric information. Thus, the current study examined test-retest reliability, practice effects, and base rates of significant performance change for the computerized ANAM General Neuropsychological Screening (GNS) battery

Participants and Methods: Participants (N=94) were individuals with no objective cognitive impairment recruited from the community ranging in age from 18 to $82(40.7 \pm 17.8$ years $)$. ANAM-GNS was administered and then repeated (alternate form) after 30 days. Reliability was estimated using intraclass correlations. To determine the presence of practice effects, paired t-tests were used to examine differences between the two administrations. Base rates of significant performance change across administrations were computed using reliable change indices adjusted for practice effects $(90 \% \mathrm{CI})$.

Results: Scores were significantly higher on 10 of 15 tests after 30 days. Although consistent with practice effects, effect sizes were small for all but two tests. Test-retest reliabilities ranged from .51-.94. Significant performance change was seen for $90.7 \%$ of the sample on at least one test and $69.3 \%$ on $2+$ tests. Only $4 \%$ showed performance change on $6+$ tests.

Conclusions: Findings indicate that the majority of ANAM-GNS tests demonstrate good reliability and minimal practice over a 30 day interval. It was common for this presumably healthy sample to demonstrate significant performance change on some tests in this large battery; 
however, it was highly unusual for individuals to demonstrate significant performance change on 6 or more tests.

Correspondence: Hannah Tyler, Master of Science, Cognitive Science Research Center, University of Oklahoma, 3200 Marshall Avenue, Suite 260,Norman,OK 73072.E-mail: hannah.tyler@ou.edu

A.L. FERNANDEZ \& R.L. FULBRIGHT. Construct and Concurrent Validity of the Spanish Adaptation of the Boston Naming Test.

Objective: In 1996 a Spanish edition of the Boston Naming Test was published. Changes to the original version were introduced but without an empirical foundation supporting these modifications. This version was later adapted by re-ordering the items according to their difficulty frequency in a Spanish-speaking population, and norms were developed from this version of the test. However, no validity studies were performed with this new version to determine if the changes introduced had impact on the test's validity. The current study was performed in order to assess the construct and concurrent validity of the Spanish-language version of the Boston Naming Test (SV-BNT).

Participants and Methods: The SV-BNT was administered to 23 patients with Alzheimer's disease (AD) and 36 normal controls. Groups were age and education matched.

Results: AD patients showed a significantly lower mean score than the controls on the SV-BNT, with a large effect size, which demonstrates adequate construct validity. However, sensitivity was $39 \%$ and specificity $89 \%$.

Conclusions: The very low sensitivity shows that the concurrent validity of the test is poor and many patients with $\mathrm{AD}$ can score within the normal range. The SV-BNT does not guarantee a proper identification of naming difficulties and therefore its clinical use should not be recommended.

Correspondence: , . E-mail:

B.L. FISCHER, R. STEIDL, C. GLEASON, A. SPANNER, T.C. SWADER, S. MONSON, T. HOWELL, M. HEIM, L. WELCH, C. HERMANN, R. SCHROEDER, A. BRIDGES \& S. BARCZI. TeleNeuropsychology at the Madison VA Hospital: An Extension of GRECC Connect.

Objective: Almost 30\% of Veterans live in rural areas, and of these, two thirds are aged 55 or older. Although subjective cognitive complaints are common, many older rural Veterans have limited access to neuropsychological evaluations, and are unable to travel the long distances to major hospitals for cognitive assessments. The Tele-Neuropsychology program was developed in conjunction with our multi-disciplinary GRECC Connect team, to provide improved access to innovative dementia care.

Participants and Methods: Ten veterans (mean age 78.5) have been evaluated via Tele-Neuropsychology to date. Veterans presented to their local clinics and were administered a standard battery of tests by the Neuropsychologist from the Madison VA Hospital over Clinical Video Teleconferencing (CVT), assisted by a local tele-conferencing technician.

Results: By driving to their local clinic rather than the main hospital, Veterans saved a total of 1372 driving miles, \$569 in mileage costs, and 29 hours of driving time. Importantly, Tele-Neuropsychology improved timely access to service. Based on wait-list times at referral, Veterans served by Tele-Neuropsychology were seen at least 10 weeks earlier than they would have had they been evaluated in our hospital's standard Memory Assessment Clinic. While the most common diagnosis has been Alzheimer's Disease, other diagnoses have included Huntington's Disease, Cognitive Disorder due to alcohol abuse, and Cognition within normal limits. Each veteran evaluated to date has expressed a preference for the CVT format rather than traveling to the main hospital. Data collection is ongoing.

Conclusions: Tele-Neuropsychology is a promising means to improve access to multi-disciplinary cognitive assessments for older rural Veterans who have suspected cognitive deficits. In conjunction with a multi-disciplinary team, the Tele-Neuropsychological evaluation provides comprehensive dementia care to Veterans living in rural communities.

Correspondence: Barbara L. Fischer, Psy.D., GRECC, Wm. S. Middleton Memorial VA Hospital, 2500 Overlook Terrace, GRECC, Madison, WI 53711. E-mail: barbaralfischer@gmail.com

B. YOCHIM, M. YUTSIS, N. RAYMOND \& K. BARRERA. A Verbal Naming Test for Use with Older Adults: Development and Initial Validation.

Objective: Neuropsychologists assess naming or word-finding in a typical evaluation, particularly in evaluations for older adults, but there are currently no measures that can be used for older adults with visual impairment. This study presents a verbal, non-visual measure of naming for use in the evaluation of older adults.

Participants and Methods: Stimuli were chosen based on their frequency of usage in everyday spoken language. A 60 -item scale was created and given to 131 older Veterans (mean age $=74.9, \mathrm{SD}=11.6$ ). Rasch analyses were conducted and differential item functioning assessed to eliminate poorly-performing items.

Results: The final 55-item scale had a coefficient alpha of 0.84 and was found to correlate with the NAB Naming test, $r=0.84, p<.01$, D-KEFS Category Fluency, $r=0.45, p<.01$, D-KEFS Letter Fluency, $r=0.40$, $<.01$, and with other measures in the expected direction. ROC analyses found the measure to have sensitivity of $79 \%$ and specificity of $85 \%$ in the detection of anomia. Patients with anomia performed worse on the measure than did patients with intact word-finding, $\mathrm{t}(84)=8.2, \mathrm{p}$ $<.001$. Patients with no cognitive impairment performed significantly better than patients with MCI, who performed significantly better than patients with dementia.

Conclusions: This new measure shows promise in the neuropsychological evaluation of word-finding ability in older adults with or without visual impairment. Future directions include the development of a shorter version and the generation of additional normative data.

Correspondence: Brian Yochim, Ph.D., Psychiatry, VA Palo Alto Health Care System, National Jewish Health, 1400 Jackson St., B134, Denver, CO 80206.E-mail: yochimb@njhealth.org

M.C. NATELSON LOVE \& D.S. GELDMACHER. Level of Impairment in Instrumental Activities of Daily Living Predicts Alabama Brief Cognitive Screener Scores in a Memory Disorders Clinic.

Objective: To determine relationships between scores on the Alabama Brief Cognitive Screener (ABCs), a newly developed cognitive screening instrument for clinical use, and scores on the Functional Activities Questionnaire (FAQ), a caregiver-reported instrumental activities of daily living (IADL) scale, in patients attending an academic Memory Disorders Clinic.

Participants and Methods: Following IRB approval, data were obtained from the electronic medical record for all patients attending the UAB Memory Disorders Clinic from 4/30/2012 to 4/30/2013 who had $\mathrm{ABC}$ s and $\mathrm{FAQ}$ recorded and were assigned a diagnosis of memory loss (ICD-9-CM 780.93), mild cognitive impairment (331.83), neurodegenerative cognitive impairment (331.9), or Alzheimer's disease (331.0) by the treating clinician. Complete data were available on 165 patients $(99$ women (60\%), 66 men (40\%); mean age 73.4). Based on FAQ scoring standards, mean ABCs scores were compared by Kruskal-Wallis tests for patients with FAQ $<9$ (not meaningfully impaired; $n=37$ ) and FAQ $\geq 9(\mathrm{n}=128)$.

Results: Mean ABCs score was 25.4 for subjects with FAQ $<9$ and 21.4 for $\mathrm{FAQ} \geq 9(\chi 2=16.38 ; \mathrm{P}<.0001)$. A secondary analysis was therefore conducted to assess for ABCs differences between low, moderate (FAQ 9-19; $\mathrm{N}=82$ ) and high (FAQ 20-30; N=46) levels of impairment. Differences in mean $\mathrm{ABC}$ s were identified across all three groups $(\chi 2=32.41$; $\mathrm{P}<.0001)$.

Conclusions: The anticipated relationship between IADL impairment and lower ABCs score was demonstrated. This finding supports the 
utility of the ABCs for predicting IADL impairment in dementia-related conditions. It shows that the ABCs' psychometric properties align with other bedside screening instruments like the MMSE and MoCA. Correspondence: Marissa C. Natelson Love, M.D., Neurology, University of Alabama Birmingham, 620D Sparks Center, 17207 th Avenue South, Birmingham, AL 35294.E-mail: marissa.natelson@gmail.com

C. TYRRELL, K.A. O'MALLEY \& B.E. GAVETT. Demographic Variables Relating to Scores on the Trail Making Test in MiddleAged and Older Adults.

Objective: The Trail Making Test (TMT) has been demonstrated to be useful in identifying cognitive impairment, such as that associated with Alzheimer's disease (e.g., Chen et al., 2000, 2001), and has also been found to be influenced by demographic variables (e.g., Tombaugh, 2003). The purpose of this analysis is to add to the existing normative data and replicate past findings on the influence of participant characteristics on the TMT. In addition, normative data for a particular subgroup is presented and other participant characteristics related to performance in this group are examined.

Participants and Methods: Data from 502 middle-aged and older adults (ages 50 to 94 ) are presented in relation to performance on the TMT. Participants in this study were community-dwelling adults (173 men and 328 women) who sought cognitive testing in an outpatient clinic and consented to have their data used in research. This cognitive testing was done in one session, typically lasting from 30 to 60 minutes. The TMT was one of several cognitive tests administered as a part of this battery, and demographic information was collected in addition to cognitive performance data.

Results: Participant characteristics from this sample that were significantly correlated with performance on both TMT Part A and Part B include age and level of education. Therefore, TMT scores presented are separated by age groups $(50-59 ; 60-69 ; 70-79 ; 80-89 ; 90$ and up) and by years of education (up to 12; higher than 12). Other participant characteristics associated with TMT performance include subjective health ratings and self-reported difficulties with retrospective memory. Conclusions: Significant associations with age and level of education are consistent with prior research on TMT performance (e.g., Hester et al., 2005). TMT performance also correlated with subjective health and memory ratings, indicating that it is related to self-reported difficulties in this sample.

Correspondence: Caitlin Tyrrell, MA, Psychology, University of Colorado Colorado Springs, 1420 Austin Bluffs Pkwy, Colorado Springs, CO 80918.E-mail: caitlintyr@gmail.com

S. LEVY, J.J. TANNER \& C. PRICE. Assessing Executive Function in Parkinson's disease: A Comparison between Sample-Matched Norms and Population-Based Norms.

Objective: Working memory, abstract reasoning, and processing speed are areas of executive impairment for Parkinson's disease (PD). Normative considerations are relevant to understanding PD progression and treatment (e.g.., DBS; Zahodne, 2011). We examined PD based executive impairment standardized on two distinct normative comparison pools: 1) well known standardized norms (Wechsler; Heaton); 2) local age and education matched non-PD peers.

Participants and Methods: Forty participants with idiopathic PD (mean age $=67.5, \mathrm{SD}=5.47)$ were matched with cognitively intact non-PD controls on age, education, gender, handedness, health comorbidities, general cognition, and estimated premorbid intellectual abilities. All resided in North Central Florida. Performances from the non-PD peers were stratified by age (i.e., 60-64, etc) to match Heaton age groupings. Using three representative measures by domain, standardized composites were created for processing speed, working memory, and abstract reasoning. Paired samples t-tests compared PD scores based on our local non-PD peers relative to popular normative references (Wechsler; Heaton).
Results: Local norm PD and Wechsler/Heaton composite scores were significantly correlated. For PD, composite scores were significantly different based on normative method (Local norm scores $<$ Wechsler/ Heaton) for working memory $(\mathrm{t}(38)=-21.59, \mathrm{p}<.001)$ and abstract reasoning $(\mathrm{t}(39)=-5.79, \mathrm{p}<.001)$. Composite processing speed scores were not significantly different based on normative method $(t(38)=$ $-1.70, \mathrm{p}=.10)$.

Conclusions: Use of population-based normative data may underestimate cognitive deficits observed in PD. Due to known influences from regional location and/or health comorbidities, matched controls are an important consideration relative to the normative standard. NINDS R01 NS082386 (CP) and K23NS60660

Correspondence: Shellie-Anne Levy, Ph.D., Clinical and Health Psychology, University of Florida, $4872 \mathrm{NW} 42$ nd Rd, Apt. 104, Gainesville, FL 32606.E-mail: stlevy@phhp.ufl.edu

S.S. AHN. The Relationship between Social Desirability and Subjective Self-Reported Cognitive Functioning in patients with Bipolar Disorder and Healthy Volunteers.

Objective: Social desirability (SD) refers to the propensity to present oneself in a positive manner. Accordingly, SD could lead a respondent to bias self-reported responses in an effort to be viewed favorably. Social desirability bias (SDB), therefore, has the potential to influence self-reports of subjective cognitive functioning which are frequently obtained in neuropsychology. The purpose of this study was to investigate the relationship between SD and subjective self-report of cognitive functioning in patients with bipolar disorder and healthy volunteers.

Participants and Methods: Forty-five euthymic outpatients with Bipolar Disorder (BD) and 40 healthy community volunteers completed self-report measures of SD (Marlowe-Crowne Social Desirability ScaleSDS) and subjective cognitive functioning (Cognitive Failures Questionnaire-CFQ). Group differences on SDS and CFQ were analyzed using t-tests, and pearson correlations were used to assess the association between these measures within each group.

Results: Healthy volunteers reported significantly higher SD (M=19.7, $\mathrm{SD}=5.2)$ than $\mathrm{BD}$ patients $(\mathrm{M}=16.2, \mathrm{SD}=5.9), \mathrm{t}(83)=-2.92(\mathrm{p}<.01)$, and reported significantly lower scores on the $\mathrm{CFQ}(\mathrm{M}=25.5, \mathrm{SD}=10.2)$ than $\mathrm{BD}$ patients $(\mathrm{M}=37.7, \mathrm{SD}=16.5), \mathrm{t}(1,83)=4.1, \mathrm{p}<.001$. Scores on the SDS and CFQ were significantly negatively correlated in both BD patients $(\mathrm{r}=-.59, \mathrm{p}<.01)$ and healthy volunteers $(\mathrm{r}=.-.31, \mathrm{p}<.05)$.

Conclusions: Higher SD was associated with lower subjective ratings of cognitive problems in both patients and healthy volunteers. Consistent with this, healthy volunteers showed higher SD and lower ratings of subjective cognitive problems than patients. These findings suggest that social desirability has the potential to bias self-rated measures of cognitive functioning. Therefore, assessment of social desirability may be a useful means of helping to identify distorted self-reports of subjective cognitive functioning.

Correspondence: Sharon S. Ahn, Bachelor's degree in Psychology, Department of Psychiatry, University of British Columbia, 3701-9888 Cameron st., Burnaby, BCV3J-0A4, Canada.E-mail:ahnsohy@gmail. com

P. BREWSTER, C. DECARLO, D. HALLIDAY, A. GORDICHUK \& S. MACDONALD. Telephone Administration of the Mental Alternation Test: Sensitivity to Cognitive Status in CommunityDwelling Older Adults.

Objective: We examined the utility of telephone administration of the Mental Alternation Test (MAT), an oral variant of the Trail Making Test, for predicting cognitive performance, self-reported IADLs, and cognitive status in a volunteer sample of older adults.

Participants and Methods: Adults age 65 and older were recruited from the community to participate in a study of risk factors for cognitive impairment and dementia. The MAT was administered as part of a telephone screening assessment. Eligible participants underwent comprehensive neuropsychological assessment and received a consensus 
research diagnosis of MCI, Probable AD, or No Cognitive Impairment. Performance on the MAT was not used in determining study eligibility or cognitive status. Linear regression was used to examine the association between MAT performance and in-person performance on neuropsychological tests and self-reported IADLs. Logistic regression was used to examine MAT scores in relation to 1) Cognitive status operationalized as "normal" ( $\mathrm{n}=51$ ) vs. "impaired" (MCI and AD combined; $\mathrm{n}=26$ ), and 2 ) presence vs. absence of impairment on one or more of the four executive measures in the study battery (normal $n=49$, impaired $n=14$ ). Results: MAT scores significantly predicted performance on the 3MS, performance on all neuropsychological tests in the study battery, and self-reported IADLs. Associations were strongest between the MAT and performance on Digit Symbol ( $r=0.58)$, Phonemic Fluency ( $r=0.53)$ and Trail Making Test part B ( $\mathrm{r}=-0.46)$. MAT scores also predicted cognitive status such that lower scores were associated with higher odds of cognitive impairment $(\mathrm{ROC}=0.70)$ and executive deficits $(\mathrm{ROC}=81)$. Conclusions: Results suggest that the MAT is a brief and valid measure of executive ability that has potential utility as a screen for cognitive impairment and functional deficits in aging research.

Correspondence: Paul Brewster, University of Victoria, 3800 Finnerty Rd, Victoria, BC 90101, Canada.E-mail:pbrew@uvic.ca

D.J. HARDY, B. SPIELBERG \& M.J. WRIGHT. The Concept of Workload: An Illustration with the Tower of Hanoi Test.

Objective: The concept of workload has been fruitfully employed in human factors research but largely overlooked in neuropsychology. A popular measure of workload, the NASA-TLX, is illustrated with a typical neuropsychological test, the Tower of Hanoi (TOH).

Participants and Methods: Forty-nine college students completed three counter-balanced conditions on the TOH (three-disk, four-disk, and five-disk). The NASA-TLX was administered after each TOH trial. The NASA-TLX subscales of Mental Demand and Physical Demand were examined in the current study. We posited that perceived mental demand, but not physical demand, would increase with TOH difficulty. Results: As expected, average number of moves on the TOH increased with task difficulty $(\mathrm{M}=9.3,27.7$, and 84.8 , respectively; $\mathrm{F}=78.94$, $\mathrm{p}$ $<.001, \eta 2=.62)$. As hypothesized, self-report scores on Mental Demand increased as a function of TOH condition $(\mathrm{M}=20.2,37.8$, and 54.9, respectively; $\mathrm{F}=74.59, \mathrm{p}<.001, \eta 2=.61)$. In contrast to expectations, average Physical Demand score also increased with TOH difficulty $(\mathrm{M}=$ $3.6,6.0$, and 8.8, respectively; $\mathrm{F}=10.61, \mathrm{p}<.001, \eta 2=.18$ ), although effect size and averages are considerably smaller. Looking at frequency distributions, it appears that a small subset of respondents reported anomalously high scores on the Physical Demand subscale.

Conclusions: Results conditionally support the main hypothesis that Mental Demand but not Physical Demand will increase with TOH difficulty. Extra care must be taken with instructions on the NASA-TLX to avoid anomalous responses. It is argued that workload can be a useful construct in neuropsychology, providing an additional channel of information on patient status.

Correspondence: David.J. Hardy, Ph.D., Psychology, Loyola Marymount University, 1 LMU Drive, Suite 4700, Loyola Marymount University, Los Angeles, CA 90045. E-mail: David.Hardy@lmu.edu

A.A. SEGUIN, C.M. CONSIDINE, K. ROBERTS, A. GARANT, C. ABEARE, A. DHAR \& W. RAJKUMAR. Predicting Differences in Mood, Cognitive Performance, and Cognitive-Affective Processes among "Good" versus "Bad" Sleepers Based on Subjective and Objective Reports of Sleep Quality.

Objective: Subjective (i.e., self-report) and objective (i.e., polysomnogram) measures of sleep quality may be differentially associated with variations in mood, cognitive performance, and cognitive-affective processes. This study investigates whether these constructs significantly differ between subjective (Epworth Sleepiness Scale, ESS) and objective (polysomnogram-determined Sleep Efficiency, SE) groups of "good" versus "bad" sleepers recruited from an obstructive sleep apnea referral source.

Participants and Methods: Fifty-five participants (Mage=53.35, 26 women) underwent a polysomnogram, completed sleep and mood questionnaires (Epworth Sleepiness Scale, ESS; PANAS-Negative Affect, $\mathrm{NA}$ ), and were administered a neuropsychological battery, including the Symbol Digit Modality Test (SDMT) and the Emotion Digit Coding Test (EDCT), to assess cognitive and cognitive-affective processes.

Results: In a 2 (high vs. low ESS) x 2 (high vs. low SE) ANOVA, a main effect of ESS was found for NA, F(1,48)=8.37, p<0.01, and EDCT, $\mathrm{F}(1,47)=4.97, \mathrm{p}<0.05$. There was no main effect of SE for NA, SDMT, or EDMT. ESS negatively correlated with EDCT $(r=-0.26, p<0.05)$ and positively correlated with NA $(\mathrm{r}=0.36, \mathrm{p}<0.01)$. SE positively correlated with NA $(r=0.25, p<0.05)$. All correlations controlled for gender, age and education; appropriate demographics were controlled for in subsequent analyses.

Conclusions: Subjective measures of sleep quality were better predictors of negative affect and performance on cognitive-affective processing speed tasks, compared to objective measures. These findings lend support to the importance in accounting for both objective and subjective indicators of sleep quality in clinical populations.

Correspondence: Ashley A. Seguin, B.Sc[H] Behaviour, Cognition, and Neuroscience, Psychology, University of Windsor, 166 Delmer Cres., Cottam,ON NOR1B0,Canada.E-mail:aseguin@uwindsor.ca

\section{J.W. ADAMS, B.E. GAVETT \& A. GURNANI. Preliminary Development of an Adaptive Digit Span Test: Effect of Item Presentation Order.}

Objective: The Digit Span test procedure is widely used in clinical and research settings. Digit Span sequences are customarily presented orderly, whereby each subsequent sequence is longer than the previous by one digit. This approach to test administration may be an inefficient way of measuring auditory attention span, especially for examinees with average or above-average abilities. Computerized adaptive methods may increase efficiency without a loss of information. The development of an adaptive Digit Span test first requires evidence that altering the test administration, from ordered presentation of sequences to presentation that is less predictable, does not affect performance.

Participants and Methods: Undergraduate participants $(\mathrm{N}=50)$ recited, forwards and backwards, ordered and random Digit Span sequences using a within-group design and each correct sequence recitation awarded a score of one. Following the first Digit Span recitation (either ordered or random) and prior to the second (the complement), participants completed the Brixton Spatial Anticipation Test and Delis-Kaplan Executive Function System Tower Test.

Results: Paired t-tests revealed no significant differences between ordered and random sequences for forward (ordered: $\mathrm{M}=9.90, \mathrm{SD}=2.01$; random: $\mathrm{M}=10.06, \mathrm{SD}=1.98), \mathrm{t}(49)=-0.698, \mathrm{p}=0.49$, or backward recitation (ordered: $\mathrm{M}=6.96, \mathrm{SD}=2.03$; random: $\mathrm{M}=6.96, \mathrm{SD}=2.23$ ), $\mathrm{t}(49)=0, \mathrm{p}=1$. Preliminary item response theory parameters were estimated, but stable estimates require larger samples.

Conclusions: Results from the current study suggest Digit Span performance is unaffected by random versus ordered sequence presentation. These results establish the foundation for the development of an adaptive Digit Span test, responsively generating customized sequences to more efficiently yield equally valid results. Active research efforts are aimed at identifying item response theory parameters for adaptive testing purposes.

Correspondence: Jason W. Adams, B.S., B.A., Psychology, University of Colorado Colorado Springs, Department of Psychology, 1420 Austin Bluffs Pkwy, Colorado Springs, CO 80918. E-mail: jadams2@uccs.edu 
C.D. PAUlS, S.M. MCCLINTOCK, J. PRUDIC, J. FISZDON \& J. CHOI. The Columbia Quality of Life Inventory (CQOL): A measure to assess meaningful outcomes in ECT trials.

Objective: We set out to fill the gap that presently exists in the measurement of quality of life (QOL) in depressed patients receiving electroconvulsive therapy (ECT). The Columbia Quality of Life Inventory (CQOL) was developed to provide a focal measurement of QOL that encompasses constructs outside of physical health and includes ADLs that rely on cognitive skills which can be compromised as a result of ECT.

Participants and Methods: 115 older adults were given the CQOL along with measures of cognition, physical health, depression, and ADLs at baseline, 2, 4, and 6 mo. Twenty-four participants were inpatients with major depression who received ECT, 52 participants were outpatients with a history of depression who did not receive ECT, and 39 healthy control participants without a history of ECT.

Results: ICC calculated with the control sample over the 6-mo period showed the CQOL to possess good test-retest stability (>.67) and good internal consistency $(\alpha>$.81). Convergent validity in all three samples was demonstrated between CQOL and a physical health measure (SF12) $(\mathrm{r}>32, \mathrm{p}<.03)$, a commonly used gauge of QOL in previous ECT outcome trials. Divergent validity was supported by lack of association between baseline CQOL and premorbid estimates of IQ. In order to assess predictive validity, we examined the strength of association between baseline CQOL (median split) and improvers/non-improvers on depression, ADLs and cognition. Higher baseline CQOL scores were related to improved depression levels (OR 3.92, $\mathrm{p}=.02$ ), improvement in ADL scores (OR 2.66, $\mathrm{p}=.03$ ), and no cognitive decline (OR 1.90, $\mathrm{p}=.04)$. Finally, two factors with eigenvalues $>1$ emerged from a principal component analysis of the CQOL: memory problems related to self-care and hope for the future.

Conclusions: We examined the CQOL's psychometric properties in a range of patients with/without a history of ECT, along with a healthy control sample. The CQOL is a psychometrically sound instrument which can be considered as a QOL measure in ECT outcome trials. Correspondence: Carol D. Pauls, Master's of Science, Mental Health Services and Policy Research, New York State Psychiatric Institute, 95 Parkway East, Bloomfield, NJ 07003.E-mail: paulsca@nyspi.columbia. edu

K.L. DINH, P.K. MARTIN, R.J. HEINRICHS, L.E. BAADE \& R.W. SCHROEDER. Comparing RBANS Performance Using Randolph and Oklahoma Norms in a Geriatric Inpatient Sample. Objective: When standardizing performances obtained by older adults on the Repeatable Battery for the Assessment of Neuropsychological Status (RBANS), clinicians may use either the original normative sample (Randolph, 1998) or a sample of community dwelling older adults referred to as the Oklahoma sample (Duff et al., 2003). While demographic characteristics of these samples are relatively similar, screening and exclusion criteria differed considerably.

Participants and Methods: One hundred five geriatric inpatients admitted to a psychiatric unit secondary to acute behavioral disturbances were evaluated with the RBANS. Their standardized scores were calculated via both the Randolph and Oklahoma norms. Rates of impaired performance (defined as <2nd percentile) across subtests were compared using both norming systems in patients aged 70-79 (n=57) and 80-89 $(n=48)$. Dependent samples $t$-tests were also conducted to determine if subtest mean scores differed significantly between norms.

Results: Across RBANS subtests, utilization of the Randolph norms resulted in a greater proportion of individuals classified as impaired than when the Oklahoma norms were utilized. These findings occurred across both age groups, with the most substantial difference seen on the Coding subtest in the older sample $(64.6 \%$ versus $14.6 \%$ labeled as impaired). Additionally, mean performances differed significantly between norms across subtests at $\mathrm{p}<.001$.

Conclusions: Differences in both impairment classification and mean score performances were found when comparing data via the Randolph and Oklahoma normative samples. These findings suggest substantial divergence between the compositions of these two samples and encourage the need to determine which norms may be more appropriate for specific geriatric inpatient settings.

Correspondence: Kara L. Dinh, Psy.D., Neuropsychology, University of Kansas School of Medicine - Wichita, 9911 E. 21st St. N, Apt. 308, Wichita, KS 67206. E-mail: kshaneyfelt@gmail.com

D.K. HORTON, L.S. HYNAN, J.M. GALUSHA-GLASSCOCK, S. PANDYA, H.C. ROSSETTI, L.H. LACRITZ, M.F. WEINER \& M. CULLUM. Diagnostic Accuracy of Standard and Abbreviated Forms of the MoCA.

Objective: To compare standard and shortened forms of the Montreal Cognitive Assessment (MoCA) in the detection of cognitive impairment. Participants and Methods: In preliminary work, we developed an abbreviated MoCA using item analysis. A total of 408 subjects [normal $\mathrm{n}=152$, mild cognitive impairment (MCI) $\mathrm{n}=169$, Alzheimer disease (AD) $n=87$ ] were randomly divided into test and validation samples to determine cutoff scores for both measures and evaluate accuracy in an independent sample. The test sample comprised a random selection of $75 \%$ of subjects; the remaining participants were included in the validation sample. For the purpose of analysis, MCI and AD groups were combined to form a "cognitively impaired" group. Crosstab analyses were used to determine frequencies of correctly classified participants using cut scores derived from Receiver Operating Characteristic analyses. Diagnostic accuracy of the standard and abbreviated MoCA forms was compared via Net Reclassification Improvement (NRI) analyses, which quantify differences in diagnostic classification rates between two measures.

Results: In the test sample, NRI analyses revealed no significant differences between the accuracy of the measures when distinguishing controls from those with cognitive impairment (NRI=0.02; $\mathrm{p}=.73$ ). Similarly, NRI analyses in the validation sample showed no significant differences in diagnostic accuracy between the measures (NRI=0.04; $\mathrm{p}=.68$ ).

Conclusions: NRI analyses revealed no significant differences between the full and abbreviated MoCA versions in terms of diagnostic accuracy. Results suggest that some MoCA items do not add appreciably to the clinical sensitivity of the instrument and that similar diagnostic classification rates can be achieved with an abbreviated MoCA.

Correspondence: Daniel K. Horton, University of Texas Southwestern Medical Center, 4533 Cedar Springs, \#425, Dallas, TX 75219. E-mail: dh1208@gmail.com

B. MILLER, J. ROBINSON, V. PACHECO \& R. COLLINS. Added Value of Neuropsychological Assessment to the Clinical Dementia Rating Scale in Assessing Mild Cognitive Impairment.

Objective: The Clinical Dementia Rating Scale (CDR) Sum of Boxes (SOB) has evidenced utility in staging dementia beyond the global CDR score (e.g., Lynch et al., 2005, O'Bryant et al., 2008), particularly with the 0.5 rating associated with mild cognitive impairment (MCI) (Morris, 1993). The present study investigated the added value of SOB and neuropsychological testing in diagnostic classification of CDR 0.5 patients. Participants and Methods: The study was a retrospective review of patients referred for an outpatient neuropsychological evaluation. The final sample is comprised of 85 patients with a global CDR rating of 0.5 (rated by a CDR certified neuropsychologist and CDR trained neurologist). CDR ratings were made, independent of neuropsychological data, using interview, mental status examination, and caregiver ratings when available. Neuropsychological data was subsequently used for consensus diagnosis of (a) MCI, (b) dementia or (c) normal.

Results: Of the 85 patients with CDR global rating of $0.5,60(70.6 \%)$ were classified as MCI, $9(10.6 \%)$ as dementia and $16(18.8 \%)$ as normal. Ranges for SOB scores were $0.5-2.5$ (normal), $0.5-4.0$ (MCI) and $2.5-7.0$ (dementia). Kruskal-Wallis ANOVA found significant difference for CDR SOB score for each group $[\mathrm{X} 2(2, \mathrm{~N}=85)=25.33$, 
$p<.001]$ and post-hocs revealed each group significantly differed from each other $(\mathrm{p}=.005$ to $\mathrm{p}<.001)$.

Conclusions: Results suggest that significant differences emerged between normal, MCI and dementia patients when utilizing SOB; however, notable overlap in SOB score ranges was present for the diagnostic groups. Therefore, on a case-by-case basis, the addition of the neuropsychological data resulted in $30 \%$ of the sample moving from an MCI diagnosis to either normal or dementia.

Correspondence: Brian Miller, Ph.D., Mental Health Care Line, Michael E. DeBakey VAMC, 2002 Holcombe Blvd., Houston, TX 77030. E-mail: brian.miller3@va.gov

M. CALAMIA, B.D. HILL, M. MUSSO \& R. PELLA. Factor Structure and Clinical Correlates of the Wender Utah Rating Scale (WURS). Objective: The WURS is a widely used retrospective measure of childhood ADHD symptoms. However, previous studies have shown that the total score on the WURS is not specific to an ADHD diagnosis and that specific WURS symptom factors vary in their relationship to ADHD. We investigated the factor structure of the full 61-item WURS and examined the relationship of different symptom dimensions to several clinically relevant variables.

Participants and Methods: Participants (n=932) were individuals seeking a psychoeducational evaluation at a university clinic. They ranged in age from 16 to $68(\mathrm{M}=22.7, \mathrm{SD}=6.3)$. In addition to completing the WURS, participants completed measures of cognitive functioning, academic achievement, personality, and psychological functioning. Results: Results were consistent with a five-factor model (inattention, disinhibition, negative affect, extraversion, and learning problems). The utility of this model was demonstrated through the divergent relationship of the individual factors with external correlates. For example, only inattention and disinhibition symptoms had small, but significant correlations $(p<.05)$ with variability on a computerized measure of sustained attention $(\mathrm{r}=0.10$ and $\mathrm{r}=0.16$, respectively $)$ and grade point average $(\mathrm{r}=-0.14$ and $\mathrm{r}=-0.11$, respectively).

Conclusions: These results highlight the utility of looking bevond the total score on the subset of WURS items traditionally used to measure childhood ADHD symptoms. Specific WURS symptom dimensions relevant to an ADHD diagnosis have some unique correlates not shared by other WURS symptom dimensions.

Correspondence: Matthew Calamia, PhD, Psychology, Louisiana State University, 236 Audubon Hall, Department of Psychology, Louisiana State University, Baton Rouge, LA 70803.E-mail: mcalamia@lsu.edu

D. LEITNER, M. LIBBEN, M. REYNOLDS, L. OHLHAUSER, J. KLUFTINGER, J.C. PIERCY \& H. MILLER. Examining the Convergent Validity of Neuropsychological Evaluation with Functional Outcome in a Stroke Population.

Objective: This study explored predictors of functional outcome in stroke, expanding on previous studies by measuring various domains of function, and utilizing a battery of neuropsychological tests, demographics, and stroke location as predictors of outcome.

Participants and Methods: Sixty two stroke inpatients, (Mean age $=69, \mathrm{SD}=12.6$; mean years of education $=12, \mathrm{SD}=2.0), 39$ males and 23 females, completed a comprehensive neuropsychological battery during acute inpatient rehabilitation. The Oxford Community Stroke Project (OCSP) classification system was employed to identify stroke subtype. Function was measured using the Mayo-Portland Adaptability Inventory - 4 (MPAI-4).

Results: In regression analyses, comprehensive neuropsychological evaluation (utilizing multiple measures' scores) accounted for a large portion of variance for the MPAI-4 Ability Subscale (46\%), Participation Subscale $(49 \%)$ and Total Score (49\%). Age and education accounted for $2 \%$ of variance in the MPAI-4 Total Score. No differences were found between sex for MPAI-4 scores. Utilizing the OCSP classification, on average, anterior circulatory strokes scored lower on tests of executive functioning, visuoconstructional skills, and verbal fluency. Posterior circulation strokes scored lower on tests of visuospatial abilities and memory. Lacunar strokes had the highest average scores in almost all neuropsychological tests administered.

Conclusions: Comprehensive neuropsychological evaluation has good convergent validity with the MPAI-4. Further, the OCSP classification system predicted performance on select neuropsychological tests. Demographics accounted for a small portion of variance in functional outcome during early stages of stroke. These results may have implication for directing care in the acute stages of rehabilitation, and developing treatment plans following discharge from hospital.

Correspondence: Damian Leitner, BSc. (Hons) Psychology, Kelowna General Hospital, 2268 Pandosy Street, Kelowna, BC V1Y 1T2, Canada.E-mail:Canadian_02@hotmail.com

J. SILVERMAN, J. CELLAR, S. PETERSON-HAZAN, J.J. LAH, A.I. LEVEY \& F.C. GOLDSTEIN. Evaluation of the Modified Telephone Interview for Cognitive Status as a Screen for Mild Cognitive Impairment.

Objective: The Modified Telephone Interview for Cognitive Status (TICS-m) is a commonly used screening tool for dementia. Items assess orientation, attention, language, memory, and conceptual reasoning. The purpose of the current study was to determine whether the TICS-m is a useful screening tool for mild cognitive impairment (MCI) and to identify whether certain domains and items are especially sensitive.

Participants and Methods: Fifty three participants received the TICS-m as part of their screening process for possible enrollment in the Emory Alzheimer's Disease Research Center. Once enrolled, individuals were administered a battery of neuropsychological and functional measures, and a consensus diagnosis of normal cognition or MCI was assigned without knowledge of TICS-m performance. Sensitivity and specificity were evaluated for different TICS-m cutoff scores and items. Results: Thirty one participants had normal cognition and 22 had MCI. The TICS-m total score was significantly $(p<.001)$ higher in those with normal cognition (mean= 36.5 SD=3.2) than MCI (mean= 32.7, $\mathrm{SD}=$ 2.7). However, scores on only 4 out of the 21 total questions were significantly different between the groups (repetition of a phrase, full name of Vice-President, immediate recall and delayed recall of words). By using these four questions, we found a cutoff score with a higher sensitivity and specificity $(\geq 80 \%)$ than various total score cutoffs. Items assessing language and both immediate and delayed recall memory were especially sensitive in differentiating the groups.

Conclusions: A focus on selective items of the TICS-m provides greater accuracy for distinguishing MCI from normal cognition than a reliance on the total score alone.

Correspondence: Felicia C. Goldstein, Ph.D., Neurology, Emory University School of Medicine, 1841 Clifton Rd., N.E., Atlanta, GA 30329. E-mail:fgoldst@emory.edu

J.J. MIETCHEN, S.D. GALE \& W. BADAL. Convergent, Discriminant, and Predictive Validity of the Neuropsychological Assessment Battery Shape Learning Subtest.

Objective: Examine the convergent, discriminant, and predictive validity of the Shape Learning subtest of the Neuropsychological Assessment Battery (NAB).

Participants and Methods: Fifty college students ( $54 \%$ female; M age $=20.46, \mathrm{SD}=1.78 ; 82 \%$ right-handed) completed the Shape Learning subtest of the NAB, the Brief Visuospatial Memory Test-Revised (BVMT-R), and the Rey Auditory Verbal Learning Test (RAVLT). Performance between these measures was correlated to establish convergent and discriminant validity. Predictive validity was established with regression.

Results: Immediate recall on the BVMT-R was correlated with immediate recall on the $\mathrm{NAB}(\mathrm{p}<.01)$ with $\mathrm{NAB}$ immediate recall performance predicting BVMT-R immediate recall performance $(p<.01)$, however, delayed recall was not significantly correlated between the BVMT-R and $\mathrm{NAB}(\mathrm{p}=.36)$. The RAVLT trials $1-5$ was not significantly correlated 
with the NAB immediate recall for trials $1-3(p=.12)$ or the BVMT-R immediate recall for trials $1-3(\mathrm{p}=.16)$.

Conclusions: The NAB appears to have predictive validity in association with the BVMT-R, and exhibited only a small correlation with a measure of verbal memory, thus establishing discriminant validity. Convergent validity of the NAB immediate recall was confirmed. However, the lack of correlation between the NAB and BVMT-R on delayed recall suggests these tests may be measuring different aspects of nonverbal memory.

Correspondence: Jonathan J. Mietchen, BS Psychology, Department of Psychology, Brigham Young University, 1001 SWKT, Provo, UT 846025543. E-mail: jonathan.mietchen@gmail.com

\section{H. TYLER, T. ROEBUCK-SPENCER \& A. VINCENT. Comparability of In-Person versus Remote Proctoring for Neuropsychological Test Administration.}

Objective: There is a growing need for telemedicine models of cognitive testing that conform to the standards for educational and psychological testing. The validity of remote testing can be compromised if the testing process cannot be appropriately observed and appropriately proctored. The present study examined the comparability of data obtained from a computerized cognitive test battery, ANAM, when testing is proctored in-person versus remotely.

Participants and Methods: Participants $(\mathrm{N}=50)$ were students recruited from a university setting. The sample was primarily non-Hispanic $(91 \%)$ white $(78 \%)$ females $(68 \%)$ with an average age of 19.0 \pm 1.4 years. Following informed consent, participants were randomly assigned to one of two groups and administered ANAM individually. In Group 1, a test proctor was present during testing. In Group 2, the test proctor was in an adjacent office (simulating a remote testing situation) and monitored the test-taker using desktop sharing software and telephone. Independent sample t-tests were conducted to compare resulting ANAM test scores.

Results: Group composition did not differ according to key demographic factors. Performance of the two groups did not differ for any of the ANAM tests (all $\mathrm{p}>.05$ ).

Conclusions: Results suggest that comparable data can be obtained using remote proctoring approaches. With the vast expansion of internet-based assessment tools and the increased need for telemedicine models of neuropsychological test administration, it is important to have robust test administration procedures available that yield data comparable to norms. Future studies should examine additional technologies and technical solutions to establish utility in the remote testing environment. Correspondence: Hannah Tyler, Master of Science, Cognitive Science Research Center, University of Oklahoma, 3200 Marshall Avenue, Suite 260, Norman,OK 73072.E-mail: hannah.tyler@ou.edu

\section{S. HAJ-HASSAN, D. LIU \& A.L. JEFFERSON. Empirically-Derived Subtypes of Mild Cognitive Impairment: The Vanderbilt Memory} \& Aging Project.

Objective: With limited empirical validation, recent efforts have dichotomized mild cognitive impairment (MCI) severity to include early MCI (i.e., subjective cognitive decline with normal neuropsychological performance) and late MCI (i.e., subjective cognitive decline with impaired neuropsychological performance). This study aimed to statistically derive MCI subtypes using an innovative analytical approach, latent class analysis (LCA), which empirically assesses the structure of heterogeneous syndromes.

Participants and Methods: MCI participants from the Vanderbilt Memory and Aging Project ( $\mathrm{n}=109,73 \pm 7$ years, $42 \%$ female) completed a neuropsychological protocol, including California Verbal Learning Test-II, Biber Figure Learning Test, Boston Naming Test, Animal Naming, WAIS-III Digit Symbol Coding, DKEFS Number Sequencing, DKEFS Letter-Number Sequencing, DKEFS Tower Test, and DKEFS Color-Word Interference Test. MCI participant raw test performances were converted to $\mathrm{z}$-scores based on cognitively normal participant test performances ( $\mathrm{n}=104,71 \pm 7,49 \%$ female). Using these $\mathrm{z}$-scores, LCA was performed for the MCI sample.

Results: Three empirically-based MCI subtypes were identified, aligning with three cognitive severity classes, including early $(n=28)$, middle $(n=49)$, and late stage MCI $(n=32)$ as evidenced by performance differences in the expected direction (e.g., early>late) on all neuropsychological tests (all p-values $<0.001$ ). The three groups differed on age $(p<0.0001$, early $=69 \pm 5$ years, middle $=74 \pm 7$ years, late $=76 \pm 9$ years $)$ but not sex $(p=0.72)$ or education $(p=0.53)$.

Conclusions: Our findings suggest that MCI severity subtypes can be empirically derived. Future studies are needed to assess biomarker and risk profile differences among early, middle, and late stage MCI subtypes as well as longitudinal outcomes, such as diagnostic conversion.

Funding: K24-AG046373, R01-AG034962, IIRG-08-88733, UL1-TR000445

Correspondence: Shereen Haj-Hassan, Vanderbilt Memory and Alzheimer's Center, 8300 Sawyer Brown Rd. Apt. \# L-304, Nashville, TN37221.E-mail: shereen.haj-hassan@vanderbilt.edu

K. BEITZ, F. KELLER, C. WILKINS, E. SASSENFELD \& H.P. DAVIS. Performance and Convergent Validity across the Life Span for a Computerized and a Manual Trails Making Test.

Objective: The Trail Making Test (TMT), widely used in neuropsychological assessment, was originally devised in 1938 as the "Partington's Pathways Test" or the "Divided Attention Test" (Partington \& Leiter, 1949), and it was initially part of a battery of tests (Army Individual Test Battery) used to assess intellectual functions. The test is typically administered in its original paper and pencil form. We report the development of a computerized TMT with life span norms and convergent validity with the manual version.

Participants and Methods: Participants (Total N $=601 ; 5-17$ yrs $\mathrm{N}=113 ; 18-59$ yrs $\mathrm{N}=396 ; 60-89$ yrs $\mathrm{N}=111$ ) were administered a standard paper and pencil TMT and a highly flexible computerized Trail Making Test with an inter-administration time of 3-7 days.

Results: The Pearson correlation coefficients for the manual Trails A and $B$ with the computerized Trails A and B version for 5 to 17 year olds were .58 and .71 , for 18 to 59 year olds .27 and .51 , and for 60 to 89 years were .55 and .64 , respectfully. Next participants were divided into 10 age groups $(5-9,10-14,15-19,20 \mathrm{~s} \ldots .80 \mathrm{~s})$ and a repeated measures ANOVA detected a significant age effect $\mathrm{F}(9,591)=67, \mathrm{p}<.001$, with the children (5-14) and elderly (60-89) having the longest times. All participant age groups had faster Trials A and B times for the computer version than the manual version with the exception of the 60 year olds on Trails B

Conclusions: The manual and computerized Trails test demonstrate convergent validity and the correlations for Trails B show a moderate to strong relationship.

Correspondence: Hasker P. Davis, Ph.D., Psychology, University of Colorado Colorado Springs, Department of Psychology, 1420 Austin Bluffs Parkway, Colorado Springs, CO 80918.E-mail: hdavis@uccs.edu

J.M. BAILIE, W.R. COLE, B. IVINS, S. ASMUSSEN, M. COTTINGHAM \& K. SCHWAB. Exploratory and Higher-Order Factor Analysis of the Automated Neuropsychological Assessment Metrics V4.0 (ANAM4).

Objective: The Automated Neuropsychological Assessment Metrics version 4.0, Traumatic Brain Injury (TBI) MIL Battery (ANAM4-TBI Mil) is widely used in pre-deployment, post-deployment, and post-injury cognitive screenings with the primary goal of aiding in the diagnosis and triaging of mild TBI. The ANAM4-TBI Mil includes seven subtests, each of which provides three primary scores (21 primary scores total). The current study provides an updated factor analysis of the instrument to determine the feasibility of factor scores to appropriately summarize performance.

Participants and Methods: Data were gathered from two samples of participants who completed the ANAM4-TBI Mil. The mild TBI sample 
included 95 male soldiers who sustained a mild TBI within the prior three months. The control sample included 872 male healthy controls with no history of TBI. Each participant completed the ANAM4-TBI Mil. A principal component analysis was used to determine first order factors. The Schmid-Leiman solution (SLS) was used to interpret the presence of a higher-order factor structure.

Results: Exploratory higher-order factor analysis supported a General aggregate factor and four primary first-order factors: Reaction Time, Learning Efficiency/ Working Memory, Math, and Accuracy. The four first order factors accounted $65.6 \%$ of total variance. The General factor accounted for $53.3 \%$ of common variance (35\% total variance).

Conclusions: The results support a two-order factor structure and indicate empirically derived summary scores may be an appropriate method to interpret performance on the ANAM4-TBI MIL. Summary scores may be more psychometrically sound and easier to interpret than the 21 individual ANAM4-TBI MIL scores currently utilized.

Correspondence: Jason M. Bailie, Ph.D., Defense and Veterans Brain Injury Center, 34730 Bob Wilson Drive, Ste 201, San Diego, CA 92019. E-mail:Jason.Bailie.ctr@med.navy.mil

\section{A. GURNANI, B.E. GAVETT \& L. ASHENDORF. Base rates for annual neuropsychological test score changes in the Uniform Data Set.}

Objective: Reliable change indices (RCIs) applied to neuropsychological tests can help identify true changes in cognition by accounting for factors such as practice effects, regression to the mean, and measurement error. However, "reliable" changes in test scores may be of limited clinical significance if they occur frequently in healthy samples. Thus, the purpose of the present study was to present base rates of longitudinal change in a robust cognitively healthy sample to identify the relative frequency of change of a given magnitude for the neuropsychological tests used at Alzheimer's Disease Centers (ADCs) in the United States (US).

Participants and Methods: Archival data were obtained from 3695 cognitively normal individuals who had completed at least 3 annual evaluations at one of the ADCs throughout the US. In order to examine the frequency with which raw scores changed from baseline to follow-up, cumulative percentages for raw scores changes of each observed magnitude for each test in the Uniform Data Set (UDS) were derived.

Results: Cumulative frequencies for change in scores on all UDS neuropsychological tests are provided.

Conclusions: Base rates of change scores can help augment the interpretation of RCIs

Correspondence: Ashita Gurnani, University of Colorado at Colorado Springs, 1510 Dublin Blvd, Apt 160, Colorado Springs, CO 80918. E-mail: agurnani@uccs.edu

\section{A. GURNANI, B.E. GAVETT \& L. ASHENDORF. Reliable change} on neuropsychological tests in the Uniform Data Set.

Objective: Neuropsychological tests are used to track annual cognitive changes in older adults, but longitudinal normative data from individuals without neurodegenerative disease is sparse. Distinguishing between usual amounts of change and change due to pathological processes requires attention to factors such as practice effects, regression to the mean, and measurement error. The purpose of the present study was to develop reliable change indices (RCIs) that can assist with interpretation of the magnitude of test score changes relative to a healthy sample of older adults believed to be free from cognitive impairment for at least one year after the collection of follow-up data.

Participants and Methods: Participants were 3695 individuals who had completed at least 3 annual evaluations and were judged to be $\operatorname{cog}$ nitively normal at each visit at one of the Alzheimer's Disease Centers throughout the United States. RCIs were calculated using two different methods for 11 neuropsychological variables at baseline and one-year follow-up visits.

Results: Baseline test score, predicted follow-up test score, and $95 \%$ confidence intervals for change scores are provided for each neuropsychological variable in the Uniform Data Set (UDS). Eight out of the eleven tests possess test-retest reliability below .7.

Conclusions: Digit Symbol, Trail Making Test-B, and Boston Naming Test are the only UDS neuropsychological test variables that possess adequate test-retest reliability. Low test-retest reliability limits the ability of neuropsychological variables in the UDS to detect change reliably, which suggests caution when these tests are used to track cognitive changes in older adults.

Correspondence: Ashita Gurnani, University of Colorado at Colorado Springs, 1510 Dublin Blvd, Apt 160, Colorado Springs, CO 80918. E-mail:agurnani@uccs.edu

L.M. BENSON, M.A. YOUNG \& N. PLISKIN. Does the Boston Naming Test - 2 Function Equivalently for African American and Caucasian Adults?

Objective: The Boston Naming Test - 2 (BNT-2) is one of the most widely used neuropsychological measures for detecting naming deficits. However, there are few studies that have investigated its item-level psychometric properties, particularly in ethnic minorities, including African Americans. The present study examined the BNT-2 for the presence of ethnicity-based differential item functioning (DIF) in a sample of diverse cognitive ability.

Participants and Methods: 744 African American and Caucasian adults were recruited from a neuropsychology outpatient clinic in Chicago. Using a two-parameter Item Response Theory (IRT) framework, difficulty and discriminability estimates were generated for items 16 through 60 .

Results: DIF was detected for 10 items ("globe," "beaver," "dominoes," "unicorn," "accordion," "latch," "tripod," "yoke," "trellis," and "palette"), indicating that these items do not function equivalently between groups. Post hoc analyses examined age, years of education, and literacy as factors to account for DIF. The three variables provided partial explanations for the DIF, although none could entirely account for the observed findings.

The IRT-estimated rank ordering of item difficulty was similar in both groups, and comparable to those initially assigned by Kaplan, Goodglass, and Weintraub (2001). Correlations between BNT-2 scale scores and IRT-based scale scores were very high, suggesting that the overall scoring of the BNT-2 was not substantially biased by ethnicity, despite the identified DIF and small fluctuations in item rankings.

Conclusions: Results indicate that the BNT-2 is a valid measure for use in African Americans, although future consideration should be given to revision or omission of those items identified as having DIF.

Correspondence: Laura M. Benson, Ph.D., Psychiatry and Behavioral Sciences, Roosevelt University, 909 Davis Street, Suite 160, Evanston, IL 60201. E-mail: lbenson@northshore.org

N.E. CARLOZZI, S. GOODNIGHT, A. KRATZ, J. MINER, N. DOWNing, J. PAUlsen, P. DAYAlu, S. PERlman, J. PERLMUTTER, C. ROSS, M. MCCORMACK, K. QUAID, H. MARIN, M. NANCE, M. GESCHWIND, A. AHMED, J. LAI, E.A. HAHN, R. GERSHON \& D. CELLA. Psychometric support for the validity of PROMIS and Neuro-QOL in Huntington disease. Objective: Commonly used measures of health-related quality of life (HRQOL) for use in Huntington disease (HD) are typically not comprehensive and do not fully account for clinically meaningful changes in functioning. HD is a debilitating genetic disease that impacts physical, behavioral, emotional, cognitive, and social health. New measures of HRQOL (Neuro-QOL, www.neuroqol.org; PROMIS, www.nihpromis. org) address several of these shortcomings, but have not yet been validated in HD.

Participants and Methods: 512 individuals with HD completed 14 HRQOL measures (12 PROMIS/Neuro-QOL, EQ-5D and WHODAS $2.0)$ and clinician-rated measures of motor, emotion, cognitive, and functional status (UHDRS and PBAs). To establish validity for PROMIS/ Neuro-QOL, we examined: correlations with other validated measures 
of HRQOL, the ability to differentiate between prodromal, early and late HD (multivariate analyses), and the ability to differentiate between individuals known to be high vs. low on the trait being measured (univariate analyses).

Results: Generally, PROMIS/Neuro-QOL measures had moderate to high correlations with the generic self-report measures ( $r$ 's ranged from .29 to .72 ). Most PROMIS/Neuro-QOL measures were able to differentiate between prodromal, early and late HD $(\mathrm{p}<.03$; except Positive Affect, Depression and Anxiety). Univariate analyses indicated that all PROMIS/Neuro-QOL measures were able to differentiate between individuals who were high or low on the trait being measured (all $\mathrm{p}<0.0001$, e.g., PROMIS Anxiety differentiated between individuals with high vs. low clinician-rated anxiety).

Conclusions: Results provide support for the construct validity of PROMIS/Neuro-QOL measures in evaluating HRQOL in HD. Future work is needed to determine the sensitivity of these measures to change over time.

Correspondence: Noelle E. Carlozzi, University of Michigan, 2800 Plymouth Road, Ann Arbor, MI 48109.E-mail: carlozzi@med.umich.edu

\section{J. DONDERS, Y. OH \& J. GABLE. Discrepancies between Self and Informant Reports on the BRIEF-A after mTBI.}

Objective: To determine correlates of self and informant reports on a standardized rating of executive functioning in persons with mild traumatic brain injury (mTBI).

Participants and Methods: Retrospective case series review of 100 referred persons who met criteria for mTBI; i.e., time to follow commands $<30$ minutes, post-traumatic amnesia $<24$ hours and Glasgow Coma Scale score $>12$. All participants and their informants completed the Behavior Rating Inventory of Executive Function - Adult Version (BRIEF-A) during outpatient neuropsychological evaluations within 30-360 days post injury.

Results: Participant and informant BRIEF-A ratings were strongly correlated but participants rated themselves as worse than informants did. Regression analysis revealed that higher levels of education and presence of intracranial neuroimaging findings were associated with better BRIEF-A ratings, whereas worse BRIEF-A ratings were associated with longer time since injury and prior psychiatric treatment. BRIEF-A ratings were not correlated with laboratory measures of executive functioning.

Conclusions: Subjective perceptions of executive dysfunction during the first year after mTBI are driven primarily by premorbid factors and do not reflect acquired cerebral impairment.

Correspondence: Jacobus Donders, Mary Free Bed Rehabilitation Hospital, 235 Wealthy, S.E., Grand Rapids, MI 49503. E-mail: jacobus. donders@maryfreebed.com

T. HILL-JARRETT, J. HAMILTON, J. MANLY \& R.M. BAUER. Measurement Invariance of the Brief Test of Adult Cognition by Telephone (BTACT) in Caucasian and African American Adults.

Objective: Establishing measurement invariance is required when comparing neuropsychological test scores across racial groups. We examined the psychometric invariance of a neuropsychological battery administered by telephone in African American and Caucasian adults.

Participants and Methods: Participants were 3697 Caucasian and 437 African American adults enrolled in the Midlife in the United States (MIDUS)-II Cognitive study. All completed the Brief Test of Adult Cognition by Telephone (BTACT), a 15-minute neuropsychological battery. Factor structure of the test battery was validated in each group against a hypothesized two-factor structure (executive function, episodic memory) based on published exploratory factor analyses (EFA). Upon validation, a multi-group confirmatory factor analysis (CFA) was conducted to test hierarchically for full configural, metric, and scalar invariance across racial groups.

Results: Overall fit of the single-group CFA based on the hypothesized factor structure was poor for both groups. When Digit Backward was allowed to load onto multiple factors, model fit improved in Caucasians $(\mathrm{RMSE}=.060, \mathrm{RMSEA}=.981)$ and African Americans (RMSEA=.061, CFI=.973). Multi-group CFA revealed full configural (RMSEA $=.042$, $\mathrm{CFI}=.980)$ and metric invariance (RMSEA $=.042, \mathrm{CFI}=.977)$. Full scalar invariance was not supported, but significant model improvement occurred when the intercept of one test (Number Series) was allowed to vary across groups (RMSEA $=.055, \mathrm{CFI}=.950$ ).

Conclusions: While the latent factor structure of the BTACT is supported in both Caucasian and African American adults, there were racial differences in average performance on Number Series (a measure of inductive reasoning). Demographically-adjusted BTACT norms should include possible interactions with race.

Correspondence: Tanisha Hill-Jarrett, M.S., Clinical \& Health Psychology, University of Florida, 3301 SW 13th Street Apt D146, Gainesville, FL 32608.E-mail: thilljarrett@phhp.ufl.edu

\section{K THIRUSELVAM, E. VOGT \& J. HOELZLE. Application of Item Response Theory (IRT) to Trial 1 of the California Verbal Learning Test - Second Edition.}

Objective: This study uses item response theory (IRT) methods to investigate if item parameters of the California Verbal Learning Test-Second Edition (CVLT-II; Delis, Kramer, Kaplan, \& Ober, 2000) vary as a function of the serial position effect.

Participants and Methods: Archival data from 577 individuals (ages 18 to 70 ) were obtained for this study. Of these, 338 (58.6\%) were research data obtained from college students and 239 (41.4\%) were clinical data obtained from a neuropsychology assessment clinic. A 2-parameter IRT model was used to establish the discrimination and difficulty parameters of each of the 16 words in CVLT-II Trial 1.

Results: Consistent with the serial position effect, CVLT-II Trial 1 items in the middle of the list were more difficult ( $\beta=0.55$ to 2.11$)$ than items at the beginning ( $\beta=-1.83$ to 1.77$)$ and end $(\beta=-2.03$ to 1.54$)$ of the list. Items at the end of the list were most accurate at discriminating between memory abilities that fell within the average range (between standard scores of 103 and 105), whereas items at the beginning of the list were most accurate at discriminating between memory abilities that fell within the average to high average range (between standard scores of 105 and 110).

Conclusions: Performance on CVLT-II Trial 1 appears to be influenced by memory and attention. The clinical utility of the CVLT-II as a measure of episodic memory is likely to improve if clinicians carefully consider how the serial position effect impacts the likelihood of word retrieval.

Correspondence: Indrani K Thiruselvam, MA, Psychology, Marquette University, 751 W. Bode Circle, Unit 209, Hoffman Estates, IL 60169. E-mail: tindrani@gmail.com

I. K THIRUSELVAM, J. HOELZLE, L.D. NELSON \& M. MCCREA. Application of Item Response Theory (IRT) to the Standardized Assessment of Concussion.

Objective: The Standardized Assessment of Concussion (SAC; McCrea Kelly, \& Randolph, 2000) is a 30-item measure utilized to identify effects of mild traumatic brain injury (mTBI). SAC items are equally weighted in generation of a Total Score. This study utilized item response theory (IRT) methods to investigate how respective items function.

Participants and Methods: Xcalibre (Assessment Systems Corporation, 2012) software was used in a two-parameter logistic IRT model analysis to investigate performances of 313 individuals (160 participants sustained an mTBI; 153 matched controls). Participants were either evaluated in an athletic context $(\mathrm{n}=200 ; \mathrm{M}$ age $=17.48[\mathrm{SD}=$ 1.98]) or Emergency Department $(\mathrm{n}=113$; M age $=29.11$ [SD = 7.34]). The sample was predominantly white $(67.1 \%)$ and male $(66.5 \%)$. Results: Non-linearity at the upper end of the ability distribution suggests decreased discrimination among high-functioning individuals. All items had very low difficulty parameters (average item $\beta=-2.32$ ). Items involving concentration for $4(\beta=-1.24), 5(\beta=0.12)$, and 6 digits $(\beta$ 
$=1.05$ ), and delayed memory of words ( $\beta$ range $=-0.59$ to -1.78 ), were among the most difficult SAC items.

Conclusions: SAC items do not function uniformly in differentiating individuals with high and low neurocognitive ability. Many items function in such a manner that a person with very low ability is likely to respond correctly. Results should be considered when developing brief psychometrically sophisticated tools or weighted scoring systems that are sensitive to cognitive symptoms associated with mTBI.

Correspondence: Indrani K Thiruselvam, MA, Psychology, Marquette University, 751 W. Bode Circle, Unit 209, Hoffman Estates, IL 60169. E-mail: tindrani@gmail.com

R. LANGE, T.A. BRICKELL, J.M. BAILIE, D. TULSKY \& L.M. FRENCH. Clinical Utility and Psychometric Properties of the Traumatic Brain Injury Quality of Life (TBI-QOL) Scale in U.S. Military Service Members.

Objective: The Traumatic Brain Injury Quality of Life (TBI-QOL) scale is a recently developed self-report measure designed to provide a comprehensive assessment of Health Related Quality of Life (HRQOL) in persons following traumatic brain injury (TBI). The purpose of this investigation is to examine the clinical utility and psychometric properties of the TBI-QOL in a U.S. military population.

Participants and Methods: Participants were 152 U.S. military service members (Age: $\mathrm{M}=34.3, \mathrm{SD}=9.4 ; 89.5 \%$ male) prospectively enrolled from Walter Reed National Military Medical Center and other nationwide community outreach initiatives (e.g., military events, social media). Participants included 99 service members who had sustained a mild TBI and 53 injured or non-injured controls without TBI ( $\mathrm{n}=29$ and $\mathrm{n}=24$ respectively). Participants completed the TBI-QOL and five other behavioral measures, on average, 33.8 months post-injury (SD=37.9). Results: The internal consistency reliability of the TBI-QOL subscales ranged from $\alpha=.91$ to $\alpha=.98$. The convergent and discriminant validity of the 14 TBI-QOL subscales was high. The mild TBI group had significantly worse scores on 10 of the 14 TBI-QOL subscales when compared to the control group (range; $p<.001$ to $p=.043$ ). Effect sizes ranged from medium to very large $(\mathrm{d}=.35$ to $\mathrm{d}=1.13)$. The largest differences were found on the Cognition-General Concerns ( $d=1.13$ ), Executive Function $(\mathrm{d}=.94)$, Grief-Loss ( $\mathrm{d}=.88)$, Pain Interference $(\mathrm{d}=.83)$, and Headache Pain $(\mathrm{d}=.83)$ subscales.

Conclusions: These results provide support for the use of the TBI-OOL as a measure of HRQOL in a military population. Additional research is recommended to further evaluate the clinical utility of the TBI-QOL in both military and civilian settings.

Correspondence: Rael Lange, DVBIC, WRNMMC, Defense and Veterans Brain Injury Center, Walter Reed National Military Medical Center, Building 8, Room 2264, 8901 Wisconsin Avenue, Bethesda, MD 20814. E-mail: rael.t.lange@gmail.com

E.F. DAY, B. SWAnSBORO, E. GOBBI \& M. AEBI. The Five Point Test: Age and Education on Test Taking Strategy.

Objective: The current study examined the effects of drawing strategy when performing a nonverbal fluency test, the Five Point Test (FPT) (Regard, Strauss, and Knapp,1982). We hypothesized that particular types of strategies are more conducive for the production of unique designs (UD). We also predicted that the number of UD will be susceptible to aging, due to general decline in motor speed. In addition, certain strategies will correlate better with educational background than age. Participants and Methods: Regard's (1982) 5PT was administered to 426 healthy adults from the general population ages 17-92 (M=34.9, $\mathrm{SD}=22.2)$. A computerized system endorsed by Regard utilized a predetermined algorithm to score the tests based on the number of designs created, number of repetitions, number of UD, and the addition, rotation, and deletion strategies.

Results: A multiple regression analysis evaluated whether the three strategies predict UD. Rotation was significantly related to $\mathrm{UD}, \mathrm{R}=.82$, $\mathrm{R}$ squared $=.68, \mathrm{~F}(1,423)=887.71, \mathrm{p}<.001$, suggesting that it is the best strategy for UD creation. Addition was the best predictor for number of repetitions, $\mathrm{R}=.50$, $\mathrm{R}$ squared=.25, $\mathrm{F}(1,423)=141.18$, $\mathrm{p}<.001$, suggesting it is an ineffective strategy. UD had a significant relationship with age, $\mathrm{R}=.39, \mathrm{R}$ squared $=.15 \mathrm{~F}(1,423)=73.95, \mathrm{p}<.001$. This unexpected finding suggests that the number of designs produced may be due to graphomotor speed instead of executive function and education may correlate with strategic predictors. Rotation and education were significantly related, $R=.16, R$ squared $=.01, F(1,423)=5.69, p=.018$, suggesting that rotation is affected by higher order processing prevalent in educated individuals.

Conclusions: The hypotheses were supported and the results showed that age may suggest how many UD are created on the 5PT. This ability may require motor speed that declines with age. The most effective strategy of the 5PT may be rotation, which requires higher order processing that is often refined through education.

Correspondence: Ellen F. Day, B.S., Psychology, Cleveland State University, 7657 Brecksville Road, Independence, OH 44131. E-mail: e.f.day@vikes.csuohio.edu

R.J. PIERS, K.N. DEVLIN, R. SWENSON, B. WASSERMAN, L.R. SILVA, Y. LIU, S. SESHADRI, P.A. WOLF, M. LAMAR, R. AU \& D.J. LIBON. Clock Drawing in a Healthy Community Sample: A Principal Component Analysis.

Objective: The Clock Drawing Test (CDT) is a widely used, popular test because of its sensitivity in detecting cognitive impairment. Traditional scoring systems can be laborious and imprecise. Digital technology permits precise assessment that is objective and reproducible requiring only minutes to administer and score. The current research describes factor structure for novel digital CDT measures.

Participants and Methods: Approximately 900 stroke/dementia-free participants from the Framingham Heart Study (mean age $=59.50+/-$ 14.23: mean education $=15.35+/-3.00: 48.43 \%$ women) were administered the digital CDT to command and copy with hands set for " 10 after 11". A core group of 30 variables measuring size, location/position on the page, and latency of all clock elements were subjected to a Principal Component Analysis (PCA) with varimax rotation.

Results: The command PCA yielded a 10-factor solution accounting for $74.66 \%$ of variance; the copy PCA yielded an 11-factor solution accounting for $77.13 \%$ of variance. In the command condition the factor structure could be reduced to four constructs - (1) position/ location of the drawing; (2) size/ length of all clock elements; (3) total drawing time to completion: (4) graphomotor precision. In the copy condition the factor structure could be reduced to five constructs - (1) position/ location of the drawing; (2) size of clock face/ digits; (3) total drawing time to completion; (4) position of the clock hands; (5) graphomotor precision. Conclusions: Digital technology yields a psychometrically objective assessment of constructs underlying clock drawing behavior, maximizing the use of this test for research and potentially increasing sensitivity for screening for emerging cognitive impairment.

Correspondence: Ryan J. Piers, Neurology, Framingham Heart Study, 19 Harvard Avenue, Brookline, MA 02446.E-mail: rpiers@bu.edu

\section{K. OMALLEY, C. TYRRELL \& B.E. GAVETT. Impact of Demographic Variables on the Brief Visuopatial Memory Test- Revised.}

Objective: This study examined whether demographic variables affected visuospatial memory abilities in a sample of community dwelling older adults who voluntarily sought cognitive testing due to memory concerns. Current BVMT-R normative data includes age, sex, and education but does not provide data on race. Given the growing diversity of the aging population, examining differences in performance for different racial groups may provide useful information for interpretation of performance. This study aimed to examine sex and ethnicity differences on the BVMT-R and broaden the range normative data available for a sample of older adults. Understanding differences or similarities in a 
help-seeking sample is important because feedback regarding cognitive performance is based on normative samples.

Participants and Methods: A sample of $\mathrm{N}=254$ (age $\mathrm{M}=70.5$, SD $=9.15$ ) community dwelling older adults (88 men and 166 women) seeking a memory assessment was used to examine the impact of age and race on BVMT-R performance. Individuals engaged in a 45-60 min memory screening which provided a brief evaluation of cognitive functioning.

Results: A significant difference in total recall scores between men (M $=15.44, \mathrm{SD}=7.57)$ and women $(\mathrm{M}=17.82, \mathrm{SD}=8.38)$ was found, $\mathrm{t}$ $(250)=-2.22, p=.03, \eta 2=.02$. A statistically significant difference by race was found $\mathrm{F} 6,243)=2.791, \mathrm{p}=.01, \eta=.06$.

Conclusions: Demographic variables impact on BVMT-R total recall performance suggest that normative data should be expanded to include race and that demographic information should be considered carefully when interpreting test results.

Correspondence: Kelly OMalley, MA, Psychology, University of Colorado Colorado Springs, 4863 N Nevada, Colorado Springs, CO 80918. E-mail:komalle2@uccs.edu

\section{Cognitive Intervention/Rehabilitation}

H. LUU, L. BALABAN \& L. LAATSCH. The influence of demographic variables on executive functioning after cognitive rehabilitation. Objective: Few studies in the current literature have looked at the effects of cognitive rehabilitation therapy (CRT) on executive functioning (EF). This retrospective study evaluated if changes occurred on neuropsychological measures of EF following CRT. Additionally, we determined whether demographic variables influenced EF post CRT. Participants and Methods: Demographic variables used included age at time of injury, months post injury before treatment, gender, and educational history. The data $(\mathrm{N}=43)$ included mixed neurological patients ( $44 \%$ moderate to severe TBI) seen for an initial evaluation, individualized CRT, and post-treatment neuropsychological evaluation. The number of CRT sessions ranged between 7-31 (mean=15, SD=6). The EF measures used included Stroop Color-Word Test (Color-Interference score) and Trail Making Test Part B. Significant change in performance was defined as an improvement of 10 or more T score points.

Results: Correlation analyses revealed that Stroop Interference scores post CRT had a significant relationship with years of education $(\mathrm{p}<0.05)$. No other significant relationship existed between EF measures and demographic variables. Paired sample T-tests indicated that performance on Trails B were significantly improved post CRT $(p<0.001)$. This trend was absent for Stroop performance. Two groups were created based on whether a significant change was found on either Stroop Interference or Trails B. A logistic regression analysis determined that included demographic variables did not significantly predict improvements on EF measures post CRT.

Conclusions: Results indicated that while individuals seeking CRT may be diverse, these factors do not play a large role on outcomes post CRT. Additional studies examining impact of CRT on different EF measures is warranted.

Correspondence: Hien Luu, Adler School of Professional Psychology, 17 N. Dearborn, Chicago, IL 60602.E-mail: hluu@my.adler.edu

S. MALIHIALZACKERINI. Meta Cognitive Training (MCT) to improve neuropsychological functioning in patients with surgically treated frontal lobe tumor and in patients affected by schizophrenia.

Objective: Recently, several studies have shown that Meta Cognitive Training (MCT) may enhance neurocognitive functioning in patients with schizophrenia. This paper describes a randomized controlled trial to investigate the efficacy of MCT to improve neuropsychological and cognitive functioning in patients treated for a brain tumor and schizophrenia. We compared the performance of patients with frontal lobe surgery in meta cognitive training, with the performance of people with schizophrenia.

Participants and Methods: A total of 30 brain tumor survivors and 30 schizophrenic patients in the age range of 22 to 40 years are recruited. Also a group of 30 healthy subjects be included as the control group. Wechsler Adult Intelligence Scales (WAIS), Wechsler Memory Scales (WMS) and Subjective Neurocognitive Inventory (SNI) administered to the patients to evaluate pre- and post- intervention functioning.

Results: We found similar cognitive profile between patients with prefrontal lobe lesions and schizophrenic subjects in terms of understanding of cognitive- intellectual, executive functions and in using metacognitive training intervention.

Conclusions: We suggest effectiveness of meta cognitive training (MCT) in cognitive - executive functioning in patients with frontal lobe lesions and schizophrenia; this can be a valuable addition to the scarce interventions available to improve neurocognitive and neuropsychological functioning of brain tumor survivors and schizophrenics.

Correspondence: ,. E-mail:

L. TAYLOR \& K. MULLIGAN. The Effects of Vitamin D Supplementation on Cognition in a Rescreened Sample.

Objective: To examine the impact vitamin D supplementation has on cognition following treatment for vitamin D deficiency in a rescreened sample.

Participants and Methods: Archival participant data from the East Central Florida Memory Disorder Clinic was utilized for this study. Participant test scores on a Brief Neuropsychological Evaluation (BNE) were compared pre- and post-treatment of low vitamin D levels from 12 older adults with a mean age of 82 . The domains assessed included attention/concentration, executive functioning, motor/processing, visuo-spatial skills and learning/memory. All participants underwent a comprehensive medical memory evaluation, including physical and neurological examination, BNE, case conference, feedback, vitamin D supplementation and BNE re-administration. Participants with kidney problems, diabetes and epilepsy were excluded from the study due to their impact on vitamin D concentrations.

Results: The paired samples t-test yielded no significant results for the attention/concentration, executive functioning, and visuo-spatial skills domains. The motor/processing speed domain $(\mathrm{M}=3.17 \mathrm{SD}=$ .835) elicited a statistically significant decrease in a participant's motor/ processing speed following vitamin $\mathrm{D}$ supplementation compared to the domain score prior to treatment $(\mathrm{M}=3.67 \mathrm{SD}=.49), \mathrm{t}(11)=2.569$, $\mathrm{p}<.026$. Lastly, the learning/memory domain $(\mathrm{M}=1.83 \mathrm{SD}=.937)$ elicited a statistically significant decrease in a participant's learning/ memory following vitamin $\mathrm{D}$ supplementation compared to the domain score prior to treatment $(\mathrm{M}=2.83 \mathrm{SD}=.718), \mathrm{t}(11)=3.071, \mathrm{p}<.011$. Conclusions: It appears that vitamin D supplementation does not improve cognition as measured by the BNE in patients diagnosed with vitamin D deficiency.

Correspondence: Leanne Taylor, M.S., Clinical Psychology, Florida Institute of Technology, 55 W Church St, apt 1614, Orlando, FL 32801. E-mail:leanne2010@my:fit.edu

H.J. MENDOZA, H.J. GRAY, D. DAVID, B. FELLEMAN, K.M. MURPHY, A. SINGH, K. ANDERSON, M.K. ASKREN, C. HIGANO \& M. CHERRIER. Attention Concerns of Cancer Survivors Participating in a Cognitive Training Intervention.

Objective: Cancer survivors with cognitive impairment concerns frequently report difficulty with attention. However, the role that attentional abilities play in overall cognitive impairment as well as how these abilities respond to a cognitive training intervention is unknown. This study examined both self-report (Attention Function Index-AFI) and objective (Continuous Performance Test-CPT) measures of attention prior to (pre) and following (post) a cognitive training intervention.

Participants and Methods: Participants were cancer survivors with cognitive symptoms who completed treatment at least six months in the 
past. Participants were randomized to treatment (tx) or control (ctl) and completed the AFI, CPT and other measures pre and post a cognitive training intervention.

Results: Participants $(\mathrm{N}=51)$ with a mean age of $59.2(\mathrm{SD}=10.3)$ years, $16.5(\mathrm{SD}=2.4)$ years of education, and $4.6(\mathrm{SD}=5.6)$ years since treatment completed all study procedures. At baseline, groups (tx, ctl) did not differ on AFI and did not evidence impairments on CPT. Post intervention, the tx group improved on the AFI sub-scale of interpersonal effectiveness compared to baseline $(\mathrm{p}=.011)$ and compared to ctl, $\mathrm{F}(1,49)=5.3, \mathrm{p}=0.25$. No significant changes were observed on CPT or other AFI sub-scales.

Conclusions: Cancer survivors reporting attention difficulties as measured by the AFI did not demonstrate impaired performance on the CPT at baseline. However, selective aspects of the AFI did improve in response to a cognitive training intervention, suggesting that cognitive training may provide symptomatic relief for select attention symptoms in cancer survivors.

Correspondence: Haley J. Mendoza, Psychology, University of Washington, $1700 \mathrm{~N}$ 80th St, Apt 3, Seattle, WA 98103. E-mail: mendozahaley@gmail.com

M. NEUGNOT-CERIOLI, C. GAGNER \& M.H. BEAUCHAMP. A game-based approach to cognitive remediation in children with borderline intelligence.

Objective: Borderline intelligence is defined as an intellectual quotient one to two standard deviations below average (70-84) and is present in $12-18 \%$ of the population (DSM-IV), often leading to school drop-out and poor vocational outcome. Developing cognitive interventions that improve reasoning skills could help reduce the performance gap between children with borderline intelligence and their peers. The goal of this study was to develop and validate two game-based approaches, one aiming to potentiate verbal reasoning and the other visuo-perceptive reasoning. Games were chosen since they are stimulating for youth and carry an intrinsic motivation.

Participants and Methods: 32 participants $(M=13.11, S D=1.2$ years) with borderline intelligence $(\mathrm{VIQ}=74.41 \pm 8.63, \mathrm{PIQ}=79.91 \pm 15.01)$ were recruited from a special education school. The participants were randomly assigned to either the verbal intervention (12), visual intervention (12) or control group (8). The "GAME" intervention consists of a program of board, computer and video games eliciting verbal abstraction and expression or deductive reasoning and logic (16 hours over 10 weeks). Individual structured feedback and strategies were provided by a neuropsychologist. Participants completed a battery of pre- and post-intervention cognitive tests (WISC-IV/WASI, TONI).

Results: Results indicate a domain-specific improvement in verbal comprehension and expression for the verbal intervention group ( $p=.005)$, but not for the visual group.

Conclusions: The GAME verbal reasoning program appears to offer a promising avenue for improving cognitive function in adolescents with borderline intelligence. The advantages of the game-based approach include cost-efficiency, availability of commercial games, and ease of administration in school or home settings.

Correspondence: Mathilde Neugnot-Cerioli, University of Montreal, Pavillon Marie Victorin F-473, 90 avenue Vincent d'Indy, Montreal, OCH2V 2S9, Canada.E-mail: mathilde.neugnot@gmail.com

E. KINNE, P. GARCIA \& C. NOGUES. Rehabilitation Needs of Female Veterans with Post-Traumatic Stress Disorder and Mild Traumatic Brain Injury.

Objective: The number of women in military service and deployed in combat zones has risen dramatically in the past decades. With this increase there comes a demand for services for female veterans. This poster's purpose is to evaluate the gender differences between male and female veterans with PTSD and mTBI, and how these differences contribute to a need for specialized care for female veterans.
Participants and Methods: A literature review was conducted to gather research surrounding veteran gender differences for trauma, mental disorders, symptoms, current female veteran care, and physical conditions that contribute to a need for specialized care. While there is a lack of research in this area, research is currently growing as veteran gender differences become more defined. Databases include: ProQuest Central, ProQuest Natural Science Collection, PsychINFO, PsychARTICLES, PubMed, ERIC, and PILOTS: Published International Literature on Traumatic Stress. A total of 22 articles were reviewed for possible inclusion. Exclusion criteria included articles not published within the last ten years, articles not related to veteran PTSD/mTBI care, and non-peer reviewed articles.

Results: Much of the literature has found that women are significantly more susceptible to PTSD than male veterans. Women are not only more likely to develop PTSD, but also more likely to experience military sexual trauma, depression, substance abuse, and physical conditions subsequent to trauma.

Conclusions: The literature shows that there is currently a greater demand for mental health services for female veterans. However, despite growing research to support an increased demand for specialized female veteran care, many female veterans are being underdiagnosed and undertreated.

Correspondence: Erica Kinne, M.S., Carlos Albizu University, 2173 NW 99th Ave, Miami, FL 33172. E-mail: ekinne4000@gmail.com

S. CHOWDHRY, H.M. LINDSEY \& G.T. VOELBEL. Adults with Severe Traumatic Brain Injury Show Greater Cognitive Improvements than Adults with Mild/Moderate Traumatic Brain Injury on Computerized Cognitive Training: A Pilot Study.

Objective: Processing speed, working memory, and attention deficits are some of the most common cognitive deficits associated with traumatic brain injury (TBI). Cognitive rehabilitation has been proven to be an effective method to remediate cognitive deficits in adults with TBI; however, little research has considered the effects of TBI severity on such interventions. The present study examines changes in cognition following a computerized training program in adults with mild/moderate TBI and adults with severe TBI.

Participants and Methods: Fourteen participants (ages 26-68; $\mathrm{M}=45$, $\mathrm{SD}=13.95$ ) with a history of TBI were separated into a mild/moderate group $(n=6)$ and a severe group $(n=8)$. Over a period of 12 weeks, participants underwent 40 hours of computerized cognitive training using Brain Fitness Program (POSIT Science) software. Baseline and follow-up neuropsychological assessments were administered to all participants.

Results: Neuropsychological scores were submitted to a $2 \times 2$ mixed design ANOVA, in which severity group (mild/moderate versus severe) served as the between-subjects variable and time (baseline versus follow-up) served as the within-subjects variable. Results indicate significant group differences on various cognitive measures including memory composite, $\mathrm{F}(1,11)=7.06, \mathrm{p}=.022$; visual memory, $\mathrm{F}(1,12)=8.13$, $\mathrm{p}=.015$; working memory, $\mathrm{F}(1,12)=4.93$, $\mathrm{p}=.046$; continuous performance task, $\mathrm{F}(1,12)=5.24, \mathrm{p}=.041$; and shifting attention test, $\mathrm{F}(1,12)=8.85, \mathrm{p}=.012$.

Conclusions: Our findings suggest that cognitive rehabilitation techniques may be especially beneficial to adults with severe TBI. Following administration of a computerized training program, adults with severe TBI showed significant improvements in cognitive performance relative to adults with mild/moderate TBI.

Correspondence: Saba Chowdhry, New York University, 250 Mercer Street, C610, New York, NY 10012. E-mail: sac589@nyu.edu

K.A. KERNS, S.J. MACOUN, J. MACSWEEN, J. PEI \& M. HUTCHISON. Efficacy of the "Caribbean Quest" Computer Intervention in Children with ASD and FASD.

Objective: This study investigated the efficacy of the Caribbean Quest (CQ) computer game intervention designed to improve attention and 
working memory. The CQ was delivered by trained educational assistants (EAs) within schools to children with either Fetal Alcohol Spectrum Disorder (FASD) or Autism Spectrum Disorders (ASD).

Participants and Methods: We investigated whether children with either FASD or ASD could benefit from a process specific intervention approach using the CQ. The CQ intervention activities are designed to target attention and working memory skills with hierarchically designed games. Children advance through self-adjusting games based on performance accuracy so that children work to their cognitive capacity without experiencing long periods of failure. Within the school day, trained EAs delivered the intervention to 17 children with ASD or FASD (ages 6-13) providing support with metacognitive strategies and encouragement. Participants completed a minimum of 12 weeks of intervention training twice per week for 30 minutes.

Results: Analyses provide support for significant improvement on several non-trained measures of working memory, including auditory and visual tasks. Children also made significant improvements in performance on a curriculum based reading fluency measure. Exit interview with EAs revealed children enjoyed the games and transferred strategies learned in game play into the classroom providing evidence of far effects (generalization).

Conclusions: Results provide preliminary support for the use of the CQ as part of a cognitive intervention program for children with ASD and FASD. Some children showed greater benefit from the intervention, possibly related to pre-existing profiles. Overall, the study suggests that children with significant neurodevelopmental conditions can benefit from cognitive training interventions.

Correspondence: Kimberly A. Kerns, PhD, Psychology, University of Victoria, PO Box 3050, Stn CSC, Victoria, BC V8W 3P5, Canada. E-mail:kkerns@uvic.ca

K.N. MORGAN, M. SHEAN, K. SHADA \& B. SCOTT. Can Lumosity Assessment Tools Determine Functional Change? An Independent Investigation of Reliability.

Objective: Developers recently presented reliability data of Lumosity's Brain Performance Test, a battery of cognitive tasks allowing users to monitor functional change over time ( $\mathrm{n}=985$, BPT $\mathrm{r}=0.89$; Sternberg, Hardy, Katz, Ballard, \& Scanlon, 2012). Participants included Lumosity subscribers who were free to train with the program between the 10-week pre- and post-test period. Researchers collected reliability data for six of the BPT tests. The present study explored test-retest reliability for these and three additional BPT tests in a sample of neurologically healthy adults who did not train between the pre- and post-test sessions. Participants and Methods: A total of 25 neurologically healthy adults between the ages of 18 and 35 completed the battery on two occasions, with one to four weeks between pre- and post-tests. Participants were recruited at a private college in the Pacific Northwest. The BPT battery included Wordy Equations, Memory Span, Reverse Memory Span, Digit-Symbol Coding, Grammatical Reasoning, Divided Visual Attention, Go/No-Go, and Trail-making A and B.

Results: Pearson product-moment correlations were calculated between pre- and post-test scores on each measure. Results reflected large significant correlations between scores at pre- and post-test on WE, SM accuracy, DS Coding accuracy, DS Coding reaction time, and GR reaction time $(p<0.01)$. SM Span, RM Span, RM accuracy, G/N-G, and TMA speed demonstrated moderate significant correlations $(\mathrm{p}<0.05)$. Relationships between scores on DVA accuracy, GR accuracy, and TMB speed at pre- and post-test were insignificant.

Conclusions: Although the majority of relationships between the BPT measures at pre- and post-test were significant, only a portion of the tasks demonstrated large correlation coefficients, which is desirable for measuring clinically significant improvement. Based on initial data for this pilot sample, not all BPT measures were adequately reliable. A larger sample size is necessary to better predict reliability of BPT measures.
Correspondence: Kelly N. Morgan, MS, Psychology, Pacific University, School of Professional Psychology, 5518 N. Haight Ave., Portland, OR 97217. E-mail: morg5891@pacificu.edu

K.R. THOMAS, C.Z. BURTON, L. VELLA, R.K. HEATON, D.V.JESTE \& E.W. TWAMLEY. Compensatory Cognitive Training for People with Severe Mental Illness.

Objective: Cognitive impairment is associated with poor everyday functioning and disability in people with severe mental illness (SMI). We aimed to determine if a 12 -week Compensatory Cognitive Training (CCT) intervention improved cognition. The CCT intervention included compensatory cognitive strategy training in four domains that are frequently impaired in people with SMI: prospective memory, attention, learning, and executive functioning.

Participants and Methods: Participants included 153 unemployed outpatients with SMI (95 with severe mood disorders and 58 with schizophrenia/schizoaffective disorder) who stated a goal of obtaining work. All participants received supported employment and were randomized to also receive CCT (delivered by the employment specialist) or additional supported employment sessions for the first 12 weeks of the supported employment program. Participants were administered cognitive measures at baseline and 3-, 6-, and 12-month follow-ups. Cognitive composite scores, based on the MATRICS Consensus Cognitive Battery (MCCB), in the domains of processing speed, working memory, learning, and executive functioning, were examined as outcomes. Results: Hierarchical linear modeling using all available data found that CCT was differentially associated with greater improvement in learning performance over 12 months $[\beta=0.10, t(84.77)=2.08, p=0.041]$. This differential trajectory was particularly prominent among the participants with severe mood disorders $[\beta=0.17, t(60.67)=2.92, p=0.005]$. The treatment groups did not differ on other cognitive composite outcomes.

Conclusions: CCT has the potential to improve learning in outpatients with SMI, even when compared to a rigorous contact-control condition. Future work will examine whether the improvement in learning is associated with improved work outcomes.

Correspondence: Kelsey R. Thomas, MS, Clinical and Health Psychology, University of Florida, 1225 Center Drive, PO Box 100165, Gainesville, FL 32608. E-mail: krthomas@phhp.ufl.edu

K.R. THOMAS, M. MARSISKE, J. JONES, D. STEINDLER, B.A. REYNOLDS \& D. BOWERS. Effects of Wii, Aerobic Exercise and Cognitive Interventions on Older Adults' Self-Evaluation of Intellectual Aging.

Objective: This study investigated whether elder's beliefs about intellectual aging were improved following a 16-week intervention.

Participants and Methods: Fifty-one healthy older adults (mean age $=78.56$ years, mean education $=16.96$ years) received computer-based visual speed-of-processing training (Insight) plus either 16-weeks of aerobic exercise $(n=19)$, Wii Sports $(n=17)$, or Insight training alone $(\mathrm{n}=15)$. Participants were given the Personality in Intellectual Aging Contexts (PIC) questionnaire at baseline, posttest, and 3-month follow-up. The PIC has six subscales: locus of control (internal, external, and chance), anxiety, achievement motivation, and attitude about intellectual aging.

Results: Repeated measures ANOVAs, controlling for group differences in gender, revealed significant intervention group by occasion effects for the Attitude $\left[\mathrm{F}(4,92)=3.78\right.$, partial $\left.\eta^{2}=0.139, \mathrm{p}<0.01\right]$ and Internal Control subscales $\left[F(4,92)=2.69\right.$, partial $\left.\eta^{2}=0.103, p<.05\right]$. For the Attitude scale, all groups showed improvement at posttest, but the Wii group had greater continued gain through the 3-month follow-up than the aerobic group, which returned to their baseline level. For the Internal Control scale, a different pattern emerged such that the aerobic group showed improvement during the intervention phase and maintained this improvement through follow-up. The Wii group had an initial 
breakdown in internal control during the intervention, followed by an improvement from posttest to follow-up.

Conclusions: During the post-intervention phase, the Wii group demonstrated growth for both the Attitude and Internal Control scales. These findings suggest that the novel and engaging $W$ ii intervention, traditionally perceived as youth-focused, can breakdown stereotypes and improve morale about one's own cognitive aging. Simultaneously, the Wii intervention seemed to highlight elders' control limitations, though this breakdown was temporary and participants ultimately recovered. Supported by McKnight Research Foundation award (DB,MM) Correspondence: Kelsey R. Thomas, MS, Clinical and Health Psychology, University of Florida, 1225 Center Drive, PO Box 100165, Gainesville, FL 32608.E-mail:krthomas@phhp.ufl.edu

B. SHARMA, J.C. TOMASZCZYK, D.R. DAWSON, G. TURNER, B. COLELLA \& R. GREEN. Feasibility and Preliminary Efficacy of an Online Cognitive Environmental Enrichment Intervention for Patients with Moderate-to-Severe Traumatic Brain Injury.

Objective: Recent research has documented progressive cognitive and neural decline in the chronic stage of moderate-to-severe traumatic brain injury (TBI). Offsetting neurodegeneration offers a new target for cognitive neurorehabilitation. Cognitive environmental enrichment (C-EE; continuously novel, challenging, and engaging cognitive stimulation) delivered early post-TBI has been negatively correlated with bilateral hippocampal atrophy from 5 - to 28-months post-injury. Some online "brain games" offer intensive cognitive stimulation that meet the criteria for C-EE.

Participants and Methods: Case-series of 11 moderate-to-severe TBI patients. The feasibility of administering online "brain games" (12 weeks, 60 minutes/day, 5 days/week) was examined.

Results: Weekly adherence rates were modest (range: 7.7-90.1\%), with most cases clustering around $50 \%$. Good acceptability was evidenced by retention of $7 / 11$ patients, with attrition attributable to non-intervention factors in 3/4 cases (i.e., return-to-school, loss of Internet, return-towork). Data available for six patients showed similarly good acceptability, with, for example, $6 / 6$ patients endorsing post-training increases in Sense of Accomplishment and Confidence, and 5/6 endorsing reductions in Boredom. Neuropsychological findings (available for 8 patients) supported feasibility, with pre-post changes in the expected directions: 5/8 achieved gains of $\geq 0.5 \mathrm{Z}$-scores in at least one cognitive domain.

Conclusions: Online, self-administered cognitive training in an intensive 12 -week program showed adequate feasibility (with good acceptability, modest adherence and expected neuropsychological findings) in a series of moderate-severe TBI patients. Protocol modifications (e.g., provision of daily reminders to participate in training) may increase adherence. Our findings support the feasibility of online cognitive training. Studies on the efficacy of daily on-line brain-games in offsetting neurodegeneration and improving cognitive outcomes are warranted. Correspondence: Bhanu Sharma, MSc, Toronto Rehab, 550 University Avenue, Toronto, ON M5G2A2, Canada.E-mail:bhanu.sharma@mail. utoronto.ca

M. SÉGUIN, A. LAHAIE \& M.H. BEAUCHAMP. Ready! Set? let's Train! : Efficacy of an intensive attention training program and its association with executive functioning after childhood traumatic brain injury.

Objective: Executive dysfunctions following traumatic brain injury (TBI) frequently complicate return to activities of daily living and negatively influence socio-academic reintegration (Galvin \& Mandalis, 2009). Yet, clinicians in rehabilitation settings have limited access to cognitive remediation protocols for which efficacy has been demonstrated (Slomine et al., 2009). The aim of this study was to evaluate whether an intensive attention process training program « Ready! Set? Let's Train!» (Séguin, Lahaie \& Beauchamp, 2012) is associated with improvements in executive functioning.
Participants and Methods: Over five weeks, 17 participants $(\mathrm{M}=$ 14.70; $\mathrm{SD}=2.17$ years, 11 males) who sustained TBI resulting in attention deficits received either an attention process training intervention (n $=8$, experimental group) or homework assistance ( $\mathrm{n}=9$, control group). Results: Following the intervention, the experimental group improved on executive function tasks including working memory $(\mathrm{t}(9.84)=2.56$, $\mathrm{p}=0.03)$, inhibition $(\mathrm{t}(15)=2.20, \mathrm{p}=0.04)$, mental flexibility $(\mathrm{t}(15)=$ $2.08, \mathrm{p}=0.05)$ and visuoconstructive planning $(\mathrm{t}(15)=-2.13, \mathrm{p}=0.05)$ Conclusions: The "Ready, Set? Let's Train!" attention remediation program appears to be associated with improvements in executive functioning. Given their role in activities of daily living, academics and social skills (Anderson \& al., 2002), improvements in executive functions are likely to positively influence prognosis and quality of life in youth with TBI who suffer from attentional problems.

Correspondence: Marilou Séguin, PhD, neuropsychology, University of Montréal, 215 appt 4 rue Pelletier, Boisbriand, QC j7g 1g5, Canada. E-mail:marilou.seguin@hotmail.com

J. KANG, K. FREDRICKSON, J. MOORER, S. THIELKE \& E.H. TRITTSCHUH. Memory Skills for Older Adults with PTSD: Update on a Clinical Demonstration Project for Veterans.

Objective: Older adult Veterans with PTSD are at a higher risk for dementia than those without (Yaffe, et al, 2010) and having PTSD often is associated with cognitive symptoms. Early identification and monitoring of Veterans with PTSD and cognitive concerns allows for treatment and other intervention that may improve quality of life. The Memory Skills for Older Adults with PTSD (MSOAP) protocol is an eight-session psychoeducational group therapy experience developed at the VA Puget Sound Health Care System, began in 2011, and was first reported at INS in 2012.

Participants and Methods: Veterans are identified through Providers. Inclusion criteria include: age $>50$, diagnosis of PTSD, and subjective complaints and/or mild objective cognitive decline. Methods consisted of 8 weekly hour long training group sessions which utilized a manual that was adapted to meet the needs of these Veterans. Pre- and post-measurements aimed at identifying quality of life variables, symptom severity, and activities of daily function.

Results: By 2015, over 60 Veterans will have participated in the group. The manual has been refined to accommodate Group Leaders from a variety of disciplines. In the 2012 pilot study, analyses of the QA/ QI measures indicated significant improvement in Veterans' sense of self-efficacy as well as ratings of improvement in quality of life. In addition, a significant reduction in the number of depressive symptoms was found. No significant effect on the report of PTSD-specific symptoms was established. The new data continue to support these findings and a model for exportation to other sites will be presented.

Conclusions: This Memory Skills group was associated with post-group report of improved self-efficacy and quality of life, and with reduced endorsement of depressive symptoms. Further directions might address the potential mechanisms by which this group might provide benefit and prospective tracking of participants could provide richer outcome data and whether continuity of care was increased.

Correspondence: Emily H. Trittschuh, PhD, Psychiatry/GRECC, UW/ VAPSHCS, 1660 S. Columbian Way, S-182-GRECC, Seattle, WA 98108. E-mail: etritt@uw.edu

D. MECHANIC-HAMILTON, F. GREENFIELD, S. NEGASH, S. REID, S.C. SELIGMAN, M. LIVNEY, P.J. MOBERG \& S. ARNOLD. The Cognitive Fitness Program: A Novel and Comprehensive Approach to Successful Cognitive Aging.

Objective: The Cognitive Fitness Program (CogFit) is a comprehensive clinical intervention designed to put common recommendations regarding compensatory strategies and wellness behaviors into action. This study aimed to evaluate the impact of CogFit on cognition, emotional functioning, and behavior among older adults who completed the program. 
Participants and Methods: Seventy subjects with no cognitive complaints, subjective cognitive complaints (SCI), or MCI completed CogFit. $\mathrm{CogFit}$ is an eight-week group intervention in which participants meet two times per week for three hours. Participants learned compensatory strategies for memory and organizational difficulties, engaged in computerized brain training, and learned how to improve wellness behaviors associated with better cognitive health. Pre and post-CogFit, participants completed measures of neuropsychological functioning and wellness behaviors, self and clinician reports of emotional functioning, and self and partner reports of daily functioning.

Results: There was significant improvement in memory measures in the no cognitive complaint and SCI group (repeated-measures t-test). Selfand clinician-report of symptoms of depression and anxiety decreased in the MCI group $(p<0.05)$. Additionally, care partners reported fewer symptoms of everyday memory failings in the MCI group $(p=0.05)$. Participants reported an increase in their use of tools acquired from CogFit.

Conclusions: $\operatorname{Cog}$ Fit is a comprehensive clinical intervention to maintain or increase pro-successful aging behaviors in older adults. Programs like CogFit can serve to support positive and sustained behavior change. Future directions include examination of which populations are best served with this type of intervention and sustainability of effect. Correspondence: Dawn Mechanic-Hamilton, PhD, Department of Psychiatry, University of Pennsylvania, 1012 Fitzwater Street, Philadelphia,PA 19147.E-mail: dawn.mechanic@uphs.upenn.edu

L.M. ERCOLI, K. VAN DYK, L. PETERSEN, S.A. CASTELLON, B. KAHN-MILlS, P.A. CERNIN, L. EMBREE \& P.A. GANZ. Association of baseline cognition and change in verbal memory among breast cancer survivors receiving a cognitive rehabilitation intervention.

Objective: Some long-term breast cancer survivors (BCS) demonstrate objective cognitive difficulties. Cognitive interventions may improve memory in BCS (Ercoli et al. 2014). We examined whether baseline cognition predicts memory change following a cognitive rehabilitation intervention for BCS with cognitive complaints.

Participants and Methods: Twenty-eight female BCS completed a cognitive training intervention focusing on executive, attention and memory functions. Participants underwent cognitive testing at baseline (T1), immediately, (T2) and 2-months (T3) post-intervention. Changes in the Rey Auditory Verbal Learning Test from T1 to T2 and, T1 to T3, were the outcomes. To reduce the number of comparisons, we chose one test of attention (continuous performance test) and one executive measure (shifting-attention test), as these are areas of difficulty in BCS. We examined baseline correlations between concentration and shifting-attention and memory change. Then, with age as a covariate, both variables were entered into multiple regression equations predicting memory change immediately and 2-months post-intervention.

Results: Only baseline concentration significantly correlated with memory change at T2 and T3 $(r=.37 ; \mathrm{p}=.046 ; \mathrm{r}=.41, \mathrm{p}=.03$, respectively). In multiple regression, age, concentration, and shifting-attention significantly predicted memory change at T3 (Rsquare $=.30 ; p=.03$ ). Partial correlation coefficients were negative for age, attention-set-shifting, and positive for concentration, but not significant ( $p$-value range .10 to .06). Conclusions: Those who are younger, have better sustained attention, and worse attention-shifting prior to the intervention showed more memory improvement. We suggest that those with room for improvement in executive function and those with better attention may maximally benefit from the intervention.

Correspondence: Linda M. Ercoli, PhD, Psychiatry, UCLA, 760 Westwood Plaza, rm 38-239, Los Angeles, CA 90095.E-mail: lercoli@ mednet.ucla.edu
J. JONES, J. HELPHREY, K.R. THOMAS, M. MARSISKE \& D. BOWERS. Influence of Everyday Walking on Cognition: Multilevel Modeling Analyses Results from the Village Interactive Training and Learning (VITAL) study.

Objective: Participation in cognitively stimulating activities and aerobic exercise has been shown to be associated with improved cognitive functioning. However, results have been conflicting regarding the utility of cognitive training and exercise as an intervention for cognitive decline. One confounding factor may be participant involvement in physical activities outside of the intervention. The current study examined the influence of walking on cognitive performance among individuals participating in a cognitive training and exercise intervention study

Participants and Methods: Participants ( $N=73$ ) included older adults participating in the Village Interactive Training and Learning study (VITAL; Supported by Mcknight Research Foundation (DB, MM). Cognitive measures included the Hopkins Verbal Learning Test-II (HVLT-II) and the Flanker Task. Participants were given pedometers to assess their amount of walking (average number of steps taken per week) outside of the intervention. Cognitive and pedometer data were assessed at 6 time periods: baseline, 5 weeks, 9 weeks, 13 weeks, 17 weeks and 28 weeks. Multilevel modeling analyses were computed to examine the influence of age, gender and walking (within-person and between-person) on cognitive performance.

Results: Between-person differences in walking showed a trend relationship toward HVLT-II delayed recall scores ( $\beta=.233$; $p=.076)$, but not Flanker Task performance. Specifically, individuals who walked more obtained better recall scores compared to individuals who walked less. Within-person differences in walking were not related to either cognitive task. The relationship between walking and cognition did not interact with intervention group or a linear-slope term.

Conclusions: Findings have implications for exercise-based interventions regarding the importance of monitoring participants' physical activity outside of the intervention.

Correspondence: Jacob Jones, MS, CHP, University of Florida, 3901 SW 20th ave apt 206, Gainesville, FL 32607. E-mail: jacob.jones126@ ufl.edu

J.A. LAFO, D. BOWERS, M. MARSISKE, D. MARRA, K.R. THOMAS, J. JONES, C.J. HASS, B.A. REYNOLDS, D. STEINDLER \& R.M. BAUER. Effects of Combined Cognitive Training and Physical Exercise on Spatial Navigation and Learning in Older Adults.

Objective: The 'C-G Arena' is a computer-based human analog of the Morris Water Maze, a paradigm used in the neuroscience literature to measure spatial navigation through the development of cognitive maps. Preclinical models have implicated the role of hippocampal mechanisms in cognitive mapping and navigation. The current study investigated whether a combined cognitive training-physical exercise intervention would improve navigational learning in older adults compared to cognitive training alone.

Participants and Methods: Participants included 32 cognitively normal older adults (mean age $=76.50$, edu=16.90) who were randomly assigned to one of three 16-week interventions. The interventions involved computer-based visual cognitive training (InSight) combined with: a) aerobic exercise $(n=12), b)$ Wii sports $(n=11)$, or $c)$ no other activity $(n=9)$. The Wii condition served as an activity control for aerobic exercise. All participants underwent baseline neuropsychological testing. The Arena was given before and after intervention. A composite Arena variable reflecting overall learning was used as the dependent variable. Data were analyzed using repeated-measures ANOVA.

Results: The intervention groups did not differ in demographics, baseline neuropsychological or Arena performance. Of the 3 interventions, only the combined Wii-cognitive training group demonstrated significant improvement in Arena performance from baseline to post-intervention testing.

Conclusions: Findings suggest that Wii-training, rather than aerobic exercise, distinctly influences spatial navigation and memory. Possible 
mechanisms could include a) Wii-training increases familiarity with virtual environments and visuomotor skills specific to virtual tasks, and/or b) the novelty and "fun" associated with immersive gaming may trigger mechanisms supporting neuroplasticity and subsequent enhancement of hippocampal functioning. Future studies should include visuomotor control tasks without a memory component and examine effects of specific neuroplastic processes.

Correspondence: Jacob A. Lafo, B.A., University of Florida, 550 NE 6th Ave., Gainesville, FL 32601. E-mail: jlafo@phhp.ufl.edu

\section{K. SOL, H. BELANGER \& G. CURTISS. Somatization Predicts Self- Efficacy in mTBI, and Both Predict Post-Concussion Symptom Reporting.}

Objective: Self-efficacy (SE; belief in one's ability to accomplish a goal) is important in adjustment to disease and disability. However, somatization (SOM; experiencing psychological discomfort as physical discomfort) may adversely affect SE. These concepts have not yet been examined within the context of post concussion symptom (PCS) reporting after mild traumatic brain injury (mTBI). This study examined the roles of self-efficacy and somatization in PCS severity after mTBI. Participants and Methods: Participants with history of mTBI and PCS were recruited. PCS was measured using the Neurobehavioral Symptom Inventory (both at baseline and 6 months); SE was measured by the Self Efficacy Symptom Management Scale; and SOM was measured by the eight item version of the Health Attitude Survey. Hierarchical regression analyses examined the predictive ability of SOM on SE, after controlling for demographic variables and time since injury. Another hierarchical regression examined the influence of both SOM and SE on change in PCS over time, as well as their interaction.

Results: 158 eligible individuals completed baseline questionnaires, 139 completed all follow up questionnaires. After controlling for demographics, SOM significantly predicted SE $\left(\Delta F[5,152]=11.71, p<.001 ; \Delta R^{2}=\right.$ $.07)$. In the second regression, SE and SOM were both centered. SE and SOM were significantly predictive of change in $\operatorname{PCS}(\Delta F[6,132]=14.57$, $\left.p<.001, \Delta R^{2}=.16\right)$, after controlling for demographics. However, the interaction term (SOMxSE) was not significant $(p=.22)$.

Conclusions: These results suggest that somatization impacts self-efficacy. In turn, self-efficacy and somatization influence PCS over time. As such, screening for self-efficacy and somatization is important within the context of ongoing PCS following mTBI.

Correspondence: Ketlyne Sol, M.A., Mental Health and Behavioral Sciences, James A. Haley Veterans' Hospital, 13000 Bruce B. Downs Blvd, Tampa, FL 33612. E-mail: ketlyne.sol@va.gov

T. NOVAKOVIC-AGOPIAN, A. CHEN, G. ABRAMS, M.R. MADORE, F. LOYA, G. CARLIN \& K. LAU. Goal-Oriented Attentional SelfRegulation Training in Veterans with Chronic TBI - Long Term Outcomes.

Objective: Deficits in executive control functions are some of the most disabling consequences of brain injury. Goal-Oriented Attentional Self-Regulation (GOALS) training was designed to target deficits in executive control processes with attention regulation and problem solving training applied to participant-defined goals. In an initial study individuals with chronic brain injury significantly improved post GOALS, but not control training, on measures of attention/executive function, functional task performance, and goal-directed control over neural processing on fMRI (Novakovic-Agopian, Chen et al 2011; Chen, Novakovic-Agopian et al 2011). The objective of ongoing study is to assess long term effectiveness of GOALS training in Veterans with chronic TBI. Participants and Methods: 19 Veterans with chronic ( $6+$ months) TBI and mild-moderate executive dysfunction completed structured interview, neuropsychological and functional assessments, and self-report measures of emotional regulation and daily functioning 6 or more months post completion of GOALS training.

Results: Relative to their baseline performance, 6 or more months post training, participants showed significant improvements on measures of attention/executive function, complex functional task performance, and on emotional regulation and daily functioning self- report measures. Furthermore, majority reported incorporating trained strategies into their daily life.

Conclusions: GOALS training may be promising in Veterans with chronic TBI. Improving cognitive control functioning may also improve functioning in other domains such as functional performance and emotional regulation. We will discuss the challenges and importance of: a) using participant-defined goals applied to relevant training; and b) assessing change in functioning at different levels, including ecologically valid assessment measures.

Correspondence: Tatjana Novakovic-Agopian, Ph.D, Neurology, UCSF, SFVA, 661 Myra way, San Francisco, CA 94127.E-mail: tna@cns-site. com

J.E. FAIR, S.W. LIEBEL, D. BJORNN \& M.J. LARSON. Errorless and Errorful Learning in Moderate-to-Severe TBI: The Impact of Neuropsychological Functioning and Mood on Performance.

Objective: Common cognitive deficits after moderate-to-severe (MS) TBI include difficulties with attention, memory, and executive functioning. The emotional sequelae of MS TBI are also frequent and debilitating. Errorless learning (EL) is a technique used to improve retention of information in some clinical groups, with mixed support in TBI samples. We examined the impact of neuropsychological (NP) performance and mood ratings on the accuracy of MS TBI survivors completing EL and errorful (EF) memory tasks.

Participants and Methods: Individuals with MS TBI $(n=28)$ and matched controls $(\mathrm{n}=28)$ completed a word-learning task with counterbalanced EL and EF learning conditions. EL included presenting a target word and having participants repeat it. EF presented the first two letters of the target word as cues and required participants to guess the word. Mood and NP data were collected and analyzed using linear regression.

Results: Four standardized memory and executive functioning variables were used as predictors. These were significantly predictive of accuracy in the EL and EF conditions, as well as in the TBI and control groups (all p's <.05). However, NP variables were more significantly predictive of EL than EF learning (e.g., $p=.001$ vs. $p=.023$ in the TBI group; $\mathrm{p}=.000$ vs $\mathrm{p}=.012$ for controls). Three mood variables reflecting depression, anxiety, and negative affect were also used as predictors in a separate regression analysis, but these were not significantly predictive of accuracy in either condition or group.

Conclusions: Results indicate a significant predictive relationship between NP functioning and accuracy on both EL and EF learning, with higher NP functioning better predicting EL than EF. This suggests that the benefits of EL are partially dependent on level of NP functioning, a finding consistent with other research that suggests better cognition results in greater EL benefits.

Correspondence: Joseph E. Fair, M.S., Psychology, Brigham Young University, 1190 North 900 East, Provo, UT 84604.E-mail: jfairmail@ gmail.com

J. SANDRY, N.D. CHIARAVALLOTI, J. DELUCA \& J.F. SUMOWSKI. What underlies the link between cognitive reserve and long-term memory impairment? Exploratory insight from two memory impaired populations.

Objective: Neurological diseases often have devastating negative consequences on a person's ability to learn and remember information. Interestingly, there is substantial variability across individuals with similar neurological insult; some may experience impaired long-term memory while others may not. The theory of cognitive reserve has explained some of this variability by suggesting that individuals who have participated in early-life intellectually enriching activities are better able to withstand long-term memory impairment despite neurological injury. To date, there has not been much research directed at understanding the cognitive mechanisms that link cognitive reserve with long-term 
memory. Using a translational approach, a model was derived that implicates working memory capacity as a mediating cognitive mechanism in this relationship. That is, individual differences in working memory capacity may help to explain how/why cognitive reserve protects against long-term memory impairment.

Participants and Methods: Patients underwent a series of neuropsychological tests and composite scores were computed to estimate cognitive reserve, working memory capacity, and long-term memory. We highlight recent evidence supporting this model in multiple sclerosis (N=70; Sandry \& Sumowski, 2014, JINS) and present new data supporting this model in traumatic brain injury $(\mathrm{N}=50)$.

Results: The presence of mediation was tested using linear regression. Working memory mediated the relationship between cognitive reserve and long-term memory in both samples (Sobel Z, p's<.05).

Conclusions: Working memory may be one underlying cognitive mechanism supporting the relationship between cognitive reserve and long-term memory impairment in neurological populations. Working memory may be an important individual differences variable to include in long-term memory rehabilitation trials. We also offer a theoretical explanation for why treatment strategies that target working memory may lead to related improvements in long-term memory.

Correspondence: Joshua Sandry, PhD, Neuropsychology\& Neuroscience, Kessler Foundation, 443 Second Ave, Floor 2, Lyndhurst, NJ 07071. E-mail: jsandry@kesslerfoundation.org

\section{N. DEYOUNG, N. HURLESS \& B. SHENAL. Should it be done? Establishing the equivalence of a telehealth versus in-person memory workshop.}

Objective: The provision of neuropsychological services remotely over telemental health can help eliminate several barriers that often prevent patients from being able to access care. These practices need to be validated for ease of implementation, appropriateness, and patient acceptance. This project was a pilot study designed to evaluate the effectiveness and patient acceptance of telehealth cognitive rehabilitation program.

Participants and Methods: 43 veterans identified as having memory, attention, and problem solving difficulties were referred to an hour long memory workshop which covered skills for improving memory functioning. The group was offered both in-person and by telehealth to an offsite location. Pre and post ratings assessed memory knowledge and satisfaction with the group.

Results: Twenty-four veterans attended the in-person intervention while 19 opted to attend the Telehealth group. Overall group ratings indicated that the veterans found the content helpful for improving memory difficulties $(\mathrm{M}(\mathrm{SD})=4.16(0.86)$ on a $0-5$ scale $)$. Pre-post ratings showed increased knowledge and memory $(\mathrm{t}=4.04, \mathrm{p}=0.001)$ and better understanding of memory strategies $(t=5.22, p<0.001)$. There were no differences observed between telehealth and onsite groups. Veterans in the telehealth group rated the option favorably, and 91 percent of them indicated they preferred to attend telehealth groups in the future. Conclusions: Veterans rated the memory workshop favorably, and it had an immediate positive effect on perceived memory knowledge and strategies for improving memory functioning. There were no observed differences between the onsite and telehealth groups. The telehealth group increased access of care to veterans in rural areas and did not hinder its effectiveness.

Correspondence: Nathaniel DeYoung, Ph.D., MHSL, Salem Veterans Medical Center, 1970 Roanoke Boulevard, Salem, VA 24153. E-mail: nathaniel.deyoung@va.gov

N. CURCIO, J. FISZDON, P. LYSAKER, B. TWAMLEY, K. WILKINS, P. KIRWIN, D. DEVANAND \& J. CHOI. Cognitive Behavioral Therapy to Enhance Cognitive Rehabilitation Efficacy in Alzheimer's Disease.

Objective: Cognitive rehabilitation (CR) programs for dementing pathology have been shown to be moderately efficacious. However, as stand-alone treatments, they can be difficult for this group of patients, as they serve as a constant reminder of cognitive deficits, which can lead to low self-esteem and a sense of hopelessness. Because of these limitations, add-on therapies directly targeting hopelessness and self-esteem may enhance the efficacy of CR in AD.

Participants and Methods: 67 outpatients with early to moderate AD were randomly assigned to 4 months of a computer-based memory training (MT-only), or a cognitive behavioral therapy regimen in conjunction with the same MT (CBT-MT) designed to target automatic distorted schemes of hopelessness and perceptions of poor self-competency. Assessments of cognition, quality of life, neuropsychiatric symptoms, treatment adherence, depression, hopelessness, treatment motivation, and perceptions of self-efficacy were conducted at intake and immediately following treatment conclusion.

Results: Patients in CBT-MT reported less overall depressive symptoms at post treatment and reported greater willingness and efficacy to tackle the challenging exercises in the CR program. Subsequently, the CBT-MT group had less treatment attrition in CR compared to the MT-only group. Of note, for the sample as a whole, greater perceptions of self-efficacy for the training at baseline significantly predicted memory outcome, accounting for $53 \%$ of the variance followed by baseline mental flexibility, which explained another 19\%. Depression severity and neuropsychiatric symptoms did not contribute to memory outcome beyond what was found in the model.

Conclusions: Findings suggest that enhancing CR with add-on interventions directly targeting self-efficacy may increase their effectiveness. These add-on treatments may be effective by directly increasing elements of control, self-efficacy, and intrinsic motivation for treatment, leading to both psychological and neuropsychological benefits. Correspondence: Nicholas Curcio, Columbia University Medical Center, 30ManorRoad, Livingston, NJ 07039.E-mail:nec2138@columbia.edu

Y. BOGDANOVA, S. KARK, V.T. HO, M.K. YEE \& A. PASCUALLEONE. TMS Improves Cognitive and Neuropsychiatric Symptoms in Veterans with Blast TBI.

Objective: Traumatic brain injury (TBI) presents a significant concern for military personnel and veterans. The most common TBI-associated problems are cognitive deficits (executive functioning and learning) and neuropsychiatric deficits (sleep disturbance, mood and PTSD). This pilot study investigated the efficacy of a novel neuromodulation treatment, repetitive transcranial magnetic stimulation (rTMS) in TBI veterans with persistent cognitive deficits (executive function) and neuropsychiatric symptoms (PTSD, depression, and sleep disturbance).

Participants and Methods: In this double-blind randomized-controlled trial, eight OEF/OIF veterans with history of mild blast-induced TBI were divided using a blocked randomization procedure into two groups: active rTMS treatment or control (sham) rTMS. All participants received rTMS treatment (active or sham) for one week, and were administered standardized neuropsychological and neuropsychiatric measures pre- and post intervention. Sleep was assessed using a self-report questionnaire (PSQI) and actigraphy.

Results: The active rTMS treatment group reported significant improvements in executive function (DEX) and neurobehavioral symptoms (NSI). No changes on these measures were seen in the sham rTMS control group. We also evaluated the effect of rTMS on the neuropsychiatric symptoms. Treatment with active rTMS resulted in a significant reduction in PTSD, depression, and sleep problems (PSQI); while the sham control group showed no change.

Conclusions: Our results indicate that active rTMS treatment can improve cognitive function and reduce the symptoms of PTSD, depression and sleep disturbance in veterans with blast-induced TBI and associated comorbidities. These findings are the first to provide evidence that oneweek treatment of chronic mild TBI and associated neuropsychiatric symptoms with rTMS is safe and effective, as compared to sham rTMS. Further assessment of this intervention is warranted. 
Correspondence: Yelena Bogdanova, PhD, Psychiatry, Boston University, Psychology Research (151-A), 150 South Huntington Avenue, Boston, MA02130.E-mail:bogdanov@bu.edu

S.M. JURICK, M. SANDERSON-CIMINO, L. TRENOVA, B. BOYD, D.M. SCHIEHSER, L. DELANO-WOOD, R. AUPPERLE, E.W. TWAMLEY, C. RODGERS \& A.J. JAK. Mental Health Treatment Reduces Post-concussive Symptoms and Symptom Overreporting in Iraq and Afghanistan Veterans.

Objective: Symptom overreporting is a prominent concern when conducting post-concussive symptom assessments in service members with a history of mild to moderate traumatic brain injury (mTBI). The Neurobehavioral Symptom Inventory is a measure of post-concussive symptom reporting and includes an embedded validity scale (Validity-10) and four symptom subscales (vestibular, somatic, cognitive, emotional). We sought to determine the degree to which treating co-morbid mental health conditions could alter post-concussive symptom reporting and validity in Veterans with co-morbid mTBI and post-traumatic stress disorder (PTSD).

Participants and Methods: 30 Operation Enduring Freedom/Operation Iraqi Freedom (OEF/OIF) Veterans with PTSD, a history of mild to moderate TBI, and current cognitive complaints received a comprehensive assessment before and after 12 sessions of Cognitive Processing Therapy (CPT) or enhanced CPT including cognitive rehabilitation strategies.

Results: Repeated-measures ANOVAs revealed that the Validity-10 score significantly declined from pre- to post-treatment $(\mathrm{F}=6.69$, $\mathrm{p}=.015)$. Total NSI scores decreased $(\mathrm{F}=13.13, \mathrm{p}=.001)$ and specifically, the vestibular $(\mathrm{F}=4.88)$, somatic $(\mathrm{F}=7.98)$, and emotional $(\mathrm{F}=20.42)$ subscales significantly declined over the course of treatment (all p's<.05). NSI and Validity-10 scores declined significantly from mid-therapy to post-treatment (all p's<.05), but not from pre-treatment to mid-therapy, suggesting that the full course of PTSD treatment is necessary to reduce neurobehavioral symptom overreporting and achieve valid svmptom reduction.

Conclusions: Results demonstrate that post-concussive symptom response bias and vestibular, somatic, and emotional symptoms are responsive to mental health treatment. CPT and enhanced CPT may reduce symptom overerporting to allow a more valid assessment of $\mathrm{OEF} /$ OIF Veterans post-concussive symptoms. A full course of structured PTSD therapy was of particular importance to realize the full extent of improvement in neurobehavioral symptoms.

Correspondence: Sarah M. Jurick, B.A., Psychiatry, University of California San Diego, 9500 Gilman Drive, Mail Code 0603, La Jolla, CA 92037-0603.E-mail:smjurick@gmail.com

L.B. ZAHODNE, O. MEYER, E. CHOI, M. THOMAS, S.L. WILLIS \&. J. PARISI. External Control Beliefs Explain Racial Disparities in Reasoning Training Gains in ACTIVE.

Objective: Racial disparities in cognitive outcomes are widely reported and may be partly explained by differences in control beliefs. African Americans report more external locus of control than non-Hispanic Whites, and external locus of control is associated with poorer health and cognition. The aims of this study were to (1) compare cognitive training gains between African American and non-Hispanic White participants in the Advanced Cognitive Training for Independent and Vital Elderly (ACTIVE) study; (2) determine whether racial differences in training gains are mediated by locus of control; and (3) identify specific aspects of locus of control accounting for results.

Participants and Methods: The sample comprised 2,062 (26\% African American) adults aged 65 and older who participated in memory, reasoning, or speed training. Latent growth curve models were used to evaluate predictors of 10 -year cognitive trajectories separately by training group. Multiple group modeling was used to examine associations between training gains and locus of control across racial groups.
Results: No racial differences in training gains were identified for memory or speed groups. Compared to non-Hispanic Whites, African Americans evidenced less improvement in reasoning performance after training and the effect was fully mediated by locus of control. African Americans reported more external locus of control, which was associated with smaller training gains. External locus of control also had a stronger negative association with training gain for African Americans.

Conclusions: African American older adults benefited less from reasoning training than non-Hispanic Whites. This disparity appears to be influenced by higher levels of external control beliefs combined with a greater negative impact of external control beliefs on training gain among African Americans.

Correspondence: Laura B. Zahodne, PhD, Columbia University, 630 West 168th Street, P \& S Box 16, New York, NY 10032. E-mail: lbz2105@columbia.edu

K.H. WALTER, A. JAK \& E.W. TWAMLEY. Effects of Compensatory Cognitive Training for Veterans with Traumatic Brain Injuries: Do Comorbid Posttraumatic Stress Disorder and Depressive Symptoms Limit Improvement?

Objective: To investigate the influence of posttraumatic stress disorder (PTSD) diagnosis and depression symptom severity on the postconcussive symptom trajectory over the course of a one-year study period.

Participants and Methods: Fifty Operation Enduring and Iraqi Free$\operatorname{dom}(\mathrm{OEF} / \mathrm{OIF})$ Veterans with a history of mild to moderate traumatic brain injury (TBI) who were unemployed, seeking work, and who had neuropsychological impairment. Of all participants, $74 \%$ met diagnostic criteria for PTSD. A randomized controlled trial comparing Veterans who received supported employment combined with compensatory cognitive training to those who received supported employment only. All participants received supported employment and half of the sample also received Cognitive Symptom Management and Rehabilitation Therapy (CogSMART), a 12-session, manualized compensatory cognitive training intervention. Assessments were conducted at baseline, 3- (post-intervention), 6-, and 12-months. The outcome variables included the Neurobehavioral Symptom Inventory (NSI) total score and the NSI total score minus three overlapping psychiatric symptom items.

Results: Veterans with PTSD and greater depression severity endorsed significantly more severe postconcussive symptomatology at all assessment time points over the course of the study. However, the rate of CogSMART-associated improvement in postconcussive symptoms did not differ as a result of psychiatric symptomatology.

Conclusions: Study results suggest that for Veterans with a history of mild to moderate TBI, presence of comorbid PTSD or depressive symptoms should not preclude participation in cognitive rehabilitation interventions.

Correspondence: Kristen H. Walter, PhD, Research, Veterans Medical Research Foundation, 3350 La Jolla Village Drive, Building 13, 111N-1, San Diego, CA 92161. E-mail: Kristen.Walter@va.gov

\section{Cognitive Neuroscience}

\section{C.E. HARRISON \& S.A. ROGERS. Effects of Leisure on Cognition Among Older Adults.}

Objective: Studies show that pursuits of personal wellness may impact memory functioning. In the present study, authors examined the way participation in leisure activities is related to older adults' cognitive functioning.

Participants and Methods: Sixty-two older adults (M age $=77.08)$ indicated weekly participation in leisure activities and completed a comprehensive neuropsychological battery

Results: Participants engaged in 5.17 leisure activities per week (range: 0-15). Canonical correlation analyses revealed positive relationships between leisure activities and the set of neuropsychological measures that assess visuospatial functioning, $\mathrm{R}=.45, \mathrm{p}<.01$, nonverbal memory, 
$\mathrm{R}=.41, \mathrm{p}=.05$, and frontal-executive abilities, $\mathrm{R}=.41, \mathrm{p}=.05$. Subsequent bivariate analyses showed that the number of leisure activities was positively correlated with WAIS-IV Similarities, $r(49)=.43, p<$ .01 , WMS-IV Visual Reproduction I, $\mathrm{r}(49)=.38$, $\mathrm{p}<.01$, Visual Reproduction II, $\mathrm{r}(49)=.28, \mathrm{p}<.05$, Rey-O 30' Delay, $\mathrm{r}(49)=.30, \mathrm{p}$ $<.04$, and WAIS-IV Block Design, $\mathrm{r}(49)=.40, \mathrm{p}<.01$, and Picture Completion, $\mathrm{r}(49)=.36, \mathrm{p}<.02$.

Conclusions: These results suggest a significant relationship between leisure and neurocognitive functioning in later life. Leisure activities seem associated with stronger frontal-executive functions, visuospatial abilities, and nonverbal memory. There were no significant relationships between leisure and the set of variables assessing language, verbal memory, attention, or processing speed. Rather, results suggest that older adults who engage in more leisure activities each week seem to benefit from stronger visuospatial functions, nonverbal memory, and frontal-executive skills, particularly verbal abstraction. These findings suggest that leisure activities may differentially impact right relative to left hemisphere functions, as well as both prefrontal and temporal regions. They also provide support for the customization of leisure-based programs toward individual difficulties and particular cognitive syndromes. Correspondence: Cory E. Harrison, PhD, Neuropsychology, Pacific Neuroscience Medical Group, 515 W Foothill Blvd, Unit A, Monrovia, CA 91016.E-mail: cburns12@apu.edu

\section{Visuopatial Functions/Neglect/Agnosia}

J.A. RODRIGUEZ, L.N. CORREA, D.M. MOSQUERA, L. SALAZAR BEJARANO, D. LAMB, Z. SCHWARTZ, R.A. COHEN, K.M. HEILMAN \& A. FALCHOOK. Background Distraction During Vertical Character Line Bisection.

Objective: A character line is a virtual line which can be composed of a string of letters. To mark a target letter closest to the line's center, focal attention is necessary to identify the target letter, and global attention is necessary to perceive the entire line to determine the center. During line bisection, global attention may also be distributed across the page. It has been reported that if a continuous line is positioned closer to one end of the page, when normal participants attempt to bisect these lines they deviate closer to the center of the page and away from the center of the line. Since character line bisections require more focal attention than solid line bisections, character line bisections may be associated with less distraction by the page. Alternatively, since character line bisections require the allocation of focal attention, participants may have less attentional resources to allocate global attention required to perceive the entire line, and thus they may be more distracted by the page than when performing sold line bisections.

Participants and Methods: Twenty-four adults bisected vertical solid and character lines centered on the page or positioned closer to the top or bottom of the page.

Results: Solid and character lines positioned closer to the top of the page were bisected below the line's center (closer to the center of the page), while those positioned closer to the bottom of the page were bisected above the line's center (also closer to the center of the page). Distraction by the page during line bisections was greater during character line bisections than solid line bisections.

Conclusions: Compared to solid line bisections, character line bisections were associated with greater distractibility of global attention. Correspondence: Julio A. Rodriguez, PONCE SCHOOL OF MEDICINE, PO BOX 8219, Ponce 00732. E-mail: jarodriguezjr@gmail.com

D.M. MOSQUERA, J.A. RODRIGUEZ, L.N. CORREA, L. SALAZAR BEJARANO, D. LAMB, Z. SCHWARTZ, R.A. COHEN, K.M. HEILMAN \& A. FALCHOOK. The Center of Mass and the Edge of Attention.

Objective: Previous research has reported that when asked to mark the center of a line composed of two unequal segments, attention is focused closer to the center of the longer line segment - the center of mass effect. The purpose of this study was to learn if when asked to allocate focal attention to a line segment, participants may be distracted by allocation of global attention to the entire line, resulting in deviation towards the center of the line and away from the center of the longer segment.

Participants and Methods: Twenty-four healthy adults were asked to bisect lines composed of two segments of unequal length, with lines presented horizontally or vertically on different trials.

Results: In all line orientations, bisection performance deviated toward the longer line segment, thus replicating the center of mass effect. When participants were asked to bisect only the longer segment of the line, bisection performance now deviated away from the center of the longer segment and toward the center of the entire line.

Conclusions: Compared to solid line bisections, character line bisections were associated with greater distractibility of global attention.

Correspondence: Diana M. Mosquera, College of Medicine, Florida State University, 501 Blairstone Road, Apartment 2502, Tallahassee, FL32301.E-mail:dmm08@med.fsu.edu

M. DIAZ-SANTOS, B. CAO, S.A. MAURO, A. YAZDANBAKHSH, S. NEARGARDER \& A. CRONIN-GOLOMB. The Effect of Visual Cues on the Resolution of Perceptual Ambiguity in Parkinson's Disease and Normal Aging.

Objective: Parkinson's disease (PD) and normal aging have been associated with changes in basic vision and perception, including visual dependence (over-reliance on external cues to guide behavior). This raises the question of the extent to which individuals with PD and healthy older adults depend on visual cues when trying to disambiguate visual information.

Participants and Methods: Twenty-seven individuals with PD, 23 matched normal control adults (NC), and 20 healthy younger adults (YA) were presented with a modified version of the Necker cube in which one particular face (left and upper face seen as left cube, or right and lower face seen as right cube) was highlighted by thickening the lines defining the face. The hypothesis was that the visual cues would help $\mathrm{PD}$ and NC to exert better control over bistable perception. There were three conditions, including passive viewing and two volitional-control conditions (hold one percept in front; and switch: speed up the alternation between the two).

Results: Mean dominance durations (time spent on each percept) under passive viewing were comparable in $\mathrm{PD}$ and $\mathrm{NC}$, and shorter in $\mathrm{YA}$. $\mathrm{PD}$ and YA increased dominance durations in the Hold condition relative to NC. In the Switch condition, NC and YA decreased dominance durations relative to $\mathrm{PD}$.

Conclusions: The results suggest that provision of low-level cues may have effects in PD that are different from in normal aging, and that only under group-specific conditions may the use of such cues allow compensation for visuo-perceptual deficits and consequent reduction of perceptual rigidity.

Correspondence: Mirella Diaz-Santos, M.A., Dept. Psychological and Brain Sciences, Boston University, 3515 SW 39th Blvd, Apt 28B, Gainesville,FL 32608.E-mail:mdsantos.bu@gmail.com

B.C. BAUGHMAN, T. ARENTSEN, L. WRIGHT \& B. ROPER. I'm Pretty Sure I had a Stroke: A Case of Focal Posterior Hemispheric Dysfunction Overlooked as Non-Organic Pathology.

Objective: Neuropsychological screening is sensitive and specific in detecting cognitive impairments associated with dementia (Duff et al., 2008) and predictive of functional independence (Larson et al., 2005). The development of more sophisticated neuroimaging methods has reduced the need for neuropsychological procedures to identify focal lesions. However, we present an unusual case where neuropsychological findings preceded radiographic detection of progressive parenchymal change.

Participants and Methods: Case report of a 71 year-old female with a complex history of meningitis, seizure (right temporal focus), and 
surgical intervention for a right-hemispheric meningoencephalocele. 1-year post-op imaging was unremarkable; however, she had persistent cognitive, visual, and left-sided weakness complaints. Neurology felt symptoms were secondary to chronic migraine and seizure medication side-effects, as opposed to a structural lesion.

Results: The patient was given a brief screening battery consisting of the MMSE, CLOX, RBANS, GDS, and WHODAS 2.0. While her MMSE was mildly impaired (23/30), she demonstrated normal auditory-linguistic abilities (i.e., memory, speech, naming, and fluency). Visually-based functions were quite discrepant, marked by severe constructional dyspraxia, visual agnosia, and a visual field deficit.

Conclusions: Follow-up imaging 1-month post-neuropsychological exam showed diffuse atrophy in the right temporal, parietal and occipital regions, with secondary dilation of the adjacent right lateral ventricle. No recent infarction or CSF reabsorption problem was noted. The atrophy was felt to represent degenerative disease (i.e., Posterior Cortical Atrophy). Rehab therapies were ordered to address her current deficits, which are followed closely by Neurology and Neuropsychology. The present case illustrates the utility of neuropsychological testing in identifying underlying pathological changes that were initially dismissed as non-organic.

Correspondence: Brandon C. Baughman, Phd, Semmes-Murphey Clinic, 6325 Humphreys Blvd., Memphis, TN 38120.E-mail: bbaughman@ semmes-murphey.com

\section{SATURDAY AFTERNOON, FEBRUARY 7, 2015}

\author{
Paper Session 10: \\ Autism \\ 12:30-2:00 p.m.
}

\section{S. DUVALL, L. HUANG-STORMS, A. HILL \& E. FOMBONNE. Examining Gender Differences in Cognition and Phenotype in Young Children with Autism Spectrum Disorder.}

Objective: The high prevalence of autism spectrum disorder (ASD) in males raises the question of possible sex differences in autism symptoms and cognitive profiles. This project examined possible gender differences in symptoms and cognition in a large sample of young children diagnosed with ASD.

Participants and Methods: Data was collected from 18 Autism Treatment Network (ATN) sites between the years 2007 and 2013. We included 2 separate samples in this analysis: Sample 1) Mullen Scales of Early Learning (MSEL: n=1,346 children) and Sample 2) The Autism Diagnostic Observation Schedule (ADOS) Module 1 (preverbal/single words, $n=2,420$ ).

Results: Both samples were approximately 17\% female. In Sample 1 no significant difference was found between sexes for MSEL composite total scores $(p=.54)$ or subscales. We performed further analyses on cognitive scores grouped in 5 levels of IQ: $<50,50$ to 69,70 to 84,85 to 99 and 100 and over and no sex differences were found (chi2=5.3, $4 \mathrm{df} ; \mathrm{p}=.26$ ). In Sample 2, mean algorithm total scores did not differ between boys and girls for Social Affect, Restricted and Repetitive Behaviors or Total scores (all p>.50). Further analyses of Module 1 item scores showed no differences for 27/29 items.

Conclusions: In this large sample of children with ASD, no sex differences were found in overall cognitive ability or any of the MSEL subscales; this held true even after grouping by cognitive ranges. Similarly, core features of the autism phenotype as measured by the ADOS Module 1 were strikingly consistent between boys and girls in this large sample of children.
Correspondence: Susanne Duvall, PhD, Psychology, Oregon Health \& Science University, Institute on Development \& Disability, $707 \mathrm{SW}$ Gaines Street, Portland, OR Oregon.E-mail:duvall@ohsu.edu

J.J. VAN STEENBURGH, M. VARVARIS, P. CHAMBERS, T.D. VANNORSDALL, D.J. SCHRETLEN \& B. GORDON. Transcranial Direct Current Stimulation Enhances Working Memory and Selective Attention in High-functioning Autism.

Objective: To test whether transcranial direct current stimulation (tDCS) can enhance working memory and attention in high functioning adults with autism (HFA).

Participants and Methods: Twelve adults with HFA (10 men; mean age $=32$ years; mean education $=14.3$ years $)$ received separate counterbalanced sessions of anodal, cathodal, and sham tDCS over the left and right dorsolateral prefrontal cortices (DLPFC) while performing WMS-III digit span and spatial span backward tasks. After stimulation they were given a subsection of the Brief Test of Attention (BTA).

Results: Spatial span backward improved during anodal ( mean $=9.4$, $\mathrm{SD}=3.4$ ) and cathodal (mean $=8.7, \mathrm{SD}=2.5 ; \mathrm{t}=3.27, \mathrm{p}=.007$ ) stimulation compared to sham (mean $=7.1, \mathrm{SD}=2.2)$ stimulation $(\mathrm{t}=2.38$, $\mathrm{p}=.036$ ). Digit span backward did not improve significantly during anodal $($ mean $=7.8, \mathrm{SD}=2.9)$ or cathodal $($ mean $=7.9, \mathrm{SD}=3.4 ; \mathrm{t}=-0.94$, $\mathrm{p}=.365)$ stimulation compared to sham (mean $=8.4, \mathrm{SD}=2.8)$. BTA performance improved $(\mathrm{Z}=1.41, \mathrm{p}=.048)$ following cathodal $($ mean $=$ $7.8, \mathrm{SD}=2.4)$ compared to sham (mean $=6.8, \mathrm{SD}=2.6)$, but not following anodal (mean $=7.4, \mathrm{SD}=2.2)$ stimulation $(\mathrm{Z}=1.41, \mathrm{p}=.158)$.

Conclusions: Spatial working memory deficits in HFA (Steele et al., J Autism Dev Disord, 2007; 37:605) have been attributed to DLPFC hypoactivation (Luna et al., Neurology, 2002; 59: 834), while verbal working memory is intact (Williams et al., J Autism Dev Disord, 2005; 35: 757). Here, bifrontal tDCS enhanced spatial but not verbal working memory. Improved selective attention at 30 -min post-stimulation suggests spatial working memory was enhanced via increased attentional control.

Correspondence: Joseph J. van Steenburgh, Ph.D., Psychiatry, Johns Hopkins School of Medicine, 600 North Wolfe Street, Baltimore, MD 21287.E-mail: jvanste1@jhmi.edu

R. LAJINESS-O'NEILL, A.E. RICHARD, J. BRENNAN, I. KOVELMAN, R. GOODCASE, S. YOUNCE, M. UGOLINI, K. MCFARLANE, J. MORAN \& S. BOWYER. Aberrant Neural Synchrony in Autism Spectrum Disorders Revealed with Magnetoencephalography (MEG) During Resting State: Relationship with Core Behaviors.

Objective: Disrupted neural synchrony is purported to be a primary pathophysiological mechanism in autism spectrum disorders (ASD) that contributes to hyperexcitability, unstable cortical network development, and aberrant connectivity. It remains unclear how whole-brain patterns of synchrony (coherence) or specific frequencies of cortical oscillatory activity contribute to this complex pattern of altered connectivity and its relationship with core features of ASD. Decreased coherence between default mode network (DMN) regions was hypothesized to occur in ASD and to be related to social-communication.

Participants and Methods: Whole-brain patterns of coherence during rest and its relationship with social-communication and restricted behaviors (RRBs) were examined in 12 children with ASD (MAge = 8.9; MFSIQ $=102.6)$ and 13 neurotypicals $($ NT) $($ MAge $=9.3 ;$ MFSIQ $=$ 112.0) using 148-channel whole head MEG. Neuronal synchronization was quantified by calculating coherence for each pair of 54 brain regions $(\mathrm{N}=1431)$, and further inspected in alpha $(8-12 \mathrm{~Hz})$, beta $(13-30 \mathrm{~Hz})$, and gamma $(30-80 \mathrm{~Hz})$ frequencies. T-tests or Pearson correlations were computed to examine differences in coherence and relationships with psychometric measures.

Results: Decreased coherence between DMN regions (medial prefrontal, cingulate, and parietal cortices) was noted in ASD and was related to lower social-communication. Less interhemispheric and right 
intrahemispheric synchronous gamma connections were noted in ASD. Higher coherence between left caudate and left hemispheric regions in ASD was related to lower social communication and higher RRBs. Conclusions: Findings support a model of aberrant neural synchrony in ASD revealing underconnectivity in the DMN and enhanced connectivity between left subcortical and left hemispheric regions. Enhanced coherence between the latter regions may underlie the rigid social-communicative and motor behaviors in ASD.

Correspondence: Renee Lajiness-O'Neill, Ph.D., Psychology, Eastern Michigan University, 320 King Hall, Ypsilanti, MI 48197. E-mail: rlajines@gmail.com

C. LUONG-TRAN, L.G. ANTHONY, E. BAL, M. WILLS, M.A. WERNER, K. ALEXANDER, L. CANNON, J. STRANG \& L. KENWORTHY. It Takes Two to Tango: Executive Control in Students with Autism Spectrum Disorder Is Affected by Teacher Behavior.

Objective: Children with autism spectrum disorders (ASD) have executive control (EC) challenges in the classroom, which interfere with their ability to transition quickly, follow rules, participate effectively, resist getting stuck, etc. This study explores the relationship between student EC and teacher behaviors, including provision of visual supports, positive reinforcement, and modeling of EC abilities.

Participants and Methods: Sixty-seven 3rd-5th grade students $(\mathrm{M}=9.52, \mathrm{SD}=1.02)$ with $\mathrm{ASD}$ and $\mathrm{IQ}>70(\mathrm{M}=108.45, \mathrm{SD}=18.01)$ and their mainstream teachers were observed in 14 elementary schools using a Classroom Fidelity Observations Coding Form (90\% inter-rater reliability) at 2-3 time points for 10-15 minute sessions (210 total sessions). Student EC behaviors (rule abidance, transitioning, resistance to getting stuck, negativity/overload, participation), and teacher behaviors (use of praise, use of priming, modeling of flexibility) were coded. The correlation between specific student and teacher behaviors was investigated and composite scores of teacher and student behaviors with good internal reliability were generated for regression analyses.

Results: Teacher use of praise and teacher flexibility were correlated with student rule abidance, transitioning, participation, and resistance to getting stuck (rhos: .21-.37, p's<.005). Stepwise regression analysis demonstrated a bidirectional relationship between teacher behaviors and student EC. Teacher flexibility and use of praise predicted positive student $\mathrm{EC}$ behaviors ( $\mathrm{R} 2=.163, \mathrm{~F}(2,197)=19.218$, $\mathrm{p}<.001)$, while student participation and transitioning $(\mathrm{R} 2=.163, \mathrm{~F}(2,197)=19.140$, $\mathrm{p}<.001)$ predicted teacher behavior.

Conclusions: Results illustrate the bidirectional influence of teacher and student EC behaviors on one another: 1) Students can benefit from positive teacher behaviors, and 2) Teachers of children with good EC are more flexible/positive.

Correspondence: Caroline Luong-Tran, Psy.D. Clinical Psychology, Neuropsychology, Children's National Health Center, 8189 Single Leaf Lane, Lorton, VA 22079. E-mail: CLuong@childrensnational.org

D. MURDAUGH, J. MAXIMO, H. DESHPANDE, A. LEMELMAN \& R. KANA. The Impact of Reading Intervention on Brain Structure and Function Underlying Language in Children with Autism Spectrum Disorders.

Objective: Deficits in language comprehension have been widely reported in children with ASD. Neuroimaging studies have consistently found that individuals with ASD tend to rely heavily on visuospatial processing to aid in language comprehension. This study used a translational neuroimaging approach to examine the impact of an imagery-based reading intervention to improve brain circuitry underlying language processing in children with ASD.

Participants and Methods: We used functional magnetic resonance imaging (fMRI) and diffusion tensor imaging (DTI) fiber tractography to investigate brain changes as a result of intervention. A sentence task and resting state scan were presented while children underwent fMRI. 32 children with ASD (ages 8-13 years) were imaged twice, with 17 children receiving the intervention between scans (ASD-EXP), and 15 wait-list control children (ASD-WLC). In addition, 27 typically-developing (TD) children were imaged once.

Results: The ASD-EXP group showed greater activation post-intervention in response to reading sentences in posterior visual and language regions, and strengthened functional connectivity $(\mathrm{FC})$ between $\mathrm{L}$ hemisphere language areas (MTG and IFG). Correlation of FC and improvement in reading comprehension in the ASD-EXP group revealed increased FC between Broca's and RSTG, and the Wernicke's and LIFG. Lastly, after intervention, the ASD-EXP group demonstrated increased white matter tract connectivity in the $\mathrm{R}$ arcuate fasciculus and $\mathrm{R}$ thalamic radiation.

Conclusions: We found that application of a novel reading intervention aimed at using a relatively intact skill, visual-spatial abilities, to aid in boosting a relative deficit, language comprehension, in children with ASD was successful in improving reading comprehension and mediating brain changes. This supports specialized intervention for children with ASD to increase high-order learning skills, and supports the growing literature of the plasticity of the young brain in ASD.

Correspondence: Donna Murdaugh, Psychology, University of Alabama at Birmingham, 2217 Garland Dr., Birmingham, AL 35216. E-mail: dlmurdaugh@gmail.com

M. SOUTH, N. TOP, K. STEPHENSON, C. NIELSON \& B. KIRWAN. A Step Behind: Atypical Amygdala Activation during Fear Conditioning and Extinction in Autism Spectrum Disorders.

Objective: Individuals diagnosed with autism spectrum disorders (ASD) frequently suffer from associated severe anxiety. Studies of classical fear conditioning and extinction can provide important insight into the foundations that underlie anxious behaviors. This is the first fMRI study of classical fear conditioning in ASD.

Participants and Methods: Participants included 20 adults diagnosed with ASD and a control group (CON group) of 19 participants who reported no history of diagnosis of psychiatric conditions. Participants undergoing fMRI completed a fear conditioning task adapted from Holt et al. (2012) that included 2 runs of fear acquisition followed by 2 runs of extinction, using colored squares as conditioned stimuli and a blast of air to the base of the neck as the unconditioned stimulus.

Results: Repeated measures ANOVA for 2 (diagnostic group) x 2 (response to threat stimulus minus safe stimulus) indicated significantly greater right amygdala and left insula response for the CON group, during the early fear conditioning phase. In contrast, the late extinction run found greater left amygdala and right insula activation in the ASD group. Tracking of amygdala and insula across all four runs showed significant group $\mathrm{x}$ phase interactions, with the control group demonstrating consistent decrease in activation from the threat context to the safe context, while the ASD group showed delayed early response to threat that increased over time into the safety phase.

Conclusions: A delayed or decreased ASD response to fear conditioning may arise because of a ceiling effect, in that substantial everyday anxiety reflects an already over-aroused fear system that is not responsive to the relatively mild threat from our air blast stimulus. This sheds light on basic mechanisms that may influence the development of anxiety in ASD, and suggests the potential for targeted treatments to reduce baseline anxiety in ASD that will improve cognitive performance and quality of life.

Correspondence: Mikle South, PhD, Psychology and Neuroscience, Brigham Young University, 245 TLRB, Provo, UT 84602. E-mail: south@byu.edu 


\section{Symposium 15: \\ Disruption of Neural Connectivity After Traumatic Brain Injury in Children: Contribution of Neuroimaging to Understanding Long-Term Cognitive and Behavioral Outcomes}

\section{Chair: Linda Ewing-Cobbs}

\section{2:30-2:00 p.m.}

L. EWING-COBBS, E.A. WILDE, N.P. RYAN, L. EWINGCOBBS, C.P. JOHNSON \& J.M. FLETCHER. Disruption of Neural Connectivity After Traumatic Brain Injury in Children: Contribution of Neuroimaging to Understanding Long-Term Cognitive and Behavioral Outcomes.

Symposium Description: Traumatic brain injury (TBI) is the leading cause of pediatric mortality and morbidity. Children who incur moderate to severe TBI are at high risk for life-long difficulties in a host of areas ranging from adaptive behavior to academic skill development to social-emotional competence. TBI sustained during childhood and adolescence disrupts neural networks at the time of injury and may interfere with the subsequent development and refinement of these networks. This symposium will examine how different structural neuroimaging metrics, including volume, cortical complexity, lesion load, and integrity of white matter pathways, illuminate post-traumatic changes in children with TBI. We will highlight the contribution of these metrics to characterizing the consequences of TBI during early stages of recovery as well as assessing subsequent changes in network integrity and connectivity. Younger age at injury has been noted as a risk factor for poorer outcome in several domains, including general cognitive functioning, working memory, reading, and social cognition. It is unclear whether younger age at injury is a risk factor for greater disruption of neural networks. To address these issues, the influence of age at injury on brain metrics during early stages of recovery and subsequent changes in both brain and behavior are examined.

This symposium will examine long-term neuroimaging and neuropsychological outcomes in terms of the trajectory of developmental changes after injury. Both outcome domains will be examined in terms of age at injury and time since injury to characterize neural and neuropsychological development after TBI in on a continuum from early plasticity to early vulnerability.

Correspondence: Linda Ewing-Cobbs, PhD, Pediatrics and Children's Learning Institute, University of Texas Health Science Center at Houston, 7000 Fannin Suite 2401, Houston, TX 77030. E-mail: linda. ewing-cobbs@uth.tmc.edu

N. RYAN, C. CATROPA, M.H. BEAUCHAMP \& V. ANDERSON.
The Emergence of Age-Dependent Social Cognitive Deficits
after Generalized Insult to the Developing Brain: a Longitudinal
Prospective Study using Susceptibility-Weighted Imaging.
Objective: While structural correlates of Theory of Mind (ToM) are well
described in adults, less is known about the anatomical regions subsum-
ing these skills in the developing brain or the impact of cerebral insult
on the development of high-level social cognition. This study aimed to
examine the differential influence of age-at-insult and brain pathology
on ToM in children and adolescents with traumatic brain injury (TBI).
Participants and methods: Children with TBI (n=106) were categorized
according to timing of brain insult: (i) Middle Childhood (5-9 years; $n$
$=41$; ;ii) Late Childhood (10-11 years; $\mathrm{n}=39$ ); and (iii) Adolescence
(12-15 years; $\mathrm{n}=32$ ) and group-matched for age and gender to healthy
controls (n=43). Participants underwent magnetic resonance imaging
(MRI) including a susceptibility-weighted imaging (SWI) sequence $2-8$
weeks after injury and completed a ToM battery at 6 - and 24 -months
post-injury.
Results: For adolescents with TBI, social cognitive dysfunction at 6 - and
$24-$-months post-injury was associated with diffuse neuropathology and a greater number of lesions detected using SWI. In the late childhood TBI group we found a time-dependent emergence of social cognitive impairment, linked to diffuse neuropathology. The middle childhood TBI group demonstrated performance unrelated to pathology and comparable to TD controls.

Conclusions: The full extent of social cognitive deficits after TBI may not be realized until the associated skills reach maturity. Findings suggest that the integrity of an anatomically distributed network of brain regions and their connections is necessary for the acquisition and establishment of high-level social cognition.

Correspondence: Nicholas Ryan, Murdoch Children's Research Institute 50 Flemington Rd, Parkville VIC, VIC 3052, Australia. E-mail: nicholas. ryan@mcri.edu.au

C.P. JOHNSON, P. SWANK, C.S. COX \& L. EWING-COBBS. Reading Skills After Traumatic Brain Injury in Children: Relation with White Matter Pathways.

Objective: Pediatric traumatic brain injury (TBI) can result in significant long-term deficits in mastery of academic skills. This study aimed to identify white matter pathways that, when damaged, predicted reading deficits in children in the areas of individual word reading, reading fluency, and passage comprehension. The influence of age at injury was also examined.

Participants and Methods: Children ages 6 to 16 years with a history of orthopedic injury (OI, n=27) or TBI (n=29) were evaluated. Diffusion tensor imaging sequences were collected three months post-injury. Pathway integrity was estimated using fractional anisotropy derived from Tract-Based Spatial Statistics (TBSS). A battery of reading tests assessing accuracy and efficiency of decoding words and sentences as well as fluency and comprehension for oral reading of connected text was individually administered 1 year after injury.

Results: Children with TBI and reduced microstructural integrity of the superior longitudinal fasciculus demonstrated reduced sight word-reading ability and a trend towards reduced phonemic decoding. Children with TBI and microstructural changes involving the cingulum bundle demonstrated reduced reading fluency. Group by age at injury interaction effects were not significant for either reading or fractional anisotropy, suggesting that the effect of age at injury on reading outcomes and FA was similar in children with TBI or OI.

Conclusions: Results support the association of the superior longitudinal fasciculus with both sight word reading and phonemic decoding. Additionally, these findings support a novel role of the cingulum bundle in reading fluency. The impact of TBI on reading skills was similar in children and adolescents, which does not provide support for the early vulnerability hypothesis.

NIH NSR01046308.

Correspondence: Chad P. Johnson, PhD, 6655 Travis, 10th Floor, Suite 10.03, Houston, TX 77030.E-mail: Chad.P.Johnson@gmail.com

E.A. WILDE, J. FABER, G. HANTEN, L. EWING-COBBS, M. AITKEN, E.D. BIGLER, R. YALLAMPALLI, S. MULLINS, Z. CHU, X. LI, J. HUNTER, L. NOBLE-HAEUSSLEIN \& H. LEVIN. Neuroimaging and Long-term Recovery after Early TBI in Children: What can Tractography Reveal about Post-TBI Development?

Objective: To examine long-term changes in diffusion tensor imaging (DTI) and their relation to outcomes in adolescents who had sustained moderate to severe traumatic brain injury (TBI) as toddlers or young children.

Participants and Methods: DTI was performed in 21 patients (15 males/ 6 females) aged 10-18 years who had sustained early (e.g., between the ages of 1.5-8 years of age) moderate-to-severe TBI 5-15 years earlier and in a group of 16 typically-developing children (13 males, 4 females) of similar age and demographic characteristics. Quantitative tractography was used to examine major white matter pathways for DTI. Neuropsychological and other outcome measures including the California Verbal Learning Test - Child Version (CVLT-C), the Behavioral 
Rating Inventory for Executive Functioning (BRIEF) were examined in relation to imaging findings.

Results: Although we found expected relations between some DTI and outcome measures in the group-level studies (e.g., lower performance on memory measures and reduced FA in temporal pathways subserving memory, lower performance on executive functioning measures and reduced FA in the ventral striatum), we did also observe some unusual tractography results in a subset of children that challenge straightforward interpretation of brain -behavior relationships in all cases.

Discussion: Evolving neuroimaging tools including DTI may be used to demonstrate alterations in the expected course, pattern, and timing of development following pediatric TBI. Although group data is useful in understanding these development patterns, we also present case studies which reflect the dynamic and individual nature of recovery in brain structure and how this may relate to function and behavior. NIH R21 NS065937

Correspondence: Elisabeth A. Wilde, Ph.D., 1709 Dryden Rd., Ste 1200, Houston, TX77025.E-mail: ewilde@bcm.edu

L. EWING-COBBS, J. JURANEK, C.P. JOHNSON, E. MAXWELL, M. PRASAD, C.S. COX, G. DUQUE \& P. SWANK. Pathway Integrity and Neuropsychological Outcomes During the First Two Years after Pediatric TBI: Recovery or Persistent Deficit?

Objective: To examine longitudinal diffusion tensor imaging metrics and their relation to neuropsychological outcomes during the first two years of recovery after pediatric traumatic brain injury (TBI).

Participants and Methods: Neuropsychological outcomes and diffusion tensor imaging were obtained 3 and 24 months after injury in children ages 6 to 15 who were hospitalized for complicated-mild to severe TBI $(n=32)$ or orthopedic injuries (OI; $n=35)$. Tract based spatial statistics were used to characterize the integrity of 7 association and projection pathways. General linear models evaluated the effect of age at injury, time of evaluation, and group on fractional anisotropy in each pathway. The correlation between neuroimaging metrics and neuropsychological performance was examined.

Results: Age x group x time interactions were obtained for fractional anisotropy of the left corticospinal tract, superior and inferior longitudinal fasciculi, and inferior frontal occipital and uncinate fasciculi. Pathway integrity was lower in the TBI than the OI group, particularly in the youngest children with TBI. Fractional anisotropy increased more in the TBI than OI group over time; despite this increase, the TBI group had a persistent deficit 2 years after injury. Diffusion tensor imaging metrics were significantly related to functional and neuropsychological outcomes for children with TBI.

Discussion: Structural neuroimaging findings supported the early vulnerability hypothesis. The youngest children with TBI had the greatest reduction in pathway integrity. Although fractional anisotropy remained significantly reduced two years following TBI, increases were noted over time in 6 of 7 pathways after both TBI and OI suggesting continued development. NIH NSR01046308

Correspondence: Linda Ewing-Cobbs, PhD, 7000 Fannin Suite 2401, Houston,TX 77030.E-mail: linda.ewing-cobbs@uth.tmc.edu

\section{Symposium 16: \\ Exercise as Brain Medicine: State of the Science}

\author{
Chair: J. Carson Smith \\ Discussant: Stephen Rao \\ 12:30-2:00 p.m.
}

J. SMITH, K.I. ERICKSON, T. LIU-AMBROSE \& S.M. RAO. Exercise as Brain Medicine: State of the Science.

Symposium Description: A focus in Alzheimer's disease and related dementias (ADRD) research has been to identify preclinical biomarkers, including modifiable risk factors such as metabolic disease and lifestyle behaviors, in order to predict future cognitive decline and clinical manifestations of dementia. A major goal of these efforts has been to facilitate targeted intervention and prevention trials. However, a multidisciplinary panel recently concluded there is insufficient evidence to support the use of pharmaceutical agents, dietary supplements, or behavioral interventions, to prevent cognitive decline or ADRD.

Interventions targeting modifiable risk factors, even if modest in the magnitude of effects, could have a major public health impact. Exercise has received much attention as a possible intervention for ADRD, especially given its minimal side effects and its ability to modify several metabolic pathways, including those related to vascular cognitive impairment. While there is substantial evidence for the cognitive benefits of exercise in cognitively healthy older adults, there are many unanswered questions regarding the role of exercise as a viable ADRD treatment or preventative intervention, such as what type or intensity of exercise may be most effective, and whether or not the well documented neurotrophic and cognitive benefits of exercise extend to those who are at increased risk or exhibit clinical symptoms. This symposium will present the current state of the science for exercise as medicine in the primary and secondary prevention of ADRD in older adults who vary in risk for future cognitive decline (Smith), including moderators of the effects of exercise on neurocognitive function, brain structure and function (Erickson), and mobility/disability (Liu-Ambrose). We aim to stimulate discussion (Rao) regarding the key knowledge gaps that need to be addressed regarding the effects of exercise on neurocognitive and brain function along the trajectory of ADRD progression.

Correspondence: J. Carson Smith, Maryland, School of Public Health Bldg 255, College Park, MD 20742. E-mail: carson@umd.edu

\section{J. SMITH. Exercise and Physical Activity Along the Alzheimer's Risk Continuum.}

Lifestyle physical activity and exercise interventions are among the most promising preventative strategies for Alzheimer's disease and related disorders (ADRD). However, there remain many unanswered questions regarding the mechanistic pathways, as well as the efficacy and treatment potential, of physical activity and exercise to prevent or decelerate cognitive decline leading to Alzheimer's disease. A translational perspective will be presented to summarize the potential mechanisms, based on animal models, by which exercise may affect brain structure and function, and its efficacy to impact brain structure, brain function, and cognitive function in older adults, with an emphasis on those who are at increased risk for Alzheimer's disease. In addition to a brief overview of the current evidence for physical activity and exercise interventions to benefit cognition and neuroimaging measures of brain structure and function, I will discuss our recent work examining interactions between physical activity and genetic risk for AD in healthy older adults, as well as outcomes from an exercise intervention in individuals diagnosed with mild cognitive impairment.

Correspondence: J. Carson Smith, School of Public Health Bldg 255, College Park, MD 20742. E-mail: carson@umd.edu 
K.I. ERICKSON. Moderators of Exercise-Related Brain Plasticity. A growing body of evidence from neuroscience, epidemiology, and kinesiology suggests that physical activity is effective for preventing, delaying, and potentially treating neurocognitive problems throughout the lifespan. Despite the emerging recognition of physical activity as a powerful method to enhance brain health, there is continued confusion from both the public and scientific communities about what the extant research has discovered about the potential for physical activity to improve neurocognitive health and which questions remain unanswered. One of the reasons for this confusion is because of heterogeneity in the ways in which physical activity is assessed, the cognitive tests that are conducted, and individual difference variables that influence its effectiveness. From this perspective, I will discuss several potential moderators of the effects of exercise on neurocognitive function including genetic, dietary, and demographic variables. I will conclude that physical activity decreases the risk for brain diseases and disorders, ameliorates symptoms, improves function, and increases regional brain volume and that we are beginning to have a better understanding of the factors that moderate these associations. Overall, physical activity is an important modifiable lifestyle that carries significant consequences for learning, memory, and brain health for people of all ages.

Correspondence: Kirk I. Erickson, 3107 Sennott Square, Pittsburgh, PA 15261.E-mail:kiericks@pitt.edu

\section{T. LIU-AMBROSE. Neuroimaging Evidence for "Central Benefit Model" of Exercise in Falls Prevention.}

The widely accepted dogma is that improved physical function underlies the effectiveness of exercise interventions in reducing falls risk. However, evidence from our randomized controlled trials suggests that exercise may reduce falls risk via mechanisms other than improved physical function. Specifically, improved cognitive function - specifically, executive functions - and associated functional plasticity may be an important yet under-appreciated mechanism by which the exercise reduces falls risk in older adults. Specifically, we demonstrated that a home-based exercise program of balance and strength reduced falls by $47 \%$ among older adults with a significant history of falls - in the absence of significant improvement in physical function (i.e., balance and muscle strength). Notably, cognitive performance of selective attention and conflict resolution significantly improved in the exercise group compared with the control group. We also found that improved selective attention and conflict resolution secondary to 12 months of progressive resistance training was associated with improved usual gait speed. Furthermore, exercise may reduce falls risk (and improve cognitive function) by slowing down the progression of white matter lesions. Evidence from randomized controlled trials of exercise will be discussed within the context of the proposed central benefit model (Liu-Ambrose et al., 2013).

Correspondence: Teresa Liu-Ambrose, 212-2177 Wesbrook Mall, Vancouver, BCV6T 1Z3, Canada.E-mail: teresa.ambrose@ubc.ca

Poster Session 10:

ADHD/Attention, Cancer, Language/Aphasia, Learning Disabilities/Academic, \&. Memory

12:30-2:00 p.m.

\section{Assessment/Psychometrics/Methods (Child)}

A.L. FERNANDEZ, L.F. LAMAS, M. GATTÁS, D. LASA \& P. MARCHETTI. Validity of a Test for the Early Detection of Dyslexia in Spanish-speaking children: Test de Velocidad de Denominación.

Objective: The assessment of speed naming allows the early identification of children at risk of developing dyslexia. Since there was not a test for the assessment of speed naming of Spanish-speaking children the Test de Velocidad de Denominación (TVD, Naming Speed Test) was developed. In the TVD the time to produce the correct names of 50 familiar figures is registered. In this poster the results of a convergent validity study are reported.

Participants and Methods: Eighty-nine children from 1st to 3rd. grade and both sexes were tested. Children assisted to public and private schools. They were also administered the Words and Pseudowords reading subtests of the LEE battery in order to obtain convergent validity evidence. For each of the LEE subtests reading accuracy and reading speed was registered.

Results: Total naming time on the TVD correlated significantly with the following variables: word reading time $(\mathrm{r}=.68)$, pseudoword reading accuracy $(r=.53)$, pseudoword reading time $(r=.67)$ and pseudoword reading accuracy $(r=.48)$. Correlation between age (months) and total naming time on the TVD was $r=-.44$. An ANOVA showed that the 1 st grade children had a significantly lower performance than the children from 2nd and 3rd grade. There were no significant differences between the children of 2 nd and $3 r d$ grade. There were no significant differences between male and female.

Conclusions: These data indicate an adequate convergence between the performance on the TVD and the performance on a previously validated test of reading accuracy and reading time. In accordance with previous reports (double-deficit hypothesis) the highest correlation was between naming time and reading time and not with reading accuracy.

Correspondence: ,, . E-mail:

\section{ADHD/Attentional Functions}

P.G. COLE \& S.T. GONTKOVSKY. WISC-IV IQ and Index Score Variability According to Severity of ADHD Symptomatology.

Objective: Studies report mixed findings regarding the relationship between symptoms of Attention-Deficit/Hyperactivity Disorder (ADHD) and intellectual functioning. Clarification of this relationship has important therapeutic implications across settings. This study further examined the relationship between intelligence and reported symptoms of ADHD in children and adolescents, with the primary hypothesis being that more severe symptoms of inattention and hyperactivity would be reflected in lower IQ scores.

Participants and Methods: Participants were 224 individuals ages 6 through 16 years ( $\mathrm{M}$ age $=10.02$ years, $\mathrm{SD}=2.88$ ) referred to an outpatient mental health clinic for psychological evaluation. Participants completed all subtests of the Wechsler Intelligence Scale for Children-4th Edition (WISC-IV) and had completed parent and teacher ratings forms of the Attention Deficit Disorders Evaluation Scale-3rd Edition. The sample was primarily male $(67 \%)$ and White $(54 \%)$.

Results: Analyses revealed that the sample obtained scores within the Low Average range across all WISC-IV index scores, including Full Scale IQ. Teacher ratings of participants' symptoms were suggestive of significantly less severe clinical inattention and hyperactivity relative to parent ratings. Parent ratings of severity of inattention, but not hyperactivity, were significantly and negatively associated with 3/5 WISC-IV index scores. Teacher ratings of severity of inattention and hyperactivity were significantly and negatively associated with $4 / 5$ and $5 / 5$ WISC-IV index scores, respectively.

Conclusions: Both parent and teacher ratings concerning the severity of ADHD symptoms are negatively associated with intelligence in children and adolescents presenting with behavioral issues, although teacher ratings are more frequently and more strongly predictive of IQ.

Correspondence: Samuel T. Gontkovsky, Psy.D., Psychology, John F. Kennedy University, 5800 Third Street Unit 1113, San Francisco, CA 94124.E-mail: sgontkovsky@hotmail.com 
J. THOMPSON, C. NOGGLE, A. LEVINE \& J. DAVIS. ADHD and Incidental Learning: Demonstrated weaknesses on the Rey Complex Figure Test.

Objective: It has been established that completion of the Rey Complex Figure Test requires an array of neurocognitive functions including visuospatial abilities, memory, attention, planning, and working memory. Given examinees are not specifically instructed that they will have to reproduce the stimuli from memory, the RCFT can be viewed as a measure of incidental learning which is dependent upon depth of processing and higher-order attention sustainment. The current study sought to determine if ADHD, a disorder associated with significant weaknesses in sustained attention, would correspond with significant deficits in RCFT performance.

Participants and Methods: An archival data set was utilized for the current study. Participants $(\mathrm{n}=52)$ included children and adolescents diagnosed with ADHD previously evaluated at an outpatient Neuropsychology clinic. All participants completed the RCFT as part of their clinical evaluation. Group means were compared to the normative average. Results: Results demonstrated ADHD was associated with significantly lower performance on the RCFT. Specifically, individuals with ADHD performed significantly lower in both immediate memory $[\mathrm{t}(51)=$ $-10.42, p<.001]$ and delayed memory $[t(51)=-8.028, p<.001]$ on the RCFT.

Conclusions: Findings suggest ADHD may be associated with significant difficulties in incidental learning as demonstrated by their lower performance in immediate and delayed memory on the RCFT.

Correspondence: Chad Noggle, Ph.D., ABN, Psychiatry, SIU School of Medicine, 315 W. Carpentedr St., Springfield, IL 62794. E-mail: cnoggle@siumed.edu

C.K. PETRANOVICH, N.C. WAlz, M. STAAT, P. CHIU \& S.L. WADE. The Influence of Neuropsychological Aspects of Attention on Parent-reported Behavior and School Competence in Internationally Adopted Girls with a History of Institutionalization. Objective: Problems with attention, behavior, and school competence have been reported in internationally adopted children. Few studies have investigated the influence of neuropsychological aspects of attention on behavior and school competence in this population.

Participants and Methods: Participants included girls between the ages of 6-12 years old who were adopted from China $(\mathrm{n}=32)$ or Eastern-Europe $(\mathrm{n}=25)$ and a group of American-born, never-institutionalized girls $(\mathrm{n}=25)$. Children completed the Walk/Don't Walk, Score!, and Sky Search subtests from the Test of Everyday Attention for Children (TEA-Ch). Parents completed the Child Behavior Checklist (CBCL).

Results: Compared to the Chinese adoptees and the controls, the Eastern European group evidenced significantly poorer performance on the Score! subtest, a measure of auditory sustained attention, and more problems on the CBCL School Competence and attention deficit/ hyperactivity disorder (ADHD) subscales. Compared to the controls, parents in the Eastern European group reported significantly more concerns on the CBCL Total Behavior Problems subscale. Controlling for group and family income, Score! performance independently predicted parental ratings on the Total Behavior Problems $(\beta=-.76, t=-2.36, p$ $=.02), \mathrm{ADHD}(\beta=-.63, \mathrm{t}=-2.90, \mathrm{p}=.005)$, and School Competence $(\beta=.92, \mathrm{t}=3.27, \mathrm{p}=.002)$ subscales. Poorer auditory attention was associated with more problems. The remaining subtests of the TEA-Ch were non-significant predictors

Conclusions: Internationally adopted children may be vulnerable to problems with behavior and school competence. Neuropsychological measures of auditory attention may further our understanding of the impact of early institutionalization on functional outcomes.

Correspondence: Christine K. Petranovich, Dept. of Psychology, University of Cincinnati, 1 Edwards Center ML 0376, Cincinnati, OH 45221.E-mail: karvercl@mail.uc.edu
L. JASINSKI \& J. RANSEEN. The Clinical Utility of the Repeatable Battery for the Assessment of Neuropsychological Status (RBANS) in the Assessment of Adult ADHD.

Objective: The objective of this study was to explore the use of the Repeatable Battery for the Assessment of Neuropsychological Assessment (RBANS; Randolph,1998) as a potential neuropsychological screening tool for adults seeking an evaluation for possible ADHD. The RBANS is particularly appealing due to the brevity of testing time, comprehensive nature of the screening, and availability of multiple versions for repeated administration.

Participants and Methods: Participants included 85 consecutive referrals for assessment of ADHD in adulthood (M Age: 28.7 years; M Ed: 14.6 years; $63 \%$ Female). Most patients had no prior diagnosis of ADHD $(67 \%)$, and were seeking evaluation for the first time. Of those tested, six failed an assessment of effort (Test of Memory Malingering) and were excluded from analyses, leaving a final sample of 79 adults. Results: Based on a comprehensive evaluation process, including a clinical interview, self-report ratings, parent or significant other ratings, and psychological and neuropsychological testing, 61 (77\%) of the patients were eventually diagnosed with ADHD. Differences on the RBANS scales between those diagnosed with ADHD and those who were either not diagnosed or diagnosed with another psychiatric condition (e.g. anxiety) were explored. No significant differences were found on mean index scores for any of the RBANS indices between those who were and were not eventually diagnosed with ADHD. However, diagnostic utility rates were explored for each index at varying cut scores, as a potential for using the RBANS as a screening tool in maximizing sensitivity to ADHD. The semantic fluency and line orientation subtests proved most powerful in doing so.

Conclusions: It is concluded that these results are consistent with much previous literature suggesting that many neuropsychological tests are insensitive to ADHD, but continued work to identify those that might be most appropriate for screening instruments should continue. Clinical utility of the RBANS in the assessment of adult ADHD is discussed. Correspondence: Lindsey Jasinski, Ph.D., Psychiatry, University of Kentucky, 1350 Bull Lea Road, Lexington, KY 40511. E-mail: ljschi2@ uky:edu

\section{A.M.PASSAROTTI, N. TRIVEDI, M.PATEL \& S.A. LANGENECKER. Do Children with ADHD and Bipolar Disorder Differ in Terms of Executive Dysfunction?}

Objective: High comorbidity and symptoms overlap in attention deficits hyperactivity disorder (ADHD) and pediatric bipolar disorder (PBD) pose significant diagnostic and clinical challenges. While PBD is considered mainly a mood dysregulation syndrome and ADHD mainly an executive dysfunction (EF) syndrome, recent evidence suggests that both EF and affect regulation may be impaired in PBD and ADHD, although with possibly differing mechanisms. In this study we examined illness-specific differences in EF as they relate to cognitive and emotional control, attention and working memory

Participants and Methods: Sixteen adolescents with pediatric BD (Type I, Type II), 12 adolescents with ADHD, Type Combined, and 9 $\mathrm{BD}$ adolescents with comorbid $\mathrm{ADHD}$ (mean age $=12.41, \mathrm{SD}=2.12$ ) were assessed using the Behavioral Rating Inventory of Executive Functions (BRIEF), the Conners' Rating Scale (parental reports), Trails A\&B. Math and Reading Fluency, and the Digit and Spatial Span tasks. Patient groups were comparable on demographics and estimated IQ (WASI, 1999). ANOVAs and post-hoc comparisons examined potential group differences.

Results: For the BRIEF, while all groups were at clinically significant levels of dysfunction, PBD had significantly worse scores than ADHD on the Inhibition ( $p=.02)$, Emotional Control $(p=.0001)$, Shift $(p=.001)$, Planning ( $\mathrm{p}=.03)$ and Monitoring $(\mathrm{p}=.01)$ subscales, but not on Working Memory $(p=.17)$ or Organization $(p=.08)$. PBD and the comorbid group did not differ significantly. Moreover, both PBD groups had worse scores than ADHD on the Conners' oppositional sub-scale $(p=.002)$. There 
were no group differences for the cognitive tasks. Across all participants there was a significant negative correlation between the Math Fluency score and the BRIEF Inhibition ( $\mathrm{r}=-.34)$, Planning $(\mathrm{r}=-.39)$ and Monitoring ( $\mathrm{r}=-.34)$ sub-scales (all $\mathrm{Ps}<.05$ ).

Conclusions: The current results contribute new knowledge on similarities as well as domain-specific differences in executive dysfunction between ADHD and PBD, which can inform intervention.

Correspondence: Alessandra M. Passarotti, PhD, Psychiatry, University of Illinois at Chicago, $1747 \mathrm{~W}$. roosevelt Rd, Chicago, IL 60608. E-mail: apassarotti@psych.uic.edu

T. KORIAKIN, N. NOONAN, M. MAHONE \& L.A. JACOBSON. The Contribution of Sluggish Cognitive Tempo to Executive and Adaptive Functioning.

Objective: Sluggish cognitive tempo (SCT) describes symptoms of under-activity, poor initiation, and slowness. SCT is related to, yet separable from, core symptoms of ADHD, with sleepy/sluggish SCT symptoms most distinct from ADHD-Inattention. Children with ADHD manifest reduced executive function $(\mathrm{EF})$ and adaptive skills, yet the contribution of SCT to these outcomes is not clear. The present study examined the contribution of SCT to executive and adaptive skills in a clinical sample, after controlling for ADHD symptomatology.

Participants and Methods: Participants included 582 clinically-referred children, ages 6-18 (64\% male), with parent ratings of inattention and hyperactivity (ADHD Rating Scale-IV), executive function (BRIEF), adaptive skills (ABAS-II), and SCT (SCT subscales: Sleepy/ Sluggish, Low Initiation/Persistence, and Daydreamy). Hierarchical linear regressions predicting the BRIEF Global Executive Composite (GEC) and ABAS-II Global Adaptive Composite (GAC) were examined, with inattentive and hyperactive ADHD symptoms entered on step 1 and the three SCT subscales on step 2.

Results: SCT added to prediction of both GEC $(\Delta \mathrm{R} 2=.023 ; \mathrm{p}<.001)$ and GAC $(\Delta \mathrm{R} 2=.014 ; \mathrm{p}=.025)$, after controlling for ADHD symptoms. Specifically, Low Initiation SCT predicted both GEC $(\beta=-.153, p=.001)$ and GAC $(\beta=-.175, p=.006)$, whereas Sleepy/Sluggish SCT predicted executive (GEC $\beta=-.108, p=.001$ ) but not adaptive (GAC $\beta=-.037$, $\mathrm{p}=.39)$ functioning.

Conclusions: Above and beyond ADHD symptoms, SCT accounted for small but significant additional variance in executive and adaptive functioning, with a specific pattern of prediction. Low Initiation was predictive of both EF and adaptive functioning; Sleepy/Sluggish SCT, although more distinct from inattention, was associated with only EF. Reduced initiation/impersistence and under-activity/sluggishness may appear to differentially impact outcomes and may offer specific targets for intervention.

Correspondence: Taylor Koriakin, Neuropsychology, Kennedy Krieger Institute, 1750 E. Fairmount Ave, Baltimore, MD 21231. E-mail: koriakin@kennedykrieger.org

\section{A. BITTON, S. O'NEILL, D. MARKS \& J.M. HALPERIN. Executive Functioning and the Role of Basic Cognitive Processes in 8-year- olds With and Without ADHD.}

Objective: To determine whether ADHD at 8 years of age is associated specifically with executive dysfunction or whether poor performance on such measures is better accounted for by more basic cognitive deficits. Additionally, a narrow age-range was studied to assure that the sample was at a relatively homogenous stage of brain development.

Participants and Methods: Participants from a longitudinal investigation of ADHD were evaluated at 8-year-olds $(\mathrm{n}=145)$ and classified as having or not having ADHD based on parent and teacher ratings, and a semi-structured interview with parents. Participants were administered four D-KEFS subtests (Trail Making Test, Verbal Fluency, Design Fluency, Color Word Interference) that include basic cognitive conditions and systematically increase cognitive load. Data from each subtest were analyzed using 2 (Group) x 3-5 (Condition) mixed design
ANOVAs. Group x Condition Interactions were of key importance to establish executive deficit.

Results: Group x Condition interactions emerged for the Trail Making Test and Color Word Interference, clearly demonstrating that the ADHD group performed more poorly on the executive measures after accounting for more basic cognitive processes. The ADHD group also performed more poorly on some of the basic cognitive conditions of these two subtests. Fluency tasks yielded no significant differences between the two groups.

Conclusions: These data indicate that 8-year-olds with ADHD demonstrate poor inhibitory control as well as more basic cognitive deficits. Correspondence: Adina Bitton, BA, Psychology, CUNY, The Graduate Center, 100-25 Queens Blvd, Apt 6N, Forest Hills, NY 11375. E-mail: adinabitton@gmail.com

A.N. SIMONE, A. BEDARD, D. MARKS \& J.M. HALPERIN. Preschool Inattentive but not Hyperactive-Impulsive Symptoms Predict Working Memory at 8-years-old.

Objective: To determine whether inattentive and/or hyperactive symptoms in preschoolers predicts verbal working memory (WM) in schoolaged children. We hypothesized that inattention, as rated by parents and teachers, would predict later verbal WM.

Participants and Methods: Preschool children's [N=156; mean age $(\mathrm{SD})=4.25(.48)$ years] inattentive and hyperactive symptom severity were assessed via parent and teacher ratings using the Attention-Deficit/Hyperactivity Disorder-Rating Scale-Fourth Edition (ADHDRS-IV). On average, $4.30(.47)$ years later, at a mean (SD) age of 8.56 (.31), children completed the verbal WM tasks (i.e., Digit Span and Letter-Number Sequencing) from the Wechsler Intelligence Scale for Children- Fourth Edition, Integrated and the Working Memory Index was computed. Data were analyzed via linear regression. Children's socioeconomic status (SES) and time between evaluations (Time 2 Baseline) were first entered into the model; ADHD-RS-IV scores were entered in the second block.

Results: Together, SES and time between evaluations significantly predicted verbal WM accounting for $8.7 \%$ of the variance $(p=.001)$. Parent and teacher ratings of ADHD symptom severity accounted for an additional $18.6 \%$ of the variance $(p<.001)$. Notably, preschool inattentive symptom severity, as rated both by parents $(p=.01)$ and teachers $(p=.001)$, significantly predicted later verbal WM. Hyperactive-impulsive symptoms, as rated by parents $(p=.237)$ and teachers $(\mathrm{p}=.123)$ at baseline, did not predict later verbal WM.

Conclusions: Although hyperactivity-impulsivity is more common in preschoolers than inattention, it appears that only inattentive symptoms portend later neuropsychological difficulties in the form of WM impairments.

Institutional Support- Queens College CUNY and The Graduate Center CUNY

Correspondence: Ashley N. Simone, Psychology, The Graduate CenterCUNY, 335 Somerston Road, Yorktown Heights, NY 10598. E-mail: ashleyn.simone@gmail.com

M. SHIN, S. OH, H. JEON, M. KIM, T. HWANG \& M. HWANGBO. Effect of Mobile-based Neurofeedback with Cognitive Training Program for Children.

Objective: Neurofeedback (NF) could help to improve attentional and self-management capabilities in children with attention deficit/ hyperactivity disorder (ADHD). In the present study, we examined the training effect of our newly developed attention training program on school-aged children. Our program combined mobile-based NF with cognitive training (CT).

Participants and Methods: Forty children (10-12 years) with inattention, impulsivity and hyperactivity problems, defined according to the Conners Parent Rating Scale (CPRS), were randomly allocated to $\mathrm{NF}$ training group over 16 sessions $(\mathrm{n}=20)$ or a waiting-list control group $(n=20)$. Each participant of the training group was individually 
administered two NF training sessions (consisting of single block theta/ beta training) and three NF + CT (including the following cognitive subcomponents: auditory attention, visual attention, visual memory). Pre-training, post-training and 1-month follow-up assessment encompassed several neuropsychological tests (e.g., general intelligence, continuous attention, attention shifting and response inhibition test) and parent-version behavior rating scales (e.g., CPRS).

Results: Scores for several neuropsychological tests and parent's behavior rating scales showed significant improvement in the training $(\mathrm{NF}+$ CT) group, but not in the control group. A larger increase in positive reward of the NF sessions was associated with a larger improvement in neuropsychological functioning after the training.

Conclusions: This study suggests that the mobile-based NF-CT training program may improve ADHD symptoms in elementary school children. Correspondence: , . E-mail:

M. VASSERMAN, M. MAIMAN, B. KAHN, G. WITKIN \& W.S. MACALLISTER. Comparing Three Methods of Stroop Interference Calculations in Clinically Referred Children.

Objective: Poor response inhibition is considered the hallmark of ADHD. However, measures of response inhibition, such as the Stroop, produce inconsistent results. One reason may be the multiple methods for calculating interference. Differing calculations are based on differing theoretical rationales and include the "difference method," the "Golden method," and the "Chafetz method." The current study aims to compare these three methods of calculating Stroop interference and determine which is most sensitive in childhood ADHD.

Participants and Methods: 57 clinically referred children ages 7-16 were included; $58 \%$ were diagnosed with ADHD. Groups did not differ in age, IQ, or reading scores. Stroop interference scores were calculated in three different ways, the Difference method, Golden, and Chafetz methods were utilized.

Results: ADHD and non-ADHD groups did not differ on any interference scores, though the difference method approached significance $(t=-1.95 ; p=.058)$. Of the three methods of calculating interference, the Chafetz method yielded the most impaired scores. Of the three interference scores, the Chafetz method showed the most robust relations with neuropsychological measures and parent ratings (e.g., Trails A $\mathrm{r}=.43 ; \mathrm{p}=.001$, Trails B r=.41; $\mathrm{p}=.001$, Auditory Consonant Trigrams $r=.41 ; p=.02$, BRIEF working memory $r=-.28 ; p=.04)$. This said, logistic regression analyses showed that no interference scores were particularly useful in classifying children into ADHD versus non-ADHD groups.

Conclusions: Of the competing methods, the Chafetz method was most sensitive to cognitive impairment. Further, this method shows stronger relations to other measures of attention. However, while more sensitive, the Chafetz method was not specific to ADHD.

Correspondence: William S. MacAllister, PhD, Neurology, NYU, 223 East 34th St, NYU Epilepsy, New York, NY 10016. E-mail: william. macallister@nyumc.org.

M. KIM, S. LEE \& K. JANG. Deficits of decision-making in college students with ADHD traits.

Objective: Objectives: Patients with ADHD (attention deficit/hyperactivity disorder) show deficits in real-life decision making, but the nature of these deficits is not fully understood. This study investigated deficits of decision-making in individuals with ADHD traits with Iowa Gambling Task (IGT) and Prospect Valence Learning (PVL) Model.

Participants and Methods: Participants \& Methods: Based on the scores of adult ADHD self-report scale and Conner's adult ADHD rating scale, ADHD-trait $(\mathrm{n}=18)$ and control $(\mathrm{n}=20)$ groups were selected.

Measurement of decision-making: IGT was administered to evaluate the decision-making. Participants were required to select one card from four decks in each trial. Each selection resulted in monetary gain or loss. The PVL model estimates four parameters; utility shape (subjective evaluation of selection outcome based on gains \& losses), loss aversion (sensitivity to losses), recency (selection based on feedback of previous trials), and consistency (consistent selection of cards which individual prefers) parameters. PVL parameters were estimated with Markov Chain Monte Carlo (MCMC) sampling scheme in OpenBugs and BRUGS, its interface to $R$.

Results: Results: ADHD-trait group received significantly lower total net score, $\mathrm{t}(36)=-2.84, \mathrm{p}<.01$, and block net score on the 4th block, $\mathrm{t}(36)=-2.94, \mathrm{p}<.01$, and selected advantageous deck significantly less, $\mathrm{t}(36)=-2.63, \mathrm{p}<.05$, and disadvantageous deck more, $\mathrm{t}(36)=2.44$, $\mathrm{p}<.05$, than control group. The ADHD-trait group exhibited lower values on the utility-shape, loss aversion, recency, and consistency parameters of the PVL model than control group

Conclusions: Conclusions: These results indicate that individuals with ADHD trait experience deficits in decision-making possibly due to the failure of contingency and reversal learning with regard to response options.

Correspondence: Myung-Sun Kim, Ph.D, Sungshin Women's University, Sungbuk Dongsun 3ga, Department of Psychology, Seoul 136-742, Korea (the Republic of).E-mail:kimms@sungshin.ac.kr

K.M. KINGERY, M. GASPAR \& J.N. EPSTEIN. The Role of Verbal and Spatial Working Memory in Predicting Academic Performance in Children with ADHD.

Objective: Though multiple studies have reported a relationship between working memory (WM) and academic achievement, how the different components of WM relate to academic achievement in children with ADHD is not fully understood. The present study aims to examine the association between verbal and spatial WM and multiple academic measures using achievement scores, curriculum-based measurement (CBM), and parent- and teacher-ratings of academic success.

Participants and Methods: 110 children with ADHD-I (n=41) or ADHD-C ( $\mathrm{n}=69)$ completed a spatial WM (block recall) and verbal WM (Digit Span) task. Objective reading measures included standard scores of WIAT-II Word Reading and a CBM reading task, while math measures included standard scores on the WIAT-II Numerical Operations and a CBM math task. The standardized CBM writing task score served as an objective measure of writing. Parent and teacher ratings of children's impairment in reading, math, and writing were also collected. Results: Three linear mixed multivariate models using all academic measures for each domain were conducted to evaluate the relationship between spatial and verbal WM and various academic domains. Spatial but not verbal WM was predictive of performance on math and writing. For the reading model, both spatial and verbal WM were predictive of reading scores. These relationships were significant even after controlling for medication status, age, and parent-rated ADHD symptom severity.

Conclusions: Results suggest specificity in the types of WM that are most predictive of achievement in specific academic domains within a sample of children with ADHD. Verbal and spatial WM were significantly predictive of reading achievement while only spatial WM was significantly related to math and writing achievement. Clinically, results highlight the importance of evaluating multiple components of WM separately in patients with ADHD to better understand how individual's WM deficits may be contributing to ADHD-related academic deficits in reading, math and writing.

Correspondence: Kathleen M. Kingery, M.A., Psychology, University of Cincinnati, 6611 Greenfield Drive, Cincinnati, OH 45224. E-mail: kateo2004@yahoo.com

P.K. ISQUITH, L. KENEALY, R. ROTH \& G.A. GIOIA. Diagnostic Accuracy of a Revised Behavior Rating Inventory of Executive Function (BRIEF) for Children with ADHD.

Objective: A large body of research has documented good diagnostic utility of the BRIEF for use in children with ADHD (McAuley et al., 2010; Toplak et al., 2009). Parent ratings of children with ADHD were examined with a more concise revised BRIEF. 
Participants and Methods: Parents of children aged 5-13 years previously diagnosed with inattentive $(n=35)$ or combined $(n=98)$ ADHD (ADHD-I, ADHD-C) and age/gender matched controls ( $\mathrm{n}=133$ ) completed the revised 63-item BRIEF. Diagnoses were confirmed with broad-band and ADHD-specific rating scales.

Results: Profile analysis with repeated measures MANOVA revealed very large scale elevations that differed between diagnostic groups $(\mathrm{p}<.001$, eta2 $=.58)$. ADHD-I, $-\mathrm{C}$ and controls were all distinct on the Inhibit, Self-Monitor, Shift and Emotional Control scales while controls differed from both clinical groups on the Initiate, Working Memory, Plan/Organize, Task Monitor and Materials scales $(p<.05)$. Discriminant function and ROC analyses showed that a combination of Inhibit, Working Memory, Task Monitor and Emotional Control scales accurately identified $88 \%$ of children with ADHD and $88 \%$ of controls (Odds Ratio=50, Positive Likelihood Ratio=6.9). With sensitivity at .90, there was a $14 \%$ false positive rate. The Inhibit, Shift and Initiate scales separated the ADHD-I and ADHD-C groups with both sensitivity and specificity of .80 (Odds Ratio=33.5, Positive Likelihood Ratio=2.0).

Conclusions: A more concise revised BRIEF demonstrated good sensitivity and specificity, and distinct clinical profiles, for identifying the presence of attention disorders. Parent ratings of children's everyday executive function remain a powerful clinical tool for detecting children with ADHD, clarifying subtypes, and ruling out ADHD in typically developing children.

Correspondence: Peter K. Isquith, Ph.D., Psychiatry, Geisel School of Medicine at Dartmouth, 221 US Route 5S, Unit 15, Norwich, VT 05055. E-mail: peter.isquith@gmail.com

\section{G. SITARENIOS, G. JOWKAR \& G. GUNN. A Revised Continuous Performance Test (CPT) for the Assessment of Attention Processes in 4-7 year-old Children.}

Objective: It is a challenge in psychological measurement to formulate effective CPT paradigms for young children. The Kiddie Continuous Performance Test 2 (K-CPT 2 ) is a revised program created to improve the assessment of attention in children by parsing out four distinct processes: inattentiveness, impulsivity, sustained attention, and vigilance. The K-CPT 2 is described and data is used to test if the program is reliable, able to differentiate the four attention processes, and able to meaningfully distinguish between clinical and non-clinical children. Participants and Methods: Subjects completed the 7.5 minute K-CPT 2 program consisting of 200 trials depicting 1 of 10 pictures, and were instructed to respond to all pictures except the soccer ball. Normative data was collected on 320 children aged 4 to 7 . This sample was representative of the U.S. population (within $1.2 \%$ of census) in terms of race/ethnicity, region, and parental education level. Test reliability was evaluated. Group differences were investigated using 100 ADHD children, and a matched sample of 100 children from the normative data. Results: The median split-half reliabilities were $r=.87$ (normative sample), and $\mathrm{r}=.86$ (clinical sample). In addition, most K-CPT 2 scores were found to be sensitive to group differences $(\mathrm{d}=.84$ to .04$)$ and, overall, greater number and severity of problems across the four attention processes indicated greater likelihood of ADHD.

Conclusions: The results support the utility of the K-CPT 2 paradigm as a component of the assessment of attention problems for young children. However, caution is advised against using this paradigm as a standalone test.

Correspondence: Gill Sitarenios, Ph.D., Multi Health Systems, 3700 Victoria Park Avenue, Toronto, ON M2H3M6, Canada. E-mail: gill. sitarenios@mhs.com

\section{Cancer}

M.D. MCCURDY, E. TURNER, L.P. BARAKAT, W. HOBBIE, J. DEATRICK, I. PALTIN \& M.C. HOCKING. Treatment Intensity Predicts Self-Awareness of Executive Functioning in Young-Adult Survivors of Childhood Brain Tumor.

Objective: Treatments for childhood brain tumors (BT) confer a substantial risk to neurological development as survivors progress into adulthood, placing this population at risk for deficits in multiple neuropsychological domains including executive functioning (EF). However, there is evidence to suggest that individuals who experience neurological insult (i.e., traumatic brain injury) may lack insight into their neurocognitive limitations (Impaired Self-Awareness; ISA). No studies to date have examined ISA in survivors of childhood BT. The present study addresses the association between treatment intensity and ISA of EF skills in young-adult survivors of childhood BT.

Participants and Methods: Thirty-four young adult BT survivors $(52.9 \%$ female), an average of 16.1 years post-diagnosis ( $\mathrm{SD}=5.9)$, aged 18 to $30(\mathrm{M}=23.5, \mathrm{SD}=3.4)$ were administered self-report (BRIEF-SR) and performance-based measures (WAIS-IV, D-KEFS) of executive functioning. Regression analyses examined the associations between treatment intensity (Pediatric Neuro-Oncology Rating of Treatment Intensity; PNORTI) and the discrepancy between self- and performance-based levels of executive functioning.

Results: Correlations between self-report and performance-based executive skills were all non-significant (ps > .05), suggesting ISA of EF skills. PNORTI scores predicted discrepancies between BRIEF-SR and WAIS-IV Letter-Number Sequencing (R2=.36, $\mathrm{p}<.01)$ and Working Memory Index $(\mathrm{R} 2=.36, \mathrm{p}<.01)$ scores, as well as D-KEFS Number-Letter Switching $(\mathrm{R} 2=.21, \mathrm{p}<.01)$, and Tower Achievement $(\mathrm{R} 2=.26$, $\mathrm{p}<.01)$. Notably, greater treatment intensity was associated with survivor overestimations of executive skills.

Conclusions: Survivors of childhood BT are at risk for diminished self-awareness of executive skills, which may be associated with treatment intensity. Neuropsychologists should be mindful to assess executive functioning through multiple sources in this population.

Correspondence: Mark D. McCurdy, B.A., Neuropsychology, Kennedy Krieger Institute, 1750 E. Fairmount Ave., 3rd Floor, Baltimore, MD 21231. E-mail: mccurdy@kennedykrieger.org.

S. WITHROW, M. GLODÉ, A. BREGA, S. MIN, P. WALKER, L. KARSH \& J. GRIGSBY. The Association of Cognitive Changes with Quality of Life in Men with Prostate Cancer Undergoing Androgen Deprivation Therapy.

Objective: Recent research suggests an association between reductions in testosterone $(\mathrm{T})$ and declines in specific areas of cognition among prostate cancer patients undergoing Androgen Deprivation Therapy (ADT). However, different domains of cognition have been inconsistently implicated. ADT as a hormone treatment has also been associated with reductions in various aspects of Quality of Life (QOL). We tested whether QOL mediates the relationship between decreased T and $\operatorname{cog}$ nitive deficits.

Participants and Methods: 74 prostate cancer patients aged 50 to 81 undergoing continuous or intermittent ADT were recruited. We assessed working memory, information processing, verbal learning and memory, verbal fluency, verbal reasoning, spatial perception, mood, and QOL at three time points. Biologically available T levels were calculated according to the Vermeulen equation. We conducted mediation analyses using Baron \& Kenny's model and tested the significance of the indirect effect following Preacher and Haye's (2004) bootstrapping guidelines. The influence of demographic variables was assessed using correlational analyses.

Results: Level of bio $\mathrm{T}$ was positively correlated with visual working memory $(p=.03)$ and verbal fluency performance $(p=.05)$. Bio $\mathrm{T}$ was also positively correlated with impacts on energy $(p=.04)$, physical functioning $(p=.01)$, pain $(p=.02)$, and general health $(p=.03)$. 
Both verbal fluency $(p<0.001)$ and visual working memory $(p=0.03)$ performance were positively correlated with energy. However, only energy mediated the relationship between $\mathrm{T}$ and verbal fluency $(\mathrm{p}<.05)$. Conclusions: The impact of QOL on the relationship between $\mathrm{T}$ and cognition may be one potential factor explaining inconsistent findings on how $\mathrm{T}$ relates to changes in cognitive performance. Nonlinear modeling procedures (e.g., CART) may more precisely show subpopulations of ADT patients that are likely to experience cognitive symptoms.

Correspondence: Susanne Withrow, University of Colorado Denver, 5901 S Versailles St, Aurora, CO 80015.E-mail: susanne.withrow@ ucdenver.edu

M. KRYZA-LACOMBE, J. ROOT, M. HENSLEY \& D.D. CORREA. Cognitive Functions in Patients with Ovarian Cancer treated with First-Line Chemotherapy.

Objective: Objective: Women diagnosed with ovarian cancer often undergo chemotherapy involving multiple agents. However, little is known about the incidence of cognitive adverse effects of chemotherapy in survivors of this rare disease. The goal of this study was to assess neuropsychological functions in patients with ovarian cancer following first-line chemotherapy.

Participants and Methods: Participants \& Methods: Eighteen patients with ovarian, peritoneal and fallopian tube cancer and eighteen healthy controls matched for age and education participated in the study. The patients were evaluated 1-4 months following completion of first-line taxane and platinum-based chemotherapy. All study participants completed a standardized neuropsychological test battery including measures of attention, executive functions and memory.

Results: Results: The results of two sample t-tests showed no statistically significant differences between patients and healthy controls on any of the cognitive tests. Although group mean test z-scores were within the average range, $28 \%$ of patients had at least one $\mathrm{z}$-score between 1 and 1.5 standard deviations below age-corrected normative mean values. Among healthy controls, $12 \%$ had a single $\mathrm{z}$-score 1 standard deviation below the norms. These low scores were mostly on tests of attention and executive functions.

Conclusions: Conclusions: The findings suggest that a subset of patients with ovarian cancer treated with chemotherapy experience mild decrements on some aspects of attention and executive functions. These results are congruent with prior studies suggesting that subgroups of women with breast and ovarian cancer have cognitive dysfunction. Additional prospective studies are needed to further characterize the contribution of disease and chemotherapy to cognitive outcome in this clinical population.

Correspondence: Denise D. Correa, PhD, Neurology, MSKCC, 1275 York Avenue, New York, NY 10021. E-mail: corread@mskcc.org.

S. NA, B. SUN \&. T.Z. KING. Dorsolateral Prefrontal Cortex Activation in Adult Survivors of Pediatric Brain Tumors Relative to Controls on a fMRI Vigilance Task Over Time.

Objective: Behavioral studies have documented impaired attention/vigilance in childhood brain tumor survivors; however, neural mechanisms have yet to be identified using fMRI. Based on previous literature with other neurological populations, we hypothesized that the dorsolateral prefrontal cortex (DLPFC,Brodmann's area 9/46) and anterior cingulate cortex (ACC,BA32) would emerge as significantly activated on a vigilance task, especially in survivors at the start versus end of the task. Participants and Methods: A 20-minute 5-run letter n-back task was administered to 20 survivors (age $\mathrm{M}=23.1 ; \mathrm{F}=55 \%$ ) and 20 age- and gender-matched healthy controls (HC) in a 3T scanner. An F-test was conducted on the $[1-0$-back] contrast to determine areas significantly different for a group by time (first 2 runs/start vs. last 2 runs/end) interaction.

Results: There were no significant differences in main effects or interactions on task performance. Given the differences in variability in task performance between groups, accuracy was included as a covariate in the F-test. The imaging analyses showed group by time interactions in the left prefrontal cortex (BA9,10), and on the right: prefrontal (BA9, 10,45,46), parietal (BA40), motor planning (BA6) and paracingulate gyrus (BA10). Survivors evidenced significant activations in BA46 at the start, but significantly less and similar level to $\mathrm{HC}$ at the end of the task.

Conclusions: Survivors evidenced increased activation of the DLPFC relative to controls at the beginning of the task (1-0back), possibly reflecting a need for increased vigilance and recruitment of DLPFC when initially learning a task and in order to perform at similar levels to healthy controls.

Correspondence: Sabrina Na, Georgia State University, 235 Lincoln Court Avenue, Atlanta, GA 30329. E-mail:s.diana.na@gmail.com

S. NA \& T.Z. KING. Cumulative Neurological Factors Predict Longterm Outcomes in Adult Survivors of Childhood Brain Tumors.

Objective: Prior research has demonstrated the reliability and validity of the Neurological Predictor Scale in relationship to childhood brain tumor survivor outcomes; however it has not been examined in adult long-term survivors. The current study examines the reliability and utility of the Neurological Predictor Scale (NPS) in predicting long-term intellectual and adaptive outcomes in adult survivors of childhood brain tumors, relative to individual predictors alone.

Participants and Methods: Sixty-eight adult survivors of childhood brain tumors on average 24 years old $(\mathrm{SD}=4)$, almost 16 years post diagnosis ( $\mathrm{SD}=6$ ), completed intellectual evaluation (WASI). Survivors' adaptive functioning skills were assessed via informant structured clinical interview (SIB-R). NPS scores were computed from data acquired from medical records.

Results: The NPS significantly predicts intellectual $\left(\mathrm{R}^{\wedge} 2=.21, \mathrm{p}<.05\right)$ and adaptive outcomes $\left(\mathrm{R}^{\wedge} 2=.30, \mathrm{p}<.05\right)$, over and above individual risk factors. Approximately $18 \%$ of long-term survivors were identified as impaired on intellectual outcomes, whereas $29 \%$ were identified as impaired on adaptive functioning in everyday life skills.

Conclusions: The NPS is a useful and easy measure to employ in clinical research that is interested in quantifying the neurological risk factors associated with long-term intellectual and adaptive functioning outcome in adult survivors of childhood brain tumors. This measure quantifies the cumulative and interactive effects of treatment and neurological sequelae experienced by both short and long-term survivors of childhood brain tumors and takes into account the complexity of neurological risk profiles individual survivors may have that contribute to their long-term outcomes.

Correspondence: Sabrina Na, Georgia State University, 235 Lincoln Court Avenue, Atlanta, GA 30329. E-mail: s.diana.na@gmail.com

K. VAN DYK, L.M. ERCOLI, L. PETERSEN, S.A. CASTELLON, B. KAHN-MILLS, P.A. CERNIN, L. EMBREE \& P.A. GANZ. Association of Self-Reported Cognitive Complaints and Neuropsychological Performance Among Younger and Older Breast Cancer Survivors.

Objective: Cognitive changes following cancer treatment are a growing concern for breast cancer survivors (BCS). Self-report measures of cognitive complaints capture changes consistent with objective cognitive testing by domain (Ganz et al., 2013). Cognitive complaints may vary across age, as found in non-clinical samples (Begum et al., 2014). We examined age-related differences in the relationship between complaints and cognitive performance in a sample of BCS.

Participants and Methods: Forty-eight women with a history of breast cancer were recruited as part of a cognitive intervention trial. Controlling for time since diagnosis, partial correlations were performed with baseline data between neuropsychological test scores of verbal and visual memory (learning and delayed recall), an executive function domain score, and self-reported cognitive complaints measured by Patient's Assessment of Own Functioning (PAOFI) Total, Memory, and Higher Level Cognition (HLC) scores. Correlations were performed 
cross the whole sample and within age groups: a Younger age group $(\leq 50, n=21)$ and an Older age group $(51+, n=27)$.

Results: Across the whole sample, there were significant associations between both the visual total recall and the visual delayed recall scores, and PAOFI Total, Memory, and HLC. Within age groups, PAOFI Memory was associated with visual delayed recall in the Younger group and visual learning in the Older group. PAOFI Higher Level Cognition was associated with visual learning in the Older group. There were no associations with the PAOFI and verbal memory or executive function. Conclusions: Findings suggest that cognitive performance associated with complaints may vary across age. Visual memory functions may be more sensitive to subjective awareness of cognitive difficulties in BCS. Correspondence: Kathleen Van Dyk, PhD, Psychiatry, UCLA Semel Institute for Neuroscience and Human Behavior, 760 Westwood Plaza, 37-360B,Westwood, CA 90095. E-mail: kathleen.van.dyk@gmail.com

\section{A. APPLE, A. RYALS, J. VOSS \& L. WANG. Hippocampal shape deformity associated with cognitive variability in breast cancer patients.}

Objective: $\mathrm{Up}_{\mathrm{p}}$ to $75 \%$ of breast cancer patients receiving chemotherapy exhibit cognitive impairment. Neuroimaging studies of this population suggest volume loss in several brain regions including parietal and occipital lobes, yet few studies have performed detailed analysis of the hippocampus, which shows volume loss in animal models of chemotherapy effects. We therefore examined hippocampal shape between individuals with breast cancer who received chemotherapy coupled with estrogen therapy and healthy controls. We also tested relationships between these shape abnormalities and cognitive ability.

Participants and Methods: 3T MPRAGE MRI images were acquired from 16 pre-menopausal breast cancer patients and 18 controls. Breast cancer patients had undergone chemotherapy within the eighteen months prior to the study, and they were all presently receiving estrogen-blockade therapy. Hippocampal surfaces were generated using high-dimensional deformation mapping. Average deformation from the population average as a reference across all surface vertices was calculated and compared between groups. The NIH Toolbox Cognition Battery was used to assess neuropsychological performance.

Results: Groups differed significantly in hippocampal shape $(\mathrm{p}=$ $0.034)$, observed predominantly as bilateral inward deformity of the mid-hippocampal body in patients relative to controls. Increased severity in hippocampal shape deformity was associated with worse performance on the Flanker Task for patients but not for controls $(\mathrm{r}=0.53$, $p=0.044$, uncorrected).

Conclusions: The severity of changes in hippocampal shape for patients was related to difficulty on a standardized task measuring attention and inhibition. Potential mechanisms for these structural changes and their relationship to other cognitive changes will be discussed.

Correspondence: Alexandra Apple, Department of Psychiatry and Behavioral Sciences, Northwestern University Feinberg School of Medicine, 710 N. Lake Shore Drive, Abbott Hall, suite \#1306, Chicago, IL 94301. E-mail: alexandraapple2013@u.northwestern.edu

A.R. GIOIA, S.A. HOSTETTER, N. DWARKA, K.S. WALSH \& K.K. HARDY. Relationships between Parent-, Teacher-, and SelfReported Behavior Ratings and Neuropsychological Outcomes in Childhood Cancer Survivors.

Objective: Survivors of pediatric cancer are at risk for psychosocial and neuropsychological late effects. Clinical and research evaluations typically include questionnaire data from multiple informants as well as neuropsychological testing; however, it is unclear whether clinically meaningful relationships exist between reports of behavior across raters and neuropsychological performance in this population.

Participants and Methods: Data from neuropsychological evaluations of 163 childhood cancer survivors (54\% brain tumor, 36\% ALL, $10 \%$ other cancer) aged $3-19$ (mean age $=10.3 \pm 4.15,58 \%$ male) was reviewed. Relationships between responses on the CBCL ( $\mathrm{n}=163)$, TRF $(\mathrm{n}=134)$, YSR $(\mathrm{n}=75)$ and IQ testing were analyzed.

Results: Inter-rater correlations of survivor's behavior were the highest between teachers rating the same student $(0.46<\mathrm{r}<0.83)$, followed by self-parent ratings $(0.4<\mathrm{r}<0.66)$ and parent-teacher ratings $(0.27<\mathrm{r}<$ $0.50)$. With the exception of attention problems $(\mathrm{r}=0.28)$, responses on the TRF and YSR were not significantly correlated. Teacher reports of somatization $(-0.36<\mathrm{r}<-0.26)$, social problems $(-.40<\mathrm{r}<-0.28)$, and attention problems $(-0.30<\mathrm{r}<-0.19)$ were highly correlated with survivor performance across all IQ indices. Parent ratings were less correlated with IQ performance, and there were virtually no significant correlations between self-report and IQ.

Conclusions: Teachers' views of survivors' behavior were the most related to each other and to children's neuropsychological performance. On the other hand, survivors themselves had the most divergent views of their behavior, as well as the lowest association with neuropsychological performance, which may indicate a lack of self-awareness of their own difficulties. This study highlights the importance of collecting behavior ratings of this population from multiple informants who interact with the child across different settings.

Correspondence: Sarah A. Hostetter, BA, Neurology, Children's National Medical Center, 111 Michigan Ave NW, Washington, DC 20010. E-mail: shostett@childrensnational.org

M. STOUTEN-KEMPERMAN, M. DE RUITER, M. CAAN, W. BOOGERD, L. RENEMAN \& S. SCHAGEN. Late Effects of Cancer Treatment on Cognitive Function and Brain White and Gray Matter in Testicular Cancer Survivors.

Objective: Chemotherapy is associated with adverse effects on cognition. Only few studies have investigated cognition in testicular cancer (TC) patients and studies on very late effects of CT on cognition are absent. Further, brain changes in relation to treatment have not been investigated in TC. The objective of the present study is to examine cognitive performance and brain structure $>10$ years following CT for TC, compared to surgery (S) only.

Participants and Methods: Twenty-eight CT (43.1 7.5 yrs) and 23 S-only (48.2 $\pm 9.5 \mathrm{yrs}) \mathrm{TC}$ survivors were assessed using neurocognitive tests and 3T MRI (Diffusion Kurtosis Imaging (DKI) and T1). An overall cognitive performance score (Mahanalobis distance) was calculated to indicate the grade of cognitive performance. Kurtosis parameters and gray matter (GM) and white matter (GM) volume were calculated.

Results: Significant overall lower cognitive performance was found after CT $(5.35 \pm 1.7)$ vs. S-only $(4.4 \pm 0.9)$. Further, DKI revealed a significantly higher radial kurtosis (RK) after CT in several anterior and posterior brain areas, which was not correlated with cognition. No other differences in kurtosis measures and overall GM/WM volume were found.

Conclusions: It seems that men receiving CT for TC are at risk for lower cognitive performance even $>10$ years post-treatment. Although differences in WM integrity were found, this was not related to overall performance. The underlying tissue microstructure measured by DKI is incompletely understood. More research is warranted to give more insight into the histological underpinnings of this finding and to investigate other risk factors in relation to lower cognitive performance in this patient group.

Correspondence: Myrle Stouten-Kemperman, MSc, Radiology, AMC, Meibergdreef 9, Amsterdam 1105 AZ, Netherlands. E-mail: m.kemperman@nki.nl

A. LUCCHETTI, N. AROLA, M. GOLDWIN, T. DROSSOS \& K. AFZAL. Comparisons of Parent and Child Reports of Pediatric Pain, Coping, and Psychosocial Functioning in Newly Diagnosed Pediatric Cancer Patients.

Objective: Detrimental effects of pediatric cancer include poor health-related quality of life, psychological distress, and chronic pain (Sawyer et al., 2004), which are related to deficits in neurocognitive 
functioning (Nicholson, 2000). Parents of chronically ill children are good reporters of quality of life and overall functioning but are less attuned to socio-emotional functioning (Eiser \& Morse, 2001), highlighting the importance of multiple reporters. This study examines parent-child agreement about pain, coping, and psychosocial functioning amongst newly diagnosed pediatric cancer patients.

Participants and Methods: Participants include 21 newly diagnosed cancer patients ( $48 \%$ female, $6-23$ years old) and their parents. Measures include parent and child versions of the Pediatric Quality of Life Inventory (Generic Core, Cancer Module [Varni et al., 2001; 2002]), Pediatric Pain Coping Inventory (Varni, 1998) and Pediatric Pain Questionnaire (Varni et al., 1996).

Results: Correlational analyses were run to examine parent-child agreement. Results revealed significant positive relationships for present pain $(\mathrm{r}=.69, \mathrm{p}=.001)$, pain over past week $(\mathrm{r}=.51, \mathrm{p}=.02)$, health-related quality of life $(r=.59, p=.005)$, and physical $(r=.54, p=.011)$, emotional $(\mathrm{r}=.57, \mathrm{p}=.007)$ and psychosocial functioning $(\mathrm{r}=.61$, $p=.004)$. No significant relationships emerged for coping strategies. Conclusions: Children and their parents had similar reports of psychosocial and physical functioning. Reports of coping strategies were unrelated, suggesting parents may have less insight into this aspect of illness experience. Given the importance of coping on overall functioning, further examination of this discrepancy is imperative.

Correspondence: Amanda Lucchetti, MA, Psychiatry and Behavioral Sciences, Children's Hospital Colorado, 5100 Leetsdale Drive, Unit 401, Denver, CO 80246.E-mail: amandalucchetti@gmail.com

J. GRIECO, C. EVANS, T.I. YOCK, K. KUHLTHAU, S. MACDONALD, N. TARBELL \& M. PULSIFER. A Matched Comparison Study Assessing Longitudinal Outcome of Children with Posterior Fossa Syndrome versus those Without Complication Following Posterior Fossa Tumor Resection and Proton Radiation Therapy. Objective: Posterior Fossa Syndrome (PFS), characterized by mutism, ataxia/hypotonia, and emotional lability, can result in long-term cognitive deficits following resection of posterior fossa tumors. This study compared longitudinal cognitive outcomes of children who underwent proton radiation therapy (PRT) following resection, comparing children with PFS to those without complication.

Participants and Methods: 36 patients, ages 1-15 years (Mean=7.26; $\mathrm{SD}=4.42$ ) were evaluated at baseline (before/during radiation) and at least 1-year post-radiation (Mean=3.26 years;SD=2.24). 18 PFS patients were matched to 18 controls by tumor location, age, gender, and handedness; there were no significant group differences at baseline for demographic/clinical variables. Measures assessed: intelligence, language, visuomotor integration, fine-motor speed. Parent ratings assessed: mood, behavior, executive functioning.

Results: Subjects- $52 \%$ male; $86 \%$ medulloblastoma/14\% ependymoma; whole $[86 \%] /$ partial $[14 \%]$ brain radiation; 86\% chemotherapy. No significant differences between mean test scores at baseline across measures [ $>>0.13]$, except slower fine motor speed for PFS group- $[\mathrm{p}<0.05]$. Mean change scores revealed no significant differences on any measures at follow-up $[p>0.10]$. All mean scores were within the normal range-(1 SD), except slower fine motor speed for both groups at follow-up. Special education rate was equal for both groups at follow-up.

Conclusions: 3 years after PRT, children with/without PFS did not differ significantly. Children performed at an age-expected range on cognitive and emotional/behavioral measures; fine motor speed was below age-expectation for all children, independent of PFS history. Long-term outcomes following PRT were favorable.

Correspondence: Julie Grieco, PsyD, Harvard Medical School/ Massachusetts General Hospital, 2970 N. Sheridan Rd., Apt. 827, Chicago, IL 60657.E-mail: jgrieco@my.adler.edu
J. GRIECO, T.I. YOCK, C. EVANS, K. KUHLTHAU, S. MACDONALD, N. TARBELL \& M. PULSIFER. Intellectual and Executive Functioning in Pediatric Brain and CNS Tumor Patients After Proton Radiation Therapy.

Objective: Radiation is integral in treatment of brain and central nervous system tumors. However, photon radiation is associated with negative long-term cognitive sequelae in intelligence and executive skills. Because proton radiation irradiates less normal brain than photon techniques, neurocognitive outcomes may be better. This study examined changes in cognitive functioning in pediatric brain tumor patients treated with proton radiotherapy (PRT).

Participants and Methods: 35 patients, ages 7-20 (Medi$\mathrm{an}=12.75 ; \mathrm{SD}=3.52$ ) were evaluated at initiation of radiation and at least 1.5 years after radiation (Median=3.0 years; $\mathrm{SD}=1.8$ ). Measures administered included a Wechsler intelligence scale, Continuous Performance Test-II, Trail Making, Wisconsin Card Sort, Verbal Fluency, Behavior Rating Inventory of Executive Functioning, and Behavior Assessment Scale for Children-2.

Results: Subjects were 37\% medulloblastoma, 14\% craniopharyngioma, $11 \%$ ependymoma, $37 \%$ other; $42 \%$ received whole brain radiation; $60 \%$ chemotherapy; $50 \%$ were infratentorial. IQ was stable (Baseline Mean=106.68;SD=12.23/Follow-Up Mean=104.14;S$\mathrm{D}=11.94[\mathrm{~ns}])$. Direct assessment of executive functioning remained largely unchanged and in the average range on most measures, including sustained attention, working memory, inhibition, and problem-solving (ns). Decreases were in processing speed $(p<0.01)$ and speeded cognitive flexibility $(p<0.05)$, but remained accurate. Parent ratings revealed no change in attention or executive functioning, but had increased working memory difficulties at follow-up $(\mathrm{p}<0.05)$.

Conclusions: At 3 years after PRT, IQ was unchanged and executive functioning remained intact and essentially stable. Declines were on timed motor-based executive functions that require the ability to efficiently process novel material and think flexibly during an ongoing activity. Early neurocognitive outcomes after PRT are encouraging. Correspondence: Julie Grieco, PsyD, Harvard Medical School/ Massachusetts General Hospital, 2970 N. Sheridan Rd., Apt. 827, Chicago,IL 60657.E-mail:jgrieco@my:adler.edu

W. LEWIS, F. ZHANG, R. OJHA, J. LANCTOT, T.M. BRINKMAN, W. CHEMAITILLY, C. KIMBERG, D. SRIVASTAVA, L. ROBISON, M.M. HUDSON \& K.R. KRULL. Association of Caloric Intake with Executive Function and Attention in Long-Term Survivors of Childhood Cancer.

Objective: Long-term survivors of childhood cancer are at risk for neurocognitive late effects attributable to chemotherapy and cranial radiation. Poor dietary habits have been independently associated with neurocognitive impairment in non-cancer populations. We examined the association between caloric intake and neurocognitive outcomes in adult survivors of childhood cancer.

Participants and Methods: We used data from 1,708 adult survivors enrolled in the St. Jude Lifetime cohort study who completed neuropsychological testing and a self-administered food frequency questionnaire assessing habitual dietary intake over the previous year. Multivariable logistic analysis, adjusted for sex, race, age at diagnosis, time since diagnosis, and stratified on treatment exposures was used to examine the association between total caloric intake (quartiles) and neurocognitive impairment. Prevalence odds ratios and $95 \%$ confidence intervals were calculated with impairment defined as $<1$ SD below national norms.

Results: Median caloric intake was 1869 calories for the sample population, the lowest quartile (reference group) ranged from 405-1378 calories, and the highest quartile ranged from 2591-8720 calories. Survivors in the highest quartile of caloric intake had $70 \%$ increased odds of impaired working memory (Q4 vs. Q1: OR=1.70, 95\% CI: 1.21-2.38), $50 \%$ increased odds of impaired verbal fluency (Q4 vs. Q1: OR=1.50, 95\% CI: 1.09-2.06), and 69\% increased odds of impaired sustained 
attention (Q4 vs. Q1: OR=1.69, 95\% CI: 1.20-2.37) compared with survivors in the lowest quartile.

Conclusions: Our results suggest that adult survivors of childhood cancer with high caloric intake have increased odds of neurocognitive impairment on measures of attention and executive function. Our findings warrant assessing whether the reduction of caloric intake among survivors with high caloric intake may be an approach for improving neurocognitive function.

Correspondence: William Lewis, Epidemiology and Cancer Control, St. Jude Children's Research Hospital, 262 Danny Thomas Pl, MS 735, Memphis,TN 38105.E-mail: will.lewis@stjude.org

\section{HEFLIN, M. GATZ \& W. MACK. Longitudinal Neuropsychological Functioning in Cancer Patients Using a Pre-Cancer Baseline Neuropsychological Assessment.}

Objective: Longitudinal studies comparing cancer patients' cognitive functioning before and after treatment have found cognitive deficits at pre-treatment baseline assessments, thereby challenging the validity of baseline assessments that may be influenced by cancer-related stress or biological processes. The present study compared neuropsychological functioning in cancer patients to cancer-free individuals using baseline assessments obtained before cancer diagnosis.

Participants and Methods: Participants included 20 individuals diagnosed with cancer during their participation in an Alzheimer Disease Research Center (ADRC) longitudinal cognitive functioning study, and a matched group of 20 ADRC participants without a cancer history. Cancer diagnosis was confirmed by the California Cancer Surveillance Program. None had dementia prior to cancer diagnosis. The most common cancers were colorectal (25\%) and prostate (25\%). Mean baseline age was 77 years; $70 \%$ were male. Participants completed a cognitive assessment before cancer diagnosis and at least 1 after, with measures of depression, verbal fluency, confrontation naming, verbal memory, constructional skills, and attention/working memory. Cancer survivors' trajectories of neuropsychological functioning were compared to cancer-free individuals using linear mixed models and ANOVAs.

Results: Cancer patients showed poorer semantic fluency $(\mathrm{p}<0.05)$ and more depression symptoms $(\mathrm{p}<0.001)$ overall than individuals without a cancer history (group effects). Cancer patients showed better verbal learning at baseline $(p<0.01)$, but a steeper rate of decline $(p<0.05)$, than the comparison group.

Conclusions: Results suggest that some cognitive deficits, and higher depression, preceded cancer diagnosis, suggesting diagnosis-related stress alone may not explain pre-treatment cognitive problems. Faster decline in verbal learning suggests cancer treatments may negatively affect verbal learning.

Correspondence: Lara Heflin, PhD, Social \& Behavioral Sciences, New Mexico Highlands University, 28 Encantado Rd, Santa Fe, NM 87508. E-mail:Iheflin@nmhu.edu

\section{MARIANI \& M. COLLINS. Executive functioning and motor deficits in WHO grade 4 primary brain tumours.}

Objective: Primary brain tumours are associated with a range of neuropsychological deficits and reduced survival. The aim of this study was to examine the impact of WHO tumour grade on neuropsychological test results, the classification value of test results, and their predictive utility for survival time.

Participants and Methods: Thirty-five outpatients being treated for WHO Grades 2, 3 and 4 primary brain tumours underwent a full neuropsychological battery as part of their routine workup. The data from groups consisting of Grades $2 \& 3$ tumours overlapped significantly and were subsequently pooled. Test results from individuals with Grade 2/3 tumours were compared to those from individuals with Grade 4 brain tumours. The variables used in analyses included Digit Span, Letter-Number Sequencing and Digit-Symbol Coding, Hand Dynamometer, Grooved Pegboard, Trails Making Test A \& B, Lexical Fluency,
Semantic Fluency, and Wisconsin Card Sorting Test. Date of death was determined from medical charts and obituaries.

Results: Individuals with Grade 4 tumours scored significantly lower than those with Grade 2/3 tumours on measures of motor and executive functioning. Binary logistic regression with adjusted cut-offs based on ROC analyses determined a combination of these variables found to correctly classify $79.2 \%$ of the cases. Preliminary Kaplan-Meier survival curves did not significantly predict survival, but the trends observed were consistent with the extant literature.

Conclusions: Individuals with Grade 4 brain tumours perform worse than those with Grade 2/3 tumours on tasks involving motor function, divided attention, and concept formation. Further research is required to determine the predictive value of neuropsychological performance on survival.

Correspondence: Mark Collins, Master of Psychology, Supportive Care, Health Sciences North, Health Sciences North, 41 Ramsey Lake Road, Sudbury, ON P3E5J1, Canada.E-mail: mcollins@hsnsudbury.ca

C. KIMBERG, H.M. CONKLIN, T.M. BRINKMAN, W. LIU, Y. MZAYEK, D. SRIVASTAVA, C. PUI, M.M. HUDSON \& K.R. KRULL. End-of-Therapy Attention Predicts Long-Term Executive Function Outcomes in Survivors of Childhood Acute Lymphoblastic Leukemia (ALL).

Objective: To determine if attention problems at the end-of-therapy are associated with executive dysfunction during long-term survivorship.

Participants and Methods: Long-term survivors of childhood ALL $(\mathrm{N}=104$; mean[SD] current age $=14.3[4.8]$ years; time since diagnosis $=7.7[1.7]$ years) treated on a chemotherapy-only protocol completed assessment of attention at the end-of-therapy (Conners' Continuous Performance Test [CPT] ) and executive function during long-term follow-up (DKEFS: Color-Word Interference, Trail Making Test, Verbal Fluency). Children who relapsed, were treated with cranial radiation, had an unrelated neurodevelopmental condition, or experienced an unrelated brain injury were excluded from the analyses. Survivor performance was compared to normative data using one sample t-tests. General linear models, adjusted for time since diagnosis, were used to examine associations between end-of-therapy and long-term neurocognitive function.

Results: At the end-of-therapy survivors differed from the normative sample on indices of attention, including response speed $(p<0.001)$, reaction time variability $(p<0.001)$, and target detection $(p<0.01)$. At long-term follow-up, survivors demonstrated lower inhibitory control $(p<0.001)$, flexibility $(p<0.001)$ and fluency $(p<0.001)$. Poor target detection and response speed at the end-of-therapy were associated with lower inhibition $(\beta=0.18, p=0.04 ; \beta=0.10, p=0.04$, respectively $)$ and lower verbal fluency $(\beta=0.21, p=0.01 ; \beta=0.12, p=0.02$, respectively $)$ during survivorship. Increased reaction time variability at end-of-therapy was associated with lower cognitive flexibility $(\beta=0.18, p=0.02)$ at long-term follow-up.

Conclusions: Survivors with attention problems at the end-of-therapy are at risk for problems with cognitive flexibility, fluency and inhibition during long-term survivorship. Interventions to address inattention, introduced prior to the completion of therapy, may promote the development of higher order cognitive skills.

Correspondence: Cara Kimberg, St. Jude Children's Research Hospital, 262 Danny Thomas Place, MS 735, Memphis, TN 38105. E-mail: cara. kimberg@stjude.org

J.M. ASHFORD, H.M. CONKLIN, V.M. CRABTREE, L.M. JACOLA, M.S. WISE, B.N. MANDRELL \& T.E. MERCHANT. Identifying Risk Factors for Adaptive Functioning Deficits among Children Diagnosed with Craniopharyngioma.

Objective: Survivors of childhood craniopharyngioma treated with conformal radiation therapy have demonstrated difficulty in adaptive functioning. Given tumor proximity to critical neural and vascular structures, impairment can manifest prior to adjuvant therapy. The current 
study sought to characterize adaptive functioning in a homogeneous sample of brain tumor patients and identify risk factors predictive of difficulties before beginning proton beam radiation therapy.

Participants and Methods: Prior to proton therapy, parents of children and adolescents diagnosed with craniopharyngioma $(\mathrm{N}=58 ; 9.63 \pm 4.8$ years old) completed the Adaptive Behavior Assessment System-2nd Edition (ABAS-II) and Behavior Rating Inventory of Executive Function (BRIEF). A majority $(n=46)$ completed overnight polysomnography and multiple sleep latency testing.

Results: Despite mean scores in average range, patients showed significantly lower adaptive skills than normative expectations on the ABAS-II Practical Index (95.95 $\pm 16.34 ; p=.021)$ and a higher percentage than expected fell below the average range $\left(25.9 \% ; \chi^{2}=3.96, p=.047\right)$. BRIEF Working Memory, Metacognition and Global Executive Composite scores were significantly correlated with adaptive functioning $(p s=.001$ .032). Children showing clinical executive dysfunction on the BRIEF also scored below average in Global and Practical adaptive scales. No relationship was found between extent of surgery and adaptive skills; however, patients with diabetes insipidus were more likely to be below average in Practical scores $(p=.028)$. Patients classified as clinically sleepy (mean sleep onset latency $\leq 10$ minutes) demonstrated impaired Global, Conceptual and Social adaptive scores ( $p$ s=.028-.044).

Conclusions: Our results suggest craniopharyngioma patients experience difficulties in adaptive functioning prior to irradiation. Disease-related factors such as endocrinopathy and sleep/wake dysfunction are potential predictors of these deficits and suggest opportunities for intervention.

Correspondence: Jason M. Ashford, MS, Psychology, St Jude Children's Research Hospital, 262 Danny Thomas Place, MS740, Memphis, TN 38105. E-mail: jason.ashford@stjude.org

K.R. NOLL, X. WANG, Q. SHI \& J. WEFEL. Neurocognitive Functioning, Gender, and Inflammatory Markers in Patients with Colorectal Cancer Prior to Chemotherapy.

Objective: Examine relationships between serum markers and neurocognitive functioning (NCF) in patients with colorectal cancer (CRC) prior to adjuvant therapy.

Participants and Methods: Fifty-two patients [M age=54.6; Edu=13.2; 64\% Male] with CRC completed cognitive testing [WAIS-III: Digit Span, Coding; Controlled Oral Word Association; Trail Making Test; Hopkins Verbal Learning Test-Revised] before adjuvant therapy. Age-adjusted $\mathrm{z}$-scores at or below -1.5 were considered impaired. Overall Cognitive Function Impairment (OCFI) was defined as two or more impaired scores or one or more at or below a z-score of -2.0. Memory complaints were also rated. Cytokines were obtained on a subset of patients $(n=30)$. Results: NCF impairment was frequent on HVLT-R Total Recall (45\%), HVLT-R Delayed Recall (31\%), TMTA (22\%), and TMTB $(21 \%)$, with $56 \%$ meeting criteria for OCFI. Performances did not differ by disease stage. Men performed worse than women on HVLT-R Total Recall [-1.54(1.37) vs. $-0.55(1.25), p=.016]$, Coding $[-0.51(1.19)$ vs. $0.30(0.88), p=.033]$, and TMTB [-0.77(1.98) vs. $0.29(1.10), p=.028]$. Cytokine levels did not differ by disease stage or sex. Males with CRC had significant correlations between sIL-6R and HVLT-R Recognition (-.57) and Coding (-.52), sTNF-RI and HVLT-R Delayed Recall (-.72), as well as sTNF-RII and HVLT-R Delayed Recall (-.70), HVLT-R Delayed Recognition (-.55), and Coding (-.52). Memory complaints were minimal (Median=0), did not differ by sex, and were not associated with performances. Complaints were associated with IL-1Ra in men only $(.55)$.

Conclusions: NCF impairment was common in CRC patients prior to therapy, with men exhibiting greater severity of dysfunction. Increased cytokine levels were associated with worse NCF performance and complaints in men only. While memory was the most frequently impaired domain on testing, complaints were minimal. Proinflammatory cytokine activity may contribute to neurocognitive dysfunction in men with CRC prior to adjuvant therapy.
Correspondence: Kyle R. Noll, Ph.D., Neuro-Oncology, UT MD Anderson Cancer Center, 1515 Holcombe Blvd, Houston, TX 77030. E-mail: knoll@mdanderson.org

C. YAO, J. RICH, I. TANNOCK, B. SERUGA \& L. BERNSTEIN. Pretreatment Reaction Time Intraindividual Variability in Women Diagnosed with Breast Cancer.

Objective: Chemotherapy has adverse effects on cognitive performance in women who have been treated for breast cancer, but less is known about the period before chemotherapy treatment. Although studies have traditionally focused on mean level of performance, there is increasing recognition that within-person variability in performance may also be an important behavioural indicator of cognitive functioning and underlying neural integrity.

Participants and Methods: We examined intraindividual variability (IIV) prior to surgery and chemotherapy treatment in 30 women with locally advanced breast cancer (mean age $=46.4$ years; mean education $=15.2$ years), and 24 healthy women matched on age and education. IIV was calculated across trials of a computerized Stroop task using several methods, including a examination of the slow and fast portions of the reaction time (RT) distribution to assess general and specific variability producing processes.

Results: Within the slow portion of the RT distribution, patients were significantly more variable than healthy controls on incongruent trials, $F(1,52)=6.25, p=.02, \eta 2=.11$, consistent with evidence that suggest IIV is driven by a specific process, such as attentional lapses. Patients and controls were equivalent on Stroop accuracy and mean reaction time.

Conclusions: These results highlight the importance of pretreatment assessment and indicate that performance variability provides information about the nature of cognitive impairment in women treated for breast cancer over and above measures of central tendency.

Correspondence: Christie Yao, M.Sc., Psychology, York University, 150 Cosburn Avenue, Apt. 1012, Toronto, ON M4J2L9, Canada. E-mail: yaoc@yorku.ca

K.P. RAGHUBAR, M. RIS, K.O. YEATES, M. MAHONE, M. MAKOLA \& K. CECIL. The Relationships among White Matter Integrity and Attention in Pediatric Brain Tumor.

Objective: The present study assessed performance on three functional components of attention - alerting, orienting, and executive among children treated with or without radiation therapy. Relations among aspects of attention and specific white matter tract regions were assessed.

Participants and Methods: The 26 participants in this study came from a sample of 59 participants in a longitudinal, prospective, multisite study (BRISC: Brain Radiation Investigative Study Consortium). Participants ranged in age from 6 - 17 years (at time 1 ) diagnosed with a brain tumor who received either RT $(n=9)$ or no RT $(n=17)$ following surgical intervention. Participants completed the Child Attention Network Test (ANT) at approximately 1 and 2 years post-surgery. Diffusion weighted images and a T1 image were collected and processed to obtain fractional anisotropy and mean diffusivity composites for the anterior internal capsule, anterior corona radiata, and splenium, regions of brain shown to be related to components of attention.

Results: On the Child ANT, pediatric survivors of brain tumor treated with RT performed similarly to those who were not. As a group, they demonstrated decreases over time in alerting attention and there was a trend for decrements in executive attention $(p<.06)$. Executive attention was also positively related to fractional anisotropy values for the left anterior corona radiata.

Conclusions: Results are discussed with reference to models of attention. Findings are compared to those from the adult neuroscience literature, relating specific white matter tract regions to aspects of attention. Implications for survivors of brain tumor treated with and without radiation are discussed. 
Correspondence: Kimberly P. Raghubar, Ph.D, Psychology, Texas Children's Hospital, 6701 Fannin Street, Houston, TX 77030. E-mail: kim.raghubar@gmail.com

J.E. SCHREIBER, S.L. PALMER, H.M. CONKLIN, D. MABBOTT, M. SWAIN, M.J. BONNER, L. CHAPIESKI, L. HUANG, H. ZHANG \& A. GAJJAR. Posterior Fossa Syndrome and Longterm Neurocognitive Problems among Children Treated for Medulloblastoma on a Multi-institutional, Prospective Study.

Objective: Patients treated for medulloblastoma who experienced posterior fossa syndrome (PFS) previously demonstrated increased risk for neurocognitive impairments at 12 -months post diagnosis. The present study examined five-year longitudinal trajectories of neurocognitive outcomes in patients who experienced PFS compared with patients who did not.

Participants and Methods: Participants comprised 29 patients (18 males) who experienced PFS and provided data at two or more assessment points and 29 comparison patients (16 males) who were matched on age at diagnosis and treatment arm (average vs. high risk) and provided data at two or more assessment points but did not experience PFS. Mean age at diagnosis was 8.5 years in both groups (PFS group $\mathrm{SD}=2.9$, Non-PFS group $\mathrm{SD}=3.0$ ). Five high risk patients were included in each group. All patients underwent serial evaluation of neurocognitive functioning spanning baseline (occurred after surgery) up to five years following treatment.

Results: Linear mixed-effect modeling revealed that the PFS group demonstrated significantly lower estimated baseline scores on measures of intellectual ability (PFS Mean=80.4, Non-PFS Mean=103.9), processing speed (PFS Mean=62.3, Non-PFS Mean=88.6), attention (PFS Mean=79.1, Non-PFS Mean=102.6), and working memory (PFS Mean=89.1, Non-PFS Mean=107.5; all p's<.001). Measures of attention and working memory significantly declined over time for both groups ( $p$ 's<.01); however, the decline was not significantly different between groups.

Conclusions: Patients who experience PFS exhibit much greater neurocognitive impairment at baseline, show little recovery over time, and may decline further in some cases. Findings highlight the particularly high risk for long term neurocognitive problems and the need for close follow up and appropriate interventions.

Correspondence: Jane E. Schreiber, PhD, Department of Psychology, St Jude Children's Research Hospital, 262 Danny Thomas Place, Mail Stop 740, Memphis, TN 38105.E-mail: jane.schreiber@stjude.org.

M. CHERRIER, M.K. ASKREN, K. ANDERSON, D. DAVID, A. SINGH, K. MURPHY, H.J. MENDOZA \& H. GRAY. Neural and Behavioral Response to Cognitive Training in Cancer Survivors with Cognitive Symptoms.

Objective: This study examined potential for training transfer on two cognitive tasks and corresponding changes in neural activation patterns as measured by fMRI prior to and following a cognitive training intervention in cancer survivors with cognitive symptoms.

Participants and Methods: Seventy five cancer (breast, uterine, ovarian, lung, prostate, colorectal) survivors (mean age: 62 years), underwent a 7-week cognitive training intervention delivered in group format. Participants were randomized to treatment or control and evaluated with a comprehensive cognitive battery (RVLT, story recall, digit span, CPT) prior to and following treatment. A subset of participants ( $\mathrm{N}=7$ ) also underwent fMRI scanning during word pair and verbal working memory tasks.

Results: Participants showed significant improvement following intervention for digit span (total and forward), and RVLT (learning over trials) as well as improvement on self-rated symptom measures. In scanner improvements in accuracy in the word pair relational condition, were observed, $\mathrm{t}(3)=5.21, \mathrm{p}<.05$. By contrast, accuracy did not increase significantly in the Item condition following intervention, $\mathrm{t}(3)=.48, \mathrm{p}=.66$.
fMRI analyses revealed activation in a frontoparietal network during both the word pairs and verbal working memory tasks.

Conclusions: Cancer survivors demonstrated improvements in both verbal memory and working memory and evidence of transfer of these memory skills on a word pair task for which frontoparietal network involvement and more efficient neural response were observed. These results suggest that cognitive training may have a beneficial impact on both behavioral measures of cognition as well as neural activation markers.

Correspondence: M. Cherrier, Ph.D., Psychiatry, University of Washington, Box 356560, Seattle,WA 98195.E-mail: cherrier@u. washington.edu

A. STUDAWAY, T.M. BRINKMAN, C. KIMBERG, Y. MZAYEK, G. ARMSTRONG, M.M. HUDSON \& K.R. KRULL. Impaired executive function and reduced social integration among adult survivors of pediatric central nervous system (CNS) tumors.

Objective: To examine associations between executive dysfunction and social isolation, community integration and social networks in adult survivors of pediatric CNS tumors.

Participants and Methods: Eighty-one adult survivors of pediatric CNS tumors (32\% infratentorial; $74 \%$ cranial irradiation; mean [SD] current age $=28.0[5.8]$ years, time since diagnosis $=18.7[6.0]$ years) completed the Wechsler Abbreviated Scales of Intelligence and measures of executive function including fluency (Controlled Oral Word Association Test), flexibility (Trails Making Test Part B), and working memory (Digit Span Backward). Age-adjusted standard scores and impairment rates ( $>1 \mathrm{SD}$ below the normative mean) were calculated. Survivors completed measures of social isolation, community integration (home integration, social integration, productivity), and social network (embedded networks, high contact networks), with higher scores indicating greater social integration. Generalized linear models were used to examine associations between executive function and social integration. Results: Fifty-one percent of survivors demonstrated impaired flexibility, 32\% impaired fluency, and 26\% impaired working memory. Survivors with any executive function impairment reported reduced social network size $(\mathrm{P}=0.003)$, fewer high contact networks ( $\mathrm{P}=0.038)$, fewer embedded networks $(\mathrm{P}=0.001)$, reduced community productivity $(\mathrm{P}=0.003)$, and greater home integration $(\mathrm{P}=0.007)$. In multivariable models adjusted for sex, current age, and IQ, impaired working memory (embedded: $\beta=-0.66, P=0.046$ ) and fluency (embedded: $\beta=-0.89$, $\mathrm{P}=0.010$; high contact: $\beta=-0.95, \mathrm{P}=0.032$ ) were independently associated with reduced social network size. Impaired cognitive flexibility was associated with reduced community productivity $(\beta=-0.84, \mathrm{P}=0.031)$. Conclusions: These findings suggest a specific contribution of executive dysfunction to reduced social integration among adult survivors of childhood CNS tumors, though longitudinal studies are necessary to better understand temporal associations.

Correspondence: Tara M. Brinkman, PhD, Epidemiology and Cancer Control, St. Jude Children's Research Hospital, 262 Danny Thomas Place, MS 735, Memphis,TN38105.E-mail:tara.brinkman@stjude.org

Z.S. SADIGHI, R. KHAN, J. ZABROWSKI, T.M. BRINKMAN, L. ROBISON, M.M. HUDSON, C. LI, D. SRIVASTAVA \& K.R. KRULL. Effect of Seizure Morbidity on Neurocognitive Outcome, Quality of Life, and Social Attainment in Adult Survivors of Childhood Central Nervous System (CNS) and Non-CNS Cancers.

Objective: Adult survivors of childhood cancer are at risk for seizures, poorer neurocognitive outcome, health-related quality of life (HRQOL), and social attainment. Severity of seizures and anti-epileptic medication use are predictors of neurocognitive decline. We propose an examination of the combined impact of cancer therapy and seizures to neurocognitive outcomes, social attainment and HRQOL in SJLIFE survivors and predict seizures will worsen neurocognitive outcome, social attainment, and HRQOL. We also predict the severity of seizure disorder will poorly impact these outcomes. 
Participants and Methods: 2,022 cancer survivors in SJLIFE treated from 1962-2002, $\geq 18$ years old at enrollment, and $\geq 10$ years from cancer diagnosis were reviewed for seizures. Multiple logistical regression modeling and Poisson regression with robust variance were used to determine these outcomes, adjusting for age, gender, and treatment. Results: $11.5 \%$ of SJLIFE cohort had seizures (54.3\% leukemia, $31 \%$ brain tumor, $6 \%$ lymphoma and $8.6 \%$ solid tumor). $63.8 \%$ had seizure resolution. Seizure and anti-epileptic medication severity were highly correlated (correlation coefficient $0.68-0.88$ ). Of seizure patients, $54.1 \%$ were married, $69 \%$ had less than college education, $56.8 \%$ had less than full-time employment, and $52 \%$ made $<\$ 40 \mathrm{~K}$ annual income. Compared to all cancer survivors, those with seizures had worse neurocognitive outcome $(12 / 13$ domains $p<0.05)$, employment status $(\mathrm{p}<0.05$, RR 1.09), and HRQOL (2/4 physical component scales $\mathrm{p}<0.05)$. Seizure severity negatively impacted neurocognitive outcome (6/13 domains $p<0.05)$, but did not affect HRQOL or social attainment. Conclusions: Seizures in cancer survivors impacts neurocognitive outcome, social attainment and HRQOL. Seizure severity only impacts neurocognitive outcome.

Correspondence: Zsila S. Sadighi, MD, Neurology, St. Jude Children's Research Hospital, 262 Danny Thomas Place, MS 220, Memphis, TN 38105.E-mail: zsila.sadighi@stjude.org

J.C. SHERMAN, M.K. COLVIN, S. MANCUSO, R. RANGAMANNAR, C. EVANS, B. YEAP \& H. SHIH. Preserved Cognitive Function following Proton Radiation in Adults with Low Grade Glioma.

Objective: Low grade gliomas (LGG) are a slow growing tumor that often occur in young adults. While these patients often survive in a stable state for several years, they may experience neurocognitive impairments. Conventional (photon) radiation therapy can positively impact survival, but has been found to negatively impact cognitive functioning. We investigated the effects of proton radiation therapy, a new form of more targeted radiation, on neurocognitive functioning in adults with LGG. Participants and Methods: 20 patients were evaluated with neuropsychological tests at baseline and at yearly intervals for five years. Standardized measures of intellectual function, attention and executive function, language, visuospatial functions and memory and a mood inventory were administered.

Results: At baseline, 3 of 20 patients had severely impaired language performance; 4 had moderately impaired verbal and visual memory and 1 had impaired processing speed. Performance in all neurocognitive domains remained stable or improved marginally over time for all patients. Notably, patients with left-sided tumors performed significantly worse than those with right-sided tumors on measures of verbal memory $(p<.01)$ and language $(p<.01)$ at baseline. However, these patients improved at a significant rate such that there was no difference between these tumor groups at year 5. Exploratory analysis also indicated that LGG patients did not exhibit typical practice effects in the areas of processing speed, executive functions, and verbal memory, although it should be emphasized that this was a statistical trend.

Conclusions: These results suggest that proton radiation therapy is not associated with cognitive decline, at least in the first 5 years following treatment. These results are encouraging, as some studies have found evidence for cognitive dysfunction in LGG patients who received conventional radiation therapy. Further follow-up is needed to determine whether proton radiation therapy reduces the potential for long-term cognitive decline.

Correspondence: Janet C. Sherman, Ph.D., Psychiatry, Mass General Hospital, 1 Bowdoin Square, 7th Floor, Boston, MA 02114. E-mail: jsherman@partners.org
P. BANERJEE, K. LEU, R.J. HARRIS, T.F. CLOUGHESY, S. BOOKHEIMER, L. LIAU \& B.M. ELLINGSON. Voxel-Based Lesion-Symptom Mapping of Expressive and Receptive Language in Brain Tumor Patients.

Objective: We examined the relationship between lesion presence and expressive \& receptive language performance on a voxel-by-voxel basis in brain tumor patients using innovative voxel-based lesion-symptom mapping (VLSM).

Participants and Methods: 98 right-handed adults with primary gliomas were administered Verbal Fluency, Woodcock-Johnson III: Word Attack, and five subtests of the Boston Diagnostic Aphasia Examination. Tasks were divided into those assessing expressive language and receptive language. Participants ranged from 18 to 80 years old $(M=47)$ with $60 \%$ males. T2-weighted images were registered to a T1-weighted MNI atlas and the tumor region was contoured using a semi-automated thresholding technique. For each voxel, patients were grouped by whether or not tumor was present, and a general linear model was performed to compare the cognitive scores of these two groups while controlling for demographics and tumor characteristics.

Results: VLSM identified several clusters of voxels with a significant relationship $(\mathrm{p}<.05)$ between lesion presence and language performance, depicted on high resolution statistical maps. Statistical maps were generated for each task as well as for composites of expressive language tasks and receptive language tasks. The three receptive language tasks showed large areas of overlap within the lateral and medial temporal lobe. In contrast, despite each of the five expressive language tasks being associated with large clusters of significant voxels in the frontal, temporal, and parietal lobes, little overlap was demonstrated on the composite map of these tasks.

Conclusions: These findings identify the key anatomic structures involved in language functioning in brain tumor patients using an innovative lesion analysis technique. The neuroanatomical correlates of expressive language tasks demonstrated little overlap, in contrast to that of receptive language, suggesting that the anatomic structures involved in expressive language are much more highly task-specific than that of receptive language.

Correspondence: Pia Banerjee, Ph.D., Neurology, University of California, Los Angeles, 760 Westwood Plaza, \#C8-746, Los Angeles, CA 90095.E-mail: pbanerjee@mednet.ucla.edu

A. AMIDI, M. AGERBAK, A. LEEMANS, L. WU, A.D. PEDERSEN, M. MEHLSEN \& R. ZACHARIAE. Brain Connectivity and Neuropsychological Functioning in Testicular Cancer Patients exploring the effect of chemotherapy.

Objective: There is evidence to suggest that the use of chemotherapy (CT) may be associated with reduced brain connectivity and impaired neuropsychological functioning. The aim of the present study was to compare brain white matter connectivity and neuropsychological functioning in testicular cancer (TC) patients who had received CT and a control group of TC patients who had not received CT.

Participants and Methods: Sixty-five men recently treated for TC (range: 4-7 months) participated in this study. Twenty-two men had received CT and surgery and 43 had been treated with surgery only. All participants underwent magnetic resonance imaging of the brain including a high-resolution T-1 weighted structural scan and a 32-directional diffusion-weighted scan. Participants also completed a comprehensive battery of standardized neuropsychological tests assessing different cognitive domains. Voxelwise statistical analysis was performed using tract-based spatial statistics (TBSS) with 5000 permutations to test for group differences in mean values of fractional anisotropy (FA) in white matter tracts. Group differences in neuropsychological outcomes were tested with ANCOVAs.

Results: TC patients who had received CT were younger (mean age=31.9; $\mathrm{SD}=9.4)$ than the surgery-only group (39.6; $\mathrm{SD}=10.7$ ) $(\mathrm{p}<0.01)$. Age-adjusted TBSS analyses revealed no statistically significant between-group differences in FA values in brain white matter tracts 
(corrected for multiple comparisons). Furthermore, group comparisons of neuropsychological test results, adjusted for age and premorbid intellectual functioning, did not reveal any significant between-group differences ( $\mathrm{p}$ (range): 0.14-0.90).

Conclusions: Our results suggest that TC patients who had received chemotherapy did not differ in brain white matter connectivity compared with control patients. Furthermore, no between-group differences were found in neuropsychological functioning.

Correspondence: Ali Amidi, MSc Psychology, Aarhus University, Bartholins Alle 9, Aarhus C 8200, Denmark.E-mail: ali@psy.au.dk

\section{Language and Speech Functions/Aphasia}

\section{J. MCCULLAGH \& M. PURDY. Dichotic Listening Training for Auditory Processing Deficits in Aphasia.}

Objective: Dichotic listening deficits are often observed in aphasia and may be characterized by difficulty hearing in background noise or when more than one person is speaking at the same time. The purpose of this study was to determine whether dichotic listening training that altered the intensity of the stimuli to bias the weaker ear in order to "force" it to process auditory information would result in gains in overall auditory processing as well as in specific language functioning.

Participants and Methods: Three individuals with chronic mild aphasia and dichotic listening deficits, documented through standardized audiological assessments, participated in auditory training for $60 \mathrm{~min}$ utes per day, 3 days per week, for 4 to 6 weeks. Dichotic Interaural Intensity Difference (DIID) training was completed using dichotic letters, numbers, words, and sentences. Training included both binaural integration and separation tasks with intensity bias for the weaker ear. Comprehensive audiological, auditory processing, and cognitive-linguistic assessment batteries were administered pre-and post- DIID training. Results: Although specific performance patterns varied among the participants following treatment, performance on the Dichotic Digits test indicated improvements in the weaker ear. Performance on the Dichotic Rhyme test indicated slight improvement in the weaker ear and stable performance in the stronger ear. Gains for all participants were also documented on story recall, verbal learning, and verbal working memory tests. Responses were more prompt, resulting in increased efficiency of processing.

Conclusions: These results indicate that DIID training may be a useful treatment for auditory processing deficits in aphasia.

Correspondence: Mary Purdy, Ph.D., Communication Disorders, Southern Connecticut State University, 501 Crescent Street, New Haven, CT 06515. E-mail: purdym1@southernct.edu

\section{A. STRASSER, D. KOO, K. GARRIDO-NAG \& L. PICK. Paired- Associate Learning in Deaf Readers.}

Objective: Prior research with hearing individuals demonstrates reading ability is associated with paired-associate learning (Hulme, Goetz, Adams \& Snowling, 2007). The deaf population also shows significant variance in their reading ability, as well as verbal memory assessed via the English language. The Signed Paired Associate Test (SPAT) was developed to assess signed paired-associate learning in native deaf signers (Pollard, Rediess, \& DeMatteo, 2005). The current study investigated signed paired-associate learning in proficient versus developing deaf readers. Demographic factors known to impact reading ability were examined in relation to SPAT performance.

Participants and Methods: 42 undergraduate deaf signers completed a demographic questionnaire, the WASI-II, the WRMT-III Reading Comprehension Cluster, and the SPAT. Participants were divided into developing and proficient readers based on their WRMT-III performance. Results: Developing readers performed significantly worse on the first trial of the SPAT compared to proficient readers $(p=.031)$. With practice, there were no differences between learning trials 2,3 and 4 , or the delay trials $(p>0.05)$. There were no relationships between SPAT performances for either reading group and the following demographic factors: parental hearing status, use of hearing assistive devices, additional support services, and years of education.

Conclusions: Developing deaf readers struggled to acquire paired-associates during initial trial of a Signed Paired Associate Test, but improved with repetition. Their learning was similar to proficient deaf readers on later learning trials and delayed recall. Paired-associate learning was not associated with the selected demographic factors. These results parallel established learning patterns in hearing readers.

Correspondence: Amanda Strasser, B.S., B.A, Clinical Psychology, Gallaudet University, $4749 \mathrm{~W}$ Braddock Rd. Apt. 20, Alexandria, VA 22311. E-mail: amanda.strasser@gallaudet.edu

M.C. STOOP \& L.J. ALTMANN. Susceptibility to Phonological Interference in a Spoonerism Elicitation Task.

Objective: Spoonerisms, the switching of the initial sounds in two adjacent or nearby words, are a common type of speech error. Two very divergent populations, older adults and people with dyslexia, are particularly susceptible to spoonerisms. This susceptibility has been attributed to weaknesses in phonological processing in both groups. We tested an alternate possibility, that susceptibility to spoonerisms in normal readers was related to cognitive abilities that are impaired in both groups: attention, working memory (WM), and executive functions. We hypothesized that inhibition would be the primary predictor of spoonerisms.

Participants and Methods: Fifteen undergraduate students completed a battery of cognitive tests, including a Go-No Go task (vigilance), the Stroop XXX and Color Words (inhibition), Trails A and B (switching), Digit Symbol Substitution (speed of processing), Digit Ordering (WM-resequencing), Digit Span Forward (verbatim memory) and Backward (WM-sequence encoding). Also, they completed a version of the Motley-Baars (Motley \& Baars, 1976) SLIP task with 5 pairs of stimuli/ trial (1 real-, 4 non-word pairs). The words in each stimulus pair in a trial started with the same 2 different sounds, but the order varied.

Results: Contrary to expectations, only Digits Backward and Digit Ordering, which were uncorrelated, were associated with number of spoonerisms produced.

Conclusions: These results suggest that spoonerisms may result from limitations in different aspects of working memory. Specifically, WM sequence encoding and WM resequencing may both play a role in spoonerism frequency. These findings indicate that future research on speech errors in aging, dyslexia and other populations should include cognitive measures.

Correspondence: Lori J. Altmann, PhD, Speech, Language \& Hearing Sciences, University of Florida, 336 Dauer Hall, Box 117420, Gainesville,FL32611-7420.E-mail: laltmann@ufl.edu

J.L. REHMEL, L.K. PAUL \& W.S. BROWN. Comprehension of Common \& Uncommon Proverbs in Individuals with Agenesis of the Corpus Callosum.

Objective: Persons with agenesis of the corpus callosum (ACC) with normal intelligence exhibit deficiencies in interpreting non-literal language. This research used the Common and Uncommon subtests from the Proverbs Test of the Delis Kaplan Executive Function System (D-KEFS) to assess the impact of common usage on non-literal language comprehension in ACC.

Participants and Methods: Fourteen adults with ACC (FSIQ 80 - 129; age 18 - 52) were compared to 15 age and IQ-matched neurotypical controls (FSIQ 84 - 116; age 20-45) on Common and Uncommon Proverbs scores of the Free Response subtest. Education, the Verbal Comprehension Index (VCI), the Perceptual Organization Index (POI), and Literal and Non-literal items from the FANL-C were all used as covariates in post-hoc analyses.

Results: A repeated measures ANOVA (Group by subscales: Common and Uncommon) revealed significant effects of proverb type $(\eta \mathrm{p} 2=.33$, $\mathrm{F}(1,27)=13.03, \mathrm{p}=.00)$ and group $(\eta \mathrm{p} 2=.36, \mathrm{~F}(1,27)=14.91, \mathrm{p}$ $=.00)$, but no group-by-proverb type interaction. Univariate ANOVAs 
showed a significant group differences on both common proverbs ( $\eta$ p2 $=.29, \mathrm{~F}(1,27)=11.20, \mathrm{p}=.00)$ and on uncommon proverbs $(\eta \mathrm{p} 2=$ $.29, \mathrm{~F}(1,27)=11.15, \mathrm{p}=.00)$. Covarying VCI, education, and the non-literal scale of the FANL-C individually resulted in a reduction in group distinctiveness; conversely, covarying POI and the literal items from the FANL-C made the groups more distinct.

Conclusions: Reduced interhemispheric connectivity has a significant impact on ability to comprehend the meaning of proverbs, but this effect is not related to whether proverbs are common or uncommon.

Correspondence: Warren S. Brown, Ph.D., Travis Research Institute, Fuller Grad Sch of Psych, 180 N. Oakland Ave., Pasadena, CA 91101. E-mail: wsbrown@fuller.edu

\section{K.P. RAGHUBAR, J. OGHALAI, R. WILLIAMSON \& S. CAUDLE. Neurocognitive Predictors of Academic Functioning in Children with Cochlear Implants.}

Objective: Children with cochlear implants often demonstrate continuing weakness in language functioning, which underlies academic skills such as reading and writing. Not surprisingly, children with cochlear implants often struggle academically. The present study investigated academic functioning including single word reading, math, and reading comprehension in school-aged children with cochlear implantation. Participants and Methods: Participants included 28 children with a history of bilateral hearing loss, treated with cochlear implantation, with the majority receiving unilateral implants, ranging in age from 6 to 17 years of age at the time of the assessment. The average length of implant use was under 6 years. Children completed academic measures of word reading, math computation, and sentence comprehension. Neurocognitive skills commonly associated with academic learning and achievement were also assessed including verbal and visual working memory, and vocabulary knowledge.

Results: As a group, children with cochlear implants performed below age-expected levels on measures of academic functioning. Age at evaluation, gender, age at implant, and maternal education were not significantly associated with academic outcomes and were not included as predictors in regression models. Vocabulary knowledge predicted performance on measures of single word reading. There was a trend for verbal working memory to predict reading comprehension and visual working memory to predict math computation.

Conclusions: Overall, children with cochlear implants demonstrated academic skills that fell somewhat below expectation for age. Findings are discussed with reference to academic models of ability and disability. Correspondence: Kimberly P. Raghubar, Ph.D, Psychology, Texas Children's Hospital, 6701 Fannin Street, Houston, TX 77030. E-mail: kim.raghubar@gmail.com

\section{A. GARCIA, R. BINNEY \& J. REILLY. Neural Correlates of Semantic Processing Across Input Modalities.}

Objective: The embodied cognition perspective predicts that concepts are encoded in a distributed network of motor and perceptual cortices, which are both necessary and sufficient for semantic conceptualization. Other theoretical perspectives claim the necessity of an additional polymodal cortical hubs for higher order convergence of information. We evaluated these hypotheses by using fMRI to examine activation during naming of both newly learned and familiar items. We hypothesized that if 'hubs' are necessary for recall of semantic information, then they could be identified by greater activation for familiar relative to newly acquired concepts.

Participants and Methods: 18 young adults were trained to name six novel items with distinctive sounds, patterns of motion and visual appearance. During fMRI, participants named these items and six familiar counterparts from their static visual form, their sound, or a point-light presentation of their motion. Contrasts between novel and familiar items were performed for each presentation modality. Region of interest analyses were performed at sites purported to be cortical candidates for polymodal hubs; the angular gyrus (AG), posterior middle temporal gyrus (MTG), and lateral anterior temporal lobe (ATL).

Results: The lateral ATL, but not the left AG or left MTG, showed a significantly increased BOLD response for familiar concepts relative to novel concepts in all three presentation modalities. Similar increases were observed in unimodal association cortices.

Conclusions: Our findings are consistent with the hub and spoke model of semantic memory, supporting a role for the ATL as a substrate for the convergence of polymodal information to form modality invariant concepts.

Correspondence: Amanda Garcia, Clinical and Health Psychology, University of Florida, 3461 SW 2nd Ave. \#404, Gainesville, FL 32607. E-mail:amgar@phhp.ufl.edu

J. BALDO, C. LUDY, S. PAULRAJ, V. PIAI \& N.F. DRONKERS. A Voxel-based Lesion Analysis of Reading in a Large Cohort of Left Hemisphere Stroke Patients.

Objective: The goal of the current study was to identify the neural correlates of distinct aspects of reading, using a statistically-based lesion analysis tool, voxel-based lesion symptom mapping (VLSM).

Participants and Methods: We analyzed data from 111 individuals who suffered a single left hemisphere stroke and who met strict inclusion and exclusion criteria. Individuals were tested on the reading subtests from the Western Aphasia Battery, including sentence-level reading, single word-object matching, and single word-spoken word matching. Reading performance was scored and entered along with individuals' lesion reconstructions from high-resolution MRI or CT scans. VLSM was used to relate performance on the distinct reading subtests to brain regions by performing t-tests at every voxel, comparing reading scores in patients with versus without a lesion in each voxel.

Results: The VLSM analysis of the sentence-level reading subtest identified a critical region in left anterior-mid superior temporal gyrus, with some extension into the middle temporal gyrus. Single word-object matching was associated with left posterior, inferior temporal cortex, including lingual and fusiform gyri. Last, single word-spoken word matching correlated with a region in the mid portion of the left middle temporal gyrus, with some extension into the left superior temporal gyrus.

Conclusions: This large-scale, voxel-based lesion analysis of reading identified a network of regions critical for different aspects of reading, with single-word reading relying most critically on left posterior-inferior temporal cortex near classically-defined visual word form areas, and reading that involved sentence comprehension relying more critically on the left superior temporal gyrus.

Correspondence: Juliana Baldo, Ph.D., Research, VA Northern California Health Care System, 150 Muir Road (126R), Martinez, CA 94553.E-mail: juliana@ebire.org

A. AILION, T.Z. KING \& B. CROSSON. Long-Term Survivors of Cerebellar Tumors: Impact of Processing Speed and Working Memory on Phonemic and Semantic Fluency Performance.

Objective: Populations with cerebellar insults show difficulty with Phonemic Fluency (PhF) over other fluency tasks. Researchers have suggested lower PhF may be due to slowed speech, strategy generation, impaired initiation, or task difficulty. To test each of these theories we explored the number of words on each time block of fluency measures and the influence of oral processing speed (OSDMT) and working memory (ACT).

Participants and Methods: DKEFs PhF and Sematic Fluency (SemF) measure speed of word generation and were administered to 28 cerebellar tumor survivors and 28 controls. Survivors were on average 15 years from diagnosis and matched with controls on age (24 years (5)) and education (14(2.5)). A 2 (Group=Control, Survivor) $x 4$ (Time $=15$ sec blocks) ANOVA was conducted with number of words generated. Regression analyses were conducted with z-scores to determine whether OSDMT and ACT explained variance in each fluency measure. 
Results: ANOVA revealed a significant main effect of group for $\mathrm{PhF}$ $(F(1,54)=8.67, p<0.01)$ but not $\operatorname{SemF}(F(1,54)=10.72, p=.09)$. Contrasts indicated that survivors generated on average 2.41 less $\mathrm{PhF}$ words than controls $(\mathrm{p}<.01)$. In regression analyses, OSDMT predicted variance in $\mathrm{PhF}$ and SemF for survivors $(\mathrm{B}=.67, \mathrm{SE}=.12, \mathrm{p}<.01 ; \mathrm{B}=.55$, $\mathrm{SE}=.12, \mathrm{p}<.01)$ as well as SemF for controls $(\mathrm{B}=.04, \mathrm{p}=.03)$. In contrast, ACT only predicted variance in SemF for survivors $(B=.44, \mathrm{SE}=.14$, $\mathrm{p}<.01)$.

Conclusions: Survivors evidenced lower PhF, and only trend with SemF, thus slowed speech is unlikely to explain these results. Similar pattern of production over time rules out impaired initiation. In survivors, processing speed uniquely explained $48 \%$ of variance in $\mathrm{PhF}$, and $27 \%$ in SemF. Implications of these findings and additional theories will be discussed.

Correspondence: Alyssa Ailion, B.S., Georgia State University, 934 Bridgegate Drive, Marietta, GA 30066.E-mail: aailion1@student.gsu. edu

N.F. DRONKERS, J. BALDO, A. TURKEN \& R. KNIGHT. Grey and White Matter Involvement in Classic Aphasia Syndromes.

Objective: To evaluate the brain lesions associated with the major aphasia syndromes in a large group of chronic aphasic stroke patients. Participants and Methods: 104 patients suffered a single left hemisphere infarction with a residual speech or language deficit. All were right-handed, native English-speaking, with no prior neurological or psychiatric history. All were evaluated with the Western Aphasia Battery more than one year post onset. Patients' lesions derived from structural neuroimaging were computer-reconstructed and normalized into MNI space. Patients who classified into the different aphasia types were grouped and their lesions overlapped to yield common areas of infarction. Fiber tract probability maps were also overlaid to determine which major fiber tracts were also involved.

Results: Lesions in chronic Broca's aphasia involve primarily Broca's area, insula, anterior arcuate fasciculus, superior longitudinal fasciculus, and possibly extreme and external capsules. Lesions in chronic Wernicke's aphasia involve the middle temporal gyrus and numerous underlying white matter tracts, and often the superior temporal gyrus. In chronic conduction aphasia, the area of common infarction is the inferior parietal and posterior superior temporal cortex, with portions of the ascending arcuate fasciculus.

Conclusions: These results suggest important differences in the lesion patterns between acute and chronic patients that can significantly alter our perceptions of the key brain areas involved in the different aphasia syndromes. Since most patients' classifications change during the course of recovery, we suggest that brain-behavior relationships are best mapped once the behaviors have stabilized.

Correspondence: Nina F. Dronkers, Ph.D., Center for Aphasia and Related Disorders, VA Northern Cal Health Care System/UC Davis, 150 MuirRoad (126R), Martinez,CA 94553.E-mail:dronkers@ucdavis.edu

S.B. PILLAY, T.A. HAMMEKE, D.S. BOOK \& J.R. BINDER. Neural Correlates of Impaired Definition Naming Relative to Picture Naming Using Voxel-Based Lesion-Symptom Mapping.

Objective: Both picture naming (PN) and definition naming (DN, also called auditory naming) are used during pre-surgical cortical stimulation mapping to identify regions critical for naming. Anterior temporal lobe (ATL) stimulation impairs DN more often than PN, yet paradoxically ATL resection typically impairs PN more than DN. We sought to clarify the neural correlates of DN impairment, relative to PN impairment, using voxel-based lesion symptom mapping (VLSM) in a series of chronic stroke patients.

Participants and Methods: Participants were 40 right-handed native English speakers with chronic left-hemisphere stroke. Patients performed an 80-item DN task in which they heard a definition ("Bait used for fishing") and had to name the object described ("worm"). Patients also performed an 80-item PN task. Lesions were labeled on high-resolution T1-weighted MRI volumes. VLSM examined lesion correlates of DN impairment independent of PN impairment.

Results: After controlling for PN accuracy, age, education, and total lesion volume, DN impairment was correlated with damage in the mid-portion of the middle temporal gyrus and anterior superior temporal gyrus. No regions were correlated with PN impairment independent of DN impairment.

Conclusions: The temporal regions specifically necessary for DN are superior and posterior to temporal regions typically resected during ATL surgery. FMRI studies suggest that these regions support auditory word and sentence recognition, a process critical for DN but not PN. Naming deficits after ATL resection are likely unrelated to this auditory comprehension process, and may result from resection of more ventral (e.g., fusiform gyrus) areas involved in object recognition.

Correspondence: Sara B. Pillay, Rosalind Franklin University of Medicine and Science, 6015 W. Wells St., Wauwatosa, WI 53213.

E-mail: sara.berentsen@gmail.com

\section{Learning Disabilities/Academic Skills}

\section{T. YOUN \& H. JUN. A Comparison of Quantitative EEG Between Cooperative Learning And Lecture-Based Learning In Adult.}

Objective: The purpose of this study is to investigate the teaching method to respect the learning instinct of

human being in $\operatorname{HRD}$ (human resource development). To investigate the effective teaching method, QEEG(Quantitative electroencephalography) was measured for 12 new employees in a company, while they were in both cooperative learning and lecture-based learning.

Participants and Methods: Twelve right-handed new subjects (male 12 , mean age $28.08 \pm 1.44$ years) were performed QEEG recordings under the international 10-20 system. The subjects didn't have any previous or current history of neurological or psychiatric abnormality. A 32-channel digital signal processing hardware and software package from Twin system (GRASS, USA) were used in the acquisition and storage of EEG data. EEG was recorded from 20 electrode site according to international 10-20 system. Impedance of all electrode was kept below $5 \mathrm{~K} \Omega$. The sample rate was $1000 \mathrm{~Hz} /$ channel and the band-pass was $0.05-100 \mathrm{~Hz}$.

Results: The result shows that the alpha absolute powers differ from cooperative learning and lecture-based learning in Pz electrode. Beta relative powers between in cooperative learning and lecture-based learning differ in Fp1, C4, Cz and O1 electrodes. Interhemispheric asymmetry of beta absolute power was observed in Fp1-Fp2 electrodes and delta absolute power was also observed in T5-T6 and 01-02 electrodes.

Conclusions: An increased activity of beta relative power during cooperative learning could be understood that

participants'cognitive functions are more activated than during lecture-based learning.

Moreover, concentration and arousal function are also increased while participants are in cooperative learning

rather than in lecture-based learning. Cooperative learning method would better to be encouraged to fulfill the learning instincts of human beings in HRD. Through the cooperative learning, people would feel and be touched, because they are respected within the interactive and communicative learning.

Correspondence: Tak Youn, MD, PhD, Neuropsychiatry, Dongguk University Medical Center, 814 Siksa-dong, Ilsandong-gu, Goyang city 410-773, Korea (the Republic of).E-mail: tyoun@duih.org

M. HARRELL, A.R. LOUGHAN, J. HERTZA \& M. COHEN. Children with Identified Reading Weakness: Significant Deficits in Phonological Processing?

Objective: Dyslexia is one of the most widely researched learning disorders. The key deficits associated with dyslexia are difficulty in word decoding and encoding, and the common variable associated with both 
is poor phonological awareness. However, there is limited research available on reading weakness. This study explored the phonological pattern of children with reading weaknesses, as defined by achievement on a single word reading measure (WIAT-III/WRAT-4) at least 10 points below their FSIQ, when compared to controls.

Participants and Methods: Participants included 88 children $(72 \%$ boys; 91\% Caucasian; Mage $=9$, range $5-15$ yrs; MFSIQ $=97$, range 77-130) who participated in a neuropsychological evaluation. Groups included those defined with reading weakness $(n=28)$, diagnosed with dyslexia $(\mathrm{n}=32)$, and controls $(\mathrm{n}=28)$. Controls had no discrepancy between FSIQ and reading ( $<9$ points). Data was analyzed via SPSS. Analyses included descriptive, frequencies, ANOVA's and correlations. Results: Results trended towards significance between groups in phonological awareness $(\mathrm{F}=2.594, \mathrm{p}=.081)$. However, as the discrepancy between IQ and reading weakness increased, there was no correlation with phonological processing scores $(r=-.011, p=.920)$. However, of the 28 children with a reading weakness, $50 \%$ displayed a phonological weakness (phonics scores at least 10 points below their FSIQ). Sixty-seven percent of the children with dyslexia also displayed phonological weakness.

Conclusions: Children with weaknesses in reading may not have clinically discrepant difficulties in phonological awareness when compared to controls. However, $50 \%$ did experience weakness in phonological awareness. It is possible that significant deficits in phonological awareness only become evident at the level of a true reading disorder. Other deficits (i.e., reading fluency, reading comprehension, working memory) may contribute to reading weaknesses and need further exploration. Correspondence: Ashlee R. Loughan, Ph.D., NeuroBehavioral Associates, 1355 Independence Dr, Augusta, GA 30901.E-mail: aspicer88@yahoo. com

\section{N. CROCKER \& S.N. MATTSON. Evaluation of Symbolic and Nonsymbolic Magnitude Judgment and Its Relationship with Mathematics Achievement in Children with Heavy Prenatal Alcohol Exposure.}

Objective: Fetal alcohol spectrum disorders are associated with a range of neurobehavioral consequences including academic difficulties. Mathematics has emerged as a specific area of weakness, though little is known about the nature of these deficits. To evaluate processes that underlie mathematics difficulties, the current study examined the relationship between symbolic and nonsymbolic magnitude judgment (MJ) and mathematics achievement in children with histories of heavy prenatal alcohol exposure (the $\mathrm{AE}$ group) and controls (the CON group). Participants and Methods: 59 children (27 AE, 32 CON) were administered the Calculation, Math Fluency, Applied Problems, and Quantitative Concepts subscales of the WJ-III Tests of Achievement and an experimental task used to measure symbolic and nonsymbolic MJ. Dependent variables for the MJ task were reaction time (RT) and accuracy. Groups were similar on SES, sex, race, and ethnicity.

Results: Data were analyzed using mixed model ANOVA. The AE group had significantly slower RT, but was not less accurate than the CON group on the MJ task. The AE group also performed significantly more poorly than the CON group on all four WJ-III measures. To evaluate the relationship between MJ and mathematics achievement, MJ outcomes were analyzed separately for each WJ-III measure using hierarchical linear regression. MJ was entered on step 1, followed by group on step 2 , and the interaction terms on step 3. For all WJ-III measures, model 1 accounted for a significant amount of variance in mathematics achievement, however model fit improved with the addition of group on step 2. The interaction terms did not account for additional explained variance. Conclusions: These findings confirm the presence of deficits in mathematics achievement in children with histories of prenatal alcohol exposure. These impairments are accounted for, at least in part, by MJ, although additional study, testing the specificity of these effects is needed. Supported by NIAAA Grants U01 AA014834 and F31 AA020142.
Correspondence: Nicole Crocker, Ph.D., San Francisco VA Medical Ceneter, 664 8th Avenue, San Francisco, CA 94118. E-mail: nicole. crocker@gmail.com

Y. MIYAKE \& H. KUMANO. Systematic review of N400 waveform processing of semantic words in specific language impairment.

Objective: Specific language impairment (SLI) is a developmental disorder in the ability to process language. Children with SLI have deficits compared to typically developed children (TD), especially in aspects of semantics, phonology, and syntax. Researchers have studied children with SLI processing semantics using $\mathrm{N} 400$ which show inconsistent results. This study identifies effective elements of the difference in N400. Participants and Methods: A systematic review of literature was performed using PubMed, PsycINFO, and Web of Science. Inclusion criteria for articles included the following: children with SLI as study participants and the use of $\mathrm{N} 400$ and related semantic deviant tasks. Studies with infant participants were excluded and some data was also excluded after intervention.

Results: After a detailed inspection of title and abstract, eight records (28 hits initially) met the inclusion criteria. Comparison of each study's results of N400 in children with SLI and TD revealed that their amplitude of N400 shows differences when they participate in experiments combining nouns and verbs. However, their amplitude is the same when experiments involved labeling pictures. In addition, these tendencies are clear when participants are 6 to 10 years old but not older.

Conclusions: This systematic review of literature revealed that age and task type affected the difference of N400 among TD and SLI, and that young children with SLI may have difficulty connecting correct verbs to the noun. Next, we should confirm the effects of learning combinations of verbs and nouns for children with SLI in primary school's lower grades.

Correspondence: Yuka Miyake, Humanscience, WasedaUniversity, 2-579-15 Mikajima, Tokorozawa-shi 359-1192, Japan. E-mail: miyake-yuka@akane.waseda.jp

J.B. HALE, H.A. KUBAS, K.M. JONES \& J.W. WITZKE. Neuropsychological and Behavioral Profiles of Written Expression SLD Subtypes: Implications for Differentiated Instruction.

Objective: Children with specific learning disabilities (SLD) have different neuropsychological processing deficits that lead to impairments in academic achievement and psychosocial functioning. This study was designed to examine the neuropsychological and behavioral characteristics of written expression SLD subtypes defined using the concordance-discordance model (C-DM) of SLD identification.

Participants and Methods: WISC-IV/WJ-III C-DM analyses of 89 girls and 194 boys, aged 6 to 16 , referred for comprehensive neuropsychological evaluation in school settings, yielded a No SLD group $(n=66)$, and Left Hemisphere (LH-SLD; $n=18$ ), Right Hemisphere (RH-SLD; $n=$ 25), Working Memory (WM-SLD; $n=46$ ), Processing Speed (PS-SLD; $n=81$ ), and Executive (EX-SLD; $n=47$ ) SLD subtypes.

Results: SLD subtypes differed in prevalence of WJ-III Spelling, Writing Fluency, and Writing Samples deficits ( $\chi^{2}$ range 49.00 to 88.44, $p<$ $.001 ; \phi$ range .45 to .66). The EX-SLD, PS-SLD, and WM-SLD subtypes were more likely to have WJ-III Spelling impairment, the LH-SLD, EX-SLD, and PS-SLD showed the most Writing Fluency deficits, and no clear pattern was found for Writing Samples. Analysis of variance using several neuropsychological executive function, memory, and psychomotor measures (D-KEFS, WRAML-2, VMI) revealed processing deficit differences ( $F$ range 2.41 to $5.67, p$ range .040 to $<.001$ ) among written expression SLD subtypes, and among the SLD subtypes and the No SLD group. BASC-2 behavioral differences were found mostly on externalizing measures ( $F$ range 2.22 to 2.74 , $p$ range .050 to .022 ). Conclusions: Children with written expression SLD are a heterogenous group, with different subtype neuropsychological processes explaining their WJ-III Spelling, Writing Fluency, and Writing Samples deficits. Implications for neuropsychological assessment of written expression 
subtypes, discriminating subtypes from low written expression achievement, and subtype differentiated instruction will be addressed. Correspondence: James B. Hale, PhD, Paediatrics, Psychiatry, School and Applied Child Psychology, University of Calgary, EDT 538, 2500 University Drive NW, Calgary, AB T2N 1N4, Canada.E-mail: halejb@ ucalgary.ca

C. FRITZ \& R. MORRIS. Differential Relation between Reading Comprehension and Neurocognitive Constructs for Dyslexic and Typical Readers at Different Ages.

Objective: Research on the relation between reading comprehension (RC) and word decoding (WD) skills suggests that as typical children get older, the correlations between RC and measures of phonological awareness (PA) and WD decrease. When students are first learning to read they are primarily dependent on decoding skills in order to read and comprehend text. Yet, as students get older, they automatically decode many of the words they comprehend in oral language. As a result, language comprehension (LC) skills play an increasingly important role in RC. Although there seems to be developmental changes in the relationships between RC, WD, PA and LC for typical readers, it remains unclear how these interrelationships between core reading and neurocognitive variables vary for dyslexic readers at different ages. Participants and Methods: Nineteen studies, which reported correlations between $\mathrm{RC}$ and at least one neurocognitive construct (LC, WD, PA), were included in the analyses. Mean sample ages ranged from 6 to 37. The bivariate correlations were weighted by number of participants and plotted separately for dyslexic and typical readers.

Results: Analyses revealed significant negative correlations between $\mathrm{RC}$ and WD with age for typical $(\mathrm{r}=-.56 ; \mathrm{p}=.001)$ and dyslexic readers $(r=-.43 ; p=.001)$. The relation between $\mathrm{RC}$ and $\mathrm{PA}$ with age was negative for typical $(\mathrm{r}=-.76 ; \mathrm{p}=.001)$ and not associated for dyslexic readers $(\mathrm{r}=.08 ; \mathrm{p}=.006)$. The correlation between $\mathrm{RC}$ and $\mathrm{LC}$ with age was non-significant for typical $(r=.014 ; \mathrm{p}>.05)$ and negative for dyslexic readers $(\mathrm{r}=-.75 ; \mathrm{p}=.001)$.

Conclusions: In this mini meta-analysis, the relation between RC and WD decreased significantly with age for typical and dyslexic readers. Interestingly, however, the relation between RC and PA decreased significantly with age for the typical readers, but was not correlated with age for dyslexic readers. In contrast to expectations, the relation between RC and LC with age was non-significant for typical readers, but actually decreased with age for dyslexic readers.

Correspondence: Cortney Fritz, Georgia State University, 870 Inman Village Pkwy NE, Apt 529, Atlanta, GA 30307.E-mail: cortney:fritz@ gmail.com

\section{T. PRESTON, A. DACOSTA \& W.S. MACALLISTER. A comparison of Woodcock Johnson III and WIAT III scores in clinically referred children and adolescents.}

Objective: To determine the relationship, in clinically referred children, between scores on comparable subtests of the Woodcock Johnson Achievement Battery, Third Edition (WJ III) and Wechsler Individual Achievement Test, Third Edition (WIAT III).

Participants and Methods: Participants were 25 clinically referred children and adolescents who received 5 subtests on both the WIAT III and WJ III. Most participants were referred for evaluation of attention/ executive and learning disorders, and in most cases children were seen because of parental concern that a possible learning problem was being missed by the school. Tests were administered within 6 months of each other. In most cases, the WJ III was administered first.

Results: WJ III and WIAT III subtests were strongly correlated with each other with coefficients ranging from .76 to $.94(\mathrm{p}<001)$. In four of five subtests - Word Reading vs Letter Word Identification, Pseudoword Decoding vs Word Attack, Numerical Operations vs Calculations, and Math Problem solving vs Applied Problems - mean WIAT III scores were lower than mean WJ III scores. Mean WJ III Passage Comprehension Scores were lower than mean WIAT III Reading Comprehension scores.
However, differences between mean scores were generally small and nonsignificant, ranging from 2 to 4 points.

Conclusions: Clinicians often compare the WIAT III and WJ 3 in their discussions of preference for one test or the other. This sample size is small, but findings do suggest that while WIAT III scores were generally lower, the level of difficulty for each of the paired subtests was essentially equivalent. As the WJ IV is now available, it will be important to compare it with current and future editions of the WIAT in referred children.

Correspondence: William S. MacAllister, PhD, Neurology, NYU, 223 East 34th St, NYU Epilepsy, New York, NY 10016. E-mail: william. macallister@nyumc.org

V. PLOURDE, M. BOIVIN, M. BRENDGEN, F. VITARO \& G. DIONNE. Neuropsychological Mediators of the Association between Inattention Symptoms and Reading Abilities in Childhood.

Objective: The association between inattention symptoms and reading abilities has been empirically supported by multiple studies but the etiological mechanisms underlying this association have been less studied. The objective of the present study is to test potential neuropsychological mediators of the association between inattention and reading abilities in primary school, specifically aiming to determine if the mediators differ for decoding skills and reading comprehension.

Participants and Methods: Participants were twins from the Quebec Newborn Twin Study (QNTS; 660 families initially enrolled). Kindergarten and first grade teachers rated inattention symptoms and children were assessed at age 8 with objective measures of decoding skills, reading comprehension, as well as measures of neuropsychological processes: rapid naming (colors and numbers), auditory and bimodal temporal processing, phonological analysis and vocabulary skills. Sex and nonverbal abilities were controlled.

Results: Bimodal temporal processing, phonological analysis and vocabulary were significant mediators of the association between inattention and decoding skills while rapid naming, phonological analysis and vocabulary were significant mediators of the association between inattention and reading comprehension.

Conclusions: This study supports a multiple deficit model at the basis of the inattention-reading association in early primary school. Additionally, some neuropsychological mediators of this association appear to vary depending on which reading ability is assessed.

Correspondence: Vickie Plourde, B. A., Psychology, Laval University, 2360 Nicolas-Pinel street, app. 202, Quebec City, QC G1V 4G6, Canada.E-mail: vickie.plourde.1@ulaval.ca

E.A. HUSTON-WARREN, P.T. CIRINO, T.D. TOLAR \& L.S. FUCHS. Path Models for Middle School Mathematical Outcomes.

Objective: There is a strong research base for early developing math skills, implicating both domain specific (e.g. numerosity) and domain general (cognitive/neuropsychological) factors. Fewer studies have focused on later developing skills, when basic math skills have ideally been mastered. Here we focus on broad, school-based assessments of math performance, as well as the key skills of fractions and proportional reasoning. We examined the direct and indirect contributions of each type of predictor, expecting a hierarchy of skill development with predominantly indirect effects of cognitive factors.

Participants and Methods: Sixth graders (N=162) completed cognitive measures of working memory, vocabulary, and spatial skills; symbolic and nonsymbolic measures of number; and graduated math skills (basic facts to procedural computations to fractions/proportional reasoning to broad school outcomes). We employed a path analytic approach to determine direct and indirect contributions.

Results: Path analyses showed good overall fit for the model $(\chi 2(30)=$ 43.662; RMSEA = .054; CFI = .975). Results controlling for age showed that the combination of cognitive, number, and arithmetic variables cumulatively accounted for $57 \%$ of the variance in the state test, with 
the strongest direct results for proportional reasoning $(p<.001)$. Other direct effects included nonsymbolic comparison, number line estimation, and procedural arithmetic (each $\mathrm{p}<.05)$. The effects of working memory and vocabulary were mediated by symbolic comparison, with working memory also mediated by nonsymbolic comparison. Vocabulary was additionally mediated by number line estimation and proportional reasoning.

Conclusions: There was clear support for a prediction model that emphasized a hierarchical progression from domain general cognitive/ neuropsychological skills through number and arithmetic skills to proportional reasoning to high stakes school outcomes. Results also suggest that number skills, but not cognitive skills, had direct impacts on broad outcomes.

Correspondence: Emily A. Huston-Warren, B.A., Psychology, University of Houston, 8383 El Mundo St., Apt. 411, Houston, TX 77054. E-mail: emily.hustonw@gmail.com

\section{Memory Functions}

E.R. APPLEMAN, B.R. KING, G. ALBOUY, A. CRONIN-GOLOMB \&. J. DOYON. Effects of Sleep Quality, Assessed by Actigraphy, on Motor Learning in Healthy Young Adults.

Objective: The purpose of this study was to use actigraphy to identify sleep-quality variables that relate to success of motor learning in adults without sleep disorders. Actigraphy is an ecologically valid, minimally invasive measure of sleep quality that allows assessment of the effects of usual sleep patterns on learning processes.

Participants and Methods: 52 healthy adults (32 women; mean age 24.1 years, SD 3.6) were characterized as having normal sleep on the Pittsburgh Sleep Quality Index and St. Mary's Hospital Sleep Questionnaire. Participants wore a wrist actigraph for four consecutive nights to collect data on usual sleep patterns; the next day they were trained on a motor sequence learning task comprising 14 blocks of practice of a 5 -item sequence on a keypad. Motor skill performance was measured as speed (block duration). Actigraphy data were averaged across all four nights of sleep. Sleep quality variables were correlated with learning abilities, i.e., gains in performance speed observed between the first and last two blocks of the training session.

Results: The amplitude of motor learning was significantly correlated with each of several sleep variables as assessed by actigraphy, including Wake After Sleep Onset, which is the total amount of time awake after the participant initially fell asleep, and average percent time spent asleep.

Conclusions: Our results indicate that scores on actigraphic indices of sleep quality may predict gains in learning a motor sequence task, even in young adults with putatively normal sleep. Individual variability in sleep quality may significantly impact learning ability.

Correspondence: Erica R. Appleman, M.A., Psychology, Boston University, 147 Trenton Street, Unit 2, Boston, MA 02128. E-mail: ericaa@bu.edu

M. Plessers, I. VAN HERZEELE, F. VERMASSEN \& G. VINGERHOETS. The Link Between $S 100 \beta$ and Audioverbal Memory Perfomance in Patients Undergoing Carotid Revascularization.

Objective: S-100 $\beta$ has shown to be a sensitive marker of clinical and subclinical cerebral damage in a variety of clinical settings, such as stroke, mild traumatic brain injury, .... In this study we try to reveal the link between S100B and postoperative cognitive impairment in patients undergoing carotid revascularization by using the audioverbal learning test (AVLT). The AVLT has shown to be a sensitive measure for cognitive changes following carotid revascularization.

Participants and Methods: Blood samples were taken in 31 patients undergoing carotid revascularization (15 carotid endarterectomy, 6 carotid stenting with cerebral protection device, and 10 carotid stenting with flow reversal) pre-operatively, perioperatively, and 2, 6, and 24 hours postoperatively. The serum S100 $\beta$ was measured using S100 Cobas ${ }^{\circledR}$. All patients received cognitive testing one month postoperatively. Results: For S100 $\beta$, repeated measures ANOVA show significant within subjects differences, $F=19,64, p<0,001$. Because the two hours postoperative $\mathrm{S} 100 \beta$ resulted in the highest peak value, we used this measure to correlate to AVLT measures. There was no relation between the sum of the five encoding trials $(r=-0,172, p=n s$.$) , but the long term recall$ showed a non-significant trend $(\mathrm{r}=-0,338, \mathrm{p}=0,078)$. Higher $\mathrm{S} 100 \beta$ values are associated with lower long term audioverbal memory scores. Conclusions: In this study $\mathrm{S} 100 \beta$ follows the typical increase early postoperatively, as shown in other studies. The magnitude of this increase seems marginally correlated to memory performance one month postoperatively. S100 $\beta$ may therefore have a predictive value for longer lasting cognitive impairments.

Correspondence: , . E-mail:

A.C. ZANINOTTO, J. VICENTINI, T.T. SILVA, V.P. GUIRADO, M.A. OLIVEIRA, F. FELTRIN, G.G. BENUTE, M.S. DE LUCIA \& W.S. PAIVA. Improvement on Visuospatial Memory Test After Diffuse Axonal Injury: One-Year Follow Up Study.

Objective: The aim of the study was to compare episodic visuospatial memory performance through Rey-Osterrieth Complex Figure (ROCF) test 6 and 12 months after the trauma in the same group of patients with DAI.

Participants and Methods: 40 patients with moderate to severe DAI were submitted to neuropsychological and behavioral assessment and the results were compared over time. Due to the potential influence of executive functions in ROCF, in addition to the traditional scoring system we also used Savage scoring system.

Neuropsychological assessment included: ROCF, Digit span test (WAISIII), Grooved Pegboard Test, Estimated IQ (Vocabulary and Matrix Reasoning - WAIS-III), Beck depression scale and State scale from StateTrait Anxiety Inventory

Results: ROCF immediate recall, a visual memory performance, has statistically significant difference over time $(\mathrm{p}=0.013)$, with no improving of the bias that could be considered confounders: motor coordination, global comprehension, depressive/anxious symptoms and executive functioning.

Conclusions: Our results suggest that there is a natural improvement of visual memory post-trauma. Further, the present findings may be useful in the construction of a management plan for long-term TBI rehabilitation.

Correspondence: „, E-mail:

\section{K. OMALLEY, C. TYRRELL \& B.E. GAVETT. Effects of Age and Sex on Verbal Memory.}

Objective: This study examined whether verbal memory in a sample of community dwelling older adults with memory concerns differed from normative samples and if there is a need to expand the normative data available for older adults. The HVLT-R manual provides normative data for, sex, age, and education but data for the oldest is old is not distinguished from $80+$. This study examines differences in verbal memory performance among the oldest old by providing more detailed normative data for 2 additional age groups, those 80-89 and 90+.

Participants and Methods: $\mathrm{A}$ sample of $\mathrm{N}=178$ (age $\mathrm{M}=70.9, \mathrm{SD}=$ 9.05) community dwelling older adults (61 men and 117 women) was used to examine study aims. Individuals engaged in a 45-60 min memory screening which provided a brief evaluation of cognitive functioning. Results: Statistically significant differences in total recall scores were found for sex, men $(M=21.10, S D=6.51)$ and women $(M=23.89$, SD $=6.38), \mathrm{t}(176)=-2.75, \mathrm{p}=.07, \eta 2=.04$. A statistically significant difference of age was also found $\mathrm{F}(4,173)=10.29, \mathrm{p}=.001, \eta 2=.19$. Conclusions: Variables of sex and age have medium to large effects on HVLT-R total recall performance. Therefore, further examination of the 
difference in verbal memory performance in the oldest old, and if these differences are diagnostically important, is warranted.

Correspondence: Kelly OMalley, MA, Psychology, University of Colorado Colorado Springs, 4863 N Nevada, Colorado Springs, CO 80918. E-mail:komalle2@uccs.edu

C.E. PRINCE \& M.S. DANIEL. Neuropsychological Performance and Response to Prompting for Delayed Visuospatial Memory.

Objective: The current study explored the non-memory neuropsychological performance of patients that did and did not benefit from verbal prompts for information not recalled during standard administration of the Visual Reproduction II subtest (VR II) of the Wechsler Memory Scale, Fourth Edition (WMS-IV).

Participants and Methods: Participants were 75 patients who underwent neuropsychological evaluation at a neurology/neuropsychology clinic, including measures of language, visual-spatial ability, attention, memory, and executive functions. Participants were divided into three groups based on the necessity and effectiveness of providing prompts after standard administration of VRII: 1.) No Cue patients ( $\mathrm{N}=22$; mean/SD: age $=52.32 / 9.40$. education $=16.05 / 2.79)$ remembered at least part of all designs in standard administration and received no prompting; 2.) Cue Improved patients $(\mathrm{N}=26$; mean/SD: age $=$ $52.35 / 12.85$, education $=13.12 / 2.05)$ did not remember all designs in VRII and remembered additional designs with prompting; and 3.) Cue Not Improved patients ( $\mathrm{N}=27$; mean/SD: age $=58.11 / 12.14$. education $=12.89 / 2.38$ ) did not recall all designs in VRII and did not remember additional designs with prompting.

Results: There were significant differences between the groups on visual-spatial / construction tests ( $p$ 's <.001), most executive function tests ( $p$ 's <.01), and processing speed $(p<.001)$ using ANCOVA analyses. Differences on language tests approached significance $(p=.09)$. On all non-memory tests, the No Cue group obtained the best performance. The Cue Improved group performed better than the Cue Not Improved group on 15 of 18 non-memory tests.

Conclusions: These findings indicate that lack of improved recall on VRII following prompting is associated with greater impairment in non-memory cognitive domains in a heterogeneous group of patients. Correspondence: Carolyn E. Prince, Pacific University, PO Box 28116, Portland,OR 97228.E-mail: prin9087@pacificu.edu

D.M. ANDERSON, A. DAUGHERTY, D. BRUSH, J. PIERCY, A. HEITZER, S. RAZ \& N. OFEN. Age-Related Differences in Free Recall and Mnemonic Strategy Use.

Objective: The use of mnemonic strategies improves memory recall. While both mnemonic strategy use and memory recall increase with age, it is unclear how they interact across development and whether children and adults similarly benefit from mnemonic strategy use. We studied the relationship between unprompted mnemonic strategy use and free recall using the California Verbal Learning Test- Children's Version (CVLT-C).

Participants and Methods: In this study, 105 participants, ages 5-25 years $(M=14.69, \mathrm{SD}=5.87)$, studied a list of 15 words from three semantic categories five times, with each repetition followed by a recall phase. The number of words recalled and the number of semantic and serial clusters were calculated for each repetition.

Results: Recall increased with age in the first repetition $(F(1,103)=30.43$, $\mathrm{p}<.001)$. In addition, semantic $(\mathrm{F}(1,103)=17.42, \mathrm{p}<.001)$, but not serial $(F(1,103)=0.65, p=.421)$, strategy use increased with age. Critically, the age-related increase in semantic strategy use mediated the age-related increase in recall $(\mathrm{F}(1,101)=8.68, \mathrm{p}=.004)$. Learning was tested across the first three repetitions to minimize the expected effects of near-ceiling performance by adults in the last two repetitions. Learning through repetitions improved with age $(\mathrm{F}(1,102)=5.18, \mathrm{p}=.025)$. Whereas, the use of semantic strategy across the first three repetitions increased independent of age $(F(1,102)=1.66, p=.171)$. Furthermore, the use of both semantic $(F(1,100)=16.87, p<.001)$ and serial $(F(1,100)=5.96$, $\mathrm{p}=.016)$ strategies correlated with better learning.

Conclusions: Although semantic strategy use mediates age-related improvement in recall, children benefit from increase in strategy use through repetition similarly to adults. Follow-up research will include examination of MRI-based neuroanatomical correlates of developmental effects and individual differences in recall and mnemonic strategy use learning.

Correspondence: Dana M. Anderson, Clinical Psychology, Wayne State University, 500 W. Willis, Apt. B1, Detroit, MI 48201.E-mail:fj3939@ wayne.edu

B.E. GAVETT, S.E. JOHN, J.W. ADAMS, C.A. BUSSELL, J. GRIFFIN \&. J. SAURMAN. The Effects of Age on the Learning and Forgetting of Primacy, Middle, and Recency Components of a Multi-Trial Word List.

Objective: The serial position effect reveals that recall of a supraspan list of words follows a predictable pattern, whereby words at the beginning (primacy) and end (recency) of a list are recalled more easily than words in the middle. This effect has typically been studied using single list learning trials, but in neuropsychology, multi-trial list learning tests are more commonly used. The current study examined trends in learning for primacy, middle, and recency effects across multiple trials in younger and older age cohorts.

Participants and Methods: Participants were 158 volunteers, including 79 adults aged 17-36 ("younger" group) and 79 adults aged 54-89 years ("older" group). Each participant completed four learning trials and one delayed (5-10 min) recall trial from the Memory Assessment Scales List Learning test. Scores were divided into primacy (first four words), middle (middle four words), and recency (final four words) scores for all five trials. For list acquisition, mixed effects modeling examined the main effects of and interactions between learning slope (logarithmic), age group, and serial position. For retention over delayed recall, a mixed effects model examined the main effects of and interactions between serial position and age group.

Results: Rate of learning increased logarithmically over four trials and varied by serial position, with growth of middle and recency word acquisition increasing more rapidly than recall of primacy words; this interaction did not differ by age group. Retention differed according to age group and serial position; both older and younger adults demonstrated similar retention for primacy words, but older adults showed reduced retention for middle and recency words.

Conclusions: Although older adults acquired less information across learning trials, the reason for this reduced acquisition was related to initial learning, not to rate of learning over time. After a delay, older adults forgot more information than younger adults from the middle and recency portions of the list.

Correspondence: Brandon E. Gavett, Ph.D., Psychology, University of Colorado Colorado Springs, 1420 Austin Bluffs Parkway, Department of Psychology,Colorado Springs, CO 80918.E-mail:bgavett@uccs.edu

\section{A. FLORES. The Effects of Negative Mood and Rumination on} Specificity of Autobiographical Memory.

Objective: Individuals with depressive symptoms report less specific autobiographical memories than healthy individuals. Depressed rumination is commonly seen in this population and evidence suggests it may interfere with autobiographical memory retrieval by depleting executive functioning resources used to retrieve memory, thus explaining the decreased rates of specific memories. The current study sought to investigate whether relationships between depressive symptoms and overgeneral autobiographical memory seen in trait-like measures of depression were also seen during an induced negative mood state, and whether rumination moderated these relationships. Furthermore, the current study sought to expand on the current literature by measuring memory content using traditional criteria (temporal categorization; valence), as well as two additional criteria (detail given and trajectory). 
Participants and Methods: In total, 160 participants were randomly assigned to an induced mood condition (negative, neutral) as well as an attentional task condition (self-focused rumination, distraction). A computerized (E-prime) version of the Velton Mood Induction Procedure was used to induce mood, followed by attention prompts modeled after Nolen-Hoeksema (1991).

Results: Results supported the predicted significant main effect of mood on rates of specific memories. Rumination was not a significant moderator of specific memory rates. However, rumination significantly moderated degree of detail when cue words were positive by impeding retrieval of details given relative to distraction. Additionally, there was an overall significant, positive linear trend of rates of specific memories across cues.

Conclusions: The results indicate that some, but not all, relationships between depressive symptoms and overgeneral autobiographical memory seen in trait-like measures of depression, are also seen during a state-like manipulation of negative mood. The moderating effects of rumination seem to mostly be reflected when negative mood is not present. Correspondence: Ana-Mercedes Flores, Psychology, Eastern Michigan University, 509 West Forest Ave., Apt 319, Ypsilanti, MI 48197. E-mail: aflore14@emich.edu

A.L. MAULE, P.A. JANULEWICZ, M. KRENGEL, R.F. WHITE, J. CIRILLO, S. JUDD \& K. SULLIVAN. A Meta-analysis of Selfreported Neurological and Neuropsychological Symptoms in Gulf War Veterans.

Objective: Gulf War veterans (GWV) have reported excess rates of health symptoms, collectively referred to as Gulf War Illness (GWI), compared to non-deployed veterans of the same era. Papers published over the past 20 years on GWI have assessed different cohorts of GWV and controls and have used different questionnaire methods, resulting in varying conclusions about the key symptoms that GWV experience. In order to identify the neurological and neuropsychological symptoms most commonly reported by different populations of GWV (cases) compared to non-deployed $\mathrm{GW}$-era veterans (controls), meta-analytic approaches were used to analyze published data on GWI.

Participants and Methods: Nineteen peer-reviewed studies were identified in PubMed and secondary referencing. Studies qualified for inclusion if they presented symptoms reported post-deployment and if self-reported symptom frequency data were reported for both cases and controls. DerSimonian and Laird random-effects models, which control for heterogeneity between studies, were used to calculate summary odds ratios for symptoms reported in at least three studies.

Results: Compared to controls, GWV had significantly increased odds of reporting headaches ( $\mathrm{OR}=2.26,95 \%$ CI: $1.9,2.7)$, migraines $(\mathrm{OR}=1.94$, 95\% CI: 1.6, 2.3), numbness/tingling ( $\mathrm{OR}=2.72,95 \%$ CI: 2.2, 3.3), dizziness (OR=2.93, 95\% CI: 2.5, 3.3), tremors/shaking ( $\mathrm{OR}=3.02$, 95\% CI: 2.7, 3.6), as well as forgetfulness ( $\mathrm{OR}=3.31,95 \%$ CI: $2.2,5.0)$, loss of concentration (OR=3.35, 95\% CI: 2.7, 4.2), memory problems $(\mathrm{OR}=4.05,95 \%$ CI: $3.2,5.1)$ and speech problems (OR=3.23, 95\% CI: $2.4,4.5)$.

Conclusions: GWV had significantly higher odds of all self-reported neurological and neuropsychological symptoms in this meta-analysis of 19 studies and is consistent with GWI literature showing a strong central nervous system component to the illness.

Correspondence: Alexis L. Maule, Environmental Health Department, Boston University School of Public Health, 715 Albany Street, Talbot Building, 4W, Boston, MA 02118. E-mail: lex0210@bu.edu

C.B. DUNN, T. MILLS, E. KING \& R.M. BAUER. Spatial Learning and Memory: Visually Scanning the Environment Predicts Performance on a Virtual Water Maze.

Objective: Visual exploration of an environment facilitates the encoding of a cognitive map. We evaluated environmental visual scanning behavior on a virtual Morris Water Maze as a component of spatial navigation performance in aging, mild cognitive impairment (MCI), and Alzheimer's Disease (AD).

Participants and Methods: Participants were 23 younger and 28 older healthy adults, 10 with amnestic MCI, and 8 with $\mathrm{AD}$. Using the $\mathrm{C}-\mathrm{G}$ Arena (Jacobs, et al. 1997), scanning of the environment was measured during search and during a brief period between target acquisition and the beginning of the next trial. Spatial memory performance was quantified as a composite score derived from path length, number of targets found, and percent of time spent proximal to the target on the probe trial.

Results: Group differences in the amount of visual scanning while on the target were significant $[H(3)=30.716, p<.001]$. Healthy young and healthy older participants scanned more frequently than aMCI and $\mathrm{AD}$ groups (all $\mathrm{p}<.01$ ) but were not significantly different from each other $(p=.169)$. Scanning on the target was positively correlated with performance on the composite index in only young healthy participants $(\mathrm{r}=0.423, \mathrm{p}=.022)$. There were no significant group differences in scanning during search, and it was not associated with performance. Conclusions: Visual scanning of the environment is reduced in individuals with cognitive impairment. Performing more scanning while on the target was associated with better performance among young participants. Older adults may not use scanning behavior to strategically explore the environment or are less effective at doing so.

Correspondence: Callie B. Dunn, MS, Department of Clinical and Health Psychology, University of Florida, PO Box 100165, Gainesville, FL 32610-0165. E-mail: cbeck@phhp.ufl.edu

\section{A.H. PANOS, R.W. MANGUM, L.K. PAUL \& W.S. BROWN. Memory in Left and Right Hemispherectomy.}

Objective: The long-term consequences of childhood hemispherectomy on adult learning and memory are not well established. The current study assessed memory in two young adults with hemispherectomy using the California Verbal Learning Test II (CVLT-II) and the Wechsler Memory Scale III (WMS-III).

Participants and Methods: Two adult females were tested, one left hemispherectomy (LH, age 22) and one right hemispherectomy (RH, age 28). Both had onset of intractable seizures at age 5 (RH from Rasmussen's syndrome, and LH from intrauterine stroke) leading to hemispherectomy at age $8(\mathrm{RH})$ and $9(\mathrm{LH})$. RH and LH were similar with respect to education (RH 14yrs; LH 16yrs) and FSIQ (LH 93; RH 84). Results: These individuals performed similarly and in the normal range on several WMS-III subtests: Logical Memory I, Logical Memory II, Visual Reproduction I, and Visual Reproduction II. RH, but not LH, performed in the abnormal range on Family Pictures I (5th \%ile) and II (1st \%ile) and Verbal Pairs I (1st \%ile) and II (2nd \%ile). Similarly, on the CVLT-II, LH scored in the normal range on all subtests, but RH was impaired on Trial 5 of learning (2nd \%ile), Short Delayed Cued Recall (1st \%ile), Long Delayed Cued Recall (7th \%ile), and False Positives (2nd \%ile).

Conclusions: With respect to these two cases, right hemispherectomy due to Rasmussen's syndrome had a significant impact on adult memory not seen in the patient with left hemispherectomy from intrauterine stroke that was evident in learning verbal pairs, memory for pictures, and cued memory retrieval.

Correspondence: Warren S. Brown, Ph.D., Travis Research Institute, Fuller Grad Sch of Psych, 180 N. Oakland Ave., Pasadena, CA 91101. E-mail:wsbrown@fuller.edu

J. HAMILTON, H.M. CONKLIN, Z.S. SADIGHI, N.D. SABIN \& C.M. HARTFORD. Isolated Memory Impairment following Bilateral Hippocampal Damage Secondary to Human Herpes Virus 6 Encephalitis (HHV6) in the Context of Bone Marrow Transplant for Pediatric Leukemia.

Objective: The case study of HM, an adult patient who suffered severe anterograde amnesia following bilateral medial temporal lobectomy, indicated the importance of the hippocampus in memory functioning. 
A pediatric oncology patient developed bilateral hippocampal lesions secondary to HHV6. Focal lesions are rare in pediatric populations; thus, this patient provides a unique opportunity to examine the role of the hippocampus in pediatric neurocognitive functioning.

Participants and Methods: A Caucasian male who was diagnosed with acute myeloid leukemia at age 7 was evaluated at age 11 . He received chemotherapy, total body irradiation, and four bone marrow transplants (BMT). He experienced HHV6 following the third BMT. MRI scans indicated stable encephalomalacia involving the hippocampal complexes, compatible with chronic sequelae of prior HHV6.

Results: Results indicated average verbal and nonverbal reasoning skills (WASI-II VCI=91, PRI=107). Receptive and expressive language was intact. Visual perception, visual motor integration and fine motor dexterity were age typical. Immediate verbal recall was low average (CVLT-C Total T=36, WRAML-2 Story Memory $\mathrm{ScS}=7$ ); delayed verbal recall was impaired (CVLT-C Long Delay Free Recall $Z=-2.0$, WRAML-2 Story Memory Recall $\mathrm{ScS}=5$ ). Visual memory was impaired for immediate and delayed recall (NEPSY-2 Memory for Designs Total $\mathrm{ScS}=1$, Delayed Total $\mathrm{ScS}=1$ ).

Conclusions: The neurocognitive pattern of anterograde amnesia within the context of otherwise intact cognitive functioning is consistent with bilateral hippocampal lesions. Amnestic memory processes are well documented for humans and animals following medial temporal lobe damage, with deficits evident following time delay, secondary to interruption in memory encoding and consolidation. Findings were instrumental in correcting attributions of academic problems solely to significant school absences and in informing memory based interventions. Correspondence: John Hamilton, PhD, Psychology, St. Jude Children's Research Hospital, 262 Danny Thomas Place, Mail Stop 740, Memphis, TN, TN 38105.E-mail: jhamilton@paloaltou.edu

S. MCCAULEY, J. TRAN, D. MENEFEE, J. WANNER, J. HICKEY, W. LEOPOULOS, C. PEDROZA, R. YALLAMPALLI \& E.A. WILDE. Prospective Memory in OEF/OIF Veterans with PTSD with or without Mild Traumatic Brain Injury using Virtual Week.

Objective: This pilot study investigated how mild traumatic brain injury (mTBI) and PTSD affect prospective memory in Veterans.

Participants and Methods: Participants included OEF/OIF Veterans $($ age $=31.2+6.5 ; \mathrm{IQ}=99.6+9.1)$ with PTSD $(\mathrm{n}=11)$, PTSD+mTBI $(n=12)$, and Veteran controls $(n=16)$. The groups did not differ by age $(p=.58)$ or IQ $(p=.16)$. A three-day version of the Virtual Week prospective memory $(\mathrm{PM})$ task was administered to investigate specific PM parameters (e.g., event- and time-based, and regular and irregular events). Participants also completed the PTSD Checklist (PCL-M), Rey Auditory Verbal Learning Test, and the Color-Word Interference test. Results: Correct responses were analyzed using a $3 \times 2 \times 2$ mixed ANOVA with the fixed effect variable Group (control, PTSD, PTSD+mTBI) and random effects variables of PM Task (regular, irregular) and PM target (event-based: EB, time-based: TB). Significant differences were found for Group $(p<.0001)$, Task $(p<.05)$, Target $(p<.0001)$, and the Task $\times$ Target interaction $(p<.02)$. The groups performed significantly differently from each other (Control > PTSD > PTSD+mTBI). Decomposition of the Task $\times$ Target interaction revealed similar performance on regular and irregular EB tasks, but participants performed more poorly on irregular TB tasks compared to regular TB. Regression analyses demonstrated PTSD severity and inhibitory control, but not retrospective memory, were significant contributors to EB and TB.

Conclusions: These are some of the first data demonstrating PM deficits in Veterans with PTSD without or without $\mathrm{mTBI}$ and underscore the role of disrupted frontally-based processes, and not retrospective memory capacity, in PM performance.

Correspondence: Stephen McCauley, PhD, Physical Medicine and Rehabilitation, Baylor College of Medicine, One Baylor Plaza, BCM 637, Houston, TX 77030.E-mail: mccauley@bcm.edu
E. RHODES, T. GIOVANNETTI, K.N. DEVLIN, M. LAMAR \& D.J. LIBON. Serial Order Position Effects in Alzheimer's and Vascular Dementia: AClinical Application of Competitive Queuing. Objective: Contemporary models of memory for serial order account for a wide range of phenomena observed in healthy young adults. Specifically, examination of serial position curves for accuracy and error production has provided support for computational models utilizing a competitive queuing (CQ) framework, which includes a parallel planning level (where the individual items in the sequence are activated) and a competitive choice-level (where the precise serial order of the sequence is specified). Less is known about the application of CQ models to clinical populations.

Participants and Methods: Participants included 26 individuals with Alzheimer's disease (AD), 28 vascular dementia (VAD) and 29 healthy control (HC) participants. Memory for serial order in the visual domain was assessed using the Object Span task. Accuracy and error scores (omissions, intrusions, between- and within-trial repetitions, and transpositions) were computed.

Results: HCs were significantly younger, better educated, and had higher MMSE scores compared to dementia groups. Significant differences were obtained for total errors $(\mathrm{HC}<\mathrm{AD}<\mathrm{VAD}$, $\mathrm{p}=.030)$ controlling for age and education. Serial position analyses indicated significant group by serial position interactions on total accuracy $(p<.001)$, omissions $(\mathrm{p}<.001)$, between-trial repetitions $(\mathrm{p}=.013)$ and within-trial repetitions $(p=.06)$. Pairwise comparisons revealed significant differences between dementia groups, such that the VAD group committed more intrusion and between-trial repetition errors in serial positions 1 and 2 (p<.05, all analyses).

Conclusions: Serial position curves suggest a degradation of the primacy effect in VAD when compared to AD and healthy controls. Poor performance on memory for serial order of visual stimuli in VAD may be due to increased interference in the parallel planning layer from previously seen test items (i.e., inappropriate items are activated for the sequence). Existing CQ models fail to adequately address this phenomenon.

Correspondence: Emma Rhodes, MA, Psychology, Temple University, 1701 N 13th Street, Department of Psychology - Temple University, Philadelphia, PA 19122. E-mail: emma.rhodes@temple.edu

D.K. LEIBEL, J.E. KISSER, D. SHAKED, S.R. WALDSTEIN, M.K. EVANS \& A.B. ZONDERMAN. Higher Levels of Perceived Stress Are Associated with Better Verbal Memory Performance in Older Men.

Objective: Examine potential interactive relations of perceived stress, age, and sex to neuropsychological functioning.

Participants and Methods: Participants were 1,462 African-American and White, urban-dwelling adults (mean age $=47.5$ years; $43 \%$ female; $57 \%$ White; $44 \%$ below the poverty line) from the Healthy Aging in Neighborhoods of Diversity across the Life Span (HANDLS) study who completed the four-item Perceived Stress Scale (PSS), and the California Verbal Learning Test (CVLT), Benton Visual Retention Test, Trail Making Test, and WAIS-R Digit Span. Multiple regression analyses examined relations of perceived stress, age, sex, and their interactions with neuropsychological functioning controlling for race, poverty status, literacy, and symptoms of depression.

Results: A significant three-way interaction among PSS score, age, and sex was found such that, in male participants, CVLT Learning Slope ( $\beta$ $=.514, \mathrm{p}=.015)$ and CVLT Long Delay Free Recall scores $(\beta=.567, \mathrm{p}$ $=.002)$ increased as a function of higher PSS scores and increased age. Greater perceived stress benefited older men more than middle-aged or young men. Additionally, a significant two-way interaction was observed between PSS and age for CVLT total scores $(\beta=.279, p=.021)$; CVLT performance increased as a function of greater PSS and greater age. Older individuals (64 years) benefited more from this effect than middle-aged ( 46 years) and younger ( 30 years) individuals. 
Conclusions: Perceived stress may have beneficial effects on verbal learning and memory skills for older adults, particularly men. Correspondence: Daniel K. Leibel, Clinical Psychology/Behavioral Medicine track, Psychology, University of Maryland, Baltimore County, UMBC Department of Psychology, 1000 Hilltop Circle, Math/Psychology Building Room 312, Baltimore, MD 21250.E-mail: dleibel1@umbc.edu

C.R. NICHOLAS, M.N. DOWLING, J.M. OH, C. ILLINGWORTH, B.P. HERMANN, B.B. BENDLIN, M.A. SAGER \& S. JOHNSON. Longitudinal assessment of self-reported memory function in cognitively normal, middle-aged adults: Findings from the WRAP study.

Objective: There is evidence that subjective memory complaints may be associated with early changes in cognitive function and biomarkers of neurodegenerative disease. The purpose of this study was to model the longitudinal trajectory of self-reported memory function and its relationship to risk factors of Alzheimer's disease (AD).

Participants and Methods: The study included 284 cognitively normal adults (mean age $=54$ ) from the Wisconsin Registry for Alzheimer's disease Prevention (WRAP), an enriched cohort of individuals with a parental family history of AD. All subjects completed the Memory Functioning Questionnaire (MFQ) at three time points over a four-year period. Latent growth curve models were conducted using age, education, depression (CESD), and familial risk factors (FH $\mathrm{F}, \mathrm{APOE} 4 \pm$ ) to estimate the longitudinal trajectory of scores on the MFQ. Cross-sectional analyses were also conducted to examine the relationship between scores on the MFQ and measures of neuropsychological performance and depression.

Results: Latent growth curve modeling revealed MFQ scores were inversely associated with the CESD and education at baseline, however, these variables did not predict longitudinal change on the MFQ. Modeling also revealed that MFQ scores did not significantly change over time and were not associated with any other variable. Cross-sectional analyses indicated that MFQ scores were significantly associated with mood and not associated with performance on neuropsychological measures nor familial risk factors.

Conclusions: In a cohort of relatively young, cognitive normal adults, self-reported memory function does not significantly change over time and was not related to familial risk factors. Consistent with other studies, depression is strongly associated with self-reported memory function, however, in this cohort, depression was not found to be a predictor of change in self-reported memory function.

Correspondence: Christopher R. Nicholas, Ph.D., Geriatrics, William S. Middleton VA, 1503 Morrison St, Madison, WI 53703. E-mail: crnichol@medicine.wisc.edu

R. FAMA, S.A. SASSOON, C. HARDCASTLE, T. ROHLFING, E.V. SULLIVAN \& A. PFEFFERBAUM. Temporo-parietal Contribution to Visual Episodic Memory Performance in HIV Infection.

Objective: HIV infection affects brain structure and component memory processes. Here, we examined visual and verbal episodic memory (immediate and delayed) in individuals with HIV and healthy controls and cortical and subcortical correlates of these processes.

Participants and Methods: Structural MRI and memory testing in 67 individuals with HIV, with and without concomitant alcoholism, and 45 uninfected, age-range matched controls, enabled examination of brain volume correlates of visual and verbal, immediate and delaved episodic memory, assessed with the Wechsler Memory Scale-Revised (WMS-R) and Rey-Osterrieth Complex Figure (Rey-O). Regional cortical and subcortical tissue volumes were derived from T1-weighted MRI data, following automated parcellation and segmentation using the SRI24 brain atlas.

Results: HIV subjects were impaired on all memory tests and had smaller lateral frontal, temporal, parietal, and thalamic volumes than controls. Principal component analysis of WMS-R memory subtests and
Rey-O yielded 2 factors: Visual (VIS; Figural Memory, Visual Reproduction, and Rey-O) and Verbal (VER; Logical Memory). All test scores were standardized, and subtest $Z$-scores were averaged within each factor to yield a composite score. In HIV, VIS was significantly correlated with parietal $(\mathrm{r}=.31, \mathrm{p}=.01)$, temporal $(\mathrm{r}=.44, \mathrm{p}=.0002)$, and precuneus $(r=.45, p=.0001)$ volumes with no significant VER-brain correlations. Multiple regression indicated that precuneus $(p=.016)$ and temporal $(p=.029)$ volumes were significant predictors of VIS over and above parietal lobe volume $(\mathrm{p}=.58)$, each accounting for approximately $33 \%$ of the VIS variance. CD4 cell count $(\mathrm{p}=.05)$ but not lifetime alcohol consumption contributed uniquely to VIS.

Conclusions: These results are consistent with animal and human studies demonstrating the relevance of the precuneus in visuospatial memory and provide evidence for contribution of a temporo-parietal structural brain network to performance on visually-based episodic memory tasks in HIV.

Support: AA017347, AA017168

Correspondence: Rosemary Fama, Ph.D., Department of Psychiatry and Behavioral Sciences, Stanford University School of Medicine, 1901 Emory Street, San Jose, CA 95126. E-mail: rfama@stanford.edu

H.B. ENGLAND, M. GILLIS, C. FYOCK \& B.M. HAMSPTEAD. Modulating Categorical and Coordinate Spatial Processing Using Transcranial Direct Current Stimulation.

Objective: Two forms of spatial processing are involved in object location memory. Coordinate processing uses a fine-grained code to provide exact knowledge for the location and is believed dependent on the right posterior parietal cortex (PPC). Categorical processing relies on spatial relationships between objects and is believed dependent on the left PPC. We used transcranial direct current stimulation (tDCS) to test these brain-behavior relationships during the encoding of object location associations.

Participants and Methods: Twelve right-handed, healthy young participants received 20 minutes of tDCS $(2 \mathrm{~mA})$ during distinct conditions over three separate sessions: anodal ("excitatory") stimulation of right PPC with concurrent left PPC cathodal ("inhibitory") stimulation $(\mathrm{R}+\mathrm{L}-)$, the reverse montage ( $\mathrm{R}-\mathrm{L}+)$, and sham stimulation. Participants completed different versions of the Object Location Touchscreen Task (OLTT) during each session, which assesses coordinate (recall of the location without the environmental context) and categorical processing (recall of the location with the environment present). Encoding occurred during the last five minutes of stimulation, while the delay phase occurred fifteen minutes after stimulation.

Results: Participants performed more accurately on the coordinate task following R-L+stimulation when compared to R+L-performance. There were no significant within-group differences in performance on the categorical task.

Conclusions: Findings suggest two possibilities that will be examined in future studies: 1) The R-L+ facilitates a categorical processing approach that persists even when in the absence of environmental details - possibly via mental imagery. 2) Cathodal stimulation decreased spurious noise in the right PPC thereby allowing for more efficient processing by the "critical" neuronal populations.

Correspondence: Heather B. England, M.A., Emory University School of Medicine, 115 112th Ave. NE, apt. 601, St. Petersburg, FL 33716. E-mail: hnbengland@gmail.com

C.P. MCFARLAND, J. CLARK, L. LEE, L.J. GRANDE, B. MARX \& J. VASTERLING. Prospective Memory among Veterans: The Role of PTSD in Forgetting Intentions.

Objective: Posttraumatic stress disorder (PTSD) has been linked with a variety of functional impairments, including in academic and occupational domains and has been associated with neuropsychological dysfunction in several areas. One domain of cognitive functioning that may be particularly relevant to the functional impairment associated with PTSD is prospective memory (PM), or remembering to complete an 
intention in the future. Studies revealing a relation between anxiety and PM deficits lend support to the hypothesis that PTSD, a disorder with prominent anxiety features, would also be associated with impaired PM. This study was designed to determine whether a relation exists between PTSD and PM. We hypothesized that PTSD symptom severity would be inversely related to PM performance. We were also interested in whether PTSD symptom clusters would be differentially related to PM.

Participants and Methods: Forty veterans completed self-report measures of PTSD (PCL-C) and depressive symptoms (BDI-II), along with measures of retrospective memory (CVLT-II). They then completed an event-based PM task.

Results: The results of a hierarchical regression analysis revealed that symptoms of PTSD accounted for $16 \%$ of the variance in PM performance $(\mathrm{F}(3,36)=3.47, \mathrm{p}<.05)$, after controlling for age and retrospective memory. Additionally, each of the three PTSD symptom clusters was related with PM performance.

Conclusions: The results of this study suggest that the more severe a veteran's experience of PTSD, the more likely he or she is to experience PM difficulties. This association between PM and PTSD may have substantial clinical implications, as PM failure could lead to poor treatment adherence (e.g., missed appointments, poor follow through on treatment related goals), occupational difficulties, and strained relationships. Future work should continue to explore cognitive mechanisms underlying the PM deficit in PTSD, with an eye towards tailoring interventions to assist veterans with day-to-day functioning.

Correspondence: Craig P. McFarland, Psychology, University of Montana, Skaggs Bldg Room 202, Missoula, MT 59812. E-mail: craig. mcfarland@umontana.edu

M. ROSSETTI, Y. CAMPOS, A.J. MENDEZ, C.B. WRIGHT \& B.E. LEVIN. The Relationship among Adiponectin Isoforms, Memory Performance, and Gender.

Objective: BMI in midlife has been shown to increase the risk of dementia later in life, independent of other cardiovascular comorbidities. However, this association reverses direction later in life (age 60-70 years) such that older individuals with dementia have lower BMIs than those without dementia. Adiponectin, an anti-inflammatory cytokine released by adipose tissue, is negatively correlated with body weight and BMI. Adiponectin exists in low molecular weight (LMW) and high molecular weight (HMW) isoforms. It has been proposed that LMW may have central effects. This study examined the association of circulating adiponectin isoforms with BMI and memory performance in older individuals with memory complaints.

Participants and Methods: Ninety-two participants (Mage=75, SD=9) from the University of Miami Memory Disorders Clinic consented to donation of a blood sample and were included for analyses in the present study. Adiponectin immunoassays for adiponectin isoforms were developed with monoclonal antibodies. Memory was measured with the Logical Memory test from the Uniform Data Set (UDS) of the National Alzheimer's Coordinating Center. Scores were adjusted for demographic factors.

Results: Linear regression analyses revealed that BMI predicted total adiponectin $(b=-.06, p<.05)$ and LMW adiponectin $(b=-.02, p<.05)$. Elevated levels total and LMW adiponectin significantly predicted poor immediate memory performance, but only for women $(p<.05)$. We found no significant association with delayed verbal memory. Conclusions: This study provides preliminary evidence that LMW may exert central effects and serve as an important biomarker of the aging process and cognition. This investigation also highlights an important gender difference in biomarkers of neurodegenerative disease. Further research in larger samples is needed.

Correspondence: M. Agustina Rossetti, M.S., Neurology, University of Miami, 1 Hermann Museum Circle Drive, Apt. 3073, Houston, TX 77004.E-mail: agustinarossetti@gmail.com
Y.T. QUIROZ, F. LOPERA, K.V. PAPP, L. VELILLA, A. BAENA, D. GOMEZ, J. RENDON \& D.M. RENTZ. Adaptation and validation of the Spanish version of the Face Name Associative Memory Exam (FNAME) in cognitively normal older individuals.

Objective: Face Name Associative Memory Exam (FNAME) is a challenging paired associative learning task that involves learning of facename pairs as well as their free recall and cued recall (Rentz et al. 2011). FNAME has been validated for clinical use with English-speaking older adults, and has proved useful in detecting subtle cognitive changes in the preclinical stages of Alzheimer's disease. In this study we adapted to Spanish the 12-item version of FNAME, and evaluated the validity and reliability of the Spanish version (S-FNAME) in a Colombian sample. Participants and Methods: Fifty cognitively-normal older adults (5080 years old) from community senior centers in Medellin (Colombia) participated in the study. Subjects were clinically normal as defined by their Mini-Mental State Examination (MMSE) score, and a review of their medical history. None of the participants had a history of alcoholism, drug abuse, or head trauma, or current serious medical or psychiatric illness. All participants completed the S-FNAME, as well as a multidomain neuropsychological evaluation. The external validity, internal consistency and reliability of S-FNAME were assessed.

Results: S-FNAME subtests showed high internal consistency. Correlations between S-FNAME subtests and another episodic memory measure were significant $(\mathrm{p}<0.05)$. The results indicated strong test-retest reliability for all S-FNAME subtests.

Conclusions: The Spanish version of FNAME is a reliable instrument for assessing memory function in Spanish-speaking older adults. Normative data stratified by age and education will be generated as we increase our sample size.

Correspondence: Yakeel T. Quiroz, PhD, Neurology, Massachusetts General Hospital, One Bowdoin Square, Boston, MA 02111. E-mail: yquiroz@mgh.harvard.edu

D.P. SHEPPARD, S. WOODS, K.L. DOYLE, H.M. HOLDEN, E. PIROGOVSKY TURK, S. TIERNEY, J. COREY-BLOOM \& P. GILBERT. A Comparison of Prospective Memory Capacity in Huntington's Disease and HIV Infection.

Objective: HIV infection is associated with deficits in the strategic aspects of prospective memory (PM), which are hypothesized to reflect prefrontostriatal circuit dysregulation. The present study evaluated this hypothesis by comparing the PM profiles of HIV infection and Huntington's disease (HD), which is a prototypical subcortical disease.

Participants and Methods: Participants included 20 individuals diagnosed with mild-moderate HD, 80 persons infected with HIV, and 34 healthy adults. All participants completed the Memory for Intentions Screening Test (MIST), which includes 2- and 15-minute delay scales for both time- and event-based PM.

Results: A mixed effects model with gender as the covariate revealed main effects of group and PM cue, as well as a significant group by PM cue interaction ( $\mathrm{ps}<.05)$. Planned post-hoc analyses showed no between-groups differences on the least strategically demanding 2-minute delay event-based scale. However, both HD and HIV+ groups performed below healthy adults on the more strategically demanding 2 - and 15-minute time-based condition scales (ps<.05). On the 15-minute delay event-based scale, only the HIV+ group performed below the healthy adults, who did not differ from the HD group.

Conclusions: Consistent with the study's hypotheses, both HIV and $\mathrm{HD}$ were associated with deficits in strategically demanding time-based PM. However, the finding that only HIV was associated with long-delay event-based PM deficits suggests that HIV+ individuals may be impaired in their capacity to retain event cue-intention pairings. Such long-delay event-based PM deficits may reflect increased retrospective memory load exacerbated by extended monitoring and retention demands, perhaps indicating greater medial temporal lobe dysfunction in HIV. 
Correspondence: David P. Sheppard, San Diego State University, 2111 Holly Hall St. Apt \#3902, Houston, TX 92107.E-mail: dsheppard.uh@ gmail.com

C.A. BAKER, M. WELSH, E. PETERSON \& J. KOLE. Musical Working Memory in Musicians and Non-musicians within Baddeley's Multicomponent Working Memory Model.

Objective: The current study examined in musicians and non-musicians the possibility of a subsidiary tonal system, in addition to the phonological loop and visuospatial sketchpad, for processing musical information within Baddeley's multicomponent working memory model.

Participants and Methods: Three primary working memory tasks (tonal, verbal, and visual-spatial), requiring participants to make a same/different judgment between two sequences, were each paired with four secondary suppression tasks (tonal, verbal, visual-spatial, and no suppression) in a dual-task paradigm. All 12 conditions were administered to 58 college students (27 musicians; 31 non-musicians). Reaction time and accuracy were examined in terms of the relative impact of the different types of suppression and musical experience.

Results: Visual-spatial suppression negatively impacted visual-spatial working memory and tonal suppression negatively affected tonal working memory compared to the other suppression conditions. Although tonal suppression interfered more with tonal working memory than verbal suppression, there was no differential impact of tonal and verbal suppression on verbal working memory. There were no differences between musicians and non-musicians on primary task by secondary task interactions.

Conclusions: A crossed double dissociation was demonstrated for visual-spatial working memory with both tonal and verbal working memory, and a single dissociation was demonstrated for tonal and verbal working memory, with evidence for a tonal loop on the tonal working memory task but not on the verbal working memory task. These mixed results regarding the existence of a tonal loop are interpreted within the multicomponent working memory model and current research involving the extent to which verbal and tonal working memory share resources. Correspondence: Crystal A. Baker, University of Northern Colorado, 832 Donegan Road, Unit C, Glenwood Springs, CO 81601. E-mail: crystal. spindler@gmail.com 


\title{
Author Index \\ Forty Third Annual Meeting \\ International Neuropsychological Society
}

\author{
February 4-7, 2015 \\ Denver, Colorado, USA
}

\author{
Aarsland D., 201 \\ Aase D., 105 \\ Abeare C., 27, 29, 244, 263 \\ Abell M., 87 \\ Abildskov T.J., 192 \\ Abrahams B.S., 149 \\ Abrams D.N., 242 \\ Abrams G., 275 \\ Accurso E., 187 \\ Acosta M.T., 37, 118, 237 \\ Adachi S., 237 \\ Adams J.W., 57, 263, 301 \\ Adams R., 86 \\ Adams S.W., 28, 198 \\ Adamson L.B., 242 \\ Adamson M.M., 8, 13, 30 \\ Adhimoolam B., 28, 127, 209 \\ Adluru N., 32 \\ Aebi M., 269 \\ af Geijerstam J., 183 \\ Afraz S., 167 \\ Afzal K., 289 \\ Agate F., 240 \\ Agbayani K., 15 \\ Agerbæk M., 294 \\ Aggarwal R., 220 \\ Agoston A., 182 \\ Aguerrevere L., 53 \\ Ahern D., 120 \\ Ahles T., 195 \\ Ahmed A., 267 \\ Ahmed Y., 167 \\ Ahn A., 22 \\ Ahn S.S., 262 \\ Ahronovich M.D., 101 \\ Aiken E., 129 \\ Ailion A., 296 \\ Aita S., 211 \\ Aitken M., 281 \\ Ajilore O., 248 \\ Aksan N., 50
}

Ala T., 81

Albert E., 154

Albouy G., 300

Alexander A., 32

Alexander K., 280

Alfini A.J., 24, 139, 141, 173

Ali S., 105

Alkozei A., 24, 26, 138, 245, 246, 247

Allen A., 90, 186

Allen B.L., 68

Allen G., 240

Almane D., 92

Almeida R.P., 32, 126, 179

Aloia M., 125, 221

Alosco M., 219, 228

Alterescu K., 211

Altmann L.J., 25, 216, 295

Alturki A., 44

Alverson W.A., 179

Amariglio R.E., 80, 227

Amaya S., 10, 11

Amaya-Hodges M., 236

Ambekar N., 43

Ambrose M., 129

Ambroziak A.R., 111

Amedoro S., 56

Amen D., 136

Amick M., 18

Amidi A., 294

Amorim R.L., 37

Amsbaugh H., 153

Anand A., 203

Ances B., 116

Anderson A.J., 231

Anderson A.W., 199

Anderson B., 250

Anderson D.M., 301

Anderson J.J., 50

Anderson K., 12, 44, 187, 270, 293

Anderson L.B., 27

Anderson P.J., 127, 128
Anderson S.A., 118, 119, 198, 199

Anderson S.W., 50

Anderson V., 95, 281

Anderson W., 64

Andersson S., 241

Andrade G.A., 149

Andreotti C., 101

Andresen E.N., 218

Andrews-Hanna J., 222

Annett R.D., 66

Anstey K.J., 110

Anthony L.G., 242, 280

Antigüedad A., 123

Antoine A., 39

Anton S.D., 140, 144

Antonini T., 183

Apostolova L., 75, 77, 148, 205, 207. 208

Apple A., 289

Appleman E.R., 300

Arce Renteria M., 115

Aréchiga A., 71, 73

Arenivas A., 108

Arentoft A., 72, 111, 114

Arentsen T., 278

Ares K., 236

Arfanakis K., 146

Arias F., 107

Armour A.C., 36, 189, 190

Armstrong G., 293

Armstrong J., 119

Arnett P., 117, 121, 122, 130, 131

Arnold A., 251

Arnold S., 201, 273

Arnsten J., 107

Arola N., 289

Aronova E., 25, 256

Arredondo B.C., 156

Arrington C., 167

Asarnow R., 193

Aschenbrenner A., 177 
Ash K., 246

Ashendorf L., 267

Ashford J.M., 161, 291

Ashford J.W., 8, 30

Ashourian P., 221

Asken B., 23, 172

Askren M.K., 270, 293

Aslan S., 23, 139

Asmussen S., 266

Aspelin P., 183

Asthana S., 32, 33, 126, 179

Atkins J., 229

Atri A.. 251

Attygalle S., 207

Au R., 181, 269

Audrit H., 12

Auerbach S.H., 181

Aupperle R., 277

Auslander D., 152

Avila D., 148

Avila J., 103, 174, 205

Aviv A.L., 98

Aylward S., 170, 228, 235

Ayres A., 185

Baade L.E., 264

Babakhanyan I., 198, 199

Babcock-Parziale J., 9

Babikian T., 193

Babu Henry Samuel I., 6 ?

Bäckman L., 82

Badal W., 265

Badaly D., 188

Baena A., 305

Baerresen K., 78

Bailie J.M., 15, 266, 269

Bajo S., 106

Baker C.A., 306

Baker D.A., 232

Baker E., 13

Baker L., 84, 111, 116, 145

Bal E., 280

Balaban L., 61, 270

Baldo J., 296, 297

Baldwin S.A., 245

Ball K., 206

Balota D., 77, 177

Banerjee N., 119

Banerjee N.S., 118

Banerjee P., 294

Bangen K.J., 83, 126, 178, 181, 203

Banich M., 248

Banks S.J., 17, 43, 176

Banville F., 12

Bar-Hen M., 54

Barakat L.P., 287

Barba A.M., 138
Barbosa P., 140

Barch D.M., 30

Barclay S., 251

Barczi S., 261

Bark J.S., 149

Barlow K.M., 129

Barnes A., 147, 148

Barnes M., 167

Barnett M., 84

Barnett P., 252

Baron I., 101

Baron K., 181

Baroncini M., 204

Barr T., 11

Barr W.., 90

Barrash J., 50, 260

Barrera K., 261

Barry D., 11

Barthelemy O., 248

Barton J., 118

Barton M., 239, 240, 241

Barulli D., 69

Barulli D.J., 77

Bassett D., 139

Basso M.R., 39, 53, 54, 58, 61, 119 , 151,156

Bateman R.J., 177

Baucom B.R., 202

Bauer R.M., 23, 147, 149, 172, 253 , 254, 268, 274, 302

Baughman B.C., 278

Bauldry R.M., 28

Baum C., 226

Baumann N., 217

Baxter J., 49, 72

Bayley P., 8

Baynard J., 198

Bean Jaworski J., 185

Beauchamp M.H., 95, 231, 232, 235, 271, 273, 281

Beaudoin C., 95, 231, 232, 235

Beaudreau S.A., 40

Beaulieu-Bonneau S., 12

Beaumont J.L., 102

Bech-Hansen T., 251

Becker B., 243

Bedard A., 285

Beddes L., 39

Beebe D.W., 64

Beers S., 30

Beevers C.. 214

Behrendt J., 208

Behrens S., 174

Beiser A., 181

Beitz K., 72, 266

Belanger H., 275
Bélanger S., 12

Belkonen S., 260

Bell B., 90

Bell M.D., 125

Bellec P., 252

Bellerose J., 95, 231

Bellinger D.C., 185

Benbadis S.R., 92, 94

Bender H.A., 91

Bendlin B.B., 31, 32, 33, 126, 179, 304

Benedetto A., 129

Benge J., 219

Benji A., 111

Bennett D., 146, 179

Bennett D.A., 75, 110

Bennett J., 103

Benson E., 69, 179

Benson L.M., 267

Benson R.R., 22

Bentley H., 123

Benute G.G., 300

Berg J., 43

Berger J.T., 175

Bergin S.W., 250

Bergquist T., 9

Berkner P., 229

Berl M., 2, 36, 37, 169, 189

Berlin M., 57

Bermudez C.I., 118, 119, 198, 199

Bernick C., 17

Bernier A., 231, 232, 235

Bernier F.P., 109, 127, 128, 129

Bernier R.A., 13, 135

Bernstein D.M., 74

Bernstein J., 231

Bernstein L., 166, 292

Beseler C.L., 105

Best M., 229

Bettcher B.M., 77, 148, 181

Bhattacharjee K., 200, 201

Bhojwani D., 33

Bianchini K., 52, 53

Bickford D., 142

Biedunkiewicz B., 214

Biekman B., 230

Bieliauskas L., 51

Bigler E., 66

Bigler E.D., 95, 147, 154, 172, 191 , 192, 222, 233, 241, 281

Binder J.R., 297

Binney R., 207, 296

Birath J., 10, 11, 51

Birtcher K., 145

Bishop E., 206

Biss R.K., 203

Bitton A., 285 
Bjoraker K., 120

Bjorkquist O., 155

Bjornn D., 275

Blackwell L.S., 132

Blaha R.Z., 233

Bland S., 198

Blanken A.E., 148

Blanton P.D., 38

Blinkoff D.C., 4 ?

Block C.K., 59, 80, 86

Blomsø L., 201

Blum A., 89

Blumen H., 136

Bo J., 240

Bocti C., 227

Bodapati A.S., 153, 155

Bodin D., 35

Boettcher A., 254

Boettger J., 252

Bogdanova Y., 276

Bogenschutz M., 140

Bohsali A., 14 ?

Boivin M., 299

Bokhari H.A., 25, 256

Boland D., 215

Bolzenius J., 84, 145

Bondi M.W., 21, 72, 81, 83, 85, 112 , 115, 126, 178, 180, 203, 224

Bonner M.J., 293

Bono A.D., 25, 211, 256

Boogerd W., 289

Book D.S., 297

Bookheimer S., 147, 294

Boone K.B., 51

Boop F.A., 161

Booth J.E., 88, 225

Boots E.A., 32, 126, 179

Borod J.C., 25, 211, 256

Bosenbark D.D., 240

Bosworth C., 139

Bosworth C.C., 23

Bothe D.. 184

Bott N.T., 127, 209

Bouchard A., 158

Bouffet E., 162

Bousman C., 107

Bousvaros A., 34

Boutzoukas A., 149

Bowers D., 25, 70, 76, 78, 82, 216. 220, 250, 257, 272, 274

Bowler R.M., 28, 29, 45, 105, 198

Bowler R.P., 221

Bowyer S., 279

Boxer A., 181

Boyd B., 277

Boyer K., 93
Boyle P., 75, 146

Boys C.J., 129, 182

Bozorg A.M., 92, 94

Bradbury C., 21, 31

Bradbury K., 239

Bradford L.S., 11

Bradley K.A., 166

Bradley M., 250

Bradley-Ewing A., 119

Bradstreet L.E., 144

Brand T., 52

Brandt J., 211

Branson R., 4 ?

Bratzke L., 31, 32, 245

Bray S., 128

Brearley A., 129

Brega A., 287

Breier J.I., 90

Breiter H., 246

Brendgen M., 299

Brennan J., 279

Brennan L., 215

Brenner L., 93

Brewster P., 69, 262

Brickell T.A., 12, 15, 60, 62, 269

Brickman A.M., 83, 127

Bridges A., 261

Brinker M., 183

Brinkman T.M., 33, 64, 65, 290, 291 , 293

Brooks B., 57, 229

Brooks Holliday S., 242, 259

Broshek D., 106

Brown A., 9

Brown B.L., 105

Brown D.S., 74, 121

Brown R., 32

Brown T., 235

Brown W.S., 27, 44, 295, 302

Brozaitiene J., 249

Bruce A., 119

Bruce J., 119, 120, 122

Bruce S.E., 26

Brush D., 301

Bryant K., 199

Bryant K.R., 101, 121

Bryant S.J., 104

Bryant V., 104

Bryson C.N., 257

Buchanan L., 16

Buchbinder J.. 184

Buchholz B., 170, 228

Buchholz J.L., 141, 149

Bucker J., 152

Budding D.. 10, 11

Budisin B., 21, 31
Bueler E., 258

Buetefisch C.M.. 148

Bullard S., 16

Bunevicius A., 249

Bunevicius R., 249

Bunner M., 39

Burbridge J., 8 ?

Burciaga J., 142

Burggren A., 147

Burghy C.A., 34

Burkauskas J., 249

Burke A., 154, 157

Burke K.P., 152

Burke M.M., 209, 210

Burles F., 251

Burns A., 237

Burr R.B., 86

Burrell L., 104

Burton C.Z., 159, 272

Busch R.M., 215

Bussell C.A., 301

Butler B., 257

Butterfield L.C., 76, 220

Butts A.M., 9, 35, 142, 178

Buxbaum L.J., 248

Byrd D.A., 111, 115, 162, 163

Caan M., 289

Cabeen R., 84, 145

Cabrera A., 123, 145

Cáceres-Luna G., 68, 106

Cadden M., 121, 130, 131

Cagigas X.E., 162, 163

Cagna C., 18

Cairncross M., 258

Cairns D., 151

Calamia M., 265

Calhoun V., 66, 67, 154, 155

Calvo D., 43, 218

Cammisuli D.M., 204

Camodeca A., 239

Campbell E., 46

Campbell L., 72, 81, 85

Campbell R., 14

Campbell T.A., 4

Campos Y., 305

Canas A., 120

Candilis P., 119, 156

Cannistraci C.J., 199

Cannon A.D., 108

Cannon L., 280

Cao B., 278

Capelli K., 208

Caplan A., 207

Carbine K., 245, 250

Carlew A.R., 239

Carlin G., 275 
Carlozzi N.E., 99, 225, 226, 267

Carlson K.. 17

Carlson-Green B., 162

Carlsson C.M., 32, 126, 179

Carmant L., 133

Carney P., 149

Carns D., 53

Carrathers T., 153, 170, 228

Carrier L., 133

Carrion C., 102

Carson A.M., 93

Casaletto K.B., 102, 113, 114, 225, 226

Casey J.E.. 168, 169

Casey S., 45

Casnar C.. 189

Cassedy A., 231

Cassidy A.R., 185

Cassill C., 84, 214

Castellon S.A., 146, 274, 288

Castelluccio B., 238

Castillo D.T., 224

Castillo G., 205

Castro E., 204

Catani M., 159

Catley D., 119

Catropa C.. 281

Caudle S., 183, 244, 296

Cavaco S., 158

Cavanaugh J., 254

Cazzanti S., 59

Cecil K., 292

Cella D., 267

Cellar J., 259, 265

Cellard C., 155, 157

Cerbone B., 210, 211

Cernin P.A., 274, 288

Cervantes Y.. 10, 51

Chakravarty M., 124, 137

Chamard E., 16, 18

Chambers P.. 279

Champoux M., 44

Chan A.. 217

Chan M.L., 206

Chang C.H., 71

Chang K., 170

Chapieski L., 86, 87, 92, 93, 293

Chapman S., 19, 21, 94

Charbel F.T., 188

Charlton R., 248

Chase K.A.. 155

Cheema S. 166

Cheiffetz R.T., 257

Chelune G.J.. 202

Chemaitilly W.. 290

Chen A., 275

Chen D., 88, 89
Chen G., 192

Chen S., 113

Cheng J., 8, 30

Cheng Y., 36

Cheong J., 128

Cherner M., 107, 115, 162

Cherrier M., 270, 293

Cherry B.J., 217

Chertkow H., 227

Chester S., 184

Cheung Y., 33

Chiaravalloti N.D., 6, 7, 15, 18, 118 , 119,275

Child A., 45, 4 ?

Chin E., 93, 114

Chiou K.S., 7

Chiu P., 100, 132, 284

Chodosh J., 197

Choi A., 140

Choi E., 277

Choi J., 125, 264, 276

Choi S., 38, 158

Chon D., 250

Chowdhry S., 271

Christ S.. 142

Christensen B.K., 22, 255

Christensen Z., 172

Christian B., 32

Chu Z., 230, 281

Chung C., 166

Chute D., 227

Chute M., 80

Cimino C., 28, 47, 220

Cirillo J., 302

Cirino P.T., 35, 44, 45, 47, 164, 166. 167, 168, 299

Clark A.. 47

Clark A.L.. 19, 20, 21, 81, 224

Clark J.. 304

Clark L.R., 31, 33, 115

Clark R.. 52

Clark U.S., 84, 111, 135

Clark V.P., 155

Clawson A., 245, 250

Clayson P.E., 245, 250

Clem M., 175, 209

Cleveland M., 219

Clifford D., 115

Clifton W.E.. 92

Cloughesy T.F., 294

Coats S.M., 192

Cobia D., 148

Cochrane B.. 82

Cochrane M., 39

Cohen J., 21, 183, 248

Cohen M., 90, 186, 297
Cohen M.L., 211

Cohen R., 91

Cohen R.A., 85, 104, 115, 117, 213. $219,228,278$

Colasurdo E.A., 20

Colbert A.M., 187

Cole P.G., 283

Cole W.R., 266

Colella B., 22, 273

Colledge M., 105

Collier A., 115

Collins M., 291

Collins R., 58, 88, 89, 225, 264

Colon C.. 224

Colon-Perez L., 145

Colvin M.K., 294

Combs D.R., 54, 119, 156

Combs H.L., 48, 231

Compas B.E., 199

Concepcion E., 5, 198, 209, 255, 259

Conklin H.M., 161, 291, 293, 302

Connelly J., 187

Connelly S., 129

Conner A., 186

Connery A.K., 232

Connor M.K., 197

Considine C.M., 29, 244, 263

Conturo T., 84, 145

Conway T.W., 2 ?

Cook A.H., 79

Cook C., 79

Cook D., 32, 204

Cook J.A., 14

Cook S.E., 215

Cool D.L., 184, 188

Cooley S., 84, 145

Coolidge F.L., 52

Coolin A., 74

Cooper L., 39

Cooper S.E., 215

Coopersmith L., 151

Copeland C., 59

Copeland C.T., 86

Copeland J.N., 218

Copersino M., 137

Corbett D.B., 67

Corcoran C., 174

Corey-Bloom J., 123, 207, 208, 305

Corkin S., 221

Corona M.. 10, 11

Correa D.D., 288

Correa L.N., 85, 278

Correia S., 48, 115, 117, 141

Corrigan K., 139

Corti M., 145

Cosentino S., 177 
Coskunpinar A., 258

Cosman J., 65, 66, 116

Costanzi M., 152

Costello R., 58

Cothran T.P., 19, 179

Cottingham M., 38, 266

Coulehan K., 58

Couturier C., 44

Cowles W., 25

Cox C.S., 281, 282

Crabtree V.M., 161, 291

Craggs J.G., 247

Crane N.A., 138

Cranston C.C., 38

Crapo J.D., 221

Crawford M., 78

Crawford S.G., 129

Crawley A., 31

Creek J., 217

Crocker N., 298

Cronin-Golomb A., 134, 221, 248, 278, 300

Crosby R.D., 213

Crosson B., 296

Crowe M., 206

Crowley J., 234

Crowley S.J., 145

Cuevas S., 217

Cui J., 149

Cukier Y., 23, 129

Cullum M., 23, 100, 139, 203, 264

Cummings T., 78

Cunningham C., 107

Cunningham J.M., 61

Curcio N., 276

Curtis K.L., 52, 53

Curtiss G., 275

Cutter G.R.. 210

Czemeres N., 197

Czepielewski L.S., 152

Czipri S.L., 9, 246

d'Hondt F.. 95

Dabbs K., 92

DaBreo C., 46

DaCosta A., 299

Dagley A., 80

Daianu M., 178

Dalmaijer E., 251

Daly B., 47

Daly C.J., 10

Daly M., 155

Damásio J., 158

Damewood G., 129

Daniel M.S., 301

Dasher N., 112

Daugherty A., 301
David D., 270, 293

Davidson L.L., 150

Davidson R.J., 34

Davis E., 161

Davis H., 4

Davis H.P., 72, 243, 266

Davis J., 150, 213, 255, 284

Davis R., 19

Dawson D.R., 47, 273

Day E.F., 269

Daya H., 57

Dayalu P., 267

de Guise E.. 12, 16, 44

De Gyves G., 168

De La Garza R., 106

de la Monte S.. 117

de la Vega A., 248

De Leon F., 77, 207, 208

de Lucia M.S., 37, 300

De Marco A., 106

de Reus M., 252

De Ruiter M., 289

Dean P.M., 101

Deatrick J., 287

DeBastos A.. 186

Debska-Slizien A., 214

Decarlo C., 262

Deck B., 80

Deck B.L., 206

Dekhtyar M., 80

Del Piero L.B., 27

Delano-Wood L., 19, 20, 21, 33, 72. 81, 83, 85, 126, 178, 180, 181. 203, 224, 277

DelBene V., 58, 149

Deldonno S.R., 138, 244

Delgado S.R., 118, 119

Delgaty L.E., 39

Delis D.C.. 207

DeLuca J., 119, 253, 256, 275

DeMarco A.T., 116

Demers L.A., 24, 25, 26, 141, 149

Demian M., 212

Demireva P., 73

DenBoer J., 14

Denburg N.L., 260

Denckla M., 159, 160

Denney D.A., 40

Denning J.H., 61

Dennis M., 35, 95, 164, 165, 166, 167 . 168, 192, 233

Deoni S., 48, 141

Deshpande H., 143, 280

Desjardins M., 12

Dettmer J., 51

Deutsch R., 115, 116
Devanand D., 125, 276

Devlin K.N., 117, 269, 303

Devlin M., 213

Dewey D., 109, 127, 128, 129

Dewey R., 40

DeYoung N., 200, 260, 276

Dhall R., 207

Dhar A., 29, 244, 263

Diaz-Santos M., 278

Didiano R., 229

Díez-Cirarda M., 145

Diggins A., 104

DiLuna M., 229

DiMuzio J., 258

Ding M., 212

Dinh K.L., 190, 264

Dinh V.T., 179

Dionne G., 299

DiQuattro M.E., 186

Disbrow E.A., 217

Divatia S., 24, 25, 26, 149

Dixon R.A., 82, 110

Dockstader C., 162

Dodd J.S., 251

Dodds A., 217

Dodge H.H., 176

Doesburg S., 162

Dolan S.L., 157

Donders J., 268

Doniger G., 54

Donofrio M.T., 36, 189, 190

Doody R., 172, 179

Dorflinger J., 170, 228, 235

Dotson V.M., 140, 144, 211

Doucet G., 252

Dougherty R., 32

Dowling M.N., 179, 304

Downing M., 17

Downing N., 26 ?

Doyle K.L., 112, 113, 305

Doyle L.W., 128

Doyon J., 300

Dresden A., 145

Dreyer W., 183

Driscoll A., 12, 62

Dronkers N.F., 296, 297

Drossos T., 187, 289

Duckworth J., 15

Duda B., 104, 135

Duda J.E., 215

Dudley K.M., 24, 242

Duff K., 80, 106

Duffield T., 241

Duffy J.R., 178

Dufort P., 4

Dufour C.A., 112 
Duggan E.C., 44, 48

Dumas B., 84

Dumont-Mathieu T., 239

Duncan C.C., 88

Duncanson H., 87, 258

Dunlosky J., 42

Dunn C.B., 259, 302

Duong P., 151

Duque G., 282

Durant J., 176

Durgerian S., 75, 76, 142, 205

Duvall S., 100, 279

Dwarka N., 289

Dye R., 78

Dykstra J., 232

Dykstra J.B., 88

Eagan D., 41, 116, 146

Eastvold A., 41

Ebert P., 206

Echlin H.V., 244

Eckerle J., 129

Edelstein K., 166

Edland S.D., 178

Edmonds E.C., 33, 72, 83, 85, 126. 178, 180, 203

Edwards D., 32

Edwards M., 4 ?

Ehana J., 119

Ehrler M.R., 104

Eich T.. 74

Eichstaedt K.E., 92, 94

Eid B., 249

Eigsti I., 238

Einerson J., 32

Eisengart J., 183

Eisman G., 38

El Hajj S.A., 37

Elashoff D., 148

Elias J., 120

Elias L.. 197

Eliassen C.F., 180, 201

Elizabeth H.M., 215

Ellefson S.E., 184

Ellingson B.M., 294

Ellis M., 193

Ellis M.U., 72

Ellis R., 107, 115

Ellison R.D., 199

Embree L., 274, 288

Embry L., 66

Engelbrecht S., 116

England H.B., 304

Eppig J., 72, 81, 85

Epstein J.N., 286

Ercoli L.M., 274, 288

Erdfelder E., 74
Erdodi L., 59

Erickson K.I., 282, 283

Erickson K.T., 170

Erickson L., 177

Erickson S.J., 100, 170

Ertelt T., 169

Erten-Lyons D., 176

Eskes G., 257

Espeland M.A., 82

Essex M.J., 34

Estevis E., 54, 156

Etherton J., 52

Ettenhofer M., 11

Euler M., 250

Evankovich K.D., 120

Evans C., 290, 294

Evans C.T., 9

Evans D.A., 179

Evans J., 77, 207, 208

Evans M.K., 5, 104, 303

Evans S.A., 22

Everest S.D., 215

Ewing-Cobbs L., 164, 165, 281, 282

Faber J., 281

Fair J.E., 245, 275

Fairchild J.K., 8, 30

Falchook A., 85, 278

Falola M., 210

Fama R., 304

Farley S.V., 75, 174, 176

Farrell E., 90

Farrell M., 177

Farrer T.J., 48, 141, 192, 233

Fatoorechi S., 51

Fausett J.K., 48, 133

Fayad A., 185

Fazeli P.L.. 112. 113, 114, 115

Feder A., 49

Fedor A., 74, 79

Fee R.. 109

Fee R.J., 108, 109

Feger D., 135

Feierstein R.E., 150

Feigon M., 49, 219

Fein D., 63, 238, 239, 240, 241, 242

Feinstein A., 2, 3, 124

Feld J., 215

Feliciano L., 217, 243

Felleman B., 270

Feltrin F., 37, 300

Ferenc L., 169

Fernandes J., 158

Fernandez A.L., 162, 163, 261, 283

Fernandez V., 167

Fernau A., 217

Feyz M., 12, 44
Field J., 64

Figueroa C., 142

Filley C.M., 159, 160

Filoteo V., 200, 205, 208, 209, 210

Fink B., 129

Fiocco A.J., 70

Fischer B.L., 261

Fischer M., 220

Fischer M.P., 183

Fisher A., 96

Fisk J., 206

Fiszdon J., 125, 264, 276

Fitch R., 181

Fitzer K.R.. 34

FitzGerald D., 147, 149

Fitzsimmons-Craft E., 187

Fladby T., 201

Flannery J., 258

Flaro L., 168

Fleischman D., 146

Fleming D.E., 105

Fleming J.C., 143

Fletcher J.M., 35, 44, 166, 167, 168 , 191, 281

Floden D.. 215, 218

Flodmark O., 183

Flores A., 301

Flores I., 102

Flores R.A., 252

Flowers A.T., 103, 174, 205

Flynn S., 208

Foldi N.S., 113, 175

Fombonne E., 279

Fong C.H., 200, 209, 210

Fong M.W., 108

Ford A., 145, 149

Ford J., 124

Forte M., 118, 119, 198, 199

Fortier C.B., 18, 144, 224

Foster N.L., 106

Fox M.E., 34

Foxe J.J., 149

Franchow E.I., 73

Francis T., 39

Franco C., 113

Franklin D., 115

Franz C., 78

Franzen M., 6

Frasnelli J., 44

Frässle S.. 137

Fredrickson K., 273

Freeman M., 17

French J., 13, 135

French L.M., 12, 15, 60, 62, 88, 269

Frias A.L., 191

Fridman A., 25 
Frim D., 184

Fritz C., 299

Frost R.B., 14, 154

Fuchs K., 75

Fuchs K.L., 174

Fuchs L.S., 299

Fuentes A., 115

Fuerst D., 22

Fuglestad A., 129

Fuimara M., 217

Fujita I., 237

Fujita T., 134

Fukunaga R., 141

Fulbright R.L., 261

Fuller B.E., 106

Furda M., 234

Furst A., 30

Fuster J., 10, 11, 51

Fyock C., 304

Fyock C.A., 202

Gabilondo-Cuéllar I., 123

Gable J., 268

Gabriel L., 151

Gabrieli S., 222, 223

GadElkarim J., 248

Gafford J., 51

Gagner C.., 231, 232, 271

Gagnier S., 12

Gair J., 208

Gajjar A., 293

Galasko D., 126, 138, 178, 180, 181

Gale S.D., 86, 134, 177, 230, 265

Galioto R., 89

Gallagher C.L., 179

Gallegos Rodríguez Y.E., 158

Gallo B., 198, 199

Gallo J., 227

Galusha-Glasscock J.M., 100, 121, 264

Galvin J.E., 210, 211

Gama C.S., 152

Gamber R., 47

Ganguli M., 71

Ganz P.A., 274, 288

Garant A., 29, 244, 263

Garcia A., 296

Garcia P., 118, 271

Garcia R., 28, 29

Garcia S., 74, 79

Garcia-Barrera M.A., 39, 44, 48

Garner A.A., 64

Garofano J., 64

Garrett K.D., 97, 98

Garrett R., 207

Garrido-Nag K., 41, 295

Gartner R., 12, 62

Gaspar M., 286
Gattás M., 283

Gattu R., 22

Gatz M., 291

Gauvreau S., 162

Gavett B.E., 20, 57, 173, 193, 194, $262,263,267,269,300,301$

Gazzaley A., 9, 246

Gazzaniga M.S., 124

Geck R., 64

Geldmacher D.S., 261

Gelman B., 115

Geneser A.C., 75, 174, 176

Genova H.M., 18, 118

Georgieff M., 129

Gerhardt C.. 95, 192, 233

Gershon R., 102, 225, 226, 267

Gerst E.H., 44, 47, 87, 92

Gertsberg A.G., 110

Geschwind M., 26 ?

Gess J.L., 48, 133

Geyer S., 252

Gfeller J.D., 60, 121

Ghilain C.S., 198, 199

Gibson J., 10

Giese E.M.. 50

Gifford K., 69, 179

Gilbert P., 67, 83, 112, 305

Gilbert P.E., 207, 208

Giles N., 145

Gill S.K., 151, 259

Gillis M., 304

Gioia A.R., 289

Gioia G.A., 171, 230, 233, 234, 236, 237, 286

Giovannetti T., 80, 202, 206, 303

Giza C.. 193

Glass L., 130, 187

Glazewski K., 27

Gleason C.. 261

Gleitsmann K., 17

Glenn T., 10, 11, 51

Glisky E., 78

Glodé M., 287

Glover N.M., 51

Glusman M., 119, 122

Gnoffo M., 204

Gobbi E., 269

Gocheva V.V., 105

Godfrey M., 69, 179

Godwin D.W., 4

Goeggel Simonetti B., 229

Gogel H., 26, 149

Goggin K., 119

Gogoi V., 81

Gokhale A., 234

Goldani A.A., 152
Goldberg C., 188

Golde S.D., 25, 256

Golden C., 76, 136

Goldsmith A., 226

Goldstein D.S., 53

Goldstein F.C., 259, 265

Goldstein J., 147

Goldstein M., 82

Goldsworthy R., 49, 197

Goldwin M., 289

Gollan T.H., 180

Golub S.A., 46, 113

Golzad M., 54

Gómez A., 123

Gomez D.. 305

Gomez R., 50, 151

Gonçalves A., 158

Gongvatana A., 115, 117

Gontkovsky S.T., 283

Gonzales M., 41, 136, 214

Gonzalez C.. 133

Gonzalez D., 220

Gonzalez J., 199

Gonzalez R., 113

Goodale G., 158

Goodcase R., 279

Goodell D., 198

Gooding A., 93

Goodman M., 166

Goodnight S., 226, 267

Gordichuk A., 262

Gordon B., 279

Gorgens K.A., 51

Gorlyn M., 154, 15 ?

Gorman Barry P., 39

Gorman M., 87

Gottuso A., 217

Gouaux B., 112, 123

Goukasian N., 75, 148

Goverover Y., 15, 256

Gowhari M., 49

Gowins J.R., 138

Grabyan J., 89

Grabyan J.M., 172

Grace E., 97

Grace E.S., 98

Grady C., 222, 223

Graham A., 12, 62

Graham D.M., 187

Grajzel K., 20, 57

Granader Y., 24, 242

Grand R.J., 34

Grande L.J., 224, 304

Grange D.K., 142, 190

Granholm E.L., 158

Grant I., 107, 112, 113, 115, 116 
Grant M., 78, 220

Gravano J., 83

Gravel J., 95, 231, 232, 235

Gray H., 293

Gray H.J., 270

Green C., 9

Green E., 140

Green R., 21, 22, 31, 255, 273

Greenberg B., 121, 185

Greenberg D.A., 88

Greene J., 171

Greenfield F., 273

Gregory A., 224

Greif S.M., 191

Greiner J., 236

Gretencord A.A., 170, 228, 235

Gretler J., 55

Greve K., 52, 53

Grieco J., 290

Griffin J., 301

Griffith E., 83

Grigsby J., 49, 72, 287

Grilli M.D., 78, 247

Gross A.C., 182

Gross E.. 135

Gross E.Z., 76

Gross P.L., 216

Grote C.. 35

Gu S., 139

Gu Y., 74, 127

Guardino D., 41

Gubanich P., 231

Guirado V.P., 37, 300

Guise B.. 11

Gullett J., 149

Gullett J.M., 147

Gundermann D., 67

Gunn G.. 287

Gunstad J., 42, 43, 74, 79, 135, 213 , 218, 219, 228

Gunther S., 40

Gupta A.K., 43

Gur R., 110

Gurnani A., 57, 173, 263, 267

Gustafson E.L., 43, 103

Guth L.M., 139

Guzman V.A., 83, 127

Haacke E., 22

Haaland K.Y., 224, 253

Haarbauer-Krupa J., 231

Hagberg J.M., 139

Hahn E.A., 267

Hahn-Ketter A., 56

Haisley L.D., 241

Haj-Hassan S., 266

Hajek C., 232
Halahalli H.N., 43

Hale J.B., 34, 298

Haley A., 41, 84, 116, 136, 146, 214

Haley W., 220

Hall T.A., 183

Halliday D., 262

Halperin J.M., 285

Hamberger M.J., 93

Hamilton J., 268, 302

Hamilton R., 201

Hamiwka L., 91

Hamlet A., 129

Hamm A., 92

Hamman R., 49, 72

Hammeke T.A., 9, 192, 297

Hammers D.B., 106

Hamsptead B.M., 126, 202, 304

Han D., 11, 146, 178, 231

Han X., 69, 179

Hancock L., 120

Hangal S., 247

Hanna S., 44, 201

Hannay J., 166

Hansen C., 242, 259

Hanson E.. 78

Hanson K.L.. 19, 20

Hanson R.S., 20

Hanten G., 148, 230, 281

Hantke N., 8, 40, 142

Hanulik I., 207

Harciarek M., 7, 214

Hardan A., 170

Hardcastle C., 304

Harder L., 185

Hardy D.J., 10, 11, 38, 51, 111, 263

Hardy K.K., 36, 37, 65, 66, 118, 161 . 162, 171, 185, 188, 189, 289

Hardy S.J., 185, 188, 189

Haren E., 71

Hargrave D.D., 90

Harik L., 165

Harley A., 151

Harmelink O.L., 50

Harms V., 197

Harrell K.M., 197

Harrell M., 186, 297

Harrington D., 205

Harris J.G., 100

Harris O., 17

Harris R.J., 294

Harrison C.E., 228, 277

Harrison T.M., 147

Hart J., 203

Hartford C.M., 302

Hartley N., 236

Hartman E.R., 48, 141
Harvey P.D., 159

Hasher L., 203

Hashimoto K., 237

Hass C., 70, 78

Hass C.J., 216, 274

Hassenstab J., 177

Hauger R., 78

Haut M. W., 38, 42, 101, 148

Hawkins K., 101

Hawkshead B.E., 84, 104, 135

Hayashi C.Y., 37

Hayes S.M., 228

Hays C., 7, 18, 138, 181

Haywood J., 151

Hazamy A.A., 25, 216

Hazlett K.E., 138, 142

He A., 208

He H., 155

Heaps J.M., 84, 116, 145

Heather F., 96

Heaton R.K., 99, 102, 107, 115, 225, 226, 272

Heaton S.C., 191

Hecht E., 144

Hedden T., 81

Hedges D.W., 177

Heffelfinger A., 114, 238

Heflin L., 291

Hegde K., 174

Heilman K.M., 7, 27, 85, 159, 160, 278

Heim M., 261

Heinemann A.W., 99, 225, 226

Heinks T., 229

Heinonen K., 241

Heinrich K., 187

Heinrich K.P., 188

Heinrichs R.J., 264

Heitzer A., 99, 186, 301

Heller W., 249

Helms M., 189

Helphrey J., 274

Henderson C., 208

Hendricks A., 13

Hendrix T., 116

Henkle L., 258

Hennig B.L., 110

Henry B.L., 112

Henry L., 232, 235

Hensley M., 288

Herbener E., 155

Herholz P., 137

Herlihy L., 241

Hermann B.P., 2, 32, 33, 90, 92, 126, 179, 304

Hermann C., 261

Hernandez B., 158 
Herndon A., 207

Herrst C., 212

Hershaw J., 11

Hershey T., 142

Herting N., 169

Hertza J., 90, 186, 297

Hertzing W., 116

Hessen E., 201

Hickey J., 46, 152, 154, 303

Hicks R.L., 101

Higano C., 270

Higginson C.I., 217

High W.M., 9

Highsmith J., 220

Higuchi Y., 153

Hildebrandt T., 152

Hildenbrand A.K., 47, 220

Hileman J.D., 67, 83

Hill A., 279

Hill B., 77

Hill B.D., 254, 265

Hill K.R., 16

Hill S., 153

Hill-Jarrett T., 268

Hillary F.G., 13, 66, 67, 134, 135

Himali J.J., 181

Hinkin C., 146

Hinkle C., 93

Hinkle C.D.. 114

Hinton V.J., 108, 109

Hiploylee C., 4

Hizel L., 141

Ho V.T., 276

Hoare J., 116

Hobbie W., 287

Hocking M.C., 287

Hodges E.K., 188

Hoecker H.. 129

Hoelzle J., 268

Hoffmeister J., 86

Hogg J., 61

Hohman T., 69, 179

Hoida E., 205

Hokkanen L., 35, 36, 125

Holcomb M., 59

Holcombe B.D., 41

Holden H.M., 67, 207, 208, 305

Holdnack J., 33

Hollis A., 258

Holm K.E., 221

Holmeide C., 201

Holness A., 73

Holtzer R., 136

Homaifar B.Y., 4

Homeidan A., 118

Hood A., 142, 190
Hoodin F., 187

Hoogs M., 9

Hope O.A., 90

Hopkins J., 235

Hopkins R., 145, 216

Horner M.D., 62

Horning S., 197

Horowitz T., 65, 66, 195

Horton D.K., 100, 185, 209, 264

Hoshino T., 153

Hoskin J., 250

Hosseini B., 123

Hostetter S.A., 37, 162, 188, 289

Hoth K.F., 221

Hovda D., 10, 11, 51

Hovi P., 241

Howard C.. 39, 246

Howell T., 261

Howerin D., 201

Howieson D., 85, 176

Hsiao S., 6

Hsu D.T., 138

Hu X., 148

Huang H., 123, 212

Huang L., 293

Huang S., 6

Huang-Storms L., 279

Hubbard N., 123

Huber J., 166

Huckans M., 106

Hudson M.M., 33, 64, 65, 290, 291 , 293

Hugenschmidt C., 69

Hugentobler J., 231

Hughes H., 121

Hughes J., 219, 228

Hughes S., 121, 185

Hughes T.F., 71

Hummer B., 52

Hunt I., 83, 245

Hunt J., 82

Huntbach B.A., 41, 254

Hunter J., 281

Hunter M., 155

Hunter S., 43, 55, 103, 236

Huremovic D., 203

Hurewitz F., 240

Hurless N., 276

Huston J., 13

Huston-Warren E.A., 47, 299

Hutaff-Lee C., 120

Hutchison J., 164, 165

Hutchison M., 271

Hwang K., 75

Hwang T., 285

Hwangbo M., 285
Hynan L.S., 74, 100, 264

Iampietro M., 55

Iaria G., 251

Ibarretxe-Bilbao N., 123, 145

Ichise M., 127

Ikanga J.N., 215

Ikeda Y., 237

Illingworth C., 304

Inder T.E., 128

Infante M., 130

Ingles J., 206

Innes A., 109, 128

Inoue K.. 134

Insel P., 142

Iovino I., 86

Ip R., 152

Irish M., 222, 223

Isaacson A., 203

Isquith P.K., 24, 169, 171, 236, 237. 286

Iudicello J., 115

Iudicello J.E., 112, 113

Iverson G.L., 229

Iverson K., 13

Ivins B., 266

Ivnik R.J., 178

Jackson D., 92

Jacobson A., 140

Jacobson L.A., 187, 285

Jacola L.M., 161, 291

Jagid J., 198, 199

Jagust W.J., 94, 95

Jaimes S., 80

Jak A., 7, 277

Jak A.J., 18, 19, 72, 81, 85, 224, 277

Jakobsen E., 252

James A., ?

James B., 146

James G., 48, 133

Janecek J., 49, 188

Janes A.C., 134, 137

Jang K., 158, 286

Janicki S., 127

Janowich J., 246

Jansen A., 136, 137

Jantz T., 19

Janulewicz P.A., 8, 14, 302

Jarrell S., 217

Jasinski L., 284

Jefferson A.L., 69, 179, 266

Jefferson S., 174

Jeffrey M.G., 20

Jegathesan T., 166

Jenkins L.M., 138, 244

Jennette K.J., 175

Jeon H., 285 
Jerard T., 151, 188

Jerskey B.A., 84

Jessen F., 194

Jeste D.V., 112, 113, 272

Jethva R., 220

Jimenez E., 178

Jivan N., 5 ?

Jivani S., 192, 233

Joannette M., 227

Joeris A., 229

John J.P., 43

John S.E., 57, 301

Johns P.A. 191

Johnson B., 60

Johnson C.P.. 165, 281, 282

Johnson E., 235

Johnson K.A., 80, 227

Johnson P.L., 28

Johnson S., 31, 32, 33, 126, 179, 245 , 304

Johnson T., 172

Jones A., 260

Jones J., 69, 70, 76, 78, 90, 92, 216, 272,274

Jones K.L., 130

Jones K.M., 298

Jones R., 71

Jones R.D., 50

Jones S., 257

Jordan L.L., 70

Josephs K.A., 178

Josephson R., 219, 228

Joska J., 116

Joubert S., 227

Jowkar G., 287

Judd S., 302

Jun H., 297

Jung R., 252

Jung S., 6

Juranek J., 35, 166, 167, 168, 191, 282

Jurick S.M., 180, 224, 277

K Thiruselvam I., 268

Kaemmerer T., 102, 184, 246

Kahn B., 55, 91, 286

Kahn-Mills B., 274, 288

Kaijser M., 183

Kais L.A., 249

Kajantie E., 241

Kakavand H., 10

Kalamangalam G.P., 90

Kalechstein A.. 106

Kamat R., 113, 115

Kamath V., 110

Kamper J., 64, 156

Kana R., 280

Kandah C.. 142, 205
Kang J., 273

Kansagara D., 17

Kao A., 181

Kaplan D., 16

Kaplan L., 175

Kaplan R., 110

Karandikar N., 17

Karantzoulis S., 210, 211

Karimian A., 72, 111

Kark S., 276

Karlson C.W.., 189

Karnik N., 43, 103, 236

Karpiak S.E., 111

Karpouzian T., 246

Karray S., 236

Karsh L., 287

Kassel M., 138

Katzen H.L., 118, 119

Kaufman D.A., 77, 98

Kaup A.R., 9

Kaur S., 41, 84, 116, 146

Kauwe J.S., 177

Kauzor K., 205

Kay C., 92, 142, 205

Kaye J.A., 85, 176

Kaylegian J., 43, 103

Keatley E., 27

Keenan P., 151

Keener W.. 56, 233

Keilp J.G., 154, 157

Keller A., 181

Keller F., 72, 266

Keller J., 50

Keller J.E., 224

Kelley E., 238

Kellogg E.J., 28

Kelly D.A., 205

Kemmotsu N.. 140

Kemp S., 33

Kenealy L., 286

Kennedy J., 15, 134

Kennedy Q., 13, 243

Kennedy T., 189, 190

Kentor R., 187

Kenworthy L., 24, 190, 242, 280

Kerce V., 53, 151

Kernan C.. 193

Kerns K.A., 105, 271

Kerr J., 138

Kersey P., 87

Kertesz A.. 159, 160

Kessels R.P., 125

Keutmann M., 113

Khan R., 293

Khurshid K., 46

Kiefel J., 186
Kielb S., 79, 181

Kiely T., 50

Kiewel N.A., 218

Killgore W.D., 24, 25, 26, 138, 149, $245,246,247$

Kim M., 285, 286

Kim R.T., 19, 20, 21, 224

Kim W., 32

Kim Y., 105, 198

Kimberg C., 33, 64, 65, 290, 291, 293

Kimura D., 134

King B.R., 300

King E., 302

King J.H., 14

King T.Z., 34, 144, 231, 288, 296

Kingery K.M., 286

Kinne E., 271

Kinney D., 52

Kinoshita L., 8, 30, 158

Kintzel F., 257

Kintzing R., 200, 201

Kinzer A., 79, 182

Kipman M., 26, 149

Kirkwood M., 29, 232, 233, 235

Kirton J.W., 211

Kirwan B., 145, 280

Kirwin P., 125, 276

Kisser J.E., 5, 104, 303

Kizling I., 183

Klein R., 257

Klein-Tasman B.P., 110, 189

Kleineidam L., 194

Kleinhans N., 19, 223

Klimek P., 152

Klipfel K., 31, 42

Kluftinger J., 259, 265

Kluger B., 67

Knevelbaard A., 71

Knight L., 6

Knight R., 297

Knoch K., 241

Knox S., 61

Kohlrus S., 81

Kole J., 306

Kondo K., 17

Kong J., 8, 30

Kontos A., 232

Koo D., 41, 295

Koop J., 93, 238

Kopelman M., 253, 254

Koppara A., 193, 194

Korcarz C., 32

Koriakin T., 285

Korinek L., 97, 98

Kornak J., 207

Kornblith E.S., 28, 45, 105 
Kornhuber J., 194

Koscik R.L., 33, 179

Koshimori Y., 21, 31

Kosik K., 181

Kovach S., 156

Kovelman I., 279

Kowalczyk W.J., 113

Kowalski G., 148

Kozora E., 221

Krach L.E., 31

Kramarski B., 100

Kramer J.H., 77, 148, 181, 206, 207

Kratz A., 267

Krauskopf E.E., 216

Krawczyk D., 19, 23

Kremen W., 78

Krengel M., 8, 13, 14, 302

Kretzmer T., 64

Krishnan K., 23, 139

Kritikos L., 206

Krivitzky L., 36, 37

Kriz D., 91

Kromash R., 234

Kroupina M., 129

Krull K.R., 33, 64, 65, 290, 291, 293

Kryza-Lacombe M., 288

Kubas H.A., 298

Kubu C.S., 215

Kuhlthau K., 290

Kuhn T., 149

Kulesz P.A., 167, 168

Kulkarni A., 117

Kumano H., 298

Kumar A., 248

Kumar D., 43

Kunin-Batson A., 65

Kuo C.. 96, 97

Kupzova S., 192

Kurczewski J., 80, 202

Kurowski B., 132, 231

Kushnir E.. 192

Kuttesch J.F., 199

Kwon Y., 38

L Alvarez T., 118

La D.D., 158

Laasonen M., 33

Laatsch L., 270

Labbe D., 84

Lace J., 228

Lacritz L.H., 40, 74, 121, 175, 203 , 209. 264

Lacy M., 108, 184, 257

Ladd L.. 113

Lafo J.A., 250, 274

Lague-Beauvais M., 44

Lah J.J., 259, 265
Lahaie A., 273

Lahti-Nuuttila P., 33

Lai J., 267

Laidlaw D., 84, 145

Laird A.R., 1

Lajiness-O’Neill R., 187, 241, 279

Laliberte' C., 57

Lalik A.M., 217

Lalonde G., 235

Lam M., 247

Lamar M., 21, 248, 269, 303

Lamas L.F.. 283

Lamb D., 7, 85, 278

Lamberty G.J., 151

Lamoureux J., 12, 44

Lancaster M., 142, 205, 234

Lanctot J., 290

Landers-Ramos R.Q., 139

Landry-Roy C., 231, 232

Lane E.M., 69, 84, 145, 179

Lane T., 155

Lange R., 12, 60, 62, 269

Lange R.T., 15

Langenecker S.A., 138, 151, 244, 284

Langenkamp M.M., 50

Langevin L., 128, 129

Langlois R., 227

Lansing A.E., 157

Laperriere N., 166

Lapin J., 11

LaPoint M., 80

LaPorte D., 6

Lara-Ruiz J., 10

Larson E.B., 19

Larson M.J., 16, 83, 245, 250, 275

Larson-Prior L.J., 77

LaRue A., 179

Lasa D., 283

Lassen-Greene C.L., 206

Lassonde M., 18, 95

Last B., 127

Lau K., 275

Lau L., 54, 156

Laughlin S., 162

Lawrence I., 80, 206

Lazosky A., 258

Lazzeroni L., 158, 243

Leaf F.O., 71, 73

Leaffer E.B., 108, 109

Lebel C.. 34

LeBlanc J., 12, 44

Lee A., 141

Lee C., 220, 240, 249

Lee E., 219

Lee G., 20

Lee J., 38
Lee L.. 304

Lee S., 143, 286

Leemans A., 294

Lefebvre G., 16, 18

Legarreta M., 258

Leger G.C., 176

Lehmann M., 203

Leibel D.K., 104, 303

Leininger S., 141

Leitner B.P., 139

Leitner D., 5, 198, 209, 255, 259, 265

Lemay J., 129

Lemelman A., 280

Lemons A., 8, 14

Lengenfelder J., 15, 18

Leon A., 102

Leonardo S.M., 37

Leopoulos W., 46, 152, 154, 303

Leow A., 21, 248

Leppo R., 53

Lequerica A., 6

Leroux J., 133

Leschke L., 238

Lessig S., 210

Lessig S.L., 209

Letendre S., 112, 113, 115

Letzen J.E., 247

Leu K., 294

Levan A., 86

Levav M., 88

Leveroni C., 87

Levey A.I., 259, 265

Levin B.E., 118, 119, 198, 199, 305

Levin H., 5, 134, 147, 148, 230, 281

Levine A., 150, 213, 255, 284

Levy R., 251

Levy S., 262

Lewine J.D., 14

Lewis W., 290

Leyton M., 250

Lezak M.D., 105

Li C.. 293

Li S., 192

Li S.T., 91

Li W., 82

Li X., 147, 148, 230, 281

Li Z., 12, 62

Liau L., 294

Libben M., 265

Libon D.J., 143, 202, 248, 269, 303

Libove R., 170

Lichtenstein J., 59

Liebel S.W., 84, 104, 275

Lieberman A., 207

Light S.N., 51

Lim A.F., 133 
Lim K.O., 31

Lima E., 71

Limkeman R.K., 68

Lin S., 172

Lin W., 6

Lin X., 134

Linck J., 13, 22, 57, 59

Lindsey H.M., 271

Lippa S.M., 57

Lippé S., 133

Lisdahl K., 144

Little S., 113

Litvan I., 209, 210

Liu D., 69, 77, 179, 266

Liu I., 251

Liu W., 33, 291

Liu Y., 269

Liu-Ambrose T., 282, 283

Livney M., 273

Llamas V., 78

Lobdell D.T., 28, 29, 45, 105, 198

LoBue C., 203

Locascio G., 56

Locke D., 201

Locke J., 239

Lockwood C.A., 242, 259

Loftis J.M., 106

Logan D.M., 16

Logan L., 69, 179

Logan R., 79

Logue E., 80, 86

Logue M., 78

Loinel A.C., 128

Lojek E., 153

Loman M., 93

Long E., 56, 233

Long J., 14, 154

Lopera F., 305

Lopes Costa S.M., 118

Lopez E., 111

Lopez F., 61

Lopez N., 68

Lopez W.D., 10, 38

Loring D., 2

Lotze T.E., 120

Loughan A.R., 90, 186, 297

Louise-Bender Pape T., 9

Low T., 57

Lowe D.A., 154

Lowe J.R., 100, 170

Lowe V., 178

Loya F., 275

Lu L.H., 9, 246

Lu P.. 174, 205

Lubomski M., 211

Luc N., 20, 21, 81, 83, 224
Luca C., 198, 199

Lucas-Jiménez O., 145

Lucchetti A., 187, 289

Luci K., 260

Ludy C., 296

Lukas S., 137

Lukose A., 43

Lundberg-Love P., 257

Lungren E., 184

Luong-Tran C., 242, 280

Lust B., 208

Luu H., 53, 58, 151, 270

Luu P., 96, 97

Ly J.J., 113, 175

Lyketsos C.G., 174

Lynch J., 57, 59, 61, 62

Lynch S., 119, 122

Lyons M., 78

Lysaker P., 276

Ma S., 18

Mabbott D., 161, 162, 293

MacAllister W.S., 55, 58, 90, 91, 286. 299

MacDonald S., 262, 290

Machado A.G.. 215

Macher R.. 55

Machulda M.M., 178

Macina L.O., 175

Mack W.. 291

MacKay-Brandt A., 40

MacKillop J., 104

Mackin R., 142

MacLean P.C., 170

MacMullen Freeman L., 239

Macoun S.J., 271

MacSween J., 271

Madore M.R., 162, 163, 275

Maganti R.K.. 90

Maharajh K., 107

Mahmood Z., 72, 111, 114

Mahone M., 169, 285, 292

Mahoney J.J., 59, 80, 86, 106

Maier W., 194

Maiman M., 55, 58, 90, 91, 286

Main K.L., 30

Maisel M., 238

Major P., 133

Make B.J., 221

Maki P., 113

Makola M., 292

Maksakova O., 192

Maleki M., 44

Maletsky K., 34

Malfait D., 133

Malihialzackerini S., 270

Malloy P.F., 48, 141
Mallya S., 70

Mancuso S.. 294

Mandell D., 239

Manderino L., 213

Mandrell B.N., 161, 291

Mangal P., 250

Mangalonzo A., 107

Mangassrian S., 51

Mangum R.W., 302

Manini T.M., 67, 140, 144

Manly J., 83, 127, 268

Mann J., 154, 157

Manning C.A.. 75, 174, 176

Manning E., 189

Manning K.J., 110, 227

Mannino C., 10, 11

Manrique A., 106

Manzel K., 50

Maple K., 144

Marceaux J., 220

Marchant B., 72

Marchetti P., 283

Marcopulos B.A., 156

Marcotte T., 116, 123

Marcoux J.. 44

Mareci T., 143, 145, 147

Margolin G., 27

Margulies D.S., 252

Mari Z., 211

Mariani M., 291

Marin D., 209

Marin H., 267

Marion S., 193

Markowski S., 24, 247

Marks D., 285

Marquez de la Plata C., 23, 117, 139

Marquine M.J., 102, 112, 115

Marra C.. 115

Marra D., 274

Marreiro C.L., 173

Marshall C.C.. 128

Marshall K.K., 257

Marsiske M., 67, 70, 76, 81, 82, 216. 272,274

Marson D.C., 206, 210

Martersteck A., 79

Martin C.S., 67

Martin E., 113

Martin G., 80, 202

Martin P.K.. 264

Martin R., 13, 22

Martin R.C., 143, 210

Martin S., 49

Martin-Cooper J., 46

Martinez A., 193

Martinez F., 11 
Marx B., 304

Mason L.H., 231

Mason W., 166

Masse B., 227

Massey Z., 107

Massman P., 112, 172, 175, 179

Masson M., 15 ?

Massuda R., 152

Mathias J.L., 236

Mathur A., 247

Matsui M., 153

Mattek N., 85, 176

Mattingly M.L., 92, 94

Mattson S.N., 130, 187, 298

Maule A.L., 302

Mauro S.A.. 278

Mavis C., 119, 122

Maximo J., 280

Maxwell B., 229

Maxwell E., 164, 282

May P.E., 69

Maye J.E., 70, 81, 82

Mayer A.R., 14, 252

Mayeux R., 127

McArthur D., 10, 11, 51

McArthur J., 115

McAuley T.L., 29

McCaffrey R., 57, 59, 61, 62

McCallum K.E., 257

McCarthy M., 64

McCauley S., 46, 147, 148, 152, 154. 303

McClintock S.M., 215, 264

McCormack M., 267

McCormick C.L., 4

McCoy K., 81, 220

McCrae C., 81

McCrea M., 192, 268

McCullagh J., 295

McCurdy M.D., 47, 287

McCutchan J.A., 115

McDonald S., 95, 96

McDonald S.D., 4

McDonnell M., 71, 73

McElwee C.., 10, 38

McFall G., 82, 110

McFarland C.P., 304

McFarlane K., 279

McGill C., 233, 234, 236

McGlade E., 104, 258

McGlinchey R., 18, 144, 224

Mcgrew C., 14

McHenry C., 227

McInerney K., 71, 73, 79

McIntosh E., 140

McKee A.C., 181
McKenzie D., 17

McKinstry R.C., 142, 190

McKittrick K.J., 91

McLaren D.G.. 251

McLaren M.E., 140, 144

McMains J.T., 84

McMillan B.F., 51

McNally K., 91

McNally K.A., 88, 232

McNeely J., 84, 146, 214

McPherson T.O., 210

McWilliams K., 195, 196

Mechanic-Hamilton D., 201, 273

Medaglia J.D., 139

Medina L.D., 77

Mednicoff S., 17

Mehlsen M., 294

Meister J., 43

Melah K., 32, 245

Melinder M., 61

Mellott E.M., 191

Meloy M.J., 138, 181

Melrose R.J., 178

Menard C., 166

Menatti A.R.. 73. 79

Mendes A.. 158

Mendez A.J., 305

Mendez M.F., 178

Mendibe Bilbao M., 123

Mendoza H.J., 270, 293

Menefee D., 46, 152, 154, 303

Meneide T., 80

Menezes N., 21

Merchant T.E., 161, 291

Merickel M., 116

Merkitch D.V., 179

Merkley T.L., 147, 148

Merritt V.C., 130, 131

Mervis C.B., 110

Merz Z., 6

Meschede K., 221

Messer M., 171

Mesulam M., 79

Meterko M., 13

Meyer J., 130, 131

Meyer O., 69, 277

Meyer R., 123

Meyers K., 22, 135, 138, 244

Michalowski J., 214

Miciak J., 47

Mickey B.J.. 138

Mietchen J.J., 230, 265

Migliorini R., 130

Mikos A., 78

Mikulis D., 21, 31

Milberg W.P., 18, 144, 224
Millar B., 166

Miller A.K., 54, 119, 156

Miller B., 13, 22, 28, 57, 58, 59, 127. 206, 209, 225, 264

Miller G.A., 249

Miller H., 5, 198, 209, 255, 259, 265

Miller I.N., 151

Miller J., 43, 108

Miller J.B., 54, 176

Miller J.S., 78, 111, 217

Miller K.J., 78

Miller L., 135

Miller M.M., 64

Miller N., 129

Miller N.K., 48, 133

Miller S., 14, 202

Miller Z.A., 181

Mills M.S., 98

Mills T., 302

Min S., 287

Minassian A., 107

Miner J., 267

Minhajuddin A., 74

Minich N., 132, 165, 235

Miranda C.. 115

Miranda L., 106

Mirsky A., 88

Miskey H.M., 3, 4, 61, 132, 216

Mitchell J.E., 213

Mitchell M.B., 251

Mitchelson B., 46

Mitic L., 181

Miura K., 153

Miyake Y., 298

Mizrahi R., 180

Mizuki B.M., 75, 174, 176

Moberg P.J., 110, 215, 273

Moelter S., 227

Moes E., 243

Mok J., 255

Moldovan C.P.. 71, 73

Molholm S., 149

Mollo J., 230

Molokie R., 49

Moncrief G.G., 42

Monson S., 261

Montague E., 56, 233

Montalvo M., 106

Montgomery V., 102, 188

Montoya J., 112

Moon C.. 245

Moon S., 158

Moore C.. 78

Moore D.J., 102, 112, 113, 114, 115

Moore E.M., 130

Moore R.C., 112 
Moore W.R., 44

Moorer J., 273

Morais H.B., 48, 133

Moran J., 279

Moran M.T., 42, 101

Moreau K.L., 221

Moreira I., 158

Morelli D.K., 20

Morgan B., 73

Morgan E.E., 112, 113, 115

Morgan K.N., 272

Morgello S., 111, 115

Moriyasu M., 29

Morley J., 215

Mormino E.C., 227

Morris J.C., 177

Morris R., 299

Morris R.E., 40

Morris S., 113

Morrow L., 30

Morse C., 110

Morton J., 217

Mosca S.J., 128

Moser D.J., 221

Moses J., 259

Mosquera D.M., 85, 278

Moss N.C., 100, 170

Motu'apuaka M., 17

Moulton E., 240

Mrakotsky C.. 34

Mueller B.A., 31

Müller U., 48

Mulligan B.P., 105

Mulligan K., 270

Mullins S., 281

Mungas D., 69, 102, 173

Munro C., 80

Murdaugh D., 280

Murphy C., 140

Murphy H., 91

Murphy K., 293

Murphy K.J., 203

Murphy K.M., 270

Murray E.M., 211

Murray L., 99, 200

Musil S., 258

Musso M., 265

Mzayek Y., 64, 65, 291, 293

Na S., 288

Nadeau S.E., 222

Nagarajan R., 146

Nagel C., 151

Nagumo M., 37

Naigles L., 238

Nakase-Richardson R., 64

Nakatani K., 134
Namazi S., 242, 259

Nance M., 267

Narad M., 235

Narkhede A., 83, 127

Natelson Love M.C., 261

Nation D.A., 83, 126, 178, 181, 203

Naughton M., 82

Navia B., 115, 117

Naylon K., 151

Neale A., 49

Neargarder S., 221, 278

Negash S., 201, 273

Neidinger S., 119, 122

Nelson C., 142

Nelson L.D., 268

Nelson Schmitt S., 141

Nesbitt P., 13

Ness A., 119

Ness K.K., 161

Netson K.L., 46, 190

Neudecker J., 61

Neugnot-Cerioli M., 271

Neumann J., 185

Neville I.S., 37

Newburger J.W., 185

Newman J.. 186

Newman K., 183

Newman S., 200

Newsome M., 134

Newton T., 106

Nguyen L., 209, 210

Nguyen P., 141, 145

Niccolai L.M., 210

Nicholas C.R., 304

Nicholls E., 47

Nichols L., 56

Nickerson L.D., 26

Nicodemus N.E.. 85

Nielson C., 280

Nielson K.A., 75, 76, 135, 141, 142. 173, 205

Niermeyer M., 250

Nikelski J., 227

Nitch S., 52

Nitzan-Tamar O., 100

Noble-Haeusslein L., 281

Noda A., 30, 158, 243

Noe O.B., 64

Noggle C., 150, 213, 255, 284

Nogues C., 271

Noll K.R., 292

Noll R., 66

Noonan N.. 285

Norman A.L., 75, 76, 135

Norris-Brilliant A., 152

Norseth C.H., 71, 73
Norton M.C., 172

Notoya M., 134, 237

Novakovic-Agopian T., 275

Novitski J., 258

Nunan-Saah J., 151

Nussbaum N., 89, 92

Nyenhuis D., 49, 219

Nyman H., 183

O' Shea D.M., 74, 77

O'Brien T., 11

O'Bryan S., 119, 122

O'Bryan S.R., 122

O'Connor M.G., 253, 258

O'Malley K.A., 262

O'Neil R., 49

O’Neill S., 285

O'Rourke J., 220

O'Shea A., 140, 144

O'Shea C., 52

Oasay L., 123

Ofen N., 301

Ogg R.J., 161

Oghalai J., 244, 296

Oh H., 139, 173

Oh J.M., 179, 304

Oh S., 285

Oh Y., 268

Ohlhauser L., 5, 198, 209, 255, 259. 265

Ohls R.K., 100, 170

Ohm D., 79

Ojeda N., 123, 145

Ojha R., 290

Okonkwo D., 30

Okonkwo O.C., 31, 32, 33, 126, 179. 206

Okun M.S., 78, 216, 220, 250

Oliveira M.A., 300

Olsen D.C., 18

Olsen E., 155

Olsen P., 107

Olson E.A., 26, 149

Olson K., 185, 188, 189

OMalley K., 269, 300

ONeil M.E., 17, 42

Oquendo M., 154, 157

Orcutt S., 198

Ord A.S., 200, 201

Orff H.J., 7, 18, 19, 224

Ortega M.R., 118, 119

Osipowicz K., 195, 196, 197

Osmon D.C., 50

Otsuka S., 153

Ott B.R., 219

Ouellet M., 12

Ouyang A., 123 
Owen A.M., 258

Pabón R., 201

Pacheco V., 58, 264

Pacileo A., 16

Padgett L.S., 65, 195

Padula C.B., 52, 82

Paek E., 99, 200

Page M.J., 239

Pagulayan K., 19, 20, 223

Paik A.M., 147

Paiva W.S., 37, 300

Palcher J., 60

Pale M., 24

Palko A.. 71

Palma I.A., 73

Palmer R.F., 193, 194

Palmer S.L., 293

Paltin I., 287

Pandya S., 175, 209, 264

Panizzon M., 78

Panos A.H., 302

Panoskaltsis-Mortari A., 65

Pantelyat A., 215

Papp K.V., 80, 227, 305

Parboosingh J.S., 109, 128

Pardo S., 40

Parikh S.A., 58

Parisi J., 277

Park S.E., 50

Parker P., 146

Parks A.C., 60

Parks N., 96

Parmenter B.A., 223

Parrish T.B., 179

Parsons J.T., 46

Parsons T.D., 84, 239

Parvateneni H., 212

Pascual-Leone A., 276

Pasqualetti F., 139

Passarotti A.M., 284

Pastorek N.J., 5, 13, 22, 57, 59, 134. 225

Patel K., 79

Patel M., 284

Patel N., 40, 148

Patel S., 66

Paterson T.S., 213

Patrick K., 195, 196, 240

Patrick R., 62

Patten C.. 145

Patterson T.L. 159

Pattock A., 60

Paul L.K., 27, 44, 295, 302

Paul R., 84, 116, 145

Paulraj S., 151, 296

Pauls C.D., 264
Paulsen J., 267

Pavisian B.. 124

Pavlos S., 129

Peach R.K., 182

Pearce K., 232, 235

Pearson M.M., 199

Pedersen A.D., 294

Pedroza C., 46, 152, 303

Peery S., 184

Pei J., 271

Pella R., 265

Peltz C.. 9

Peña J., 123, 145

Penderville J., 30

Penn M.S., 219

Pennington B.F., 1

Pennington C.R., 257

Perlman S., 267

Perlmutter J., 267

Perlstein W.M., 83

Perry C.E., 172, 177

Perry R.I., 107

Perry W., 97, 98

Persad C., 212

Peskind E., 223

Peskind E.R., 19, 20

Pesonen A., 241

Pestilli F., 30

Peter S., 244

Peters B., 99, 186

Petersen L., 274, 288

Petersen N.J., 5

Petersen R.C., 178

Peterson A., 258

Peterson E.. 306

Peterson R.L., 232, 233

Peterson-Hazan S.. 265

Petranovich C.K.. 100, 284

Petrauskas V., 169

Petrides M., 252

Petrie E., 19, 223

Petrie J., 105

Petros T., 169

Pfaller J., 90

Pfefferbaum A., 304

Phillips J., 170, 200, 201

Phillips S., 84

Phoong M.A., 184

Piai V., 296

Pick L., 41, 295

Pickett T.C., 4

Pierce C., 54

Piercy J., 99, 301

Piercy J.C., 5, 186, 198, 209, 255, 259. 265

Pierpont R., 183
Piers R.J., 269

Piguet O., 96

Pillay S.B., 297

Pilon R., 227

Piper L., 49, 102, 219

Pirogovsky Turk E., 83, 205, 208, 209. 210,305

Piscione J., 162

Piscitello J., 177

Pisner D., 138, 246

Pizzano B., 12, 62

Plante W.. 157

Plenger P., 117

Plessers M.. 300

Pliskin N., 49, 102, 105, 205, 219, 267

Ploetz D., 57

Plotkin R., 53

Plourde V., 299

Pogoda T., 13

Pommy J., 50, 140, 252

Ponce F., 207

Ponsford J.L., 17, 35

Poorzand P., 127, 209

Porat S., 75

Porges S., 7

Porter J.B., 113

Porter R., 49

Posecion L., 151, 158

Possin K., 209

Pothier W., 155

Potts G., 28, 4 ?

Potvin D., 118

Poulsen C., 96, 97

Prasad M., 282

Preer L., 26, 149

Prelec D., 221

Presson N.. 30

Preston T., 299

Price C., 22, 140, 141, 143, 145, 212 , 262

Price J.S., 137

Prieur M., 213

Primosch M., 250

Prince C.E., 301

Prince E., 212

Pritchard A.E., 169, 187

Pritchard P., 87

Proto D., 57, 89

Prudic J., 264

Pruitt D., 12, 62

Przybelski S., 178

Pugliese C., 242

Pui C., 33, 64, 65, 291

Pulsifer M., 290

Pulsipher D.T., 14, 56, 233

Purdie R., 156 
Purdy M., 295

Putcha D., 134

Pyhälä R., 241

Quaid K., 267

Qualls D., 117

Quezada M., 10

Quigley T., 260

Quinn T., 217

Quintanilla J.P., 106

Quintin E.C., 39

Quiroz Y.T., 305

Quittner Z., 55

R.-Mercier A., 157

Raak J., 42, 49

Rabinovitz B., 23

Racine A., 32

Rademaker A., 79

Radke A., 127, 209

Radke J., 129

Raghubar K., 244

Raghubar K.P., 292, 296

Rai J.K., 168

Räikkönen K., 241

Rainville C., 227

Rajaram S., 71, 73

Rajkumar W., 29, 244, 263

Rakic J., 19

Ramífez-Flores M., 24

Ramig L.O., 211

Ramírez-Flores M., 168

Rammohan K., 118, 119

Ramon D., 140

Ramoskaite R., 43

Randolph J., 124

Rangamannar R., 294

Rankin K.P., 28, 127, 209, 222

Ranseen J., 284

Ransom D., 233, 234

Rao J.A., 138

Rao R., 108

Rao S.M., 75, 76, 135, 142, 205, 282

Raskin S., 129

Rau H., 223

Rau H.K., 20

Rau S., 187

Rauch S.L., 26, 149

Ray C., 119, 122

Raymond N., 261

Raz S., 99, 186, 301

Razani J., 103, 174, 205

Razlighi Q., 77, 127

Ream D., 100

Reckziegel R., 152

Reddy V., 250

Reed B., 173

Reesman J., 53
Regner M.F., 107

Rehmel J.L., 295

Reiber G., 82

Reid S., 273

Reilly E., 124

Reilly J., 246, 296

Reineberg A., 248

Reinhard M., 242, 259

Reinvang I., 201

Reiter K., 141, 173

Reiter M., 19, 223

Reither K., 135

Ren X.. 248

Rendon J., 305

Reneman L., 289

Renn B.N., 217, 243

Reno A., 106

Renshaw P., 104

Rentz D.M., 80, 227, 305

Reusser C., 46

Revueltas A.M., 24

Reynolds B.A., 272, 274

Reynolds G.O., 221

Reynolds M., 5, 198, 209, 255, 259, 265

Rhodes E.. 303

Rice L., 39, 53, 56, 58, 61, 151

Rice M., 212

Rich J., 292

Richard A.E., 241, 279

Richard Y., 12

Richman M., 110

Richmond C.E., 156

Rider G., 101

Rider R., 252

Ridge B.E., 257

Rieger R.E., 100, 170

Riesgo V., 118, 119

Riley A., 46

Riley E.P., 130

Riley T., 85

Rilo Cano O., 123

Ringe W., 139

Ringman J., 77, 148, 205, 207, 208

Ris M., 292

Riskin-Jones H.H., 84

Rissling M., 82

Rivera F., 73

Rivera K., 189

Rivera P.M., 118

Rivera R., 68

Rivera-Mindt M., 107, 115

Rivkin M.J., 34

Robbs R., 81

Robe P., 251

Roberg B., 119, 122
Roberg B.L., 122

Roberts K., 29, 244, 263

Roberts R.M., 236

Roberts S., 236

Robin K.J., 113

Robins D.L., 144, 242

Robinson J., 1, 58, 88, 89, 264

Robinson K.E., 95, 199, 232

Robinson M.E., 144, 224, 247

Robison L., 290, 293

Rochette A.D., 219

Rodgers C., 277

Rodgers-Neame N.T., 92, 94

Rodrigues P.A., 37

Rodriguez G., 77, 118, 119, 207, 208

Rodriguez J.A., 85, 278

Rodriguez R., 78

Roebuck-Spencer T., 260, 266

Rogalski E., 79, 94, 181

Rogers S., 68, 71, 154

Rogers S.A., 85, 228, 277

Rohlfing T., 304

Rohrbacher C., 45

Roman C.A., 122

Romanowska-Pawliczek A.M., 166, 167

Romero E., 10, 11

Romero K., 124

Romesser J., 13, 22, 57, 59

Root J., 288

Ropacki S.A., 17

Roper B., 39, 53, 58, 61, 151, 278

Rose S.E., 236

Roseberry J., 153

Rosen A., 55

Rosen A.C., 82, 247

Rosen C., 155

Rosen H., 207

Rosenqvist J.E., 33

Rosenthal E., 166

Rosenthal T., 123

Ross C., 267

Ross L.A., 149

Ross R.S., 134

Ross S., 117

Rossetti H.C., 203, 264

Rossetti M., 305

Rosso I.M., 141, 149

Rotblatt L.J., 83

Roth R., 286

Rotheram-Fuller E., 239

Rothlind J., 142

Rothong N., 61

Rowe G., 203

Rowland J.A., 3, 4, 61, 132

Rowley H.A., 179

Roy A., 13, 135 
Roy M., 155

Royall D.R., 193, 194

Roye S., 72

Ruberg F., 69, 179

Rubin K., 95, 192, 233

Rubinfeld L., 25 ?

Rushby J.A., 95, 96

Rusin T., 60

Russell M.E., 48

Rutkowski B., 214

Ryals A., 289

Ryan A.B., 219

Ryan K., 151

Ryan N., 281

Ryan N.P., 281

Ryman S.G., 50, 252

Rypma B.. 123

Saavedra F., 183, 244

Sabartinelli A., 72

Sabaté J., 71, 73

Sabbah L.E., 243

Sabin N.D., 33, 302

Sabino J.P., 37

Sadek J.R., 224

Sadighi Z.S., 293, 302

Sady M.D., 233, 236

Saenz N., 107

Safren S., 87

Sager M.A., 32, 33, 126, 304

Saheb S., 139

Sak T., 53, 61, 151

Sakamoto M., 116

Salama C.H., 184

Salat D.H., 144, 224

Salazar Bejarano L., 7, 85, 278

Salazar R., 220, 248

Salins N., 207

Salloway S.P., 48, 141

Salminen L., 84, 116, 145

Salmon D.P., 126, 178, 180

Salonen E., 183

Salorio C.F., 184

Samuels L., 69, 179

Sanchez Diaz D., 21

Sanchez-Ramos J., 220

Sanders C.L., 174

Sanderson-Cimino M., 72, 81, 83, 85,

$$
277
$$

Sandry J., 7, 275

Sands K., 121

Sands S., 66

Santos O.A., 50

Sanyal R., 154

Sanz J.. 36, 189, 190

Sapkota S., 82

Sarma M., 146
Sarokhan A.K., 221

Sasidharan A., 43

Sass S.M., 249

Sassaman S., 47

Sassenfeld E., 266

Sassoon S.A., 304

Sathian K., 126

Saurman J., 301

Sautter S., 200, 201

Saxbe D., 27

Sayegh P., 72, 111, 114

Saykin A.J., 227

Scanlon B.K., 178

Scarisbrick D.M., 80, 86

Scarmeas N., 74

Schachar R.J., 191

Schaefer L.A., 203

Schaffer S., 23

Schagen S., 289

Schatzberg A.F., 50

Scheibel R.S., 5, 134

Schell E., 80

Scherer S.W.. 128

Scherzer C.R., 221

Schiehser D.M., 19, 20, 21, 200, 209, 210, 224, 277

Schmalfuss I., 212

Schmidt A., 230, 257

Schmidt E., 12, 62

Schmidt T., 172

Schmitt A.L., 257

Schmitter-Edgecombe M., 204

Schneider H., 169

Schneider W., 30

Schoenberg M.R., 92, 94

Schofield H., 234

Schonfeld D., 114, 146

Schraegle W.A., 89

Schreiber J.E., 293

Schreiner T., 120

Schretlen D.J., 279

Schroeder R., 261

Schroeder R.W., 59, 264

Schryver H., 250

Schuh J., 238

Schultheis M., 7

Schultheis M.T., 227, 240

Schultz A., 80, 81

Schultz R., 238

Schultz S.A., 31, 32, 126, 179

Schumacher L.. 249

Schupf N., 83, 127

Schuster V., 137

Schwab K., 266

Schwab N., 140, 141, 212

Schwab Z., 26
Schwartz D., 64

Schwartz J., 87

Schwartz S., 174

Schwartz Z., 85, 278

Schwarz G., 110

Schwarz N.F., 84, 104, 135

Schwehm A., 46

Schweiger A., 54

Scoggins M.A., 161

Scorpio K., 211

Scott B., 42, 272

Scott B.M., 70, 82, 257

Scott C.. 113, 123

Scott J., 80, 86

Scott M.N., 55

Scott S.E., 84, 145

Scott T.M., 107

Scruggs J., 71

Seedat S., 116

Seelye A., 85

Segerstrom S.C., 48

Seguin A., 29, 244

Seguin A.A., 263

Séguin M., 273

Seibert L., 15

Seichepine D.R., 8, 14

Seidenberg M., 75, 76, 92, 135, 142 , 205

Seider T., 115

Seligman S.C., 80, 202, 206, 273

Semla M., 61

Sepeta L., 169

Seppä E.R., 241

Seruga B., 292

Seshadri S., 181, 269

Sevel L.S., 247

Severson J., 116

Sévigny-Dupont P., 227

Shabo L., 208

Shada K., 272

Shah R., 39

Shah R.C., 179

Shaked D., 104, 303

Shane B., 245

Shany-Ur T., 127, 209

Shapiro E., 259

Shapiro R., 212, 213

Sharan A., 252

Sharland M.J., 60

Sharma A., 72

Sharma B., 21, 31, 255, 273

Sharma R., 155

Sharp C., 164

Sharrette J., 183

Shaw E.E., 80, 81

Shdo S.M., 28 
Shean M., 272

Shear P., 220

Sheikh M.O., 210, 211

Shelach S., 106

Shenal B., 200, 260, 276

Sheng T., 8

Sheppard D.P., 112, 305

Sherman J.C., 208, 294

Shevtsova T., 192

Shi Q., 292

Shieh B., 37

Shih H., 294

Shih S. 169

Shimony J., 142

Shimony J.S., 190

Shin B.C., 148

Shin D., 181

Shin M., 285

Shirk S.D., 251

Shofer J.B., 20

Shollenbarger S.G., 144

Shore J., 100

Shura R.D., 3, 4, 61, 132, 150

Siebenmorgen M., 46

Sikora I., 10, 11

Silk-Eglit G., 57, 59, 61, 62

Silton R.L., 249

Silva L.R., 269

Silva T.T., 300

Silver J.M., 1

Silverman J., 265

Sim A., 13, 22, 57, 59

Simic N., 164, 165, 167

Simone A.N., 285

Simpson D., 115

Simpson R., 166

Singer C., 198, 199

Singer E.J., 111, 114, 146

Singh A., 270, 293

Singh V., 32

Sinopoli K., 164

Sitarenios G., 287

Skeel R.L., 42, 49, 197

Skelly C., 187

Skidmore C., 252

SKillicorn K., 91

Skocypec R., 136

Slater J.D., 90

Slater M., 260

Sligar K., 84

Slogar S., 200

Slomine B., 184

Slone E., 251

Small B.J.. 71

Small G.W., 78, 14 7

Smith A., 15
Smith A.L., 217, 243

Smith B., 9

Smith B.M., 10, 11

Smith C.. 109

Smith D., 43, 103, 113, 236

Smith G., 201

Smith J., 12, 24, 62, 75, 76, 135, 139 , 141, 142, 146, 173, 205, 282

Smith J.M., 132

Smith K., 69, 111

Smith K.R., 106

Smith S., 23, 172

Smith T.J., 24, 139, 141, 173

Smith Watts A.K., 48, 141

Smith Z., 64

Snitz B.E., 71

Snyder A., 23

Snyder A.R., 172

Soares S., 183

Soble J.R., 94

Sodos L., 55

Sodré L.A., 152

Sol K., 275

Solomon S., 227

Solowij N., 63

Solvason B.H., 50

Soman S., 30

Song D., 209, 210

Song W., 205

Sordahl J., 220

Sorg S., 19, 20, 21, 224

Soto-Añari M., 68, 106

Sotomayor J., 234, 236, 237

Sotomayor J.A., 230

Soubbotina M., 169

South M., 238, 250, 280

Souza A.L., 152

Spangenburg E.E., 139

Spanner A., 261

Spat J., 42, 101

Spaulding W.D., 158

Spechler P., 24

Spence J., 23, 139

Sperling M., 252

Sperling R.A., 80, 81, 227

Sperling S.A., 75, 174, 176

Spielberg B., 263

Spielman J.L., 211

Spittle A., 128

Spitz G., 17

Spitznagel M., 43, 213, 218, 219, 228

Sprich S., 87

Sriram D., 234, 236

Srivastava D., 33, 290, 291, 293

Srnka K.D., 92

Srour M., 133
Staat M., 100, 284

Stabler A.R., 257

Stacy M., 215

Staffaroni A.M., 50, 207

Stafford R., 25, 211, 256

Staines R., 124

Stancin T., 95, 132, 165, 192, 233, 235

Stanczak J., 153

Stanford L.D., 56, 233

Stapleton-Kotloski J., 4

Starkman M.N., 244

Starosta A., 54

Stebbins G.T., 179

Steed D., 14, 202

Steele N., 224

Stefanatos A.K., 92

Stefanatos G., 249

Steffensen S., 105, 250

Stegemöller E., 216

Steidl R., 261

Stein D., 116

Stein J., 32

Stein T., 181

Steinberg J.L., 134

Steindler D., 272, 274

Steiner A., 72, 111

Steinlin M., 229

Stenclik J., 59, 61, 62

Stephens M., 77

Stephenson K., 238, 280

Stern C.E., 134

Stern Y., 30, 69, 74, 77, 83, 127, 177

Stevens M., 238

Steward K., 39, 84, 116, 146

Stewart C.C., 75

Stewart J.J., 76, 136

Stewart J.L., 249

Stika M.M., 53, 142, 205

Stiles-Shiels C.. 187

Stinson J.M., 89

Stitt A., 200, 201

Stodola D.E., 34

Stolzmann K., 13

Stoop M.C., 295

Storandt M., 177

Storbeck J.L., 25, 256

Storzbach D., 17, 42

Stouten-Kemperman M., 289

Strain G., 213

Strand E.A., 178

Strang J., 242, 280

Strasser A., 295

Stringer A.Y., 126

Strober L., 118, 119

Strutt A.M., 257

Studaway A., 293 
Studer M., 229

Stuebing K., 35

Stuss D., 50

Suarez P.A., 107, 162, 163

Suchy Y., 41, 73, 202, 250, 254

Sudikoff E.L., 98

Sufrinko A., 232, 235

Sugarman M.A., 75, 135, 142, 205

Suhr J., 71, 73, 79, 182

Sullan M., 49, 72, 14 ?

Sullivan E., 84

Sullivan E.V., 304

Sullivan K., 8, 14, 302

Sullivan T., 220

Sumida C., 67, 83

Sumner E.C., 69, 179

Sumowski J.F., 275

Sun B., 288

Sun-Suslow N., 198, 199

Sunahara N., 134

Sundaram S.E., 123

Sunderaraman P., 7

Susanne M., 142

Suskauer S., 184

Susmaras T., 258

Suzuki M., 153

Swader T.C., 261

Swain M., 293

Swaine B., 12

Swan N., 43

Swank P., 281, 282

Swansboro B., 269

Sweet L.H., 84, 104, 135, 219, 228

Swenson R., 248, 269

Switalski K.A., 191

Szeles D., 27

Sztainer M., 187

Szymkowicz S.M., 140, 144

Tabak A., 92

Taber K.H., 3, 4

Tahsin S., 39

Takeda C.. 134

Tan A., 39, 214

Tanabe J., 107

Tanaka H., 41, 136

Tandon N., 90

Tang R., 225

Tangeman L., 122

Tangen R.B., 184

Tanner J.J., 22, 140, 141, 143, 212 , 262

Tannock I., 292

Tapert S., 63, 64, 130

Taran A., 60

Tarbell N., 290

Tarhan L.Y., 248
Tart-Zelvin A., 195, 197

Tarumi T., 136

Tashima K., 115, 117

Tator C., 4

Taylor A.M., 132

Taylor H.G., 95, 132, 165, 191, 192, 233, 235

Taylor J., 231

Taylor J.A., 254

Taylor J.L., 30, 243

Taylor L., 270

Taylor N., 252

Taylor P., 167

Teague A., 55

Ten Eycke K.D., 127, 128

Terwilliger V.. 189

Thaler N.S., 72, 80, 86, 111, 114, 146

Thames A.D., 72, 111, 114, 146, 162

Thamilavel S., 89

Thebault-Dagher F., 95

Theoret H., 16, 18

Thibodeaux D., 118

Thielke S., 273

Thieman T., 71

Thoma R.J., 14, 50, 140, 154

Thomas A.M., 146

Thomas K.. 70

Thomas K.R., 70, 76, 272, 274

Thomas M., 69, 277

Thompson A., 188

Thompson D., 128

Thompson J., 134, 150, 213, 255, 284

Thompson L.I., 46

Thompson P., 178

Thorgusen S.R., 202

Thornton A.E., 74

Thornton S.K.. 127. 128

Thornton W.L.. 74, 212, 213

Tierney S., 207, 208, 305

Till C.. 123

Timmons B., 162

Timpano Sportiello M.R., 204

Tinawi S., 12

Tingus K., 207

Titus J., 87

Tkachenko O., 26, 149

Tocchini S., 204

Tolar T.D., 299

Toledo R., 139

Tomassilli J.C.. 113

Tomaszczyk J.C., 22, 255, 273

Tomaszewski Farias S., 173

Toofanian-Ross P., 52

Top N., 280

Topping K.M., 243

Torstrick A., 46, 129
Tosto G., 127

Tosun D., 142

Tracy J.I., 252

Trahan D.E., 55

Tran J., 46, 152, 154, 303

Tranel D., 50, 256

Travis Seidl J.N., 175

Treble-Barna A., 165, 168, 232

Tregellas J., 107

Tremblay S., 155

Tremont G., 89

Trenova L., 277

Trettin L.. 50

Triebel K.L., 210

Trifan G., 22

Trifilio E., 70, 257

Triplett W., 145

Trittschuh E.H., 273

Trivedi M.A., 179

Trivedi N., 284

Troche M., 25

Trontel H., 241

Tröster A.I., 207, 218

Trotter B.B., 144

Troyanskaya M., 5, 134

Troyb E., 241

Truong J.H., 146

Truran-Sacrey D., 142

Tsai Y., 6

Tsapanou A., 74

Tschanz J.T., 172, 174

Tsui-Caldwell Y., 172

Tsui-Caldwell Y.H., 83

Tucholka A., 133

Tudor B., 86

Tulsky D., 60, 99, 225, 269

Tulsky D.S., 225, 226

Tun S., 42

Tunney M., 246

Tupler L.A., 4

Turetsky B., 110

Turken A., 297

Turkstra L., 191

Turner E., 47, 287

Turner G., 31, 273

Turner J., 154

Turner J.A., 144

Twaite J.T., 25, 211, 256

Twamley B., 276

Twamley E.W., 7, 18, 159, 224, 272 , 277

Tyler H., 260, 266

Tyrrell C., 262, 269, 300

Tyson B., 124

Uderman J., 23

Udhnani M., 191 
Ugolini M., 279

Ukueberuwa D., 117, 130, 131

Umekubo K.A., 157

Umlauf A., 102

Upshaw J., 5, 198, 209, 255

Urgesi C., 33

Vaccariello E., 250

Vakhtin A.A., 252

Vakil E., 100

Valcour V., 116

Valdez D.. 11, 51

Vale F.L., 92, 94

Valle M.. 93

van den Heuvel M., 252

van Dulmen M., 42

van Dyck C., 125

Van Dyk K., 113, 175, 274, 288

Van Etten E.J., 67, 83

Van Herzeele I., 300

van Huiden S., 48

Van Moorleghem K., 40

Van Patten R., 77

van Steenburgh J.J., 279

van Zandvoort M.J., 251

Vannasing P., 95

Vannatta K., 95, 192, 233

Vannorsdall T.D., 279

Vanuk J.R., 24, 25, 26

Vardhan S., 21

Vargas G., 234

Varvaris M., 279

Vas A., 21

Vasserman M., 55, 58, 91, 286

Vassileva J., 113

Vasterling J., 3, 304

Vaughan C., 230, 233, 234

Vaurio L., 90

Vega C., 93

Velilla L., 305

Vella L., 272

Venkatesan U.M., 134

Ver Hoef L., 143

Verber M., 24

Verfaellie M., 247, 253

Vermassen F., 300

Vernale M., 49, 219

Verney S.P., 103

Vespa P., 10, 11, 51

Vicentini J., 300

Victor T.L., 38

Vik P.. 112

Vila-Castelar C., 113, 175

Vila-Chã N., 158

Vincent A., 260, 266

Vingerhoets G., 300

Vitaro F., 299
Voelbel G.T., 271

Vogt E., 268

Voss J., 289

Waber D., 34, 93

Wade S.L., 100, 132, 165, 231, 233, 235, 284

Wadley V.G., 206

Wagner A.E., 240

Wagner G.M., 67, 83

Wagner M., 87, 194, 213

Wald D., 78, 172

Walder D.J., 155

Waldstein S.R., 5, 104, 303

Walker K.A., 46, 129

Walker L.A., 141

Walker P., 287

Walker W.C., 4

Walsh K.S., 36, 37, 41, 66, 118, 171 , 289

Walter K.H., 277

Walton M., 34

Walz N.C., 100, 284

Wamboldt F.S., 221

Wang A.Y., 106

Wang J., 36

Wang L., 289

Wang T., 71

Wang X., 292

Wanner J., 46, 152, 154, 303

Ward A., 256

Wardin L., 56

Ware A.L., 90, 16 ?

Waring S., 60

Warner E., 141

Was C., 42

Washington K., 55

Wasserman B., 269

Watson C.. 148

Watson C.E., 248

Watson C.G., 34

Weakley A.M., 204

Weber E., 151

Weber M., 26, 149

Wefel J., 292

Weijer C., 258

Weinberger H.D., 221

Weiner M., 142

Weiner M.F., 74, 100, 264

Weintraub D., 215

Weintraub S., 79, 181

Weise R.M., 73

Weisenbach S., 138, 151

Weiss B.A., 101

Weiss L., 139, 141

Weiss L.R., 24, 173

Weissberger G., 180
Weitekamp M., 149

Weitlauf J., 82

Welch L., 261

Wellington R., 46, 129

Wells B.E., 46

Welsh M., 39, 45, 306

Welsh R., 138

Wengreen H., 174

Werhane M.L., 20, 181, 203

Werner M.A., 280

Wertheimer J.C., 217

Wertz C., 252

Wesonga E., 142, 190

Westervelt H., 120

White D.A., 142, 190

White R.F., 302

Whitehead N., 104

Whiteside D., 53, 54, 56, 58, 119, 156

Whiteside D.M., 39, 61, 151

Whiting S., 189

Whitney J., 170

Whitney K., 79

Whitsel N., 18, 181

Whitwell J.L., 178

Wiebe S.A.. 110

Wiener J.R., 38, 101

Wierenga C.E., 138, 181

Wiggins E., 69, 179

Wigman S., 80

Wild K., 85

Wilde E.A., 46, 66, 67, 134, 147, 148 , $152,154,230,281,303$

Wilhalme H., 148

Wilkins C., 266

Wilkins K., 125, 276

Wilkins S.S., 197

Wilkinson A., 164, 165

Wilkinson C.W.., 20

Wilkinson J., 215

Willeumier K., 136

Williams B., 122

Williams C.. 112

Williams I., 75

Williams J., 195, 196, 197, 204

Williams V., 167, 168

Williams V.J., 35

Williamson J.B., 7, 214

Williamson R., 244, 296

Willis S.L., 277

Wills M., 280

Wilson C., 42, 101, 199

Wilson E., 177

Wilson K.I., 114

Wingate M., 61

Wischnewski M., 148

Wisdom N.M., 88, 225 
Wise J., 231

Wise M.S., 161, 291

Wise S., 185, 188, 189

Wishart H., 124

Wisniewski K., 69, 179

Withrow S., 287

Witkiewitz K., 50

Witkin G., 55, 286

Witzke J.W., 298

Wojtowicz M., 229

Wolf P.A., 181, 269

Wolfe K.R., 91, 232

Wolfsgruber S., 194

Wolfson T., 123

Womack K., 203

Wonderlick J.S., 221

Wong A., 99, 226

Wong D., 10

Wong Gonzalez D., 16

Woo E., 10, 11, 51, 75, 77, 148, 205 . 207, 208

Woo Y., 149

Woodard J.L., 22, 69, 75, 76, 135, 142. 205

Woods A., 140, 144

Woods C.. 116

Woods S., 112, 113, 114, 115, 119. 207, 208, 305

Woolverton C.B., 78

Woon F.L., 175, 209

Wozniak J.R., 31, 129

Wright C.B., 305

Wright C.W., 28, 105, 198

Wright L., 278

Wright M., 12, 43, 62

Wright M.J., 10, 11, 38, 51, 77, 207. 208, 263

Wu L.. 294

Wu T.C.. 148
Wyatt G., 154

Wylie G., 18, 66, 67

Wyman-Chick K., 59, 218

Wynn M., 148, 181

Xian H., 78

Xu J., 210, 211

Xu X., 84, 135

Xu Y., 71, 73

Yackley B., 155

Yaffe K., 9, 82

Yakobson M., 46

Yallampalli R., 46, 152, 281, 303

Yamamoto D.. 107

YANG C., 6

Yantz C.. 16

Yao C.. 292

Yazdanbakhsh A., 278

Yeager C., 169

Yeap B., 294

Yeates K.O., 95, 127, 132, 165, 192 , 233,292

Yeatman C.. 193

Yee M.K., 8, 14, 276

Yen K., 214

Yeo R.A., 50, 159, 160, 252

Yesavage J.. 30, 158, 243

Yoash-Gantz R.E., 3, 4, 61, 132

Yochim B.. 261

Yock T.I., 290

York C., 9

Yoshida H., 44

You Y., 6

Youmans R.J., 133

Youn T., 297

Younce S., 279

Young E., 166

Young M.A., 267

Yu L., 75, 146

Yu S., 32, 126, 179
Yuan J., 136

Yurgelun-Todd D., 2, 63, 104, 258

Yutsis M., 8, 17, 261

Zabel T., 53, 187

Zabrowski J., 293

Zachariae R., 294

Zackey D., 209

Zafonte R., 229

Zahodne L.B., 69, 83, 277

Zaldivar R.A., 111

Zang H., 165

Zaninotto A.C.. 37, 300

Zanto T., 75

Zaremski J., 23, 172

Zayat M., 234

Zec R.F., 81

Zeisel S., 129

Zenisek R., 54

Zettel-Watson L., 217

Zetterberg H., 32, 126

Zhang F., 290

Zhang H., 293

Zhang N., 165

Zharikova A., 192

Zhavoronkova L.. 192

Ziegler D., 221, 246

Ziegler R.S., 183

Zilberfayn I., 154

Zimmerman M.E., 127

Zimmermann K.M., 136

Zimny S., 6

Zitzelberger T., 176

Zizak V., 11

Zlatar Z.Z., 138, 181

Zobel H., 249

Zonderman A.B., 5, 104, 303

Zubieta J., 51, 138, 244

Zuccato B., 27

Zupek S.J., 50 


\title{
Keyword Index \\ Forty Third Annual Meeting \\ International Neuropsychological Society
}

\author{
February 4-7, 2015 \\ Denver, Colorado, USA
}

academic achievement, 35, 43, 44, 45, 85, 86, 87, 92, 168, 169, 187, 234, 280, 281, 286, 296, 297, 298, 299, 300 activities of daily living / adaptive functioning, 10, 25, 80, 86, 87, 102, 103, 114, 173, 175, 176, 191, 194, 195, 201, 205, 206, 210, 240, 245, 256, 283, 285, 288, 292 adolescence, 27, 42, 58, 64, 99, 106, 129, 187, 199, 231, $233,236,237,271,285$

affective processing disorders, 12, 25, 51, 96, 118, 244, 263 affective processing, normal, 24, 25, 26, 27, 28, 29, 34, 138, 244,247

aggression, 42, 199

aging disorders, 9, 68, 69, 71, 72, 74, 75, 78, 80, 83, 93, 98,

$112,126,127,138,142,148,172,173,175,178,180$, $182,202,204,205,207,208,209,211,214,223,227$. $259,261,263,302,305$

aging, normal, 11, 39, 48, 50, 67, 68, 69, 70, 71, 72, 73, 74, $75,76,77,78,79,80,81,82,83,84,85,86,94,95,98$, $110,115,135,136,137,139,143,145,146,147,148$, $154,174,182,203,211,227,228,243,246,251,260$, $266,269,270,273,274,275,277,278,301,303,304$ alcohol, 13, 20, 46, 105, 112, 129, 144

anomia, 200, 261

anosognosia, 89, 153

anterior cingulate, 34

anterior communicating artery aneurysm, 254

anxiety, $7,11,20,28,40,56,85,175,232,233,236,237$. 239,280

apathy, 15, 47, 76, 114, 159, 175, 211, 212, 220

apnea, 244, 263

apolipoprotein E, 32, 76, 86, 116, 179, 194

apraxia, 160, 249

assessment, 13, 44, 46, 49, 58, 86, 98, 132, 133, 153, 169 ,

$170,171,172,187,194,198,209,225,226,227,237$.

$239,255,256,258,259,260,261,262,263,264,265$,

$269,283,284,287$

asymmetry, 198

attention deficit hyperactivity disorder, 24, 40, 45, 50, 55, 99 118, 169, 186, 188, 189, 191, 230, 265, 284, 285, 286, 287 attention, 11, 19, 22, 66, 77, 84, 86, 88, 90, 99, 106, 113 , 119, 124, 155, 161, 165, 168, 175, 182, 188, 191, 192. 214, 215, 218, 241, 246, 247, 251, 257, 271, 273, 278, 279, 283, 284, 285, 286, 287, 291, 293, 299

auditory processing disorder, 249, 295

autism spectrum disorders, 24, 166, 170, 237, 238, 239, 240, $241,242,251,280$

autism, 24, 238, 239, 242, 279

autoimmune disorders, 114, 185

awareness, 16, 25, 153, 159, 164, 178

basal ganglia, 38

bilingualism, 38, 44, 102, 180

bipolar disorder, 169, 170, 262, 285

bone marrow transplant, 187, 303

brain damage, 8,145

brain development, 170, 238, 252

brain disorder, 87, 214

brain function, 21, 39, 95, 96, 127, 128, 134, 137, 138, 140 . 212, 223, 282

brain injury, $6,8,9,14,16,19,21,66,96,123,192,224$, $226,229,256,273,275$

brain plasticity, 141, 273, 282, 283

brain structure, 77, 128, 142, 145, 147, 149, 160, 253, 289 , 304

brain tumor, 161, 162, 166, 185, 200, 270, 287, 288, 290, 291, 292, 293, 294, 297

breast cancer, 274, 289, 292

cancer, 41, 65, 66, 171, 195, 271, 288, 289, 290, 291, 292. 293, 294, 295

cannabis, 55, 63, 64, 104, 123, 124, 144

cardiovascular disease, 15, 21, 41, 78, 82, 85, 178, 185, 188 , $190,219,228,245,249$

caregiver stress/burden, 13

cerebellum, 16, 137, 140, 184, 192, 297

cerebral blood flow, 136, 139, 181, 203

cerebrevascular accident/stroke, 16, 255, 259, 265

cerebrovascular disease, 127, 182, 188, 194, 203, 220

chemotherapy, 33, 65, 66, 274, 289, 292

child brain injury, 31, 134, 165, 172, 192, 230, 231, 232 , $235,236,237,281,282$ 
child development disorders, 35, 40, 55, 127, 128, 129, 142 , $169,184,187,237,242,272,285$

child development, normal, 24, 34, 40, 168, 301

childhood maltreatment, 47, 157

chronic pain, 52, 53, 187, 217, 247, 258, 259, 290

chronic stress, 148

clinical trials, 130, 177

cocaine, 113

cognitive control, 27, 40, 115, 134, 138, 246, 250

cognitive course, $32,114,116,228,294$

cognitive functioning, 5, 7, 9, 20, 21, 34, 41, 42, 45, 48, 50, $67,68,69,70,71,72,73,74,75,76,81,82,85,98$,

$100,104,105,106,110,112,117,119,121,123,124$, 129, 133, 146, 148, 151, 152, 154, 156, 158, 159, 172 , 174, 175, 177, 183, 184, 185, 201, 210, 213, 214, 215 , $216,219,220,227,228,231,240,243,245,248,257$, 260, 266, 269, 270, 275, 278, 288, 289, 290, 291, 292 . 297

cognitive neuroscience, 48, 70, 75, 97, 117, 118, 135, 160, $243,244,245,247,251,252,269,303$

cognitive processing, 25, 29, 77, 85, 214, 217, 244, 247, 251. 258, 263, 277, 299

cognitive rehabilitation, 12, 17, 18, 37, 69, 87, 98, 126, 153, 162, 173, 249, 256, 270, 271, 272, 273, 274, 275, 276, 277, 282, 293

cognitive reserve, 39, 42, 69, 70, 82, 83, 116, 127, 136, 147, 179, 211, 276, 282

cognitive screening, 23, 52, 73, 82, 90, 98, 122, 176, 197 , $216,217,259,262,264,269$

cognitive style, 100

cognitive, 6, 16, 20, 21, 46, 66, 76, 79, 118, 126, 132, 146 , $193,204,210,211,221,243,244,248,279,292,293$. 296

computerized neuropsychological testing, 20, 66, 73, 99, 100 , 102, 150, 183, 216, 225, 226, 227, 233, 236, 248, 261. $263,266,267,272$

concussion, 5, 6, 13, 14, 16, 17, 18, 20, 23, 28, 56, 131, 132, 148, 149, 171, 192, 224, 229, 230, 231, 232, 233, 234, $235,236,237,269$

confabulation, 254

congential disorders, 37, 183, 186, 216

corpus callosum, 27, 45, 148, 167, 198, 296

cross-cultural issues, 34, 35, 36, 101, 102, 103, 111, 163 , $164,166,277$

decision-making, 13, 27, 39, 43, 45, 46, 76, 119, 146, 168 , $222,258,286$

deep brain stimulation, 198, 199, 212, 215, 218

dementia with Lewy bodies, 216

dementia, Alzheimer's disease, 32, 33, 48, 54, 58, 59, 75, 86, 116, 126, 127, 136, 141, 172, 173, 174, 175, 176, 177 . $178,179,180,181,182,193,194,201,202,203,205$, $206,261,262,276,282,303$

dementia, other cortical, 29, 120, 174, 200, 201, 204, 205, 207, 209, 210, 254, 279

demential, subcortical, 15, 202, 211, 257 demographic effects on test performance, 43, 68, 101, 104, 109, 226, 304

depression, 4, 9, 11, 28, 49, 51, 73, 75, 106, 114, 119, 121, 122, 123, 131, 138, 142, 145, 150, 151, 152, 153, 154, 155, 156, 157, 173, 202, 217, 219, 220, 233, 264, 272, 302,304

diabetes, 140, 217, 243

dichotic listening, 167, 295

disinhibition, 4, 46 driving, 64, 71, 74, 79, 110, 123, 219, 227, 259

dyslexia, 99, 283, 298

eating disorders, 152

ecological validity, 6, 98, 168, 254

effort, 51, 52, 53, 54, 55, 56, 57, 58, 59, 60, 61, 62, 91, 224 . 225,263

electroencephalography, 28, 38, 84, 88, 96, 97, 105, 193, 251 ethnicity, 102, 103, 108, 199, 267

event related potentials, 16, 47, 84, 97, 158, 193, 214, 249 . $250,251,298$

everyday functioning, 19, 24, 29, 39, 49, 72, 88, 112, 113 , 114, 119, 165, 171, 202, 206, 213, 231, 240, 259, 268

executive abilities, abnormal, 3, 4, 33, 36, 37, 38, 39, 40, 41. $42,45,46,47,48,49,50,51,70,72,91,92,102,103$, 106, 107, 108, 110, 111, 114, 129, 133, 142, 151, 152 , 155, 157, 158, 165, 168, 169, 170, 172, 182, 186, 202 , $224,225,230,236,239,241,242,253,259,270,280$, 285, 287, 293

executive abilities, normal, 9, 28, 38, 39, 42, 43, 44, 45, 46, 47, 48, 49, 50, 51, 70, 72, 73, 82, 100, 103, 136, 214. 218, 250, 254, 258, 266, 285, 295, 302

face processing, 24, 27, 136, 138, 187

facial affect, $7,18,24,105,209,212$

fatigue, $19,68,76,117,121,146,302$

fetal alcohol syndrome, 106, 130, 168, 182, 184, 187, 298

fluency, 17, 38, 41, 94, 104, 295, 297

fluid intelligence, 27, 256

forensic neuropsychology, 51, 52, 53, 60, 62

frontal lobes, 38, 39, 41, 44, 50, 117, 134, 141, 206, 210. 228, 252, 253, 254, 273, 288

genetic neuropsychology, 82, 107, 108, 109, 110, 111, 116 , 127, 128, 129, 131, 132, 149, 175, 177, 222

geriatric depression, 112, 141

head injury, closed, 4, 6, 14, 18

hippocampus, 17, 116, 143, 145, 180, 196, 197, 252, 303

HIV/AIDS, 111, 112, 113, 114, 115, 116, 117, 304, 306

hormones, 20, 152

Huntington's disease, 110, 207, 208, 268, 306

hydrocephalus, 167, 203

hypertension, $69,70,79,140,145,214$

hypothyroidism, 183

hypoxia, 221, 253

information processing speed, 77, 214, 220

informed consent, 156

inhibitory control, 28, 38, 40, 43, 49, 74, 93, 99, 128, 130 . 197, 216, 218, 292

intellecutal functioning, 46, 92, 199, 271, 283 
intelligence, 25, 26, 27, 65, 167, 193, 194, 195, 213, 245 , 252. 288

language, 21, 25, 38, 40, 41, 89, 97, 157, 174, 186, 191, 209 , 237, 252, 294, 295, 296

language: aphasia, 178, 182, 207, 258, 295, 297

language: development, 280, 298

language: second/foreign, 295

laterality, 94, 198, 199, 302

learning disabilities, 130, 133, 167, 283, 299

learning, 7, 25, 43, 200, 221, 240, 246, 295, 297, 300, 301

leukemia, 33, 65

low birth weight, 186

M.D. Event, 35, 164, 165, 166, 167, 168

magnetic resonance imaging, diffusion tensor, 18, 21, 22, 31,

$34,35,84,115,117,122,134,143,144,145,149,178$. $179,190,196,224,230,236,238,252,281,282,295$, 297

magnetic resonance imaging, functional, 18, 23, 24, 26, 27 ,

$34,48,63,67,81,84,97,107,133,134,135,136,137$.

138, 155, 178, 179, 181, 192, 212, 246, 252, 288, 296

magnetic resonance imaging, structural, 23, 34, 79, 81, 83 ,

$96,115,130,140,141,143,144,145,146,147,148$,

149, 206, 233, 245, 248, 281, 283

magnetoencephalography, 280

malingering, 54, 56, 57, 58, 59, 60, 61, 62, 157

mathematics ability, 87, 164, 298, 300

medical illness, 34, 80, 111, 187, 189, 212, 213, 214, 215, 221

memory complaints, 8, 71, 75, 76, 78, 90, 151, 157, 176 ,

178, 180, 203, 211, 215, 224, 259, 265, 304

memory disorders, 10, 11, 34, 54, 83, 90, 92, 93, 130, 143,

149, 152, 174, 176, 181, 184, 196, 197, 205, 207, 219.

$220,247,253,254,261,276,300,302,303,304,305$

memory training, 126, 153, 158, 274, 276

memory, implicit, 203, 246

memory, normal, 28, 67, 68, 69, 72, 78, 86, 135, 143, 196,

207, 248, 249, 280, 301, 302, 304, 305

memory, prospective, 10, 16, 44, 120, 152, 208, 303, 305 .

306

metacognition, 71, 114, 159, 301

methamphetamine, 105, 107, 184

mild cognitive impairment, 59, 72, 75, 78, 80, 82, 112, 126 ,

146, 176, 180, 201, 202, 203, 204, 205, 206, 207, 208,

$209,210,218,227,257,265,266,273,274,302$

mild traumatic brain injury, $3,4,5,7,8,9,11,13,14,15$,

$16,18,20,21,28,31,39,42,47,53,57,59,61,147$.

148, 151, 152, 183, 224, 229, 230, 232, 234, 267, 271,

277,303

minority issues, 101, 116, 189

motivation, 55, 126

motor function, 31, 45, 73, 97, 105, 128, 129, 148, 150, 198 . $226,248,255,283$

motor speed, 255

multiple sclerosis, 117, 118, 119, 120, 121, 122, 123, 124, 131, 132

muscular dystrophy, 109 naming, 101, 248, 297

neglect, 251, 278

neural circuitry, 135, 136, 137, 155, 192, 246, 248, 252, 280

neurocognition, $14,51,65,72,80,110,113,114,134,153$,

$156,157,178,212,291,294,304$

neurofeedback, 96, 286

neurofibromatosis, 118, 171, 189, 237

neuroimaging, functional, 4, 20, 26, 64, 66, 97, 104, 107, $123,126,133,134,135,136,137,138,139,140,160$, 161, 162, 173, 196, 197, 223, 247, 248, 280, 283, 293

neuroimaging, structural, 30, 31, 64, 66, 75, 81, 83, 96, 120 . $124,127,130,139,140,141,142,143,144,145,146$, 147, 148, 149, 165, 167, 179, 192, 197, 203, 279, 280, 281, 283, 289, 294, 296, 297

neuropsychological assessment, 6, 8, 13, 38, 39, 42, 43, 47. $52,54,55,56,57,59,60,61,74,75,84,90,91,98$,

$103,112,116,120,150,151,155,156,157,161,172$, 174, 176, 180, 182, 183, 184, 186, 188, 199, 206, 207. $211,213,215,216,217,219,225,241,243,255,256$, $257,258,259,261,262,264,265,266,267,268,269$, $270,289,299,300,301,305$

neuropsychological outcome, 3, 16, 22, 30, 47, 52, 80, 81 , $111,115,116,120,129,157,185,187,228,265,281$, 282, 286, 291, 300, 302

neurotoxicity, 28, 29, 45, 105, 117, 198, 215, 295

neurotransmitter systems, 243

nonverbal learning disability, 24

normative data, 56, 260, 262, 264

occipital lobes, 26

olfaction, 4, 44, 254

outcome, 7, 13, 18, 87, 185, 198, 226, 235, 252, 259, 268

parietal lobes, 249, 304

Parkinson's disease, 22, 25, 40, 79, 134, 142, 143, 146, 159 , 198, 199, 200, 201, 204, 206, 207, 208, 209, 210, 211, $212,215,216,217,218,219,220,221,222,248,250$, 262. 278

pediatric neuropsychology, 36, 37, 54, 55, 59, 66, 93, 102 , 108, 111, 114, 118, 120, 133, 161, 162, 169, 183, 184, 186, 189, 190, 191, 200, 229, 230, 232, 233, 235, 239, $240,242,272,289$

perinatal factors, 186

personality, 50, 62, 68, 77, 119, 151, 258, 260

phenylketonuria, 190

planning, 133, 242, 248

positron emission tomography, 51, 227

post traumatic stress disorder, 3, 4, 7, 8, 10, 14, 26, 47, 61, $132,134,141,147,150,152,154,158,225,233,271$, $273,277,303,305$

prematurity, 99, 100, 102, 128, 170, 241

psychometric constructs, 3, 8, 121, 172, 193, 194, 195, 236, $254,261,267,268,269$

psychopathology, other, 5, 7, 15, 53, 132, 151, 156, 202 , $223,245,257$

psychopathy, 213

psychopharmacology, 140, 152

psychosis, 203 
pulmonary disorders, 221

quality of life, 5, 9, 15, 19, 23, 29, 45, 100, 201, 207, 210, 217, 219, 228, 264, 278, 288

radiotherapy, 166, 290, 294

reaction time, 76

reading disorders, 34, 48, 296, 298, 299

reading, normal, 41, 101, 231, 299

schizophrenia, 44, 50, 64, 150, 152, 153, 154, 155, 156, 158, 253, 272

schizotypy, 50, 155, 157, 158

seizure disorders / epilepsy, 56, 86, 87, 88, 89, 90, 91, 92, 93, $94,149,170,294$

seizure disorders / epilepsy, surgical treatment, 90, 92, 93, 94, 96, 302

seizures, psychogenic, 88, 257

self-esteem, 275

self-monitoring, 48, 89

self-report, 4, 5, 12, 89, 113, 218, 235, 256, 262, 273, 287. 289,302

semantic processing, 205, 296, 298

sickle cell disease, 49, 186, 188, 189, 220

sleep disorders, 25, 29, 64, 71, 74, 186, 245

sleep, 6, 19, 25, 43, 64, 65, 74, 82, 85, 108, 144, 147, 149 , $154,158,161,229,236,247,300$

social cognition, 24, 27, 29, 39, 74, 87, 93, 95, 96, 101, 105 .

122, 127, 134, 144, 155, 170, 182, 213, 230, 281, 293

spina bifida, 168, 188

spinal cord injury, 22, 31

sports-related neuropsychology, 14, 22, 23, 131, 149, 230, 231,235

stimulants, 99, 107

strategic processing, 70, 269 stroke recovery, 5, 37

substance abuse treatments, 104, 108

substance abuse, 104, 105, 106, 113, 115, 137, 155

temporal lobes, 29, 210, 249, 253

test development, 89, 117, 133, 172, 261, 263, 264, 267

test reliability, 137, 197, 257, 261, 267, 272

test theory, 241, 260, 268

test validity, 53, 57, 60, 61, 255, 257, 262, 263, 265, 266 , 283

thalamus, 124, 253

theory of mind, 95, 122, 144, 232

transdisciplinary research, 65

traumatic brain injury, 3, 4, 6, 7, 8, 9, 10, 11, 12, 13, 14, 15, $16,17,18,19,20,22,23,30,31,38,44,52,55,60,64$, 67, 95, 96, 117, 132, 133, 135, 139, 164, 165, 193, 204, 209, 213, 223, 224, 225, 226, 230, 231, 232, 233, 235 .

$237,256,268,269,271,273,275,277,300$

treatment outcome, 12, 17, 34, 41, 47, 69, 108, 138, 152, $153,175,182,200,216,220,225,270,271,275,276$, 277,288

vascular dementia, 172, 174, 220

verbal abilities, 52, 76, 100, 104, 157, 218, 221, 244, 245, $256,301,304$

verbal auditory agnosia, 249

visuoconstruction, 142, 156, 184

visuospatial, 17, 27, 138, 185, 209, 241, 247, 252, 260, 270, $275,278,279,300,302,304$

vocation, 23

William's syndrome, 110

working memory, 7, 43, 99, 104, 109, 111, 123, 153, 162 , 189, 223, 246, 255, 276, 279, 285, 286, 291, 295, 306 writing, 254 